\title{
DE91 014075
}

titLe hUMAN RETROVIRUSES AND AIDS, $1991 \quad$ :..

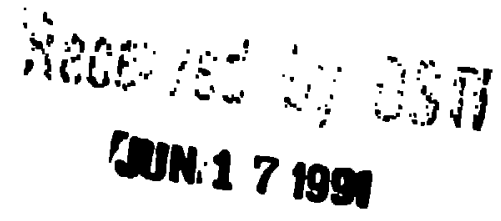

\author{
AUTHOR(S) GERALD MYERS \\ BETTE KORBER \\ JAY A. BERZOFSKY \\ RANDALI. F. SMITH \\ GEORGE N. PAVLAKIS
}

SUBMITTED to BOOK: to be published by Theoreticel Biology and Biophysics

LOS Alemos Nationel Laboratory

\section{DLSCLAIMER}

\begin{abstract}
This report wa prepared as an recownt of work aponevred by an agency of the United Silucea Government. Neither the Unilod Sialea (iuvernment nor any apercy thereof, mor any of thair emphoyees, makes any wurranty. express or umpliod, of anumes any legal liability or reaponsibility for the excuracy, completeneas, of unfulnew of iny information, apparatun, product, or

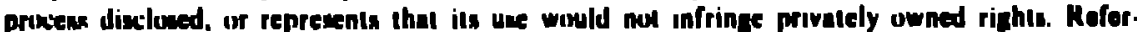

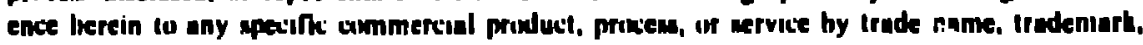

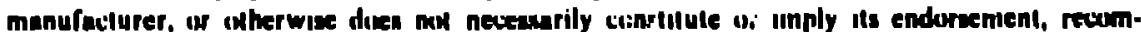
mendatum, or favoring by the linited States fivivernment or any aconcy therear. The view

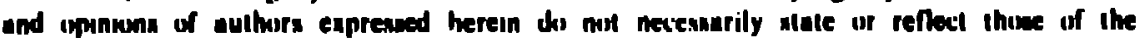
Unilod Siluten (jovernment or any agoncy thereed
\end{abstract}

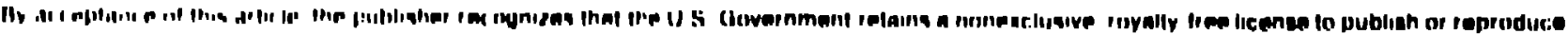

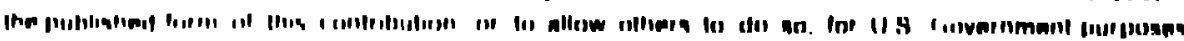

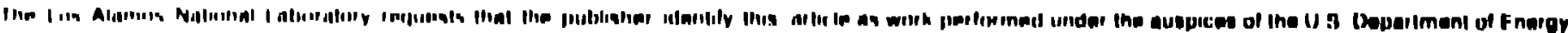






\section{HUMAN RETROVIRUSES and AIDS \\ 1991}

A COMPILATION AIVD ANALYSIS

OF NUCLEIC ACID

AND AMINO ACID SEQUENCES

\section{Edited by}

Gerald Myers

Belte Korber

Theoretlcal Division

Los Alamos National Laboratery

Theoretical Dlvision

Jay A. Berzotsky

Natlonal Cancer Insiltule

Randall F. Smith

MBCRR/DFCl, Harvard Untverstty

George N. Pavlakls

Frederlck Cancer Research Facllity

National Cancer Institule

Databace and Analyale Staft

April Gifford, Jellrey Lawrence, Rhoshel Lenrool, Kersll 'Aacinnes

Theoretical Division

Les Alamos Natlonal Laboratory

This Publication is being funded by the Division of AIDS, National Inatliute of Allorgy and Infectlous Dlsoases, through en interagency agreoment with the U. S. Departm int of Fnorgy.

Published by Theorotical Blology and Biop lyeica

Group T. 10, Mall Siop KTIO

Los Alnmos Nalional Laboratory, Los Alamos, Now Aloxico 67545 USA

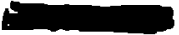




\section{CONTENTS}

More derailed tables of contents are to be found within the various parts of the compendium. The following provides merely an overview.

ACKNOWLEDGMENTS ................... i

INTRODUCTION .................... . . . . . . . .

glosSARY . . . . . . . . . . . . . . . . . vi

PART I. HIV and SIV NUCLEIC ACID SEQUENCES

A. HIV-1 Sequences

Introduction and Contents . . . . . . . . . . . . . . . . . I-A-1

Nucleodde Alignments and Consensus Sequences . . . . . . . . . . . 3

Entries . . . . . . . . . . . . . . . . . . . . . . . . .70

B. HIV-2/SIV Sequences

Nucleotide Alignments and Consensw Sequences . . . . . . . . . . . . I-B-1

Entries . . . . . . . . . . . . . . . . . . . . 58

C. SIVAGM and SIVMND Sequences

Nucleotide Alignments and Consensus Sequences . . . . . . . . . . . . I-C-I

Entria . . . . . . . . . . . . . . . . . . . . . 22

PART II. HIV and SIV AMNO ACID SEQUENCES

Introduction and Contents . . . . . . . . . . . . . . . . . II-1

Alignments and Consensus Sequences/Pautem Catalogs . . . . . . . . . . . 5

PART III. ANALYSIS

Introduction and Contents . . . . . . . . . . . . . . . . . . . III-I

VPT Coding Sequences . . . . . . . . . . . . . . . . 2

Procetn Information Summary . . . . . . . . . . . . . . . .4

Analysis of the HIV-1 V3 Loop . . . . . . . . . . . . . . . . . . .6

Gregory HIV.2 and SIV gpl20 models (updale) . . . . . . . . . . . . . . 23

Sequence Distancrs and Phylogenetic Trees . . . . . . . . . . . . . . . 25

PART IV. RELATED SEQUENCES

Introduction and Contents . . . . . . . . . . . . . IV-1

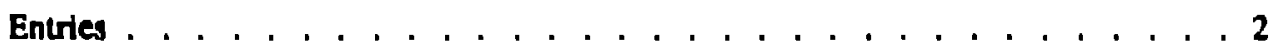

PART V. DATADASE COMMUNICATIONS

Introxlucuion . . . . . . . . . . . . . . . . . . . . . V-I

Moppy Diskettes 


\section{ACKNOWLEDGMENTS}

We thank our former editors Flossie Wong-Staal, Temple Smith and Amold Rabson for their advice and contributions to the database project since 1986 . We will alsc miss the services of Jeff Lawrence, a graduate researc 1 assistant who has worked on the project since 1988.

The computer-generated models of the HIV-1 principal neutralization determinant presented on the cover (also known as the V3 loop) were kindly provided by Goutam Gupta, Theoretical Division. Los Alamos National Laboratory. Chuck Calef, of Information Services, Los Alamos National Laboratory, is responsible for the typesetuing of this edition.

As ever, we are grateful to the Vaccine Development Branch of the NIAID/NIH and to the Los Alamos National Laboratory for their support and guidance. 
INTRODUCTION

This compendium and the accompanying floppy diskettes are the result of an effort to compile and rapidly publish all relevant molecular data conceming the human immunodeficiency vinuses (HIV) and relaced retroviruses. The scope of the compendium and database is best summarized by the five parts that it comprises: (I) HIV and SIV Nucleotide Sequences; (II) Amino Acid Sequences; (III) Analyses: (IV) Related Sequences; and (V) Database Communications. Information within all the parts is updared at least twice in each year, which accounts for the modes of binding and pagiration in the compendium.

While this publication could take the form of a review or sequence monograph, it is not so conceived. Instead, the litcrature from which the database is derived has simply been summarized and some elementary computational analyses have been performed upon the data. Interpretation and commentary have been avoided insofar as possible so that the reader can form his or her own judgments concerning the complex information. In addition to the general descriptions below of the parts of the compendium, the user should read the individual introductions for each part.

Part I. HIV and SIV Nucleotide Sequences. Annotated nucleic acid sequences of HIV and SIV are presented in a form close to that of the GenBank Sequence Library. Our lew modifications of standard GenBank format were instituted wo better serve the particular community for which this database is intended.

The LOCUS name or identifier of an entry may differ slightly from that found in the GenBank or EMBL Librarles, but the ACCESSION numbers are identical for entries in all three databases. Thus each entry is universally and uniquely traceable. The SOURCE line provides some information about the infectivity or biological activity of the molecular clone from which a sequence has been derived. REFERENCES are limited to literature or personal communications having authority for the original sequence data; references that review sequence information, or that shed light light upon the function or variation of coding and regulatory sequences, are now listed in Part V.

Entries in Par I are annotated within the sequence, while their GenBank or EMBL-formatted versions on the floppy diskettes make use of FEATURES tubles. The hard-copy winnotation includes coding regions, regulatory structures, splice sites, and other features of functional sigi:íicance. The authority for this annotation is largely invariance, the recurrence of patterns such as TATA and AATAAA. Although our practice has been w conservautvely announce, we caution the user against docillty: sequence information regarding transcripts, for example, is far from sadisfactory or complete at this ume.

Previous releases of the dumbase included reference sequences, or consensus sequences to replace those. Because many researchers find the nucleotide sequence alignments helpful, we are now electing to publish those ir. Part I. For further information about these alignments and their consensus sequences, refer to the introduction to Pan I.

Part II. Amino Acld Sequences. This soction contains in alignment the amino acid sequences of all known coding regions and open reading frames of HIV-1, HIV-2 and SIV's. Consensus sequences and AACC consensus-like pattems are given with their respective alignments. Protein proxessing sites are annolated when known. Again, the reader should consult the introduction to Part II for further expianation of the presencation and annotation of the amino acic' sequences.

Purt III. Anulysls. This section is open-ended with the constraint that the sequence analyses and compilations be basic and of interest wo the diversity of users. Currently, the analveses include new daw peraining (6) coling regions and transcripts not heretofore reported; summuries of protein variability; and molecular erolutionary patlems of the various regions of the IIIV and SIV genomes. A large survey of protein similarities and homologies is planned for the summer release in 1990 . 
Part IV. Related Sequences. With the exception of the coding sequence for the human T4 surface receptor, this section contains nucleic acid sequences of lentiviruses and other retroviruses related to HIV and SIV. Entries are presented in the same format as Par I entries.

Part V. Database Communications. This part sonsists of a printed supplemental reference list and diskettes. The supplemental reference list contains so-called "secondary references" that review sequence information and shed light upon the function or variation of coding and regulatory sequences. With this edition, the list has been expanded 10 include literature pertaining to the immunology of HIV. We welcome user input to help ensure the completeness of this list.

The floppy diskettes contain the nucleic acid sequences from Part I and Part IV and the translated arnino acid sequences of coding regions. For the most current informacion regarding database files, see the READ.ME file on each diskette. Nucleotide entries are presented in GenBank format for North American users and in EMBL format for European users (unless otherwise requested). Similarly, amino acid sequences are in either PIR or Swiss-Prot format The diskettes themselves are either 5.25" IBM-DOS format or 3.5" Macintosh format, depending upon what has been requested. If there is any trouble using these files with software designed 10 work with the format we have sent, please let us know the name of the program you are using and the file that it could not handle.

We are prepared to quickly enter both protein and nucleotide sequences into this database and, in the case of nucleotide sequences, oversee their entry into the GenBank and EMBL libraries. Submission of unpublished sequences is inviced and encouraged. Sequence dala or inquiries regarding the database should be addressed to

\author{
Gerald Myers \\ Theoreucal Division \\ $\mathrm{T} \cdot 10$, MS K710 \\ LANL \\ Los Alamos, NM 87545
}

(505)-665-0480; fax (505)-665-3493

c-mail: glm\%life@lanl.gov

A shor glossary follows. 

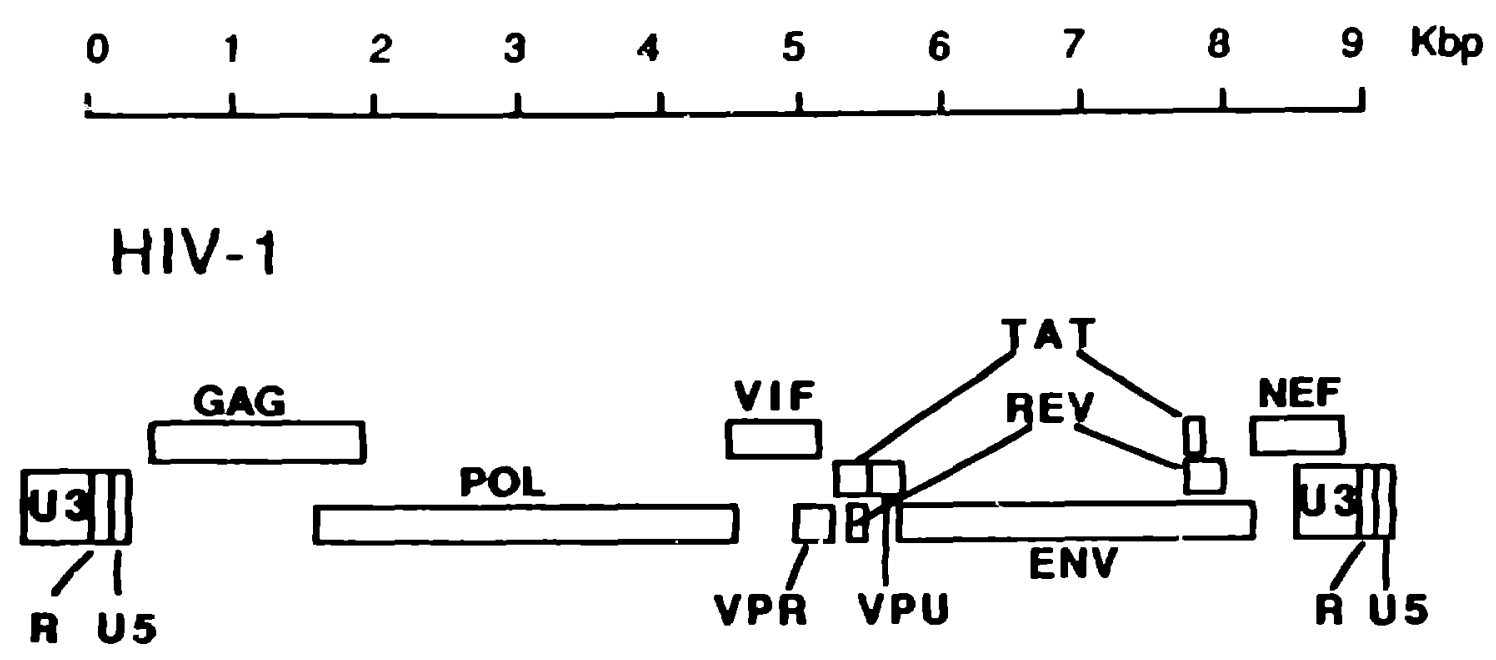

8

HIV-2

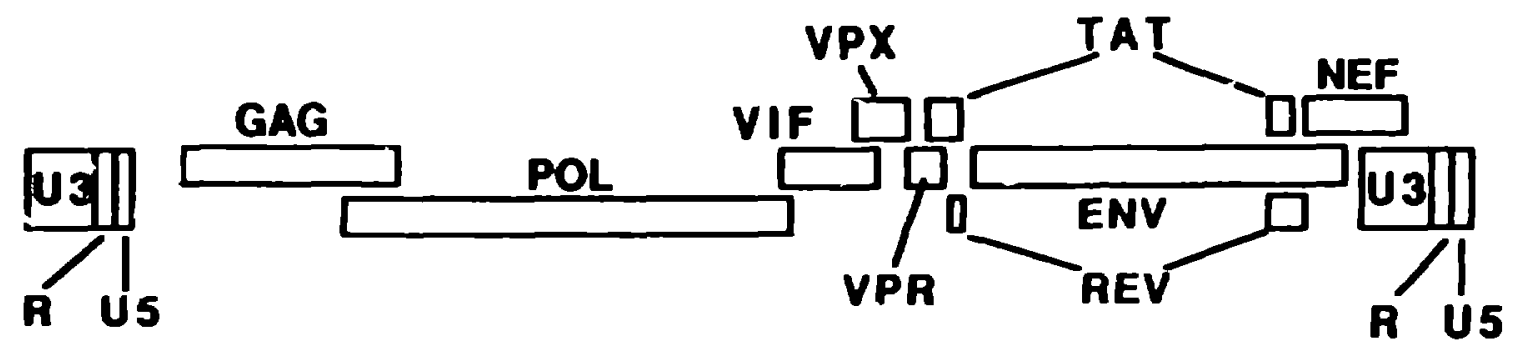




\section{GLOSSARY \\ HIV/SIV PIROTEINS}

\begin{tabular}{|c|c|c|c|c|}
\hline Name & Typical Size & Function & Localization & Immunogenicity \\
\hline GAG & $\mathrm{p} 55 / \mathrm{p} 17 / \mathrm{p} 24 / \mathrm{p} 7$ & $\begin{array}{l}\text { structural capsid } \\
\text { proteins }\end{array}$ & virion & H \\
\hline POL & $\mathrm{p} 66 / \mathrm{p} 51 / \mathrm{p} 32$ & viral enzymes & virion & H \\
\hline ENV & $\mathrm{gp} 120 / \mathrm{gp} 41$ & $\begin{array}{l}\text { external viral } \\
\text { glycoproteins }\end{array}$ & $\begin{array}{l}\text { plasma membrase. } \\
\text { virion envelope }\end{array}$ & H \\
\hline TAT & p16/p14 & $\begin{array}{l}\text { viral } \\
\text { transactivawr }\end{array}$ & $\begin{array}{l}\text { primarily in } \\
\text { nucleolus/nucleus }\end{array}$ & L \\
\hline REV & pl9 & $\begin{array}{l}\text { RNA transport, } \\
\text { stability and } \\
\text { utilization factor } \\
\text { (phosphoprocin) }\end{array}$ & $\begin{array}{l}\text { primarily in } \\
\text { nucleolus/nucleus }\end{array}$ & L \\
\hline VIF & p23 & $\begin{array}{l}\text { promotes virion } \\
\text { infectivity }\end{array}$ & Golgi? & L \\
\hline VPR & p10-15 & $\begin{array}{l}\text { unkonown, a virion } \\
\text { protein also called } \\
\text { 'ray' (rapid) }\end{array}$ & virion & $\mathbf{M}$ \\
\hline VPU & p16 & $\begin{array}{l}\text { promotes extra- } \\
\text { cellulur release } \\
\text { of viral particles } \\
\text { (phosphoprotein) }\end{array}$ & $\begin{array}{l}\text { integral } \\
\text { membrane protein } \\
\text { only found in HIV.1 }\end{array}$ & $\mathbf{M}$ \\
\hline NEF & $p 27-p 25$ & $\begin{array}{l}\text { unknown } \\
\text { (myristoylated } \\
\text { precin) }\end{array}$ & $\begin{array}{l}\text { cyloplasm, } \\
\text { plasma membrane }\end{array}$ & H \\
\hline VPX & p12-16 & $\begin{array}{l}\text { unknown, a virion } \\
\text { protein }\end{array}$ & $\begin{array}{l}\text { virion, only found } \\
\text { in HIV-2 and SIV }\end{array}$ & L \\
\hline TEV & p28 & $\begin{array}{l}\text { Uiparite tat-env-rev } \\
\text { prciein (found in LAV/ } \\
\text { IIIb) }\end{array}$ & $?$ & $?$ \\
\hline
\end{tabular}




\section{HIV GENOMIC STRUCTURAL ELEMENTS}

LTR - long terminal repeat, the DNA sequence flanking the genome of integrated proviruses; contains important regulatory regions, especially those for transcriptional start and polyadenylation.

TAR - target sequence for viral transactivation, the binding site for TAT protein and possibiy cellular proteins; consists of approximately the first 45 nucleotides of the viral mRNAs in HIV.1 (or the first 100 nuc eotides in HIV-2 and SIV). TAR forms a hairpin stem-loop structure with a side bulge; the bulge is necessary for TAT binding and function.

RRE - (also known as CAR) REV responsive element, an RNA element encoded within the ENV region of HIV-1, consisting of approximately 200 nucleotides. The RRE is necessary for REV function: approximately seven binding sites for REV exist within the RRE RNA. Other lentiviruses (HIV-2, SIV, visna) have similar RRE elements in similar locations within ENV, and HTLVs have an analogous RNA element (RXRE) serving the same purpose within their LTR; RRE is the binding element for REV protein, and RXRE is the binding element for the Rex protein. RRE and RXRE thus form complex secondary structures important for specific protein binding.

CRS - cis-acting repressive sequences postulated to inhibit structural protein expression in the absence of REV. Their exact locations and function have not beer. Jefined; splice sites have been postulated as CRS sequences.

INS - "inslability" RNA sequences found within the structural genes of HIV-1 and of other complex retroviruses. Multiple elements appear to exist within the genome; ont of the best characterized elements spans nucleotides 414 to 631 in the OAG region of HIV-1.

\section{GENES AND GENE PRODUCTS}

GAG - group-specific antigens or capsid proteins; the procursor is the p55 myristoylated protein. which is processed to p17 (MAtrix) p24 (CApsid) and p7 (NucleoCapsid) proieins by the viral protease. Other small proteins are generated from the gag polyprotein.

POL - generates the viral enzymes protease, reverse transcriptase and integrase after the processing of a GAG-POL precursor polyprolein by the viral protease; $O A G \cdot P O L$ precursor is produced by ribosome frumeshifting.

ENV - viral glycoproteins produced as a precursor ( $(\mathrm{ppl60}$ ) and processed to the extermal glycoprotein gp 120 and the transmembrane glycoprotein gp4l. The mature proteins are held together by noncovalent interactions; as a result, a substantial amount of $\mathrm{gp} 120$ is released in the medium. Gp120 contains the binding sito for the CD4 receptor.

TAT - transaculvator of HIV gene expression; one of the two necessary viral regulatory facLors (TAT and REV) for HIV gene expression. Two forms are known, TAT-lexon (minor form) of 72 amino acids, and TAT-2exon (major form) of 86 amino axids. The electrophoretic mobility of these two forms in SDS gels is axomulous: they are approximately $16 \mathrm{kD}$ and $14 \mathrm{kD}$ in weight. Low levels of both proteins are found in persistendly infected cells. TAT is localized primarlly in the nucleolus/nucleus; it acts by binding to the TAR RNA element and activating transcription from the LTR promocer. Post-uranscriptional effects of TAT huve been postulated. 
REV - the second necessary regulatory factor for HIV expression. A 19kD phosphoprotein localized primarily in the nucleolus/nucleus, REV acts by binding to RRE and promoting the nuclear expon, stabilization and utilization of the viral mRNAs containing RRE.

VIF - viral infectivity factor, typically $23 \mathrm{kD}$; required for the efficient transmission of cellfree virus in tissue culture. In the absence of VIF, the produced viral particles are defective, while the cell-to-cell transmission of virus is not affected significandy. It has been reported that the cellular localization is in the Golgi (VIF is not found in the virion).

VPR - virion-associated protein of unknown tunction found in HIV-1, HIV-2, SIVmac, and SIVmnd; typically 15kD. May be homologous to VPX. Also called 'RAP' for rapid.

VPU - "oUt" protein that promotes extracellular release of viral particles. Found only in HIV1. Integral membrane phosphoprotein of 16kd; similar to $\mathrm{M} 2$ protein of influenza virus. It may be involved in ENV maturation. It is not found in the virion.

NEF - approximately $27 \mathrm{kD}$ non-virion protein found in the cytoplasm of infected cells. Potentially myrisloylated and associated with the inner plasma membrane. One of the first HIV proteins to be produced in the infected cells, it is the most immunogenic of the accessory proteins and may be used in the future for diagnosis and staging of the discase. NEF is dispensable and probably suffers counter-selection during ex vivo viral propagation. Recent evidence suggests that SIV NEF is required for viral propagation in vivo.

VPX - virion procein of $12 \mathrm{kD}$ found only in HIV-2/SIV and SIVAGM; not in HIV-1 or SIVmnd. Function unknown. VPU/VPX may be used to distirgiish between HIV-1 and HIV-2 infection. (VPX may have some homology with VPR.)

TEV - (alsc known as tnv) a tripartice $28 \mathrm{kD}$ viral phosphoprotein produced very early in infection by some HIV-1 strains. Found primarily in the nucleolus/nucleus. TEV contains the first exon of TAT, a small part of ENV and the second exon of REV. It has both TAT and REV functions in the same polypeptide and can functionally replace both essential regulatory proleins of HIV-I.

STRUCTURAL PROTENS - the products of GAG, POL and ENV genes, which are essential components of the retroviral particle.

REGULATORY PROTEINS - TAT and REV proteins of HIY/SIV and TAX and REX proteins of HTLVs; essential for viral expression in infected cells.

ACCESSORY PROTENS - additional (non-regulatory) virion- and non-virion-associated proteins produced by HIV/SIV retrovinuses: VIF, VPR, VPU, VPX, and NEF. Although the accessory proteins are not necessary for viral propagation in thsue culture, they have been conserved in the different isolates; this conservation and experimental observations suggest that their role in vivo is very importanc

COMPLEX RETROVIRUSES - retroviruses regulating their expression via viral factors and expressing additional proteins (regulatory and accessory) essential for their life cycle. The following figure shows the extent of this complexity as manifested in multiple transcripts and altemative forms of tal and rev proteins; for a fuller presentation of the informatior in this figure. sec 1Il-22 of SEP 9 (). 


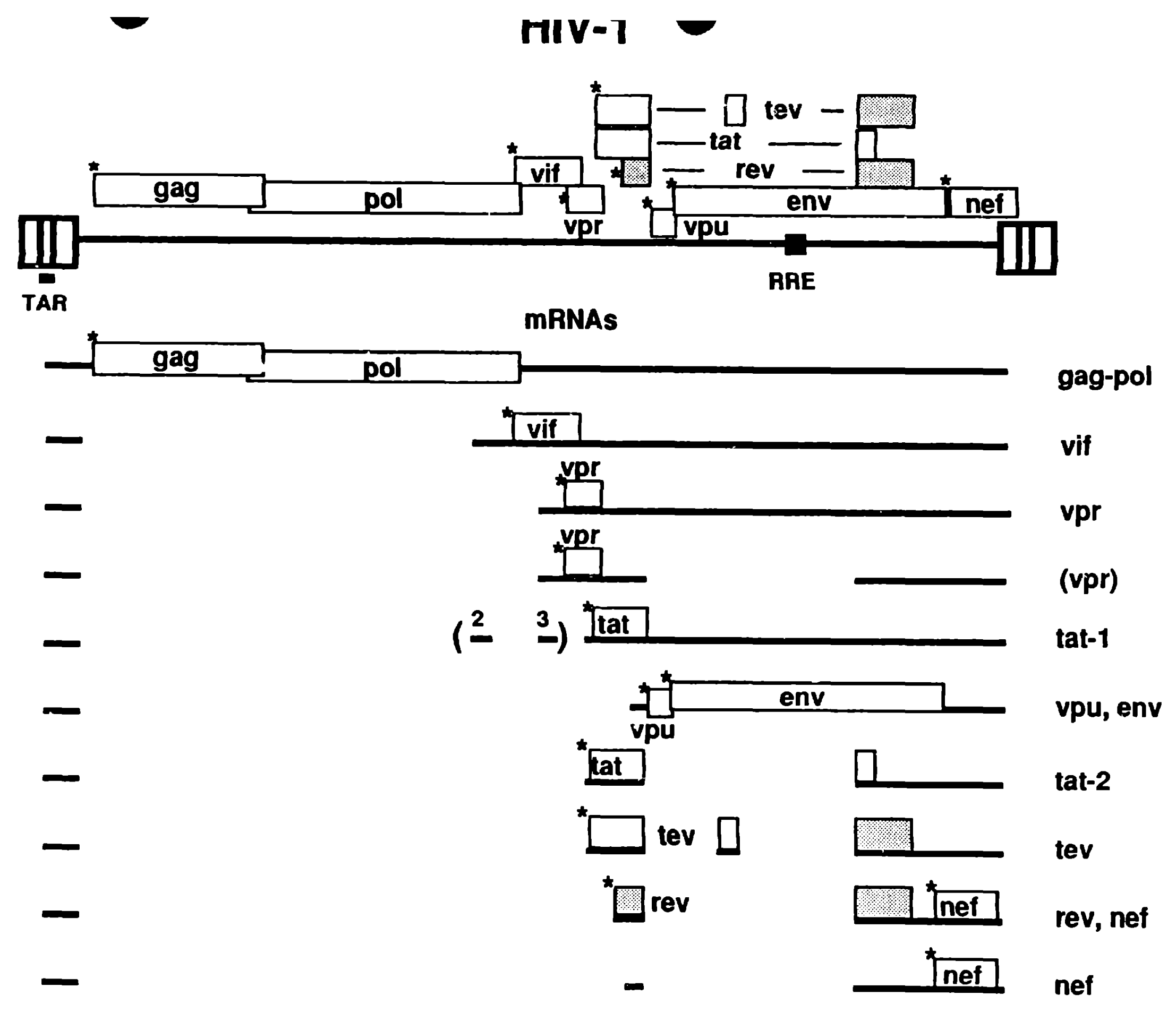


Nucleotide sequence alignments were generated using the PIMA program developed by Randy Smith and Temple Smith, MBCRR, Dana Farber Cancer Institute, Harvard School of Public Health, Boston, MA 02115, (617)-732-3746. By this approach, a protein pattern is created which then serves as a template for the nucleotide sequence alignment Hand-editing, when required, was done with the MASE program (also provided by the MBCRR; see K. F. Smith and T. F. Smith, PNAS 87: 118-122 1990).

With a few exceptions, only full-length coding sequences were included in these alignments. Mixed case consensus sequences are used as the reference sequences for each alignment. Upper case letters indicate $100 \%$ conservation of nucleotide bases in a given position of the alignment, and lower case letters represent bases conserved in at least $50 \%$ of the sequences. To reduce the alignment uncertainty with the large HIV-1 set, sub-groups of North American and African sequences were constituted and consensus sequences were generated for each. The highly divergent SIV-cpz sequence (Huet et al., Nature 345: 356-359 1990) was not included in the consensus sequences for the HIV-1 alignments. Large deletions, if present in only one sequence (i.e., the HIV2nihz nef coding region sequence), were also excluded from the consensus sequences. The co isensus sequences in this edition take intw account two unpublished sequences which were not preser tited in the alignments.

The authority for some of the annotation is limited largely $\omega$ invariarice-the recurrence of patterns such as AATAAA, for example. The reader should be cautior's in drawing upon this information. In particular, the annotation of splice sites is tentative; therr is a flurry of work going on now in this area (see the GLOSSARY and also III-2I and followin's pages of SEP 90).

Only new and revised sequences are repored as entries in this section. Otherwise, sequence entries from previous years are presented in the 1990 compendium and in the new GenBank format on the floppy diskettes. 
PART 1. HIV and SIV Nucleic Acid Sequences

Introuluction . . . . . . . . . . . . . . . . . . . . . . . . . . . I - A - 1
Contents . . . . . . . . . . . . . . . . . . . . . . . . . I - A - 2

A. HIV.1 Sequences

Nucleotide alignments of HIV-1 sequences . . . . . . . . . I - A - 3

HIVLAI (KC2013; M64186-190; M64194-198; M64203-223) . . . 70

HIVNL43 (M19921) . . . . . . . . . . . . . . . . . . . 76

HIVJB01 (X57447) . . . . . . . . . . . . . . . 80

HIVJB02 (X57450) . . . . . . . . . . . . . . . . . . 83

HIVJB03 (X57465) . . . . . . . . . . . . . . . . . 89

HIVBAL (M68893) . . . . . . . . . . . . . . . . . . . 92

HIVADA (M60472) . . . . . . . . . . . . . . . . . . . . 95

HIVSWB40 (M27951; M27946) . . . . . . . . . . . . . . 96

HIVSWB88 (M27952; M31462; M27947; M58206) . . . . . . . 97

HIVM77, HIVV77, HIVT77 (Pending) . . . . . . . . . . . . . 100

HIVM82, HIVV82, HIVT82 (Pending) . . . . . . . . . . . 103

HIVNJA (M61028 - M61045) . . . . . . . . . . . . . . . . 117

HIVU455 (M62320) . . . . . . . . . . . . . . . . . 122

HIVSF170 (M66535; M66533) . . . . . . . . . . . . . . 126

HIVCBL4 (M63474; M63475) . . . . . . . . . . . . . . . . 129

B. HIV-2 and Related SIV Sequences

Nucleotde Alignments of HIV-2 and SIV Sequences . . . . . I - B - 1

HIV2D194 (J04542; X52223) . . . . . . . . . . . . . . . . 58

C. SIVAGM and SIVMND Sequences

Nucleotide Alignments of AOM Sequences . . . . . . . . . I - C - I

SIVAGM677 (M66437) . . . . . . . . . . . . . . . . . . . 22

SIVMND121 (M62883; M62884) . . . . . . . . . . . . . 26 
Authority for the annotation 19 glven on pages I-A-4 through I-A-9 of APR 90.

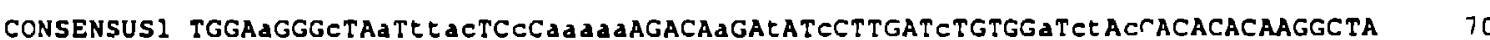

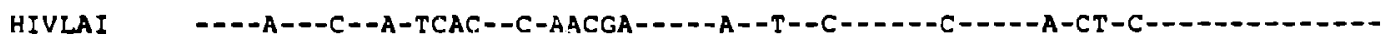
HIVHXB2R - ---A---C--A-TCAC--C-AACGA-----A--T- C-1----C----A-CT-C-1HIVMT HIVBRVA HIVSC

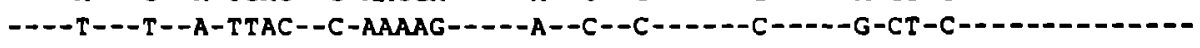

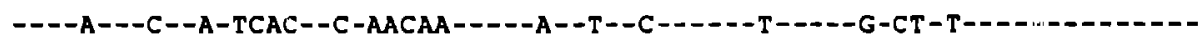
HIVJH3 HIVBAL1 HIVJRCSE HIVJRFL HIVOYI HIVSE 2 HIVNYSCG HIVNL4 3 HIVSE 162 HIVCDCA HIVSF 33 HIVHAN HIVSW 1 व̃स HIVRF

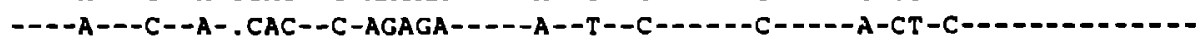

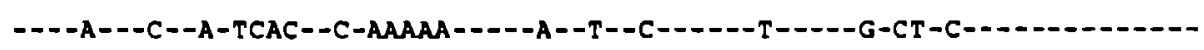

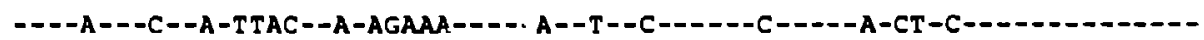

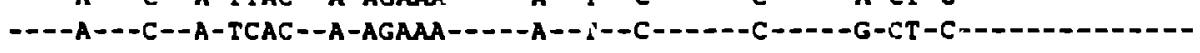

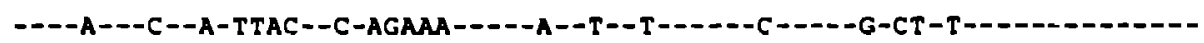

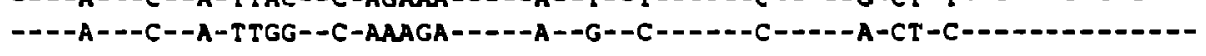

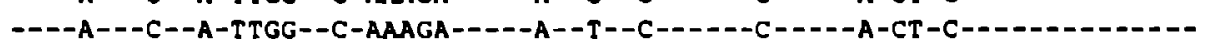

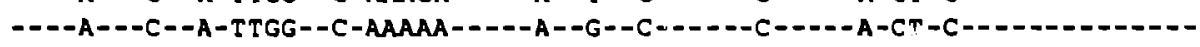

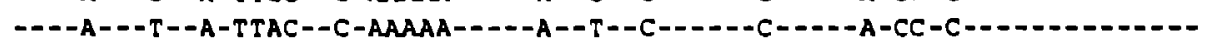

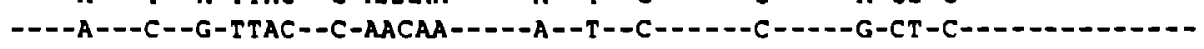

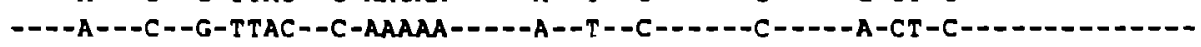

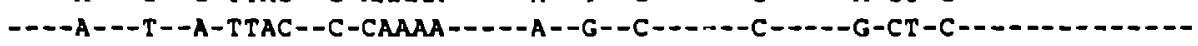

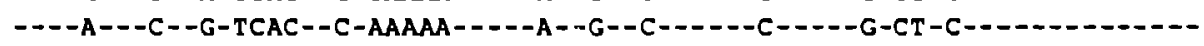

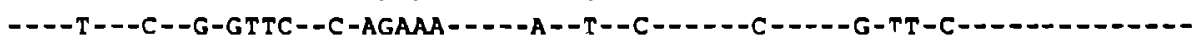

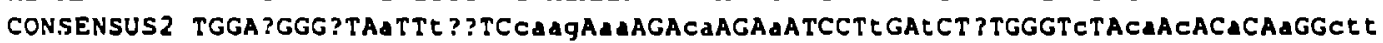
HIVELI I ----A---C--A--TGG--CAAA-AG---CA-- -G-----T--T--T-----C--CA-C--A--A--CAT HIV 2226 HIVNDK HIVMAL HIVU 455 HIVSF 170 HIVZ321 HIVANT 70 $---A--C--A--T G G--A A A A-G A--C A---C----T--T-=T----C--C A-C--A--A--C A T$ $---A--C--A--T G G--C A A G-A A---C A---G----T--T--T-----C--C A-C--A--A--C A T$ $---T=-T--G--T G G--C C C A-A A---C A---A-----T--T--G=---C--C C-C--A--A--C T A$ $----T---T=-A--C A C--A C A G-A A---C A---A-----T--T--G-----C--T C-C--A--A--A T T$ $---T--T--A--T A C--C A G G-G A=-C A---A----T--T--G=--C--2 A-T--A--A--C T A$ $---T--C--A--T A C--C A A G-A A---C A---A----T--C--G-----C--T C-T--A--A--C T T$ SIVCPZ $---A---T--A \cdot-T A C--C C A T-A A---G C---A-----G--T--T-----G--T A-C--T--G=-A T T$ $----A---T--G--T A C--C A G G-G A---C A---G-----T--C--C-----C--T C-C--A--A--C T T$

$$
\text { - AP-1 - }-A P-1-
$$

CONSENSUS1 cTtCCCTGAT TgGCAGAACTACACACCAGGgCCAgGgatCAGATatCCiActgacett TGGATCigTGCTt HIVLAI HIVHXB2R HIVMN HIVBRVA HIVSC HIVJH 3 HIVBALI HIVJRCSF HIVJRFL HIVOYI HIVSF 2 HIVNYSCG HIVNL4I HIVSF 162 HIVCDCA HIVSF] 3 HIVHAN HIVSWBOB 4 IIIVRT

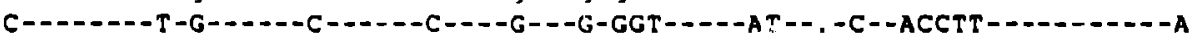

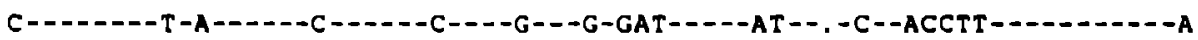

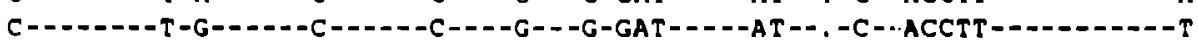

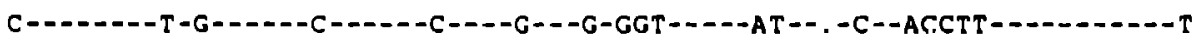

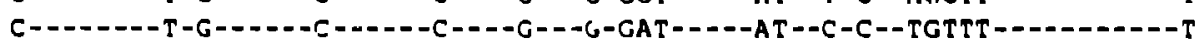

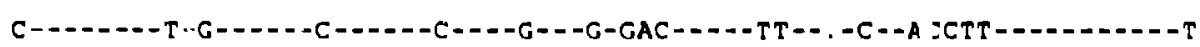

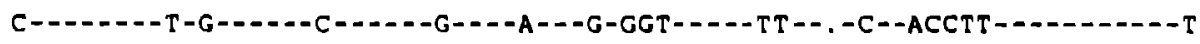

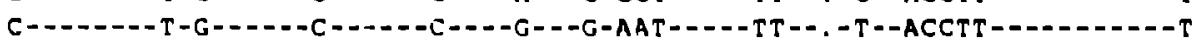

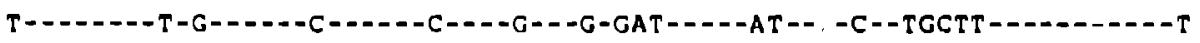

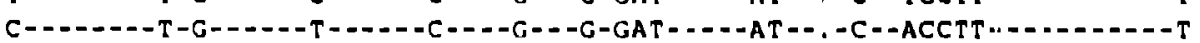

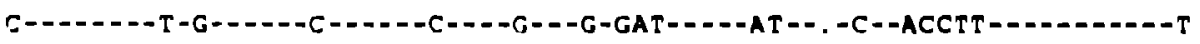

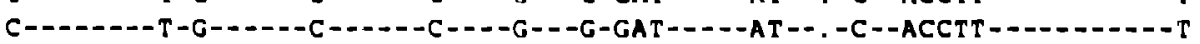

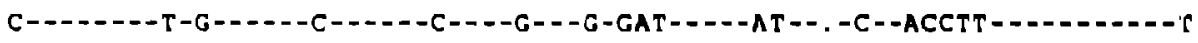

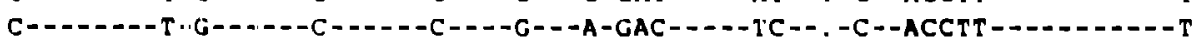

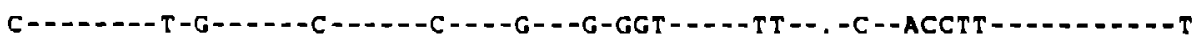

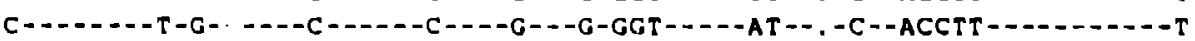

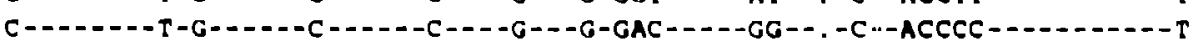

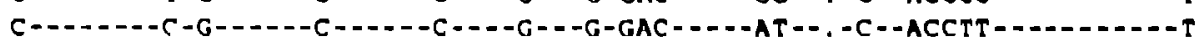
CONSENSUS2 CTTCCCLGATTGGCAgAACTACACACCAGGqCCAGGqATCAGATACCC. ACTqACCTTEGGATGGTqCTE HIVELI HIVZ2Z6 IIIVNDK HIVMAL -

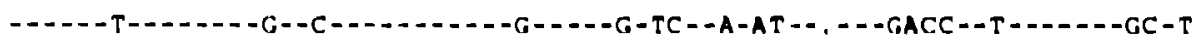

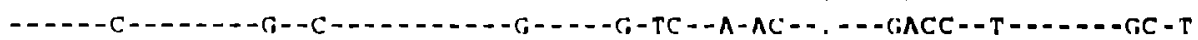

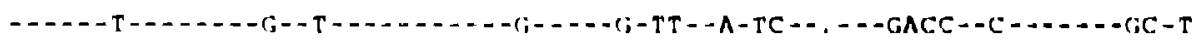

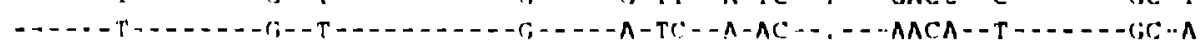
- - - - - - - - - - - HIVU 155

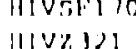
IIVกNT 10 - - - - T. - - - - - -

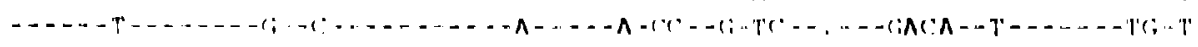
:ivc.?

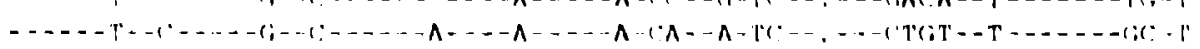


CONSENSUS1 CAAGCTAGTACCAgT... tgAgCCAGagaagg agaagaggccaAgaAggAGAGAACaaCaGct TGTtA IIVLAI HIVHXB 2R II IVMN HIVBRVA HIVSC HIVJH 3

HIVBALI HIVJRCS? HIVJREL HIVOYI HIVSF 2 HIVNYSCG HIVNL 43 HIVSE 162 HIVCDC 4 HIVSE 33 HIVHAN HI'SWB884 HIVRE CONSENSUS2 HIVELI HIVZ226 HIVNDK HIVMAL HIVU455 HIVSF 170 HIVZJ2! if IVANT 70 SIVCR 2

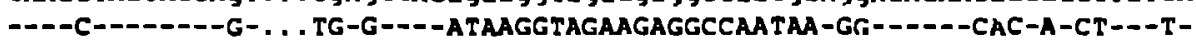
----C--.---G-. . . TG-G----AGAAGTTAGAAGAAGCCAACAA-GG------CAC-A-CT---T-

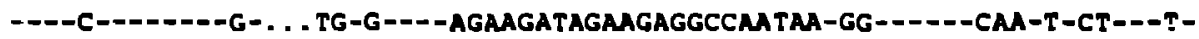

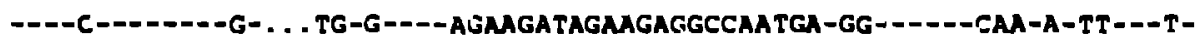
$---C------G-\ldots$ TA-A----AGAAGATAGAMGAAGCCAATGA-GG------CAA-A-CT-- T-

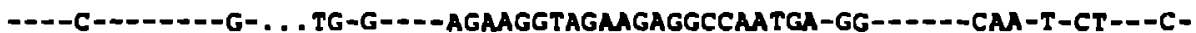

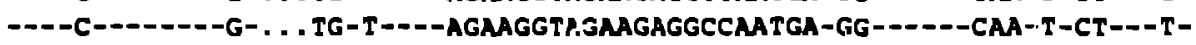

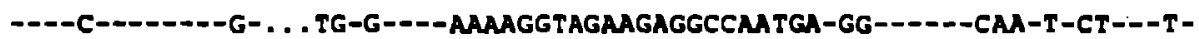
----C-------G- . . TG-G----AGAGGTAGNAGAGGCCNATGA-GG------CAA-A-CT--T-

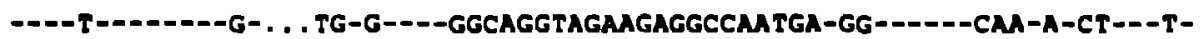

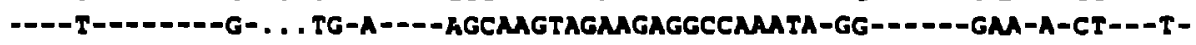

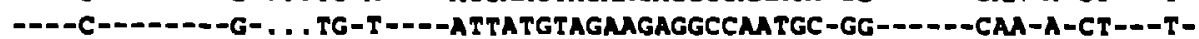

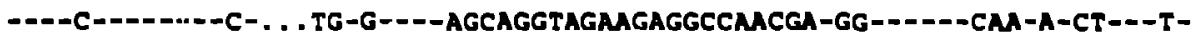

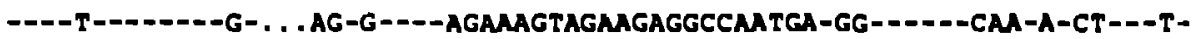

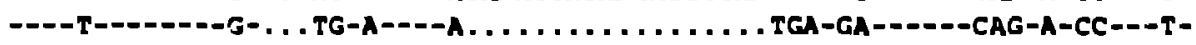
-.-C--_---G- . . CG-G---AGAAGGTAGAAGAGGCCAATGA-GG-----CAA-A-CT -.-T-

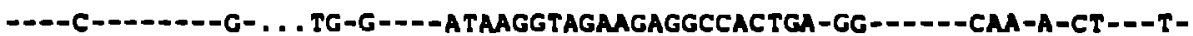

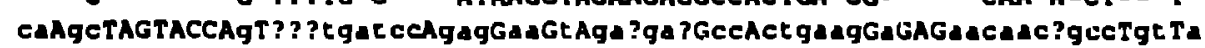
CG-GC-----G- . . . TGATCC-CAG-AG-T-GAAGAA-AC-CTGAAG-A---ACCAACAGCT-GT-A CG-GC------G- . . TGATCC-CGG-AG-T-GAAGAG-CC-CTGAG-A---ACCAACTGCT-GT-A CC-GC------G- . . TGATCC-CAG-AG-T-GAAGAG-Cr,-CTGMA-A---GACAACTGCT-GT-A TA-GT--.--A- . . GAGTCC-GAG-AA-T-GAGGAG-CC-ATGAG-A---ACAACTGTC-GT-A CA-GC--_---G- . . TGATCC-GCT-AA-T-GAGGA-CT-CTGGAG-A---ARCAACAGTC-f._-A CA-GC------G- . . TGATCC-GAG-GA-T-GAGAAG-AC-CGGTGG-A---ACCAACAGCC-AT-A CA-GC----1-G- . . TGATCC-AGA-AA-T-GAGGAA-CC-ATACAG-A---MACAACTCCC-G!-A TA-AC-----G-GTCAGMAGA-GAG-CA-A-AGACTA-GA-ATACAT-T---AGGGCTMATC-CC-G CA-AC----G--CC-GACAGA. . , GGAGCAG-T-GNACAA-CC-ATGAG-A--TAACAACTGCC-GT-G NFAT -1

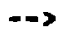

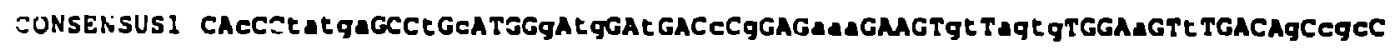
HIVLAT HIVHXB2R HIVMN HIVBRVA

II IVSC iI VJH 3 IIIVIIAL.! IIV.JRCSF IIIY.JRI"?. IIIVOY I !! IVSF2 II: VNY SCO [I : VNL, 4 ] IIIVSF 162 II VCDC 4 iI [ V 5 B.] IIIVIIAN IIIVGWBGA III VHF --C--TGTGA---T-C----A-TG--T---C-T---AGA----GT-AGAG - --G--T----G-CGC-

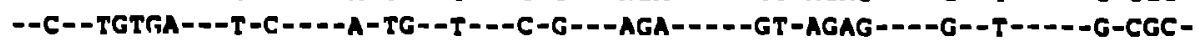
--C--TATGA---A-C---- . -TG--T---C-G---AGA-----GT-AGTG----A--C-----G-CAC--C--TATGA---A-C----A-TG--T---C-G---AGA-----GC-GCAG----G--T-----G-CGC$--C--G A T G A---T-C----G-$ TG--G---C-G---AGA-----GT-AGAG---.G--T-----A-CGC-

- -C--TATGA---T-C----G-TG--T---C-G-- -AAA-----GT-AGCA----A--T-----G-AGC--C--TATGA---A-C----A-TG--C---C-A---AAG-----GT-AGTG----A--T-----G-AAG--C--TATGA---A-C----G-TA--G---C-G---AAG-----GT-AGAG----G--T-----G-AAG--T--TATAA---T-C----G-TG--T- -C-A---AAA-----GT-AGTG----A--T-----G-CGC--C--TATGA---T-C----G-TG--G---G-G---AMA-----GT-AGTG----G--T-----G-AAA--C--TATGA---A-C----G-TG--G---C-G---GGA-----AT-AGTG----A--T-----G-CTC--C--TATGA---A-C----G-TG--G---C-G---GGA-----AT-AGTG----A--T-----G-CTC--C--TATGA---A-C----G-TG--T---C-G---AAA-----AT-AGTG----G--T-----G-CGC--r.--TATGA---A-G----G-TG--T---C-A---AAA-----GT-AGTG-D-A--T-----G-CTC--C--TATGA---T-C----G-1G--G---G-G---AAA-----GT-AGTG----A--T-----G-CAC-

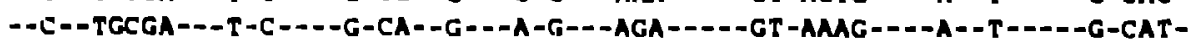
--C--TATGA---T-C----A-TG--G---C-G---AMA-----GT-AGTG----A--T-----G-AGC- -C--TATAT---T-C-- - -G-TG--T---C-A---MAA-----GT-AGTG----A--T-----G-CGC-

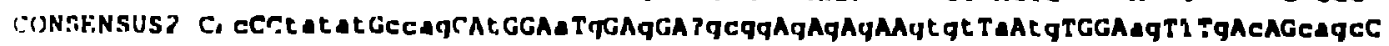
HIVLLI --L--TATAT-CCAG--T---A-G--G--CCCGG-G-G-C--GTGT-A-AA----GA--A-A-C--CAGAIIVZ2Z6 - C--TGTAT-CCAG--T---A-G--G--CACGG-G-G-G--GTGT-A-AG----GA---A-C--CAGAII: VNUK II: VMAI. i:ग!1, $\because \because$ III $: \because, \because,:$

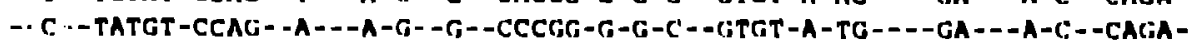

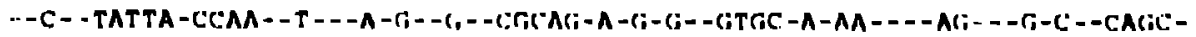

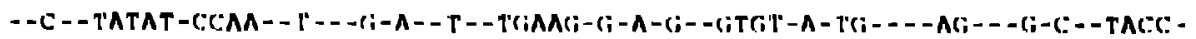

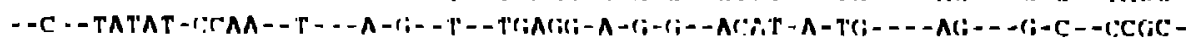

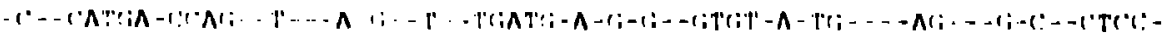

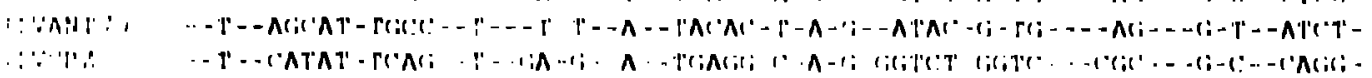




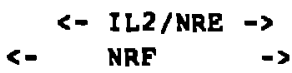

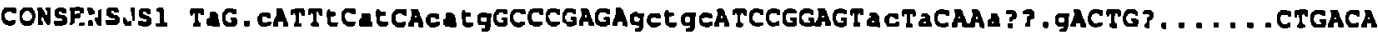

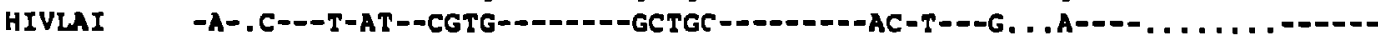

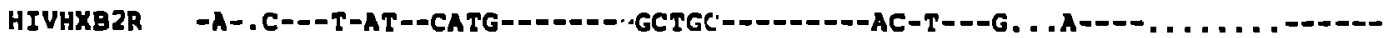
HIVNN -A-.C---T-AG--TTAT-------GCTGC--------AC-A---G...A----.......---HIVBRVA HIVSC HIVJH3 HIVBAL1 HIVJRCSF HIVJRFL HIVOYI HIVSF2 HIVNYSCG HIVNL 43 HIVSF 162 HIVCDCA HIVSF 33 HIVHAN HIVSWB884 HIVRF

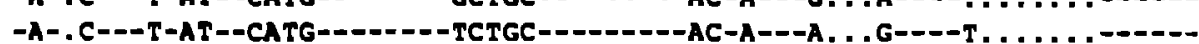

-A-.C---C-AT--CGTG--------A

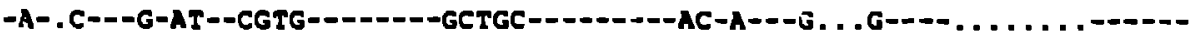

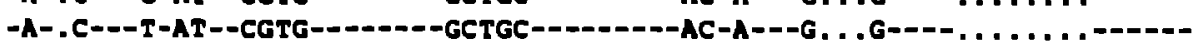

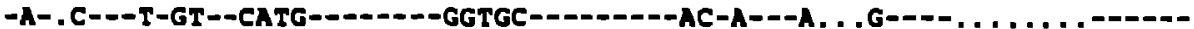

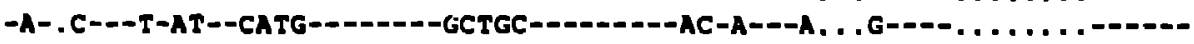

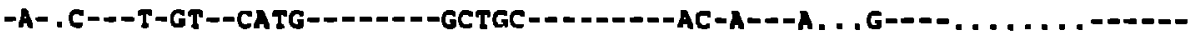

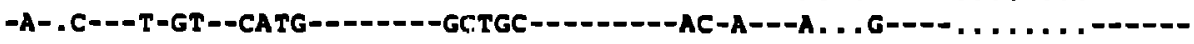

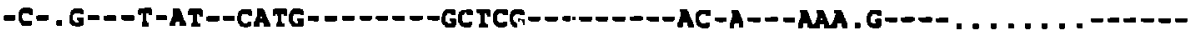

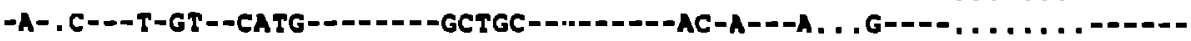

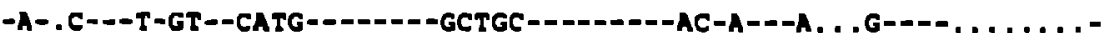

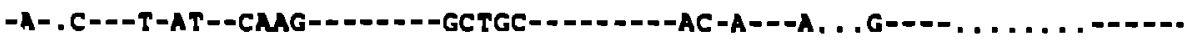

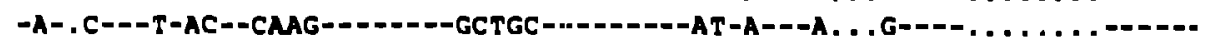

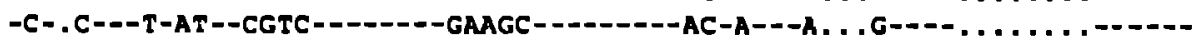

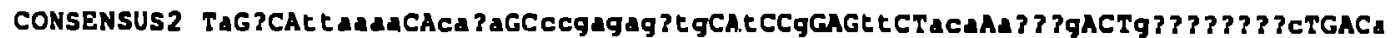
HIVELI HIVZ226 HIVNDK HIVMAL HIVUA55 HIVSF 170 HIVZ321 HIVANT70 SIVCP 2

-A-. --TTTGAG--CAAG--CCGAGAGATG--T--G---TT--ACA-A. . .A---G........A----A -A-. --TTTTGA--CAMG--CCGAGAGCTG--T--G---TT--ACA-A. . G---G....... C----A -A-. --CTTGAG--CAAG--CCGAGACICTG--T--G-.-TT--ACA-A. . G---G....... . ----A -A-. --CTMAGA--CAGA--CAGAGACAA--T--G---TA--ACA-A, . G---G........---A -G-. --TTAMA--CAGA--TTATGAGCTG--T--G---TT--ATA-A. . .G--GAMAGACTGC----A -G-. --TTAMA--CAGA--CCMAGAGATF--T--G---TT--ACA-A. . .G---G......... --A -A-. --CGMAA--CCTA--CCGAGAGATG--T--G---TT--ACA-A. . .G---. MAGACTGE----A -A-G--ACAC.C--TGTT--TATGATAACT--C--A---CT--TCC-GMAGG--- . MAMACTGC----C -G-. -CTTMGA--TATT--CAGAGMACA--T--G---TA--ACA-A. . G---GACTITAGAC--G-G

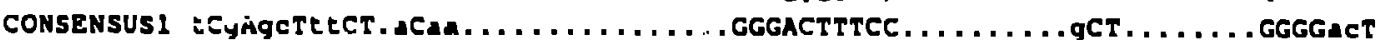

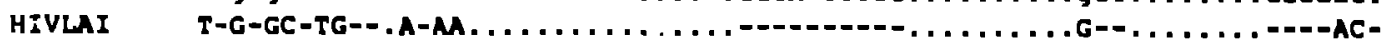
HIVHXB2R T-G-GC-TG--A-A. . . . HIVMY

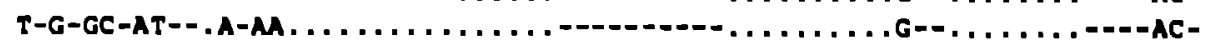
HIVBRVA HIVSC HIVJH3 HIVJRCSF HIVJRFL HIVOYI HIVSF2 HIVNYSCG HIVNLA] HIVSFI 62 HIVCDCA IIIVHAN HIVSWB884 HIVRT

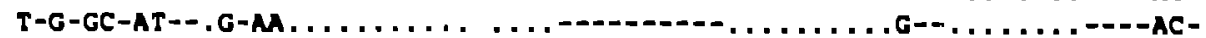

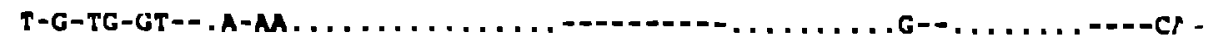

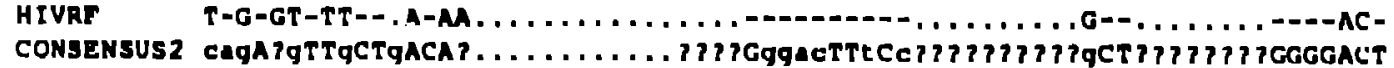
HIVELI HIVZ226 IIIVNDK IIIVMAL. IIIVU455 IITVSFI10 IIIVZIZI HIVANT IO :IVI:P':

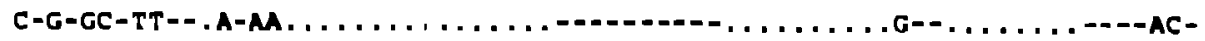

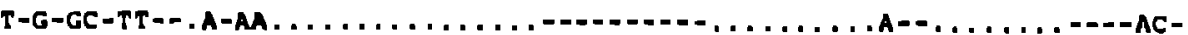
T-G-GC-TT--.A-AG............-.............G-......-AC-

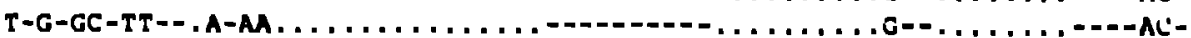

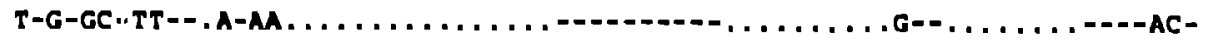

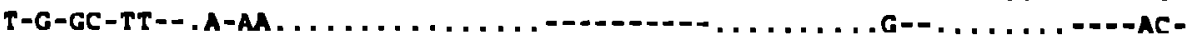
$T-$

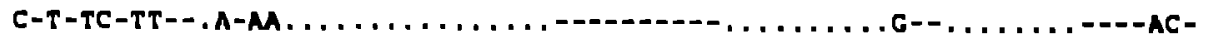
C-G-GT-TT-- .A-AA...........,

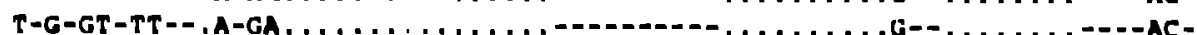

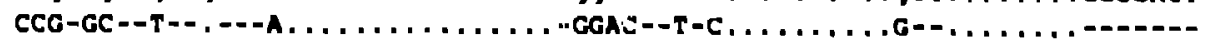

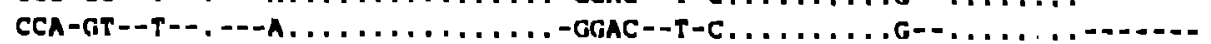

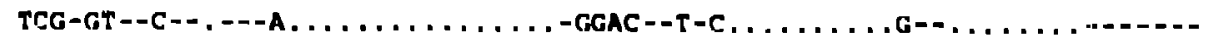

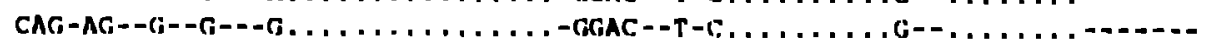

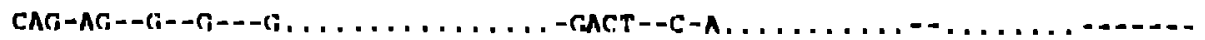

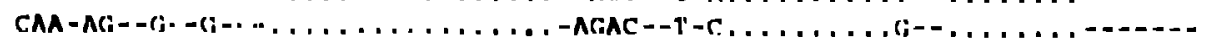

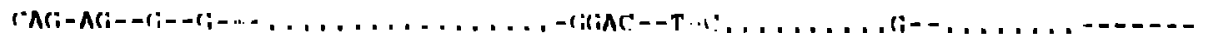

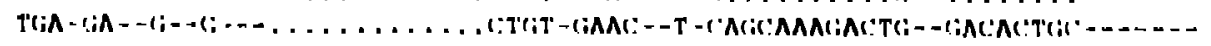

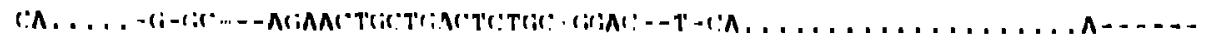


c.0.-) Sp-1 Sp-1 Sp-1

CONSENSUSI TTCAAG.GG ?AggCgtGgCCTGGGCGGGACEGGGGAGTGGCg? ? ? ? ? ? ? ? ? ? AgCCCTCAGATgCTgCA

HIVLAI --C--....GGCGT-G-C-..

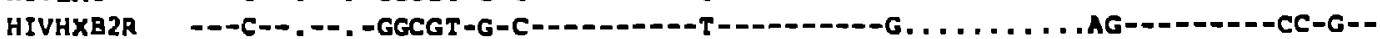

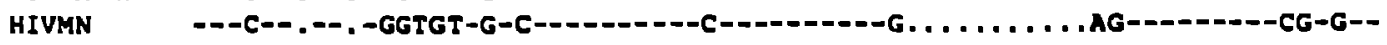

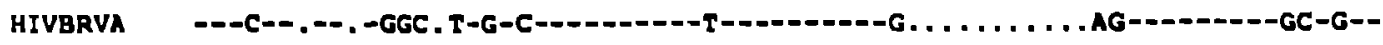

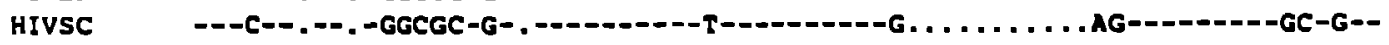

HIVJHJ

HIVJRCSF

HIVJRFL

HIVOYI

HIVSF 2

HIVNY5CG

HIVNL 43

HIVSE1 62

HIVCOCA

HIVHAN

HIVSWB8B 4

HIVRF

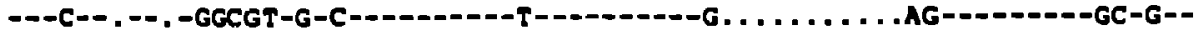

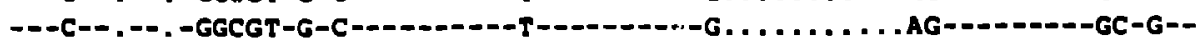

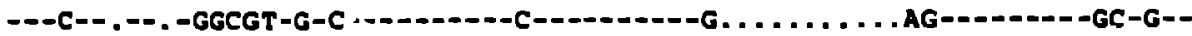

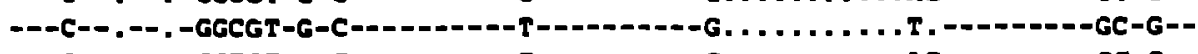

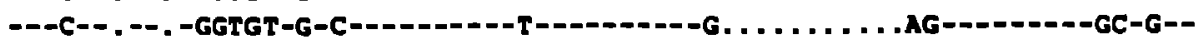

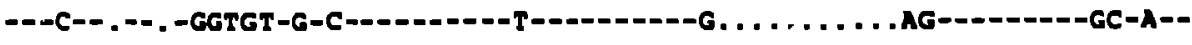

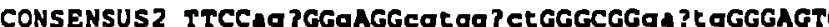

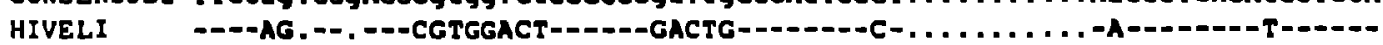

HIVZ226

HIVNDK

HIVMAL

HIVU 455

HIVSF 170

HIVZ321

HIVAIS: 70

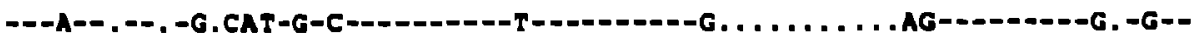

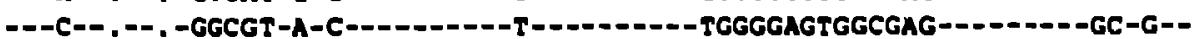

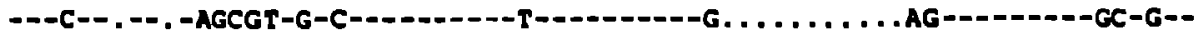

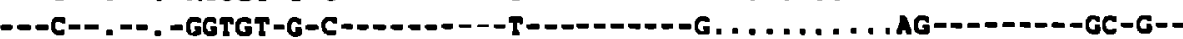

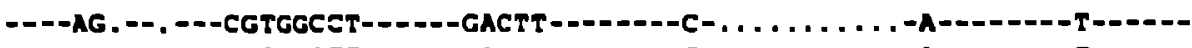

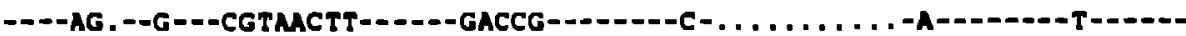
----GA. --G---TGTGGTTP------AGTTG-------C-..........-G-------T-----

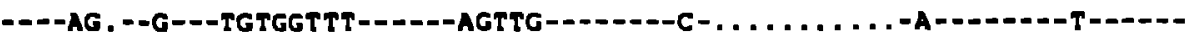

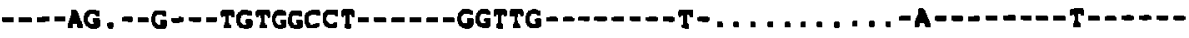

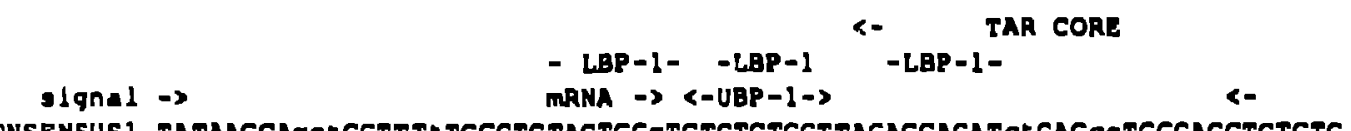

CONSENSUS1 TATMGCAgCEGCTTTETGCCTGTACTGGgTCTCTCTGGTTAGACCAGATCEGAGCeTGGGAGTCTCTG

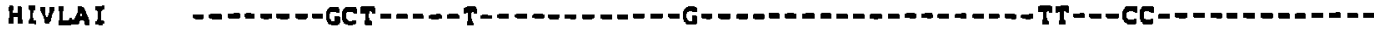

HIVHXB2R - - -

HIVMN

HIVBRVA

HIVSC

- - -

IIIVJHJ

HIVJRCSF

-

HIVJRFL

-

HIVCYI

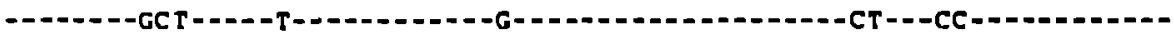

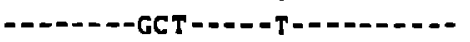

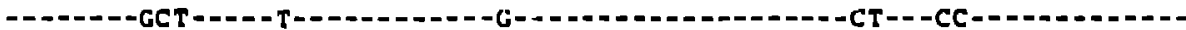

IIVSF

HIVNYSCG

HIVNLA3

IIIVSF 162

IIIVCDCA

IIIVHAN

IIIVSWBBE 4

IIIURF

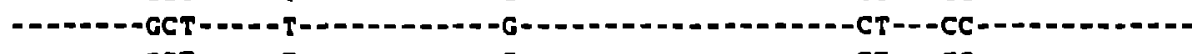

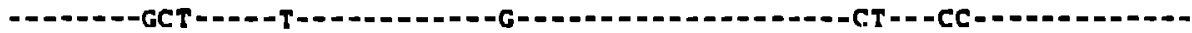

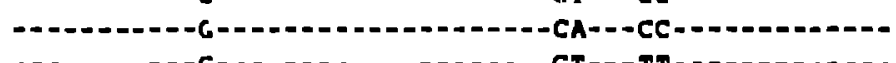

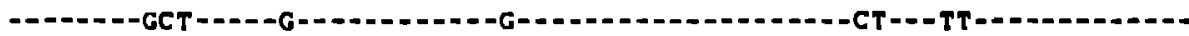

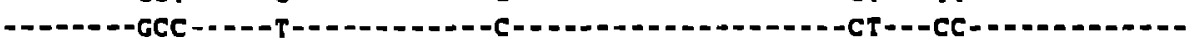

-

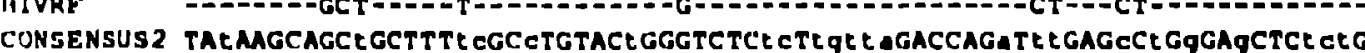

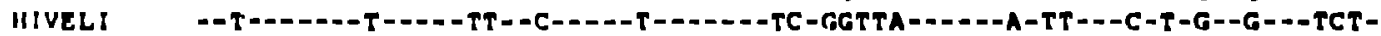

HIVZ2Z6

:IIVNDK

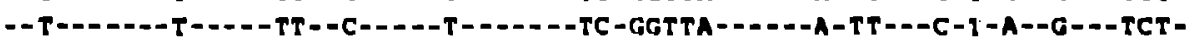

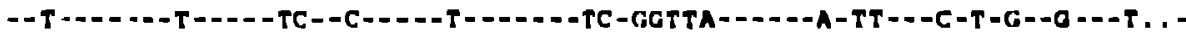

IIIUMAL

IIVUAS5

11\%:31\% 10

IIIV: 121

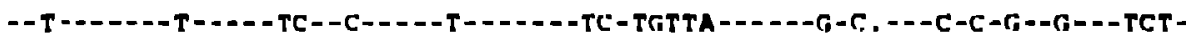

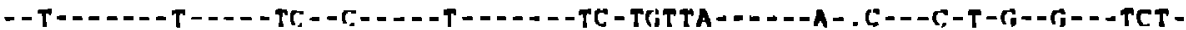

1: : : ANT in

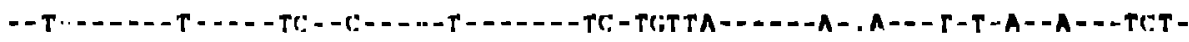

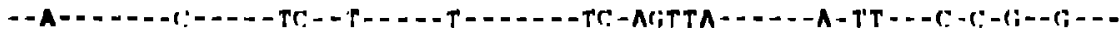

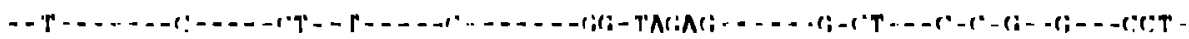


TAR CORE $\rightarrow$

UBP $-1 \rightarrow 2$

gignal $\rightarrow$

$<-$ mRNA

CONSENSUSI G.CTa ?CTAGGG. AACCCACT?GCTTAAGCCTCAATAAAGCTTGCCTTGA.GTGCTLCAAGTA.GTGTGT HIViAI HIVHXB2R HIVHN

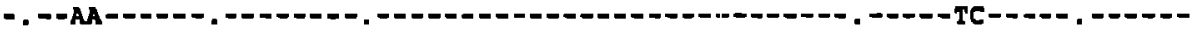
HIVJH3

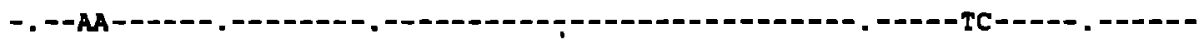
-. --MA--2-1. - - -

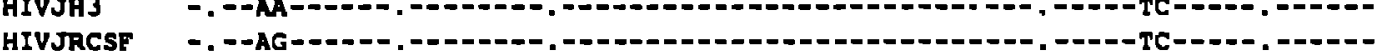
HIVJRFL HIVOYI HIVSF 2 HIVNYSCG HIVNL43 HIVSF 162 HIVCDC4 HIVHAN HIVSWB884 HIVRF

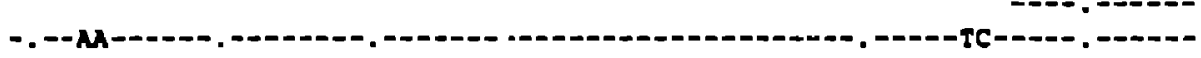
-. --AG--1-_.

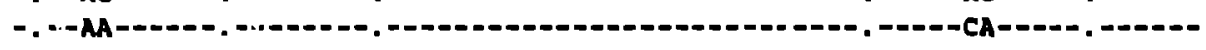

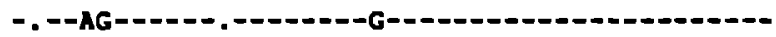

- . --AG--1---. -. --AG-1-1-- , -, --AG-1-0. CONSENSUS2 GrCTAgCEAggg?AACLCACT.gCTTAAgCCTCAATAMAGCTTGCCTTGA ?GEGCE EAMGEA.GTGTGT HIVELI HIV2226 HIVNDK HIVMAL HIVU455 HIVSF 170 HIVANT70 SIVCP 2

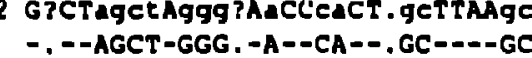

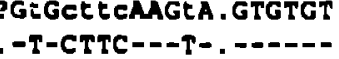

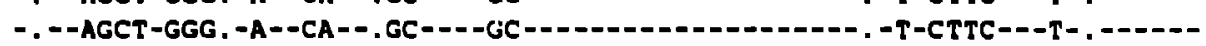

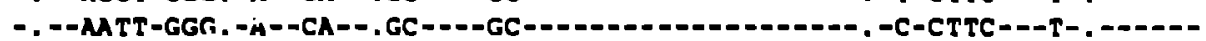

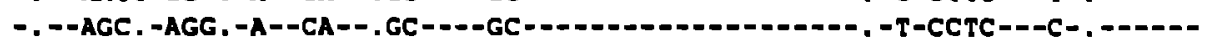

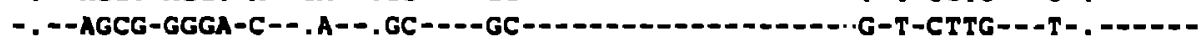

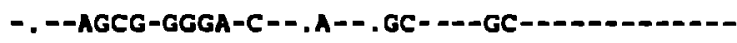

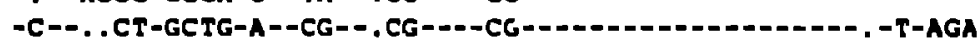

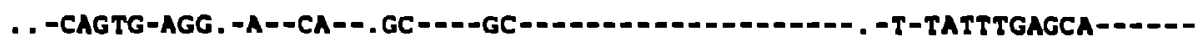

\section{HIVLAI}

HIVHXB2R

HIVMN

HIVJHJ

HIVJRCSF

HIVJRFL

HIVOYI

HIVSF 2

HIVNYSCG

H[VNLA]

HIVCDCA

HIVHAN

HIVSWBOB 4

HIVAF CONSENSUS
HIVELI

HIVELI
HIVZ226

HIVNDK

HIVMAL

HIVUA55

s IVCP 2
CONSENSUS I GCCCgTCTGTTGTGTGACTCTGGTAACTAGAGATCCCTCAGACCCL TTTAGLCAGEGTGGAAATTCTCT

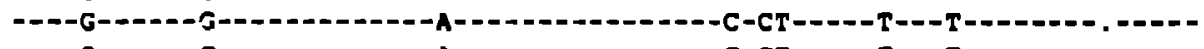

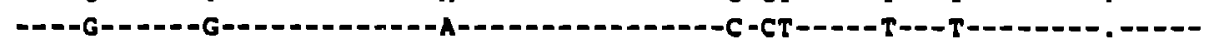

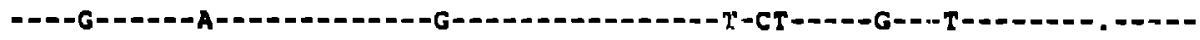

-

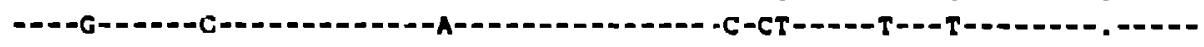

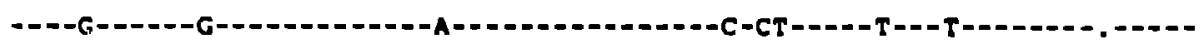

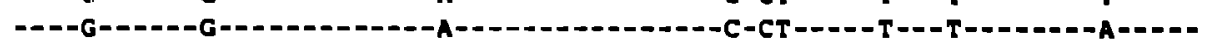

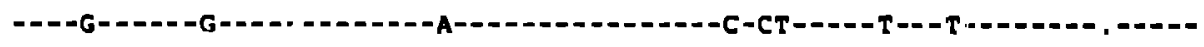

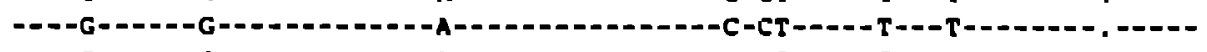

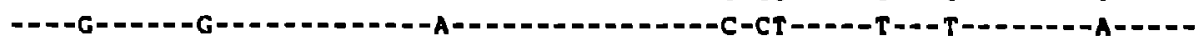

-

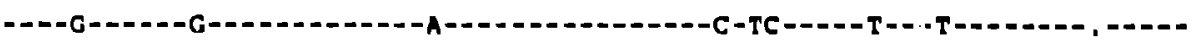

629

630

630

629

144

630

495

532

630

630

630

629

623

562

630

616

630

6.30

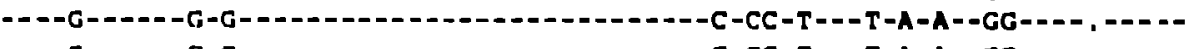

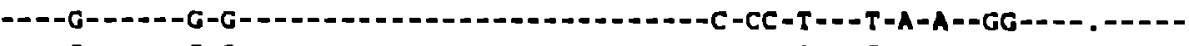

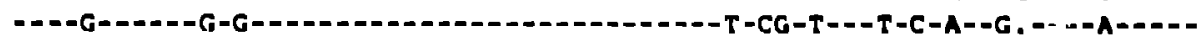

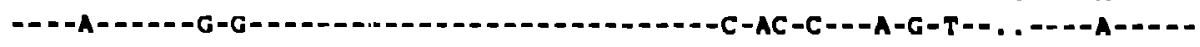

- - -G- - - - A-T-1.

$62 \mathrm{~B}$

629

638

049 


\section{HIV-1 LTR}

$<-$

$$
\text { <- LYS-LRNA PbI }
$$

CONSENSUS1 AGCAGTGGCGCCCGAACAGGgACtLgAaAgCGAAAGtaA . . . . . . . . . . . . . .

HIV!AI

HIVHXZ2R

HIVHN

HIVJH3

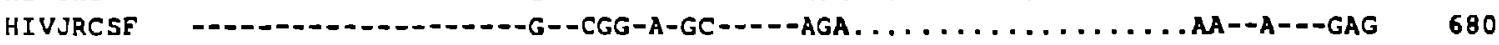

HIVOYI $\quad-1 .-. .-1 .-1 \ldots \ldots A--A---G A G \quad 5 B 2$

HIVSE2

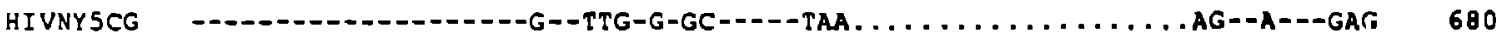

HIVNL43

HIVCDCA

HIVHAN

HIVRF $\quad$ -

CONSENSUS2 AGCAGTGGCGCCCGAACAGGGACCLgAAAGCGAAGTAg???????????????????AACCAGAGAAG 666

HIVELI

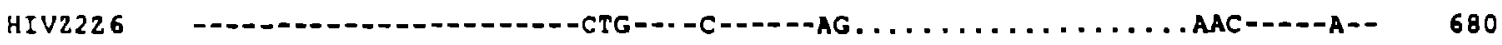

HIVNOK

HIVMAL

HIVU455

SIVCPZ

CONSENSUSI cTCTCTCGACGCA.GG̈̈CTCGGCTTGCTgAAGCGC? ?GCaCgGCAAGAGGCGAGGGGcGGCGactGGTGA 746

HIVLAI C--_-1-1--.

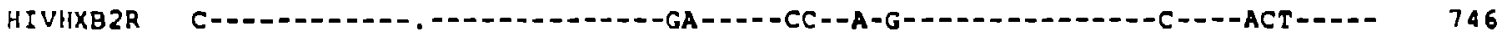

IIIVMN CDN C

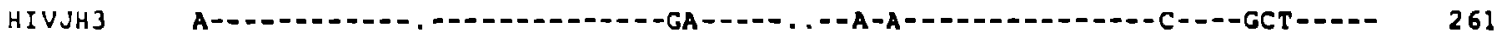

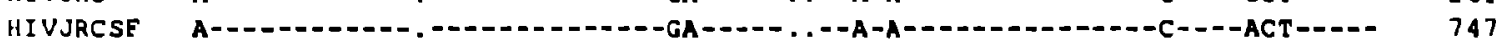

HIVJRFL

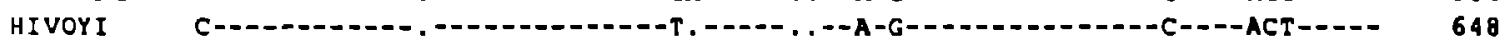

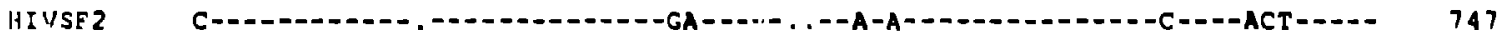

HIVNYSCG A-1.-1-2-_.

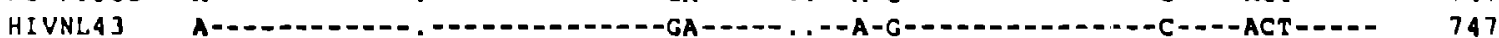

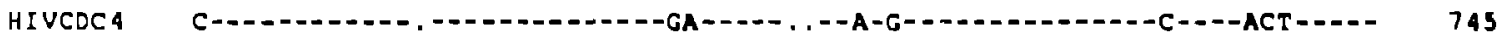

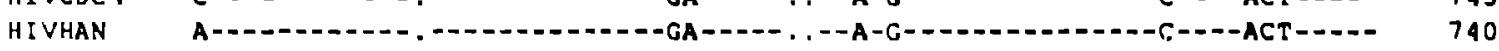

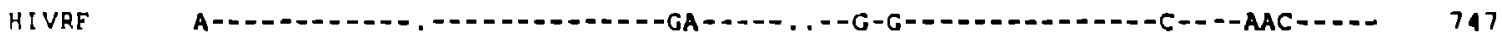

C.DNSENSUS2 7TCTCTCGACGCA.GGACTCGGCTTGCTGA \& qCGCqCACa ? ?GCAAGAGGCGAGggqCaGCGACtGGTGA 732

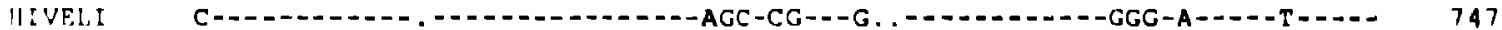

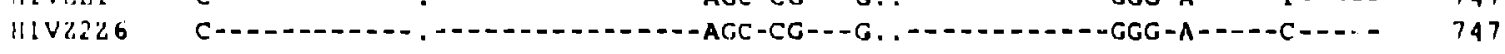

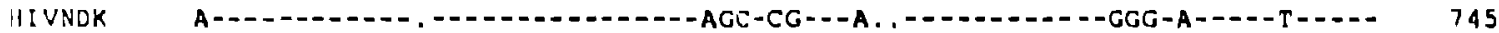

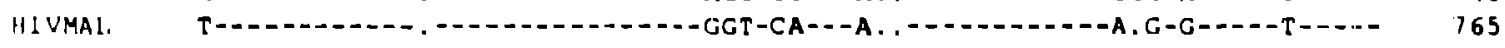

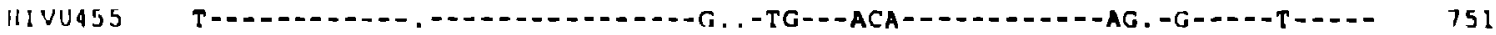

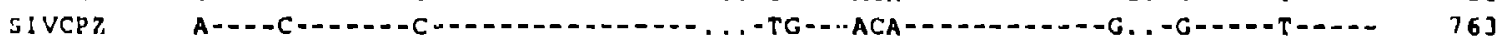


HIV-1 LTR

I/ major 5'sj gag cds $\rightarrow$

CONSENSUS1 GTACGCCaaaatTt?.gACTAGCGGAGGCTAGAagGagagagag 788

HIVLAI -

HIVHXB2R - 2 -

HIVMN $\quad-1-(-$-AAAAA--CTT.

HIVJH3 - 3 - 303

HIVJRCSE -

HIVJRFL - -.---AAAAA- - TT ...-- 598

HIVOY I _-

HIVSF2 --.-....AA--TTT.--_- 789

HIVNYSCG -

HIVNL4 3 -

HIVCDCA -

HIVHAN -----.AAAA--TT...--- 782

HIVRF

CONSENSUS2 GTACGCE TAAAATTTIL PGACTAGCGGAGGCEAGAAGGAGAGAgA 775

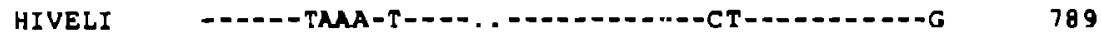

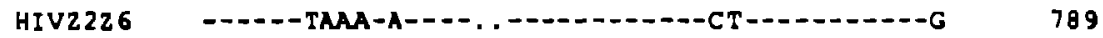

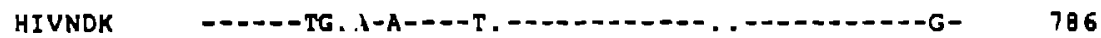

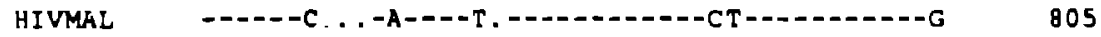

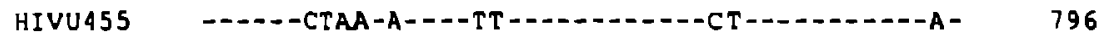

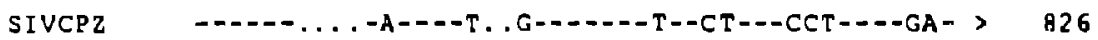


CONSENSUS1 ATGGGTGCGAGAGCTCaGTAT TAAGCGggGGAgAATT agALAa ?'GGGAaAAAT'TCGGTTAAGGCCAg

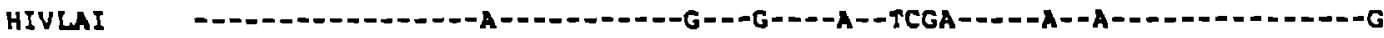
HIVHXB2R HIVMN HIV HIVJH3

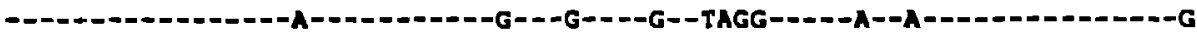
HIVJRCSF HIVOYI HIVSF2 HIVNY5CG -

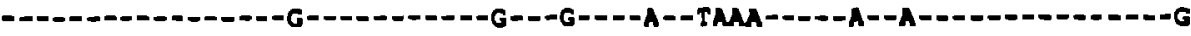
HIVNL43 HIVCESA HIVHAN -_-1 HIVRF CONSENSUS2 ATGGGTGCGAGAGCGTCAGTATTMGCGGGgMAMTTAGAT PCATGGGAAAMATTCGGTIACGGCCAG
HIVELI Hives2 HIVNDK HIVMAL HIVU455 SIVCP 2

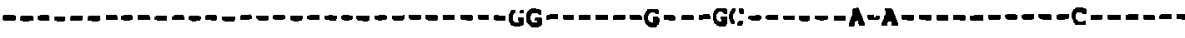
- - - - - - - - -

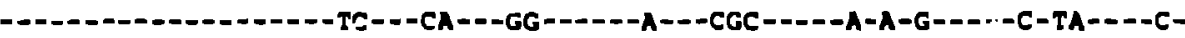

CONSENSUS1 GgGGAMGMALAATATAat TMMACATATAGTATGGGCAGCAGGgAgCTAGACGATTCGCagTEMA HIVLAI HIVHXB2R HIVMN HIVJH3 HIV JRCSF HIVOYI HIVSF2 HIVNYSCG HIVNLA3 HIVCDCA HIVHAK HIVRF

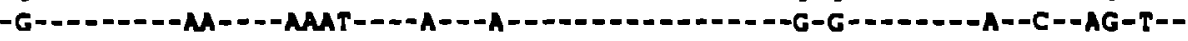

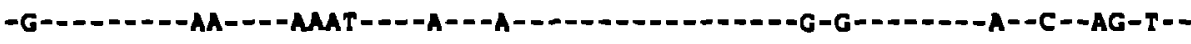

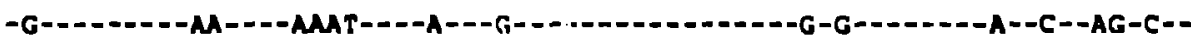

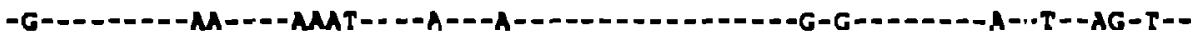

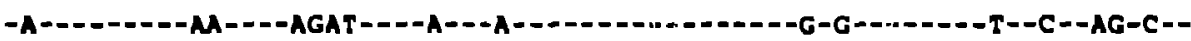

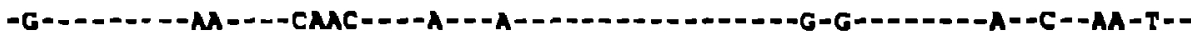

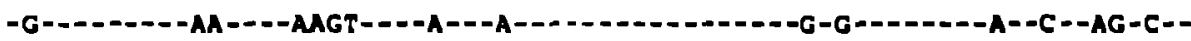

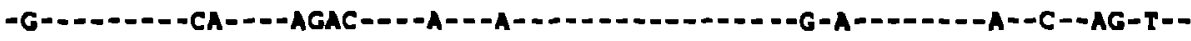

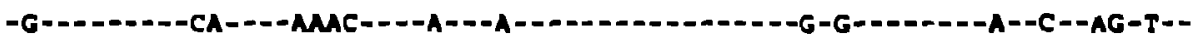

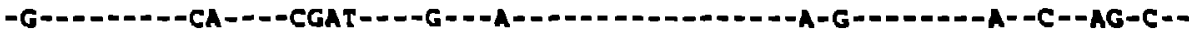

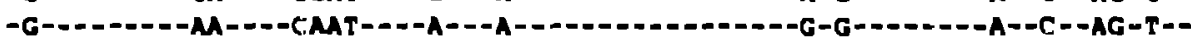

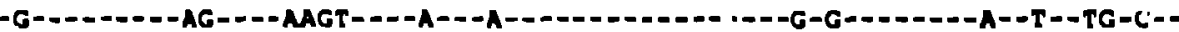
CONSENSUS? GAGCMAGRMMMATAT HIVELI HIVZ226 HIVNDK HIVMAL HIVU 455 SIVCP 2

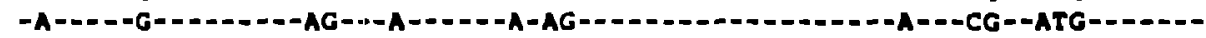

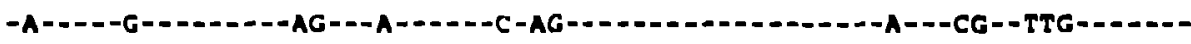

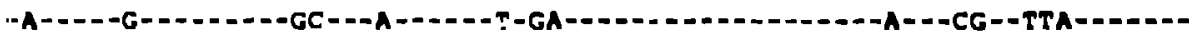

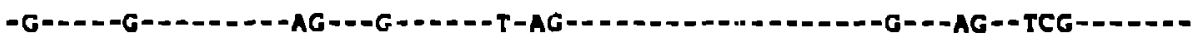

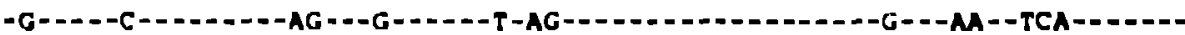

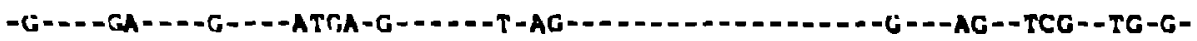

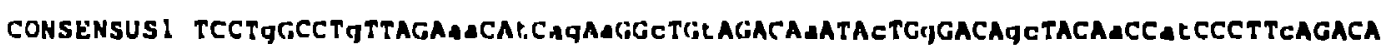

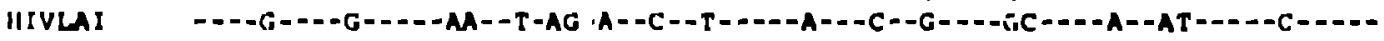
IITVHXBZR HIVMN

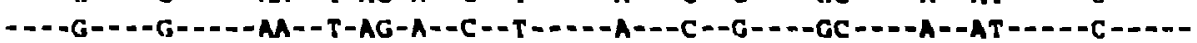

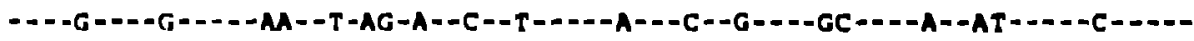
iIVJHJ HIVJRCSF HIVOYI IIIVSF 2 HIVRYSCG HIVNLAJ IIIVCDCA IIIVHAN IIIVHr

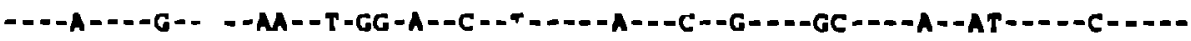

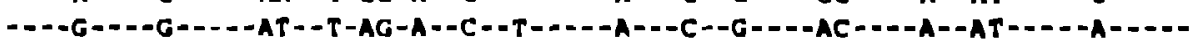

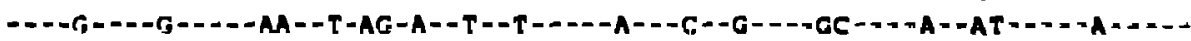

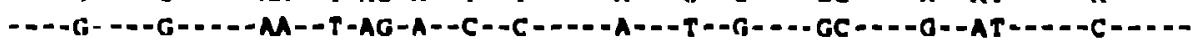

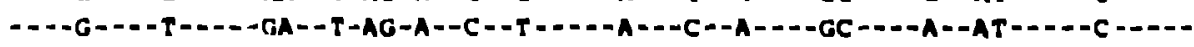

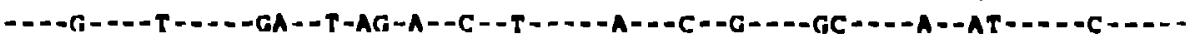

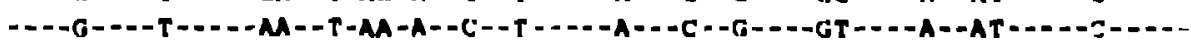

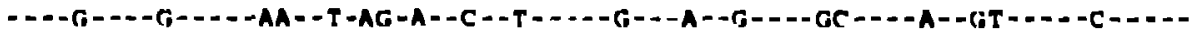

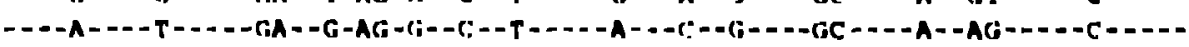

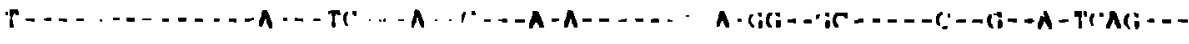

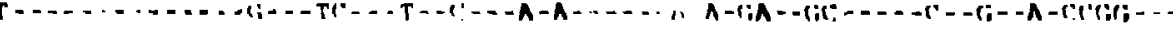


TONSENSUS.L GGATCAGAAGAACL TAgATCATTAT T TAATACAgTAGCAACCCTCTAT TGTGTgCATCAAAggATAGAgg

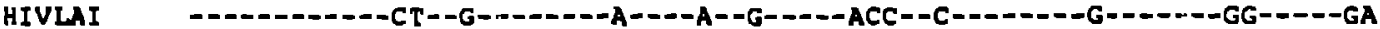
HIVHXB2R - -

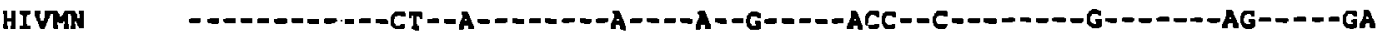
HIVJH3 HIVJP.CSF -..-_HIVOYI

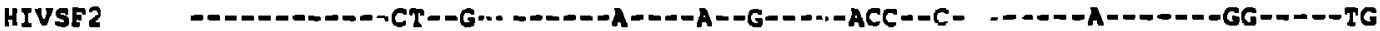

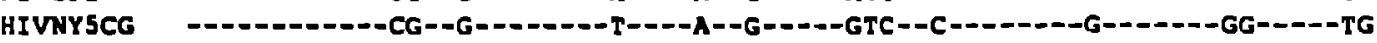
HIVNL43 HIVCDC4 HIVHAN HIVRF -

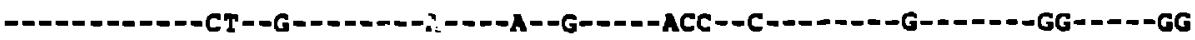

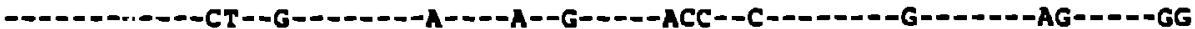

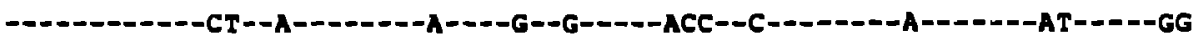
CONSENSUS2 GGALCAGMGMCTTAgATCATTATATMTACAGTAGCAACCCTCTATTGTGTACAT ?AAGgATAGAEG

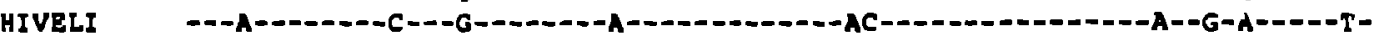
HIV2226 HIVNDK HIVMAL HIVU455 SIVCP2

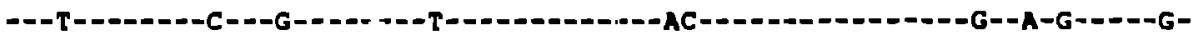

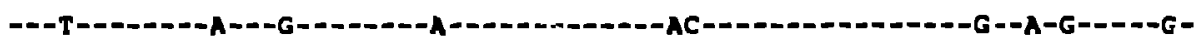

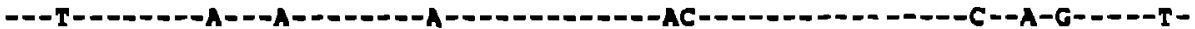

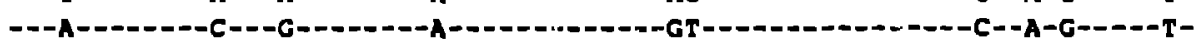
$--C T-----G-C-G C G G--C--G-T---C--$ TC-G-- GTA--G-GG- CA-----AGTGAC--CACT-

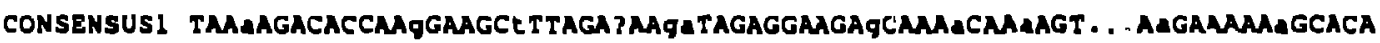

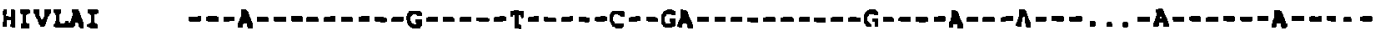

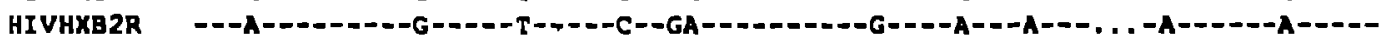

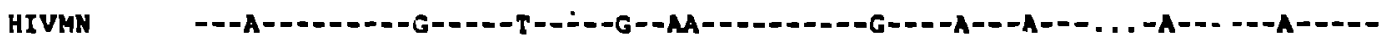

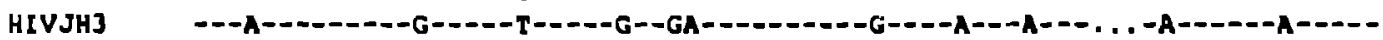

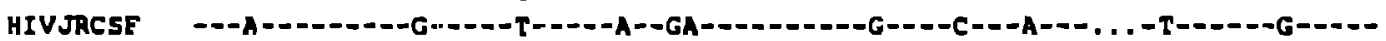

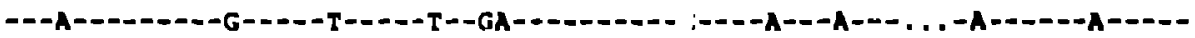
HIVSE 2 -

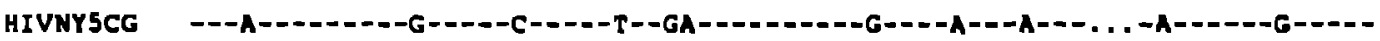

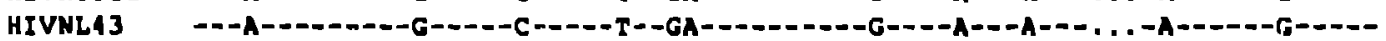
HIVCDC4 HIVHAN

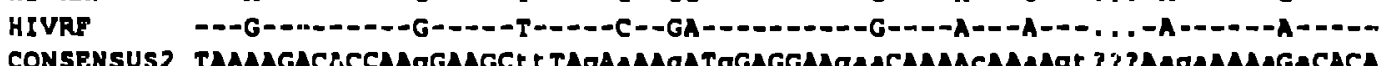

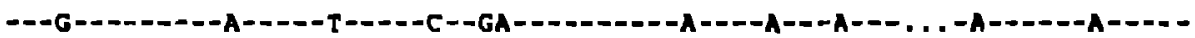

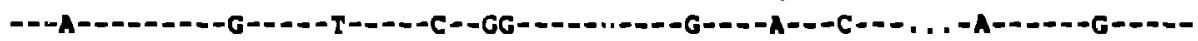
HIVELI HO

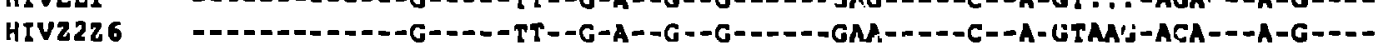
HIVMY

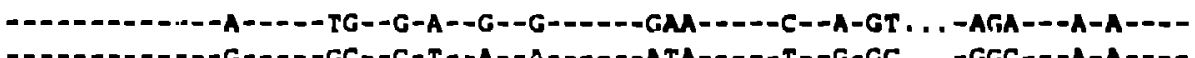

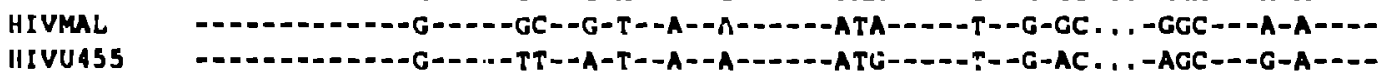
IIIVU 455 -
SIVCPZ

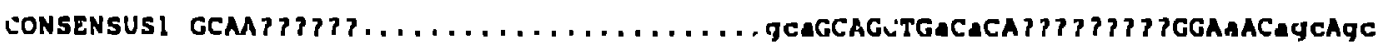
-......................GCA-.-.--A-A-.......--C--AGC-GC

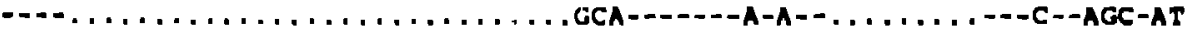

IIIVJH 3 IITUJRCSE HIVOY I HIVSE? IIIVNYSCG HIVNL 3 HIVCOCA II UHAN II UAF

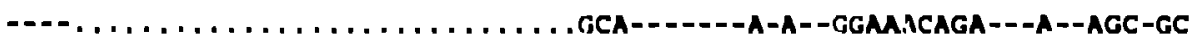

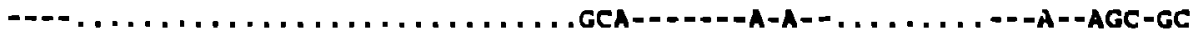

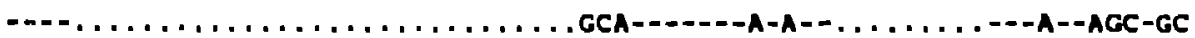

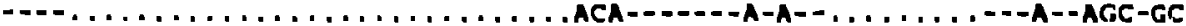

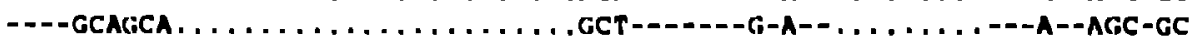

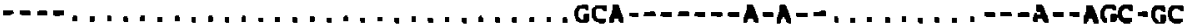

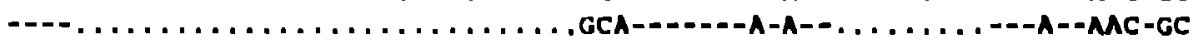

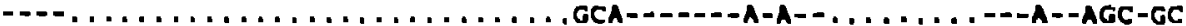

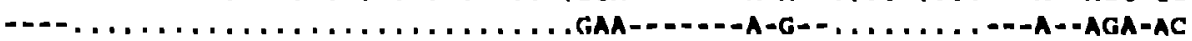

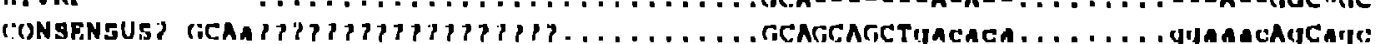
IIIVHILI IIIV7iz.6 IIIVNISH - -A. . . . . . . . . . GiAAAC-A-AGC

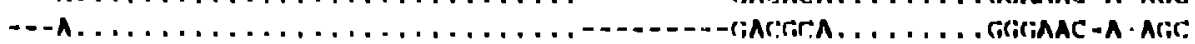

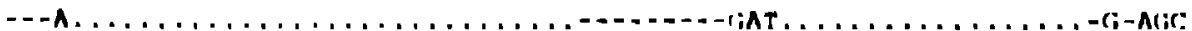
IIIVMAI. IIIVI,4',

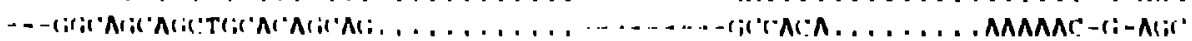

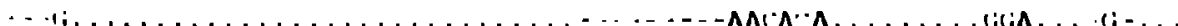
; 1 VII': 
CONSENSUS1 CAgGTCAGCCAAAATTACCCLATAGTgCAgAACCT? CAGgGgLAATJGTACATCAggCCATATCACCTA

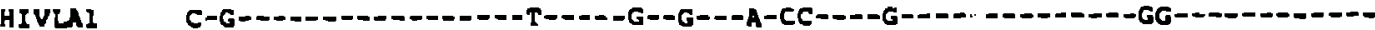

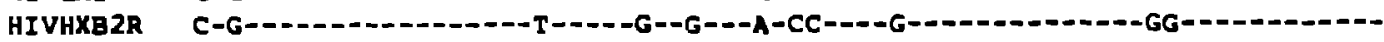

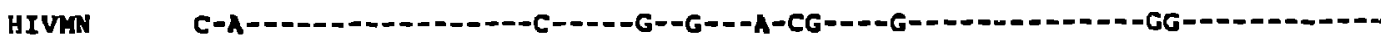

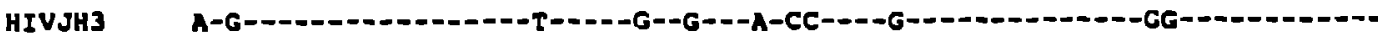

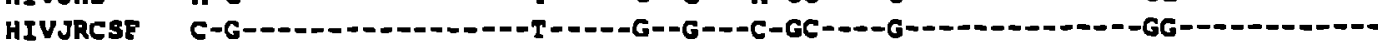

HIVOY

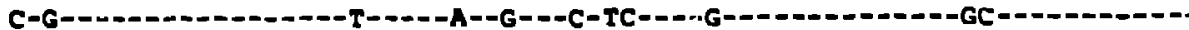

HIVSF 2

HIVWYSCG

HIVNL43

HIVCDRA

HIVHAN

HIVR:

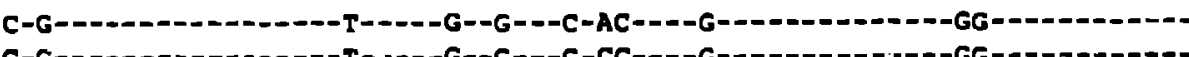

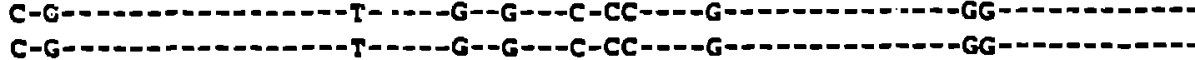

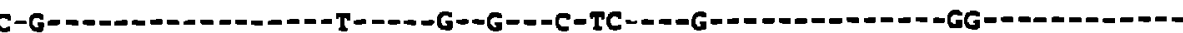

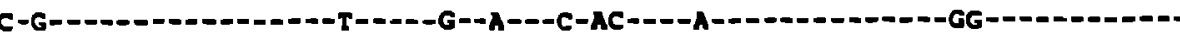

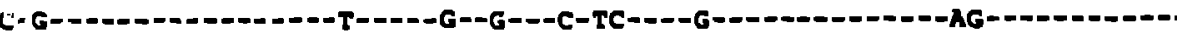

CONSENSUS2 CAggECAGCCMNKTTACCCEATAGTGCAgMACCEACAgGGGCMAT ggTACAECAGGCCATATCACCTA

HIVELI

IIIV2226

HIVNDK

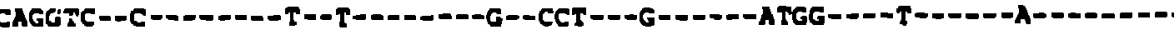

HIVML

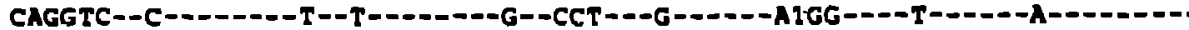

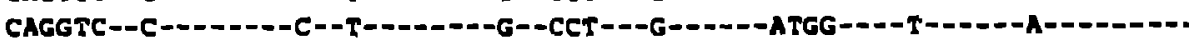

HIVU455

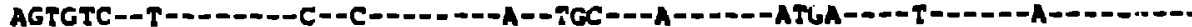

SIVCP2

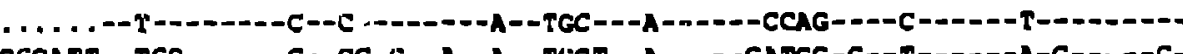
GGCATT - TGS--.---C--CC-C--A--A--TGCT--A-----GATGG-G--T------A-C--..--C-

CONSENSUS1 GACTTTMATGCATGGGTAMAGTAGTAGAGAgAAGCTTTCAGCCCAGMGTAATACCCATGTTTEC

HIVLAI

HIVHXD2R

HIVHA

HIVJH3

HIVJACSF

HIVOYI

بIVSF 2

HIVNYSCG

HI JNL43

HIVCDCA

HIVHAN

HIVRF

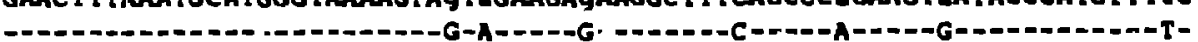

-

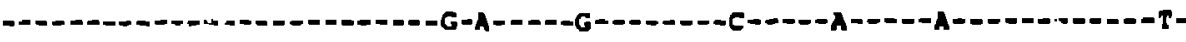

-

-

-

-

-

-

-

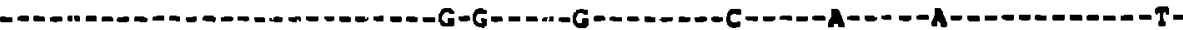

CONSENSUS2 GACT TTCMACGCATGGGTAMAGTA TAGMGA MMGGCETTCAGCCCACMGT AATACCCATGTTETC

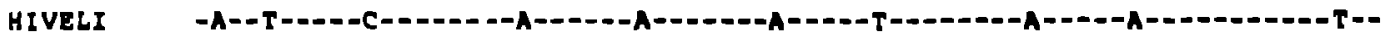

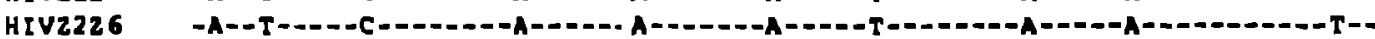

HIVMDK

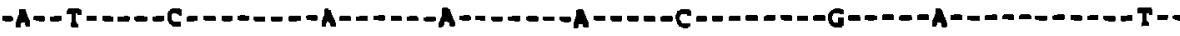

HIVMRL

HIVU455

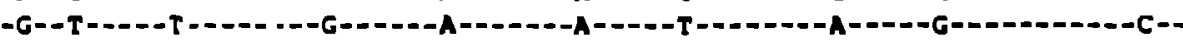

SIVCP2

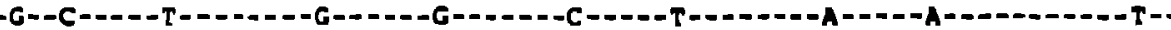

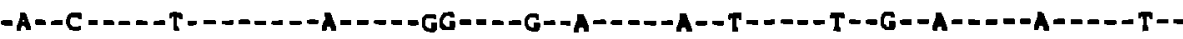

CONSENSUS1 AGCATTAECAGAGGaGCCACCCCaCAAGATt TAAACACCATGCTAMCACAGTGGGGGACATCAGCA HIVLAI HIVHXB2R HIVMN

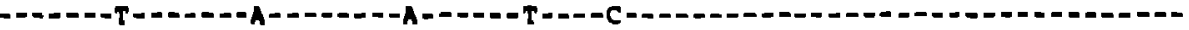
- - - HIVJIIJ IITUJRCSF HIVOYI IIIVSF2 HIVNYSCG HIVNLA3 HIVCDCA HIVHAN HIVHF -

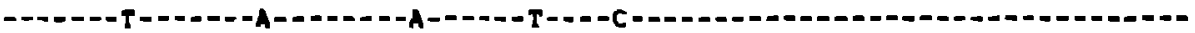

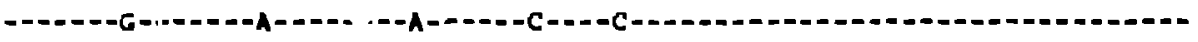

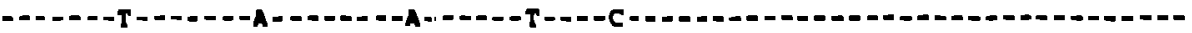

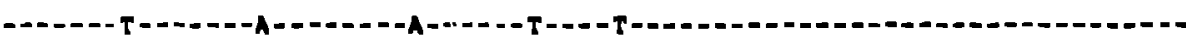

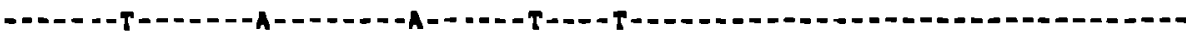

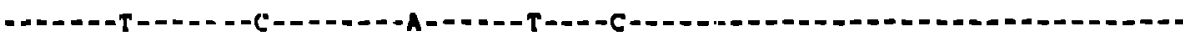

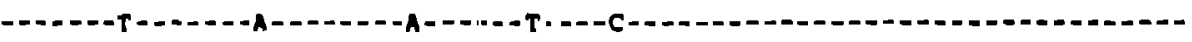

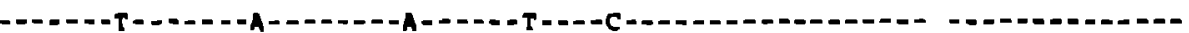

CONSENSUS? AGCATTATCAGAACGAGCCACCCCACAAGATTTAAATACCATGCTAAACACAOTGGGqGGCATCAAGCA HIVELI IIIVZ226 IIIVNIDK HiVMAI.

- - - - - - -

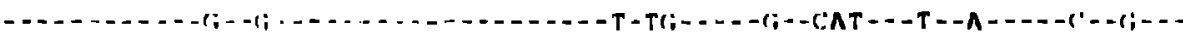

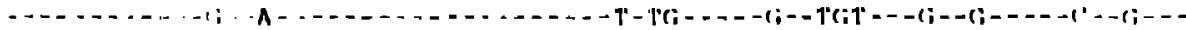
: : V V'P' 
CONSENSUS1 GCCATGCAAATGL TAAAAGAgACCATCAATGAGGAAGCTGCAGAATGGGATAGATTGCAtCCAGtgCATG

HIVLAI

HIViXB2R

HIVRN

HIVJH3

HIVJRCSE

HIVOYI

HIVSE2

HIVNY5CG

HIVNL 43

HIVCDCA

HIVHAN

HIVRF

CONSENSUS2

HIVELI

HIV222 6

HIVNDK

HIVIAL

HIVU455

SIVCPZ

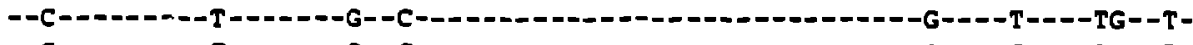

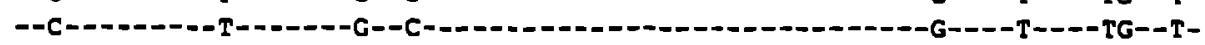

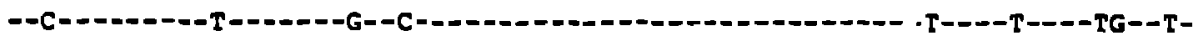

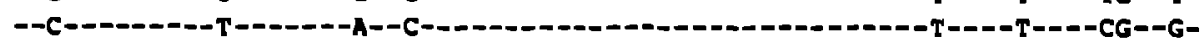

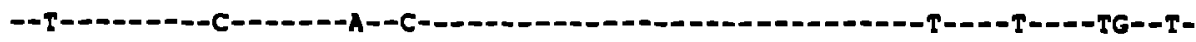

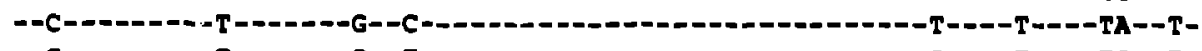

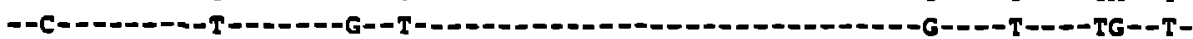

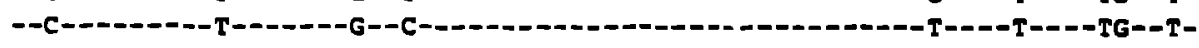

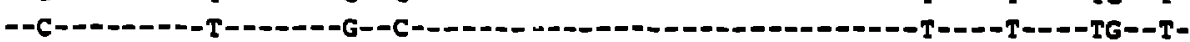

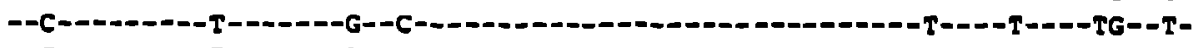

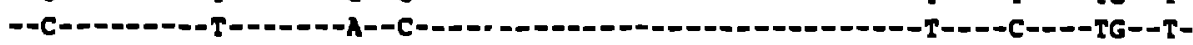

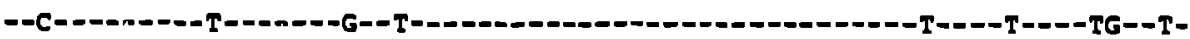

--C--

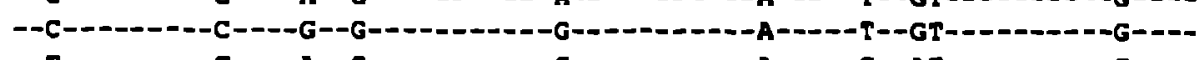

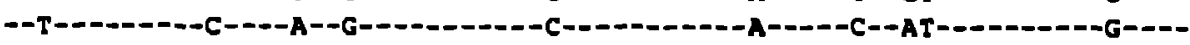

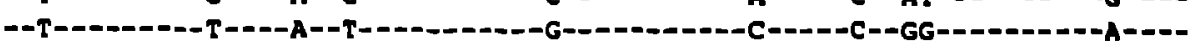

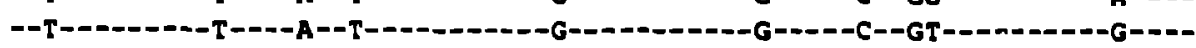

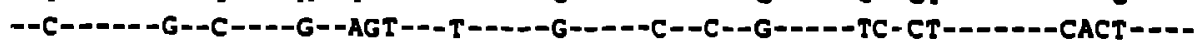

CONSENSUSI CAgGgCCTATTgCACCAGgcCAgATGAGAGAACCAAGgGagtGaCATAGCAGgaCtACTAGTACCCT

HIVLAI

HIVHXB2R

HIVAN

HIVJH3

HIVJRCSE

HIVOYI

HIVSF 2

HIVNYSCG

HIVNL43

HIVCOC4

HIVHAN

HIVRP

CONSENSUS2

HIVELI

HIVZ22 6

HIVNDK

HIVMAL

HIVU455

SIVCP:

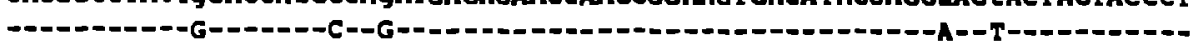

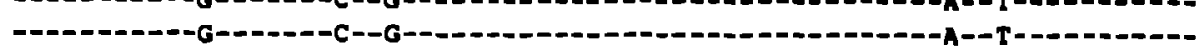

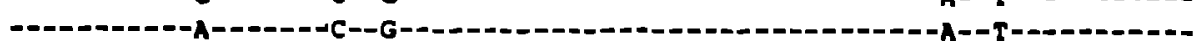

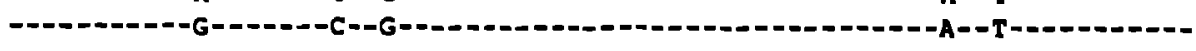

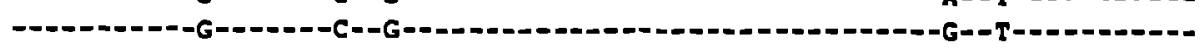

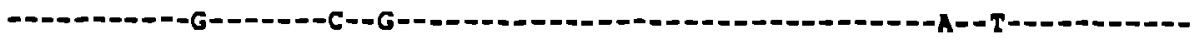

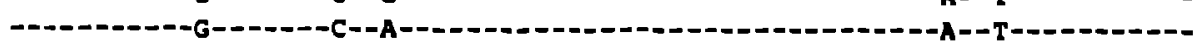

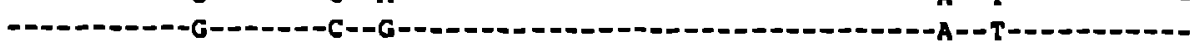

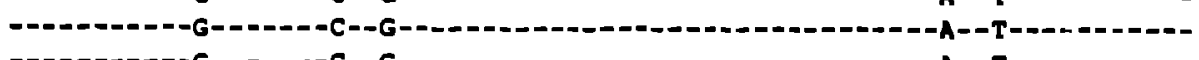

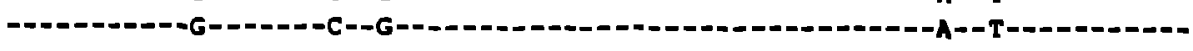

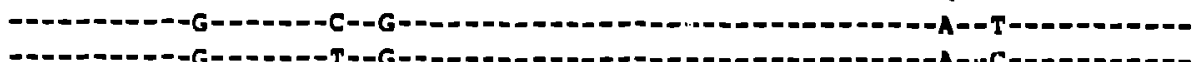

CAGgGCCT atTgCaCCAGGCCAgATGAGAGAACCAAGgGGAGTGATTACCAGGACTACTAGtACCct -

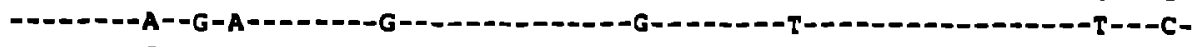

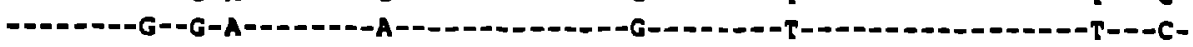

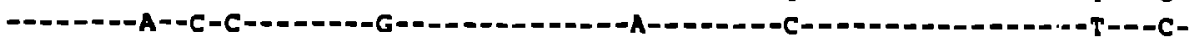

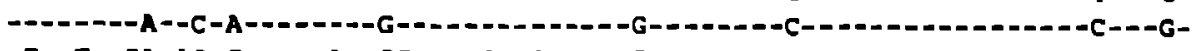

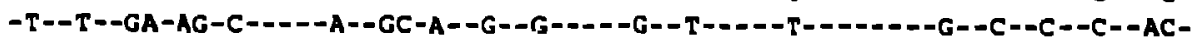

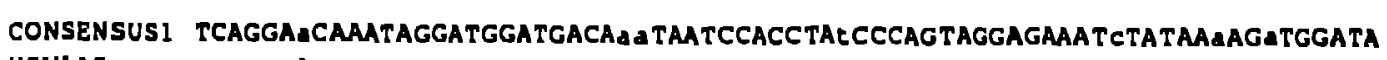

HIVLAI

HIVHXB2R

HIVHN

HIVJH3

IIIVJRCSF

H I VOYI

HIVSF 2

HIVNY 5 CG

hIVNLA3

HIVCDC4

II IVHAN

HIVRE

CONSENSUS 2

IIIVELI

HIVZ226

II I VNDK

II I VMAL

III Vu45.5

:i) VCP'
TCAGGA САA TAGCATGGATGACA

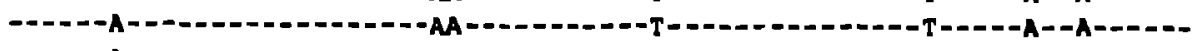

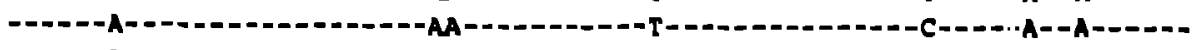

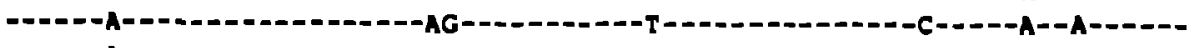

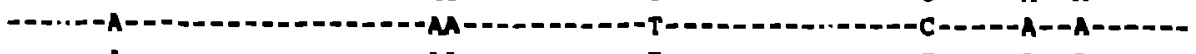

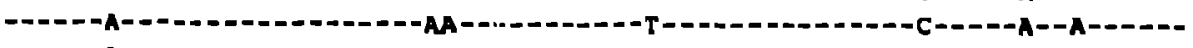

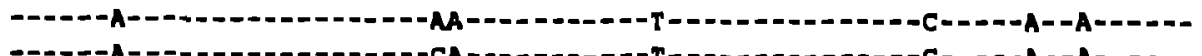

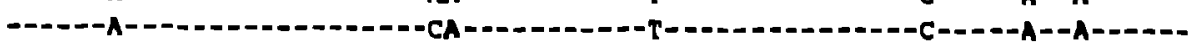

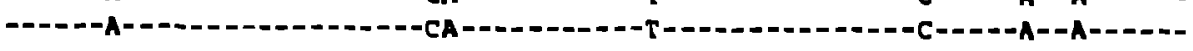

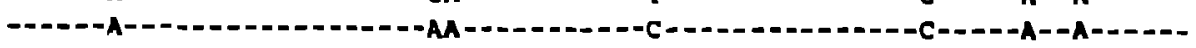

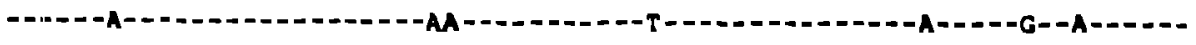

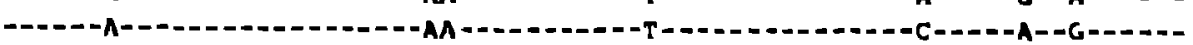

TCAgGAACAAATAGCATGGATGACAaGcAAcCCACCTATCCCAgT gGGAGAaATCTATAaAAGATGGATA

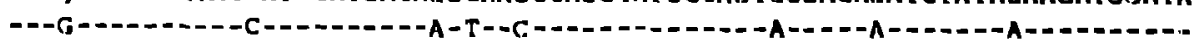

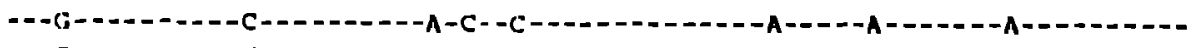

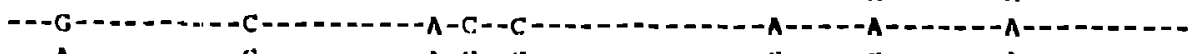

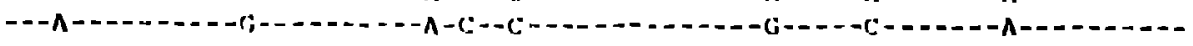

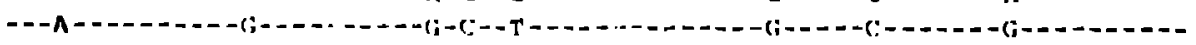

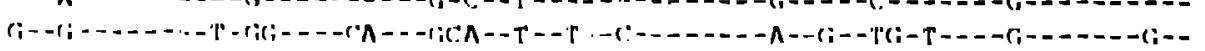




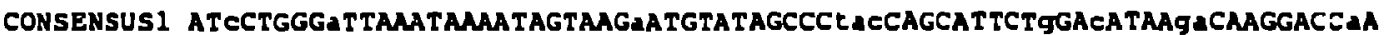

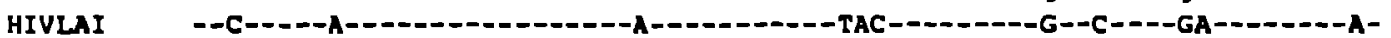

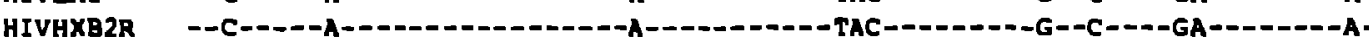
HIVMN HIVJH3 HIVJRCSF HIVOYI HIVSF 2 HIVNY5CG HIVNL43 HIVCDCA HIVHAN HIVRF - -C-1--A-_-_-

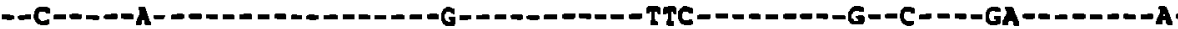

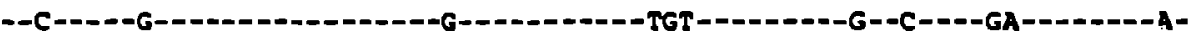

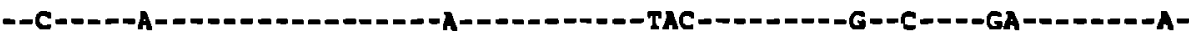
--C----A-D-.---C-----A-1--_-

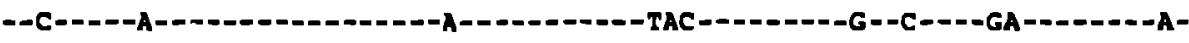

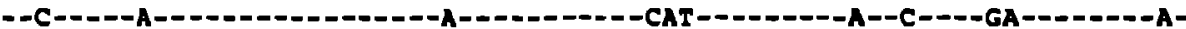
--C----A-_CONSENSUS2 A TCCTGGGATTMATAMATAGTAGMATGTA TAGCCCTGTCAGCATtTGGACATAGACAgGGaCCA HIVELI HIV2226 HIVNDK HIVMAL HIVU 455 SIVCPZ

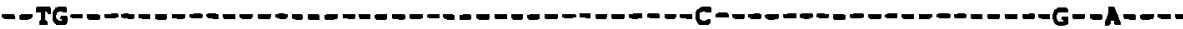

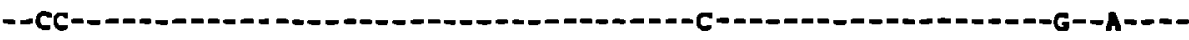

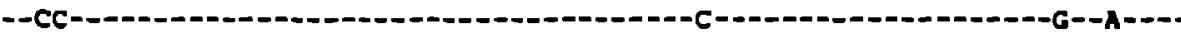

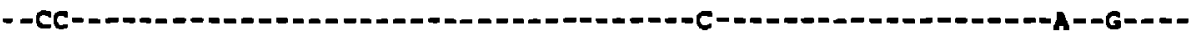

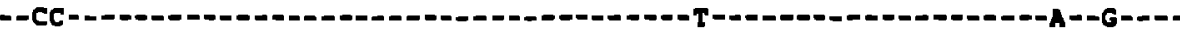

CONSENSUSI AgGMCCCTTTAGAGACTATGTAGACGgTTCTATAAACECTAGAGCCGACAMGCTECACAgGAgGT HIVLAI HIVHXB2R HIVMN HIVJH3 HIVJRCSF HIVOYI HIVSE2 HIVNY5CG HIVNL43 HIVCDC4 HIVHAX HIVRF

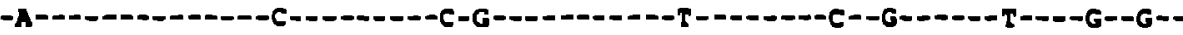

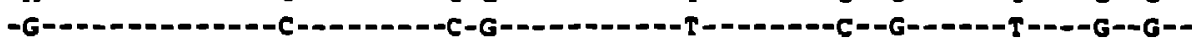

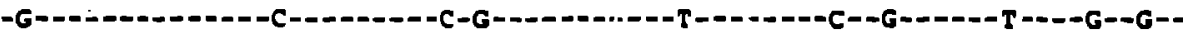

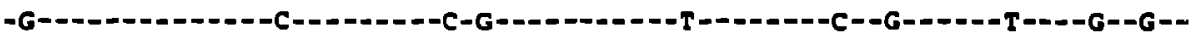

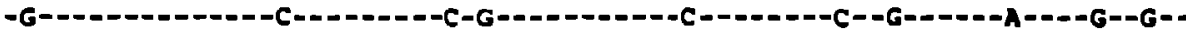

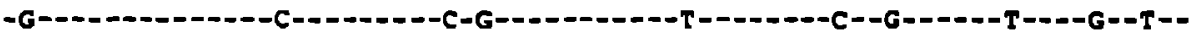

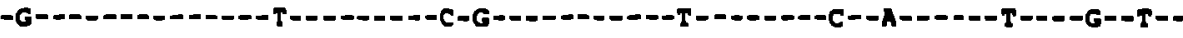

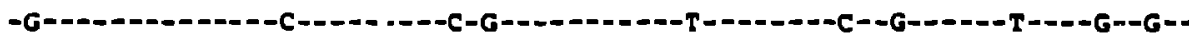

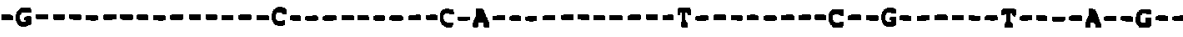

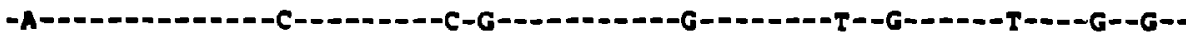

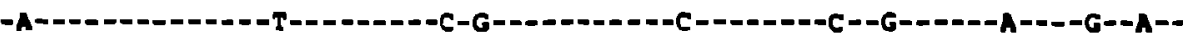

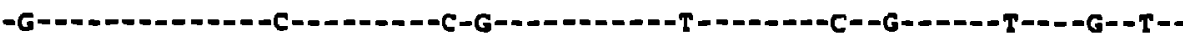
CONSENSUS2 AgGACCETTEAGAGACTATGTAGACCGTTCTATAAA TCT AAGAGCCGAGCAAGCTECACAGGATG HIVELI HIVZ226 HIVNDK

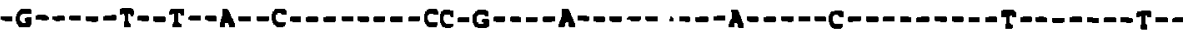

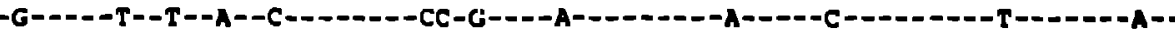

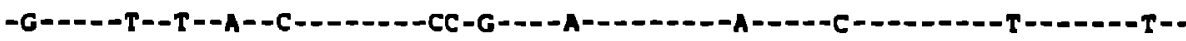
HIVMAL HIVU 455 SIVCP2

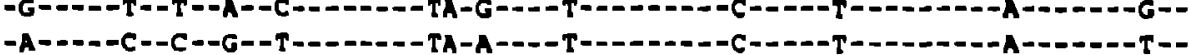

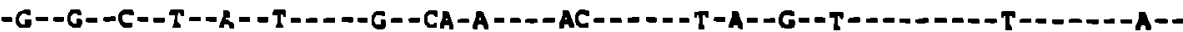

CONSENSUS 1 MAAMALGGATGACAGAACCTTgL TGGTCCAAATGCgMACCCAGATTGTAAgACTATTTTAAMGCA HIVLAT HIVHXB2R HIVMN HIVJH3 HIVJRCSF HIVOYI HIVSF HIVNYICG HIVNL4J HIVCDCA HIVHAN HIVRF

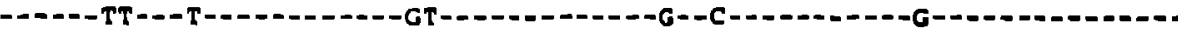

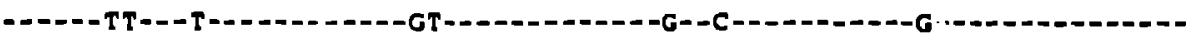

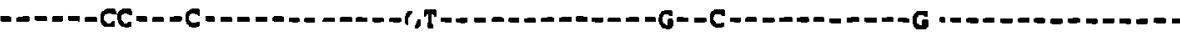

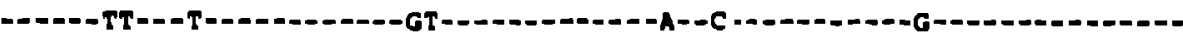

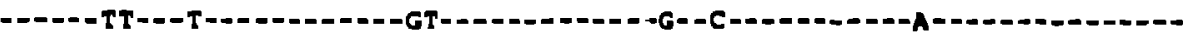

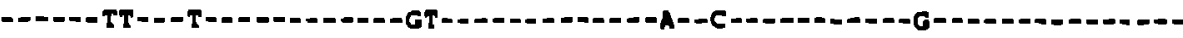

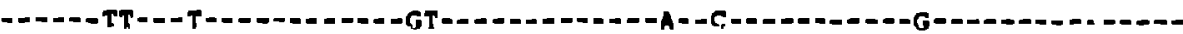

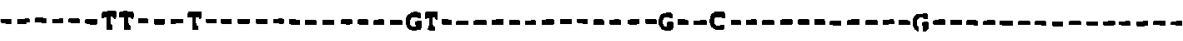

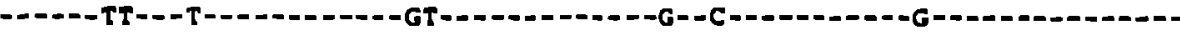

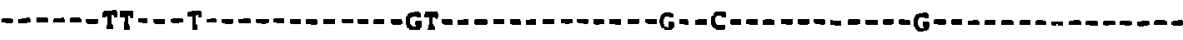

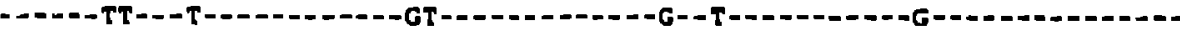

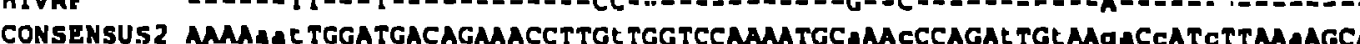
HIVELI HIVZ 226 IIIVNDK IIIVMAL IIIVUS455 GIVCP

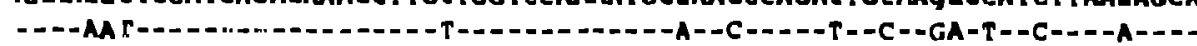

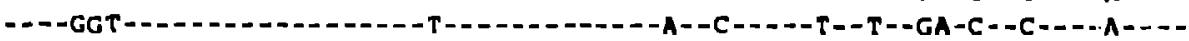
- - -AAC--_-_-_-_-

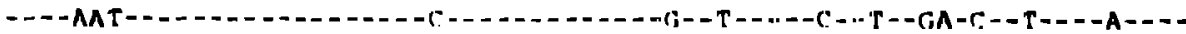

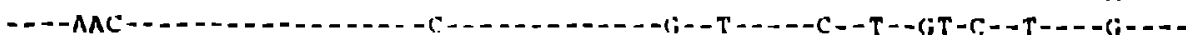

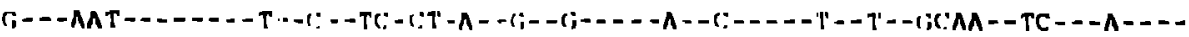


CONSENSUS1 TTgGGACCaGCaGC , ChCTAGAAGAAATGATGACAGCATGTCAGgGAGTygGgGGACCegGcCATAAag HIVLAI HIVHXB2R HIVMN HIVJH3 HIVJRCSE HIVOYI HIVSF 2 HIVNY5CG HIVNL 43 HIVCDCA HIVHAK HIVRF HIVELI HIV2226 HIVNDK HIVMAL HIVU455 SIVCPZ

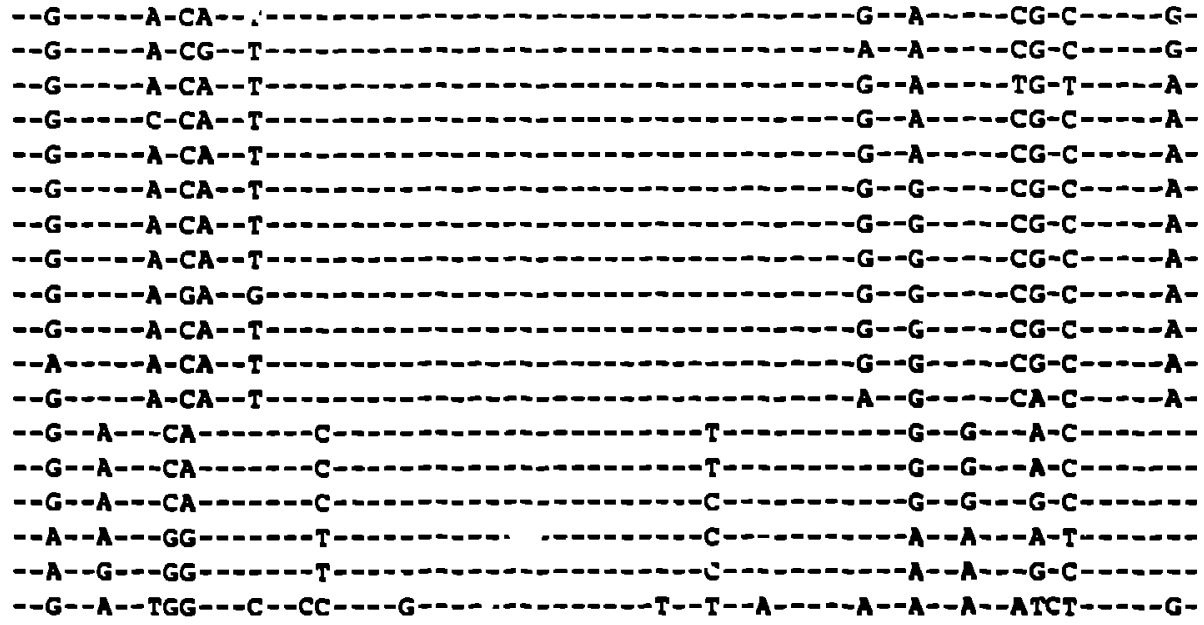

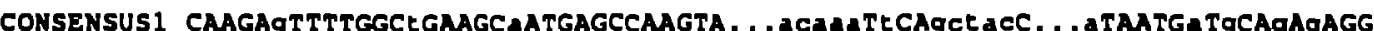
HIVLAI HIVHXB2R HIVMN HIVJH 3 HIVJRCSF HIVOYI HIVSE2 HIVNY 5 CG HIVNL43 HIVCDC4 HIVHAN HIVRF CONSENSUS HIVELI HIV2 226 HIVNDK HIVML HIVU455 SIVCPZ

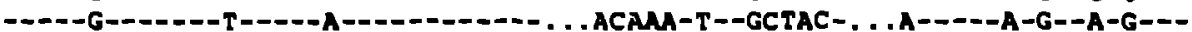
-

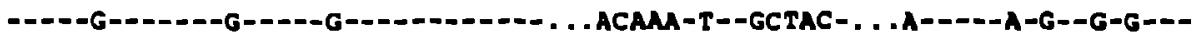

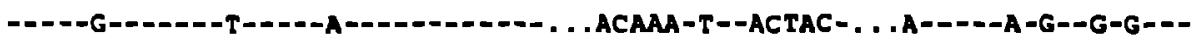

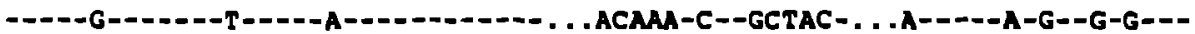

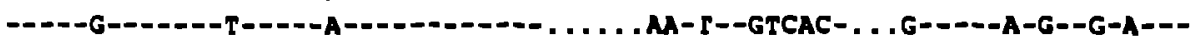
-

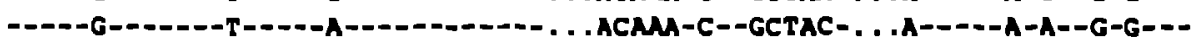

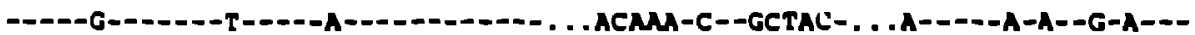

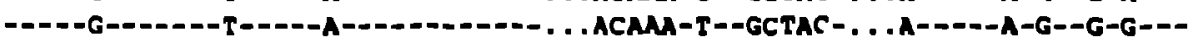

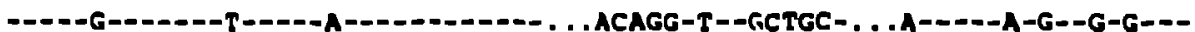

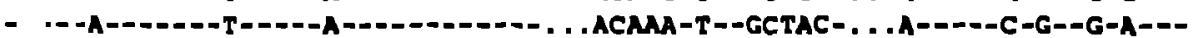
C.' BAGTTt TGGCTGAGGCAATGAGcCAAGcA ? ? acaAat caget aCtgeagtaATGATGCAGAGAGG

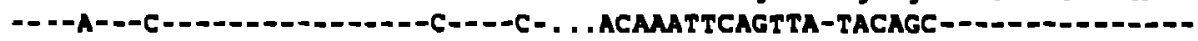

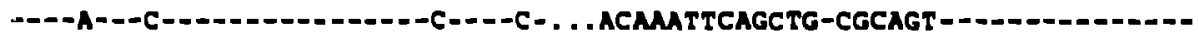

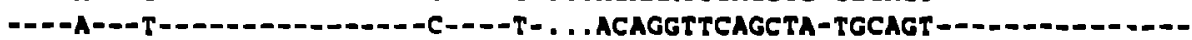

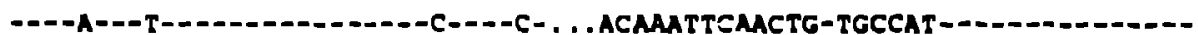

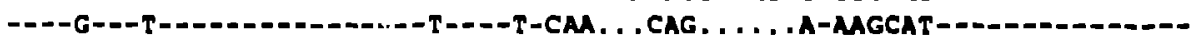
----G---C-A-----A--C--. TCAATG-TTCAGAATCAAGGGAGAG-AGATGTTT-CT-C----AG--

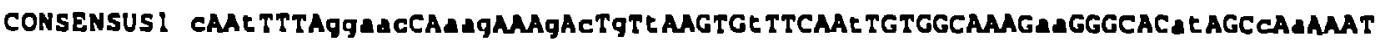
HIVLAI HIVHXB2R HIVMN HIVJH3 HIVJRCSF HIVOY I HIVSF: HIVNYSCG HIVNL 33 HIVCDCA HIVHAN II VRF CONSENSUS2 IIIVELI HIVZ226 IIIVNDK HIVHAL IIVUas5 s I verd

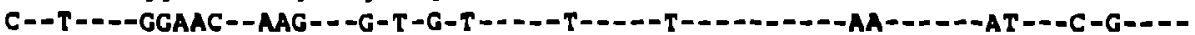

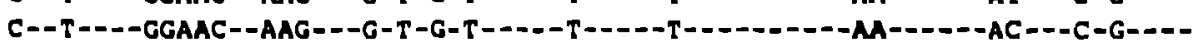

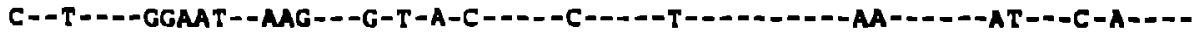

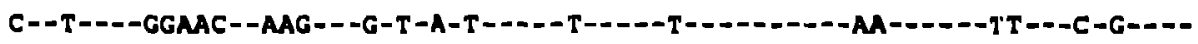

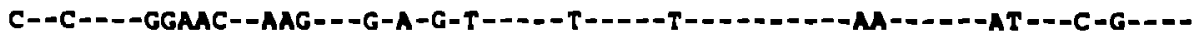

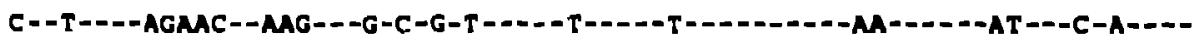

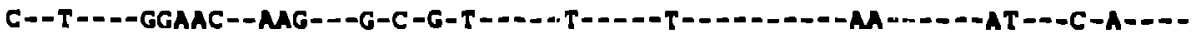

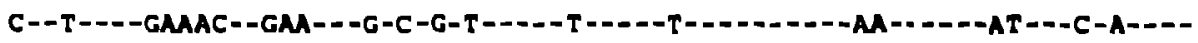

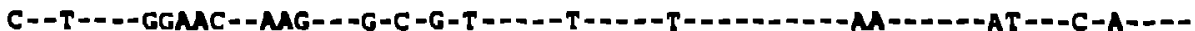

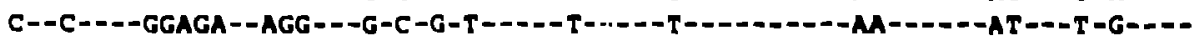

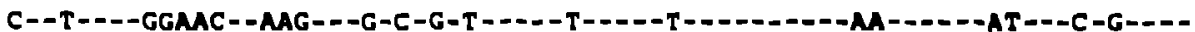

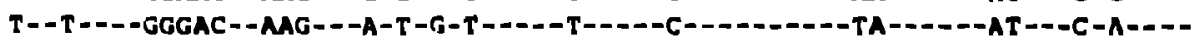


CONSENSUS1 TGCAgGGCCCCTAGGMAAAGGGCTGTTGGAATGTGGMAGGAGGACACCMATGMAGAT TGEACLg

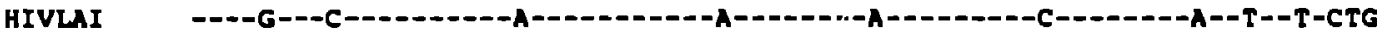
HIVHXB2R ----G---C---1-1--A-_-

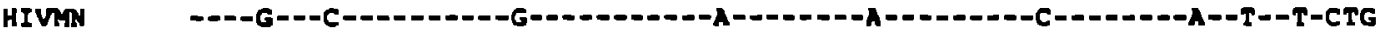
HIVJH3 HIVJRCSE HIVOYI HIVSF 2 HIVNYSCG HIVNLA3 HIVCDC4 HIVHAM HIVRT

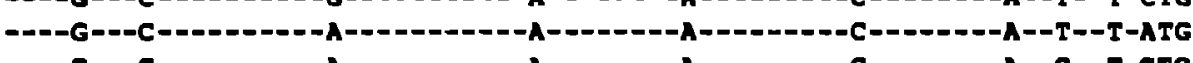

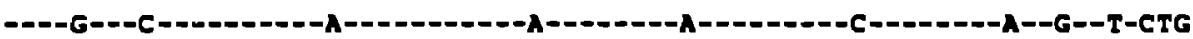

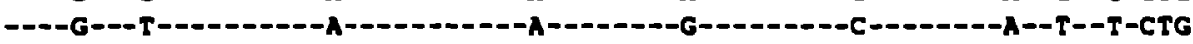

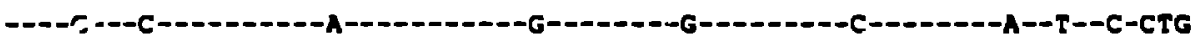

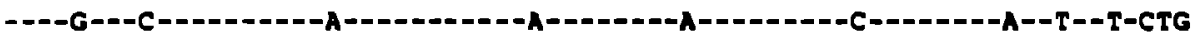

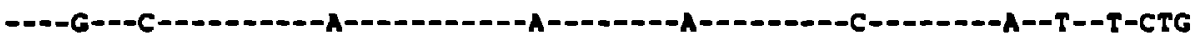

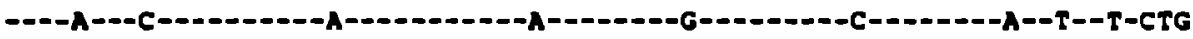

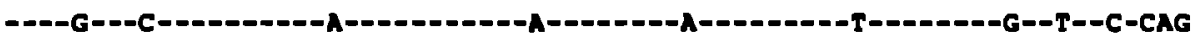

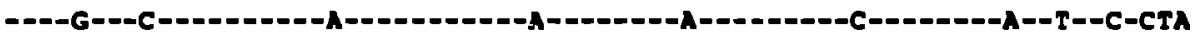
CONSENSUS2 TGCAGGGCCCCTAGgAANMGGGCTGTTGGAAATGEGAA GGMAGGACACCMATgMAGAE TGCACTG HIVELI HIVZ22 6 HIVNDK HIVIAL HIVU455 SIVCP2

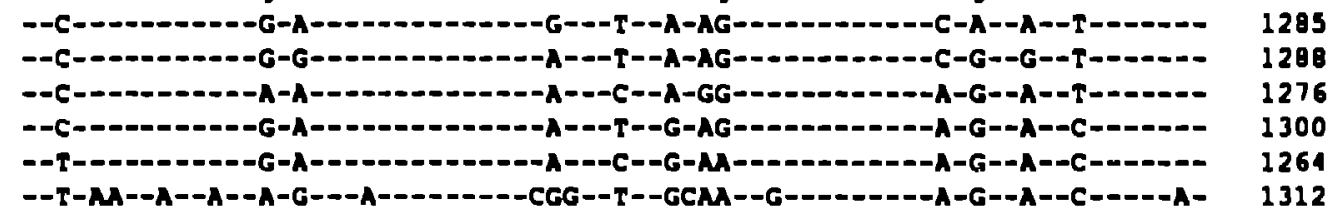

pol cds $\rightarrow$

CONSENSUSI Ag? ? ? ? AGACAGGCTMATTTTTAGGGMGATCTGGCCTTCCCACMGGGAAGGCGAGGGME ? ? ? HIVLAI HIVHXB2R HIVIN HIVJH3 HIVJRCSF HIVOYI HIVSF2 HIVNYSCG HIVNL43 HIVCDCA HIVHAN HIVRF

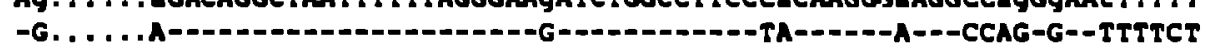

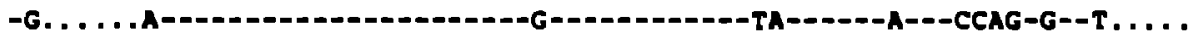

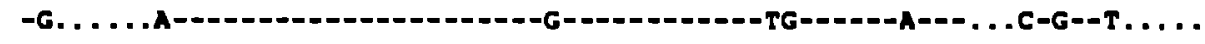

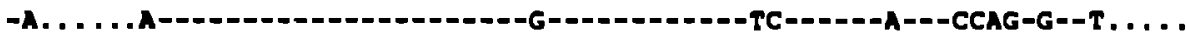
-G. . . . . A-1-1 -G......A--G. . . ...A-1...................

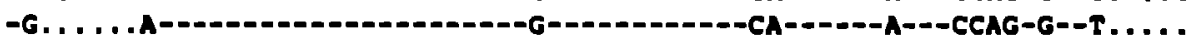
-G. . . . . A--.-. -G. . . . . A A-

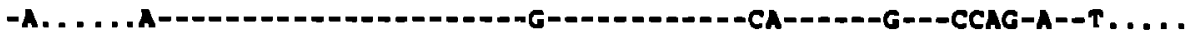

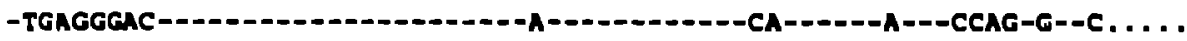
CONSENGUS2 Aq.......AGACAGGCTMATTTTTAGGGANATITGGCCTTCCCACMGGGAAGGCCgGGGAL.... . HIVELI HIV2226 HIVNDK HIVMAL HIVUA5S -G. . . . . . . -A. . . . . . -

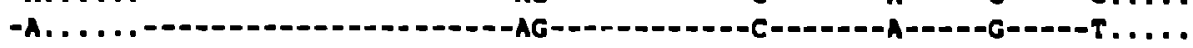
-G. . . . . . SIVCPZ

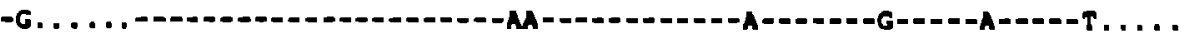

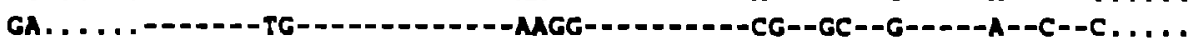

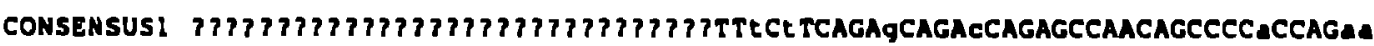
HIVLAI HIVHXB2R HIVHN

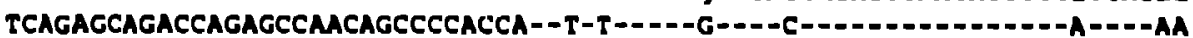

HIVJH3

HIVJACSF

IIIVOYI

HIVST2

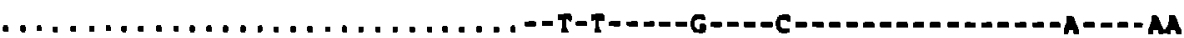

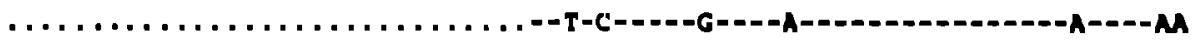

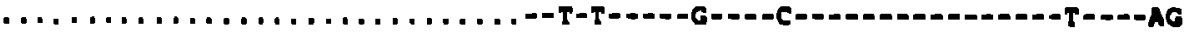
.......................

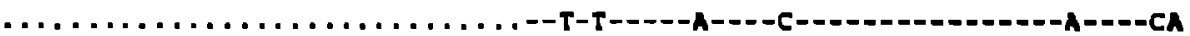

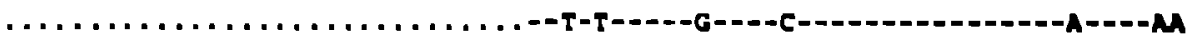

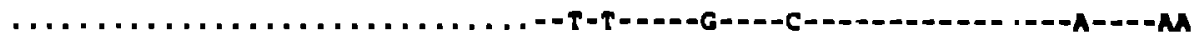

HIVNL4 3 HIVCDCA HIVHAN .........................

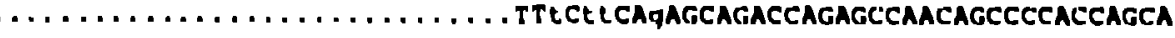


CONSENSUS1 GAGAGCTTCAGGTETGGGgaGAgaCAaCAACECCCLCTCAGAAGCAGGAG???????????CCGAEAG HIVLAI HIVHXB2R HIVMN

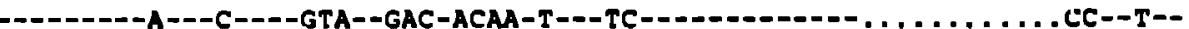

HIVJH3 -

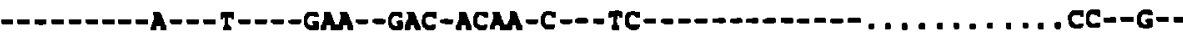

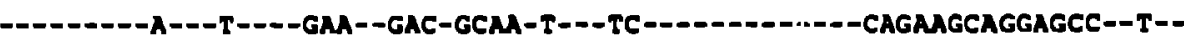

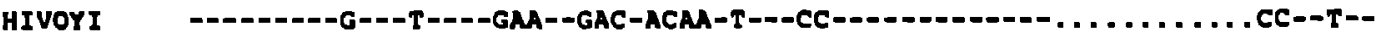

HIVSF2

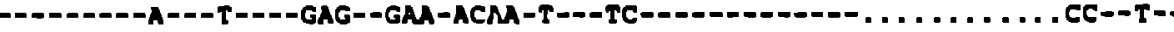

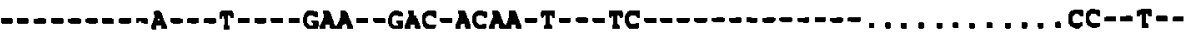

HIVNY5CG

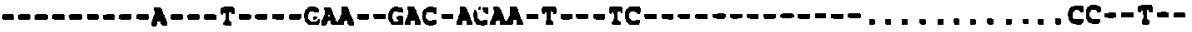

HIVNL \& 3

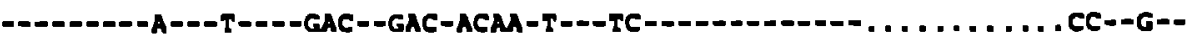

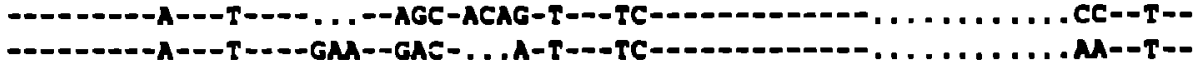

HIVHAN

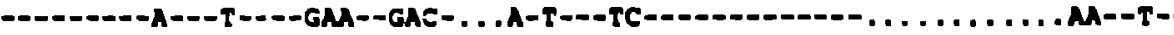

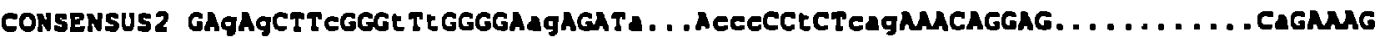

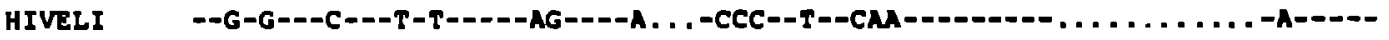
HIV2226 HIVNDK

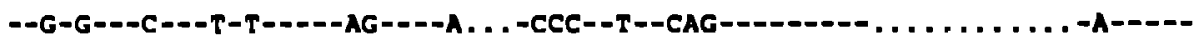

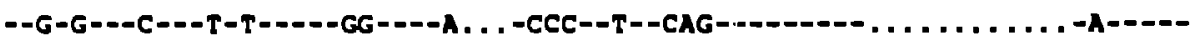
HIVML

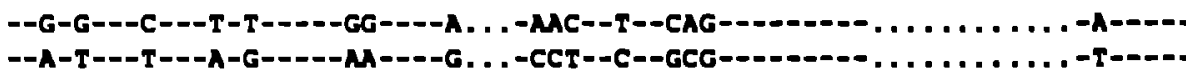

HIVUA53

SIVCPZ

--G-GT-AT---TACCA---GG-AGAG. . .-AGAG-CAGGAG--GA-A-.-..........GGA....

1435

1441

1436

1438

1441

1429

1453

1417

1462

CONSENSUS1 ACAMGGACTGTATCCETTAgCt TCCCTCAgaTCACTCTTTGGCAACGAZCCCT CGTCACA."*

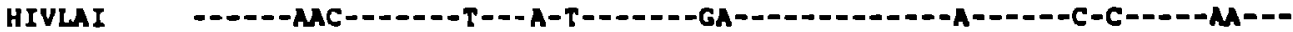

HIVHXB2R - -

HIVMN

HIVJH3

HIVJRCSF

HIVOYI

HIVST 2

HIVNYSCG

HIVNLA3

HIVCDCA

HIVHAN

-----ACC

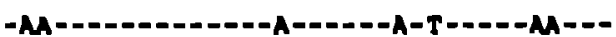

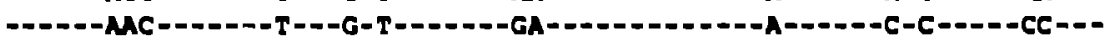

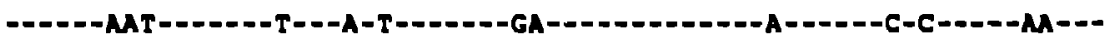

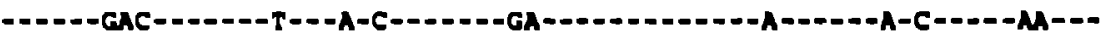

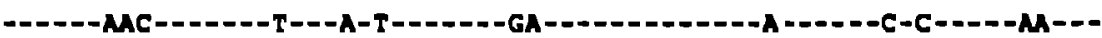

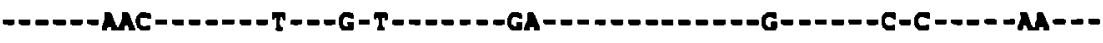

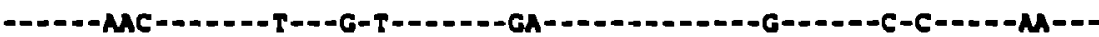

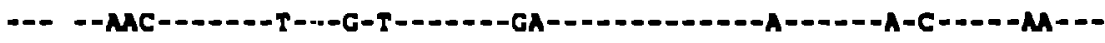

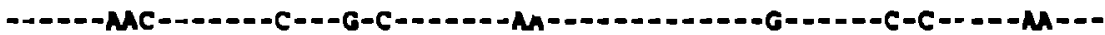

HIVRF

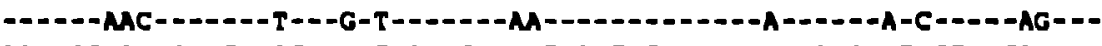

CONSENSUS2 ACAAGGACLGLATCCTTEAgCTECCCTCAMACACTCTTTGGCMACGACCCETEGTCACAA---

HIVELI

HIV2226

HIVNDK

HIVHAL

HIVUA55

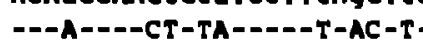

--A--_-CT-ta--2--C-AC-G--

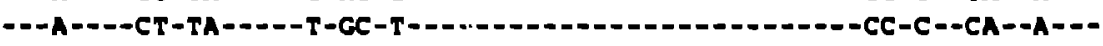

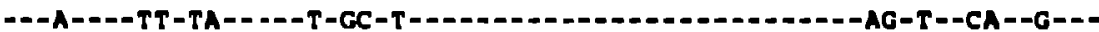

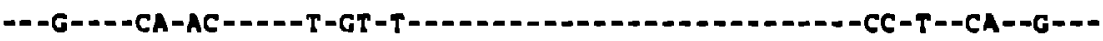

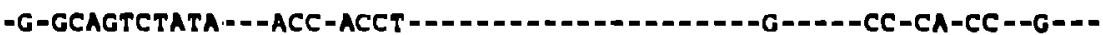

1539

1503

1521

1503

1515

1500

1509

1503

1503

1503

1500

1506

1501

2503

1506

1494

1518

1482

152\% 
gag $\rightarrow$

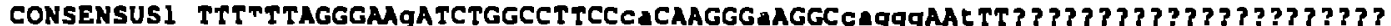
HIVIAT HIVHXB2R

HIVIN HIVJRCSF HIVOYI

HIVSF 2 HIVNYSCG HIVNLI HIVHAN hIVRF CONSENSUS2

HIVELI HIV2226 HIVNDK HIVMAL HIVUASS

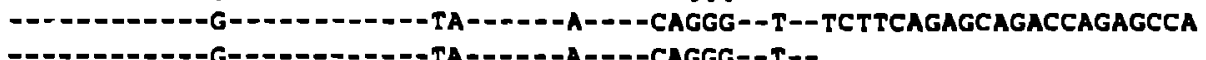
-

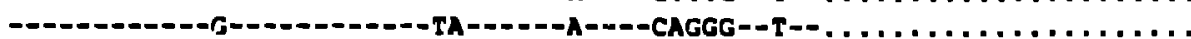

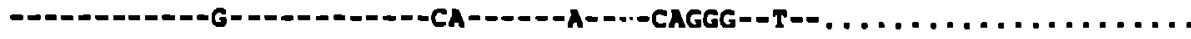
-

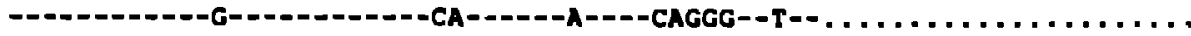
-

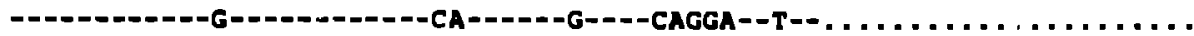

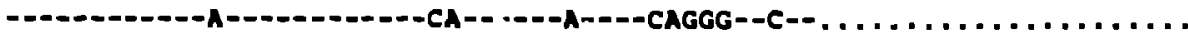

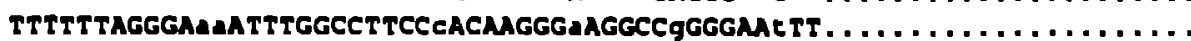
- -

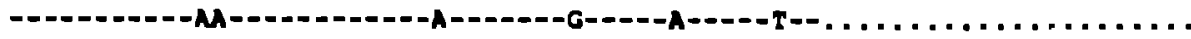
SIVCPz -AMGG-

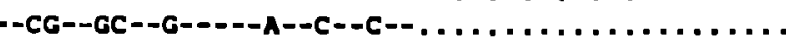

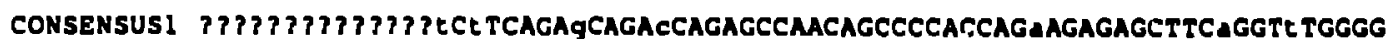
HIVLAI HIVHXB2R HIVHA

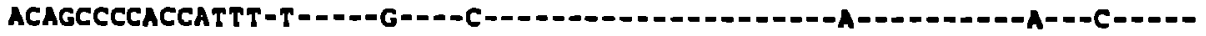

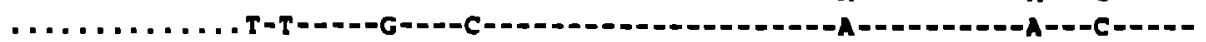

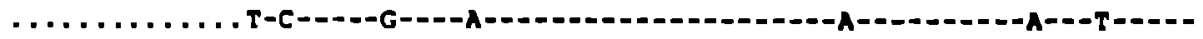

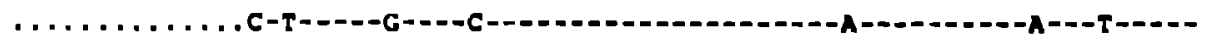

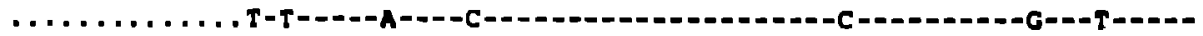

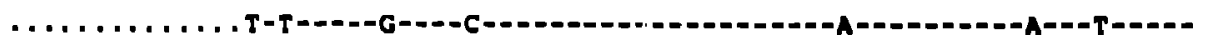

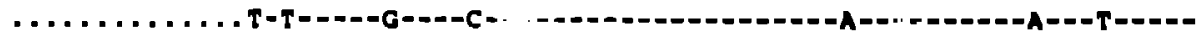

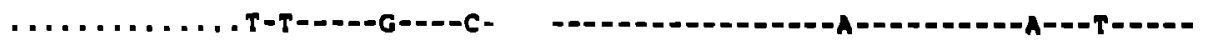

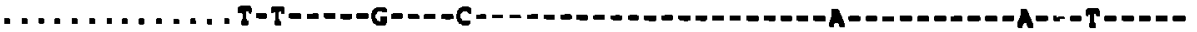

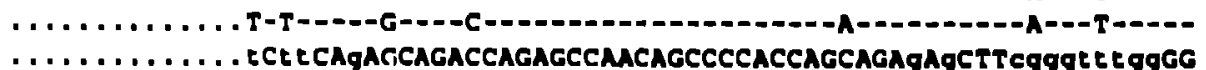
HIVOYI

HIVSF? HIVNYSC6 HIVNLA3 HIVHAN HIVRF $\begin{array}{lll}\text { Consengus2 } & \ldots \ldots \ldots \\ \text { HIVELI } & \ldots \ldots \ldots \ldots\end{array}$ HIVZ226 HIVNDK . . . . . . . . . T-TT--G-_._.-.

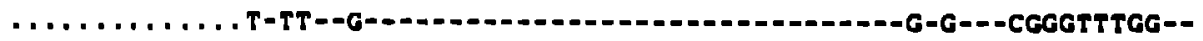

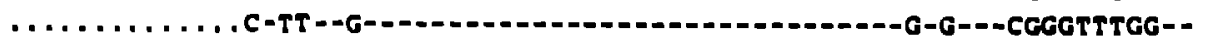
HIVMAL HIVU4Ss

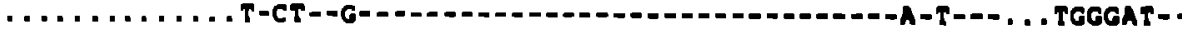
SIVCPz

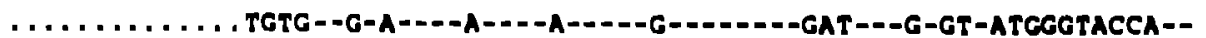

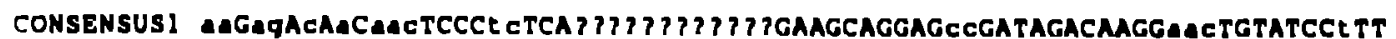

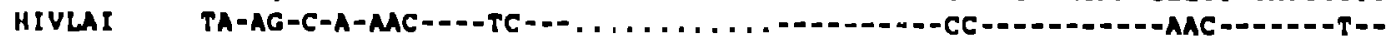

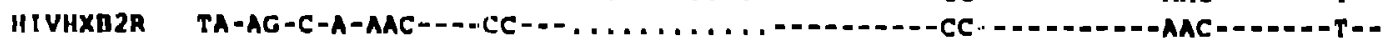
HIVMN IIIVJRCSF HIVOY!

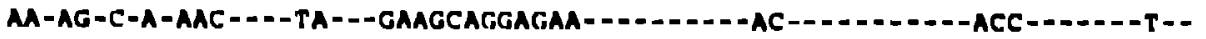

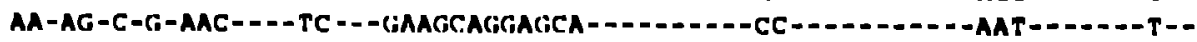

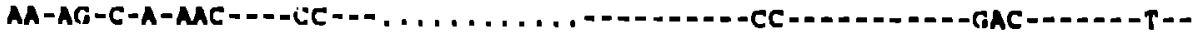
AG-AG-A-A-AAC---TC..- . . . . . . . . Hivst 2 HIVNYSCG IIVNL4J HIVHAK М-AG-C-A-AAC-D-TC--., , . . . .

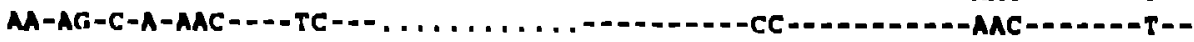

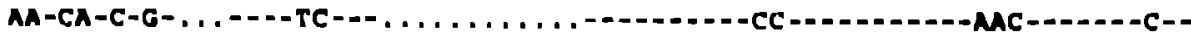

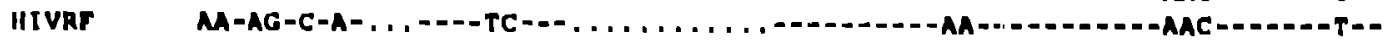

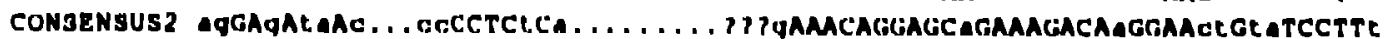
HIVELI IIIVZ226 HIVNI)K IIIVML.

IIIVU45s

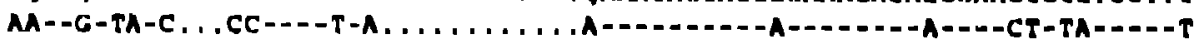

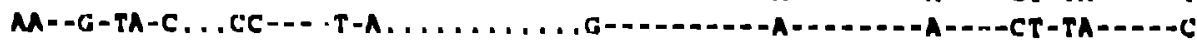

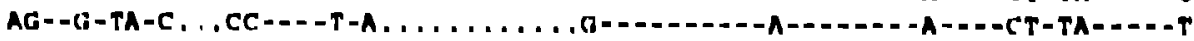

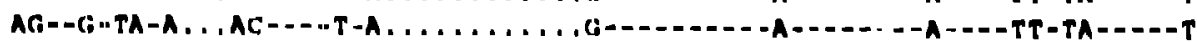

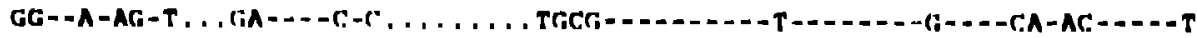
s I ve.lin

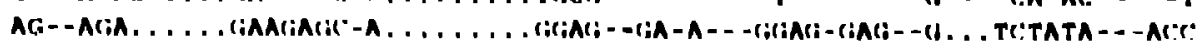


HIV-1 POL

$\checkmark$ proteage <- yag cds

CONSENSUSI AgCETCCCTCAgaTCACTCTTTGGCAaCGACCCCTCGTCACAaTAAaGATAGGGGGGCAACTAAAgGAaG HIVLAI -A-T-----GA--

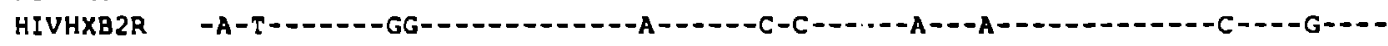

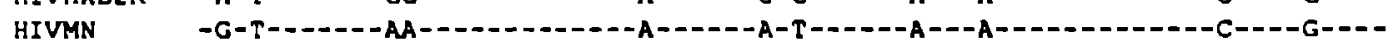

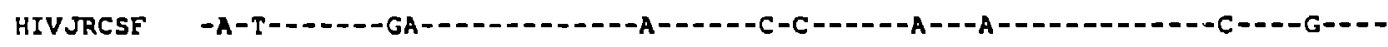

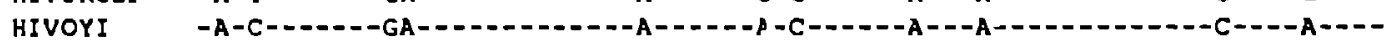

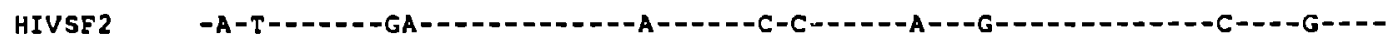

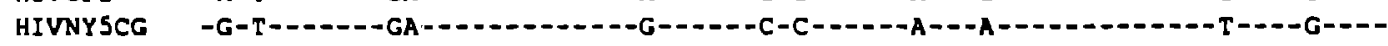

HIVNL43 -G-T-D--GA-- 3 -

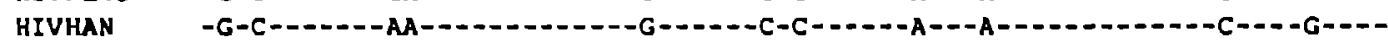

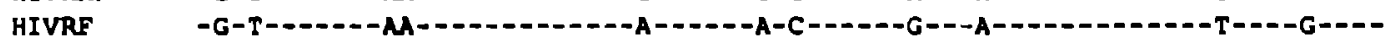

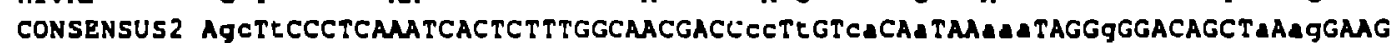

HIVELI -AC-T-.--

HIV2226

HIVNDK

HIVMAL

HIVU4S5

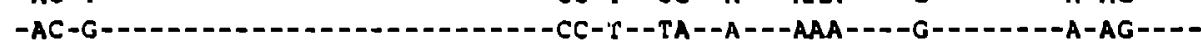

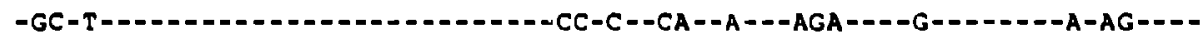

-GC-Y-1-10

SIVCP 2

-GT-T -

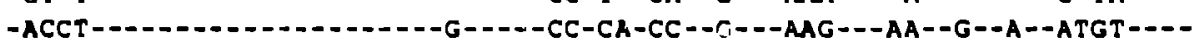

CONSENSUS I CTCTATTAGATACAGGAGCAGATGATACAGTALTAG $2 A G A \_A T G \perp$ TTTGCCAgGAAgATGGAAACCAAA HIVLAI HIVHXB2R

HIVMN HIVJRCSF

HIVOY I

HIVSF' 2

HIVNYSCG

HIVNL43

HIVHAN

HIVRF

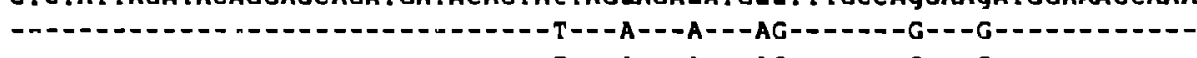

CONSENSUS2

HIVELI

HIV 2.226

HIVNDK

HIVMAL

HIVUASS

-

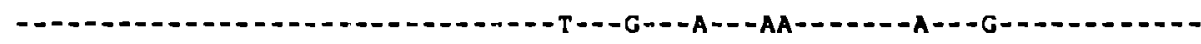

-

-

-

-

-

-

- -

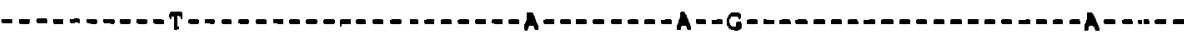
SIVCPZ

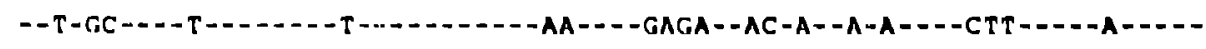

CONSENSUS] AATGATAGGQGGATTGGAGGTTTTATCAMAGTAAGACAGTALGATCAGATACt CATAGAAATCTGLGGA IIVLAI IIIVHXB2K HIVMN HIVJACSF HIVOY! MIVSE2 HIVNYSCG HIVNI,A] IIIVIIAN HIVRT -

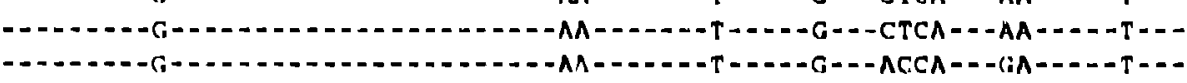
-

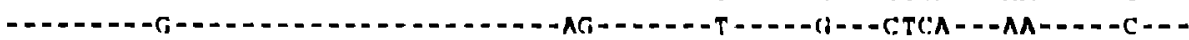
HIVELL IIIVIZZG HIVNIIX HIVMAL. IIIVIISs

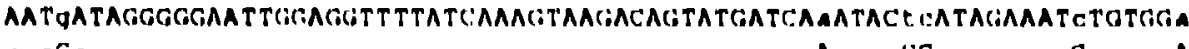

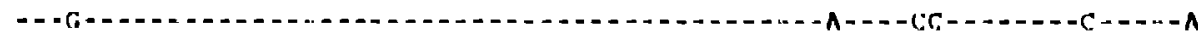

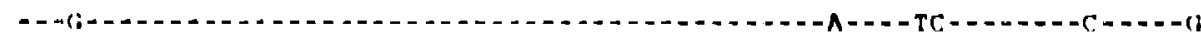

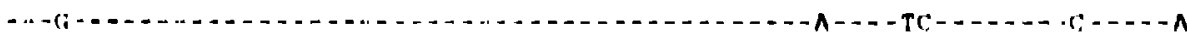

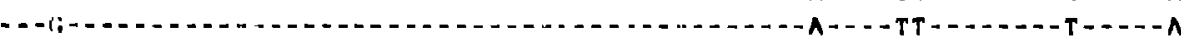
- - - 
CONSENSUS 1 CATAAGCTaTAGGTACAGTATTAGTAGGACCTACACCTGTCAACATAATTGGMGAATCTGTTGACTC HIVLAI HIVHXB2R

HIVHN HIVJRCSF

HIVOYI

HIVSF 2

HIVNYSCG

HIVNL43

HIVHAN

HIVRF

CONSENSUS 2

HIVELI

HJV7.226

HIVNDK

CATMAC

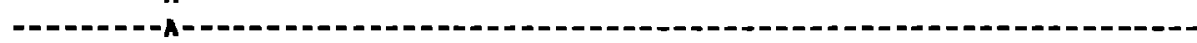

-

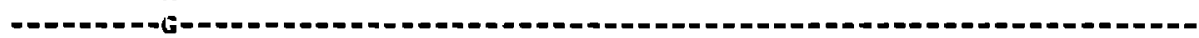

-

- -

- -

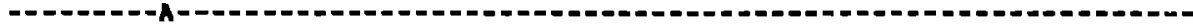

-

-

HIVMAL

HIVU455

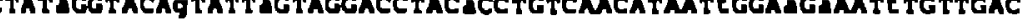

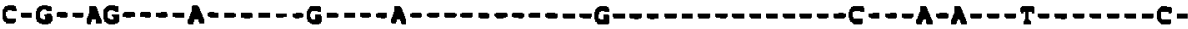

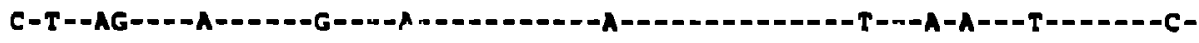

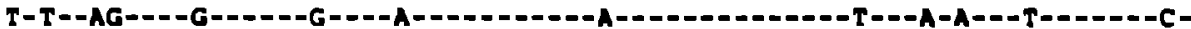
SIVCPZ

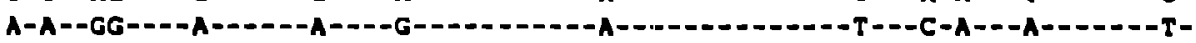

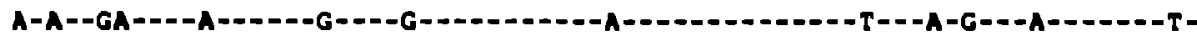

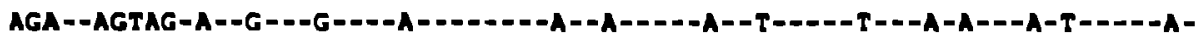

protease \/ p66, p51

CONSENSUS I AGATTGGETGCACTTTMATTTECCCATTAGECCTATTGAAACTGTACCAGT AMATTANAGCCAGGMT

HIVLAI

HIVHXB2R

HIVMN

HIVJRCSF

HIVOYI

HIVSF?

HIVNYSCG

HIVNL43

HIVHAN

HIVRT

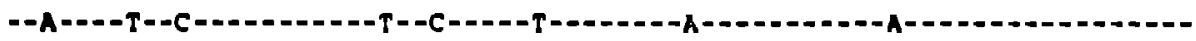

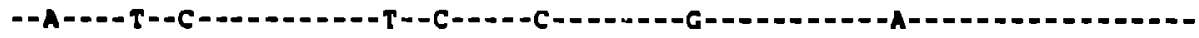

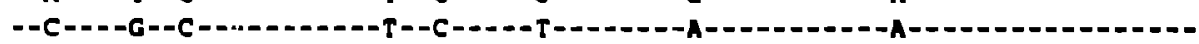

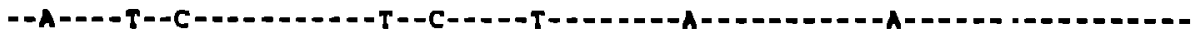

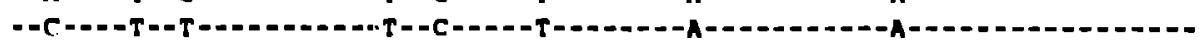

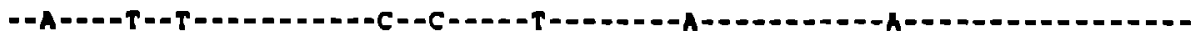

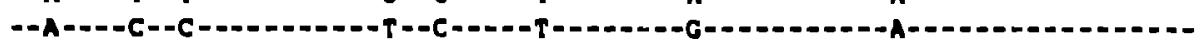

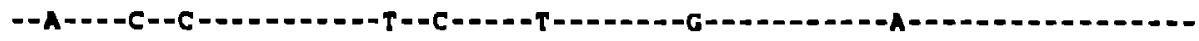

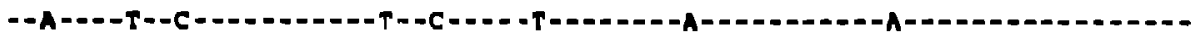

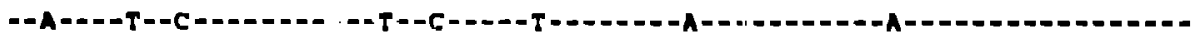

CONSENSUS2 AGATTGGCTGCACTTTALATTTTCCMATTAGTCCTATTGAAATGTACCAGTMAMTTMAgCCAGgaAT

HIVELI

HiVZ226

HIVNDK

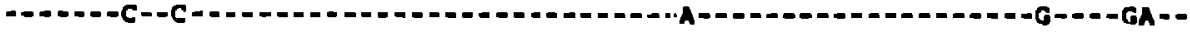

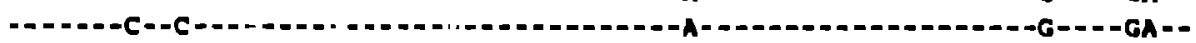

- -

-

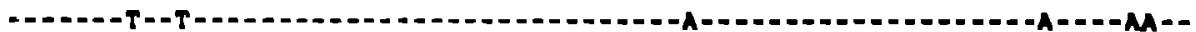

ilverass

SIVCP 2

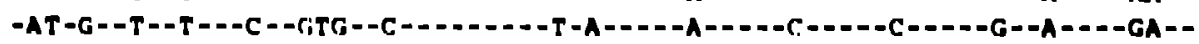

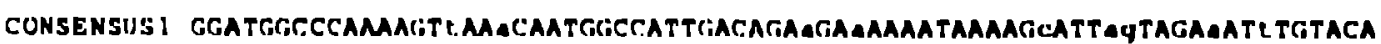

IITVIAI

IIIVIXIBJH

IIIVMN

IIIV.JRCSF

IIIVort

HIVsrz

HIVHYSCE

IIIVI.4]

IIIVHAN

HIVAR

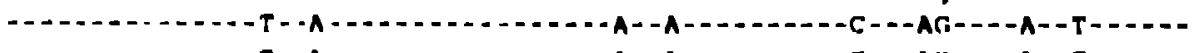

-

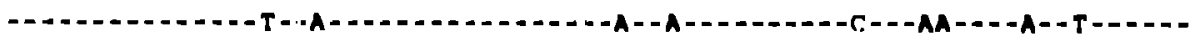

-.

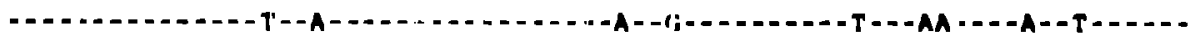

- - -

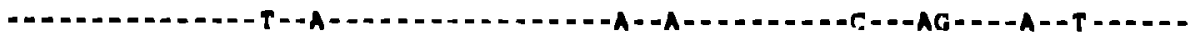

- = -

-

- -

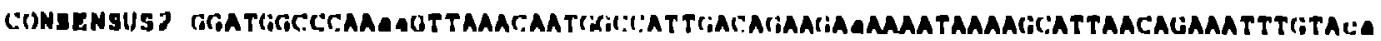

IIIVri.1

HIV7.226

IIIVNI)K

IIIVMaL.

IIIVI) 53

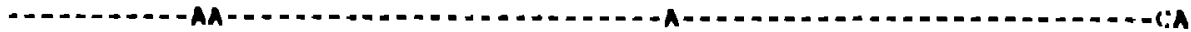

-

-.

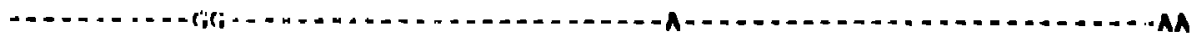

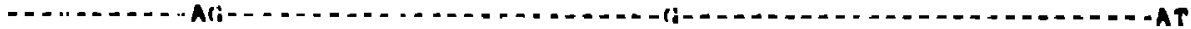

הIVIV 2$$
\text { -...... - T- - A }
$$

AT

512

548

512

521

524

512

512

512

512

509

509

307

509

509

509

509

509

506

582

6.8

58.1

391

594

अ 1 2

582

582

$30 \%$

319

319

311

519

310

319

318

319

i/s 
HIV-1 POL

COHSENSUSI GAaATGGAAAGGAGGgAAaATTCANAAATGGGCCTGMAATCCATACAATACTCCAGTATTTGCCA

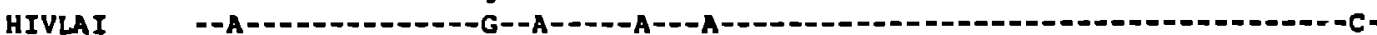

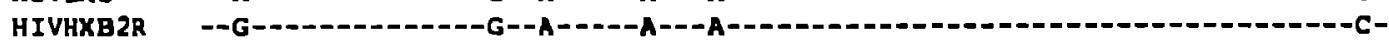

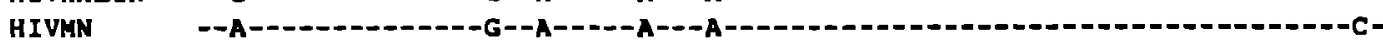

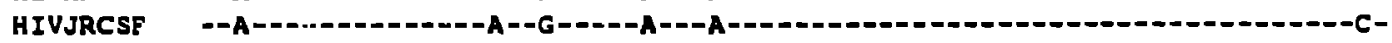

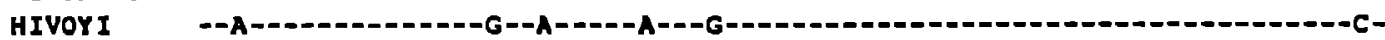

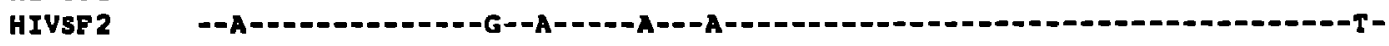

HIVNYSCG --A--1-1

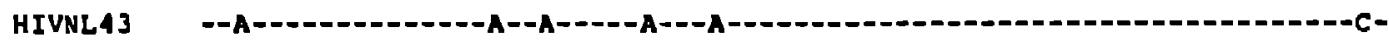

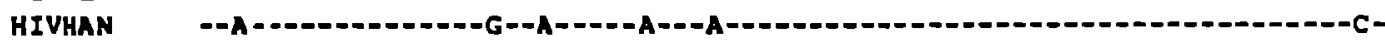

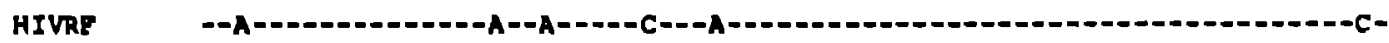

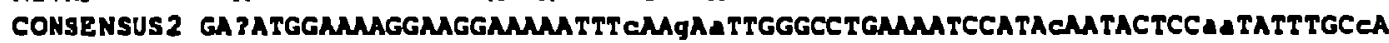

HIVELI -T-T--

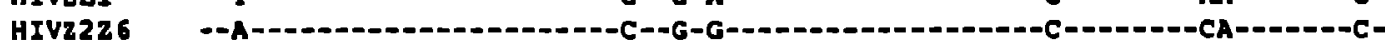

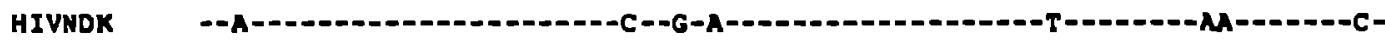

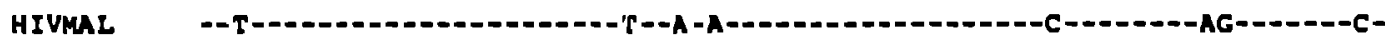

HIVU455 -G-G...

SIVCP Z -

CONSENSUS I TAAAGAMAAAGACAGTACTAAATGGAGAAALTAGTAGATTTCAGAGACTTMATAAgAqAACTCAAGA

HIVLAI

HIVHXB2R

HIVMN

HIVJRCSF

HIVOYI

HIVSF 2

HIVNY 5CG

HIVNLA3

HIVHAN

HIVRF

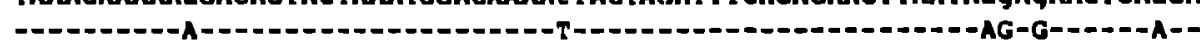

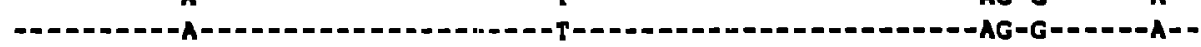

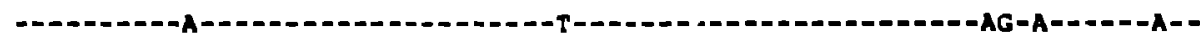

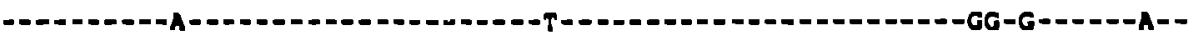

-

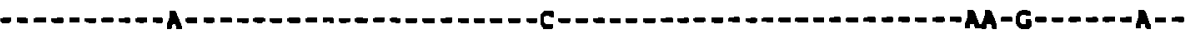

-

-

- - -

-

CONSENSUS 2 TMAGMAMAGACAGEACCMAgTGGAGAAMATTAGTAgATTTCAGaGAACTEMATAAgAGACTCAAGA

HIVELI

HIVZ226

HIVNDK

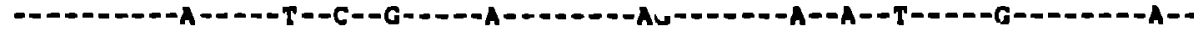

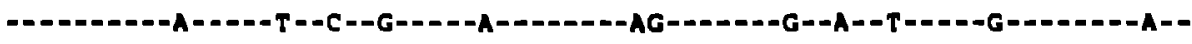

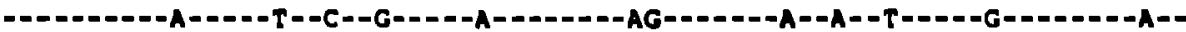

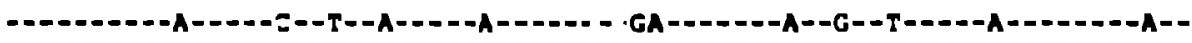

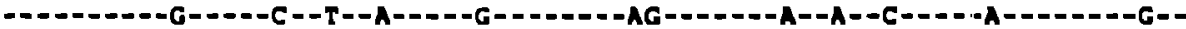

HIVu45s

SIVCP Z

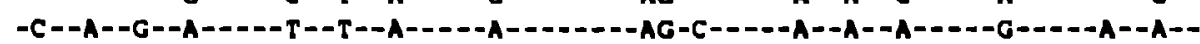

:ONSENSUS 1 CTTCTGGGAAGTECAATTAGGAATACCACAECC ?GCAGGGTTAAMAARAAAAAAATCAGTAACAGTACTG

MISLA I I

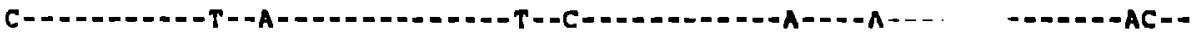

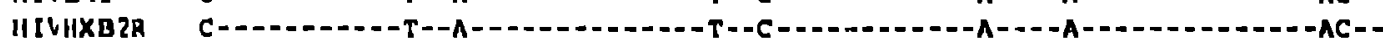

MIVMN

IIIVJHCSF

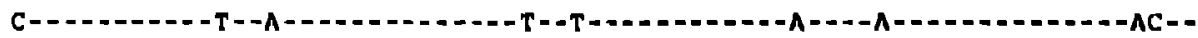

IIIVOYI

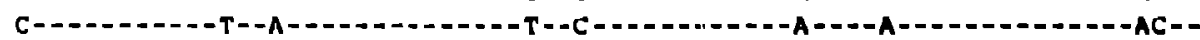

IIIVSF 2

HIVHYSCG

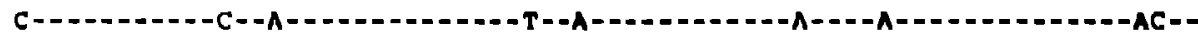

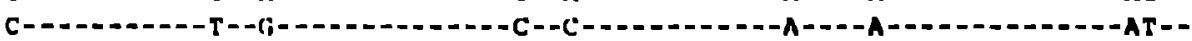

HIVNLA]

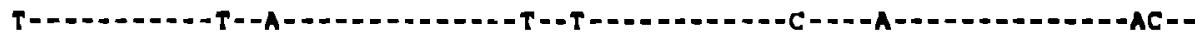

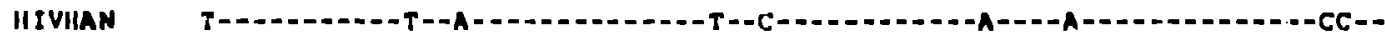

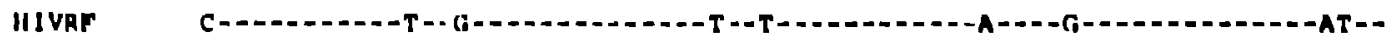

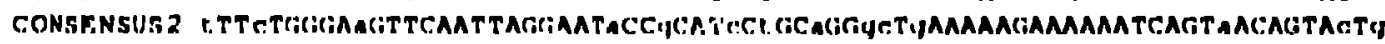

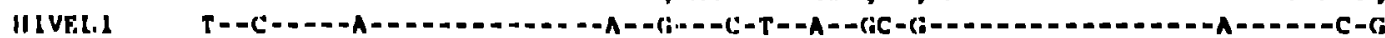

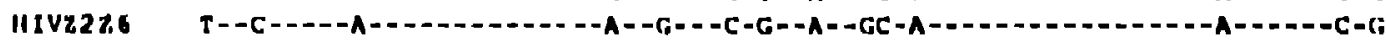

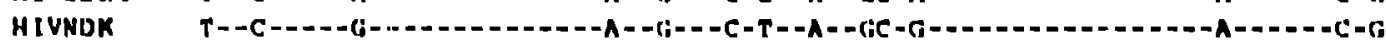

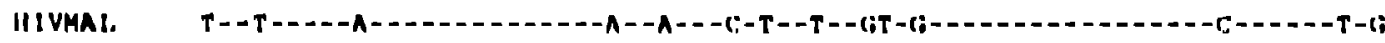

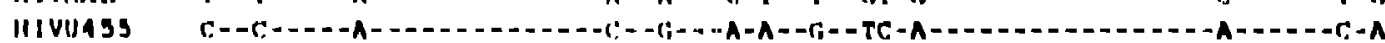

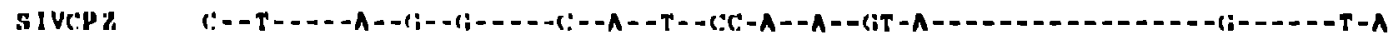


CONSENSUS I GATGTGGGLGATGCATALTTTTCAGTTCCCLTAGATAAAGACTTCAGgAAgTATACTGCATTTACCATAC HIVLAI - - -

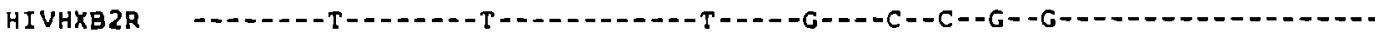
HIVMN HIVRRCSE HIVOYI HIVSF2 HIVNYSCG HIVNL4 3 HIVHAN HIVRF

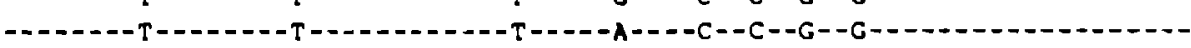

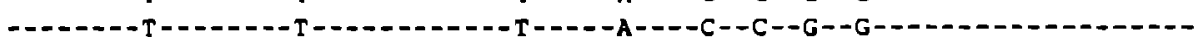

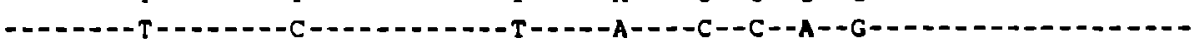
CONSENSUS2 GATGTGGGTGALGCATATTTL TCAGTECCCTTAGATgAMgatTTEAGgAaATATACEGCATTCACCATAC

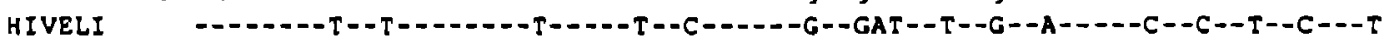
HIV222 6 HIVNDK HTVMAL HIVU 455 SIVCP 2

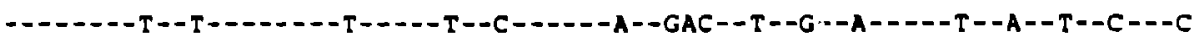

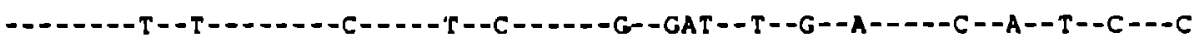

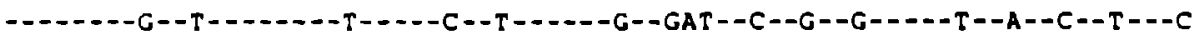

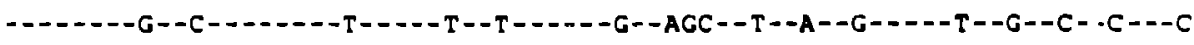
$----A--A--T--C--C--C--T T G T--C C-G=--A--G A T--C--G--A---C-T--A--T--A--T C$

CONSENSUSI CTAGTAEAAACAATGAgACACCAgGGATTAGATATCAGTACAATGTCCTECCACAgGGaTGGAAGGATC HIVLAI HIVHXB2R HIVMN HIVJRCSE HIVOYI HIVSE 2 HIVNY 5 CG HIYNL4 3 HIVHAN HIVRF

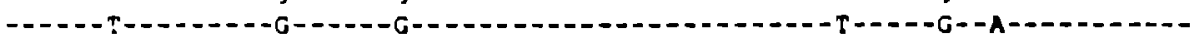

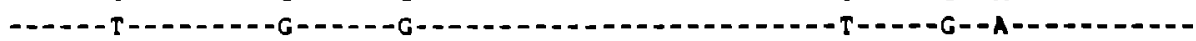

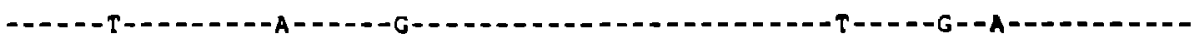

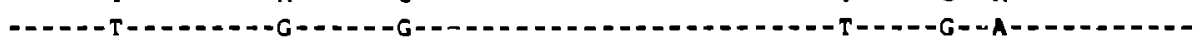

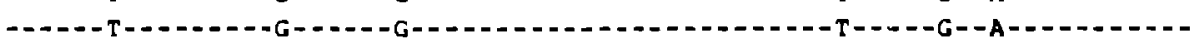

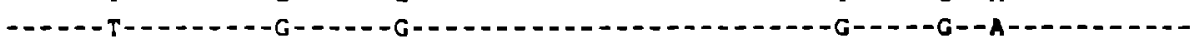

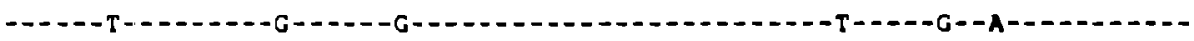

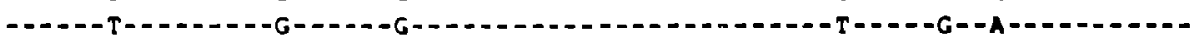

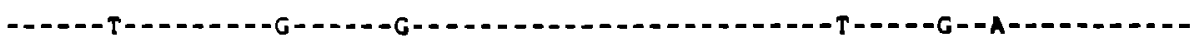

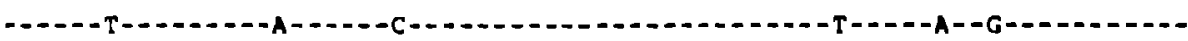

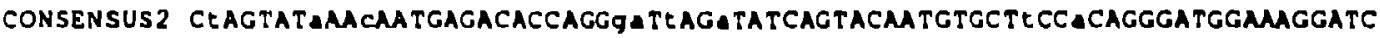
HIVELI HIVZ2Z6 H I VNDK HIVMAL HIVUA5 5 S I VCPz -T-- - -A- -C - - - - - - - - - -GA-T - -A- - - - - - -

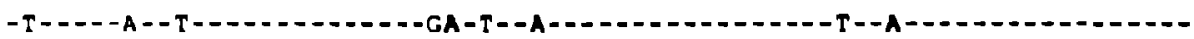

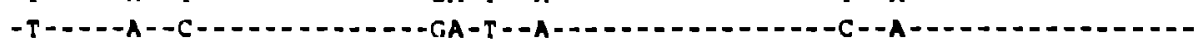

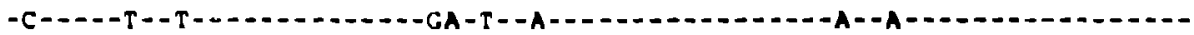

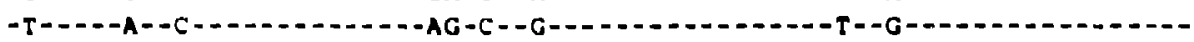

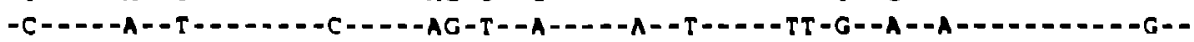

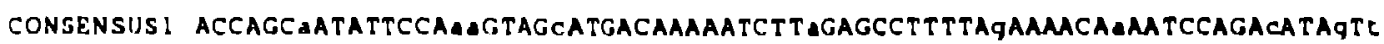
IIIVIAI IIIVHXB2R II IVAN IIIVJHCSF IIIVOY! IIIVSF 2 IIIUNYSCG II [VNLA] IIIVHAN II I VHF CONSENSUS HIVELI II 1 V.27.6 II I VNDK IIIVMAL. III Vu45s 5! VC'PZ

1001 1030 1002 1011 1014 1002 1002 1002 1002 999 999 796 999 299 999 979 499 276 
CONSENSUSI ATCTA2CAA TACATGGATGATT TGTATGTAGGATCTGACTTAGAAATAGgGCAGCATAGAaCAAAAATAG HIVI.AI HIVHXB2R

HIVMN HIVJRCSF

HIVOYI

HIVSE 2

HIVNY5CG

HIVNL43

HIVHAM

HIVRF

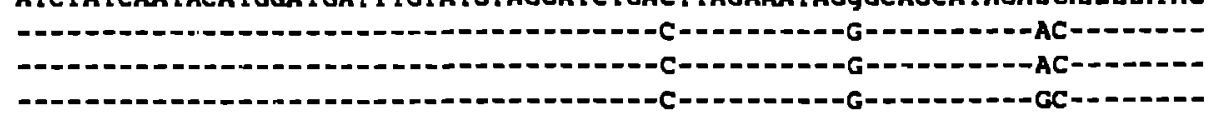

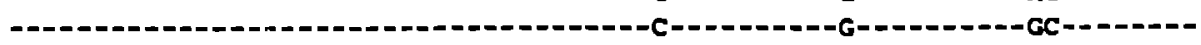

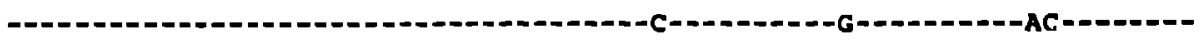

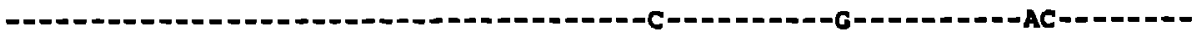

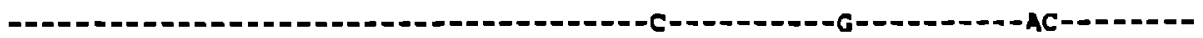

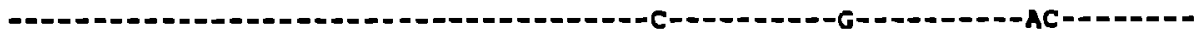

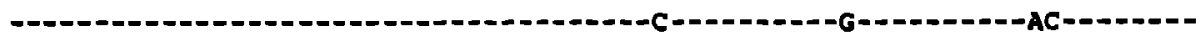

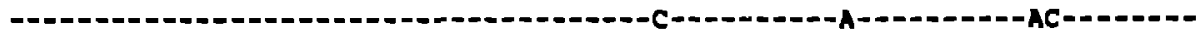

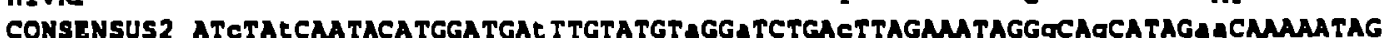
HIVELI HIVZ2z6 HIVNDK HIVMAL HIVU455 ATCTAECNATACATGGA TGAT TTGTATGTAGGaTCTGACTTAGMMTAGGgCAgCATAGA CNAAATAG

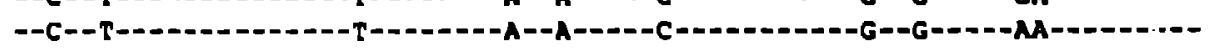

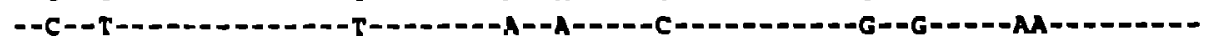

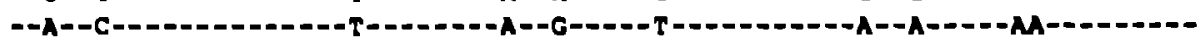
SIVCP2

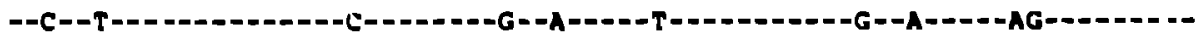

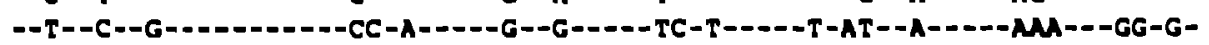

CONSENSUS1 AGGAaCTGAGACA CATCTGTTgAgGTGGGGatTTACCACACCAGACAAANACATCAGMAGMCCTCC HIVLAI HIVHXB2R HIVMN HIVJRCSF HIVOY I HIVSF? HIVNY5CG HIVNLA 3 HIVHAN HIVRF

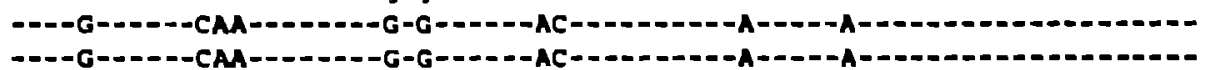

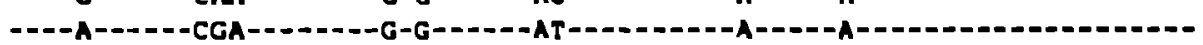

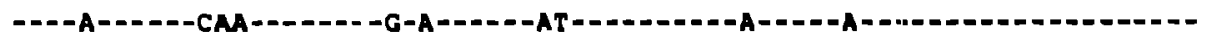

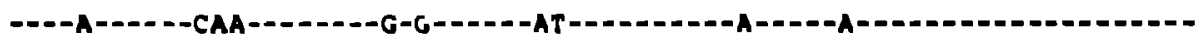

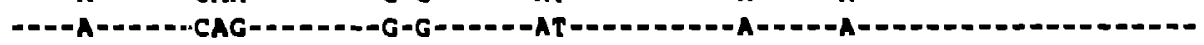

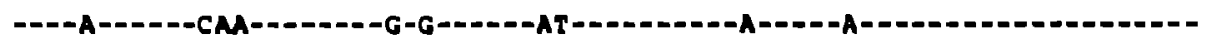

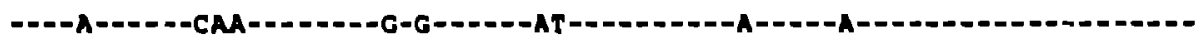

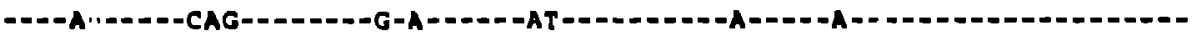

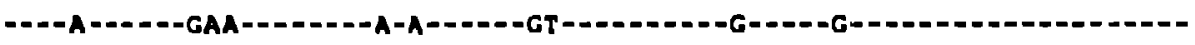
2066

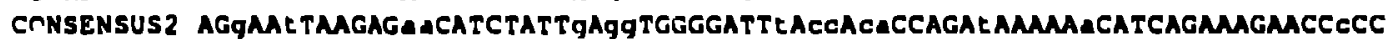

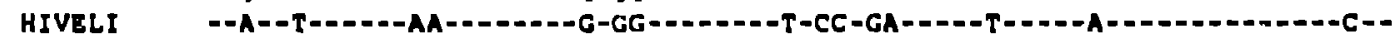

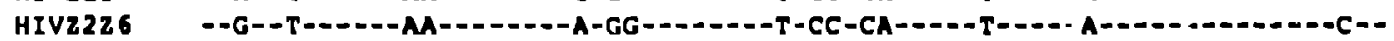
HIVNDK HIVMAL HIVU455

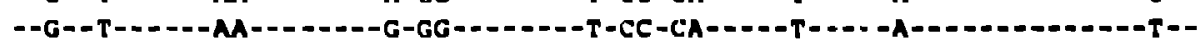

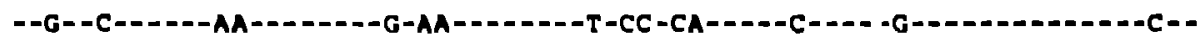
SIVCPZ

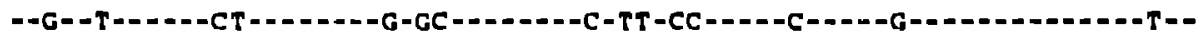

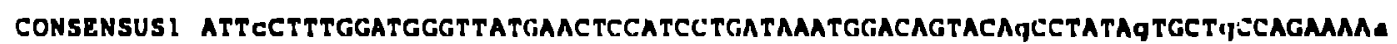
HIVLAI IIIVIIXI'?R IIIVMN IIIVJRCEP IIIVOY! HIVSE 2 MIVNY SCr II IVNLA] IIIVIIAN HIVRF AтTсСтTТССА

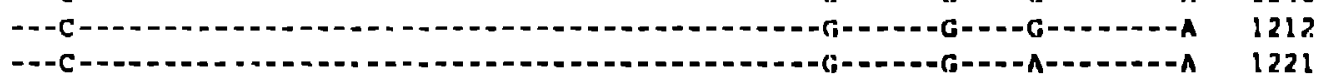

- - C -

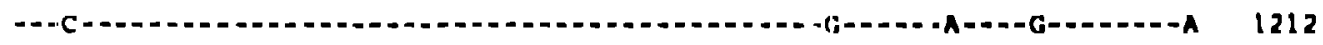

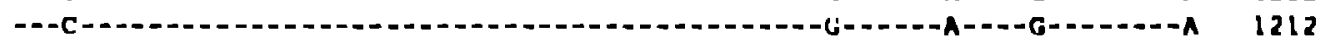

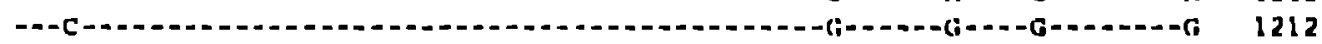

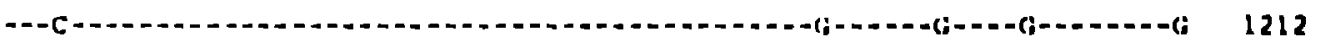

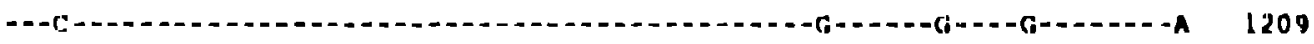

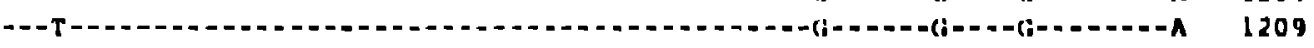

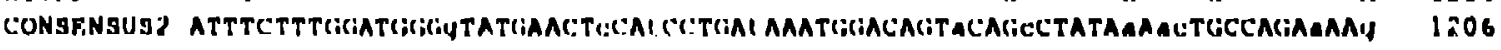
IIVEL? IIIV7225 IIIVNDK IIIVMAL. IIIVU4.5 AтT 1209

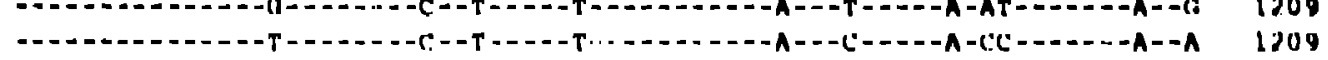
H..._. i I Vil'

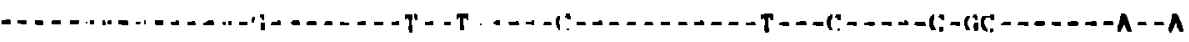

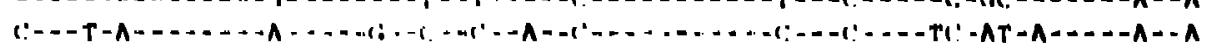

1209 
CONSENSUS1 GACAGCTGGACTGTCAATGATATACAGAAgTTAGTGGGaAAAT TAATTGgGCAAGTCAgATE TALgCAG HIVLAI

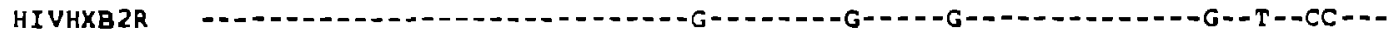

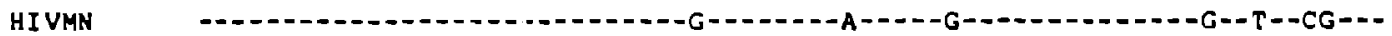
HIVJRCSF - - HIVOYI HIVSF 2 HIVNYSCG HIVNL4 3 HIVHAN HIVRF

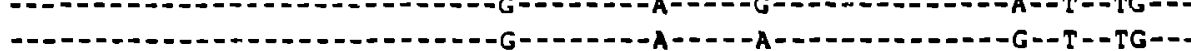

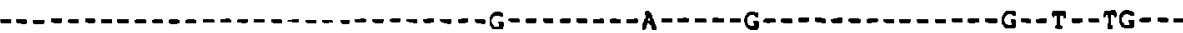
-

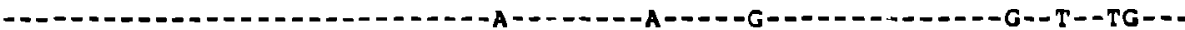
- - - - - - - -

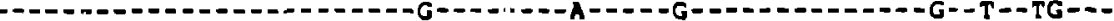
CONSENSUS2 GA?AGCTGGACTGTCAATGATATACAGAATTAGTgGggAaA TAMATGgGCAAGcCAGATtTATCCAG HIVELI HIV2226 HIVNDK HIVMAL HIVUASS SIVCP 2 - G-

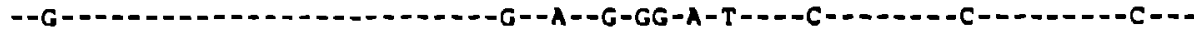

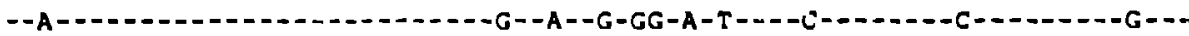

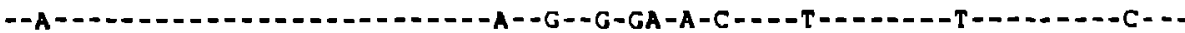

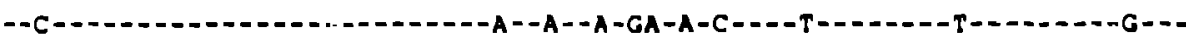

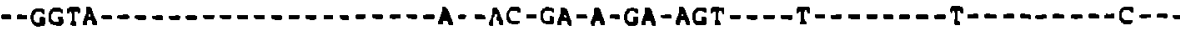

1281

1310

1282

1291

1294

1282

1282

1282

1282

1279

1279

1274

1279

1279

1279

1279

1279

1276

CONSENSUS] GGATTAAAGTAMAGCAATTATGTAAACTCCTTAGgGGA CCAAAGCACTAACAGAAGTA TACCACTAAC HIVLII HIVHXB2R HIVMN HIVJRCSF HIVOYI HIVSF 2 HIVNYSCG HIVNL43 HIVHAN HIVRF CONSENSUS2 HIVELI HIVZ22 6 HIVNDK HIVMAL HIVUA35 SIVCPZ -

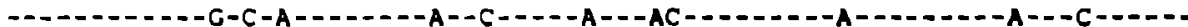

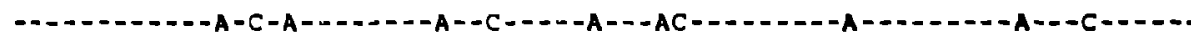
-

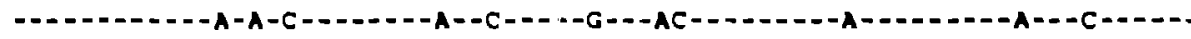

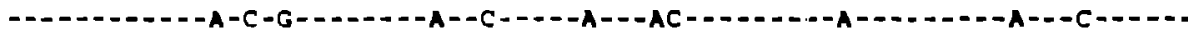

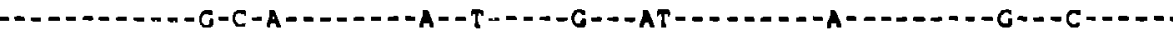

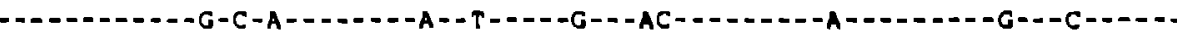

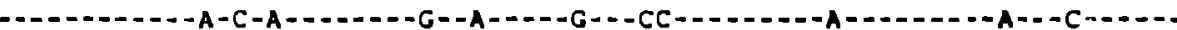

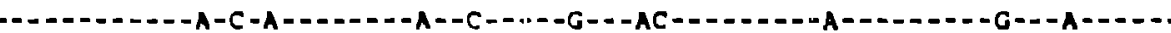

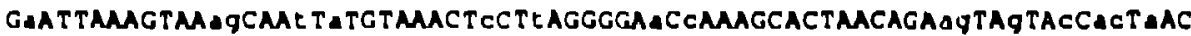

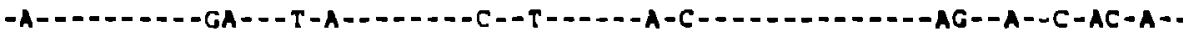

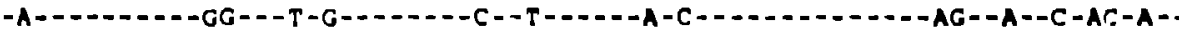

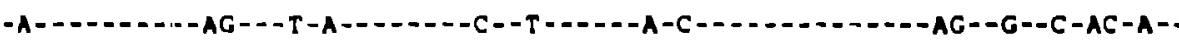

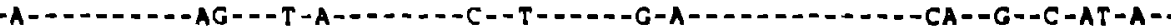

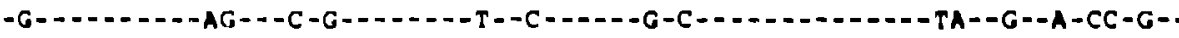

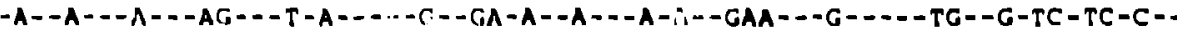

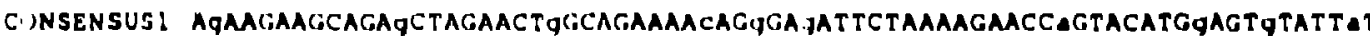
IIIVIAI HIVHXB2R HIVHN IIIVJHCSF HIVOYI IIIVSF 2 IIIVNYSCG II [VNLA] II! VIIAN HIVRF CONBENSUE? MIVRLI IIIVI.22,6 IIIVNUK IIVMAL II VIISs I เ

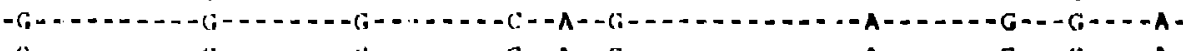
- ;

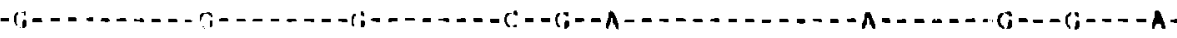

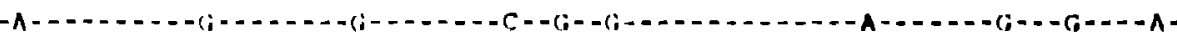

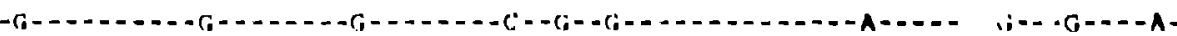
-G- - -...-- -

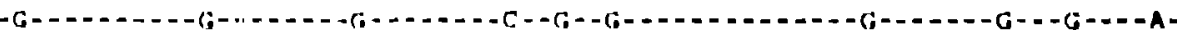

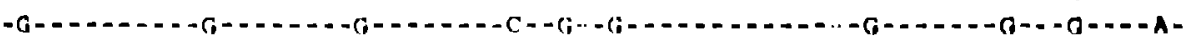

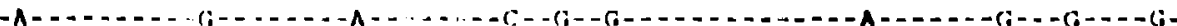

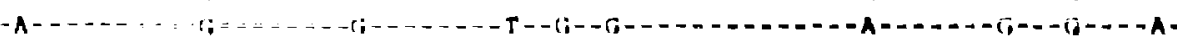

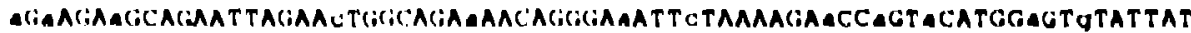

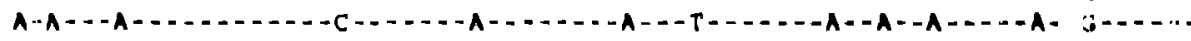

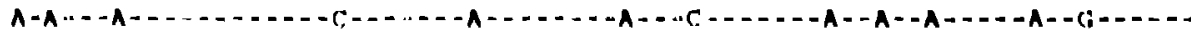

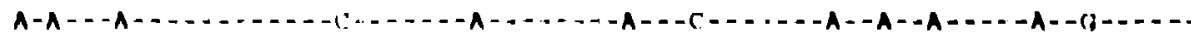

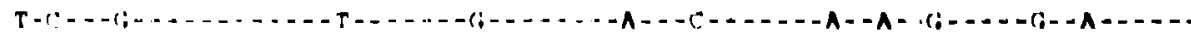

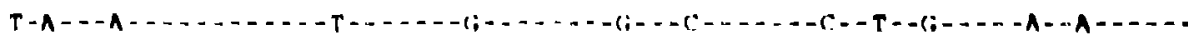

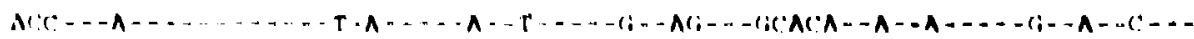


HIV-1 POI.

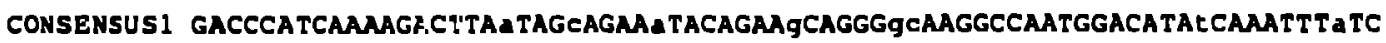
HIVLAI - -

HIVHXB2R -

HIVMN -

HIVJRCSE

-

HIVOYI

HIVSF 2

HIVNYSCG

HIVNL43

-1
-1

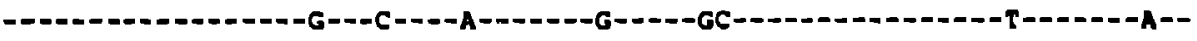

-

HIVHAN

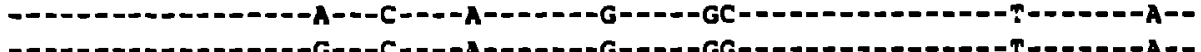

HIVRF

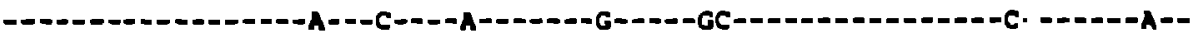

CONSENSUS2 GACCCATCAMMGACETAATAGCAGMATACAGMACAAGGGCACGgCCATGGACATACCMATt TAEC

HIVELI

HIVZ226

HIVNDK

-

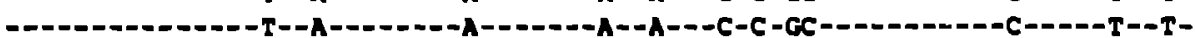

HIVMAL

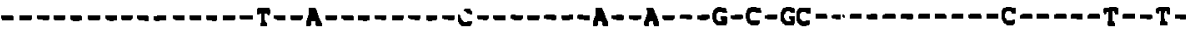

HIVUASS -

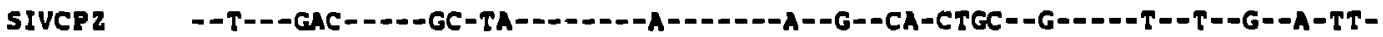

CONSENSUSI MAGAgCCATTTMAAALCTGAAACAGGAMgTATGCAMgAEGAgGGGTCCCCACACTAMTGATgTAM

HIVLAI

HIVHXB2R

HIVMN

HIVJRCSE

H:VOYI

HIVSF 2

HIVNLAJ

HIVHAN

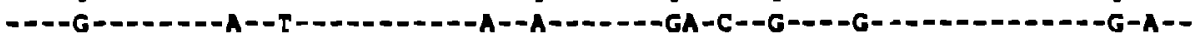

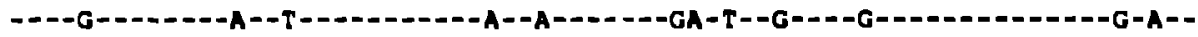

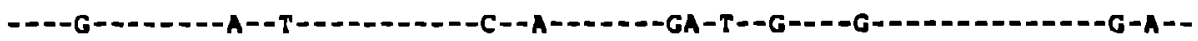

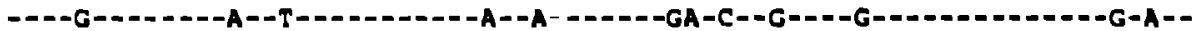

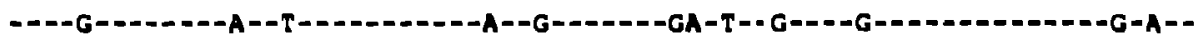

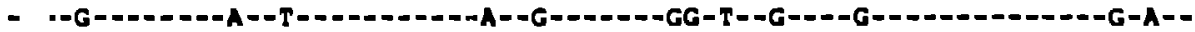

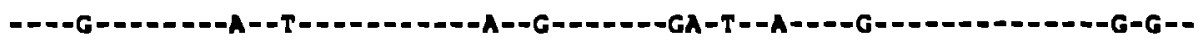

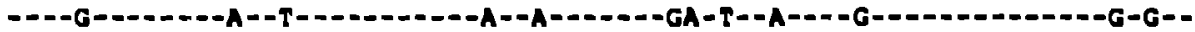

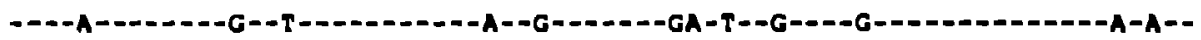

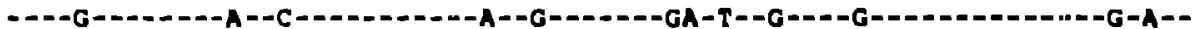

HIVRF

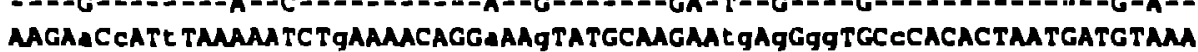

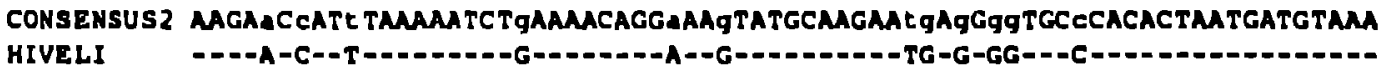
HIVZ226 HIVNDK HIVMAL HIVU455

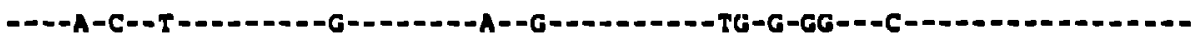

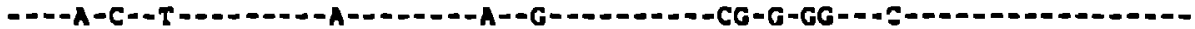

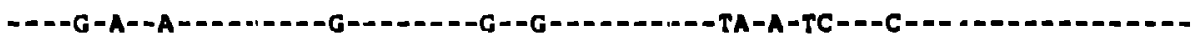

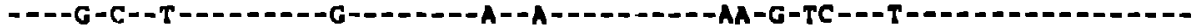
SIVCP2
HIVNYSCG

1561 1598

1562 1571

1574

1562

1562

1562

1562

1559

1559

1554

1559

1559

1559

1559

1559

1556

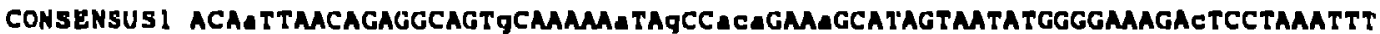
HIVLAI

HIVHXB2R

IIIVHN

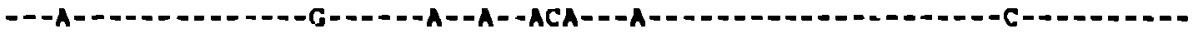

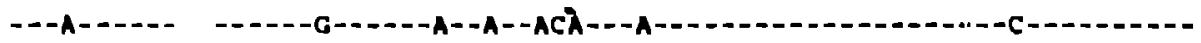

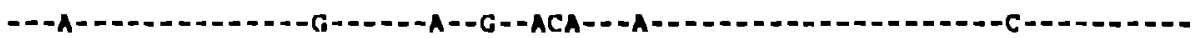

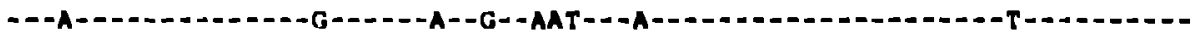

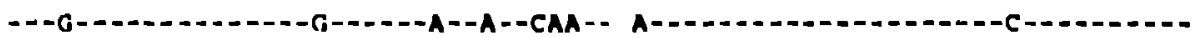
- - - ; - - -

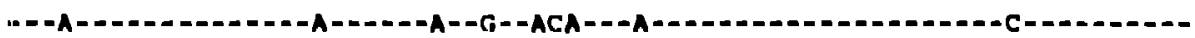

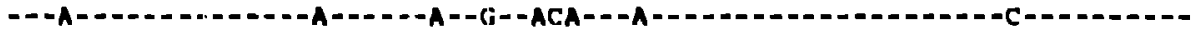

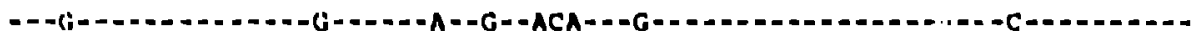

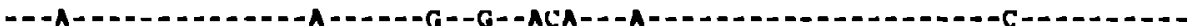

HIVST2 HIVNYSCO

HIVNLAJ

MIVIIAN

IIIVHF

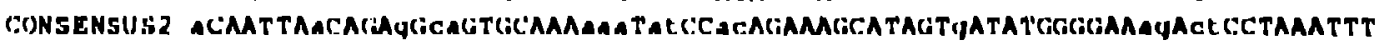
IIIVELL IITVZ2Z6 IITVNDK

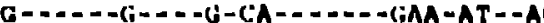

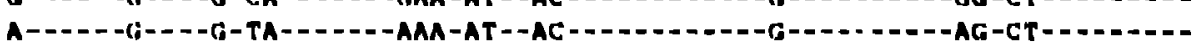

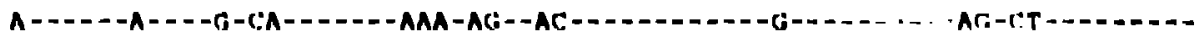
HIVMAL,

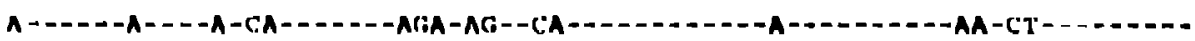

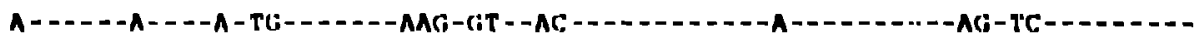
ilrvilas

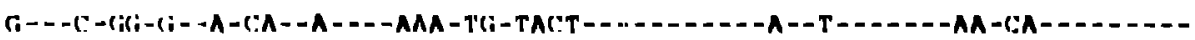

1631

1668

1632

1641

$16+4$

1632

1632

1632

1632

1629

1629

1624

1629

1629

1629

1629

1629

$162 \mathrm{~h}$

I.A.25 
CONSENSUS1 AaACTACCCATACAAAGGAAACATGGGAagCaTGGTGGACAGAgTATtgGCAAGCCACCTGGATTCCTG

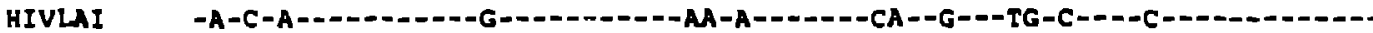

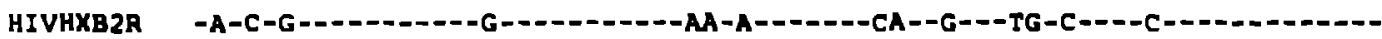

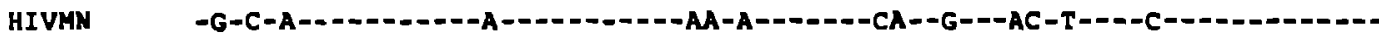
HIVJRCSF -A-T-A-_-HIVOYI -A-C-A-_HIVNYSCG

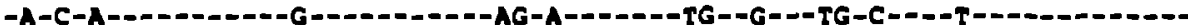
HIVNL43

HIVHAN

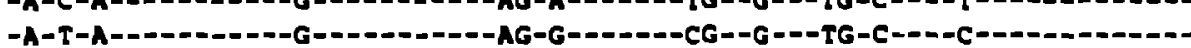

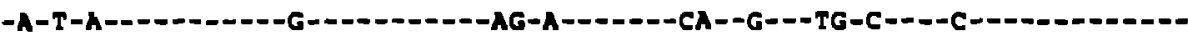

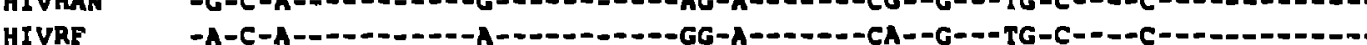

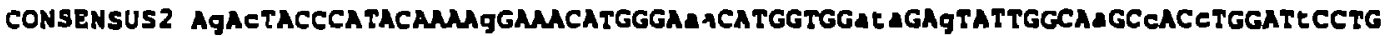
HIVELI -G-C-D-_-_HIV2226 HIVNDK HIVMRL

HIVU455

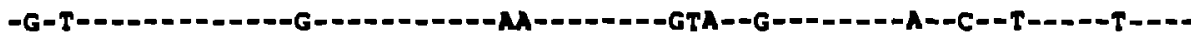

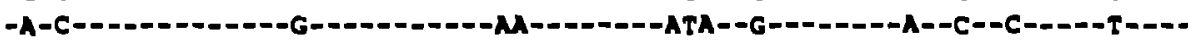

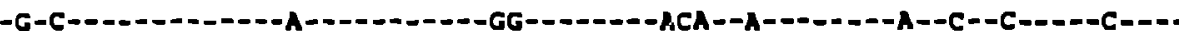

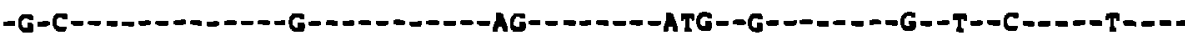

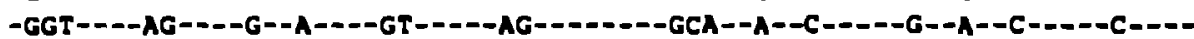

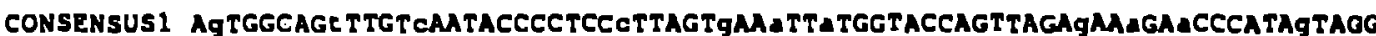
HIVLAI HIVHXB2R HIVMN

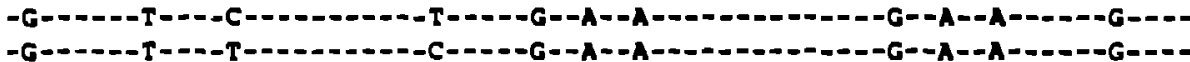

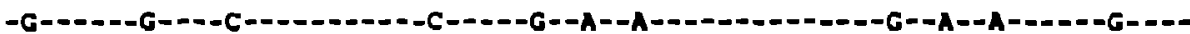
HIVJRCSP HIVOYI HIVSF2 HIVNYSCG HIVNL43 HIVHAN HIVRF

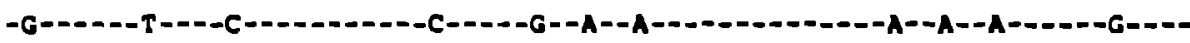

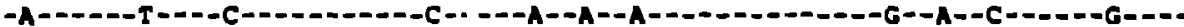

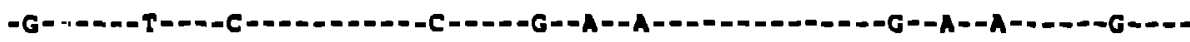

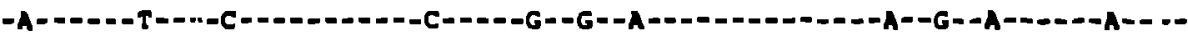

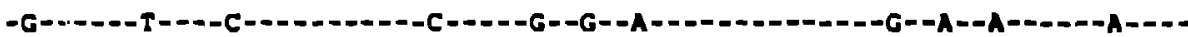

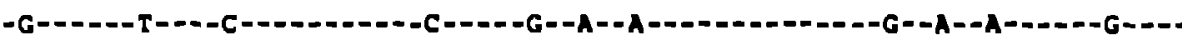

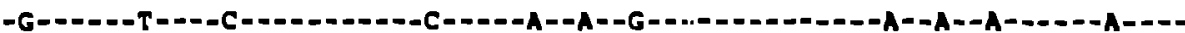

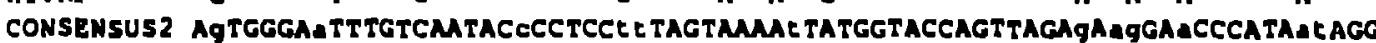
HTTELI HIVZ226 HIVNDK HIVARL HIVUA55 SIVCPZ

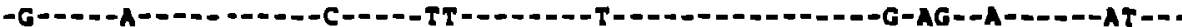

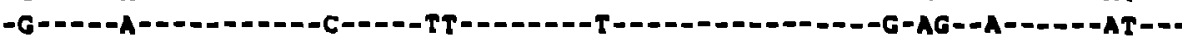

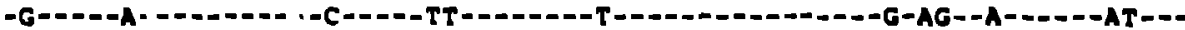

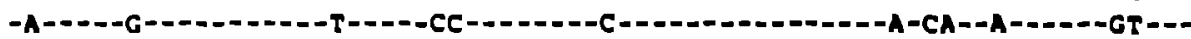

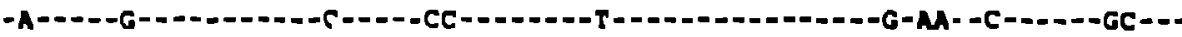

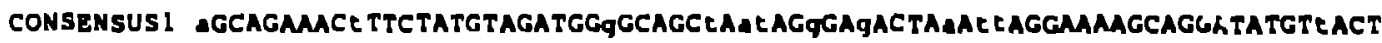
HIVLAI A-D---G-G---IIIVHXB2R HIVMN IIIVJRCSF HIVOYI HIVSF 2 HIVNYSCG IIIVNLA3 HIVHAN IIIVRF

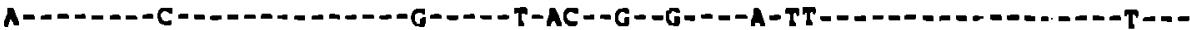
T-1-

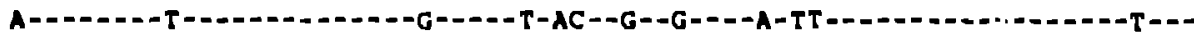

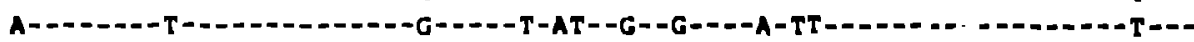

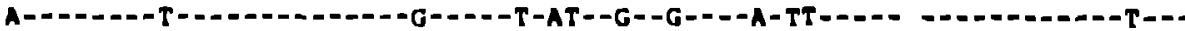
A-_-

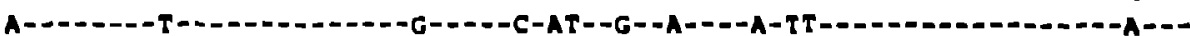

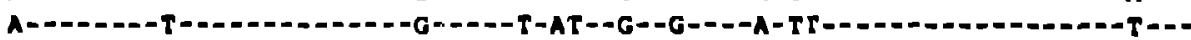

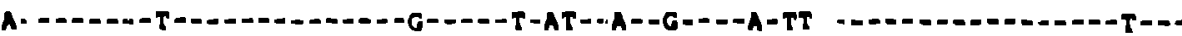
HIVEL.I II) V2226 II IVNDK HIVAAL HIVUASS -

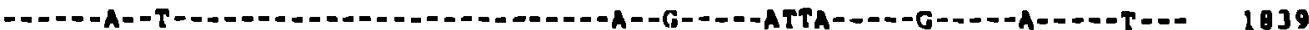

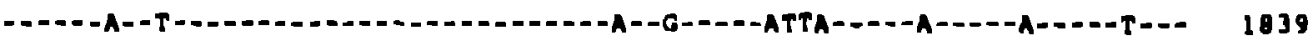

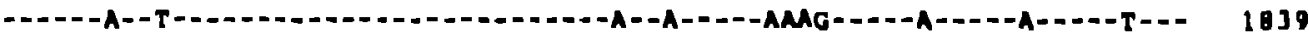

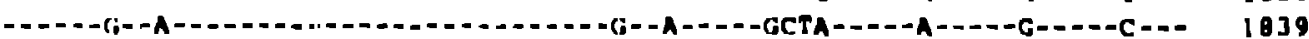
sIVCP2 
CONSENSUSI gaCAGaGGaAGACAaAAaGTTGT CLCCCTAaCtGACACAACAAATCAGAAgACTGAgTTACAAGCAATTC

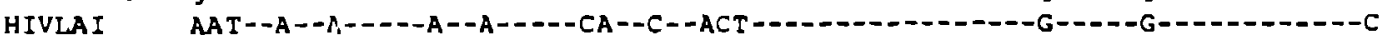
HIVHXB2R AAT--A--A-----A--A-----CA--C--ACT----

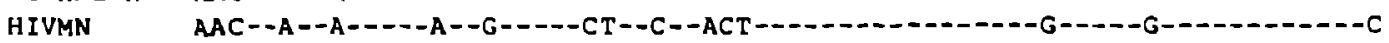

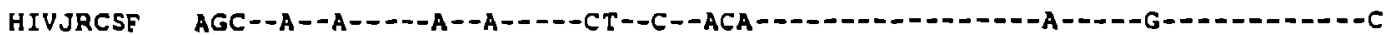
HIVOYI GAC--A--A-----G--A-----CT - C - ACT -

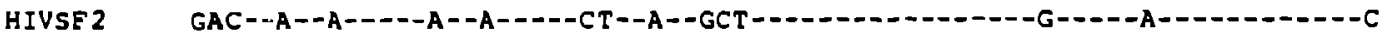
HIVNY5CG GAC--A--T----A--A----CC--T--ACG-1-1 HIVNL43 GAC--A--A-----A--A--1--CC--C--ACG--_- 3

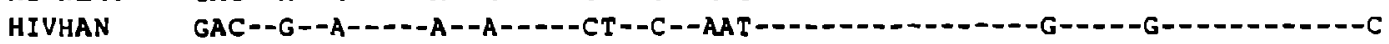

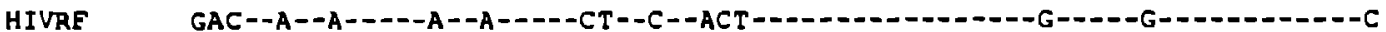
CONSENSUS 2 GACAGAGGAAGACAgAAaGTTGT CCCETT ?ACTGA?ACaACAAATCAgAAGACTGAgTTACAaGCAATta

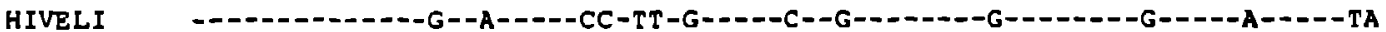

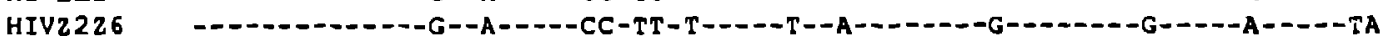
HIVNDK - HIVMAL HIVU 455 SIVCP Z ---A---G-A---A--AA-CA-TAGCT-AGAAA-T--C--T----GC-AG-A--A---A-G- -TT -GC

1911 1948 1912 1921 1924 1912 1912 1912 1912 1909 1909 1902 1909 1909 1909 1909 1909 1906

CONSENSUS I ALCTAGCTETGCAgGATTCgGGat TaGAAGTAAACATAGTAACAGACTCACAATATGCATT AGGAATCAT HIVLAI HIVHXB2R HIVMN HIVJRCSF HIVOYI HIVSF 2 HIVNY SCG HIVNL43 HIVHAN HIVRE

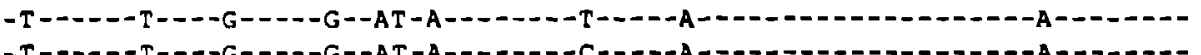

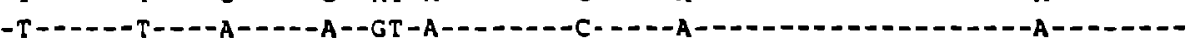

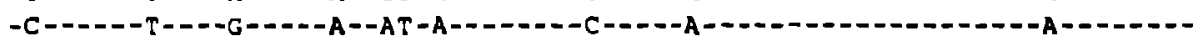

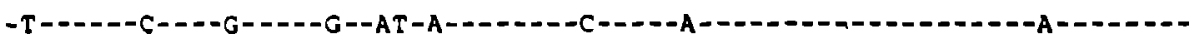

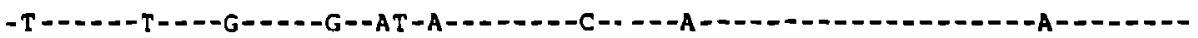

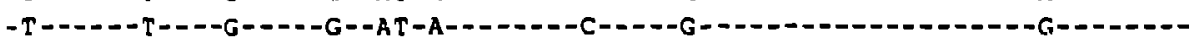

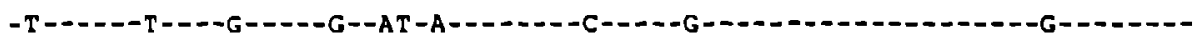

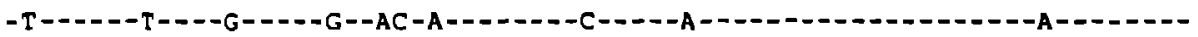

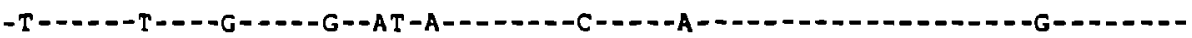
CONSENSUS 2 AL CTAGCETTgCAGGATTCgGGATtAGAAGTAAACATAGTAACAGAT TCACAATATGCAT TAGGAATCAT

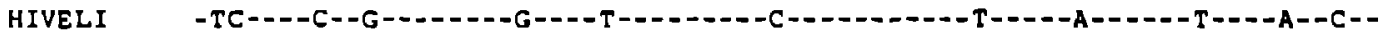

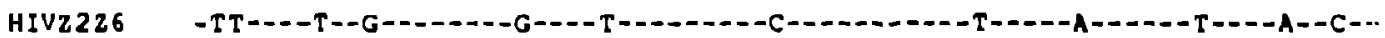
HIVNDK -TC-D-T--A-D-C-G-

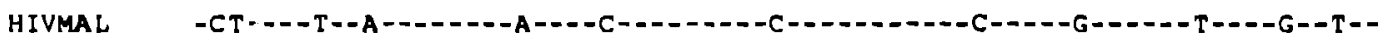

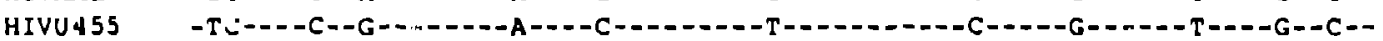

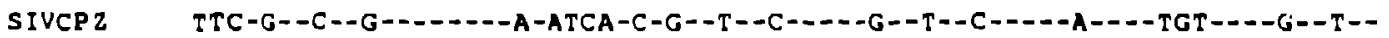

CONSENSUS 1 TCAAGCACAACCAGAT $\triangle A A G T G A A T C A G A G T T A G T C A g T C A a A T A A T A G A g C A G t$ TAATAAAAAAGGA HIVLAI HIVHXB2R HIVMN HIVJRCSF HIVOYI HIVSE 2 HIVNY SCE HIVNL4] H: VHAN -

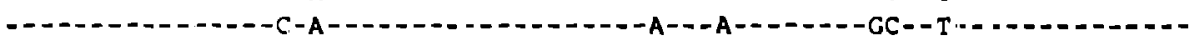

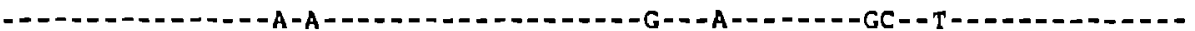

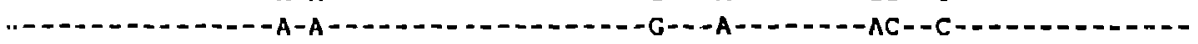

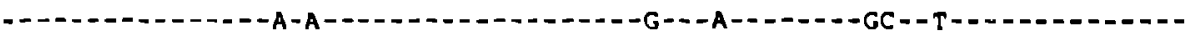
- - - - -

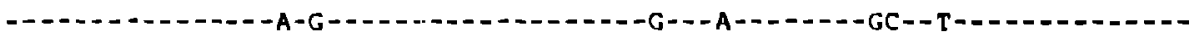

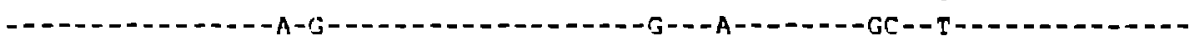

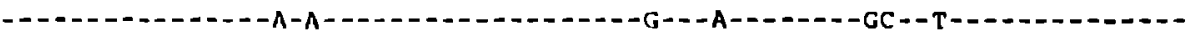
HIVRF

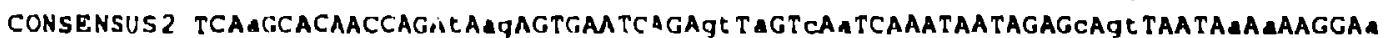

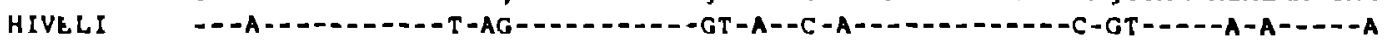
HIVZ226 HIVNDK

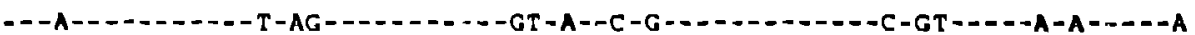

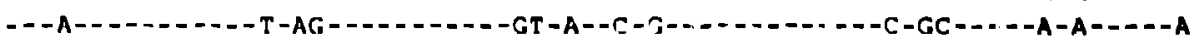

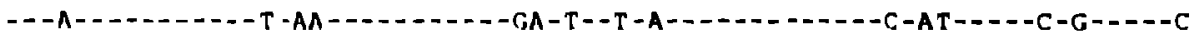

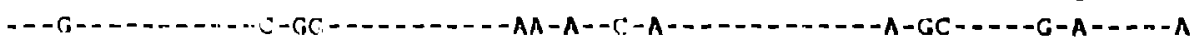

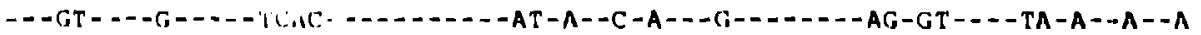




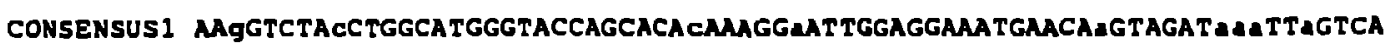

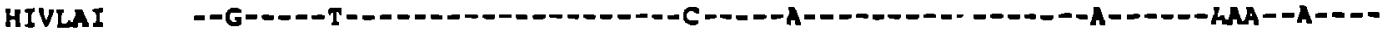
HIVHXB2R - GHIVMN HIVJRCSE HIVOYI HIVSE 2 HIVNY5CG HIVNL43 HIVHAN HIVRF

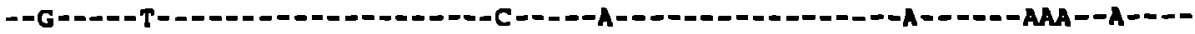

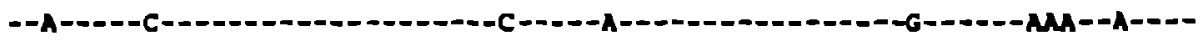

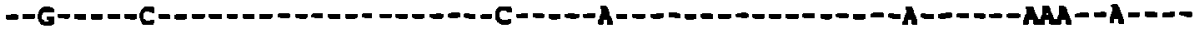

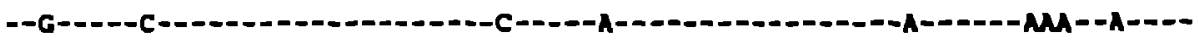

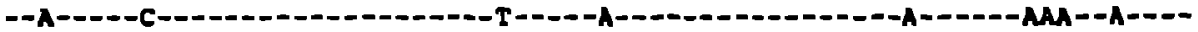

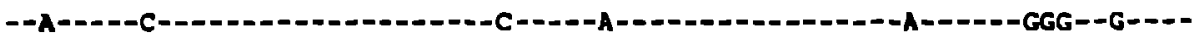

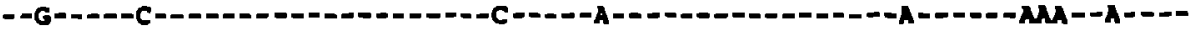

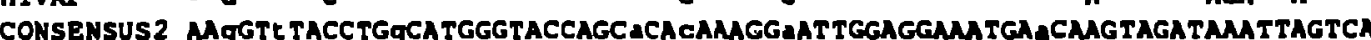
HIVELI HIVZ226 HIVNDK HIVMAL HIVU455 SIVCP2 MAGTL TACCTGgCATGGGTACCAGCaCACAMAGGAATTGGAGGAMTGACAMGTAGATAMATTAGTCA

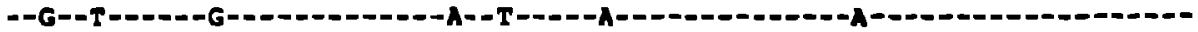

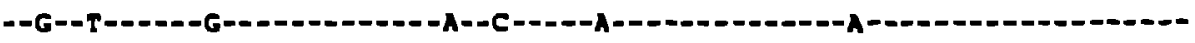

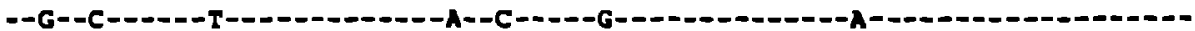

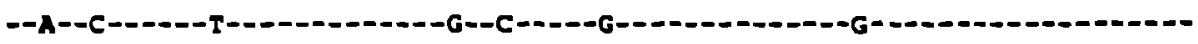

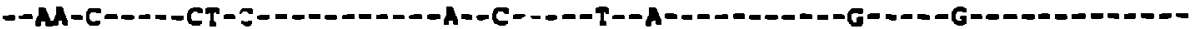

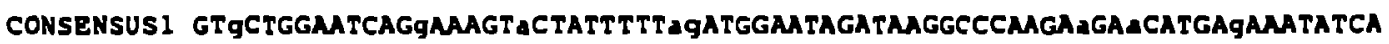
HIVLAI HIVHXP2R HIVIN HIVJRCSF

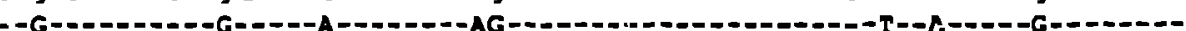

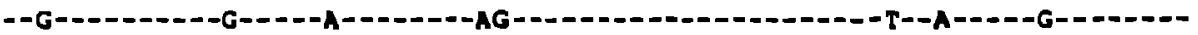

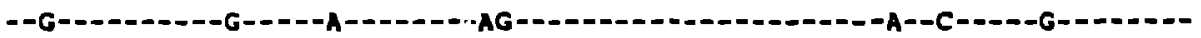
HIVOYI

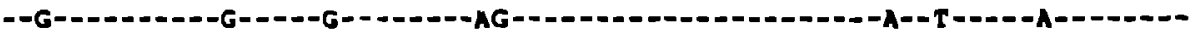

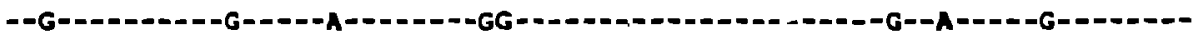
HIVSF2 HIVNY5CG HIVNL43

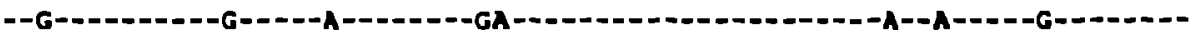

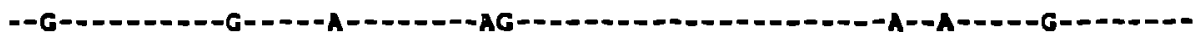

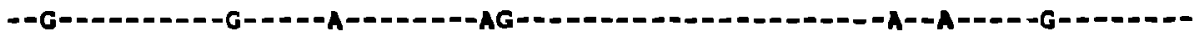
HIVHAN

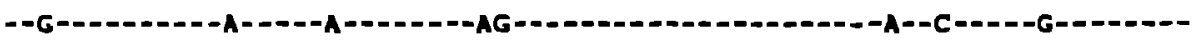

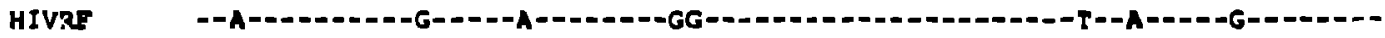

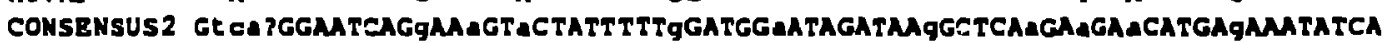
HIVELI HIVZ226 HIVNDK

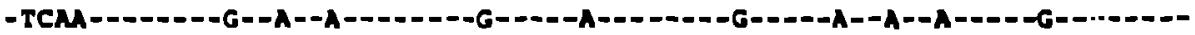

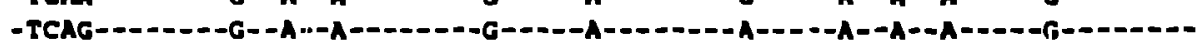

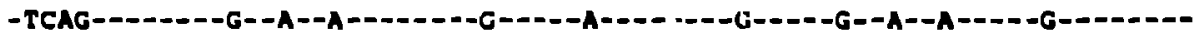
HIVAR

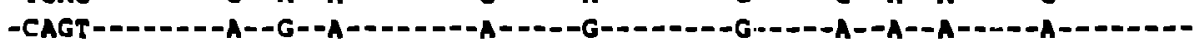
HIVU 455

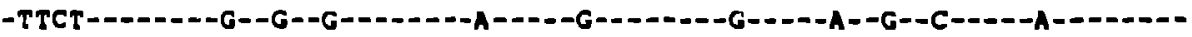
SIVCPZ

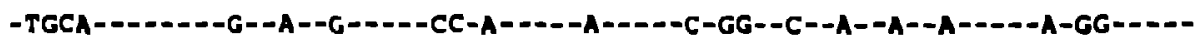

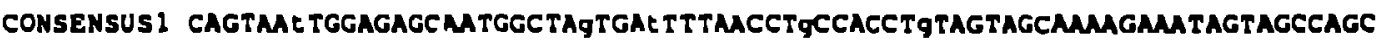

HIVLAI HIVHXB2R

HIVMN HIVJRCSF HIVOYI HISSF 2 HIVNYSCG HIVNL43 HIVHAN HIVRE

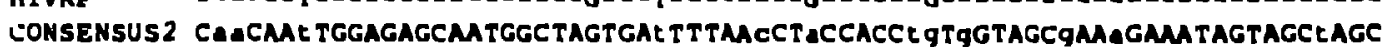
HIVELI HIVZ226 HIVNDK HIVMAL HIVU 455 SiVCP 2

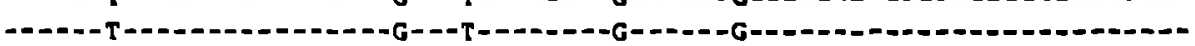

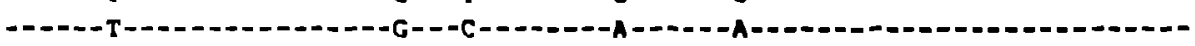

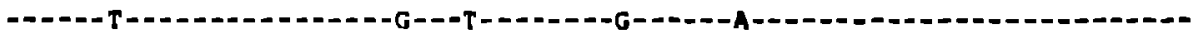

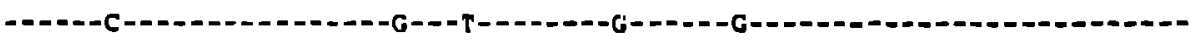

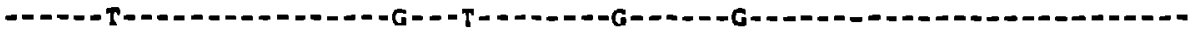

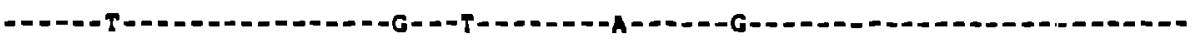

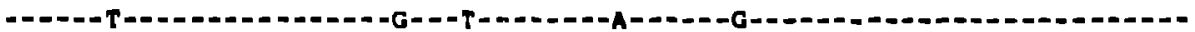

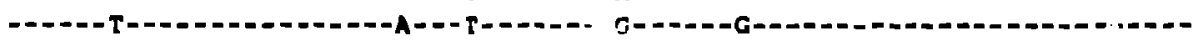

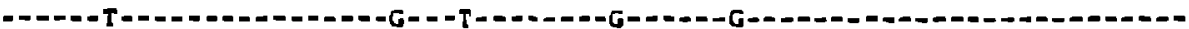


HIV-1 POL

CONSENSUSI TGTGATAAATGECAGCTAAAAGGGAAGCCATGCATGGACANGTAGACTGTAGLCCaGGAATATGGCAaC HIVLAI - HIVHXB2R HIVMN HIVJRCSE HIVOYI

HIVSF2 HIVNYSCG HIVNL43 HIVHAN

HIVRF - - - - - - - -

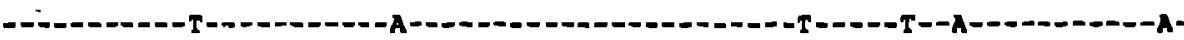
CONSENSUS2 TGTgATANATGTCAgCTAAMGGAGMGCCATGCATGGACAGTAGACTGTAGTCCAGGAATATGGCNAT HIVELI HIVZ226 HIVNDK HIVMAL HIVU455 SIVCP2

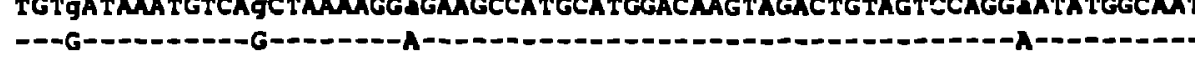

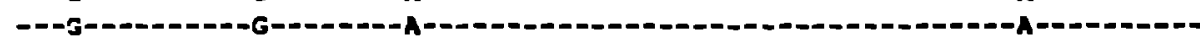

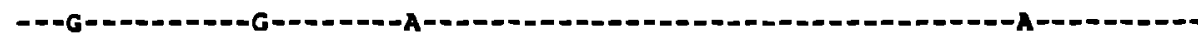

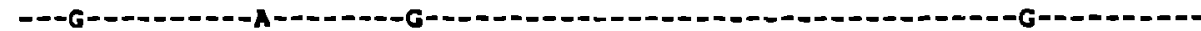

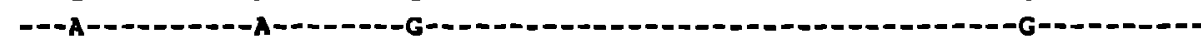

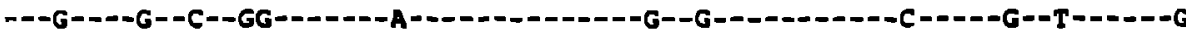

CONSENSUSI TAGATTGTAEACATETAGAGGAAAgTTATCCTGGTAGCAGTLCATGTaGCCAGTGGatAtATAGAAGC HIVLAI HIVHXB2R HIVMN HIVJRCSF HIVOYI HIVSF2 HIVNY5CG HIVNLA3 HIVHAN -

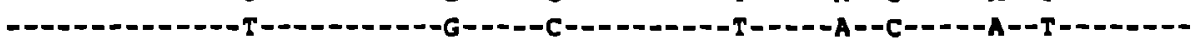

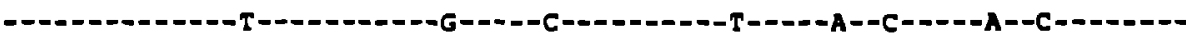

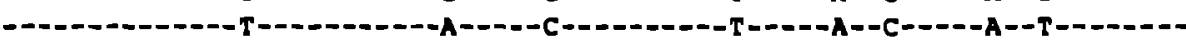

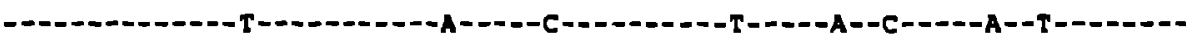

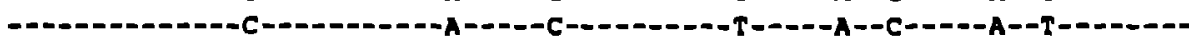

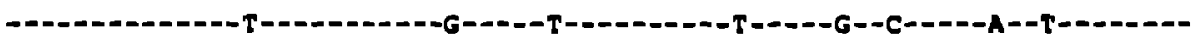

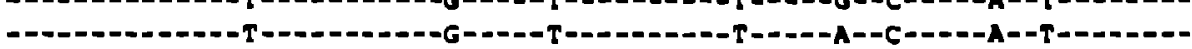

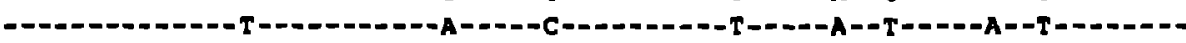
HIVRF

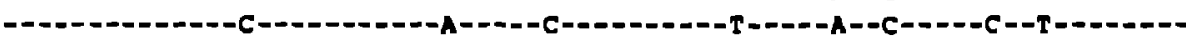

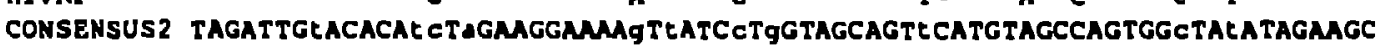
HIVELI HIVZ226 HIVNDK HIVMAL HIVU455

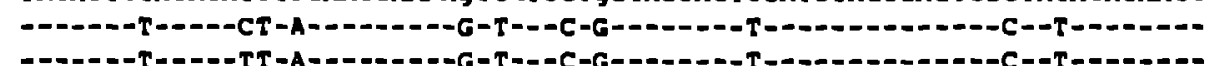

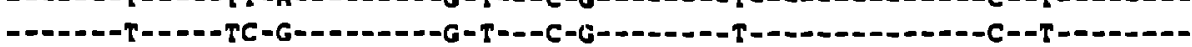

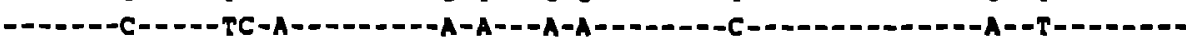
SIVCPZ

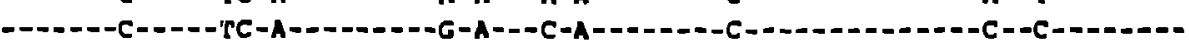

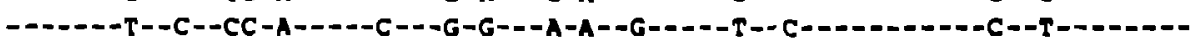

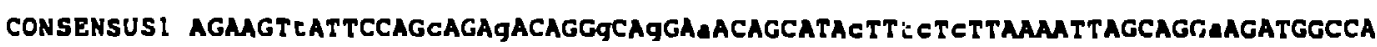
HIVLAI HIVHXB2R HIVMN HIVJKCSE HIVOYI HIVSF 2 HIVNYSCG HIVNL4] HTVHAN HIVRF

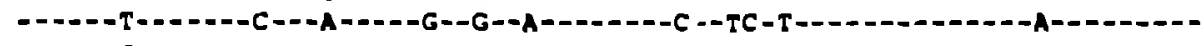

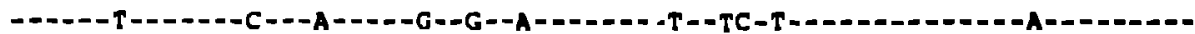

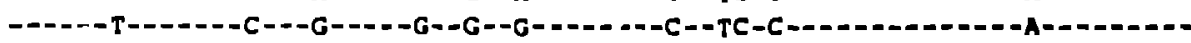

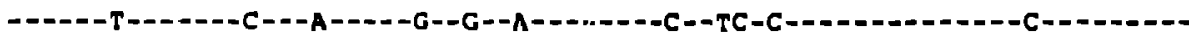

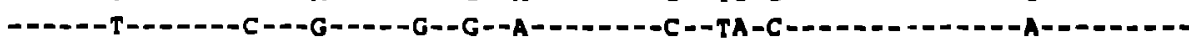

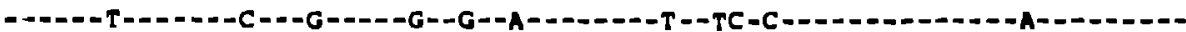

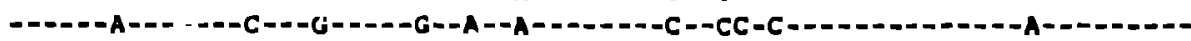

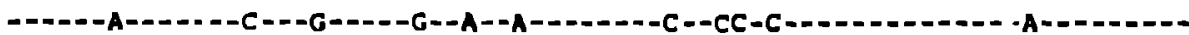

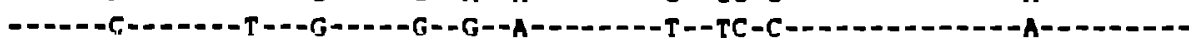

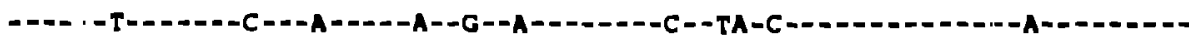
CONSENSUS2 AGAATTATtCCAGCAGAAACAGgqCAgGAaACAGCATACTTTAT ?t TAAATTAGCAGGAAGATGGCCA HIVELI HIV2226 HIVNDK HIVMAL HIVU453 SIVCPZ

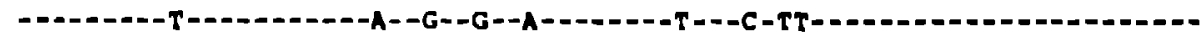

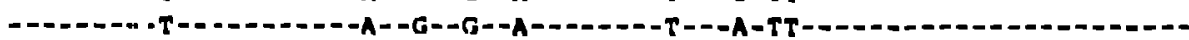

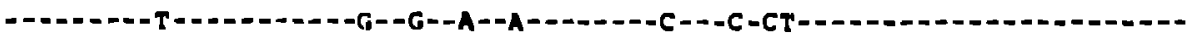

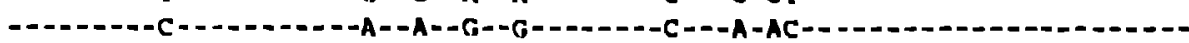

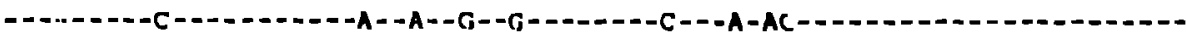

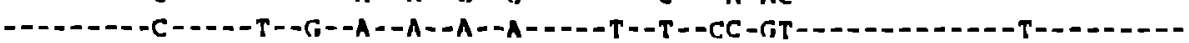


CONSENSUSI GTAAaAaCAaTACALACaGACAATGGCagCAATTTCACCaGTaCTACgGTTAagGCCGCCTGTTGGTGGg HIVLAI HIVHXB2R HIVMN HIVJRCSF HIVOY: HIVSE 2 HIVNY5CG HIVNL 43 HIV:IAN HIVRF

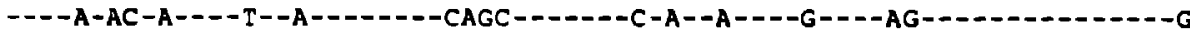

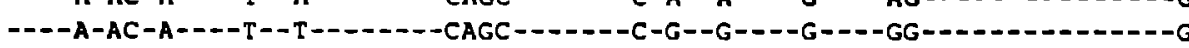

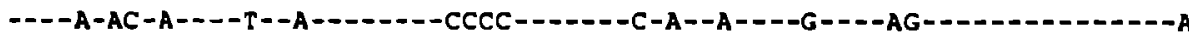

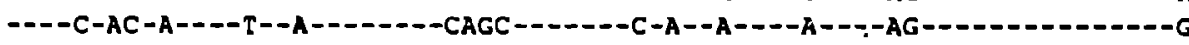

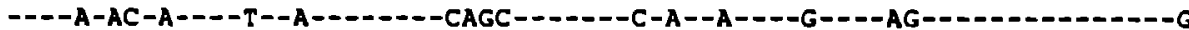

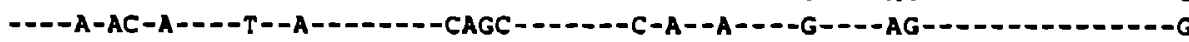

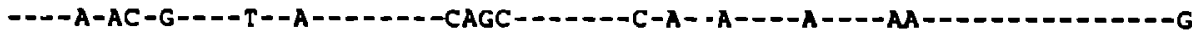

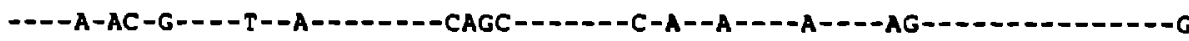

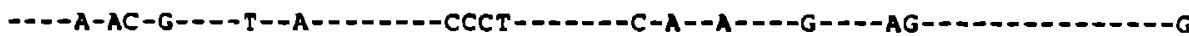
$\begin{array}{ll}\text { HIVRF } & --A-G T-A---T-A-A \\ \text { CONSENSUS2 GTAAAATTAgTACALACAGACAATGGCAGCAATTTCACCAGLGCTgCAGTTAAgGC ?GCCTGTTGGTGGG }\end{array}$ HIVELI HIVZ226 HIVNDK HIVMAL HIVU455 SIVCP Z

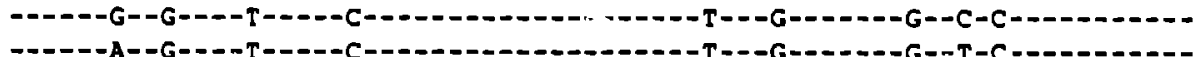

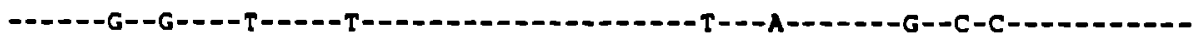

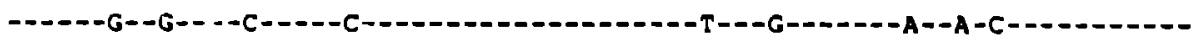

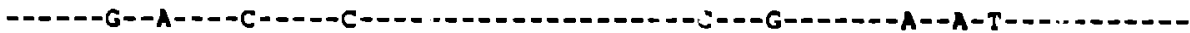

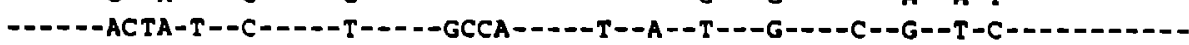

CONSENSUS1 C?GGgATCAAGCAGGA ATTGGCATTCCCTACAATCCCCAAAGTCAAGGaGTAgTAGAATETATGAATAA HIVLAI HIVHXB2R HIVMN -G--A-12536

HIVJRCSF

HIVOYI

HIVSF 2

HIVNY SCG

HIVNL 4

HIVHAN

HIVRP

CONSENSUS:

HIVELI

HIV2226

HIVNDK

HIVMAL

HIVU455

SIVCPZ

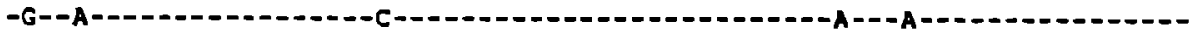

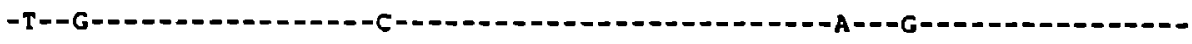

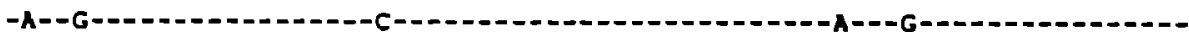

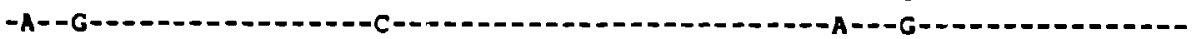

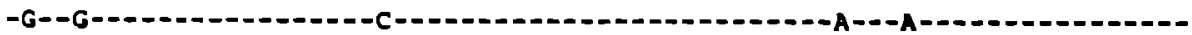

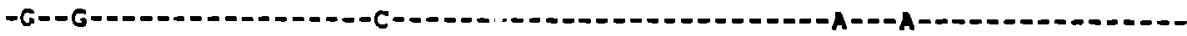

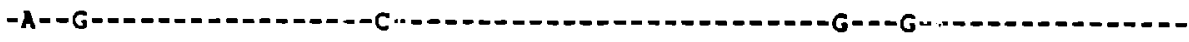

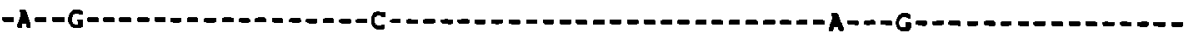

GATCAMACAGGAATTTGGATtCCCTACAAtCCCCAAAGTCAMGGAGTAGT GAATCEATGALAA

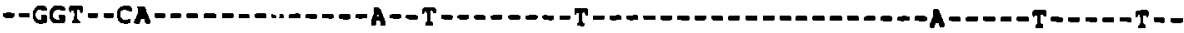

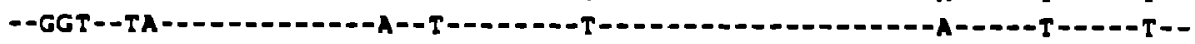

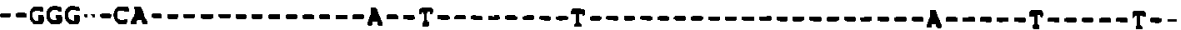

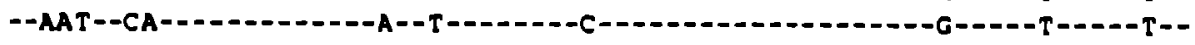

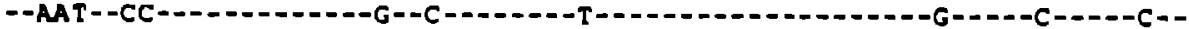

CONSENSUS1 IgAatTAAAGaAATTATAGgaCAgGTAMGAGATCAgGCTGAACALCTTAAGACAGCAGTACAMATGCI.

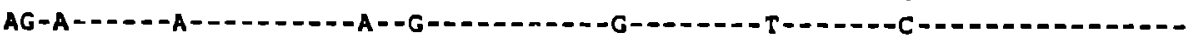

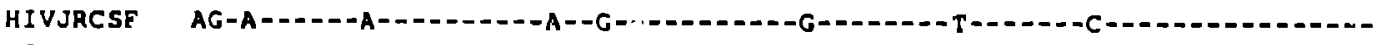

AG-A-C-A HIVHAN AG-G-1-1-A-1-1 
CONSENSUSI GTATTCATCCACAATTTIAAAAGAMAGGGGGGATTGGGGGTACAGTGCAGGGGAAAGAATAgTAGaCA HIVLAI HIVHXB2R HIVMN HIVJRCSF HIVOYI HIVSF 2 HIVNY5CG HIVNL43 HIVHAN HIVRF -

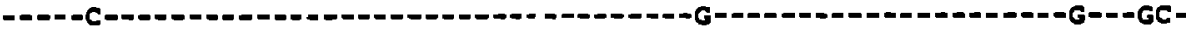
- - - T-O-_-

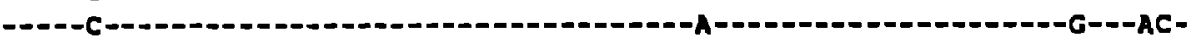

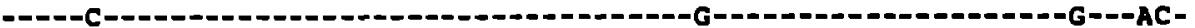

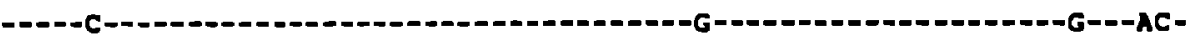

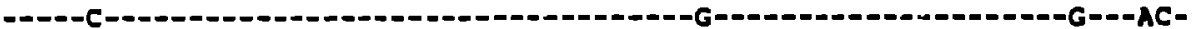
GENSU2 GTATTCATCCACMATTTIMAMGMAAgGGGGGATTGGGGaTACAGTGCAGGGGAAGMATAATAGACA HIVELI HIV2226 HIVNDK GTATTCATCCACNATTTINAMGMAAgGGGGGATTGGGGGATACAGTGCAGGGGAANGMATAMTAGACA

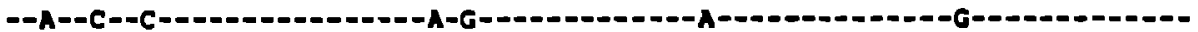

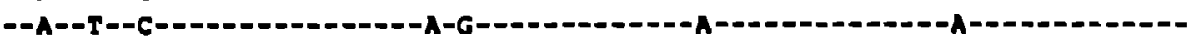
HIVMAL

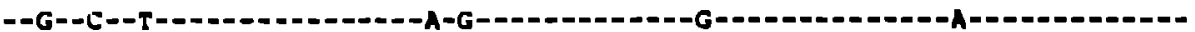
HIVU 455 SIVCP Z

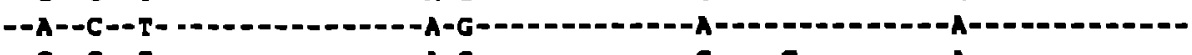

CONSENSUS1 TMATAGCAACAGAGATACAMACTMAGMA TACMMA_CAAATTACAAAATTCAAMTTTTCGgTTTA HIVLAI HIVHXB2R HIVMN -

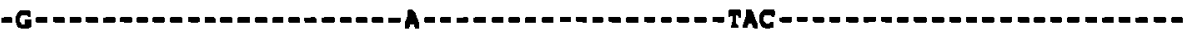

SIVCP 2

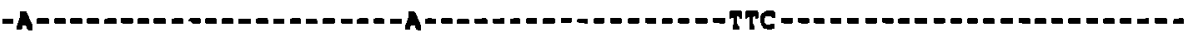

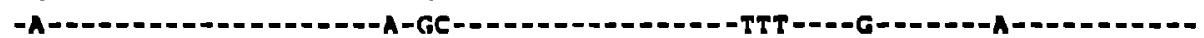

2831

2834

2822

2822

2822

2822

2819

2019

2809

2019

2819

2819

2819

2819

$1 / 31$ -

5. $.51 /$

CONSENSUS I TTACAGGGACAgCAgAgAE CCACTTTGGAAGGACCAGC MAAGCTECTCTGGMAGGTGAAGGGGCAGTA

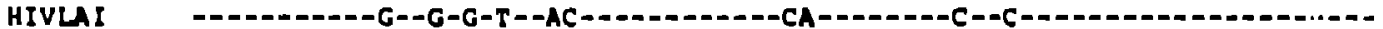
HIVHXB2R HIVMN HIVJRCSF HIVOYI HIVSF 2 - 2 (1)

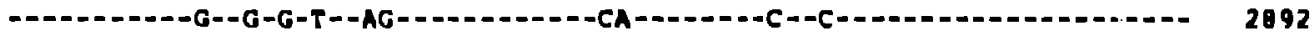
HIVNLA]
HIVIIAN

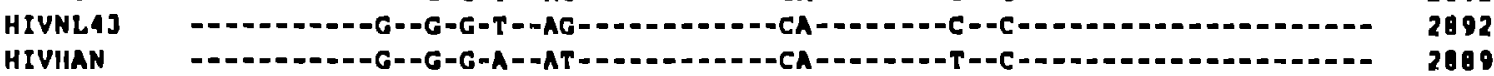
HIVRT
HIVIAN CONSENBUS2 TTACAGqGACAqCAGAGALCCAATTTGGMAGGACCAGCAAAqCT7CTCTGGAMAGGTGAAOGGOCAGTA HIVELI

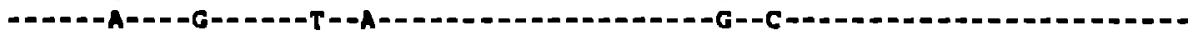
HIVZ 226

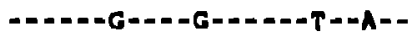


HIV-1 POL

vlf eds $\rightarrow$

CONSENSUSI GTMTACAMGATARTAgTGACATAAMAGTAGTGCCAMGAGaAMGCAMAgaTCATEAGGGATTATGGAA

HIVLAI

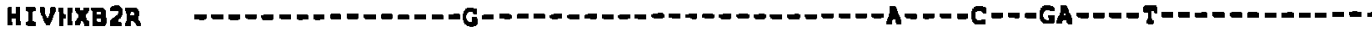

HIVMN

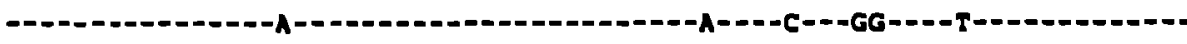

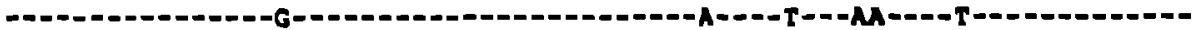

HIVOYI

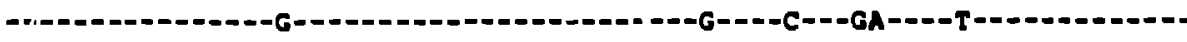

HIVSF 2

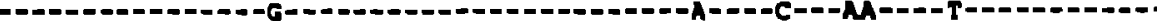

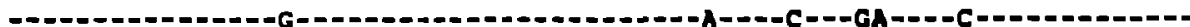

- -

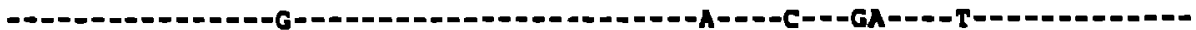

HIVNL43

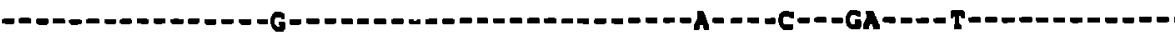

HIVHAN

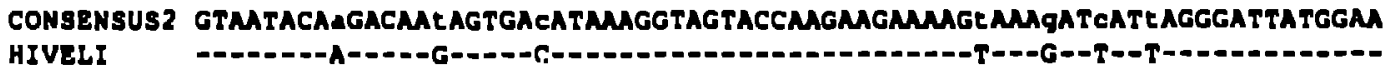

HIV7226

HIVNDK

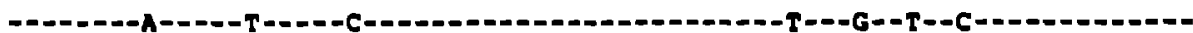

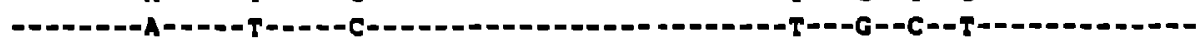

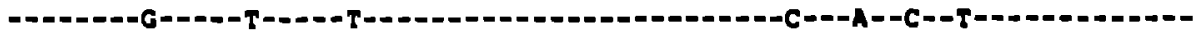

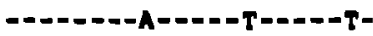

2962

2959

HIVUA55

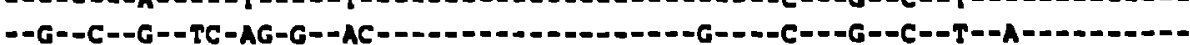

2948

2959

2959

2959

2959

SIVCP2

consensus l mCAgAcggaggtgatgattgtgtggcmatagacaggatgaggat** 3010

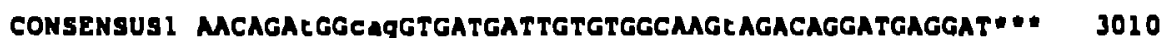

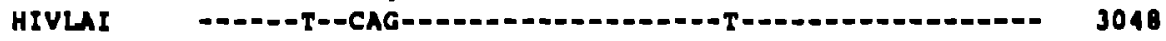

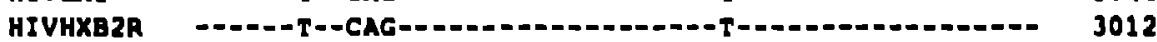

HIVHN HIN

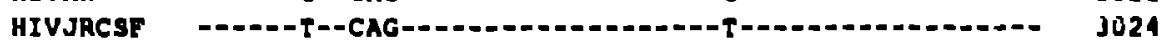

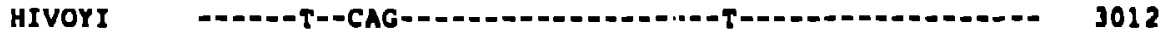

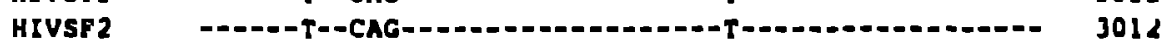

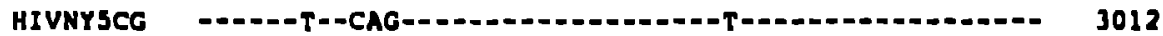

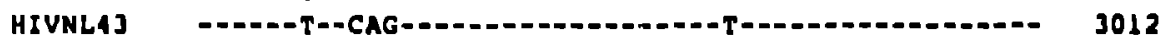

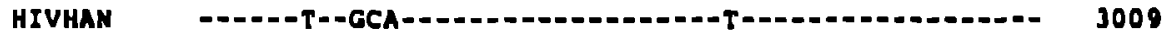

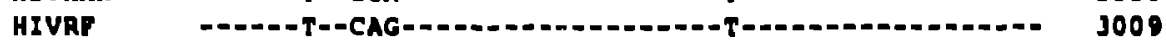

CONBENBUS2 MCAGATGGCAGGTGATGATTGTgTGOCAGGTGGCAGGATGAqGAT-2- 2990

HIVELI

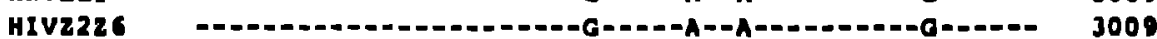

HIVNDK HIXN J009

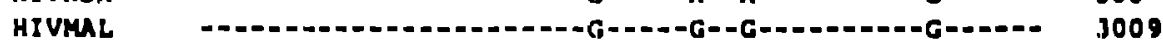

HIVU4SS

SIVCPZ 
<- pol cd:

CONSENSUS I ATGGAAACAGAEGGCAgGTGATGATTGTGTGGCAAGEAGACAGGATGAGGAitAgAACATGGMAAGt

HIVLAI

HIVHXB2R

HIVMN

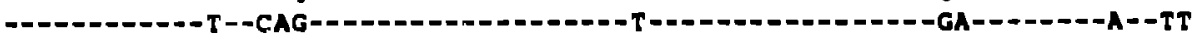

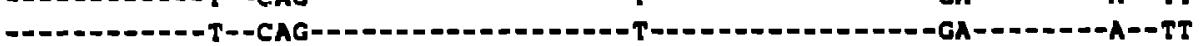

-

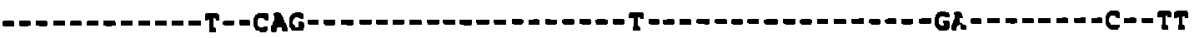

HIVJRCSF

HIVOYI

HIVSF 2

HIVNYSCG

HIVNL 43

HIVHAN

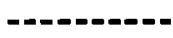

-

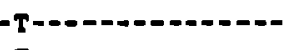

-GA-D-D--DA--TT

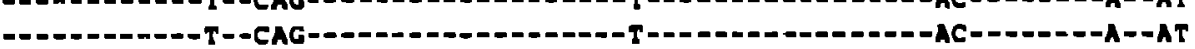

-

HIVRF

-T--CAG-

$-4-2$

GA-D-D--DA--TT

CONAENSUS 2 ATGGAMACAGATGGCAGGTGATGATTGTqTGGCA_GTaGACAGGATGAgGATAgAACATGGAAAGTT

HIVELI

HIV222 6

HIVIDK

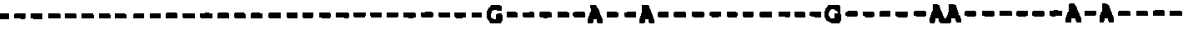

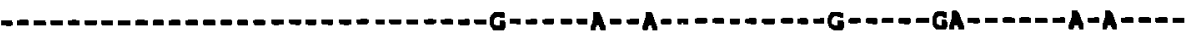

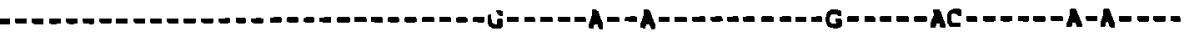

HIVMAL

HIVUASs

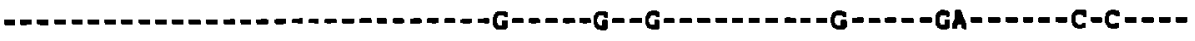

- - - - - -

SIVCP Z

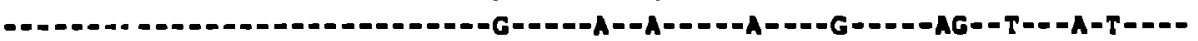

CONGENSUSI TAGTAAMCACCATATgTATATTTCAGGMACCTAMggatGGLETTATAgACAECACTATGMAGGAC

TaGT

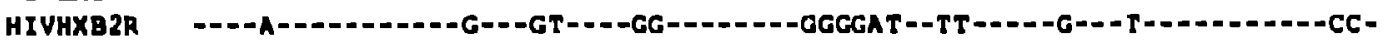

HIVHN

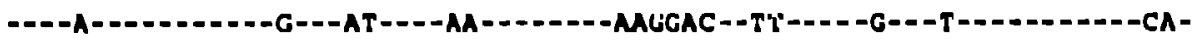

HIVJRCSE

HIVOYI

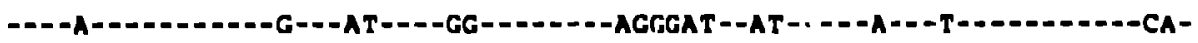

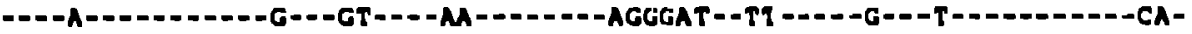

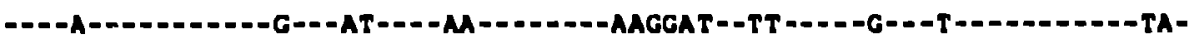

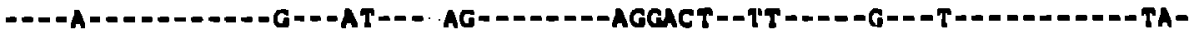

HIVWYSCG

HIVHLAJ

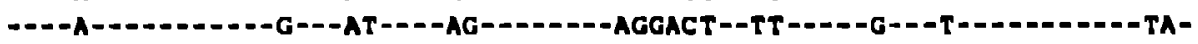

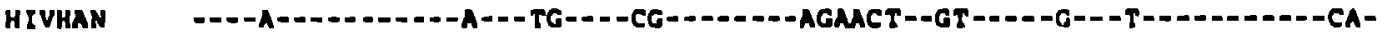

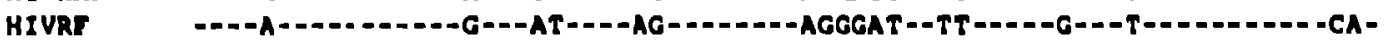

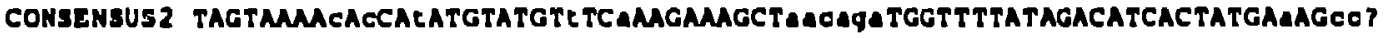

HIVELI - 2 I

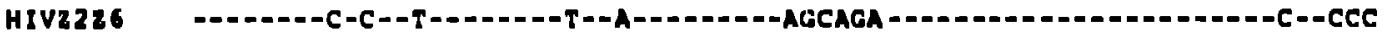

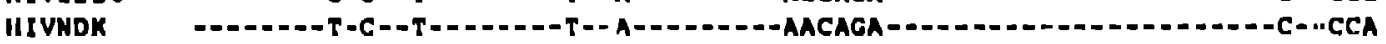

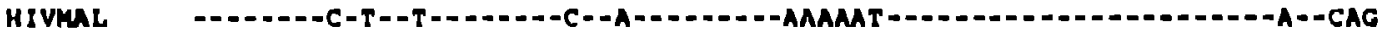

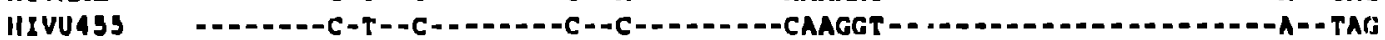

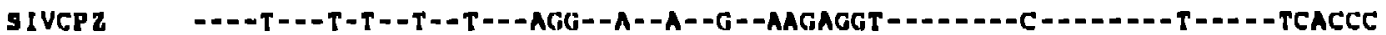

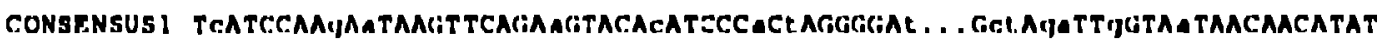

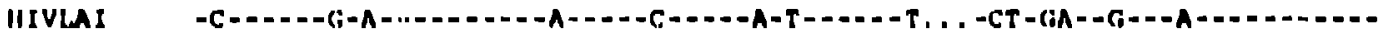

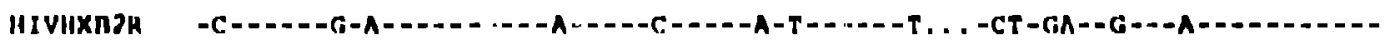
IIIVMN IIIVJResr IIIVOYI IIIVarz HIVWYSCE IIIVNLA3 IIIVIIAN -C . - - - -

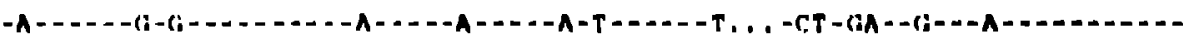

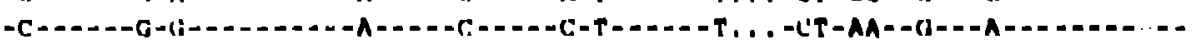

-

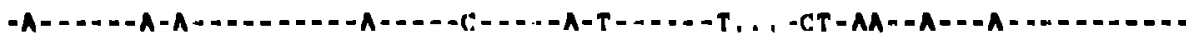

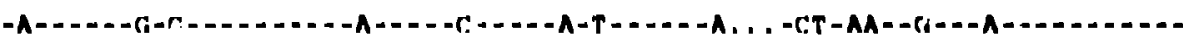
201

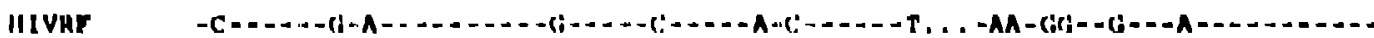

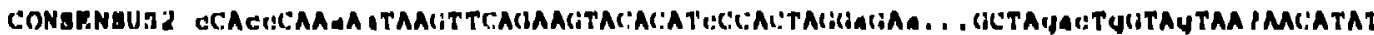
IIIVRLI IIIV827.6 IIIVMDK IIIVMAL. IIIVUass

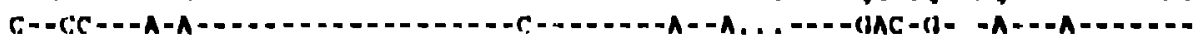

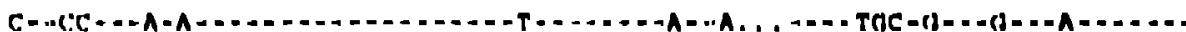

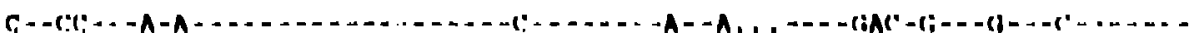

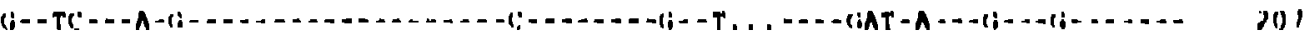
: I IVi:l' z. 
CONSENSUSI TGGGGTCTGCATACAGGAGMAGAGA TGGCATETgGGECAGgGAGtCtCCATAGARTGGAGGAAMAGa

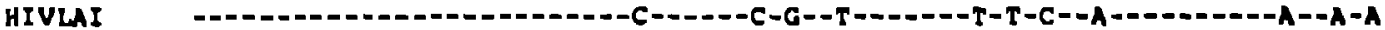
HIVHXB2R HIVMN HIVJRCSF - HIVOYI

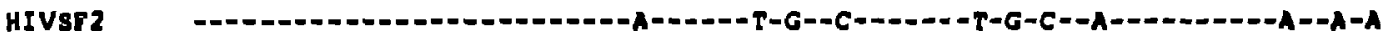
HIVNYSCG - HIVNL 13 - 3 HIVHAN HIVRF

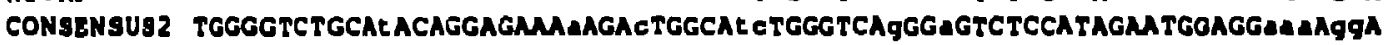
HIVLLI

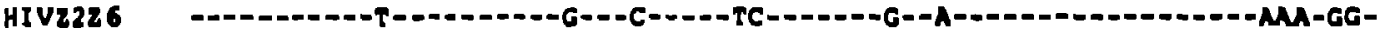

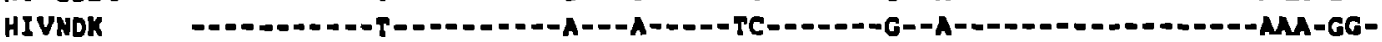

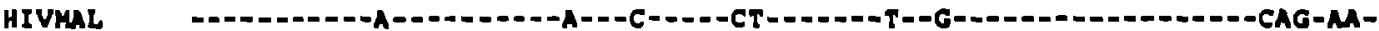

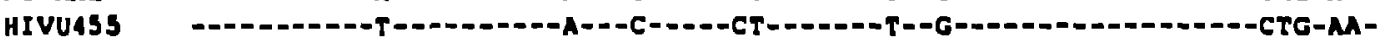

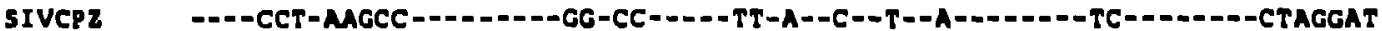

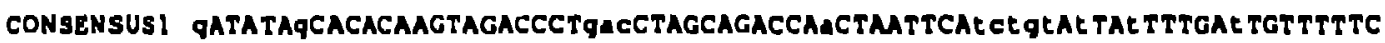

\section{HIVLAI}

HIVHXB2R

HIVMH G--D-G--_-_-

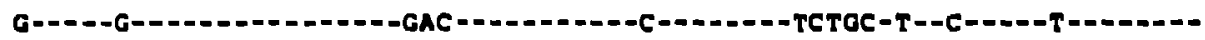

HIVOYI

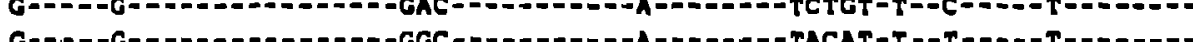

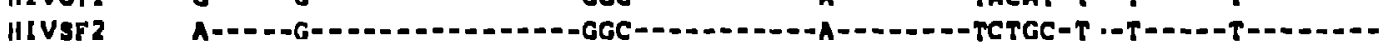

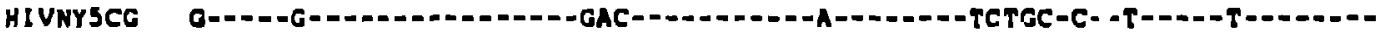

HIVNLA3

IIIVIIAN

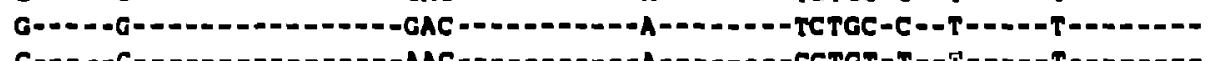

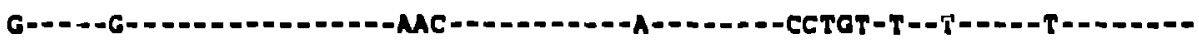

HIVRT

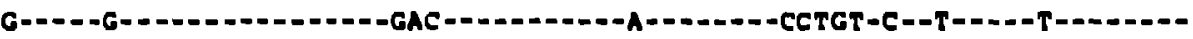

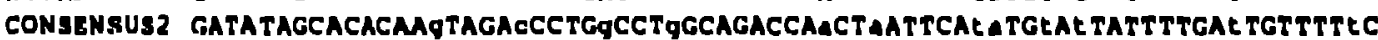

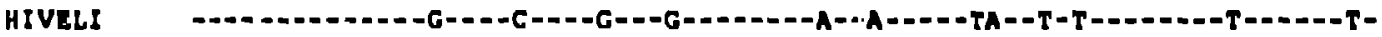

IIIVZ226

HIVNOK

HIVML

HIVUASS

sIVCpz

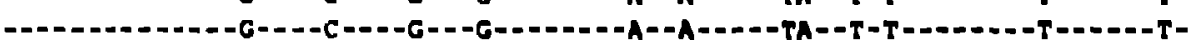

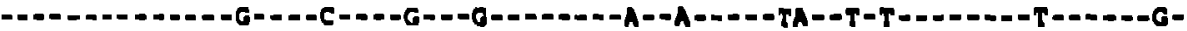

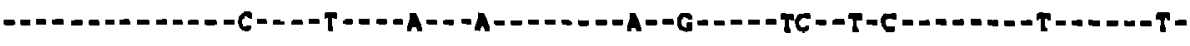

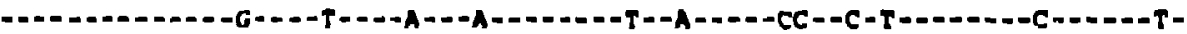

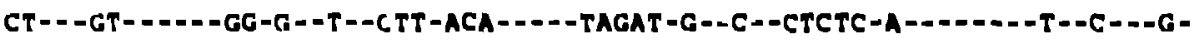

$1 / 3,-1$

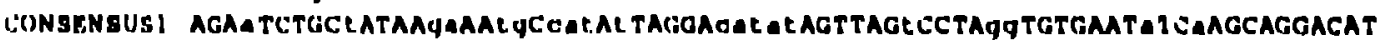
IIIVIAI IIVIXH2K III VMN

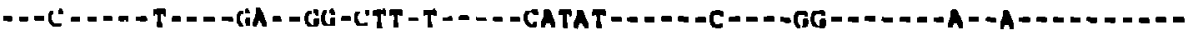

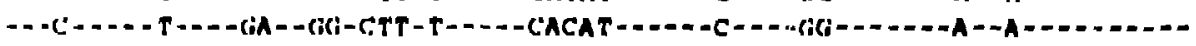

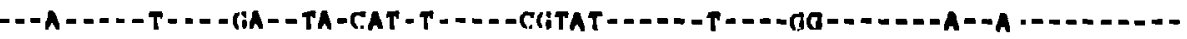

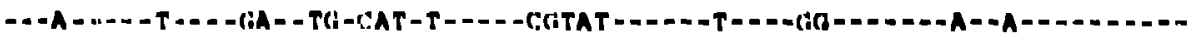

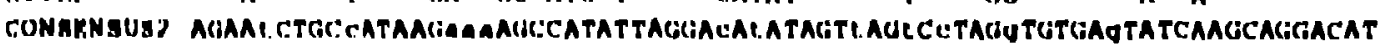


5' 3小/

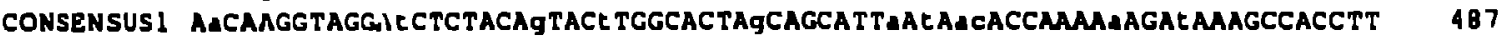

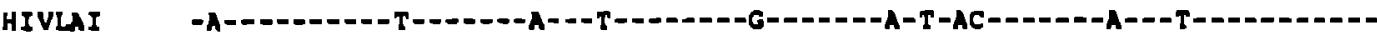

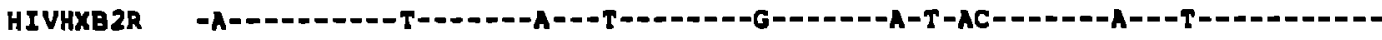

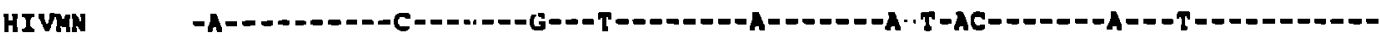

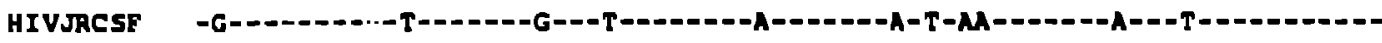

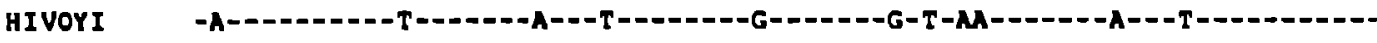

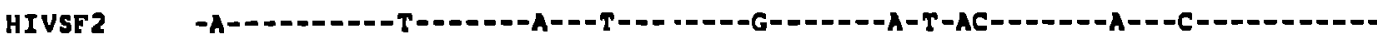

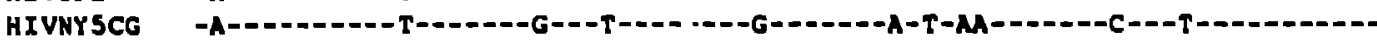

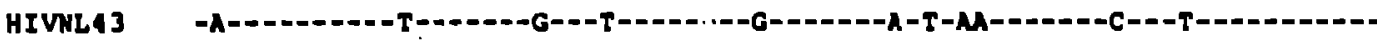

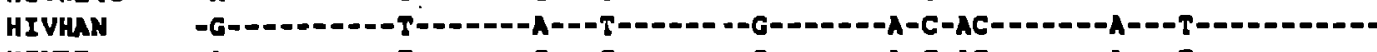

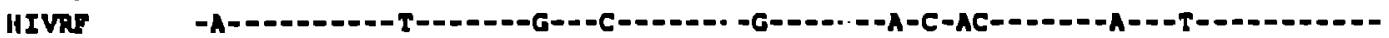

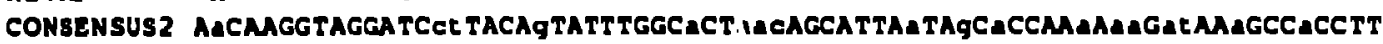

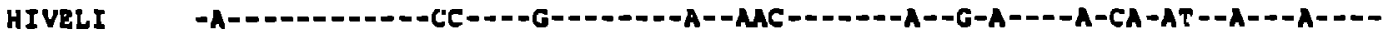

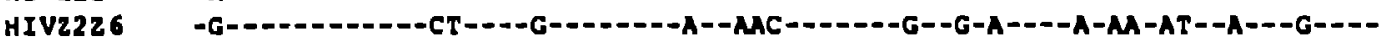

HIVNDK

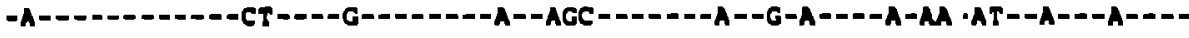

HIVMAL

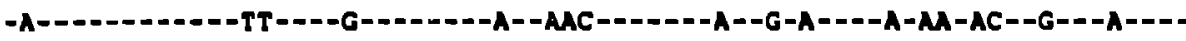

HIVUA55

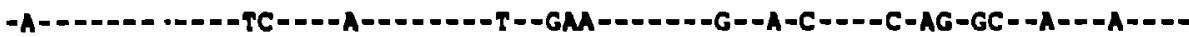

SIVCP Z

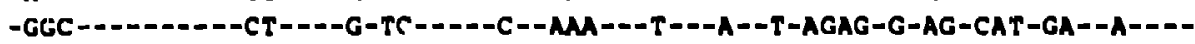

vpR cds $\rightarrow$

CONSENSUSI TGCCTAgTGTTA TgAACTgACAGAgGALAGATGGMCAAgCCCCAGMGACCMGGGCCACAGAGGgAG

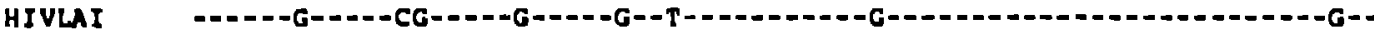

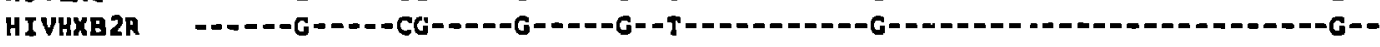

HTMY

HIVJRCSF

HIVOY I

HIVSF 2

HJVHYSCG

HIVHLA J

HIVHAN

HIVRF

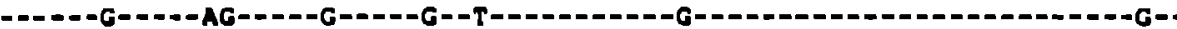

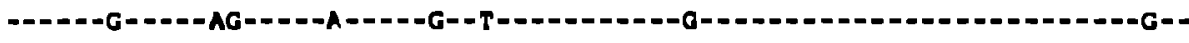

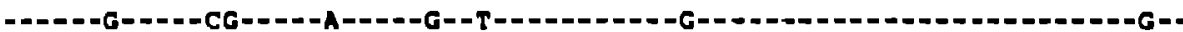

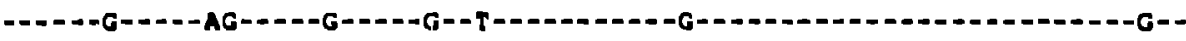

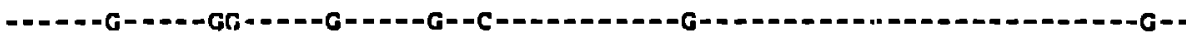

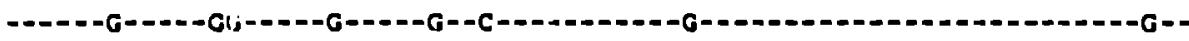

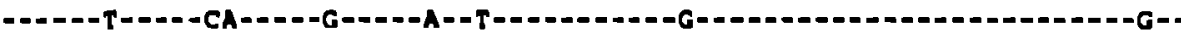

CONSENSUS2 TGCCTAGTGTTAGGAGCTMACAGAGATAGATGGAACAAGCCCCAGAAGACCAAGGGCCACAgAGGGAG

HIVELI

HIVZ226

HIVHDK

HIVMAL

IIIVUass

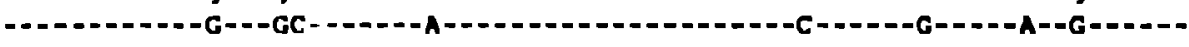

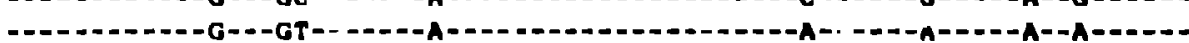

-

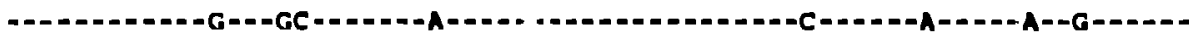

SIVCPz

-

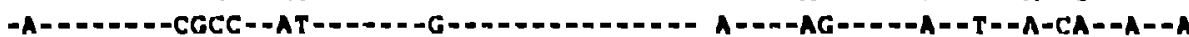

CONGENSUS 1 cCatacantqaATGGaCAC".e S/8

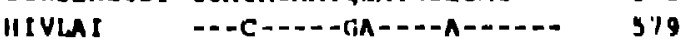

HIVIIXB2H

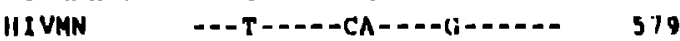

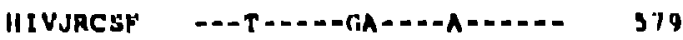

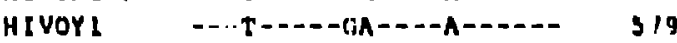

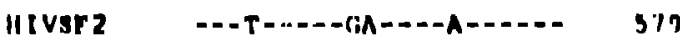

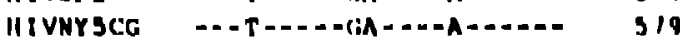

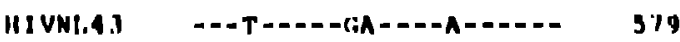

IIIVIIAN

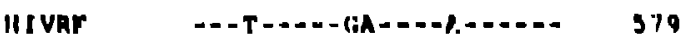

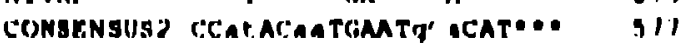

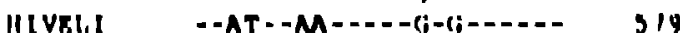

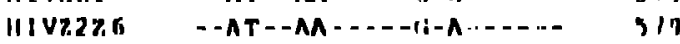

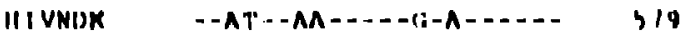

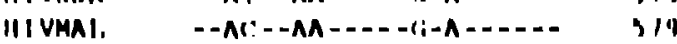

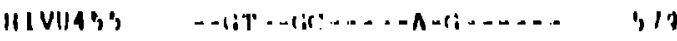

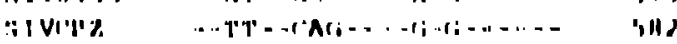




\section{HIV-1 VPR}

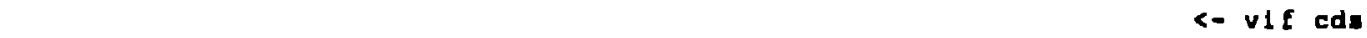
ATGGACAAGCCCCAGAGACCAAGGCCACAGAGGgAGCCALACAATgaATGGACACTAGAGCTTTTAg HIVLAI HIVHXB2R -

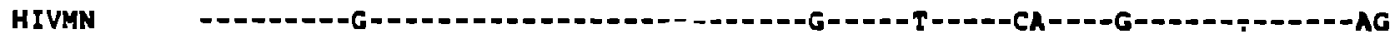
HIVSC - HIVJACSF HIVOYI - HIVSE2 - 2 HIVNY5CG - HIVNLA] -

HIVHAN
HIVRT

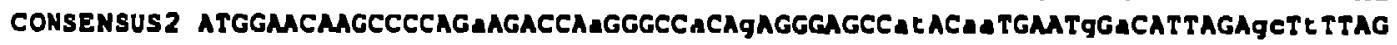

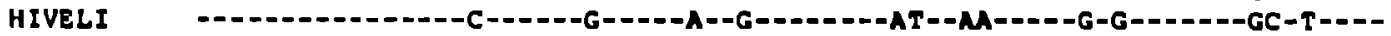
HIV222 6 - HIVNDK

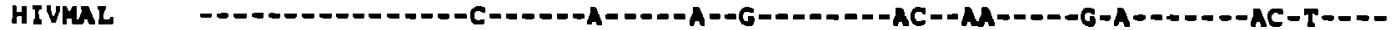

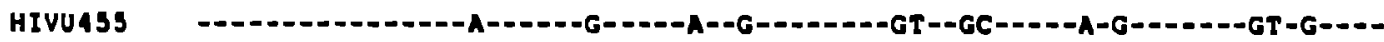
5 IVCPZ ---O------A----AG------A--T--A-CA--A--A--TT--CAG----G-G-------GACC--G-

CONSENSUSI AGgAgCTTAAGAgLGMGETGTEAGACATTTTCCEAGataTGGCTCCATAgCTTAGgaCAACATATCTA

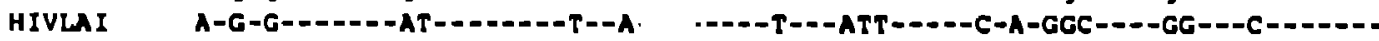

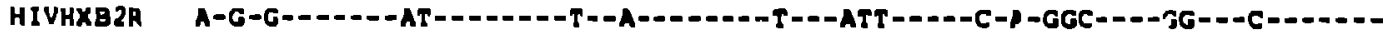

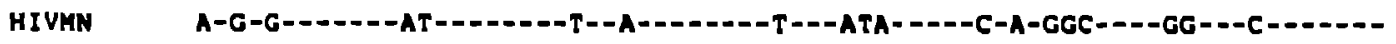

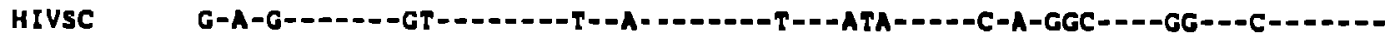

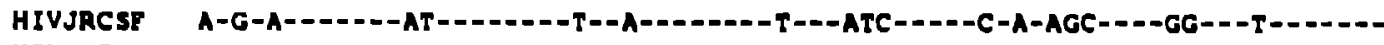

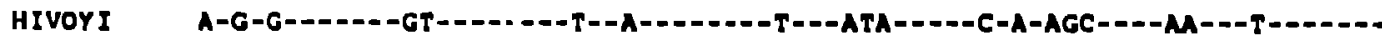

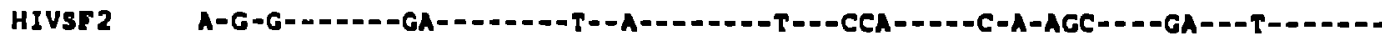

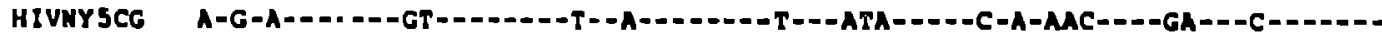

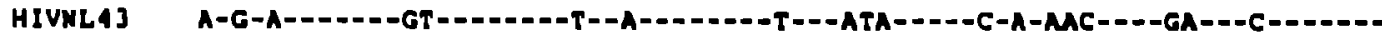

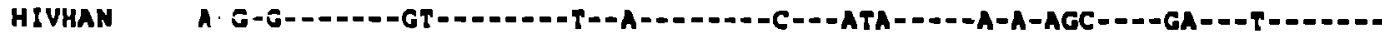

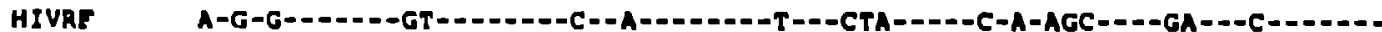
CONSENSUS 2 AYGAGCTTMGAgtGMAGCTGTI AGACAE TTTCCTAGGatATGGCTCCATAGCTTAGGACAMCATATCTA

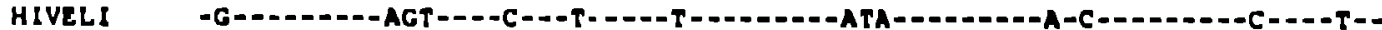

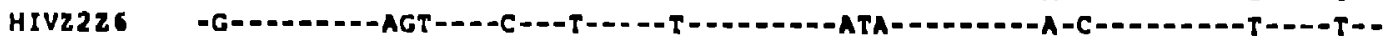

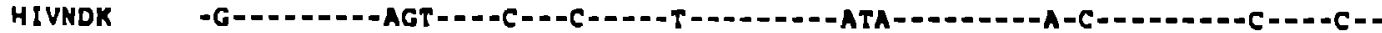

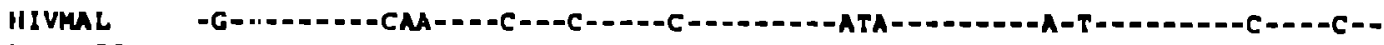

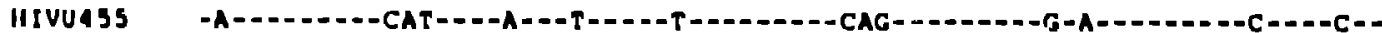
SIVCPZ -A--AT-A--AAAT----CA--A-----T--C-----ACCA---T-G--CAA----D---TTC--T--

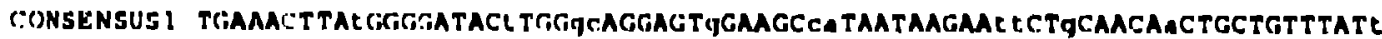

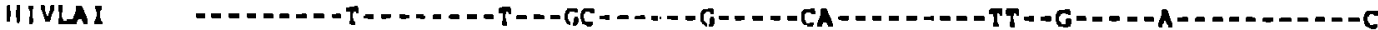
HIVHXB2R HIVMN IIIVge IIIVJRC.SF HIVOYI HIVsr 2 IIIVNYSCG HIVNLA3 IIIVHAN

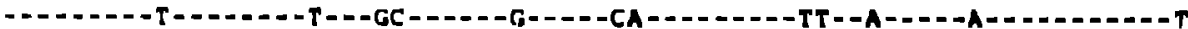

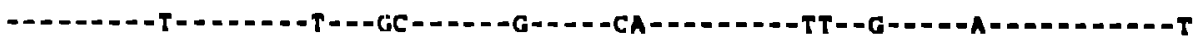

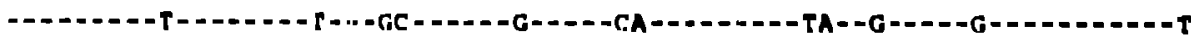
- .

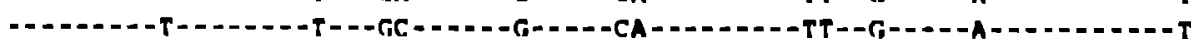
-

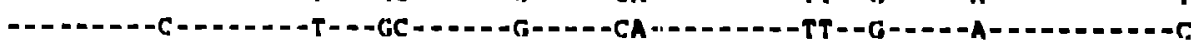

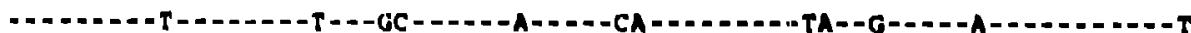

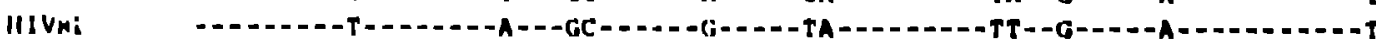

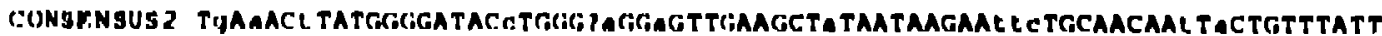
Hivili

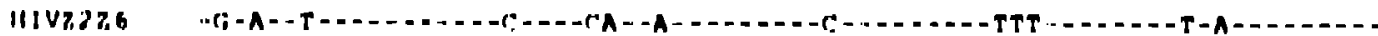

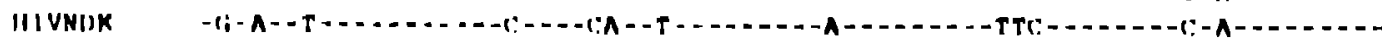

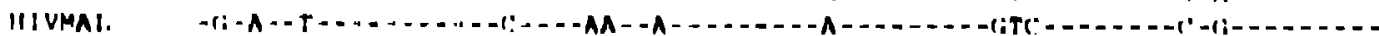

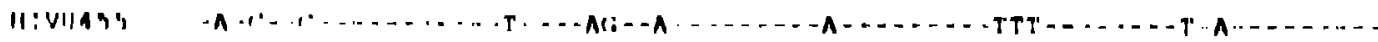

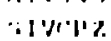


HIV-1 VPR

$1 / 3^{\prime}=1$

tat cds $\rightarrow$

\begin{tabular}{|c|c|c|}
\hline & 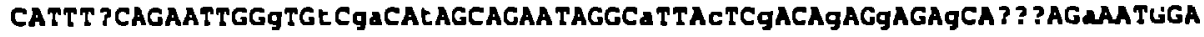 & 276 \\
\hline IVLAI & 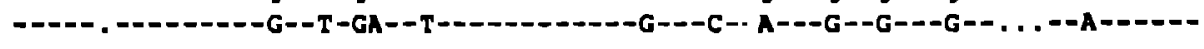 & 276 \\
\hline CVHXB2R & 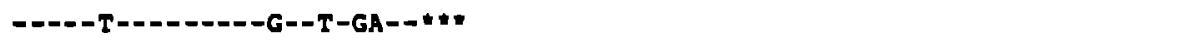 & 236 \\
\hline IVMN & $---A---2--G---G--G---C$ & 276 \\
\hline IVsc & 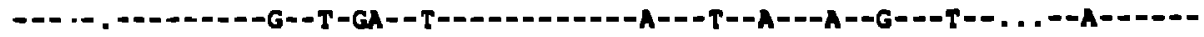 & 276 \\
\hline IVJRCSF & 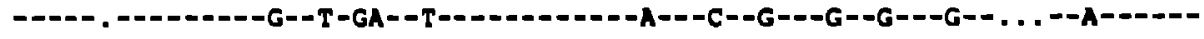 & 276 \\
\hline IVOY I & 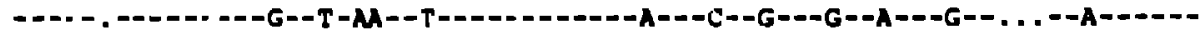 & 276 \\
\hline IVSF 2 & 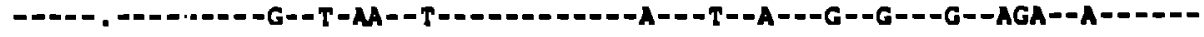 & 279 \\
\hline [VNYSCG & 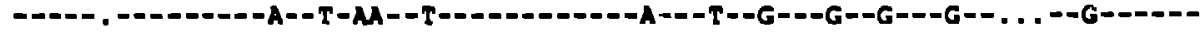 & 276 \\
\hline IVNL43 & $-G--T-G A--T-=--$ & 276 \\
\hline IVHAN & $---G--c$ & 276 \\
\hline IVRT & 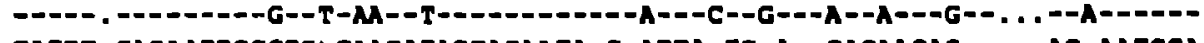 & 276 \\
\hline ONSENSUS2 & TTGGGTGECMACATAGCAGAATAgGC & 275 \\
\hline IVELI & - & 276 \\
\hline IV2226 & 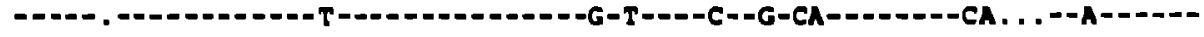 & 276 \\
\hline HIVNDK & 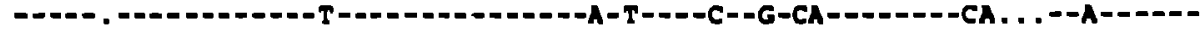 & 276 \\
\hline IIVMAL & - & 276 \\
\hline IVU455 & 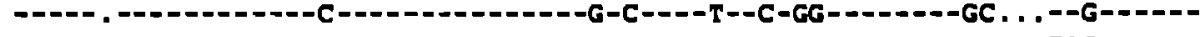 & 276 \\
\hline SIVCPZ & $-G-C--\operatorname{cCTC}-C-C N A--2--A$ & 276 \\
\hline
\end{tabular}

CONSENSUS1 gCCAGTAGatCC*"* 291

HIVLAI G-......... 291

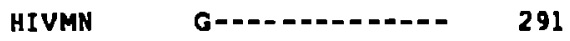

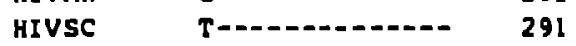

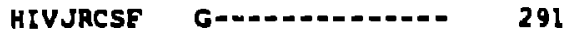

HIVOYI G-.-Den 291

HIVSP 2 G-_.-_...... 294

HIVNYSCG G-_. 291

HIVNLA3 G-_......... 291

HIVHAN G-... 291

HIVRF G-...-.......- 291

CONSENSUS2 tCCAGTAGATCC". 290

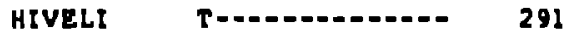

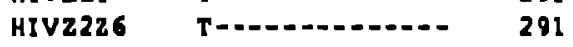

HIVNDK T-D.-De-1 291

HIVMAL T-1.-291

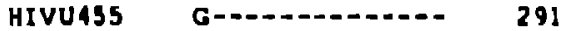

SIVCPZ T-.A-...... 291 
<- UPR

CONSENSUS1 ATGGAgCCAGTAGATCCTAgACTAGAGCCCTGGMAGCATCCAGGMGECAGCCTAAgACEgCTEGLACCA HIVHXB2R HIVNL43 HIVMN

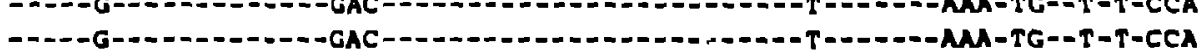

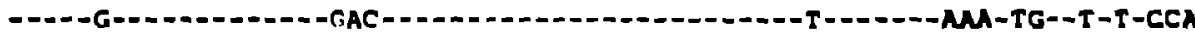

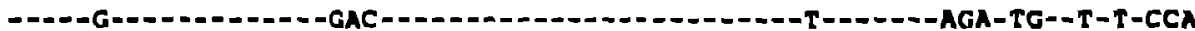

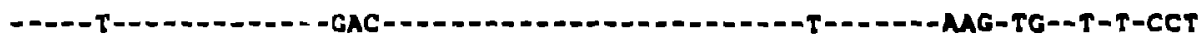
-

HIVBALI HIV JRCSF HIVOYI

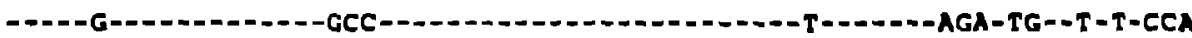

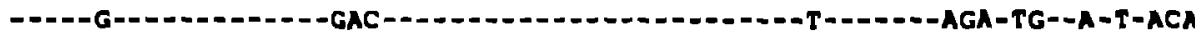

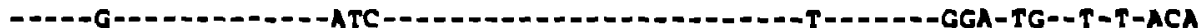

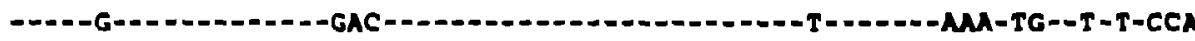

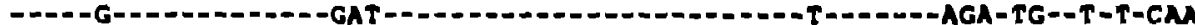

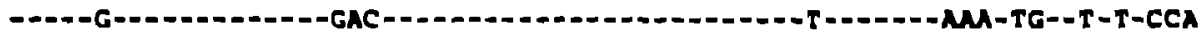

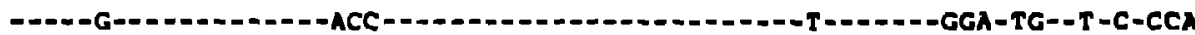

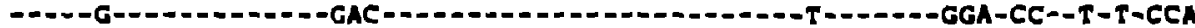

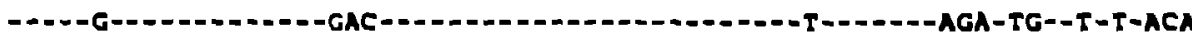
HIUSF162 HIVCDC4 HIVSF 33 HIVHA CONSENSUS: ATGGAECCAGTAGATCCTAACCTAGAGCCCTGGAACCALCCAGGAAGTCAGCCTAgGACL gCTTGTAACA

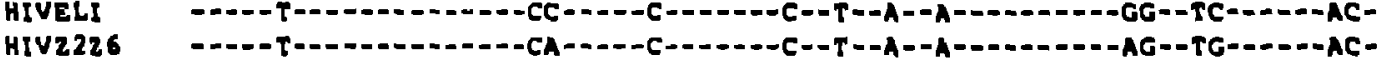
-

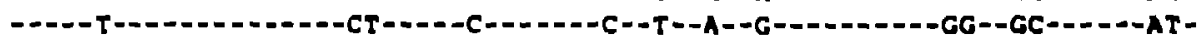

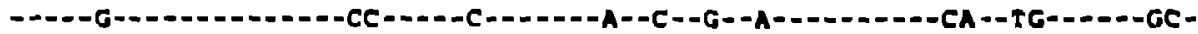
HIVMAL Hivuass

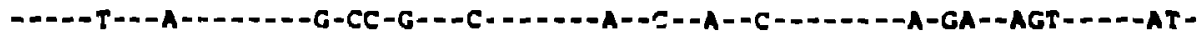

$1 / 3 \cdot-j / 3,=1$

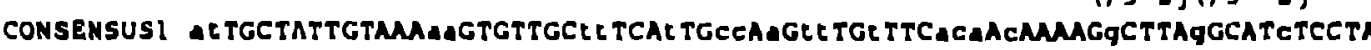

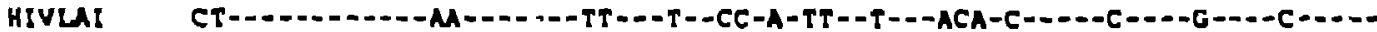

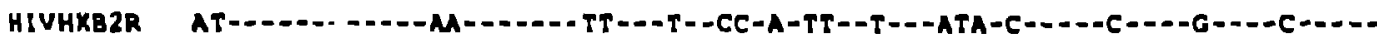

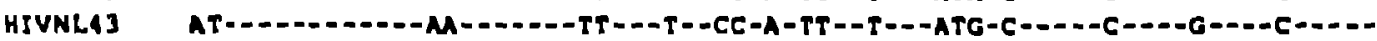
HIVHY

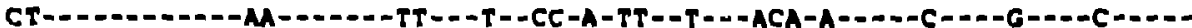

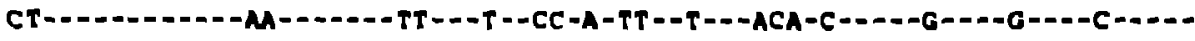

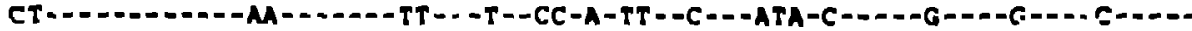
HIVSC HIVBALI HIVJRESF HIVOY I

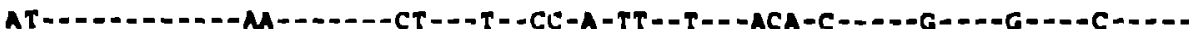

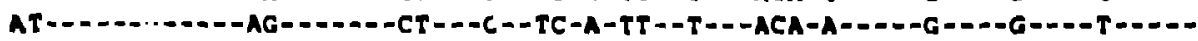

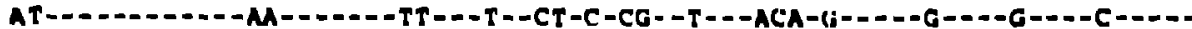

IITU5T2 HIVNYSCG HIVSF 162 HIVCDCA

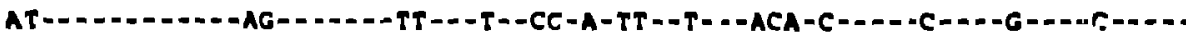

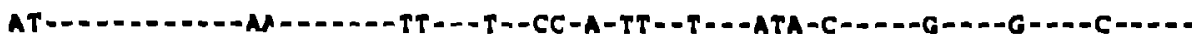

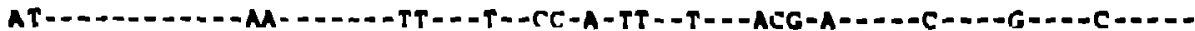

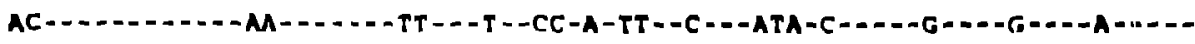

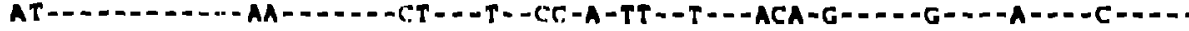
IIVIIAN IIIVRT AT - - - . -

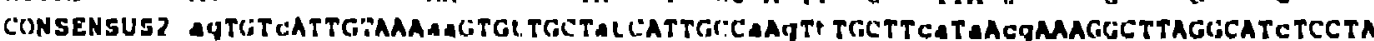
IIIVELI IIIVZ226 IIIVNOK IIIVIAL

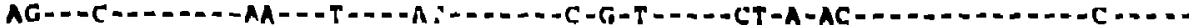

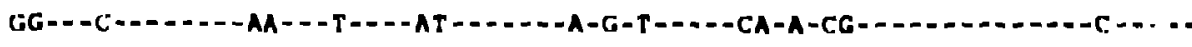

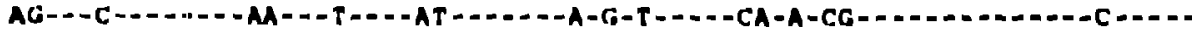

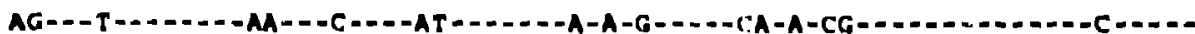
IIIVuass

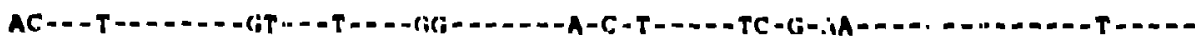

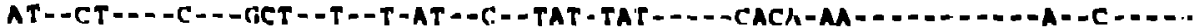


rev eds $\rightarrow \quad 1 / 3$, is

CONSENSUS1 TGGCAGGAAGAGCGGAGaCAG. . CGAggaaGAgCTCCTCaAgaCAGT CAGaCTCATCAAGEtTCTCTA HIVLAI -----------C----A---....--CGAA--C---C-CAAGG----C--AC--.--.--TT---C--

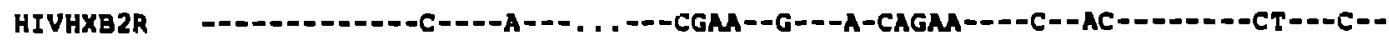

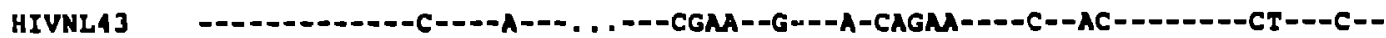
HIVMN HIVSC

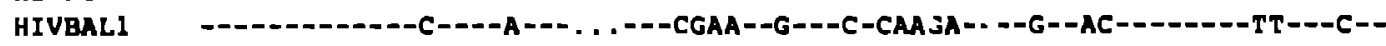

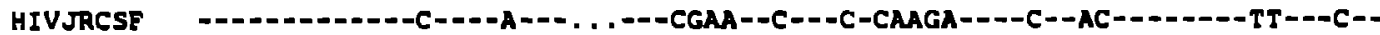

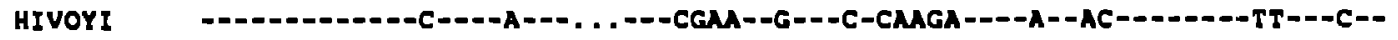
HIVSP2 -

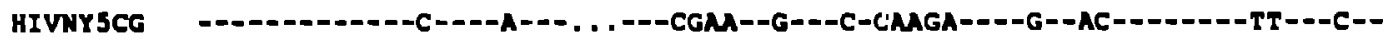

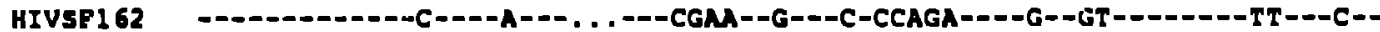

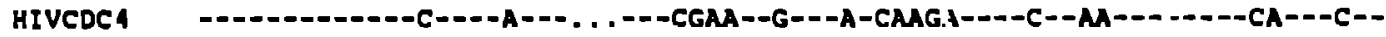

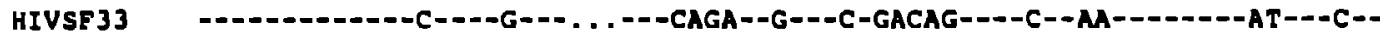
HIVHAN -----------C----A---...--CGAA--G---C-CAAGA----C--AC--1----TT---C-HIVRF CONSENSUS2 TGGCAgGMGAGCGGAgACAg. . . CGACGAAgACCTCCTCAAGGCggTCAGgeTCATCMAg?TCcTaTA

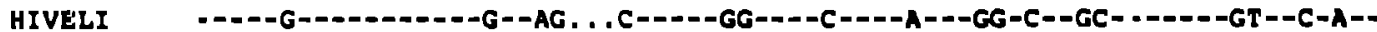

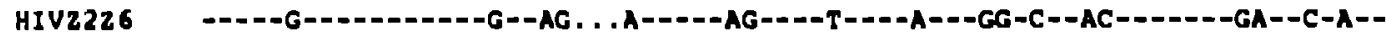

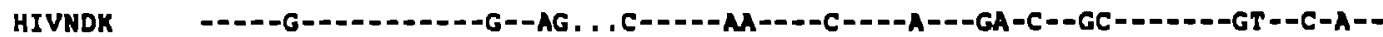

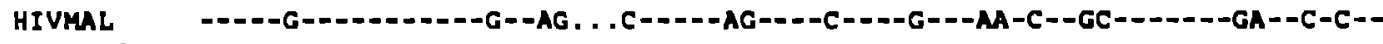

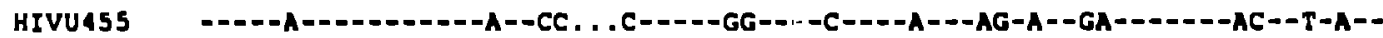
SIVCPZ --_--G--2.-1--A-CCACAAGAC----CACGG--C--GCA---AG-A-NAA-A-----GA-TC-A-exon \/exon

1- env $\rightarrow$ (for more Information, sae the HIV-1 ENV cDS that follows)

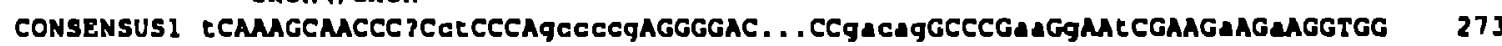

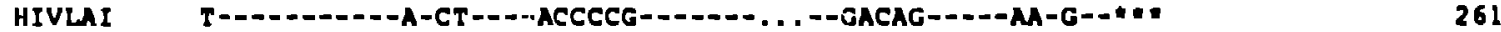

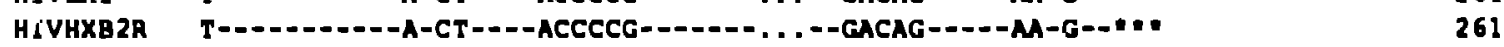

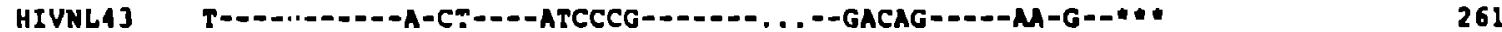

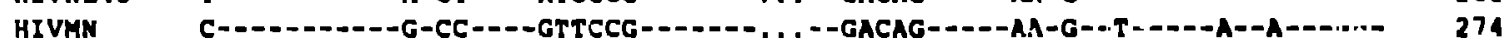

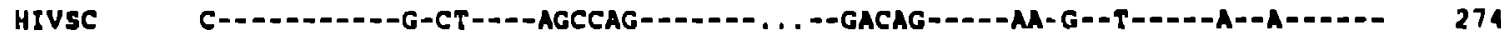

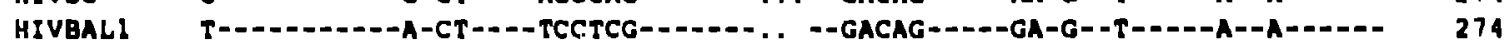

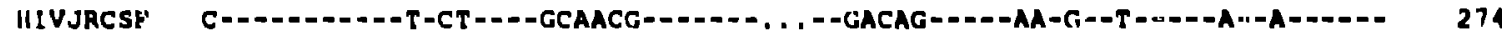

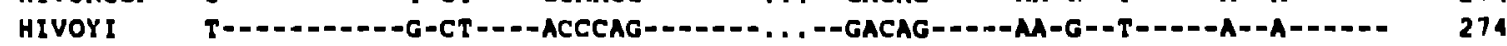

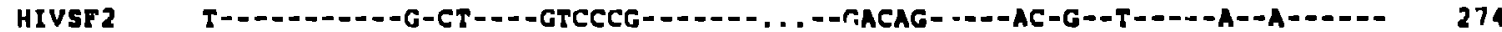

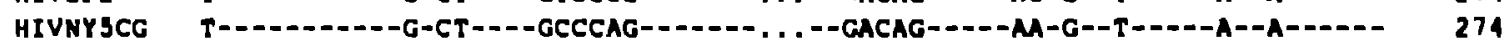

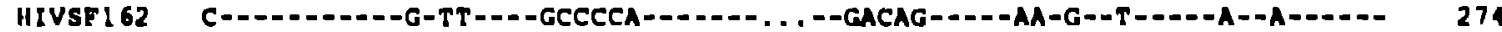

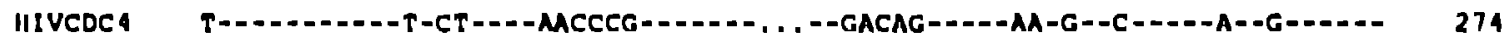

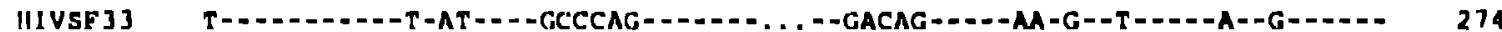

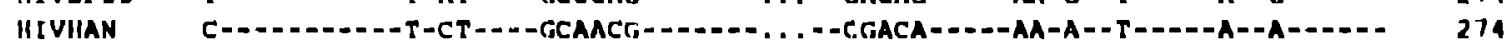

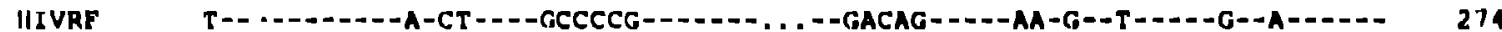

CONSENSUS2 CCAAAGCAACCCTCCECCCAYCCCCYAgGGGAC? ?CCGACAGGCCCGAAggAM? ?GAAGAAGMGGTGG $2 \%$

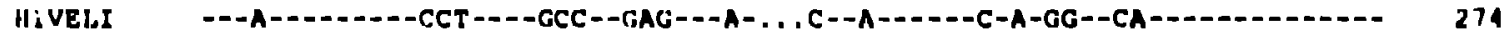

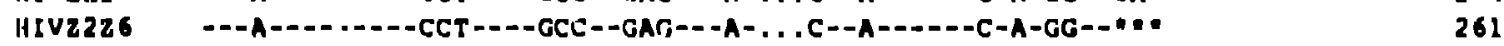

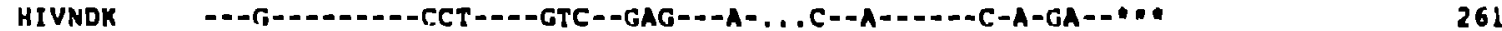

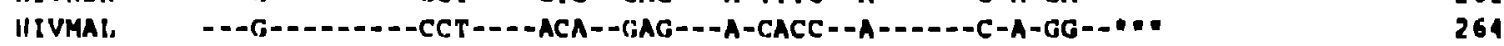

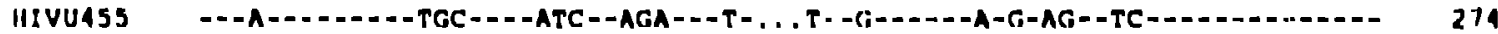

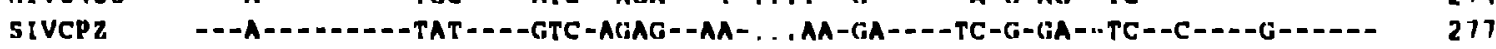




\section{HIV-1 TAT}

CONSENSUSI AGaGAGAGaCAGAGaCagATCCggtcgat??? 307 HIVMN $\quad$-A-----A----A-AC---GGTCGAT"* 306 HIVSC $\quad-$ A-D---A-----A-AG---GGTCGAT*\# 306 HIVBALI - A-----A----A-AG---GGTCCAT*\# 306

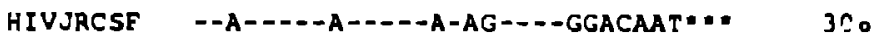
HIVOYI --A----A-----A-AG---GGAAGAT*\# :06 HIVSE 2 - A-----A---..A-A/,---GTTCGAT" " 306 HIVNYSCG --A----A----A-AG---GGGCCAT"* 306 HIVSF 162 --A----A-----A-AG----AGTCCAT"\#" 306 HIVCDC4 --A-----G----A-GG---ACTCGAT"* $3: 6$ HIVSF 33 --A-----A----A-AG----ACTCGAT** 306 HIVHAN --A-----A-----G-AG----GTTCGAT*" 306 HIVRF --A-----A-----A-AG----GGCGGTGCAG**" 309 CONSENSUS2 AGAGC?AGGCA?AGACAGATC????? 298

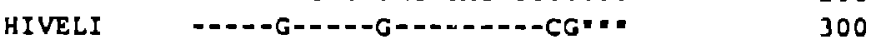

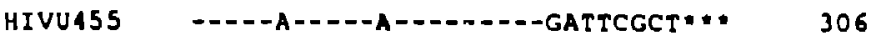

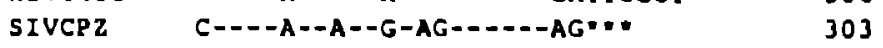


tat $\rightarrow \quad \backslash / J^{\prime}, j$

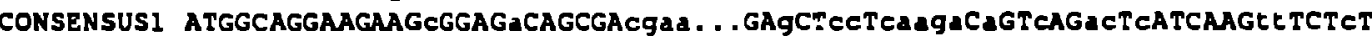

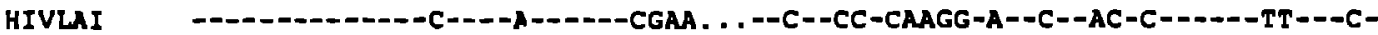

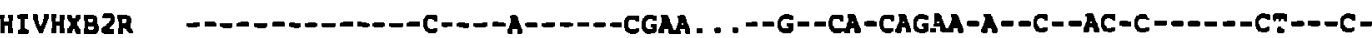
-

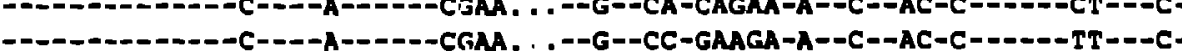
--G--CC-GAAGA-A--C--AG-C------TT---CHIVBRVA HIVSe HIVJH3 HIVEALI HIVJRCSE HIVOYI HIVSF 2 HIVNY5CG HIVSF162 HIVCDC4 HIVSE33 HIVHAN HIVRF

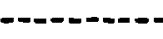
-

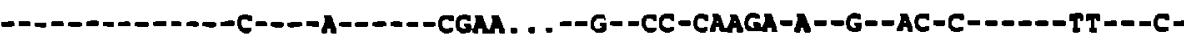

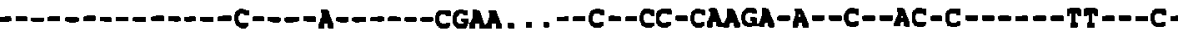
-

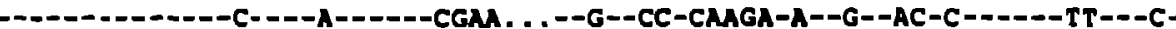

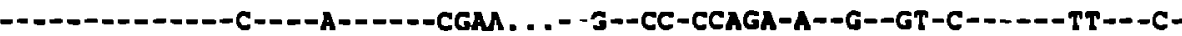

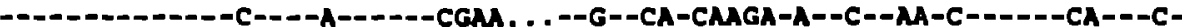

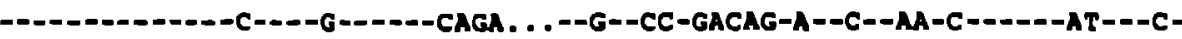

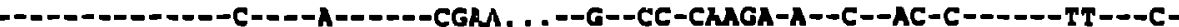
CONSENSUS2 ATGGCAgGNAGNAGCGGAgACAgCGACGA . . . gACCTCCTCAAGGCggTCAGgCTCATCAAg?TCeTaT HIVELI _HIV2226 HIVNDK HIVMAL HIVU455

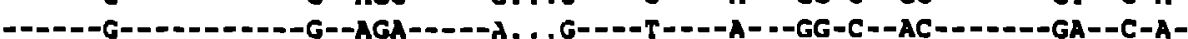

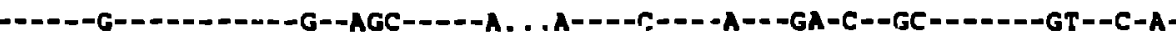

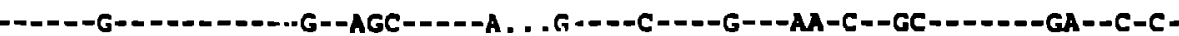

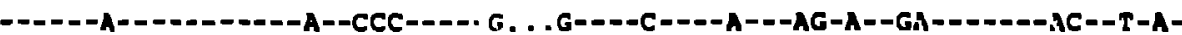
SIVCP 2

1- onv $\rightarrow$ (for more Intormation, sen the HIV-1 ENV COS that follows) exon \/exon

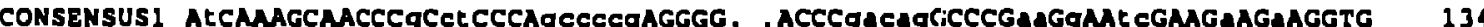

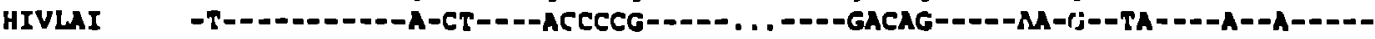

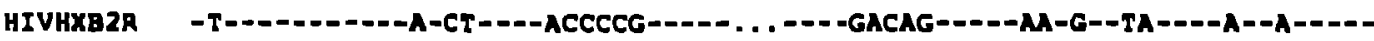

HIVNL 43

HIVMN

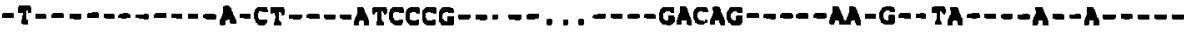

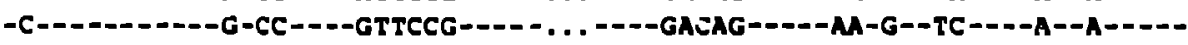

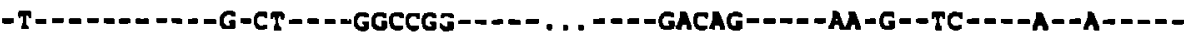

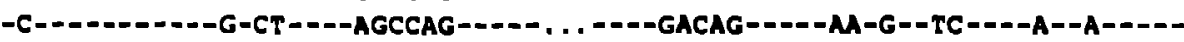

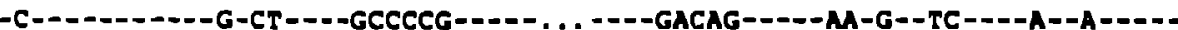

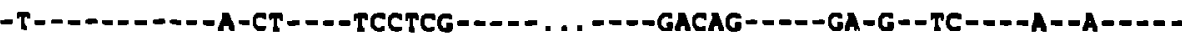

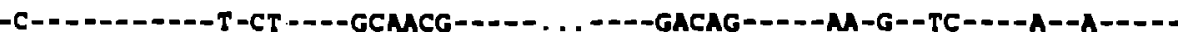

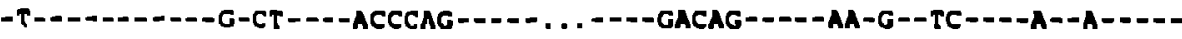

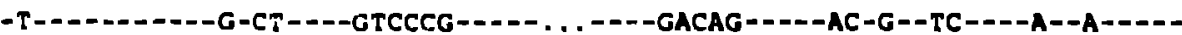

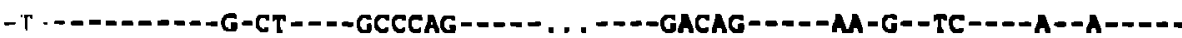

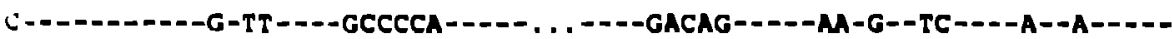

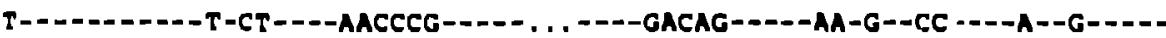

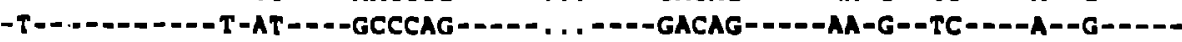

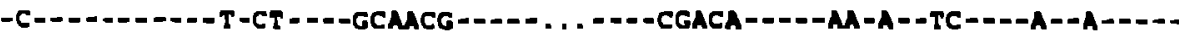

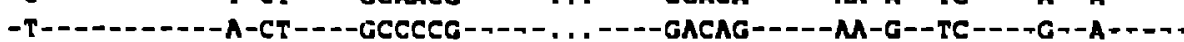


CONSENSUS1 GAGagaGAGaCAGAGACagATCCggtegattAGTgaatGGaTtcTragCactLaTCTGGGacGALCtgeG HIVLAI ---AGA---A-----A-AG----ATTCGATT---GAAC--A-CC--AG-AC-TA------AC--TCTGCHIVHXB2R ---AGA---A-----A-AG----ATTCGATT---GMAC--A-CC--GG-AC-TA---_--AC--TCTGCHIVNLA 3 ---AGA---A-----A-AG----ATTCGATT---GAAC--A-CC--AG-AC-TA---.- -AC--TCTGCHIVHN ---AGA---A-----A-AC----GGTCGATT---GCAT--A-TC--AG-MA-TA------TC--CCTGCHIVBRYA ---AGC---A----A-AG----AGTCCCTT---GGAT--A-TC--AG-AC-TT------TC--TCTGCHIVJH3 HIVEALI HIVJRCSE HIVOYI HIVSF 2 HIVNY5CG HIVSF 162 HIVCDC4 HIVSF 33 HIVHAN HIVRF --AGA--A----A-AG---GGTCGAT---GGAT--A-TC--AG-AM-TA---.--TC--CTAGC---ACA---A--_--A-AG----GGTCMATT---GGAT--A-TA--AG-MA-TA-_-_-_-TC--CCTGC---AGA---A-----A-AG-_--GGTCCATT---GAAC--A-TC--GG-GC-TA------TC--TCTGC---AGA---A-----A-AG----GGACAATT---GAAC--A-TC--AG-AC-TA------TC--CCTGC---AGA---A-----A-AG----GGMAGATT---GGAT--A-TC--AG-AC-TA------AC--TCTG=---AGA---A-----A-AG----GTTCGATT---GGAT--A-TC--AG-AC-TA--_---AM--TCTGC---AGA---A-----A-AG----GGACCATT---GAT--A-TC--AG-AC-CA--_-_-TC--TCTGC---AGA---A-----A-AG----AGTCCATT---GCAT--A-TA--AG-AC-CA------AC--TCTAC---AGA---G-----A-GG----ACTCGATT---GCAT--C-TC--AG-AC-TG------AC--TCTGC---AGA---A-----A-AG----ACTCGRTT---GAAC--A-TC--AG-AC-GT------AC--TCTIC---AGA---A-----G-AG----GTTCGATT---GAGC--A-TC--AG-AC-TT------AC--CCTGC---AGA---A-----A-AG----GGCGGTGC---GAAT--A-TC--GA-AC-TA------AC--TCTGTCONSENSUS2 GAGAGCgAGGCAgAGaCAGATCCATt CGATTggTGACGGATTCTCAGCACTTATCTGGACGACCTGaG

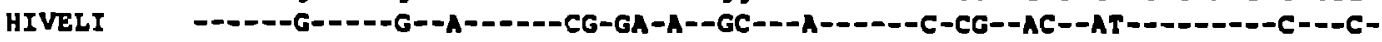
HIVZ226 HIVNDK HIVMAL HIVU45S

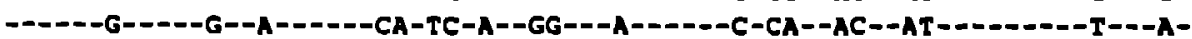

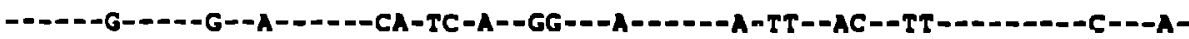

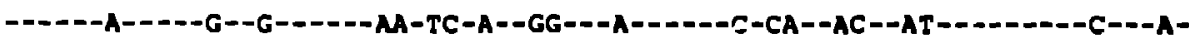

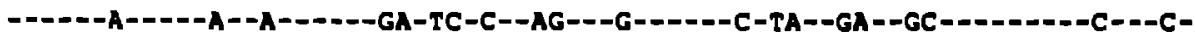
SIVCP z $-C---A--A--G-A G-----A G-G A-A--A G--G A A--G-G C-T G C-A C--A T-\ldots-----C--C C-$

CONSENSUS1 GAgCeTGTgCctCTTCAGCTACCACCgCttgAgAGACTTACTCTrGAtGgagegAgGAt TGTGGACt T HIVLAI HIVHXB2R HIVNL 43 HIVMN HIVBRVA HIVSC HIVJH3 HIVBAL1 HIVJACSE HIVOYI HIVSF 2 HIVNYSCC HIVSF 162 HIVCDCA HIVSF 33 HIVHAN II URE - -G-_.-G-CT-_- - -

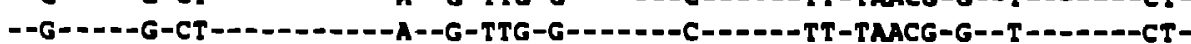

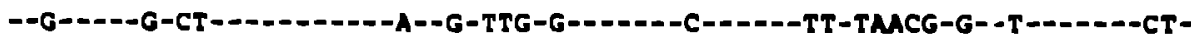

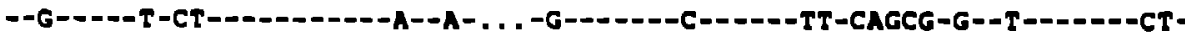

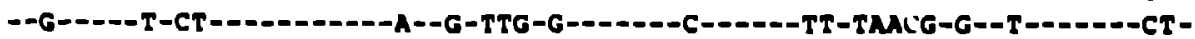

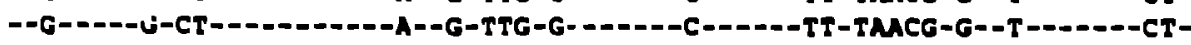

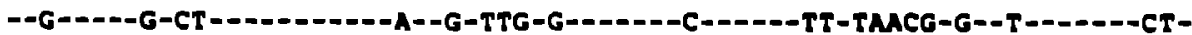

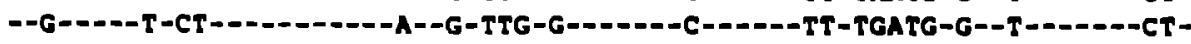

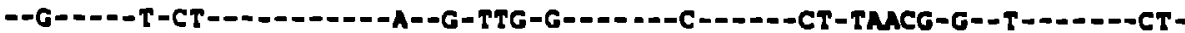

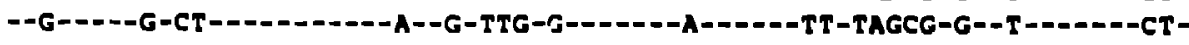

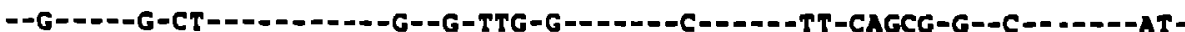

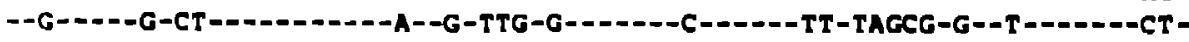

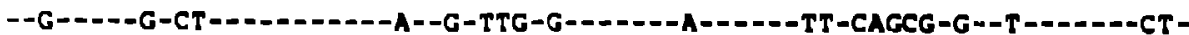

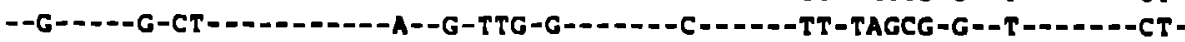

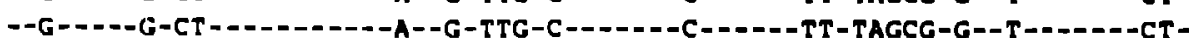

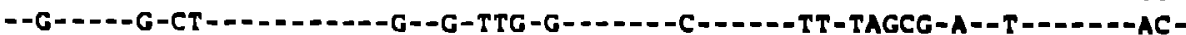

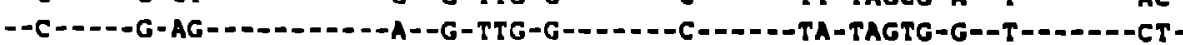
CONSENSUS2 GAACCTGTGCCTCTTCAGGTACCACCGCTTGAGAGACTEAACTTAATGCAgCGAGgAETGTgGAACTT

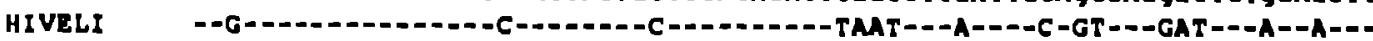
HIV2226 HIVNDK HIVMAL HIVU455

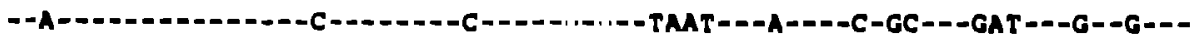

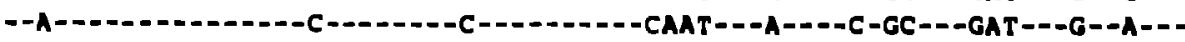

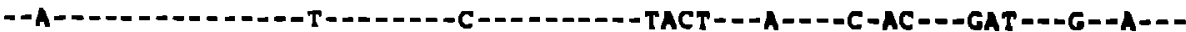

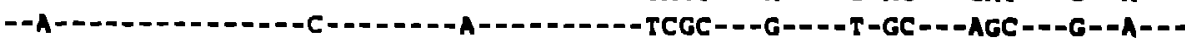
5 IVCP 2 
CONSENSUS1 CTGGGaCgCag. ............... ? ?GGGGTGGGAgCCCTCMATALTGgtGCAATCTCCT PC

HIVLAI

HIVHXB2R

HIVNL43

HIVAN

HIVBRVA

HIVSC

HIVJH3

HIVBAL1

HIVJRCSF

HIVOYI

HIVSE 2

HIVIY 5 CG

HIVSF1 62

HIVCDCA

HIVSE 33

HIVHAN

HIVRF

CONSENSUS2

HIVELI

HIV2226

HIVNDK

HIVMAL

HIVU 455

SIVCPZ
-

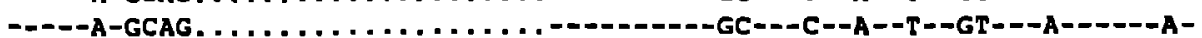
-----A-GCAG. . . . . . . . . . . . . . . - - ------GC---C--A--T--GT---A------A-

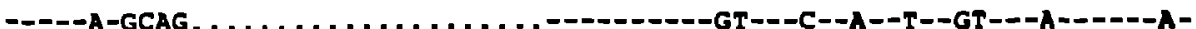

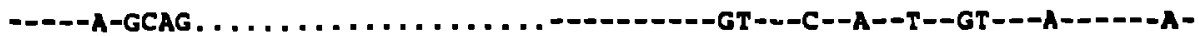

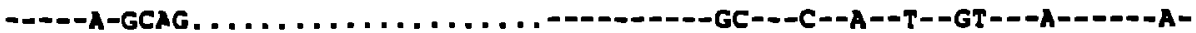
-.--A-GCAG. . .................-2.

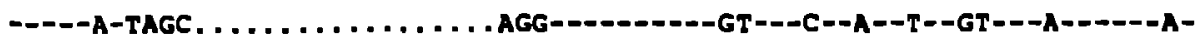

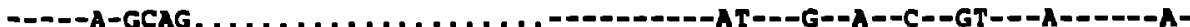

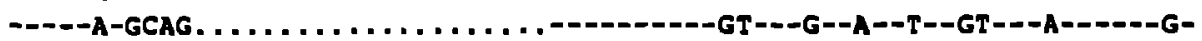

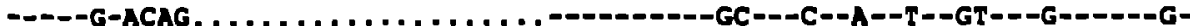

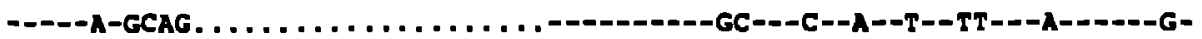

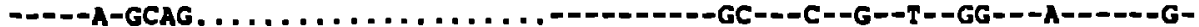

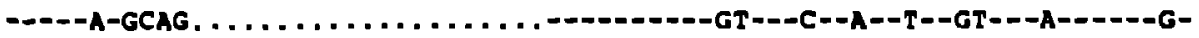

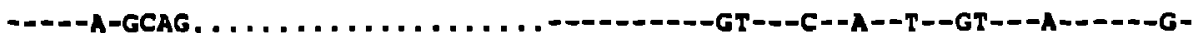

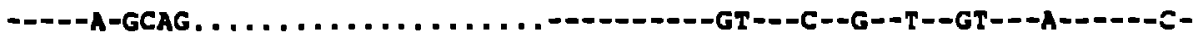

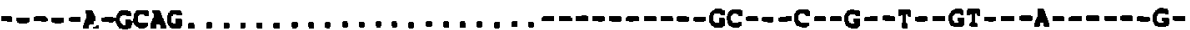

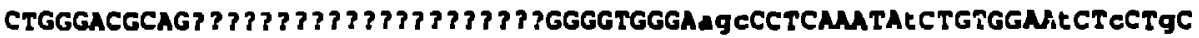

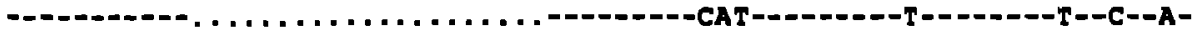

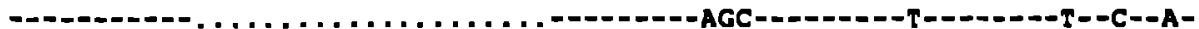

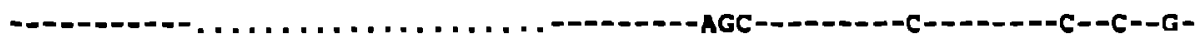
-

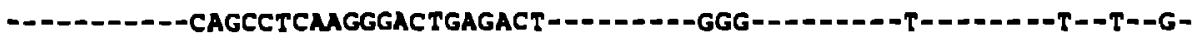
T------ATCTAATACTTCACAGCCTCAGACT-CTAC----GAGA--GTGCCTG---G--GGM-TA-T-

CONSENSUSI agTATTGGagtCAGGACTAAaGA ** 350

HIVLAI AG------AGT-----A----A--A--- 351

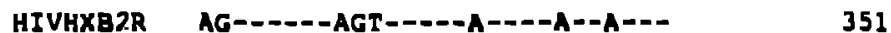

HIVNL 43 AG------AGT----A--D-A--A--- 351

HIVMN AG------AGT-----A----A--G--- 348

HIVBRVA AG-D-A-AGT-D-A-A-D-A-A-D- 321

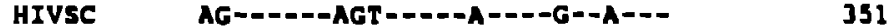

HIVJH3 MA------AGT-----A----A--A--- 342

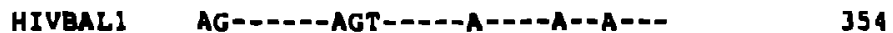

HIVJACSF AG-_-_-AGT-ב-A-A-A-A-A-D 351

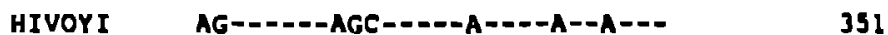

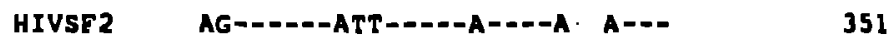

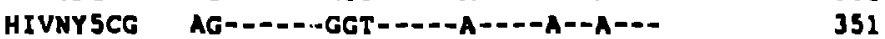

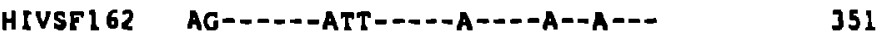

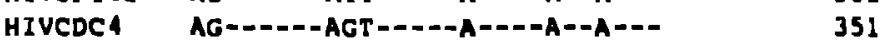

HIVSE 33 TG-D---AGT-D---A---A-A-A-D 351

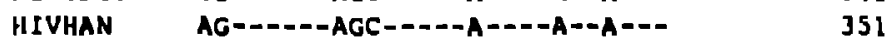

HIVRF AG---.-AGT--_-G-1--A--A--- J51

CONSENSUS2 AgTATTGGaGTCAGGAACTqAagAa PAGTGC*." 355

HIVELI AG------A---A------G-GG-AC-------- J57

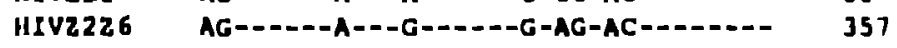

HIVNDK AG------A---A------G-GG-A**" J51

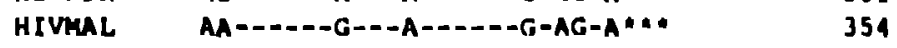

HIVUA55 TG------G---G-----A-AA-T*A 372

SIVCPZ M--N--G-MAA---G--A-M-T*** 375 
CONSENSUSI ATGCAACTTTICAAaTAT ?agCaAAGEAgCATTAGTAGTAGCAgCAATAdTAGCMATAgTTGTgTGg.

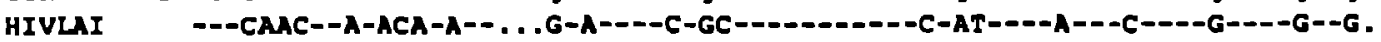

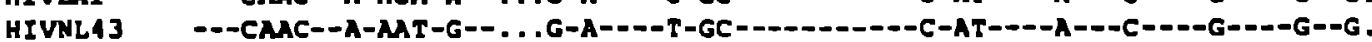

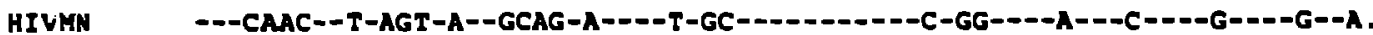

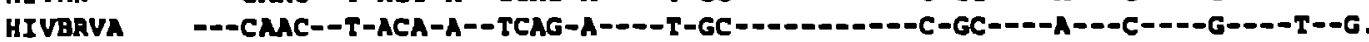
HIYSC

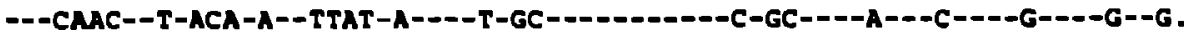

HIVJH3 HIVBALI HIVSE2 HIVJRCSE HIVNYSCG HIVSE 162 HIVHAN HIVRF

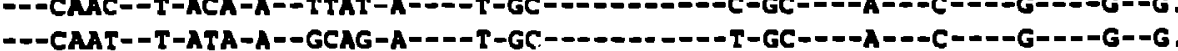

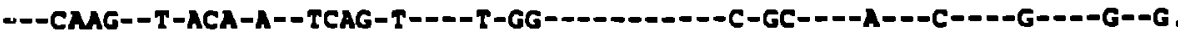

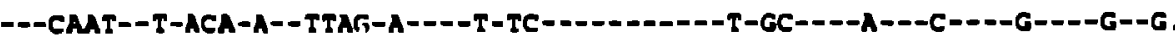
---CAAC--T-ACA-A--TTAG-A----T-GC-------D---C-GG----A---C----A----G--G.

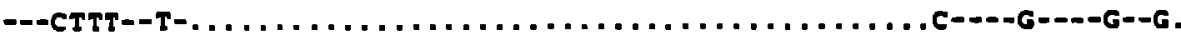

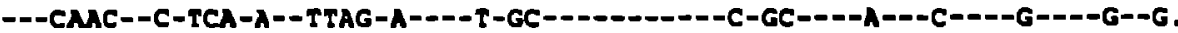
---TTAT--T-ACA-A--GCAG-A----T-GC-----------C-GC----A---T---G----G--G. ---CAAT--T-AGA-A-- TTAG-A----T-GC-----------C-GC----C---C---G---G--G. CONSENSUS2 ATgCAACCTTtAgtGATAatAGCMTAGcAGCATTAGTAGTAgCaAtATAatAGCAATAGTTGTGTGG. HIVELI HIV2226 HIVNDK HIVAL SIVCPz ATgCACCT TEAgEGATAATAGCNTAGCAGCATTAGTAGTAgCAATNATAaTAGCAATAGTTGTGTGG.

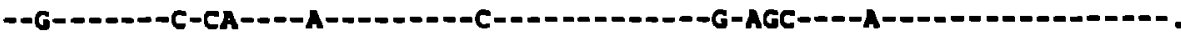

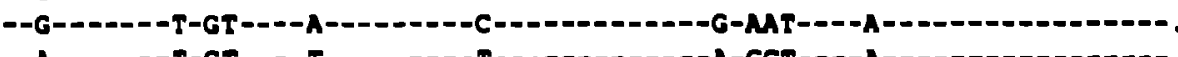

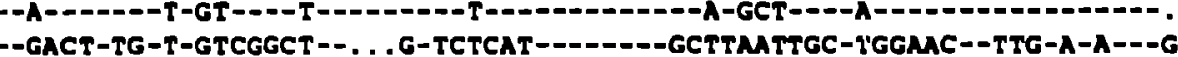

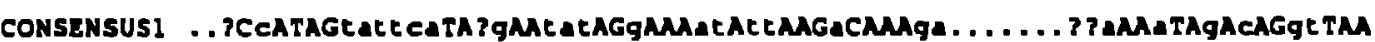
HIVIAI HIVNL4 3 HIVIN HIVaRVA HIVSC HIVJH 3 HIVBAL1 HIVSP 2 HIVJRESF HIVAYSCG HIVSF1 62 HIVHAN H:ERF

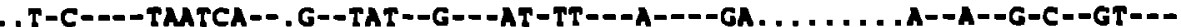
. T-C---TATCA--.G--TAT--G---AT-TT---A--O-GA. . . . . . .A--A--G-C--GT-.-

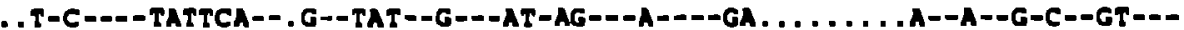
. T-C----CATTAC--. G--TAT--A---TT-TT---A----GG. . . . . . .A--A--G-C--GT--. .T-T----TATTCA--TT--TAT--G---AT-TT---A----GA . . . . . .A--A--G-C--GT---

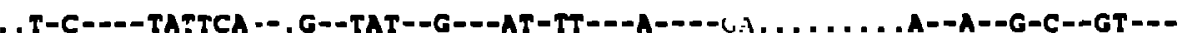
. A-C----TATTCA-- , G--TAT--G---AT-TT---G----GA . . . . . . .A--A--G-C--GT-.. .A-C--.-TACTCA-- , G--TAT--G---AT-TT---A----GA. . . . . . . .-A--G-C--AT-..

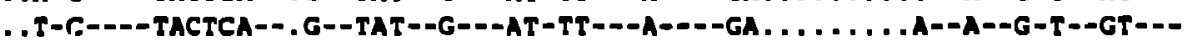

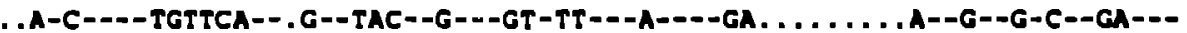
. A-C-... TGTACA-- . S--TAC--G---AT-TT---A----GA . . . . . . A-A-A--G-T--GT-.-

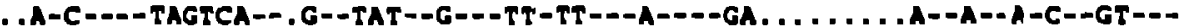
. .A-C----tTGGCA--. G--ATT--G---AC-TT---G----AA.... . . . AMA--A--G-C--GT---

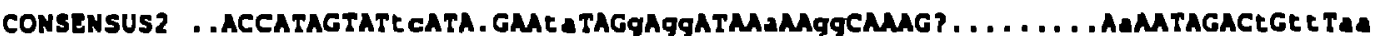

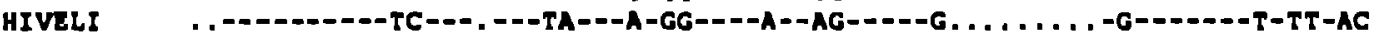

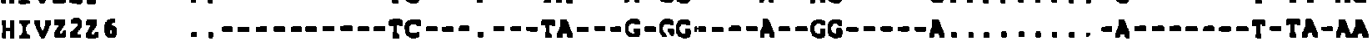

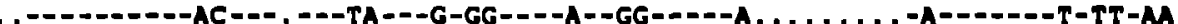

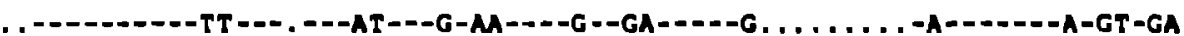
HIVMAL SIVCP2 GATA---TA-TMATGG. -G-TA---A-GGTAT-A--GA--T--GCTTGAGACAGA---T--GA-AC-TA

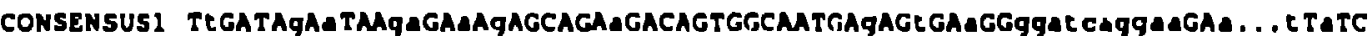

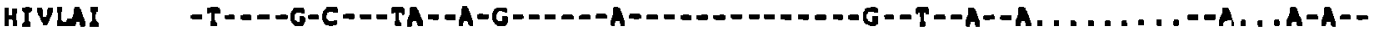

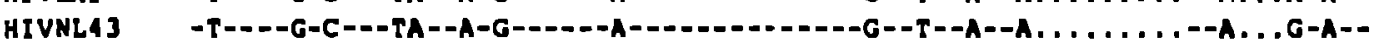
HIVIN HIVBRYA HIVSC НรบงH3 HIVBAL1 HIVSF2 HIVJRCSF HIVNY3CG HIVSFI 62 H IVHAN HIURF

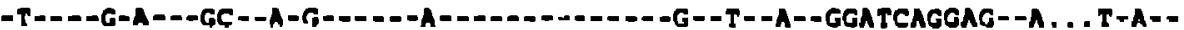
-A-_--G-A---GA--A-G-_-_-A--_-_-_-_--G--T--A--GGATCMAGA--A . . T-A--

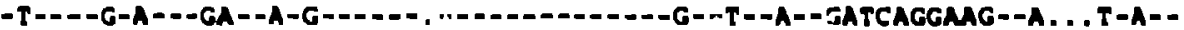

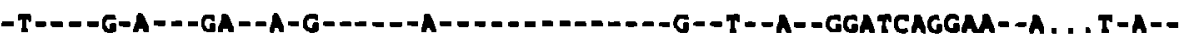

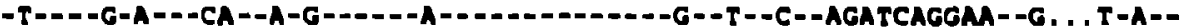

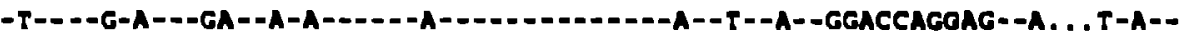

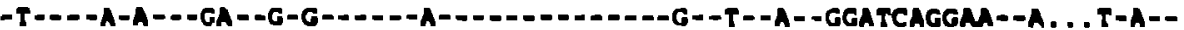

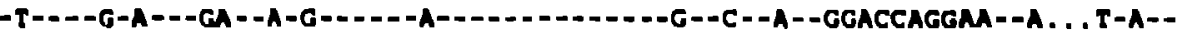

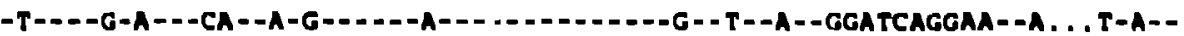

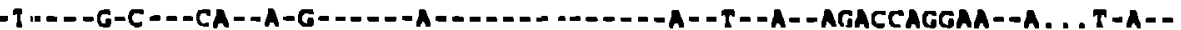

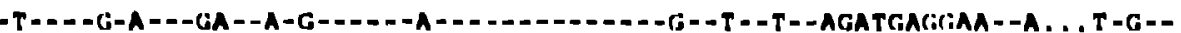

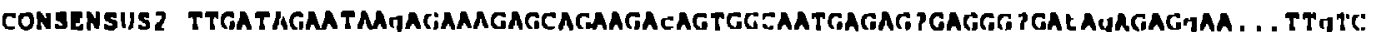
H IVI:L.I IIIVZ226

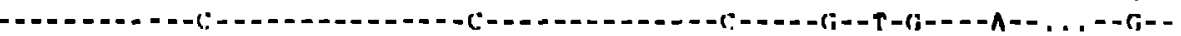
- - - - - - - - - - II I VNDK IIIVMAL

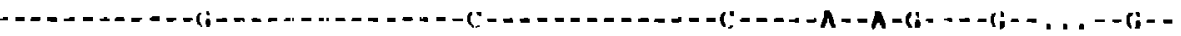
- - - - - - - - $\because$ IVi:P 2. 


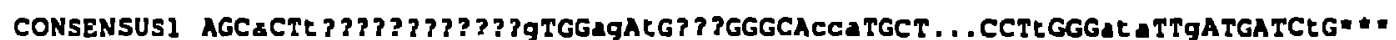
HIVLAI HIVNL43 HIVAN HIVBRVA HIVSC

HIVSH3

HIVBAL1

HIVSF 2

HIVJRCSF

HIVNY 5 CG

HIVSF 162

HIVHAN

HIVAF

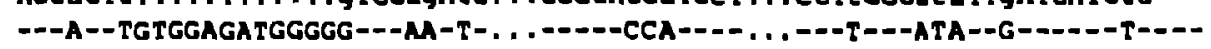

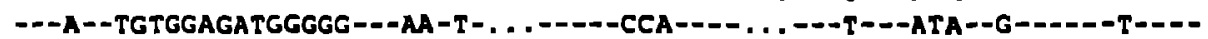

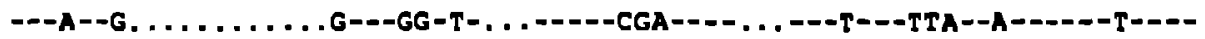

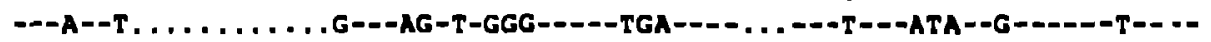

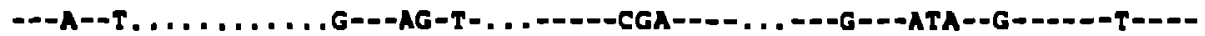

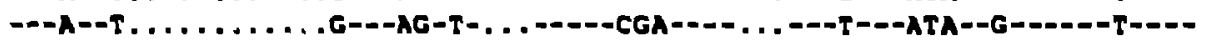

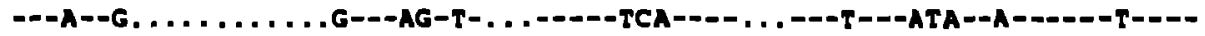

---A--T. . . . . . . . .G---AG-T-....----CCT--.... ---T---ATG--G------T--.-

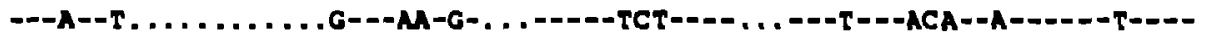

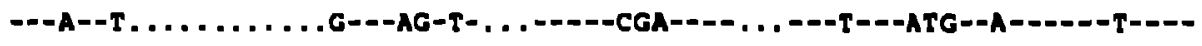

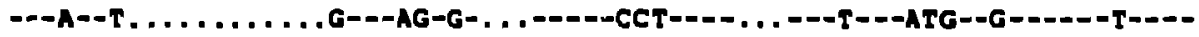

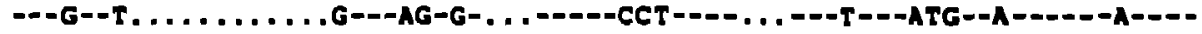

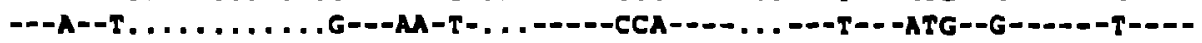

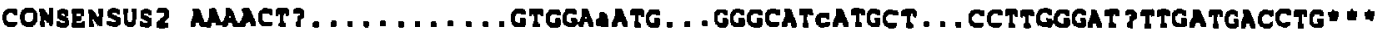

HIVELI

HIVZ226

HIVNDK

-..--G.............---A-_. . .

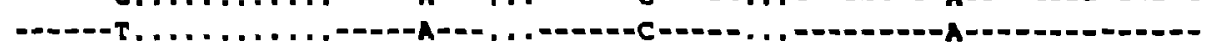

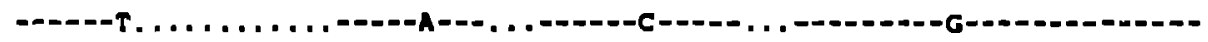

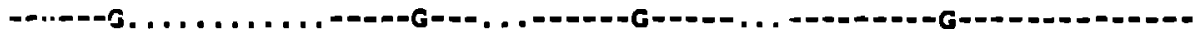




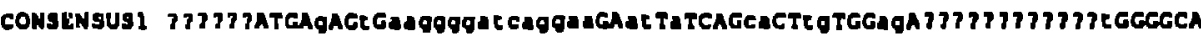
HIVLA

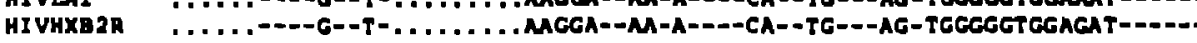

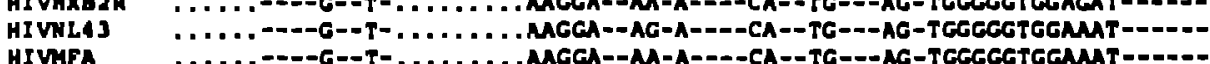
HIVI:

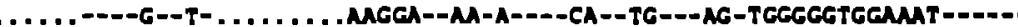

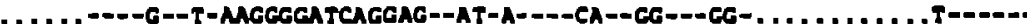

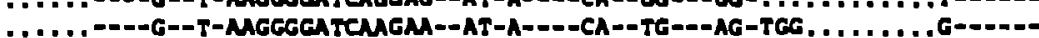
…

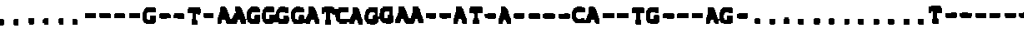

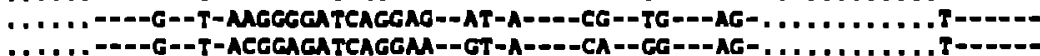

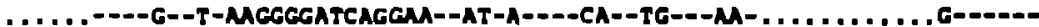

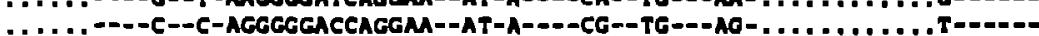

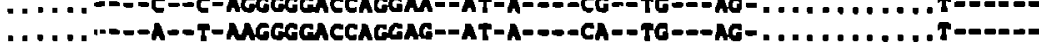

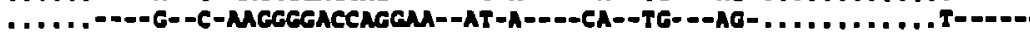

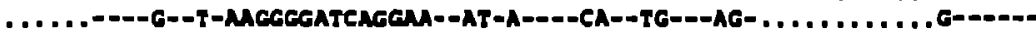

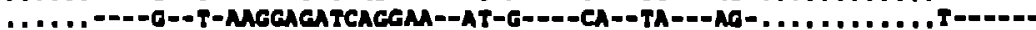

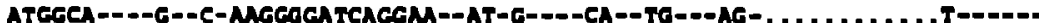

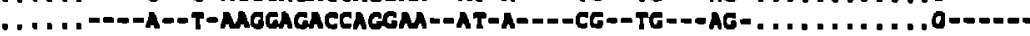

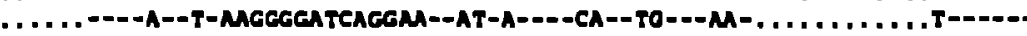
. . . . .---G--t-MGGGCATCATGAG--AT-G----CA--TG---AT- . . . . . . . . . T-.....

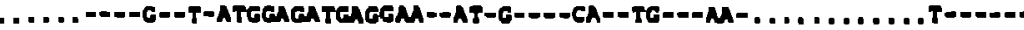

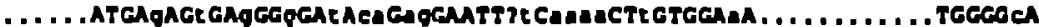

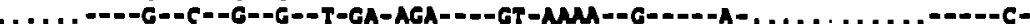
$\because$. . .

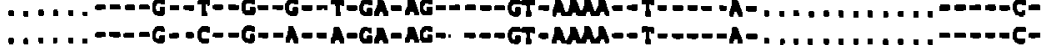
…

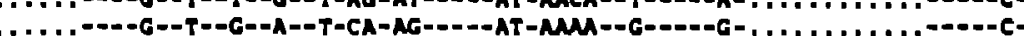

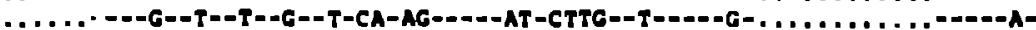

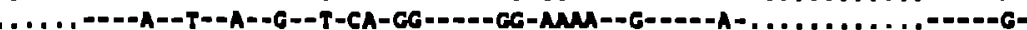

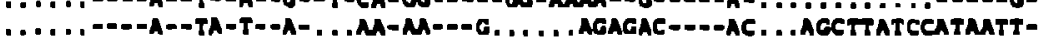
algnal popela l/ gplat

$$
\text { <- vpU }
$$

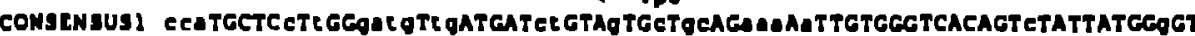

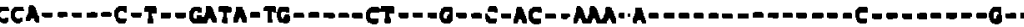

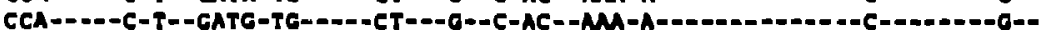

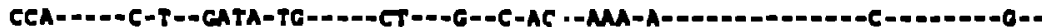
TCA-CA-

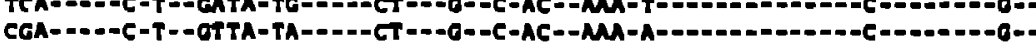

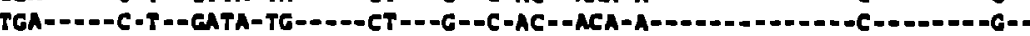

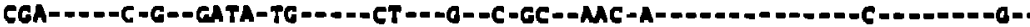

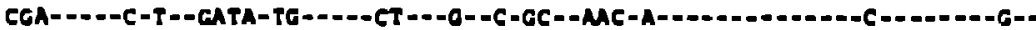

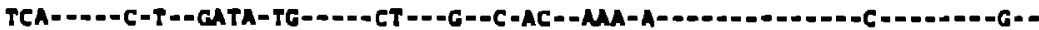

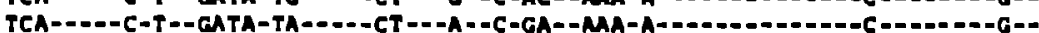

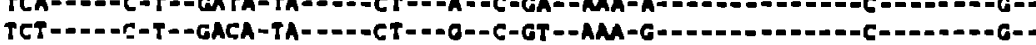

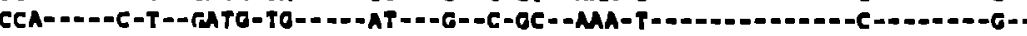

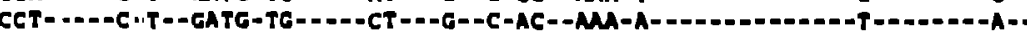

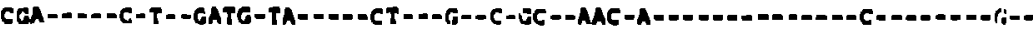

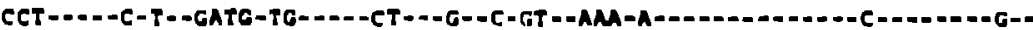

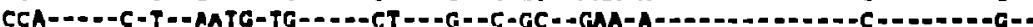

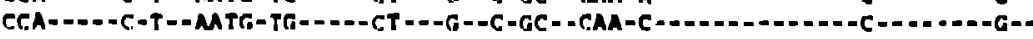

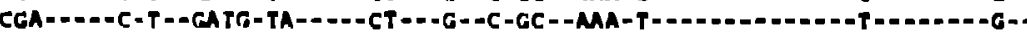

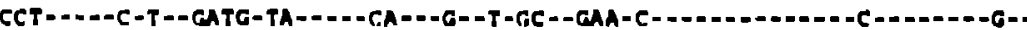

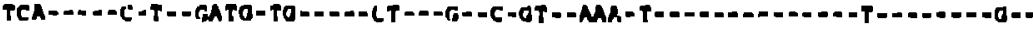

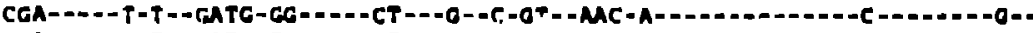

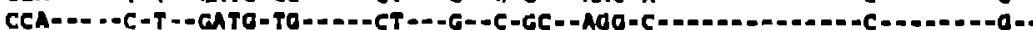

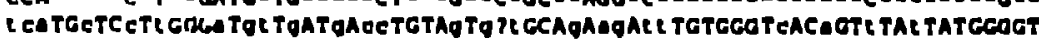

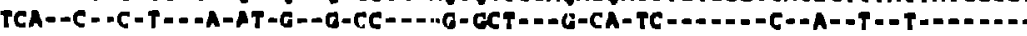

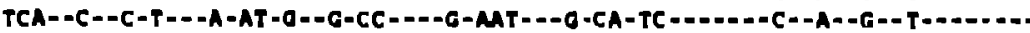

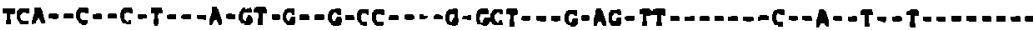

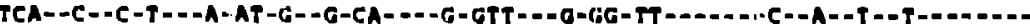

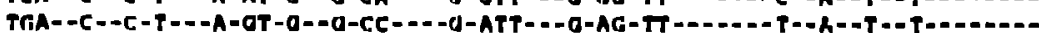

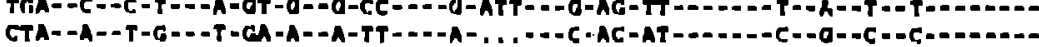

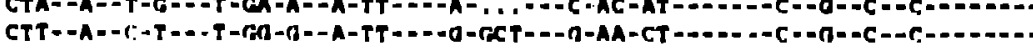

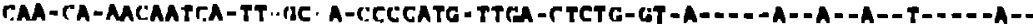


HIV.1 ENV

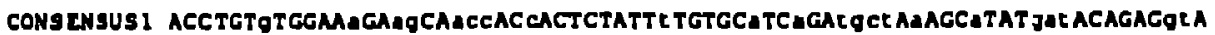

HIVLAI

HIVHXB2R

HIVNLAJ

HIVAFA

HIVAN

HIVBRVA

HIVSC

HIVJH3

HIVALAI

HIVBAL!

HIVJRCST

HIVoYI

HIVAF2

HIVIYSce

HIVSTIG?

HIVSTL

HIVedeA

HIVIFJ3

HIVHAN

HIVADA

HIVWM2

HIVRF ACCTCTOTCGNAGAngCA acCAC CACTCTATTETGTCCaTC

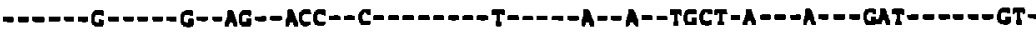

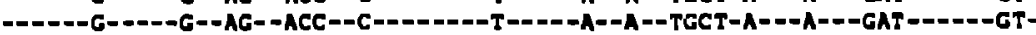

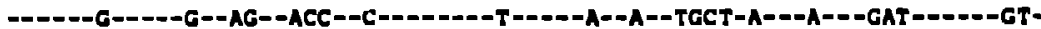

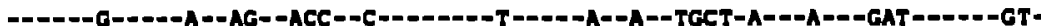

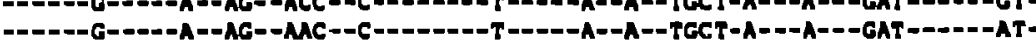

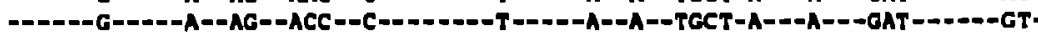

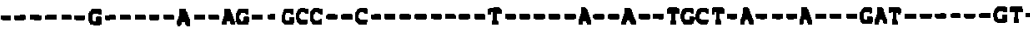
-1---G--1-A--AG--ACC--C---D--

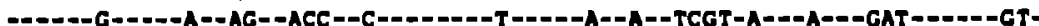

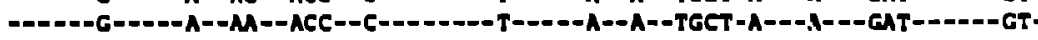

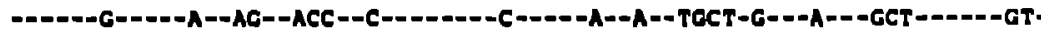

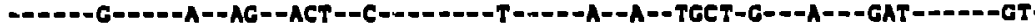

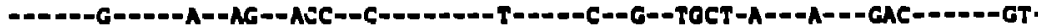

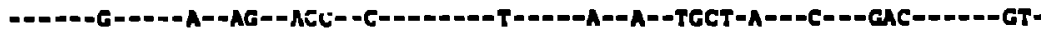

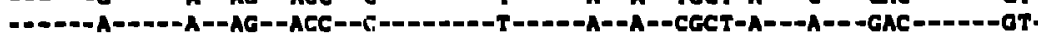

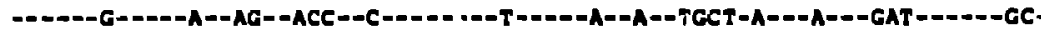

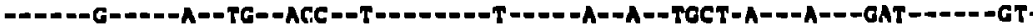

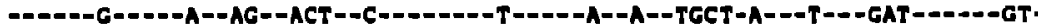

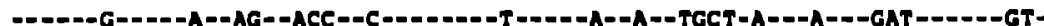

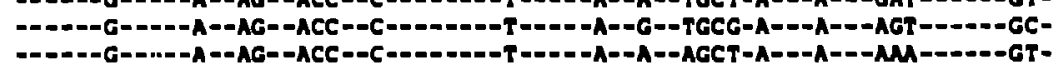

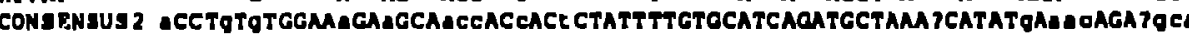

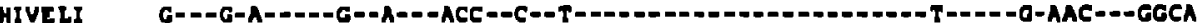
HIVI220 HIVNDK HIVJY! G-

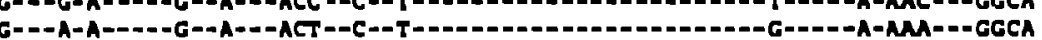

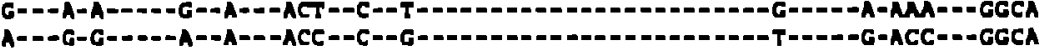

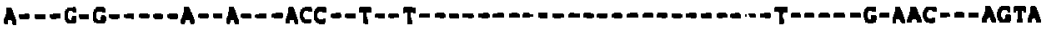

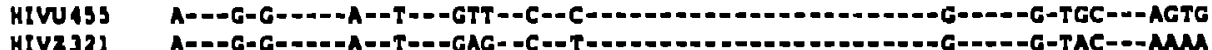

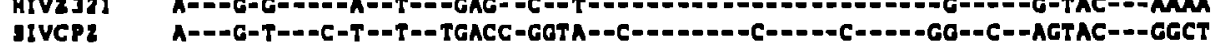

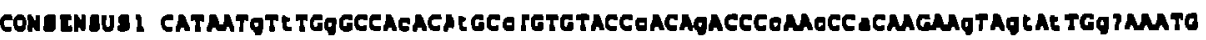
HIVLAl

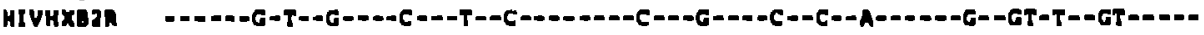

AIVILIS -

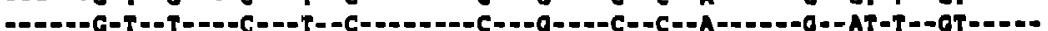

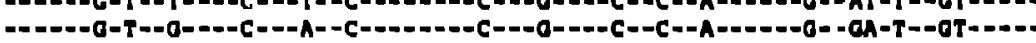

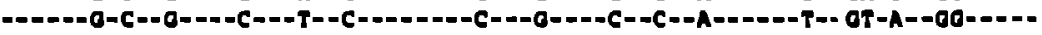

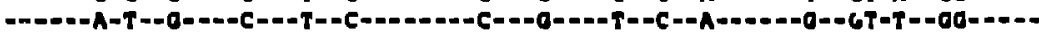

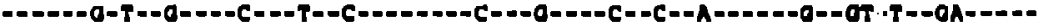

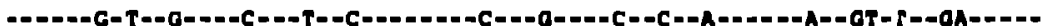

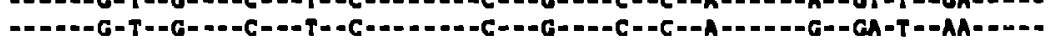

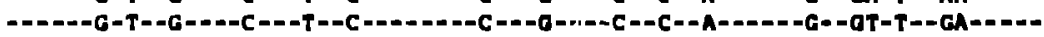

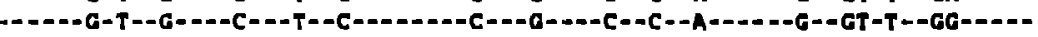

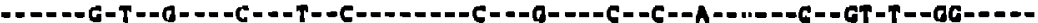

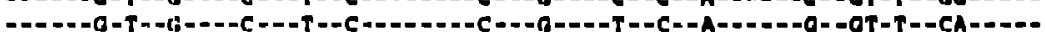

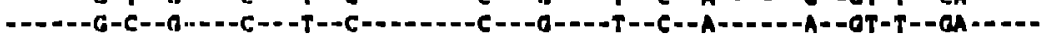

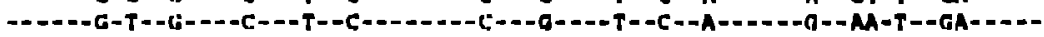

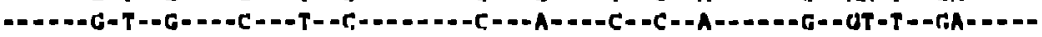

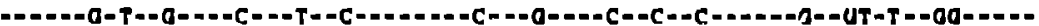

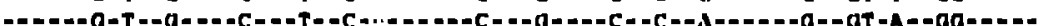

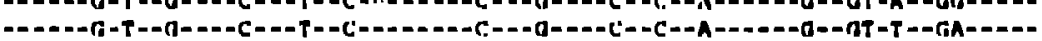

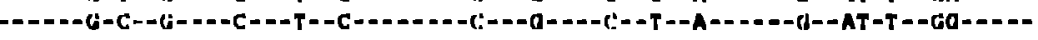

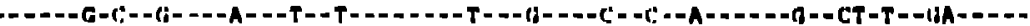

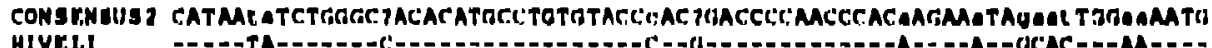
HIYTLLI

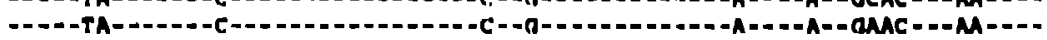

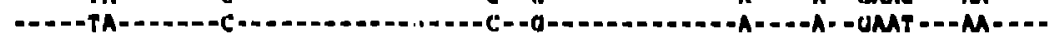

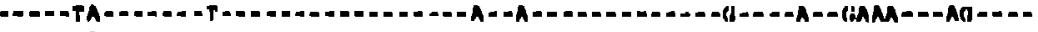
- _. - - 


\section{HIV-I ENV}

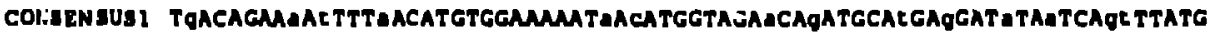
HIVLAI HIVHXB2月 HIVWLAJ

KIVAFA

HIViN

HIVany

Hivic

HIVSH3

HIVaAL2

HIVJACsr

HIVOYI

Hivar?

HIVwrsce

HIVAri 62

HIVJTL

HIVCDCA

hivarj3

hIVIAN

HIVADA

HIVind?

Hivir

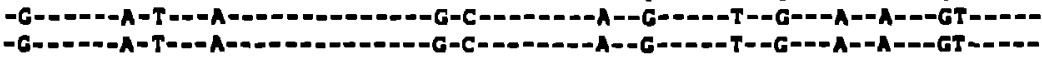

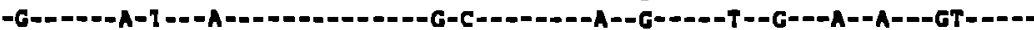

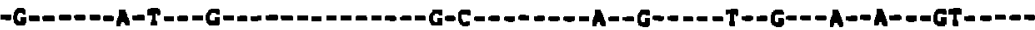

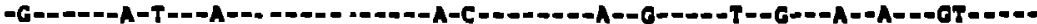

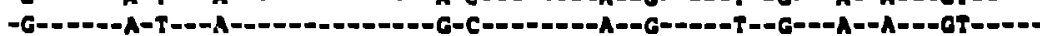

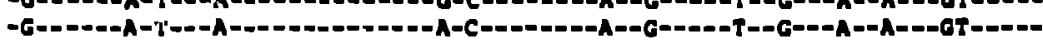

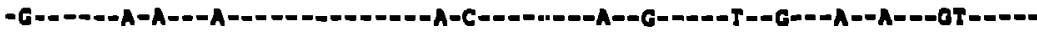

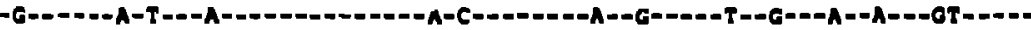

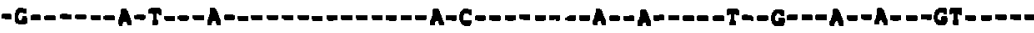

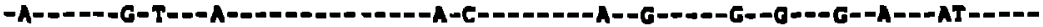

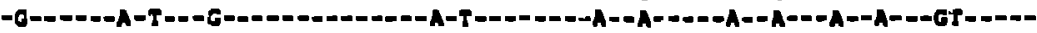

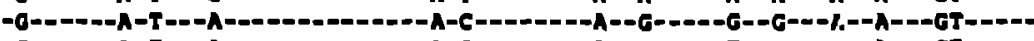

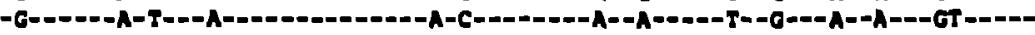

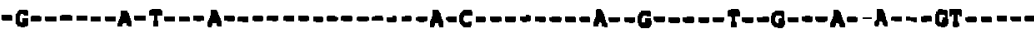

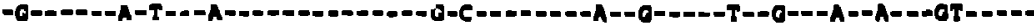

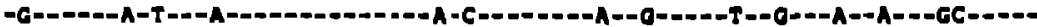

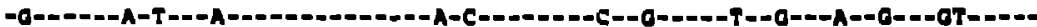

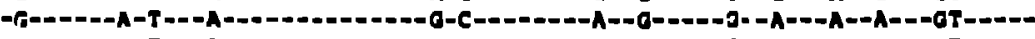

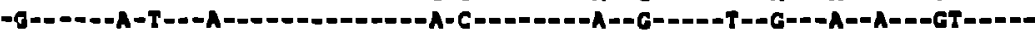

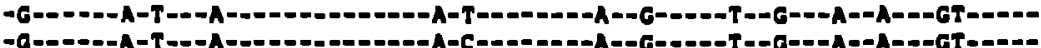

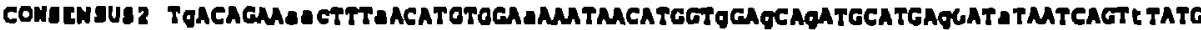

HIVELI HIV2226

HIVNOK -GACACA

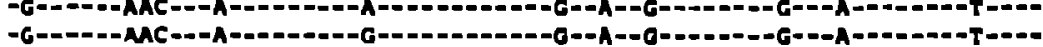

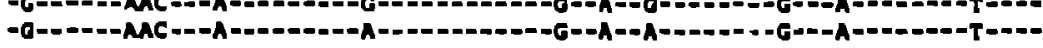

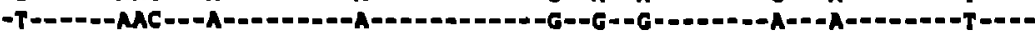

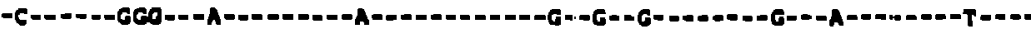

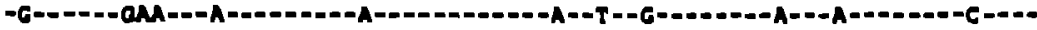

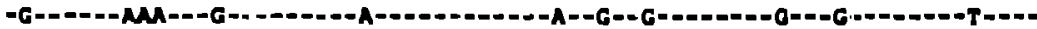

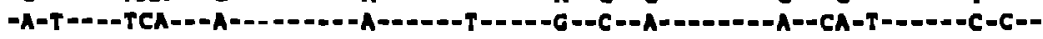

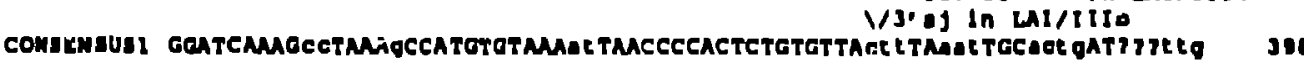

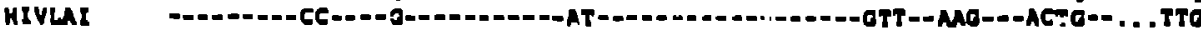

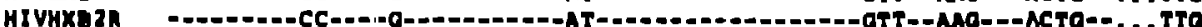

HIVIus

MImara

HIVAN

MIVanu

HIVIC

HIVJHJ

HIVALAI

HIVIALI

hivjncer

hivori

llivira

Hivirsed

livarisz

HIVJrL

II IVCDCA

IIIVITJ J

IIVHAN

MivaUa

III VMIJ 2

hIVAr

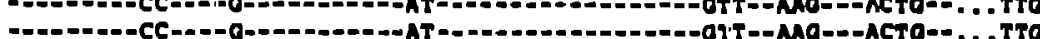

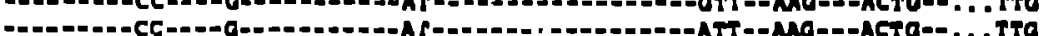
-

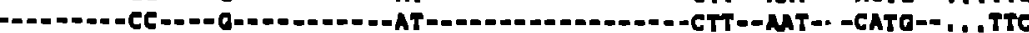

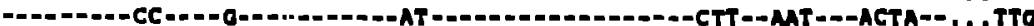

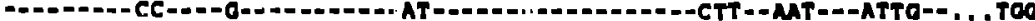

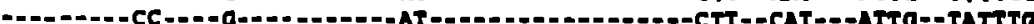

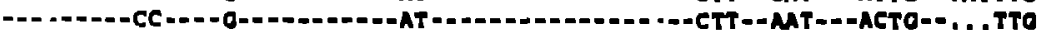
-

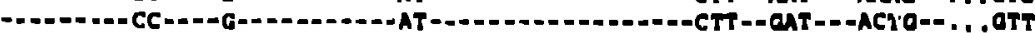

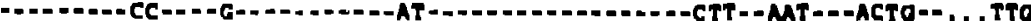

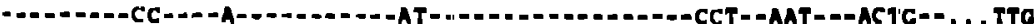

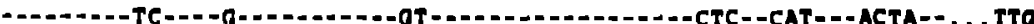

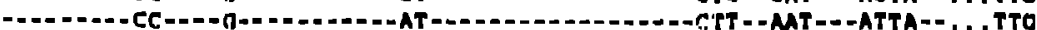
-

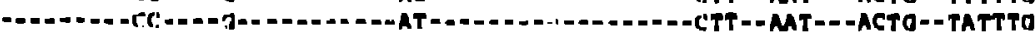
-

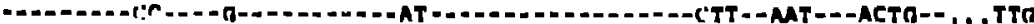

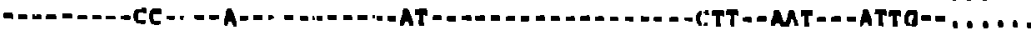

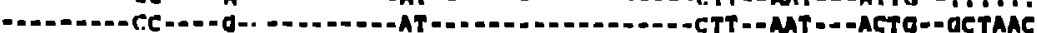

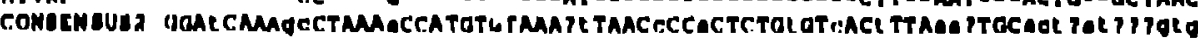

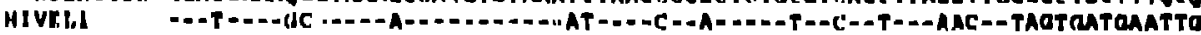
HIVII2: IIVTBK

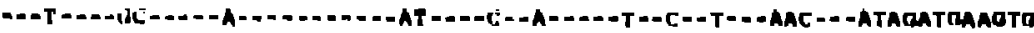

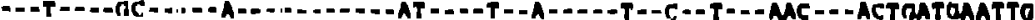

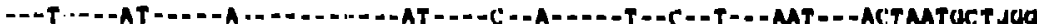
HIVuL
HIVWGS
HIVI3gi

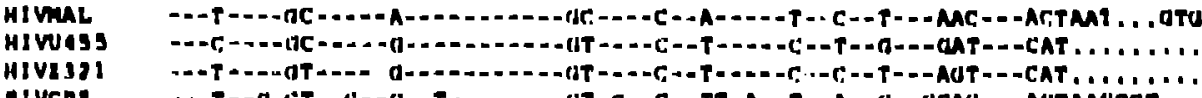

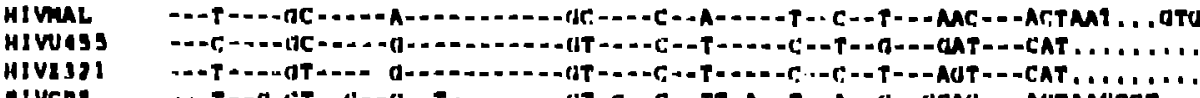


HIV-I ENV

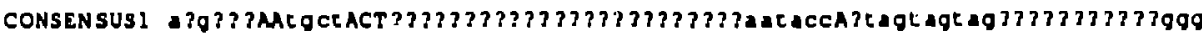
HIVLAI GGG. ..-TGCT-.-AMTACCAMTAGTAGT. ....... MATACC-ATAGTAGTAGC........GGGG

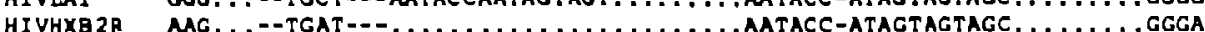

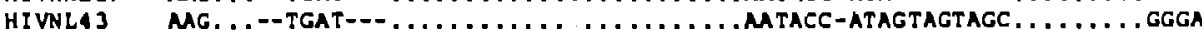

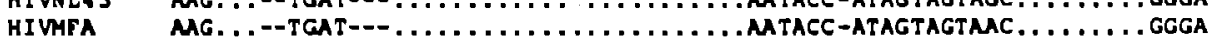
HIVMN AGG...-TACT---AMTACCAMTATAGT...ACTGCTMTAAC-ATAGTATAGC........GAGG

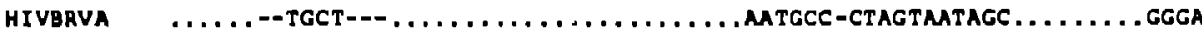
HIVSC AGG...-TGAT---AGCACCAATGCTACT........ MATACC-CTAGTAGTMT........ CGGG

HIVJH3 HIVALAI HIVBALI HIVJRCST HIVOYI HIVIs: HIVNYSCG HIVNYSCG HIVSFI 62 HIVSTL HIVCDCA HIVSFJ 3 HIVHAN HIVHA HIVADA HIVHAJ
HIVRT

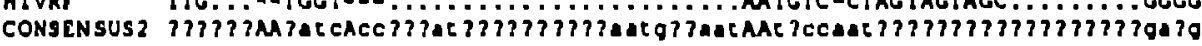
HIVELI

HIV2226

HIVNDK

HIVJYI

HI VHAL

HIVuAss

HIVZ32I

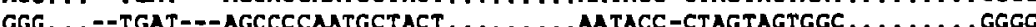
GG. . - TGCT-..

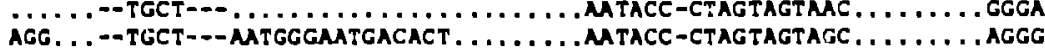
......-TGCT--........................ .....-TACC---AGTAGTAGTTTGAGGAATGCTACTMATACC-CMGTAGTAGT........ TGGG

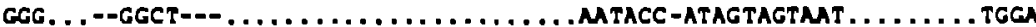
ACG. . --TGCT-1...TAT. ...........GCC... MATGGT-GTAGT...............

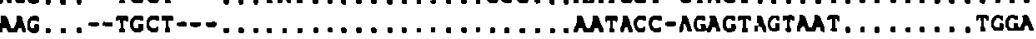

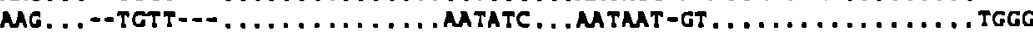
MTACT--TMT---ACT . . . AATACTACT. . . . . .GMCTATC-ATM

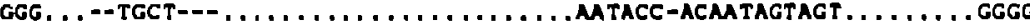

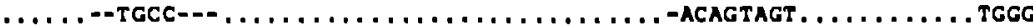

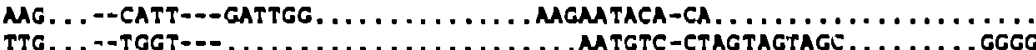
AGGAAC--TGGC-CT . . .ATG...........GGGMC- -TGTCACTACA. . . . . . . . . . .

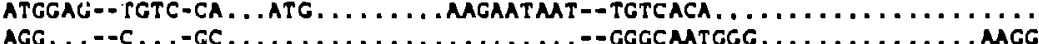

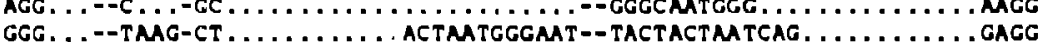

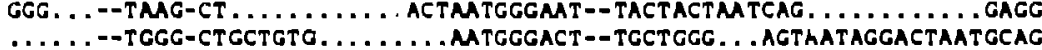

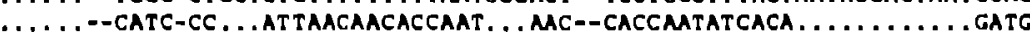

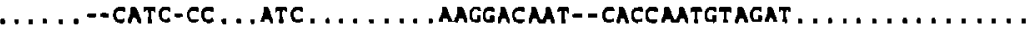
.....--CTTT-GC...... CAGGCAMMMCCTAMCA--CCAGACATCTTCT.

tov or cnv In Lal/IIIb $\rightarrow$

\section{S. $1 /$}

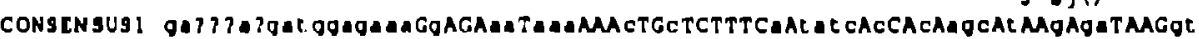
HIVLAI AMAT HIVNLA] GAATGATAATGGAGAMA-G---GA-MA---C--C-_.--A-TATC-G--C-AGC-T--G-GA---GT HIVITA HIVBR HIVSe HIVJH3 HIVALA)

HTVALLI HIVJACST HIVOY! HIVAT HIVHYSCS

HIVSFI62

HIVJPL

MiVCDCA hiverj]

HIVIIAN

MIVADA

HIVMAJ

hivar

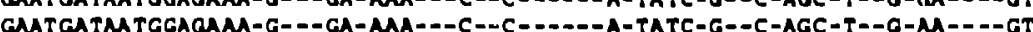
GA. ACMTMMGGGA-G- --M-GAA-- -C--C-----A-TATC-C--C-AGC-T--G-GA-D--AT M... ATGATGGAGGGA-G-D-M-GAA---C--C-----A-TATC-C--C-AGC-T--G-GA-D--AT

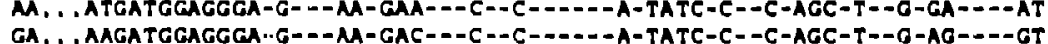
GA. . AAGATGGAGGGA.G---AA-GAC--C--C--2-A-TATC-C--C-AGC-T--G-AG----GT

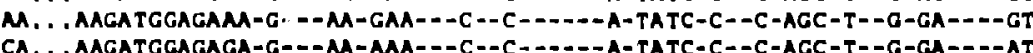

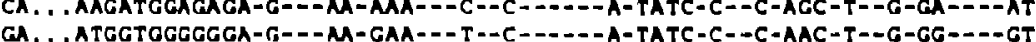
GA. ATGATGGAGAGA-O---MA-AM---C-C-

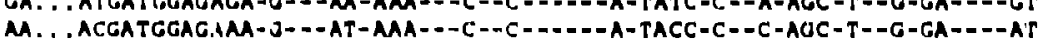
MGRACAMATA... AMA-G---AA-AAA---C--C--.--A-TATC-C--C-AGC-ף'-G-GA---AT

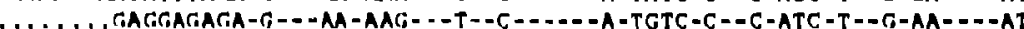
AAGAG. ATGGACACA-G-.--MA-AMA---T--C-----A-GGTC-C-OC-AGC-T--G-AA-D--AT GAMG...ATGGAGGAA-G---AA-AAA---C--C-..--A-TACC-C--C-AGC-T--A-MA-..-AT MACAA CGGGGTAAA-G---AA-CiAC:--C--T------A-TATC-C--C-AGC-T--G-GA----GT

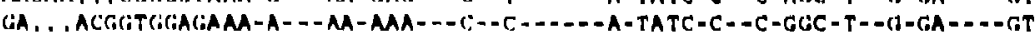

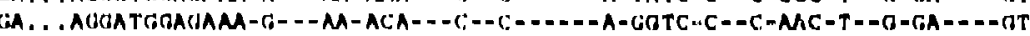

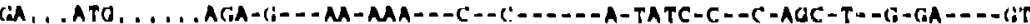

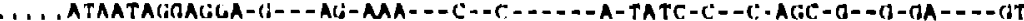
BAAC'AATCIATGGACAAAC-(1---MA-AAA-..-C--C;------C-AGTT-C--C-AUT-6--C1-CA----AC

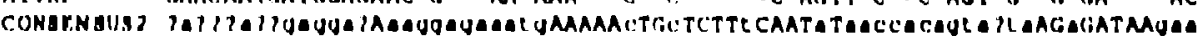

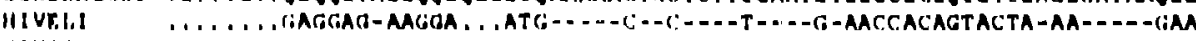

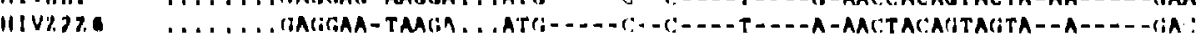

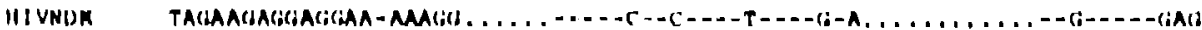
IIIYJYI

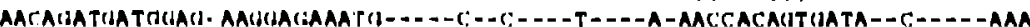

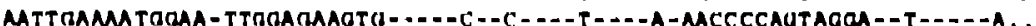

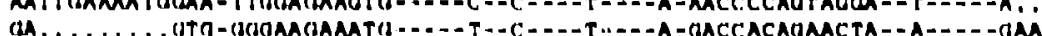

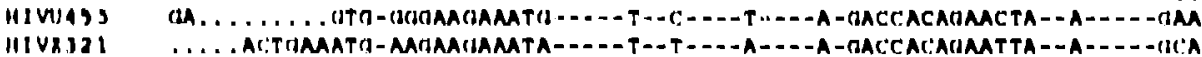

IIVRIJ2
GIVEDz 


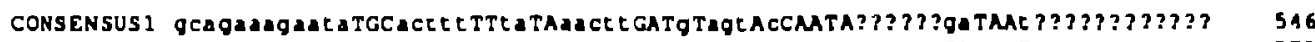

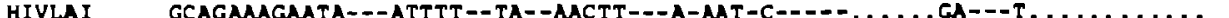

HIVHXB2R GCAGAMGMATA---ATTTT--TA--MACTT--A-AAT-C-.-.....GA--T........

HIVNLA] GCAGAAGMATA---ATTCT--TA--AACTT---A-AGT-C-.-......GA--.T........

HIVAFA GCAGMAGAATA---ATTCT--TA--MACTT---A-MAG-C-.-......GA--.T........

HIVHN GCAGMAGATA---ACTTC--TA--AACTT-.A-AGT-T-........GA---T........ 573

HIVBRVA GCAGMAGMATA---ACTTT--TA--MACTT--A-AGT-C-._.....GA-.-TGAT.......

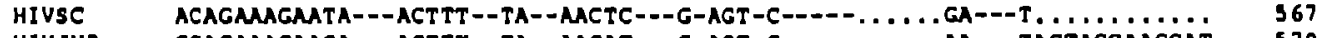

HIVJHJ GCAGAAGMCA---ACTTT--TA--MCAT--G-AGT-C-_-_......M--TAGTACCMGGAT 579

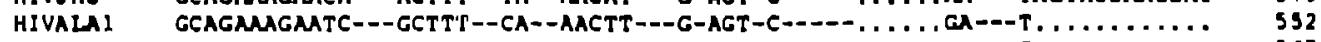

HIVBAL1 GCAGMLGATA---ACTTT--TA--MACTT---A-AGC-C-_-_.....GA-.-T......... 567

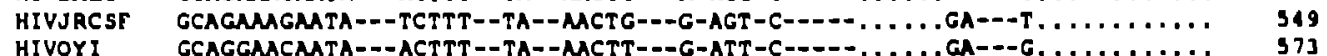

HIVSF 2 TCAGMAGAAA--ACTTT--CG--ACCTT-_-G-AGT-C-_-_....GA---TGCTAGTACT... S61

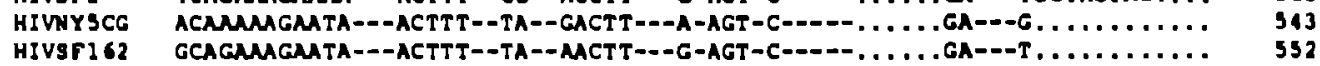

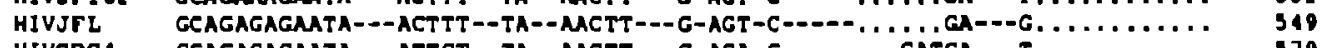

HIVCDC4 GCAGAGAGMTA---ATTGT--TA--MACTT--G-AGA-C-1....GATGA---T.........

HIVSF33 ACAGMGGCATA---ATATT--TA--AACTT-_-G-AGT-C-_--GATGATGA--T.......... 561

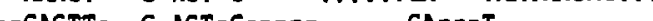

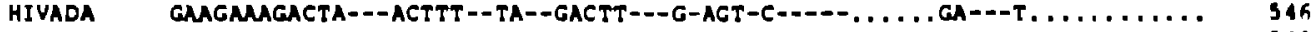

HIVMMJ 2 GCATMAGMTA---ACTTT--TA--MACTT-_-G-AGT-C---_-MGGGTGA--T........ S49

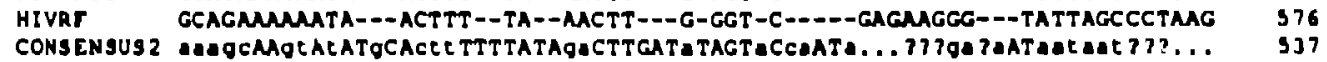

HIVELI GCAGC--GT-T--G--CTT--1

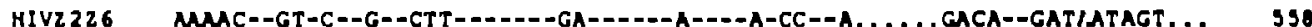

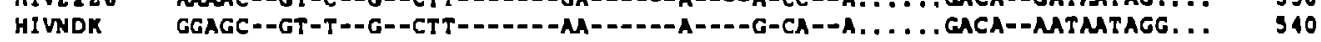

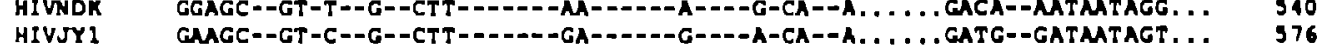

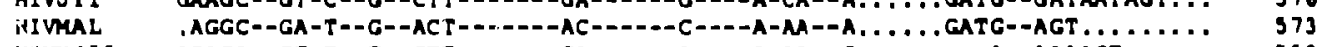

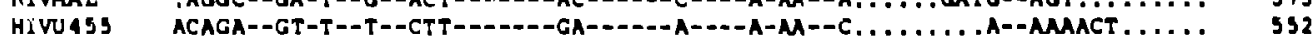

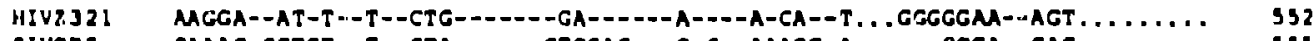

SIVCP 2 GAMC-GGTCT--T--CTA-..--GTGGAG--G-G--MACC-A.....GGGA--GAG........ 33S

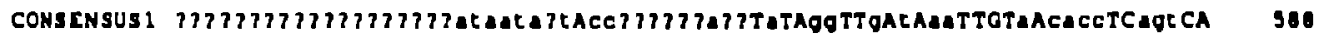

HIVIAI $\ldots \ldots \ldots \ldots \ldots \ldots \ldots$ GAT,..ACT-CC.....AGC-A--CG--G-C-AG-.--A-CACC--AGT-- 616

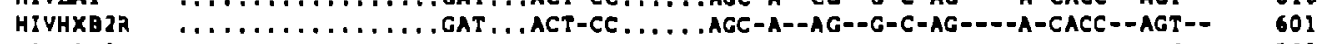

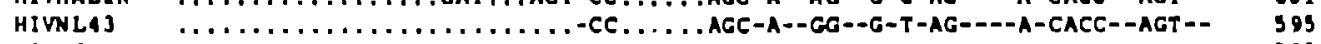

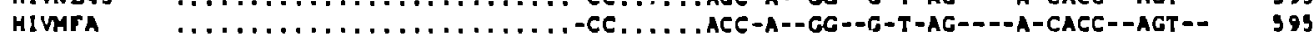

HIVMN $\quad \ldots \ldots \ldots \ldots \ldots \ldots \ldots$ GAT...AGT-CC.....AGC-A--GG-G-T-AG----A-TACC--AGT-- 616

HIVBRYA $\quad . \ldots \ldots \ldots \ldots \ldots \ldots \ldots$ AMACTAAT-CT......AGA-A--GG--G-T-AG----A-TACC--AGT-. 601

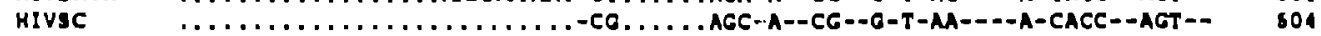

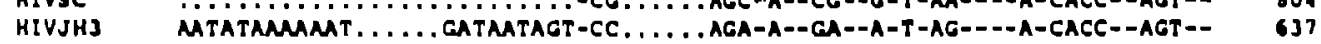

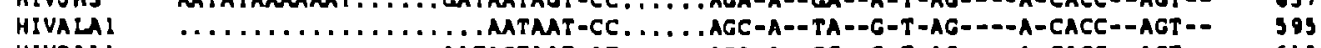

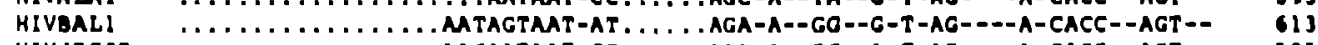

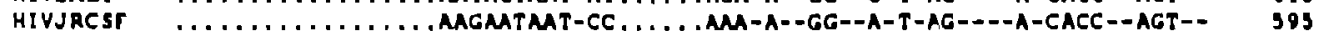

HIVOYI

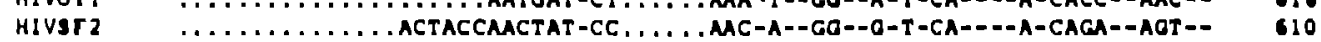

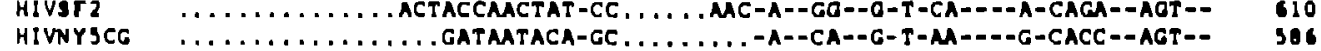

HIVIrigz $\ldots \ldots \ldots \ldots \ldots \ldots \ldots$. . .

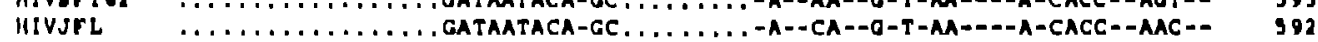

HIVCOCA ........MMAATACTACCAACAAC-CC .....MA-A--GG-O-T-T-AA---A-CACC--AGT-- 634

HIVST 33 .

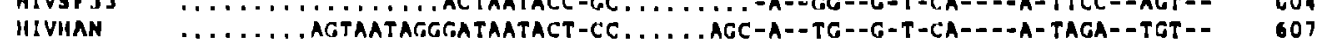

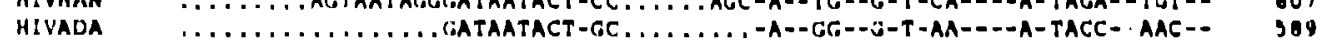

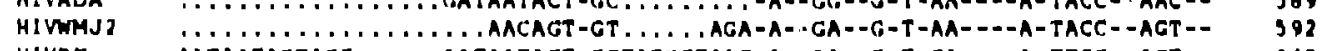

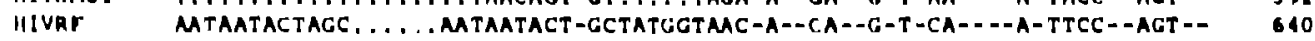

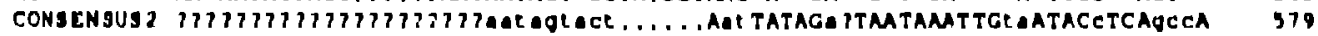

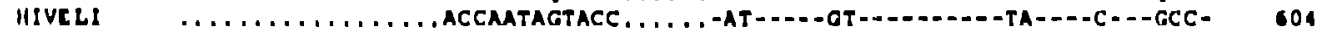

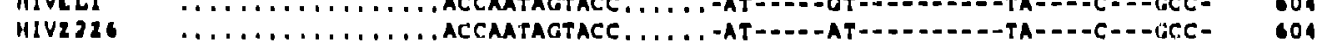

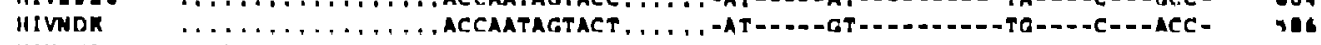

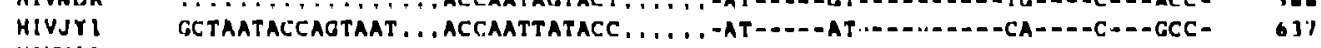

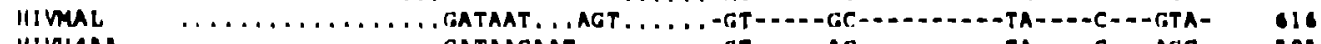

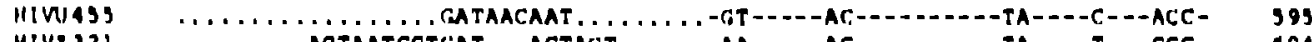

HIVZ321
SIVCTz 
HIV-1 ENV

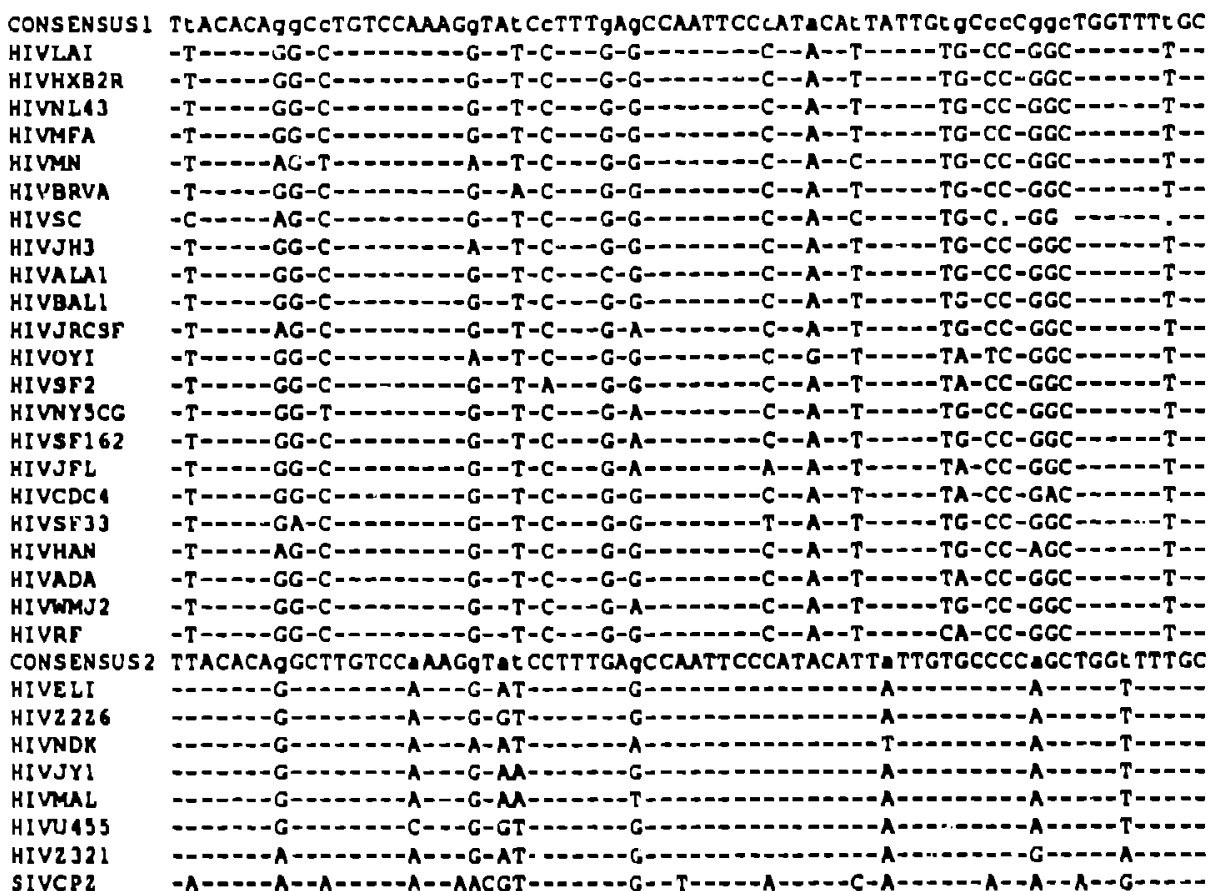

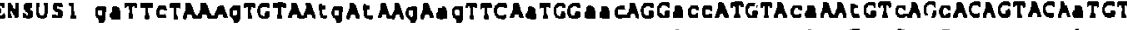

HIVLAI GA--C----A-----TA-T--G-CG----A---AAC---ACC-----CA--T--C--C-------A---

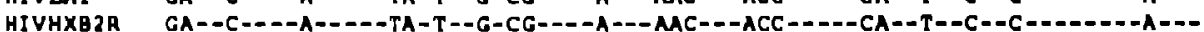

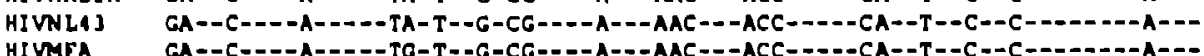
Hivisa

HIVMN

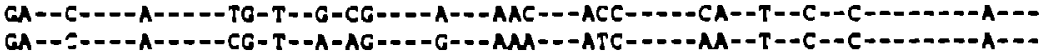

HIVSC

HIVJHI

HIVALAI

HIVBALl

HIVJRCSF

HIVOYI

HIVST?

HIVNYSCG

IIIVSri62

HIVJPL

HIVCOCA

HIVSFJ3

HIVIIAN

IITVAOA

Hivar

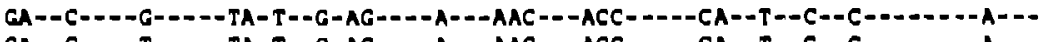

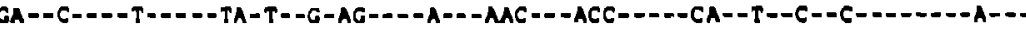

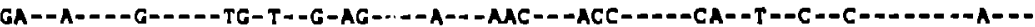

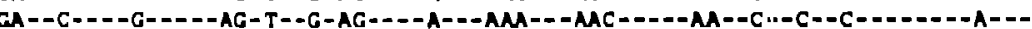

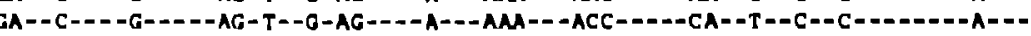

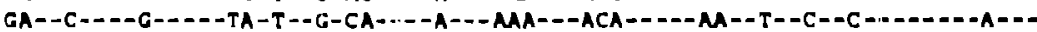

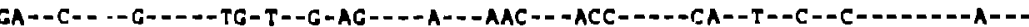

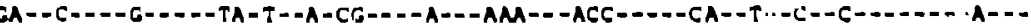

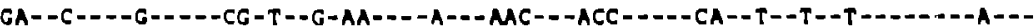

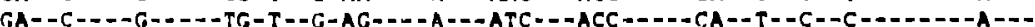

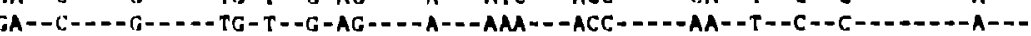

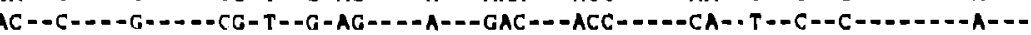

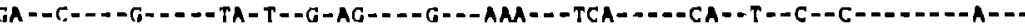

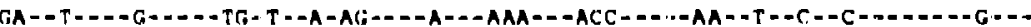

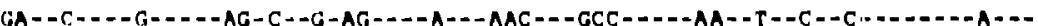

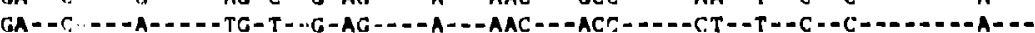

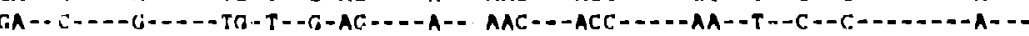

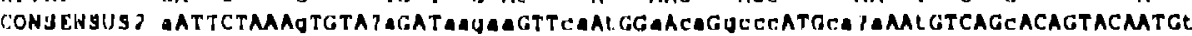

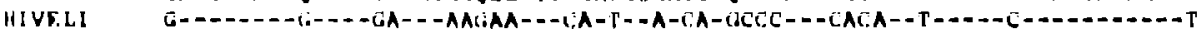

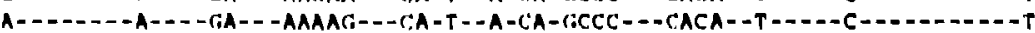

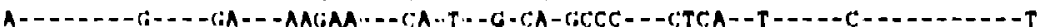
A - A-19-1

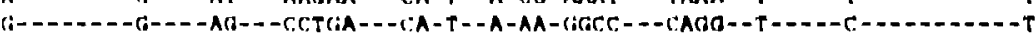

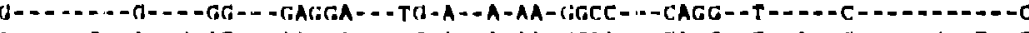

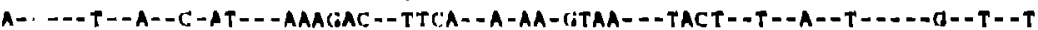


HIV.1 ENV

CONS ENSUS I ACACATGGMTE AggCCAgT_GTgTCAACTCAACTgCTGL TaMATGCAGE CTACCAGMgaAgaggt ag

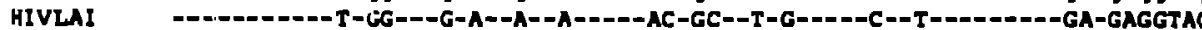

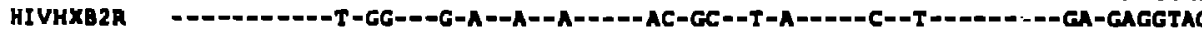

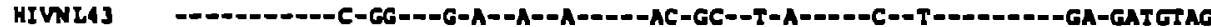
HIVAF" HIVMN HIVDRVA HIVIC -. -

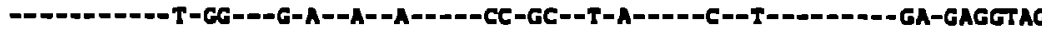

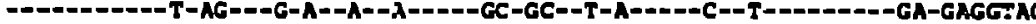

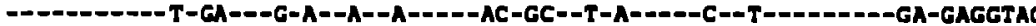

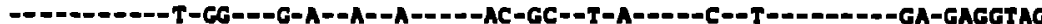

HIVAIAI

HIVBALI

HIVIncer

HIVOY!

HIVsF2

HIVNYSCG ---

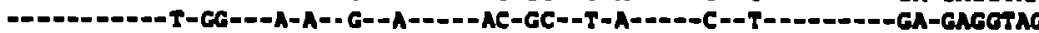

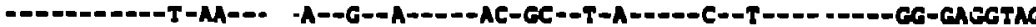

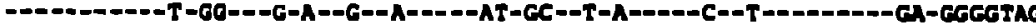

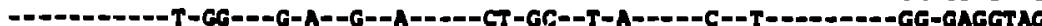

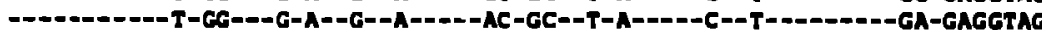
-

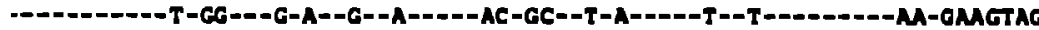

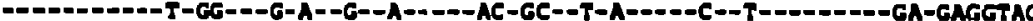

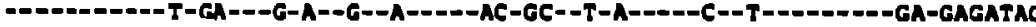

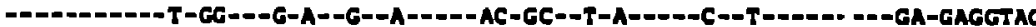

HIVJFL

HIVEDEA

HIVHAY

HIVINA?

H.TVRF

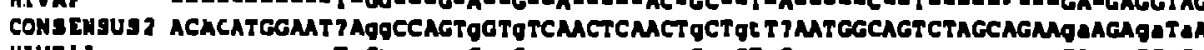

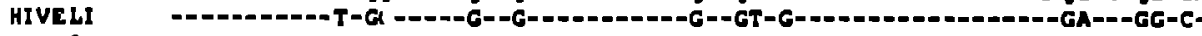
HIVZ2Z6

HIVIDK

HIVJY1

HIVML

hIV0453

HIVZJ21 -

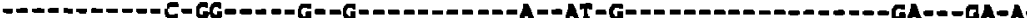
$-10=0$ $-1-0-10-0$

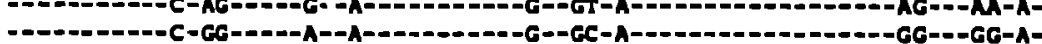
-

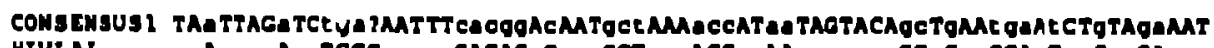
HIVLAI --A---A--TGCC--_-CACAG-C--CCT--ACC--AA-_-_-GC-G--CCA-T--G--GA---

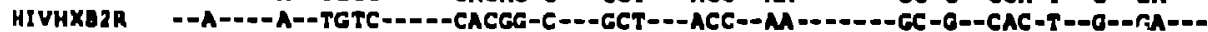
HIVIS

Hivisa HIVAM HIVORYA HIVIS

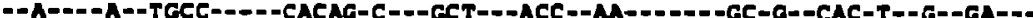

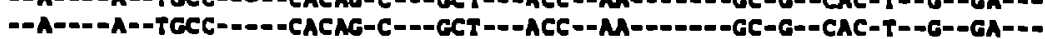

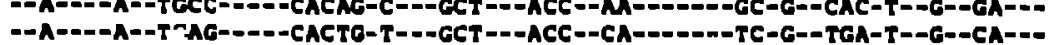

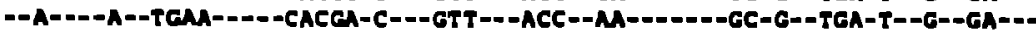
--C--.-G- -TCAA--_--CACCG-C---GCT---ACC--M----D--GC-G--AGA-G--G--GA--.

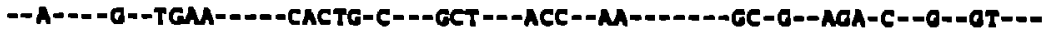

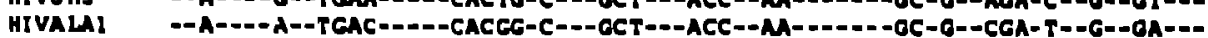

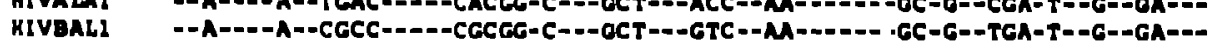

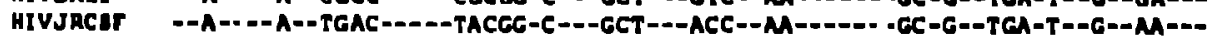

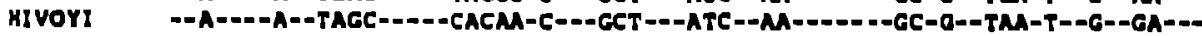

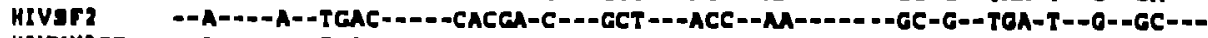

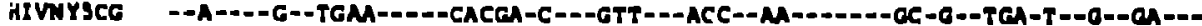

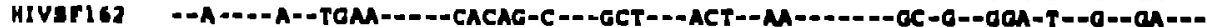

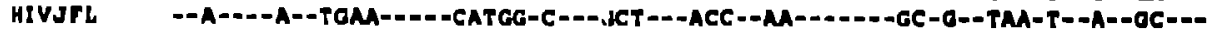

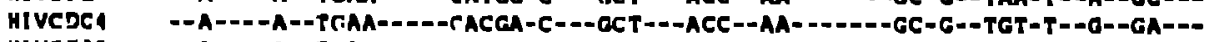

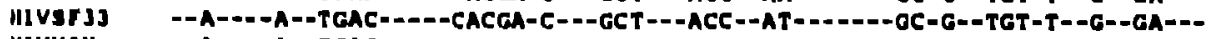
HIVHAN --A---A--TGAC-----CACAG-T--ACT--ACC-AA------TC-O--CGA-T--G--GA-HIVADA --A---A--TAGT-D--CACAG-C--GCA---AMC--AA------OT-G--AGP-T--G--GA--. HIVIMJ2 --A--.-A--TGA--_-CACAG-C--GCT---ACC--MA------CC-G--TGA-T--G--GA---

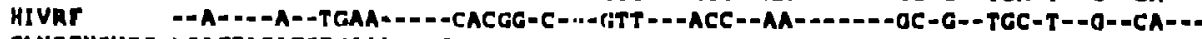

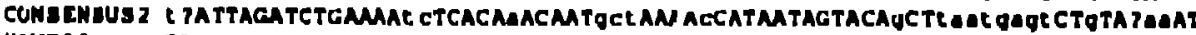

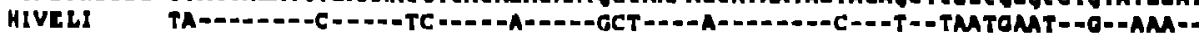

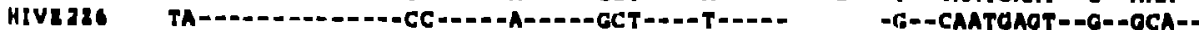

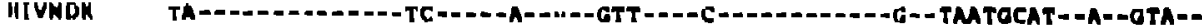

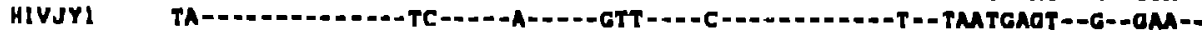

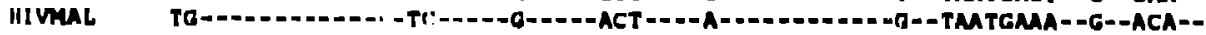

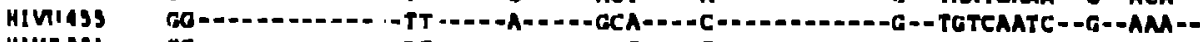

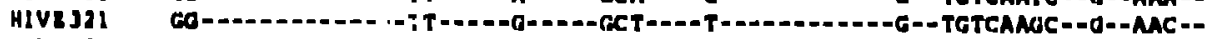
SIVCD CTO-A---GTO--O -TAMOT-A-A---ACTO-TOT-TCO---D---Q--AOTAOMA-AO-TAOTC- 
HIV-1 ENV

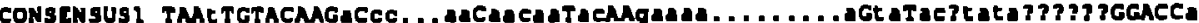
HIVI HIVLAI

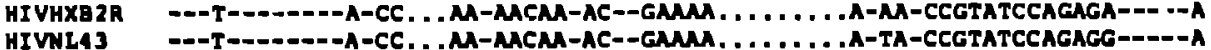

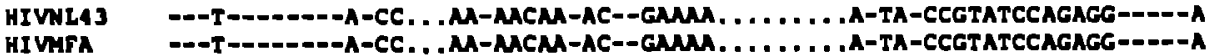
HIVBRYA HIVIC HIVJH3 HIVALA1 HIVBAL HIVJACST HIVOYI
HIVST2 HIVirscg HIVAr1 62 HIVJTL HIVCDCA HIVSTJ3 HIVHA HIVNDA KIVIMJ2 HIVRF

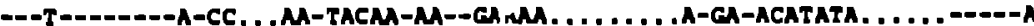

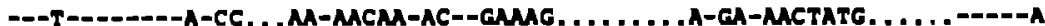

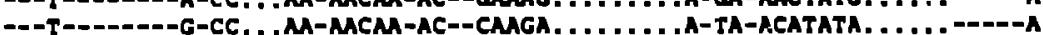

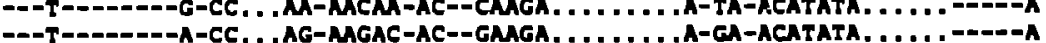
---T-------A-CC. . . MA-ATCTA-AG--MGGA. . . . . . . . .A-GA-ACATATA. . . . . .---A

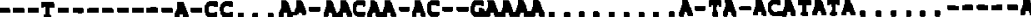

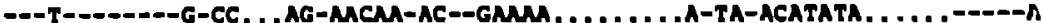
--T---_-A-CC. . M-MCM-AC--GMMC. . . . . . . .A-GA-ATCMATA. . . . . -----A - - -

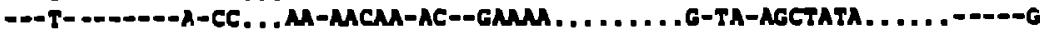

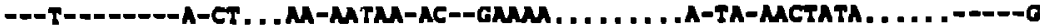

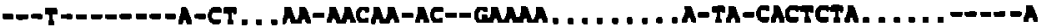

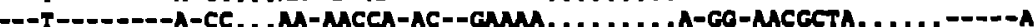

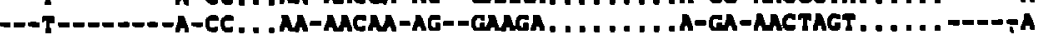

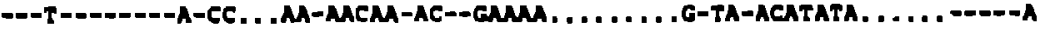

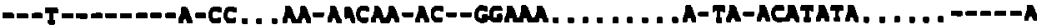
-.T-T-.---A-CE. . . TA-MACM-GT--GMOA. . . . . . . .A-TC-ATCTATA. . . . . . .-.-A

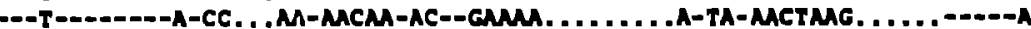

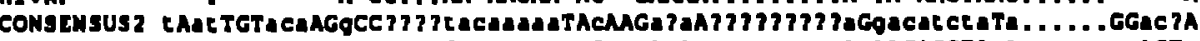
HIVLLI T-CC-_-GCA--G--C... TATCAMA--C-.-ACA-........A-ACACCTA-A......-ACTHIV2226 T-AC---ACA--G--C...TACAGAM--T---ACA-.........A-GACATCTA-A.....--ATTHITOE HIVSY HIVLL HIVuss HIV3321 T-AT-D-ACA-G--C . TACMMTA--C--GCA- 0 -GACATCGA-A. . . .-ACTT-AT---АCA--G--CGACACAMAT--C---ACA-.........А-TACACCTA-A. ......-АCTT-AT---ACA--G--T. . . GGMACAA--C---AAG- . . . . . . G-GATACATT-C. . . . . --CCCC-AT---TCC--A--T. . . TAC. . . M--C---MM-MATATACGTA-GTATAGTA-A. . . . . --ATC-

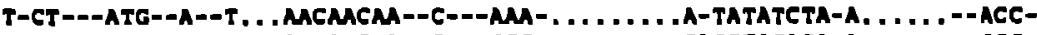
A-AT-_-CAT--A--A. . . GGMACMA--C---GGG- . . . . . . GAGGTGCACA-A. . . . . . .GCC-

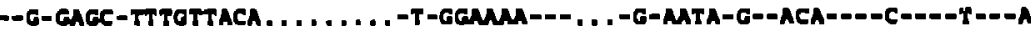

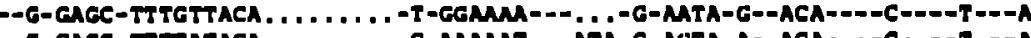

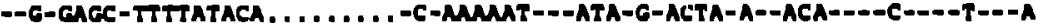
--G-GAGT-TATTATACA . . . . . -C-GGACMA---ATA-G-GATA-A--ACG----C----T---C

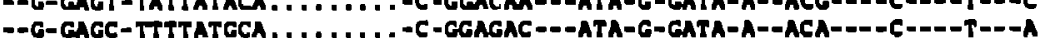

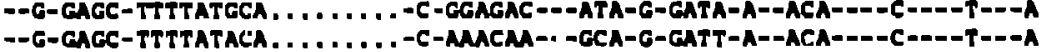
--G-GACC-TTTCATACA . . . . . . . . -C-ACACM---ATA-A-MTTA-A--ACA----C----T---A --C-GACC-TTTTATACA . . . . . . . -C-GCACMA---ATA-G-GATA-A--ACA----C----T---C

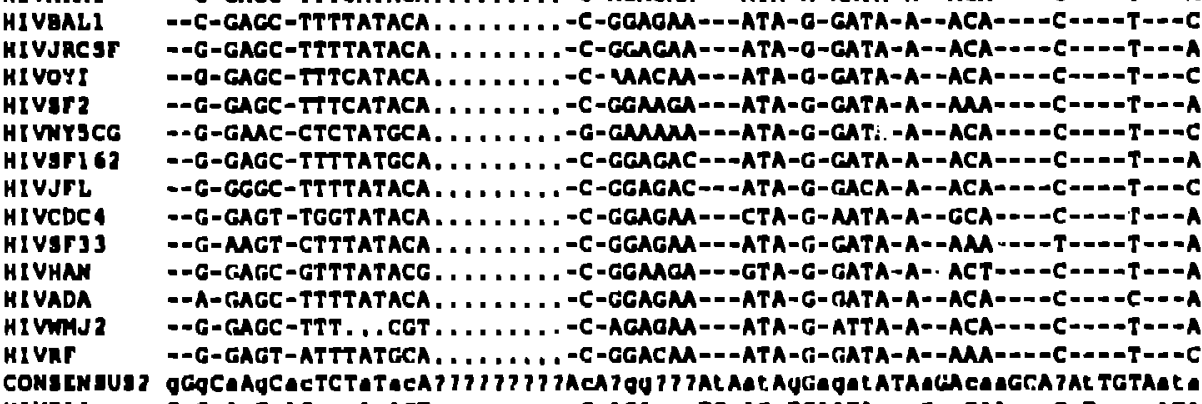

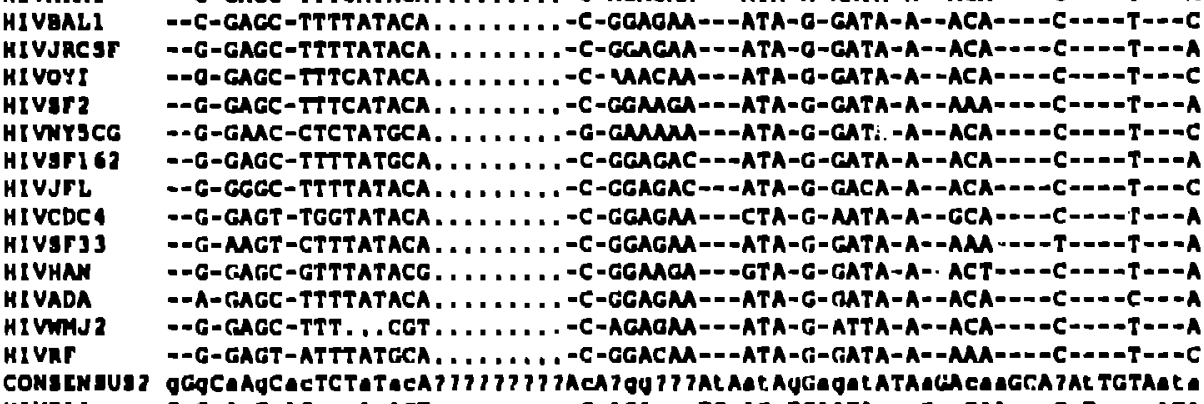

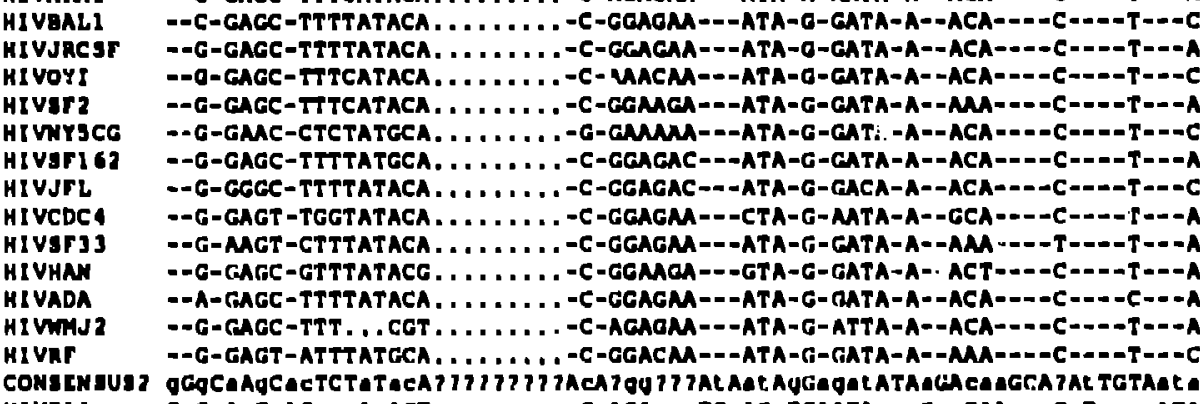

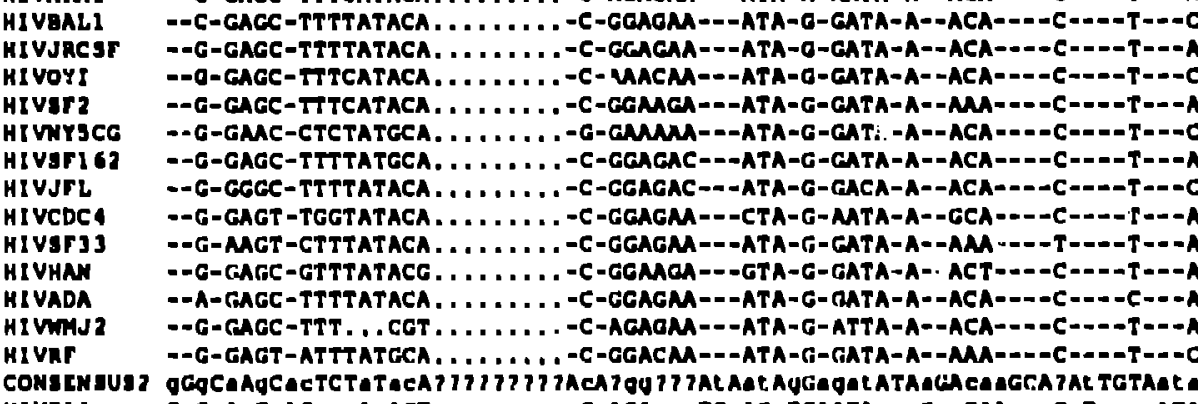

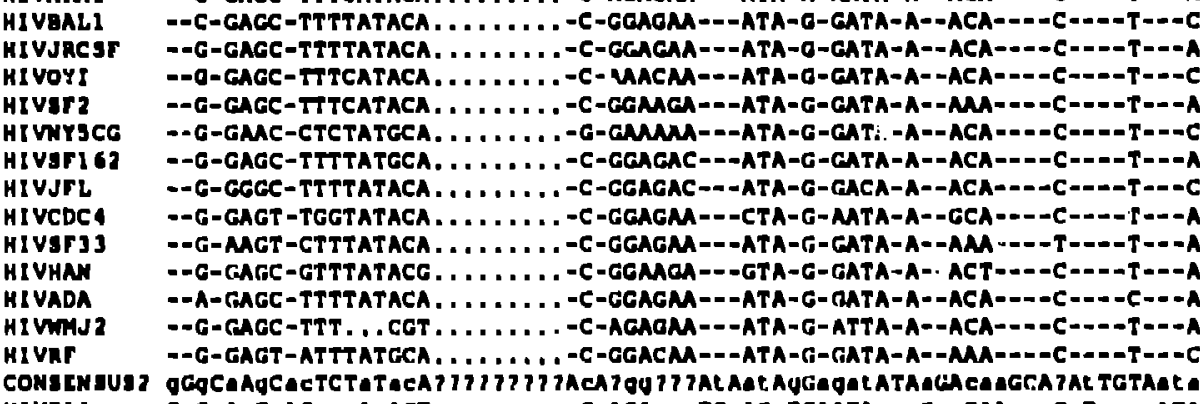

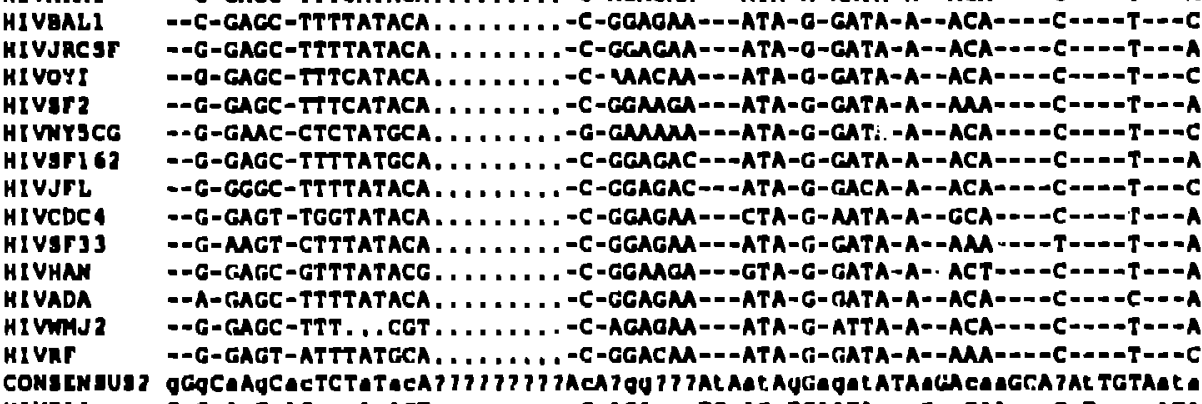

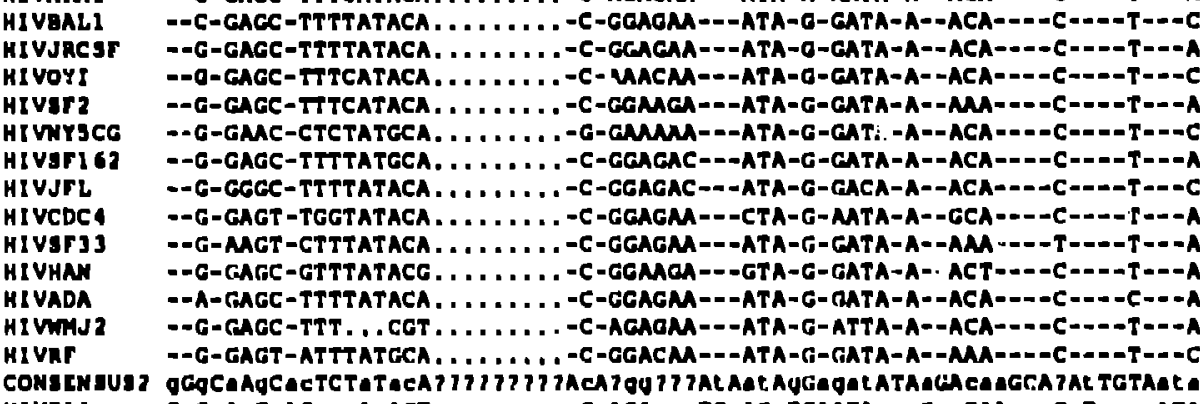

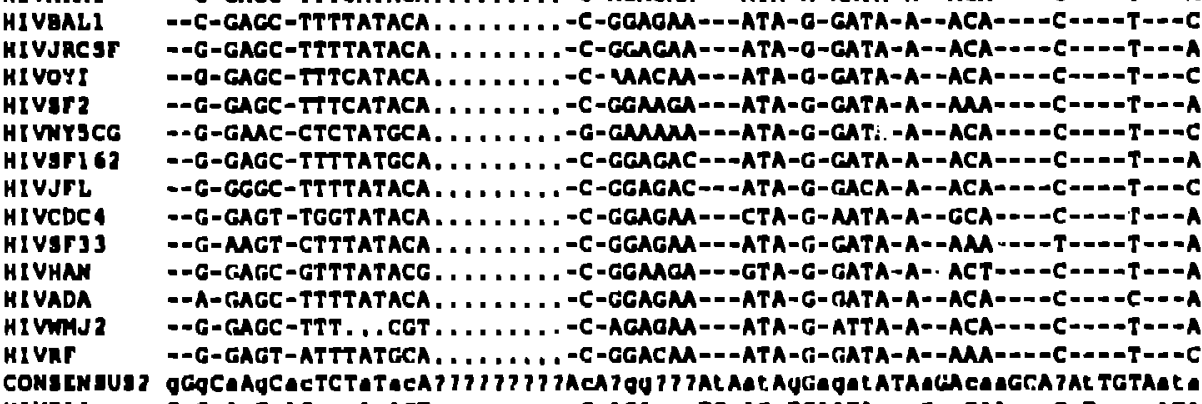

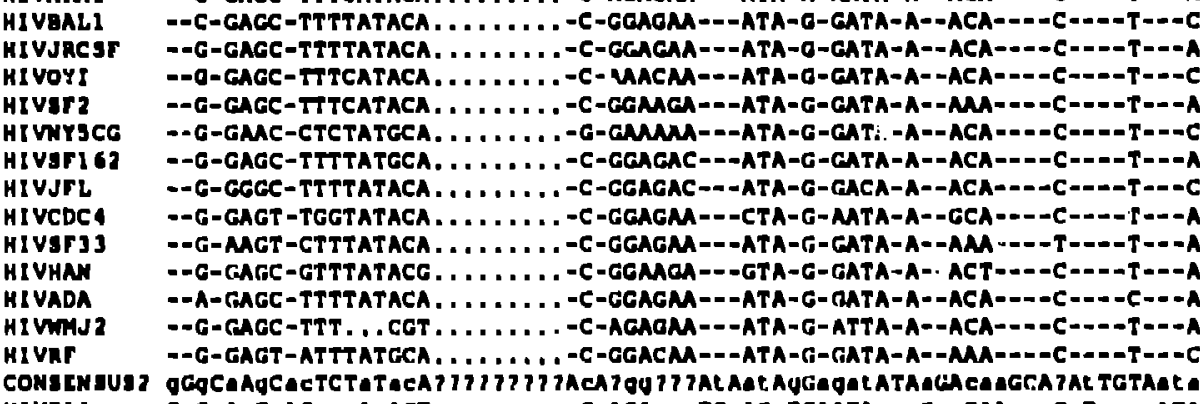

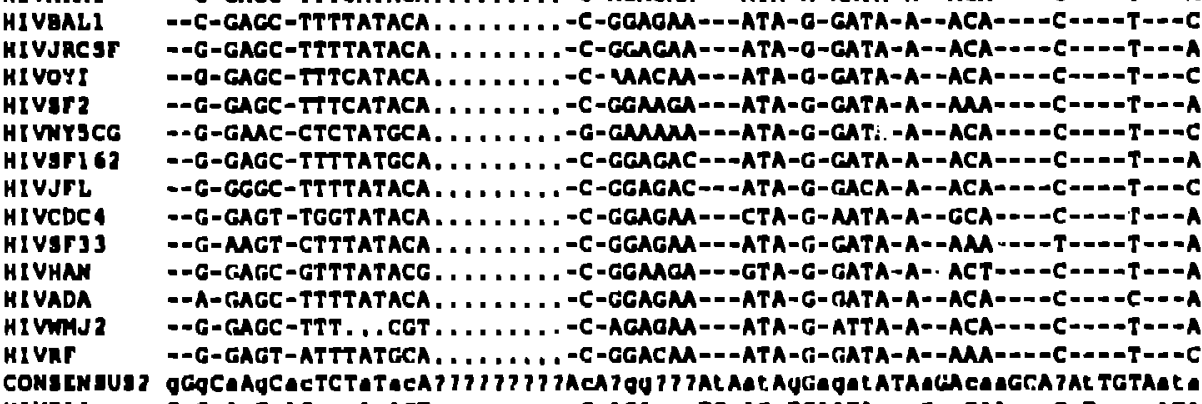

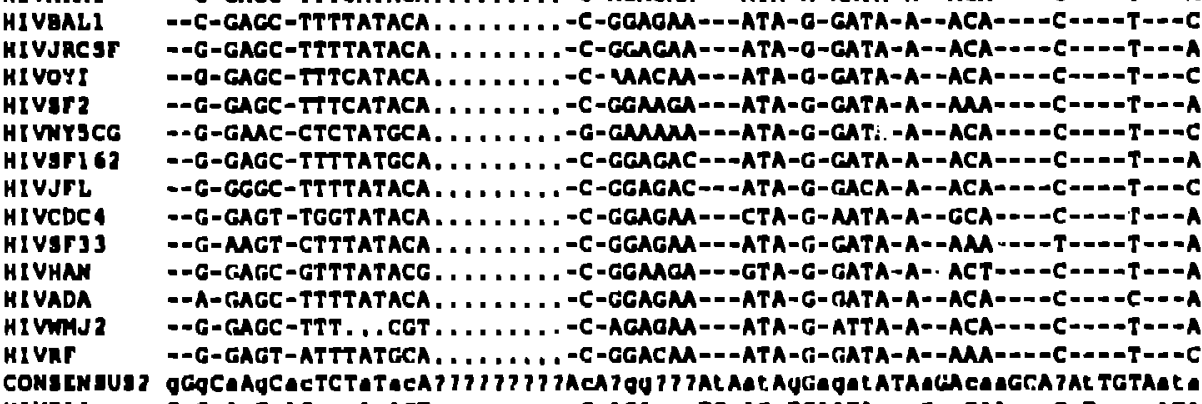

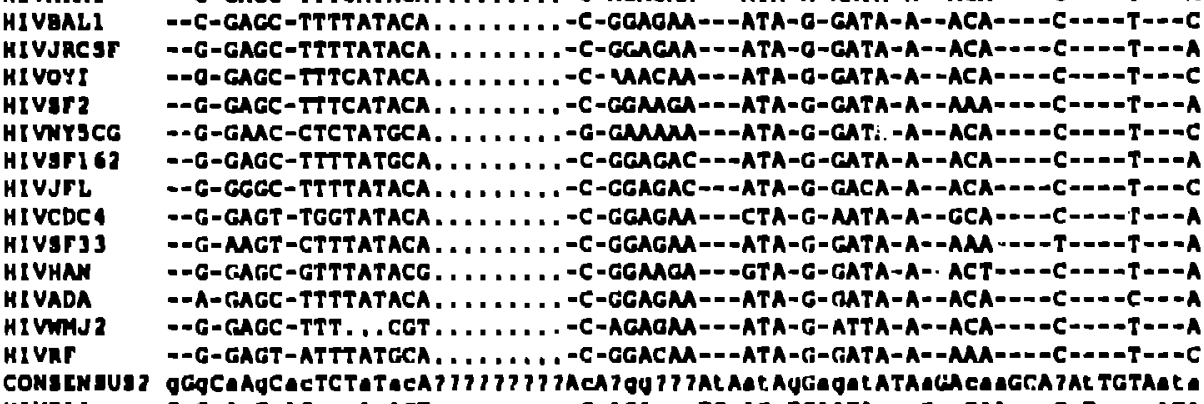

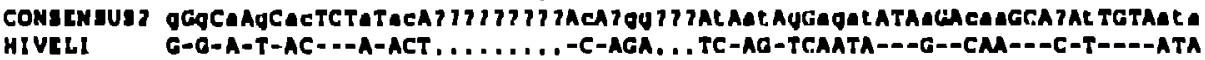
TV2) Hivise HIVIDK HIVJY) HIVAL

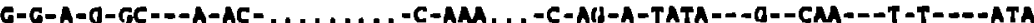
A-G-A-T-AC---A-AC-ATAACAGCA-A-MACAAG-A-AC-G-ATAC---G--CMA---C-T----AMA G-G-A-G-AC-.-A-AC-........-C-AGG...-T-AA-G-AGAT---A--CA-D-T-T-D-ATA

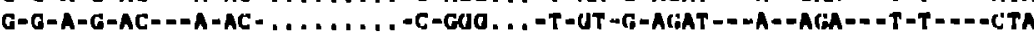
G-A-A-G-AT---A-GT-. . . . . . . -C-GGTAMA-T-AT -G-GCAT---A--CAA-- -C-T--_-ATG

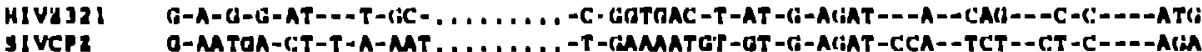




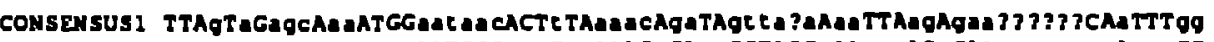
HIVLAI ---G-A-AGC-A-_--AMTGCC---T--MAC-GA--GCTAGC-M---AG-CAN. . . . . --A---GG HIVHXE2R ---G-A-ACC-AM-_--AATMC-_-T--AMC-GA--GCTAGC-M-_-AG-GM.....--A-.-GG

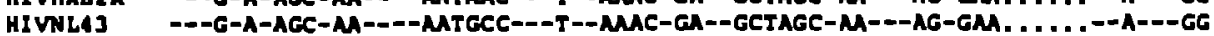
HIVAFA ---G-A-AGC-M----ATGTCC---T--MAC-GA--GCAMGC-MA---AG-GM......--A---GG HIVT) HI VBRVA HIVSC HIVJH3 HIVALAI HIVJRCSP HIVOYI HIVST HIVSTI62 HIVJT: HIVJTL hIVIT33 HIVST33
HIVHAY HT VADA HIVIN2 HIVRT consensus HIVILI HIV2226 HTVNDK HIVSY1 4I VML. HIVuass HIV2321 siverz - G-A-AGC-A M---G-A-ATC-M-D-GAGMC---T--MMC-GA-GTTACA-M---AG-GTA. . . . . .-A-.-... --G-A-ATC-M---GAGMC---T--MAC-GA-GTTACA-M--AG-GTA. . . . .-A--. . $--A-A-A G C-A G---A$-ATCCC---T--MAC-GA--GTTGGA-M---AG-AM . . . . . --A---GT ---G-A-ACC-M----GAGGAC--- T--MMC-GA--GTTGM-M---AG-GM. . . . . ,--A--- . . ---G-A-ACC-M-O--MATCAC---T--MTA-GA--GTTATA-M---AG-GM . . ...-A---GC ---G-A-ACC-CA----MTAAC-.-T--AMC-GA--GTTGM-M---AG-GM. . . . . --A---.. --G-A-AGC-AC-_-GAGMA---T--GMC-GA--GCTACA-M---AG-MM.

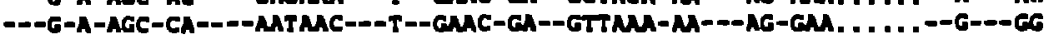
---G-A-ACC-M----MATAC---T--MMC-GA--GTIACA-M---M-CM . . . . . . --A---AG ---G-G-ACA-M----MTMA---7--MMC-GA-GTTACA-M---CA-GCA . . . . . --A---CG

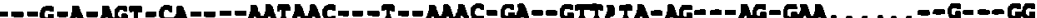
-G-A-ACC-CA-D-MAMC--T--CMC-GA--CCTACA-CC---AG-GM --G-A-AGC-M----MTMA-- T--GMC-CG--CCTACA-M---AG-GM.

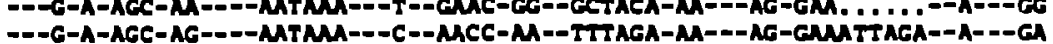

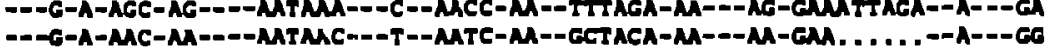

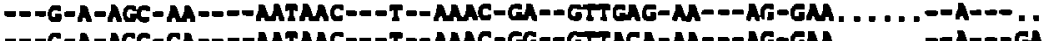

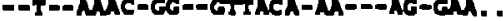

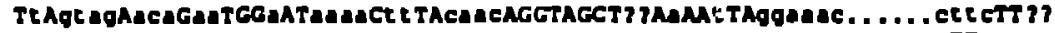
-T-GTAG-GCACM---AG-AMA-TT--CMC-A------AG-A. -T--GEMCC. . . . . , CTTC-- . -T-GTMA-MT-AM---A--AMCA-AT--CMAC-_-_-AT-A--P--CGMAC. . . . . . CTTC-. . -T-GCAG-GCA-AM---A--MMG-TT--CMAC---.---AC-A--C--GGMAC. . . . . CTTC-- .

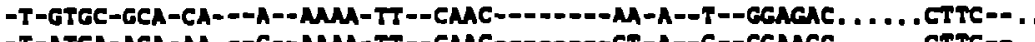
-GA-C--T--MGMA. . . . . MAGT-I. -C-GTAG-ACA-A---A--GACA-TT--TCCA--.-.--GC-C--T--AGMA. . . . . .САCT--GT -А-ATGGGACCACA---A--CGM-GG-GGMAG-A---MMMGGCTT-. GCAACC . . . . . . . . TC

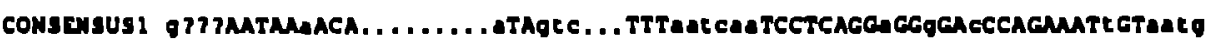
HIVIAI HIVHXอ2R HIVILA HIVira HIVir. HIVanva HIVanva
hIVse HIVJH3 HIVALAI

HIVBALI HIVJRCSF hIVOYt HIVoYt
hIVSr? hIVirseo HIVSTI 62 HIYJPL HIVCDCA Hivst3 3 HIVIYTS YI UADA HIVMu? IIIVAT

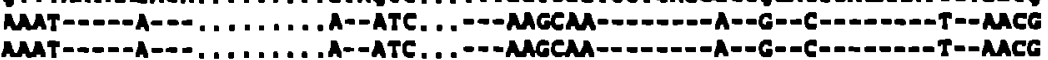

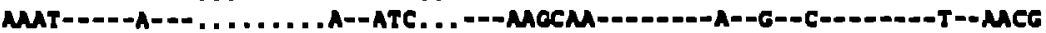
MAT-D--A--1 NAT--DA-

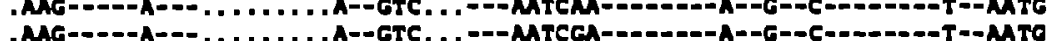

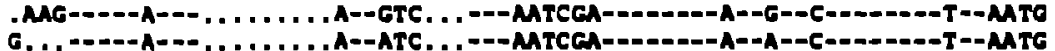

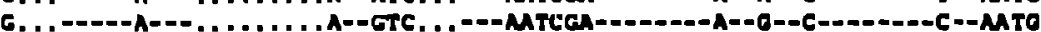

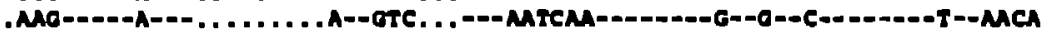

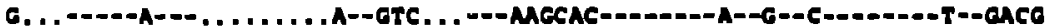
Мiт-...-A-_..........A-GTC

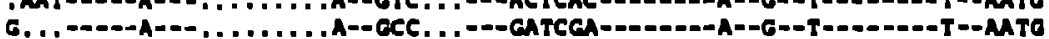

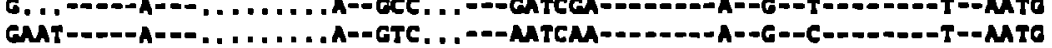

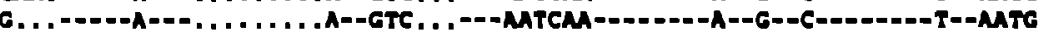

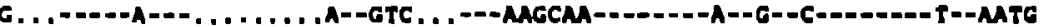

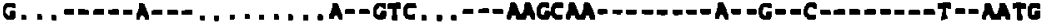
G. . -

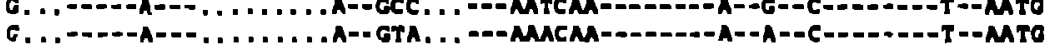

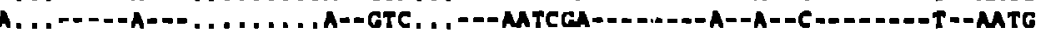

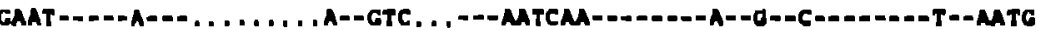

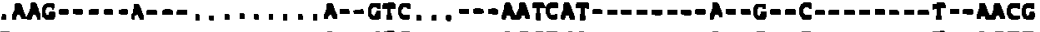
C. -0...A--.

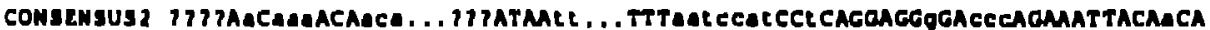

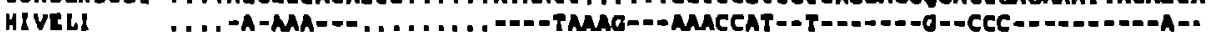

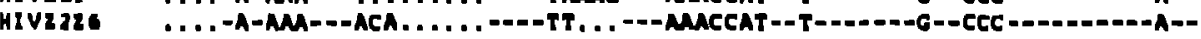
HIVTSR HIVJYI HIVML hivis HIV2.121 HIV2.12I
SIVC.P2

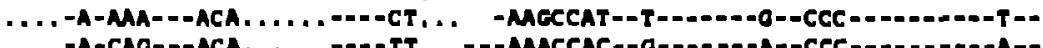

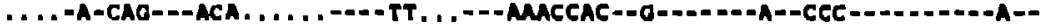

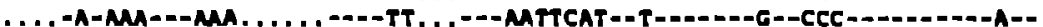

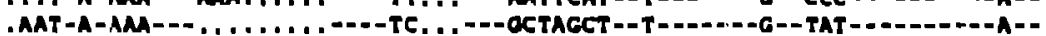

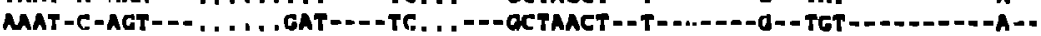
ATCA-ATCGA-_-CCAGCCAC---_CA. . . C-CMACAGAO--T-T-_-_-A--TCC-_-GO-C-_-CAT 
HIV-1 ENV

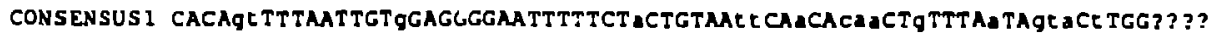
HIVLA HIVHXB28 HIVNL43

HIVMFA

HIVHN

HIVBRVA

HIVSC

HIVJH3

HIVALAI

HIVBAL]

HIVJRCSF

HIVOY

HIVSF?

HI VNYSCG

HIVSFI 62

HIVJFL

HIVCDC 4

AIVSF3

HIVHAN

HIVADA.

HIVRF

CONSENSU

HIVELI

HIV2226

HIVNDK

HIVJYI

HIVUA.

HIVU 455

HIVZ321

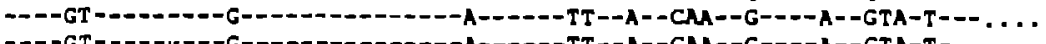

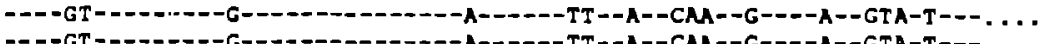
-

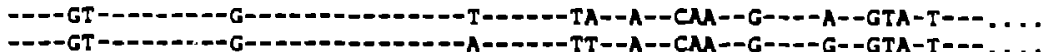
- - GT -

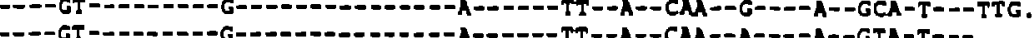
--GT--

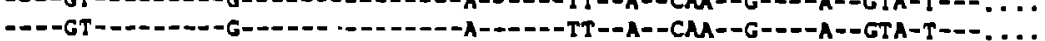
-

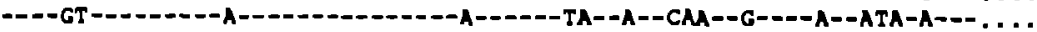

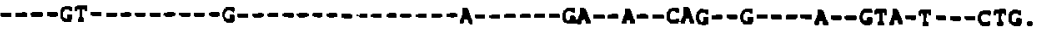

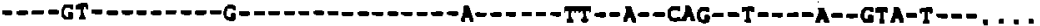

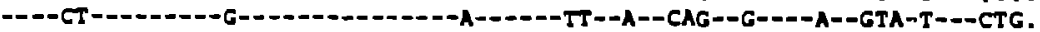

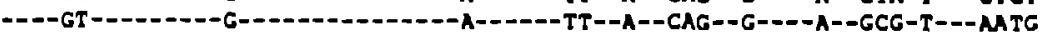

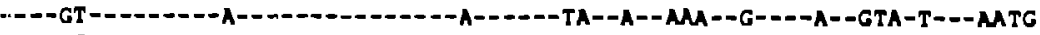

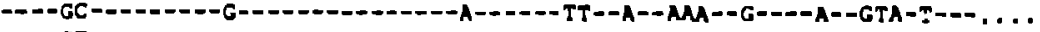

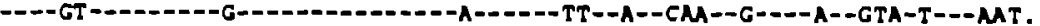

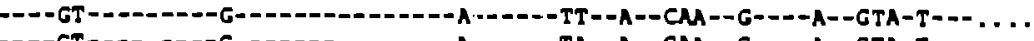
CA CACAgtETTAATTGTgGAGGgGALTTLTTCTACTL?ALACATCA ?gACTGTTTAMTaGEACaTGG? ? ?

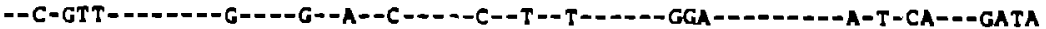

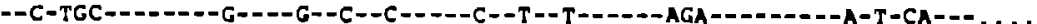

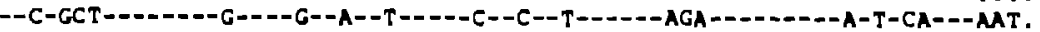

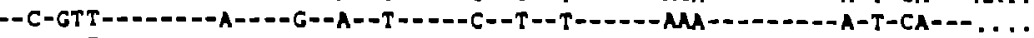

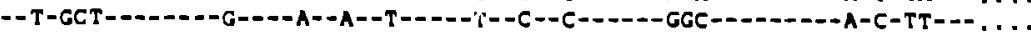

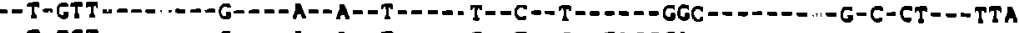

stvep?

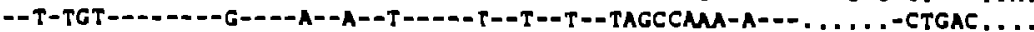

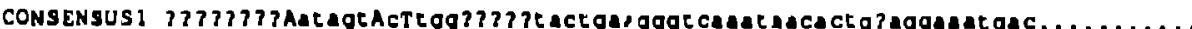
.... TTt-ATAGT-C-tGg . . AGTACTGMGGGTCMMATMCACTGMGGAGGTGAC . . . . . . . . . 1251

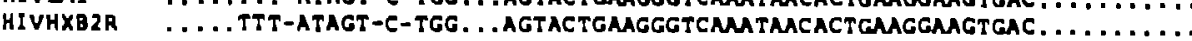
HIVI:

HIVMFA . . . .TTT -ATAGT-C-TGG. . . AGTACTGAAGGGTCAMATMCACTGMAGGMGTGAC... . . . . .... TTT -ATAGT-C-TGG... AGTACTGAAGGGTCAMTMACACTGAMGGAMGTGAC......... GT .....-ATAMT-C.TGGMATATACTACAGGGTCAMATMC ................

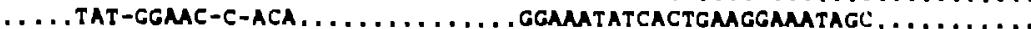

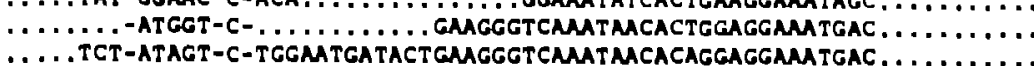
.... TCT-ATAGT-C-TGCMATCATACTCMAGGGTCMATMCACAGGAGGMMTGAC........

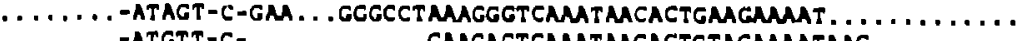

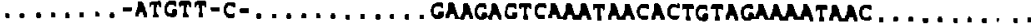
.......-ATGAT-C-........... GMMAGTCMGGTGCACTGAAGGAMTCAC.........

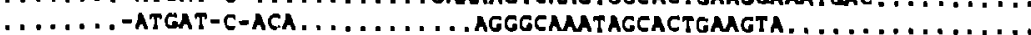

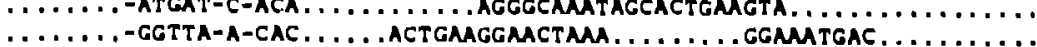

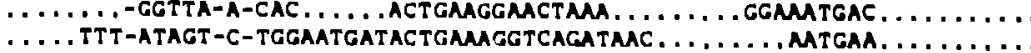

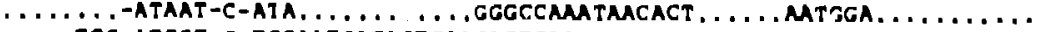

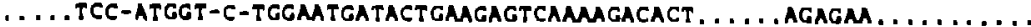

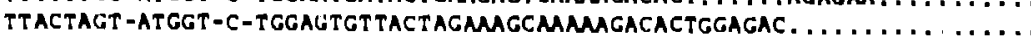

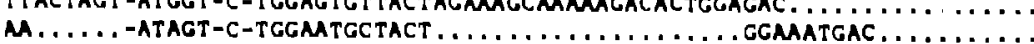

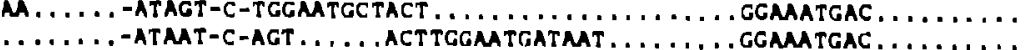

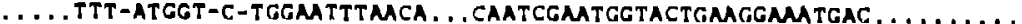

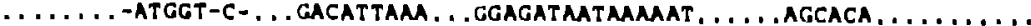

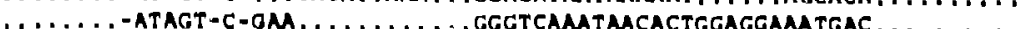




\section{HIV.1 ENV}

CONGENSUS1 . AC?ATCACACTCCEATGCAgMTMMMCMATTATMACAEG. TGGCAGgAAGTAGGARAAGCARTGTA

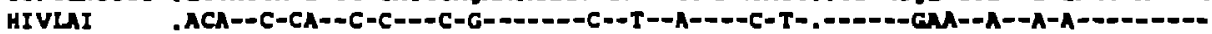

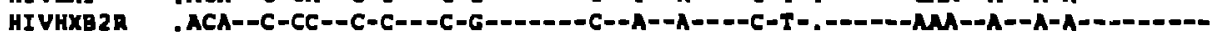

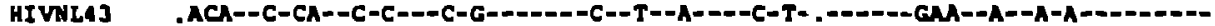

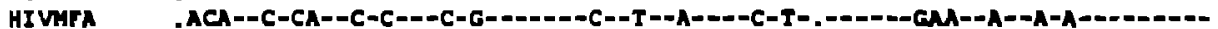

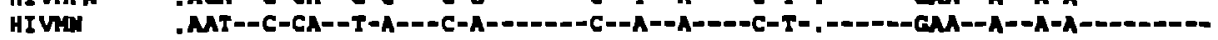

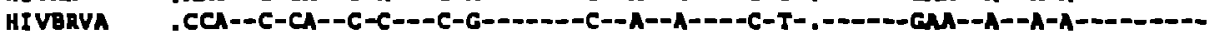

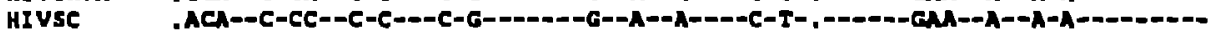

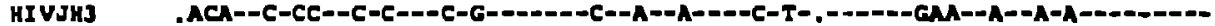

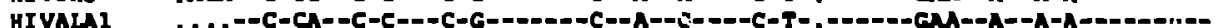

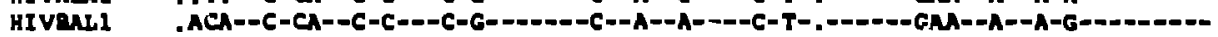

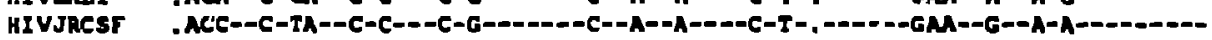

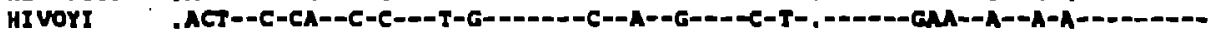

HIVIT2

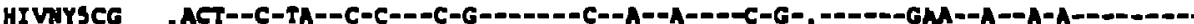

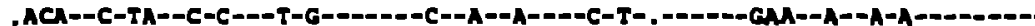
HIVSR1 62 . ACT--C-CA--C-C--C-G--

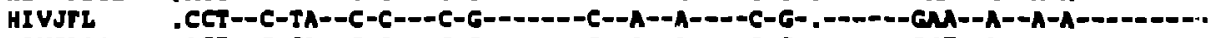

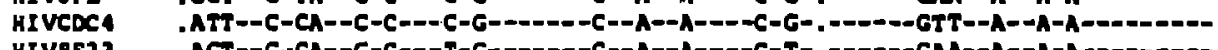

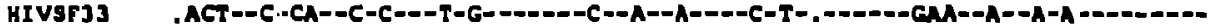
HIVTN HIVAOA HIVIW2 .CTC--C-CA--C-C--C-G-

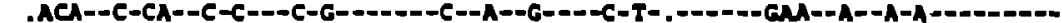

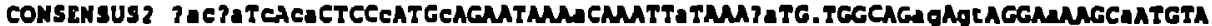

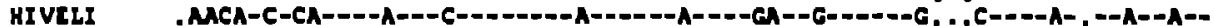

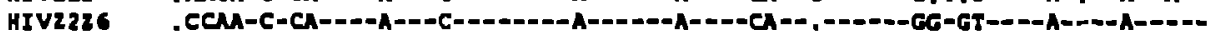

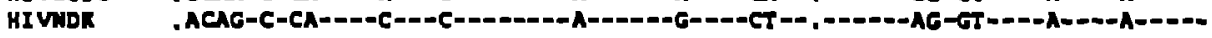
HIVJYI

HIVine

HIVuiss

HIVZ32I

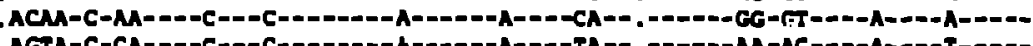
ACTA-A-CT--D-A -0.

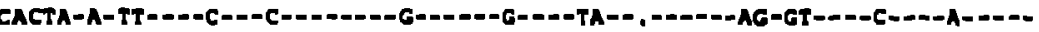
. ATTA-A-TA--G-C---C-----T-GA--G--AG-T-GTTCA.---AT-AGGGT----AG--GA--A--

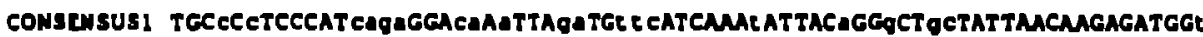

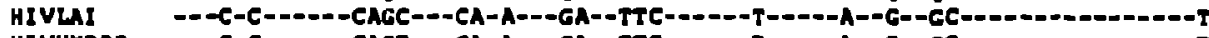

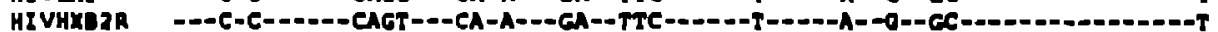

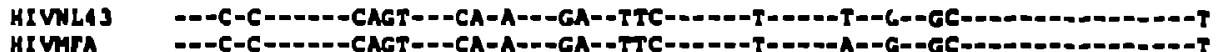
HIVHF HIVU HIVARVA

HIVIC

HIVJH3

HIVALA

HIVARL!

HIVJnest

HIVor?

HIVIr?

HIVWYSCG

HIVari 62

HIYJTL

HIVCDCA

HIVST3

HIVHAN

HIVADA

III VMW ?

HIVAr

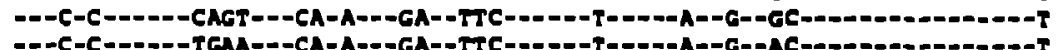

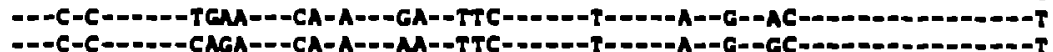

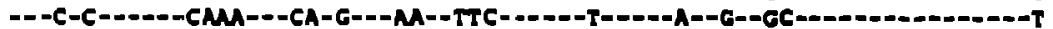

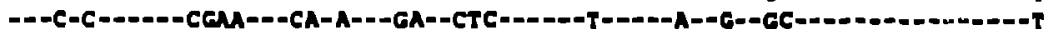

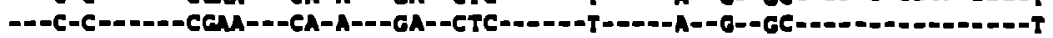

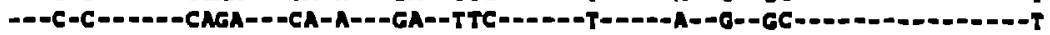

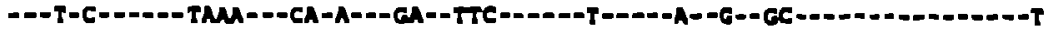

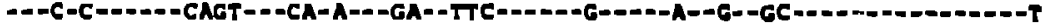

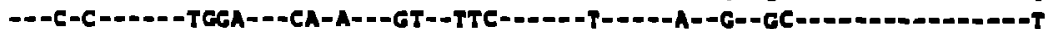

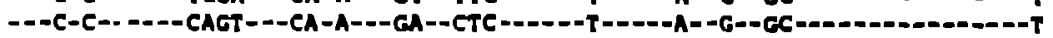

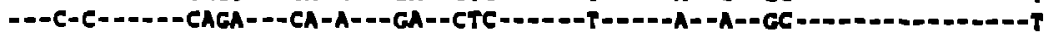

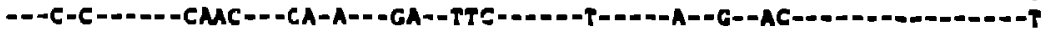

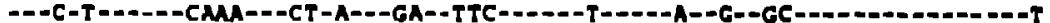

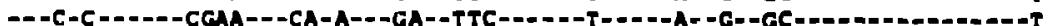

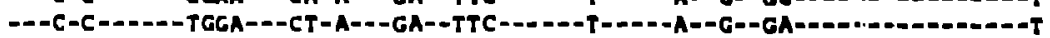

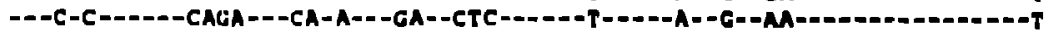

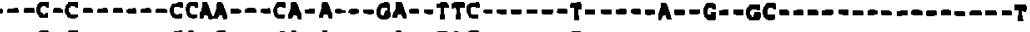
HIVLLI HIVI226

HIVION

HIVJYI

IIIVAL

HIVU⿺s

HIVI32I

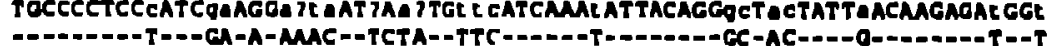
-

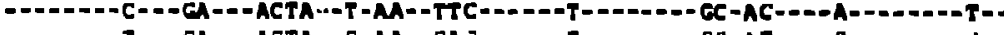

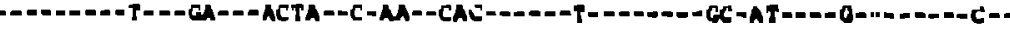

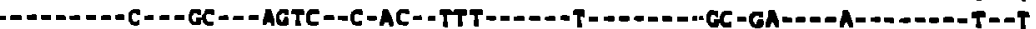

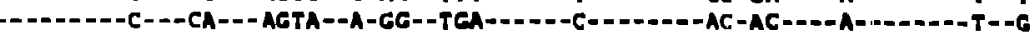

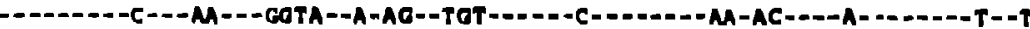
$---A--C--A---A G---M A C--C-C T--C A A T--T--C--D-T--A C-T C-C--A--T T C--C A C T$ 
HIV.1 ENV

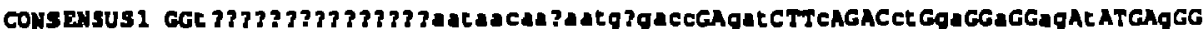

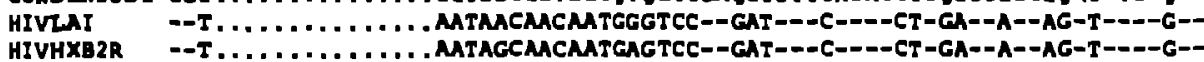

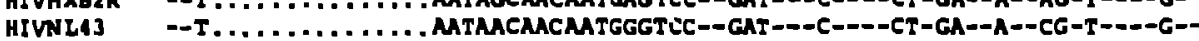

HIVAFA

-T.

HIVAF

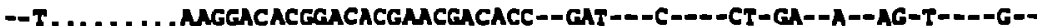

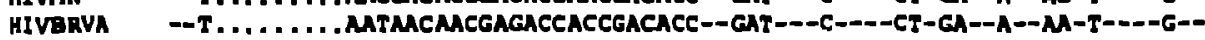

HIVSC

HIVJH3

HIVALAI

HIVBALI

HIVJResr

HIVOYI

HIVAr2

HIVTYSCe

hivaric2

HTVSTl

HIVCDCA

HIVST33

HIVHAN

HIVADA

HIVW152

HIVRF

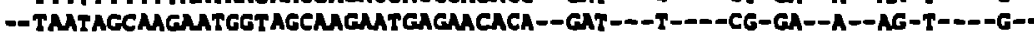

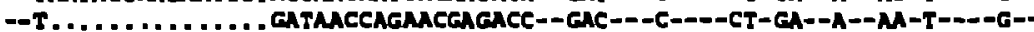
--T. . . . . . . . . . . MTAGCAGCMACMCACE--GAT---C----CT-GA--A--AG-T---G--T.

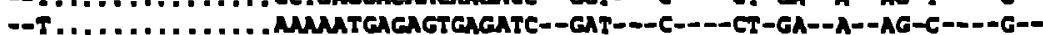

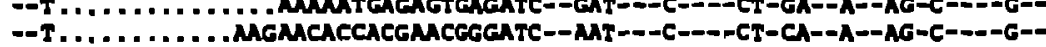

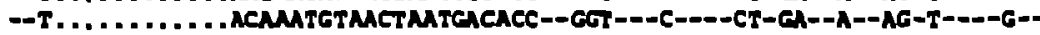
--T. . . . . . . . . . GATMMCAGMCAGTACCACC--GAT--C----CT-CA--A--AG-T--G-

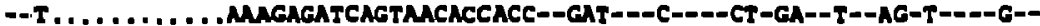

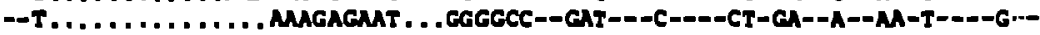
--T. . . . . . . . . . . GGTGAGACCACACCACC--GAT--T--.-CT-GA--A--AG-T---G-

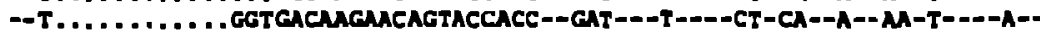

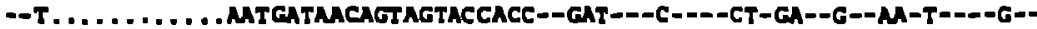

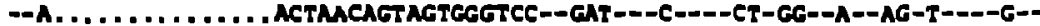

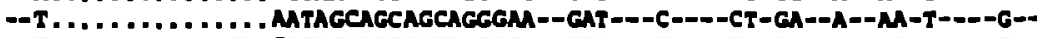

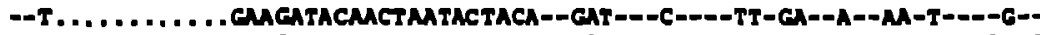

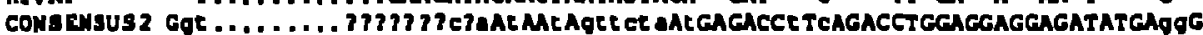

HIVELI

HIVZ226

HIVNDK

HIVJY1

HTVLL

HIVUass

HIV2J21

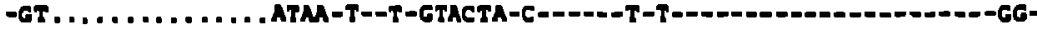

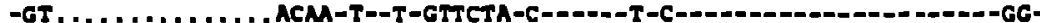

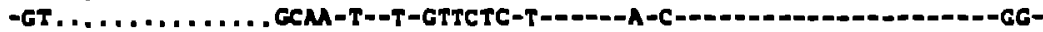

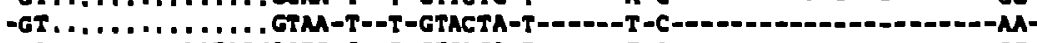

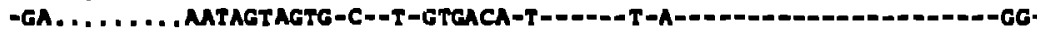

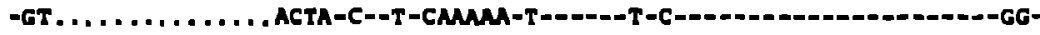

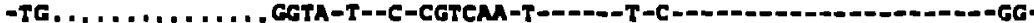
CCT. . . . . . . . GTGACTA-C--C-GTGGTA-CCT--AT-T-2.--AC-_-_-_-A-C--.-AA-

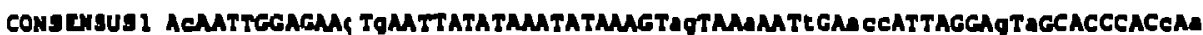

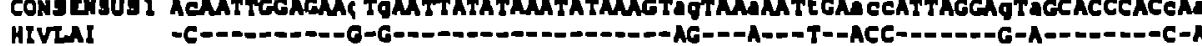

HIVHXE2R -C-

MIVNLA3

HTver

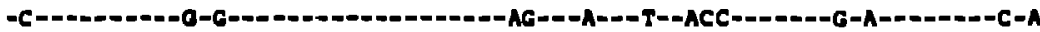

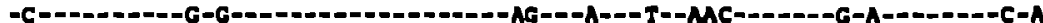

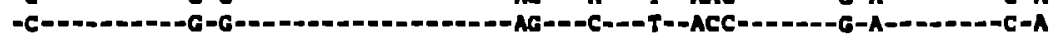

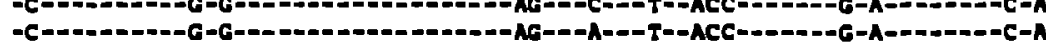

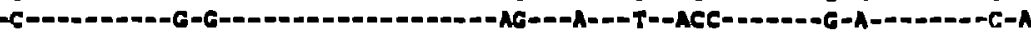

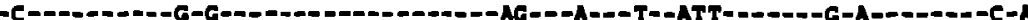

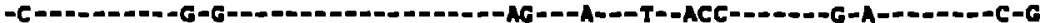

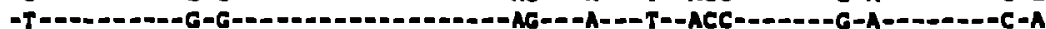

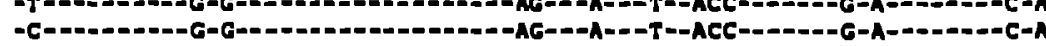

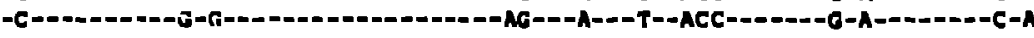
-C-O-_-

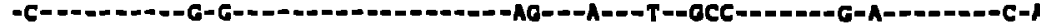

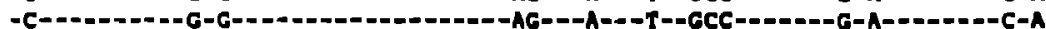

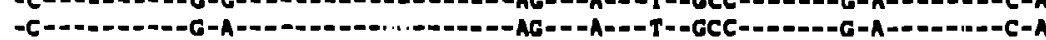

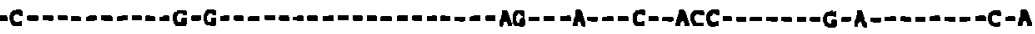

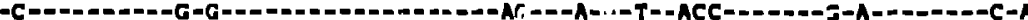

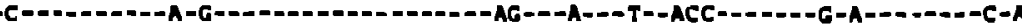

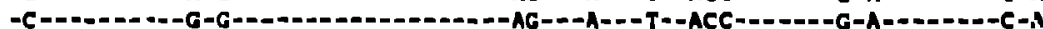

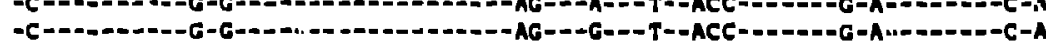

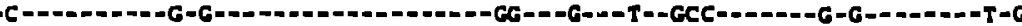

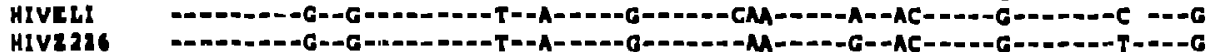

HIV2226

HIVNDK

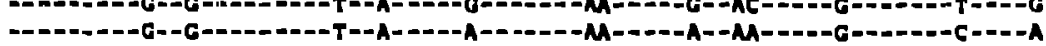
-

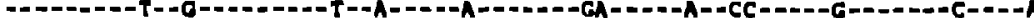
- - 
HIV-1 ENV

T5120 \/ gp41

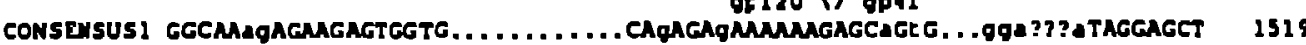

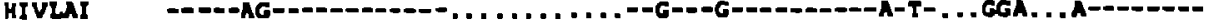

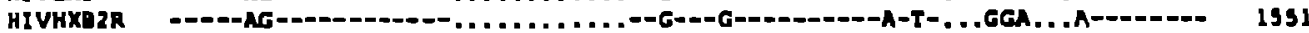

HIVTL3

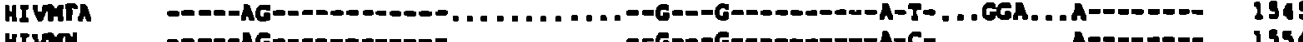

HIVI

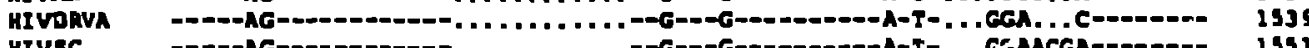

HIVSC

HIVJK3 -

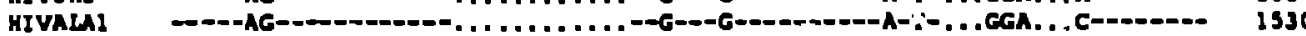

HIVDALl _.

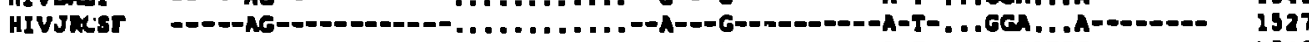

HIVOYI - -

HIYSF2 -

HIVWYSCG -

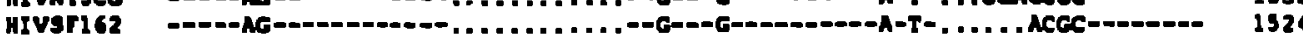

ITVJT

HIVJPL

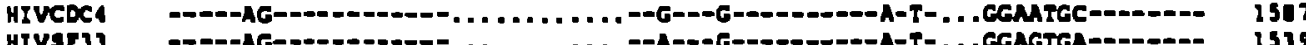

HIVSFJ3

HIVHN

HIVNAA -

HIVwid2

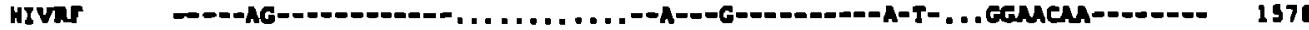

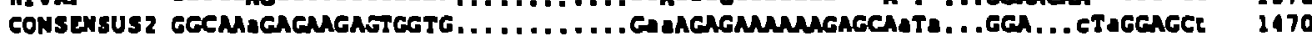

HIVELI

HIV22L6

HIVNDR

HIVJYI

HIVRA

HIVUASS

HIVz321 -

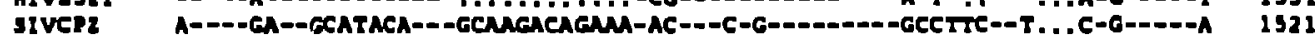

<- anc or can

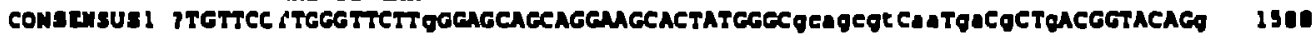

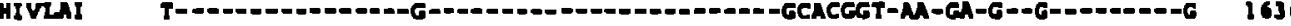

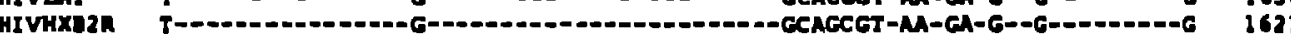

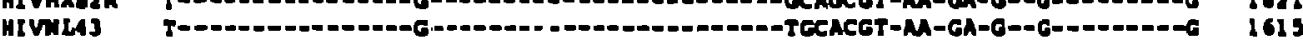

HIVILAJ
KIVHIA

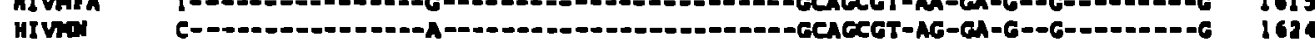

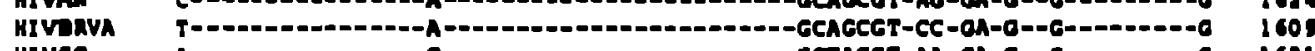

HIVSe A

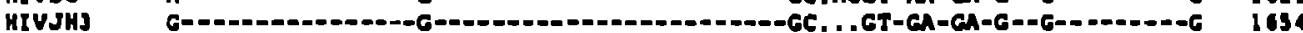

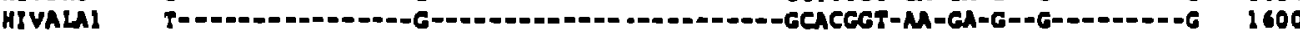

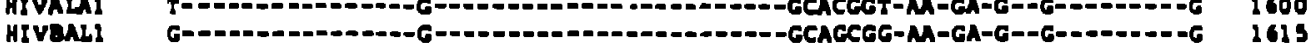

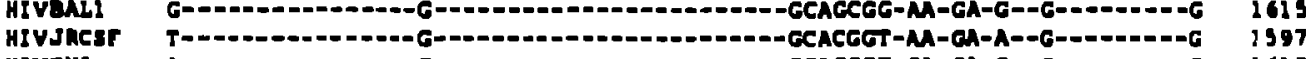

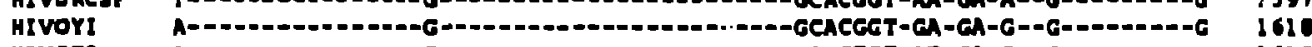

HIVST? A-_._.

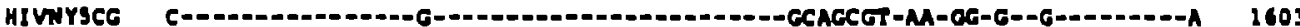

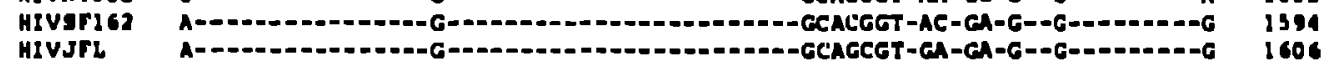

HIVCDCA A- A.

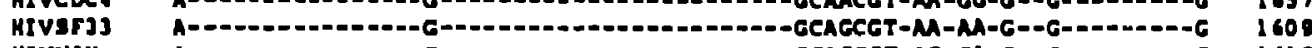

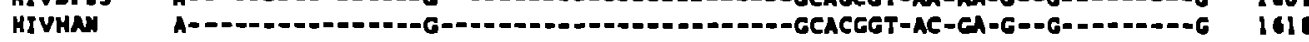

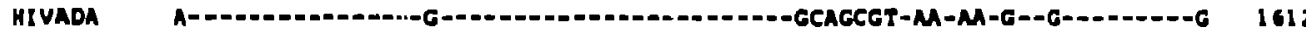

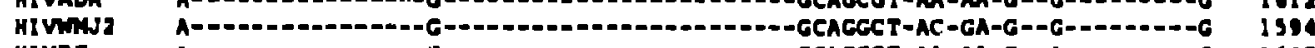

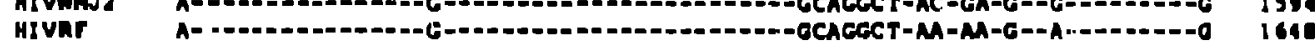

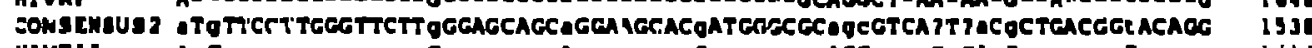

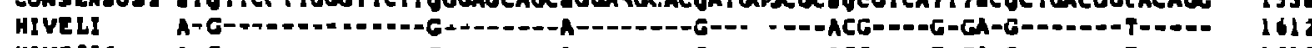

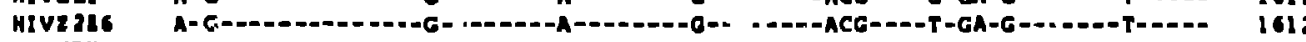

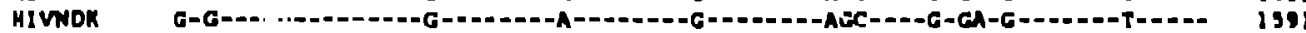

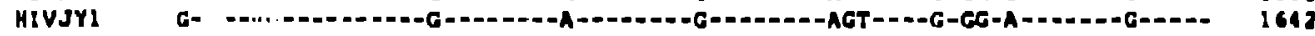

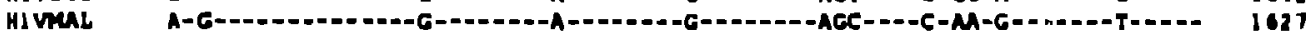

HIVUASS A-C-1.-1,

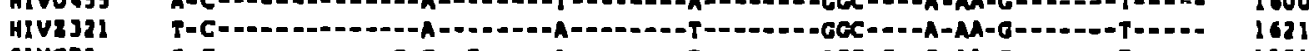

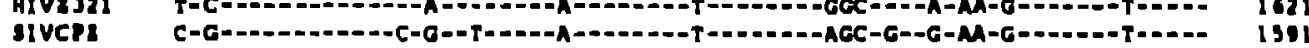


HIV-1 ENV

CONSENSUSI CCAGACaATALTGTCTGgTATAGTGCAMCAgCAgMACMLETGCTGAggGCLATTgAgGCgCMACAgCA HIVLAI HIVHXB 2R

HIVNLAJ

HIVMre

HIVAFA

HIVBRVA

HIVSC

HIVJH3

HIVALAI

HIVBALI

HIVJRCSF

HIVOYI

HIVSF?

HIVNYSCG

HIVSF 162

HIVJFL

HIVCDCA

HIVSFJ?

HIVHAN

HIVADA

HIVMAJ 2

HIVRF

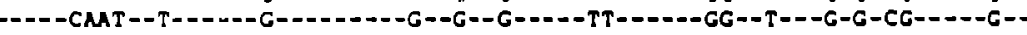

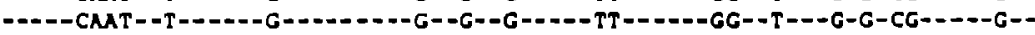

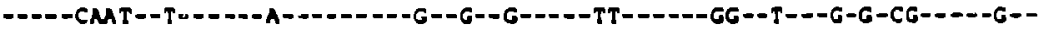

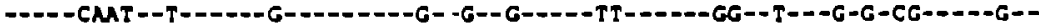

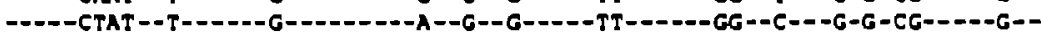

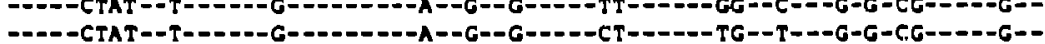

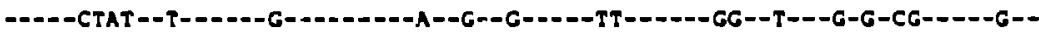

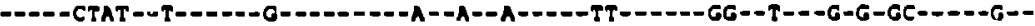

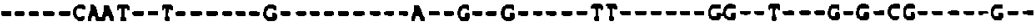

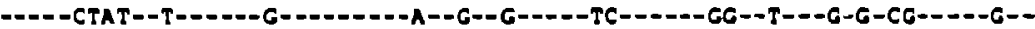

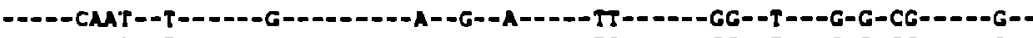

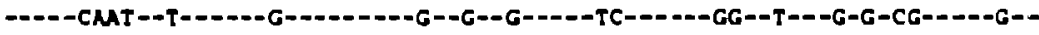

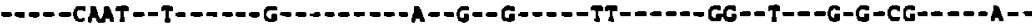

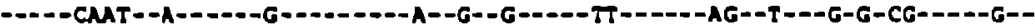

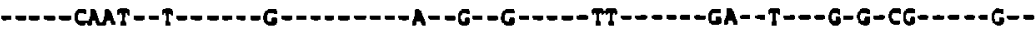

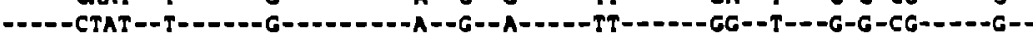

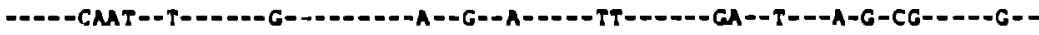

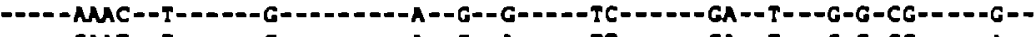

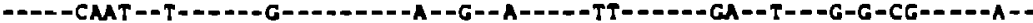

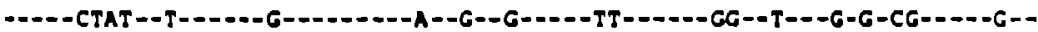

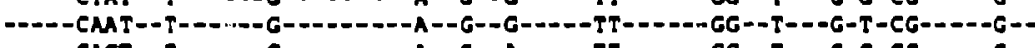

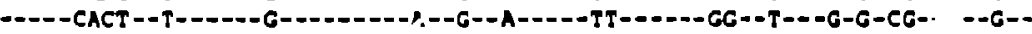

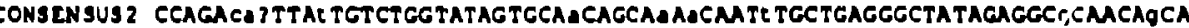

HIVELI

HIV2226

HIVNDK

--_--CM---A--- -

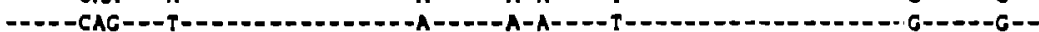

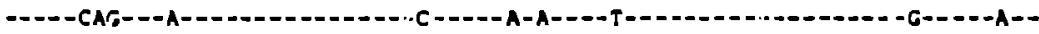

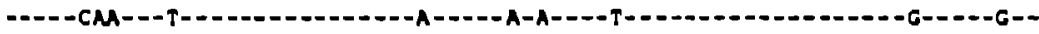

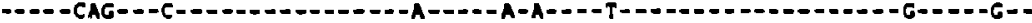

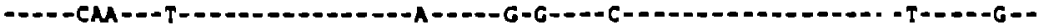

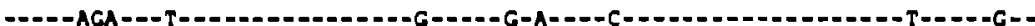

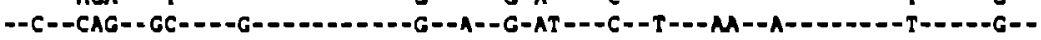

RRE OR CAR $\rightarrow$

CONSENSUS 1 C CTGTTGCAACT CACAGTCTGGGGCATCAMgCAGCTCCAGGCMAGAgTCCTgGCTgTgGMAGATACCTA

HIVLAI

HIVHXB 2R

HIVWLA J

HIVATA

HIVMM

HIVBRVA

HIVSC

HIVJH3

HIVALAI

HIVBALI

HIVJRCSF

HIVOYI

MIVSF?

HIVTYSCG

HIVSTIG

HIVJFL

HIVCDCA

HIVST 3

HIVHAN

HIVADA

HIVAMS

HIVRT

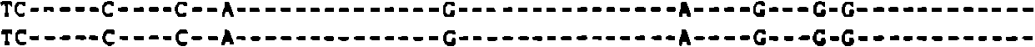

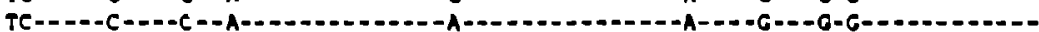

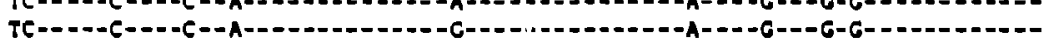

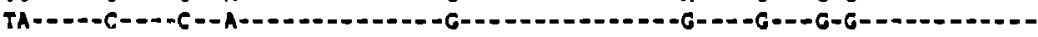

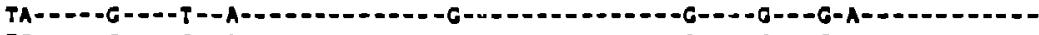

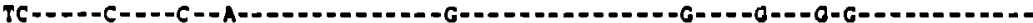

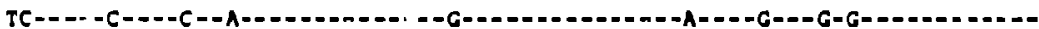

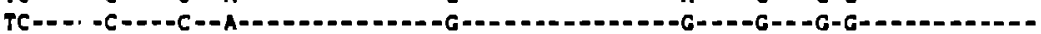

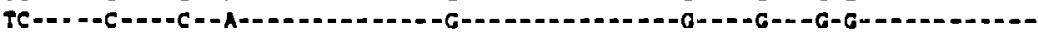

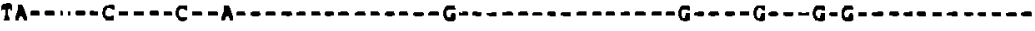

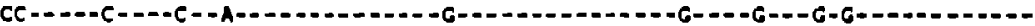

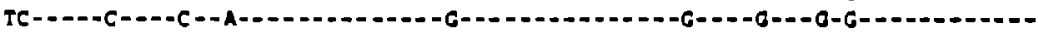

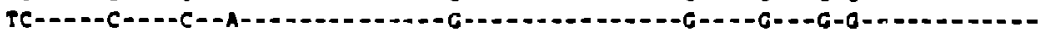

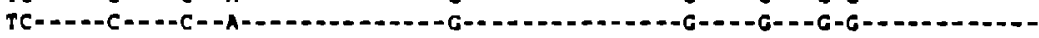

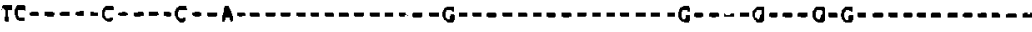

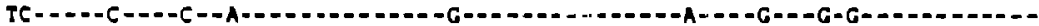

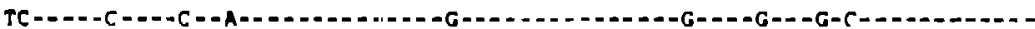

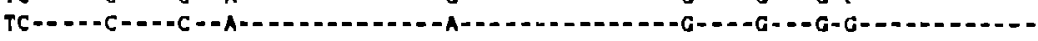

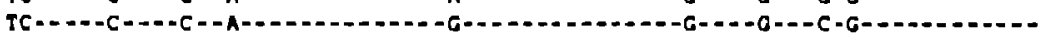

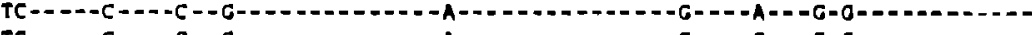

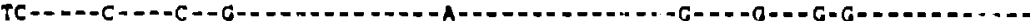

CONIEN SUS 2 TCTGTTGCAACTCACUOTCTGGGGCATTMACAGCTCCAGGCMGAgTCCTGGCTGTgGAAAGTACCTA HIVTILI

HIVIZZ

IIIVNDK

IIIVJYI

HIVML.

Hivu'ss

HIVI321

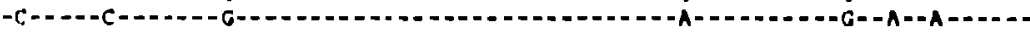

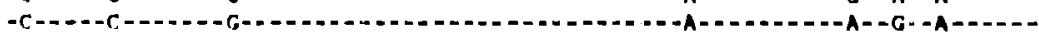

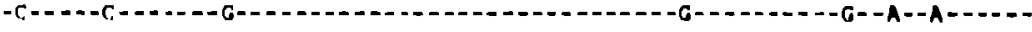

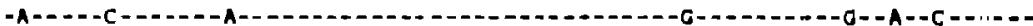

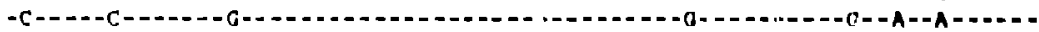

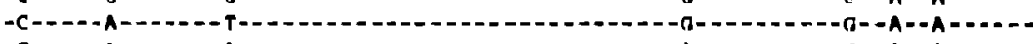

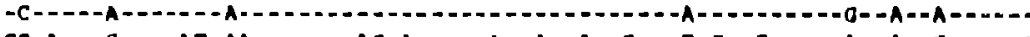

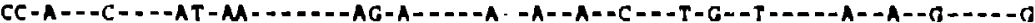


HIV-1 ENV

CONSENSUS1 agGALCAACAGCTCCTgGgga TTTCGGGLTGCTCTGGMMaCTCATE TGCACCACTgCTGTGCCTTCGA HIVLAI MG--T-1-1

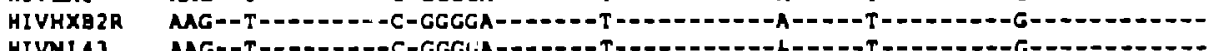

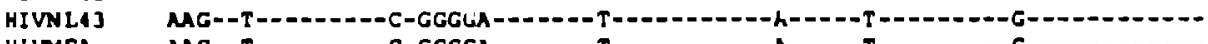
HIVAN HIVBRVA HIVSC HIVJH3

HIVALAI

HIVBAL HIVJRCST HIVOY! HIVST? HIVWYSCG

HIVSTI62 HIVJFL HIVCDCA HIVSTJ3 HIVHN HIVADA HIVIMJ? HivRF HIVRF

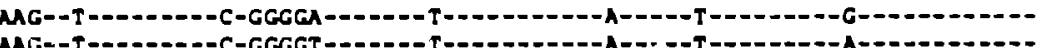

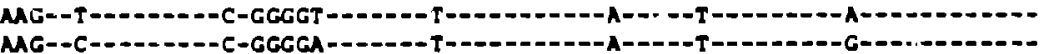

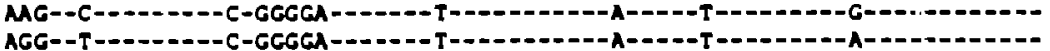

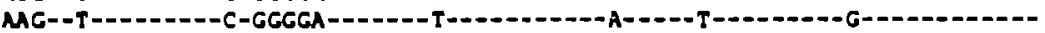

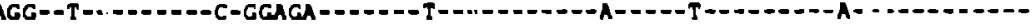

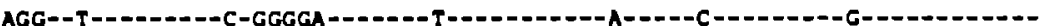

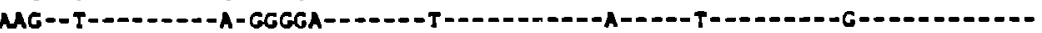

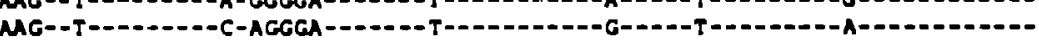

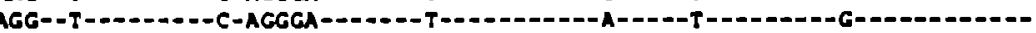

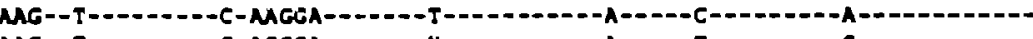

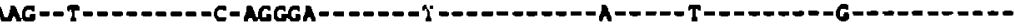

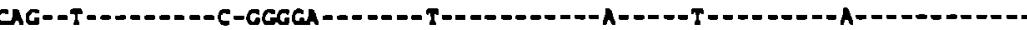

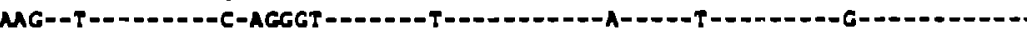

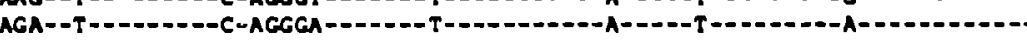

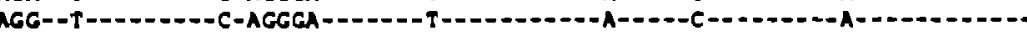

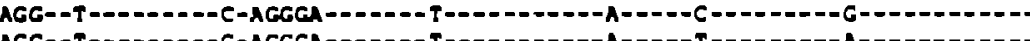

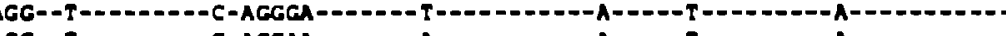

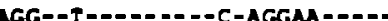
CONSENSUS 2 aGGATCAACAGCTCGTAGGMTt TGGGGt TGCTCTCGMAA JCATETCCACCACE ACTCTgCCETCCA HIVELT HIV2 226 HIVNDK HIVJYI HIVAAL HIVU455 HIVZ321 SIVCP 2

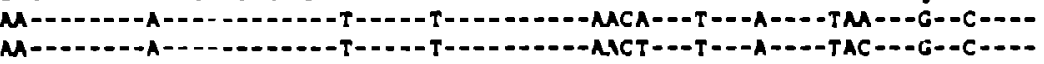

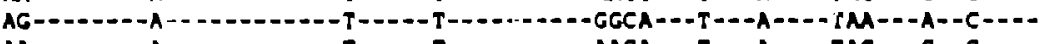

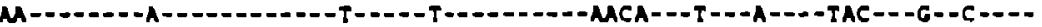

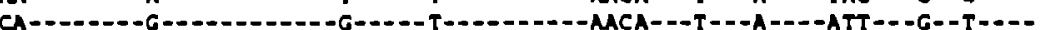

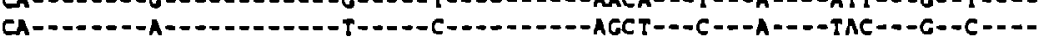
MA-10.-1-

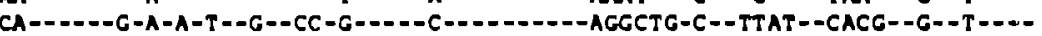

CONSENSUSI AE gCTAGTTGG. . AGTMATMAC CL CTGOAT rogatTTggaATaACOL GACETGGATggAGTGGGAaAg

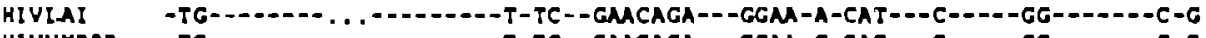

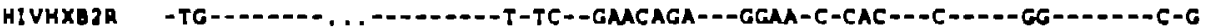
HIVNLAJ
HIVMTA HIVMFA HIVAN HIVBAVA HIVSC

HIVSHJ HIVALAI HIVDAL

HIVJRCST HIVOYI -TG--O-... . .

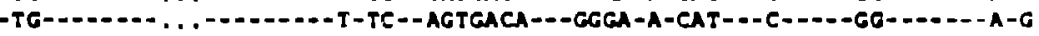

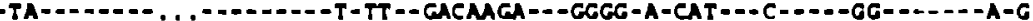

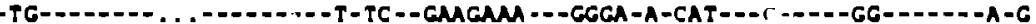

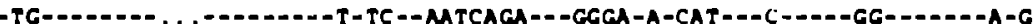

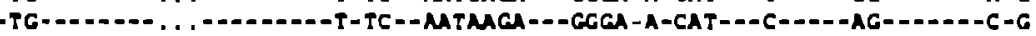

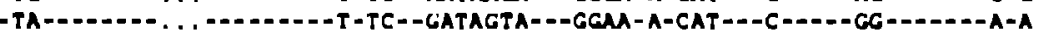

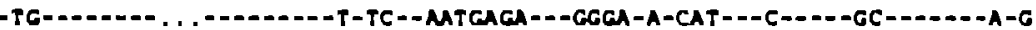

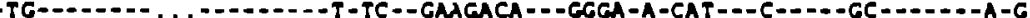

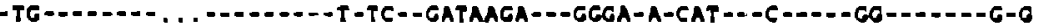
-TG-_..... . . - TG-1.2.-. . . -TG-_...- . . .TA-_-1.-. . . .

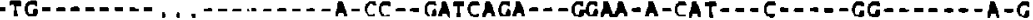
hIVIFj] HIVHAN TIVADA HIVADA HIVINJ
HiVAT

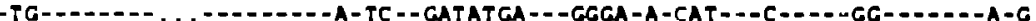

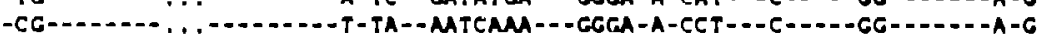

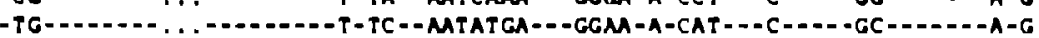

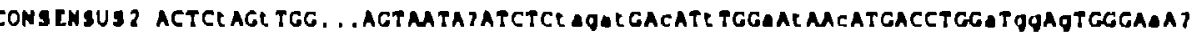

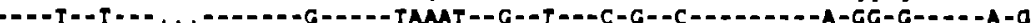
HIVI2 25

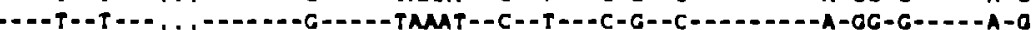

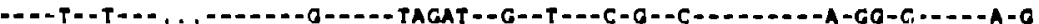

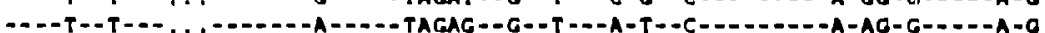

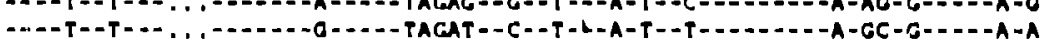

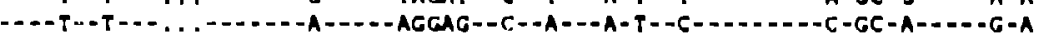

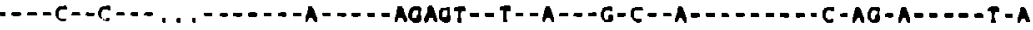
HIVALL hinusg 
HIV.I ENV

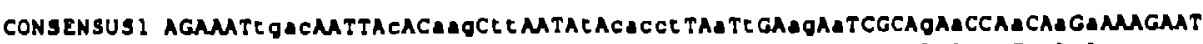

HIVLA?

HIVHXB2R

HIVN L4 3

HIVMrA

HIVMN

HIVBRVA

HIVSC

HIVJH3

HIVALAI

HIVBALI

HIVJRCSF

HIVOYI

HIVST?

HIVNYSCG

HIVSTIG2

HIVJTL

HIVCDCA

HIVSFJ

HIVHAN

HIVADA

HI VWAJ 2

HIVRT

CONSENSUS 2

HIVELI

HIVZ226

HIVNDK

HIVJYI

HIVMAL

HIVUAS:

HIV2J2 I

SIVCPZ

CONSENSUS!

HIVLAI

HIVHXB2R

H[VNLA]

HIVMFA

HIVMN

HIVBRVA

HIVSC

HIVJHJ

HIVALAI

HIVBAL!

HIVJRCSF

HIVOY!

HIVST?

hiveyse.

HIVET 162

HI'SJFL

HIVCDCA

HIVSTJ

HIVHAN

HIVADA

HIVNAJ 2

IIIVRT

CONSCNSUS 2

HIVELI

IIIY2 216

HIVNDK

HIVJYI

HIVAat

IIIVUas

HIVZ32I

sivep?

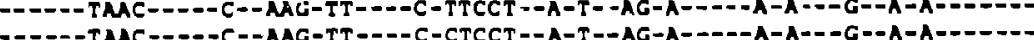

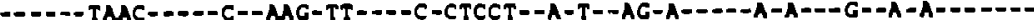

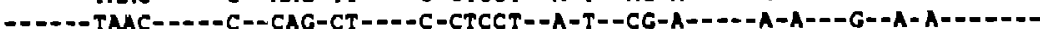

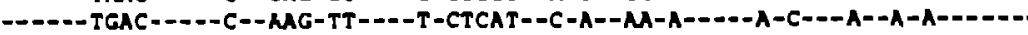

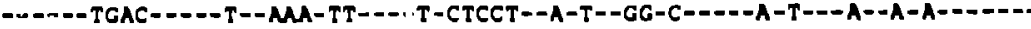

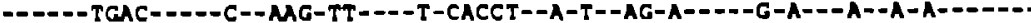

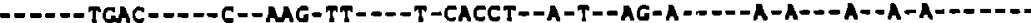
-

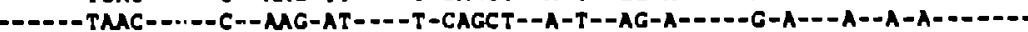

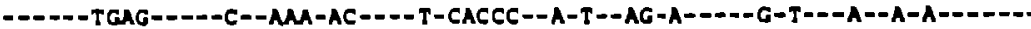

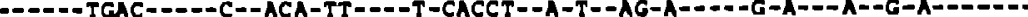

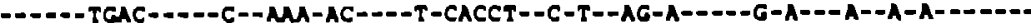
-

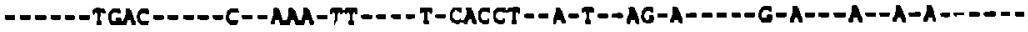

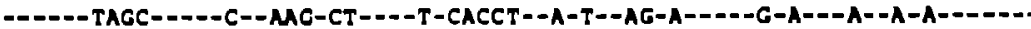

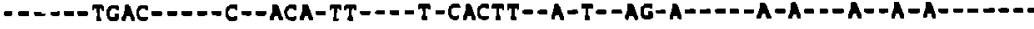

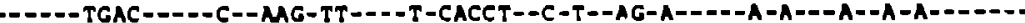
-

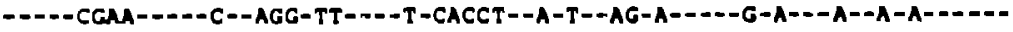

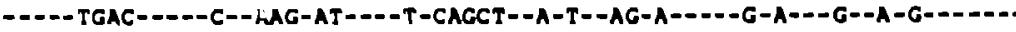

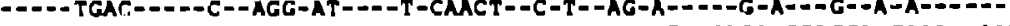

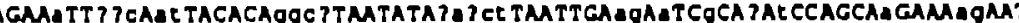

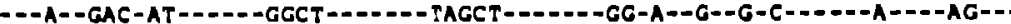

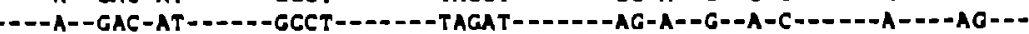

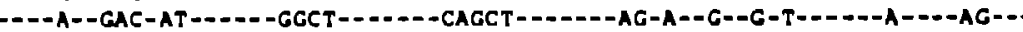

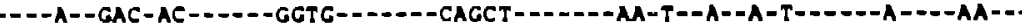

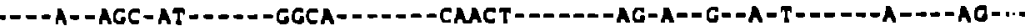

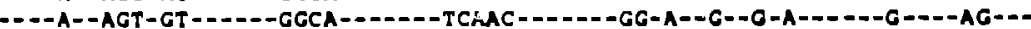

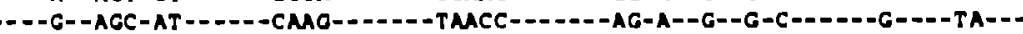
-TT-C-GTCT-AC-_-_-_GGGM---T-TTGGTC-GT-A--GG-AG-A--ATCA--A--A-DG-AA-_.

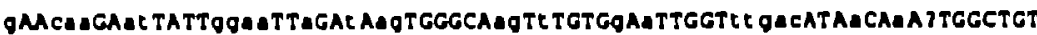

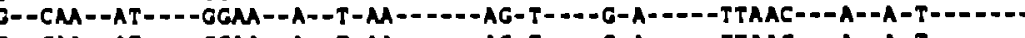

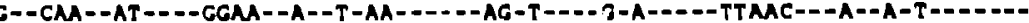
G--CM--AT----GGMA--A--T-AA------AG-T----G-A---D-TTAAC-D-A--A-T------

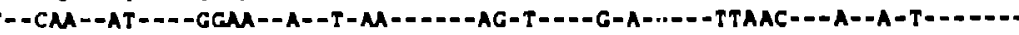

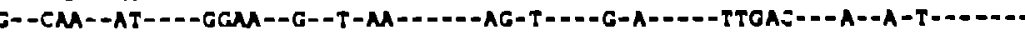

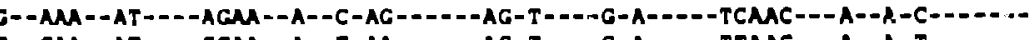

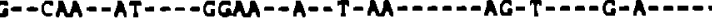

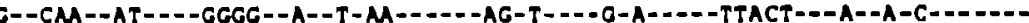

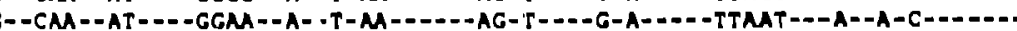

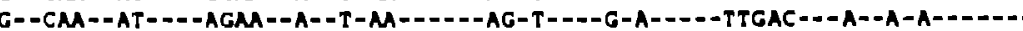

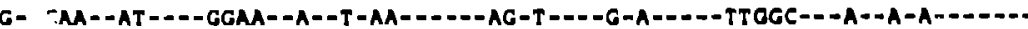

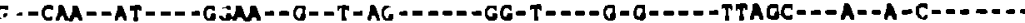

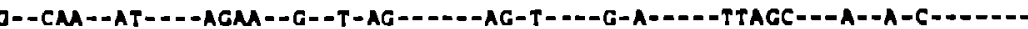

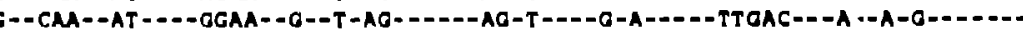

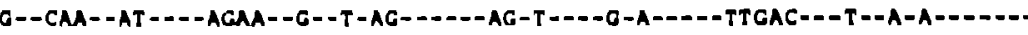

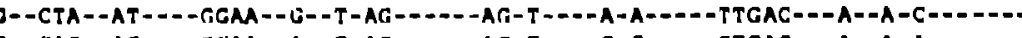

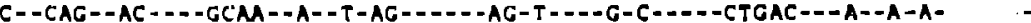

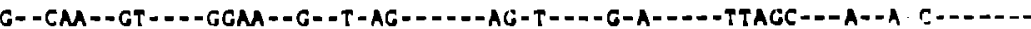

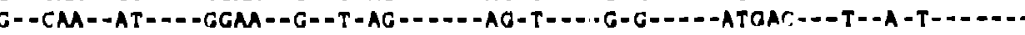

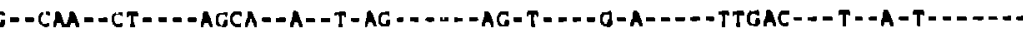

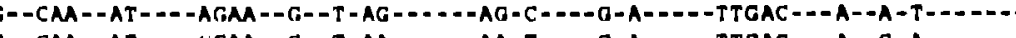
-

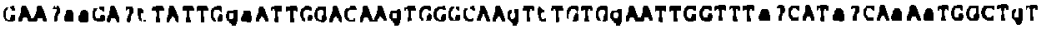

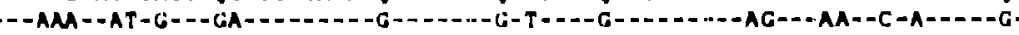

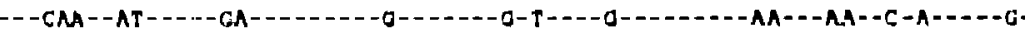

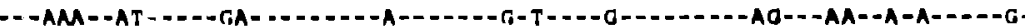

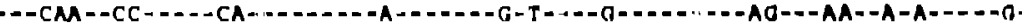

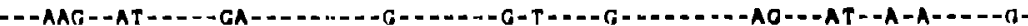

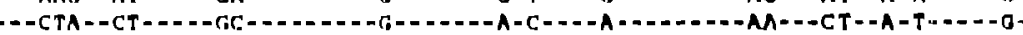

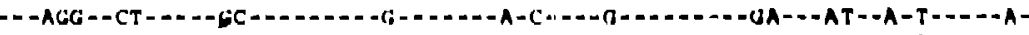

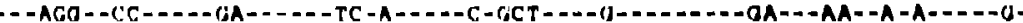

1934

1983

1968

1962

1962

1971

1956

1968

2001

1947

1962

1944

1950

1941

1953

1965

1941

1995

1079

1959

1959

1938

1909

1974

1947

1961

2003

2053

2038

2032

2032

2041

2026

2071

2017

2032

2014

2035

:0:1

2023

<4)

2021

20J3

2029

2011

2069

1941

2024

2029

2008

2058

2044

$201 \%$

2010

2011

|.A.61

MAY YI 


\section{IIV.I ENV}

CONSENSUSL GGTATATMAAATATTCATMATATAgTAGgAGGCTTgGTAGGTETMOAATAgTTTTTOCTOTACTTIC HIVLAI HIVHX82R

HI VAFA

HIVMN

HIVBRVA

HIVSC

HIVJHJ

hIVALAI

HIVBALI

HIVJRCSF

HIVOY!

HIVSr?

HIVNYSCO

HIVSFI 62

HIVJTL

HIVCDC4

HIVST33

HIVHAN

HIVADA

HIVIMJ 2

HIVAT

CONSENgus

HIVELI

HIVI226

HIVJYI

HI VMLL

HIVUAs

HIVZ321

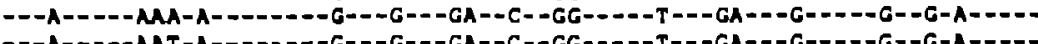

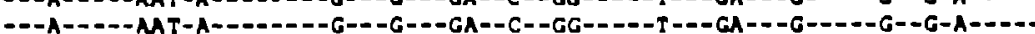

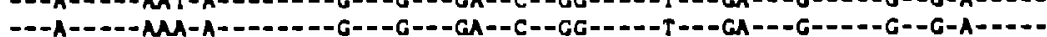

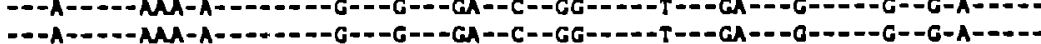

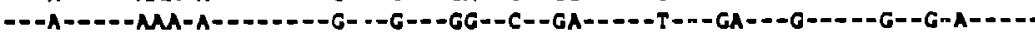

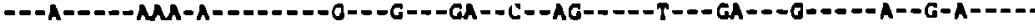

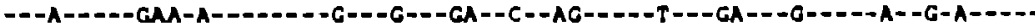

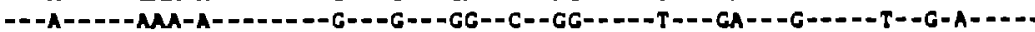

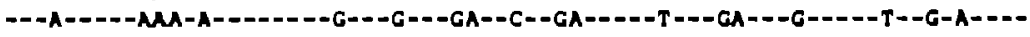

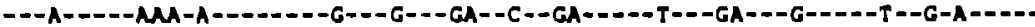

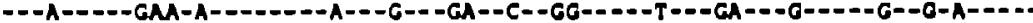

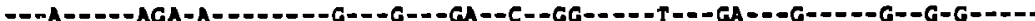

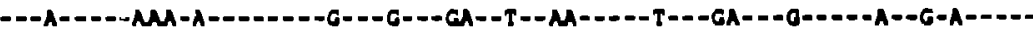

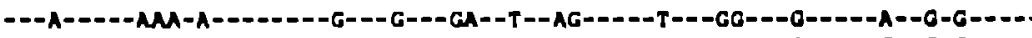

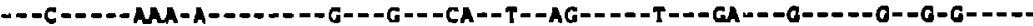

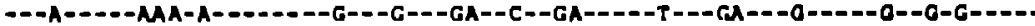

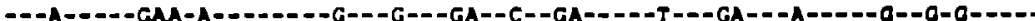

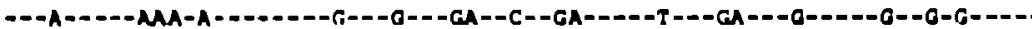

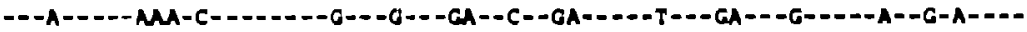

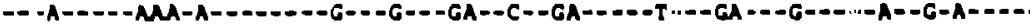

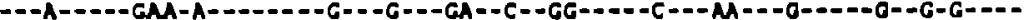
GGTATATAAAAATATTCOTAATgATAGTAGGAGGCETgATAGGLTTMAGATAgTTTTTgCEGTGCTTTC

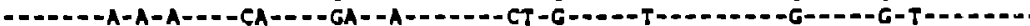
-

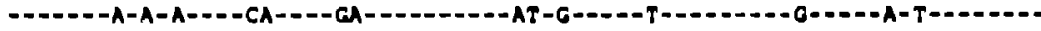

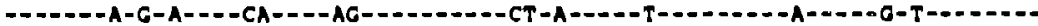
-

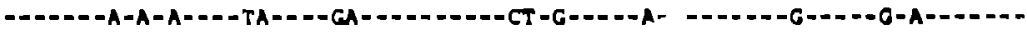
-

Y/J' (m/nor)

1/3. j (minor)

V/3' of (ma for)

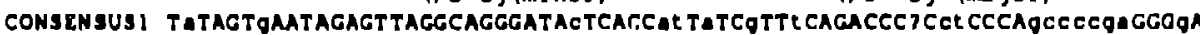
HIVLA! HIVHXO2R

HIVNLA]

HIVMFA

HIVMN

HIVIRYA

HIVIS

HIVJHJ

KIVALA!

HIVEALI

HIVJACST

HIVOYI

HIVST?

HIVNYSCO

HIJST162

IIIVJTL

IIIVCDCA

H[VIT]J

HIVHAN

HIVADA

HIVMMJ2

HIVAT

HIVELI

HIVI2ZO

IIIVNON

HIVJYI

HIVMAL

HIVU45

IIIVI,12!

sivep:

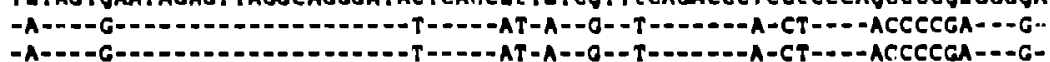

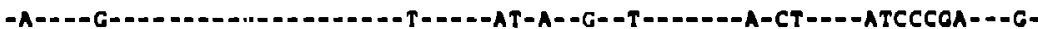

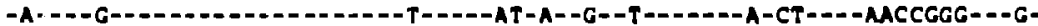

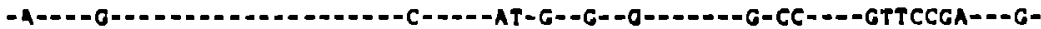

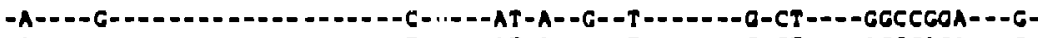

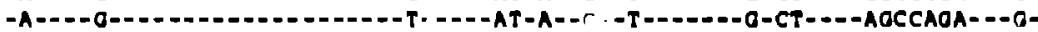

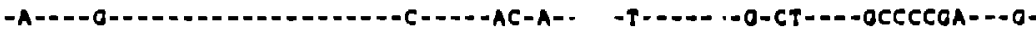

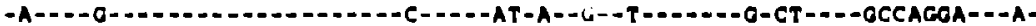

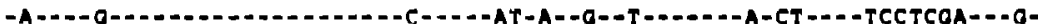

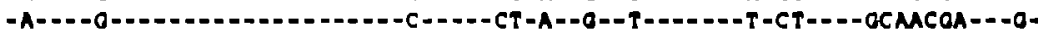

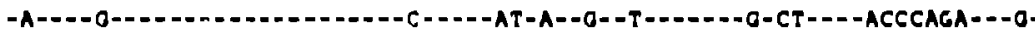

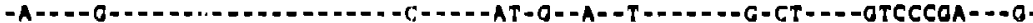

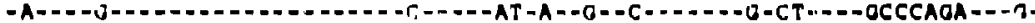

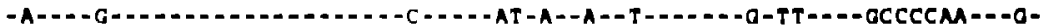

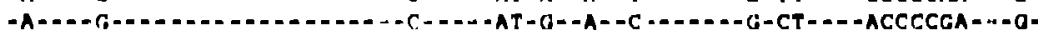

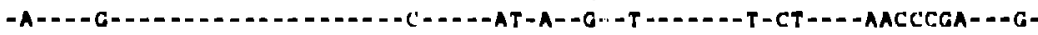

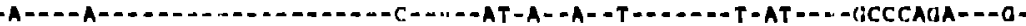

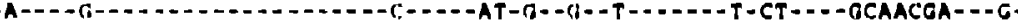

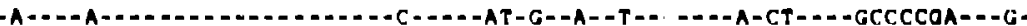

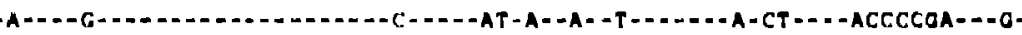

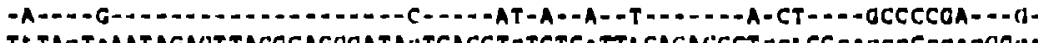

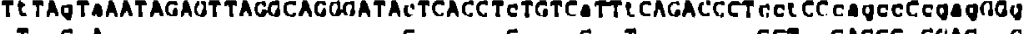
T- - G-A-2.--

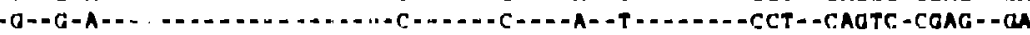
-T- -

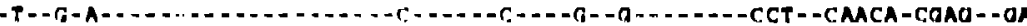

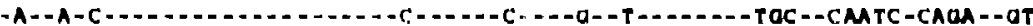

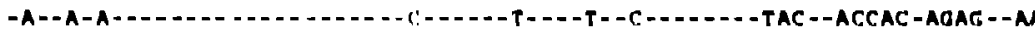
AO--O-C-GG-2.- 
HIV-1 ENV

$<-t a t$

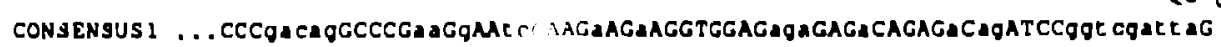

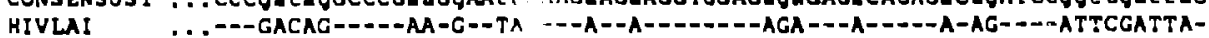
HIVHXB2R $\quad . .--$-GACAG-----AA-G--TA----A--A----.--AGA---A-----A-AG----ATTCGATIA-

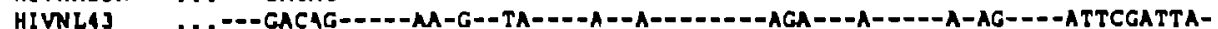

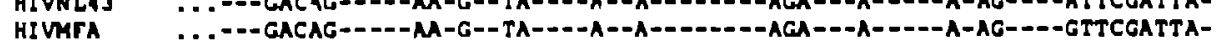

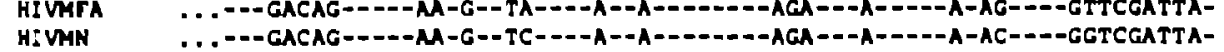

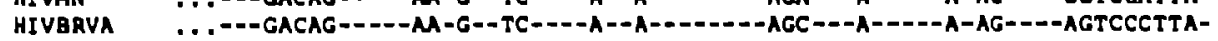

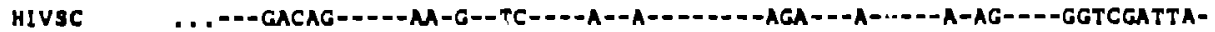

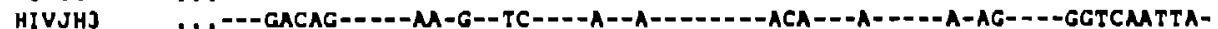

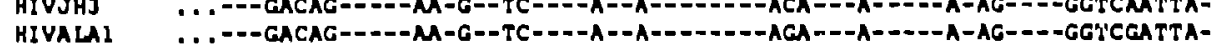

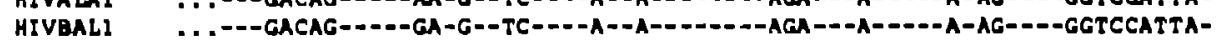

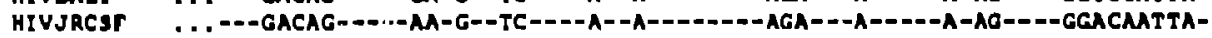

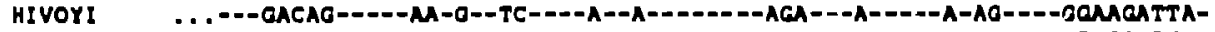

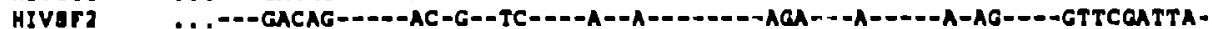

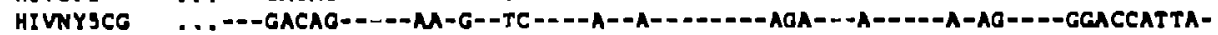

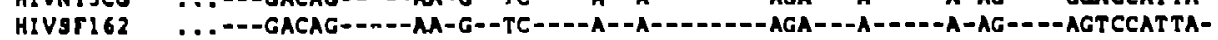

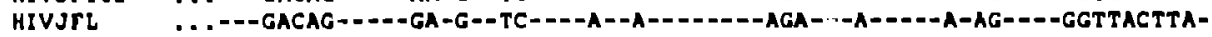

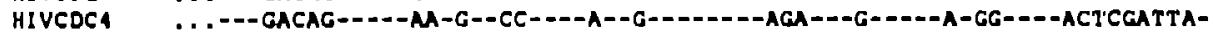
HIV3T33

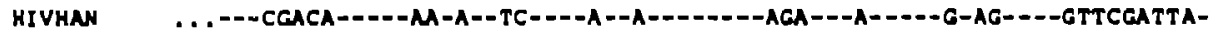

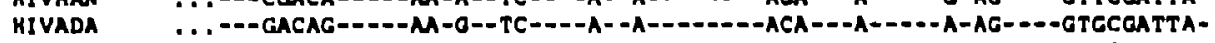

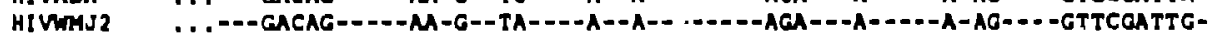

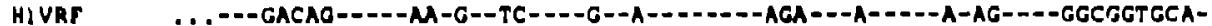
CONSENSUS 2 ?7CCCGACAGGCCCGAAggMTAGAAGAGAAGGTGGAGAGCaAGgCAgALACAGATGCATTCGaTTgG

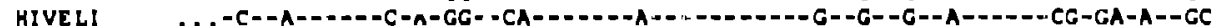

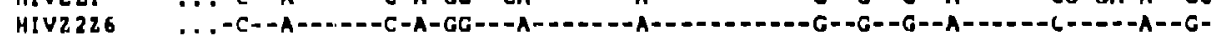

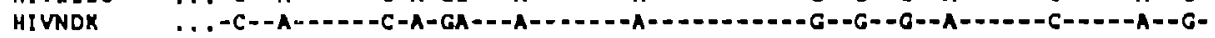

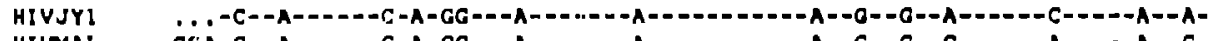

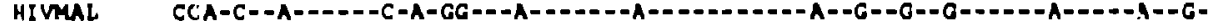

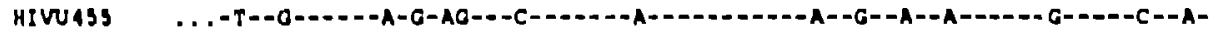

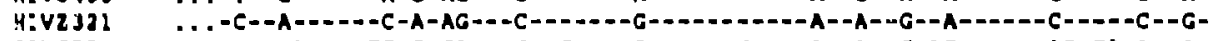

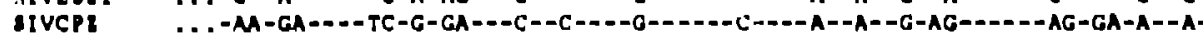

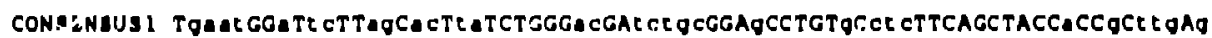

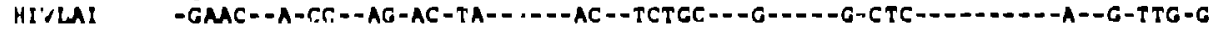

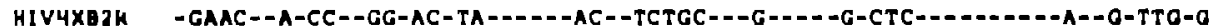

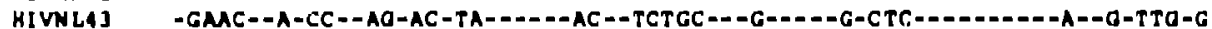

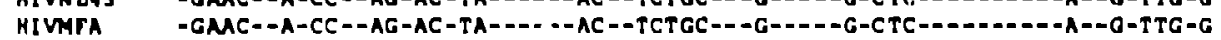
KIVMA HIVARVA

HIVIC HIVJHJ HIVALAI HIVBALI HIVJRCA! HEVOYI HIVar? HIVNYSCG HIVSTIR] HIVJrI. HiVCDCA MIVITJ. IIVIIAN iIIVADA MIVIMJ? IIVur

\section{CONARE} Hivri.l III Yzaz HIVNDK IIIV.IYI IIIVMAI. H:VU43) IIY212!

I IVCEz 


\section{HIV-1 ENV}

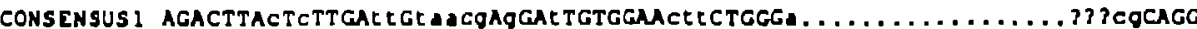
HIVLAI
HIVHXB2R
HIVILA3 HIVHXB2R
HIVNLA3 Hing HIVMA HIVBRYA HIVSC HIVJH3 HIVALA! HIVBALI HIVJRCSP HTVOY RIVOY HIVWYSCE HIVWYSCG HIVJTL HIVCDCA HIVSFJ J HIVHAN HIVAOA HIVกMJ2 HIVRT -

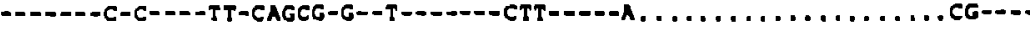

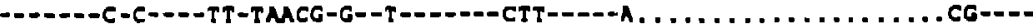

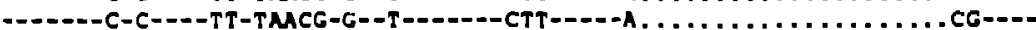

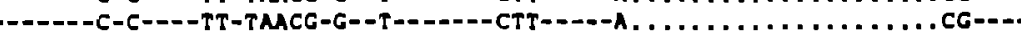

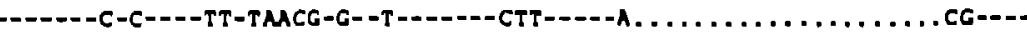
-

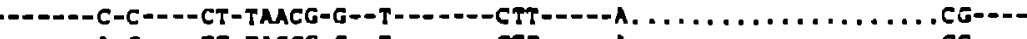

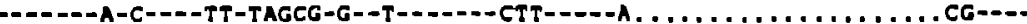

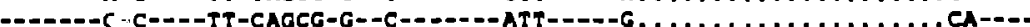

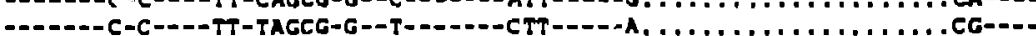

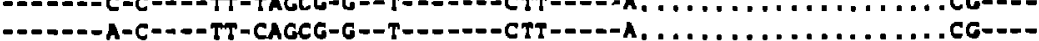

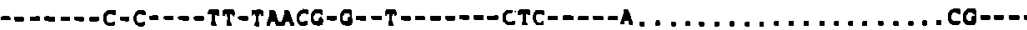

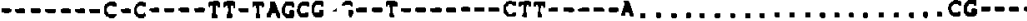

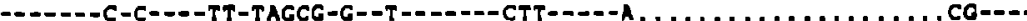

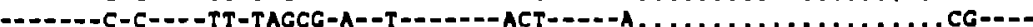
-

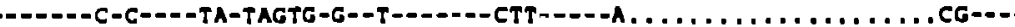

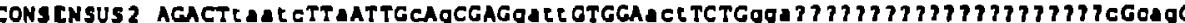

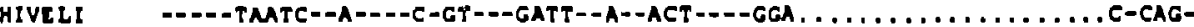
HIVI226 Hindon MIVJYI HIVIAL HIVUASS

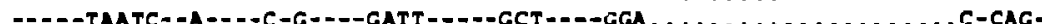
- -

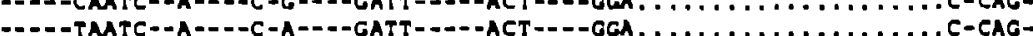

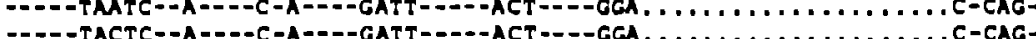

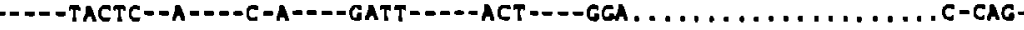
---TCGCC--G---T-G----AGCT--.--ACT----GOACGCAGCAGCCTCAAGGGACTGA-ACT-..-GCGCA-G---C-G---GATC----GAC-.-.............. ATCC-CAG-AG--TAGCC-GC-A--TGTG---ACAAC-CA-GACGT--CCACATCTMTACTTCACAGCCTCA-ACT-

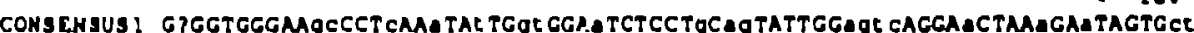
HIVLAI -.

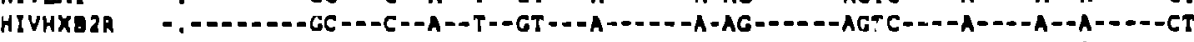

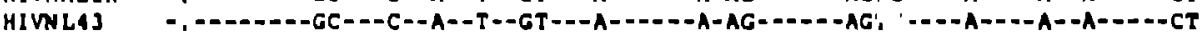
HIVATA HIVMY HIVERVA HIVIC HIVJHJ HIVALA! HIVBAL! IIIVJRCST HIVoY! hivarz HiVNYICO hivisice Hiv.JF?. Hiveriat IIVsrj j IIVIIAN IIIVIIAN IIIVTMJ HIVRT

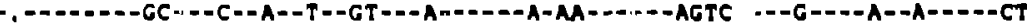

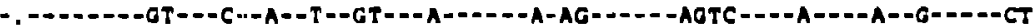
-

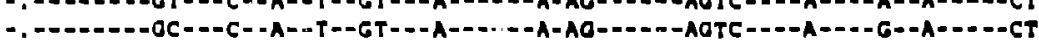

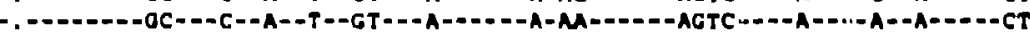

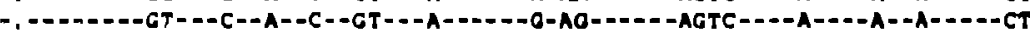

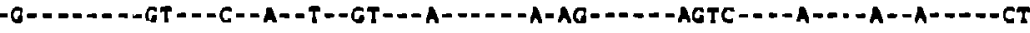

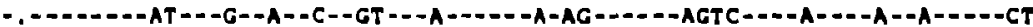

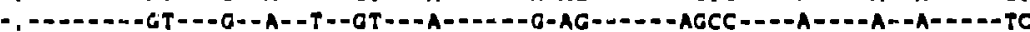
$\therefore$ -

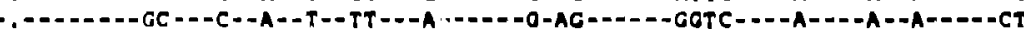

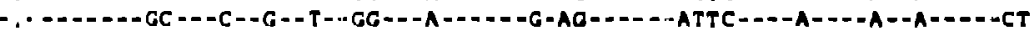

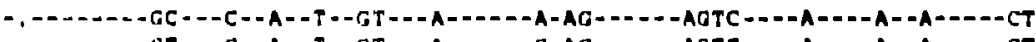

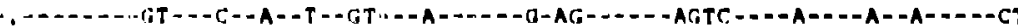
-

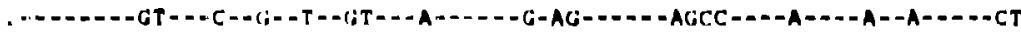

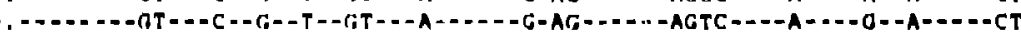

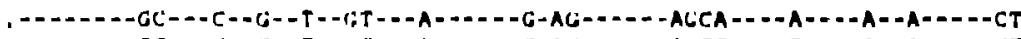
,

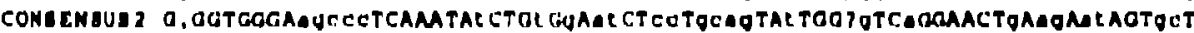

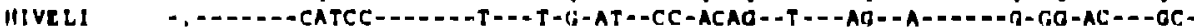
Hivez26 IIVNDK HIVJYI III VMAL IIIV4)

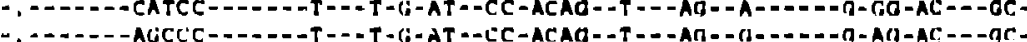

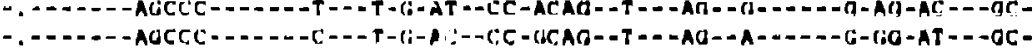

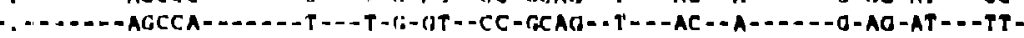

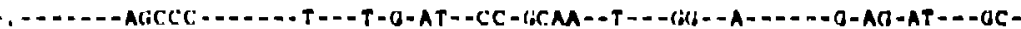

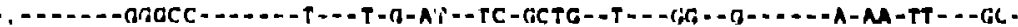

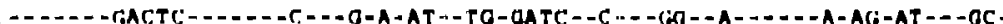

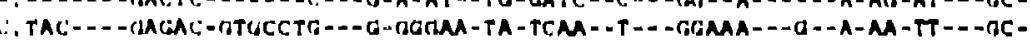


HIV-I ENV

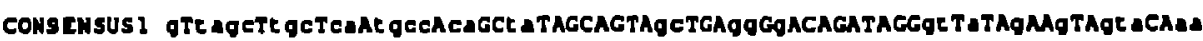
G-TAGC-TGC-CA-TCCC-CA--CA--------GC---GG-G---------GT-A--G--G--GTA--AG HIVHXB2R G-TAGC-TGC-CA-TGCC-CA--CA-------GC---GG-G--0-----GT-A--G--G--GTA--AC HIVHXBR GR

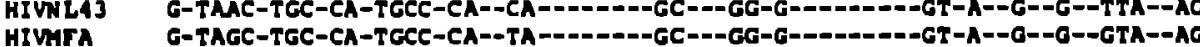

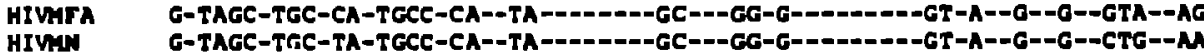

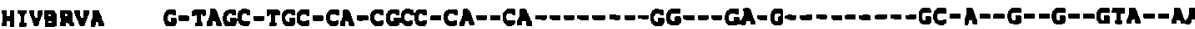

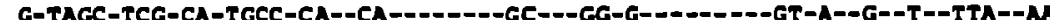
HIVSU

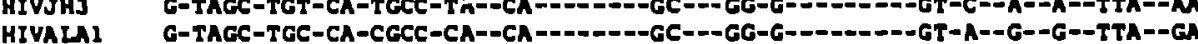

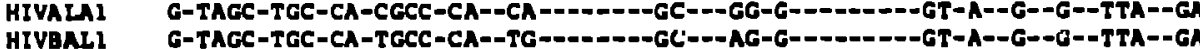

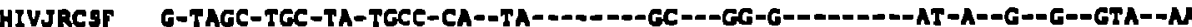

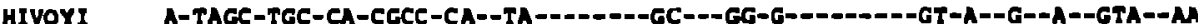

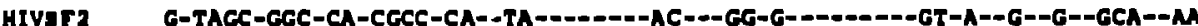
HIVIYSCG A-TAGT-TGC-TA-TCCC-CA--TG--_---GC--GG-G-1-1-1-GT-A--G--G--GCA--M

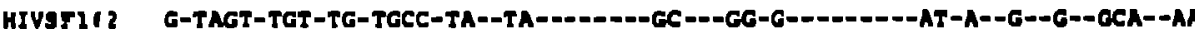

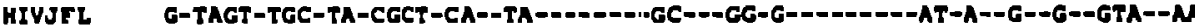

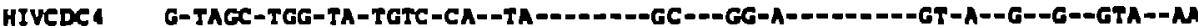

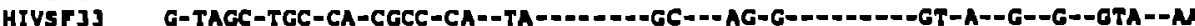

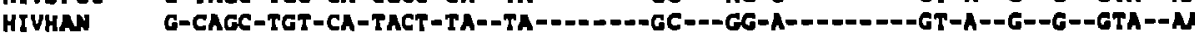
HIVADA G-TACC-TGC-TA-TCCC-CA--TA---1--GC--GG-G--0--1--GT-A-G-G-G-GTA--M

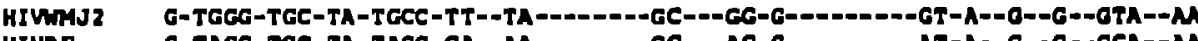

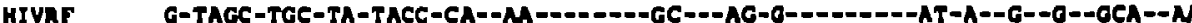

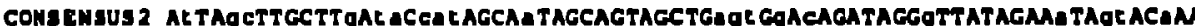

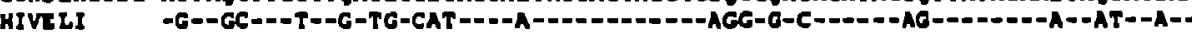

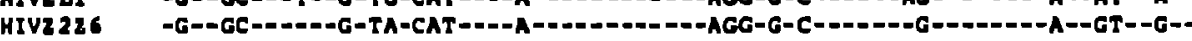

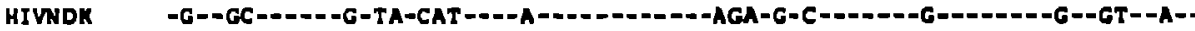

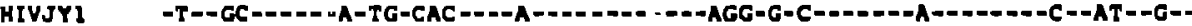

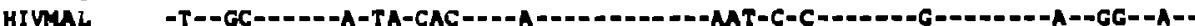

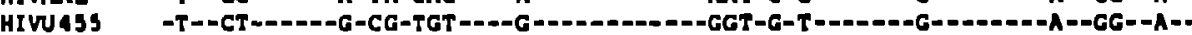

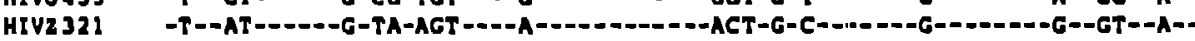

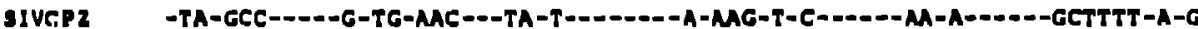

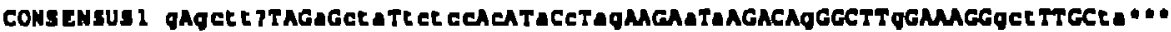

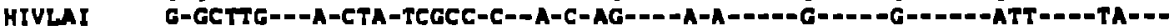

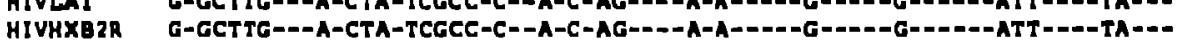

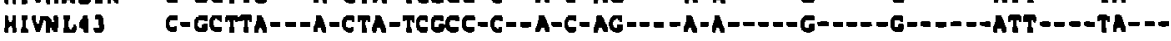

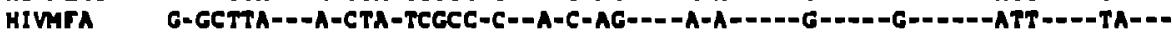
HIVAN HIVERVA HrVse

HIVJHJ

HIVALA

HIVBALI

HIVJRCgr

HIVOYI

hIVST2

HIINYSCO

HIVITIG

HIVJFL

IIIVCOC4

IIIVISJj

IITVIIAN

HIVADA

IIIVMMJI

HIVRT

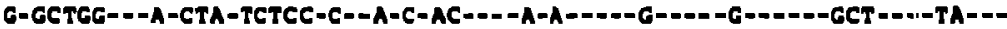

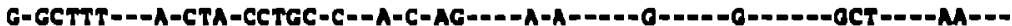

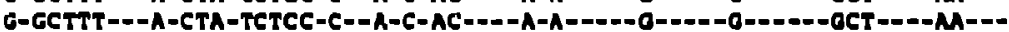

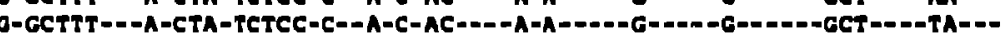

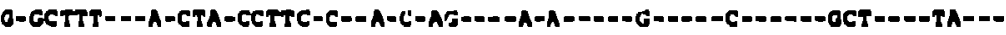

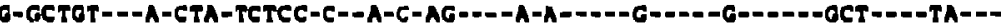

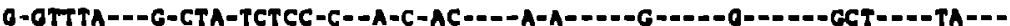

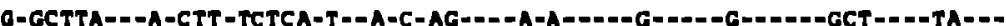

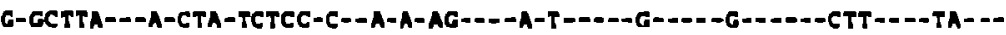

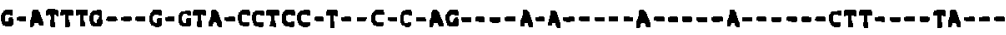

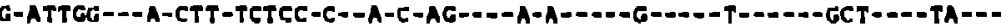

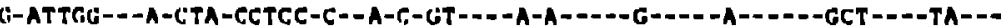

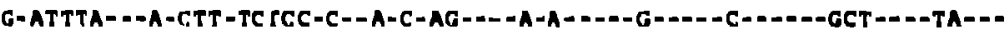

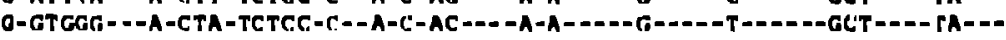

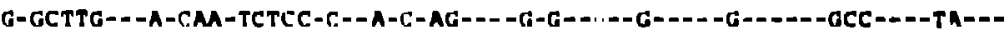

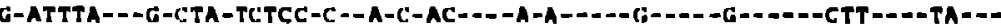

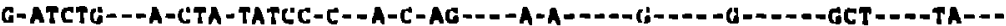

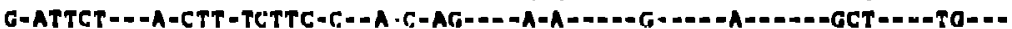

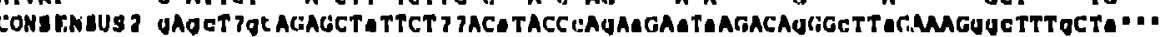

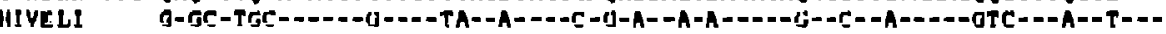

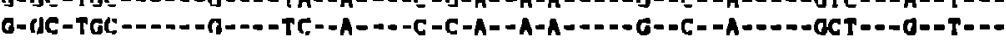

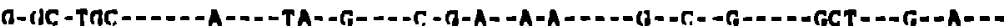

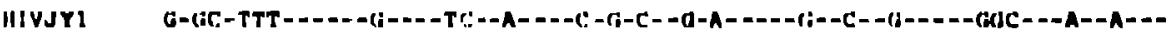

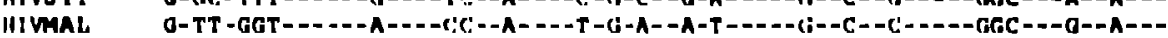

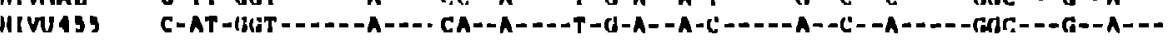

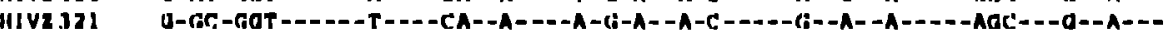

sive'p TTAC:AC:TT-D-AT-A--ACIAA--A-C:--T-CIGC-C:A-A-----A--1LC-1;-----AGC---A--T--- 
CONSENSUSI ATGGGEGCAAGTGGTCAAA ?gtaJtatgg?tGgATGGCCEgCtgTAAGGgMAGAATIgaCgAgCTg HIVIAI -

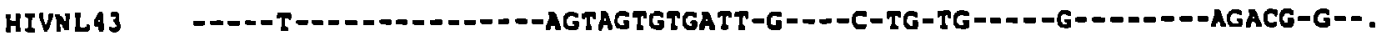

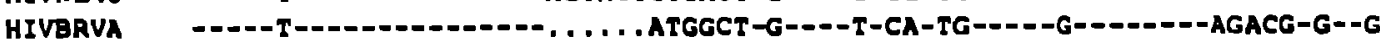
HIVBAL1 HIVJRCSF HIVOYI HIVSF? HIVSF 162 HIVSF 33 HIVHAN HIVRF

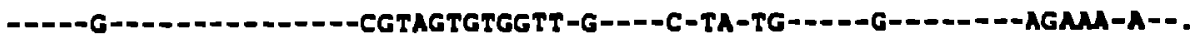
-

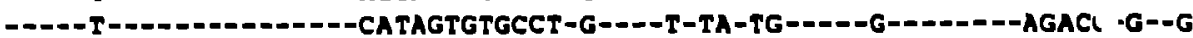
-

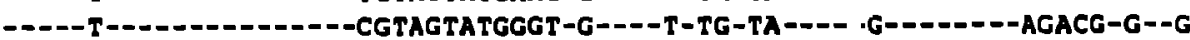
-

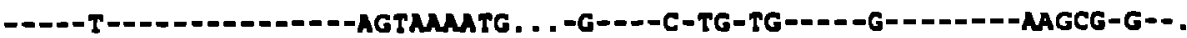
-

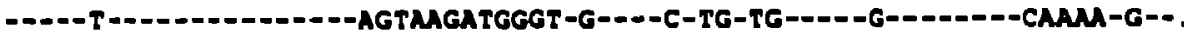

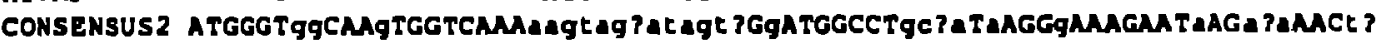

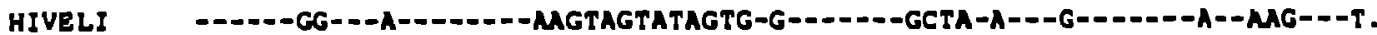

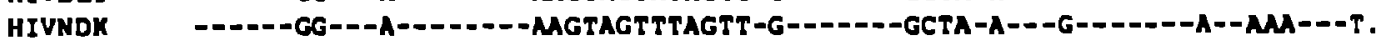
HIVMAL HIVU455 HIV2321

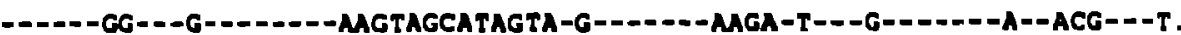

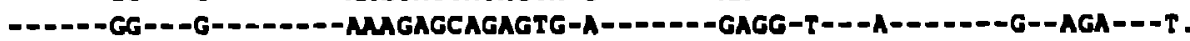
SIVCP 2

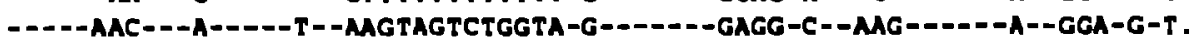

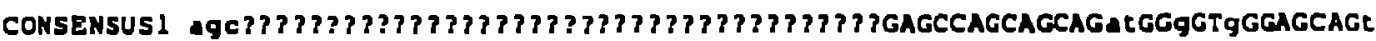
HIVLAI HIVNL43 HIVBRVA

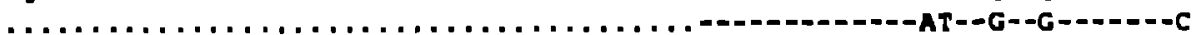

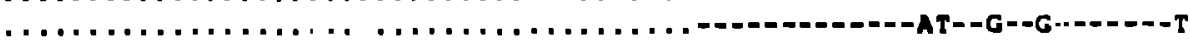

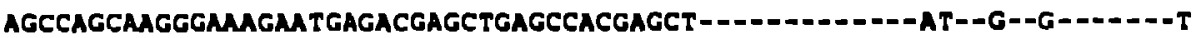
HIVSC HIVBAL1 HIVJRCSF HIVOYI HIVSF 2 HIVSF 162 HIVSF33

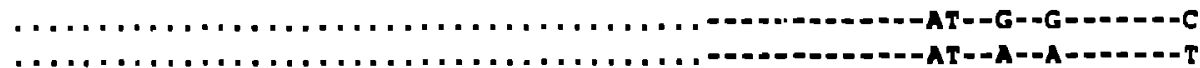

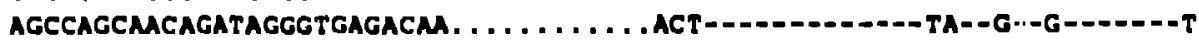

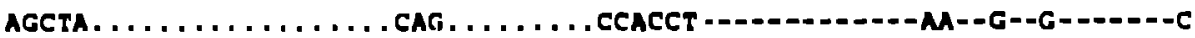

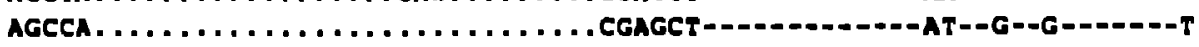

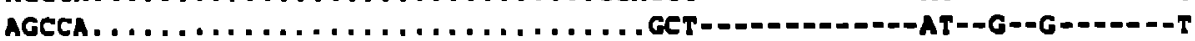
HIVHAN

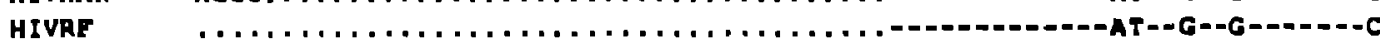

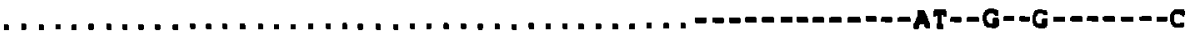

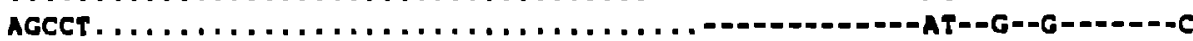

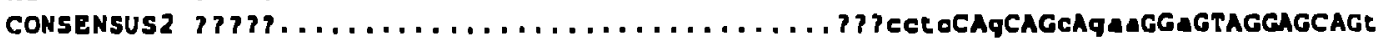

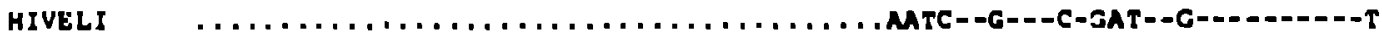

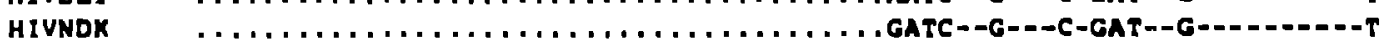

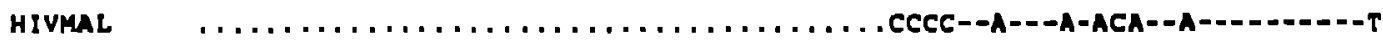

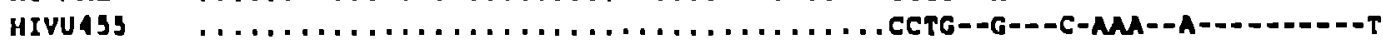

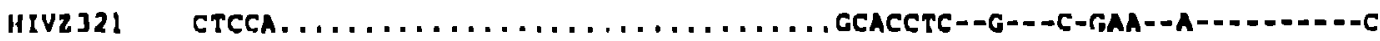

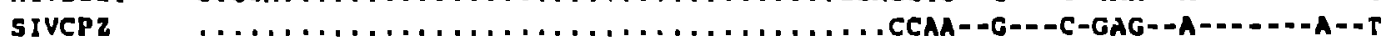

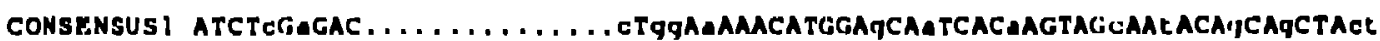

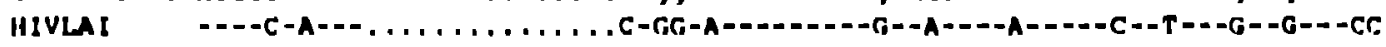
HIVMLAJ HIVBRVA HIVSC IIIVIBALI HIVJRCSF IIIVOY I IIIVSF? HIVSr 162 IIIVSF 3 HIVHAK

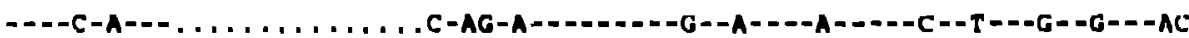

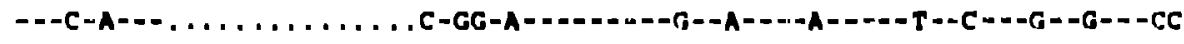

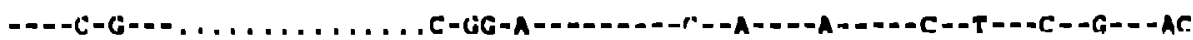

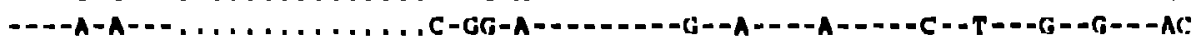

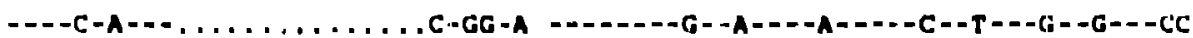

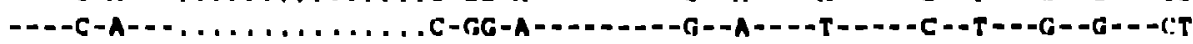

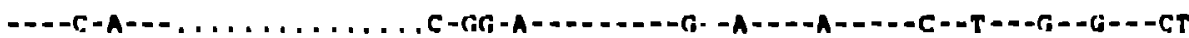

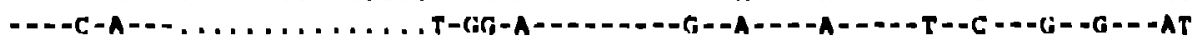

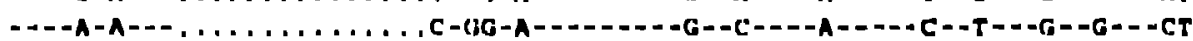

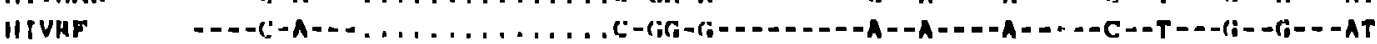

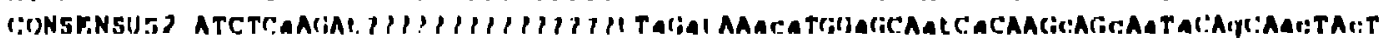

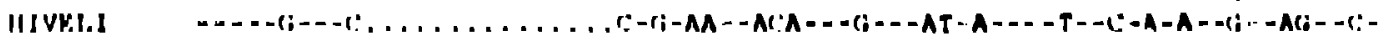
IIIVNDK $\quad$-...--1;-.-1:.............

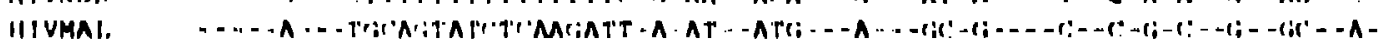
IIIII4) III\%)

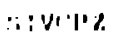

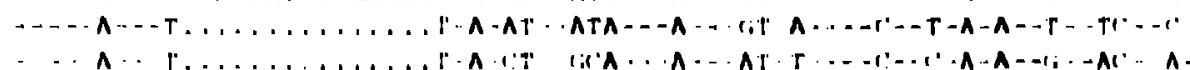
inger 
CONSENSUS1 MALGCTGATTGLgCCTGGCTAGMAGCACAA ? ? ?? ?GAGGA ?GAgGAgULGGGtTTTCCAGTCAgACCLC

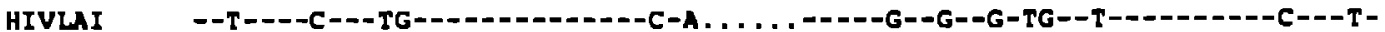

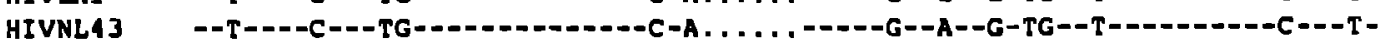

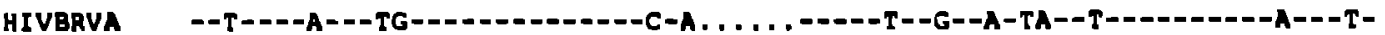
HIVSC HIVBAL1 HIVJRCSE HIVOYI HIVSF 2 HIVSF 162 HIVSF 33 HIVHAN HIVRF

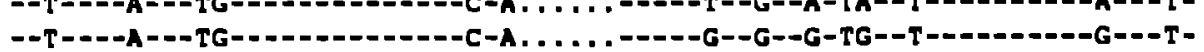

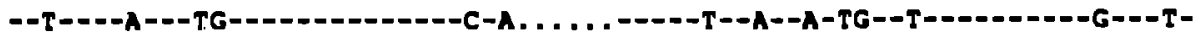

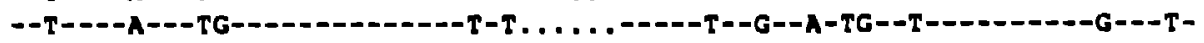

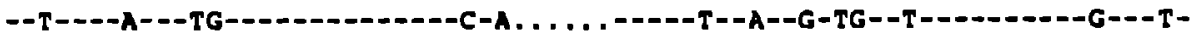

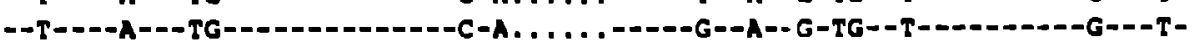

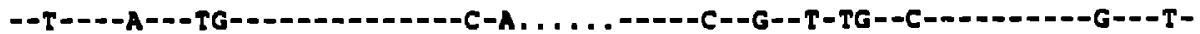

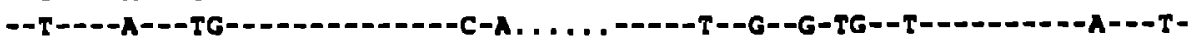

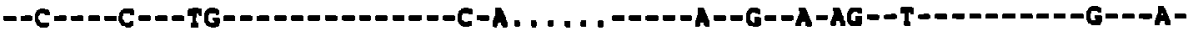
CONSENSU52 MATgGta rETGTgCCEggCtgGMaCaCaAga? HIVELI ---GCTGAC---GCCTGGCTA---G-A-A-GM....-GAGC-AC--G--G-----T-----CA-A--CHIVNDK ---GATACC---GCATGGCTG---G-A-A-GAG... .-GAGC-AG--G--G-----T-----TA-A--THIVMAL HIVU455 HIV2321 ---GCTAGT-..........--C-A-C-GM... .-AGAG-AG--G--A----T-----CC-T--T-..GCCAGT---GCCTGGCTG---G-G-A-......, --GGM-GA--C--A-----C-----TA-G--A-

SIVCPZ ---CCTGAT---GCCTGGTTG---G-A-A-GAG... .-GTCA-AG--G--A-----T-----CA-A--A---CAAACTCTAGCTTGGCTA--GGMATG. ......--CAAT-AA--A--A--A--C-----AA-A--A-

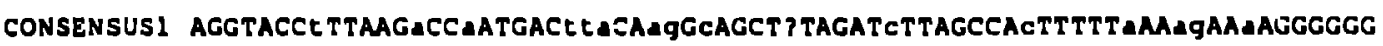

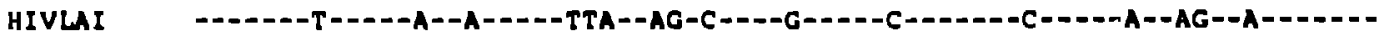

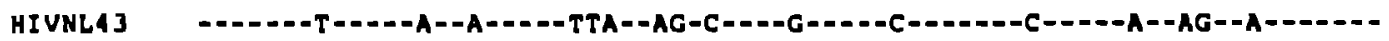
HIVBRVA HIVSC HIVBAL1 HIVJRCSE HIVOYI HIVSF 2 HIVST 162 HIVST3 3 HIVHAN

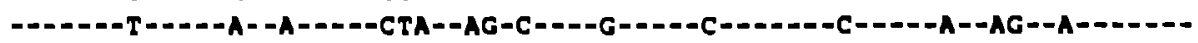

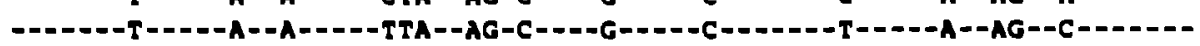

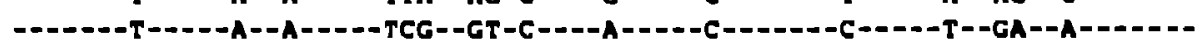
-

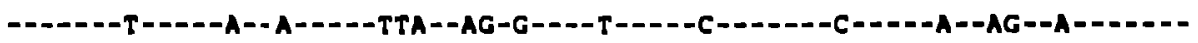
- -

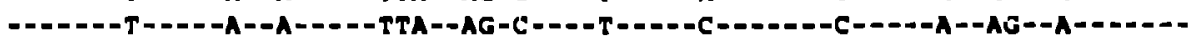

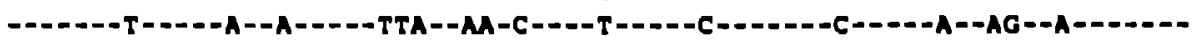
HIVRF

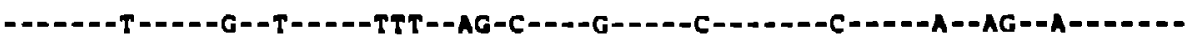
CONSENSUS2 AGGTACCTE TAAGACCAATGACE TATMAG PGCTE TEGATCTCAGCCACTTTTTAAAMGAAAGGGGG HIVELI HIVNDK HIVMAL

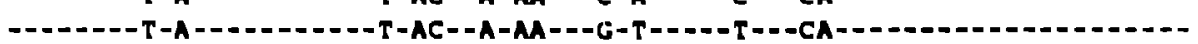
-

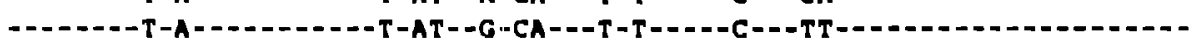
HIVUASS IIIVZJ? I

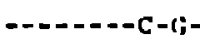
$-C-T T--G-G T-B-T-T-C$ $-\mathrm{T}-\mathrm{-TT}-\mathrm{T}-\mathrm{s}$

J' LTR $\rightarrow$ (for more Information, sen the liTh allynment)

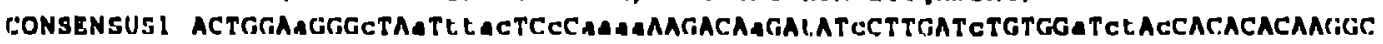
HIVIAI HIVNLA] IIIVกhVA IIIVSC IIIVIALI IIIVJACLiF IIIVOYI IIIVsr2

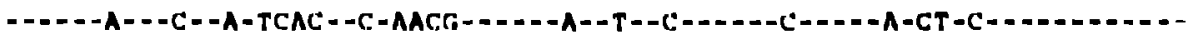

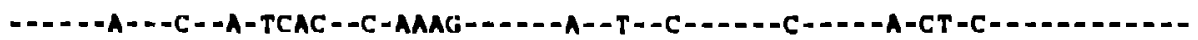
-----A---C--A-TCAC-- -

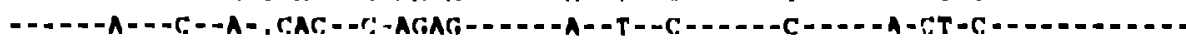

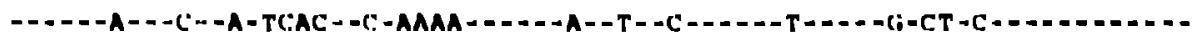

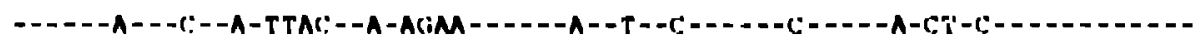

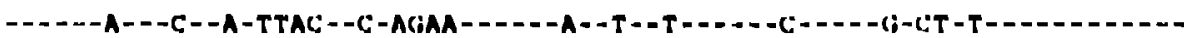

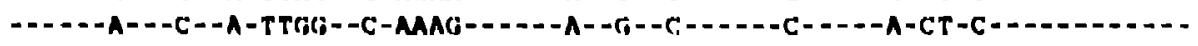

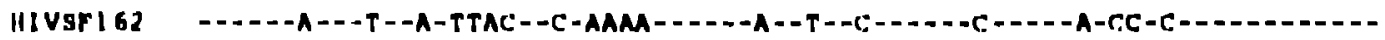

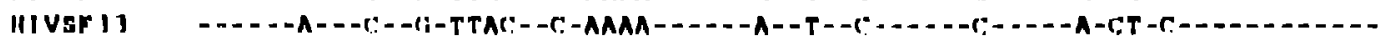

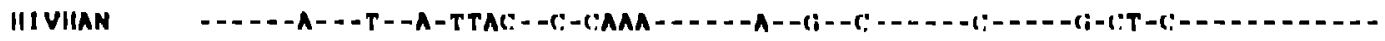

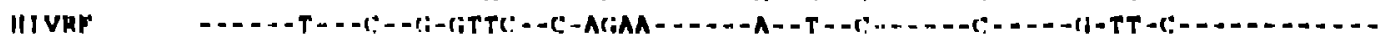

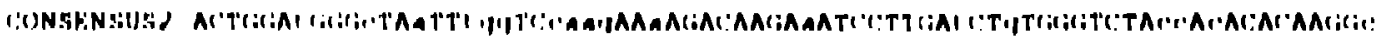

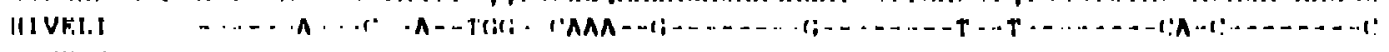
III VNIIK IIIVMAI. IIIVIIA' IIV)I :I Vill

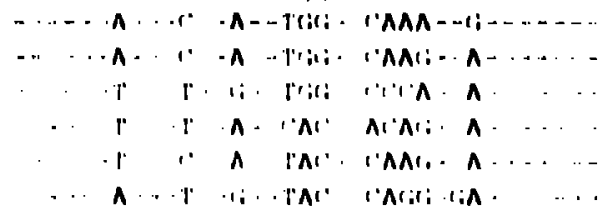

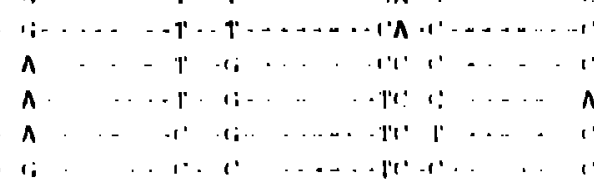


CONSENSUSL TACTTCCCTGATTGGCAGACTACACACCAGGgCCAGGGATCAGATAT PCCACTGACCTTTGGATGGTGC

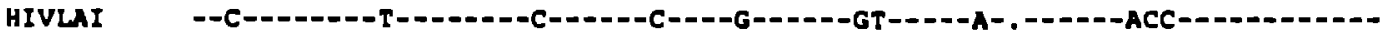

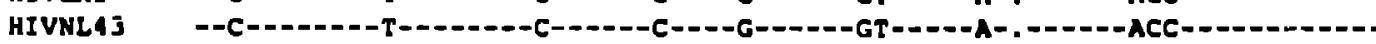

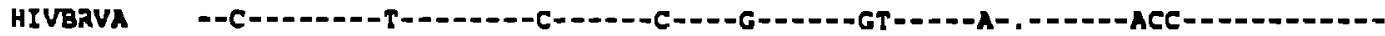

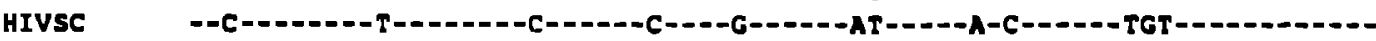
HIVBNL1 HIVJRCSF HIVOYI HIVSF? HIVSF 162 HIVSF 33 HIVHAN HIVRE

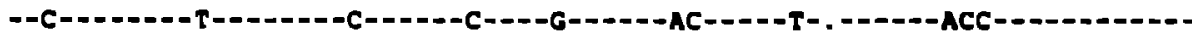

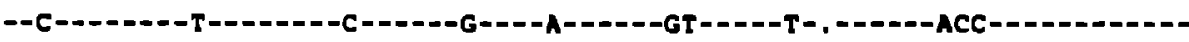

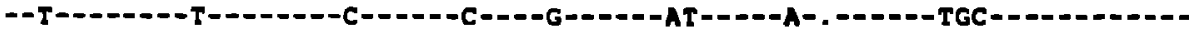

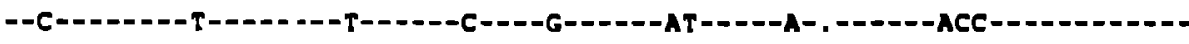

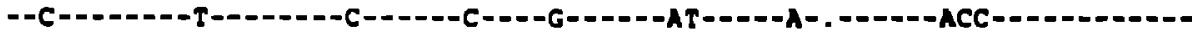

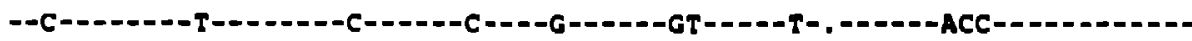

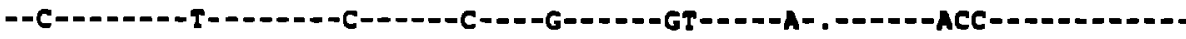

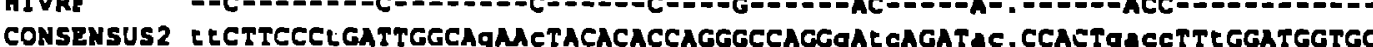
HIVELI HIVNDK HIVMAL HIVU455 HIVZ321

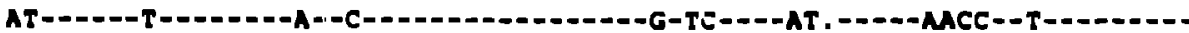

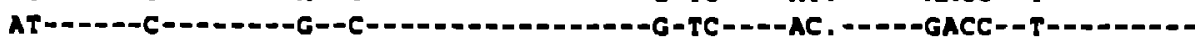

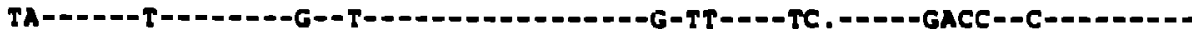

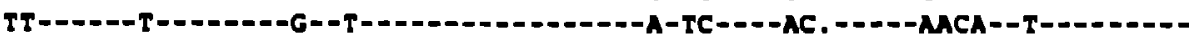

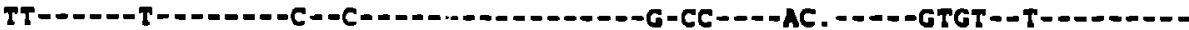
SIVCP2

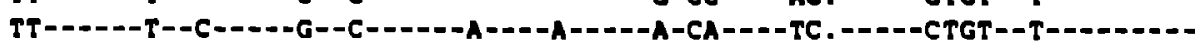

C- NFAT-1

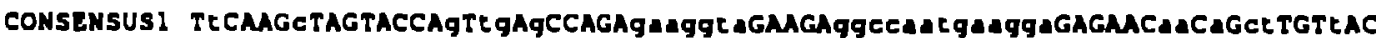

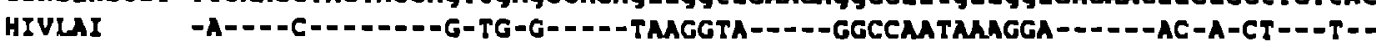

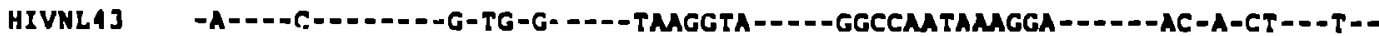

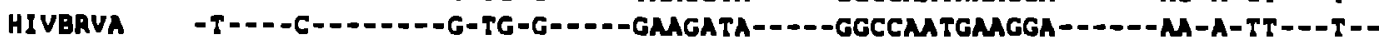
HIVSC HTVBALI HIVJRCSF HIVOYI HIVSF 2 HIVSF 162 HIVSF 3 HIVHAN HIVRF

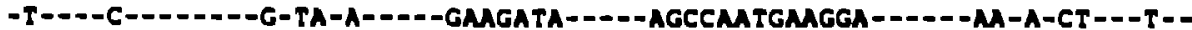

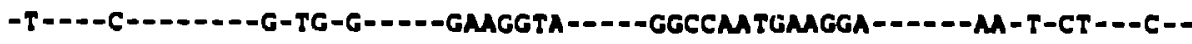

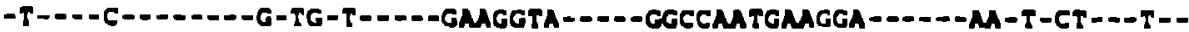

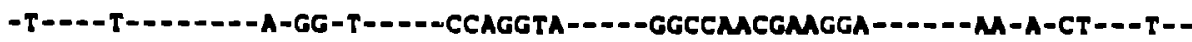

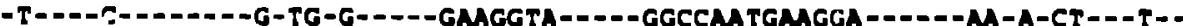

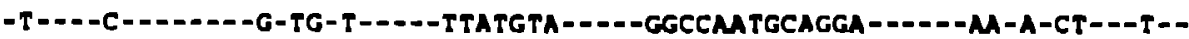

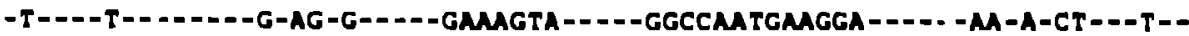

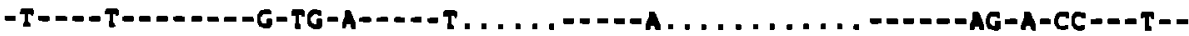

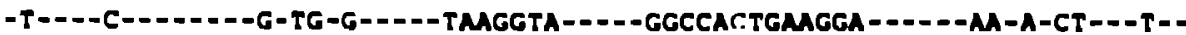

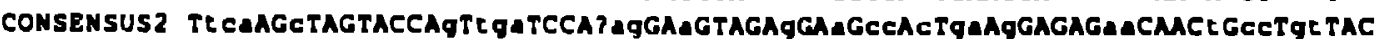
HIVELI -ACG--C-.-----G-TGA----CAT--G-----A--A-AC-C-GA-G----AC----A-CT-GT---

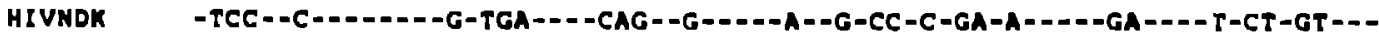
HIVMAL -TTA--T-------A-GAG---GAG--A-----G--G-CC-A-GA-G-----AA---T-TC-GT-D-

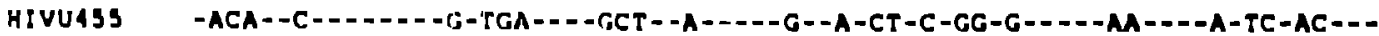

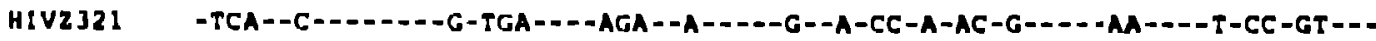

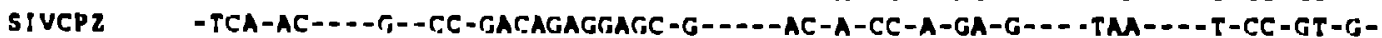


NFAT-1 $\rightarrow$

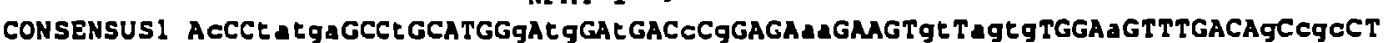
HIVLAI -C--TGTGA---T------A-TG--T---C-T----GA-----GT-AGAG----G--------G-CGC--

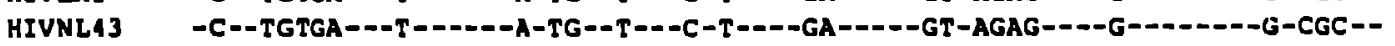
HIVBRVA HIVSC HIVBALI HIVJRCSF HIVOYI HIVSF2 HIVSF162 HIVSF33 HIVHAN HIVRF

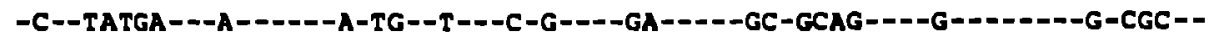
-C--GATGA---T------G-TG--G---C-G----GA-----GT-AGAG----G--------A-CGC--८--TATGA---T------G-TG--T---C-G----AA-----GT-AGCA----A-------G-AGC--C--TATGA---A------A-TG--C---C-A----AG--.--GT-AGTG----A-------G-AAG--

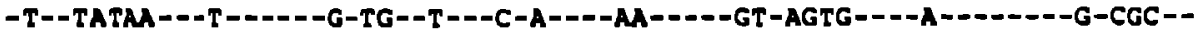
-C--TATGA---T------G-TG--G---G-G----AA-----GT-AGTG----G--------G-AAA--

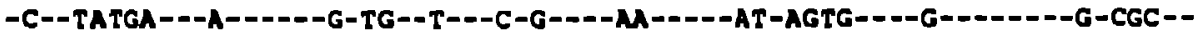
-C--TATGA---T------G-TG--G---C-G----AA----GT-AGTG--.-A--------G-CAC--C--TGCGA---T------G-CA--G---A-G----GA--_--GT-AMAG----A--------G-CAT--C--TATAT---T------G-TG--T---C-A----MA-----GT-AGTG----A--1--1--G-CGC-.

CONSENSUS2 ACCCEAT? GCCAgCALGGAATGAgGACgC ?GAgAgAgMAGTGETALGTGGA ITTIGACAGCAgCCT HIVELI HIVNDK HIVMAL HIVU 455 iIIVZ321 SIVCP2 ---T--AT----G--T---A-G--G--CCCG--G-G-C-----T---AA----GA---A----CAGA-----T--GT----G--A---A-G--G--CCCG--G-G-C--1--T---TG---OGA---A--1- CAGA-- --T--TA----A--T---A-G--G--CGCA--A-G-G--D--C---AA----AG---G----CAGC--

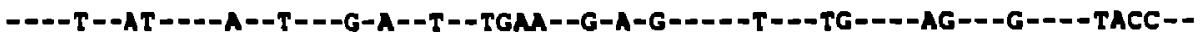

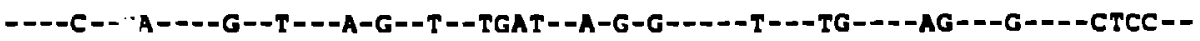
-T--C- I-T--G--T--GA-G--A--TGAG--C-A-G-G--CT-GGTC---CGC---G----CAGG--

$$
\text { <- } \begin{gathered}
\text { IL2/NRE } \\
\text { NRF }
\end{gathered}
$$

CONSENSUSI AGCATTLCATCACAEgGCCCGAGAgCEGCATCCGGAGTACTACAAAACTGC?? 621

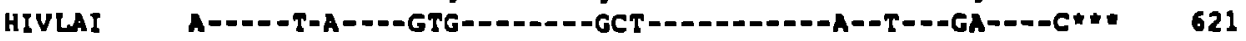
HIVNL43 A-----T-A----GTG-------GCT-----------A--T---GA----C*\#" 621

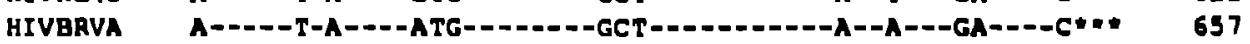
HIVSC HIVBALI HIVJRCSF HIVOYI HIVSF2 HIVSF 162 HIVSF 33 HIVHAN HIVRT

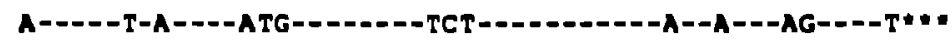
A-----C-A----GTG--------A=": 


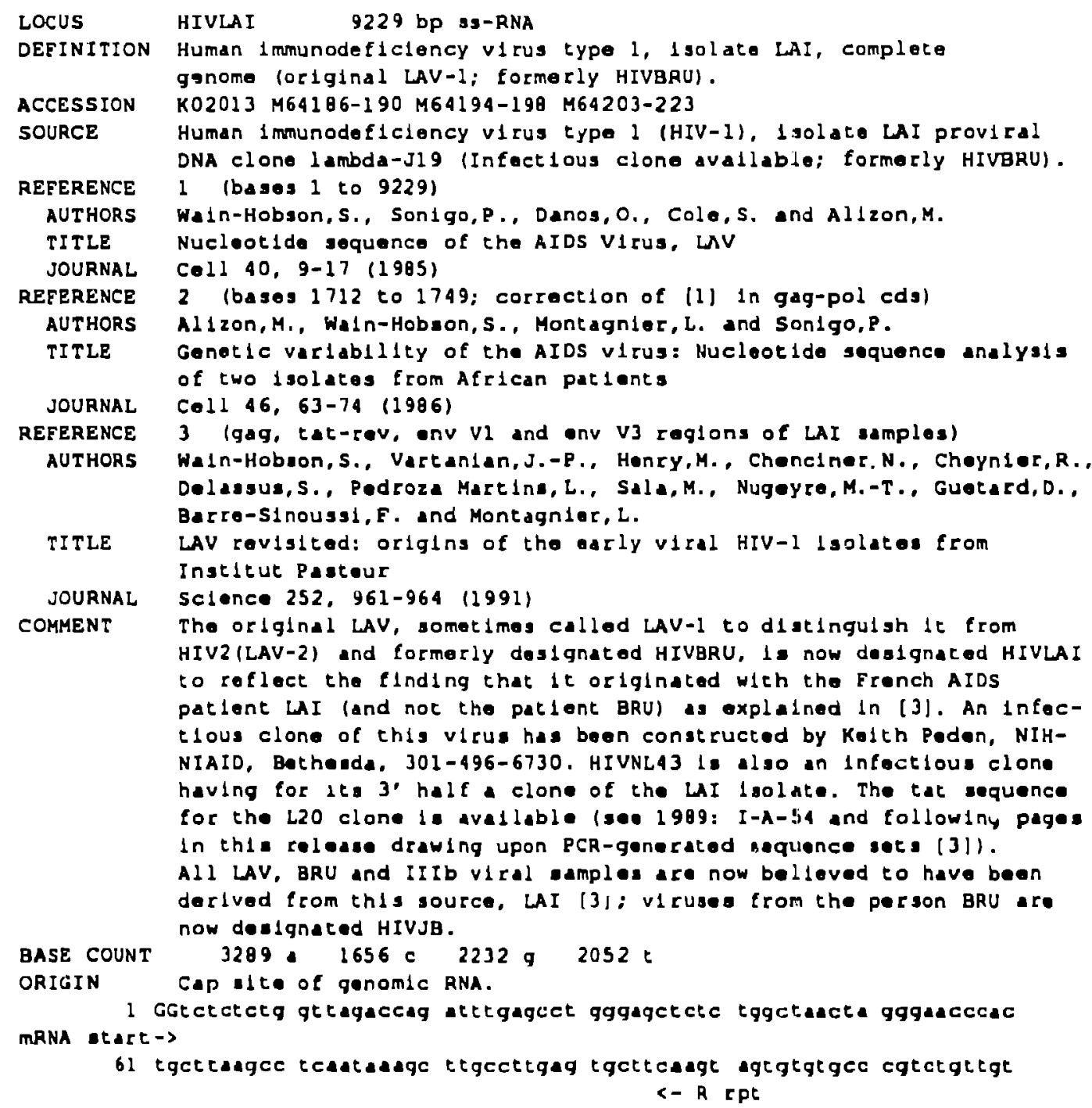

121 grgactetgg taactagaqa tccctcagac ccttetagtc agtglggaa atctctagca

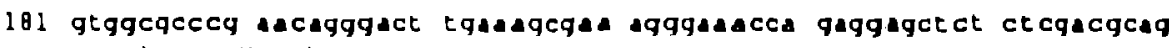
<- LYS-CRNA pbs

241 gactegqete getgaagcge gcacggcaaq aggeqagggg aggcgactge Tgagtacgce major $5^{\circ}, 1 /$

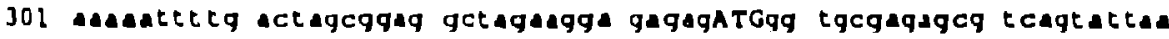
gag atart $\rightarrow$

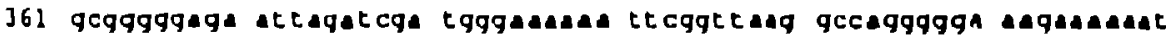

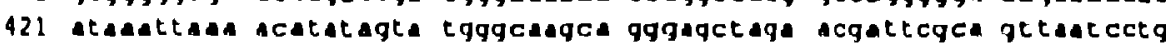

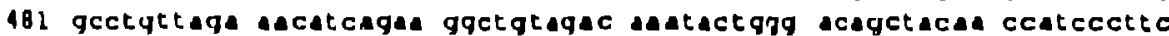

341 agaceggatc aqaagaact agatcattat ataatacagt agcauccetc tatgtgtgc

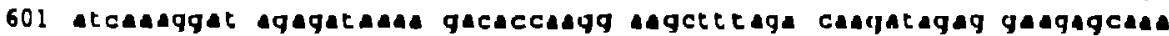

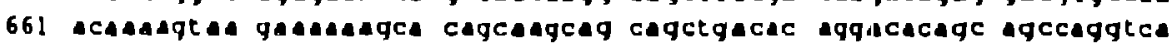

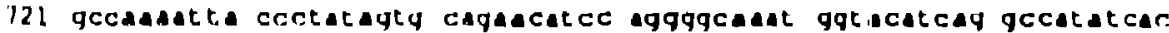

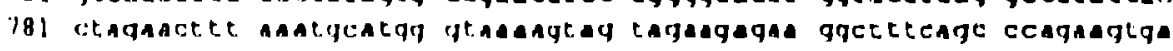

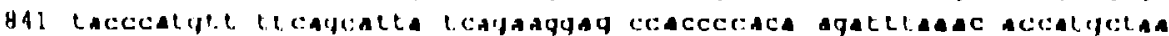

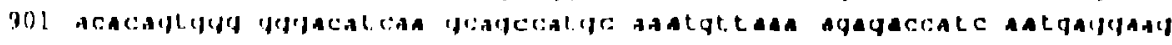

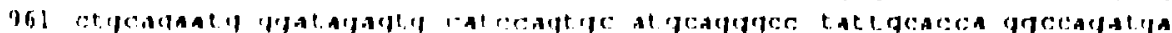

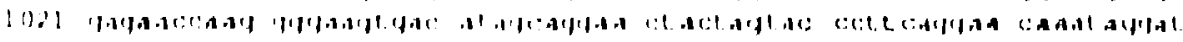

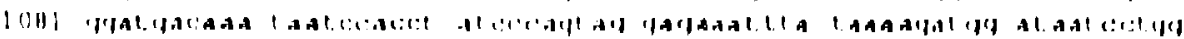

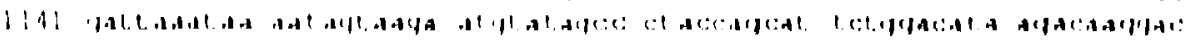

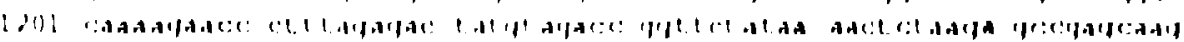

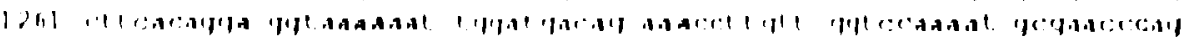

I-A-70

MAY 91 
1321 attgtaagac tattthaaa geattggoac cagcagctac actagaagaa atgatgacag

1381 catgteaggg agtgggagga cecggccata aggcaagagt tttggctgaa gcaatgagce

1441 agtaacaa ttcagctace ataatgatgc aagaggcad ttttaggaac caagaaaga

1501 trgttangtg tttcauttgt ggcaagaag ggcacatage cagaattgc agggececta

1561 ggaaanggg ctgttggaa tgtggaagg aggacacca aatgaajgat tgtactgaga

1621 gacaggcTAA ctttttaggg adgatctggc ettcetacaa gggaaggcca gggaattetc pol orf $\rightarrow$ (NH2-terminus uncertaln)

1681 tecagagcag accagageca acagceceac cattectca gagcagacca gagccascag

$\rightarrow$ beses added in revision [2]

1741 ceccaccaga agagagctec aggtetgggg tagagacasc actecctet cagaageagg $\rightarrow$

1801 agecgataga caggaactg tatecttad ctcectcag atcactctet ggeacgace 1861 cetcgtcaca arNagatag gggggeadet aaggagget ctattagata caggagcaga <- gag and

1921 tgatacagta ttagaagada tgagtttgcc aggaagatgg adaccasaa tgataggggg 1981 attggaggt tttatcaag taagacagta tgatcagata ctcatagaa tctgtggaca 2041 taagctata ggtacagtat tagtaggace tacacctgte adeatadtg gaagaatet 2101 gttgactcag attggttg a cttadatte teccattagt cetattgaa ctgtaccagt

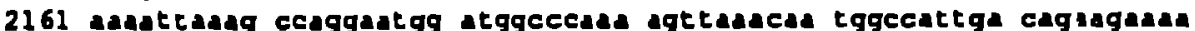

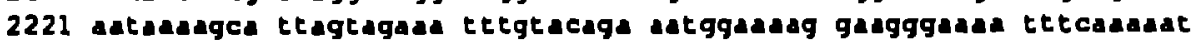

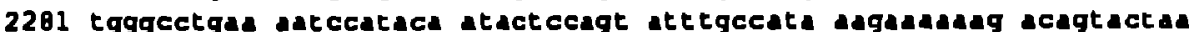
2341 atggagaaa ttagtagat tcagagaact tadeagaga acteagact totgggaagt 2401 tcattagga ataccasatc ccgeagggtt daadagad aatcagtad cagtactgga 2461 tqtgggtgat geatattttt cagttccett agatgadac ticaggaagt atactgcate 2521 taccatacet agtatadaca atgagacace agggattaga tatcagtaca atgtgctece

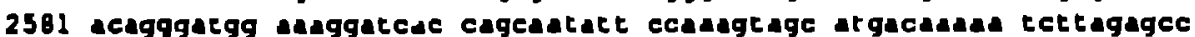
2641 ttteagaaa caaatccaq acatagttat ctaccatac atggatgate tgratgtagg

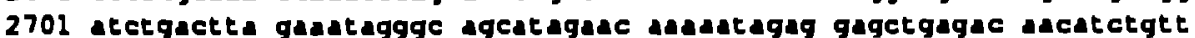
2761 gaggtgggga cteaccacac cagacauad acatcagada gadcctccat tcctetggat 2821 gqgttatgaa ctccatcctg dtaatggac agtacagect atagtgetgc cagaadaga

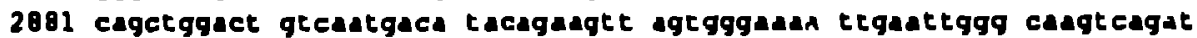
2941 ttacecaggg attaagta ggcastatg taactcct aquggadeca agcactaac

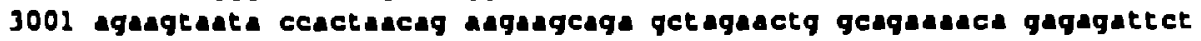
3061 aargacea gtacatggag tgtattatga cccatcaua gacttautag cagaataca 3121 gaagcagggg caggccaut ggacatatca attetaca gagecatte auatctgaa

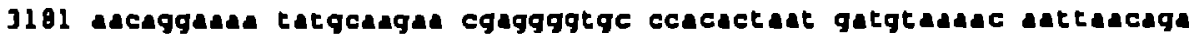

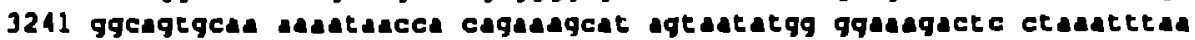
3301 actacccata caaaggaa catgggaace atggtggaca gagtattgge aggceacctg 3361 gatectgag tqgyagtttg teatecece tecttigtg aattatggt accagt taga 3421 gaagaacce atagtaggag cagaeacgt ctacgtagat ggggcagcta gcagggagac

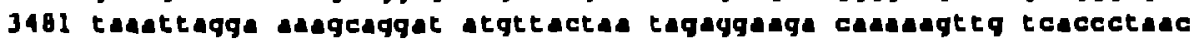

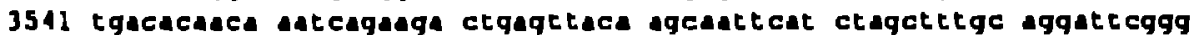
3601 attagasgta datatagtad cagdetcaca atatgcate gqaicatec agcacaacc

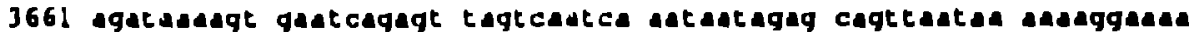
3721 ggtetatctg geatgggtac caqcacacas aggaattgga gquatgaac asgtagata 3781 attagtcagt qctgganca ggaagtact atttetagat gqaatagaca aggcocaaga

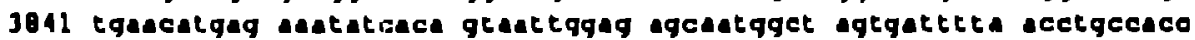
3901 tgtagtagca asagaatag tagccagctg tgatauatgt cagctaanac gagaagerat 3961 gcatgqacas qtagactgta qtccagqaet atggcaacta gattqtacac attegaagg

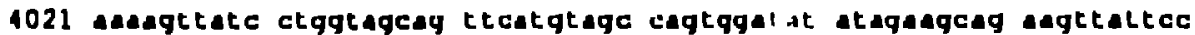

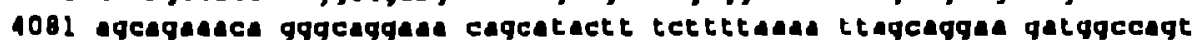
1141 aneacate catacagaca atggcagcas tttcaceagt actacqgtta agqceqcetg

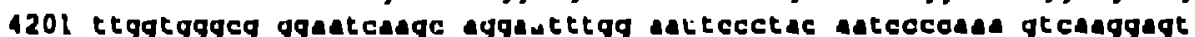

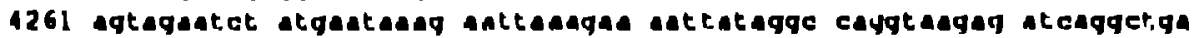
4321 acatct

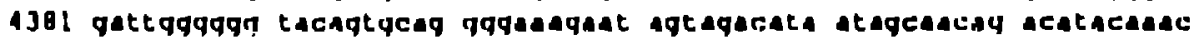

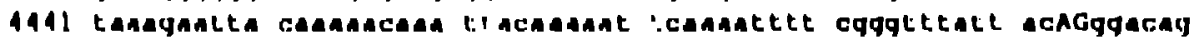
II 101

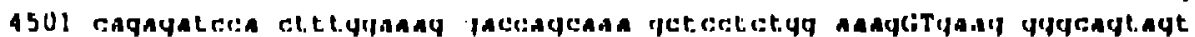
J' 1 八

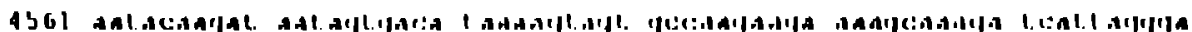

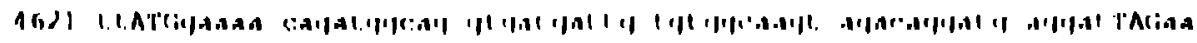
vit $\rightarrow$

$\langle-10):$

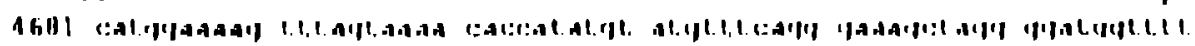


4741 aragacatca ctatgaaagc cctcatccad gaataggttc agadgtacac atcceactag

4801 gggatgctag atcggtada acacatatt ggggtctgca tacaggagad agagactggc

4861 atctgggtca gggagtctce atagadgga ggadasga atatagcaca cadgtagacc

4921 ctgaactagc agaceadeta attcatctgt attacttiga ctgtttttca Gactetgcta 八了'sj

4981 tagaadgc cttattagga catatagtta gccetaggtg tghatatcad geaggacata

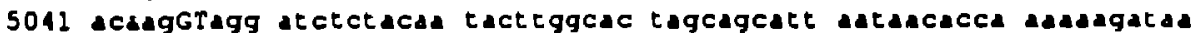
$5 \cdot 3 \mathrm{~s}$

5101 agccacettr. gcctagtgtt acgaadetga cagaggatag ATGgaecasg ceccagaaga vpR otart $\rightarrow$

5161 ecaagggcea cagagggagc cacacalga alygacacta Gagcttetag aggagctta $<-v i f$ end

5221 gaatgaget gttagacate ttcctaggat ttggctceat ggct agggc aacatateta 5281 tgaadetet gqggatactt gggcaggagt ggaagccata atagaatec tgcacadet

5341 getgtetatc catttcAGa ttgggtgtcg acatagcaga ataggcgeta crcaacagag 八 3'sj

5401 gagagcaaga aATgagcea gtagatccta Gactagagce ctggaagcat ceaggadte tat start - > <- vpR end

5461 agcetaade tgctggtacc actggctatt gtauaagtg ttgctttcat tgceadgtt 5521 gettcacade aaagcetta ggcatctect ATGgeAGgaa gaagcggaga cagegacga rov atart $\rightarrow \quad / 3$ is

5581 gacctcetca aggeagtcag actcatcad tttetctatc aagcagTa gtagtacatg 5. I 11

5641 taATGcasce tatacadata gcaucagcag cattagtagt agcautaata atagcaatag vpU atart $\rightarrow$

5701 ttgtgtggtc catagtaatc atagaatata ggaeatat asgacaaga aaatagaca 5761 ggttaatiga tagactaata gaadagcag adgacagtgg CaArGagagt gaaggagaae onv start $\rightarrow$

5821 tatcagcact tgtggagatg ggggtggaad tggggctica tgctccttgg gatattgatg 5881 atctgTAGtg ctacagada attgtgggtc acagtctatt atggggtace tgtgtggaag $<-v p U$

5941 gasgcasca ccactctatt tegtgcatca gatgctadag catatgatac agaggtacat 6001 atgtttggg ccacacatgc ctgtgtaccc acagacccea acccacaaga agtagtattg 6061 gtaatgtga cagasatt tacetglgg auastgace tggtagaeca gatgcatgag 6121 gatataatca gtt:atggqa tcaagceta agccatgtg taaattade cccactctgr 6181 gttagttea agtgcactga ttgggggat gctactaata ceatagtag taataccast 6241 agtagtagcg gggaatgat gatggagaa ggagagata adacegctc tttcatate 6301 agcacagca taagaggta ggtgcagaa qaetagcat tetcttata actggatata 6361 ataccastag ataugatac taccagctat negttgacas gttgtaacac ctcagtcatt

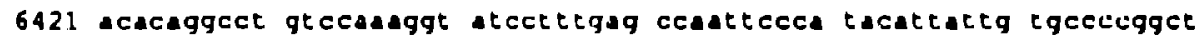

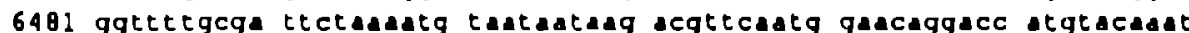

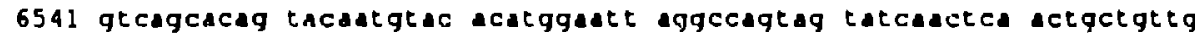
6601 atggcagtc tagcagaaga agaggtagta attagatctg ccatettcac agacatggt

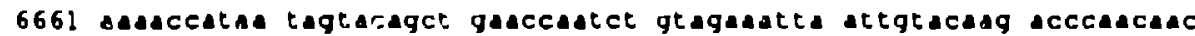

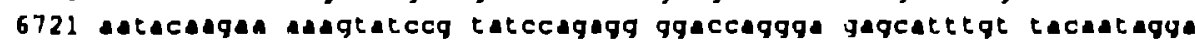
6781 aAataggas atatgagaca agcacattgt accatagta gaycauatg gaetgccact

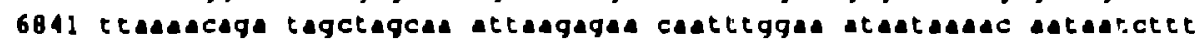
6901 aegcarcet eaggagggga cecagaate gtaecgcaca gttetastg tggaggggaa 6961 tettecact gtastcas acastgtte atagtact ggtteatag tactggagt

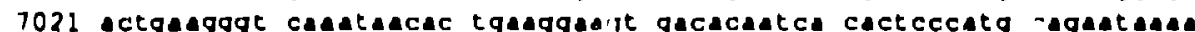
7081 caatetata acatgtggca ggaagtagqa asagcatgt atgcecctcc catcagcgga 71 il caattagat getcatcaa tattacaggg ctgctatta caugagatgg tggtautac 7201 aecatgggt ccqagatct eagacetgqa ggaggagata tgagggaca ttggagaagt

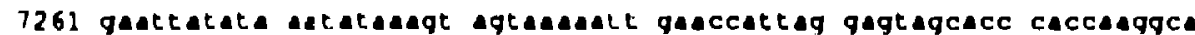

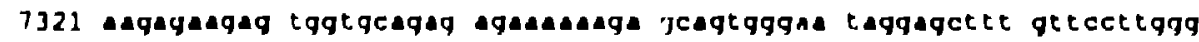
7381 tecteqqqaq caqcagqaaq cactatqqqc qcacgqtcia tqacqctqac qquacaqqce

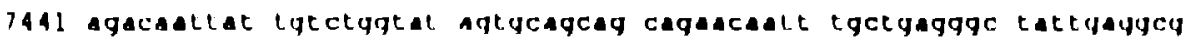

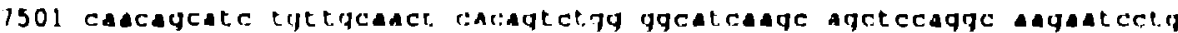

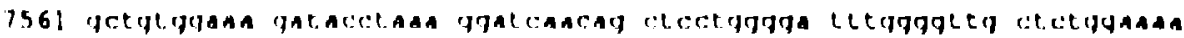

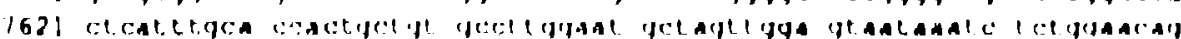

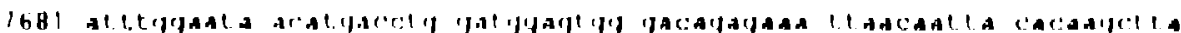

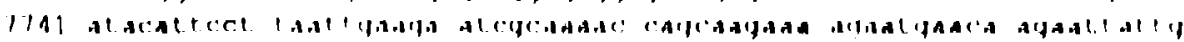

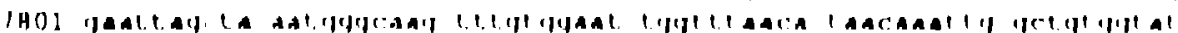

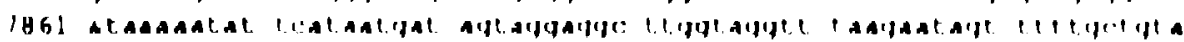


7921 ettectatag tgaatagagt taggcaggga tatteaceat tategttteA Gacccacctc 八 J'j

7981 ccauccecga ggggaccega caggccega ggaatagadg aagaaggtgg agagagagac c- cat end

0041 agagacagat ccattcgate agtgaacgga tectegeac ttatctggga cgatctgcgg 8101 agcetgtgec tctecageta ceacegettg aqagactede tettgattgt acgaggatt

8161 gtggaetre tgggaegeag ggggtgggaa gccetcaat attggtgqaa tctcetacag

0221 tactggagte aggaactaa gaaragtget gteagettgc teatgceac agceatagea <- rev

0201 gtagergagg ggacagatag ggttatagaa gtagtacag gagettgtag agctattege 8341 cacataccta gaagatiag acagggcttg gaaggatt tgctaragh rgggtggcaa L- env

nef atart $\rightarrow$

8401 gtggtcaua agtagtgtgg ttggatggce tactgtaagg gaagatiga gacgagctga B461 gecagcagea gatggggtgg gagcagcatc tegagacetg gainacatg qagcaucac

8521 agtagcat acagcagcta ccalgctgc tegtgcetgg ctaquagcae agaggagga

0581 ggaggtgggt tetccagtca cacctcaggt acctetaga ceatgact acaggragc

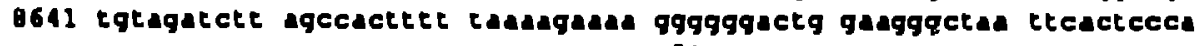
3. LTR $\rightarrow$

8701 acgaagacas gatatcettg atctgtggat ctaccacaca canggetact tecctgattg

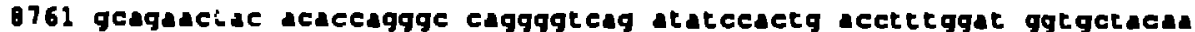
B821 getagtacea gttgagceag ataggtaga agaggceas anaggagaga acaccagcte B88I gttacaccet gtgagectgc atggaatgga tgacectgag agagaagtgt tagagtggag B94l gtetgacagc egcctagcat tecacacge gqecegagag ctgcatcegg agtackteas

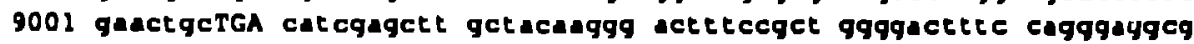
<- nel

9061 tggectggge $999 a c t 9999$ agtggegage cetcagatge tgcatatAag cagctgctet ignal $\rightarrow$

9121 tegcetglac tgGGetcte tggttagace agattgage ctgqgagcte tetggctaec $R$ rpt -2

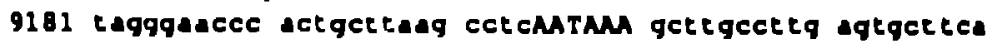
slqnal $\rightarrow$
c- maNa and 
HIVLAI. The following sequences ropresent the V3 Lonp coding sequencer from LAI, a roported In Waln-Hobson ot al., Sclence 252, 961, 1991 (f1g.1).

\begin{tabular}{|c|c|}
\hline 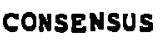 & CANCAATACAaGMAMAGTATeCgTATCCAgagaGgaCCAgggAGagCA \\
\hline L7 & - \\
\hline L3 & --1-1-1 - \\
\hline L2 & ---CAMT---A-----A-----CCG---CCAGAGA-GA---GGG--AG-----GT-A \\
\hline L6 & ---A----CCG---CCAGAGA-GA---GGG--AA-----GT-A \\
\hline L9 & ---CAАT---A------A-----CCG---CCATAGA-GA---GGG--AG-----TA-A \\
\hline L4 & 作 \\
\hline L20 & 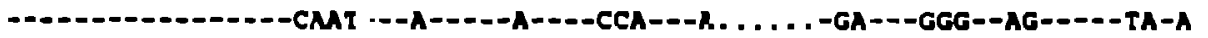 \\
\hline L16 & --CAMT---A-----A----CCG---CCAGAGA-GA---GGG--AG----TA-A \\
\hline E70 & --A-----CCG---CCAGAGA-GA---GGG--AG------GT-A \\
\hline Eาg & -- CAMT---A-----A----CCG---CCAGAGA-GA---GGG--AG-----GT-A \\
\hline E69 & ---CART---A-----A----CCG---CCAGAGA-GG---GGG--AG-----GT-A \\
\hline E86 & ---CAAT---G-----A----CCG---CCAGAGA-GA---GCG--AG-----GT-A \\
\hline E74 & --TAAT---A-----A----CCG---CCAGAGA-GA---GGG--AF-----GT-A \\
\hline M401 & $--A----$ CCG---CCAGAGA-GA---GGG--AG-----GT-A \\
\hline M415 & --A----CCG---CCAGACA-GA---GGA--AG-----GT-A \\
\hline M416 & 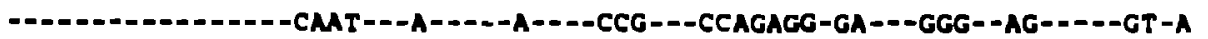 \\
\hline $\mathbf{K} 110$ & --A-----CCG---CCAGAGA-GA---GGG--AG-----GT-A \\
\hline K139 & ---CAAT---A------A----CCG---CCAGAGA-GA---GGG--AG-----GT-A \\
\hline K138 & --CAMT $---A--\cdots--A---$-CCG---CCAMGMA-MA---MAG--AG-----GT-A \\
\hline K145 & -- CMAT---A-----A----CTG---CCAGAGA-GA---GGG--AG----GT-A \\
\hline $\mathbf{K 1 4 7}$ & 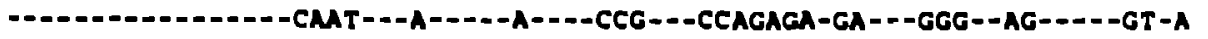 \\
\hline K135 & ---CAAT---A-----G----CCG---CCAGAGA -GA---GGG--AG--.--GT-A \\
\hline K\144 & --CMAT---A-----A----CCG---CCAGAGA 'BA---GGA--AG-----GT-A \\
\hline K152 & 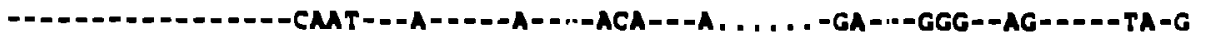 \\
\hline CO & -.CAAT---A-----A----CCG---CCAGAGA-GA---GGG--AG----GT-A \\
\hline CS & -- CAAT---A------A----CCG---CCGGAGA-GA---CGGG--AG-----GT-A \\
\hline G9J & --CAAT---A-----A-----CCG---CCAGAGA-GA---GGG--AG-----GT-A \\
\hline G104 & --- CAAT --G-----A----CCG---CCAGACG-GA---GGG--AG-----GT-A \\
\hline 11117 & :МАT---A-----A----CCG---C'CAGAGA-GA-- -GGG--AG-----GT-A \\
\hline H1116 & 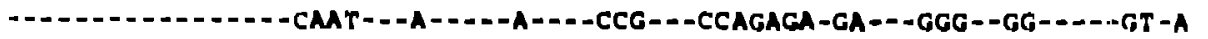 \\
\hline 1121 & 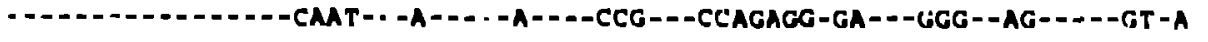 \\
\hline 11130 & - -A-----CCG---CCAGAGA-GA---GGG--AC,------GiT -A \\
\hline H1 J3 & -A----CCG---CCAGAGG-GA---GGG--AG-----GT-A \\
\hline
\end{tabular}

\begin{tabular}{|c|c|c|}
\hline NSF & 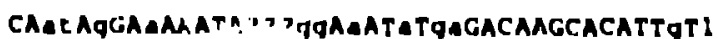 & 100 \\
\hline 1.7 & 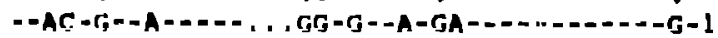 & 100 \\
\hline L.] & 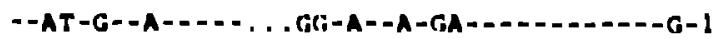 & \\
\hline 1.2 & 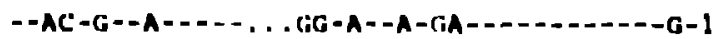 & 100 \\
\hline 1.6 & 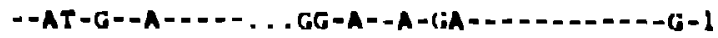 & 108 \\
\hline L9 & 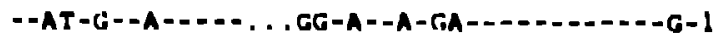 & 100 \\
\hline L4 & $--A C-G--G----A T A G G-G--A-A A-\cdots--------G-1$ & 105 \\
\hline 1.20 & 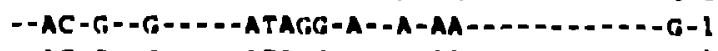 & 103 \\
\hline I.16 & 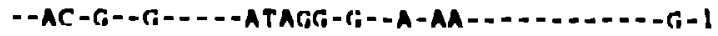 & 111 \\
\hline 10 & -АТ - & 100 \\
\hline 19 & 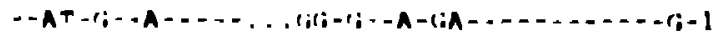 & $10 n$ \\
\hline ' & 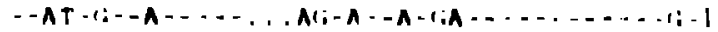 & 10 n \\
\hline Wh & A: " - , & : :H \\
\hline$\cdot 4$ & $\ldots$ & $: \cdot \mathbf{H}$ \\
\hline
\end{tabular}




\begin{tabular}{|c|c|c|}
\hline M401 & 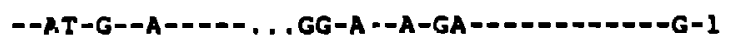 & 108 \\
\hline M415 & 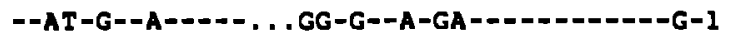 & 108 \\
\hline M416 & $--G T-G--A----.$, GG-A--A-GA----- & 108 \\
\hline $\mathbf{K} 140$ & 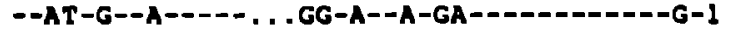 & 108 \\
\hline K139 & 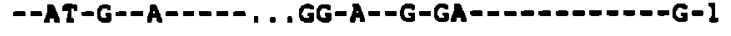 & 100 \\
\hline K138 & 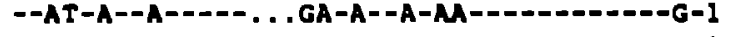 & 108 \\
\hline K145 & $--A T-G--A----., G G-A--A-G A----------G-1$ & 108 \\
\hline K147 & $--A T-G--A-----\ldots, G G-A--A-A A--D----D---A-1$ & 100 \\
\hline K155 & $--A T-G--A---D, \ldots, G G-A--A-G A---1$ & 108 \\
\hline K144 & 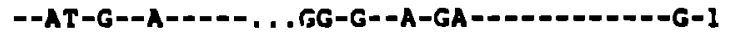 & 108 \\
\hline K152 & 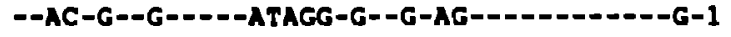 & 105 \\
\hline $\mathrm{C \theta}$ & 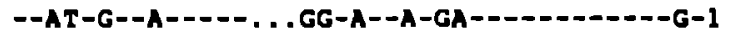 & 108 \\
\hline c5 & 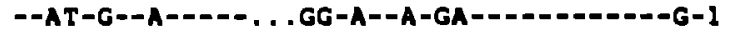 & 108 \\
\hline G93 & 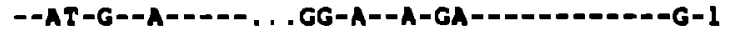 & 108 \\
\hline G104 & 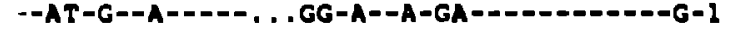 & 108 \\
\hline H117 & 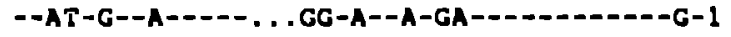 & 108 \\
\hline H116 & 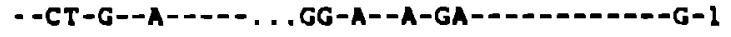 & 108 \\
\hline H127 & $--A T-G--A-\ldots-, \ldots G A-A--A-G A-\ldots-------G-1$ & 108 \\
\hline H135 & 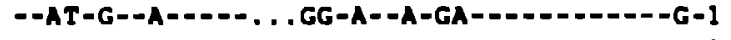 & 105 \\
\hline K152 & $--A C-G--G----A T A G G-G--G-A G-\ldots \ldots$ & 105 \\
\hline C5 & 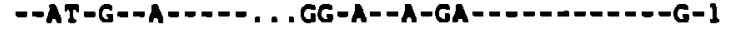 & 100 \\
\hline $\mathbf{C B}$ & $--A T-G--A---D-\ldots G G-A--A-G A-------$ & 108 \\
\hline H130 & 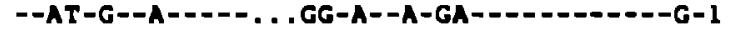 & 100 \\
\hline
\end{tabular}




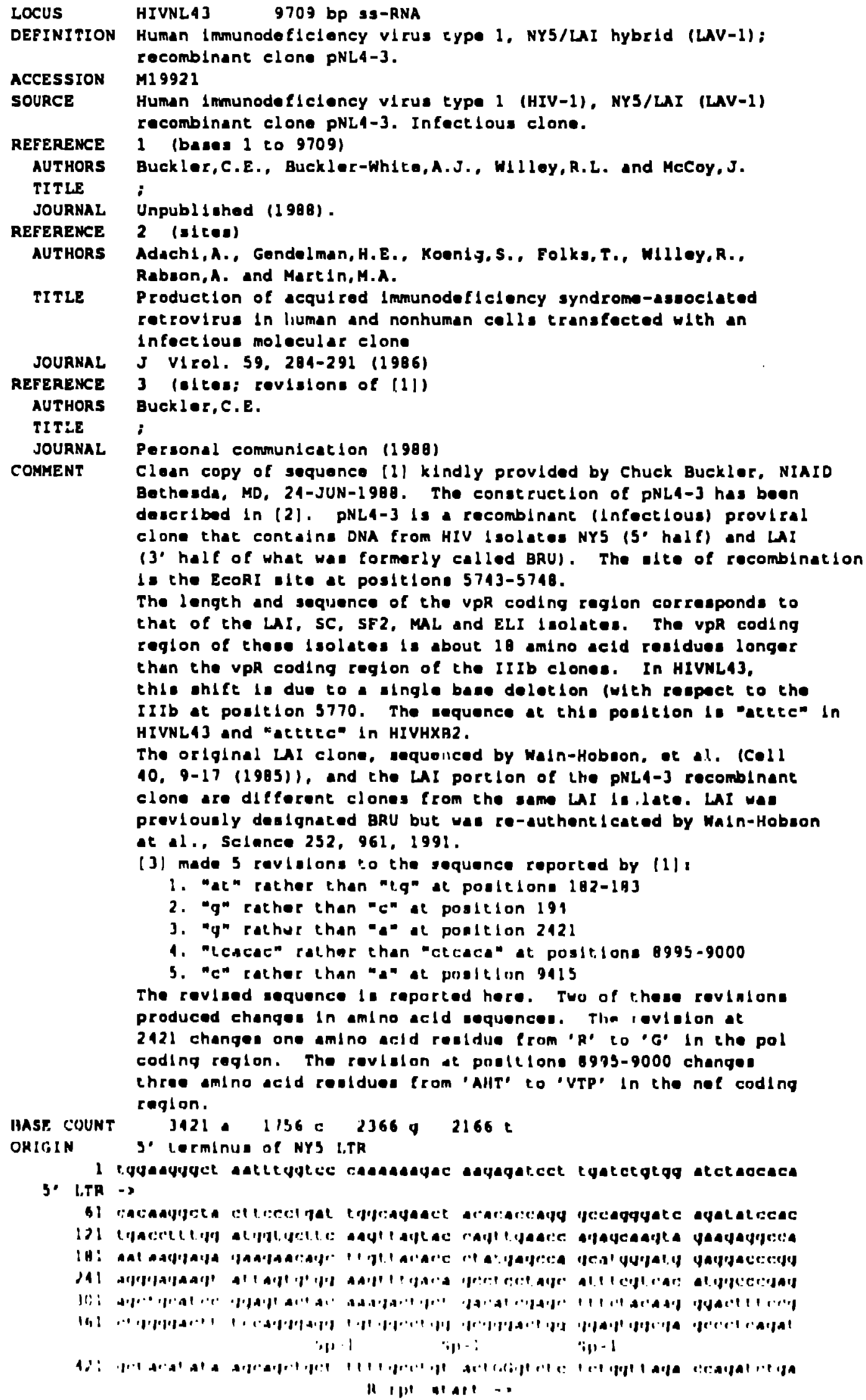


mRNA DEart $\rightarrow$

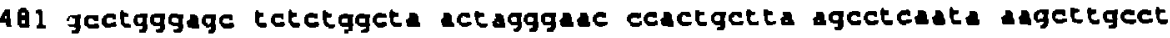
541 tgagtgetca aAgtagtgtg tgecegtetg ttgtgtgact ctggradeta gagatecete <- $R$ rpt and

601 agacecttt agtcagtgtg gaadictet agcagtggeg cecgadeagg gacttgaad

$$
\begin{aligned}
& \text { <- 54 LTA } \\
& ->\text { Lys-tRNA pbs <- }
\end{aligned}
$$

661 egaugtau gecagaggag atctetegac gcaggacteg gettgetgad geqegeargg

721 casgaggega ggggeggega ctgGTgagta cgccasast tttgactage ggajgetaga major 5' eplice junction 八

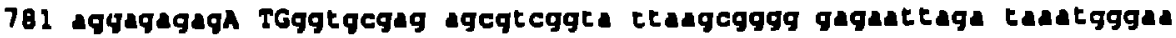
gag cds stert $\rightarrow$

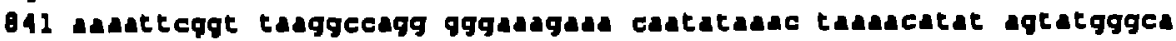

901 agcagggaye tagaacgate egcjgttaAt ectggectt tagagacatc agaaggetgt

961 agacaatac tgggacaget acasceatce ettcagacag gatcagaqa acteagated

1021 teatatade castagcagt cetetattgt gtgcatcas ggatagatgt auagacace

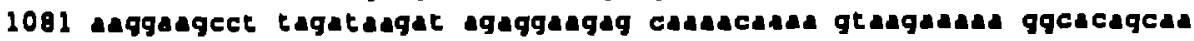

1141 geageagetg acacagqua cascagecag gteagceas attaccetat agtgeaganc

1201 ctccaggggc asatggtaca tcaqgecata tcacetagas ctteastgc atgggtauda

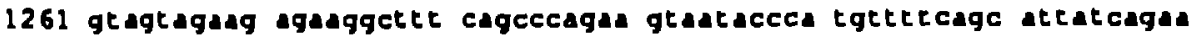

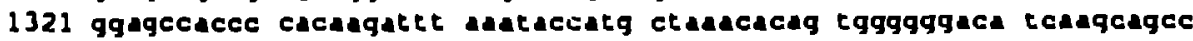

1381 atgcanatgt tasagagac catedatgag gaugctgcag astgggatag attgcatcea

i411 gtgcatgcag gqcetattgc accaggecag atgagagade cagggydag tgacatagca

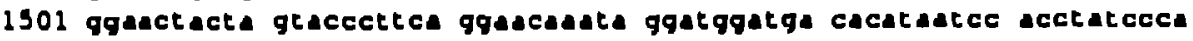

1561 gteggagae tctitauag etgqatale etgggatta acuanagt agantgtat

1621 agcectacea geatetgga cataugaca ggaceasagg eacretterg agactalgta

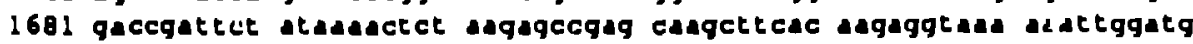

1741 acagasect tgteggtcce asatgeganc ceagattgta agactattet auagcattg

1801 gqaccagqaq cqacactaga agasatgatg acagcatgke agggagtggg ggricecggc

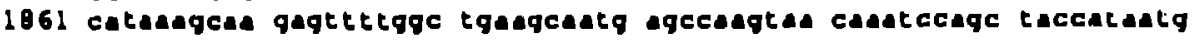

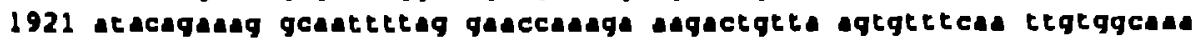

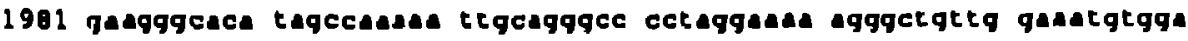

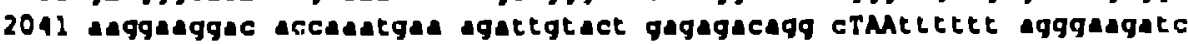
pol ede atart (NH2-termlnue uncertaln) $\rightarrow$

2101 tggectetcc acasgggang gccagggat tetcttcaga gcagaccaga gccacagec

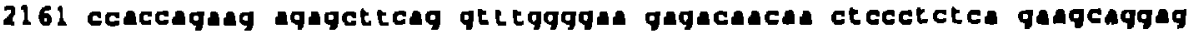

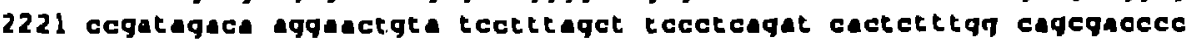

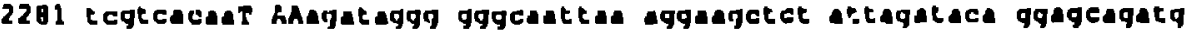
C- gaq cdu end

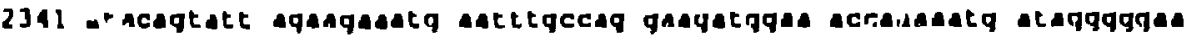

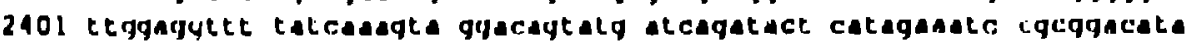

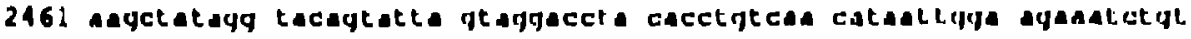
2521 tgectcagat tggetgcact teantettc ccattaqtec lettgagact gtaccertes

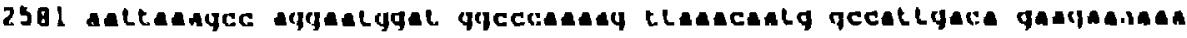

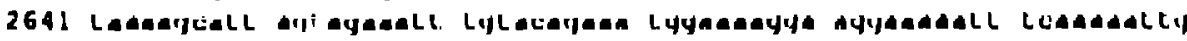

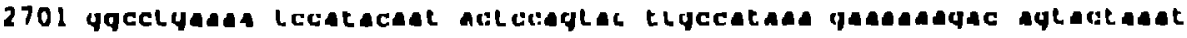

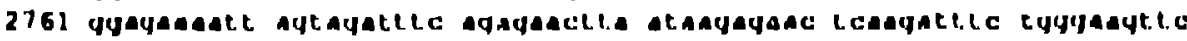

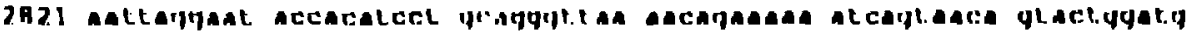

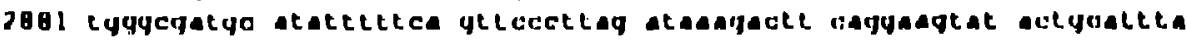

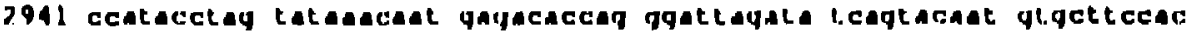

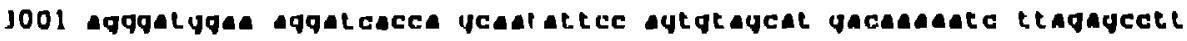

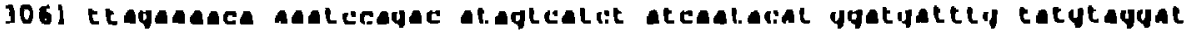
3121 ct.yect

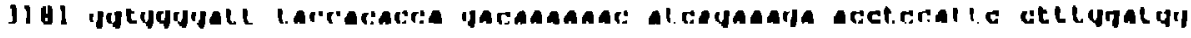

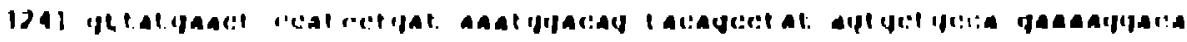

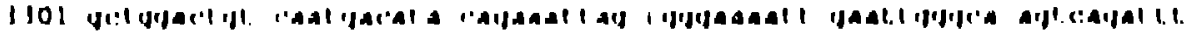

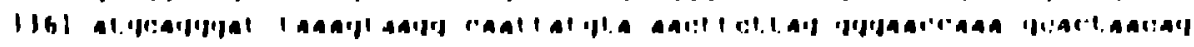

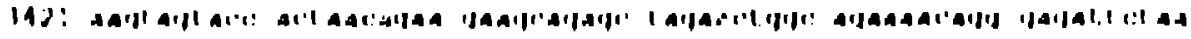

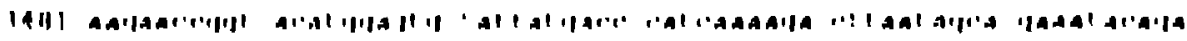

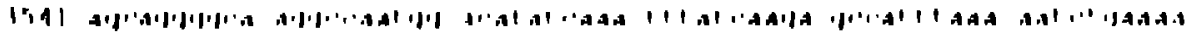

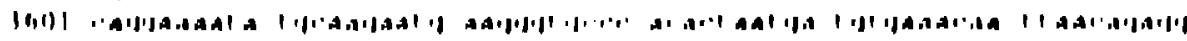

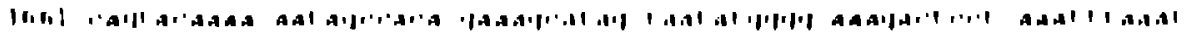

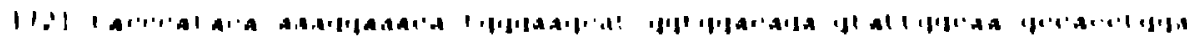

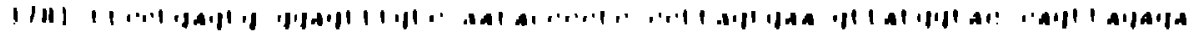


3841 adgaacecat autaggace gaactetet atgtagatgg ggcagccast agggnacta 3901 asteaygada agcaggatat gtaactgaca gaggaagaca adadgttgte cccetaacgg 3961 acasacada tuagadact gagttacad cadtcatct agcttggedg gaticgggat 4021 tagasgtada catagtgace gactcacas atgcattggg atcattcas gcacascag 4081 atasgagtga atcagagtte gtcagtcasa tastagagca qteateasa asggadadag 4141 tetacetgge atgggtrcca gcacacasa gastggagg auatgasad gtagatgggt 4201 tggteagtgc tggadtcagg aadgtactat ttetagatgg adeagatadg gcccadgadg 4261 aceatgagaa atatcacagt adtggagag catggctag tgattctac ctaccacetg 4321 tagtagcasa agaatagta gccagctgtg ataAutgted getadaggg gadgcedigc 4381 atggacaagt agactgtagc ccaggasat ggcagctaga ttgtacocat itagaagga Q441 augteatet ggtagcagte catgtageca gtggatatat agasgeaga gtaatceag 4501 cagagacagg gcasaauca gcatacttcc tettaaatt agcaggaaga tggceagtaa 4561 aacagtaca tacagacast ggcagcast tcaccagtac lacagttadg gecgectgtt 4621 ggtgggeggg gatcasgcag gasttggca ttcectacas tecceasat casggagtad

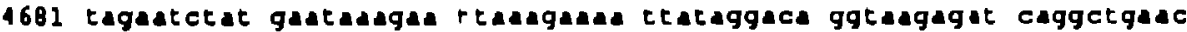
474 atctrasjac agcagtacas atggcagtat ccatcracas ttteasaga aagggggga 4801 ttggggggta cagl.gcaggg gaadgatag tagacatad agcadegac atacasecta

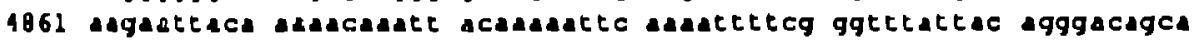
4921 gagatecagt ttggaagga reagcaadgc tectch7jad aggtgasgg gcafitagtad 4981 tacagatan cagtgacata augtagtge cadadagaua agcaagatc at jeyggatt 5041 ATGgaaaca gatggcajgt gatgattgtg tggcastag acaggatgar gat TAAcaca vif ecs $\rightarrow$ $<-$ pol

5101 eggaaugat tagtauaca ccatatgtat attecaagga asgetaaga ctggttteat 5161 agacatcact atgasajtac tadecasa atasttcag agtacacat cccactaggq 5221 gatgctadat tagtadeac adcatattgg ggtctgcata caggagaag agactggrat 5281 t.tgggteagg gagtctccat agaarggagg auagagat atagcacaca agtagacect 5341 gacctagcaq accactas tcatctgcac tattetgate gttetccaga atetgctata \$401 agaatacea tattaggacg tatagtege octaggtgtg autatcago aggacataa 5461 aggtaggat etctecagte cttggeacta gcagcattes teaseceas acagateag 5521 ceacettze ategrgttag gaactgaca gaggacagAT Ggaceagce ccagargace VpR cd. utart $\rightarrow$

5501 idgggccace gagggagcce tacastaet ggacactaGa gcteteagag gascteaga <- vif cdn and

5641 gtgaagetgt tagacatet ectaggatat ggctccata cteagacas catatctatg

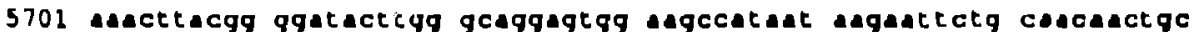
IIIV-1 NYS DNA $八$ HIV-I LAV (LAI)

5761 tgtteatcca teteAGate gggtgtcjac atagcagat aggcgtcact egacagagya 八了. I

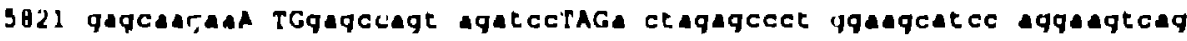
tat cda otart $\rightarrow$ r vpR cda und

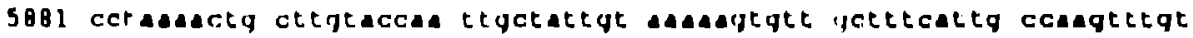

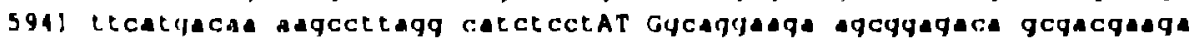
rev rdo or art $\rightarrow$

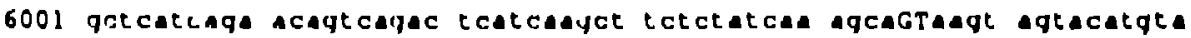
(tat, rav, nef) gi if $/$

6061 ATGcasccte teategtagc eategtagca thagtegtag caetaateat eqcaetegt.t vpll cde $\rightarrow$

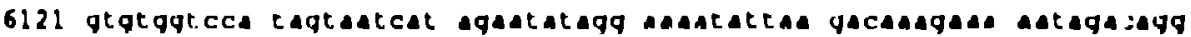

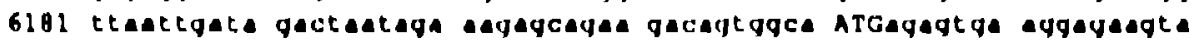
env cds it art. $\rightarrow$

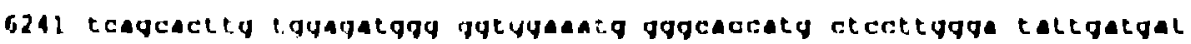

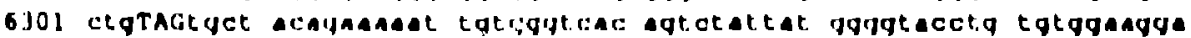
<- vpl cod and

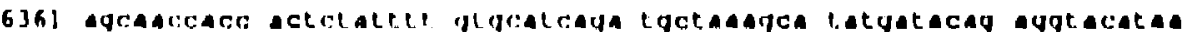

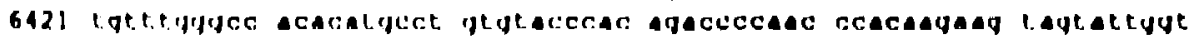

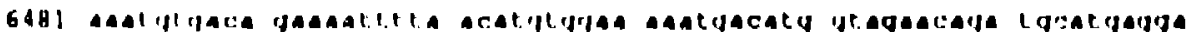

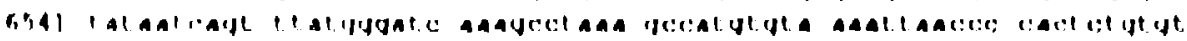

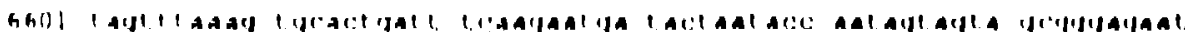

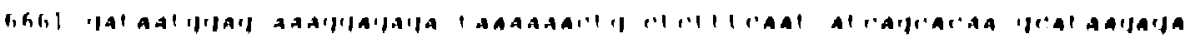

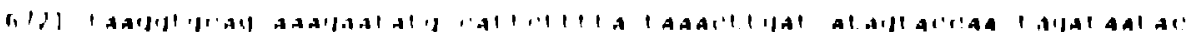

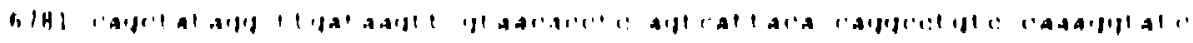

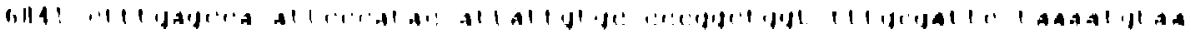

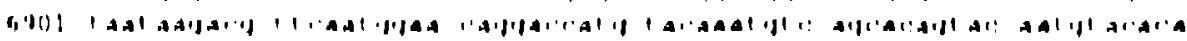

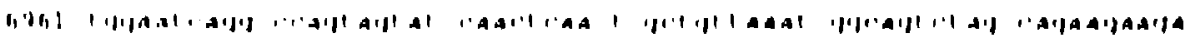

I.A.78

MAY 91 
7021 tgtagtaat agatctgcca atttcacaga cargctada accatading tacagctgaa

7081 cacatctgta gaateate gtaceagacc caucacal acadaaad gtatccgtat

7141 ccagagggga ccagggagag cattegttac adaggaad ataggaata tgagacaagc

7201 acattgtac attagtagag caadigga tgccacteta adacagatag ctagcaate

7261 agagancaa tttggaada atadacad atctteag caacctcag gaggggacce

7321 agaattgta acgcacagt

7381 actgtttad agtacttggt ttadigtac teggaglact gaagggtcad atadeactga

7441 aggagtgac acalcacac tcccatgcag ataaacad tetataaca tgtgycagga

7501 agtaggaad gcadigtatg cecctcccat cagtggacad attagatget catcaatat

7561 tactgggctg ctattaacad gagatggtgg tadiacaac autgggtceg agatcteag

7621 acctggagga ggcgatatga gggacaatg gagaggtgad teatataat ataagtagt

7681 auadtgaa ccattaggag tagcacccac caaggcaag agagagtgg tgcagagaga

7741 aaaagagca gtgggaatag gagctttgte ccttgggtt= itgggagcag caggaagcac

7801 tatgggctgc acgtcaatga cgcigacggt asaggceaga caateattgt ctgatatagt

7861 gcagcagcag aacaattggc tgagggctat tgaggegcad cagcatctgt tgcadetcac

7921 agtctggggc atcaacagc tccaggcaag atcctggct gtggaagat acctaagga

7981 tcaecagctc ctggggattt ggggtegctc tggaaacte attegcacea ctgetgtgce

B041 ttggaatgct agt tggagta atadatctct ggaacagate tggadaaca tgacetggat

0101 ggagtgggac agagaatta acautacac agcteata cactccteda tegaagatc

0161 gcauaccag caagaaaga atgaacaaga attattgga teagataat gggcagttt

8221 gtggaatggg tetaacatad caattgget gtggtatata aateattea tatgatagt

0281 aggaggctig gtaggettaa gaatagtett tgctgtactt tctatagtga atagagttag

834] gcagggatat ccaccattat cgtttcaGac ccacctcced ateecgaggg gaceggacag

$$
\text { 八 J of (tat, rev, nef) }
$$

8401 gcccgaagya aTAGadaag a aggtggaga yagayacaga gacagatcca ttcgattagt $<-t a t$ cda and

B461 gaacggatcc ttagcactta tctgggacga tctgeggagc otgtgectct teagctaoca B521 cigcttgaga gactiactct tgattgtaac gaggattgtg gaacttctgg gacgcagggg B581 gtgggaagcc ctcaatatt ggtggaatct cctacagtat tggagtcagq aactaaaga 0641 TAGtgctg:t a actigctca atgccacagc catagcagta gctqagggga cagatagggt c- rev cda end

8701 tatagaagta ttacaagcag cttatagagc tactcgccac atacctagaa gaataagaca

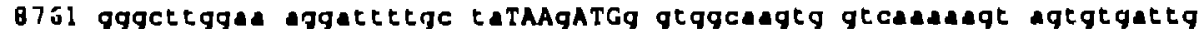
c- eny eds end

nef cde etart $->$

8021 gatggectge lqtaugqgad agatgagac gagctgagce agcagcagat ggggtgggag

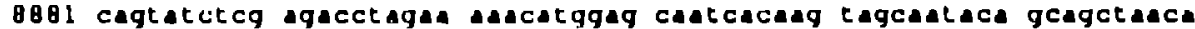

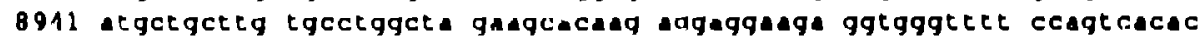
9001 ctcaggtace tttaagacca utyacttaca aggcagctgt agatctagc cactetea

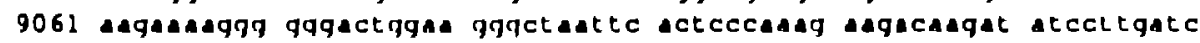
3. LTP $\rightarrow$

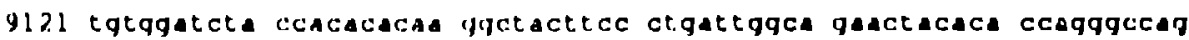

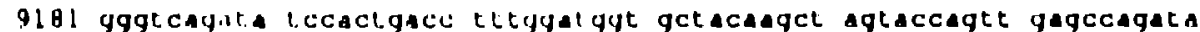
9241 aqgtaqaada yqucaalaa qqaqaqaaca ccaqcteqte acaccctqtg aqcetgcatg

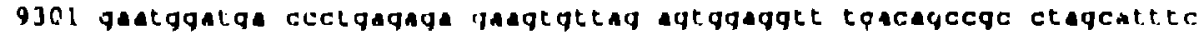

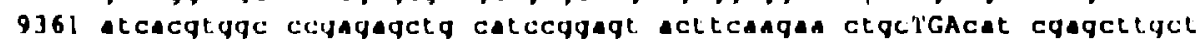
i. - net eds end

9421 acaugqaet teccgetqqq yactetccag quaqgeqtqg cetqugcqqq actqqgqaqt

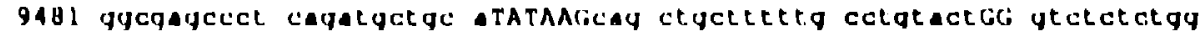
-lynal -> 4 rpt itart $\rightarrow$

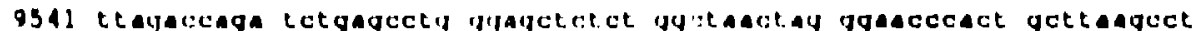

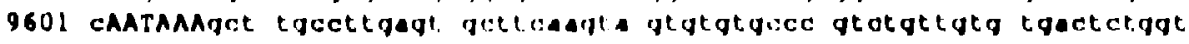
lqnal $\rightarrow$ $<$ - mRNA end $r-H$ rut. and

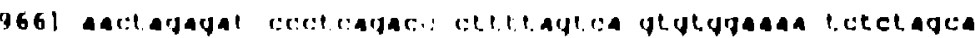
C- J' LTH 


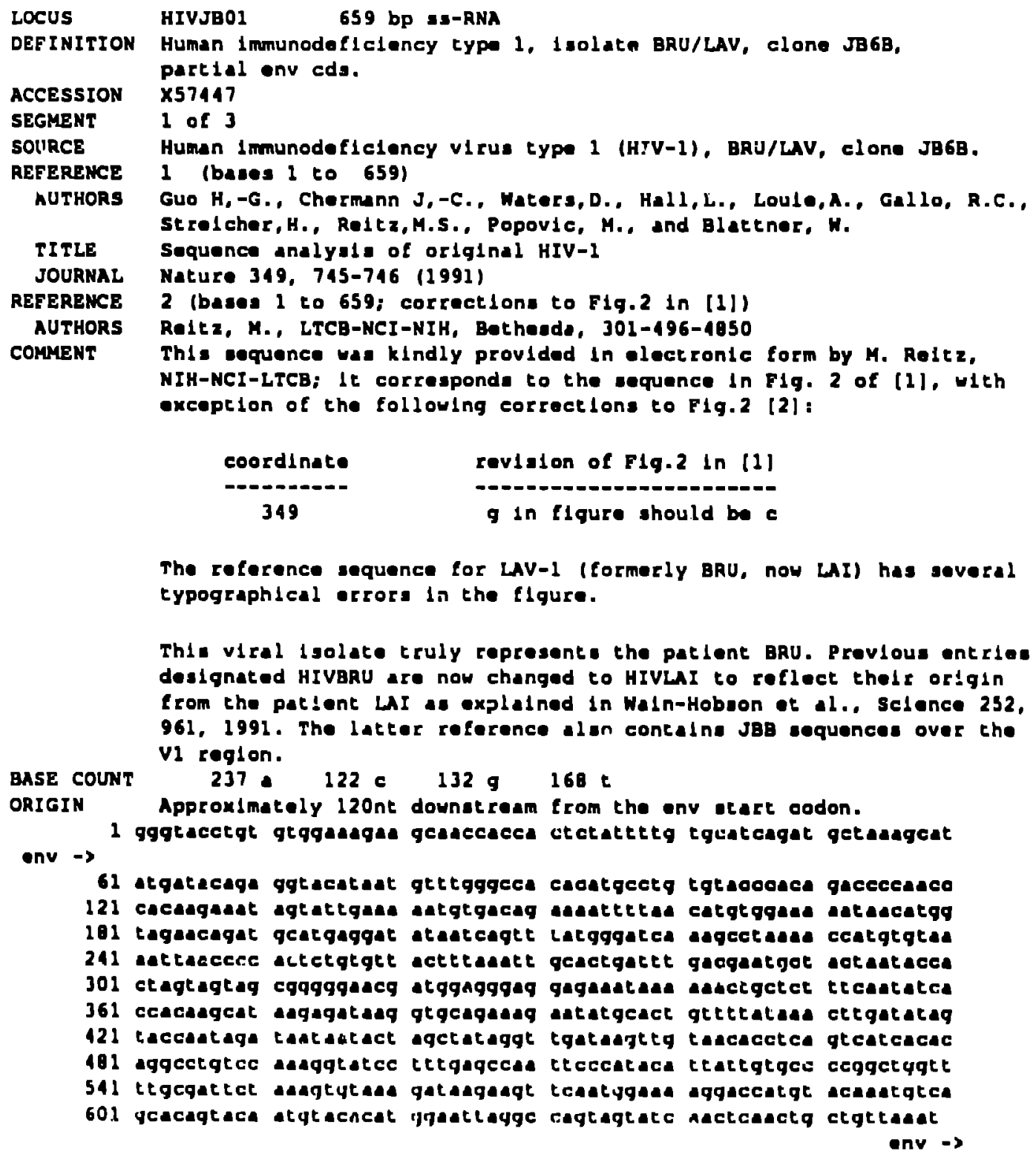


HIVJB01. Guo et al., Nature 349, 745, 1991. The original LaV eequence, now designated LAI, is shoun for comparison. These sequences start at about 1.20 nt downstream from the env start codon. See also 〈HIVJBO2> and 〈HIVJBO3s.

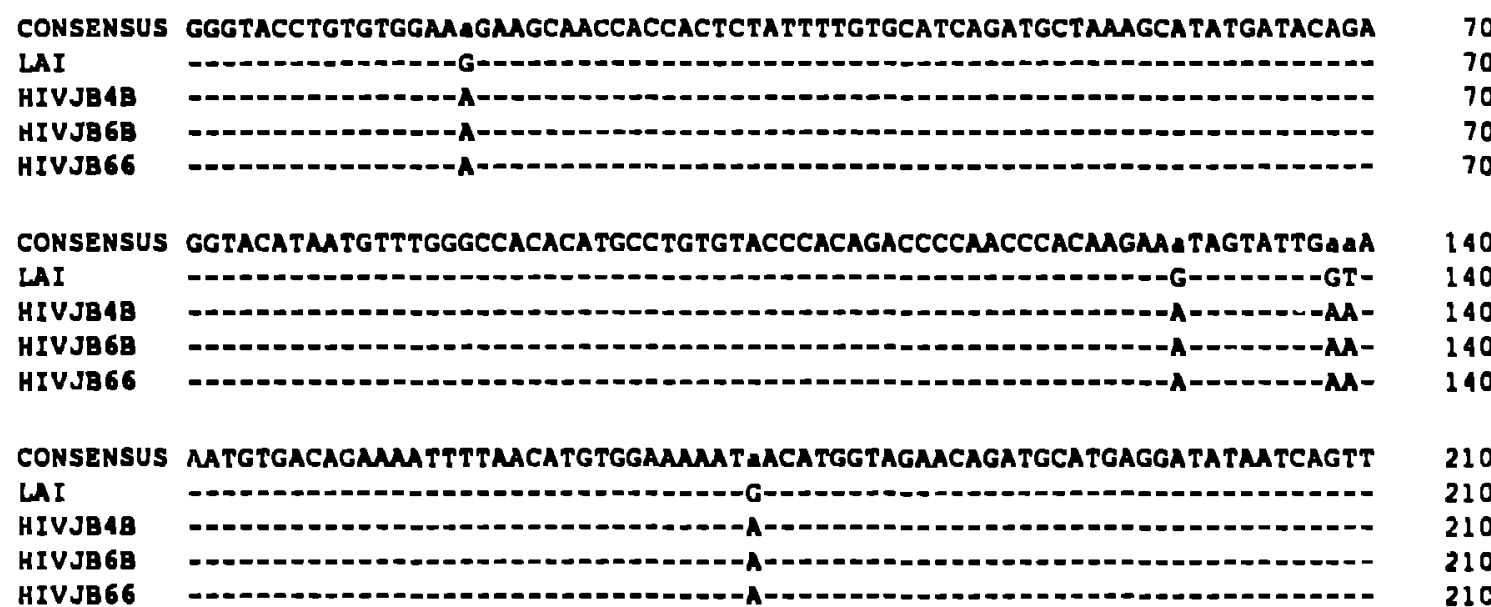

CONSENSUS TATGGGATCAAGCCTMA CCATGEGAMATTAACCCCACTCTGTGTTACTTTAAAL TGCACTGATTT

LAI

HIVJBAB

HIVJB6B

HIVJB66

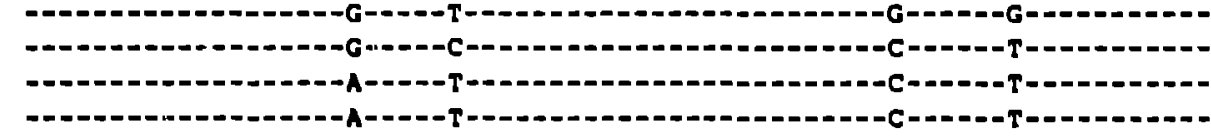

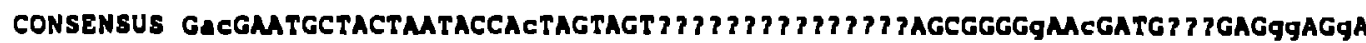

LAI

HIVJB4B

HIVJB6B

HIVJB66

CONSENSUS

LAI

HIVJBAB

IIIVJB6B

HIVJBG6

CONSENSUS

IAI

IIIVJBAB

HIVJH6H

IIIVJB66

CONgENSUS

IAI

HIVSBAB

HIVJHGh

HIVJA6 6

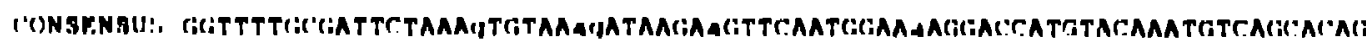

$$
\text { I.A I }
$$

IIIV.JHAh

IIIV IIInI

IIIVIIITh

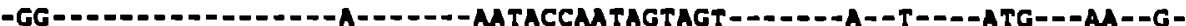

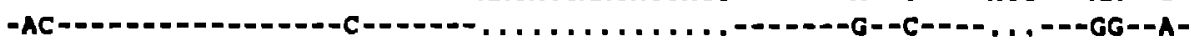

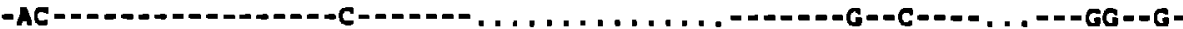

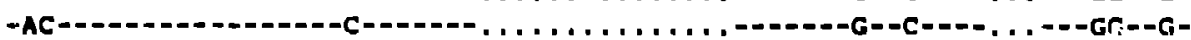

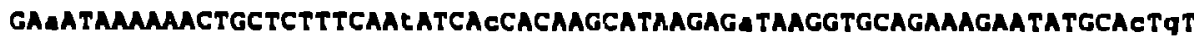

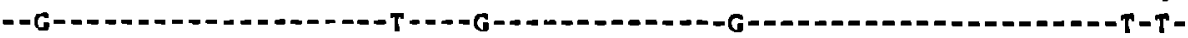

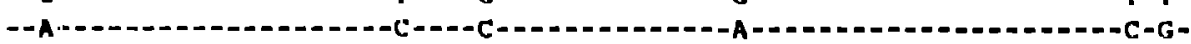

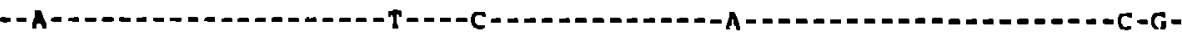

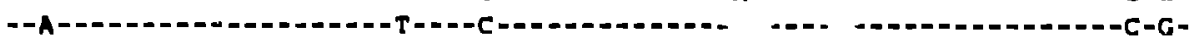

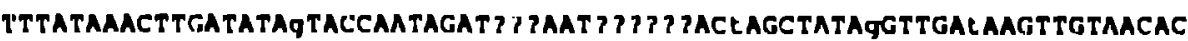
-

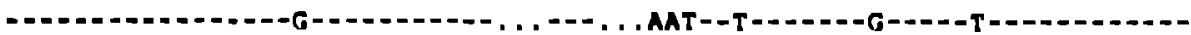

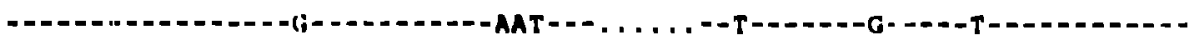

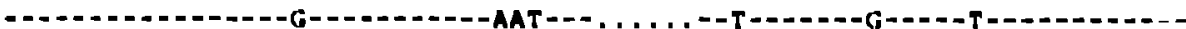

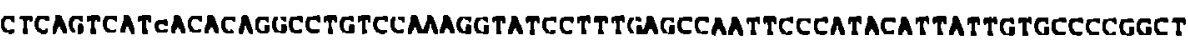

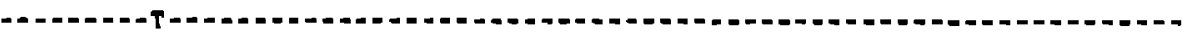

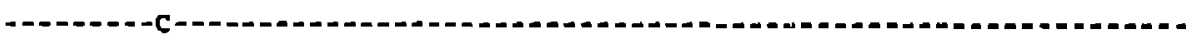

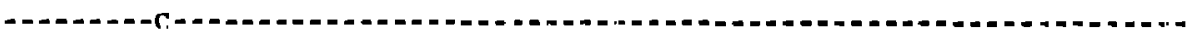

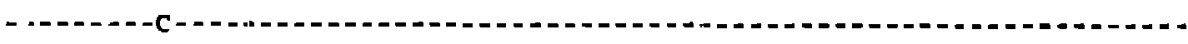
- - - - - - - - - - - - - - - -

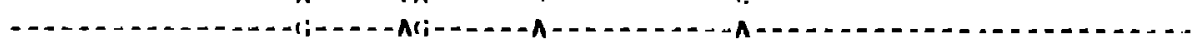

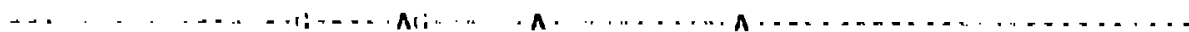
$\boldsymbol{\Lambda}$ 
HIVJB01

CONSENSUS TACAATGTACACATGGAATTAGgCCAGTAGTATCAACTCAACTGCTGTT IMAT

655

LAI

680

HIVJB6B

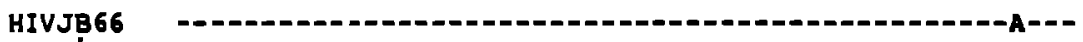

659 


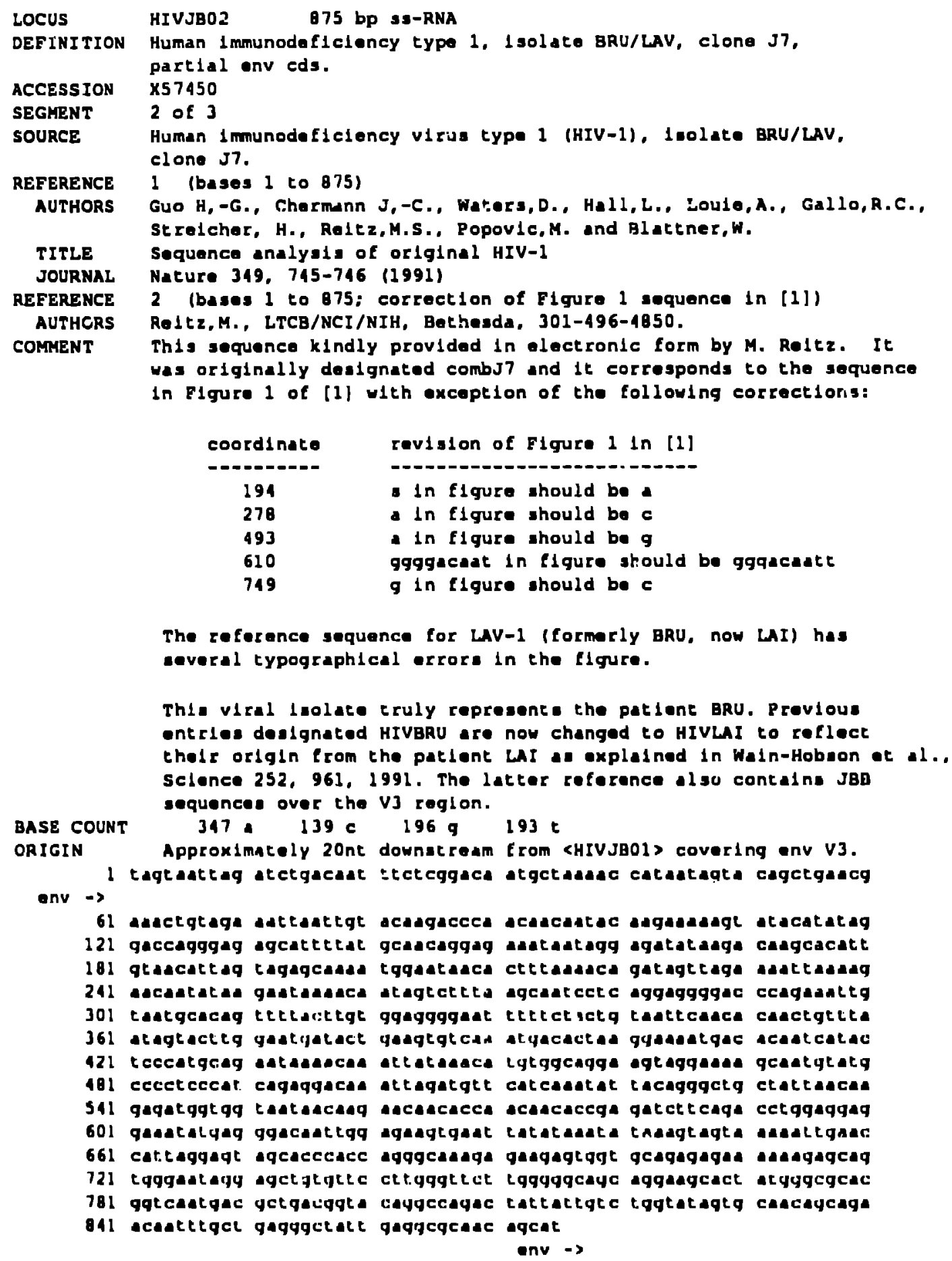

coordinate
194
278
493
610
749

The zeforence sequence for LAV-1 (formerly BRU, now LAI) has eneral typographical errore in the flgure.

Th1. viral leolate cruly represente the patient BRu. Previous entrles dealgnated HIVBRU are now changed to HIVLAI to reflect thals orlgln trom the patlent hal a explalned in Wain-Hobson ot al.. sclence 252, 961, 1991. The latter reference also contalna JBB sequences over the Vu reglon. 
HIJJB02. These sequences start approximately $20 \mathrm{nt}$ downtream from <HIVJBO1> In the env eds; they cover the VJ region. LAI, the original LAV, is shown for compasison.

\begin{tabular}{|c|c|c|}
\hline CONSENSUS & ГAGTAATAGATCTGACATTTCtCggACAATGCTAMACCATAATAGTACAGCTGAMCgAhaCTGLAGA & 70 \\
\hline WA & 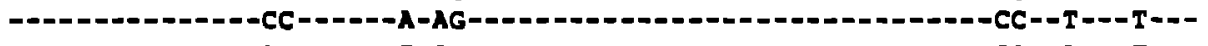 & 70 \\
\hline HIVJB61 & 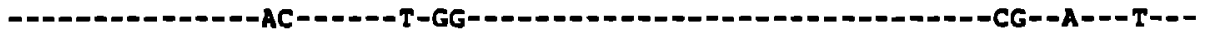 & 70 \\
\hline HIVJB7 & 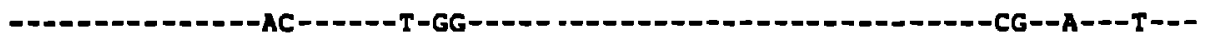 & $r$ \\
\hline HIVJBH41A & 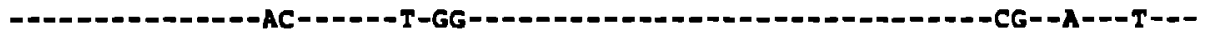 & 76 \\
\hline HIVJBH4R & 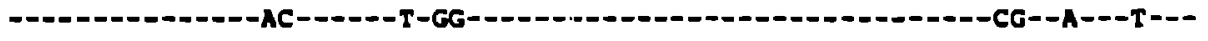 & 10 \\
\hline HIVJB5I & 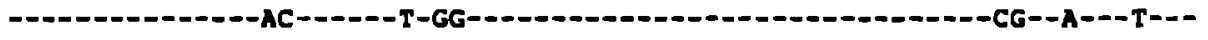 & 70 \\
\hline H:VJBD 4LL & & \\
\hline HIVJB31 & $--C G--A---T---$ & 70 \\
\hline IVJB21 & & \\
\hline HIVJB1 1 & & \\
\hline IVJBH $14 \mathrm{~A}$ & $--C G--A---T---$ & 70 \\
\hline HIVJBD221 & $--N G--C---N-=-$ & 70 \\
\hline IVJB71 & & \\
\hline HIVJBC 306 & & \\
\hline SONSENSUS & 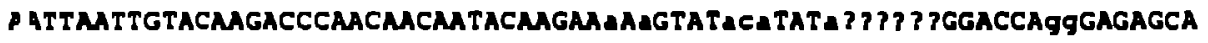 & 134 \\
\hline LAI & 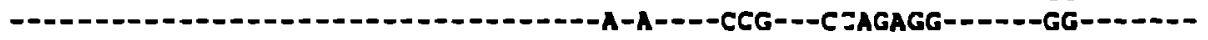 & 1.40 \\
\hline HIVJB 61 & - - - - - - & 134 \\
\hline HIVJB7 & 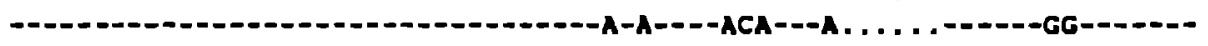 & 134 \\
\hline HIVJBH41A & 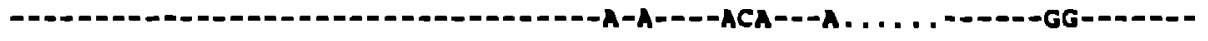 & 234 \\
\hline HIVJBH4R & 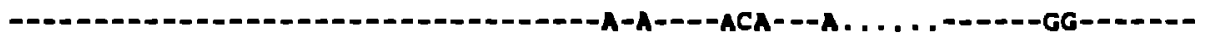 & 234 \\
\hline HIVJB5I & - & 134 \\
\hline HIVJBDALL & & \\
\hline HIVJB3I & 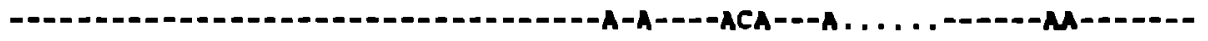 & 134 \\
\hline HIVJB2I & & \\
\hline HIVJB 11 & & \\
\hline HIVJBH 14 A & 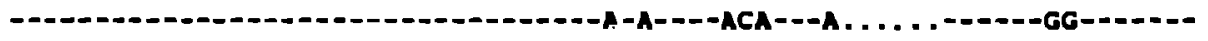 & 134 \\
\hline HIVJBD221 & 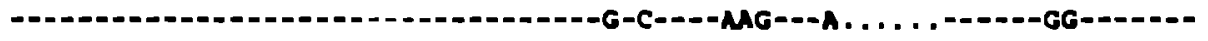 & 134 \\
\hline HIVJB7 I & & \\
\hline HIVJBC 306 & & \\
\hline cONSENSUS & 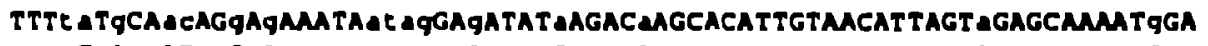 & 204 \\
\hline LAI & 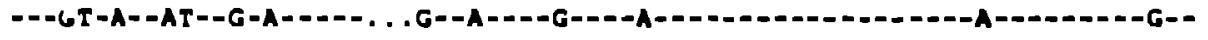 & 207 \\
\hline HIVJB6I & 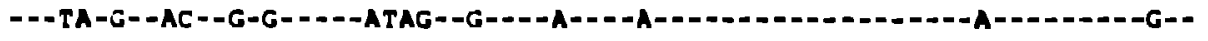 & 204 \\
\hline HIVJB 7 & 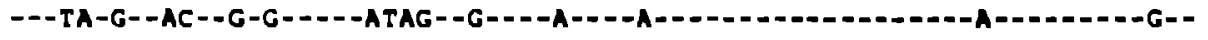 & 204 \\
\hline HIVJBHAIA & 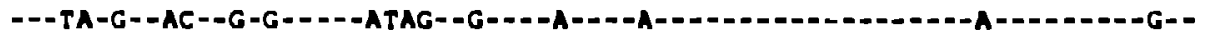 & 204 \\
\hline HIVJBHAR & 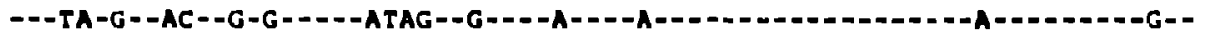 & 204 \\
\hline HIVJBSI & 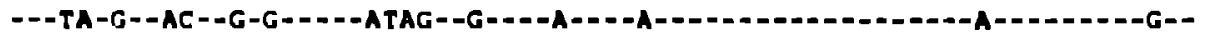 & 204 \\
\hline HIVJBDALL & & \\
\hline HIVJA31 & 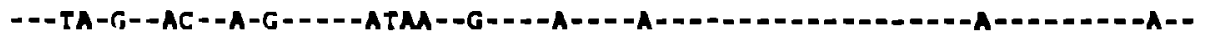 & 204 \\
\hline HIVJB2I & & \\
\hline HIVJBII & & \\
\hline HIYJBHI 1A & 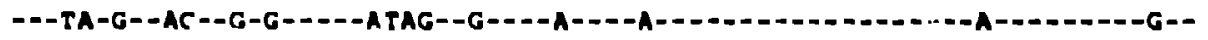 & 204 \\
\hline HIVJBD22I & 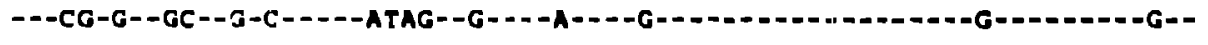 & 204 \\
\hline IYJB $7 !$ & & \\
\hline IYJBCJO & & \\
\hline
\end{tabular}




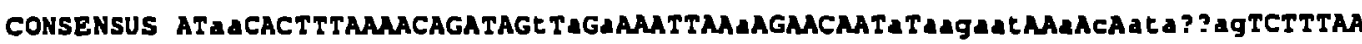

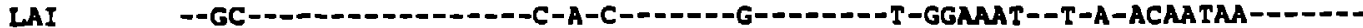

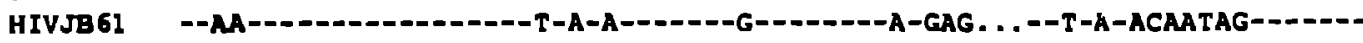

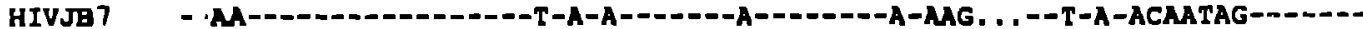

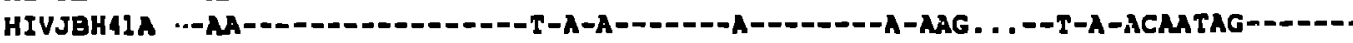

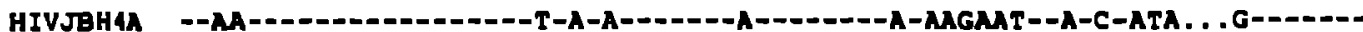

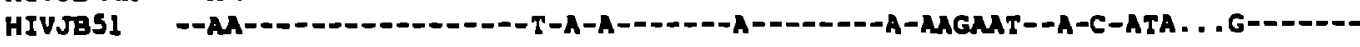
HIVJBD4LL HIVJB31 HIVJB21 --MA--_HIVJA1I

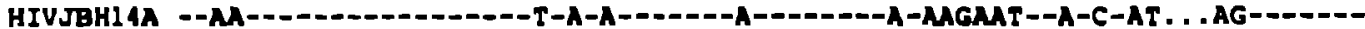

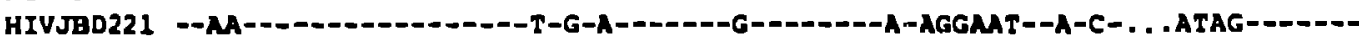
HIVJB7I HIVJBC306

CONSENSUS gCAMTCICAGGAGgGGACCCAGAAATTGTALGCACAGTTTTACTTGTgGAgggGAATTTTCTACTGT

LAI

HIVJB61

HIVJB 7

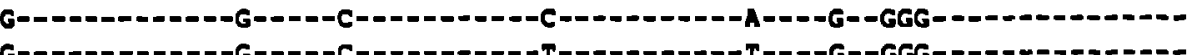

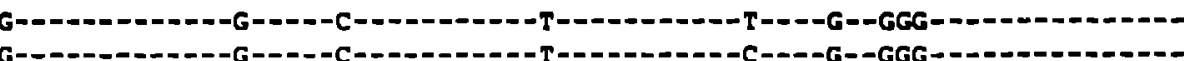

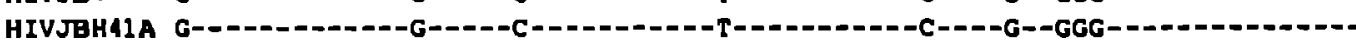

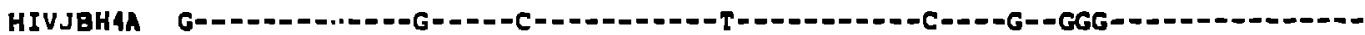

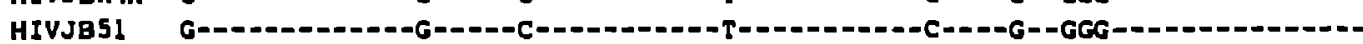

HIVJBDALL

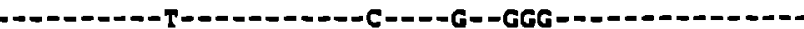

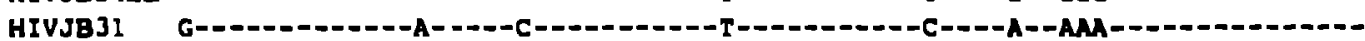

HIVJB21

HIVJB11

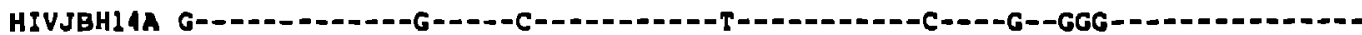

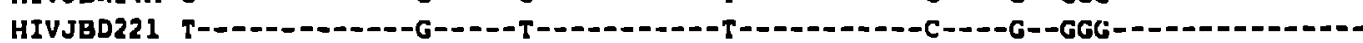

HIVJB 71

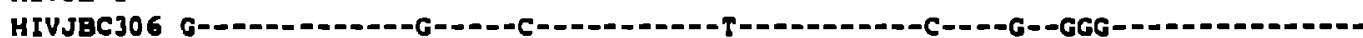

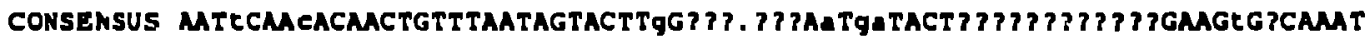

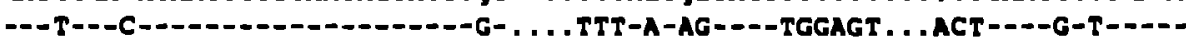

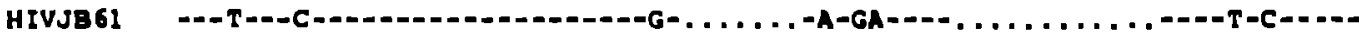

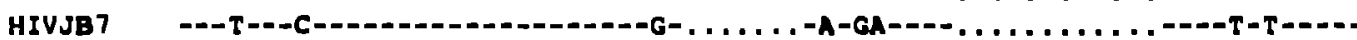

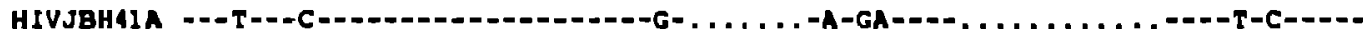

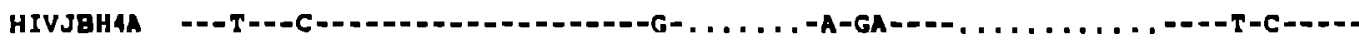

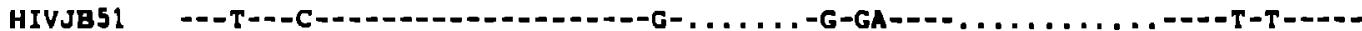

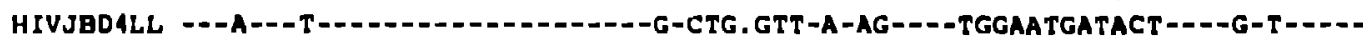

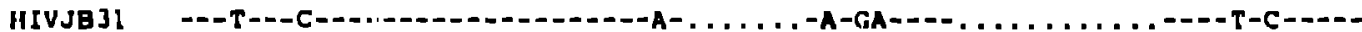
HIVJB21

HIVJBII

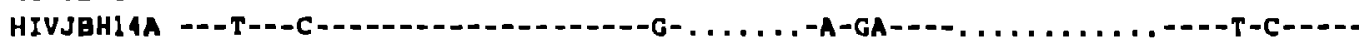

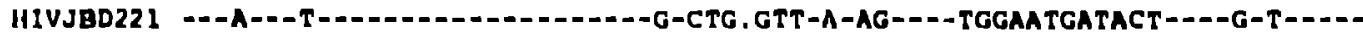
HIVJB71 
CONSENSUS aACACLAatgaaALgACACAATCALACTCCCATGCAGAATAAAACAA TT TIAAACATGTGGCAGGAAG LAI A---CTGAAGGAAGTG--

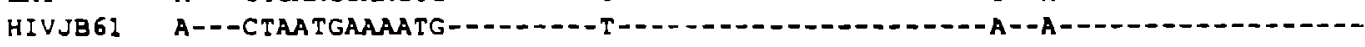

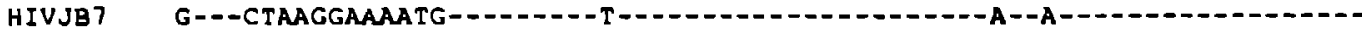

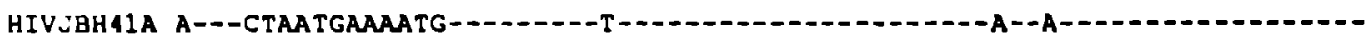

HIVJBH 4A A---CTAATGAAAATG---0---T

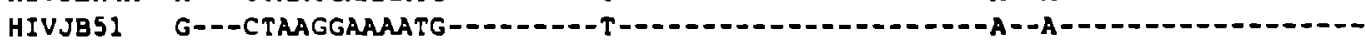

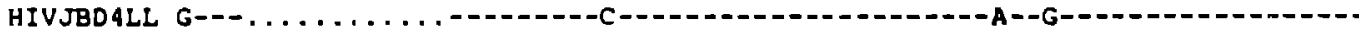

HIVJBJ1 A--CTANTGAAAATG-_-_-

HIVJB21

HIVJBII

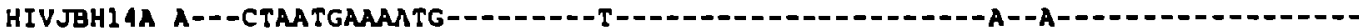

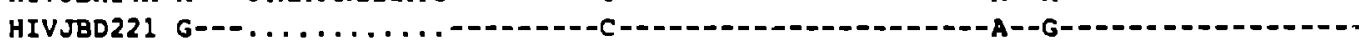

HIVJB71 G---CTAAGGAAATG--.

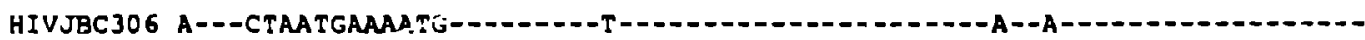

CONSENSUS TAgGAAAACCATGTATGCCCCTCCrATCAgagGACAAATTAGATGTTCATCAAATATTACAGGGCTGCT

LAI --G--0--10

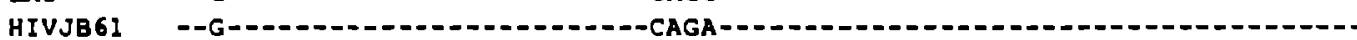

HIVJB7 - -G-

HIVJBH41A --G-_-_._.

HIVJBHAA --G-1--

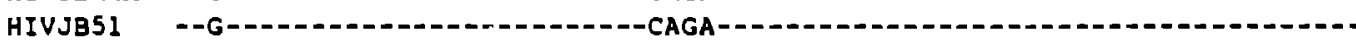

HIVJBDALL --G-1.-1

HIVJB31 --A

HIVJB21

HIVJB11

HIVJBHI 4A --G---1

HIVJBD221 --G-1-

HIVJB71 --G-1...-

HIVJBC306 - -G-

CONSENSUS ATTHACAAGAGATgG; JGT?? ?AATAACAAqMACACACCAACA???ACACCGAGATCTTCAGACCTgGA

LAI

...VJB61

HIVJB?

HIVJBH41A

HIVJBH4A

HIVJBSI

HIVJBDALL

HIVJB 3 I

HIVJB2I

HIVJBII

HIVJBHI $4 A$

HIVJBD221

HIVJB?I

HIVJBCJO6

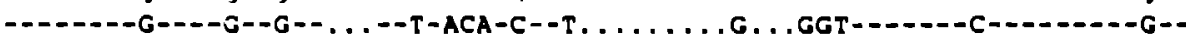

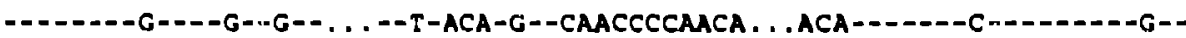

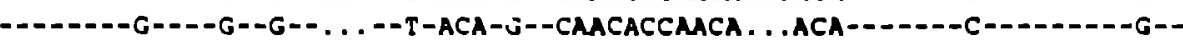

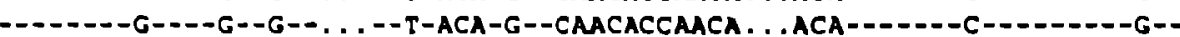

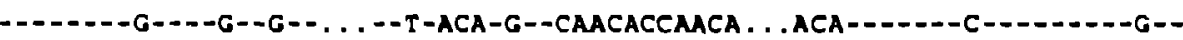

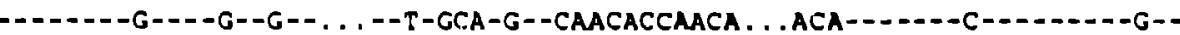
---.-G----r,-G--...--T-ACC-G--CAACACCAATAATGATA-------T--------G-- - 
CONSENSUS gGA TGAaATATGAggGACAAT TgGAGAGTGATTA: ATAAATATAAAgTAGTaAAAATGAACCATTAG

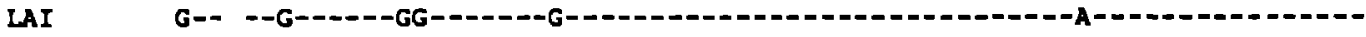

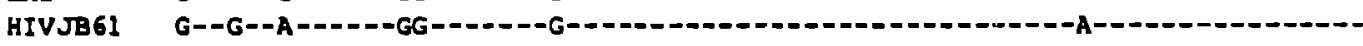

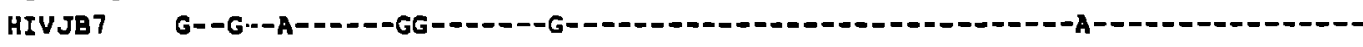

HIVJBH41A G--G--A---D-GG--1-- -G-

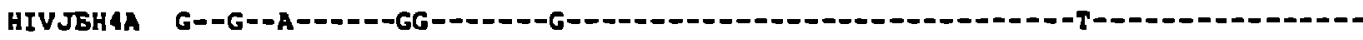

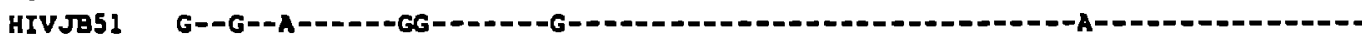

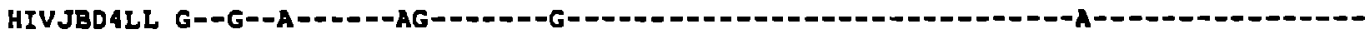

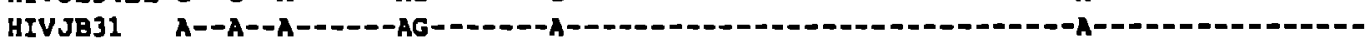

HIV J821

HIV JB11

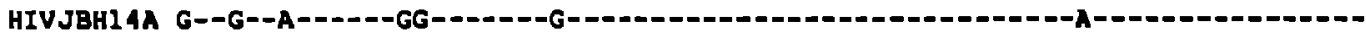

HIVJBD221 G--G--A---D-AG----D--G-- 630

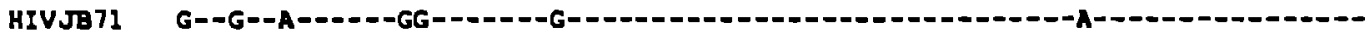

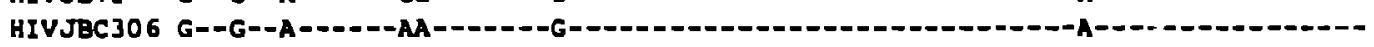

CONSENSUS GACTAGCACCCACCAgGGCAAGAGAMGAGgGtGCAGAGAGAAAAAGAGCAGTGGGAATAGGAGTgT

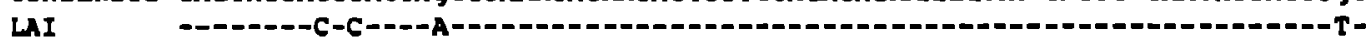

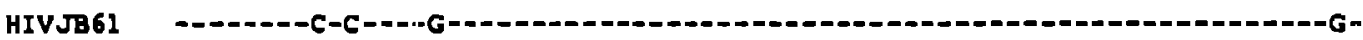

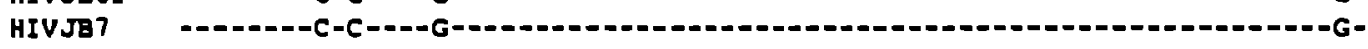

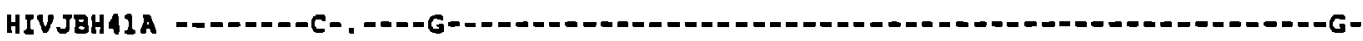

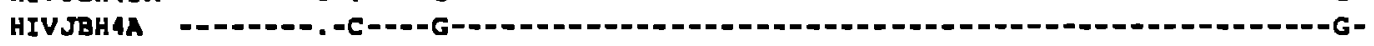

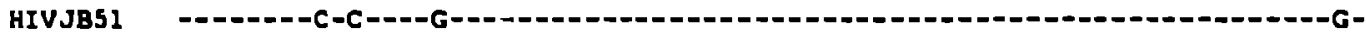

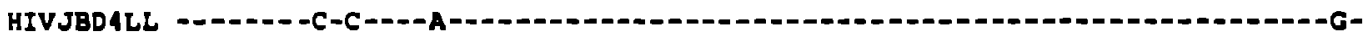

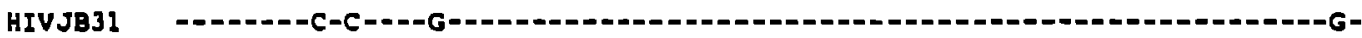

HIV TB21

HIV JB1 1

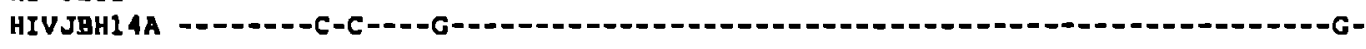
HIVJBD221

HIVJB71

6

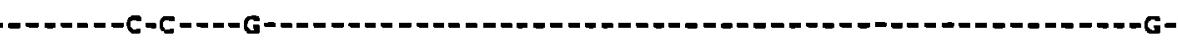

HIVJBC306

$-\mathrm{C}-\mathrm{C}-\mathrm{-}-\mathrm{-G}-\mathrm{G}$

$-G=$

CONSENSUS GTTCCTTGGGTTCTTGGgGCAGCAGGAAlicACTATGCGCgCAcggtCaATgACgCTgaCGgTACAGgCC

LAI

HIVJB61

HIVJB7

HIVJBH4IA

HIVJBH4A

HIVJBS 1

HIVJBDALL

HIVJBJI

HIVJB21

HIVJBII

ITVJBHI 4A

HIVJBD221

HIVJB71

HIVJBCjO 6

-

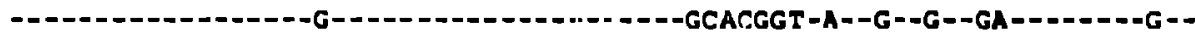

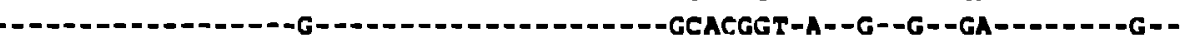

-

-

作

-

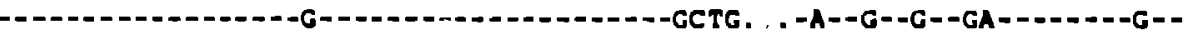

-- GCACCGT-A--G--. --GA---.----G-TTTTCGT-A--C--,--.,-------T-- GCAGGCT-A--G--G--GA--n-----G-- 


\section{HIVJB02}

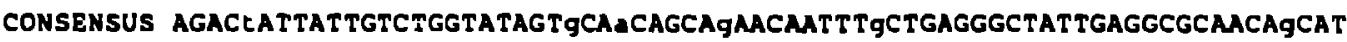
LAI

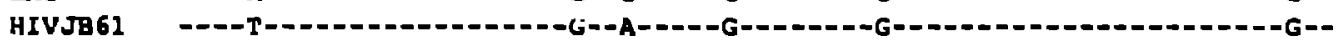

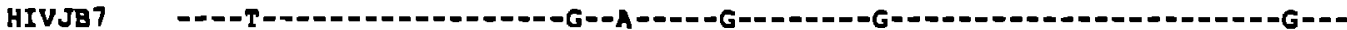

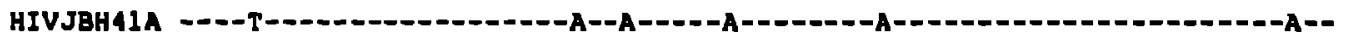

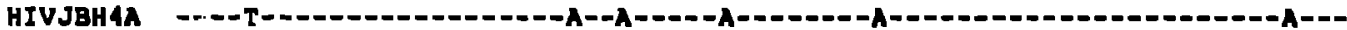

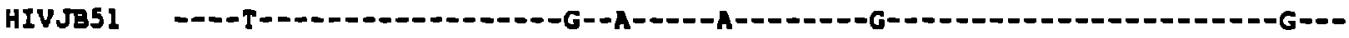

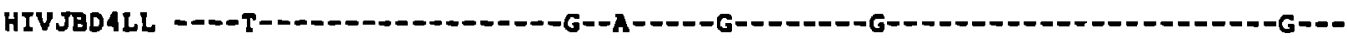

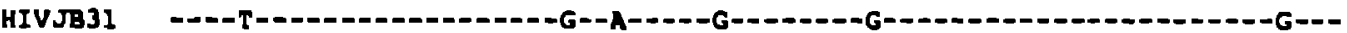

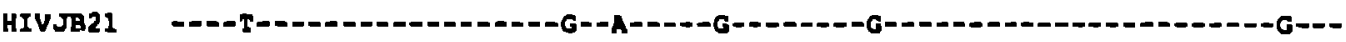

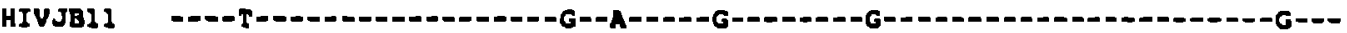

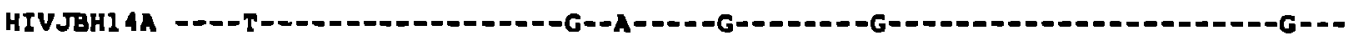
HIVJBD221

HIVJB71

- - - T- - - HIVJAC306

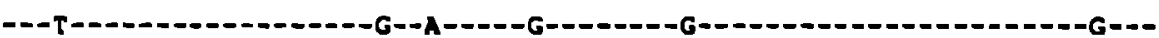




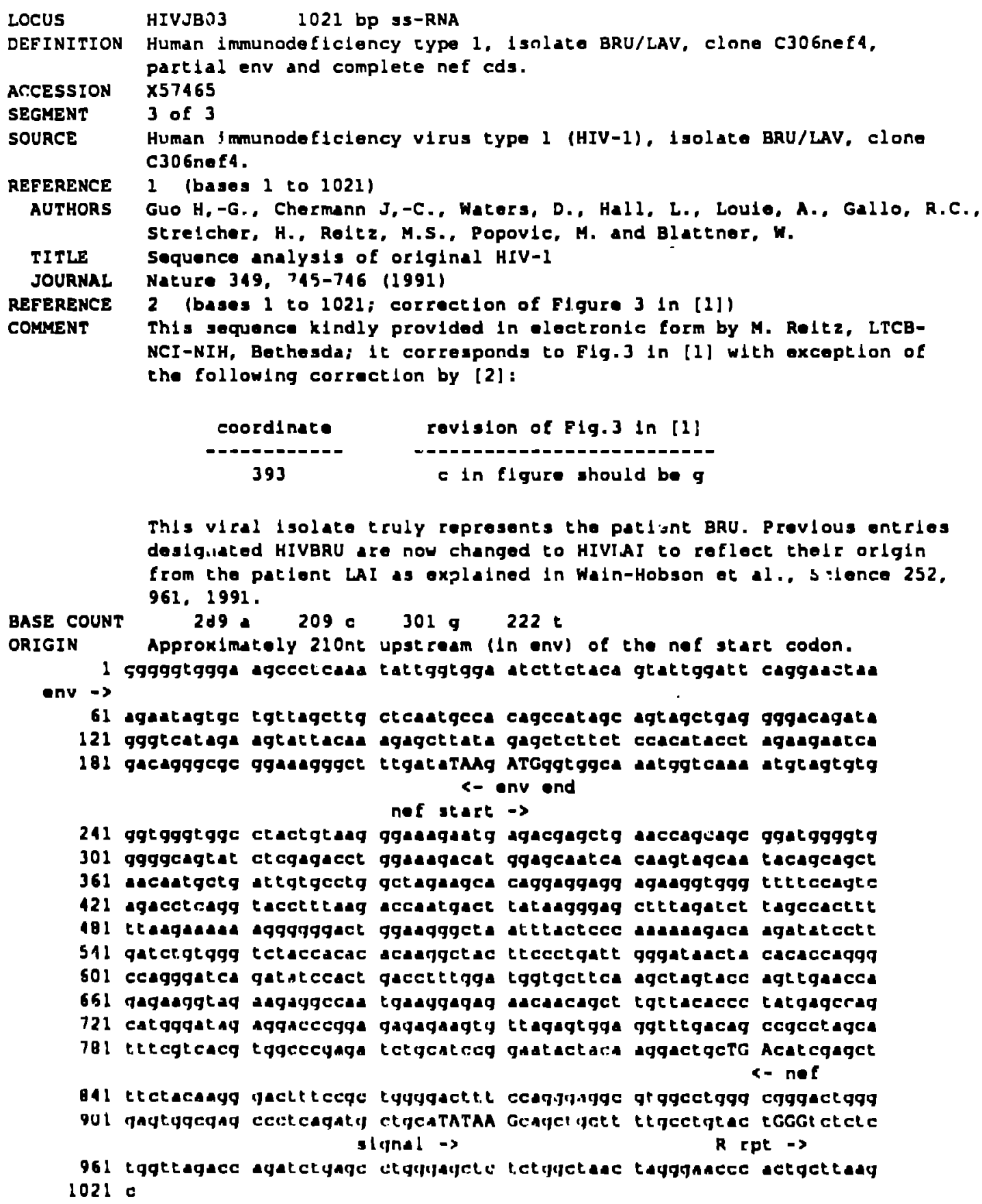

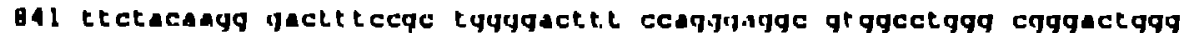

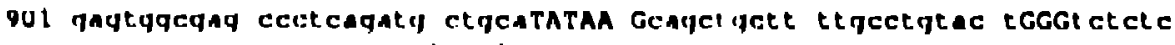
slunal $\rightarrow$ $R$ rpt $\rightarrow$

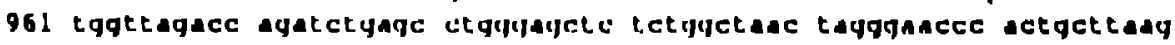
$1021 \mathrm{c}$ 
HIVJB03. The following sequence start in the onv cds approximately 210nt upgtream from the nef start codori. The LAI sequence 1s shown for comparison.

CONSENSUS GGGGGTGGGMACCCTCAAATATTGGTGGAATCTCCTACAGTATTGGAT TCAGGACTANAGAATAGTGC -

HIVJBNC31 -

HIVJBNPII

HIVJBNP41

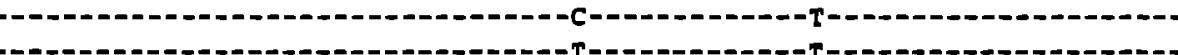

HIVJBC4

HIVJBCS

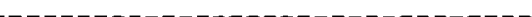

C-n-m-n-m-

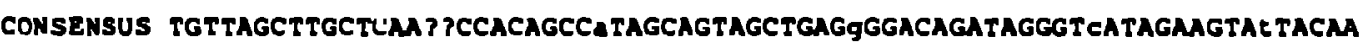

LAI

HIVJBMC31

HIVJBNP11

HIVJBHP 41

HIVJBC4

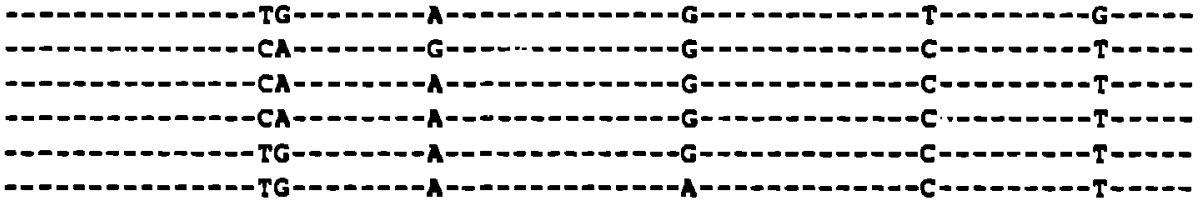

HIVJBC5

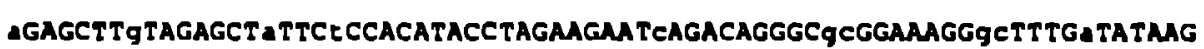

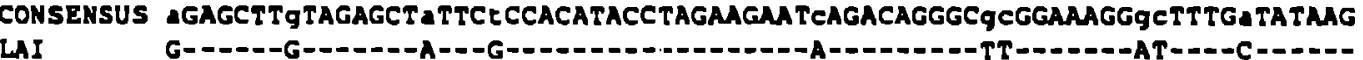

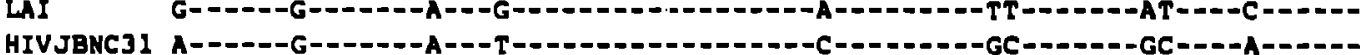

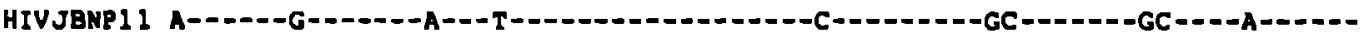

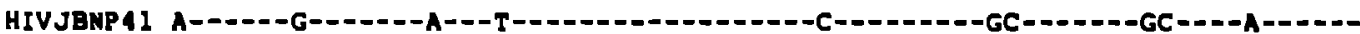

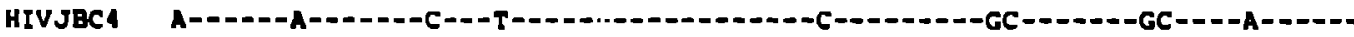

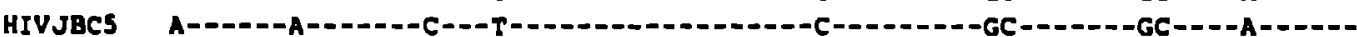

CONSENSUS ATGGGTGGCAATGGTCAAACGT PGT PTGGgTGGATGGCCTACTGTAAGGGAMGATGAGACGAGCTG

LAI

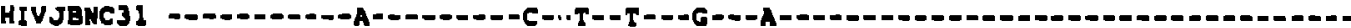

HIVJBNPL 1 -

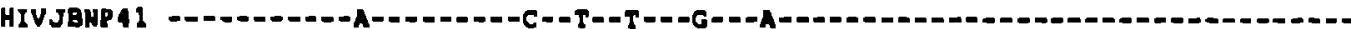

HIV J

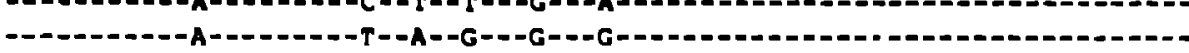

HIVJRCS

- -

CONSENSUS AaCCAGCAGCgGATGGGGTGgGaGCAGLAtCE Cgagacet ggaeagacatggegcalt cacaagtagcM

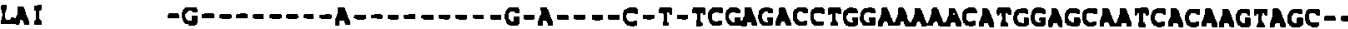

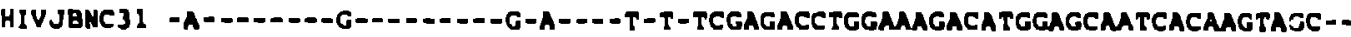

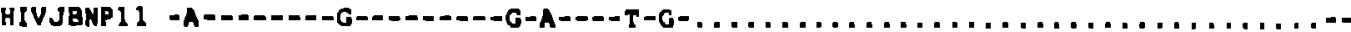

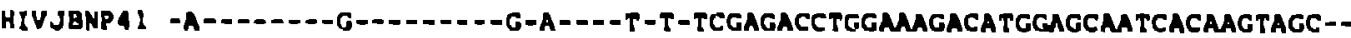

HIVJBCA -A---.----G---------G-G----T-T-TCGAGACCTGGAMGACATGGAGCAATCACAAGTAGC--

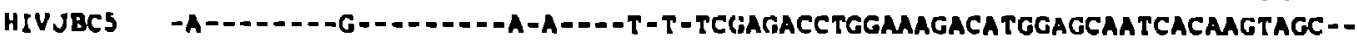

CONSENSUS TACAGCAGCTAACATGCTGATTGTGCCTGGCTAGAACACAqGAGGAGGAGGAGGTGGGTTTCCAGTC

LAL

HIVJBNCJ1 -. -

HIVJENPI 1 - - -

HIVJBNPA1 -.

-

HivJBCS

CONSEMSUS AqAC-TCAGGTACCTTTAAGACCAATqACTTAEAAGGYAGCTETAGATT,TTAqCCACTTTTTAAGAgAAA

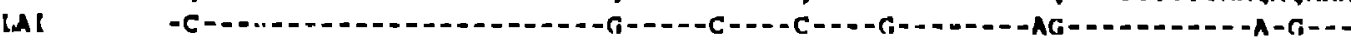

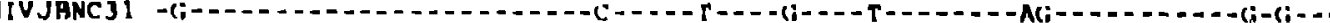

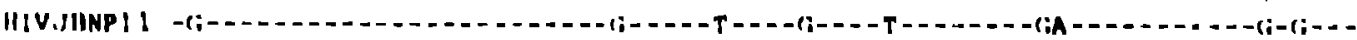

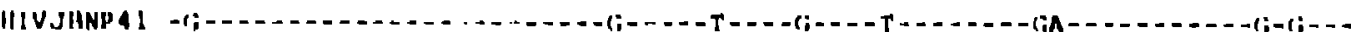

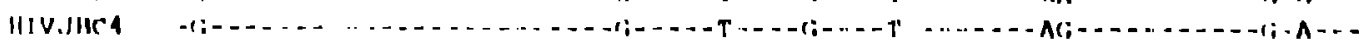

IIIV.ils."

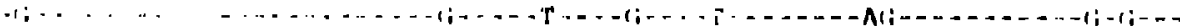

416

420

420

383

120

120

120 
CONSENSUS AGGGGGGACTGGAAGGCTAATTLACTCCCA? aAAAG?CAAGATATCCTTGAT ?TGTGGgTCTACCACAC LAI HIVJBNCJI

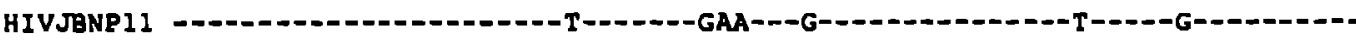
HIVJBNP 41 HVIB HIVJBC5

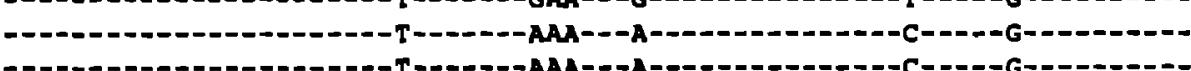
CONSENSUS ACA?GGCTACTTCCCTGATTGGgAtACTACACACCAGgGCCAGGGATCAGATATCCACTGACCTTTGGa

LAT ACA

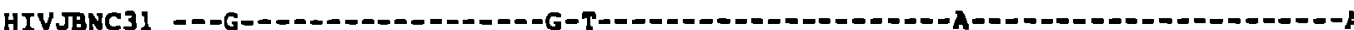

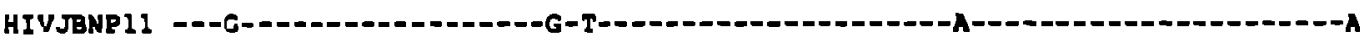
HIVJBNP 41 - - G- HTV

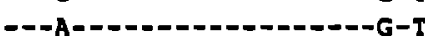

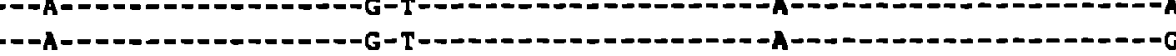

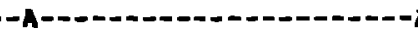
HIVJBC5

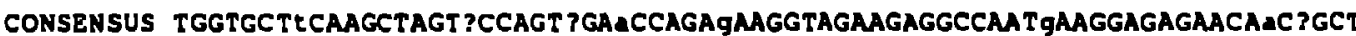
LAI -

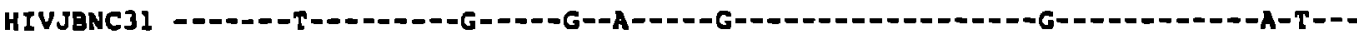

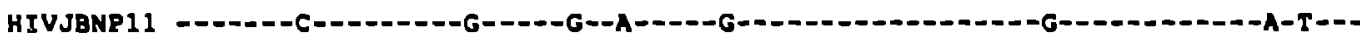

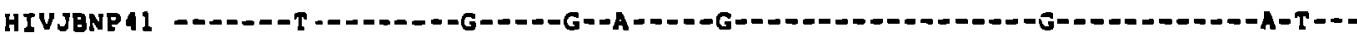
HIVJBC 4 - -

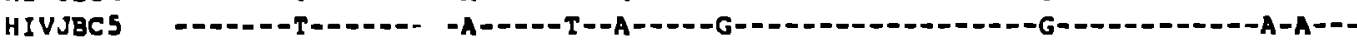

CONSENSUS TGTTACACCCTATGAGCCAGCATGGATgGAtGACCCgGAGAGAGAhGTGTTAG PGTGGAGG!TTGACAG LA I -

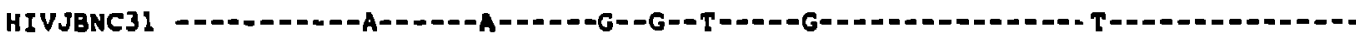
HIVJBNPII - 1 -

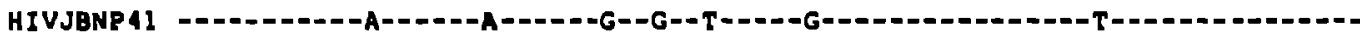
HIVJBC 4 -

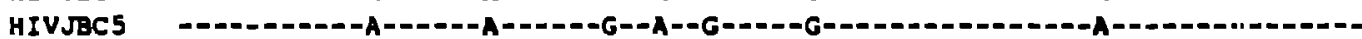
CONSENSUS CCGCCTAGCATTTCgTCACGTGGCCCGAGAg ? ?CATCCGGAqTACTACAAGgACTGCTGACATCGAGCT LAI
HIVJBNCJ1 -

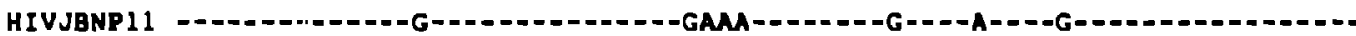
HIVJBNP 41 HIVJBC4 HIVJBCS

CONSENSUS TtCTACAAGGGACTTTCCGCTGGgGACTTTCCAGGGAGGCGTGG TCTGGGCGGGACTGGgGAGTGGCGAq

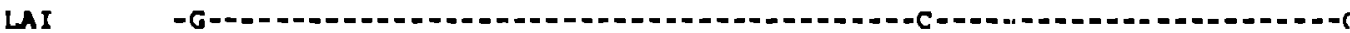

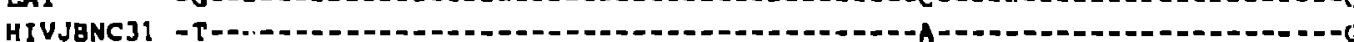

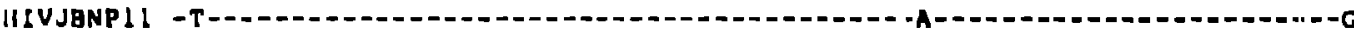

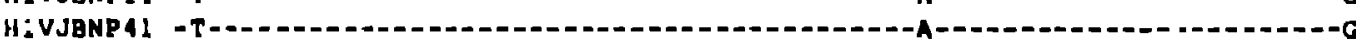

HIVJBCS -T-D.-1.

CONSENSUS CCCTrAGATGCTGCATATAAGCAGCTGCTTTTEGCCTGTACTGGGTCTCTCTGGTTAGACGAGATE TGAG LAI

HIVJBNCJI

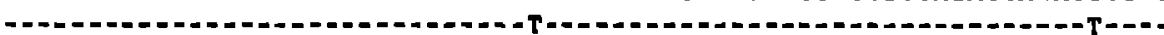

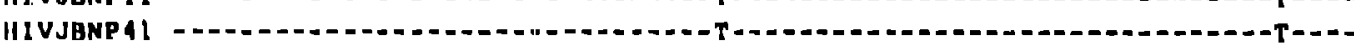

HIVIRC4

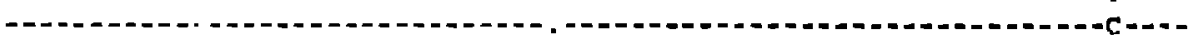

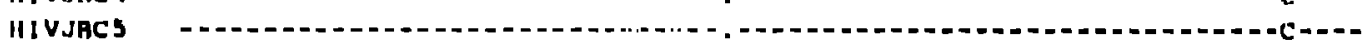

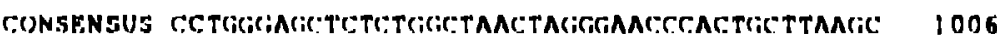

I.AI

IIV.JHNC:\| -

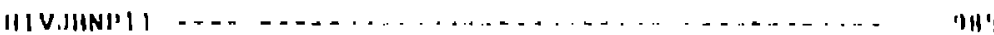

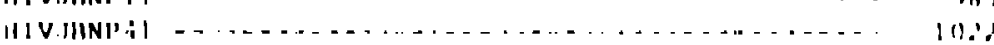

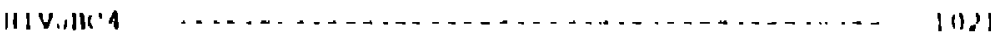

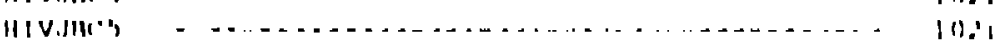


HIVBAL

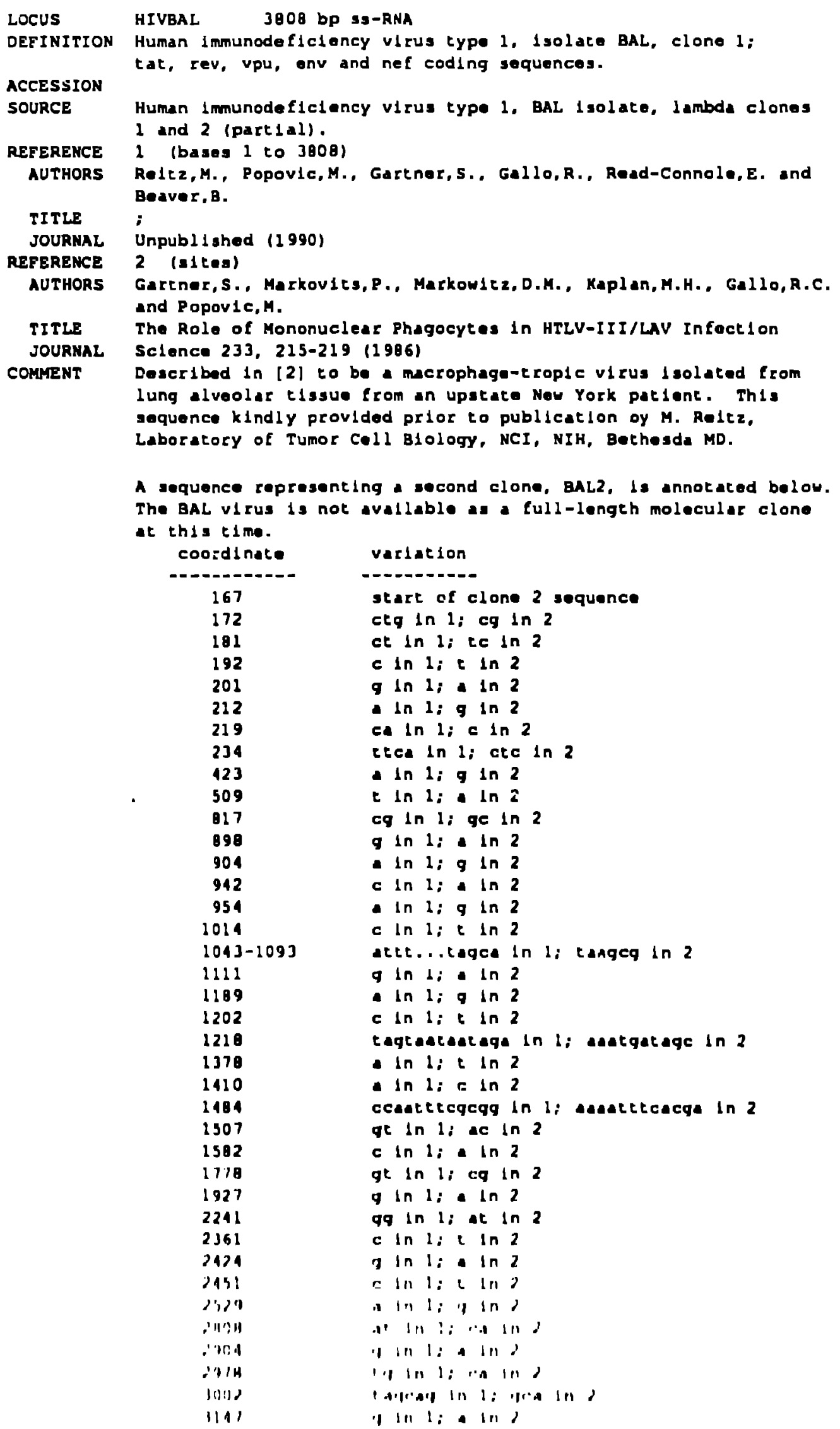




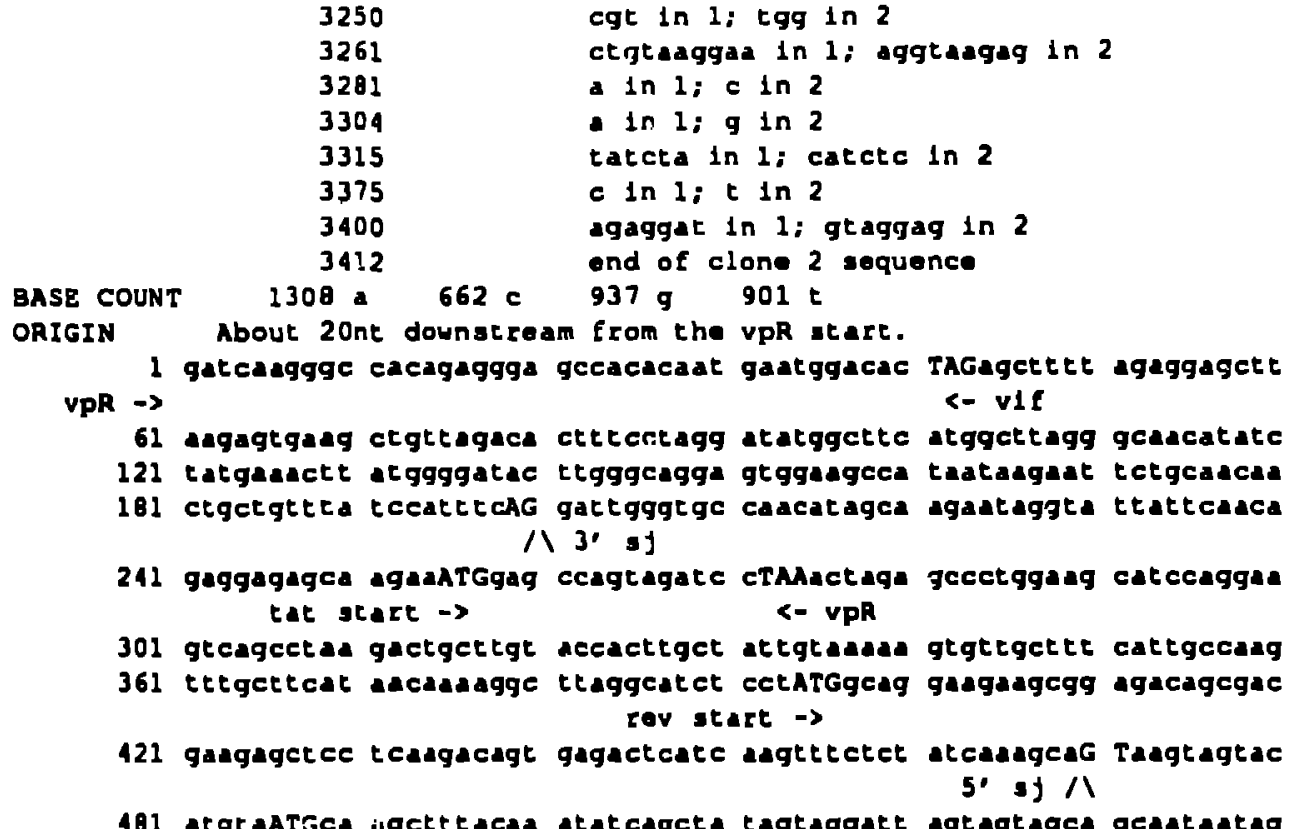

481 atgtaATGCa igctteacas atatcagcta tagtaggatt agtagtagca geastaatag vpU start $\rightarrow$

541 catagttgt gtggaccata gtattcatag astatagga antattagg cauagaasa

601 tagucaggt attggataga atadeagasa gageagaaga cagtggeaAl Gagagtgacg onv start $\rightarrow$

661 gagatcagga agagttatca geactggtgg agatggggca ceatgctcet tgggatatea

721 atgatctgTA atgetgaaga aaattgtgg gtcacagtet attatggggt acctgtgtgg S- vpU

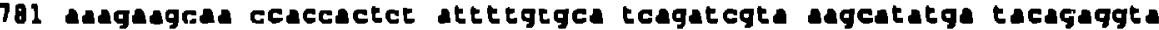

841 catartgtt gggccacaca tgcetgtgta cccucagace ceascceca agastagas

901 tryauaAatg tgacagada tettaucatg tggaaAata acatggtaga acauatgcat

961 gaggatatad tcagtteatg ggatcadajc ctasagccat gtgtaaate aucccacte

1021 tgtgttact taatlgcac egattega'jg astgctacta atgggadga cactaatacc

1001 ectagtagta geaggggat ggtgggggya qgagasatga anattgete tetcastate

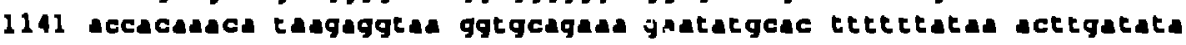

1201 gcaccatag atautatag taatautaga tatag7ttga taagtegta cacctcagtc

1261 attacacagg ectgtecana ggtatcette gagceattc ceatacatta tegtgceceg

1321 getggttetg eqattctada gtgtaAsat adast tca atgqaasagg accatgtaca

1381 atglcagca cagtacentg tacacatgga attaggceag tagtatcaec tcastgetg

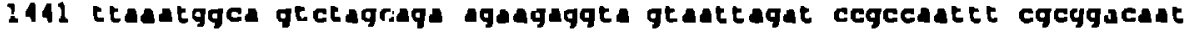

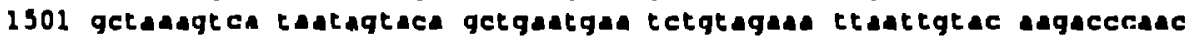

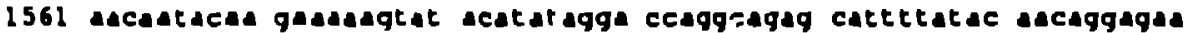

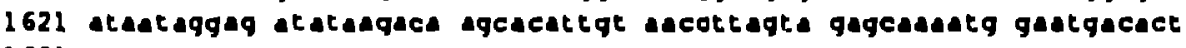

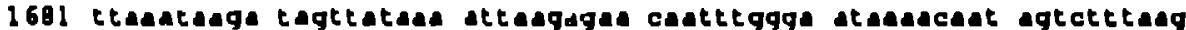

$1 / 41$ cactcctcag gagqggacec agaeattgtq acgcacagtt teattgtgg aggggatte

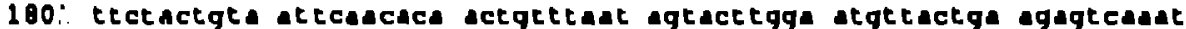

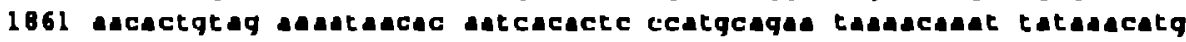

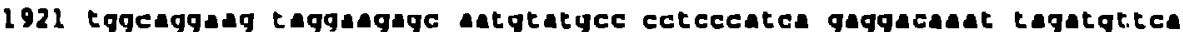

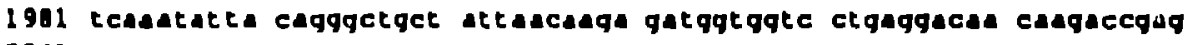

2041 gtctecaguc et qgaggagg agalatgayg gateattqge gasgtgeatt atateselat

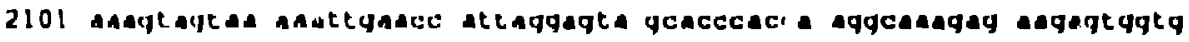

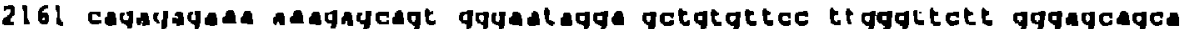

22.21 qqanycarta tgq7eycarjc gycantigacq ot qacgqeac aygecagact attategtet

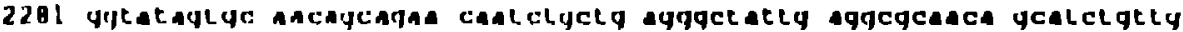

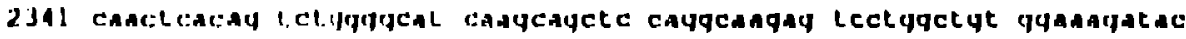

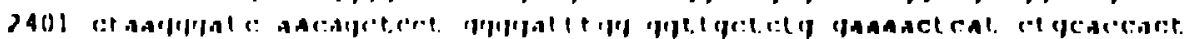

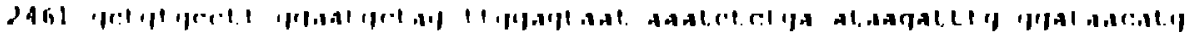

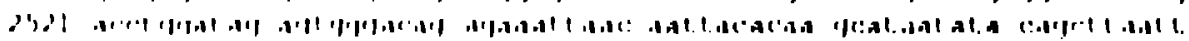

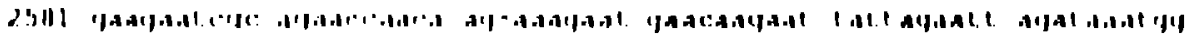

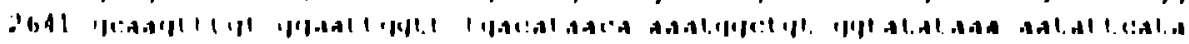

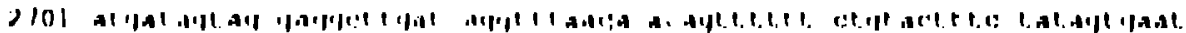

I.A-93

MAY 9! 


\section{HIVBAL}

2761 agagttaggc agggatactc accattatcg tttcagacce accteccatc etcgagggga 八 $3 \cdot 35$

2821 cccgacaggc ceggaggaat cgaagaagaa ggtggagaga gagacagaga cagatccggt 2881 ccatTAGtga acggattct ggcgettatc tgggtcgatc tgcggagcet gtecctettc <- $t$ at

2941 agctaccacc gettgagaga cttactcttg attgtgatga ggattgtgga acttctggga 3001 ctagcagggg ggtgggadgt cctcadatat tggtggadc tcctacagta ttggagtcag 3061 gadetadaga aTAGtgctgt =agcttgctc aatgccacag ctgtagcagt agctgaagg <- rev

3121 acagataggg ttatagaagt attacagaga gctgetagag ctattctcca catacctaga 3181 agateagac agggctegga aaggettrg ctatAAgATG ggtggcaagt ggtcaaaag nef

$$
\text { <- onv }
$$

3241 tagtatagtc gtatggcetg ctgtaaggaa agaatgaga agaactgagc cagcagcaga 3301 tggagtagga gcagtatcta gagacctiga daacatgga geatcaca gtagcaatac 3361 agcagctaac aatgctgate gtgcetggct agasgcaca gaggatgaag aagtgggttt 3421 tccagtcaga cetcaggtac ctetaagace altgactcge agtgcagcta tagatctag 3481 ccactetete aagaaaagg ggggactgga agggetadt cacteccada adagacaga 3'LTR start $\rightarrow$

3541 tatectggat tegtgggtct dccacacaca aggetacttc cctgattggc agadetacac 3601 accagggcea gggaccagat ttccactgac cttgggatgg tgcttcaagc tagtaclagt 3661 tgagceagag aaggeagaag aggccaitga aggagagad adctgettgt cacaccetat 3721 gagectgcat gggatggatg acceggagaa agaagtgtta gcatggaagt ttgacagcag 3781 cctagcattc catcacgtgg cccgagad

$$
\text { nef } \rightarrow
$$


LOCUS HIVADA 2562 bP SA-RNA

DEEINITION Human immunodeficiency virug type 1 monocyte-replicative 1solate ADA, complote env cos.

ACCESSION M60472

SOURCE Human 1mmunodeficiency virus eype 1, SacI/SacI molecular clone from the ADA isolate.

REFERENCE 1 (bases 1 to 2562)

AUTHORS Westervelt,P.. Gendelman, H.E. And Ratner, L.

TITLE Identification of a determinant within the HIV-l surface envelope glycoprotein critical for productive infection of primary menocytes journal Proc. Natl. Acad. Se1. U.S.A. 88, 3097-3101 (1991)

COMYENT Hardcopy of sequence in [1] kindly providod by Ratner, 10-FEB-1989. studies reported in [1], using chimerie molecular clones with the ADA viral fragment, suggest that the HIV-1 onv V3 region contains determinants for productive infection of primary monocytes.

BASE COUNT 093 a 425 c $604 \mathrm{~g} 640 \mathrm{t}$

ORIGIN Env cds start. env siart $\rightarrow$

1 ATGaagtga aggggatcag gaagaatiat cagcactigt ggaatgggg catcatgete

61 cttgggatgt tgatgatctg TAGtgetgta gaaatetgt gggtcacagt tcattatggg $<-$ vpu

121 gtacetgtgt ggaagaage auccaccact ctattetgtg catcagatge taasgeatat 181 gatacagagg tacatatgt ttgggceaca catgectgtg tacccacaga ceceauccea 201 caagaegtag tattggaaa tgtgacagad autttaaca tgtggaaad teacatggta 301 gaacagatgc atgaggatat aatcagtte tgggatcaad gcctaadgce atgtgtaad 361 ttascecac tetgtgttac ttraattgc actgatttga ggastgtic tautatcaut 421 aatagtagtg agggaugag aggagaate aaaactget ctttcatat caccacage 481 atagagata aggtgaagas agactatgea ctttettata gactggatgr agtaccata 541 gatargate atactagct taggttgata attgtata cetcaccat tacacaggec

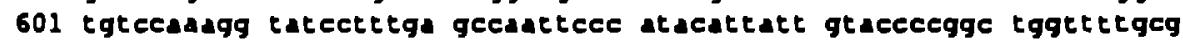
661 attchaagt gtaagacad gaagttcaut ggaacagggc catgtadaa tgtcagcaca 721 gtacaugta cacatggaat taggccagta gtgtcactc auctgctgtt autggeagt 78: etageagaag augaggtagt astagatet agtauttea cagacadge auadacata B41 atagtacagt tgaagaute tgtagaatt autgtaca gacecacas cautacaagg 901 a inagtatac atataggace aggaagagca ttttatacas caggagaat adaggagat

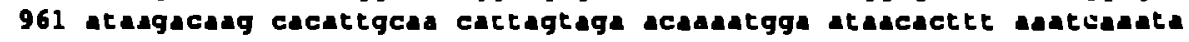

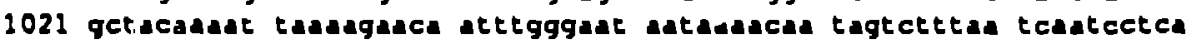
1081 ggaggggace cagadattgt autgcacagt tttaattgtg gaggggast tttetactgt

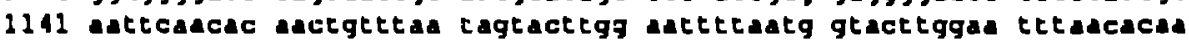
1201 tegaatggta etgadggada tgecactate acactcceat gtagaatada acaanteata 1261 adatgtgge aggaagtagg adagchatg tatgcecte ccatcagagg acauatcaga 1321 tgetcatcas atattacagg getaatatta acaagagatg gtggadcta cagtagtggg 1381 tecgagatct tcagacetgg gggaggagat atgagggaca atrggagaag tgatetatat

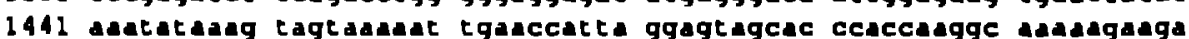
1501 gtggtgcaga gagaasaag agcagtrgga acgetaggag ctatgttect tgggtectg 1561 ggagcagcag gaagcactat gggcgcagcg tcautacge tgacggtaca ggecagacta 1621 ttattgtetg gtategtgca acagcagace atttjetgu gggctattga gqcgcases 1681 catitgttge adetcacagt ctggggcatc ageagctec aggeagagt cetggetetg 1741 gaasgatsce tasgggatca acagctceta gggatteggg gttgctctgg ausuctere 1801 tgcaccactg ctgtgecteg gaatgetagt tggagtaata auctctgga tatgattegg 1861 gatadetga cetgqutgga gtgggaaga gadatcgaa attacacagg cttadatac

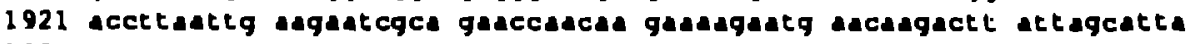
1981 gataagtggg casgtttgtg gastggtte gacatatca attggetgtg gtatataua 2041 atcticata tgatagtagg aggcttgata ggtteagaa tagttettac tgtactetct 2101 atagtaate gagtaggce gggatactca ccattgtcat ticAGaccea cetcceagec 八了's]

2161 ecquggggac cegacaqgec cquaggaste gasgaqaag gtggagacaq agacagaque 2221 dqatcegtge gat TAGtgga tggattctle gedettelet gqgacgacet geggagectq <- tat

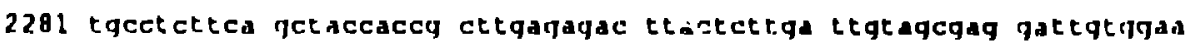

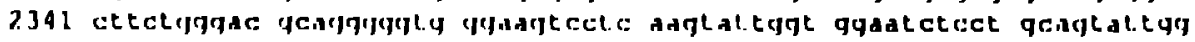

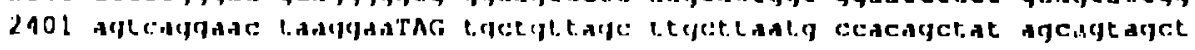
$\therefore \operatorname{rov}$

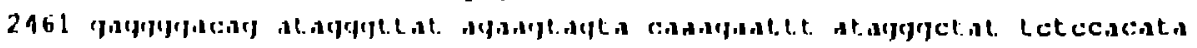

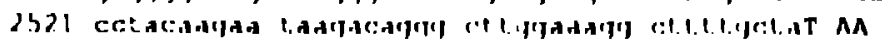

$$
\text { c- onv }
$$




\section{HIVSWB40}

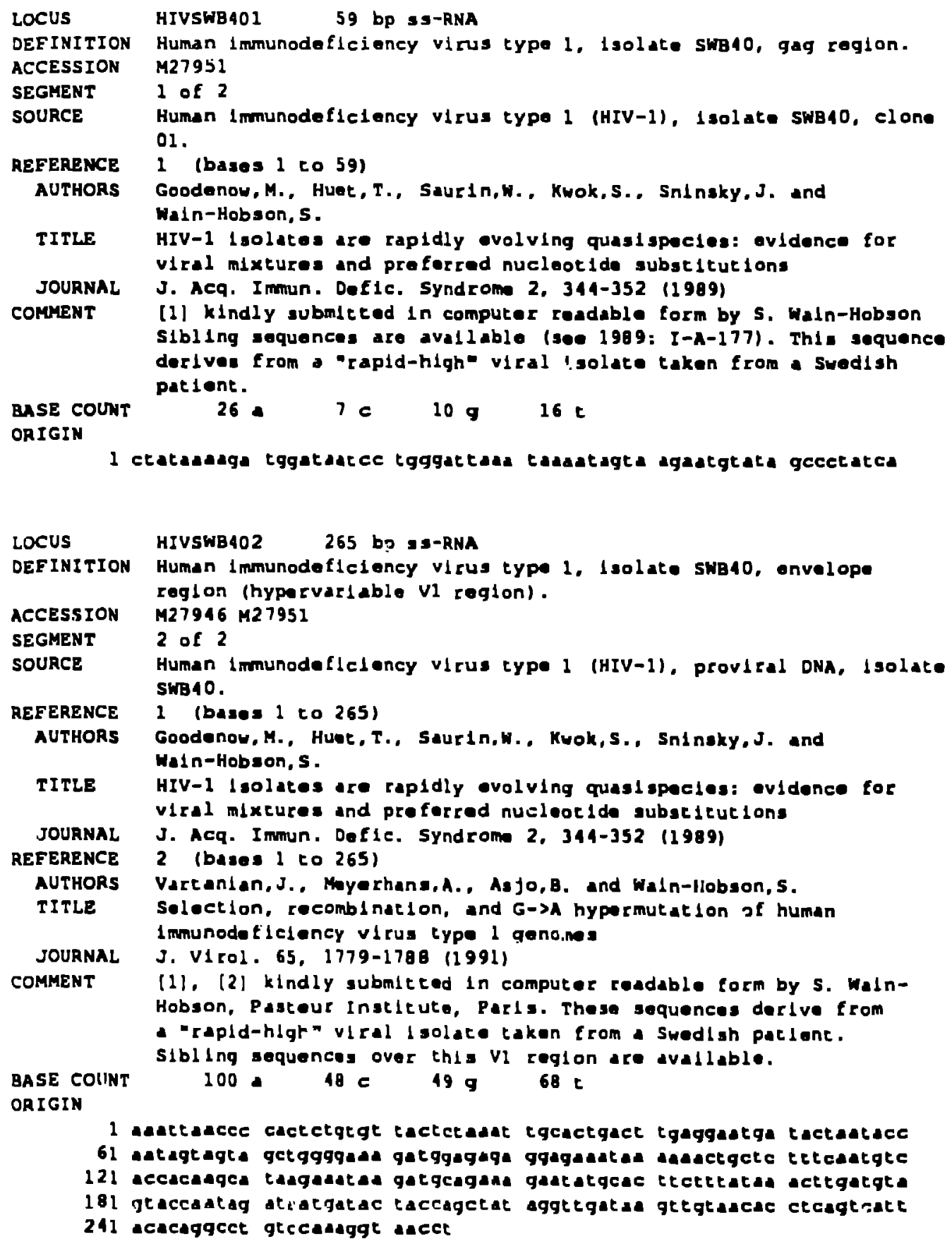




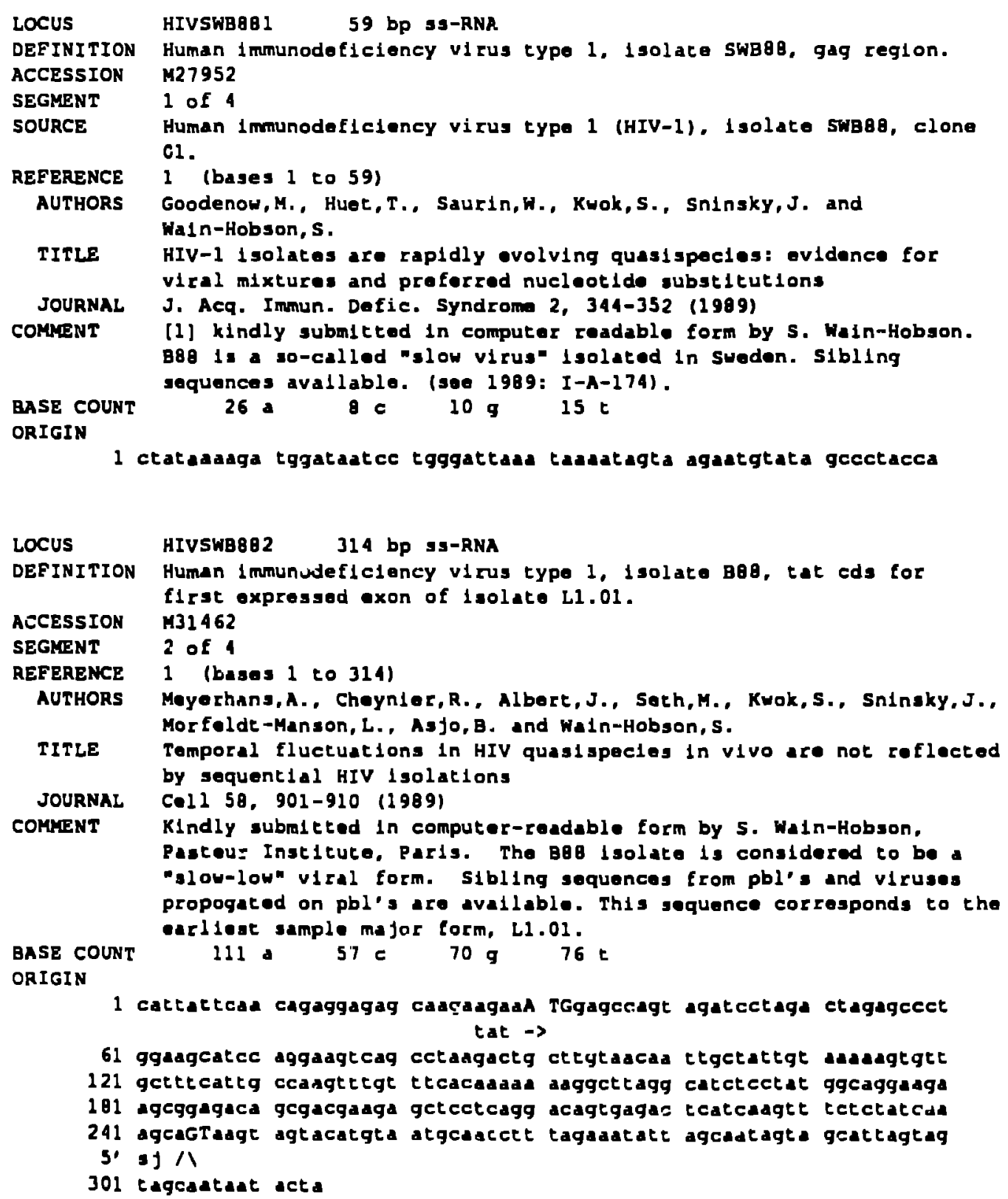


HIVSWB883

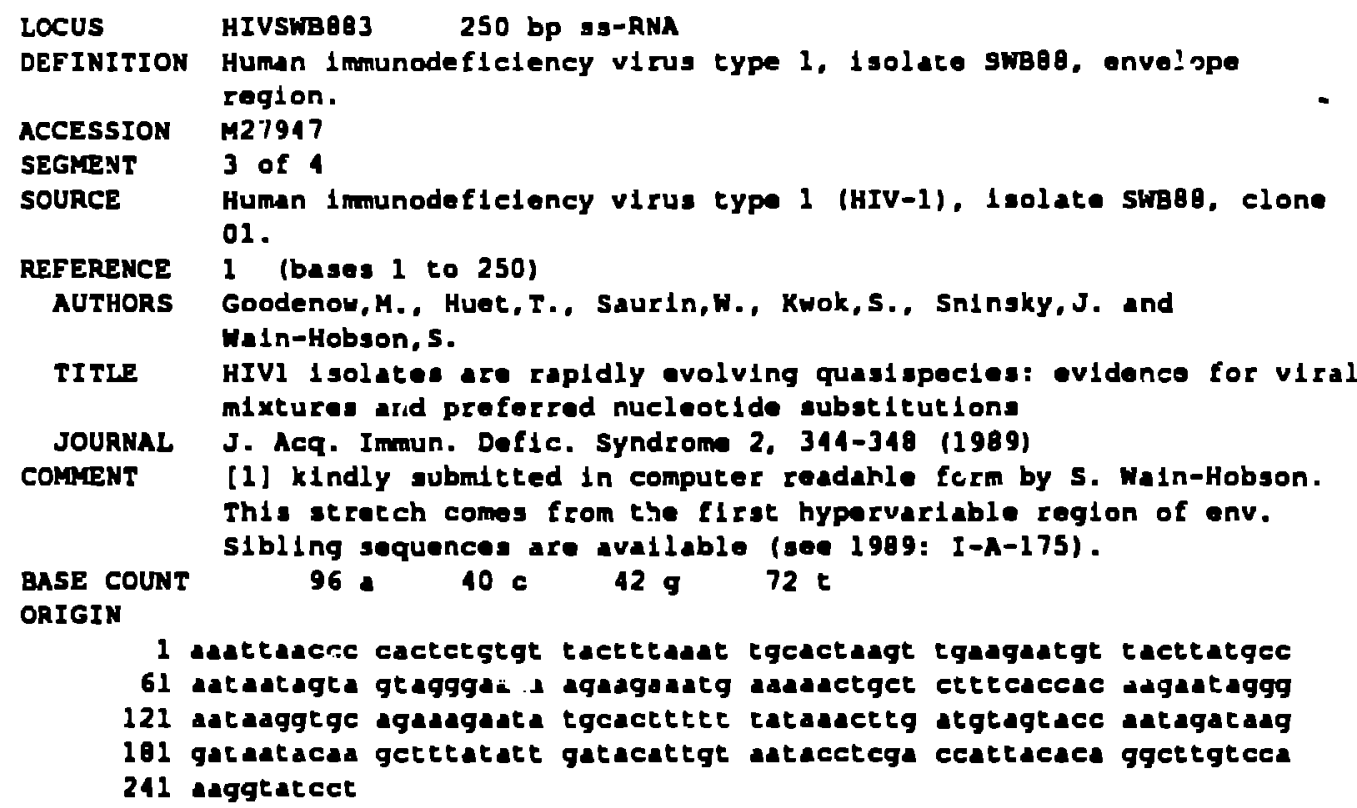




\footnotetext{
LOCUS HIVSNB884 851 bP SS-RNA

DEFINITION Human dmmunodefielency virus type 1, isolate swB8g, nef,ltr reglons. ( nef cds for lyolate Ll.14).

ACCESSION M58206

SEGMENT 4 of 4

REFERENCE 1 (bases 1 to 051)

AUTHORS Delassus, S., Cheynler, R. and Wain-Hobson, S.

TITLE Evolution of human 1mmunodeflclency virus type 1 nef and long

cerminal repeat aequences over 4 years in vivo and in vitro

JOURNAL J. V1rol, 65, 225-231 (1990)

corager [1] kindly submitted in computer readable form by s. Wain-Hobson, Parteur Inatitute, Parla. Sibling sequences of this laolate

(L1.14) are avallable.

BASE COUNT 237 a $186 \mathrm{C} \quad 251 \mathrm{~g} \quad 177 \mathrm{C}$

ORIGIN

I ATGggtggca agtggtcad agtagkatg agtggatgge ctgctataag ggaagaatg nef atart $\rightarrow$

61 aacagetg agecageage agatggggtg ggagcagtat ctcgagacet ggaaaacat

121 ggagcautca cadgagcaa cacagcagct actartgetg attgtgcetg getagaagea

181 caagaggagg aagaggtggg ttttccagte agacetcagg tacctteag accautgact

241 cgcagggcag ctatagatce tagccactet ttagagaa aggggggact ggagggcta 3'LTR $\rightarrow$

30.6 gttcactcec auaagaca agagatcett gatetgtggg tetaccacac acasggetac

361 ttccetgatt ggcagaucta cacaccaggg ecagggace gatggeract gacecetgga

421 tggtgeteca agctagtace agtcgageca gagaaggtag augaggcead tgaggagag

481 adeacaget tqteacacce tatgagcetg catggatgg aggacecgga gaagaagtg

541 teagtgtgga agtttgacag eagectagca ttlcaccaca aggeccgaga gctgcatceg

601 gagtatedea agactgetG Acaccgagte tectacgagg gactetccge tggggactet

S- net

661 ccagggange geggectggg eqggactggg gagtggegag cecteagatg ctgcatArA algnal $\rightarrow$

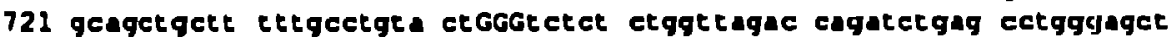
$R$ seart $\rightarrow$

781 etctggetaq ctagggace eactgctta gectedarA Agctggect gagtgcteca

841 agtagrgtgt 9

signal $\rightarrow$
} 


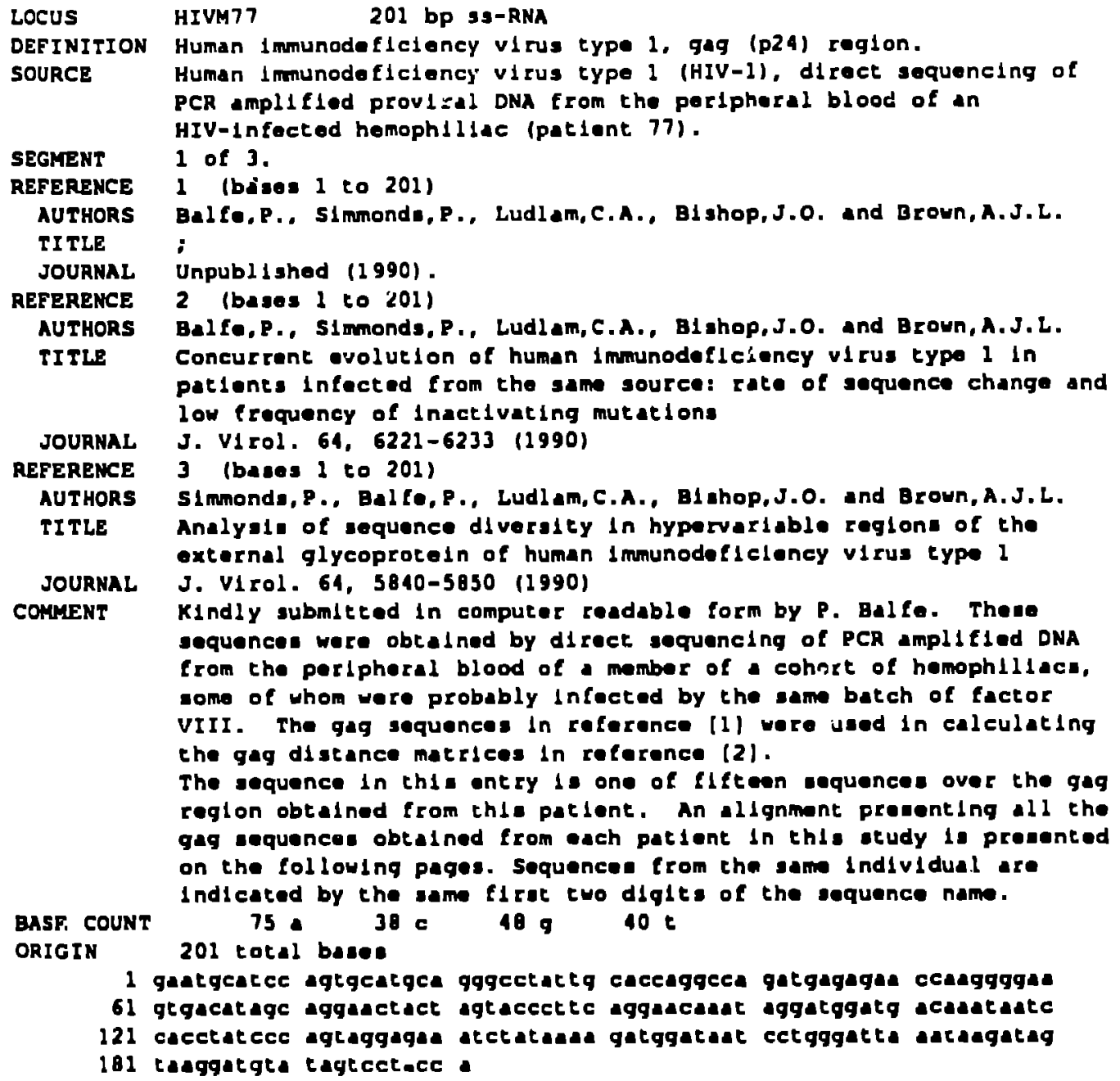

1 gaatgcatce agtgeatgea gggectattg caceaggeea gatgagagan ccasgggas

61 gtgacatage aggauctact agtacecte aggaucaut aggatggatg acasatate

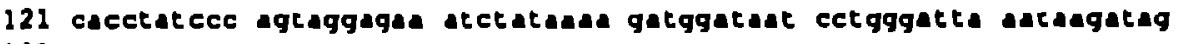

181 tasggatgta tagtcctace a 


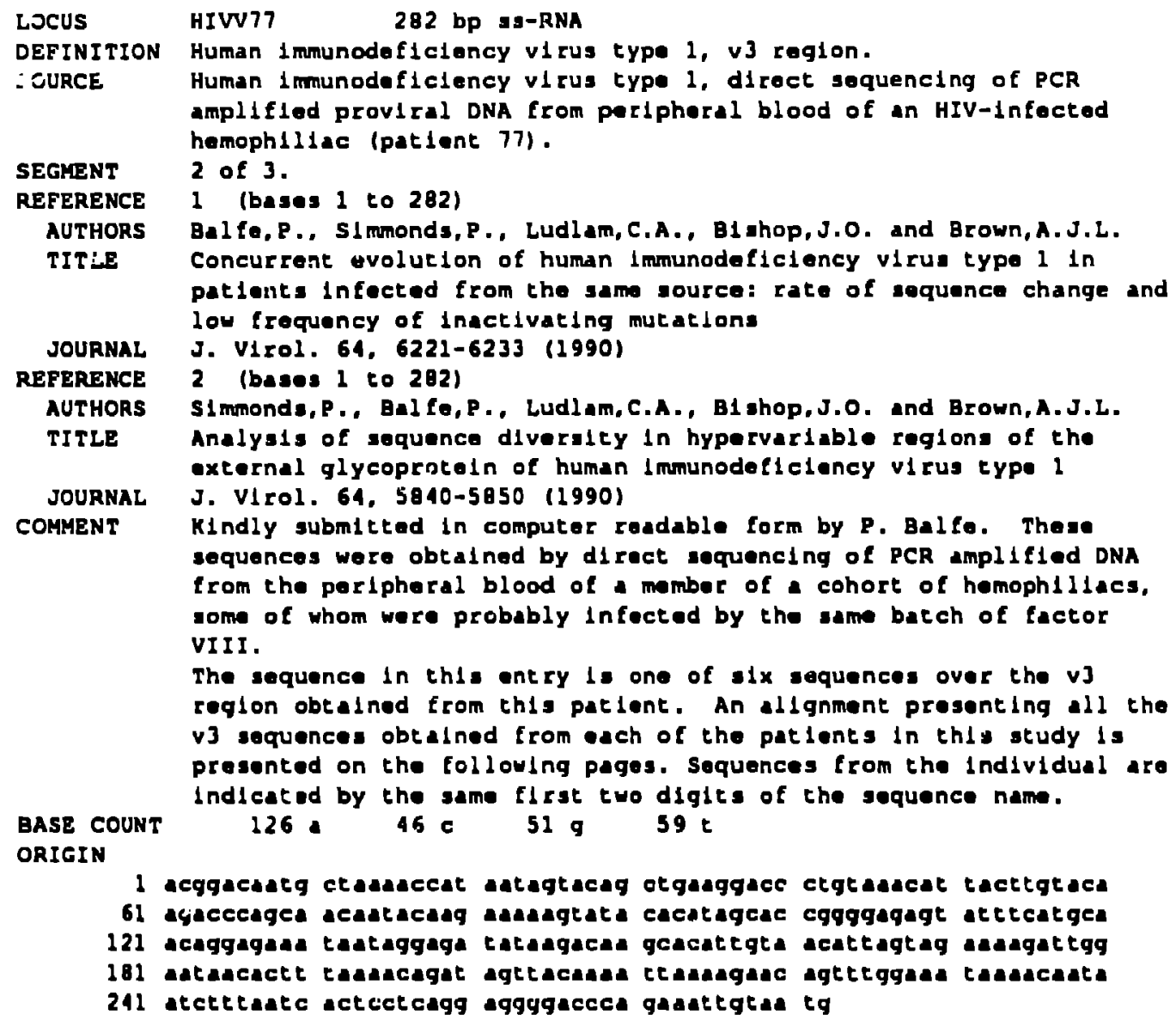

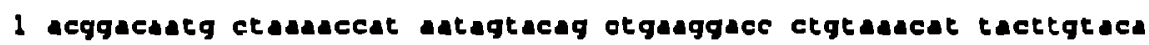

61 agacceagea acautacas auadagtata cacatagcac cggggagagt attcatgea

121 acaggagaa taataggaga tatagaca gcacategta acattagtag arasattgg

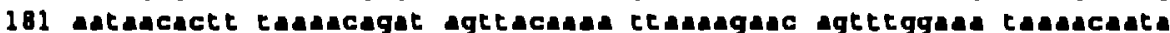

241 atctetare actectcagg aggggaccea gaattgtas tg 


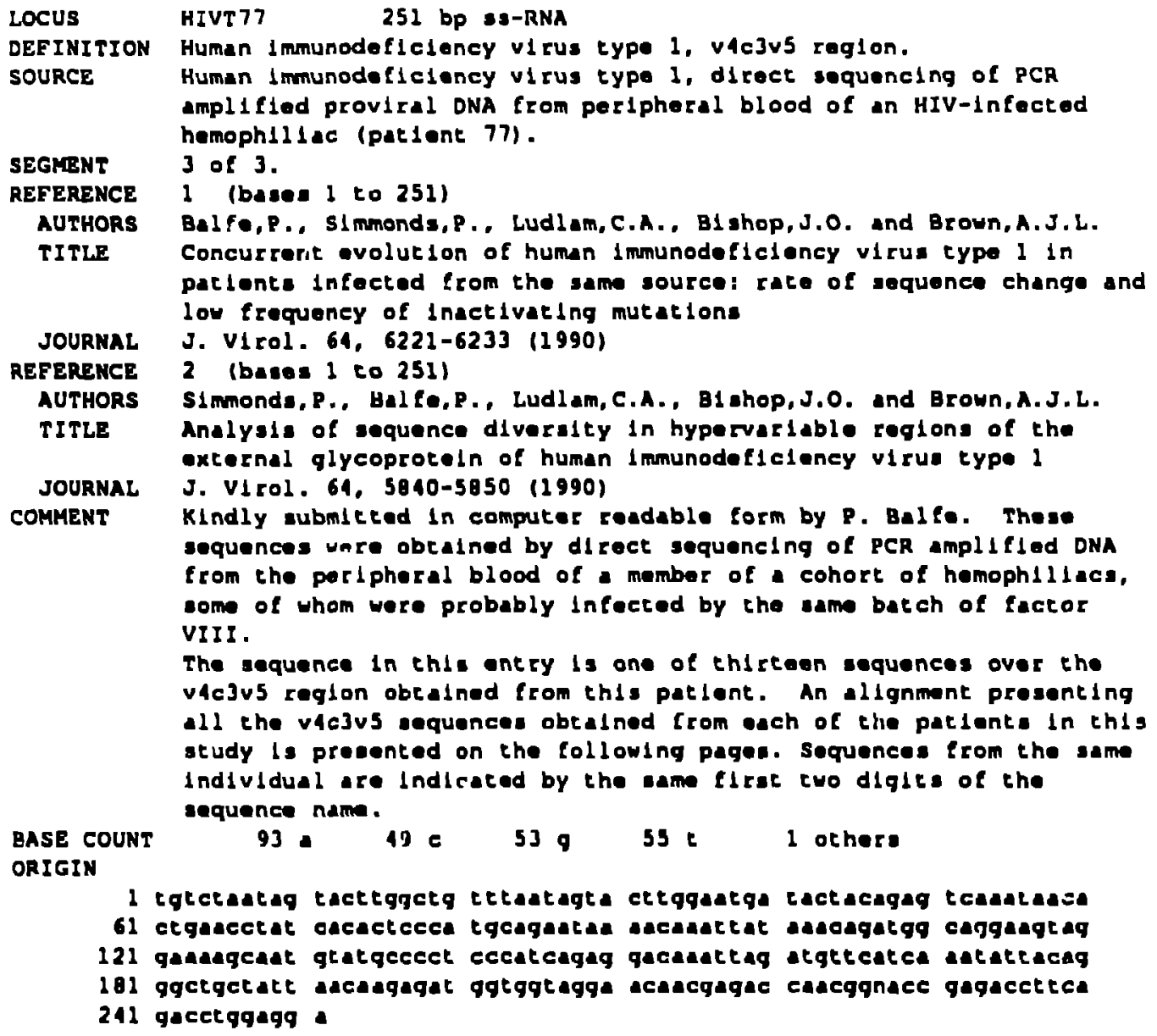




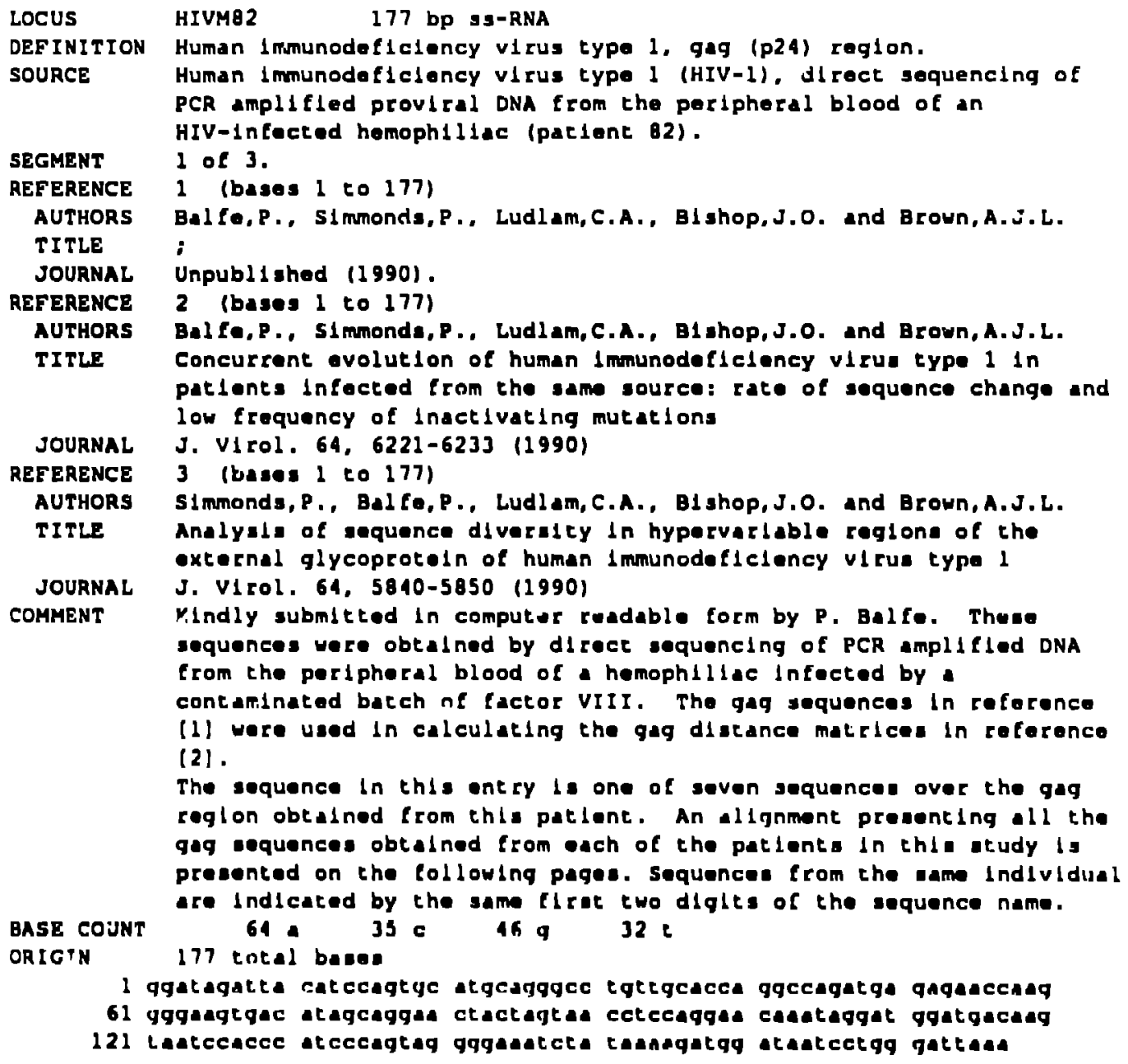

1 qgatagatta catccagtge atgeagggce tqtegcacca gqceagatga qagaecauq

61 gqgagtgac atagcagga ctactagta cetceaggae casataggat ggatgacag

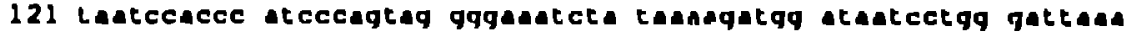




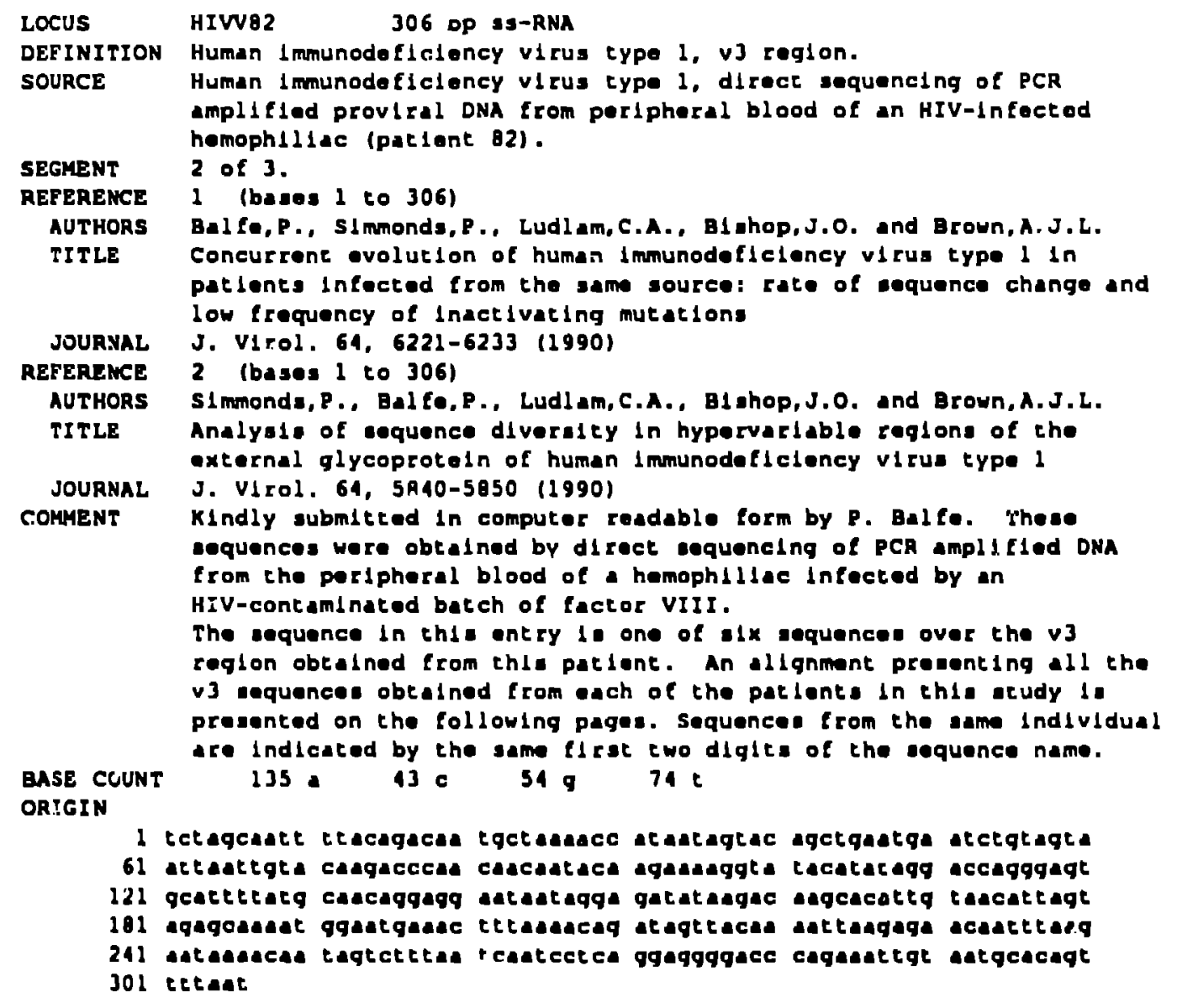




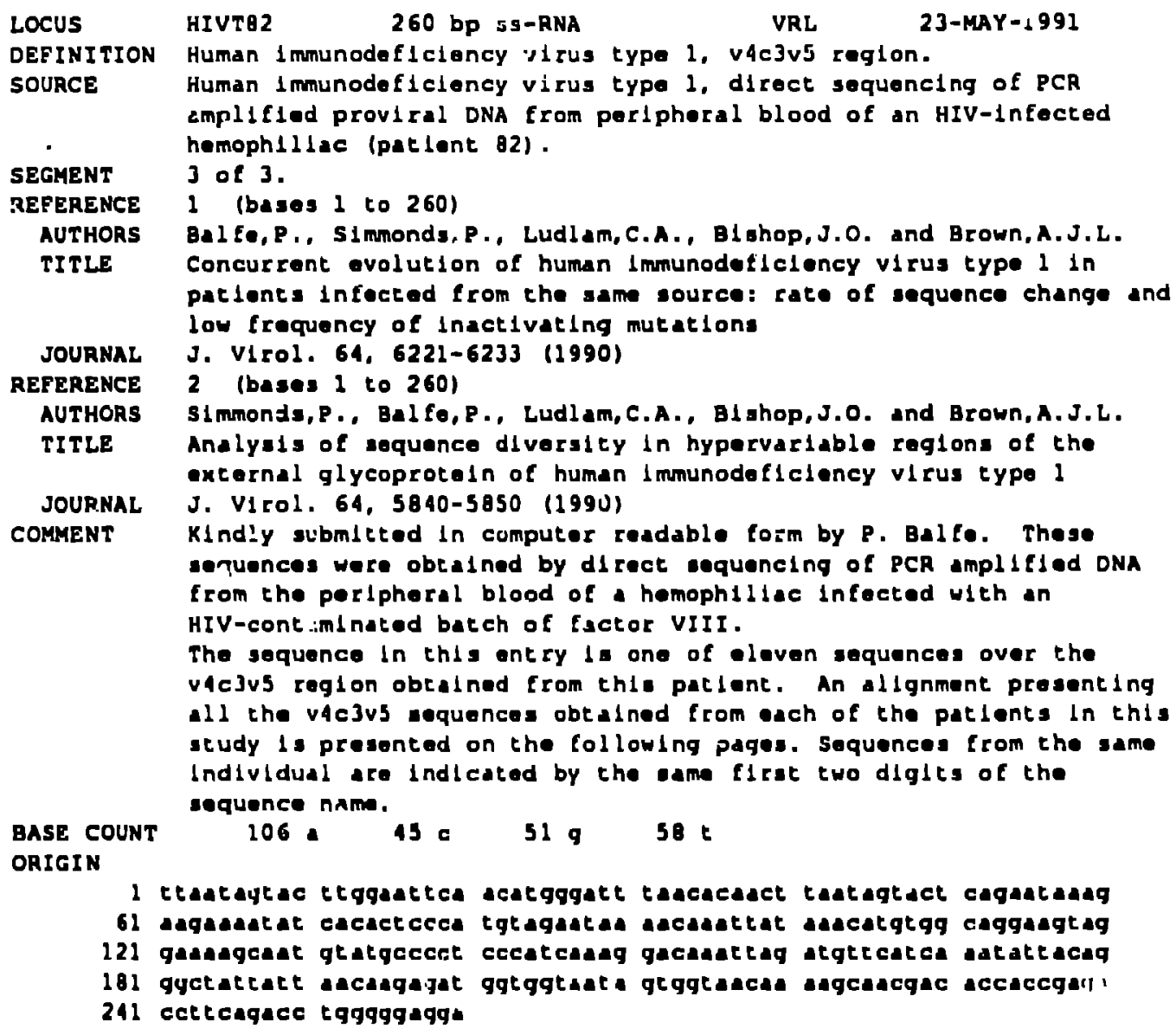


$\cos 21307$

ต71307

$\min 1392$

M7171

ตาว 172

wr:74

ตijis

ต7:76

a7ias

m7:04

ต714

פด

ต7189

ת:

mi:

ריב

7953

meste

messe

ripes61

ceszose

xu32001

4032002

me32085

432122

$3 \div \quad$ Ma3226

103227

1032378

x18234

m42351

met235e

mist

074166

m4167

nesie

mes1s

rite76

m56s5

misss

m61303

ח76230

76697

mesie

meswo

mes.2

M2597

Me2533

$M 2600$

$\operatorname{mes} 603$

M2600

พ112612

Me26:5

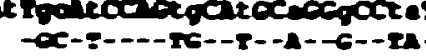

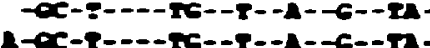
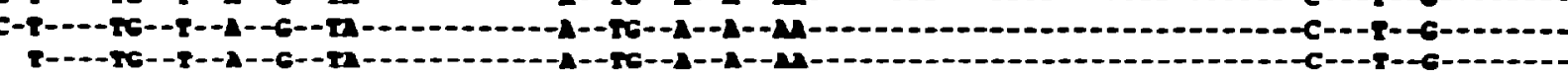

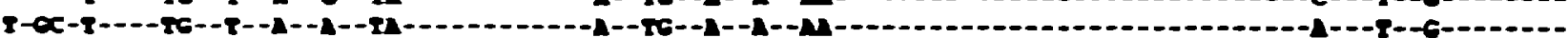

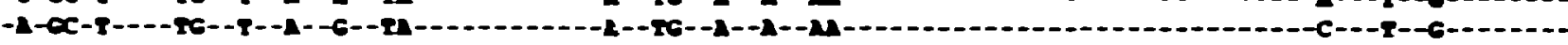

-

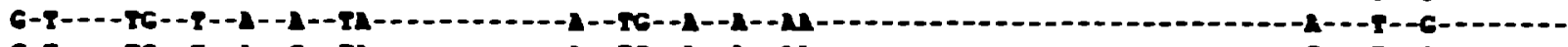

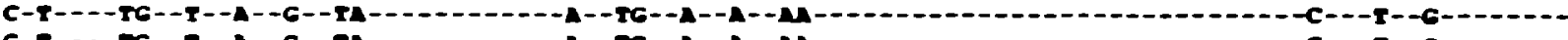

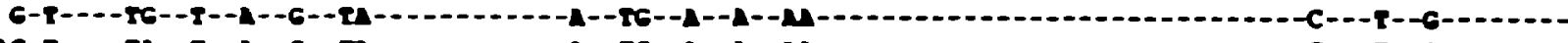

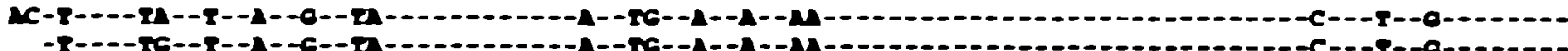

C-

0

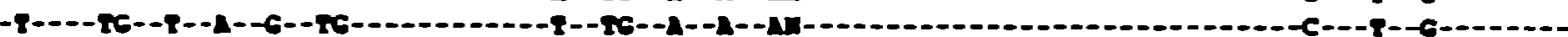

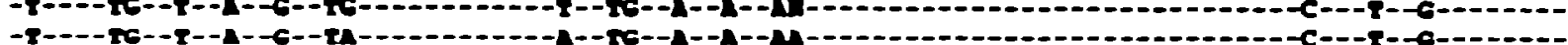

-

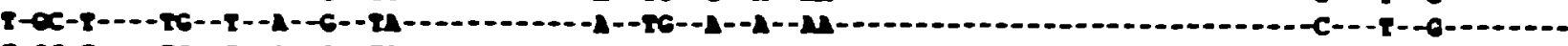

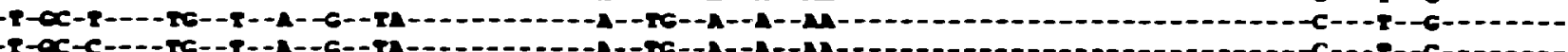

r-cer-

A-C-TA-

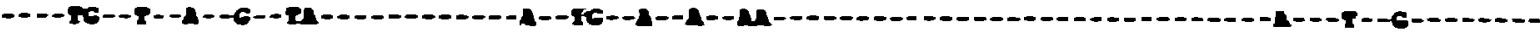

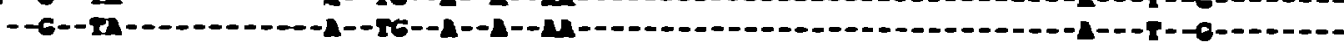

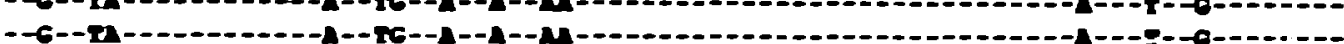

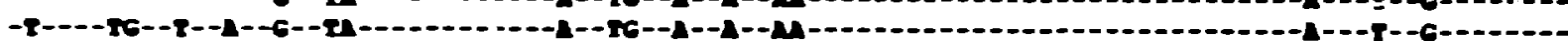

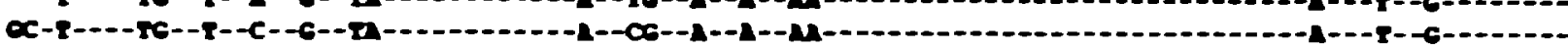

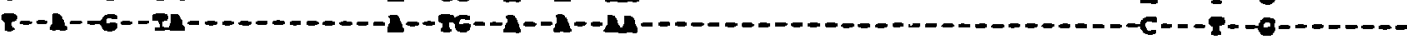

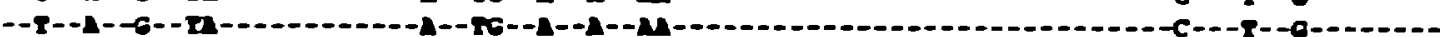

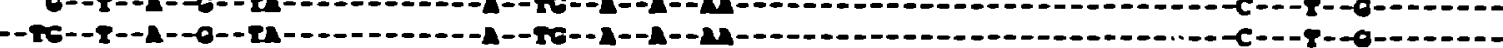

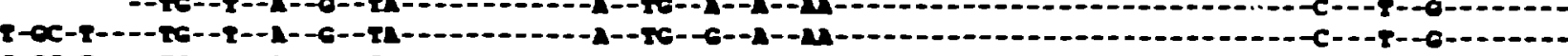

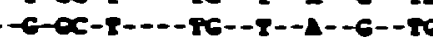

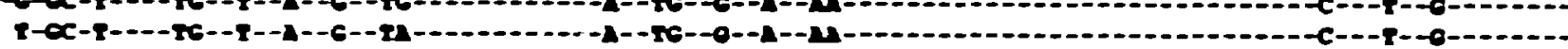
- ra-10.0.

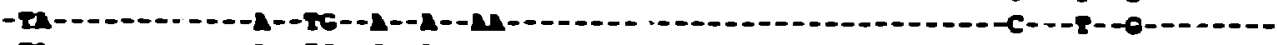
-ra-

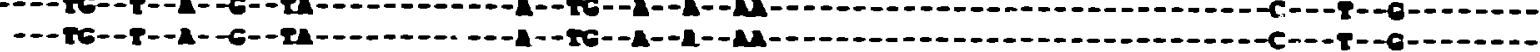
-

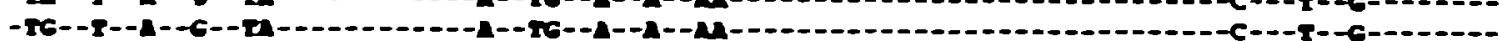

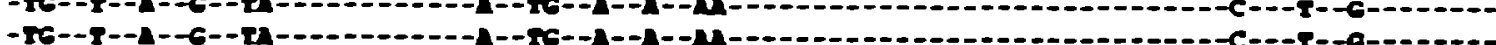

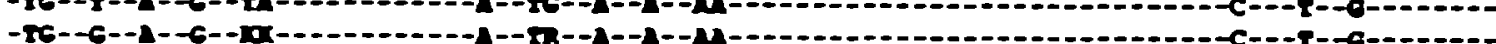

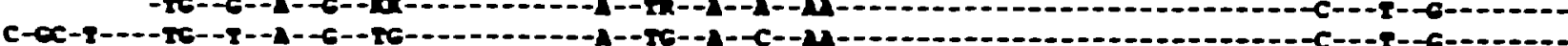

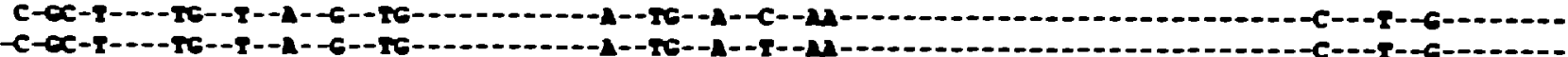

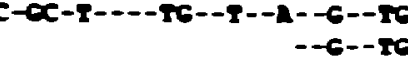
$-6-10$

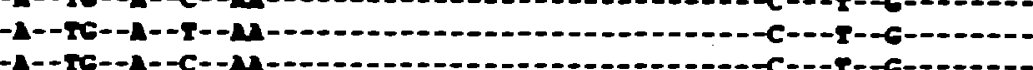
-

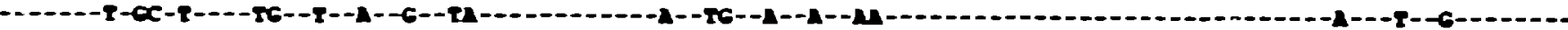

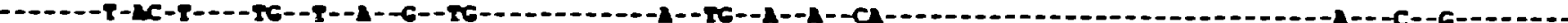

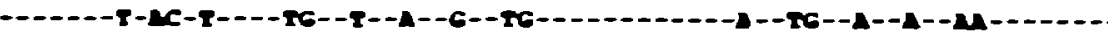
-

C- $-\mathbf{A}--6--7 \mathrm{C}$

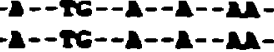

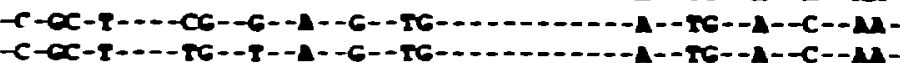




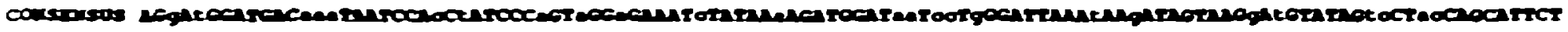

- C-

ח7172

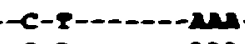

m7174

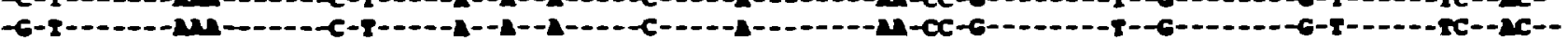

177175

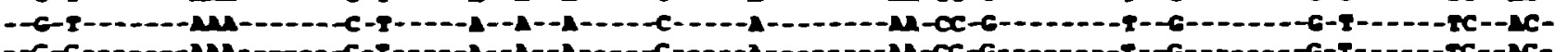

กา176

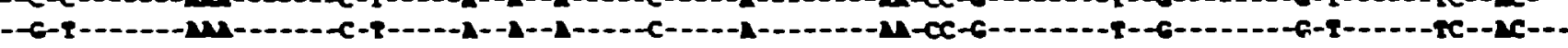

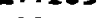
- -

ח7187

mines

mises

เกา1

1791401

I781403

ח2537

nose6

nos 61

m32020

mes2001

- C-

- C-8-

-C-

- -

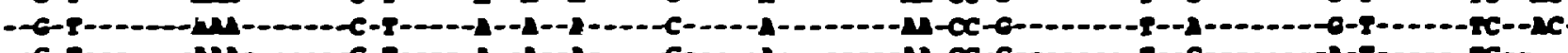

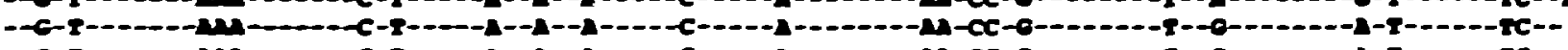

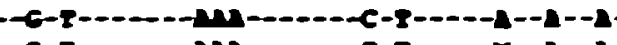

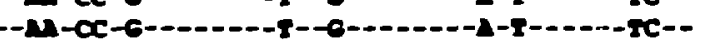

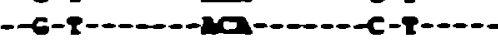

- C-5 -

4032002

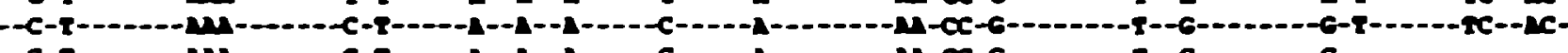

C-

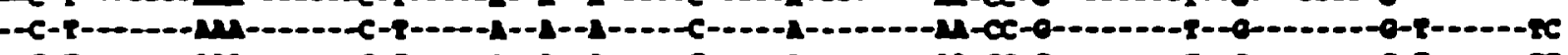

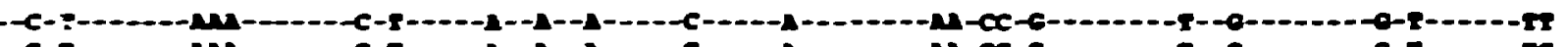

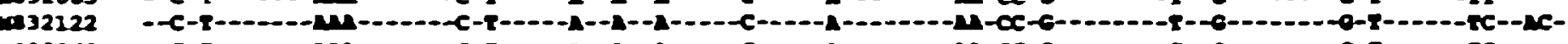

032269

$-\mathbf{C - T}$

032374

202349

1012351

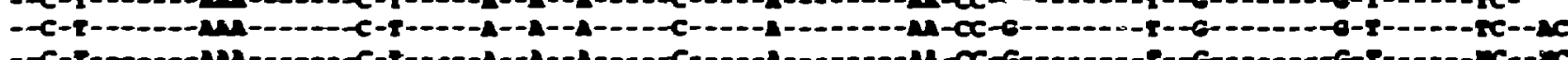

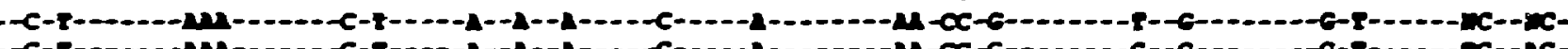

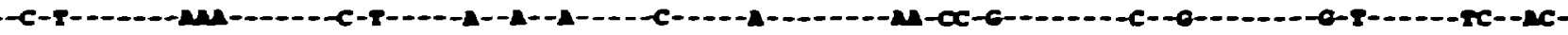

-

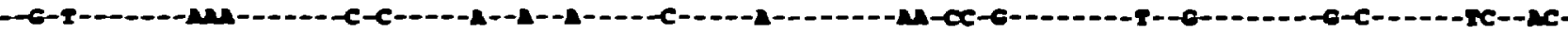

G-7....--- $1 M$

Mil6s

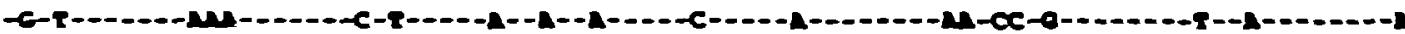

174167

mi614

me615

Me617

70676

moses

m62383

n61384

I76697

rin698

mes74

nes 90

M70592

402597

M02599

$\min 2600$

$\mathrm{M2} 2 \mathrm{CO}$

me260s

502612

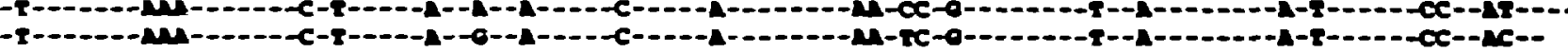

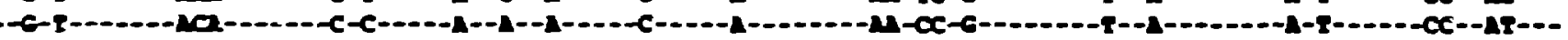

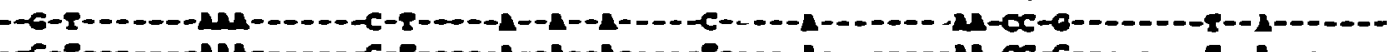

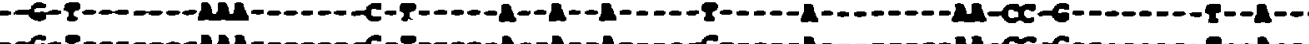

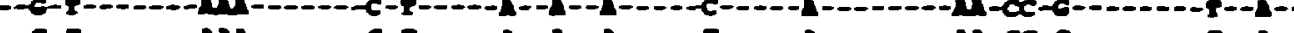

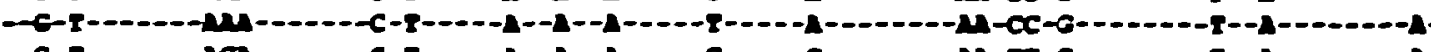

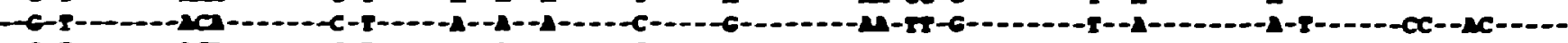

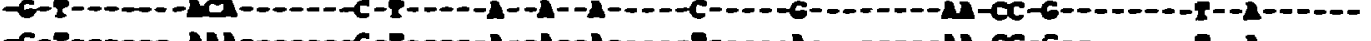

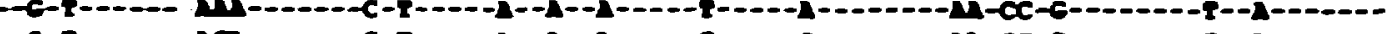

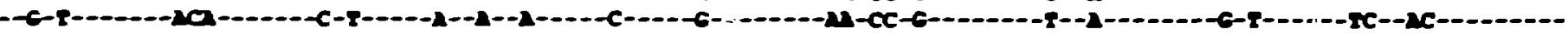

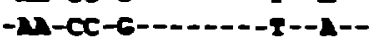

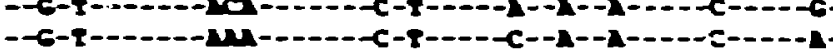

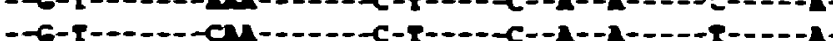

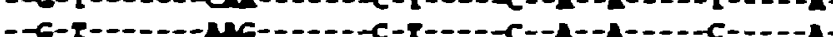

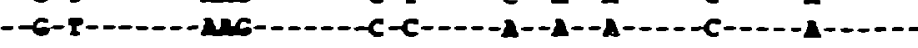

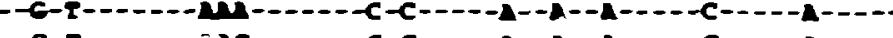

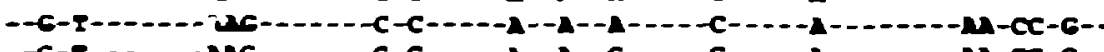

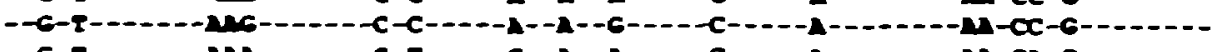

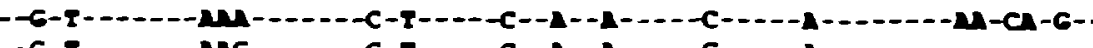

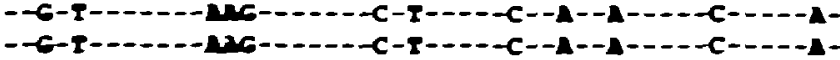


comsmers: กา171.778 จ7172.77. 77173.771 87174.77d จ7175. 770 77176.77 a 002711.20 T02711. प्र०2712.2b 02713.021 ve2716.e2g กอ2า1 VezTl. 7072420.07. V072421.078 v072422.071 7072423.07b v272424.871 v172426.7g vo72.27. $\mathrm{m}$ ve7240.10 ve72020.07\% 7672430.07 vicos.910

3 - V\$1611.910 $2 \geq$ Fo1613.910 $-8 \nabla 261.10$ 72036.120 12037.120 72010.120 nilut 74 nit6s .74b nit67.74 nil67.74 nel6s.7e 70179.74 nele2.74 702711.20 V02712.028 V02712.021 ro2714.22e ve2ni.e2d vo2710.020

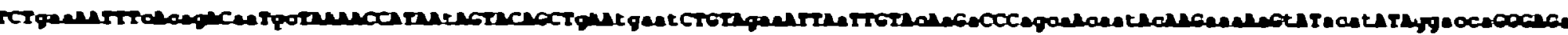

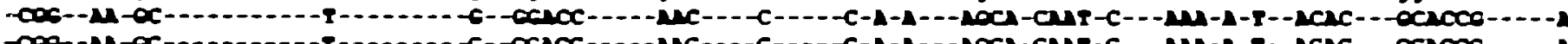

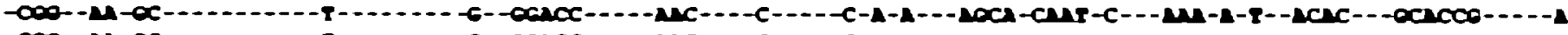

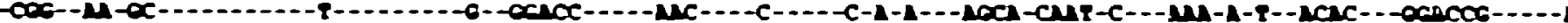

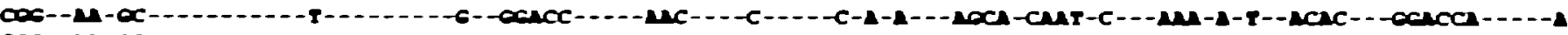

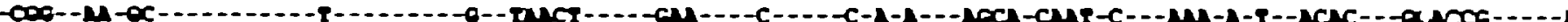
c-ac-

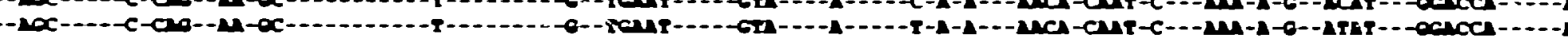

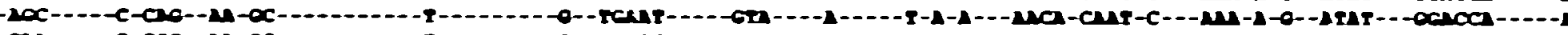
-

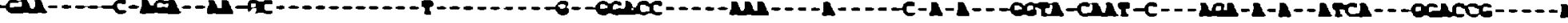

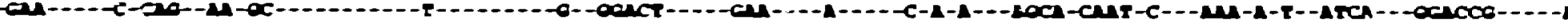

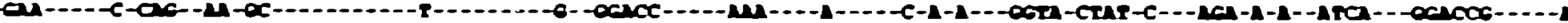

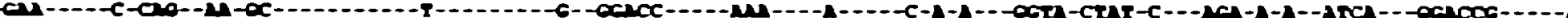

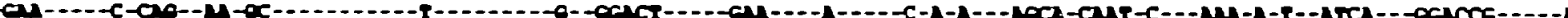
-

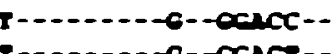




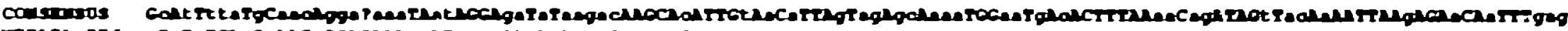

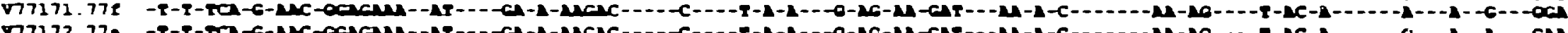

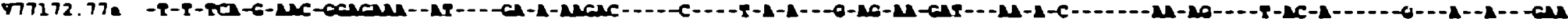
จ7173. 771 จ7174.77d 07175.77 viา176. 370 va2711. 20 va2711.02c จ०2712.02b पष2713.021 V.2714.021 ve2715.029 702717.02d vi271:.020 ve72420.7.

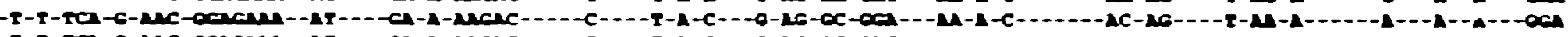

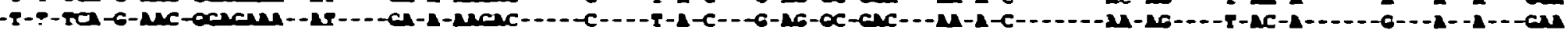

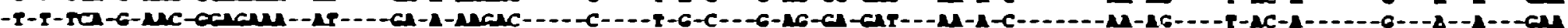

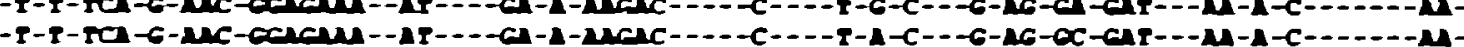

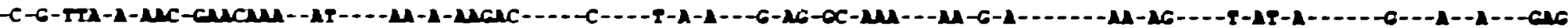

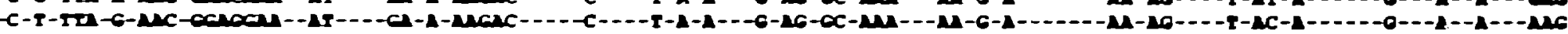

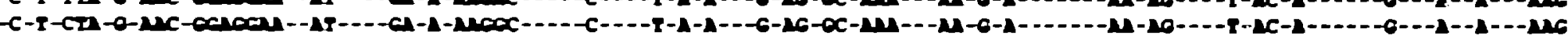

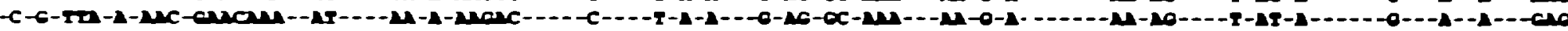

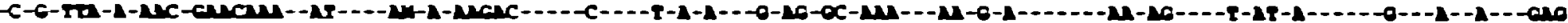

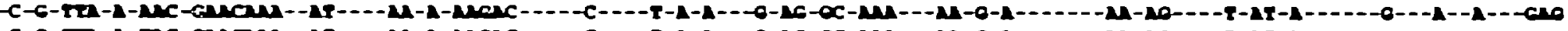

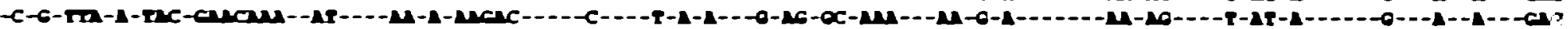

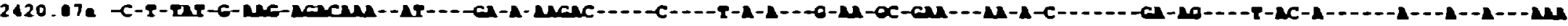

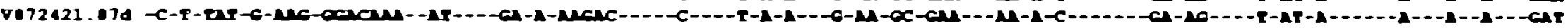

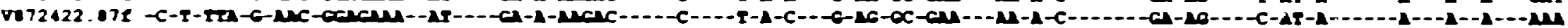

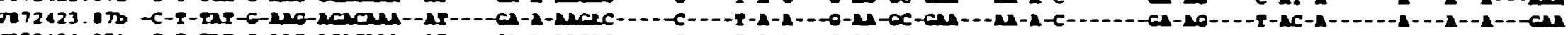

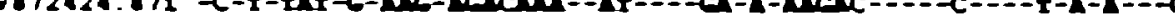

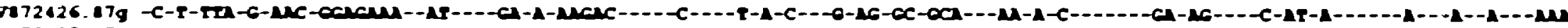

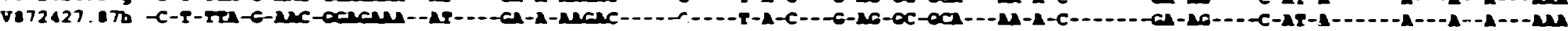

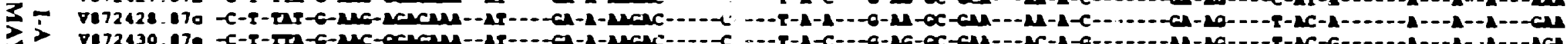

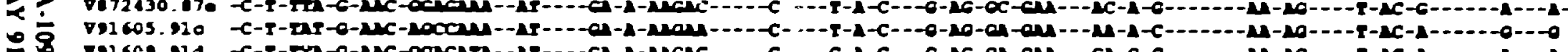

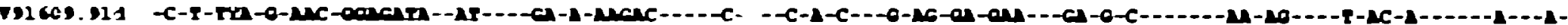

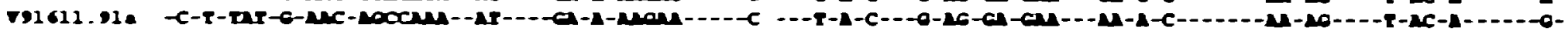

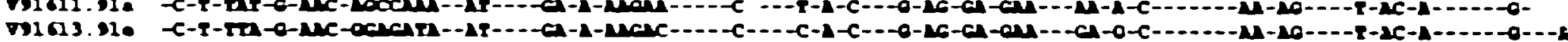

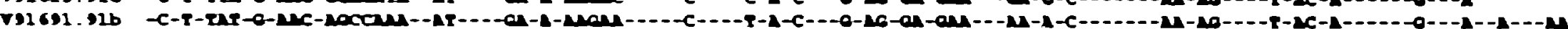

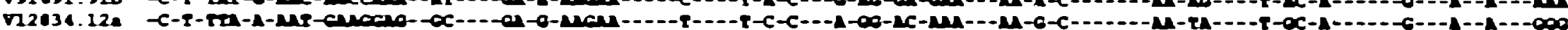

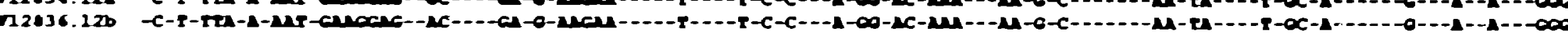

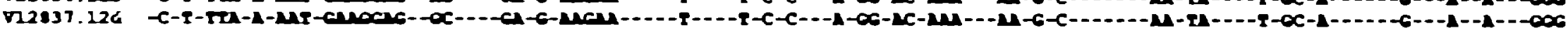

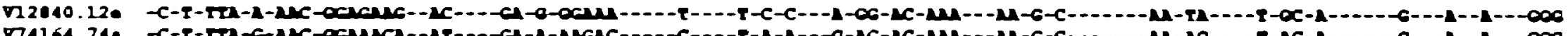

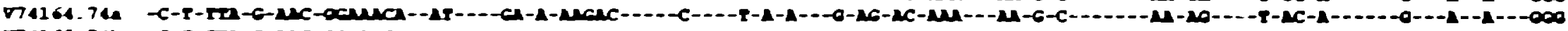

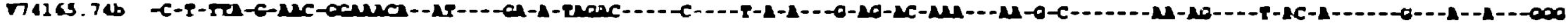

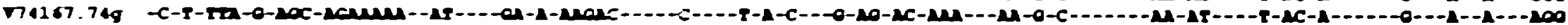

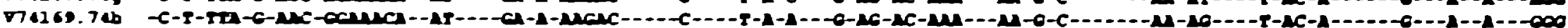

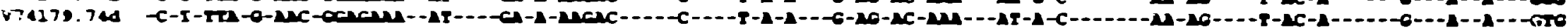

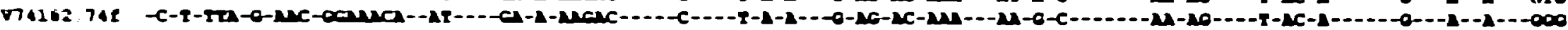

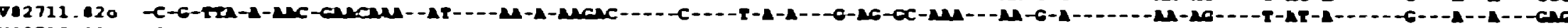

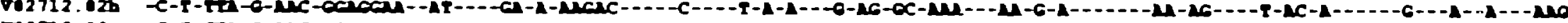

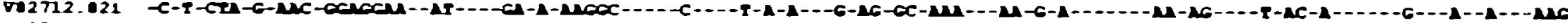

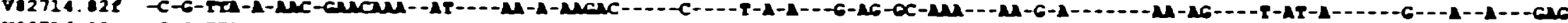

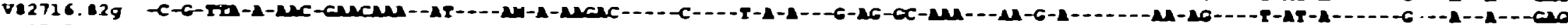

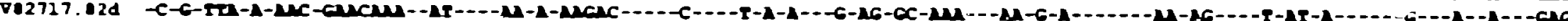

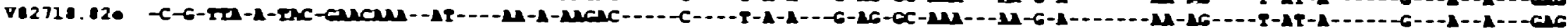




\begin{tabular}{|c|c|c|}
\hline & Mra. & \\
\hline 17171.778 & $---M M-\Delta-T-\Delta T C-D M T-M--C T--$ & 202 \\
\hline 17172.79 & 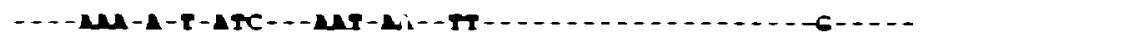 & 202 \\
\hline 7173.771 & 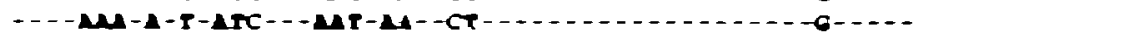 & $2 \bullet 2$ \\
\hline $1714.77 d$ & 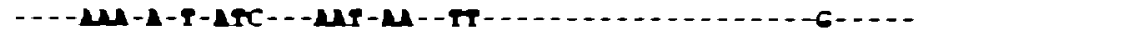 & 202 \\
\hline ש7. & $---M M-\Lambda-t-\Lambda T C--M T-M--T T-1$ & $2 \bullet 2$ \\
\hline .2711 .20 & $-\ldots M M-1-T-G T C---M E-M A-C T-\ldots$ & 302 \\
\hline $2712 \cdot 2 \mathrm{~b}$ & 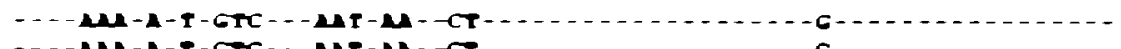 & 306 \\
\hline 182713.021 & $---\operatorname{Mn}-\mathbf{A}-\mathrm{t}-\mathrm{GTC}-\mathrm{MMT}-\mathrm{M}-\mathrm{CT}---$ & 306 \\
\hline 102714.28 & 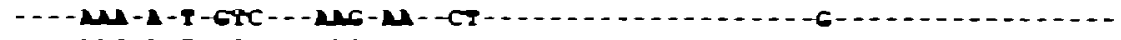 & 306 \\
\hline 02716.029 & 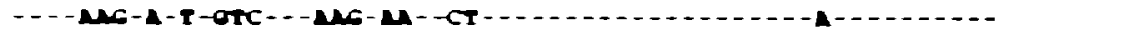 & 290 \\
\hline $702717.02 \mathrm{~d}$ & 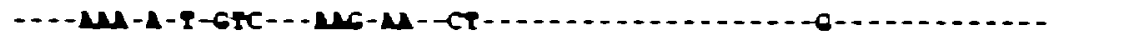 & 302 \\
\hline$\bullet 271 \bullet .20$ & 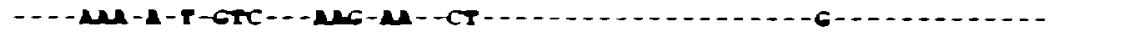 & 302 \\
\hline 12420.7. & $---M-\Delta-T-\Delta T C--M T-M--C T--$ & 200 \\
\hline $072421 \cdot 94$ & 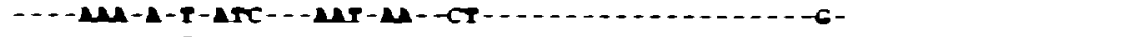 & 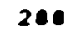 \\
\hline 072422.78 & $\Delta-\mathbf{t}-\Delta \pi--\mu \mathrm{\mu}-\mu-\infty-$ & 200 \\
\hline 072423.10 & $--M M-\Lambda-T-\Lambda T C--M P-M-C T--$ & 240 \\
\hline 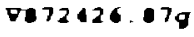 & 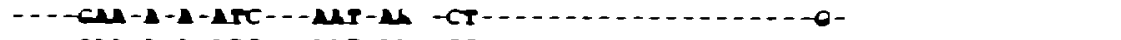 & 201 \\
\hline $012127.07 \mathrm{~b}$ & $-\cdots-G M-\Lambda-\Lambda-\perp T---M T-M-C T-$ & 200 \\
\hline $\begin{array}{l}07208.170 \\
072430.70\end{array}$ & 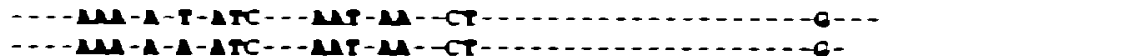 & $\begin{array}{l}280 \\
200\end{array}$ \\
\hline 12834.12 & 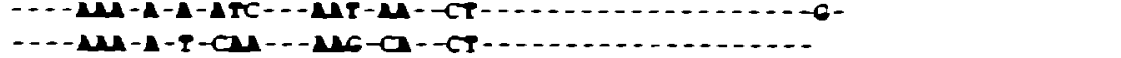 & 272 \\
\hline 12036.120 & 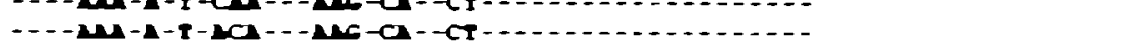 & 272 \\
\hline $12037.12 \mathrm{~d}$ & 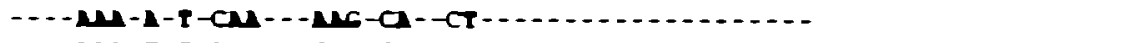 & 273 \\
\hline 2040.120 & 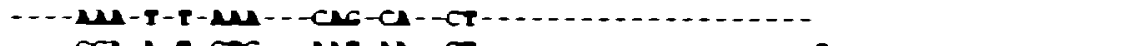 & 273 \\
\hline 1164.746 & $---\infty C-\Lambda-T-G T C---M T-M-C T-$ & 303 \\
\hline 14165.746 & 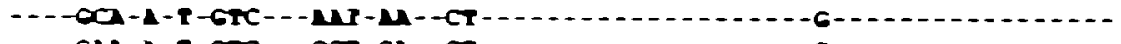 & 306 \\
\hline 14167.74 & 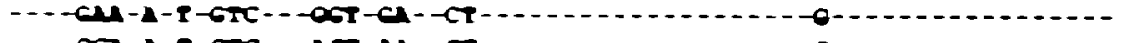 & 306 \\
\hline 14169.746 & 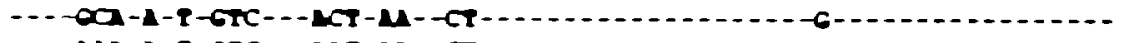 & 306 \\
\hline 7179.740 & 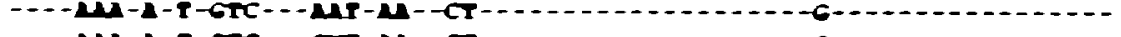 & 306 \\
\hline 1102.746 & $--M M-1-q-G T--a r-M--C T-$ & 906 \\
\hline 2721.20 & $\mathbf{T}-\mathrm{CTC}---\Delta \mathrm{M}-\boldsymbol{M}--\mathrm{CT}-$ & 302 \\
\hline 12712.26 & - -MA-A-T-GTC-CMT-M- -CT - & 306 \\
\hline 12712.021 & 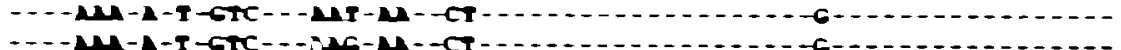 & 306 \\
\hline 02714.218 & 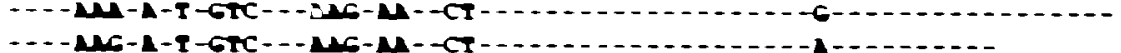 & 2306 \\
\hline 12717.29 & 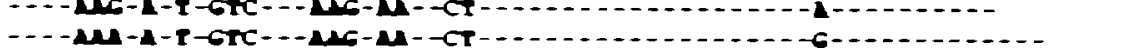 & 302 \\
\hline 12710.20 & $--M \mu-\Delta-T-C T C--M \sigma-M-C T-$ & 302 \\
\hline
\end{tabular}


vevs

comsings

$77-9$

Iי1

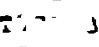

$x \div 210$

?: .75

$\therefore 1103$

ז77104

รา71:5

รา71:6

รา716

$5771 \%$

ค7า181

97935
5.937

$3 \mp \begin{array}{r}19538 \\ 579340\end{array}$

? ros4

印

T7955

T73557

T785s

77961

17962

1936s

Tej200

9032001

T132002

1032260

T032273

T०12201

$T 012218$

$\mathrm{TQ42286}$

זด

701228

T4225

T०12294

ro1223:
- TTh-tractict-c--at - ITIA- rnatert-c- ma- racer c-car TTIA- TACTACT-G--AT TIA-TMGTACT-C TTA- thetact - 6 - TTA-TACTRET-G--AT TTA-TACTATT-G--AT ITTA-TAETATT-G--AT TTI-TRETACT-G--1T TIA-TACTACT-C--Ar

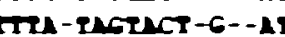

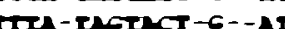

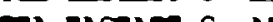
CT-O-AT coc-a- -1 TTE-TMETICT-C--AT TAFTACT-G--AT. GTNT-G--AT.

TA-TNCTLCT-A - - IT TIA-THOTACT-G--AT $C T-G--A T$
$\Lambda T-G--\Lambda T$ T-G--AT TIR-thotnct-0--AT $\mathbf{T}-\boldsymbol{Q}-\mathbf{A T}$ - TTL-TACTNT-0--1T

-. -Tru-ractsca-o- - TIta-ractact-o- - TTA- INCTACT-Q--AT. - TTA-TACTACT-G--AT

- -TTU - TACTACT-0--1T. TIA-THochet-G--AT.

TI- Enocert-G--AT.

a-trocict-c-nt trocact-o- -at. rnocacr-c--at. nes-c-at LTE-Cat T-G--AT
I-G--AT

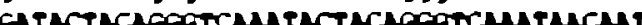
caracenchangtcmer. Macer

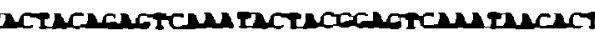

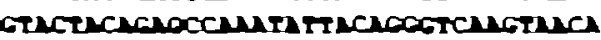

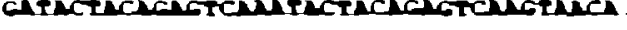

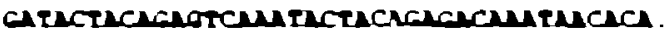

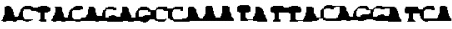

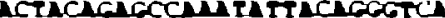

CATACTACNCAGTCMLT

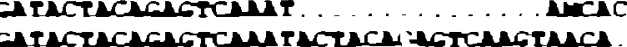

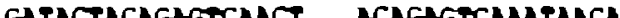

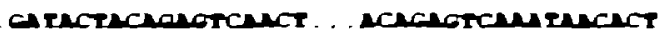
Levichanoccilientricleocta

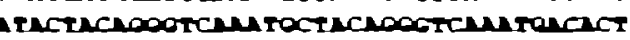

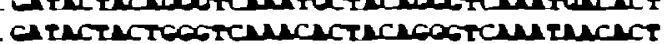
caraciacos

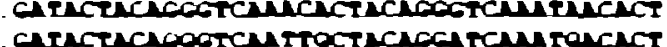

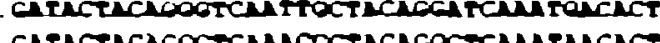

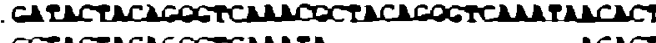
octertacicoctcrunta.

incict

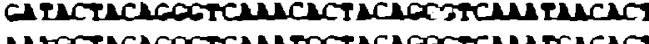

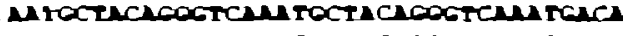

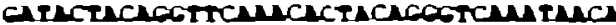

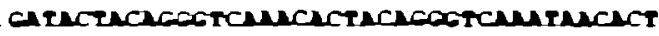

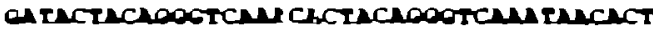

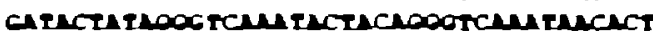

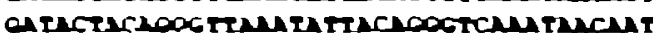

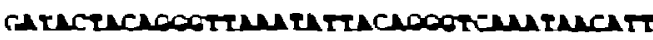
caractacacostrana

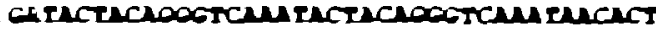

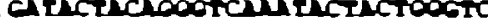

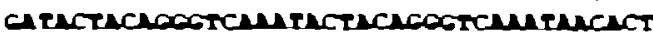

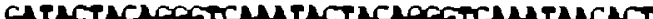

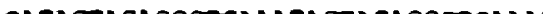

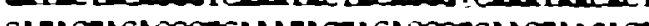

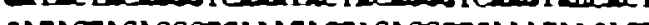

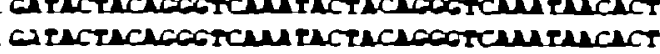

it a cuncta---c-----c--oc-ca----1--1cenccta-- -c--.--c--oc-ca--.--1--1-

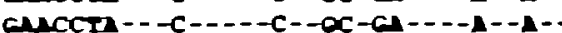

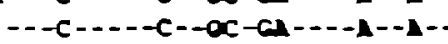
$--c----c--\infty-c----1--1-$ Giari-- $-c-1--c-\infty-c a---1--1-$

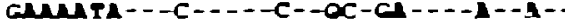

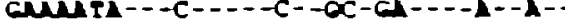

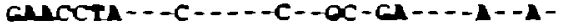

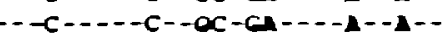

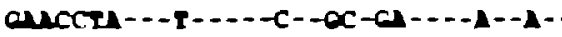

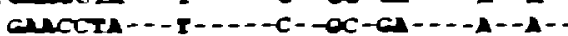

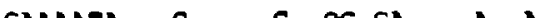

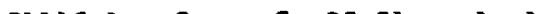

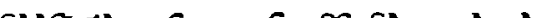

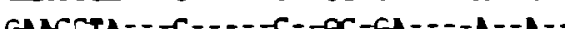

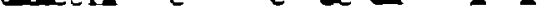

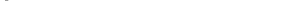

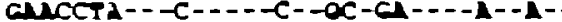
GMnctL-- -c--D--c--oc-ca----A--A-

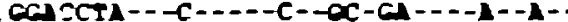
$-1-c-\infty-c a-\cdots--1--1-$

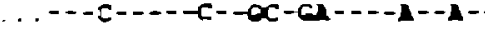
guccera- - -c-..--c--oc-ca-..--1--1onccta-- -c-----c--oc-on--.--1--1ouncta---c -...-c--oc-oa-..--1--1-

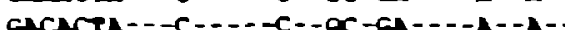

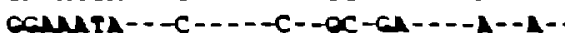
conara-c-co-co-

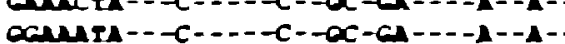

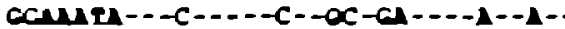

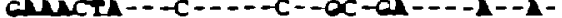

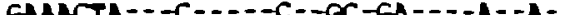
coucra--c- $c-c-c-\infty-c a-0-1-1$

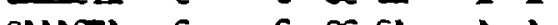

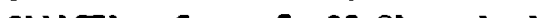

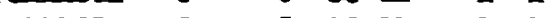
- canacra -

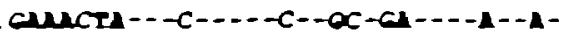

108 108 


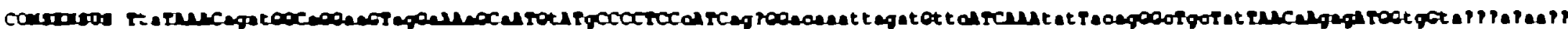

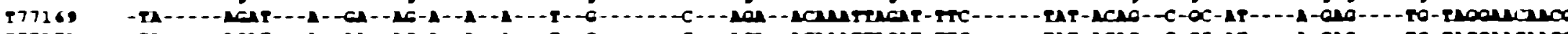

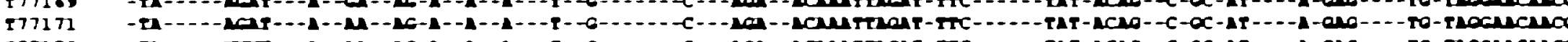

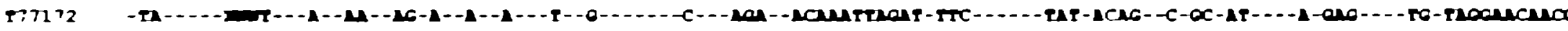

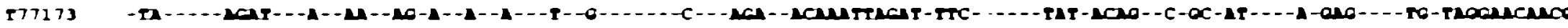

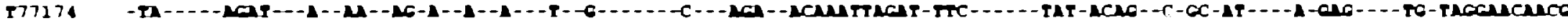
577175

27103

277100

277106

rา7105

รา1

27700

รากเา

579535

ร7953

ร79539

T79540

579511

570543

P79551

r7osse

279561

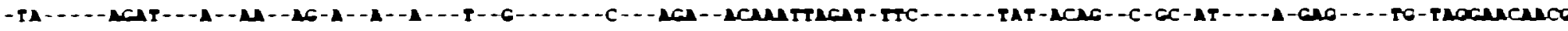

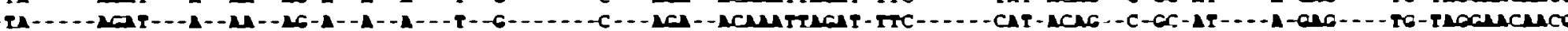
- A- -

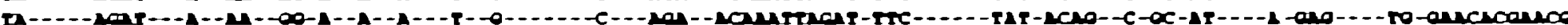

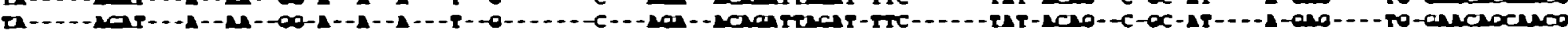

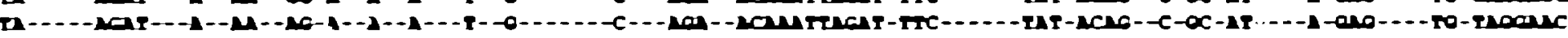

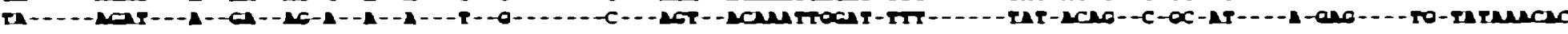

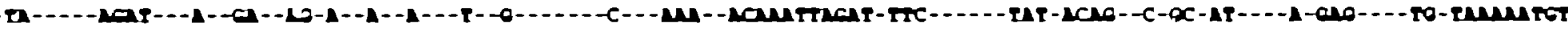

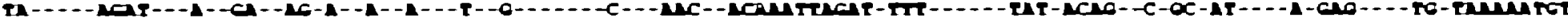

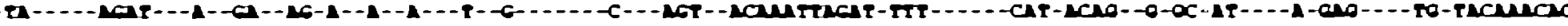

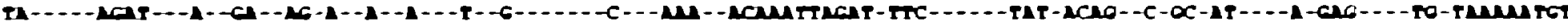

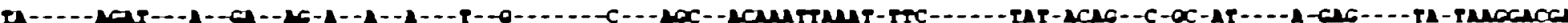

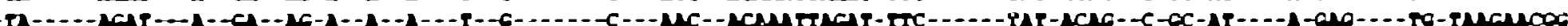

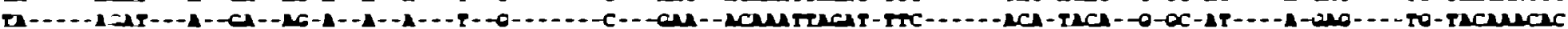

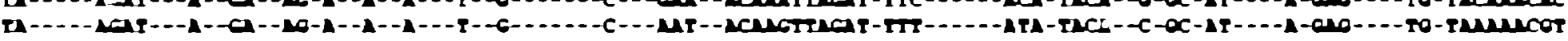

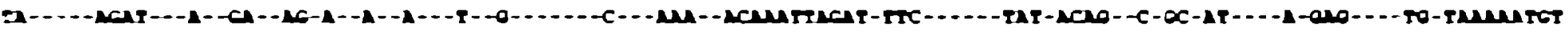

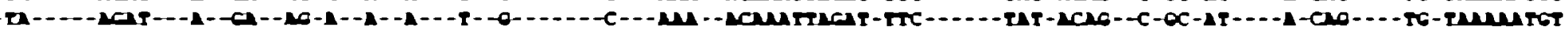

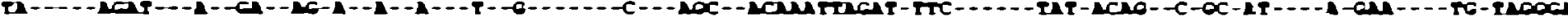

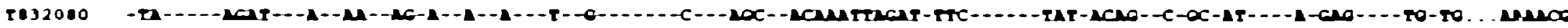
2032001

2032002

103260

r.32273

T24221

5012212

502206

I04220s

7012292

1042294

104229

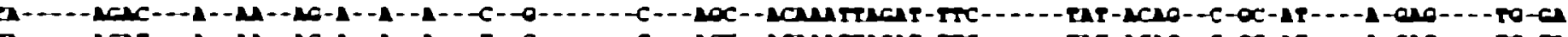

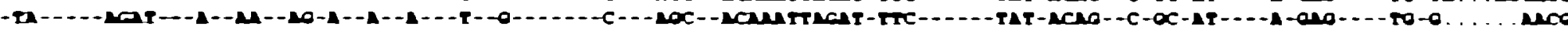

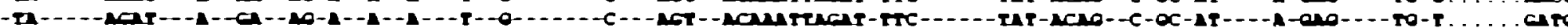

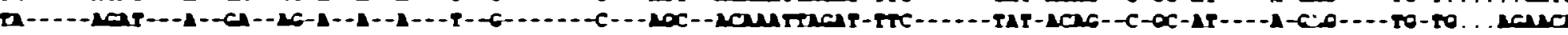

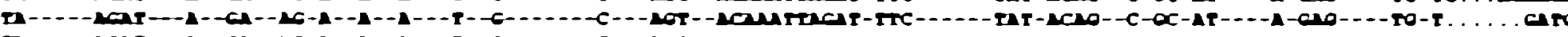

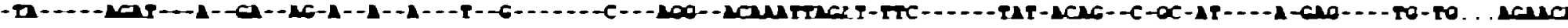

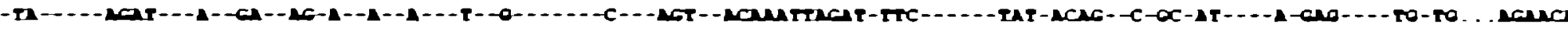

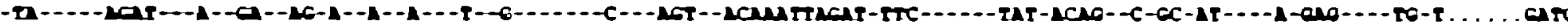

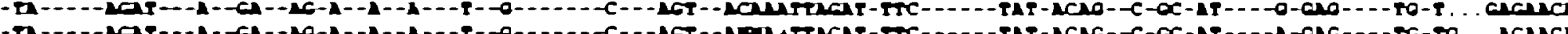




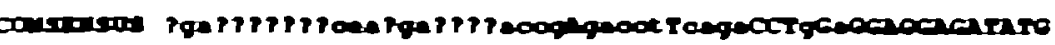
vil

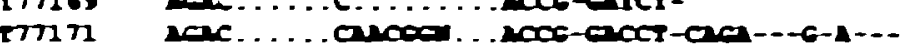

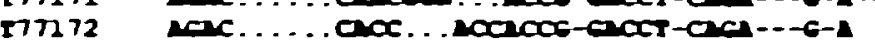

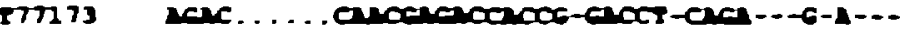

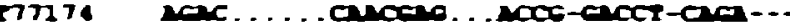

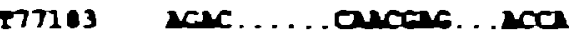

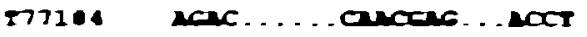

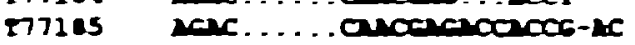

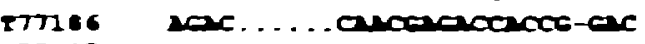

7 cac. . . . . oveoncac

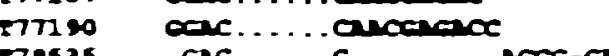

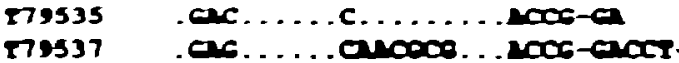

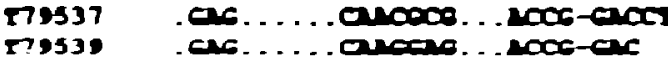

rigs39
rigs 40

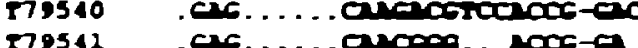

27954

79551

ross

การ55

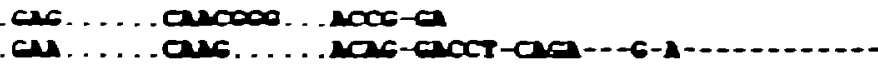

ac. . . . andons . . nace-ca

ac. . . areacroaces-a

ac. . . . antere. . noce-a

cre. . . . avacos. noce-a

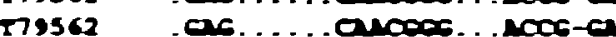

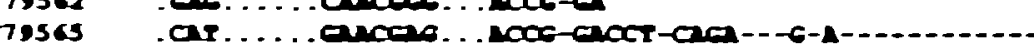

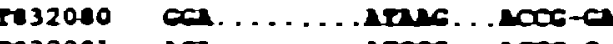

ta.

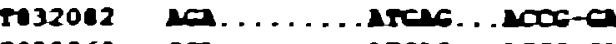

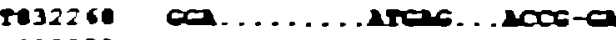

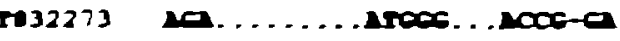

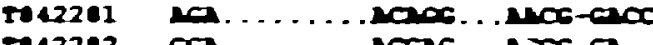

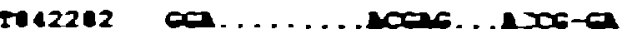

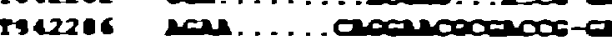

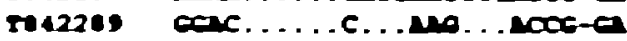

ro12292 or..........ac. . 1006-a

Te12294 val. ..... ondancanaces-a

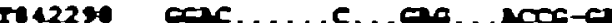

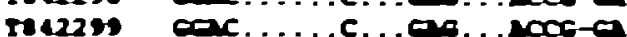




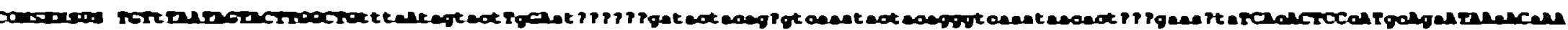

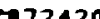

2072021

5072422

T172423

Ta72426

7072427

ra72420

ricos

rsicos

T31609

(1010

P16

2128

512832

712034

71283

712037

72040

874164

T7416

T74166

574167

Fา1160

7419

$3=\quad 2022100$

Tt22112

$0=$ raz21as

rescos

rescol

5272

Ta27

50271

5027

rean

rezn

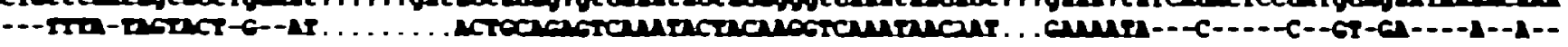

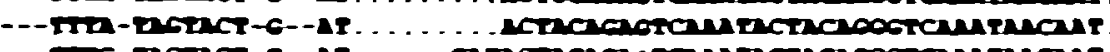

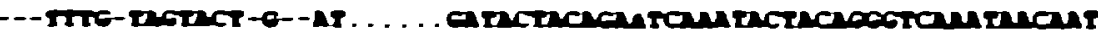

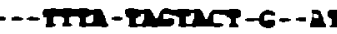
.

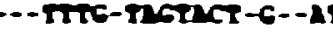

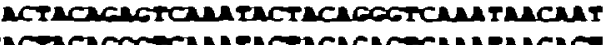

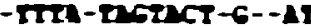

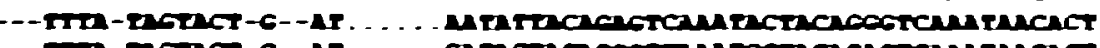

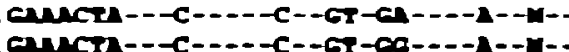

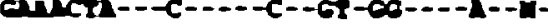

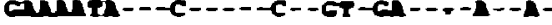

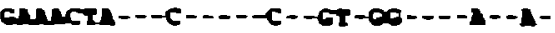

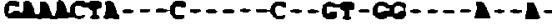

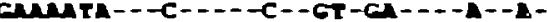

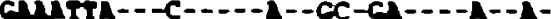

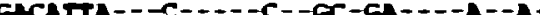

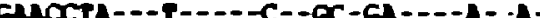
T-c--1.

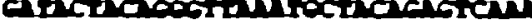

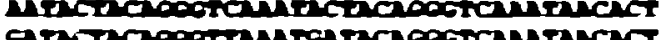

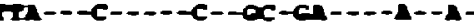

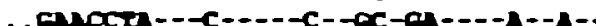

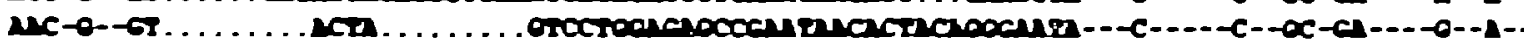

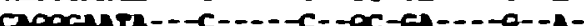

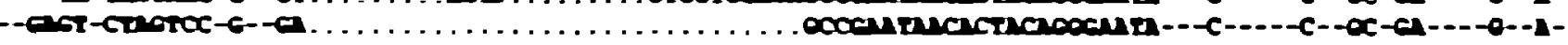

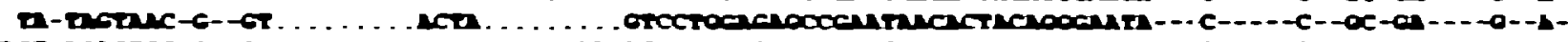

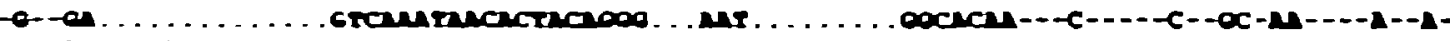

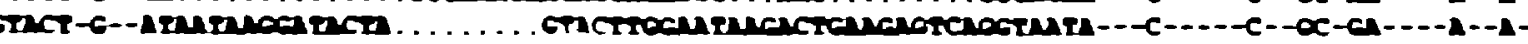

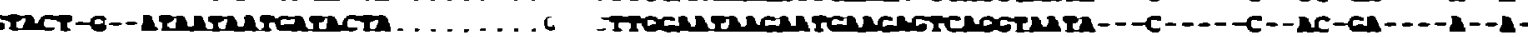

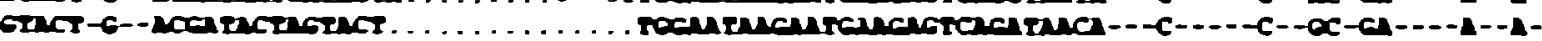

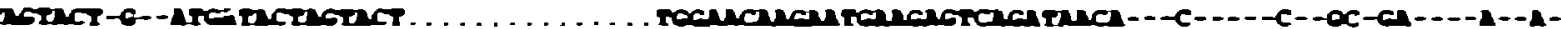

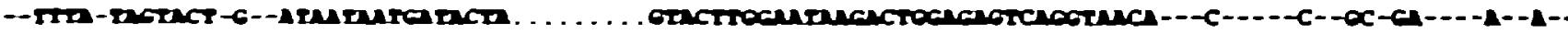

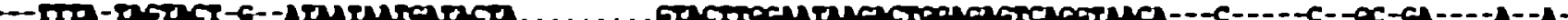
Th- -

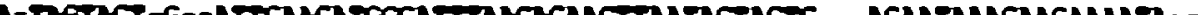
moract

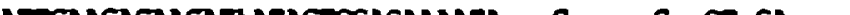

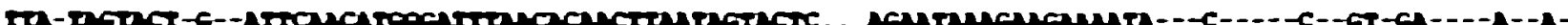

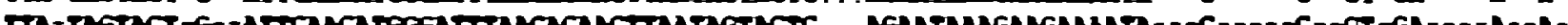

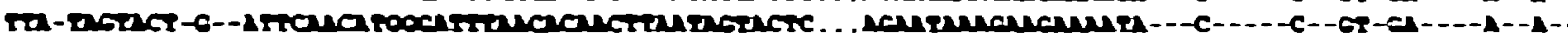

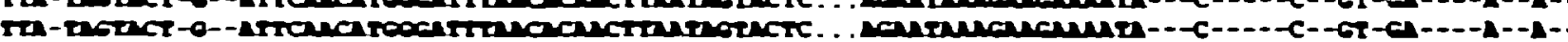

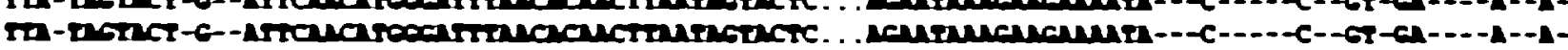

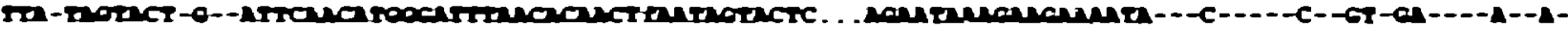

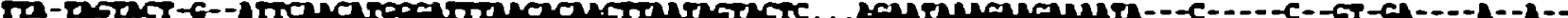


vors

CQ 77200 retance

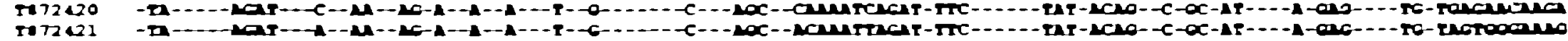

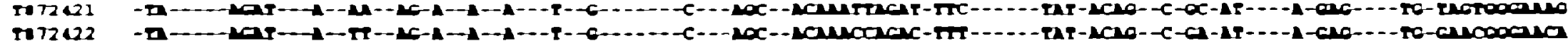

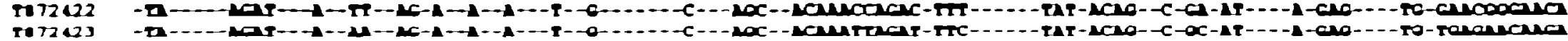
707266 - 10. TS1003

21011

3161

$\operatorname{ring}$

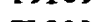

T12012

T21036

T12037

T12110

T74

T7416

F)116

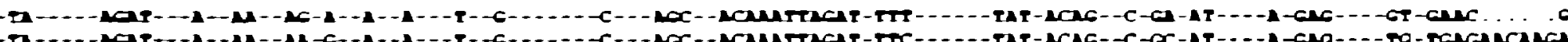

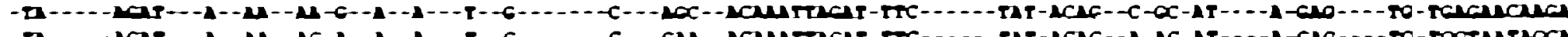

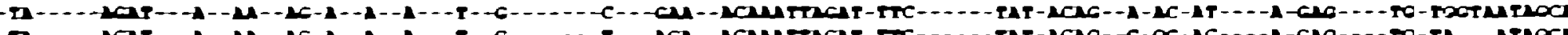
D -...- -

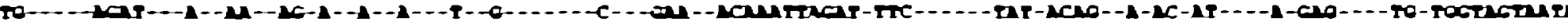
Th.... -

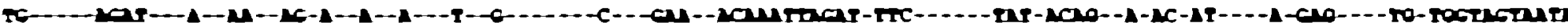

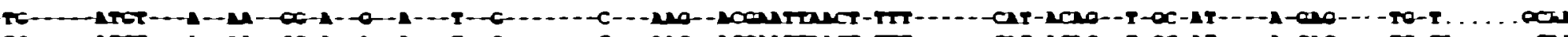

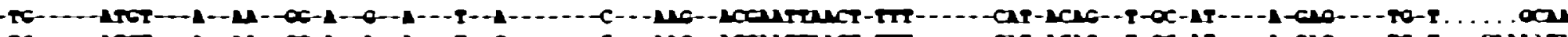

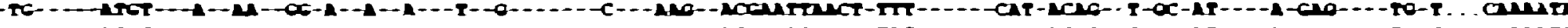

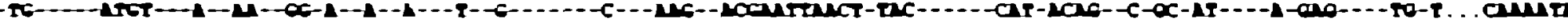

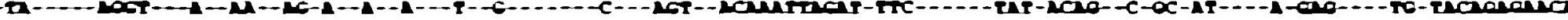

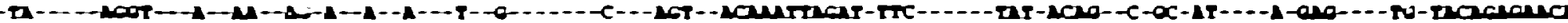

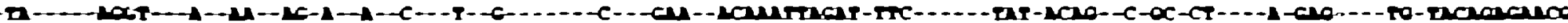

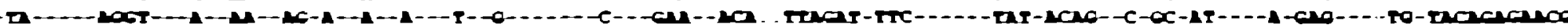

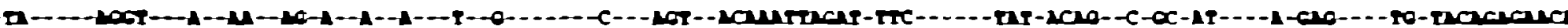
a -.

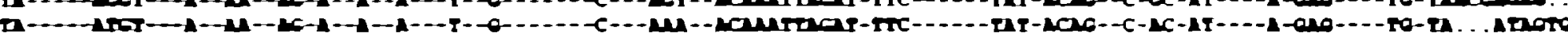
D-..-

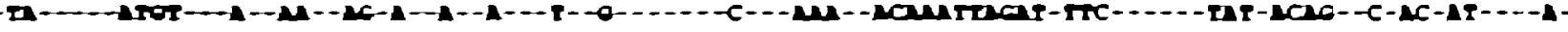

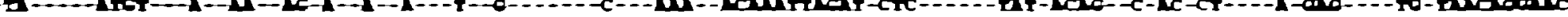

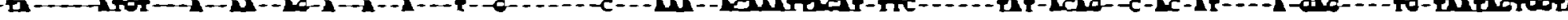

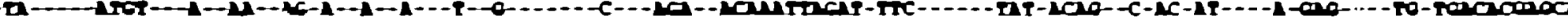

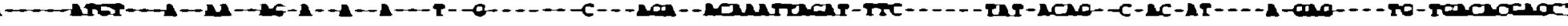

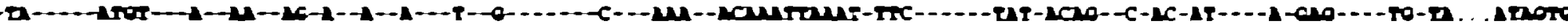

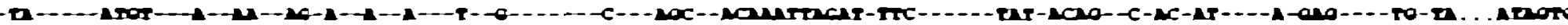

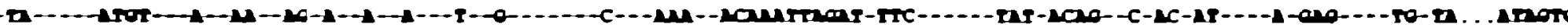

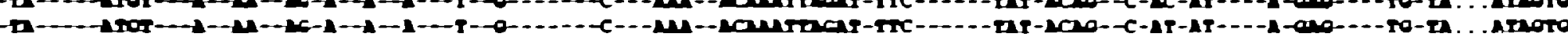




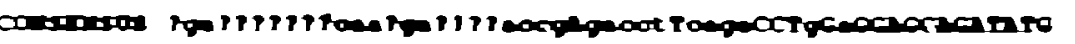

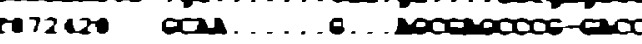

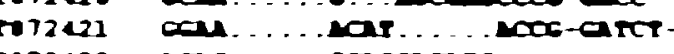

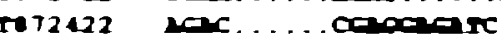

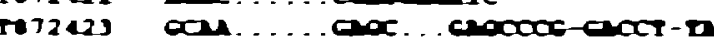

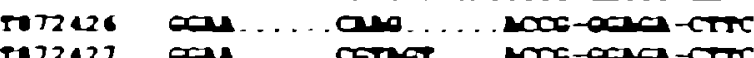

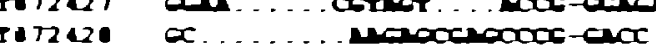

1300

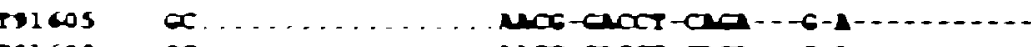

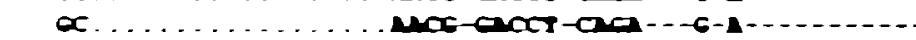

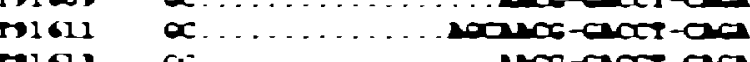

TO1610

T1631

nimo-

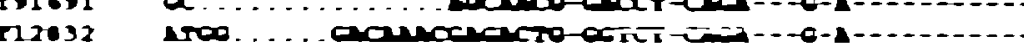

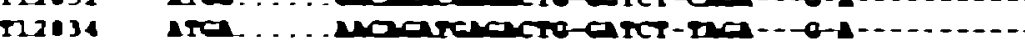

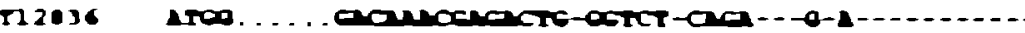

T12137 ITO . . a

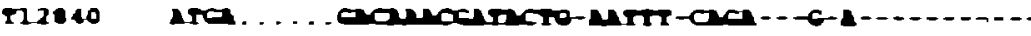

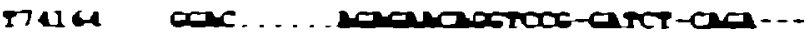

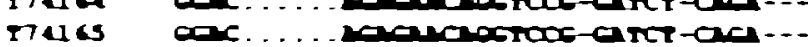

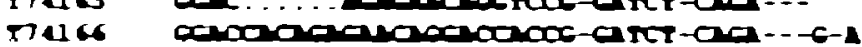

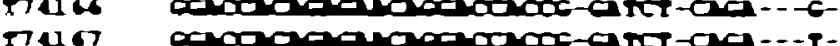

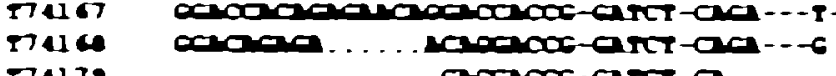

$>$ T7418

Tr22100

onvanhararacocor-a

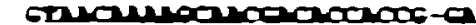

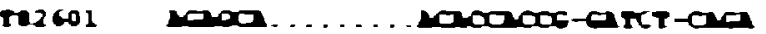

$\operatorname{tancos}$

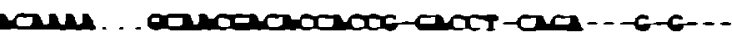

rasmis

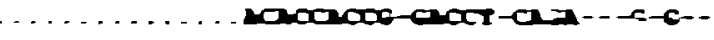

nacococ-acct-oca-- -o

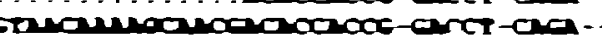

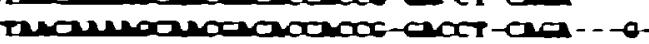

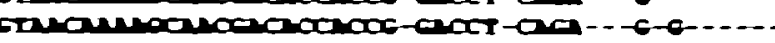

กาก

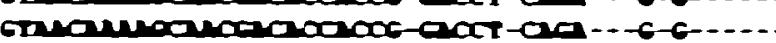

250

253

240

260 


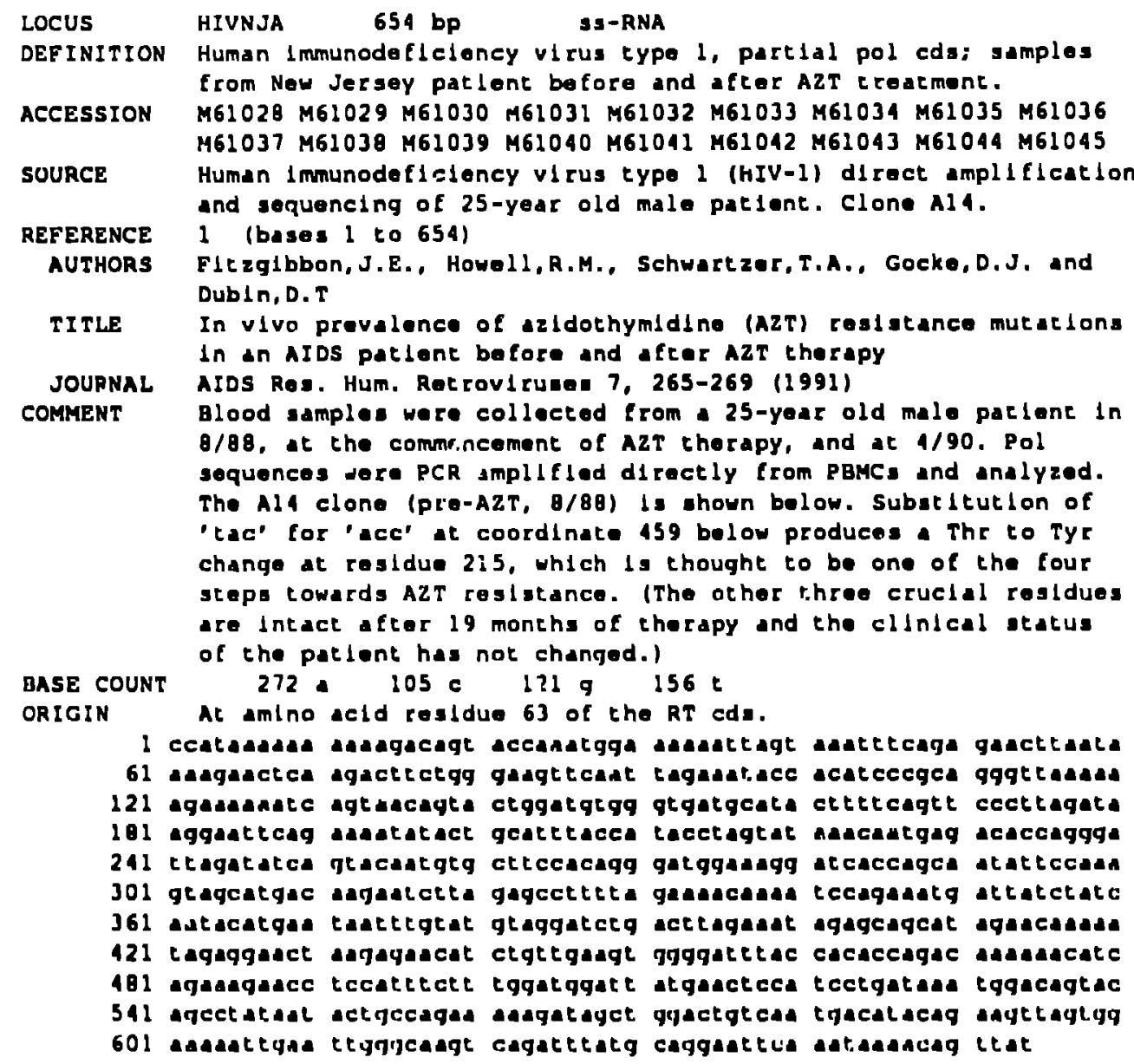


Ficzgibbon ot 1 ., AIDS Res. Hum. Recroviruses 7, 265, 1991. The flrse nine sequences are pro-A2T (M61028 is clone A14): the second nine are post-A2T.

\begin{tabular}{|c|c|}
\hline NSENSUS & CAATAAAgMAMAGACAGTAC.AAATGGAgAMATTAGTAgAT \\
\hline 61028 & 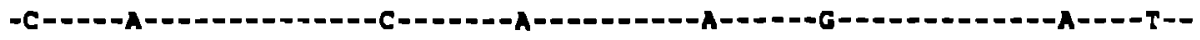 \\
\hline 61029 & 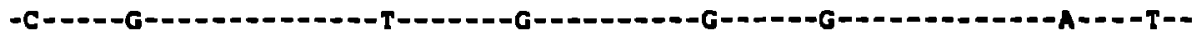 \\
\hline 61030 & 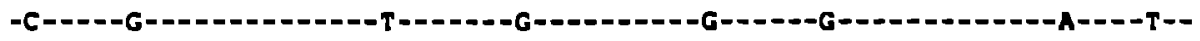 \\
\hline 61031 & 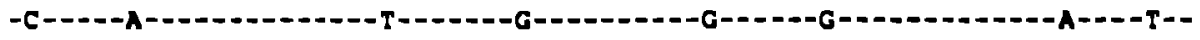 \\
\hline 61032 & 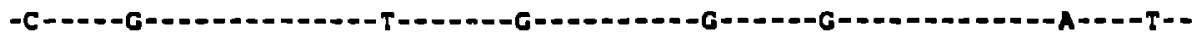 \\
\hline 61033 & 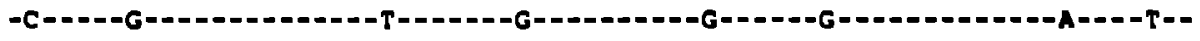 \\
\hline 62034 & 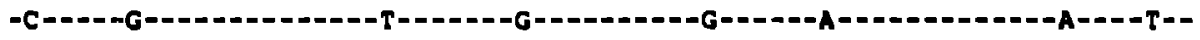 \\
\hline 51035 & 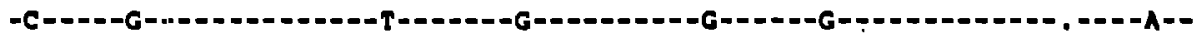 \\
\hline 61036 & 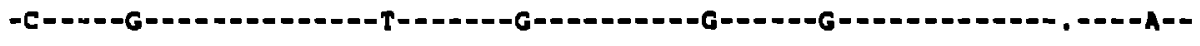 \\
\hline 61037 & 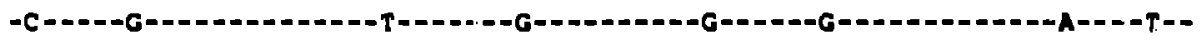 \\
\hline 51038 & 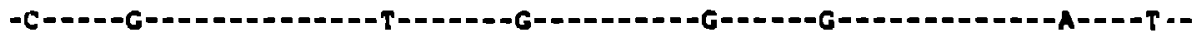 \\
\hline 61039 & - \\
\hline 51040 & 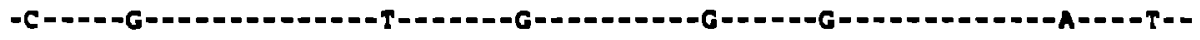 \\
\hline 61041 & 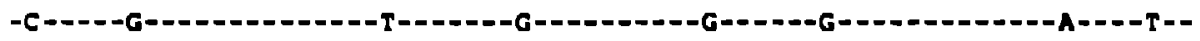 \\
\hline 61042 & 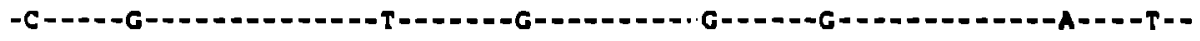 \\
\hline$\$ 1043$ & 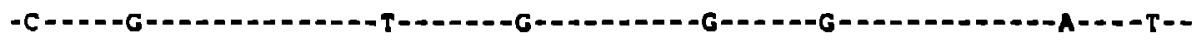 \\
\hline$\$ 1044$ & 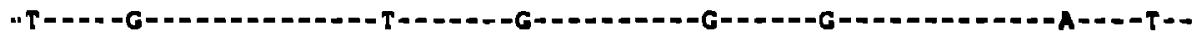 \\
\hline 1045 & 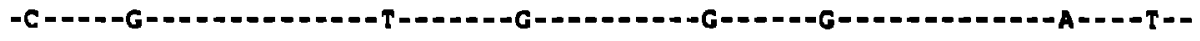 \\
\hline
\end{tabular}

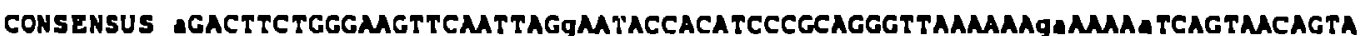
M61028

M61029

M61030

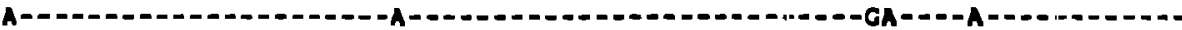

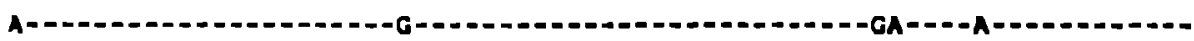

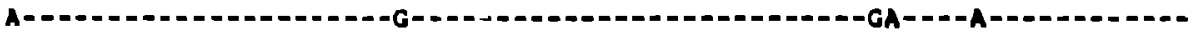
G-1-10-1-

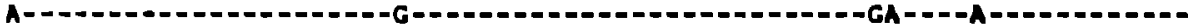

461033

M61034

$M 61035$

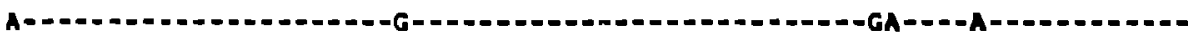

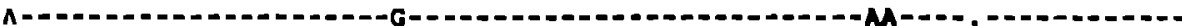

M61036

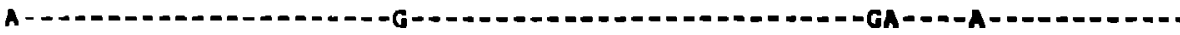

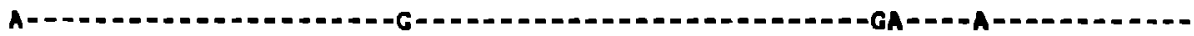

M61037

461030

M61039

M61040

M61041

961042

M6104J

M61044

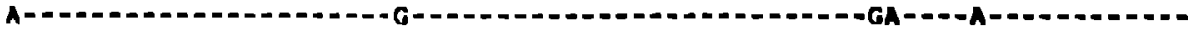

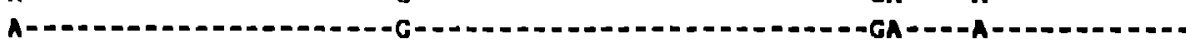

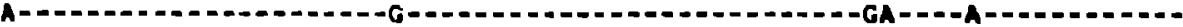

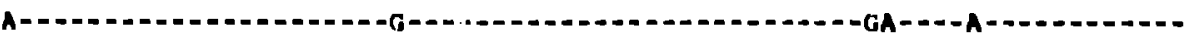

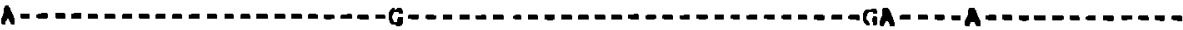

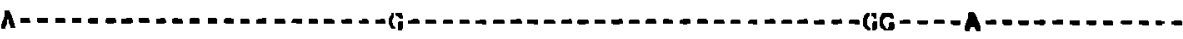

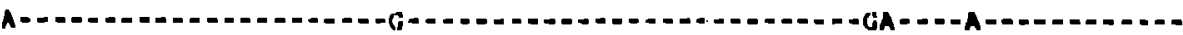

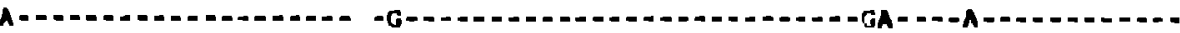

461045

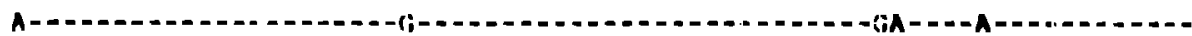

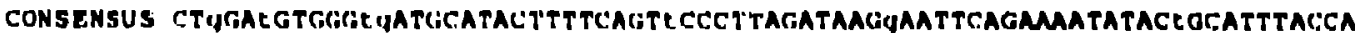
M6102I

461029

M610 0

M61031

M61032

M61013

M61019

Ma10.39

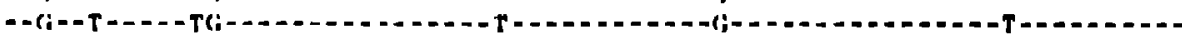

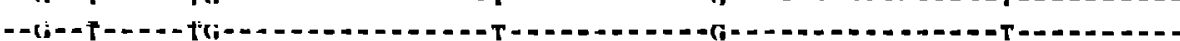

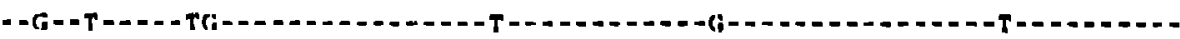

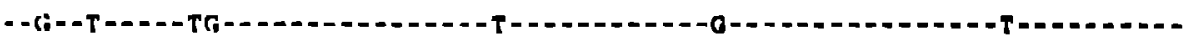

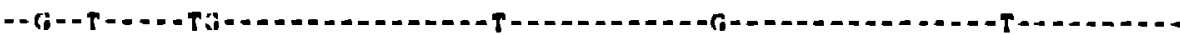

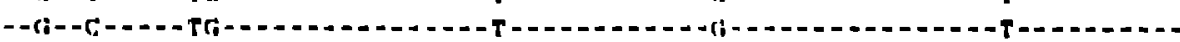

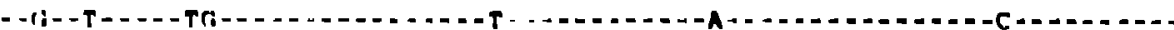

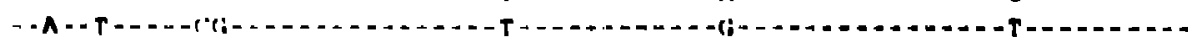

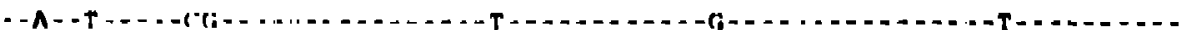




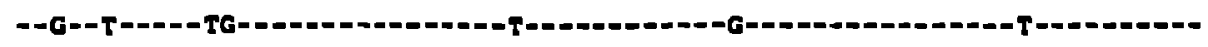

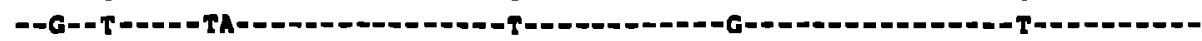

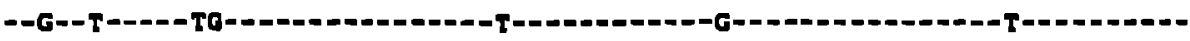

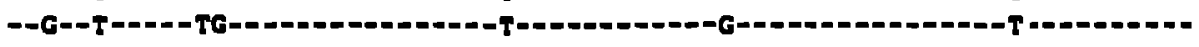

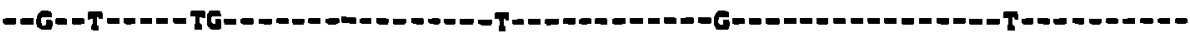

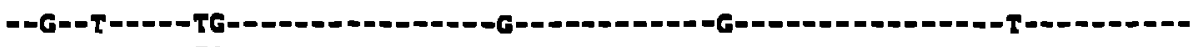

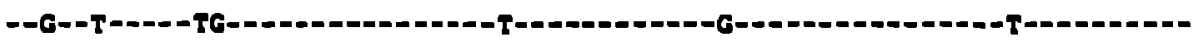

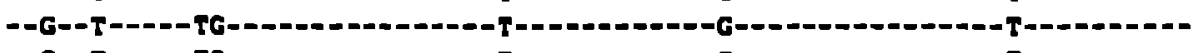

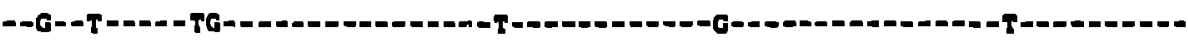

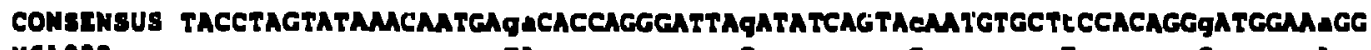
M61020

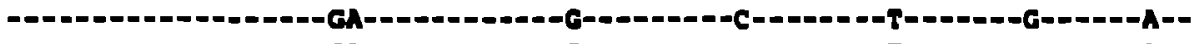
- - - - - - - - - - - - - - - -

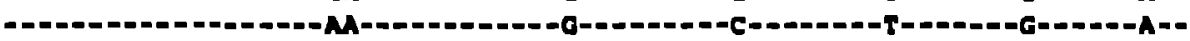

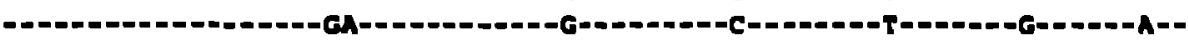

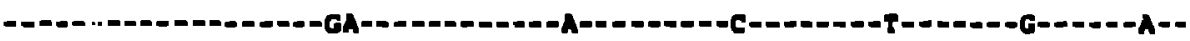

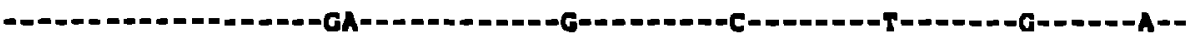

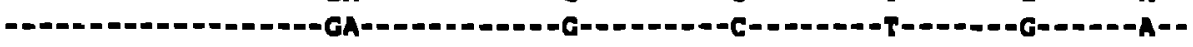

M61037

M61038

MS1039

MG1040

M61041

H61042

M61043

M61044

M61 045

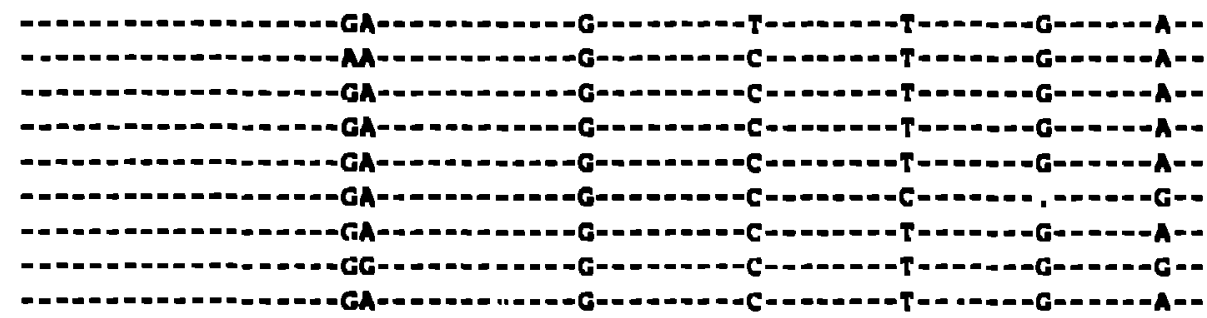

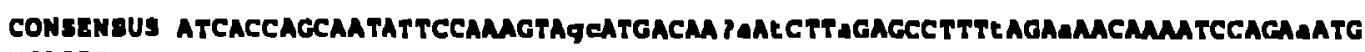

H61028

M61029

M61030

M61031

M61032

MGI0JJ

M610J4

M610Js

Mo10J6

H61011

H61010

H61019

M⿻1040

Moto4l

M61042

Ho1114 I

M61044 -

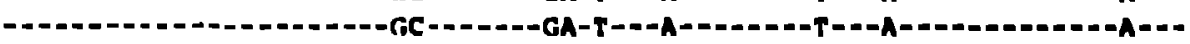

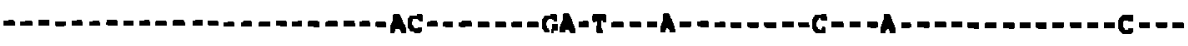

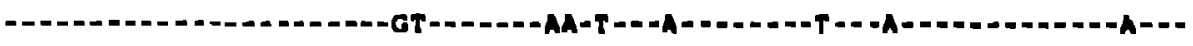

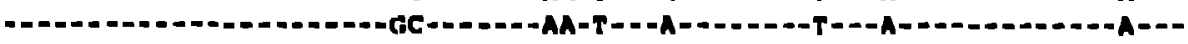

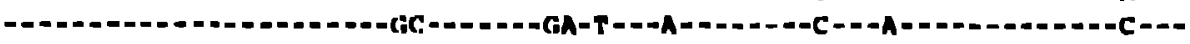

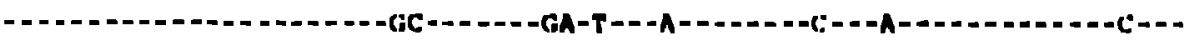

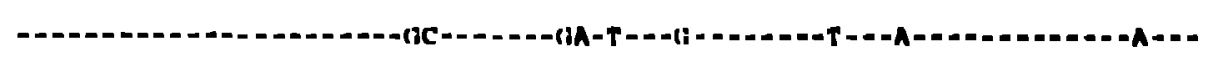

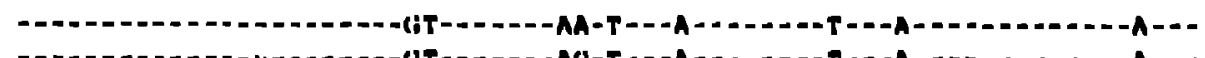

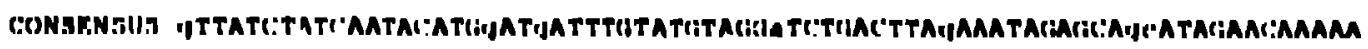

Mol $02 \mathrm{H}$

Mal1129

MG1010

Mb!III

MbIUI)

Mal1) I

Ma11) 14

עיו

MII : I

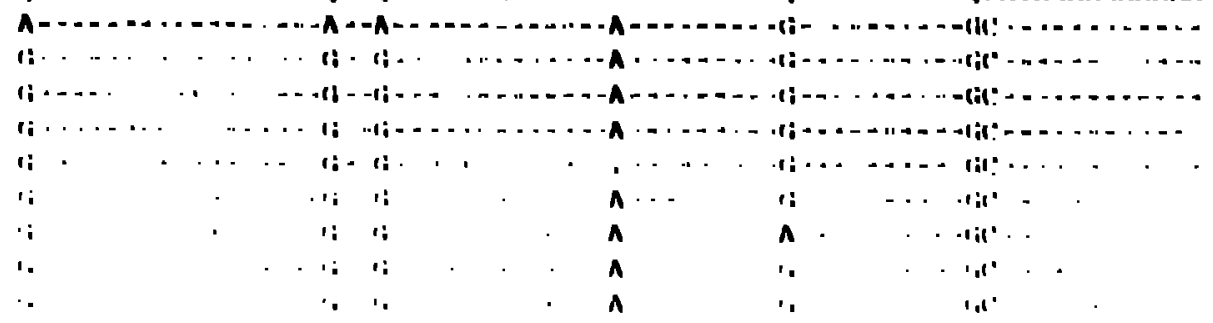




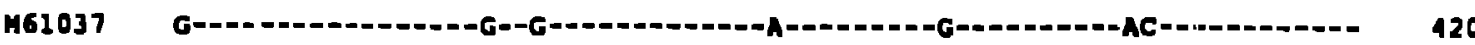

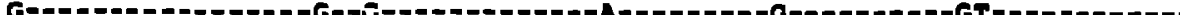

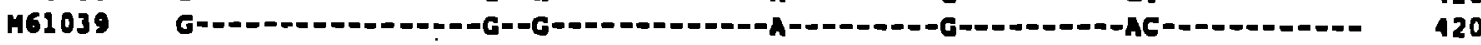

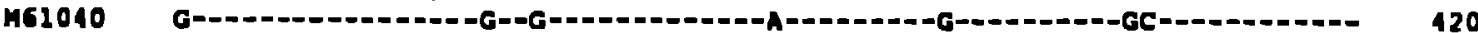

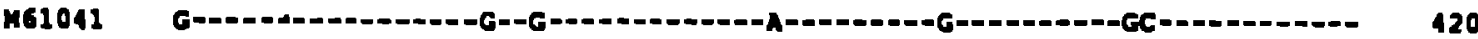

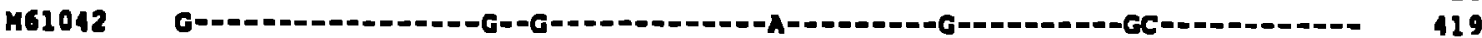

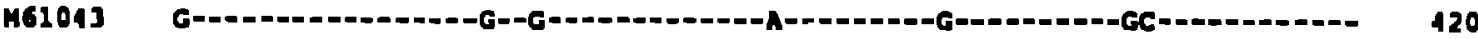

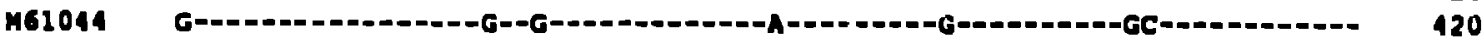

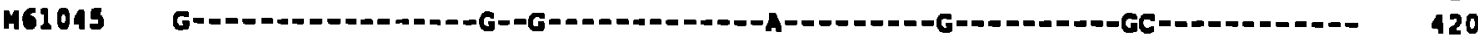

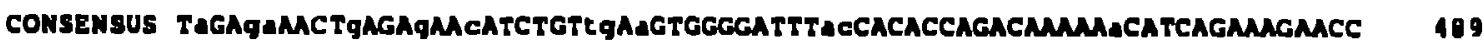

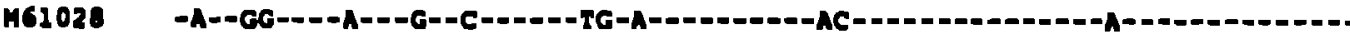

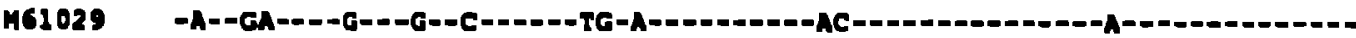

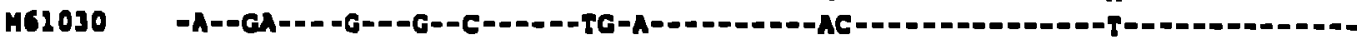

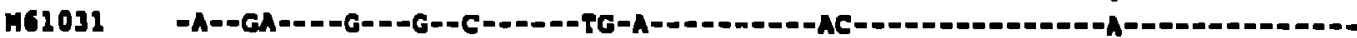

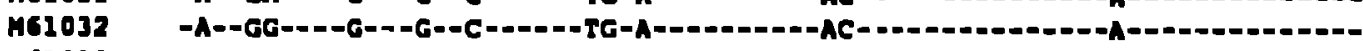

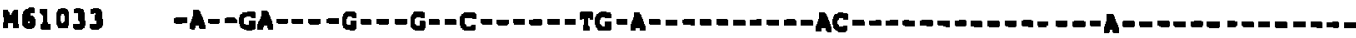

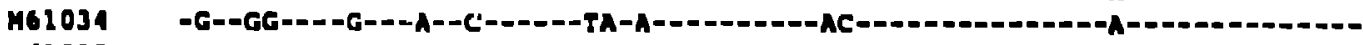

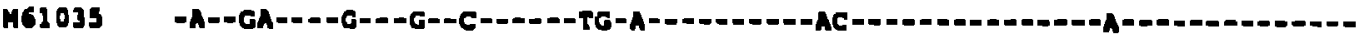

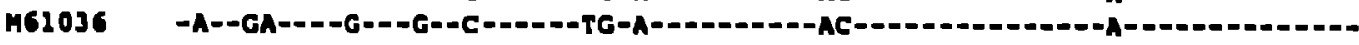

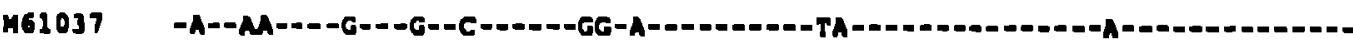

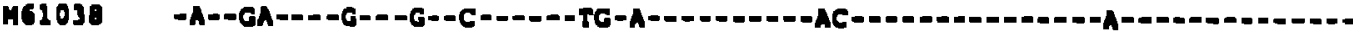

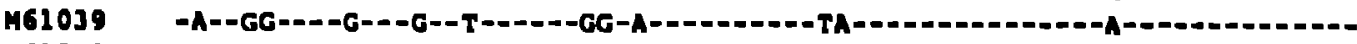

H61040

M61041

H61042

H61043

n61044

M61045

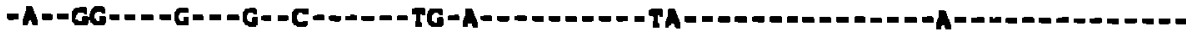

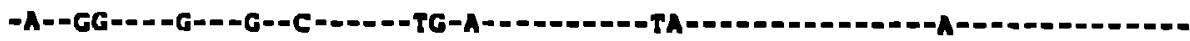

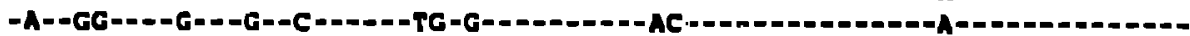

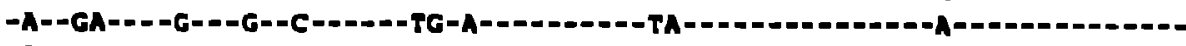

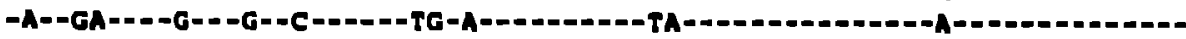

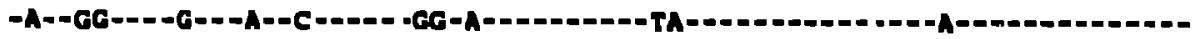

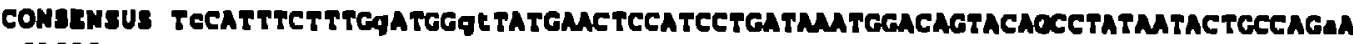

M61020

M61029

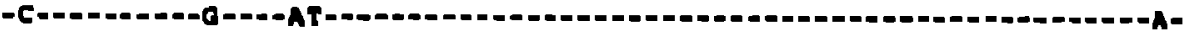

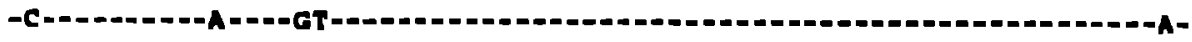

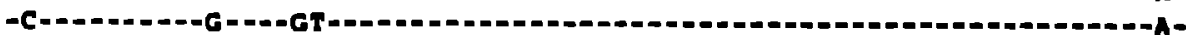

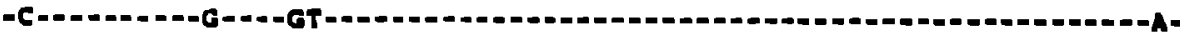

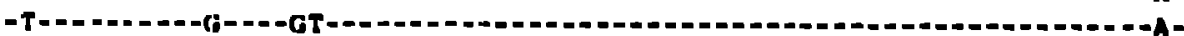

16109

1451032

M610J3

161034

M61035

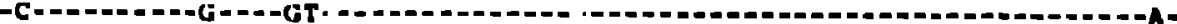

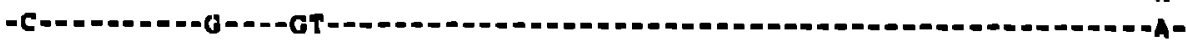

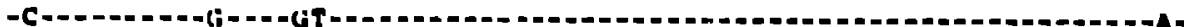

MS10.36

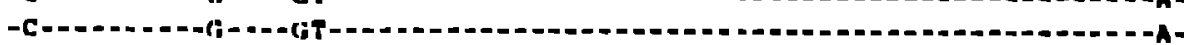

Moloj'

M61030

M61019

M61040

Ma1041

M61042

M6104]

H61044

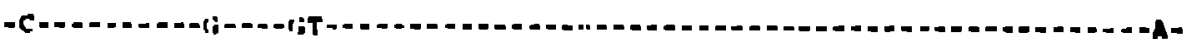

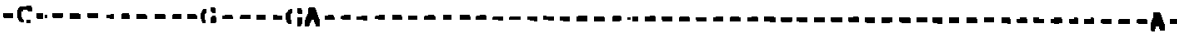

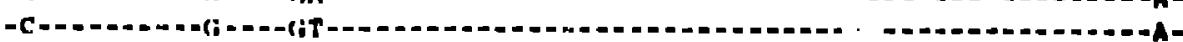

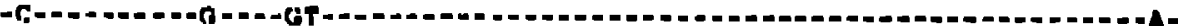

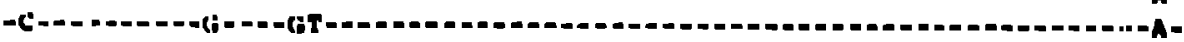

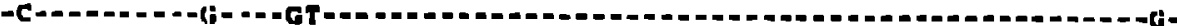

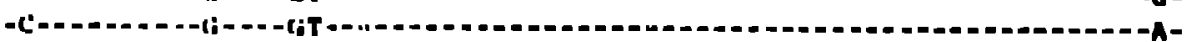

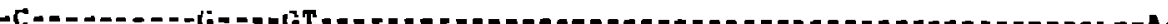

M61045

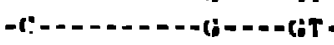

T

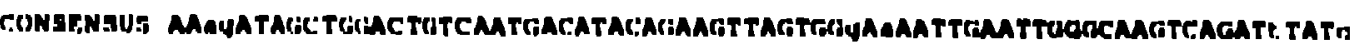

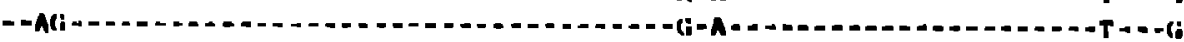

461111

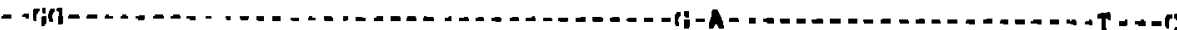

Maloid

Malnil

Mallin

MaIll',

- Ali - -

a. - n 


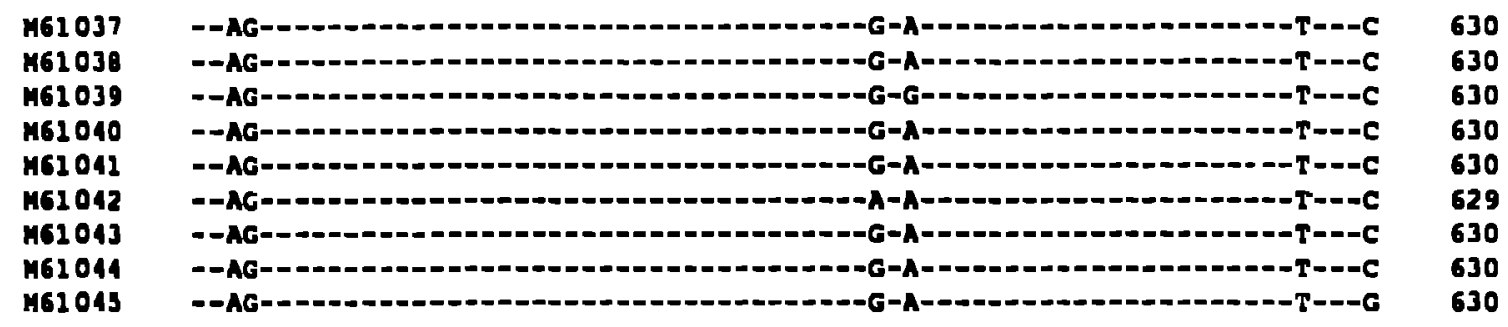

CONGEMSUS CAGGMTTAMALTMAMCAGTTATI 653

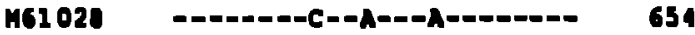

M61029

M61030 - 654

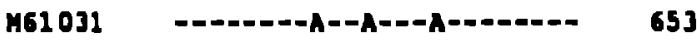

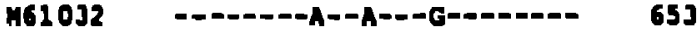

M61033 - 654

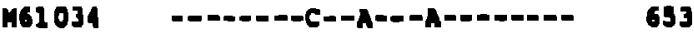

M61033 -. 653

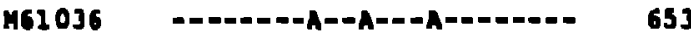

M61037 (103) 654

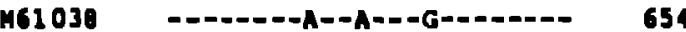

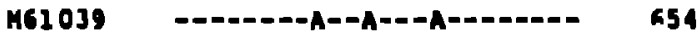

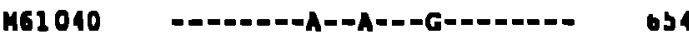

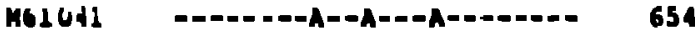

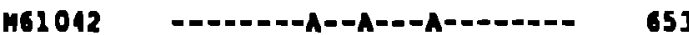

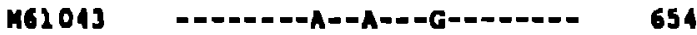

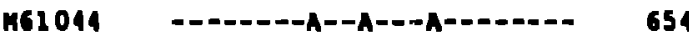

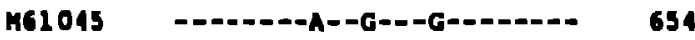


LOCUS HIVU455 9178 bP SS-RNA

DEFINITION Human Immunodeflelency vitus type 1. 1901 ate U455 from a Ugandan person (1985): complete genome.

ACCESSION M62320

SOURCE Human immunodeflclency virus typo 1 proviral DNA taken from PBLa.

REFERENCE 1 (basos 1 to 9178 )

AUTHORS Oram,J.D., Downing,R.G., Roff,M., Clogg,J.C.S., Sorwadda, D. and Caravell, J.w.

TITLE Nucleotide sequence of agandan HIV-I provirus reveala genetic diversity from other HIV-I isolater

JOURNAL AJDS Res. Hum. Retroviruses 6, 1073-1078 (1990)

COMMENT Hardeopy of sequence [1] kindly provided by J.D. Oram, 2/5/91. The U455 virus was isolated in 1985 from a 35-year old Ugandan male. The sequenced clone has in-phase stops in the vpR and env coding sequences and a frameshlft, generating a stop codon, In the vpU eds. While thls virus is related to other afrlean HIV-1 forms ( most closely to 2321.; see Part III), tho serology of the gag proteln, for example, 1s unugual according to [1].

BASE COUNT 3263 \& $1636 \mathrm{C} 2200 \mathrm{~g} 2069 \mathrm{t}$

ORIGIN

A rpt $\rightarrow$

argettgect tgagtgeteg asgtagtgtg tgecegtetg ttatt

61 gagatcecte agaccactat agaetgtgta auatetcta geagtggege eegadeggg

121 actegaage gaagttcca gagaagtect etegacgeag gactegget getgaggtge 101 acacageas aggegagage ggegactggt gagtacqeet aadattett gactagegga 211 ggetagaagg agagaAatGg gtgegagage gtcagtatta agcgggaad astagat te gag start $\rightarrow$

301 atgggagaas atteggttas qgceaggggg adacasasa tatagactga aacattcagt 361 atgggeagc agggagetgg auadtcac acteaccet ggectttag aacagcaga

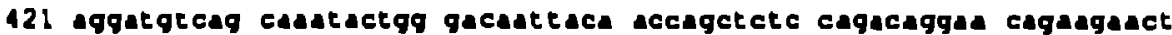

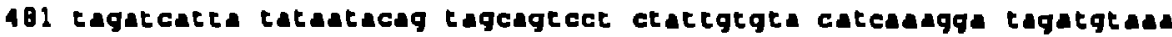

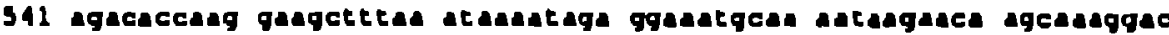
601 aca jeaggea geagctaca caggagcag tesanatede cceatagtgc aneatgcace 661 agqgeascea gtacaccagg cettatcace taggacettg autgcatgag tgaaagtagt

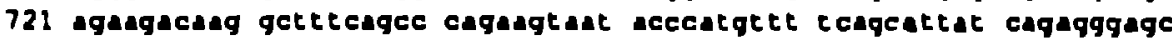
781 caccecacas gattranta tgatgctgas tgtagtgggg qgaesccagg cagctatgca 84 atcgt taAa gataceatca atgaggaage tgeagagtgg gacaggtec atecagtgca $90 \mathrm{l}$ tgcagggcee attecaceag gccagatgag agaeceasq ggangtgaca tagcagguac 961 tactagcace gttcapaac auataggatq gatgacagge antecaceta tcecagtggg 1021 agasatclat agaagatgga tastcctggg attaataad atagtadga tgtatagcec 1001 egttagcate ttggacata gacasgggce asagascce tecagggate atgtagatag 1141 attctteas acteteagaq ctgarcade tacacaggat gtausanet ggatqucaga 1201 acctegctg gtccasasg eqaatccaga ctgtangtce attetasqag cattagggce 1261 aggggctace ttagasqaas tgatgacage atgceaggqa qtgggeggac ceggccataa

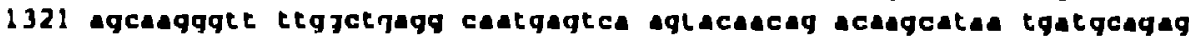

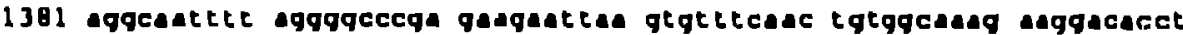

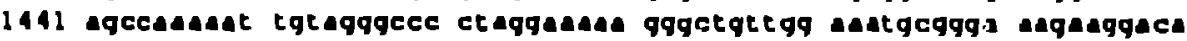

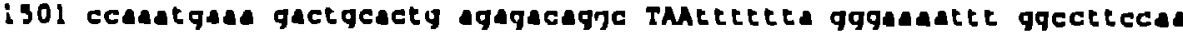
pol orf gtart $\rightarrow$

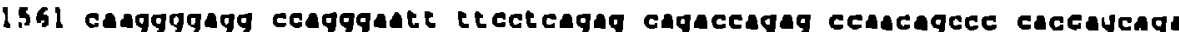

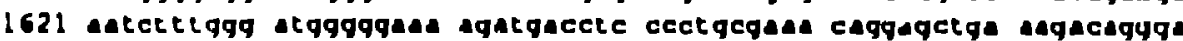

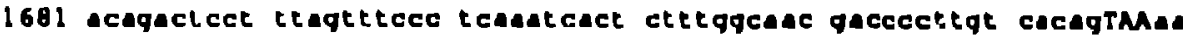

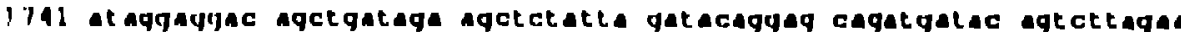

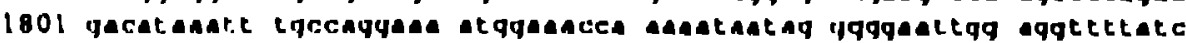

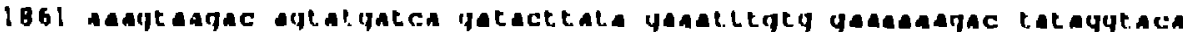

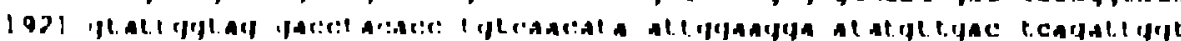

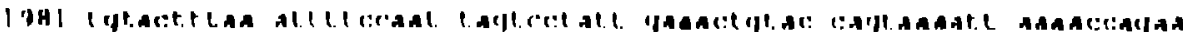

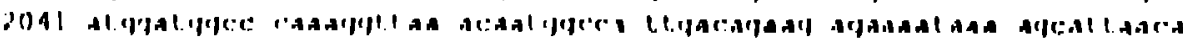

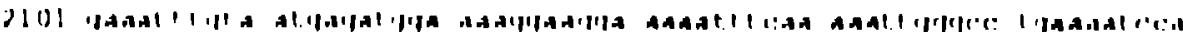

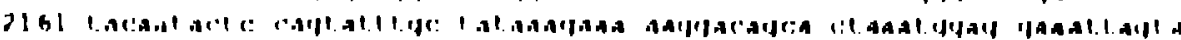

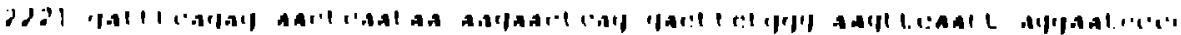

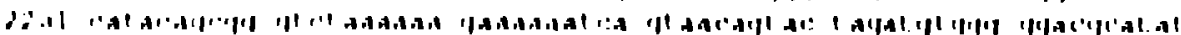

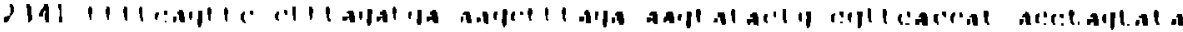


2401 aacartgaga cacciggagt caggtatcag tacalgtgc ttccgcaggg atggaadga

2461 tcaccatca tattccagag tagcatgaca daatcttag agccetteg atcacaacat

2521 ccagacatag ttatctatca atacatggat gacttgtatg tgggatctga tttagaada

2581 gggcadeata gagcadaut agaggadta agagetcatc tattgagetg gggattcat

2641 accceagaca aadagcatca gaagaacet ccattcett ggatggggta tgaactecat

2701 cetgacaat ggacagttea gectacacag ctgceagaad agacagetg gactgtcaat

2761 gatatacaga autcagragg aadactaat tgggcagtc agatttatge agggattaa

2821 graagcaac tgtgtaaet tetcagggga gecaugcac caucagatat agtaaccetg

2881 actgaagaag cagaattaga attggcagag aacagggaga ttctaaaga cectgtgcat

2941 ggagtatatt atgacceatc aaagaceta gtagcagaa tacagaade agggcaagac

3001 caatggacat atcasatt a tcaagagced tttadadc tgaaacagg adatatgca

3061 agaaaggt ctgctcacac tatgatgta aucautua cagaagtggt geadadgeg

3121 tccacagaa geatagtaat atygggaag atccetaat ttagactacc catacaaag

3181 gaacatggg aageatggtg gatggagtat tggeaggeta cetggattcc tgantgggag

3241 tttgtcata cectccect agtaaatta tggtaccagt tagagaaga ccceatagca

3301 ggagcagaya cattctatgt agatggggca gctautaggg adactaaget aggaaagca

3361 gggtacgtca ctgacagagg adgacadad gttgtttecc taactgagac adcadatcad

3421 agactgat tacatgcat ceatetagec ttgeaggate caggateaga agtaaatata

3481 gtaacagact cacagtatgc attagggatc attcaggcac aaccagacag gagtgaatca

3541 gaatagtca atcadatat agagaageta atagaaagg aadagtcta cotgteatgg

3601 gtaccagcgc acauaggat tggaggaal gagcagtag atadategt cagttetgga

3661 atcaggadg tgctattte agatgggata gataggctc agaggacca tgaaaatat

3721 cactgcadet ggagagcad ggctagtgat tttadtctgc cacetgrggt agcgaagga

3781 atagtagcca gctgtaatad atgtcacta aaggggang ceatgcatgg acadgagac

3841 tgtagtccag ggatatggca attagattgc acacatctag aggaaagt alcctagta

3901 gcagtccatg tagccagtgg ctacatagaa gcagaagte tcccagcaga aacaggacag

3961 gagacagcat actttatact aadtagca ggagatggc cagtaaagt alacacaca

4021 gacartggca gcalttcac cagegctgca gttaageag tetgttggtg ggcaatate

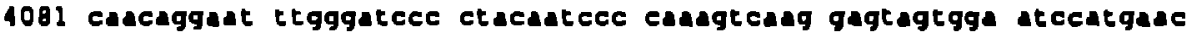

4141 aggaatta agauatcat agggeaggta agagagcad ctgaacacet taagacagca

4201 gtacadatgg cagtatclat tcacalttt aaagaaag gggggattgg gggatacagt

4261 gcaggggaa garaataga cataragca acagacacac aactaaga attacaana

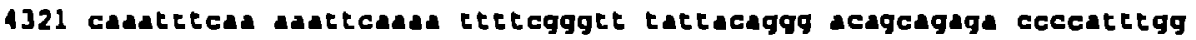

4381 aaggaccag cauactact ctggaaggt gaagggcag tagtadeca agacalagt

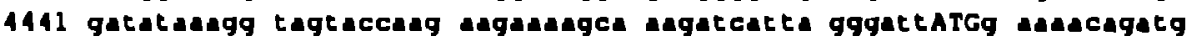
vif otart $\rightarrow$

4501 gcaggtgatg attgtatggc aggtagacag gatgaagat AGacatgga acagtteagt

$$
\text { <. pol }
$$

156? dasacatcac atgtatgtct ccasqaagc tcauygtcgg tettatagac atcactatga

1621 agtagacat tcaagagtad qteagadgt acacalccea ctaggggasg ctagattagt

4681 agtaagace lat:ggggtc tgcatacagg agaaadgac tggeactlgg gtcatggygt

474 ctceatagaa tqgaggctga augatatag cacacagta qatcetgace tagcagacca

4001 tctartcac ctgeattat ttgactgtet ttcagatct gccatagga gagccatat

4861 aggacasata qteagnceta ggtgtgade tcagcagga cataacaagg tagqactct

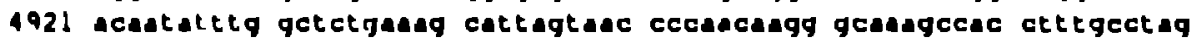

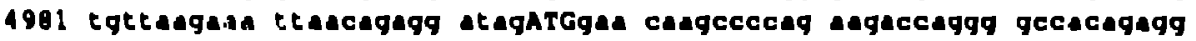
vpR $\rightarrow$

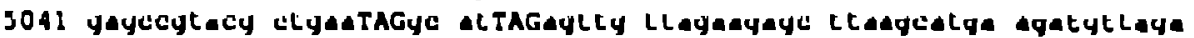
C- vph premature dtop

$$
\text { c- vir }
$$

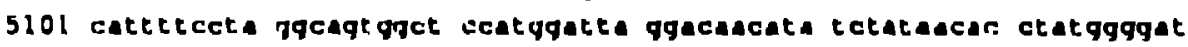

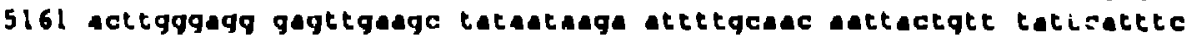

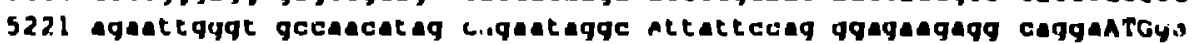
tat otart $\rightarrow$

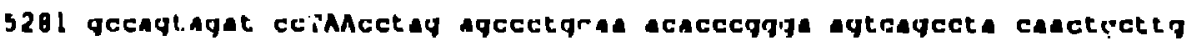
<- upR norma: : - Lup

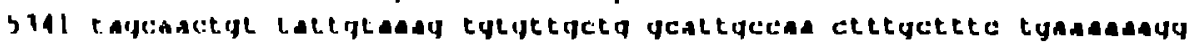

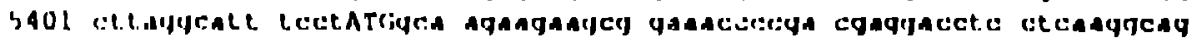
rav stiart -

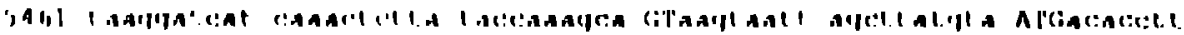

$$
\text { 5. }
$$

וינין

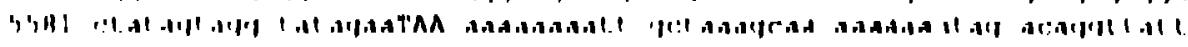
- vpll nermal at un 
5641 aAacagate agagaagag cagagacag tggcaATag agtgatgggg atacagagga env start $\rightarrow$

5701 attaccettg cttgtggaga tggggadeta tgatcttggg gt:gatasta atttglaatg 5761 cacadcadt gtgggtcacg gtctzctacg gggtacctgt gtggaagat gcagttacca 5821 ccctatttig tgcatcagat gctaagcat atgatgcaga agtgcatad gtctgggcta 5881 cacatgcctg tgtacccaca gaccccadc cacaugad agatttggte atglgacag 5941 adgatttaa catgtggaad ataacatgg tagatcagat gcatgaagat ataatcagtc 6001 tatgggacca adgcctaad ccatgtgta agt taccce tctctgcgte acgttagatt 6061 gecatadat caccattauc aacaccada acaacaccad tatcacagat ggagtgaggg 6121 aagaatgaa aattgctct ttcaatetga ccacagaact adgagatadadacagaag 6181 catatccact tcttedaga cttgatatag tacadatcad tadacegat dacadagt 6241 atagactaat aattgtaac acctcadcea ttacacaggc ttgtcccaag gegtcctteg 6301 agceaattcc catacattat tgtgccecag ctggttttgc gattctaag tgtadggatc 6361 ctgagttcaa tggaaaggg ccatgcagga atgtcagcac agtacaatgt acacatggaa 6421 teadgceagt ggtatcaact caactgctgt taatgacag tctagcagaa agagaataa

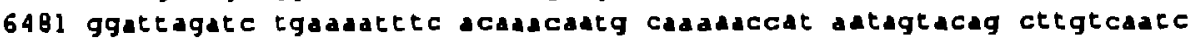
6541 ctgtadaat cadtgtccc agacctica atacagada adatatacgt aggtatagea

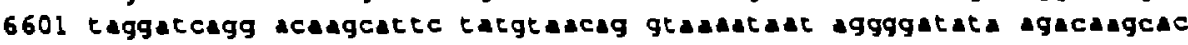
6661 attgtaatgt cagtagaagg gactggaata gaactataca acaggtagct gadcatea 6721 agdaadagt taatadeaa acaatadct ttgctagctc ctcaggaggg gatatagaaa

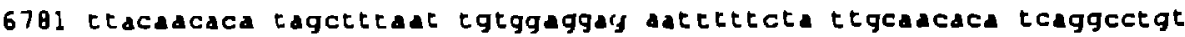
6841 thadagcat ttgqaatgqc aqcatgtcad atgacatgqg gccaatggc actataactc 6901 tccadgtag aataagcad atcatadata tgtggcagag agtaggacaa gcadgtatg 6961 cccetcccat ccaaggagta atarggtgtg atcaucat tacaggacta ctateacad 7021 gagatgyggg tactaucaet acauaaatg agaccttcag acctggagga ggagatatga

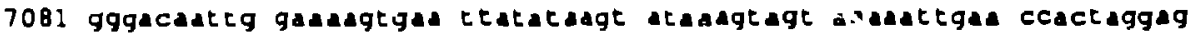
7141 tagcacccac cagggcaag agaagagtgg tggagagaga aaaagagca gttggactag 7201 gagctatct cettgggttc ttaggageag etggaagcac aatgggegeg gegtcaua 7261 cgctgacggt acaggcraga caattattgt ctggtatagt gcaacagcag agcaatctgc 7321 tgagggctat agaggctcas cagcatctgt tgaadtcac tgtctggggc attaasagc 7381 tccaggcasg ogtcctggct gtgqaaagat acctacigga tcadcagctc ctaggautt 7441 ggggetgctc tggaagctc atctgcacea ctactgtgce ctggaactet agteggagla 7501 ataatctce ggaggacata tggatauca tgacctggat gcastgggag aagaatta 756 gtagttacac aggcatalta tatcaactaa itgaggaatc gcagaaceng caggaaaga 7621 atgaactaga cttattggca teggacaat gggcaiatct gTGaattgg tetancatct <- premature env stop

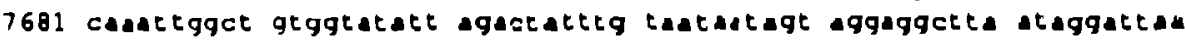
7741 gasegttet tactgtgct tctataatca atagaqteag gcagggatac tcacctotgt 7801 cqtt CAGac cettgcccea atcccagagg gtctcggcag gccaggaaga atcgaagaag 八 $3^{\prime}, 1$

7861 a agtggaga gcasggcaaa gacagarega ttcgct TAGt gagcggattc ttagcgattg $<-t a t$

7921 cetgggacga cetgeggasc etgtycetct bcagctacca cegattgaga gacttcqcet 7901 tgattgtagc gagagctgtg gaectctgg gacgcagcag cctcasggga ctgagactqg 8041 ggtgggaggg cctcastat ctgtggastc ttctgctgta ttggggtcgg gasctaasa 8101 ttagtgctat tactetgct gacgctgtag caytagcagt agctgggtgq atagataggg

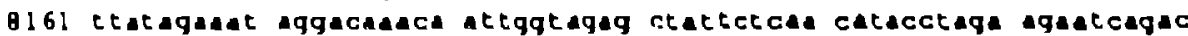

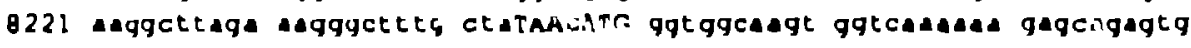

$$
r-\text { normal onv end }
$$

$$
n \in t \rightarrow
$$

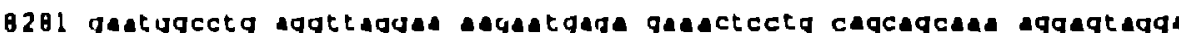

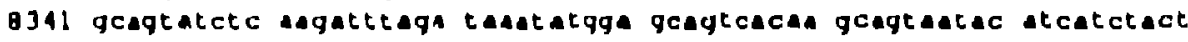
B 401 aatgecagt ytgectgget ggaegcqcas gagqaaggag acgtaggcte cccagtegg

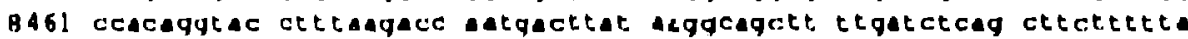

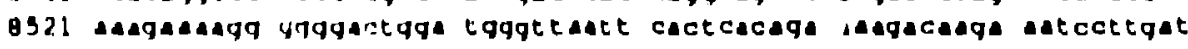
J'LTH $\rightarrow$

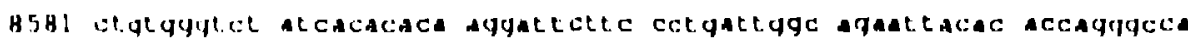

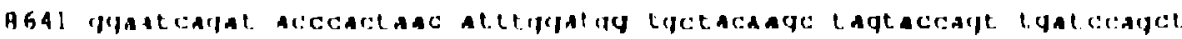

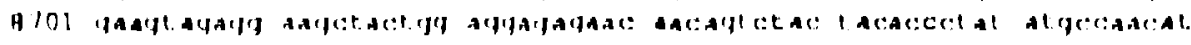

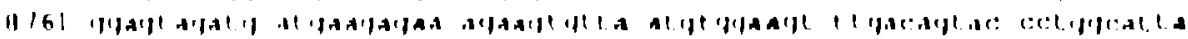

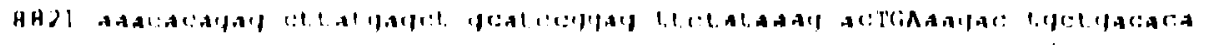
- ninl alid

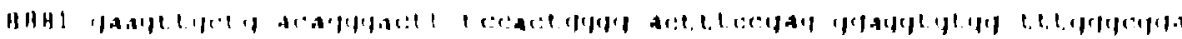
म)

I-A-124

MAY 91 
olgnal $\rightarrow$

R start $\rightarrow$

9001 Gtctctettg ttagaccaga tegagcctgg gagctctctg gctagcgagg gaacceactg 9061 cttaagcctc AATAAAgett gectigaggt guttgadgta gtgtgrgcce gtctgttatt signal $\rightarrow$

$$
<-R \text { end }
$$

9121 tgactetg' aactagagat cectcagace actatagact gtgtaaaat ctctagca 
HIVSF1701

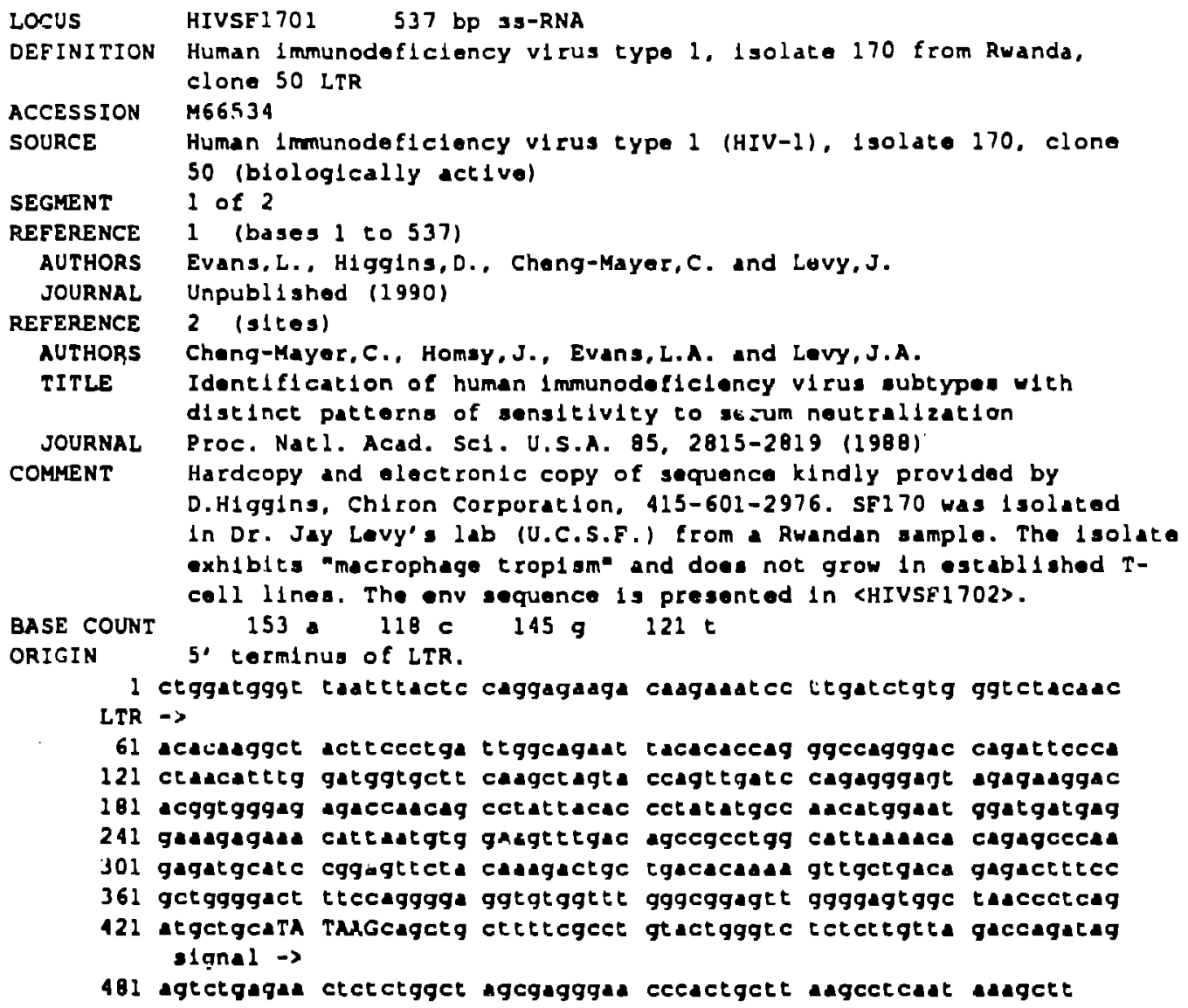

61 acacaggct actecctga teggcagat tacacaceag ggccagggac cagattccca

121 ctaacattig gatggtgcte caagctagta ccagttgatc cagagggagt agagaaggac

181 acggtgggag agaccacag cctattacac cetatatgcc aacatggaat ggatgatgag

241 gaagagade cattatgtg gadgtttgac agcegcetgg cattadaca cagagccca

301 gagatgcatc cggigttcta caagactgc tgacacaaa gttgctgaca gagacttecc

361 gctggggact ttccagggga ggtgtggtte gggeggagtt ggggagtggc tauccetcag

421 atgctgcarA TAgcagctg ctttegcet gtactgggtc tetctegtta gaccagatag sgnal $\rightarrow$

481 agtctgagaa ctctetgget agcgagggaa cceactgct aagcctcaut aagct 


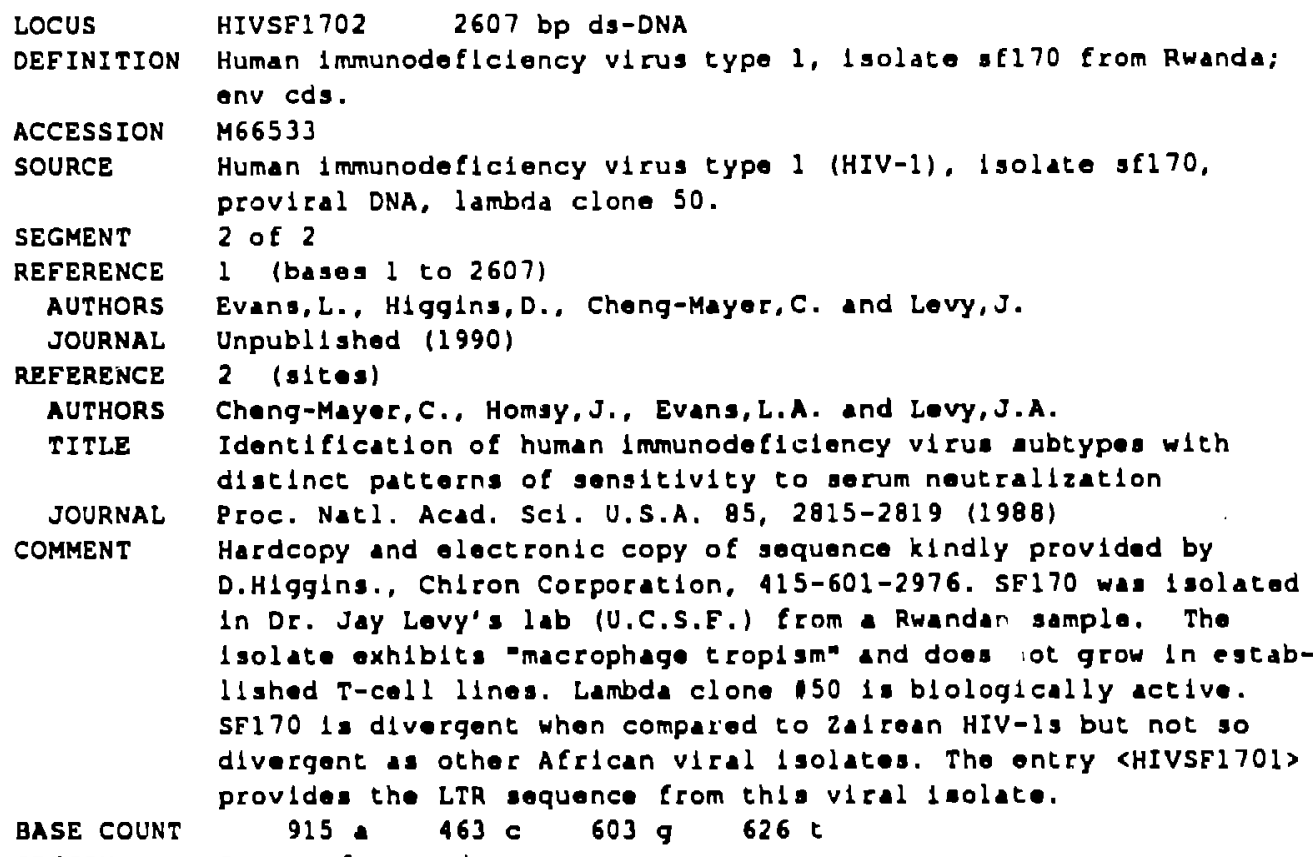
ORIGIN Stert of env cds. onv otart $\rightarrow$

1 ATGagagtga tggggataca gatgaactgt casact tat tgagatgggg auctatgatc

61 ttgggasgt taataretg tagtgetaca agcaattgt gggttactgt ctateatggg

121 gtacctgtgt qgaagacgc agagaccacc ttatttggtg catcagatgc taangatat

181 gagagagaag tgcatatgt ctgggcgaca catgectgtg taccecaga ccecgaccea

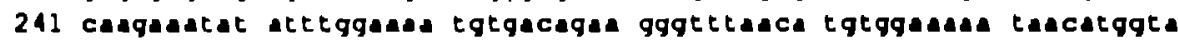

301 gagcagatgc atecagatat asteagtcta tgggaccaga gtctaeagce atgtgtaas

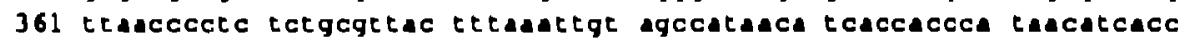

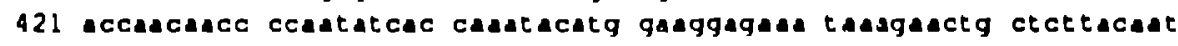

$48]$ atgaccacaj asctaaggga taagasacag aastgtate cactetetta taeactegat

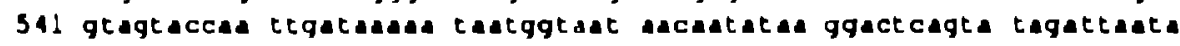

601 aattgtaata cetcagccat tacacagget tgtccaaag tatccttiga gecauttccc

661 atacattatt gtgetccage togttetgeg attctaagt gtaatgatge agagttcat

721 ggascaggge catgcasas tgtcagcace gtacatggea cacatggat caggceagta

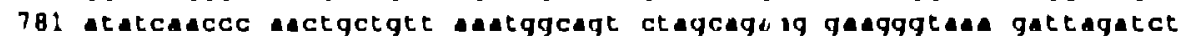

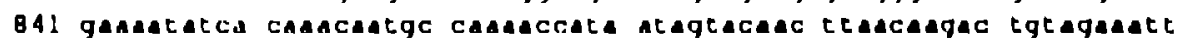

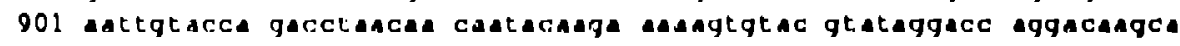

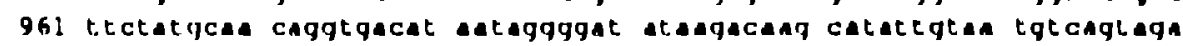

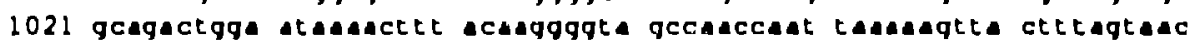

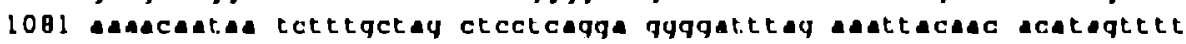

1141 astrgtggag gagaettete teattgtgat acatcaygce tgtetasag cacatyggqy

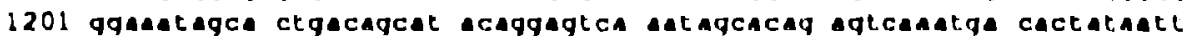

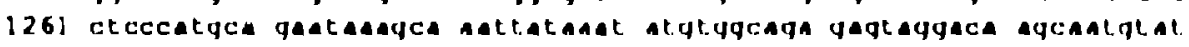

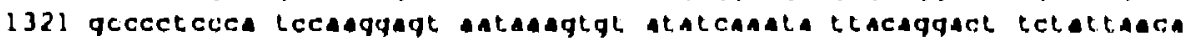

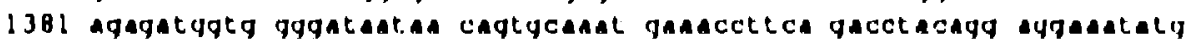

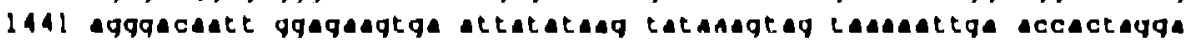

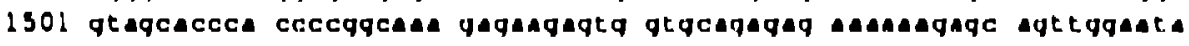

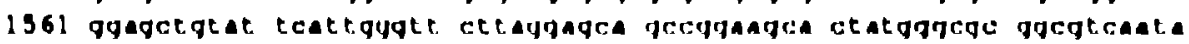

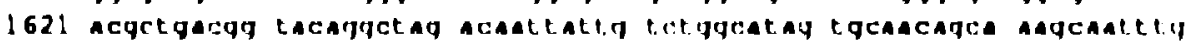

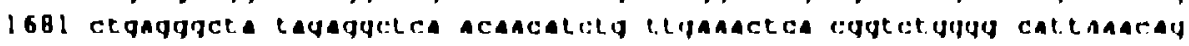

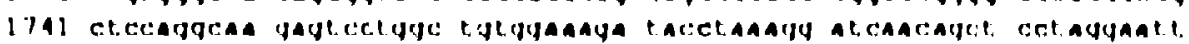

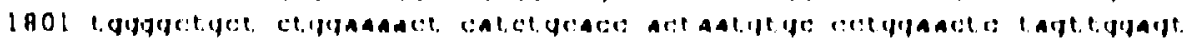

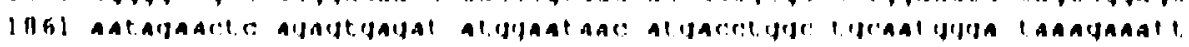

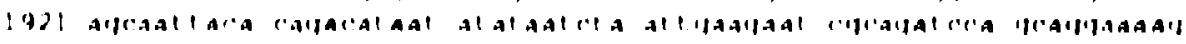

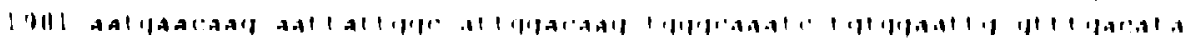

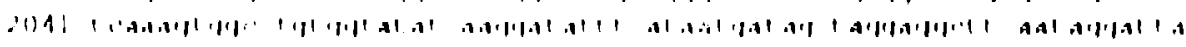

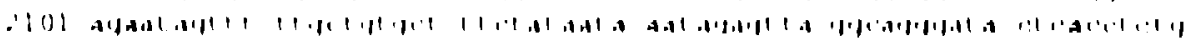

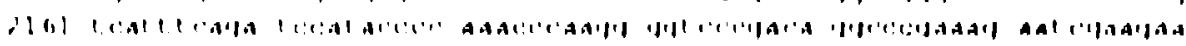

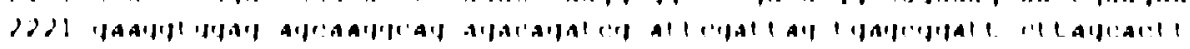




\section{HIVSF 1702}

2281 gectgggacg acetgeggag cetgtgecte treagetace accgettgag agacttcate

2341 tegategcag cgaggacegt ggaettetg ancgeagea gtetcagggg ge'gagactg

2401 gggtgggaag gectcagta cetgtggat ettetgotat attggggteg ggaactada

2461 atagtgeta ttatetigct tgataccata geadagcag tagctggetg gacagatagg

2521 gttatagaa taggacagge auttgtaga gctattetca acatacctag augatcaga

2581 cagggetteg aAgggett gteatM

<- env

I.A.12A

MAY 91 


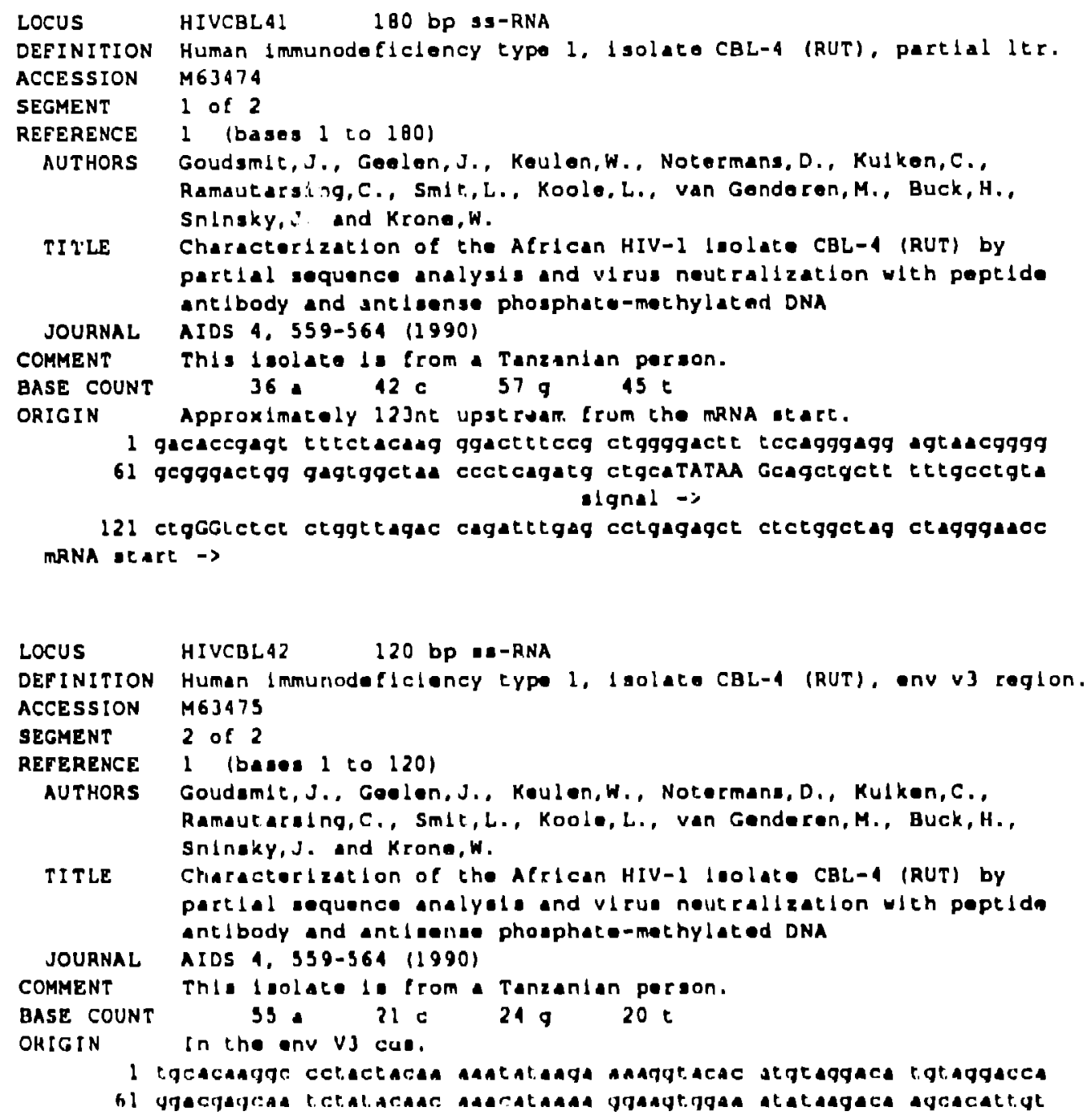


Splice sites have been determined for siv only.

net eds $\rightarrow$

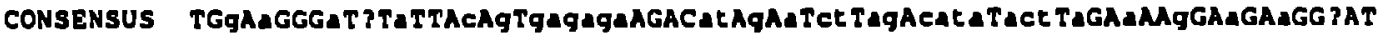
HIV2ROD HIV2NIHZ HIV2ISY HIV2ST HIV2BEN HIV2D194 HIV2GHI HIV2D203 SIVMM251 SIVMMI 42 SIVMM239 SIVMNE STVSHMHA SIVSHAPBJ --G-A---A-G-T---C-G-GMAGA----AT-A-A-CT-MA-TATA-ACT-A--A--G--A--A--G---G-A---C-G-T---T-G-CAGAGA----AT-G-A-CT-AG-CTTA-ACT-A--C--G......... --G-A---A-G-A---C-G-GAGAGA----AT-G-A-CC-AG-CATA-ACT-A--A--G--A--A--G---G-A---C-G-A---C-G-GATAGG----GT-G-G-CC-AG-CATA-ACT-A--A--G--A--G--A- --C-A---A-G-T---C-G-AGGAGG----AT-G-A-CC-AG-CATA-ACC-A--A--A--G--A--G- --G-A---A-A-T---C-G-AGGGAG----AT-G-A-CC-AG-CTTG-TCC-A--A--G--G--A--G- --G-T---A-G-T---C-G-AGGGAT----AT-G-A-CC-AG-CTTG-ACC-A--A--G--G--A--G-.--G-A---A-G-A---T-G-GAGAGA----AC-G-A-AT-AG-CACA-ATT-T--G--T--A--A--C-$--G-A---A-T-A---C-G-G C A A G A----A T-G-A-C T-A G-C A T G-A C T-A--\lambda--G--A--A--C--$ --G-A---A-T-A---C-G-GCAAGA----AT-G-A-CT-AG-CATA-ACT-A--A--G--A--A--C---G-A---A-T-A---C-G-GCAAGA----AT-G-A-CT-AG-CATA-ACT-A--A--G--A--A--C---G-A---A-T-A---C-G-GMAGA----AT-A-A-CT-AG-CATG-ACT-A--A--G--A--A--C---G-A-- -A-T-A---C-A-GAGAGA----AT-G-A-AT-AG-TATG-ACT-G--A--G--A--A--A-$--G-A---A-T-A---C-G-G A T A G A----A T-A-A-A T-A G-T C T G-A C T-A--\lambda-O G--A--A--A--$

CONSENSUS HIV2ROD

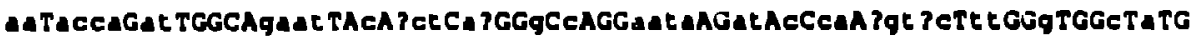
M-TGCA-AT-----GMA--C-. CT-AT--G-C---AGTA--AT-C-CA-TGTTC-TT--G---C-A-HIV2NIHZ HIVZISY HIV2ST HIV2BEN HIV20194 HIV2GHI HIV20205 SIVMM2S1 SIVMMI 42 GIVMM2J9 SIVHNE STVSMMH SIVSMMPBJ M-тCCA-AT--_-GAAC-T-. CT-AT--G-T--AGTA-GT-C-CA-TGTTC-TT--G---C-A-M-TGGA-AC----GAC--T-.CT-AT--A-C---AGTA--GT-T-CA-AGTTC-TT--G---T-A-MA-ACCA-AT--.--GAT--T-. CT-AT--G-C--AGTA--GT-C -CA-TGTAC-TC--G---C-G-M-ACCA-AT--.-GMT--T-.CT-AT--G-C---MCA--GT-C-CA-TGTAC-TC--G---C-G-MA-ACCA-AT--- -GMT--T-. CT-AT--G-C--AGTA--GT-C-CA-TGTGT-TC--G-.-C-A-. TG-GTCT-GC----MAC--T-.CT-AT=-G-C---GATA--GC-T-CC-MATAC-TT--T---C-G-СА-ACCA-AT-----GGAT--C-CCT-A. --A-C---MATT--AT-C-CA-AGACA-TT--C---C-A-СА-АCСА-AT----GA. .--C-.CT-C. --A-C---MATT--AT-C-TA-AGATG-TT--C---C-A-СА-ACCA-AT-...-GGAT--C-CCT-A. --A-C---MATT--AT-C-CA-AGACA-TT--C---C-A-СА-GCСA-AT-----GAAT--C-CCT-G. --A-C---ACCT--AT-C-CA-AGACA-TT--C---C-A-AA-ACCA-AT-----MAT--C-CAT-A. --G-C---MATT--AT-C-CT-TOCAC-AT--G---C-C-MA-ACCA-AT-----AAAT--C-CAG-A. --G-C---MTC--AT-C-CT-TGTTC-TT--A---C-C-.

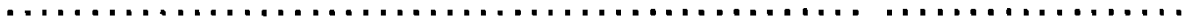

--CC-AG-AC-A---ACTG-CC--CAA-AAGGGG -G--CACT-- GACTCTC-GCT-AATG - -C -.-GC-AG-AC-A---GATG-C:C-CAA-AGGGAG. . . - T- - CAGT- . . . GACTCAC-GCT-AGTTi- - T

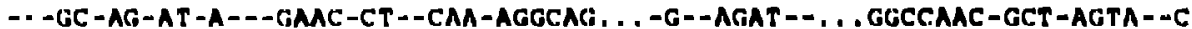
- --AC-AG-AC-A---GACA-CT - -CAA-AGGCAG . . , -G--AGTA-- . . , GACCAAC-GCT-AGTA - -C

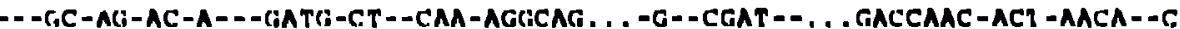

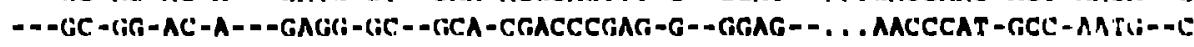
---AT-AG-CC-T---AATG-AT--CAT-AGGCAC. . . -G--GGAT - - . . GAGGCAT-ATT-AATG - -G --AT-AA-CC-T---AATS-AT--CAT-AGCCAC. . . -G--GCAT- - . . GGAGCAT-ATT-AGTG--C

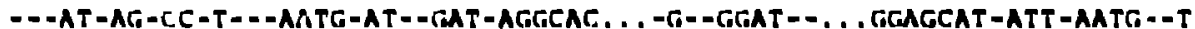

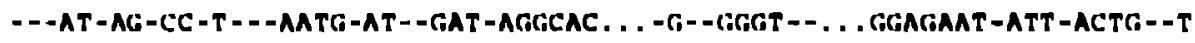

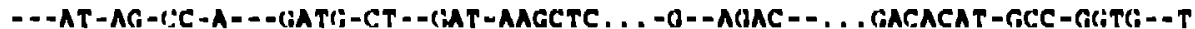




\section{IIV2/SIV LTR}

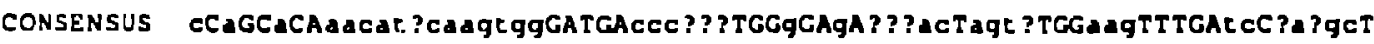
HIV 2ROD HIVZNIHZ HIVZISY HIV2ST HIV2BEN HIV2DI94 HIV2GHI HIV2D205 SI VMM 21 SIVMMI 42 SIVMM2 39 SIVMNE 5 IVSHMH 4 SIVSMMPBJ C-A--A--AACAAGCAAGTTT-----CCCGCA---G--G-. .CAC-AGTC---GAG-----TC-CTTGC-

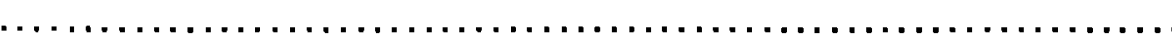
T-A--A--AGTMAGCAGATTT-_---CCCGCA---G--G-. . CAC-AGTC---MAG-_---CC-CATGC-

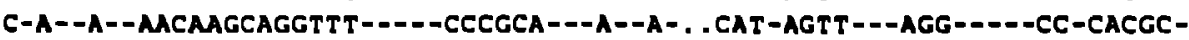
C-A--A--AACAAGCAGACAT-----TGAGCA---G--G-. . CAT-AGTG---CAG-----CT-CATGCC-A--A--MCAACCAGATAT------CGAGCA---G--G-. . CAC-AGTT---CGG-----CC-CATGCC-A--A--MACMAGCAGACAT-----CGAGCA---A--A-. . CAC-ACTT---CGG-----CC-CACGCC-G--A--GATCTCCTCATGR-----CATCCA---G--G-. . CTC-TATC---CAG-----TT-CCTCCC-A--T--AACTTCCAAGTGG-..--CCCT...---G--G-GGTTC-AGCG---MG-_---TC-MACTCC-A--T--AACTTCCEAGTGG-...-_CCT. . ---G--G-GGTTC-AGCA---MG-----TC-AACTCC-A--T--AACT TCCCAGTGG---_-CCCT. . --_-j--G-GGTTC-AGCA---MG-_-_-TC-MACTCC-A--T--AACTTCCCAGTGG-----CCCT. . ---G--G-GGTTC-AGTA---MAG-----TC-AACTCC-G--A--GACTTATCAGTGG-----CCCC. . ---G. -G-GGTAC-GGCA-.--MG-.---TC-AGMTC-A--A--AACTTCTCAGTGG-----CCCC., ---G--G-GGTAC-GGCA---MG-----TC-MAGT-

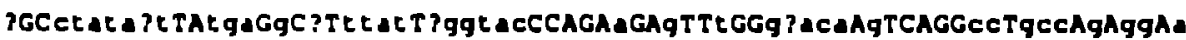
G--TTATAGT--CGA-G-T-TTAT-CGGTAC-----G--A--T--GCACA-G-----CC-GCC-G-GG-A HIVZNIHZ HIVZISY HEV2ST H!VZUEN HIV2DI 94 HIV2GHI HIV:D205 S! VMM2 5 ! SIVHH! 42 S I VMM 39 S: VMile SI VSMMHA SIVSMMPEJ ............G-T-ITAC-CTGTAC--.--G--A--T-GCATA-T-----AC-GCC-G-GA-A G--TCATGAG--CAC-A-C-TTAT-CTATAC----G-G-A--T--GCACA-G-----AA-GGA-G-AG-T A--TTTTAGC--CGA-G-C-TTAT-CGATAC-----G--G--T--GTACA-G-----CC-GCC-G-GG-T G--CTATAAC--CAA-G-C-TCAC-CTGTAC-----A--G--T--GCACA-G-----AT-GCC-G-GA-A G--CTATAGT--CMA-G-C-I'CAT-CTCCAC-----A--A--T--GCACA-G-----AT-GCC-G-GA-A G--CTATGAT--CAM-G-T-TCAT-CTGCAT-----A--G--T--GCACA-G-----AT-GCC-G-GA-A G--ATATGAT--TGT-G-T-TCMA-AGGTTT-----A--G--C--GTACC-G-----GC-ACC-G-GG-G A--CTACACT - TGA-G-A-ATGC-AGATAC-----A--G--, --AAGCA-G---.-CC-GTC-G-GG-A A--CTACACT- - TGA-G-A-ATAT-AGATAC---.-A--G--T. -AMGCA-G---.-CC-GTC-G-GA-A G--CTICACT--TGA-G-A-ATGT-AGATAC-----A--G--T--AMGCA-G-----CC-GTC-G-GG-A A--CTACACT---TGA-G-A-ATAT-AGATAC--..--A--G--T--AAGCA-G-----CC-GTC-G-GG-A A--TTATAGC-- TAA-G-A-TTAT-AAGTAC-----A--G--T--TACTA-G-----CT-GTC-G-GG-A A--TTATAAC--TAA-G-A-TTGT-GAGCAC--.--A--G--T--TAGTC-G-----CT-GTC-A-GG-A

\section{<- net}

CONSENSUS IIIVAHOD HIVON IHZ, IIIVZ:SY HIVIST II:JiHE.N 11: '习i)l 34 11: yxiall 11:20:17n: :: :4M231 :: :Јмм। 12 : : : УMMJ J : : JMNE. : : V:SMmHA

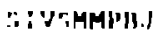

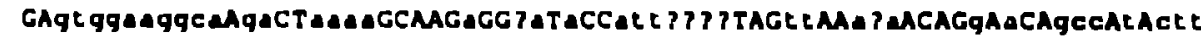
--GTGGAAGGCG-GA--GAMA-----A--MA-A--ATT....---TT--A.G----G-A--GCT-T-CTT --GTGGAAGGCG-GA--GAMA-----G--MA-A--ATT.....---TT--A.A----G-A--GCT-T-CTT --CTGGAAGGICA-AA--GAAA--- -A--GA-A--ATT. . . -.-TT--A.A----G-A--ACC-T-CTT --ATGGNAGGCA-CIA--GAAA------A--GA-A--GTT.....---CT--A.A----G-A--GCT-T-CTT - -ATGGAAGGCA-AA--GAAA---.-A--GA-A--ATA. . . - - -TG--T.A----G-A--ACC-T-CTT --GTTGGAAGGCA-AA--(iAAA-----A--GAA-A--ATA....---TG--T.A----(;-A--ACC-T-CTT

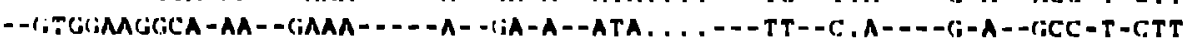

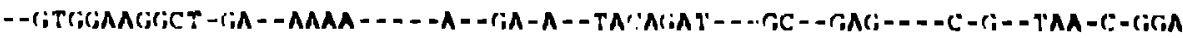

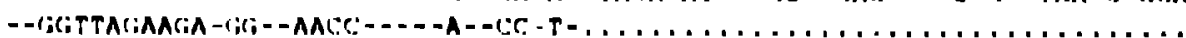

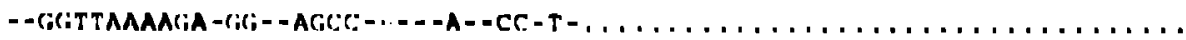

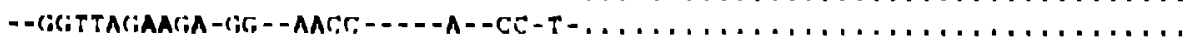

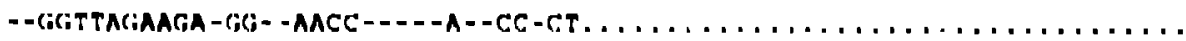

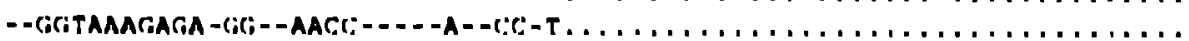

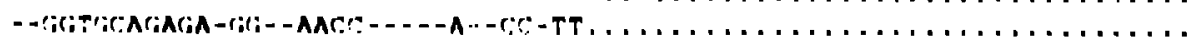

4

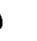

1

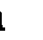

.

9

341 
enhancer

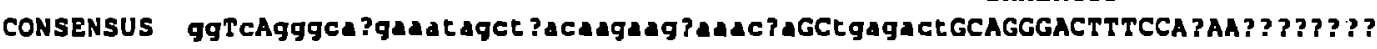
HIV2ROD HIV2NIHZ HIV2ISY HIV2ST HIV 28EN HIV2DI 94 HIV2GHI HIV20205 SIVMM251 SIVMM1 42 SIVMH239 SIVMNE SIVSMMH 4 SIVSMMPE

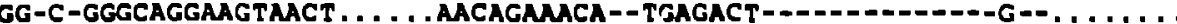

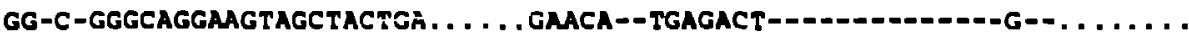

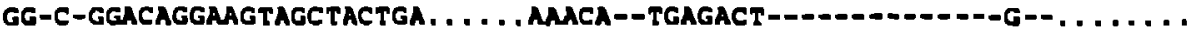
GG-C-GGGCAGGAGTARTTMCAGA . . . . ANACA--TGAGACT-_-

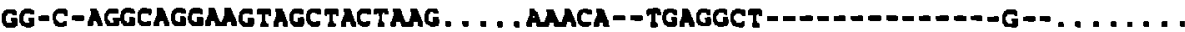

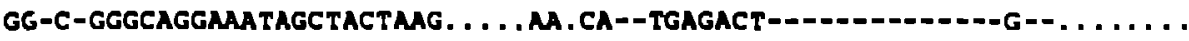

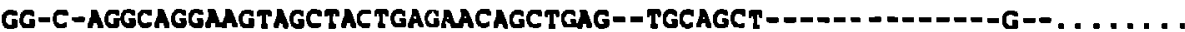

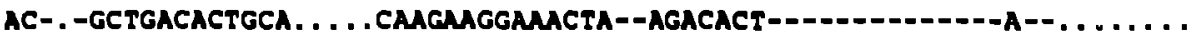

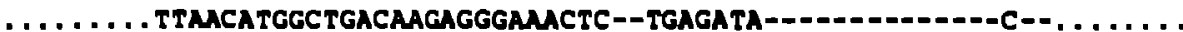

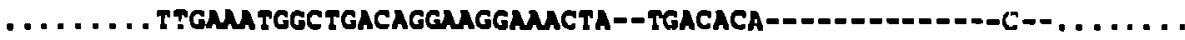

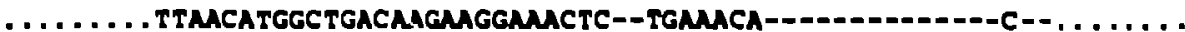

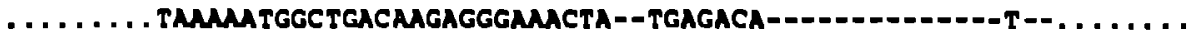

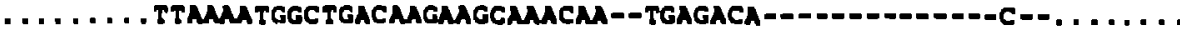

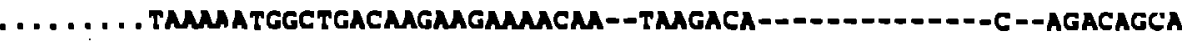

CONSENSUS HIV2ROD anhancer sp-1 5p-1 sp-1 HIVZNIHZ HIV2 IsY HIV 25T HIV 2BEN HIV 2D194 HIV2GHI HIV 20205 SIVMH251 SIVMAI 42 SIVMH2 39 SIVMNE SIVSMAHA SIVSKMPBJ

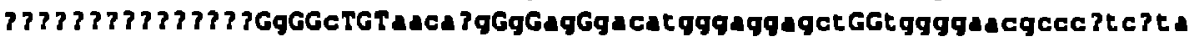

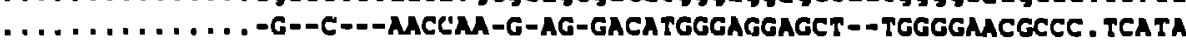
. . . . . . . . . -G--C--AACCAG-A-AG-GACATGGGAGGAGCT--TGGgGAACGCCCTTCATA . . . . . . . . . . -G--C---AACCAG-G-AG-GACAtgGGAGGAGCT--TGGGGACGCCC. TCATA . . . . . . . . . . -G--C-.-TACCAG-G-AG-GACATGGGAGGAGCC- - TGGGGACGCCC . TCATA . . . . . . . . .-G--C---AACCAA-G-AG-GACATGGGAGGAGCT--TGGGGAACGCCC. TCATA . . . . . . . . . , -G-C-.. -ACCMA-G-AG-GACATGGGAGGAGCT--TG. . . . . . . . TCATA . . . . . . . . . -G--C---AACCAM-G-AG-GACATGGGAGGAGCT--tGGGGAACGCCC . TCATA . . . . . . . . . -A--C---NACAT . -G-AG-AGCAMGGGGGGGTT- - TTGGGACGCCC . ATTAA . . . . . . . . . -G-A---T . ATG-G-AG- . . . . . . . AGCC- - TCGGGAACACCC. ACTTT . . . . . . . . -G--A---C. .ATG-G-AG-TACTGGGGAGGAGCC--TTGGGACACCC.ACTTT . . . . . . . . . . G--A--T. . ACG-G-AG-TACTGGGGAGGAGCC--TCGGGAACGCCC. ACTTT . . . . . . . . . . .G--A---C . .ATG-G-AG-TACTGGGGAGGAGCC--TCGGGNACACCC . ACTTT

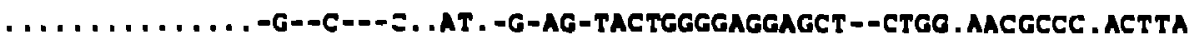
GGGACTTTCCACAA-G--A-_-T . . ACG-G-AG-TACTGGGGAGGAGCT--CTGG . MCGCCC . ACGTA Iqnal $\rightarrow$ mRNA $\rightarrow$ la- car core I

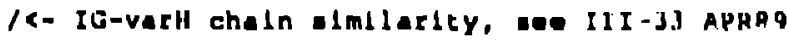

CONSENSUS HIV 2ROD cTt

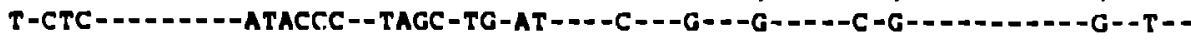
HIV2NIHZ HIV 2 ISY IIV25T HIV2BEN HTV2D194 IIV 2GII HIV 2020S SIVHA23! SIVMMI 42 II VMH? 19 S IVMNF: s [VIAMHII

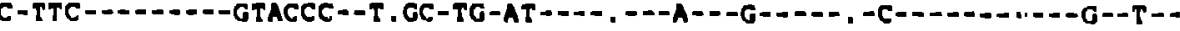

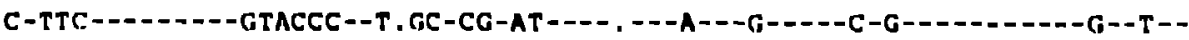

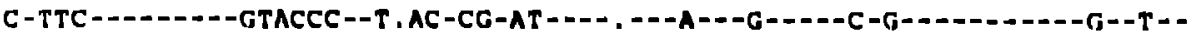

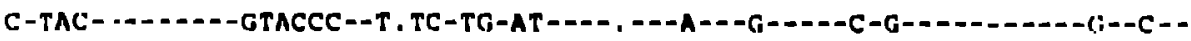

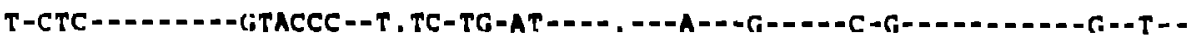

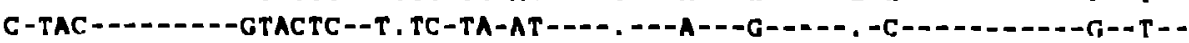

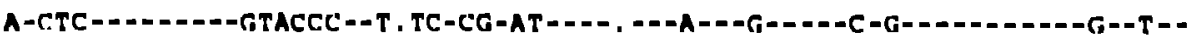

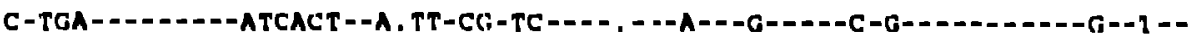

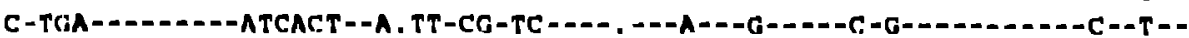

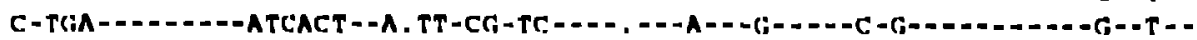

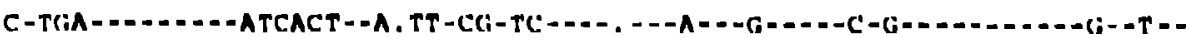
s[VIMMPI]

360 386 356 501 585 585 514 509 591 $3.16^{\circ}$ 545 5411 548 345 510 


\section{HIV2/SIV LTR}

tar core I

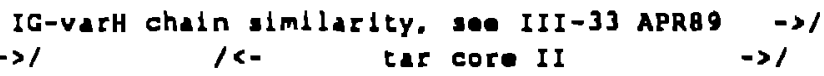

I/ 5' a

CONSENSUS GCCCTGgGAGGTTCTCTCCAGCACTAGCAGGTAGAGCCTGGGTGTTCCCTGCTAGACTCTCACCAGcaCT HIV 2ROD HIV2NIHZ IIIV2ISY HIVZST HIV2BEN HIV 2DI94 HIV 2GHI HIV 20205 SIVMM25I SIVMMI 42 SIVMM2 39 SIVMNE

SIVSMMH 4

IVSMMPBJ

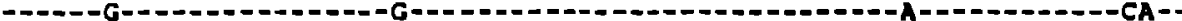

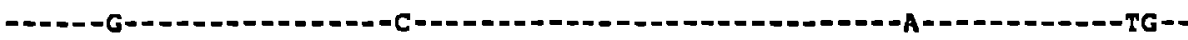

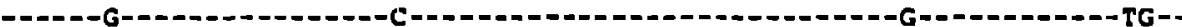

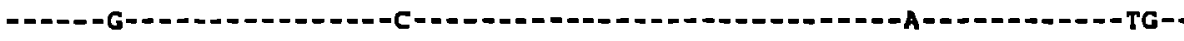

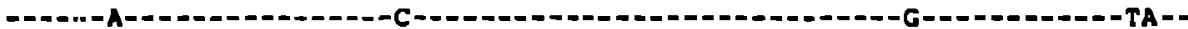

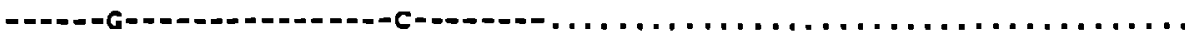

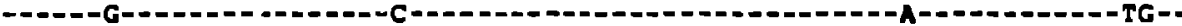

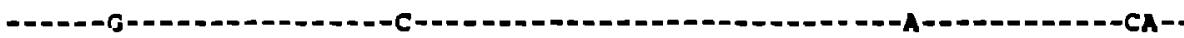

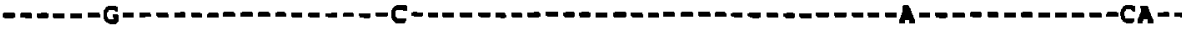

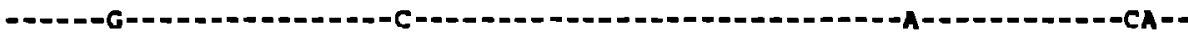

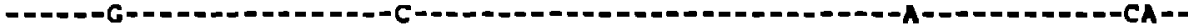

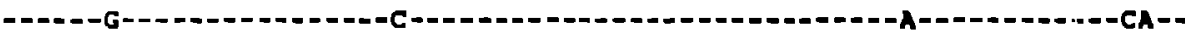

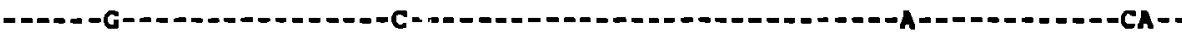

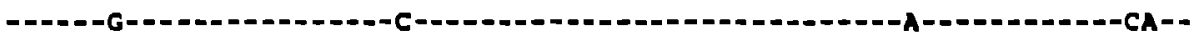

$\operatorname{lgnal} \rightarrow$

CONSENSUS tGGCCgGtgCTGGGCACA ?GgCECCACGCTTGCTTGCTTAAA gacCTCETCAATAAAgCTGCCA ?TT HIV 2ROD HIV 2NIHZ HIV2ISY HIV2ST HIV 2 BEN HIV20194 HIV 2GHI HIV20205 SIVMM25 1 SIVMMI 42 S IVMM2 J9 SIVMNE. STVSMHH4 SIVSMMPUJ

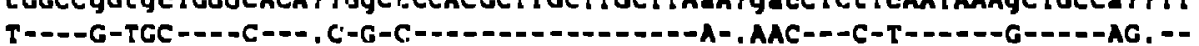

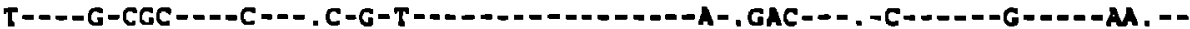

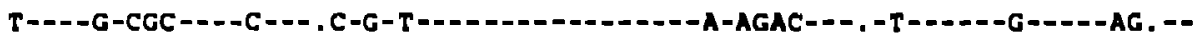

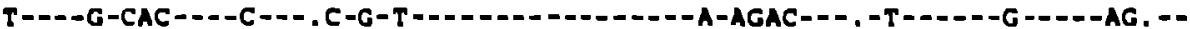

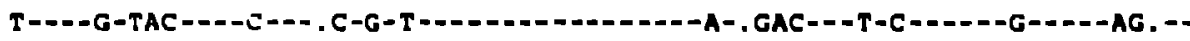

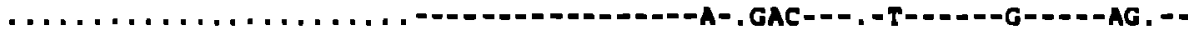

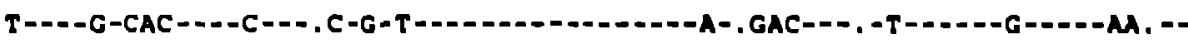

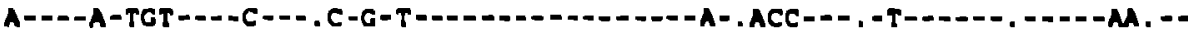

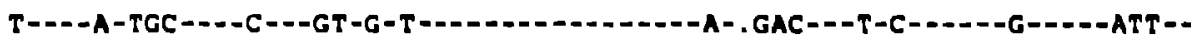

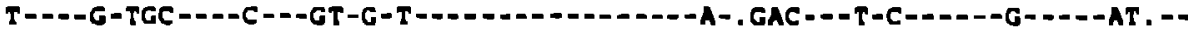

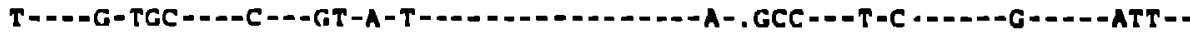

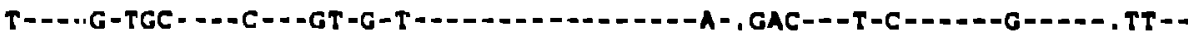

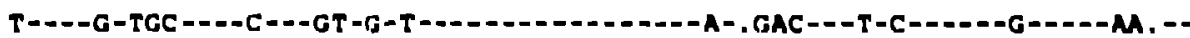

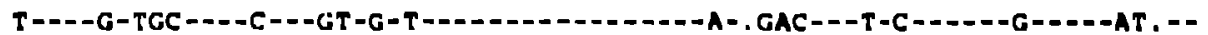

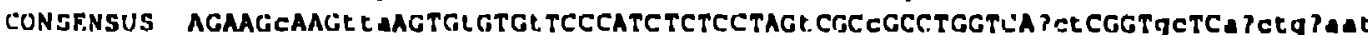
IIIVTHOD II V.NIIH\%. IIIVIISY IIVTST IIV IIIFN IIIV20194 IIIVJCII! IIV.TU20s SIVMA2SI IIVMM1 42 :IIVMM2 39 :IVMNF.

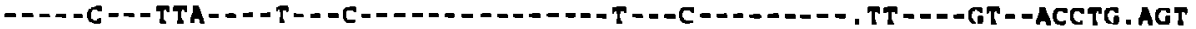

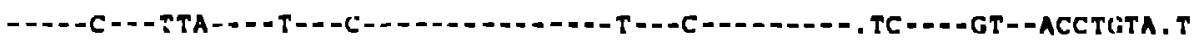

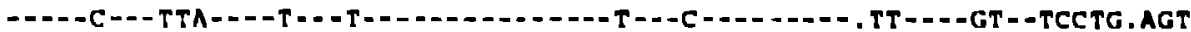

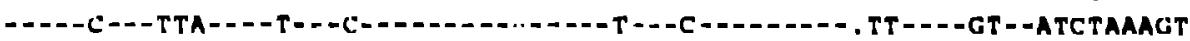

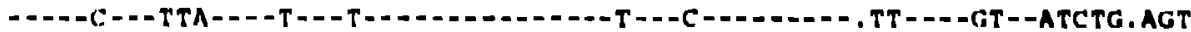

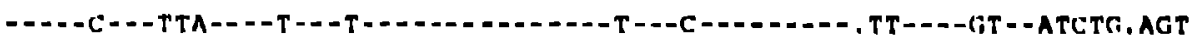

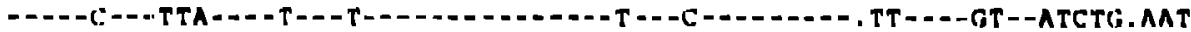

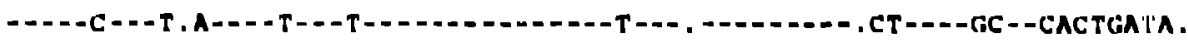

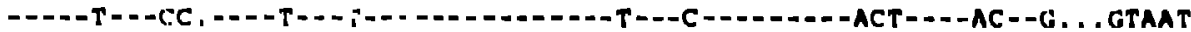

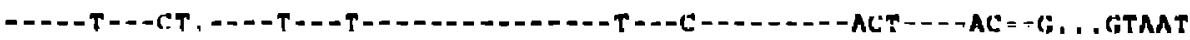

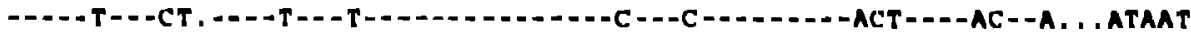

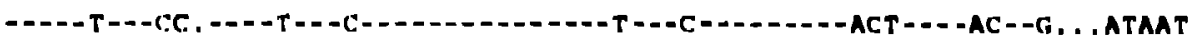

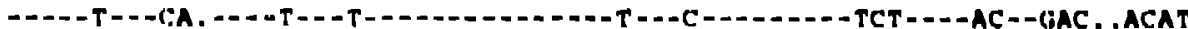




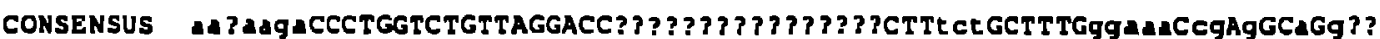
HIV2ROD HIV2NI HZ HIV2ISY HIV2ST HIV2BEN HIV2DI9A HIV2GHI HIV2D 205 SIVMA251 SIVHA142 SIVMM239 SIVMNE SIVSMMH4 SIVSMMPBJ

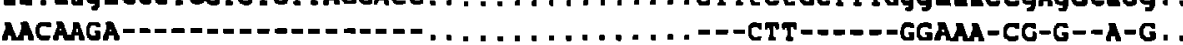

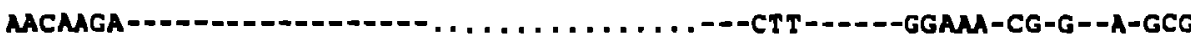

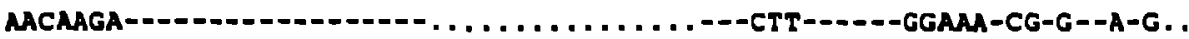

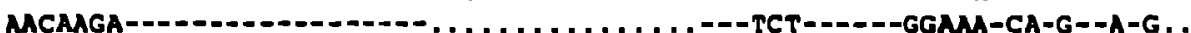

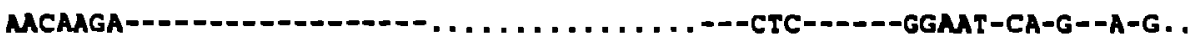

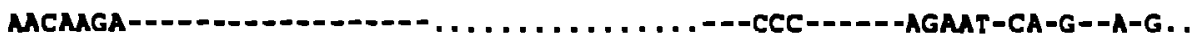

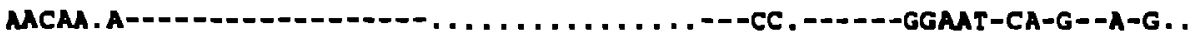

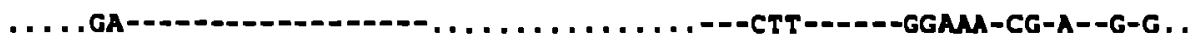

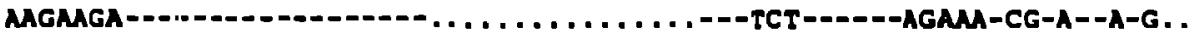

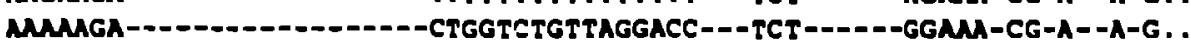
MGNGGA-2_-_-_-_-.. . . . . . . . . . . .--TCT-_-_-GGMM-CG-A--A-G. .

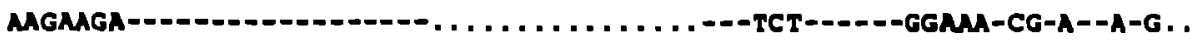

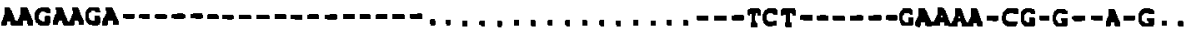

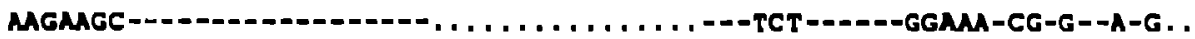

\section{<- 1ys-tRMA pbs}

CONSENSUS PMMATCCCTAGCAGqTTGGCGCCggACAGGgACtTGaggaGa ?qTGAGAgact cct ?GAgCACgG HIV2ROD HIV2NIHZ HIVZISY HIV2ST HIV2BEN HIV20194 HIVZGHI

IIV20205

SIVMM251

SIVMA 142

SIVMM239

SIVMNE

SIVSHMHA

SIVSHMPBJ G-DG. . . . . . . , - , - , . -

CONSENSUS HIVIROD

TgAGTGAAGGCAGt MAGGgCGGCAGGMACAAACCACqACGGAGtGCTCCTAgAMAgGCGCgGGccgaggt

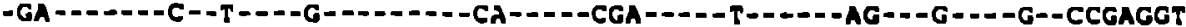
HIVIN I Hz HIVZISY HIV2:T HIV2BEN HIVZOI94 IIIV2GHI HIV2D20S SIVMM25I SIVMI4I 42 SIVMH2 39 SIVMNE SIVSHMII4 S IVSMMP|H,J

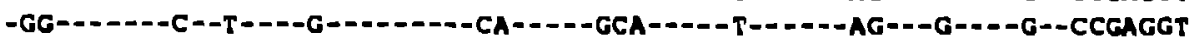

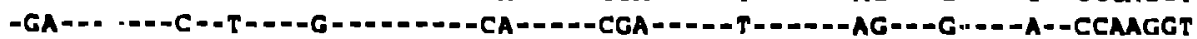

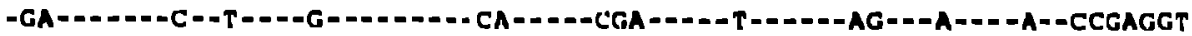

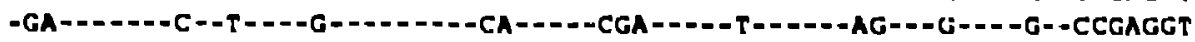

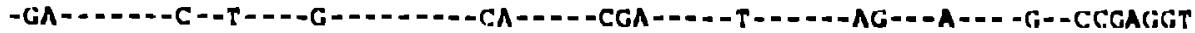

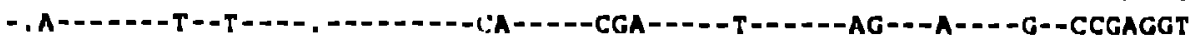

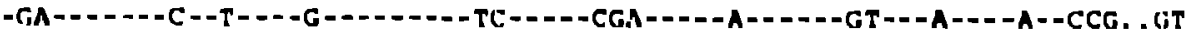

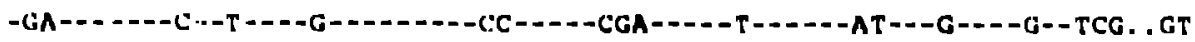

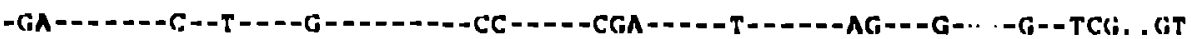

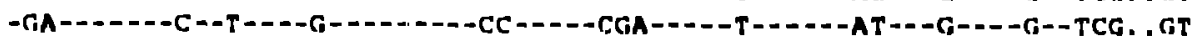

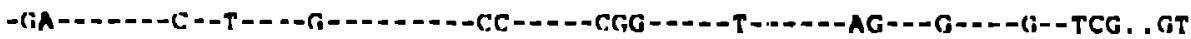

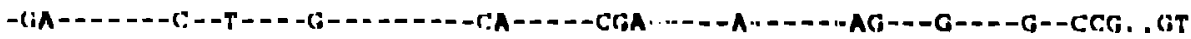




\section{IIV2/SIV LTR}

major 5' is $\backslash$

CONSENSUS ACA??ggegGCGrGaGGagcgggagt ?gaaGAGgCcTCCgGgt ?gadg?GEAAGTgC?t?C3?caAda HIV2ROD HIV2NIHZ HIV2ISY HIV2ST HIV2BEN HIV2D? 94 HIV2GH1 HIV2D205 SIVMM2SI

SIVMMI 42

SIVMM239

SIVMNE

SIVSMMH4

SIVSMMPBJ A-CAAAGGA-C-T-T--AGCGGGAGGAGAA---G-C---G-GT.GAAG. -T----ACCTACACCAAAAA
A-CAMAGCG. -C-T-T--A . CGGGAGTCGA. ---G-C---G-GT.GAGG-T----HCCTACACCAAMA A-CMMGGCG-C-T-T--AGCGGGAGTCAM. ---G-C---G-GT.GMG. -T----ACCTACACCAMAAA A-CMAGGGG-C-T-T--AGCGGGAGTGAMA---G-C---G-GT. GMG. -T----GCCTACACCAMTA A-CAMGGCG-C-T. T--AGCGGGAGTGAAA---G-- ---G-GT.GMG, -T----ACCTACACCMAAA A-CGMAG.CG-C-T-T--AGCGGGAGTGAMA---G-C---G-GT.GAAG. -T----ACCTACACCGAMA A-CAMGGCG-C-T-T--AGCGGGAGTGMAA---G-C---G-GT . GMG. -T----GCCTACACCGAAAA A-CA. .GGCA-C-T-A--AGCGGGAGGAGM---G-. ---G-GAGTAAG. -T----ACTCC. . . . . AAA A-CA. . GACG-C-T-A--AGCGGGAGAGGAG---G-C---G-TT. GCAG. -T----G. . . CAACACAAA A-CA. . GACG-C-T-A--AGCGGGGAGAGAA---G-C---T-GTTGCAG. -T----G. . . . CAACACAAA A-CA. . GACG-C-T-A--AGCGGGAGAGGM---G-C---G-TT. GCR, -T----G. .. . CAACACAAA A-CA. . GACG-C-T-A--AGCGGGAG. AGAA---G-C---G-TT. GCAG. -T----G. . . CAACACAAA A-CA. . GGCG-C-T-A--AGCGGGAGTCGGA---G-C---G-TT. GCAG. -T----G. . . CAMCAGAAA

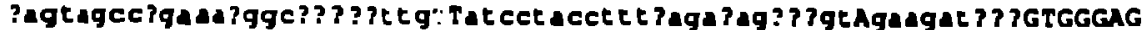
CTGTAGCC.GAMGGGC. . . . . TTGC-ATCCTACCTTT. AGACAG. . . GT-GMAGATT. .

CTGTAGCCAAAGAGGGC..... . TTGT-ATCCTACCTTT. AGACAG. . . GT-GMAGATT . - -

CTGTAGCCGGAMAGGC. . . . . TTGT-ATCCTACCTTT . AGACAG . . . GT-GAAGATT . . -...-.-

CAGTAGCCAGAA. GGGC. . . . . TTGT-ATCCTACCTT . AGACGG . . GT-GAAGATT. . . . . . . .

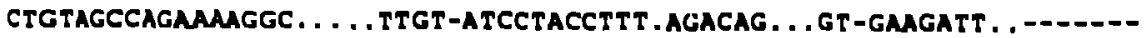

CTGTAGCCAGAAMGGC. . . . . TTGT-ATCCTACCTTT . AGACAG. . . GT-GMAGATT . . ......-

CCGTAGCCAGMAAGGC. . . . . TAGT-ATCCTATCTTTTAGACAG... .GT-GAAGATT. . -...--TTTT.GC............... AT-GTC. TACTCTMMGAGGGA . . GT -GGGCATMA. -...-.MA . . . . . . GAATAGC. TGTCTTGT-ATCC. AGGAAGG. GATA. . . . . . -TAAGATAGA-.....

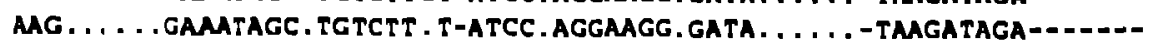
AAA .... . . GAAATAGC. TGTCTT . T-ATCC. AGGAAGG. GGTA. . . . . . TMGATAGA-...-MA ... . . . GAGATAGC. TGTCTT . T-ATCC. AGGAGG . GATA. . . . . . -TAMGATAGA-...-.. AGT. . . . . . CATAGGACTGAG. . . T-CCCT. ACtTTTGAGGAMAGA. GT-GGAGA. . . . . . ...-.

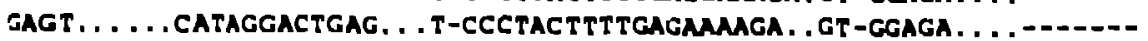




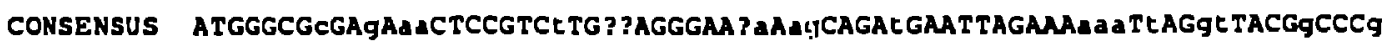
HIVZROD HIVZNIHZ HIV2ISY HIV2ST HIV2BEN HIV2D194 HIV2GHI HIV2D205 SIVMA251 SIVMM1 42 SIVMM239 SIVMNE SIVSMMH4 SIVSMMPBJ

CONSENSUS HIV2ROD HIV2NIH2 HIV2ISY HIV2ST HIV2BEN HIV2D194 HIV2GH HIV20205 SIVMM25I SIVHM142 SIVMM239 SIVMNE SIVSMHHA SIVSMMPEJ

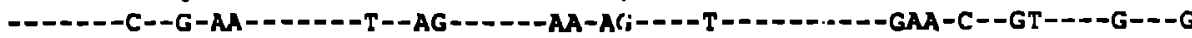

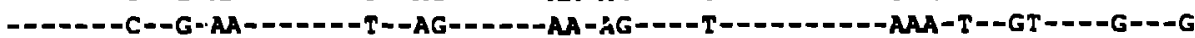

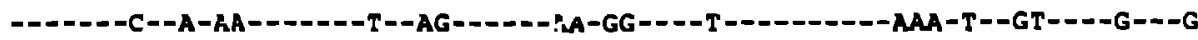

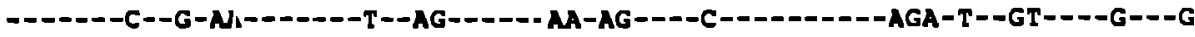

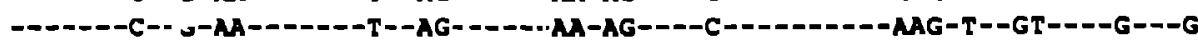

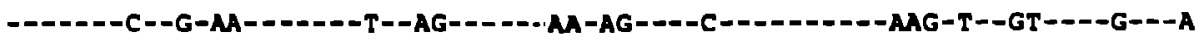

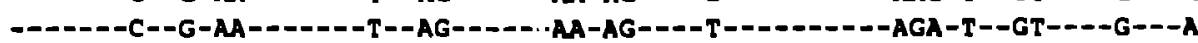

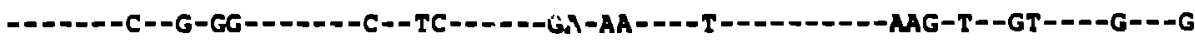

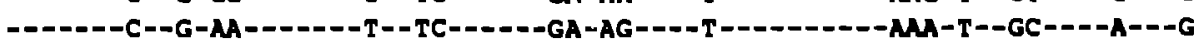

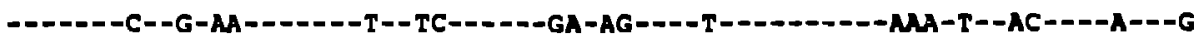

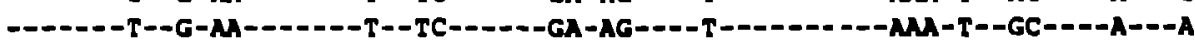

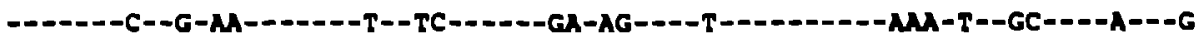

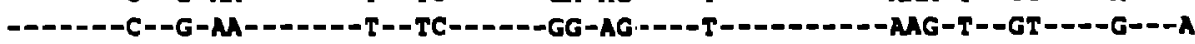

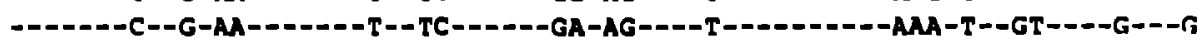

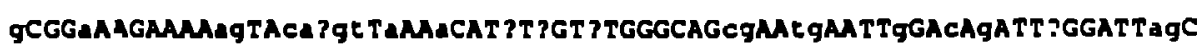

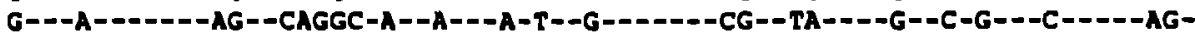
G---A---D--AG--CAAGT-A--A---A-T--G-------CG--TG----G--C-G---C-----AG-

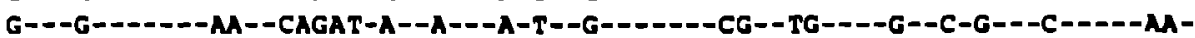
G---A-----AA--TAGGC-A--A---A-T--G-------CG--TG----G--C-G---C-----GGG---A--1---AG--CAGGT-A--A---A-T--G----1--CG--TG--2-G-T-A---C---- GGA---A------GA--CAGGT-A--A---G-T--G-------CG--TG----A--C-G---C-----GGG- -A-1----AG--CAGGC-A--A---A-T--G-------CG--CG----G--C-A---C--n-GGG---A---D-AG-- TATGC-A--A---G-A--A------TG--CG----A--C-G---T-----AG-

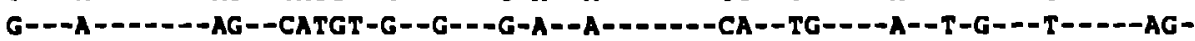

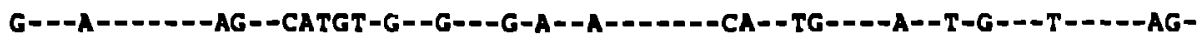
A---A---1--AG--SATGT-G--G---G-A--A-------CA--TG----A--T-G---T-----AGG---G------AG--CATGT-G--G---G-A--A-------CA--TG----A--T-G-D-T-----AGA---A-------AG--TATGT-G--G---G-A--A =------CA--TG----G--C-G---T-----AG-

CONSENSUS HIV2ROD HIV2NIH2 HIV2ISY HIV25T HIVZBEN HIV2DI 94 HIV2GHI HIV20205 SIVMM251 SIVHM142 SIVMM2J9 S.TVME SIVSHMHA G---A------GG--CCAGC-G--G---A-A--A---D--CA--TG----G--C-G---T-----AG-

AGAgAGCCTGTTGGAgtCAAAAGAGGtTGtCAMAAATt CTE ? CaGTt TTAG? CCAt TaGTaCCAACA

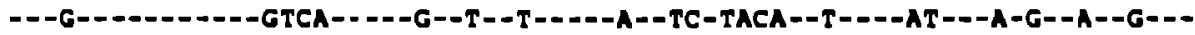

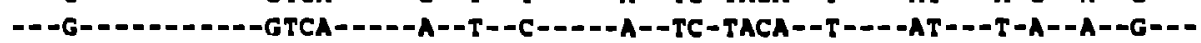

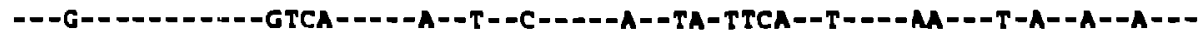

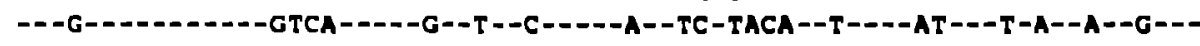

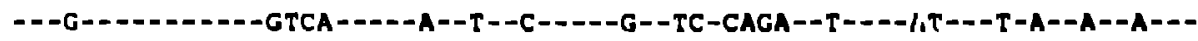

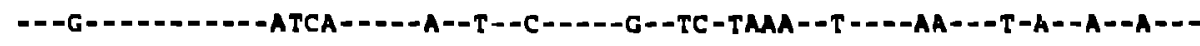

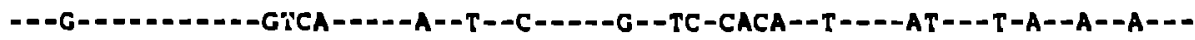

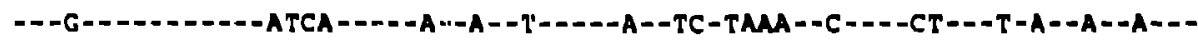

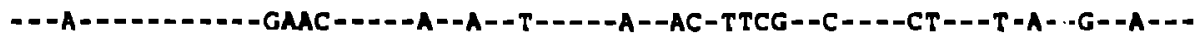

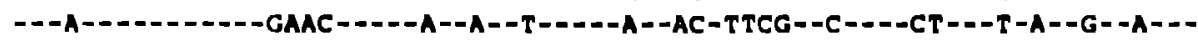

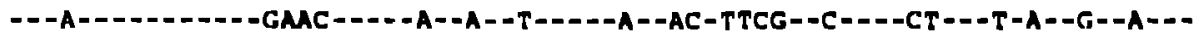

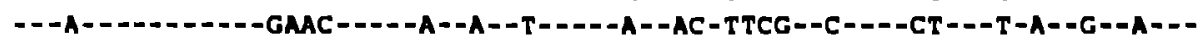


CONSENSUS HIV2ROD HIV2NIHZ HIV2ISY HIV25T HIV2BEN HIV2D194 HIV2GH1 HIV2D205 SIVia1251 SIVMMI 42 SIVTa4239 SIVANE SIVSHAH4 SIVSHAMBJ

CONSENSUS HIV2ROD HIV2NIHZ HIVZISY HIV2ST HIV2BEN HIV2D194 HIV2GH1 HIV2D205 SIVMM251 SIVMA1 42 SIVHA239 SIVMNE SIVSHMHA SIVSHAPBJ

CONSENSUS HIV2ROD HIV2NIHZ HIV2 ISY HIV2ST HIV2BEN HIV2D194 HIV?GH 1 HIV20205 SIVMH251 SIVMM1 12 SIVMH2] 9 SIVTANE SIVSMMH. SIVSHMPIIJ
GG PTCAGAAALTTAMAAGCCTTT?TAALACtgtCTGCGTCATE TggTGCAT ?CACGCAGAMGAGAMAG

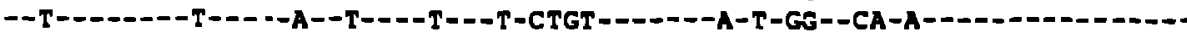

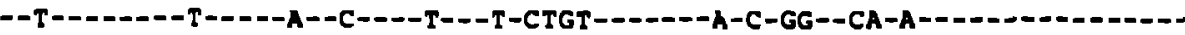

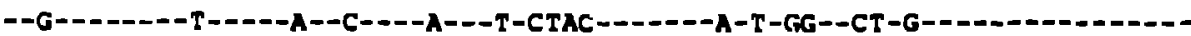

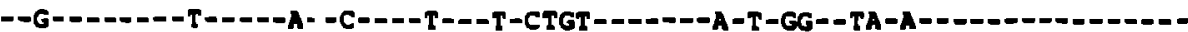

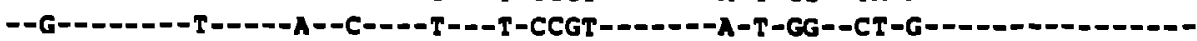

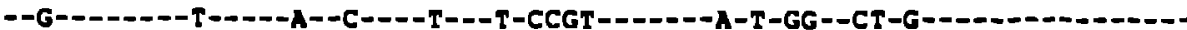

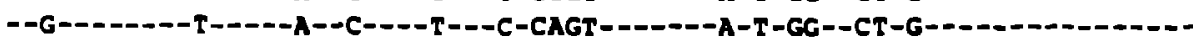

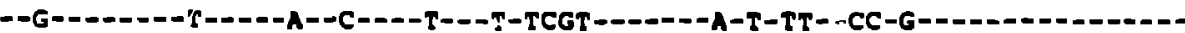

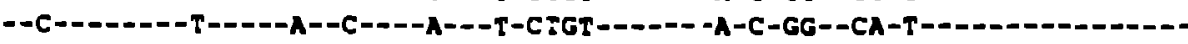

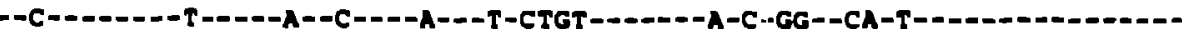

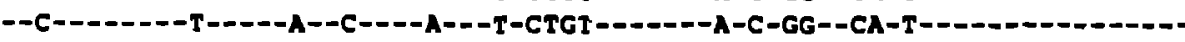

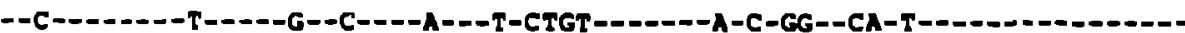

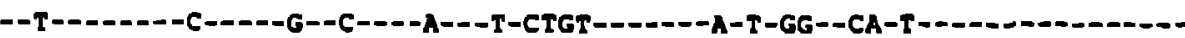

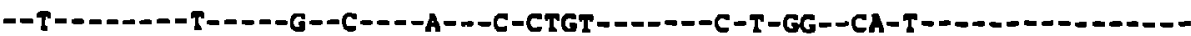

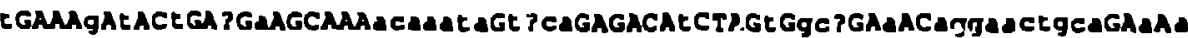
T----G-T--T--A-G------ACMMTA-TGCS---1--T----T-GCA--A--AGGMACTGCA--G-A T----G-T--T--A-G------ACMATA-TACA--.-.-T----T -GCA--A--AGGMACTGCA--G-A T----G-T--T--A-A------MGAMTA-TAGG------T----T-GCA--A--AGMACTGCA--G-A C----G-T--T--A-A-_----ACMAAG-TACA-_--_-T-.--T-GCA--A--AMACTACA--A-A T----G-T--T--G-A------GhHACTA-CACA-..-.-T-.--T-GCA--A--TGGACTGCA--G-A T----G-T--T--A-A------GAMCTA-CAEA--. .-T----T-GCA--A--AGGMACTGCA--G-A T----G-T--T--A-A------AANCTA-TACA-----T----G-GCA--A--AGGNACTGCA--A-A T----G-T--A--G-A------AMAGATA-ChCA--.-.-T--.-C-GCG--C--A.......-A-A I----C-C--T--'j-A------AChGATA-TGCA------C----T-GTG--A--AGGMCAGCA--A-C T----C-C--T--G-A------ACAGATA-TGCA------C----T-ATG--A--AGGMACAGCA--A-C T----r.-C--T--G-A------ACAGATA-TGCA------C----T-GTG--A--AGGACAMCA--A-C T----C-C--T--G-A------ACAGATA-TGCA------C----T-GTG--A--AGGMACAGCA--A-C T----C-T--T--G-A------ACMAATA-TGCA------T-.--T-GTG--A--AGGMACAGCA--C-G T----C-C--T--A-A------ACMATA-TGCA------T----T-GTG--A--AGGACAGCA--C-A

AATGCCAATACAMEAgACCMACAGCACCNCCTAGCGg a AggAggAMALTACCCAGTgCA.CAA ? ? A---. --AGC-C--G'-G------------TC---C-AGMGGGAGCA--T-AC--A--G--A--T. . .

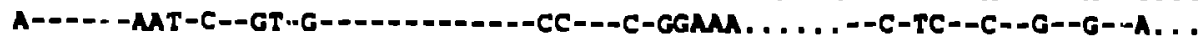
A-----AMT-T--GT-G--_-_... . --CC---C-GGAMGGGGGA--C-TC--C--G--A--A. . h-----AGT-C--GT-G-...-_-_-_---CC---C-GGMACGGAGGA--C-TC--C--A--A--A. . . A-----AMT-C--GT-G-_--_-_-_-CC---T-GGMMAGAGGA--C-AC--C--G--A--A...

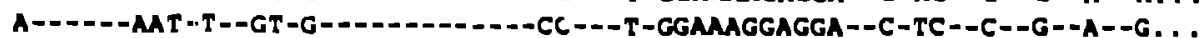

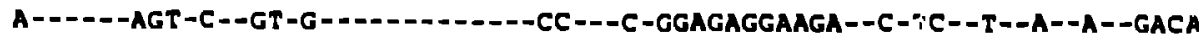
A-_---GCT-C--AT-A--1...-----CC---C-GC..... GGA--T-AT--A--G--G--A. . . T------AMA-C--GT-G---_---1----TC---C-GCAGAGGAGGA--T-AC--A--A--A--A. .

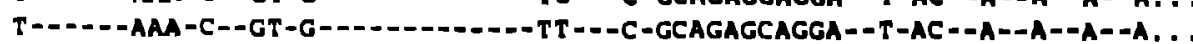
T-..--AAA-C--GT-G--.----_---TC---C-GCAGAGGAGGA--T-AC--A--A--A--A. . T--..--AAA-C--GT-G---.------TC---T-GCAGAGGAGGA--T-AC--A--A--A--A... A--.--GCA-C-..GC-G--2-1--_..---CC---T-GCAGAGGAGGA--T-AC--A--G--G--A. .

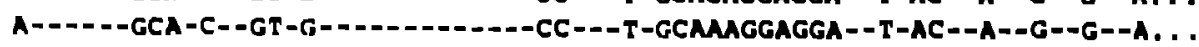


CONSENSUS gtaGgtGGcAATAtatCCAcCTgCCACTaAGcCCccGAC??TAAATGCt TGGGTAAAATagTaGAgG HIV2ROD HIV2NIH2 HIV2ISY HIV2ST HIV2BEN HIV2D194 HIV2GH1 HIV20205 SIVM425I SIVMA142 SIVI1239 SIVNRE SIVSMMH4 SIVSHMPEJ

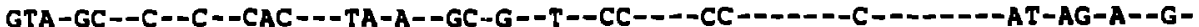

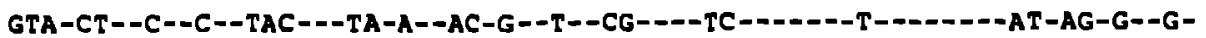
ATA-GC--C--C--TGT---TC-G--GC-G--T--CC---CC------T--D----GT-AG-A--G-

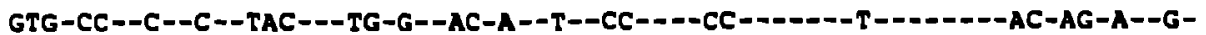

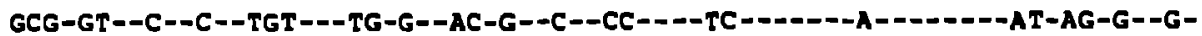
GCA-GC--C--C--TAT--TG-G--GC-G--C--CC---TC----D-T----D-AT-AG-A--GGGC-GT--C--C--TAT---CG-G--AC-G--C--CC-- - TC---1--T-D---AAT-AG-A--G-

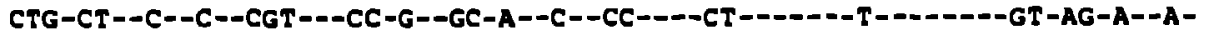

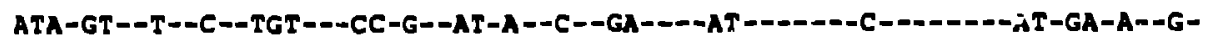

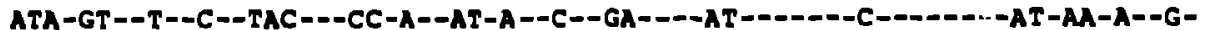
ATA-GT--T--C--TGT---CC-G--AT-A--C--GA----AT----D-C--D----AT-GA-A--GGTA-GT--T--C--TAC---CC-A--AT-A--C--GA----AT--_-_-C-D--_--AT-GA-A--G-

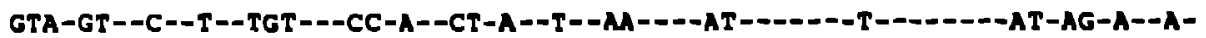

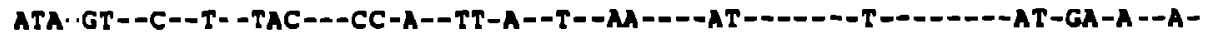

CONSENSUS HIV2ROD HIV2NI iZ HIV2ISY HIV2ET HIV2BEN HIV2DI 94 HIV2GH1 HIV2D20S SIVM2SI SIVMMI 12 SIVHA2 39 SIVMNE SIVSMMH4 SIVSMYPBJ

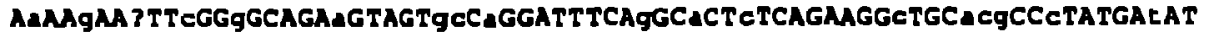

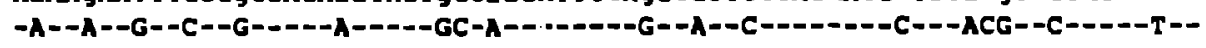

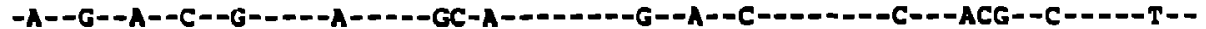

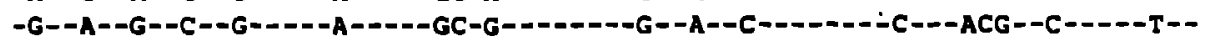

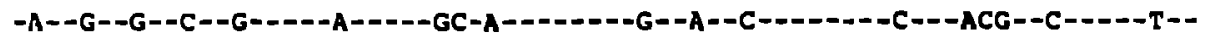

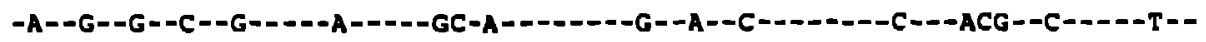

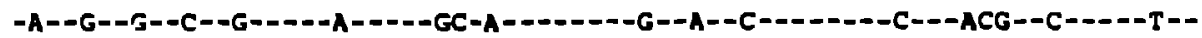

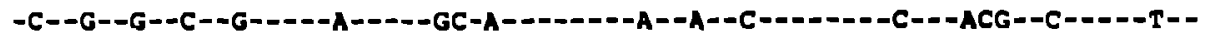

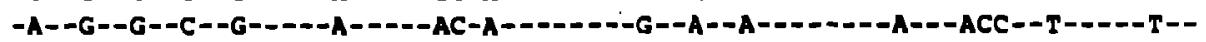

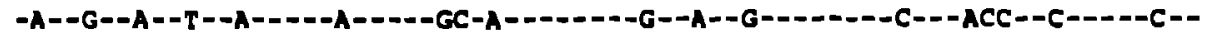

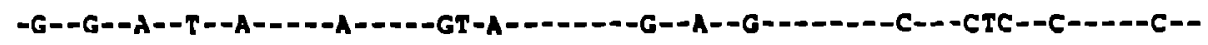

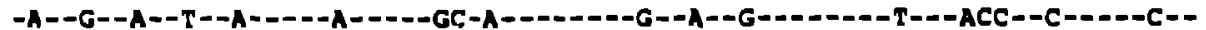

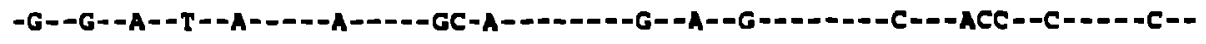

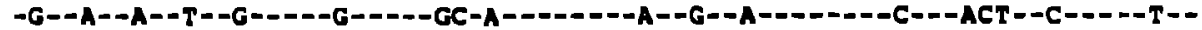

CONSENSUS HIV2ROD YIVZNIHZ HIVZISY HIV2ST HIVZUEN HIV2DI 94 HIV2GH1 HIV20205 SIVMM 251 SIVMAI 42 SIVMM 239 SIVMNE SIVSMMH4 SIVSMMPAJ

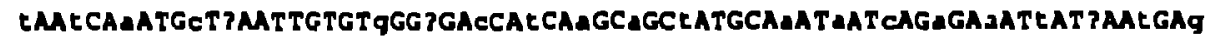

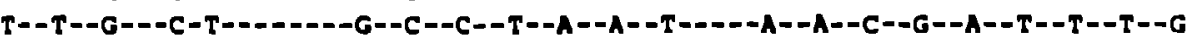

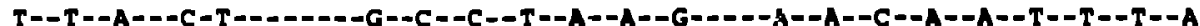

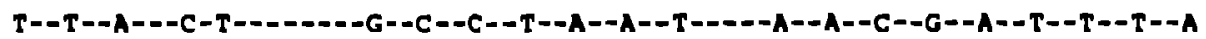

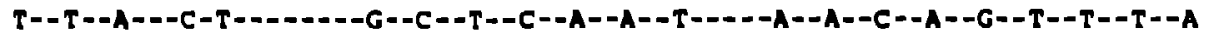

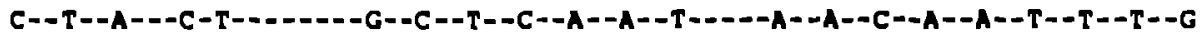

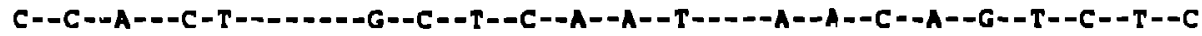
$A--T--C=-C-A--D----A--A--A--T--G--A--C-D---A--T--T--A--A--A--C--I \quad J$

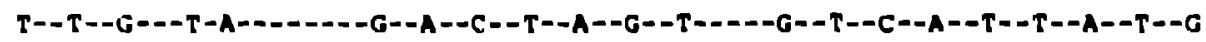

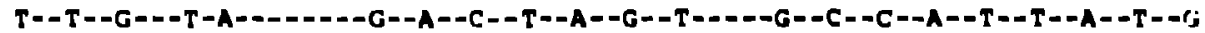

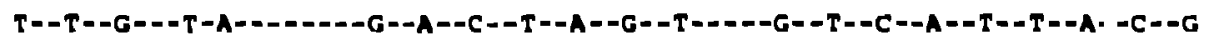

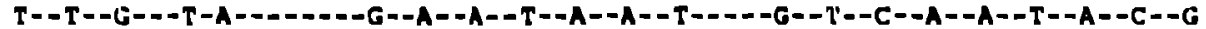

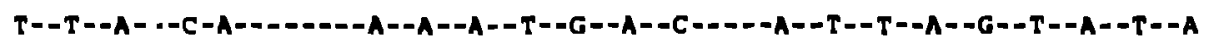


CONSENSUS GAaGCaGCaGAtTGGGettgcapCAcCCaataCcAGgecCcttaCcagcalg?CAgCTtAGaGAgCCaA HIV2ROD HIV2NIHZ HIV2ISY HIV2ST HIV2BEN HIV2D194 HIV2GH 1 HIV2D20S SIVI251 sI Vial 42 sIVIN239 s IVNE SIVSHAHA SIVSHAPBJ --A--A--A--A-----TGTGCAA--T--AATA-C--GC--CTTA-CAGCG--G--G--T--A--G--AA $--A--A--A--C--2-$ TGTTGCA--T--CATA-C--GC--CTTA-CAGCG--G--G--T--A--G--AA --A--A--A--C-----TGTACAA--T--AATA-C--GC--CTTA-CAGCG--G--G--C--A--T--AC --A--A--A--T-----CGCACNA--C--NATA-C--GC--CTTA-GAGCG--G--G--C--G--G--NA --A--A--A--C-----TTCGCAG--C--AATA-C--GC--CTTA-CAGCA--A--G--C--A--C--AA --A--A--A--T-----TGCGCAG--C--AАTA-C--GC--CTTA-CAGCA--G--G--T--A--C--AA --A--A--A--T-----TGCACAG--C--AATA-C--GC--CTTA-CAGCA--G--G--T--A--C--AA --A--A--A--C-----CCAGRAA--C--GTCA-C--GC--AATG-CGGCA--A--A--T--G--C--AA $--G--\mathrm{T}--A--\mathrm{T}----C T T G C A G--C--A C A A-C--C T--A . . .-A A C A A--A--G--T--G--G--G T$ $--G-T--A--T----C T T G C A G--C--A C A A-A--C T--A . . .-A A C A A--A--G--T--G--G--G T$

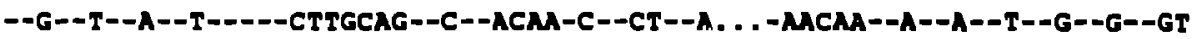
$--G--T--A--\mathrm{T}-----C T T G C A G--C--A C A A-A--C T--A . . .-M A N A--A--G--T--G--G=-G T$ $--A--\mathrm{T}-\mathrm{C}-\mathrm{T}----\mathrm{TTTACA}--\mathrm{C}--\mathrm{GCAA}-\mathrm{C}--\mathrm{GT}--A C T A-C A G C A--G--A--\mathrm{T}--\mathrm{A}--\mathrm{G}--\mathrm{AA}$ CONSENSUS HIV2ROD HIV2NIHZ HIV2ISY HIV25T HIV 2 BEN HIV2D1 94 HIV2CH1 HIV2020S SIVM251 SIVMa1 42 SIVMH239 SIVMNE SIVSMAH4 SIVSMPPB

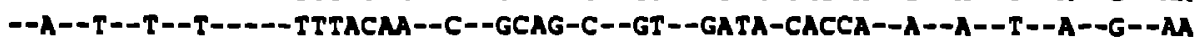

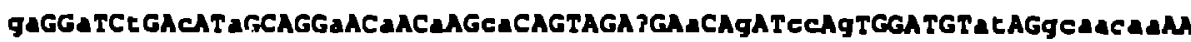
GG--A--T--C--A-----G--A--A--CA-------A--A--G--CC-G-------TT--GCCACAA--

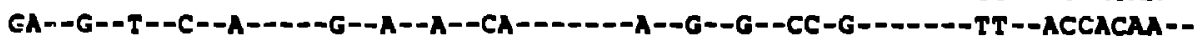
GA--A--T--C--A-----G--A--A--CA-------G--A--G--CG-A-------AT--GCMMGAA-GG--A--T--C--A--2--G--A--A--CA--1----A--G--G--CC-G-------TT-GCCACAAGA--G--T--C--A----A--A--A--CA--1----T--A--G--CC-G-------AT--GCCACAA--

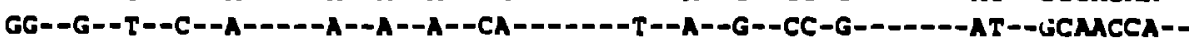
GG--G--T--C--A-----A--A--A--CA-------A--A--G--CC-G----1--AT--GCCACAA-GA--G--A--T--A-----A--C--C--CA-------G--A--G--AC-G-------AC--GGCCCNA--

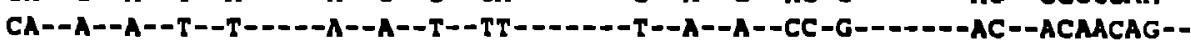
CA--A--A--T--T----A--A--T--TA-------A--A--A--CC-G-------AC--ACACAG--

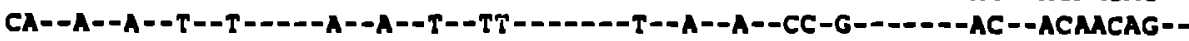
CA--A--A--C--T----A--A--T--TA-------T--A--A--CC-G-------AC--ACACAG--

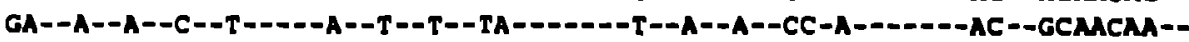

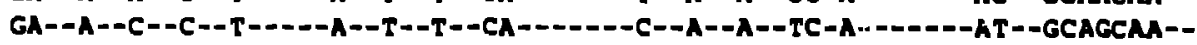

CONSENSUS HIV2ROD HIV 2NIHZ HIV215Y HIV2ST HIV2BEN HIV2D1 94 HIV2GH 1 HIV2D205 S I VMA2 51 SI VMA1 42 S IVMH2 39 SIVHNE SIVSHMH4 SIVSHHPBJ
tCCcgTaCCAGTaGGPACATCTACAG PAGaTGGATCCAaA T--TG-A--A--A--A-A---C--T--A--A-----C--GA-A--AT-G--G--G--T--CA-G-T---C T--TG-A--A--A--C-A---C--T--G--A---- - T--GA-A--AC-G--A--G--C--CA-G-T---C T--TG-A--A--A--A-A---C--T--G--A-----C--GA-A--AC-G--G--G--T--CA-A-T-- -C T--TG-A--A--A--A-G---C--T--A--A-----C--GA-A--GC-A--G--G--C--CA-G-T---C T--CG-A--G--A--G-A---C--C--A--A-- -- -C- -AA-A--GC-G--A--G--T--CA-A-A---C T--CG-G--G--A--G-A---C--C--G--A-----C--GA-A--GC-A--G--A--T--CA-G-T---C T--CG-G--G--A--G-A-- T--C--A--A-----C--GA-A--GC-A--G--G--T--CA-G-7---C T--TG-C--A--G--A-A-- T--T--A--A-----T--AT-A--AT-G--G--A--T--CC-A-T---C Z--CA-A--A--A--C-A---T--C--G--A-----C--AC-G--GT-G--A--A--T--CA-A-T---T C--CA-A--A--A--C-A---T- -C--G--A-----C--AC-G--GT-G--A--A--T--CA-A-T---T C--CA-A--A--A--C-A---T--C--G--A-----C--AC-G--GT-G--A--A--T--CA-A-T- - T C--CA-A--A--A--C-A-- -T--C--G--A---- -C--AC-G--GT-G--A--A--T--CA-A-T- - T C--CA-A--A--A--C-A---T--T--A--G-----C--AT-A--GC-G--G--A--T--AA-A-T---T

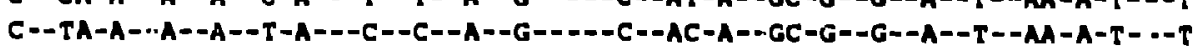

I-B.10

MAY 91 
HIV2/SIV GAG

CONSENSUS AACCCAAC?AaCATECTaGA?gTAAAACAaGGaCCAAAaGAgCCaTTCCA ?AGCTALGTAGA?AG?TTCT

HIV2ROD

HIV2NIHZ

HIV2ISY

HIV2ST

HIV2BEN

HIV2D194

HIV2GHI

HIV20205

SIVMM251

SIVTMI 42

SIVMM239

SIVINE

--C--G--C-A---CC-A--CA-A--A--G--A-----G--GC-G--C--A--C--T--A--T--A---$--T--A--C-A---C C-A--C A-A--T--A--A-----G--A C-A--C--A--C--T--A--T--G=--$ $--T--A--C-A---T C-A--C A-A--A--A--A-----A--G T-G--C--A--C--T--G--T--A----$ --C--A--C-A---CC-A--CA-A--A--G--A-----G--GC-A--C--G--T--T--A--T--A---$--C--A--T-A---C T-A--C A-A--A--G--A-----A--A C-G--C--A--C--T--A--C--G=--0$ --C--A--T-A---CT-A--TG-G--G--G--A-----A--AT-G--C--G--C--T--A--C--A---$--C--A--T-A--C T-A--C G-A--G=-G--A-----G--A C-G--C--G--C--T--G=-C--G=---$

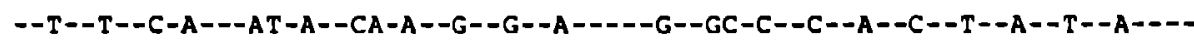
$--C--A--A-A---T C-A--T G-A--A--A--G-----A--G C-A--T--G--C--T--A--C--G----$ --C--A--A-A---TC-A--TG-A--A--A--G-----A--GC-A--T--G--C--T--A--C--G---$--C--A--A-A---T C-A--T G-A--A--A--G--.--A--G C-A--T--G--C--T--A--C--G--0$ $--C--A--A \cdot G---T C-A--T G-A--A--A--G=---A--G C-A--T--G--C--T--A--C--G----$

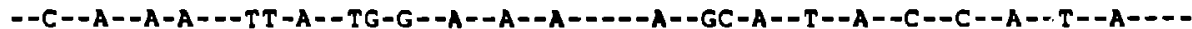

SIVSMMH

SIVSMMPBJ

$--T--A--A-A---T T-G--T G-G--A--A--A-----A--G C-A--T--A--C--T--A--T--A----$

CONSENSUS

HIV2ROD

HIV 2NIHZ

HIVZISY

HIV2ST

HIV2BEN

HIV2OI 94

HIV2GH1

HIV2D205

SIVMM25I

SIVMAI 42

S[VMM2 ]9

SIVMNE

SIVSAMHA

SIVSMMPBJ

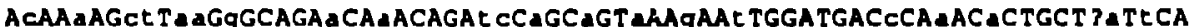
-C--A--CT-GA-G-----A--A-----TC-A--A--G--G--T--------C--A--A-----1.J-A--

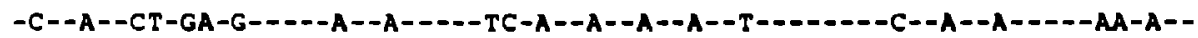
-C--A--CT-AA-G-----A--G-----TG-A--A--G--G--T--------C--G--G-----AG-G--

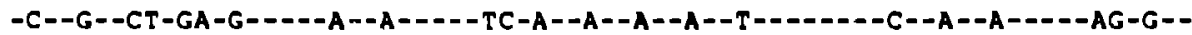
-C--A--CT-GA-G-----A--A-----CC-A--A--A--A--T--------C--A--G-----AA-A--

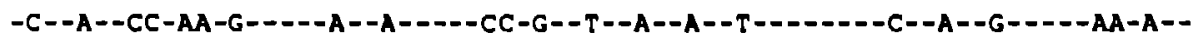
-T--A--CT-GA-G-----A--A-----TC-G--A--A--G--C--------C--A--G-----AA-A--C--A--CT-AC-G-----A--A-----CC-A--A--G--A--T-1--1--A-A-A-A---O-GA-T--

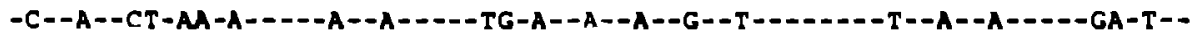

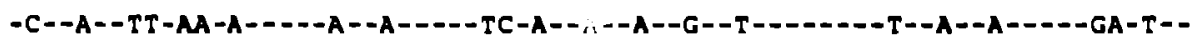

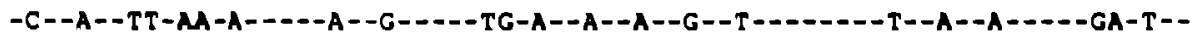
$-C--A--C T-A A-A-----A--A-----T C-A--A--A--G--T-1--1--T--A--A-----G A-T-1$

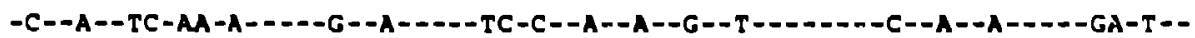

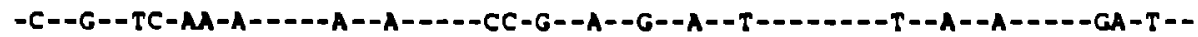

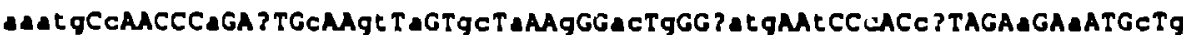
AAATG-C-----A--C--T--AT-A--GC-A--A--AC-A--GATG--C--T--CT----A--G---C-G GAATG-C-----A--T--T--AT-A--AC-A--A--AT-A--AATG--T--T--CT----A--A---C-G A...T-G-----A--C--T--GT-A--AC-A--G--AC-A--GATG--T--T--CT----A--G---C-A GAATG-C-----A--C--T--GT-A--AC-A--A--AC-A--GATA--T--T--CT----A--A---C-A GAATG-C--.--A--C--C--GT-A--AC-A--A--AC-G--GATG---7--C--CC----A--G---C-A GAATG-C--.--A--C--C--GT-A--AT-A--A--AC-A--GATG--T--C--CC----G--G---C-G GAATG-C-----A--C--C--GT-A--AC-A--A--AC-G--GATG--T--C--CC---A--G---C-G GAATG-T-----A--T--C--GT-A--GC-T--G--CT-G--AATC- -T--C--CT--- -G--A---C-A AAATG-T-D--A--T--C--GC-A--GC-G--G--GC-G--TGTG--T--C--CC----A--A---C-G AAATG-T--.--A--T--C--GC-A--GC-G--G,-GC-G--TACS- -T--C--CC----A--A---C-G AAATG-T--D-A--T--C--GC-A--GC-G--C--GC-G--TGTG--T--C--CC----A--A-- -C -G

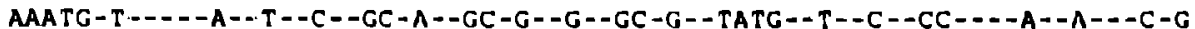

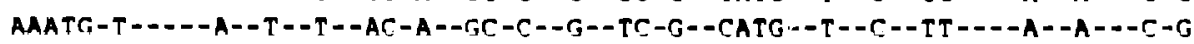
AAATG-C--.-G--C--T--AT-G--GC-C--G--TC-G--TATA--T--C--TT----A--A-D-T-G

943

977

971

977

977

977

977

980

962

974

974

974

974

977

977

1010 1047 1041 1044 1047 1047 1047 1050 1032 1044 1044 1044 1041 1047 1047 
CONSENSUS AC?gCcTGECAgGG?gTAGG?GGaCCAgGCCAgAAaGCEAGatTAATGGCaGAaGCCCT raAagAgGCCC HIV2ROD --CG-C--T--G--GG----T--G--AG-C--G--A--T--AT-------A--G--CC-G--A--G-TCA HIV2NIHZ --CA-C--T--G--GG----G--A--AG-C--G--A--T--AT-------A--A--CC-A--G--G-TCA HIV2ISY --CG-C--T--A--GA----T--A--AG-C--G--G--C--AC-------A--A--CT-A--A--G-CCA HIV2ST HIV2BEN HIV2OI94 HIV2GH1 HIV2D205 SIVMM251 SIVMMI 42 SIVMM239 SIVMNE SIVSRMH4 SIVSMMPB --CG-C--T--G--GG----T--A--AG-C--G--A--C--AT-------A--A--CT-A--G--G-CCA --CG-C--C--G--GG----C--A--AG-C--G--A--C--GC--------T--A--CC-A--A--G-CTA --TG-C--C--G--AG----C--A--AA-C--G--A--C--AC--_---.-T--A--CC-A--G--G-CTT $--T G-C--T--G--G G=--T--A--A G-C--A--A--C--A C------C--A--C C-G--G--G-C T T$ --GG-C--C--A--GA----A--C--AG-G--G--G--A--GC--_-----C--A--CT-A--A--G-CCC $--G G-T--T--A--A G---G--A--A G-A--G--G--T--A T------A--A--C C-G--A--G-C C C$ --GG-C--T--A--AG-----G--G--AG-A--G--G--T'-AT-------A--A--CC-G--A--G-CCC $--G G-T--T--A--A G----G--G--G G-A--G--G--T--A T-1-----\lambda--\lambda--C C-G--A--G-C C C$ $--G G-T--T--A--A G----A--\lambda--A G-A--A--G--A--A T-------\lambda--\lambda--C C-G--\lambda--G-C C C$ $--\lambda G-C--T--G--\lambda G----\lambda--G--\lambda G-A--\lambda--\lambda--T--G T-------\lambda--\lambda--A T-G--\lambda--G-C A C$ $--A G-C--T--G--A G----A--G--A G-A--A--A--T--A C-------A--G--A T-A--A--T-C A C$

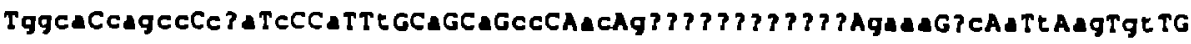
-AGGA-CTGCC-CTA-C--A--C--A--A-CC--GC-G.......... -GAMA-GC-T-T-AA-GC-HIV2NIHZ HIV2ISY HIV2ST HIV 2BEN HIV 20194 HIV 2GHI HIV 20205 SIVMM231 SIVMM1 42 SIVMM239 SIVHNE SIVSMMH 4 SIVSMMPBJ -GGCA-CAGCC-CCA-C--A--T--A--A-CC--GC-G.......... -GMA-AC-T-C-AG-GC-. -GCGA-CAGCC-CTA-C--A--T--A--A-CC--AC-G..........-AAAG-GC-A-T-AG-GT-. -GGCA-CAGCC -CCA-C--A--T--A--A-CC--AC-G......... -GAAG-AC-A-T-AG-GC-. -GGGA-CAAGC-CTA-C--A--T--A--A-CC--AC-A.......... -GAM-GC-A-T-GG-AT -. -GACG-CAGCC-CTA-C--A--T--A--A-CC--AC-A.......... -GMG-GC-A-T-GG-GT-GACA-CACEC-CTA-C--A--T--A--A-CC--AC-A......... -GAMA-GT-A-T-GG-GT-- AACA-CTGCA-CCA-A--G--T--T--C-TT--AC-AMAGCAGGGMAG-GAGG-AC-G-G-CA-GC-- CGCA-CAGTG-CAA-C--T--T- -A--A-CC- -GA-GAGGGGACCA... -GMA-CC-A-T-AG-GT- - CGCA-CAGCG-CAA-C--T--T--A--A-CC--AC-GMGGGACCA... -GMA-CC-A-T-AG-GT - . - CGCA-CAGTG-CAA-C--T-.-T--A--A-CC--AC-GAGGGGACCA. . -GMA-CC-A-T-AG-GT-- TGCA-CAGGG-CAC-C--T--T--A--A-CC--AC-GAMGGGACCA. . . -GAAA-CC-A-T-AG-GT- -GAGG-CAGAT-MCC-C--A--T--A--A-TC--AC-GAMAGGACAA. . -GGA-AC-A-C-AG-GT- ---C--T--A--GG-A--G---T-G--A-GA--A--CC-AG-A--T-----G--G--C--------G--. $---T--T--A--G G-A--A---T-G--A-G A--A--G A-C G-G=-T--O--A--G--C--1--1-G---$ $---T--T--A--G G-A--G---T-G--A-G A--A--C C-A G-G--T-1--A-A-G--C-1-1-1-A-1$

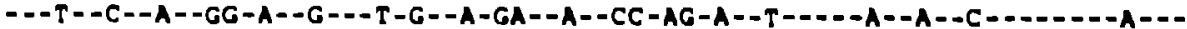

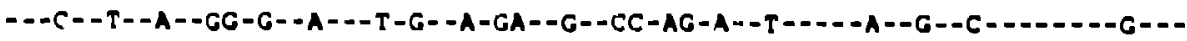
$---T--T--A--G G-G--A---T-G--G-A A--G=-C C-A G-A--C-\cdots--A--G--C=--.-1-G-.-$

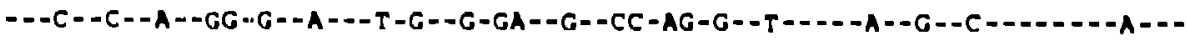

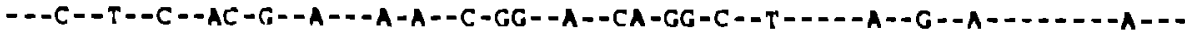
$---T--T--G--G G-G--A---T-T--A-G G--A--C A-A G-C--A-----A--G--A-\ldots--1--A-$.

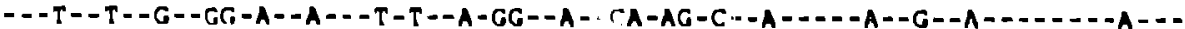
$---T--T--G=-A C_{i}-G--A---T-T--A-G G--A--C A-A G-C=-A----A--G--A-------A--$.

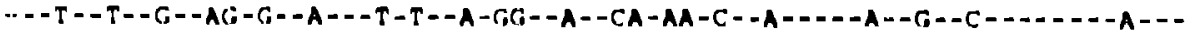
SIVSMMH4 ---T--T--A--GG-G--A---T-T--A-AA--A--CA-GG-C--T-1---A--G--C-1-1--G--SIVSMMPBS

1132

1175

1169

1172

1175

1175

1175

1178

1172

1181

1181

1181

1181

1184

1184

1202

1245

1239

1242

1243

1245

2243

1248

1242

1231

1251

1231

1751

$\lcm{254}$

1254

1.13-12 
pol ort ->

CONSENSUS GGAARACAGgaCA?GTCATGgCAMATGCCCAgAAAGACAgGCgGGTTTTTTAGGgCTEGGCCCAtGGG HIV2ROD HIV2NIHZ HIV2ISY 4IV25T HIV2BEN HIV2DI 94 HIV2GH1 HIV2D20S SIVRa4251 IIVRA1 42 SIVHA239 SIVMNE SIVSMMH4 SIVBHAPBJ

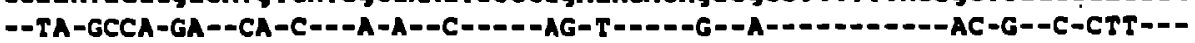

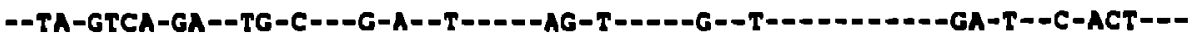

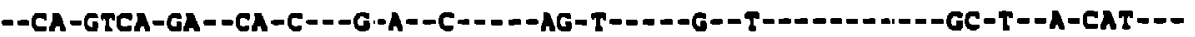

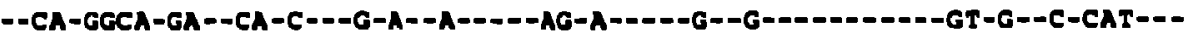

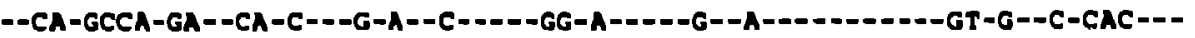

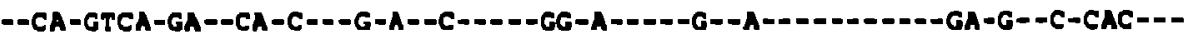
--CA-GACA-GA--CG-C---G-A--A-----AG-A-----G--A----1------GA-G--T-CAT--0

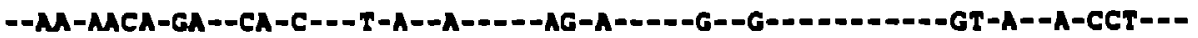

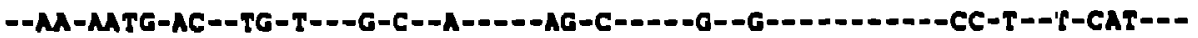

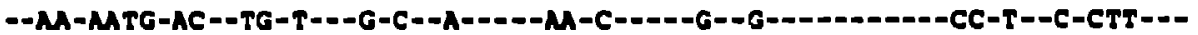

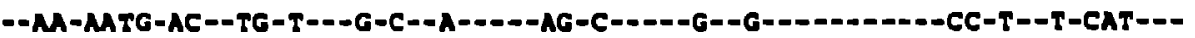

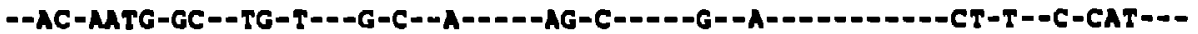

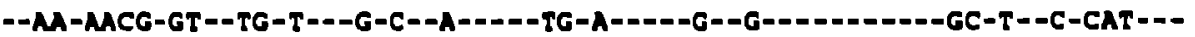

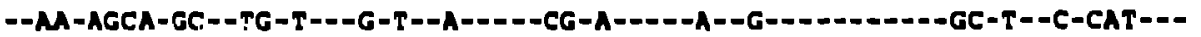

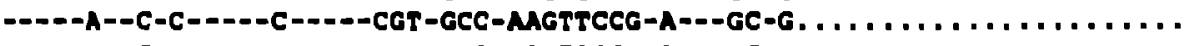

$-----A--C-C$

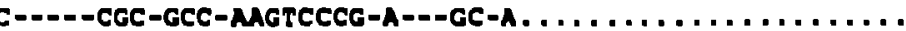

1356 


\section{IIV2/SIV GAG}

CONSENSUS CTg?AgAATTACATGCAgC?gGGgA?AAgCAgAGدGAg????????????A?CAgAgAgAgaCC?TACA HIV2ROD HIV2NIH2 HIV2ISY HIV2ST HIV2BEN HIV2D194 HIV2GHI HIV2D20S SIVRO25I SIVMMI 12 SIVMH239 SIVANE SIVSRMH SIVSHMPEJ C-GG-G-AA--T---C-GCAA--G-AAAGA--G--A--G. . CAG. . . . . . . AGAGAG-GA--A--.C-GG-G-MA--I---C-GCAA--A-AAAGG--A--G--G. . CAG. . . . . . AGAGAG-GA--G---C-GG-G-AG--C---C-GCAA--G-GAMA--G--A--G...CAG........ AGACAA-GA--A--.C-AG-G-AG--C---C-GCAA--G-AMAGG--G--A--G. . CAG........ AGAGAG-GG--A---T-GG-G-GA--T---C-GCAA--G-GAMAG--G--G--G. . CAG. . . . . . . AGGGAG-GA--A---T-GG-G-MA--T---C-GCAA--G-GAAAG--G--A--G.......... CAGAGGGAG-GA--A---T-GG-A-GA--T---C-GCAG--G-GAGAG--G--G--G. . CAG. . . . . . AGGGAG-GA--A---C-GA-A-GT--C-.-C-GATG--G-GACAA--G--A--G......... AGCCGAGAG-LA--C--.C-AA-G-AC--C---C-GTTG--C-AGCAG--G--A--A.........AGCAGAGAG-AG--T---C-AA-G-AC--C---C-CTTG--C-AGCAG--G--A--A..........AGCAGAGGG-AG--T---C-AA-G-AC--C---C-GTIG--C-AGCAG--G--A--MAGCAGAGAGAAAGCAGAGAG-AG--T---C-AA-A-AC--C---C-GTTG--C-AACAG--G--A--A......... AGCAMAGG-AG--T---C-GA-G-AT--C---A-GATG--C-GAMAG--r,--G--G......... MACAGAGAG-GA--T--.C-GA-G-AT--C---A-GATG--C-GAMGG--G--G--G......... MCAGAGAG-GA--T-...

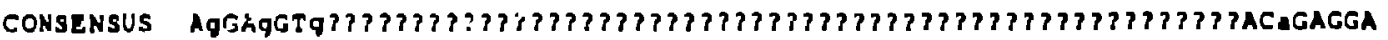
HIV2ROD HIV2NIHI HIV2I $3 Y$ HIV2ST HIV2BEN HIV2DI 94 HIV2GHI HIV2D20S SIVHM231 S IVAMI 12 SIVHM2J9 SIVHNE SIVIROAH SIVSMPPEJ

CONSENSUS HIVZROD II? 2N I HZ IIIVZISY HIVZST HIVZBEN IIIV20194 IIIv2011? ค. IV20209 उIVMM 25: SIVMMI 1 ? SITMM219 SIVMNE SIVARMII sivgMmPNJ

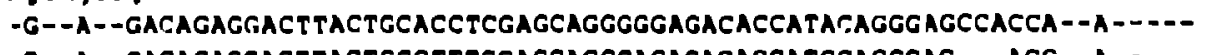
-G--A--GACAGAGGACTTACTGCGTTTCGAGCAGGCAGAGACACCATGCAGGGAG... ACG--A-.... -A--A--gacagaggacttgctgCatctcgagcahgGagagacaccacacagagag. . . ACg--A-----

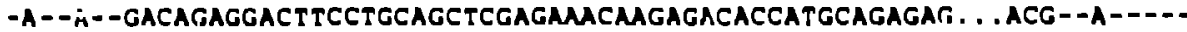

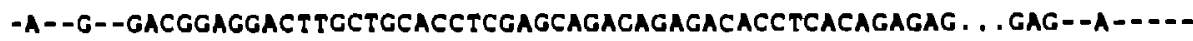

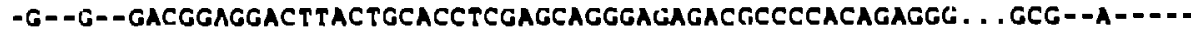

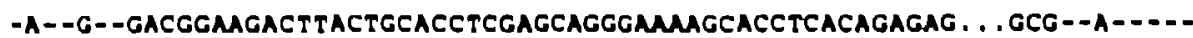

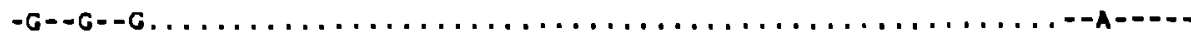

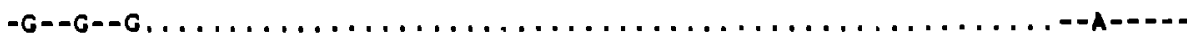

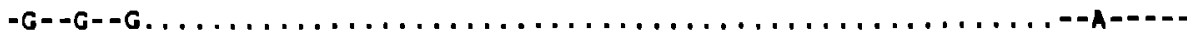

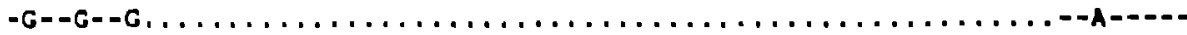

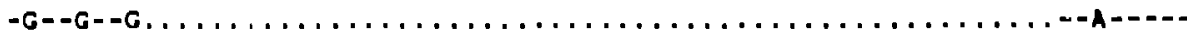

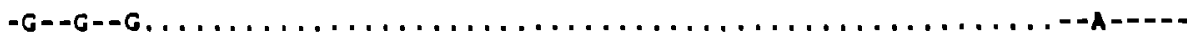

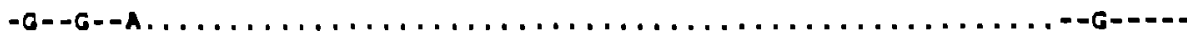


Spllce sites have been determirod for SIVs only.






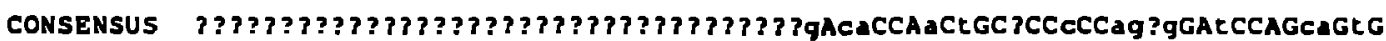
HIV2ROD HIV2NIHZ HIV2ISY HIV2ST HIV2BEN HIV2DI 94 HIV2GHI HIV2D205 SIVMA251 SIVMA1 42 SIVMm2 39 SIVNAE SIVSFAH4 SIVSHAPBJ

CONSENSUS HIV2ROD HIV2NIHZ HIV2ISY HIV2ST HIV2BEN HIV2D194 HIV2GHI HIV2D205 SIVMM2S1 SIVMMI 42 SIVMm2 39 SIVMNE SIVSMMHA SIVSMHPBJ

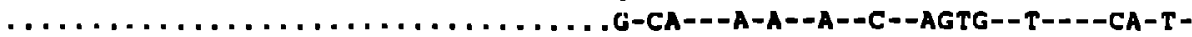
..............................A-CA--A-A--A--C--ATTG--T----CA-T-

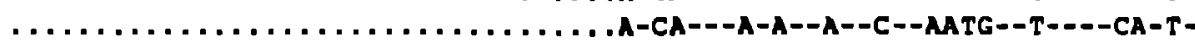
.............................G-CA---A-A--A--C--GATA--C----TA-A........................... G-TA---A-A--A--T--GGCA--T----CA-C-

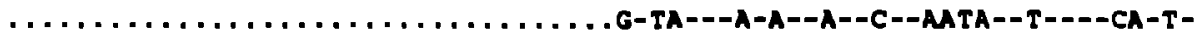
.......................... G-tA---A-A--A--C--AGCA--T----CA-TGATGAACCCAGCAGAGGGCATGACACCTCGGGGGGCG-CA--T-T--G--C--TGCA--T---CA-T-

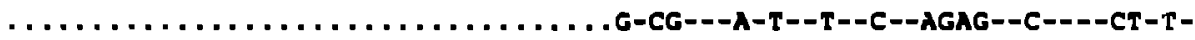
............................ . . . . . . . . . . . ...........................G-TG--A-T--T--C--AGAG--C---CT-T-

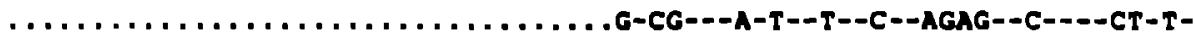
..........................G-TA---A-T--T--C--AGAG--T---CT-T-

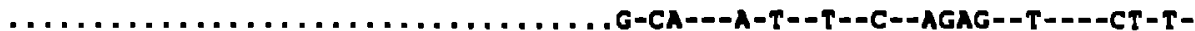

GAteTgctga agAa ?TACATGcAgctgGG ?AaAa ? ? 7 ? ? ? ? ? ?gCAgAGaGAgaacagagagAgaC --TC-AC- GGAG-NA--T---C-GCNA--G-MAG . . . . . . . . . A--G--A--GCAGAGAGAG-GA--CC-AC-GGAG-AA--T---C-GCM--A-AMG. . . . . . . . . G--A--G--GCAGAGAGAG-GA--CC-AC-GGAG-AG--E---C-GCNA--G-GNM . . . . . . . . . A--G--A--GCAGAGACAA-GA--CC-AC-AGAG-AG--C---C-GCAA--G-ANAG. . . . . . . . G--G--A--GCAGAGAGAG-GG--AC-GT-GGAG-GA--T---L-GCAA--G-GNA . . . . . . . . G-G-G-G-GCAGAGGGAG-GA--CC-GT-GGAG-AA--T---C-GCMA-G-GAM. . . . . . . . G--G--A--GCAGAGGGAG-GA--TC-AT-GGM-GA--T---C-GCAG--G-GAGA . . . . . . . . G-G-G-G--GCAGAGGGAG-GA--GA-GC-GAM-LiT--C---C-GATG--G-GACA. . . . . . . . A--G--A--GAGCcGAGAG-GA--TC-GC-AAAG-AC--C---C-GTTG--C-AGCA . . . . . . . . G--G--A--NGGAGAGAG-AG--TC-GC-AAAG-AC--C---C-CTTG--C-AGCA . . . . . . . . , G--G--A--AAGCAGAGGG-AG- -TC-GC-ANG-AC--C---C-GTTG--C-AGCAGCAGAGAGMMAG--G--A--AAGCAGAGAG-AG- -TC-GC-ANA-AC--C---C-GTTG--C-AACA . . . . . . . . . --G--A--AMGCNAMGG-AG- -TC-AC-GAAG-AT--C---A-GATG--C-GAA . . . . . . . . . G-G-G-G--GACAGAGAG-GA- -TC-AC-GAAG-AT--C---A-GATG--C-GAMG . . . . . . . G--G--G--GACAGAGAG-GA-

CONSENSUS HIVZROD HIVZNIHZ HIVZISY HIV25T HIV2BEN HIV 20194 HIV 2 GIII IIV 20205 SIVMM2 5 I SIVMHI 42 SIVMH2 39 SIVANE gIVSMHHA SIVSHMPBJ

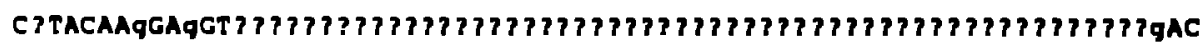
-A--2-G--A--GACAGAGGACTTACTGCACETCGAGCAGGgGGAGACACCATACAGgGAGCCACCA--G----G--A--GACAGAGGACtTAC TGCGTtTCGAGCAGGCAGAGACACCATGCAGGGAGAC . . G- -A----A--A--GACAGAGGACTTGCTGCATCTCGAGCMGGAGAGACACCACACAGAGAGAC. . . G--A----A--A--GACAGAGGACTTCCTGCAGCTCGAGMMCAAGAGACACCAтGCAGAGAGAC . . G--

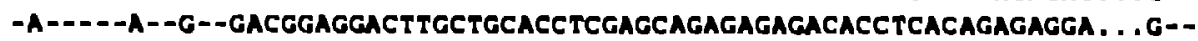

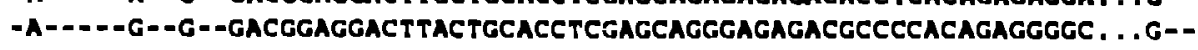

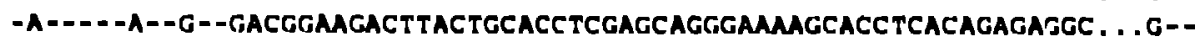

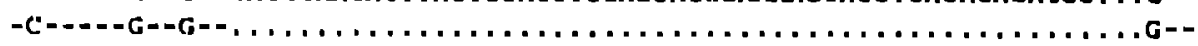

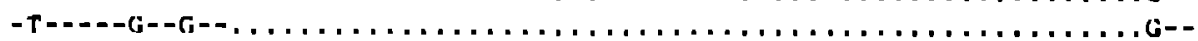

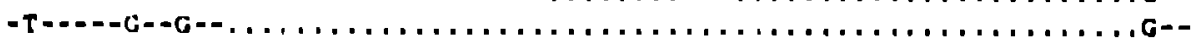

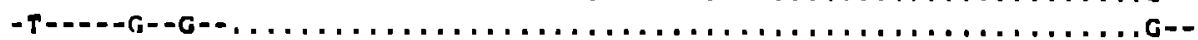

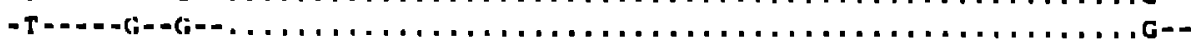

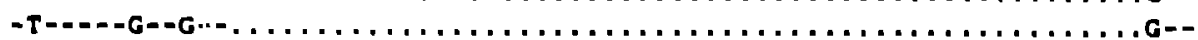
240 237 
<- gag

CONSENSUS HIV2ROD HIV2NIH2 HIV2ISY HIV2ST HIV2BEN HIV2D194 HIV2GHI HIV2D205 SIVMA251 SIVM1 42 SIVIA2 39 SIVMNE SIVSMMH 4 SIVSMMPBJ

CONSENS!'" HIV2ROD HIV2NIH2 HIV2ISY HIV2ST HIV2BEN HIV201 94 HIV2GH 1 HIV20205 sIVRa251 SIVRA1 12 SIVRA2 39 SIVMHE SIVSMMHA SIVSMMPBJ

CONSENSUS HIV2ROD HIV2NIHZ HIV2ISY HIV2ST HIV2BEN IIIV2DI 94 IIIV2GHI IIIV20205 SIVMM25! SIVMA1 42 SIVAM2 39 SIVMNF: SI VSMMHA SIVSMMPly.
AGAGGALTTGCTGCACCTCAATCTCTCTTTGGAGAএGACCAgTAGTCACAGC?t ACATEGAgGg?CAGC

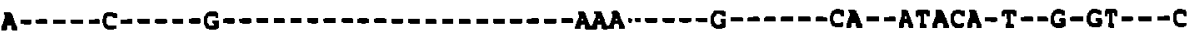

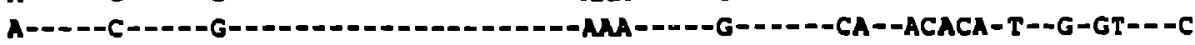

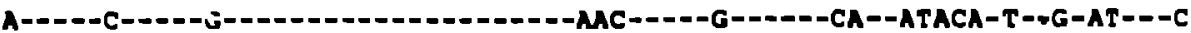

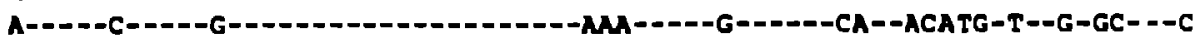

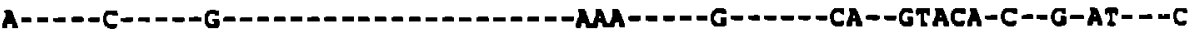

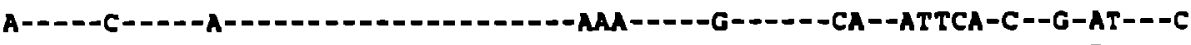

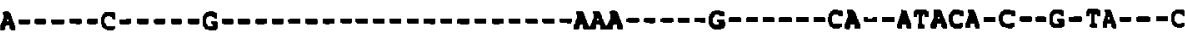

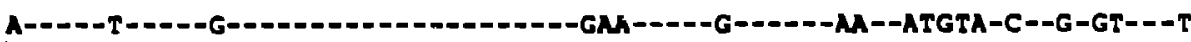

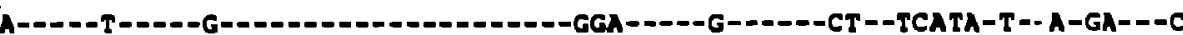

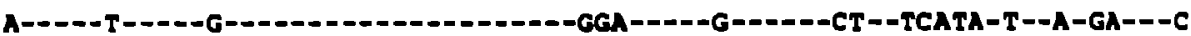

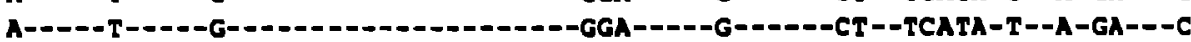

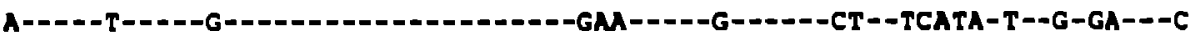

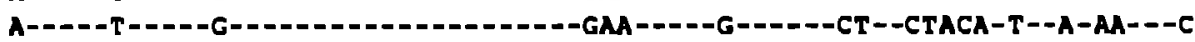

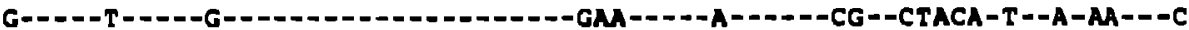

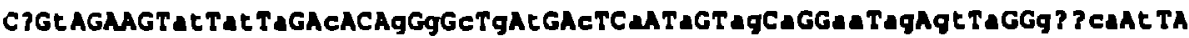
-A-T---D-CT-GT-A--C---G-G-C-G-C--C--A--A--AG-A--AA-AG-GT-A--GAACA-T- $-A-T=----T T-G T-A--C---A-G-C-A-C--C--A--A--A G-A--A A-A G-G T-A--G A G C A-T=-$ $-A-T-----T T-A C-A--C---G-G-C-G-C--C--A--A--A G-A--A A-A G-G T-A--G A G C A-T-=$ $-A-T-----T T-G C-A--C---G-G-C-G-C--C--A--A--A G-A--C G-A G-G T-A--G A G C A-T--$ $-G-T----C T-A C-A--C---G-G-C-G-T--C--A--A--A G-A--A A-A G-A T-A--G G A C A-T--$ $-G-T--0--C T-A C-A--C---G-A-C-G-T-C-C-A--A--A G-A--A A-A G-G T-A--G G A C A-T--$ $-G-T----C T-A T-A--C---G-G-C-G-T--C--A--A--G G-A--A-A C-G T-A--G G A C A-T--$ -A-T-----AT-AC-A-.C---G-A-T-G-C--C--A--A--AG-A--GA-AG-AT-A--TAGCA-T-$-T-T---D-A T-A T-G--T---G-G-C-G-T--T--T--T--A-A--A A-A G-G T-A--T C C A C-T--$

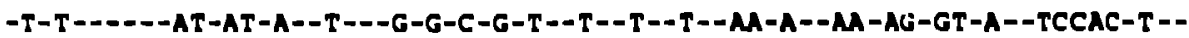
$-T-T-2--A T-A C-G--T---G-G-C-G-T--T--T--T-A-A--A A-A G-G T-A--T C C A C-T--$ $-\mathrm{T}-\mathrm{C}-----A T-A T-A--T---G-G-C-G-T--T--T--T-A G-A--A A-A G-G T-A--T C C A C-T--$ -C-T--D--AT-AT-A--T---G-G-C-G-C--T--A--T--AG-A--GA-AG-AT-G--TCCAA-T-D $-C-T--D-A T-A T-A--T--G-G-C-G-T-T--A--T-A G-A--G A-T G-A T-G--T C C A A-T-D$

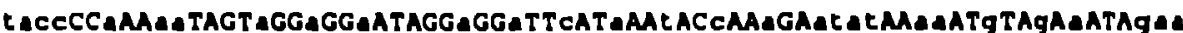
TAGC--A--AA----A--G--A-----C--A--C--A--T--C--G--ATAT--AA--G--G-A---GAA TAGT--A--AA----A--A--A-----G--A--C--A--T--C--G--ATA T--AA--G--G-A---GMA TAGT--A--AA----A--G--A-----A--A--C--A--T--C--A--ATAT--AG--G--G-A---AGA TAGT--A--GA----A--G--A-----G--A--C--A--T--C--A--ATAT--AA--G--G-A---AGA САСТ--A--AA----A--G--A-----G--A--T--A--C--C--A--ATAC--AA--G--G-A---AAA САСТ--A--AA----G--G--A-- --G-A--C--A--T--C--A--ATAT--AA--G--G-A---AAC CGTT--A--AA----G--G--A-----G--A--C--A--C--C--A--GATT--AA--A--G-A---AAA CACC--A--AA----A--A--G-----A--G--C--A--T--C--A--ATAC--AG--G--G-A---GAA

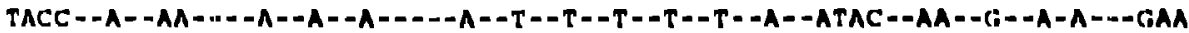
TACC--A--AA----A--A--A-----A--T--T--T--T--T--A--ATAC--AA--G--G-A-1-GAA TACC--A--AA----A--A--A-----A--T--T--T--T--T--A--ATAC--AA--G--G-A---GAA TAC'C--A--AA----A--A--A-----A--T--T--T--T--T--A--ATAC--AA--Ci--A-A---CiAA

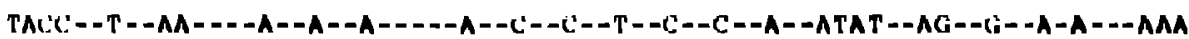

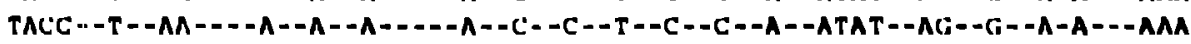




\section{HIV2/SIV POL}

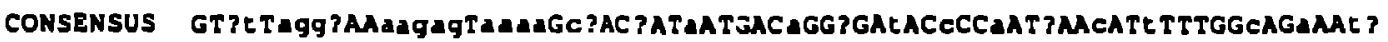
HIVZROD HIV2NIH2 HIV2ISY HIV2ST HIV2BEN HIV2DI94 HIV2GH 1 HIV2D205 SIVRo1251 SIVPA1 12 SIVHo1239 SIVRE SIVSMAH4 SIVSMRPBJ - -TC-NAT - -AAAGG-ACGG-CC--C--A-----A--C--C--C--A--C--C--T----C--A--TA --CC-AGGT--AAGGG-AAAA-CC--C--A-----A--T--T--C--G--C--C--T-----C--A--TG --GC-AAAT--ANAGG-AAGA-CC--C--A-----A--T--T--C--A--C--C--T-----C- -A--TA --AT-AMT--MAGAG-AAGA-CC--C--A----A--T--T--C--A--C--C--T--D-C--A--CA --AC-АMT--MAGAG-AAGA-CC--C--A-----A--A--T--C--A--C--C--C-----C--A--TA --AC-NMT--MGAG-NAGA-CC--C--A-----A--A--T--C--A--C--C--T-----C--A--TA --AC-AMT--MGAG-MAGA-CC--C--A-----A--A--T--C--A--C--C--C-----E--A--TA --AG-GGGA--AMGAG-AAGG-CA--T--A-----A--A--T--C--A--A--C--T-----C--A--TA - -TT-AGGC--MGGA-TAM-GG--A--C-----A--G--C--T--G--T--C--T-----T--G--TT - -TT-AGGC--AMGGA-TAAA-GG--A--C-----A--G--C--C--G--T--C--T-----T--A--TT -- TT-AGGC--AMGGA-TMA-GG--A--C-----A--G--C--C--G--T--C--T----T-T-A--TT

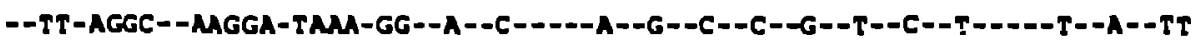

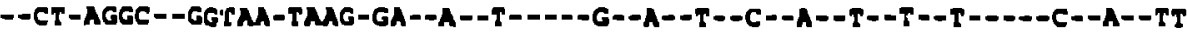
$--A T-A G G C--A G T A A-T N A G-G A--A--T-----A--A--T--C--A--T--T--T-----C--A--T C$

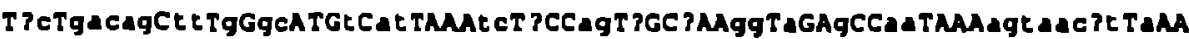
-TC-GACAG-CT-A-GC---T-AT--_-TC-A--AG-C--C--AG-A--G--AA----MATATGC-A-HIV2NIHZ HIV2ISY HIV25T HIV2BEN HIV2DI 94 HIV2GH HIV2D205 SIVMH251 SIVMal 42 S I VMA2 39 SIVMNE SIVSMMHA SIVSHAPBJ -TC-GACAG-TT-A-GC---T-AT----CC-G--AG-T--C--GA-A--A--MA----NATAATG-A--CC-GACAG-CT-G-GC---T-AT--_-TT-A--AG-C--C--AA-A--A--AG----AGTAMCAT-A--TC-GACAG-CT-A-GC---T-AT--_-TC-A--AG-C--C--GA-A--A--MA----AATAATGC-G-- TC-GACAG-CT-A-GC-- -T-AT-.--TT-A--AG-T--C--GA-A--G--A---AGTNACAT-G--TC-GGCAA-CT-A-GC-- -T-AT----CC-A--AG-C--C--GT-A--C--AA----AGTAACAT-G-CC-GACAG-CT-A-GC---T-AT-- - -TT-A--AA-C--C--GA-A--A--AA----AGTAACGT-G-ГT-ANATA-CT-G-CC---A-TT----TT-C--AG-G--A--GG-A--A--AG----AGTTGAGT-A--GC-AACAG-TC-G-GG---T-TC----TC-T--CA-A--T--GG-A--G--TG----AGTCACCT-A-. -AC-AMCAG-TC-G-GG---T-TC-_-TC-T--CA-A--T--GG-A--G-TG-.-GTCGCCCT-A--GC-AACAG-TC-G-GG--T-TC----TT-T--CA-A--T--AG-A--G--TG---AGTCGCCT-A--GC-NACAG-TC-G-AG---T-TC--- -TT-C--CA-A--T--GG-A--G--TG---AGTCACCT-A--GC-.MACAG-TA-G-GC---T-TT--_-TC-C--CA-A--T--GG-G--G--TA---AGTAACAC-A--GC-AACAG-TA-G-GT---T-TC----TC-C--CA-A--T--GG-A--G--TA---AGTAACAC-A--

CONSENSUS HIV2ROD

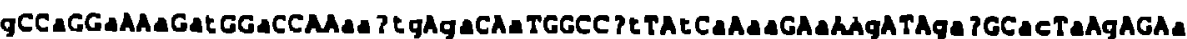
G--A--G--A-AT--A----MCTG-GA--A----CT--A-A-AA--A--A---GAA--AC-A-A---A G--A--G--A-AT--A----GACTA-AA--A---O-TT--A-A-AA--A--A---GAA--AC-A-A---A G--A--A--A-AT--G----ACAM-GA--A-----CT--A-A-GA--A--A---GMA--AC-A-G---A G--A--A--G-AT--A----ACTG-GA--A-----CT--A-A-AA--A--A---TAG--AC-A-A---G G--A--G--A-AT--A----GGCTG-AA--A-----CC--A-A-A--G--A---GAA--AC-A-A---G (1) HIV25T HIV2BEN HIV20194 HIV2GHI G--A--G--A-AT--A----GGCTG-AA--A-----CC--A-A-AA--A--A---GAA--AC-A-A---A G--A--G--A-AT--A--- -GACTG-GA--A-----CC--A-A-AA--G--A---GAA--AC-A-G---G HIV2020S S【VMM2 51 SIVMMI 42 SIVMM2 39 SIVMNE A--T--A--A-AT--G----AGATC-GA--A-----TC--T-C-GG--A--G---CTA--CC-C-A---A G--A--A--G-TT--A----AATTG-AG--G-----AT--T-A-AA--A--G---GTT--AT-A-G---A G--A--A--Gi-AT--A----AATTG-AG--G-----AT--T-A-AA--A--G---(;TT--AT-A-G---A G--A--A--G-AT--A----AATTG-.AG--G----AT--T-A-AA--A--G---GTT--AT-A-G---A G--A--A--A-AT--A----MTTG-GG--G-----AT--T-A-AA--A--G---(;TT--AT-A-G---A SIVSMMH4 A--A--A--A-AA--A----AАTTA-GA--G----GC--T-A-AA--A--G---ATT--AT-A-G---A SIJSMMPBJ A--A--A--A-AT--A-D--AATTA-GA--G----GC--T-A-AA-A-A-G---ATT--AT-G-G---A 
CONSENSUS ATCTGEGAaAAATGGAAAAGATGGCCAgCTAGAgGAaGC PCCECCAACtAATCC P TAEAALACCCCCA HIV2ROD HIV2NIH2 HIV2ISY HIV25T HIV2BEN HIV2DI 94 HIV2GH1 HIV20205 SIVM251 SIVRa1 42 SIVIN239 SIVHAE SIVSMMHA SIVSHMPBJ $--\mathrm{C}--\mathrm{T}--\mathrm{A}--\mathrm{A}-----\mathrm{A}-\mathrm{AA}--\mathrm{A}--\mathrm{C}--\mathrm{GC}-\mathrm{A}-\mathrm{-G}--\mathrm{A}--\mathrm{A}--\mathrm{T}--\mathrm{A}--\mathrm{T}-----\mathrm{T}--\mathrm{T}--\mathrm{T}--\mathrm{C}--\mathrm{C}-$

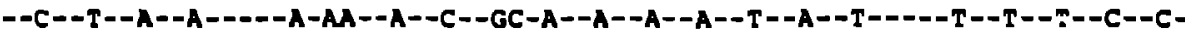
$--C=-T--A--A=----A-G A--A--T--G C-A--A--A--G--T--A--T=----C--T--T--C--T-$

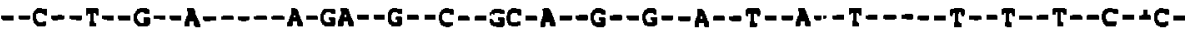
$--C--T--A--A-----A-A A--C--C--G C-A--A--G--A--T--A--T-----T--T--T--C--C-$

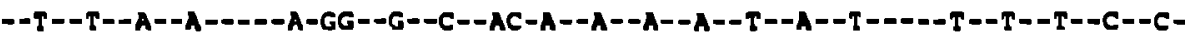

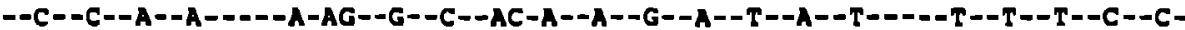
$--C--T--A--A----A-A G--G--A--A T-A--A--A--A--C--T--T-----A--C--C--A--C-$ $--C--T--A--G-----A-A G--T--T--G T-G--G--A--T--C--G--C-----A--C--C--C--C-$ --C--T--A--G- $\cdots-A-A--T--T--G T-G--G--h=-T--C--G--C-----A--T--C--C--C-$ $--C--T--A--G----A-A G--T--T--G T-G--G--A--T--C--G--C-\cdots---A--C--C--C--C-$ $--C--T--A--G-----A-A G--T--T--G T-G=-G--A--T--C--G--C-----A--C--C--C--C-$ $--C--T--A--A-----A-A A--T--C--G T-A--G--A--C-D-T-A--C-----G--T--C--C--C-$

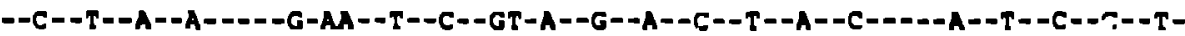
$-A-\cdot---A=-C----A--G--C--A--C--A-----A--G--A-----C--T--G--G C-A--C-A G--$ $-A-----A--T----A--G--C--A--C--A-----G--G--G=--\infty-T--T--A--A C-A--C-A G--$ HIV2ST HIV2BEN HIV20194 HIV2GH 1 HIV 20205 SIVMH251 SIVMM1 42 SIVHA2 39 SIVMNE SIVSMAHA SIVSHMPBJ

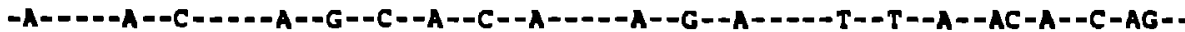
$-A----A--T----A--G--C--G--C--A-----G--G--G----T--T--A--A C-A--T-A G=-$ $-A-=--A--\mathrm{T}-----A--G--C--G--C--A-----A--G--A-----T--T--A--A C-A--C-G G-$ $-A----A--T-----A--G--C--G--C--A----G--G=-A-=---C--T--A--A C-A--C-G G--$

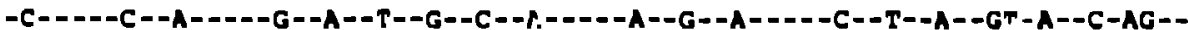
$-A----C--A--D-A--A--T--G--C--A-----A--G--G--1-T--T--G--A C-A--T-G G=-$

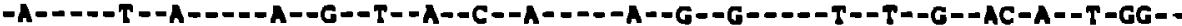

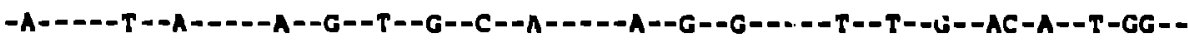
$-A-----C--A-----A--G--C--G--C--A-----A--A--G=----T--T--G--A C-A--T-A G--$

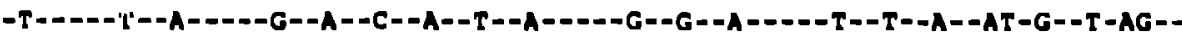
$-A----T--A-----G--A--C--G--T--A-----G--G--A-----T--T--A--A T-A--C-A G--$

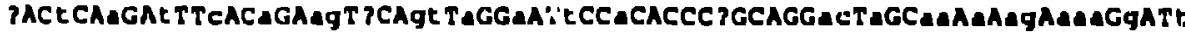
HIV2ROD HIV2NIHZ HIV2ISY HIV2ST HIV2BEN HIV2DI 94 IIIV2GIII HIV2O205 SIVHA25I SIVMH 142 S I VMM2 39 SIVMNE SIVSHMHI IIVSMMPIIJ A--T--A--T--C--A--AA-T--GT-A--A-- P--A-----A-----GT-G--CA-G-AG-GMA-A--T A--T--G--T--C--A--AA-T--GT-A--A- - T--A-----A-----AT-G--CA-A-AG-GGA-A--T A--T--A--T--C--A--GG-T--GT-A--A- -T--A-----A-----AT-A--CA-G-AA-GAA-A-D

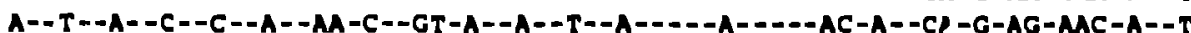
G--T--A--T--C--A--AA-T--GC-A--A--T--A-----G-----AC-A--CA-A-AG-AAA-G--C G--T--A--T--C--A--AA-T--GC-A--A--T--A-----G-----AT-A--CA-A-AG-AAA-G--T G--T--A--T--C--A--AA-T--GC-A--A--T--A-----G-----AT-A--CA-A-AG-AAA-G--C

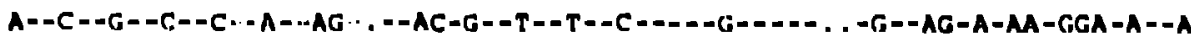
C--T--G--C--T--A--AG-C--AT-A--A--A--A--D--T-----AC-A--AA-A-GG-AAA-G- -T C--T--A--C--T--G--AG-C--AT-A--A--A--A--D-T--D--AC-A--AA-A-GG-AAA-G--T

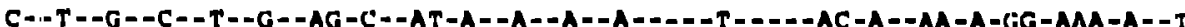
C--T--G--C--T--A--AG-C--AT-G--A--A--A-----T-----AC-A--AA-A-GG-AGA-G- -C C--T--A--C--T--A--AG-A--GT-A .. -A--A-----T-----AC-A--AA-G-CA-GGA-G--C 


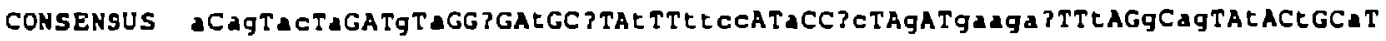
HIV 2ROD HIV 2NIHZ HIV 2 ISY HIVZST HIV2BEN HIV2O194 HIV ?GHI HIV 20205 SIVMM251 SIVHMI 12 SIVM2 39 SIVANE SIVSMHH4 SIVSMRPBJ A-TG-AC-A---G-A--G--T--T--C--TTCC--A--AC--C--GAGGAC- - T--A-CA--T--T--AA-TG-AC-A---G-A--G--T--T--C--TTCC--A--AC--C--GAGGAC- -T- -A-AG--T--T--AA-TG-GT-A---G-A--A--T--C--C--TTCC--A--CC--T--GAGGAT--T--A-AG--T--T--AA-TG-CC-A---G-A--G--T--T--C--TTCC--A--AC--C--GAGGAT-D-T-A-AG--T--T--AT-TA-AT-A---G-A--G--T--C--T--TTCC--A--AC--C--GAAGT--T--G-AG--T--T--A$A-T G-A C-A---G-A--G--T \cdot-C--C--T T C C--A--A C--C--G A A G A T--T--G-A A--T--T--A-$ A-TG-AC-A---G-A--G--T--C--C--TTCC--A--GC--C--GAAGAT--T- -G-AG--T--T--AA-AG-AA-A---G-G--A--T--C--C--CAGT--C--AC--G--CCAAC--C--A-AG--T--A--AA-AG-AC-G---A-A--T--T--A--T--CTCC--A--TC--G--GAAGAA--T--G-AG--こ--T--CA-AG-AC-G---A-A--T--C--A--T--CTCT--A--TC--G--GMGAA--T--G-AG--C--T--CA-AG-AC-G---A-A--T--T--A--T--CTCC--A- -TC--G--GAAGAA--T--G-AG--C--T--CA-AG-AC-G---G-A--T--C--A--T--CTCC--A--TC--G--GMGAA- - T--G-AG--C--T--TA-AG-AT-G---G-A--T--T--A--T--CTCC--A--TC--G--GAGGAA--C--G-AG--C--T--C$A-A G-G T-G---G-A--T--T--A--T--C T C C--A--T T--G--G A G M A--C--G-A A--C--T--C-$

CONSENSUS HIV2ROD HIV2NIHZ HIVZISY HIV25T HIV 2BEN HIV2D194 HIV2GH1 HIV2D20S 5 IVMA2 51 SIVMHI 42 SIVMi4239 SIVMNE SIVSHMH4 SIVSMMPBJ

CONSENSUS HIV2ROD HIV 2NIHZ HIVZISY HIV $2 \mathrm{ST}$ HIV 2 BRN HIV2D194 HIVZGHI HIVZO203 SIVMM 231 SIVMMI 12 SIVAM2 39 SIVMNE SIVSMMH SIVSMHPBJ

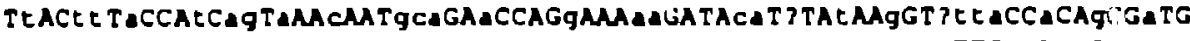

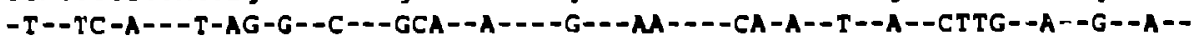
$-C--T C-A---T-A G-A--C--G C A--A----G---A A----T A-A--C--A--C C T A--A--G--G--$ - T--TC-G-- - T-AG-A--C---GCA--A----G---AA----TA-A--C--A--CTTA--A--G--A-$-T--T C-A---T-A A-A--C---G C T--A----G---A A----C A-A--T--A--C T C A--A--G=-A--$

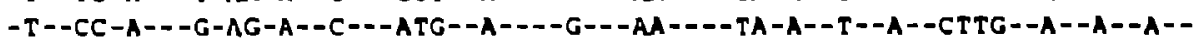
$-T=-C C-A---T-A G-A--C---G C A--G----A---A A----T G-A--T--G=-C T T A--A--A--A--$ $-T--C C-A=--T-A G-A--C---G C A--A----G---A A----T A-A--T--G--C T T A--A--A--A--$ $-C--C T-G---T-A G-A--C---G C A--G----G=-G A----C A-T--T--A--T C T A--A--A--G--$ $-T--T T-A=-T-A G-A--T---G C A--G----G---A C----C A-T--T--G--T C T G--T--G=-A--$

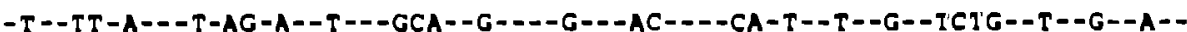

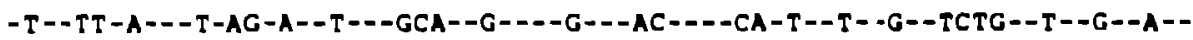

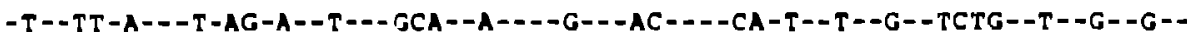
$-T--T T-A--T-A G-A--T=-G C A--A----C_{-}---A A----C A-C--T--G--A T T A--T=-A--G--$ $-T--C T-A=--T-G G-A--C---G C A--A----G---A A----C A-C--T--G--A T T A--T--A--G--$

1026 1010 1007 1007

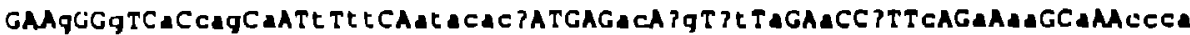
---G--A--A-CAG-A--T-TT--ACACACA-----AC-GG-AT-A--A--A--C--A-AA--A--CAAG $---G--G--A-C A G-A--T-T T--A T A C A C A----G C-G A-C T-A--A--A--C--A-A A--A=-C G A G$ $---G--G--A-C A G-A--T-T T--A T A C A C A----G C-A G-C T-A--A--A--C--A-A A--A--C C C A$ ---G--A--A-CAG-A--T-TT--GTACACA-----GC-GG-CT-A--A--A--C--A-A--A--CCCG $---G--A--A-C A G-A--T-T T--A T A C A C A----G C-A G-C T-A--A--T--C--A-A A--A--C C C A$ ---A- -A--A-CAG-A--C-TT--ATTCATG-----GC-AA-CT-A--A--T--C--A-AA--A--CCCA $-\cdots-G--A--A-C A G-A--T-T T--A C A C A C A----C C-A G-C T-A--A--T--C--A-A A--A--C C C A$ $---G--G--C-A G T-A--T-G T--A T A C T C C-----A A-G G-A T-A--T--T--T--A-A G--C--C A G C$ $---G--G--A-C A G-C--C-T C--A T A C A C T----A C-T G-G C-A--A--C=-C--G-A G--A--T C C A$ - - -G--G--A-CAG-C--C-TC--ATACACT-----AC-TG-GC-A--A--C--C--G-AG--A--TCCA -- -C--G--A-CAG-C--C-TC--ATACACT-----AC-TG-CIC-A--A--C--C--G-AG--A--TCCA ---G--G--A-CAG-C--C-TC--ACACACT----AA-TG-GC-G--A--C--C--G-AG--A--TCCA -- -G--G--A-CAG-T--T-TT--GTATACT-----AA-TG-AT-A--A--T--C--A-AA--A--TCCA -- -A--A--A-CAG-T--T-TT--GCATACT-----AA-TG-CT-A--A--C--T--A-AA--A--TCCA 
CONSENSUS HIV2ROD HIV2NIHZ HIV2ISY HIV2ST HIV2BEN HIV2D1 94 HIV2GH1 HIV2D205 SIVMM251 SIVMM1 42 SIVMM239 SIVHNE SIVSMAH 4 SIVSMMPBJ

CONSENSUS HIV2ROD HIV2NIHZ HIV2ISY HIV2ST HIV2BEN HIV2D1 94 HIV2GHI HIV2D205 SIVMA251 SIVMM1 42 SIVRM2 39 SIVMNE SIVSMMH4 SIVSHAPBJ

CONSENSUS HIV2ROD HIV2NIHZ HIVZISY HIV2ST HIV2BEN HIV2DI 94 HIV 2GH1 HIV 2D203 SIVMM2bl SIVMMI 4 ? SIVMm2 39 SIVMNE: SIVSMall SIVSMMPRJ
GAtgTCAtE ?T ?ATCCAgTACATGGATGAEATCL Ta TAGCEAGtGACAGgACaGatt TaGAaCAtGACA --TG-C-TTA-CA-T--G--C--------T--CT-AA----T--T--C--G-CA-ATT-A--A--T--T--TG-E-TTA-CA-T--G--C--------T--CT-AA----C--C--C--G-CA-ACT-A--A--T--C--TG-C-TTA-CG-T--G--C--------T--CT-AA----T--T--C--G-CA-ATT-G--A--T--C--TA-C-TTC-CA-T--G--C---D---T--CT-GA----C--C--C--G-CA-ATT-A--A--T--C--TG-C-TTC-OA-C--G--C----D---T--CT-AA----T--T--C--G-CA-GTT-A--G--T--C--CG-C-ITC-CA-C--A--C-------OT--CT-MA---OT--T--C--G-CG-GTT-A--G--T--C- -TG-C-TTC-CA-C--A--C---_---OT-OTT-MA----T--T--C--G-CA-GTT-A--G--T--C- TG-C-TTA-AA-T--G--C--.-...-C--CC-TA----A--T--C--A-GT-ATC-G--G--C--C-

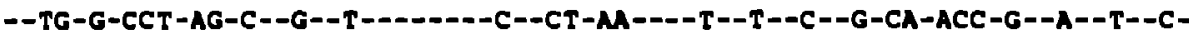
- -TG-G-CCT-AG-C--G--T-------C--CT-MA----T--T--C--G-CA-ACC-G--A--T--C-

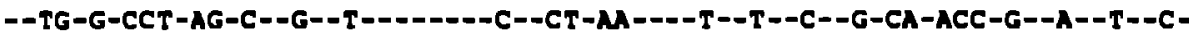
--TG-G-CCT-AG-C--G--T--------C--CT-AG----T--T--C--G-CA-ACC-G--A--T--C--TG-G-CCC-GA-C--A--C--------C--CT-AA----T--T--T--A-CA-ATT-A--G--T--C--TG-G-CCC-GA-C--A--C--------C--CT-AA----T--T--T--A-CA-ATI-A--A--T--C-

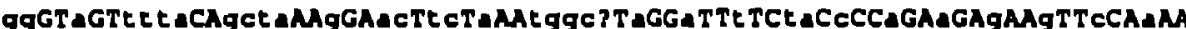
GG--A--CCTG--GCTC--G--AC-TC-A--TGGCC-A--A--T--TA-C--A--T--G--G--C--A-M--G--CCTG--GCTA--A--AC-TC-A- - TGGAC-A--A--T--CA-C--A--T--G--A--C--G-MA--A--CCTA--GCTA--G--AC-TC-A--TGGCC-G--A--T--CA-C--A--C--A--G--C--A-GA--G--TCTG--GCTA--G--AC-TC-A-TGGCC-G--A--T--CA-C--A--T--G--G--C--A-MA--G--CCTG--GCTA--A--AC-TC-A--TGGCC-A--G--T--TA-T--A--T--G--G--C. -A-AA--A--CCTG--ACTA--A--AC-TC-G--TGGCC-A--G--C--TA-C--A--T--G--G--C--A-MA--A--CCTG--GCTA--A--AC-TC-G--TCGCC-A--G--C--TA-C--A--T--G--G--C--A-GG--A--GTCC--ACTA--A--GT-AT-A--TGACA-G--A--C--TA-C--A--A--A--G--C--A-GG--A--TTTA--GCTA--G--AC-CT-A--TAGCA-A--G--C--TA-C--A--A--G--A--C--A-GG--A--TTTA--GTTA--A--AC-CT-A--TAGCA-A--G--T--AT-C--A--A--G--A--C--A-GG--A--TTTA--GTCA--G--AC-CT-G--TAGCA-A--G--T--TA-C--A--A--G--A--C--A-SG--A--TTTA--GTTA--G--AC-CT-A--TAGCA-A--G--T--TA-C--A--A--G--G--C--A-GG--A--TTTA--GTTA--G--AC-TC-G--CGGCA-A--A--C--TA-C--A--A--G--G--C--G-GG--A--TTTA--GTTG--G--AC-TC-G--CAGTA-A--A--T--CA-C--A--A--G--G--C--G--

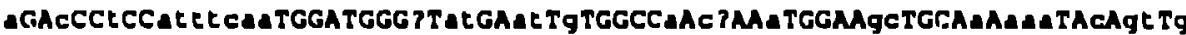
A--C--T--ATACCAC-------C-AT--AC-A-----A-CT--A-----GT----G-AMA--C-GT-G A--C--T--АTATCGC---_----C-AT--AT-A--_--A-CT--A-----GT----A-AAA--C-GC-G G--C--T--ATACCM--_-_---C-AT--AC-G-----A-CC--A-----AT----A-AMA--C-A'T-G

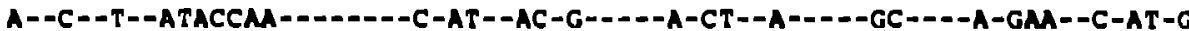

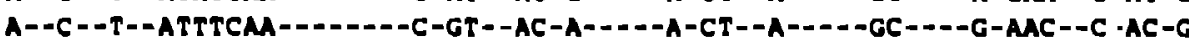
G--C - - T- -GTTTCAA-------C-AT--AT-G-----A-CT--A-----AC----G-AAA--C-AT-A

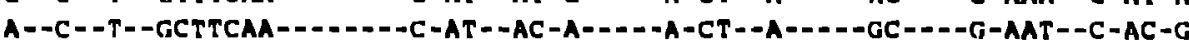
A--C--T--GTTCAMA---.---T-AT--GC-C-.---A-AA--G--.-AC----A-AAA--C-AC-G A--T--C--ATTTCAA-......--G-AC--AT-Gi-.-- -(i-CA--A-1--GT---A-AGA--G-GT-G

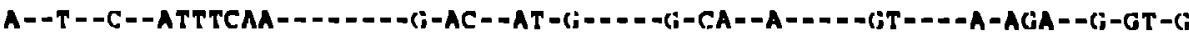

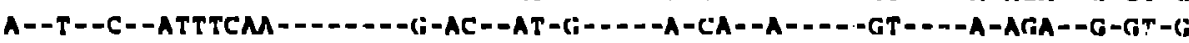
A--T--C--ATTTCAA-.-------G-AT--AT-G-----A-CA--A-----JT----A-AGA--(;-C:T-G

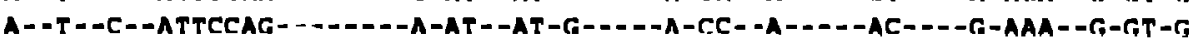

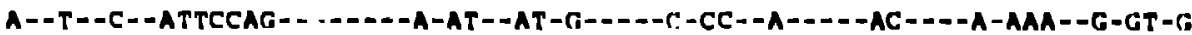

1160

1150

1147

1147

1261

1207

1261

1207

1207

1210

1210

1222

1210

1099

1108

1229

1220

1217

1217

1331

1277

1331

1277

1277

1280

1280

1292

1280

1169

1278

1297

1290

1287

1287

1402

1347

1401

1341

1347

1350

1350

1362

1350

1219

1248 
CONSENSUS HIV2ROD HIVZNIHZ HIVZISY HIV2ST HIV2BEN HIV2D194 HIV2GH 1 HIV2D205 SIVMA 251 SIVMA1 42 SIVAM2 39 SIVANE SIUSIOAH SIVSMAPBJ

CONSENSUS HIVZROD HIV2NIHZ HIVZISY HIV2ST HIV2BEN HIV2DI 94 HIV2GH HIV2D205 SIVMM 251 5 IVRA1 42 SIVHA239 SIVMNE SIUSMMHA SIVSHMPOJ

CONSENSUS HIVZROD HIV 2 NIHZ HIVZISY HIVZST HIV.:"HN HIV2DI94 HIV $2 \mathrm{GH} I$ HIV 20205 SIVMM23: 5 I UHMI 42 SI 'MAM2 39 SI VMNE SIVSMMHA SIVSMMPBJ

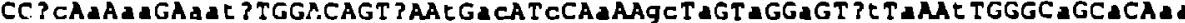

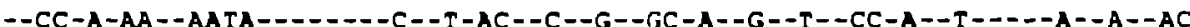
$--C C-A-A A--A G T A------C--T-A C--C--A--G C-A--G--T--C C-A--T-----A--A=-A A$ --CC-A-AG--AGTA-------T--T-AC--C--G--AC-A--G--T--CC-A--C-----G--A--AA $--C C-A-A G--A G T A-------C--T-A C--C--A--A C-G--G--T--C C-A--T-----A--A--A A$ --CC-G-AA--CATA--------C--T-AC--C--A--GC-A--G--A--CT-A--T-----G--A--A -- TC-G-AA--AATA-------C--T-AC--C--A--AC-A--A--A--TT-G--C-----G--G- -GA $--C C-G-A A--M A T A--------C--C-A C--C--G=-G C-G--G--A--C T-A--T-----G--A=-M$ $--A G-A-A A--A G T T-------G--T-C A--T--A--A C-G--A--A--A T-A--C----A--T--A C$ --AC-A-GA--GACC--- -- - -G--T-AT--A--G--GT-A--A--A--AT-A--T-----A--T--A --AC-A-GA--GACC-------G--T-AT--A--G--GT-A--A--A--AT-A--T-----A--T--MA --AC-A-GA--GACC--------G--T-AT--A--G--GT-A--A--A--AT-A--T-----A--T--A $--A C-A-A A--G A C C-------G--T-A T--A--G--G T-A--A--A--A T-A--T-----A--T--A A$

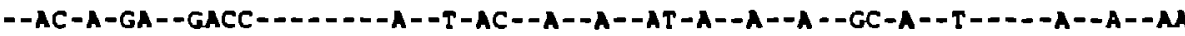

--AC-A-GA--MACC--------A--T-AT--A--A--AT-A--A--A--GC-A--T-----A--A--A

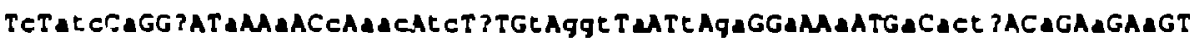
$-C-A C C-A--G--A--G=-C-A A C-C T-A--T-G G T-A--C-G A--A--A---A-A C T C--A--A--A--$ $-C-A C C-A--G--A--G=-C-A A C-C C-A--T-G G C-A--T-G A--A--A---A-A C T C--A--A--G--$ $-C-A C C-A--A--A--G--C-A A C-C T-A--T-A G C-A--T-G A--A--G--A-A C C C--G--A--A--$ $-C-A C C-A--G--A--G--C-G A A-C T-A=-T-G G T-A--C-G A--A--A---A-A C T C--A--A--G--$ -C-ATT-A--A--A--A--C-AAC-CT-A- - T-GAC-A--T-GA--A--A---A-ACTC--A--A--A--C-ATC-A--G--A--A- C-AGC-TT-A--T-AAT-G--T-GA--A--A---A-ACTC--A--G--A--C-ATC-A--A--A--A--C-AAC-TT-G--T-GGC-A--C-AA--A--A---A-ACTC--A--A--A--C-TTC-T--A--T--G--A-GGC-CA-A--C-AAC-A--T-GG--A--G---A-CCTA--A--A--A--T-ATC-A--T--A--A--C-A'C-TC-C--T-GGT-A--T-GA--A--A---A-TCTA--A--G--A--T-ATC-A--T--A--A--C-MAC-TC-C--T-GGT-A--T-GA--A--A---A-TCTA--A--G--A--T-ATC-A--T--A--A--C-A AC-TC-C--T-GGT-A--T-GA--A--A---A-TCTA--A--G--A--T-ATC-A--T--A--A--C-AA - TC-C--T-GGT-A--T-GA--A--A---A-TCTA--A--G--A-T-ATC-A--A--A--G--T-AAC-TC-T--C-GAC-A--C-GA--A--A---A-TTTA--A--A--G-$-T-A T C-A=-A--A--G--T-A A C-T C-T--C-G A T-A--C-G A--A--A---A-T T T A=-A--A--G--$

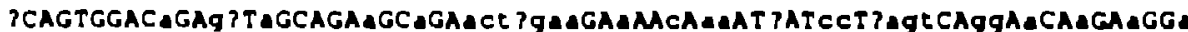
A-------A--AT-A-----A--A--GCTAGAA--A--C-GA- - T--CC-AAGC - - GG-A--A--G--A A---D---A--AT-A-----A--A--GCTAGAG--A--C-GA--T--CT-AAGC--GA-A--A- -A--A A---D---A--AT-A----A--A--GCTGGAG--A--C-AA--T--CT-MGC--GG-A-G-G- A A------A--AT-A-----A--G--ACTAGAA--A--C-M--C--CT-AAGC--GG-A--A-A--A G-------A--AC-A-----A--A--GCTAGAA--A--C-AA--T--CT-GAGS--GG-A--A--h--A A-------A--GT-A-----G--A--ACTAGAA--A--G-MA--T--CT-AAGI --GG-A--A--G--A G-------A--GT-A-----A--A--GCTAGAA--A--C-AA--T--CT-AAGC--GG-A--A--A--A A-----A--AC-A----A--A--GCTACAG--G--T-AA--C--CT-AGAA--GG-A--A--A--A T-2-1.- - T-GA-G----A--A--ATATGAG--A--T-AG--A--TC-CAGT--GG-A--A--A--A T---1--T--GA-G----A--A--ATATGAG--A--T-AA--A--TC-CAGT--GG-A--A--A--A T------T--GA-G----A--A--ATATGAG--A--T-AA--A--TC-CAGT--GG-A--A--A--A T------T--GA-G-----G--A--ATATGAG--A--T-AA--A--TC-CAGT--GG-A--A--A--A T------T--GA-G-----G--A--ATATGAA- -A--C-AG--A--TC-CAGT--AG-A--A--A--A

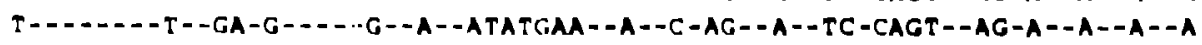


CONSENSUS HIV2ROD HIV 2 I I Z HIV2 ISY HIV2ST HIV2BEN HIV2D194 HIV2GHI HIV2D205 SIVAM 251 SIVHMI 12 SIVHM2]9 SIVHNE SIVSMOHA SIVSMMPBJ

CONSENSUS HIV2ROD HIV2NIH2 HIVZISY HIV2ST HIV2BEN HIV2D194 HIV2GHI HIV2C 205 SIVMM251 SIVMM142 SIVMM239 SIVMNE SIVSMMH4 SIVSMMPBJ

CONSENSUS HIV2ROD HIV $2 \mathrm{NIHZ}$ HIV2 IS: HIV25T HIVIBEN HIV2DI94 HIVZGHI HIV2D20S SIVMM23I S:VMM1 42 SIVMM 2]9 SIVMNE SIVSMMHA SIVSMMPHJ

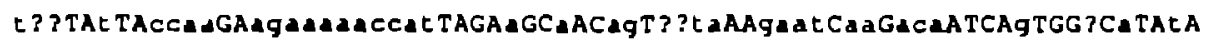
CAC--T--CCAA--AGAAAAAGAGC-----A--A--AG-CCAA--GGAT-AA-AGA----G---A-A--TCAC--T--CCAG--AGAAAAAAAGT----A--A--AG-CCAG--AGAT-AA-ACA----G---A-A--TCAC--T--CCAA--GGAAAAAGAGT----A--A--AG-TCAA--GGAT-AA-ACA----G---A-A--T TGC--T--CCAA--GGAMAAGGAGC----A--A--AG-CCAA--AGAT-AA-ACA----3---A-A--TTAT--T--CCAA--AGAMAMGMAT----G--A--AA-CCAA--AAGC-AA-GAC----A---A-A--CTCC--C--TCAG--AGAACMAGAAC----A--A--AG-CATC--AAGC-AA-ACA-.--G---G-A--CTAC--T--CCAA--AGAMAAGAAT----G--A--AA-CCAA--AAAT-AA-ACA----G---A-A--CTCC--C--CAAG--AAGGG TACCGC----A-.A--AG-ACAG--AAAC-TA-CAA----G---A-A--CTGT--T--CCAA--AGGCAAGCCAT----A--C--GG-AATA--GAGT-AG-ACA---G---T-T--TTGT--T--CCMA--AAGCAAGCCAT----A--C--GG-GATA--GAGT-AG-ACA----G---T-T--TTGT--T--CCAA--AGGCAAGCCAT----A--C--GG-MATA--GAGT-AG-ACA----G---T-T--TTGT--T--CCMA--AGGCAAGCCAT----G--C--GG-MATA--GMAT-AF-ACA----G---T-T--TTGT--C--CCAA--AGGNAAACCAA----G--A--AG-AATA--GAGT-AG-ATA----A---T-A--TTGT--C--TCAG--GGGAAAGCCAC----G--A--AG-AATA--GAGT-AG-ATA----A---T-A--T-

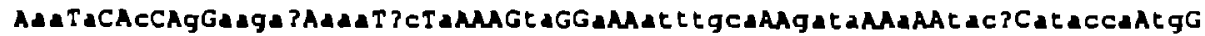
-AA-A--C--G-AAGAA-AAA-TC-A----TA--A--ATATGCA--GGTG--A--CACC-ATACCA-TG-GG-A--C--G-GGGAG-AAA-TC-C---..GT--G--AATATGC--AGAT--A--ATAC-CATACC-AC-AG-A--C--G-GAGAA-AA-TC-A----TA--A--ATATG IA--GATA--A--TACC-ATACCA-CG-GA-A--C--G-GAGGA-ARA-TC-A----TA--A--ATATGCA--GGTA--A--TACC-ACACCA-CG- AA-A--C--G-AAGAG-AAA-CC-A--.-TA--A--GTATGCA--GATA--A--TACC-ATACCA-TG-AA-A--E--G-GAGAG-GGG-TC-A----TA--A--GTATGCG--GATA--A--TACT-ATACCA-TG-AA-A--C--G-AAGAG-AAA-CC-A----TA--G--ATATGCA--GATA--A--TACC-ATACCA-TG-AA-T--T--G-GAAAT-AAG-CC-A----TA--A--ATATGCA--GGTT--A--CACG-ACACCA-CG-AA-T--C--A-AAGAC-AAA-AC-G----TA--A--ATTTGCA--GATA--G--TACA-ATACCA-TG-AA-T--C--A-AAGAC-AAA-AC-G----TA--A--ATTTGCA--GATA--G--TACA-ATACCA-TG-AA-T--C--A-AAGAC-AAA-AC-G----TA--A--ATTTGCA--GATA--G--TACA-ATACCA-TG-GA-T--C--A-AAGAC-AAA-AC-A----TA--A--ATTTGCA--GATA--A--TACA-ATACCA-TG-AA-T--C--A-AAGAC-AAG-AC-G----TA--T--ATTTGCA--GGTT--A--TACA-ATACAA-TG -AA-T--C--A-AAGAC-AMA-AC-G----TA--C--ATTTGCA--GATT--A--TACA-ATACAA-TG-

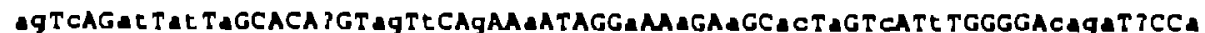
AA-C--AT-GT-A-----G--AG-T--G--A-----A--A--A--AC-A--C--T--..--CGAA-A--A GG-C--AT-GT-A-----G--AG-T--A--A-----A--A--A--AC-A--C--T------CGGA-A--A GG-C--GT-GT-G-----G--AG-T--G--A------A--A--A--AC-A--C--T------CGAA-A--A AG. C--AC-CC-A-----A--AG-T--A--A-----A--A--A--AC-A--C--T-----CGAA-A--A GG -C--AT-AC-A-.--G-G-AG-T--G--A-----A--A--G--AC-A--C--T------CGGA-A--A GG-C--AC-AC-A--- -A--AG-C--A--A----A--G--A-AC-G--C--T-----CGAG-G--A GG-C--AT-AC-A-----G--AC-T--G--A-----A--A--A--AC-A--C--C---.--CGGA-A--A GG-A--AC-AC-G--.--T--AG-T--G--A--.--C--A--A--CC-A--C--C----- $G A C A-A--A$ $A G-T--A C-A T-A-1--T--A A-A--G-A-----A--G--A--A A-A--G--C--.--C A G G-C--A$ $A G-T--A C-A T-A-----T--A A-A--G--A-----A--G--A--A A-A--G--C-----C A G G-C--A$ $A G-G--A C-A T-A----T--A A-A--G--A----A--G--A--A A-A--G--C-----C A G G-C--A$ AG-T--AC-AT-A--.--T--AA-A--G--A-----A--G--A--AA-A--G--C--.---CAGG-C--A $A G-C--A T-A C-A-----C--A G$-G--G--A-----A--A--A--AC-A--A--T------GAGG-G--A $A G-C--A T-A T-A----T--A G-G--G--A-----A--A--A--A A-A--A--T-----C A G G-G--G$ 


\section{HIV2/SIV POL}

CONSENSUS aaATCCACETACCaGTaGAgAgaGA ?atCTGGGAaCAgTGGTGGgCagAcTATTGGCAaGTaACaTGGa HIVZROD HIV2NIHZ HIV2ISY HIV2ST HIV2BEN HIV 2D194 HIV 2GH1 HIV 20205 SIVMA251 SIVMH1 12 SIVMM239 SIVMNE SIVSHMH 4 SIVSMMPBJ AAA- - T--CC-A--A--A--G-GA--AATC-----G--G-----GATA-C--C-----A--G--A---A AAA--T--CC-A--A--A--G-GA--TACC-----G--G------GATA-C--C-----A--A--A-- -A MAA--T--CC-A--A--A--A-GA--GACC-----A--G---1--GATA-C--T-----A--G--A---A MAA--T--CC-A--A--A--A-GA--TACC-----A--G------GATA-C--C-----A--G--A-- -A MA--T--CC-G--A--G--G-GA--GACC-----G--G------GATA-C--C-----A--G--A-AAA--T--CC-A--G--A--G-GA--CACC-----G--A------GATA-C--C-D--A--A--A---G AM--C--CT-A--G--G--G-GA--GACC-----G--G------GATA-C--C-----A--G--A---A GTG--C--TC-G--A--A--A-GA--GACA-----C--G------ACAG-T--C-1---A--A--C---A MA--C--CT-A--A--T--G-GG--TGTh-----A--G------ACAG-C--T----G--A--C---A MA--C--CT-A--A--T--G-AG--TGTA----A--G-----ACAG-C--T-D--G--A--C---A MA--C--CT-A--A--T--G-AG--TGTA-----A--G------ACAG-C--T-D--G--A--C---A AMA--C--CT-A--A--T--G-AA--TGTA-----A--G------ACAG-C--T-----G--A--C---A AAA--C--TI-G--A--A--A-GA--AATT-----A--A------ACAG-T--T-----A--A--C---A

CONSENSUS HIV 2ROD HIV 2 NIHZ HIV 2 ISY HIV 2ST HIV 2BEN HIV2D194 HIV 2GHI HIV20205 SIVMM251 SIVMM1 42 SIVMH239 SIVMNE SIVSMAH 4 SIVSMAPBS

CONSENSUS HIV 2 ROD HIV 2 NIHZ HIV 2 ISY HIV2ST HIV 2 BEN HIV 20194 HIV 2GH] HIV 20205 SIVMH251 SIVMMI 12 SIVMM239 SIVMNE SIVSMMHA SIVSMMPBJ

AGA--T--TT-G--A--A--G-GA--AATT----A--A---D-ACAG-T--T-----A--A--T---A

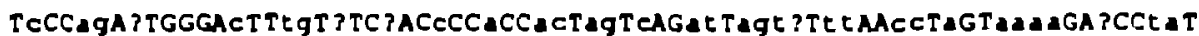
-C--AG-C------C--CG-G--T--C--A--AC-GG-C--GT-AGCG-TT--CC-G--AGGG--T--TA-C--AG-C-----C--TG-A--T--C--A--AC-GG-C--GC-AGCA-TT--CC-G--AGGA--G--TG$-C--A G-C-----C--C G-A--C--C--A--G T-G G-C--G T-A G C A-T T--C C-G--M A A--T--T A-$ -C--AG-C-----C--CA-A--T--C--G--AC-GG-C--AT-AGTA-TT--CC-G- -GAAA- - T- - CA$-C--A G-G----C--T G-A--T--C--A--A C-G G-C--G T-A A C A-T T--C C-A--A G G A--T--T A-$ $-C--A G-G=---C--C G-A--T--C--A--A C-G G-C--G T-G A C A-T T--C T-G--A G G A--T--T A-$ $-C--A G-G----C--C G-A--T--C--A--A C-A G-C--G T-A A C A-T T--C C-A--A G G A--T--T A-$ $-C--A G-G----C--T G-C--G--C--A--A T-A A-A--A C-A G C C-A C--C C-A--C A A A--C--C C-$ -A--GG-G-----T--TA-C--A--G--A--AC-AG-A--AT-AGTC-TC--TC-A--GAAG--C--TA-A--GG-A----T--CA-C--A--A--A--AT-AG-A--AT-AGTL-TC--TC-A--GAAG--C--TA-A--GG-A----T--TA-C--A--A--A--GC-AG-A--AT-AGTC-TC--TC-A--GAAG--C--TA-A--GA-A----IT--TA-C--A--A--A--AC-AG-A--AT-AGTC-TC--TC-G--MAAG--C--TA$-A--A G-T----C--T G-G--A--A--T--C T-A G-C \cdot-A T-A G T C-T C--C C-A--A M A--G--T A-$ -A--AG-A-----C--TG-G--A--A--T--CT-AG-C--AC-AGTT-TC--CC-A--AMAA--G--TA-

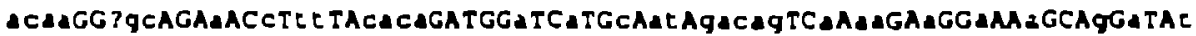
ACCA--TGC---G--C-TC--CACA--- - A--C--C-AT-GGCAA--A-AA--A--A--A---G-A--T ACCA--CGC---A--T-TC--CACA-----A--C--C-AT-GGCAG--A-AA--A--G--A---G-A--T ACCA--CGC---G--C-TC--CACG-----A--T--C-AT-GGCAA--A-AA--G--A--A---G-A--T ACTA--CGC---A--C-TC--CACA-- - --A--C--C-AT-AGCAA--R-GA--A--A--A---G-A--C ACCA--CGC---G--C-TC--CACA-----A--A--C-AT-GACAG--A-AA--G--A--A---G-A--T ACCA--CAC---G--C-TT--CACA-- - -A--A--C-AT-GACAG--A-AA--A--A--A-- -G-A--T ACCA--CGC---G--C-TC--CACA--- -A--A--C-AT-GACAG--A-AG--A--A--A---C-A--T AGAA--GAG---A--C-AC--CACA-----G--C--C-AT-GAACC--A-AG--A--A--A---G-A-DT AGAG - - AGA- - -A-- - -AT--TACA-- - -A--A--T-AT-AACAG - -A-AA--A--G--A-- G-A--T AGAG--AGA---A--C-AT--TGTA----A--A-DT-GT-AACAG--A-AA--A--A--A-- -G-A--T AGAG--AGA---A--C-AT- - TACA-----A--A-- T-AT-AACAG--A-AA--A--G--A---G-A--T AAAG--AGA---A--C-AT--TGTA-----A--A- - T-AT-AACAG--A-AA- -A--G--A---G-A--T ACAG - -AGC---A--A-TT--TGTA-----A--C- - T-AT-GGCAG--A-GA--A--A--A-- - G-C- - T 
CONSENSUS HIV2ROD HIV2NIHZ HIV2ISY HIV2ST HIV2BEN HIV2D194 HIV2GHI HIV20205 SIVRe125I SIVIM1 42 SIVM2 39 SIVINE SIVSMMH4 SIVSHAPBS

CONSENSUS HIV2ROD HIV2NIHZ HIVIISY HIV2ST HIV2BEN HIV2OI 94 HIV2GH 2 HIV2D2OS SIVIM251 5 I VMa1 42 SI VIA 239 SIVHAE SIVSHMHA SIVSHAPBJ

ZONSENSUS HIVZROD HIVZNIHZ HIVIISY HIV2ST IIIV2BEN HIV2CI 94 H I $2 \mathrm{GHI}$ HIV2D2OS S IVMA2 51 SIVMAI 42 5 I VMA2 39 5 I VMNE SIVSHMHA SIVSMMPQJ

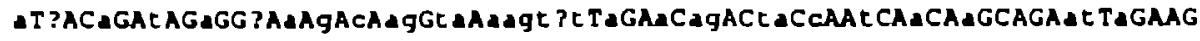
G-A--A--T--A--G-A-G-C-AG-TA-AGLAAC-A--G-AA--TA-C--T--G--A-_--AC-A-.-A-A--A--T--A--G-G-G-C-GG-TA-AAGTAT-A-DG-AA--TA-C--T--G--A-----AT-A--A-A--A--T--A--A-A-G-C-AA-TA-GGATAT-A--G-AA--TA-E--C--A--A-----AT-A---A-A--A--T--A--A-G-G-L-AG-TG-GGCTAT-A-OG-AA--CA-C--T--G--A-----AT-A---G-A--A--T--A--A-A-G-C-AA-TA-AMGTAT-A--A-MA--TA-C--T--G--G-----AT-A---G-A--A--T--A--G-G-G-C-GG-TA-GAGTAT-A--G-AA--AT-C--T--G--A-----AC-A---G-G--A--T--A--A-G-G-C-AG-TA-GAG $(A C-\lambda$. --A-GA--TA-C--T--G--A----AT-A---G-C--T--C--G--A-A-G-T-AG-TT-AAGTGT-A--A-AG--AA-A--C--A--A-----AC-T---A-C--A--T--G--C-A-G-C-MA-TA-AAGTGT-A--A-AG--TA-T--T--A--A--.--AT-A-D-. A-C--A--C--G--C-A-G-C-I.G-TA-AMGTGT-A--A-AG--TA-T--T--A--A-----AT-G---A-C--A--I--G--C-A-G-C-MA-TA-MAGTGI-A--A-AG--TA-T--T--A--A-D--AT-G--.A-C--A--T--G--C-A-G-C-AA-TA-MGTCT-A--A-AG--TA-T--T--A--A-----AT-G-..-G-G--G--T--G--C-G-G-C-AA-CA-AACTTT-A--A-AG--TA-C--C--A--A-.-.-GT-G--.G-G--A--T--A--C-G-G-C-AG-CA-AACTGC-G--A-AL--TA-C--T--A-・A-----GT-G-..-

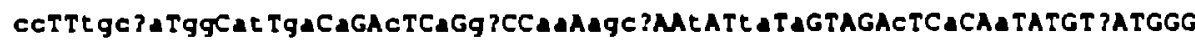
CC--TGCGA-GG-AC-AA-A--C--G-GT--AA-AGTT--T--TA-A-----C--A--G--.--A--.-CC--TGCAA-GG-AC-AA-A--C--A-GT--AA-AGCT--T--TA-A-----C--A--A-----A----CC--TGCAA-GG-AG-AA-A--C--A-GT--AA-AGTC--T--TG-A-----C--A--G-----A---. CC--TGCGA-GG-AG-AA-A--C--A-GT--AA-GGCC--C--TA-A-----C--A--A-----A----TC--TCGGA-GG-AC-GG-A--C --A-GC--AA-GGTT--T--CA-A-----T--A--G-----A---CC--TGCGA-GG-AC-GG-A--C--A-GT--CA-GGTT--T--CA-A--_--C--A--G-----A--.-CC--TGCGA-GA-AC-GA-A--C--G-GT--AM-AGTT--T--CA-A-----C--A--G-----A----CA--TGCAT-AG-AT-AA-A--C--A-AA--AC-AGTT--C--CA-A-----T--A--A-----C----CA--TCTCA-GG-AT-GA-A--C--A-GG--AA-GACA--T--TA-A--- --T--A--A-----T---CA--TCTCA-GG-AT -GA-A--C--A-GG--AA-GGCA--T--TA-A-----C--A--A-----T----D CA--TCTCA-GG-AT-GA-A--C--A-GG--AA-GGCA--T--TA-A-----T--A--A-----T-1--CA--TCTCA-GG-AT-GG-A--C--A-GG--AA-GGCA--T--TA-A-----T--A--A-----T---1. CC--CTATC-AG-CT-AG-A--T--G-GA--AA-AGCA--T--TA-A-----T--C--A-----T-----

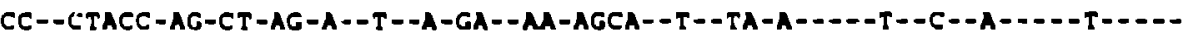

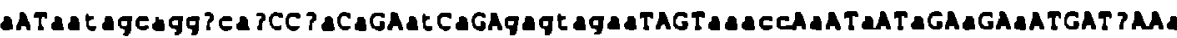
G--CAGTGCAAGCCAA--AA-A--GT-A--MAGTAMA----GMACC-G--C--A--A--A-----A--A G--AGTAGCAGGCCAA--AA-A--GT-A--GMATAGAA----MAATC-G--C--A--A--A---.--A--G A--AGTAACAGGCCMA--GG-T--AT-A--GAGTAGAA----AAATA-A--T--A--A--G-----A--A A--AGTAGCAGGCCAA--AA-A--GT-A--GAGTAAAA----AAATC-A--C--A--A--A-----A--А G--AGTAGCAGGCCAG--AA-A--GT-A--MAATAGM----GAACC-G--C--A--A--A-----A--G G--AGTAGCAGGCCMA--AA-A--GT-A--AMATAGMA----AAACC-A--C--T--G--C-----A--13 A--AGTAGTAGGLCAA--AA-A--GT-A--AAGTAGMA----AAALC-A--C--T--G--C-----A-A--AATAGCTGCACAG--AA-A--AA-A--ATCACCAA----AGCAA-A--A--T--A--A-----C--h A--AATAACAGGATGC--TA-A--AT-A--GAGCAGGC----TAACC-A--A--A--A--A-----T--A A-- $A$ TAACAGGATGC--TA-A--AT-A--GAGCAGGC--- - TAACC -A--A-..A--A--A-----C--A A--MATAACAGGATGC--TA-A--AT-A--GAGCAGGC----TMATC-A--A--A--A--A-----T--A

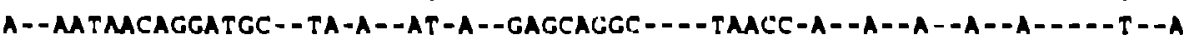
C--MATAGCAGGTCAG-. CA-T--AT-A--MAGTAGAT----AAACC-G--A--A--G--A-----T--A C--AG:AGCAGGTCMA-CA-T--AI 1--MAGTAGAT---AAACC-G--A--A--A--A-----T -A
1965 1990

1987 1987 2101 2047 2101 2047 2047 2050 2050 2062 2050 1939 1948

2031 2060 2057 2057 2171 2117 2171 2117

2117

2120

2120

2132

2120

2009

2018

2097

2130

2127

2127 2241

2187 2241

2187 2187 2190 2170

2202

2190

2079 $20 \mathrm{BA}$ 


\section{HIV2/SIV POL}

CONSENSUS AAggaAGCAATTATGT?GCATGGGT?CCAGC?CACMAGG? ATAGGaGGAHCCAgGAagTaGACCACE HIV2ROD HIV2NIHZ HIVIISY HIV2ST HIV2BEN HIV2DI94 HIV2GHI HIV2D205 SIVMa251 SIVMMI 12 SIVHM 239 SIVANE SIVSMMHA SIVSMMPBJ --GGAA-C-A-C-----T-CA-----C--A--C--C--A--CA-A--G--A--C--G--AG-A--T--TT $--A G A A-C-A-C-----T-C G----C--A--C--C--A--C A-A--A--A--C--G--A G-A--T--T T$ $--G G A-C-A-C-----T-C A-----C--G--C--C--A--C A-A--A--A--T--A--M A-T--C--C T$ - GGMA-C-A-C-----T-CA-----C--A--C--T--A--CA-A--A--A--T--G--GG-A--T--CT $--G G M-C-G-C-----T-C A-----C--A--C--T--A--C A-A--A--A--C--G--A G-A--C--T T$ --AGM-C-G-C-----T-CA-----C--A--C--T--A--CA-A--A--A--C--G--AG-A--C--TT $--A G A-C-G-C=----C-C G-----C--A--C--I--A--C A-A--A--A--C--G=-A G-A--C--T T$ --AGAG-C-G-A-----A-GA-----A--A--T--C--G--AC-G--T--T--T--G--AG-A--C--CC $--G T C A-A-A-T-----A-C A-----A--A--A--C--A--T A-A--A--A--C--A--M A-A--C--C C$ - -GACA-A-A-T-----G-CA-----A--A--A--C---A--TA-A--A--A--C--A--AA-A--C--CC $--G T C A-A-A-T-----A-C A-----A--A--A--C--\lambda--T A-A--A--A--C--A--\lambda A-A--C--C C$ $--G A C A-A-A-T-----A-C A-----G--A--A--C--A--T A-A--A--\lambda--C--A--A \lambda-A--C--C C$ $--G G A-C-A-T-----A-C A-----A--T--A--T--A--M A-A--A--C--T--A--A G-A--T--C C$ $--G G A A-C-A-T-----A-C A-----A--T--A--T--A--M A-A--A--A--T--A--A G-A--T--T C$

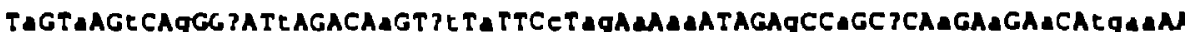
-A--G--T--G--T--C------A--GT-G---C-GG-A-AA-----G--C--T--G--A--A--TGAA--A--A--T--G--C--T-----A--AT-A---C-AG-G-MA-----A--C--G--G--A--A--TGMA-$-A--A--T--G--C--C-----A--A T-A---C-A G-G-G A-----G--C--T--G--A--A=-T G G A-D$ $-A--A--T--G--C--C----A--A T-A---C-A G-G-1 A-----A--C-T--G--G--A--T G M A-D$

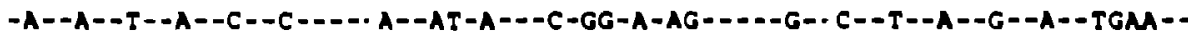

HIV2OI94 HIV2GH1

HIV20203

SIVHM231

SIVRM1 42

SIVMA239

SIVHNE

SIVSMMHA

SIVSMMPBJ -A--A--T--G--C--C-D---A--AT-A---C-GO-A-AG--D--G--C--T--A--A--A--CGAA-$-A--A--T--G--C--C-----A--A T-G---C-A G-A-G G----G--C--C--A--A--A--T G M A-D$ $-A--A--T--A--A--C-----G--C T-G---C-A G-A-M A-----A \cdots-A--C--G--A--G--$ TGMA--A--T--T--G--G--T-----A--TC-C---T-GG-A-AG---D-G--A--A--A--A--A--TGAT-D

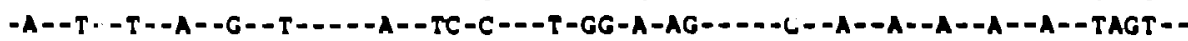
-A--T--T--A--G--T-D.--A--TC-C---T-GG-A-AG-----G--A--A--A--A--A--TGAT--A--T--T--A--G--T-----A--TC-C---T-GG-A-AG-----G--A--A--A--A--A--TGAT-

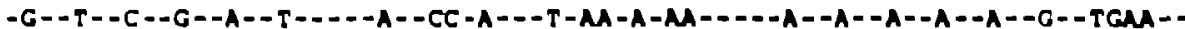

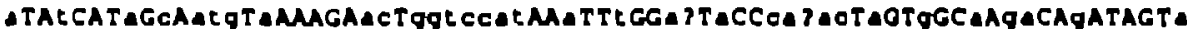
A--T---A-C-ATG-A-----AC-GTCTCAT--A--T--MA-A--CMTT-A--G--A-GG--A--.--A A--T---A-C-ATA-A-----AC-GTCCCAT--A--T--A-A--CMMGC-A--G--A-GA--A-----A A--T---A-C-ATG-A-----AC-AGCCCAT--G--T--AT-A--CAMCC-G--G--A-GA--A-----A 1--T---A-C-ATG-A--.--AC-ATCCCAT--A--T--AC-G--CMAT-A--G--A-GA--A-----A

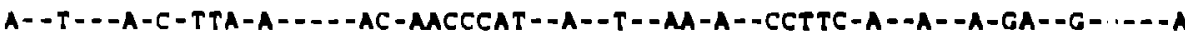
HIVIST HIVZBEN HIV2DI94 HIV2GHI HIV2O2OS SIVMA2S! A--T---A-C-ATA-A---.-AC-AACCCAT--A--T--AA-A--CCMAC-A--G--A-GA--G-----A A--T---A-C-ATA-G---D-AC-AACCCAT--A--T--AA-A--CCAAC-A--A--A-GA--C----A A--T---G-C-ATG-A-..--AC-GGTCCAT--A--C - -AA-T--ACAAT-A--G--A-AA--G---- -A A--C---A-T-ATG-A-----AT-GGTATTC--A- -T - -AT-A--CAGAA-A--G--C-GA--G-----A A-.C---A-T-ACA-A--.--AT-GGTATTC--A--T--AT-A--CAGAC-A--G--C-AA--G---- -A A--C---A-T-ATG-A-----AT-GGTATTC--A--T - -AT-A--CAGMA-A--G--C-GA--G-----A A--C---A-T-ATG-A--.--AT-GGTATTC--A--T--AT-A-DCAGAC-A--O--C-AA--G--.--A SIVMM2 ]9 SIVMNE

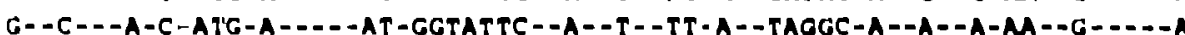


CONSENSUS HIV2ROD HIV2NI HZ HIVZISY HIV2ST HIV2BEN HIV2DI 94 HIV2GH 1 HIV2O 205 SIVID25! SIVMA1 12 SI UMM 239 SIVMNE SIVSKMHA SIVSMAPBJ

CONgENSUS HIV2ROD HIV2N I HZ HIVIISY HIV2ST HIV2BEN HIV2DI 91 HIV2GH 1 HIV2D20S SIVMA2SI SIVMMI 42 SIVMM239 SIVHAE IIVSMMHA S IVSMMPBJ

CONSENSUS HIVZROD HIVZNI HZ HIV2ISY HIVIST HIVZDEN HIV2DI 94 IIIVZOHI IIIVzDzOS SIVMH2 31 SIVMAI 42 JIVMA? 39 SIVMNY II VRMMIIA SI VSAMPIIIS

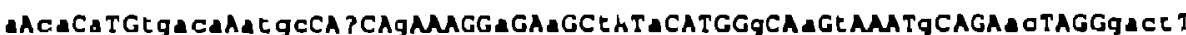
A-CT-A--TGCCC-ATGT--A--G-----G--A--T--A-----G--A-T----G----AC----CACT -

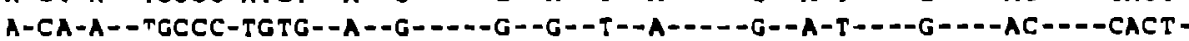
A-CA-A--TGCCC-GTGC--A--A-----G--A--T--A-1---G--A-T----G----AC----CACC-

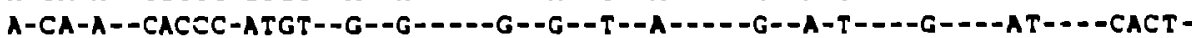
A-CT-A--TCCCC-ATGC--A--G-----A--A--C--A-1---G--A-T----G----AA----CGTT-

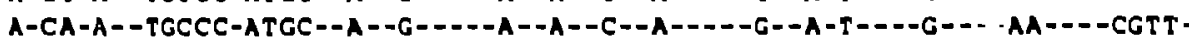
A-CA-A--TGCCC-ATGC--A--G-----A--G--C--A-----G--G-T----G----AA----TGTCA-TT-C--TGATA-ATGC--A--A-----G--A--T--T-1---A--G-T----G-D-CC-.-GACAG-CA-C--TGATA-ATGT--T--G-----A--A--T--A-----G--G-T----T----TC--D-GACT-

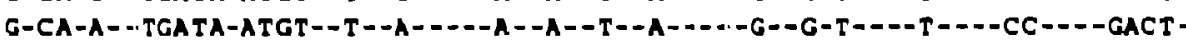

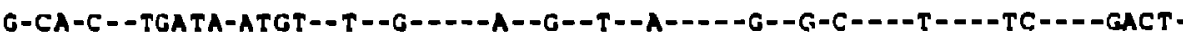

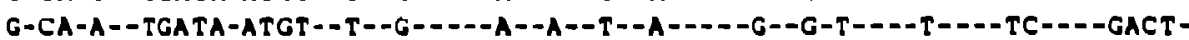

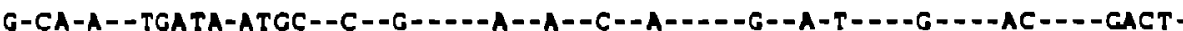

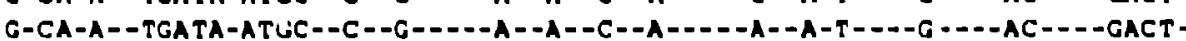

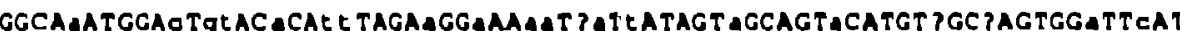

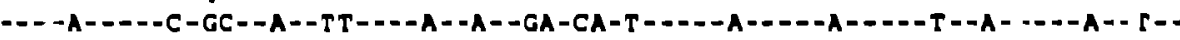

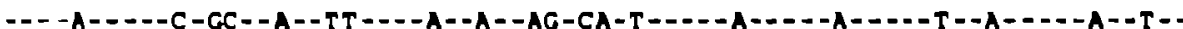
$---A-----C-G C--A--C T----A--A--A A-C A-T-----A-----A-----T--A-----A--T-$

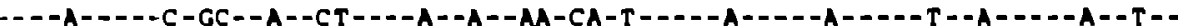
$----A-----C-A C--A--C T----A--A--A A-C A-T----A-----A----T--A-----A--C--$

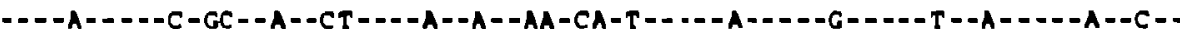

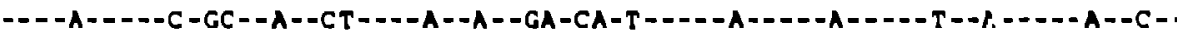

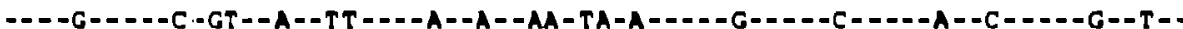

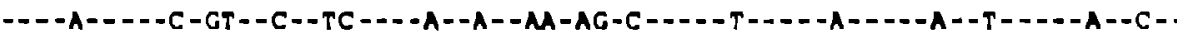

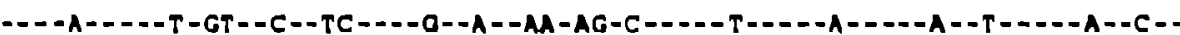

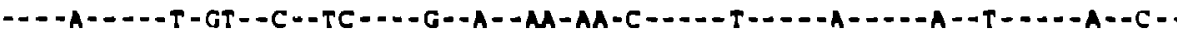

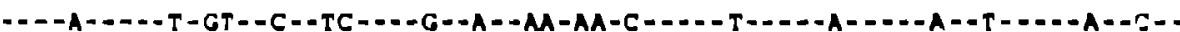

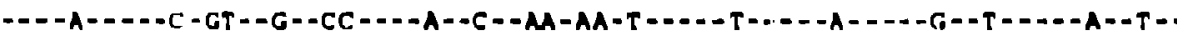

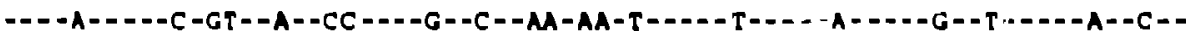

AGAAGCAGA_GTAATCCCACAYGAACAGGAAGACAYACAGCAOTPTTOOTALTAAAALTgGCCAGCAGA $---A----A--C--C--A--G=-A T-\cdots-A=-A--A--A--A C-C--C C-A T-G--A C-G--A A-T-O C$ $---G=---A--T--C--A--G--A T---A--G--A--A--A C-G--C T-A C-G--A C-G,-C A-T-G$ $---A-.--A--C--C$-A--G--AT----A--C;--A--A--AC-C--CC-AT-A--AC-C;--CA-P--G $---A-----A--C--C--A--C_{1}-A T----A--\left(;--A--C--A C-E--C C-A C-A--A C-C--C A-T--C_{3}\right.$

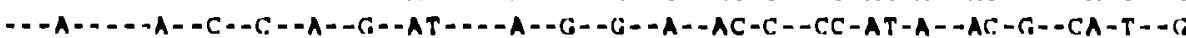

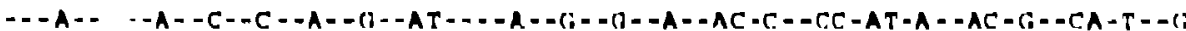

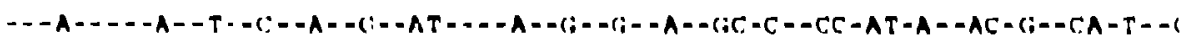
$---A--\cdots--(;--A--A--C--A--C A----A--A--(;--A--T C-C--C C-A C-A--C ; T-C)--C A-C--A$

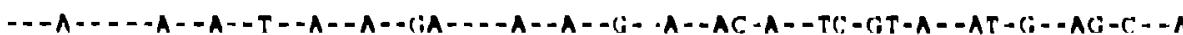
$---A-----A--A--T--A--A--A A----A--A--1 ;--A--A C-A--T C:-1: T-A--A T-6 ;--A A-1:--A$

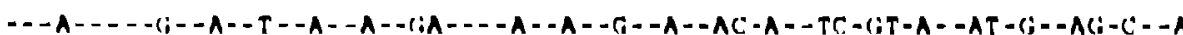

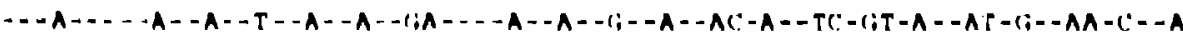

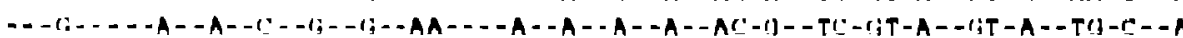

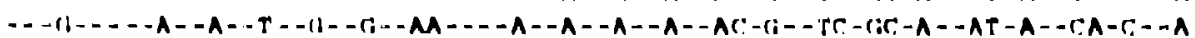

2366

2410

2407

2407

2521

2467

2921

2467

2167

2470

2470

2482

2470

2359

2368

2433

2480

2477

2477

2591

2537

259:

2537

2537

2540

2540

2532

2540

2429

2438

2502

2350

2547

2547

2661

2601

2661

2601

2601

2610

$2 \mathrm{~h} 10$

2022

2110

3499

$290 \mathrm{H}$ 


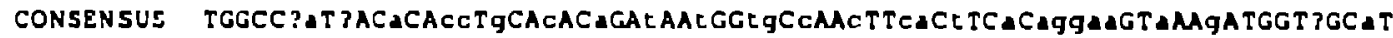
HIV2ROD HIV2NI H2 HIVIISY HIV2ST HIV2BEN HIV2OI 94 HIV2GHI HIV2020S I I VRo' 251 SIVRA1 12 IIVRA2 39 SIVHAR SIVIMHA IIVIAOPB.

CONAENEUS HIV2ROD HIV2NIHZ HIVIISY HIV2ST HIV2BEN HIV2DI 94 HIV2GH I HIV $2020 S$ $\therefore$ IVIOA 251 S I VIMI 12 IIVMI2] 9 SIVMNE SIVIMMHA II VSIAPBJ $----A$-A--A--CT-G- - T--A--T--T--TG-C--C--CA-T--A-AGGAG--G--G-----A--A$----A A-A--A=-C C-G--C--A--T--T--T G-C--C--C A-T--A-A G G A--G--A-----A--A-$ $----A A-A--A--C T-G=-C--A--T--T--T G-C--C--C A-T--A-A G G A G--A--G----A--A-$ $----A-A--A--T T-G--C--A--C--T--T G-C--C--C A-T--A-A G G A A--A--G----G--A-$ $-----M A-A--G--C T-G--C--A--C--T--C C-C--C--C A-T--A-A G G A--G--G=---G--A-$ $-----A A-A--A--C T-G--C--A--C--T--C C-C--C--C A-T--A-A G G M--G--G----G--A-$ $----A A-A--A--C T-A--C--A--C--T--C T-C--C--C A-T--A-A G G M A--G--G-----G--A-$ $---1 A-C--A--C C-A--C--A--C--C--T G-C--C--C A-C--A-C A M G T--A--G----A--C-$ $----T A-T--A--T C-A--C--A--T--T--T G-T--C--T G-C--G-M A G A--A--G=---T--A-$ $---T A-T--A--T C-G--C--A--T--T--T G-T-C--T G-T--G-M A G A--A--G=---T--A-$ $----T A-T--A--T C-A--C--A--T--T--T C-T--C--T G-T--G-M G M--A--G----T--A-$ $----T A-T--G--T C-A--C--A--T--T--T G-C-C--T G-T--G-M G A--A--G----T--A-$

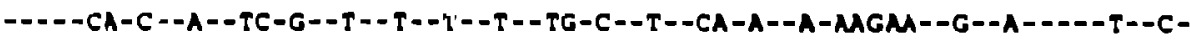

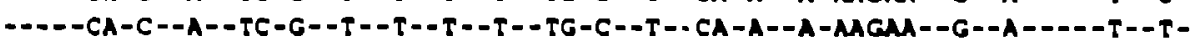

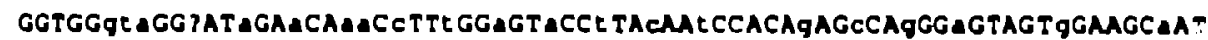
$----G T A--C--A--A--1 T-C--T--A--A--T--C--T-----A--C--G--A-----A-----A--$

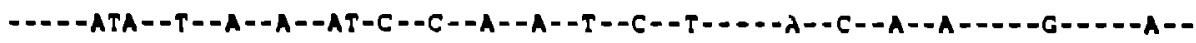
$----G T A--T--A=-A--A T-C--T--A=-A--T--C--C-----A--C--G--A----A----A--$

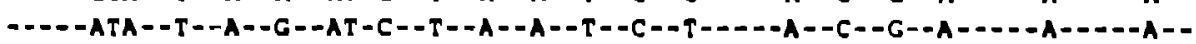

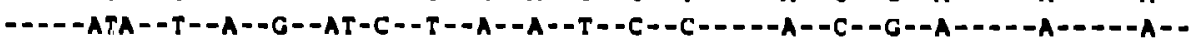

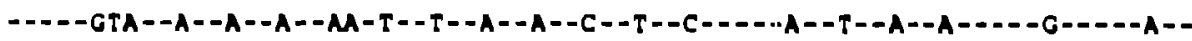

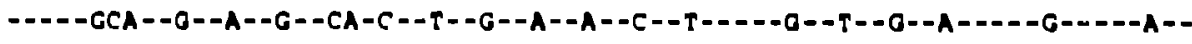

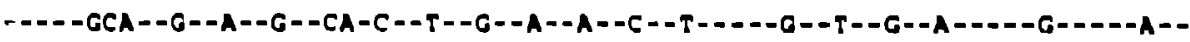

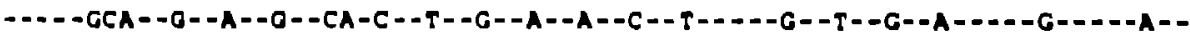

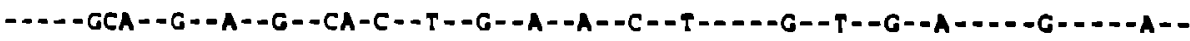

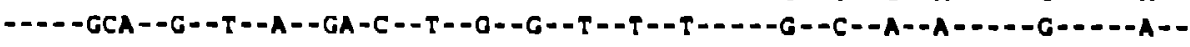

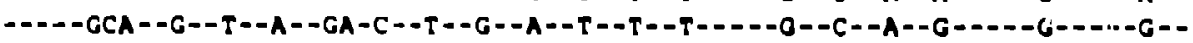




\section{HIV2/SIV POL}

CONSENSUS ATGGCAgtTCATTGCATGAATTTTAAAg_AGGGG_GGATAGGGGATATGACCCCAgCAGAAGALTAa HIV2ROD HIV2N I HZ HIV2ISY HIV2ST HIV2BEN HIV2D194 HIV 2GHI HIV20 205 SIVMM251 SIVMA1 42 SIVMA2 39 SIVMNE SIVSMMHA SIVSMMPBJ

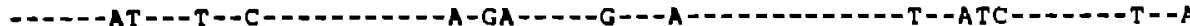
-

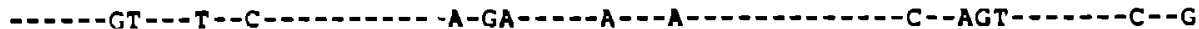

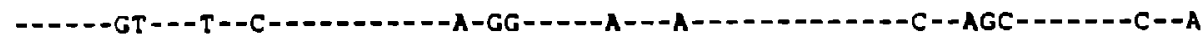

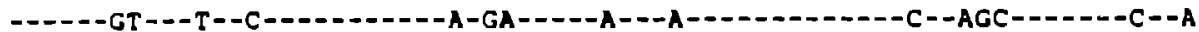

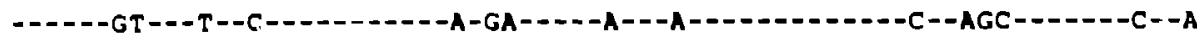

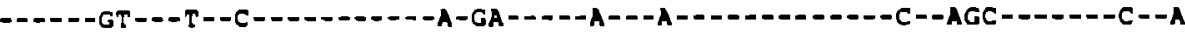

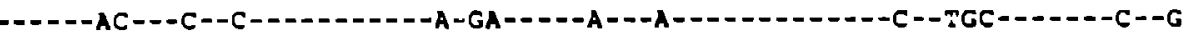
-

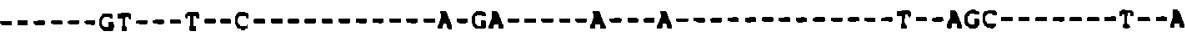

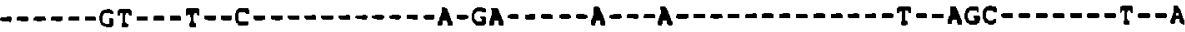

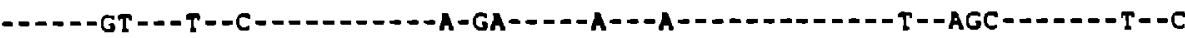

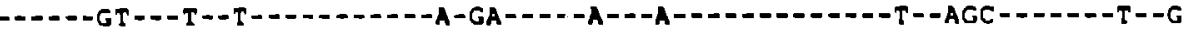

CONSENSUS HIV2ROD HIV2NIHZ HIV2ISY HIVZST HIV2BEN HIV2D194 HIV2GHI HIV2O2O5 SIVMM 251 SIVMMI 42 SIVHM2 39 SIVMNE SIVSMMHA SIVSMMPBS

CONSENSUS HIVZROD HIVINIHZ IIIVIISY HIV2ST IIIV2BEN IIIV2DI94 IIIV2GIII IIIV20209 SIVMM251 SIVMMI 12 SIVMMZ] 9 SIVMNE. SIVSMMHA GIVSMMPll

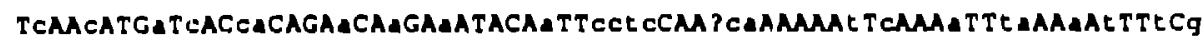
-C--T---A-C--CA----A--A--G-----A--CCTC---GCC-----T-C---A--AA--G-T--T-G $-C--T---A-C--C A----A--A--A-----A--C C T C---G C A----T-C---A--A A--A-T--T-G$

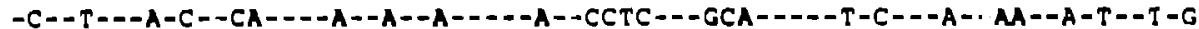
$-C=-T---G-C--T G----A--G--A-----A--C C T C---G C A-----T-C---A--A C--A-T--T-G$ $-C--C---A-T--C A----A--A--A-----A--C C T C---A G A----T-C---T--T A--A-T--C-A$ -T--C---A-C--CA----A--A--A-----A--CCTC---AGA-----T-C---T--TA--A-A--C-A $-C--C---A-T--C A---A--A--A---D A--C C T C---A G A----T-C=-T--T A--A-C--C-A$ $-T--C---A-A--C A---G--A--A-----G--C T T C---G C A----T-T---A--T C--A-T--C-A$ -T--C---A-C--TA----A--A--A-----A--TCAA---TCA-----C-C---A--TA--A-T--T-G $-T--C---A-C=-T A----A--A--A----A--T C A A---T C A----C C-C---A--T A--A-T-O T-G$ $-T--C---A-C--T A----A--A--G----A--T C A A---T C A----C-C---A--T A--A-T--T-G$ $-T--C-D-A-C--T A----A--A--A-----A--C C A A---T C A-----C-C---A--T A--A-T--T-G$ $-C--T---A-C--C A----A--A--A-----A--C C A A---T C A-----T-C---A--T A--A-T--T-G$ $-C--T---A-C--C A----A--A--A-----A--C C A A---T C A----T-C---A--T A--A-T--T-G$

\section{YJ Of (rare)}

I/ 5 of (rata)

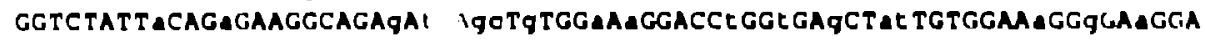

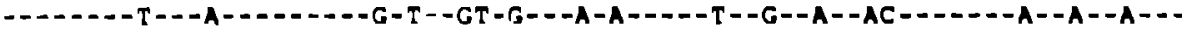

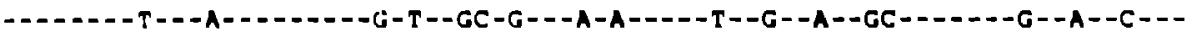

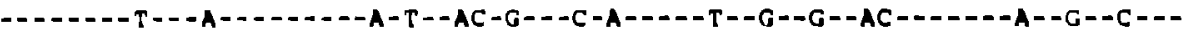

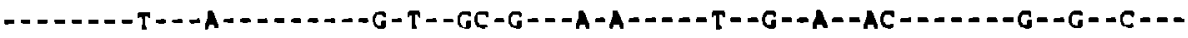

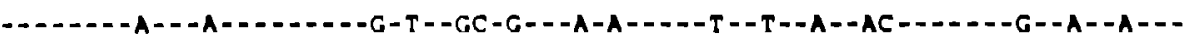

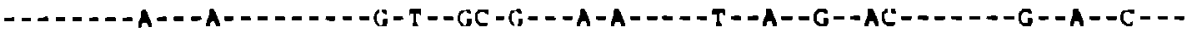

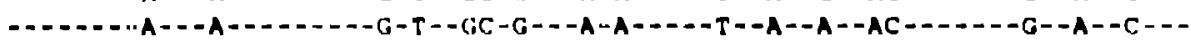

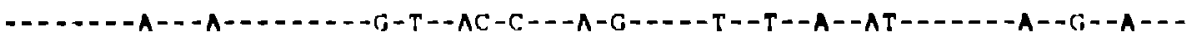

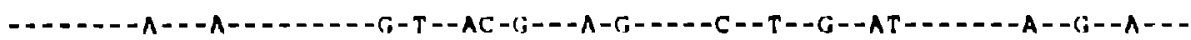

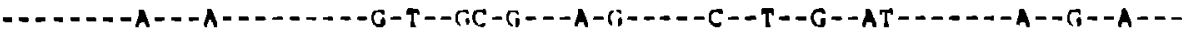

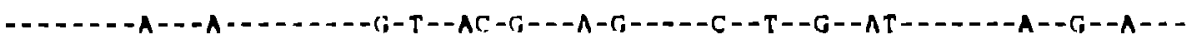

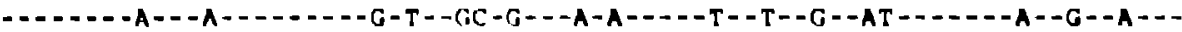
-

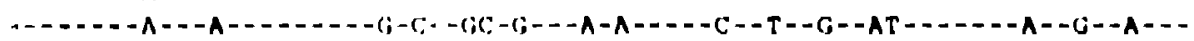

2777

2830

2827

2827

2941

2887

2941

2887

2887

2890

2890

2902

2890

2779

2788

2846

2900

2897

2897

3011

2957

3011

2957

2957

2960

2960

2972

2960

2849

2858

2916 2970

2967

2967

1081

$1: 27$

उ०O I

J02\%

3027

30.30

1030

3012

3030

2919

$292 \pi$ 
CONSENSUS GCAGTCATCgTAAAGGTAGggaCaGACAT?AAAgTAgTACCaAGaAggAgGCLAAgATtATCAaAGACT HIV2ROD HIV2NIHZ HIV2ISY HIV2ST HIV2BEN HIV2DI94 HIV2GHI HIV2D205 SIVMH 251 SIVMM1 42 SIVMM239 SIVANE SIVSHOHH SIVSMMPBJ - - - C-AG-C-------AA-A--C--A--AA--A----A--A--G--A--C--G--C--C-GAG-C----A,-AG-C------GA-A--A--A--AG--G----A--A--G--A--C--G--C--T-AAG-C$--1-A-A G-C------G A-A--T--A--A G--A----A--A--A--G--C--G--C--C-G A G-C-$ $----A-A G-C------G G-T--C--A--A A--A----A--A--G--A--T--G--C--C-A M G-C-$ --D--A-AC-C-D------GA-A--C--A--AG--G----A--A--G--G--C--G--T--C-GGG-C$----A-A G-C-------G G-G--C--A--A G--G----A--A--G--G--C--G--T--C-G G G-C-$ --D--A-AG-C--------GG-A--C--A--AG--A----A--A--G--G--C--G--T--C-GAG-C$----A-C A-A=-2---G A-A--A--C--A G--G---C--G--A--A--A--A--T--A-G G C-C-$

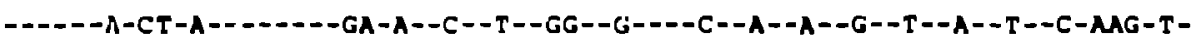
$-----A-C T-A------M A-A--C--T--G G--G----C--G--A--G--T--A--T--C-A A G-T-$ $-----A-C T-A------G A-A--C--T--G G--G---C--A--A--G--T--A--T--C-A A G-T-$ $-----G-T T-A-------G A-A--C--T--G G--G---C--A--A--G--T--G--T--C-M G-T-$ $----A-C C-A--.---G A-A--G--C--G G--G----A--G--G=-A--T--A--T--C-A A G-C-$ $-----A-C C-A------G A-A--G--C--G G--G----A--G--G--A--T--A--T--C-A M G-C-$

vif cds $\rightarrow$

CONSENSUS ATGGAggAgGaAAGag?TGGATAGt rgt tCCCAC?tGGagGataccagggAGGcTgGAGAggTGGCALA HIVIHOD HIV2NIHZ HIV2ISY - - GG-A-AC-A-AGA ---A-TGGTTC-CACCT--AG-GTGCCAGGG---A-G----AA-_---TA

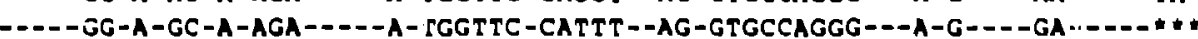
-----CC-A-GC-A-AGA-----A-CGGTTC-CACCT--AG-GTGCCAGGG---A-G----AA----TA

3110

HIV20205

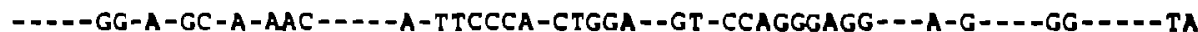

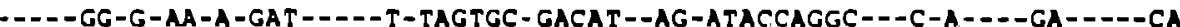

CONSENSUS HIV2AOD HIVIISY HIV2ST HIV 2 BEN HIV2OI94 HIV 2GHI HIV 20205 SI VMA2 51 SIVMMI 42 SIVMM2 39 SIVRINE g? G*⿻. $\mathrm{G} \cdots$

CC-TG-CANGTA > 3430 G** 3225 G*⿻ 3171 GT-GA-T-D政 3180 Ge.e 3174 G०. 3174 (i).. $3180^{\circ}$ G*⿻ $3 ! 74$ SIVSMMHA G*** 3063 SIVSMAPBJ G*. 3072 
Splice sitos have been determined for SIV only.

CONSENSUS ATGGAggAgGaAAGag?TGCATAgL?GTtCCCAC?TGGAGGaTaCCaGggAGG ? ?CTgGAGAggTGGC HIV2ROD ----GG-A-AC-A-AGA----AGTG--T--C-CC-----G-G--A-GG---...A-G----AA----

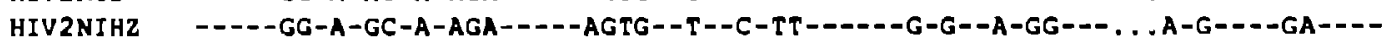
HIV2ISY HIV2ST HIV 2 BEN HIV2O194 HIV2GH1 HIV 20203 SIVMM 251 SIVMA1 42 SIVMM 239 SIVMNE SIVSMMH4 SIVSMMPBJ

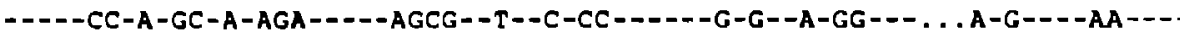
---- -GG-A-GC-A-AGA-----AGCG--T--A-CT------G-G--A-GG--- . . A-G----GG-------GG-A-AC-G-AAC-----AGTA--C--C-CC------G-G--A-GG--- . . A-G----AA-------GG-A-GC-A-AAC-----AGTA--T--C-CC------G.-G--A-GG--- . . A-G----GG----

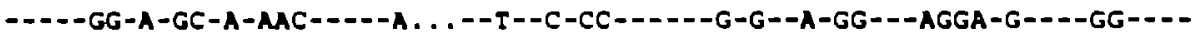
$----G G-G-M A-A-G A T----$ - TGTA--G--G-CA------A-A--A-GC--- . , C-A----GA---$----G G-G-A M-A-A G G----A G C A--T--C-C A-----A-A--G-A G---\ldots$. C-A----GG---$----G G-G-M A-A-A G A----A G T A--T--C-C A------A-A--G-A G---\ldots$. C-A----GG-----.--GG-G-AA-A-AGG----AGCA--T--C-CA-----A-A--G-AG--- . . C-A----GG---$----G G-G-A A-A-A G G----A G C A--T--C-C A------A-A--G-A G---\ldots$. C-A----GG----

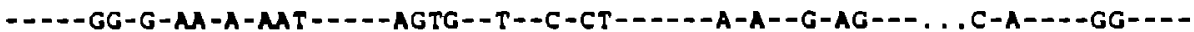

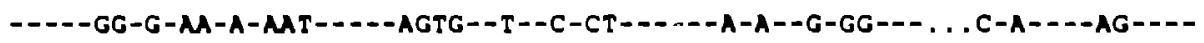

CONSENSUS HIV2ROD

$$
\text { c- pol cda }
$$

HIV 2 NHZ IIIV2 ISY .IV2ST HIV $2 \mathrm{BEN}$ HIV2D194 HIV2GHI HIV2D205 SIVHM2S1 SIVHMI 42 SIVMM239 SIVMNE SIVSHMH 4 SIVSMMPIJ

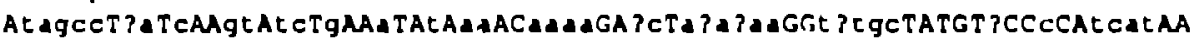
-TAGCC-TG-C--GT-TC-A--A.--C-AA--AAAG--TC-AGAAMA--TGTGC-----T--C--CCAT-- TAGCT-GG-C--GT-CT-G--A--C-GA--AAAA--CT-AGAGAA--TATGC---- -T--C--TCAT--TAGCC-TA-C--GT-TC-A--A--C-GA--AAAA--TC-AGAACA--TGCGC-----T--C--CCAT-- TAGCC-TA-C--GT-TC-A--A--C-GA--AGGA--TC-AGAGMA--TGTGC-----T--C--CCAT-- TGCCC-TG-C--GT-CC-G--A--C-GA--AAAA--CC-AGAGGA--TGCGC-----T--C--CCAC-- TAGCC-TG-C--GC-CC-G--G--C-GA--AAAA--CT-AGAGGA--TGCGC-----T--C--TCAC-- TAGTC-IG-C--GT-CC-A--A--C-GA--AAGA--CC-AGAGGA--TGCGC-----T--C--CCAC--CAGTC-GA-T--GT-TC-T--G--T-GA--AGGA--GT-GCAACA--TCTCT-----C--T--CCAC--TAGCC-CA-A--AT-TC-G--A--T-AA--TA; 1--TC-ACAAAA--TTTGC-----G--C--TTTT-- TAGCC-CA-A--AT-TT-G--A--T-AA--TAAR - TC-ACANA--CTTGC-----G--C--TCAT--TAGCC-CA-A--AT-TC-G--A--T-AA--TAAA--TC-ACANAA--TTTCC-----G--C--TTTT-- TAGCC-CA-A--AT-TC-G--A--T-MA--TAMA-- IC-ACAMA--TTTGC-----G--C--TCAT-- TAGCC-CA-C--AC-CC-G--A--T-AC- - TAAA--CC-ACAGAT--CTTCT-----G--C--TCAT--

CONSENSUS HIV 2ROD HIV2NIHZ HIVIISY IITV2ST IIIV 2 BEN IIIV20194 IIIV2GIII IIIV 20203 SIVMM251 5 IVMM1 42 SI!'MMIJ9 SIVMNF. SIVGMMIIA SIVSMMPII I

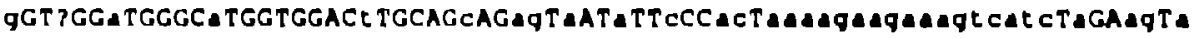

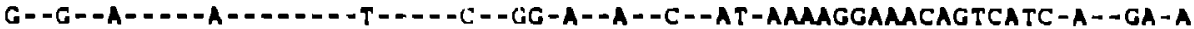
G--G--G----A-------T----C--GG-A--A--C--AC-GAMAGAGAACAGTCATC-G--GA-A G--G--G----A-------T----C--(;G-A--A--C- -AT-AMAGGAAACAGTCATC-A--(;A-A

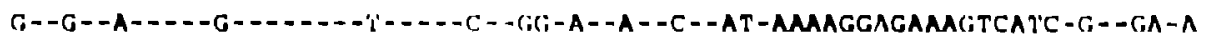

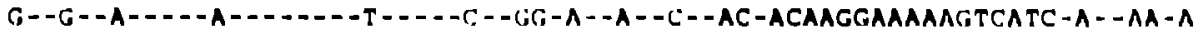
G--A--A-----A------T-----(:--1;G-A--A--C--AC-AGAAGGAGAAAGTCATC-A--6;A-A

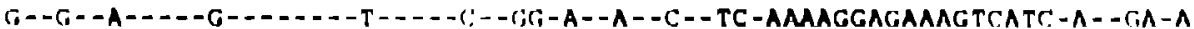

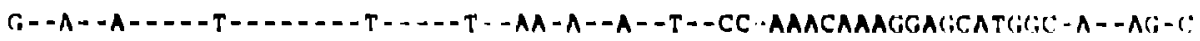

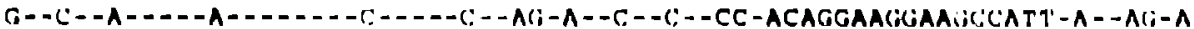
G--C--A-----A----- --C:----C--AGi-A--C--C--AC-ACAGGAAGGAAGGCATT-A--AGi-A

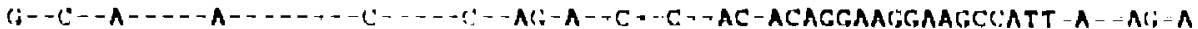
: - -

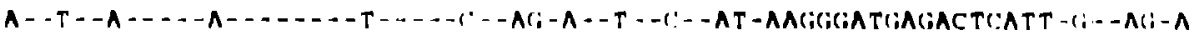

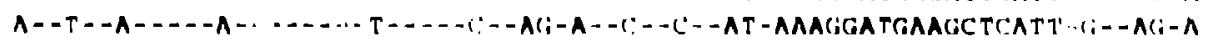

197

[-|I..]| 


\section{HIV2/SIV VIF}

CONSENSUS CAAGgaTALTGGAACtTaCACCAGAAAAGGaTggCTCagetCLtAtgCAGTgAGaATACCTGGAC? HIV2ROD HIV 2NIHZ HIV2ISY HIV2ST HIV2BEN HIV2D194 HIV2GHI HIV2D205 SIVMH251 SIVMH1 12 SIVMH239 SIVMNE SIVSHAH4 SIVSMMPBJ $--G-C A--T---A-C T-M A-A----A-A A--A-G G C-C T C C T C T T-T T-A--A--A A-A--T----C A$ $--A-C A--T---A-C T-M A-A----A-M A--A-G G C-C T C C T C T C-C T-A--G--A A-A--T----T A$ $--G-C A--T---A-C C-A A-A-----A-A A--A-G G C-C T C C T C T T-T T-A--A--A A-G--T----C T$ $--G-C A--C---A-C C-A A-A-----A-A A--A-G G C-C T C C T C C T-T T-A--A--A C-A--T----T A$ $--G-C A--T---A-C C-A-A-----A-A A--A-G G C-C T C C T C T C-T G-A--A--A T-A--C---1-T A$ $--G-C A--T---A-C C-A A-A----A-M A--A-G G C-C T C C T C T C-T T-A--A--G T-A--C-----T A$ $--G-C A--T---A-C C-A A-G-----A-A A--A-G G C-C T C C T C T C-T T-A--G--A A-A=-C-----T A$ $--A-G A--T---A-C C-A A-C-----A-G G--A-T C T-G A G C T C C T-T G-T--A--A C-A--A=---T G$ $--A-G G--T---C-T T-G A-A----A-G A--G-G G C-C A G T A C T T-T G-A--G--G A-A--C-----C T$ $--A-G G--T---A-T T-G A-A-----A-G A--G-G G C-C A G T A C T T-T G-A--G--G A-A--C----C T$ $--A-G G--T---C-T T-G A-A----A-A A--G-G G C-C A G T A C T T-T G-A--G--G A-A--C----C T$ $--A-G G--T---A-T T-G A-A-----A-G A--G-G G C-C A G T A C T C-T G-A--G--A A-A--C----C T$ $--A-G A--T---A-T T-G G-A----A-A A--A-G G C-C A G T A C T T-T G-A--A--A A-A--C----C T$ $--G-G A--T---A-T T-G A-A----G-M A--G-G G C-C A G T A C T T-T G-A--G--M-A--C-\ldots--C T$

CONSENSUS HIV 2ROD

CaAaAa 7TTCTggaCAGATGT TACACCAqACtgLGCAGACa r rTAcTgCAtaGcaCTTATTTCcCTTG CAGAMAG--C-GGA-------T--C--AG-CTGT--A--TGTCC--A-A--TA-CA-------C---CAGAMAG--C-GGA------T--C--AG-CTGT--A--TACCC--A-A--TA-CA--_-_--T--.-

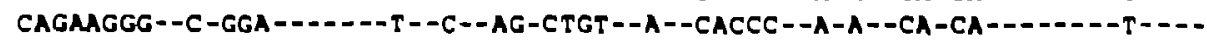

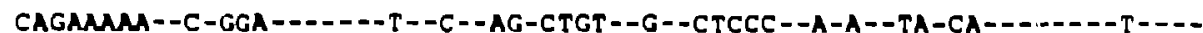

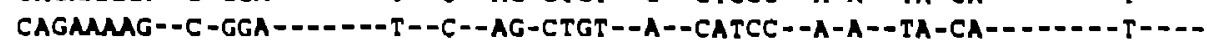
CAGMAAG--C-GGA------T--C--AG-CTGT--A--CTCCC--A-A--CA-CA-------T---

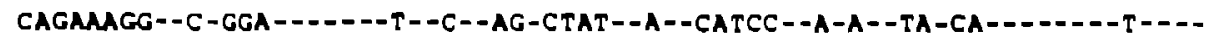
AGAGGAAC--T-ATA--.---A--A--TG-TGTG--A--CCAGC--C-G--TG-GT-------T---

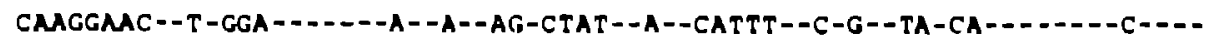

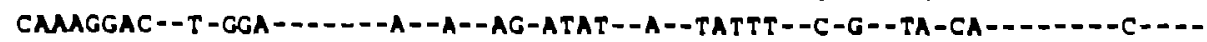
CAAAGAAC--T-GGA--_---A--A--AA-CTAT--A--CATTT--C-G--TA-CA--_----C--.CAAGGAAC--T-GGA--.---A--A--AG-CTGT--A--CATTT--C-G--TA-CA---_---C----

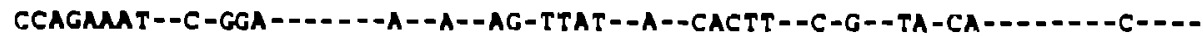
HIVIST HIV 2BEN HIV 2D194 HIV2GHI HIV 20205 SIVMH251 SIVMHI 42 SIVMH2 39 SIVMNE SIVSAMHA SIVSHAPBJ CCAGAAT--C-GGA---D---G--A--AG-TTAT--A--CACTT--C-G--TG-CA----2--C----

$$
1 / 3 \cdot 1
$$

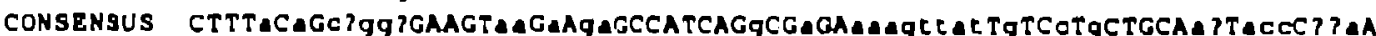
IIIV 2ROD HIV ZNIHZ HIVZISY HIVZST HIVZAEN II V2DI 94 HIV2GHI II) 20205 :IVAM251 SIVMH 12 S IVMM2 39 SIVMNE SIV SHAHA SIVGMMPUJ ---A-A-CAGGT----AA-A-GA------A--G--AMAGTTAT-G--C-G----AT-ATC-CCG-

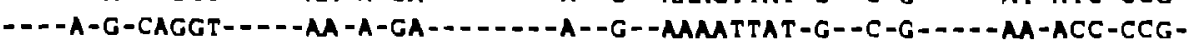
$---A-G-C A G G T----A A-A-G A------G--A--A M A G$ ICAT-G--C-G----AT-ATC-CCA----A-G-CAGGC-.---AA-A-GA---2.-A--G--AAAGCTAT-A--C-G----AC-ACC-CCA---A-G-CAGGT----AA-A-GA----2--A--G--AAAGTTAT-G--G-G----AC-ATC-CCA----A-G-CAGCT----AA-A-GA-------A--G--AAAGTTAT-G--C-G----AC-ACC-CCA----A-G-CAGC:T---.-AA-A-GA-------A--G--AAAGTTAT-G--C-G----AC-ATC-CСA-

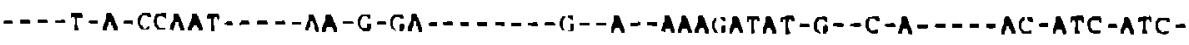

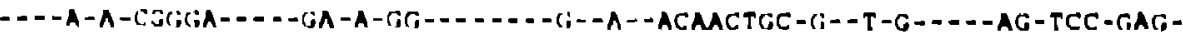

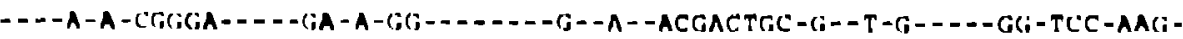

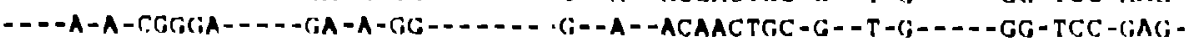

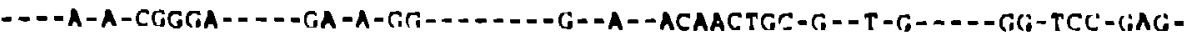

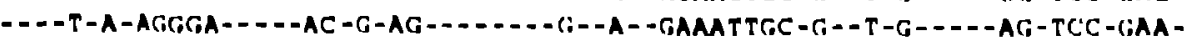

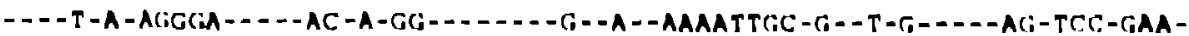


5.s] $(r+20) \backslash /$

vpX cds ->

CONSENSUS HIV2ROD HIV2NIHZ HIV2ISY HIV2ST HIV2BEN HIV2D194 HIV2GHI HIV2D 205 SIVMM25I SIVMal 42 SIVRA239 SIVMNE SIVSMMHA SIVSHMPBJ

CONSENSUS HIV2ROD HIV2NIH2 HIV2ISY HIVIST HIV2BEN HIVZOI94 HIV2GHI HIV2D 203 S IVAR2 51 SIVMMI 42 5 IVMM 239 SIVMNE SIVSMMHA SIVSMMPBJ

CONSENSUS HIV2ROD HIV2NIHZ IIIV2ISY HIV25T HIVZUEN IIIV2DI94 IIIV2GIII IIIV2O2US SIVMMZ3: SIVMMI 42 SIVMM2J9 3 IVMNE GIVSMMIIA SIVSMMPIIS

CONSENSU: IIIVZROD HIVZN IHY, IIIVZISY IIIV2ST IIIVZHF:N IIIVI)I94 IIIVacill III :IVMMय)। $\therefore$ IVMMIA. "IVMM?ll :IVMNI: :I V:AMMIIA SIV:iMMIIIII

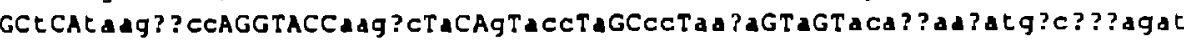
--T--TAGAGCCC--.----GTCAC-T--A-TTC-G--CT-AGTG--A--GCAACAAAATGAC . . AGAC --G--TAGATCCC------GTCAC-T--A-TTC-G--CT-AGTG--A--GCAACAAATGAC. . AGAT --C--TMATCCA-------GTCAC-C--A-TTC-G--CT-AGTA--A--ACAGCAAAATGAC. . AAAC --C--TAGTACC-------GTCAC-C--G-TTC-G--CT-AGTG--A--GCAACAAAATGGC. . AGGC --T--TAAAGCAC-------ATCAC-T--A-ACC-A--CC-AGTA--A--ACAACAAAATGAC. . AGAC --T--TAAAGCAC----.--ATCAC-T--A-ACC-A--CC-AGTG--A--GCAACAAAATGGC . . AGAC --T--TAAGTAC--2---ATCAC-T--A-ACC-A--CC-AGTG--A--GCAACAAAATGAC. . AGAC --T--CGAAGGGC-------MAGCT-A--G-TTC-A--CC-NAGG--C--ACAGGAAGGAAAAAATGGAT --T--TAGGTACC-------AGGCC-A--G-ACT-A--AC-AMA--A--A... AGCGATGTC. . AGAT --T--TAAGCACC--..---AAGTC-A--G-ACT-A--AC-GAGA--A--A... AGTCATGTC... AGAT --T--TAAGTACC-------AAGCC-A--G-ACT-A--AC-GAM--A--A. . AGCGATGTC . . AGAT --T--TAAGACCC-------AAGTC-A--G-ACT-A--AC-GAGA--A--A...AGTTATGTC. . .AGAT --T--TAAAAATC-------AAGCC-A--G-ATC-A--AC-AACA--A--A... AGTCATGTC. . AGAT --T--TAAGMATC-------MGCC-A--G-ATC-A--AC-MACA--A--G... AGCCATGTC... AGAT

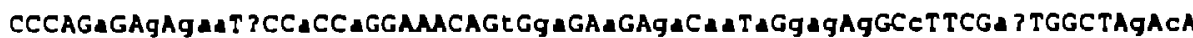
$----A--G-C A G-A--A \cdot-A---1---C-G C--A--G A-T A-C-G A G-G--C---C C--.--A-C-$ -----G--A-CAG-G--A--A--------C-GC--G--GA-TA-C-AAG-G--C----CA------G-C-

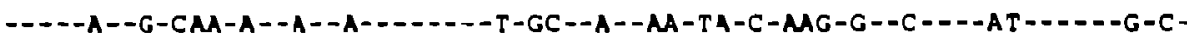
$----A--G-C A A-A-\cdot A--A---1---T-G C--A--A A-T A-C-G A G-G--C----A G-----G-C-$

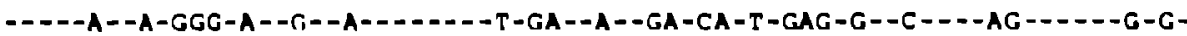
$----A--A-G G G-G--G--A-------T-G A--A--G A-C A-T-G A G-G--C----A G-\cdot---G-C-$ $----A--A-G G G-A--G--A-------T-G A--A--G A-C A-T-G A G-G--C----A G-----G-C-$ ----G--G-GAG-G--A--A---1---C-AC--A--AA-AG-A-GAG-A--A----CT------G-A$-1--G--G-G A A-C--A--T---1---T-G A--A--G A-A A-A-G A G-G--C----A A---1-A-C-$

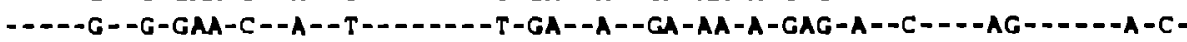
$----G--G-G A A-C--A--T-------T-G A--A--G A-A A-A-G A G-G--C----A A---1--A-C$ -

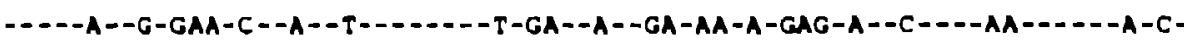
$----G--G-G G A-C--A--T---1----T-G A--A--M A-M A-A-G A A-G--C=---M A-----A-C$

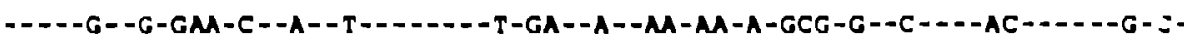

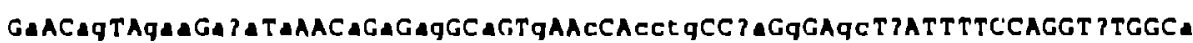
-G--AG--GAA-CCA-A---A-A-AA--A--G--T--CCTA--CC-A--AC-T----.-----G----A $-G--A G--G A G-C T A-A---A-A-A G--A--G--T--C C A C--A C-A--G C-C---1----C---A$ $-G--G G--G M A-C C A-A---A-A-A G--A--G--C--C C T G--C A-G--G C-T----------G----A$ $-G--G G--G A A-C C A-A---A-A-A G--A--G--C--C C T G--C C-A--G C-T-1-1-1 .-G--1-A$ $-G--C A--G A A-C C T-A---A-G-A G--A--G--C--T C T G--C C-A--G C-C---------G----A$ $-G,-T A--G A A-C C T-A---A-G-A G--A--G--C--T C T G--C C-A--G C-C---1-1--G---\Lambda$ $-C--T A--G A A-C C T-A---A-A-A G--A--C_{0}--C--T C T G,-C C-A--C C-C---.-1---G-1-A$ $-A--A A--A C A-A C C-C---A-C_{3}-T A--G--C--C--T T T G--C C-A--A C-T-\ldots . .-1 .-C-1-A$

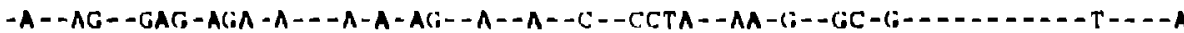

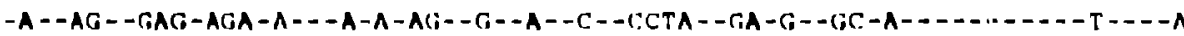

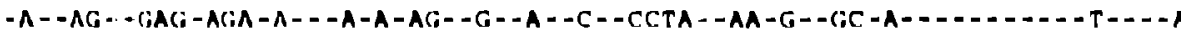

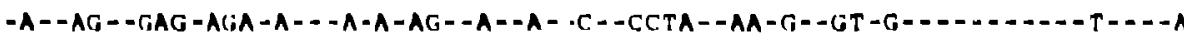

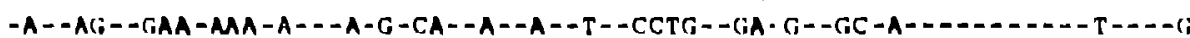

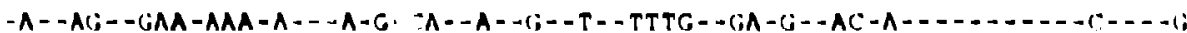

angGtcetgey tatactghea...

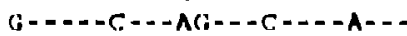

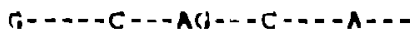

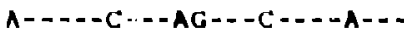

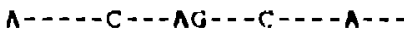

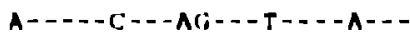

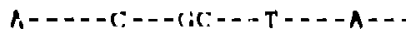

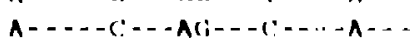
1;-

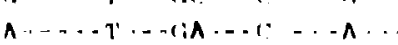

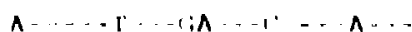
$\Lambda \cdots-T^{2} \cdots \operatorname{rin} \cdot \cdots \Lambda$ A .... . iA ,

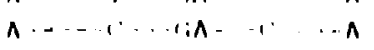

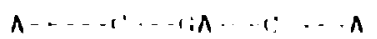

613
648
648
648
648
648
648
694
611
111
411
114
111
619
614

[.|1..1.1 
Splice sltes have been determined for SIV oniy.

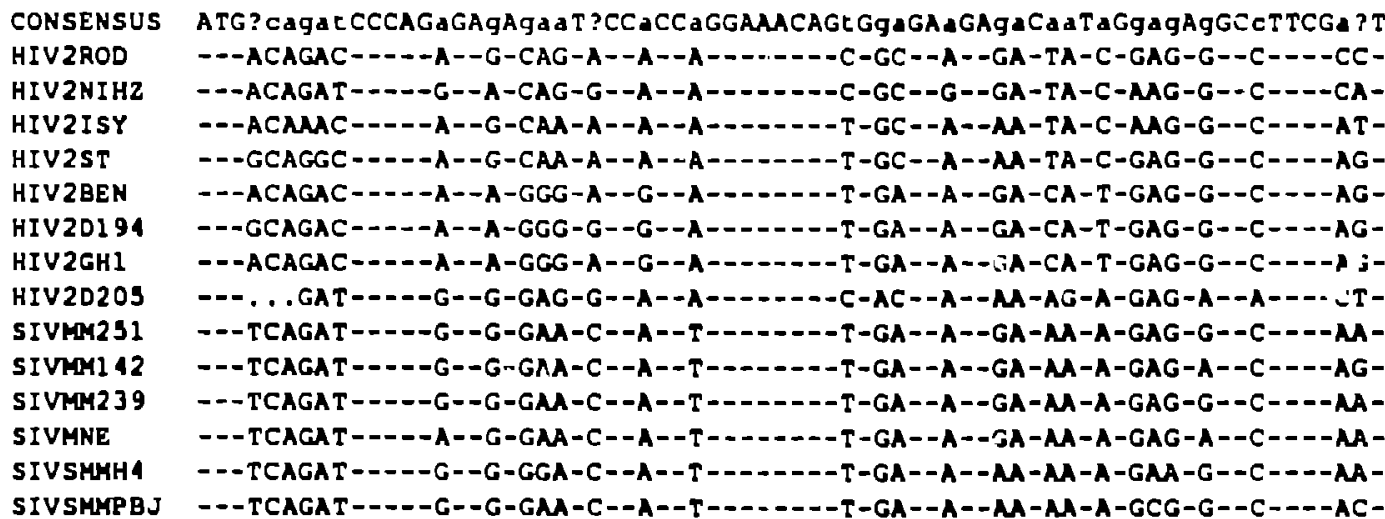

CONSENSUS HIV2ROD HIVZNIHZ HIVZISY HIV2ST HIV 2BEN HIV 2DI 94 HIV 2GH 1 HIV 2D2OS SIVMH25I SIVMMI 42 SIVMK239 SIVMNE SIVSMAH 4 SIVSMMPBJ

CONSENSUS IIIVZROD HIVINIHZ HIVZISY IIIV2ST IIIV 2HEN IIIV2DI 94 HIV $2 \mathrm{GHI}$ IIIV 20205 SIVMM23I SIVMMI 42 SIVMM2 39 SIVMNE SIVSMMHA SIVSMMPHJ

GGCTAgACAGAACAgTAgAAGA PATAMACAGAGAgGCAGTgMACACCL gCC?AGgGAgCT ?ATTTTCCA -----A-C--G--AG--GAA-CCA-A---A-A-AA--A--G--T--CCTA--CC-A--AC-T-_.-...--.--G-C--G--AG--GAG-CTA-A---A-A-AG--A--G--T--CCAC--AC-A--GC-C---1--$----G-C--G--G G--G A A-C C A-A---A-A-A G--A--G--C--C C T G--C A-G--G C-T-------$ $-----G-C--G--G G--G A A-C C A-A---A-A-A G--A--G--C--C C T G--C C-A--G C-T--------$ $-----G-G--G--C A--G A A-C C T-A---A-G-A G--A--G--C--T C T G--C C-A--G C-C-------$ $----G-C--G--T A--G A A-C C T-A---A-G-A G--A--G--C=-T C T G--C C-A--G C-C-.+\cdots-.$. $-----G-C--G--T A--G A A-C C T-A---A-A-A G--A--G--C--T C T G--C C-A--G C-C-1-1--1$ $----G-A--A--A A--A C A-A G C-C---A-G-T A--G--C--C--T T T G--C C-A--A C-T=-----$ $-----A-C--A--A G--G A G-A G A-A---A-A-A G--A--A--C--C C T A--A A-G--G C-G-------$ $----A-C--A--A G--G A G-A G A-A---A-A-A G--G--A--C--C C T A--G A-G--G C-A------$ $-----A-C--A--A G--C A G-A G A-A---A-A-A G--G--A--C--C C T A--A A-G--G C-A-1-1 .-$

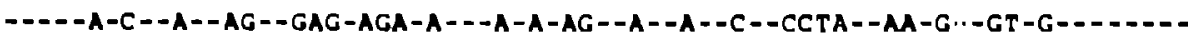
$-----A-C--A--A G--G A A-A M A-A---A-G-C A--A--A--T--C C T G--G A-G \cdot-G C-A----.--$ ----G-C--A--AG--GMA-AAA-A---A-G-CA--A--G--T--TTTG--GA-G--AC-A---.---

<- VIf

GGT ?TGGCAAGGTCCTGGQ?ATACTGGCATGAEGAACAAGGATGTCA? IAGCTACACAAATALAGA ---G----AG-----C---AG---C----A---T--ACAA--G---T-AGMA--T--CACA--G--T---

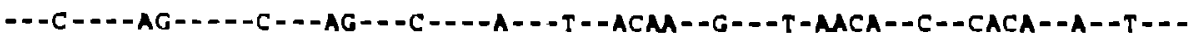

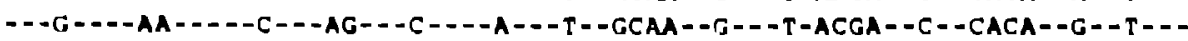
---G----AA-..---C---AG---C----A---T--ACAA--A---T-AATA--T--CACA--G--T---

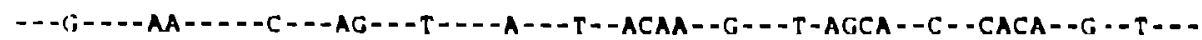

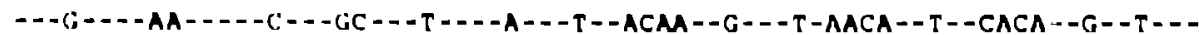

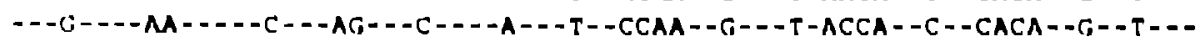

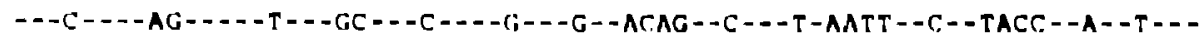

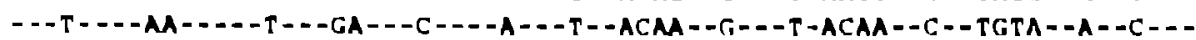

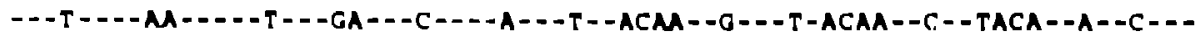

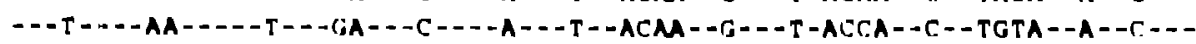

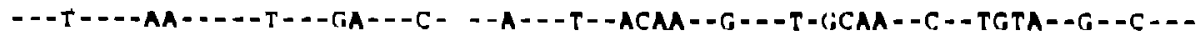

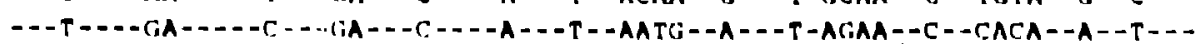

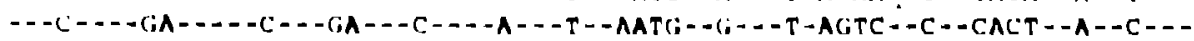


CONSENSUS TACTTGTgCtT LATACAgAaAGC ?TgTtLatgCALt?CAagAaAGgtGGAg?TGCCtgggggaGgaC --T----GCT-A--A--G-TG--AA-G-ACATG--TGCT-AG-GA-ATG-C-CT--CCTGGGGGGA-G. --T----GCT-A--G--G-AA--TG-G-TCATG--TTTC-AG-AA-GGT-C-CT--CCGGGGGGAA-GA--T----GCC-A--G--G-AA--TA-G-TCATA--TTCT-AG-GA-GGT-C-CT--CCTGGGGGGA-GAHIV 2BEN HIV2DI 94 HTV2GHI HIV20205 SIVMH251 SIVMA1 12 SIVMa1239 SIVRNE SIVSHAH4 SIVSMMPBJ --T----GCC-A--G--A-AA--TA-A-TTACA--TTTC-AG-GA-GGT-C-CT--CTGGGGG . GA-GA--T----GCA-A--G--G-AA--TG-G-ATATA--TTTC-AG-AG-GGT-C-CT--CCTGGGGAGA-GA--T----GCC-G--G--G-AA--TG-A-TTATA--TTTC-AG-GA-GGT-C-CT--CCTGGGGGGA-GA--C----TGC-A--G--G-AA--AA-G-TTGTG--CTAT-CA-AG-GCT-T-GG--CCTGCAGGAG-GC--C----GTT-A--G--A-AG- -TT-A-TTATG--TTGC-AG-AA-GCT-T-GA--TCTAGGGGAA-GA--C----GTT-A--A--A-AG--TT-A-TTATG--TTGC-AG-AA-GCT-T-GA- - TCTAGGGGAA-GA--C----GTT-A--A--A-AG--TT-A-TTATG--TTGC-AG-AA-GCT-T-GA--TCTAGGGGAA-GA--C----GTT-A--A--A-AG--TT-A-TTATG--TTGC-AG-AA-GCT-T-GA--TCTAGGGGNA-GA--C----GCT-G--A--G-MA--TC-G-TTGTG--TTGC-AG-AA-GGT-T-GG--CTTAGGAGAG-AG-

CONSENSUS HIVZROD HIVZNIHZ HIV 2 ISY HIV2ST HIV 2BEN HIV2D194 HIV 2GHI HIV 20203 SIVMH25] SIVMHI 4 ? SIVMH239 IVMNE SIVSHAHA SIVSMMPBJ
AtGGgcCaGga rGGa ? TGG ?AGA CCA ?GGaCCTCC tCCTCCTCCcCCtCCAGG ? CTaGcc ** $-\mathrm{T}--\mathrm{GC}-\mathrm{A}-\mathrm{GA} .,-\mathrm{G} .---,---\mathrm{C}--,--\mathrm{G}-----\mathrm{T}-------\mathrm{C}--\mathrm{T}----\mathrm{TC}-\mathrm{G}-\mathrm{TC}--$

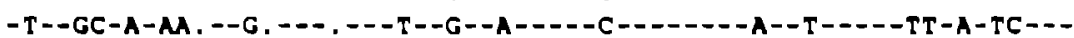

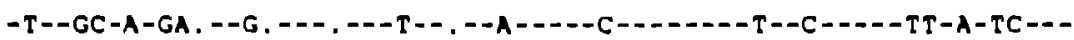

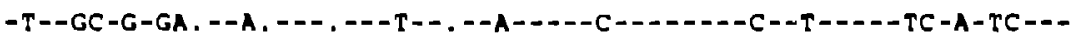

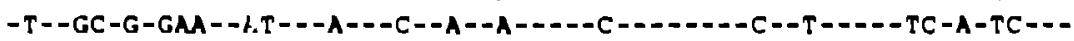

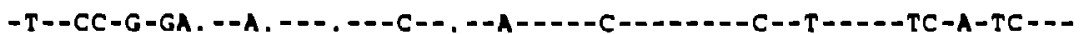
$-\mathrm{T}--A C-G-G A,--A,---,---T--,--A-----C--------C--T-----T C-A-T C---$ $-T--G C-A-G G,--A,---,---T--,--A-----T--------T--C-----C C-G-C C---$

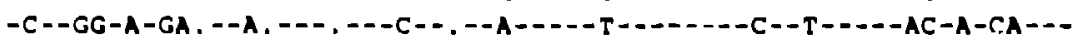
$-C--G G-A-G G,--A,---,--C--,--A---=-T------C--T----A C-A-C A--$ $-T--G G-A-G G,--A,---,---C--,--A-----T-------C--T-----A C-A-C A-=$

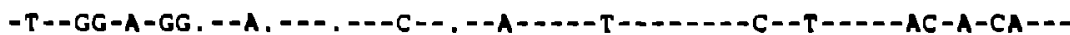
$-T--G G-A-G G,=A,---,---A--,--G----T-------C--T---A C-A-C A---$

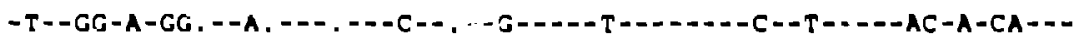




\section{HIV2/SIV VPR}

Splice sltes have been determined for SIV only.

<- vpX

CONSENSUS ATGaC?gaagCa?c??CagA?a??CCLCCaGaagAtgagacerCACagAGgGAaccatGGgatga?TGGg HI'2ROD HIV2NIHZ HIV2ISY HIV2ST HIV2BEN HIV2DI 94 HIV2GHI HIV2D205 SIVMM251 5 IVRAI 42 SIVHA 39 SIVHNE SIVSMMH4 SIVSMMPEJ

CONSENSUS HIV $2 P O D$ HIV 2 N I H2 HIV2ISY HIV2ST HIV 2BEN HIV2OI 94 HIV 2GH 1 HIV2D205 SIVMM2 51 SIVAM 12 SIVMM2 J9 SIVANE SIVSMMHA SIVSMMPBJ

CONSENSUS HIVZROD HIV2NIHZ HIVZISY HIVZST HIV 2 BEN HIV2DI94 HIV 2GHI HIV 2020S SIVMM25! SIVMMI 42 SIVMA2 39 SIVMNE SIVSMMHA SIVSMMPB
---GCTGAAGCACCAACAG-GCTC--C--G-TGG-TGGGACC- --TG--G--GCCAG--GATGAG---A

---ACTGAAGCACCAACAG-ACTC--C--G-AGG-TAGGACC----CG--G--GCCAG--GATGCA---G ---ACTGAAGCACCAGCAG-GTTT--C--G-AGG-TGGGACC----CG--G--ACCAG--GATGAG---G ---ACTGAAGCACCAACAG-GTCT--C--G-AGG-TAGGACC----CG--G--GCCAG--GATGAG---G ---ACTGLAGCACCAACAG-GTTT--C--A-AAG-TGGGACC----GG--G--CTTAG--AGTGAC---G ---ACTGAAGCACCAACAG-GTTT--C--A-AMG-TGGGACC----GG--A--GCTAG--AGTACC---G ---ACTGAAGCACCAACAG-GTTT--C--A-AAG-TGGGACC----GG--G--ACTAG--GGTGAC---G ---GCAGAAGCAGCCCCAG-GATC--T--A-AGA-CGAGAAC----AA--A--ACCGT--GAAGAG---A -.... GAA.........G-AAGA--T--A-MAA-TGMGGC -..-AA--G--ACCAT--GATGAA---G --...GAA........G-AAGA--T--A-AAA-TGAAGGC----AA--G--ACCAT--GATGAG---G --...GAA........G-AAGA--T--A-MAA-TGAAGGA----AA--G--ACCAT--GATGAA-- -G --...GAA........ G-MAGA--T--A-AAG-TGAAGGC----AA--G--ACCAT--GATGAA---G ---GCA..........G-AAGA--T--A-AAG-TGAAGCC----AG--G--ACCAT--GATGMA-- -G ---ACA. . . . . . . .G-AAGA--T--A-MAG-TGMAGCC----AG--G--ACCGT--GATGAA---G

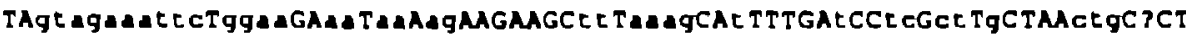
--ATAGAMATCT-GAGA--AA-AA-AG--_----TT-AAAG--T-----C-O-TC-CT-G----TTG-T--ATR AAATCC-GAGA--AA-AG-AG------TT-AAGG--T----C--TC-CT-G----CTG-T---AI AAATTC-GAGA--AA-AA-GG--_---TT-AMAG--T--.--C--TC-CT-G----CTG-T- -ATAGAAACCC-GAGA--GA-AA-AT--.---TT-AAAG--C-..--C--TC-CT-G--.-TTA-T--

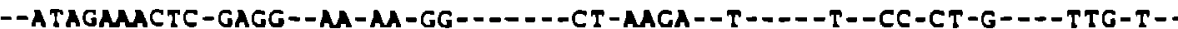

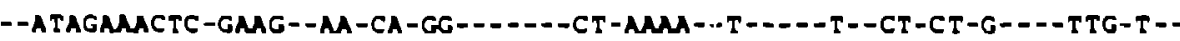
--ATAAGATTC-GGGA--AA-AM-GG-------CT-MAAG--T-----T--CC-CT-G----TCG-T--GGGGAGATCC-GGAG--AM-AA-GC--.-----CT-AMAG--T-.-.-T-T-TC-CT-G----CTG-G---GTGGAGGTTC-GGAA--AT-GA-AG-------TT-AMAA--T-----T-TC-CT-G----CTG-A--GTGGAAGTTC-GAAA--AC-GA-AG-------TT-AMAG--T--.--T-T-TS-GC-T----CCG-A--

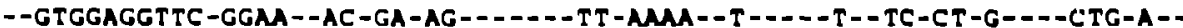
--GTGGAGGTTC-GGAG--AC-GA-AG--_----TT-AAAA--T-----T--TC-CT-G----CTG-G---GTGGAGTTC-AGAG--AT-AA-AG-------CC-GAMA--T----OT-OTC-CT-G----CTG.G-D

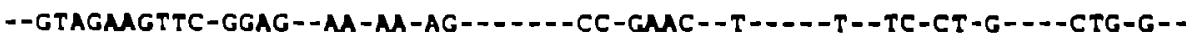

tat ede $\rightarrow$

$$
\backslash / J^{\prime} \cdot 1
$$

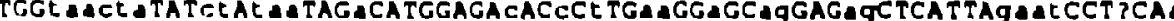

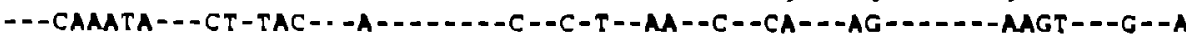

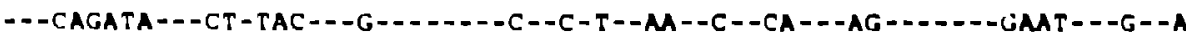

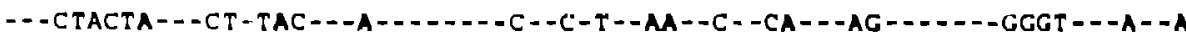

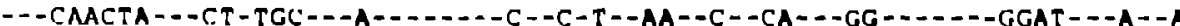

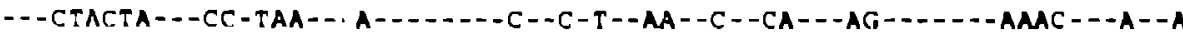

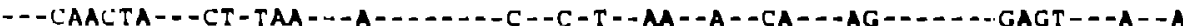

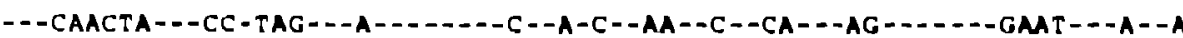

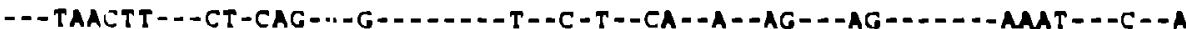
--- TAATCA---СT-TAA---A---------C--C-T--AG--A--AG---AA--.----GAAT---C- -A

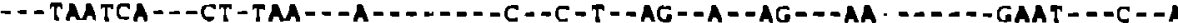

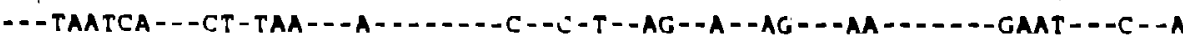

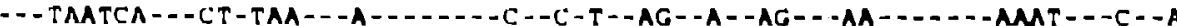

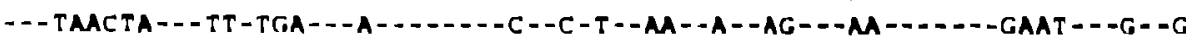
-- - TMACTA-.-CT -TGA---A-..-----C--C-T--AA--A--AG---AG-...---GAAT---C - - 


\section{HIV2/SIV VPR}

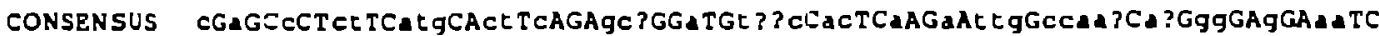
HIV2ROD C-A--C--TT--ACG--CT-C---GCA--A--TGGC-AC--A--A-TTG-C--GA-AA-GG--G--AAHIV2NI H2 C-A--C--CT--GCG--CT-C---GCA--A--TGGC-AC--A--A-TTG-C--GA-AA-GG--G--AA-HIV2ISY C-A--C--CT--ACG--CT-C---GCA--A--TGGC-AC--A--A-TTG-C--AC-AA-GG--A--AA-HIV2ST C-A--C--CC--TTG--CT-C---GCA--A--CGGC-GC--A--G-TTG-T--GC-CA-GG--C--AA-HIV2BEN C-A--C--CT--GTG--CT-C---GCG--A--TAAC-GC--A--A-TTG-C\#"

HIV2DI 94 C-A--C--CT--GTG--CA-C---GCG--A--TGAC-GC--A--A-AGG-C--AA-MA-GA--A--GC-HIV2GHI C-A--C--CT--GTG--CC-C---GCA--G--TAAC-GC--A--A-TTA-C--AA-AA-GA--A--AC-HIV20205 C-A--C--CT--CTC--CT-C---GCC--T--TCAA-AC--A--G-TTG-A--AT-AG-GG--G--AA-SIVMH251 SIVRA1 42 SIVIOA239 SI VMNE SIVSMMH4 SIVSMMPBJ C-A--G--CT--ATG--TT-T---GGC--A--CAAC-AC--C--A-TCG-C--AC-TG-GG--G--AA-C-A--G--CT--ATA--TT-C---AGC--C--CAGC-AT--C--A-TCG-C--AC-TG-GG--G--AA-C-A--G--CT--ATG--TT-C---GGC--A--CATC-AC--C--A-TCG-C--AC-TG-GG--G--AA-C-G--G--CT--ATG--CT-C---GGC--C--CACC-AC--T--A-TCG-C--AT-TG-AG--G--AA-A-A--A--CT--ATC--TT-C---AGT--A--TGCC-AC--C--A-TCG-C--AT-TA-AG--G--AA--

CONSENSUS CTCEctCA CTAtACCgcCCcCTagaggcatgc?a"* 303

HIV2ROD --CTCT--G--AT---GA--C--AGAAACATGCAA--- 318

HIV2NIHZ --CTCT--G--AT---GA--C--AGAGGCATGCAC--- 310

HIV2ISY --CTCT--G--AT---GA--C--AGAAACATGCAA--- 319

HIV2ST --TTAT--G--AT---AA--C--AGAGGCATGCGA--- 318

HIV2O194 --TGCC--G--GC-..-GA--C--AGAGGCATGCAC--- 318

HIV2GH 1 --TTCC--G--GC---GA--C--AGAGGCATGTAC--- 318

HIV20205 --CTCT--A--AT---GC--C--"* 306

SIVMM251 --CTCT--A--AT---GC--T-D\#" 294

SIVMMI 42 -CTCT--A--AT---GC--T--AGAAGCATGCTA-- 306

SIVMH2 39 --CTCT--G--AT---GC--T--AGMAGCATGCTA--- 306

SIVMNE --CTCT--A--AT---GC--T-AGAAGAATGCTA--- 306

SIVSMMHA --CTCT--A--AT---GC--T--AGAGGCGTGCTA--- 306

SIVSHMPBJ --CTCT--A--AT---GC--T--AGAGGCGTGTTA--- 306 
Splice sltes have been determined for SIV only.

CONSENSUS HIV2ROD HIV2NIHZ HIV2I $S Y$ HIV25T HIV2BEN HIV2DI 94 HIV2GH1 HIV2D205 SIVP251 SIVIRP 11 SIVHA1 42 5 I VMH2 39 SIVHNE SIVSIMHA SIVSHAPBJ

CONSENSUS HIV2ROD HIV2NI HI2 HIVZISY HIV2ST HIV2BEN HIV2DI 94 HIV2GHI HIV20205 SIVMH25I SI VRAP 11 SIVAM1 42 SIVHA2 39 SIVHNE SI VSHAHA SIVSMMPEJ

CONSENSUS HIV2ROD HIV2NI HZ HIV2ISY HIV25T HI V2BEN HIV2DI 94 HIV2GH 2 HIV20205 5 I VHH2 51 S I VMMP 11 SIVMMI 12 S [ VMM2 ]9 SIVMNE SI USMMHA SIVSMMPBJ
ATGGAGACACCCE TGaAGGaGCagGAGagCTCATTAgaacCCTCCAacGaGCeCTCE TCatgCACE TCAG $-----C--C-T--A A--C--C A=-A G------A A G T---G--A C-A-C--T T-A C G-C T-C-1$

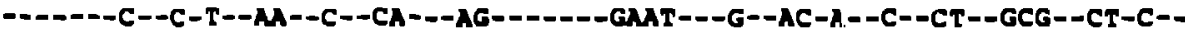

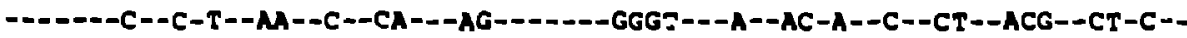
$-----C--C-T--A A--C--C A---G G=-----G G A T---A--A C-A--C--C C--T T G--C T-C-$ $-----C--C-T--A A--C--C A---A G------A A C---A--A C-A--C--C T--G T G--C T-C--$ $-----C--C-T--A A--A--C A---A G------G A G T-=-A--A C-A--C--C T--G T G--C A-C-=$ $-----C--A-C--A$-C-C-CA---AG---D---GAT---A--AC-A--C--CT--GTG--CC-C$-----T--C-T--C A--A--A G---\lambda G---0--N M T--C--A C-A-C--C T-C T C--C T-C-=$

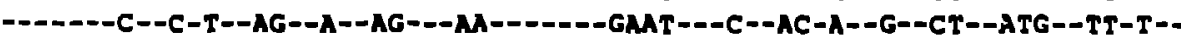

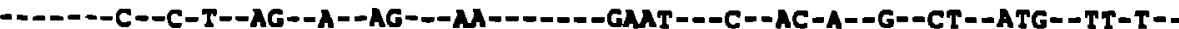

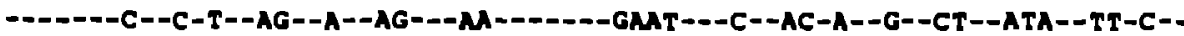
$-----C-C-T--A G--A--A G---N A------$ GAAT---C--AC-A--G--CT--ATG--TT-C-

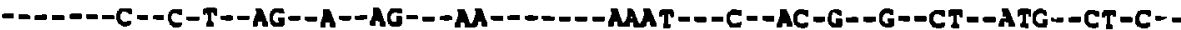
----D-C--C-T--AM--A--AG---MA------GAMT---G--GA-A--A--CT--ATC--TT-C--

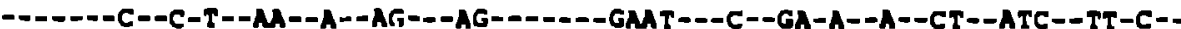

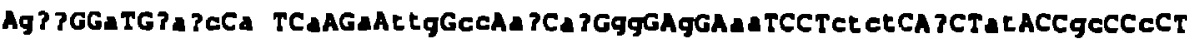
-GCA--A--TGGC-AC--A--A-TTG-CC-GA-A-GG--G--AA----CTCT--G--AT---GA--C--GCA--A--TGGC-AC--A--A-TTG-CC-GA-AA-GG--G--AA----CTCT--G--AT---GA--C--GCA--A--TGGC-AC--A--A-TTG-CC-AC-M-GG--A--M----CTCT--G--AT---GA--C--GCA--A--CGGC-GC--A--G-TTG-TC-GC-CA-GG--C--MA----TTAT--G--AT---MA--C--GCG--A--TAAC-GC--A--A-TTG-CT-AA-AA-GA--A--AC----TGCC--G--GC---GA--C--GCG--A--TGAC-GC--A--A-AGG-CC-M-A-GA--A--GC----TGCC--G--GC---GA--C-GCA--G--TAAC-GC--A--A-TTA-CC-AA-M-GA--A--AC----TTCC--G--GC---GA--C--GCC--T--TCMA-AL--A--G-TTG-AC-AT-AG-GG--G--AA----CTCT--A--AT---GC--C--GGC--A--CAAC-AC--C--A-TCG-CC-AC-TG-GG--G--AA----CTCT--A--AT---GC--T-GGC--A--CAAC-AC--C--A-TCG-CC-AC-TG-GG--G--AA----CTCT--A--AT---GC--T--AGC--C--CAGC-AT--C--A-TCG-CC-AC-TG-GG--G--AA----CTCT--A - -AT---GC--T-GGC--A--CATC-AC--C--A-TCG-CC-AC-TG-GG--G--AA---CTCT--G--AT---GC--T-GGC--C--CACC-AC--T--A-TCG-CC-AT-TG-AG--G--MA----CTCT--A--AT---GC--T--AGT--A--TGCC-AC--C--A-TCG-CC-AT-TA-AG--G--AA----CTCT--A--AT---GC--T-$-G G T--A--C A G A-A C--C--A-T C G-C C-G T-T G-A G--G--A$ - $--C T C T--A--A T---G C--T--$

$$
\text { C- vpR }
$$

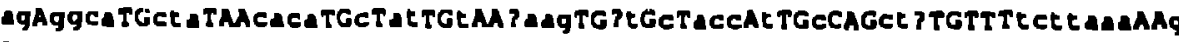
AG-AACA--CAA---CTCA--C-AT--T--GCGA--CT-C-ACC-T--T---ATG-----TCTAAC--G AG-GGCA--CAC---CTCC--C-AT--C--GAAA--CT-C-ACG-T--C---CTG---- - TTTACM- -G AG-AACA--CAA---CAAA--C-TT--T--GGGG- -CT-C-TCC-T--C---CTG---- TTTAAC- -G AG-GGCA--CGA---CAAA--T-AC--T--AAAG--CT-C-ACC-T--C---ATG-----TTTAMC--G AG-GCCA- -CAC- - - CAAA--C-AT- - T--GCGA--CA-T-TCC-T--C---CTG---- -CTCGNA--G AG-GGCA--CAC---CTCA--C-AT--T--GCAG--CA-T-ACC-T--C---CTG-----CTTGAM--A AG-GGCA- - TAC---CTCA--C-AT--T--GCAG--CA-C-TCC-T--C---CTG-----CT TGAAA--A TA-GGCA--CGA-- - TACA--C-AC--T--GAM- -CT-C-ACC-T--C---CTT---_-TCTTAM--G TG-GGCG--CTA---CACA--C-AT--T--AAAG--TT-C-ACC-T--C-- -TTT---- -TCTTAM--G TG-GGCG--CTA---CACA--C-AT--T--AAAG--TT-S-ACC-T--C---TTT-----TCTTAM--G AG-AGCA--CTA---CACA--C-AT--C--AAAG - - TT-C-ACC-T--C---TTT-----TCTTAM--G AG-AGCA--CTA---CACA--C-AT--T--AAAG--TT-C-ACC-T--C---TTT----TCTTAM--A

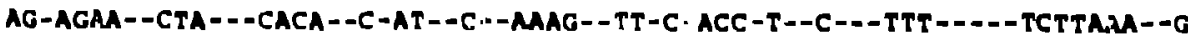
AG-GGCG--CTA---CAAG - -C-AC--T--GAGG--1'l'-C-ATC-T--T---CAT---- -TCTTAM--G AG-GGCG--TTA- - -CAAG--C-AC--T--AAGG--TT-C-ATC-T--T---CAT----TCTTAA--G 
rev eds $\rightarrow$

CONSENSUS HIV2ROD HIV2NI HZ HIVZISY HIV2ST HIV2BEN HIV2DI 94 HIV2GH1 HIV2D205 SIVHA251 SIVMAP II SIVAM1 42 SIVHA239 SIVHNE SIVSMMHA SIVSMMPBJ

CONSENSUS HIV2ROD HIV2NIHZ HIVZISY HIV2ST HIV2BEN HIV2DI 94 HIV2GH HIV2D205 SIVMM 25 SIVHMP 11 SIVMM1 42 SIVMM 239 SIVHNE SIVSMMHA SIVSMMPBJ

CONSENSUS HIV2ROD HIV2NIH? H IV2 ISY HIV2ST HIV2BEN HIV2DI94 HIV2GHI SIVMA25I SIVHMP 11 SIVHM 12 SIVMM2 39 SIVHNE SIVSMMIIA SIVSMMPBJ

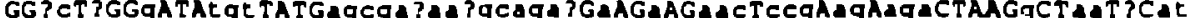
--GC-C--G---TGT----AACGAAAGGGCAGAC-A--A--GAC-CCA-AG-AMA-----A--CA-C-GT --GC-C--G---TGG----ACCGAAAGGGCAGAC-A--A--GAC-CCA-AG-MA-----G--CA-C-GT --GC-C--G---TGT----ACCGAMGGGCAGAC-A--A--GAG-CCG-AG-MM------G--CA-T-GT --GC-C--G---TGG----AMCGMMGGGCAGM-A--A--AAC-CCG-AG-AMA-----G--CA-T-GT --GC-C--A---TCA----AGCGNAGGGCAGAC-A--A--GAC-ECA-GG-AAA-----A--CC-T-GC --GC-C--G---TGG----CGCGACAGGGCAGAC-A--A--GAC-CCA-GA-MAA-----A--CA-C-GC --GC-C--G---TGG----CACGAAAAGCAGAC-G--A--AAC-CCA-GA-AGA-----A--CA-T-GT --TC-T--G---TGT----ACCGCTCG......A-A--G--ATC-GCA-MA-GAG-----A--AC-G-AC --AT-G--G---TGT----AGCAGTCACGAAGA-A--A--AAC-CCG-AA-AGG-----G--AA-A-AT --AT-G--G---TGI----AGCAGTCACGMAGA-A--A--AAC-CCG-MA-AGG-----G--AA-A-AT --CT-G--G---AGI----AGAAGTCACACAGGA-A--A--AAC-CCG-AG-AGG------G--AA-A-AT --CT-G--G---TGT----AGCAATCACGMAGA-A--A--AAC-CCG-MA-AGG-----G--MA-A-AT --CT-G--G---TGT----AGCAGTCACGCAGMA-A--A--AAC-CCG-AG-AGG--.--G--A-A-AT --TT-G--A---TGC----AGCAG. ..CAC. . . C-A--A--AAC-CCG-AG-AGA-----G--AA-C-AT --CT-G--A---TGC----AGCAGCAA. . . . . C-A--A--MAC-CCG-AG-AGA-----G--AA-A-AT

exon $2 \backslash$ /exon 3

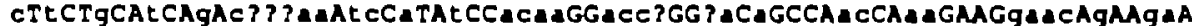
C-C--A--C--G-C...AA-TC-A--T--ACAA--ACCG--GA-A----GC--AC----AMAC-G--GAC-T--G--T--G-C...AA-TC-A--T--ACMA--ACCA--MA-A----GC--GA----AMAC-G--GAC-C--G--T--G-C...AA-TC-A--T--ACMA--ACTG--AA-A----GA--GA----AAAC-G--GAC-T--G--T--G-C...AA-TC-A--T--ACAA--ACCG--AA-A----GC--GA----AMAC-G--GAC-T--G--C--G-C...AA-TC-A--T--ACAA--ACCG--GA-A----GC--AC----GAAC-G--GAC-C--G--T--G-T...AA-TC-A--T--ACAC--ACAG--GA-A----GC--AC----MAAC-G--GAC-T--G--T--G-C...AA-TC-A--T--ACAC--ACCG--GA-A---GC--AC----GMAC-G--GA$C-T--G=-C-=+4$

C-T--G--T--A-C. . AA-CT-A--C--AACA--ACCC- -CA-T----AC--GA----GCAA-G--GGC-T--G--T--A-C. . AA-CT-A--C--AACA--ACCC--CA-T----AC--GA----GCAA-G--GGC-T--G--T--A-C. . . GA-CC-A--C--AACA--ATCC--CT-T----AC--AA----GCAA-A--GGC-T--G--T--A-C, . AA-CC-A--T--AACA--ACCC--CA-T----AC--GA----GCAA-G--AGC-T--G--T--A-C . . AA-CC-A--T--GACA--ACCA--CA-T----AC--MA----GAAC-G--GGT-C--G--T--A-C. . . MA-TC-C--T--ACMA--GCCA--AA-C----AC--MA----GAGA-G--GGC-T--G--T--G-C. . AA-TC-C--T--AGAC--GCCA--AA-T---AC--MA----GAGA-G--GG-

GACGgtgGagacageggTGG aaCagcTeeTgGCCtTGGCaga".. -AC-GTG-MAGCAACGG---AGA-AGA-AC-G---C-.--CGA..- 393 -AC-TTG-AAGCAACGG--AGA-AGA-CT-G---T---CGA--- 393 -AC-TTG-GAACGACGT---MAG-AGA-CC-G---T----CGA--- 393 -AC-TTR -AAACAGCGT---AGA-AAT-GG-G---C--.-CGA-.- 393 -AC-TCG-AG-ГCACAG---TTA-GAC-TG-G---T-.--CAA-.- 393 -AC-CCG-AGACGACAG---TTT-GGC-TG-G---T----CAC--- 393

-AC-ACA-AGACGACGA---TTA-GAC-TG-A---T----CGA--- J9]

-AC-GTG-AGAAGGCGG---.CAA-AGC-CC-C---T----AGA--- 393

-AC-GTG-AGAAGGCGG---CAA-AGC-CC-G---T---AGA--- 39]

-AC -GTG-AGGCAGCGG---CAA-AGC-CC-G---T---AGA--- 393

-AC-GTG-AGAAGGCGG---CAA-AGC-CC-G---T----AGA--- J9]

-AC-GTG-AGGCAGCGG---CAA-AGC-CC-G---T----AGA--- 393

-AC-GTG-AGACAGAGG---CAG-AGA-CT-G---T---AGA--- 387

-AC-GTG-AGGCAGAGG---CAA-AGA-CT-G---T---AGA--- 387 
exon 2\/axon 3

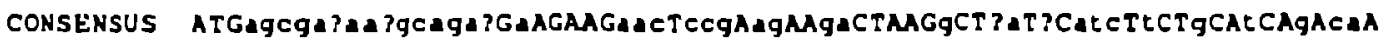
HIV2ROD HIV2NIHZ HIV2 ISY HIV2ST HIVZBEN HIV2D194 HIV2GH1 SIVM123I SIVRaP 11 SIVIM1 42 SIVMA239 SIVHNE SIVSMAHA SIVSMMPB ---MACGMAGGGCAGAC-A-----GAC-CCA-AG--MA-----A--CA-C-GTC-C--A--C--G-CA - --ACCGAMAGGCAGAC-A-----GAC-CCA-AG--AA-----G--CA-C-GTC-T--G--T--G-CA-- -ACCGMAGGOCAGAC-A-----GAG-CCG-AG--M-----G--CA-T-GTC-C--G--T--G-CA---AACGMAGGOCAGM-A----MAC-CCG-AG--AM-----G--CA-T-GTC-T--G--T--G-CA---AGCGAMGGGCAGAC-A---- -GAC-CCA-GG--AA-----A--CC-T-GCC-T--G--C--G-CA---CGCGALRSGGCAGAC-A-----GAC-CCA-GA--AA-----A--CA-C-GCC-C--G--T--G-TA---CACGAMAMGC: GAC-G-----AAC-CCA-GA--GA-----A--CA-T-GTC-I--G--T--G-CA---AGCAGTCACGNAGi -A-----MC-CCG-MA--GG----G--AA-A-ATC-T--G--T--A-CA---AGCAGTCACGAAAGA-A---- -MC-CCG-M--GG----G--MA-A-ATC-T--G--T--A-CA- --AGAAGTCACACAGGA-A-----MC-CCG-AG--GG-----G--M-A-ATC-T--G--T--A-CG---AGCAATCACGAMGA-A-----AAC-CCG-MA--GG-----G--M-A-ATJ-T--G--T--A-CA---AGCAGTCACGCAGM-A-----AC-CCG-AG--GG-----G--AA-A-ATC-T- J--T--A-CA---AGCAGCACC. . . . . -A-----MC-CCG-AG--GA-----G--M-C-ATT-C- G--T--A-CA---AGCAGCAAC. . . . .-A-----AAC-CCG-AG--GA-----G--AM-A-ATC-T--G--I--G-CA-

CONSENSUS HIV2ROD HIV2NIH2 HIV2ISY HIV2ST HIVZBEN HIV2O194 HIV2GHI SIVMM251 SIVRAP II SIVAM1 12 SIVMM239 SIVANE SIVSMAHA SIVSMMPB HIVZROD HIV2NIHZ HIVZISY HIVZST HIV2BEN HIV2DI94 HIV2GHI SIVMA25I SIVMMP II SI UMM! 12 SIVMM 39 SIVANE SIVSMMHA SIVSHAPBJ

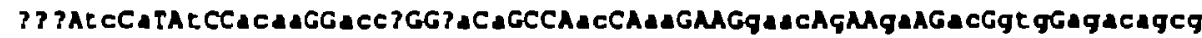
- -TC-A--T--ACMA--ACCG--GA-A----GC--AC----MMC-G--GA--AC-GTG-MAGCAACC ...-TC-A--T--ACAA--ACCA--M-A----GC--GA----MAC-G--GA--AC-TTG-MAGCAACG . . -TC-A--T--ACAA--ACTG--MA-A----GA--GA----MAC-G--GA--AC-TTG-GAACGACG ...-TC-A--T--ACAA--ACCG--AA-A----GC--GA----ANC-G--GA--AC-TTG-AAACAGCG .. -TC-A--T--ACAA--ACCG--GA-A----GC--AC----GAAC-G--GA--AC-TCG-AGGCGACA ...-TC-A--T--ACAC--ACAG--GA-A----GC--AC----MAC-G--GA--AC-CCG-AGACGACA ...-TC-A--T--ACAC--ACCG--GA-A----GC--AC----GAC-G--GA--AC-ACA-AGACGACG ...-СТ-A--C--AACA--ACCC-- CA-T----AC--GA----GCAA-G--GG--AC-GTG-AGAMGGCG . . -CT-A--C--AACA--ACCC--CA-T----AC--GA----GCA-G--GG-AC-GTG-AGAMGCO ...-СC-A--C--AACA--ATCC--CT-T----AC--AA----GCA-A--GG--AC-GTG-AGGCAGCG ...-СC-A--T--AACA--ACCC--CA-T----AC--GA----GCAA-G--AG--AC-GTG-AGAMGGCG ...-CC-A--T--GACA--ACCA--CA-T----AC--AA----GAAC-G--GG--AC-GTG-AGGCAGCG ...-TC-C--T--ACAA--GCCA--M-C---AC--M----GAGA-G--GG--AC-GTG-AGACAGAG ...-TC-C--T--AGAC--GCCA--M-T----AC--M----GAGA-G--GG--AC-GTG-AGGCAGAG

$$
<-t a t
$$

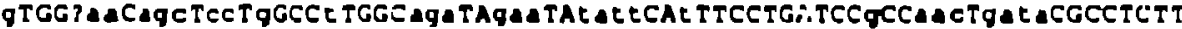

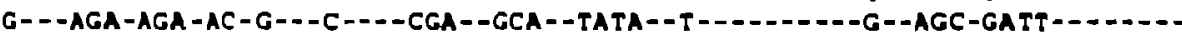

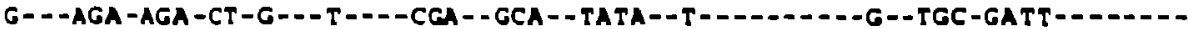

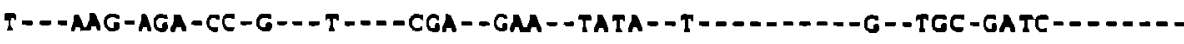

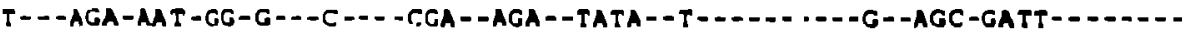

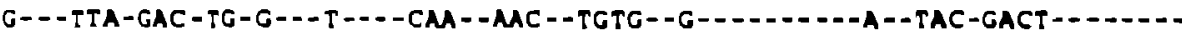

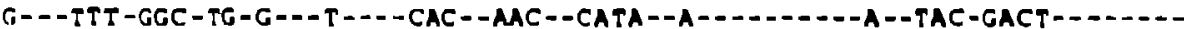

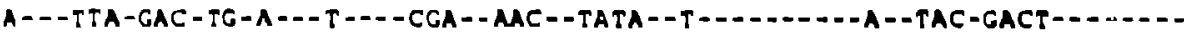

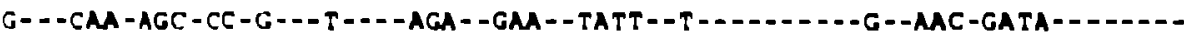

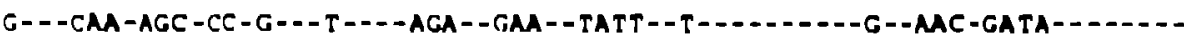

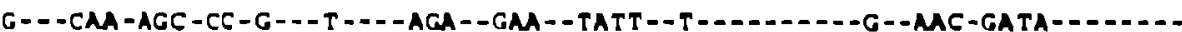

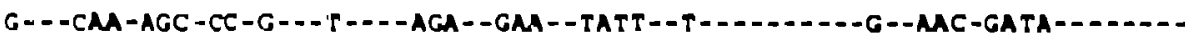

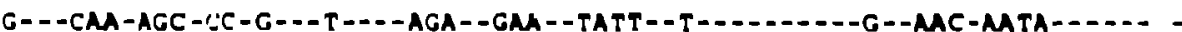

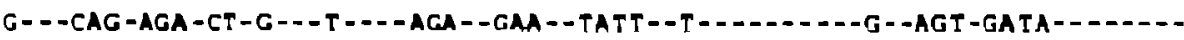

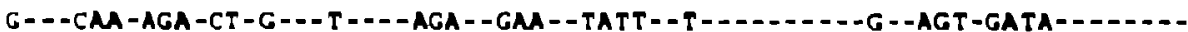


HIV2/SIV REV

CONSENSUS GACETGgCTATACAgCA?CTgCAGagaCTE?CTATCRAgga?CTaCCagAtCCtCCaACaA?tLaCag HIV2ROD HIV2NIHZ HIVIISY HIV2ST HIV2BEN HIV2DI99 HIV2GHI SIVHM2 31 SIVAP 11 SIVMH 142 5 I VIM 239 SIVANE -CCA-AC-A-A--GC-TC-G---GGAC-TA-..-CC-GGAGC-T--TG-C--T--MA-TCATCTACCAG --CCG-GC-A-A--AC-TC-G---GGAC-TA----CC-GGATC-C--CG-T--T--AA-CAATCTTCCACI --CCA-AC-A-A--AC-GC-G---GGAC-TA----CC-GACTC-A--TG-T--T--AA-CACT...... --ACA-AC-A-A--AC-TC-G---GGAC-TA----CC-GGACC-T--AG-C--T--MA-TAATCTCCCAG --TCG-GC-A-A--AC-TC-G---AGAC-TA----CC-AGAAC-C--CG-C--G--GA-TGATCTCCCAG --CCG-GC-A-A--AC-GC-G---GGGC-TA----CC-AGAAC-C--CG-C--G--GA-TGATCTCCCAG --CCG-GC-A-A--AG-TC-G---AGAC-TA----CC-CGMAC-C--CG-C--A--GA-TGATCTCCCAG --CTT-GC-A-T--GC-AC-G---AACC-TG----CG-GAGCA-A--AG-T--T--AA-CAATACTCCAG --CTT-GC-A-T--GC-AC-G---MACC-TG----CG-GAGCA-A--AG-T--T--MA-CAATACTCCAG --CTT-GC-A-T--GC-AC-G---MACC-TG----CG-GAGCA-A--AG-T--T--AA-CIATATTCCAG --CTT-GC-A-T--GC-AC-G---MACC-TG----CG-GAGTA-A--AG-T--T--MA-CMATACTCCAG --CTT-GT-A-T--GC-AC-G---AACC-TG----CG-GAGCA-A--AG-T--T--AA-CAMTATTCCAC --CTT-GC-A-T--GC-GC-G---GGAT-GG----TG-GGAGC-A--AG-T--T--AA-CAGTGCTCCAG

SIVSKMHA

SIVSMMPBJ --CTT-GC-A-T--GC-GT-G---AGAT-GG----TG-GGAC-G--LA-C--T--AG-CAGTGCTCCAG 


\section{HIV2/SIV ENV}

Splice sites have been decermined for SIV only.

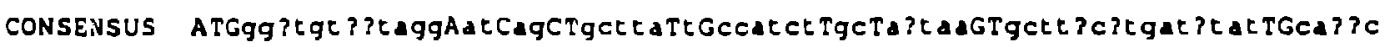
HIV2ROD -.........ATG-AT-AG--GCTTA-T-CCATTT-AT-AGCTA--GCTTGCTTAGTATAT--CACCC HIVZNIHZ --AAGGGTAGTAAG-AT-AA--GCTGA-T-CTATTG-AC-AGCTA--GCTTACCTAMTACAT--CAAGC HIV2ISY --AG...TGGTAAA-TT-AG--GCTTG-T-CCTTTC-GC-AACTA--GCTTGCTTAATATAT--CACCA HIV2ST ---TG...TGGTAGG-AT-AA--ATTTG-T-CCAGCT-GC-AGCTA--GCTTGCTTAATATAT--CGTCC HIV2BEN ---GAGCCTGGTAGG-AT-AG--GTTTG-T-TCATTT-AC-ANCAA--GCTTGCTTAGTATAT--TAGCC HIV2D194 ---GAGCCTGGTAGG-AT-AG--GCTTG-T-CCATTT-AT-AACTA--GCTTGCTTAATATAT--CAAAC HIV2GH1 ---TG...TGGTAAG-GT-TA--ATGTG-T-CCAGCT-GC-AGCTA--GCTTACTTAGTATAT--CACCC HIV2D205 ---GCATATTITAGC-GC-GC--GCCTA-T-CGCTCC-GC-TATAG--ATCAGTGGGTTIGTA--TAMC SIVMA251 ---GGATGTCTTGGG-AT-AG--GCTTA-C-CCATCT-GC-TTTAA--GTCTATGGGATCTAT--TACTC SIVMM1 42 ---GGTTGTCTTGGA-AT-AG--GCTTA-C-CCATCT-GC-TCTAA--GTCTATGGGATTTAT--TATTC SIVMM239 ---GGATGTCTTGGG-AT-AG--GCTTA-C-CCATCT-GC-TTTAA--GTCTATGGGATCTAT--TACTC SIVMNE SIVSHMH 4 SIVSMMPBJ ---GGATGTCTTGGG-AT-AG--GCTTA-C-CCATCT-GT-TCTAA--GCCTATGGGATCTAT--CATTC -.-GGATGTCTTGGG-AT-AG--GCTTA-C-CGCTCT-GC-AGTAA--GTTTTAGAGATTTGT--TGTTL

CONSENSUS HI: 100 HIV.NIHZ HIV2ISY HIV2ST HIV2BEN HIV $2 D 194$ HIV 2GHI HIV 20205 SIVMH25I SIVMMI 42 SIVMM239 SIVMNE SIVSMMH4 SIVSMMPBJ --GGATGTCTTGGG-AT-AG--GCTTA-C-CGCTCT. GC-ATTAA--GCTTCAGGGATTTAT".TGTTC

CONSENSUS HIV 2ROD HIV 2NIHZ HIVZISY HIV 2ST HIV 2 BEN HIV 20194 HIV2GH1 HIV2O205 SIVMMZSI 5 [VMMI 12 SIVMA2 39 SIVMNE

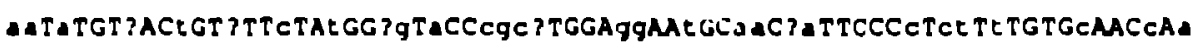
AA-A---A--T--T--C--T--CG-A--CACG----AA--T--AA-CA----C-CT-T----C---C-G

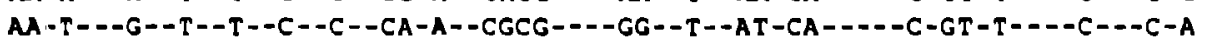
AA-A---G--T--T--C--T--AG-A--CGTG---AA--T-AT-CA----CC-CT -T----C--T-A

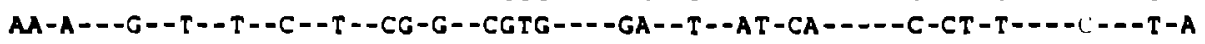
$A G-A---G--T--T--C--T--C A-A--C G C G----A A--T--A T-T A----T-A T-T---C---T-A$ AA-A---G--T--T--C--T- -CA-A--CGCG----GA--T--AT-TA--D-C-AT-T---OC-D-C-A AA-A---G--T--T--C--T--CG-G--CGTG---GA--T--AT-CA----CC-CT-T----C---T-A

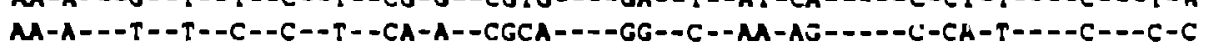

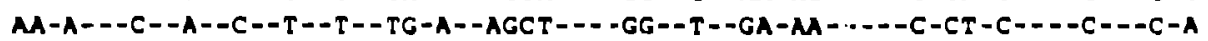
AA-A---C--A--C--T--T--TG-A--AGCT----GG--T--GA-AA-----C-CT-C----C---C-A TA-A---C--A--C--T--T--TG-A--AGCT--- -GG--T--GA-AA----C-C-CT-T----C---C-A AA-A---C--A--C--T--T--TG-A--AGCT----GG--T--GA-AA-----C-CT-C----T---C-G AA-A---A--A--A--C--T--TG-A--AGCA----AG--T--GA-AA-----C-CT-C----C---C-A AA-A---A--A--A--C--T--TA-A--ACCA----GG--T--GA-AG-----C-CT-C----C---C-A A--T--G--T--T------A--CATA--G--CTTG--T--C----A---T--TCAG--AA-MACTT-G--T A--T--A--T--T-----A--AATA--G--CTTA--A--C----A---T--TCAG--AA-AACIC-A--T A--T--A--T--T-----A--CATA--G--CTTG--A--C----A---T--TCAA--GA-ACCTT-G- - T A--T--A--T--T-----A--CATA--G--CTTG--A--C----A---C--TCAG--AA-AGCTT-A- -T A--T--A--C--T-----G--CATA--G--CTTG--A--C----A---T--TCAG--AA-AATT'I'A--T A--T--A--T--T-----G--CATC--G--CTTG--A--C----A---T--TCA.U--AA-AACCT-A--T A--C--A--T--T-----A--CATA--G--CAAG--A--C----A---T--TCAA--AA-AACCT-G--T A--C--A--C--C-----A--TGTA--G--TCTC--A--C----G---C--CACT--GA-CAGGC-A--C

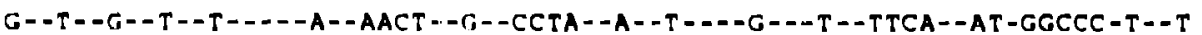
G--T--G--T--T-----A--AACT--G--CCTA--A--T-D--A---T--TTCA--AT-GGCCC-T--T

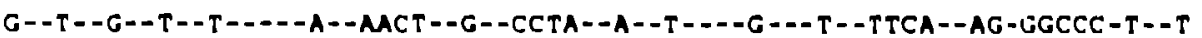

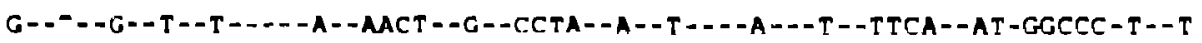
SIVSMMHA G--T--G--C--T-----A--AACA--A--CTTG--A--T-N-A-N-T--CTCA--AT-GGCAA-C--T SIVSMMPBJ G--T--G--C--T-----A--AACA--A--CTTG--A--T----G---T--CTCA--AT-GGCAA-T--T 
CONSENSUS HIV2ROD HIV2NIHZ HIV2ISY HIV2ST HIV2BEN HIV2D194 HIV2GHI HIV2D205 SIVHA251 SIVMA1 12 SIVMA239 SIVMNE SIVSMMH4 SIVSMMPBJ

CONSENSUS HIV2ROD HIV2NIHZ HIV2 ISY HIV2ST HIV 28EN HIV2D194 HIV2GHI HIV20205 SIVM4251 SIVMM1 12 SIVMM239 SIVMNE SIVSMMHA SIVSMMPBJ

CONSENSUS HIV2ROD HIV2NIHZ HIV2ISY HIV2ST HIV28EN HIV2D1 94 HIV2GH1 HIV20205 SIVMM25I SIVMMI 42 SIVMK2] 9 SIVMNE SIVSMMHA SIVGMMPUJ
gT PACAGAggCtTTEGALGCATGGgAtAATACAGT ?ACAgAACAaGCAATAGAgGALGTCTGGa ?CT ? G-A-----GGCT--T--T--A---A-T----C---A---G----A---A----A--T--C---CAT--AG-G----GGCT--C--T--A---A-T----C---A---G----A---G----G--T--C---AAT--A-

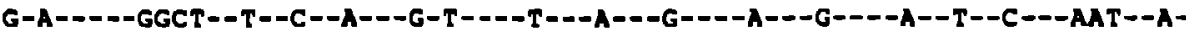
G-G-----GGCC--C--C--A---A-T----C---A---G----A---G----A--T--C---AGT--A-

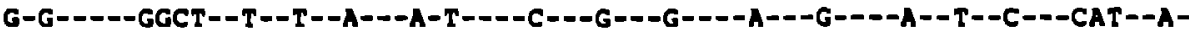
G-G-----AGCT--T--T--A---G-T----C---A---G----A---A----A--T--C---AGA--GG-G-----GGCT--C--C--A---G-T -.--C---A---G----A---G----A--T--C---AGT--A-

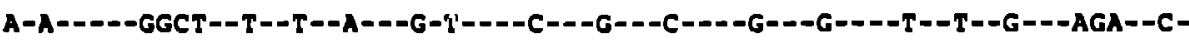

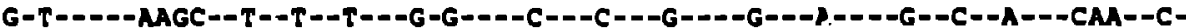

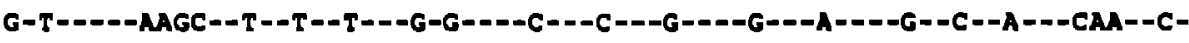
G-T-D---MAGC--T--T--C---A-T----C---C-C--G----G---A-D--G--T--A---CMA--C-

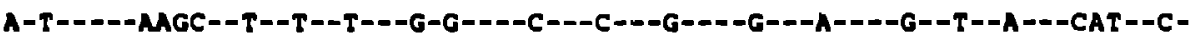

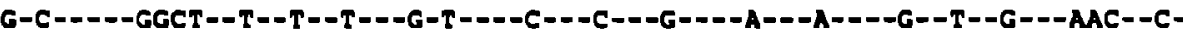

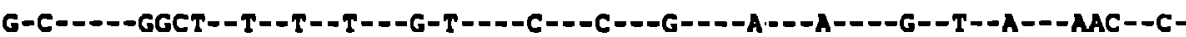

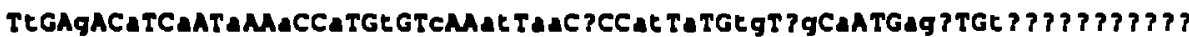
-C--G--A--A--A--A--A--T--C--AC-AA-A--TT-A--TG-AG-A---AAA--C......... -T--G--A--A--A--A--A--T--C--AT-AA-A--CT-A--TG-AG-A---MAC--TACAAGG. . . . -T--G--A--A--A--A--A--T--C--AT-AA-G--TT-A--TG-AA-A---AAC--T. . . . . . . $-T--G--A--A--A--A--A--C--C--A C-M A-A--C T-A--T G-A G-A-\ldots C G T--T \ldots \ldots \ldots \ldots$ -T--G--A--A--A--A--A--T--C--GC-MA-A--TC-A--TG-GG-A---AAT--TAGCAGGGTTCA $-T--G--A--A--A--A--A--T--C--G T-G A-G--C C-A--T G-G G-G---A M T--T . \ldots \ldots \ldots .$. -T--G--A--A--A--A--A--C--C--AC-AA-A--TC-A--TG-AG-A---AGT--T. . . . . . -T--A--C--C--A--A--A--T--C--AC-MA-C--AC-G--TG-GG-A---MAC--T. . . . . . -T--G--C--A--A--G--T--T--A--AT-AT-C--AT-A--CA-TA-T---AGA--C. . . . . . -T--G--C--A--A--G--T--T--A--AT-AT-C--AT-A--CA-TA-T---AGA--C. . . . . . -T--G--C--A--A--G--T--T--A--AT-AT-C--AT-A--CA-TA-T---AGA--C........ -T--G--C--A--A--G--T--T--A--AT-AA-C--AT-A--CA-TA-T---AMA--C........ -T--A--A--C--T--G--C--T--A--AC-CA-C--AC-A--TA-AG-A---AGA--T........ $-T=-A--A--C--T--G--C--T--A--A C-C A-C--A C-A--T A-A A-A---A G G--T, \ldots \ldots \ldots$.

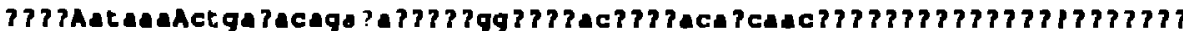

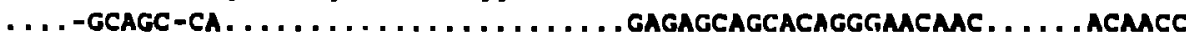

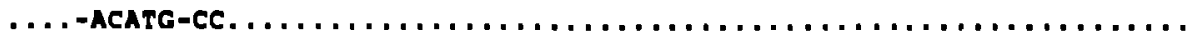

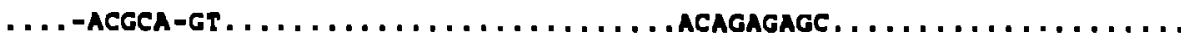

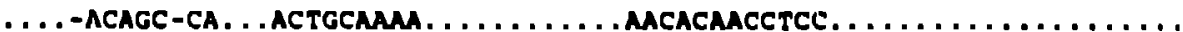

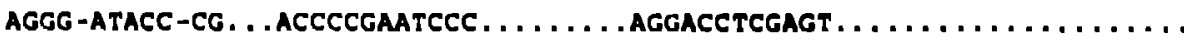

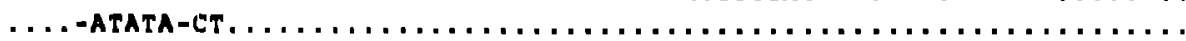

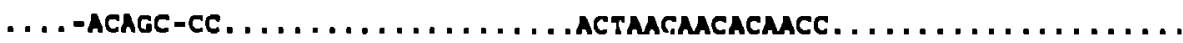

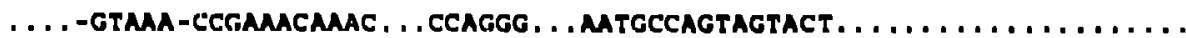

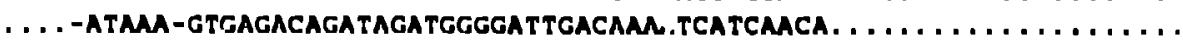

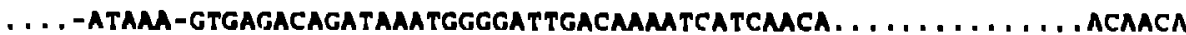

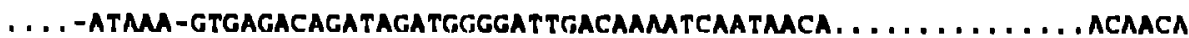

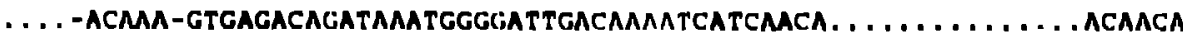

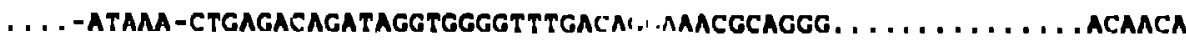

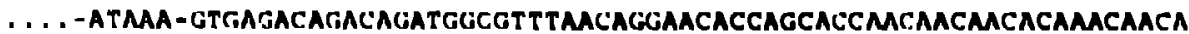




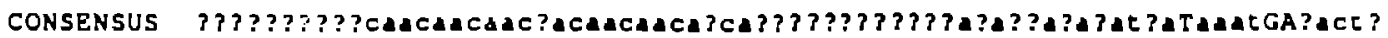
HIV2ROD HIV2NIHZ HIV2ISY HIV2ST HIV2BEN HIV2D194 HIV2GH1 HIV2D205 SIVAM25I SIVMHI 42 SIVA2 39 SIVMNE SIVSHAH SIVSHMPBJ

CONSENSUS HIV2AOD HIV2NIHZ HIVZISY HIV2ST HIV2BEN HIV2DI94 HIV2GHI HIV2D2O S I VAM2 51 SIVMHI 42 SIJHH239 SIVHNE SIVSHOAH SIVSHMPBJ

CONSENSUS HIV2ROD HIVZNIHZ HIVZISY HIVIST HIV $2 \mathrm{BEN}$ HIV 2DI94 HIV 2GHI IIIV2D205 SIVMHZ 51 SIVHMI 42 SIVMA2 99 S I VHNE SIVSHAHA SI USMMPUJ TCAAAGAGCACAAGCACAACCACAACCACACCCACAGACCAG. . . . GAGCAAGAGA-MAGT--GGATA

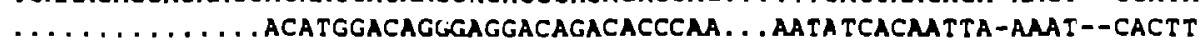

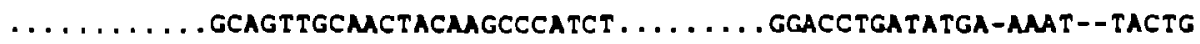

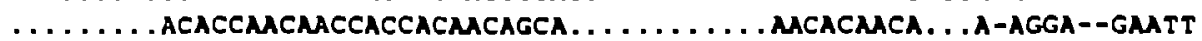

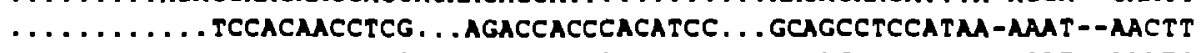

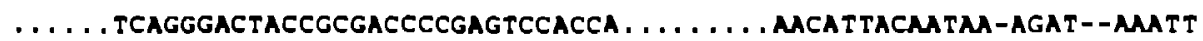

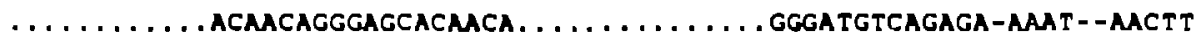
. . ACcaccacta

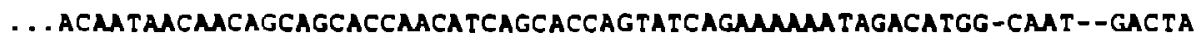
GCA. . TCAACMCAACAACAACAMCAGCAAMATCAGTA. . GAGACAICAGACATAG-CAAT--GACTA

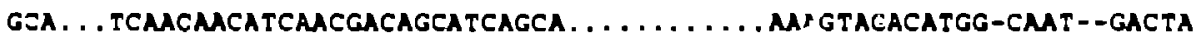
GCA. . . . .CAMCAGCA TACCAACAMAGCA . . . . . . GAGGLAA TAMAGTGG-CAAT- -GAATA ACA . . ACAGCAMTAACAACAACAGCAACACCAAGTCTA. . . . GCAGAAMATGTTA-AMAT--AAGTA

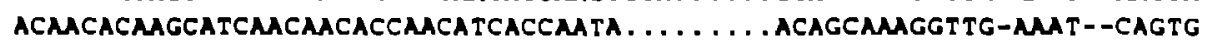

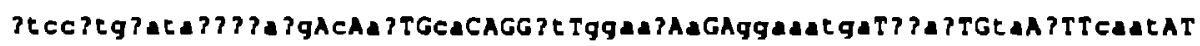
CTCCATGCGCACGCGCAC-C-AC--CT----AT-GGGAG-G--AGAMCGA-CAAT--CC-G--CAAT-C. CCAT. . GCGCGTG AG-C-AC--CA----AT-AAGG-G--AGMATGA-CGAC--TC-G--TAGT-ATCCATGCATACAATTGA-C-AT--CT----AC-GAGGG-G--AGACATGG-CCAG--TC-G--CAAT-CTTCATGCATACGCACAG-C-AC--CA---GT-GGGAG-A--AGAGATGG-CGAC--TC-G--CAAT-CTAACTGCATAGAMACA-C-CA--CG----AT-AGGCT-T--GGACATGA-GCAA--TG-G--CAMT-CTACCTGTATAGCCGACA-C-AC--CA----AT-AGGGA-A--AGAGGTGG-TGAG--TG-G--CAAT-СT. . CСATCATACTCAG-C-AC--CA----AT-GGGGA-G--AGAGATAG-CMAT--TC-G--CTAT-ACCCATGCATMANATG-C-GC--CA-.--AC-AGGAG-A--GGAMTAA-GCAA--TA-T--TAGT-GTTCTTGTATAGCTCAGA-T-AT--CA----CT-GGAAC-A--GCMATGA-AMC:--TA-A--CACC-GTCCTTGTGTAGTTCATG-T-AT--CA----CT-GGAC-A--GCCATGA-AMC - -TA-A--CMC-GTTCTTGTATAGCCCAGG - T-AT--CA----CT-GGAAC-A--GCAAATGA-AAGC--TA-A--CAAC-GTCCTTGTATAAATCATG-T-AT--CA----CT-GGAA-A--GCCATRA-AAGC--TA-A--CAM -ATCCTTGCATAMAMATA-T-GT--TG----CT-GGAAC-G--GCCCATGA-AGGT - -TA-A--TAA - ATCCTTGTATAMATTA-T-AT--TA---CT-GGAA-G--GCCCATGG-AAGT--TA-A--TAAC--

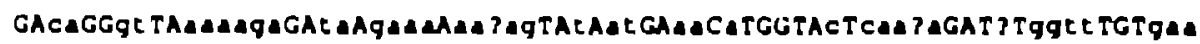
--CA--AT - -GAMAGA--TA-GAM-AACAG- -T-AT--AA-A----C-CAMA- - -G-GGTT--GAG --CA--AT--GAGAGA--CA-GAGA-AACAG--T-CT--AG-A-----C-CAMA---G-GGT - - -GAC --CA--AC- -GAGT TA--TA -GAMA-MACAG--T-GT--MA-C-----C-CAAA---G-GGTT---GMA - -CA--AT - -GAGAGG - - TA-GAAA-AACTA- - T-AT - - AA-A-----C-CAMAA- - -G-AGTC-- -GAA - -AG--GT - -GAACAA- - TA-GAAA-GEAGC- - T-AG--CA-A- - --T - TAGAA- - -G-GGTT--GAC - -CG--GC--GAACAA--TA-GAAA-GGAAG - - T-AT--CG-A-----G-CAAGA---G-GGTT---GAC - -CA--AT - - GAGAGA - - TA-GAAA-AAC AG - -T-AT--AA-A-- --C-CAAAA--G-AGTT---GAA

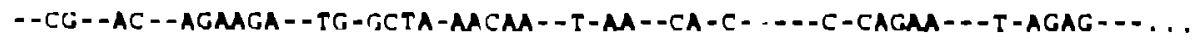

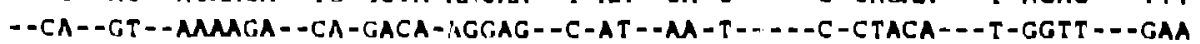

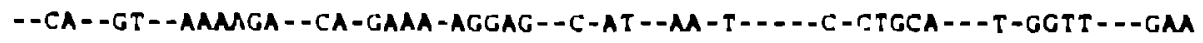
- -CA - -GT - -AAMAGA - -CA - GAAA-ARGAG - - -AT--AA - T- ---C - CTGCA - - T-GCTA - -GAA - -CA--GT--AAAAGA--CA-GAGA-GAGAG- -C-AT- -AA-T- - - -C-CTGCA- - - -GGTT-- -GAA 
CONSENSUS
HIV 2 ROD HIV 2NIHZ HIV2ISY HIV2ST HIV 2BEN HIV2O194 HIV 2GHI HIV 20205 SIVMa151 SIVMAl 42 SIVMA239 IIVMNE SIVSMMHA SIVSMMPBJ

CONSENSUS HIV2ROD HIV2NIHZ HIV2ISY HIV2ST HIV 2BEN HIV 2D194 HIV $2 \mathrm{GH} I$ HIV2D205 SIVMH23I SIVMHI 42 SIVMM2 39 SIVMNE SIVSKMHA iIVSMHPBJ

CONBENSUS HIVZROD HIV2NIHZ HIVIISY IIIV2ST HIV 2BEN IITV2DI 94 HIV 2GHI IIIV202J5 SIVMH25] SIVMHI 4 ? SIVMM2 99 SIVMNF. SIVSMHIIA SIVSMMPII.

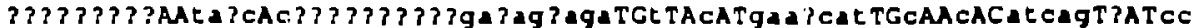
... ACAAA T--TAGC-CAAATCAGACCCAG.......--T--C.-GAACCAT--C--C--ATCAG-C--CA ........ --TAaC-CC. . TCAAGTCAGAGCAaG--T--C--GAACCAT--C--C--ATCAG-C--CA ... ICAGa T--CAGC-CAGACCGa. . . . . AaAAGa--T--C--GAACCAT--C--C--ATCAG-C--CA ...TCA... --TGAC-CCAMGAMA...GAGAAMACA--T--C--GMACCAC--C--C--ATCAG-C--CA .......--CACA-CA. ....... GCTGGCACA--T--C--GAGACAT--C--C--ATCAA-C--Ca ... AAGACA--CGGA-CA. ..........GGCACA--T--C--GAGACAT--C--C--ATCAG-C--CA ... TCAAAT--TACC-AAGATGGGMA. . AACAGA--T--C--GAACCAT--C--C--ATCAG-C--CA ........-T-TAAT-CCAGLMAGTATACCAGCAGA--C--T--AAGAACC--C--C--AACAA-T--CC CAA. . GGG--TAGC-CT. .. GATAATGAAGCAGA--C--C--GAATCAC--T--C--TTCTG-T--CC CAA. . .GGG--TAGC-CT. . .GGTAATCAMATA A--T--C--GAATCAC--T--T--TTCTG-T--CC CM... GGG--TMC-CT. . GGTAATGMAGTAGA--T--C--GAACCAC--T--C--TTCTG-T--CC CAA. . .GGT--TAGC-CT. . GAMATGAAGTAGA--T--C--GAATCAC--T--C--TTCTG-T--TC CAGTCAGCG--TGAA-GT........GAGAGTAMA--T--C--GCATCAT--T--C--CAGTG-T--TC CAMAATAGC--TGAA-ET. . . . . . . GACAGTAAA--C--T--GAACCAT--T--C--CAGTG-T--TC

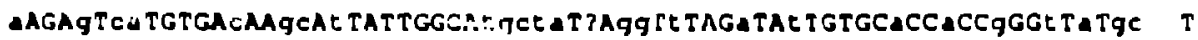
C---A-CA-----C--GC-C--------TGCTA-A-GG-T---A--C-...--A--A--G--T-A-GCCCC---G-CA-----T--GC-C- ------TGCTA-G-GG-T---A- -C-----A--A--G--T-T-GCCT-

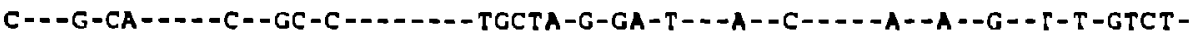

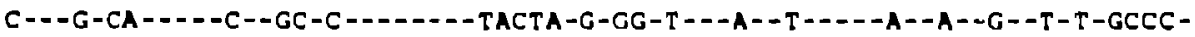

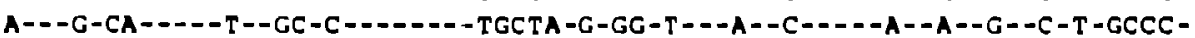

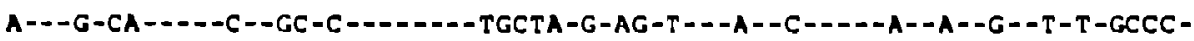
C---G-CA----T--GC-C--------TGCTA-A-AG-T---A--C----A--A--G--T-A-GCCCA---G-CA--.--C--AC-T-D.-D-CACCT-A-GG-T---G--T-D--T--C--G--G-T-TTTC-

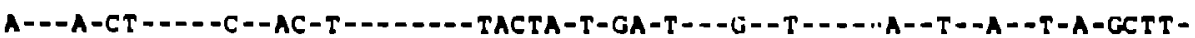

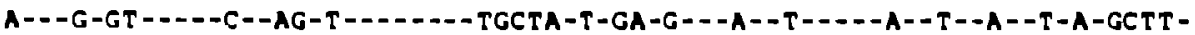

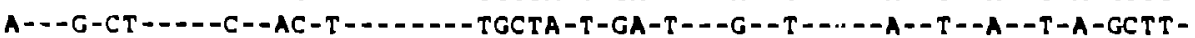
A---A-CT----C--AC-T------TGCTA-T-GA-T---G--T-----A--T--A--T-A-GCTTA---A-CC-----C--GC-T-------TGCTA-T-GA-T---A--C-----A--G--A--T-A-GCTTA---A-CC--.-C--AC-T--D---TGCTA-T-GA-T---A--T----A--G--A--T-A-GCTT-

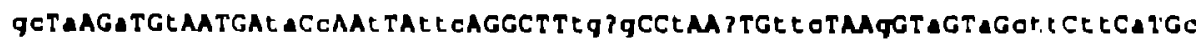
AT-A--A--?-----'ТA-C =-T--TTC----.-TGCA--C--C--TTC---A--A--A-CTT-TA-A--C AC-A--A--C----TA-C,- T--TTC----- TGCA--T--C--CTC --A--A--A-CTG-TA-A--C GC-A--G--C---.-TA-C--T--STC-D-- -TGAG--C--T--CTC-- -A--A--A-CTT-TA-A--T GC-A--A--C-----TA-C--T--TTC---.--TGAG--C--T--TTC-..-G--A--A-CTG-TA-A--T AT-A--A--T-----TA-C--C=-TTC---D--TGAA--T--G--CAC---A--A--A-CTG-TT-A--C AC-A--A--C--.--TA-C--C--TTC--.--TTIAA--T--G--CTC---A--A--A-CTG-TT-A--C

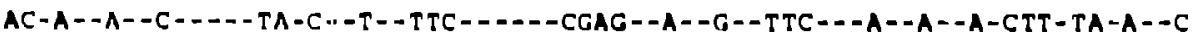
AC-A--A--T-----TA-C--C--TTC---.--CATG--C--C--CAG---G--A--A-CGT-CT-C--C

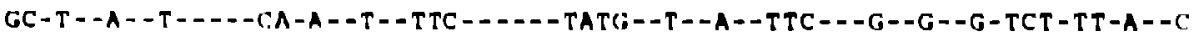

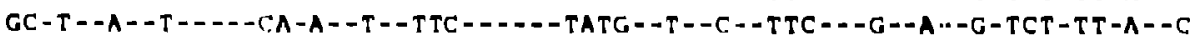
GC-T--A--T-D--CA-A--T--TTC--..-- -TATG--T- -A--TTC---G--G--G-TCT-TT-A--C

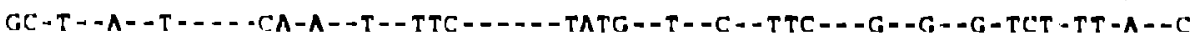

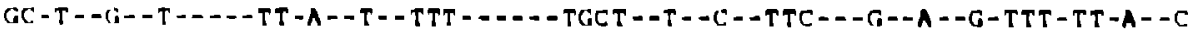

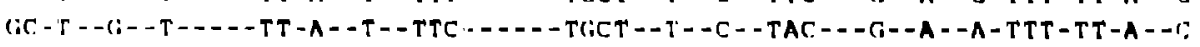

547

631

601

604

613

631

598

604

631

655

658

649

649

652

$66 \%$

616

701

671

674

683

701

668

674

701

725

728

719

719

722

737

684

771

741

744

753

771

728

744

771

725

IDA

789

1119

$72 \%$

ค01 


\section{HIV2/SIV ENV}

CONSENSUS ACAAGgATGATGGAACgCAaACtTCEACE TGGE TTGGCTTLAATGG JACtAGaGCAGA AAALAGaACaT HIV2ROD HIV2NIHZ HIV2ISY HIV2sT HIV2BEN HIV2DI94 HIV2GHI HIV2D205 SIVMR251 SIVMM1 42 SIVIO1239 SIVMNE SIUSMMH4 SIVSMAPBJ

CONSENSUS HIVZROD HIV2NIHZ HIVZISY HIV2ST HIV2BEN HIV2D2 94 HIV2GH 1 HIV2D205 SIVMM2 51 SIVHMI 42 SI VMH2 39 SIVANE SIVSHMHA SIVSMAPBJ

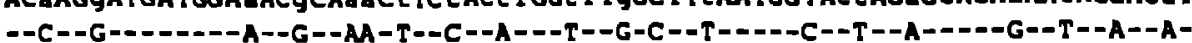

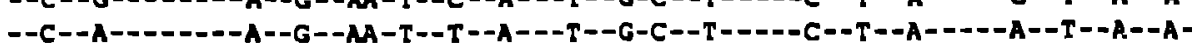
$--A--A-------A--G--A C-T--T--T---C--G-C--T---D-C--T--G---D-A--T--A--A-$

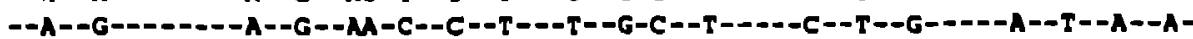

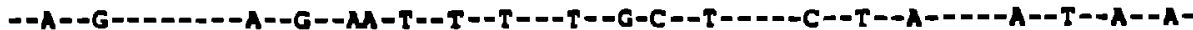

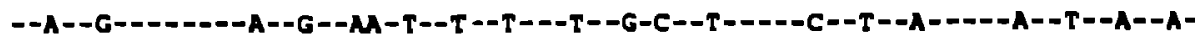

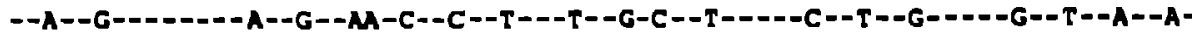
$--A--A-------A--A--G T-C--T--A---T--G-C--C-----T--A--G=----G--C--G--A-$

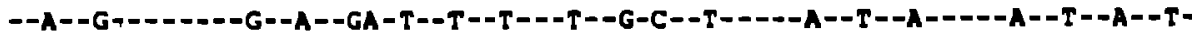

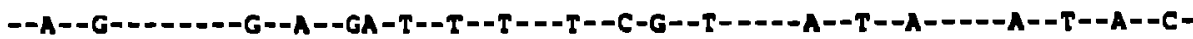

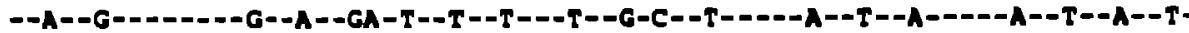

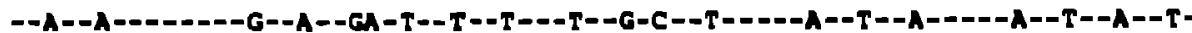
$--A--A-=-----G=-G--M-C--T--T---T--G-C--C----T--T--G---D-A--T--A--A-$

CONSENSUS HIVZROD HIV2NIHZ I:IVIISY iIVZST HIVZBEN HIV2DI 94 HIVZGH HIV 20205 SIVAm2 5 ] SI VMMI 42 S IVMM2 J9 SIVMNE SIVSMHHA SIYSMMPDJ

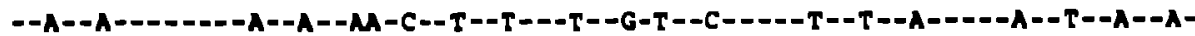

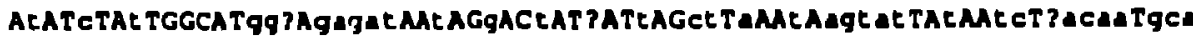
-T--C--T-----GGC-GAGAT--T--A--T--C--C--CT-A--C-AATAT--T--TC-CAGTT-GCA $-T--C--T-=--G G T-M A G A T-C--A--T--T--C-C T-A--T-A C T T T--T--T C-C A C T A-G C A$ $-T--C--T-----G G T-G G C A T--C--A--T--T--C--C T-A--C-N A T A T--T--T C-C A C C A-A C T$ -T--C--T---1--GGT-GGGAT--T--A--T--C--T--CT-A--C-AGTTT--T--TC-CACCG-ACA $-T--C--T-----G G C-G A G A T--T--G--T--C--T--C T-A--C-A G T A T--T--T C-C A C A A-G C G$ -T--C--T-----GGT-AGGAT--T--G--T--C--T-OCT-A--C-AGTAT--T-AT-CACAA-GCA -T--C--T-----GCC-GACAT--T--G--T--C--C--CT-A--C-AGTAT--T--CC-TTCTA-ACA

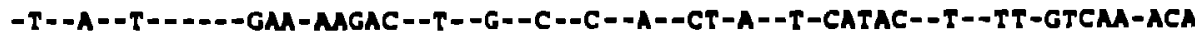
$-T--T--C-D--G G T-G G G A T--T--G--T--A--T--T T-A--T-A G T A T-T-T C-M A C A A-G M$

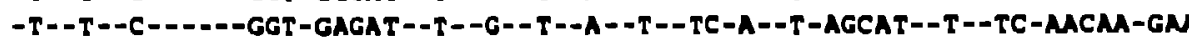
-T--T--C-----GGT-GGGAT--T--G--T--A--T-OTT-A--T-AGTAT--T--TC-AACAA-GAA -T--T--C-----AGC-AAGAT--T--G- -T--A--T--TT-G--T-AGTAT--T--TC-AACAA-GAA -C--T--T-----GGC-MAGT--T--A--C--A--T--CT-G--T-AGTAT--T-OTC-AACAA-GAG

$-C--T--T---D-G G C-G M G C--T--A--C--A--T--C T-A--T-A G T A T--T--T C-N A C M-G A G$

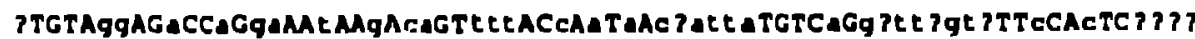
T----AG--G--A-GG--T--G-TA--GMA--A-A-A-TGCTTA----A-GACATGTG--T--C--CCAC T----AG--G--G-GA--T--G-CA--GTT--C-A-A-CATTTA----A-GGTTTAAG--T--C--C... T-.--GG--A--A-M--T--A-CA--TGT--C-A-A-CACTCA---A-GCCGCAGA--T--C--C . . T----AG--G--A-GA--C--G-CA--TGT--C-A-A-CACTCA----A-GGTTAGTG--T--C--C... T----AG--A--A-GA--T--G-CA--TTT--C-A-A-CACTTA----A-GATTAGTG--T--C--T. . T----AG--A--A-GA--1,-G-CA- - TIT--C-A-A-CACTTA----A-GGCGAAGG--T--C--T . . T----AG--A--A-GA--C--G-CA--TGT--C-A-A-CACTCA----A-GGTTAGTG--T--C--C'. . C----AG--G--A-GA--C--G-CG--TGT--C-A-A-GAACCG----A-GACTACTT--C--T--A , . A----GA--A--A-GA--T--C;-CA--TTT--C-G-C-CCATTA--.-T-GATTGGTT--C--C--A... A----GA--A--A-GA--T--C;-CA--TTT--C-G-C-CCATTA----T-CATTGGTT--C--C--A. . A----GA--A--A-GA--T--G-CA--TTT--C-G-C-CCATTA----T-GATTGGTT--C--C--A... A----CAA--A--A-CiA--T--G-CA--TTT--C-G-C-CCATCA--.-T-GATTrGGTT--C--C--A... A----GA--A--A-AA--T--G-CA--TTT--C-G-C-CCATTA-.--A-GGTTGGTE--C,--T--G... 
CONSENSUS KIV2ROD HIV2NIHZ HTV2ISY HIV2ST HIV2BEN HIV2D1 94 HIV2GH1 HIV2D205 SIVRa1251 SIVRa1 42 SIVAM2 39 SIVHNE SIVSMMHA SIVSHMPBJ

CONSENSUS HIV2ROD HIV2NI H2 HIV2ISY HIV2ST HIV2BEN HIV2DI 94 HIV2GHI HIV2D205 SIVMM23I 5 IVHMI 42 SIVMM2 39 SIVMNE SIVSMMHA SIVSMMPBJ

CONSENSUS HIV2ROD HIV2NIH2 HIV2ISY HIV2sT HIV2UEN HIV2DI 94 HIV2GHI IITV20205 SI VMM 251 SIVAMI 42 S I VMM2 39 51 VMNE S I VSMMHA SI VSMMPBJ

CONSENSUS IIIVZRON HIV2NIHZ HIVZISY IIIVZST III VZUF. IIIVZ0I 94 III varill

:I I VMM 51 : | VMMI 12 :I VMM I" :I VMNI: :I I V:AMMIIA 'i| V:IAMAMIIII

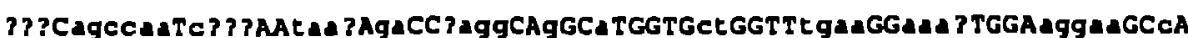
TAC-AGCCGA-C. . . - -TAMA-GA--CAGA--A--A-----CT----CAAA--CAAA----AAGAC--C ...-AGCCAG-CATC--TNA-AA--CAGG--A--A--.--TT----CGAA--CCAA----AGGAA--C... -AGMAGA-CATC--TAAA-MA--CAGG--A--A-----CC----CAMA--CGAG----GGGAA--C... -AGCCMA-C... .--TAGA-GA--CAGG--A--A-----CT----CAM--CGAG----AGGAA--C. . . -AGCCAA-C....--CACA-GG--TAGG--G--A-----CC----TGGA--CAGA----GGGM--C... -GGCCAG-CTAC--CAAA-AA--TGGG--G--A-----TT----TCMA--CMAC----TAGAA--C. . . -AGCCAA-C. . . - -TACA-GA--TAGG--A--A-----CT----CAMA--CAAG----GGGAA--C... -AGCCTA-C ... --TAAG-GA--CAGA--A--T-_-_-CT-...- VG--AAAC----CAGAA--C...-AACCM-C....--TGAT-GG--MAG--G--A-_---TT-_.-TGGA--AAAA----AGGAT--A...-AACCAG-C... .--TGAG-GG--MAG--G--A-----TA----TGGA--MAT----AGGAG--A...-AACCA-C... --TGAT-GG--AMAG--G--A----TT---TGGA--MMA----AGGAT--A... -AACCAA-C... - -TGAT-GG--MMA--G--A-----TA----TGMA--MMT----AGGAG--A... -AGCCCA-A ... --TGAG-GA--MMA--G--C-----CT----TGAA--MAGC----AAAAG--C... -AACCCA-A. .. --TGAG-GA--AAMA--G--C-...-CT--.-TGGA--AGAA----AAAAG--C-

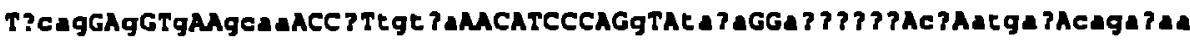
-GCAG--G--G--GGA---C-TGCAA----------G--TAGA--A, . . . . - -CC-ATGAC-CMAGGNA -GCAG--G--G--GGAG---C-TGCGA----------A--TAAA--G. . .AAC-GG-GCCGC-CAGAGAA -GCAG--G--G--ACAA---C-TGTAA----------G--TAAA--A. . . . . -CC-ATGAC-CAAATAA -GAAG--G- -G--GCTA---C-TGCAA---------G--TAAA--A. . . . . -CC-ACGAC-CAGAAA -GCAG--G--G--GCAA---C-TGTAC---------A--CAMA--A. . . . . -GCGG--G--G--GCAA---C-TGCAA----------G--CGGA--A. . . . . -ГA-ATGAT-CAGGAMA -GCAG--G--A--GCMA---C-TATAA--_---2--G--TMA--A. . . . . -CC-ACGAC-CAMAGA -AMA--G--G--AMGG---A-CATM-----_---G--TMA--AGGTGCA-MA-ATATC-CALGCGT -AAAA--G--G--ACAG---A-TGTCA--------G--TACT--A. ..... -СT-ACAAT-CTGATAA -MAA--G--G--GCAG---A-TGTCA---------G--TACT--A. . . . . -CT-ACMAT-GTGATAA -AAAA--G--G--GCAG---A-TGTCA--.-------G--TACT--A. ..... -CT-ACAAT-CTGATAA

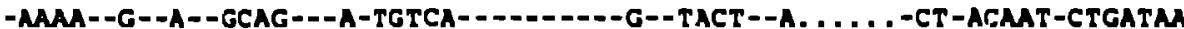

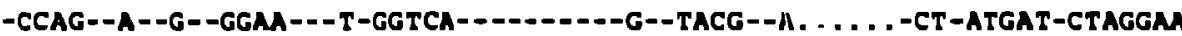
-CCAG--A--G--GGMA-.-T-GLTCA----------G--TACG--A. ..... -CT-ATMAG-CTGMACA

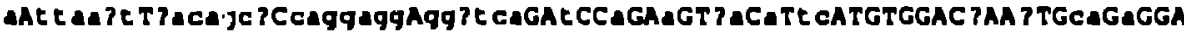

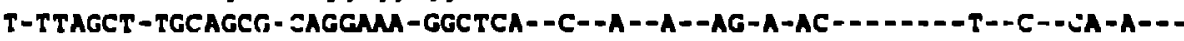
T-TTMAT-TAMGCA-CAGGGAG-GGCTCA--C--A--A--AA-A-AC--------T--C--CA-A--A-TTAACT - TACAGCA-CAGMAA-GACTCA--C--A--A--AL-A-AT--.-.---T--C--CA-A--A-TTCGTT-TATAGCG-CAGGAGA-CGCTCA--C--A--A--GG-A-AC--------T--C--CA-A--A-TTAACT - TACGAAA-CGGGAGC-GGCTCA--C--G--A--GG-A-TT---------T--C--CA-A--A-TTAACT - TACGAAG-CAGGAAT-GGT'CA--C--A--A--GA-A-AC--------T--C--CA-A--T-TTAACT - TACAAAA-CAGGAMG-GGCTCA--C --A--A--(IG-A-AC--------T--C--CA-A--A-AGTTAG-ATCAGAA-ATGGMAA-GGTTCA--T-

A-TCAATT - MACGGCT-CTGGACG-GGA. . . --T --G --A--TA-C-TC--------A--T--CA-A--A-TCAATT-GACGGCT-CTAGAGG-GGA. . . --T-T-G--A--TA-C-TC--1--.--A--T--CA-A-A-TCAATT -GACCGCT -CTGCAAGG -GGA. . . - - T--G--A--TA-C-TC--------A--T--CA-A--A-TCANTT-GACGGC'-CTCCIAGG-GGA. .. --T--G--A--TA-C-TC--------A--T--CA-A-A-TTAATC-AACAGCT .CAGICAGG-GGA. . . --T--A--A--CA-T-TT--.----A--T--TC-A-D A-TTMAGC-MACAGCT-CAGGAGG-GGA. . . --T--A--A--TA-T-TC--..---A--T--TC-A--

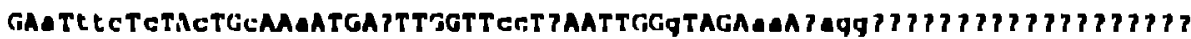

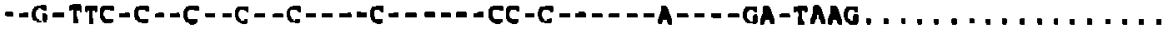

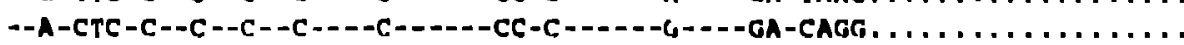

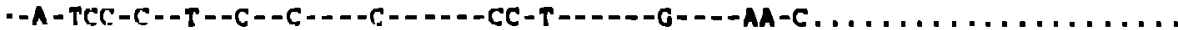

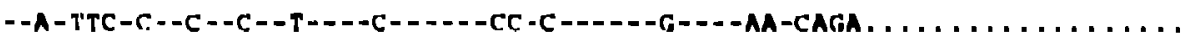

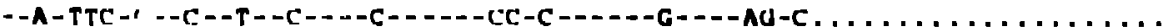

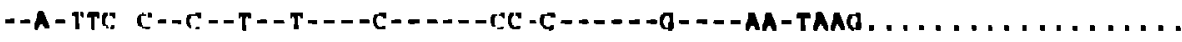

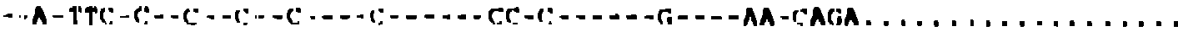

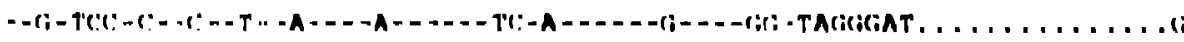
-

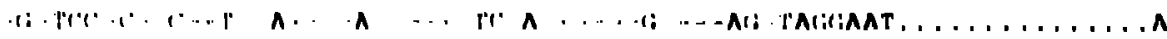

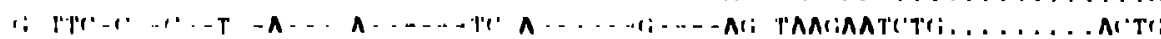

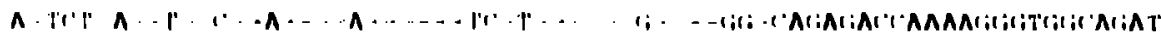
A 


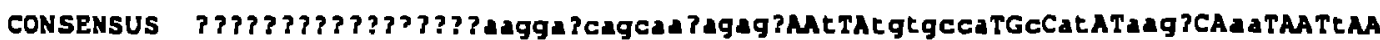
HIV2ROD HIV 2NIHZ HIV2 ISY HIV2S? HIV2BEN HIV2D194 HIV2GH1 SIVHM251 SIVMM1 42 SIVMY239 SIVRTE SIVSMMHA SIVSMAPBJ . ... . . . . . . ACGGGTCAGAMACAGCGC-- :--TGCACCG--C-GT--AMGG--AA----T-. . . . . . . . . . . AAGACGGGTCAACAGCAT--C--TGTGCCG--C-AT--AGAG--AA----T-

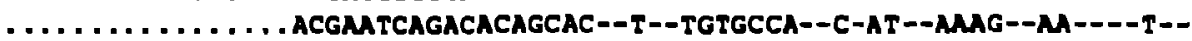
... . . . . . . . . . MAGACCAMCACGGCGC--C--T . . . . --C-AT--NMG--GA--- -T. . . . . . . . ACGAACCAMCACACCGC--C-TGCGCCA--C-AT--AAGG--GA---T-. . . . . . . . . . . . CCGM TCAGACACAGCAC-T-T-TGCGCCG--C-AT-AAGG--AA--- -T- TACTACCCAGAGGCCAMGGAACGCATAGMGG--T--CGTGCCG--T-AT--TAGA--AA----C-TACTACCCAGAGCCAMGGACGGCATAMAGG--T--CGTACCA- -T-AT--TAGA--AA----C-САGCTAACСAMAGCAMGGACACCATMMGG--T--CGTGCCA--T-AT--TAGA--AA----C-GMCTACCCAGMACCACAGGACGGCA TAMAGG--T--CGTGCCA--T-AT--TAGA--AA-- - -C--

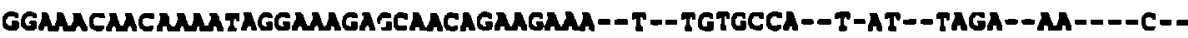
GGACAAGTCMMACCAGMAGAGCGACNMGGAGA--T--TGTGCCA--T-AT--TAGA--GA--_-C--

CONSENSUS HIV2ROD

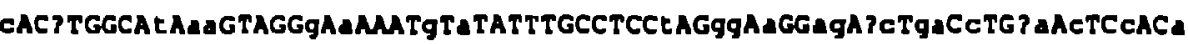
HIV2NIHZ

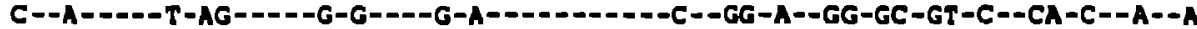

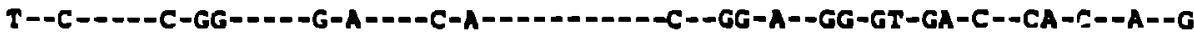

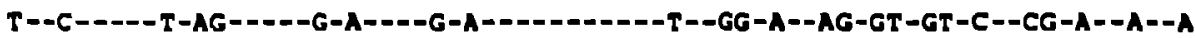

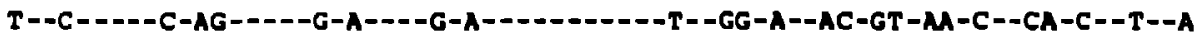
HIV2ISY HIV $25 T$ HIVZBEN HIV2D194 HIV 2 GH1 SIVHH2S1 SIVMM1 42 SIVMM239 SIVMNE SIVSMMHA SIVSIMPBJ

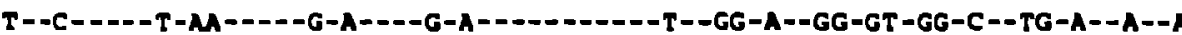

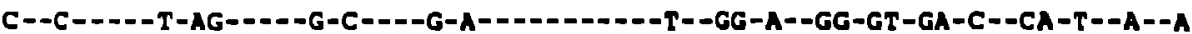

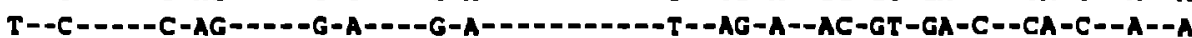

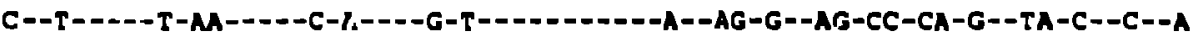
C--T--D-T-MA-----C-A----G-T----------A--AG-G--AG-CC-CA-G-TA-C--C--A

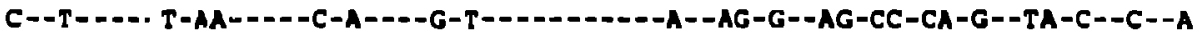

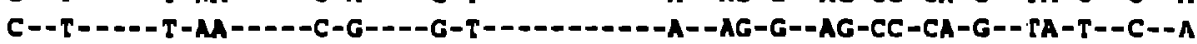

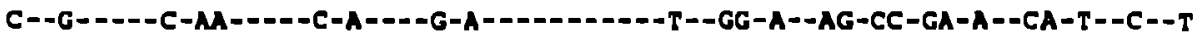

CONSENSUS HIV2ROD HIV2NIHZ HIV 2 ISY HIV2sT HIV 2BEN HIV 20194 HIV 2GHI SIVMH25I SIVHHI 42 SIVMH23s SIVHNE SIVSMMH4 SIVSAMPBJ

CONBENSUS IIIV2ROD HIV 2 NIII? HIVZISY HIV 25T HIV 2BEN IIVZDI94 HIV2GIII SIVHM2SI SIVMMI 42 SIVMH/ 19 SIVMNE: GIVIMHIIA IIVTMMI'H.J

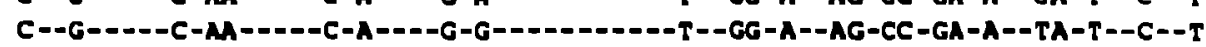

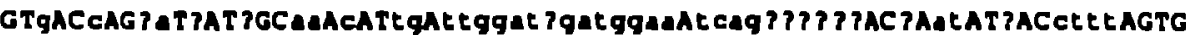
--A--C--CA-A--T--TA-C--TG-CTGGCNMMCMATA-TCAG . . . . . -A-AC--T--CTTT--. --G--C--CA-A--T--CA-C--TG-T. . . . . GCGGGAG-TCAR . . . . . --A-AT--T--CTTT-..--G--C--TA-C--T--TA-C--TG-T. . . GTTGATGGAG-TMACGG. . .--A-AT--T--CTTT-----G--C--CA-A--T--TA-C--TG-C. . , GGAGGAGAGA-CCAG . . . . .-A-AT--T--CTTT----A--C--CA-A--T--TA-C--TG-C. . . ATAGATAMA-TCGGACTCAT--C-AC--T--CTTT---- -A--C--CA-A--T--TA-C--TG-C. . TCAGATGGM-TCAG. . . . .--C-AC--T--CTTT-----G--C--CA-A--T--TA-C--TG-C . . GTAMTAGTA-CCAG . . . . --A-AT--T--CTTT-----G--C--TC-C--A--AA-C--AG-TTGGACTGATGGM-CCAA . , , . --T-GT--C--CATG----G--C--TC-C--A--AA-C--NA-TTGGACTGATGGM-CCAA , . . . - -T-GT--C- - CATG--.--G--C--TC-C--A--AA-C--AG-TTGGATTGATGGM-CCAA. . . . .--T-AT--C--CATG-----G--C--TC-C--A--AA-C--AG-TTGGATTGATGGAA-CCAA . . . . .-T-AT--C--CATG-.---A--T--TC-C--A--AG-G--AG-TTGGATCNATAGCA-TGAG. . . . . --C-AT--C--CATG--. --A--T--CC-C--A--AG-A--AG-TTGGATCAATGGCA-TGAG . . . . .-C-AT--C--CA TG--.-

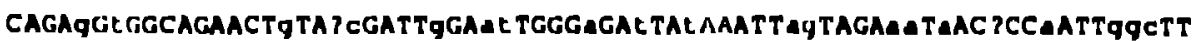

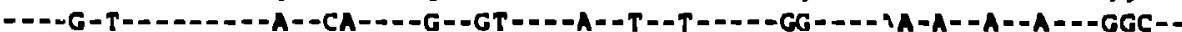

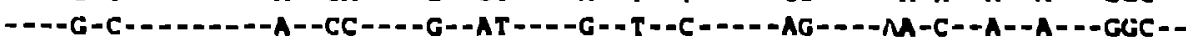

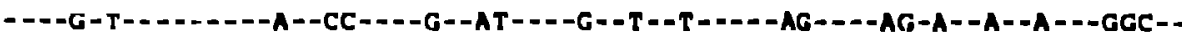

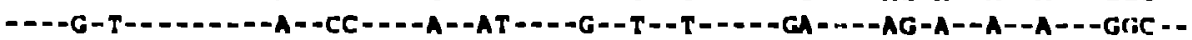

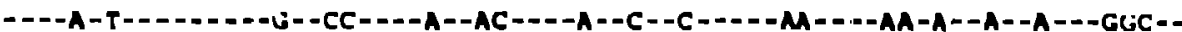

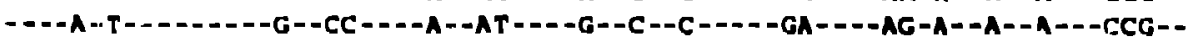

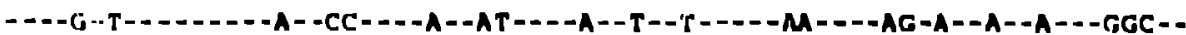

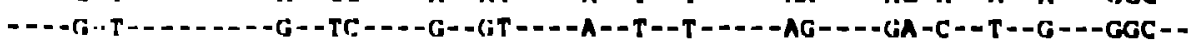

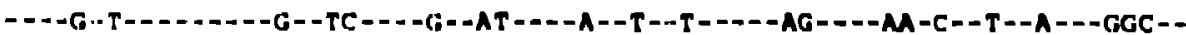
- - - -

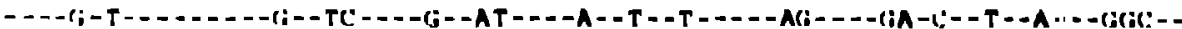

- - - - - $;$ - T- - - - - - - -

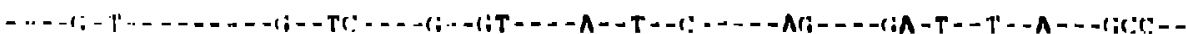

1226

1347

1326

1323

1332

1341

1320

1323

1392

1305

1386

1392

1404

1416

1284 1411

1384

1387

1393

1408

1301

1384

1456

1459

1450

1456

1468

1480

1332

1481

1454

1457

1463

$14 \%$

1451

1454

1526

1579

1520

1376

13 11

$1,1 \cdot 0$ 
CONSENSUS HIV $\angle R O D$ HIV2NIH2 HIV2ISY HIVIS: HI V2BEN HIV2D194 HIV2GHI SIVMM251 SIVMM1 42 SIVMA2 39 SIVINE SIVSMMHA SIVSAMPBJ

CONSENSUS HIVZROD HIV2N IHZ HIV2ISY HIV25T HIV2BEN HIV2D1 94 HIV2GHI SIVMM251 SIVMA1 12 SIVTM2 39 SIVMNE SIVSMMHA SIVSMMPBJ

CONSENSUS HIV2ROD HIV2NIHZ HIV2ISY HIV2ST HIV2BEN HIV2D194 HIV2GHI SIVMM25! SIVMm1 12 SIVMM2] 9 SIVMNE. SIVSMMHA SIVSMMPUS

CONGENSUS HIVZROD IIIVZHLHZ HIV2ISY HIV2ST HIVZBEN HIV2DI 94 IITV2GIII SIVMm251 SiVMK1 4) SIVMM2 I9

BIVMNF SIVGMMHA :IIVIMMMIPII.I

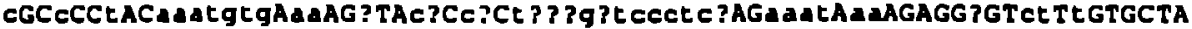
C--A--T--AAAAGAA-AA--A--CT-CT-T. . . GCTCACGGG--ACAT-CA-----T--GT-C-----C--A--T--ATCAGTA-AG--A--CT-CT-T. . . GCTCACCAG--ACAT-CA-----T--GT-T-_-..C--C--T--AGCAGM-P --A--CT-CT-T. . . GCTCCAGGG--ACAT-AG-----T--GC-T---.-. T--A--T--ACCAGTA-AA--A--CT-CT-T. . . GCTCCAGTG--GAT-MA-----T--AT-C-----C--A--T--AGATCAG-GA--G--CT-CT-A. . . ACTCCAGTG--GMAC-AA-----T--GT-C-1-_-C--A--T--AAAAGAG-AA--A--TT-CT-G. . . GCTCCAGTG--GAAC-AA-----T--GT-C-----C--A--T--GAGAGAG-AA--A--TT-CT-G. . . GCTCCAGTG--GAAC-MA-----T--GT-T--1--G--C--C--AGATGTG-AG--G--CA-TA-TGGTGGCACCTCA--AMAT-MA-----G--CT-T-----G--C--C--AAATGTG-AG--G--CA-TA-TGGTGGCACCTCA--AATT-MA-----G--CT-T-----G--C--C--AGATGTG-AG--G--CA-TA-TGGTGGCACCTCA--NMT-MA-----G--CT-T-----G--C--C--MATGTG-AG--G--CA-TA-TGGTGGCACCTCA--AMT-MA-_---G--CT-T--_-_G--C--C--MAGTGTA-GA--G--CA-CA-MCTGGTGCCTCA--MAT-AG-----G--CT-T-----C--C--C--AAGTGTA-AA--G--CA-CA-AACTGGTGCCTCA--AAMT-MA-----G--CT-T------

GGGTTCE TgGGTTT TCTCgCgACaGCAGGE ECTGCAATGgGCGCggCGTCCETGACgCTGECgGCLCAGT

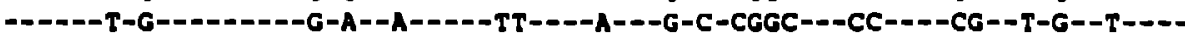

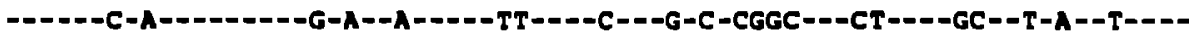

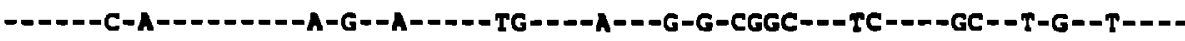

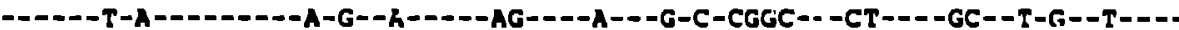
-----T-G--------G-G--A-----TT----A---G-C-CGCG---CC----GC--T-A--C----

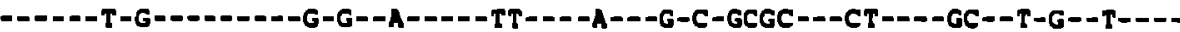

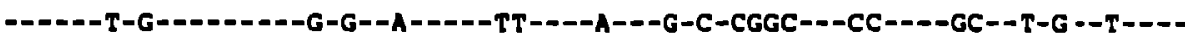

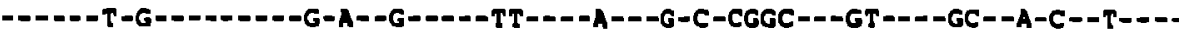

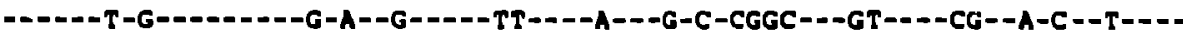

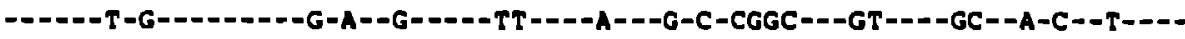

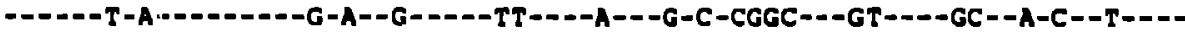

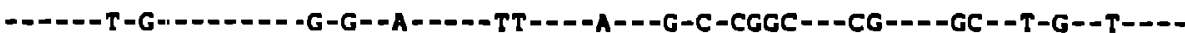

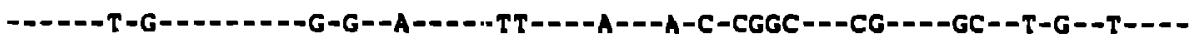

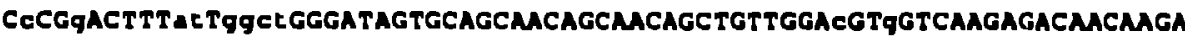

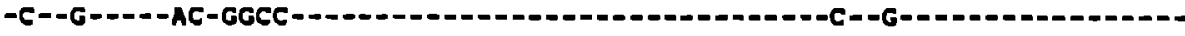

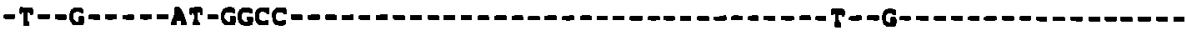

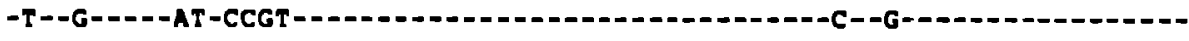

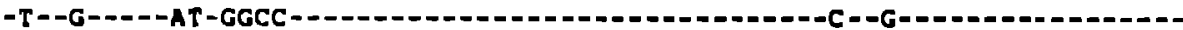

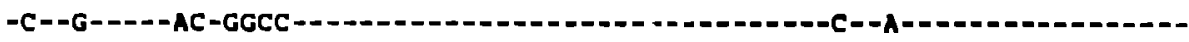

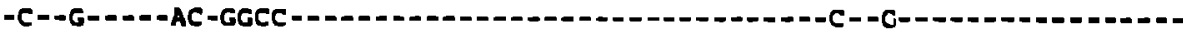

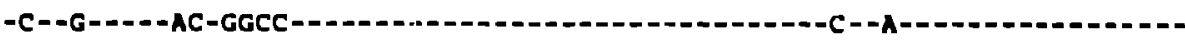

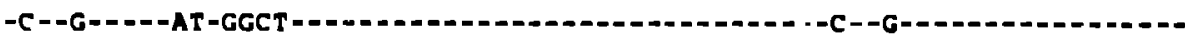

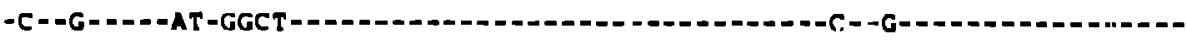

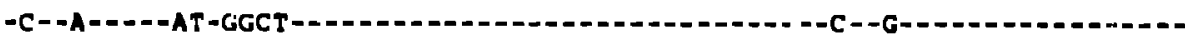

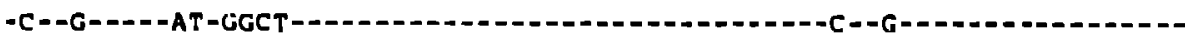
-C--G-1.-GT -GGC'1 -

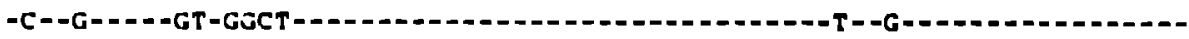

1553

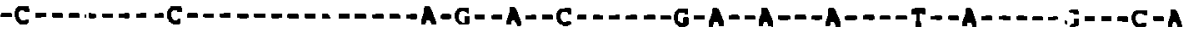

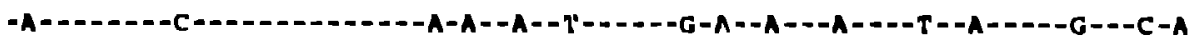

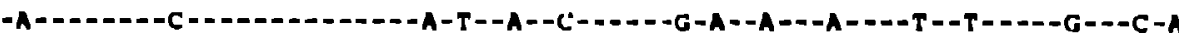

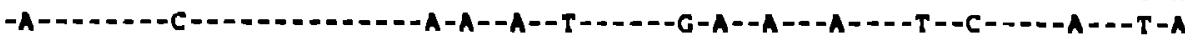

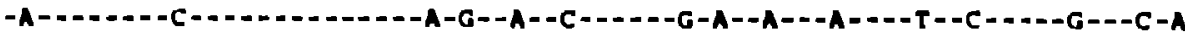

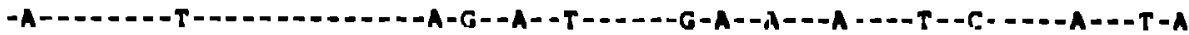

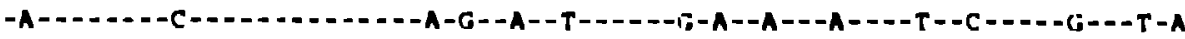

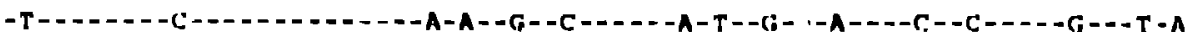

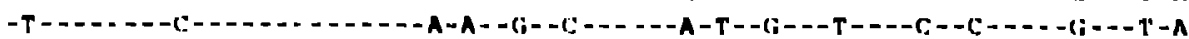

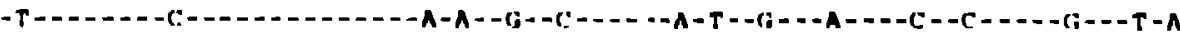

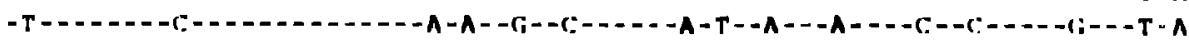

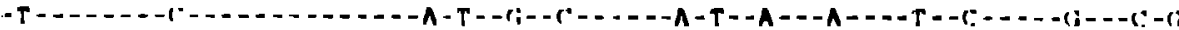




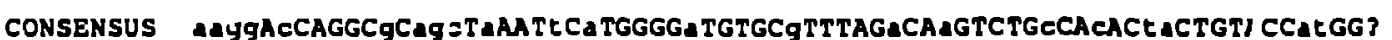
HIV2ROD HIV2NIH2 HIV2I5Y HIV2ST HIV2BEN HIV2D194 HIV2GHI SIVHM251 SIVHMI 42 SIVIM239 SIVHNE SIVSHAH4 SIVSMMPBJ

CCNSENSUS HIV2ROD HIV2NIHZ HIV2ISY HIV2ST HIV2BEN HIV2D1 94 HIV2GH1 SIVMM251 SIVIRII 12 SIVRa1239 SIVRNE SIVSMAH4 SIVSMAPBJ

CONSENSUS HIV2ROD HIV2NI HZ HIV2ISY HIV2ST HIV2BEN HIV2D1 94 HIV2CHI 5 IVHA2 51 SIVHMI 42 SIVMM2 39 SIVMAE SIVSMMHA SIVSMAPBJ CONSENSUS HIV2ROD HIVZNI HZ HIVIISY HIV2ST HIVZBEN HIVZDI 94 HIV2GH SIVMA25I SIVMAI 12 SI VMM2 39 SIVMNR GIVSMmII4 GIVIMMPB.J

CAGG-C-----G-GGC-A---T-A-----A-----G-----A--A-----C--C--TA-------AT--G

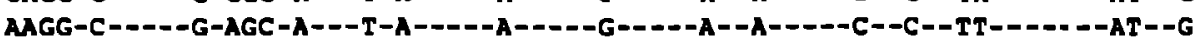
GCAG-C-----G-GAC-A---T-A-----A-----G-----A--A-----C--C--TA-------AT--G

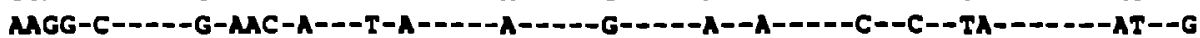

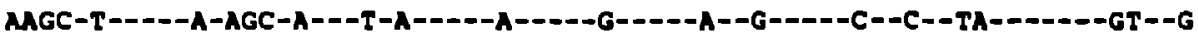

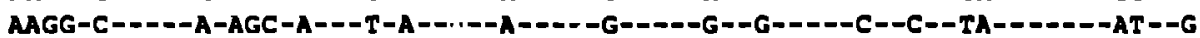

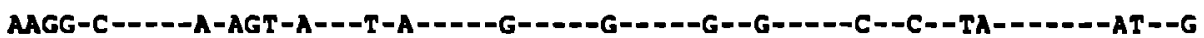

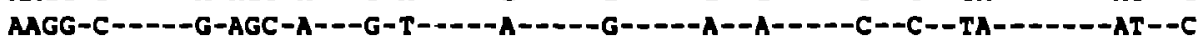

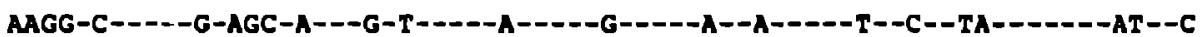

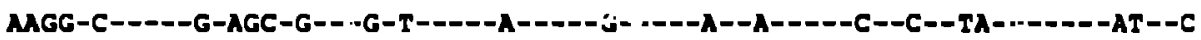

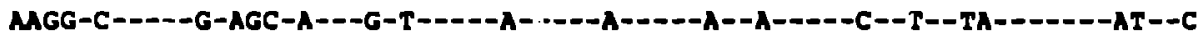

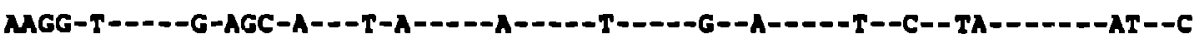

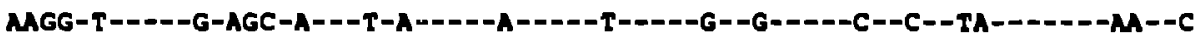

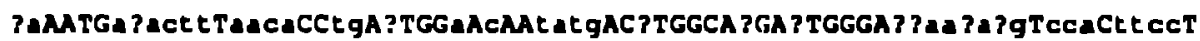
TT----ATTCCT-AGCA--TG-C---G-C--TATG--G-----G--A---.-AMACMAG-CCG-TACCTA----ATACCT-GACA--TG-T---A-C--TATG--G-----G--A-----ACMAMAG-CCG-TACCTA----ACACCT-ACA--TG-G---A-C--CATG--A-----A--A-----ACACMAA-CCG-TTCCTA----ACACCT-AACG--TG-T---A-C--CATG--A-----G--A-----GCMACGA-CCG-MACCTA----ACTCTT-ATCG--TG-C---A-A--TATG--A----G--G-----GMACMG-CCG-':ACCTA----ACTCCT-AACA--TG-C---A-C--TATG--A-----G--A-----MAACGAG-ZCA-TACCTA----AiTCTT-ATCG--TG-C---A-C--TATG--A-----G--A-----AMACMAG-CCG-TACCCA----CAAGTC-AACA--AG-C---A-C--TGAT--T--.--A-.-G----GCGAAGG-TGA-TTCTCA----CAMGTC-AACA--AG-T---A-C--TGAG--T-----A--G-----GCGGMGG-TGA- \'TCTCA----CAAGTC-AACA--AA-G---A-C--TGAG--T-----A--G-----GCGMMGG-TGA-TTCTCA----CAMATC-MCA--AA-T---A-C--TGAG--T-----A--G-- --GCGMMGG-TGA-TTCTCA----MACAT-GGTG--TA-T---A-C--TATG--T-----A--G-----MAGACAGG-TGA-TTCCCA----ACACAT-GACA-- TA-C---A-C--TATG--T-----A--G-----AMACAGG-GMA-TTCC-

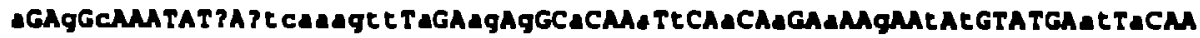
G--G-C------C-GTMMAGTT-A--AC-G--A---A-T--G--A--G--A--T-T------AC-A--G--G-C------C-GTCAMAGTC-A--AC-A--A---A-T--G--A--A--G--T-T------GC-A--. A--G-C------C-GTGAGAGTT-A--AC-G--A---A-C--G--A--A--G--T-T------GC-G--A--G-C------C-GTGAMGTT-A--AC-G--A---A-C--G--A--A--G--C-T------AC-A--A--G-C-----C-GTCMAGTT-A--AG-A--C---A-T--A--A--A--G--T-T------AT-A-DA--G-C--.---C-GTCMAAGT -A--AC-G--A---A-T--A--A--A--G--T-T--D---AC-A-DA--G-C------C-GTCMAGTT-A--AC-G--A---A-T--A--A--A--G--T-T------AT-A--G--G-A------A-CAGCCCTCF-A--AG-G--A---A-T--A--A--G--G--C-T-----AT-A-D. G--G-C-----A-CGGCCCTCC-A--AG-G--A---A-T--A--A--G--G--C-T-----AT-A--G--A-A-----A-CAGCCCTCC-A--GG-G--A---A-T--A--A--G--G--C-T-D---AT-A-D. G--G-A-----A-CGGCCCTTT-A--AG-G--A---A-T--A--A--A--G--C-T------AT-A--A--G-C---D-A-CTCAATTAT-A--AG-A--A---A-T--G--A--A--G--T-T------AT-G--A--G-C--.---A-CTCAATCAT-G--AG-A--A---A-T--G--A--A--G--T-C-----AT-G---

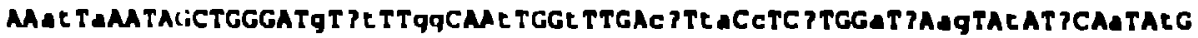
--AT-A-----------A-TT--GG - - -T---T----CT-MA-C--C---G-C-AG--T--T--A--T-

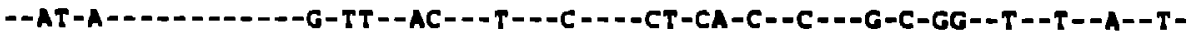

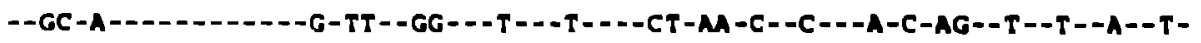

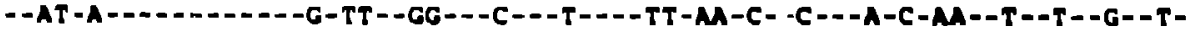

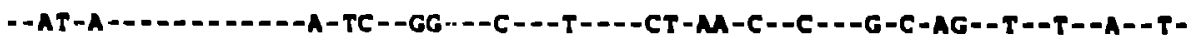

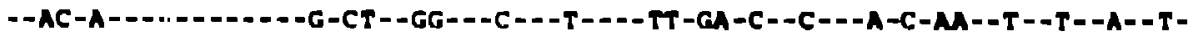

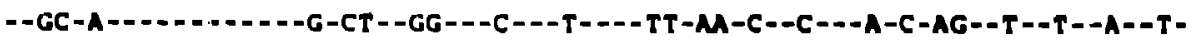

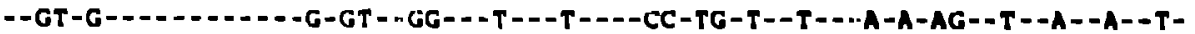

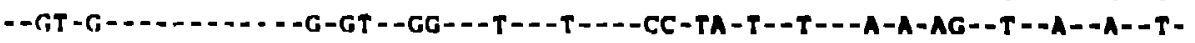

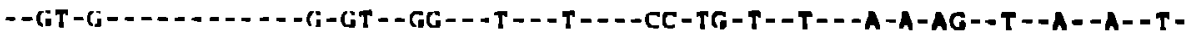

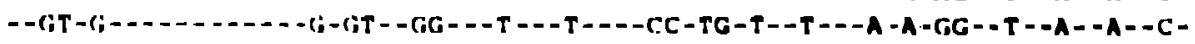

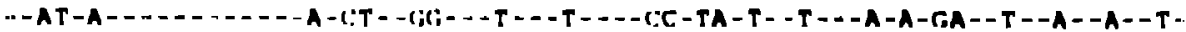
2038 2011 


\section{HIV2/SIV ENV}

CONSENSUS GagttEataTAgT?gTaGgAgTaATAgtt TaAGAaTAGLAATaTATaTAgTaCAaAGETA??TAgg?T HIV2ROD HIV2NIH2 HIV2ISY HIV2ST HIV2BEN HIV20194 HIV2GHI SIVMM251 SIVMa142 SIVMM239 SIVMNE SIVSMIH4 SIVSRMPBJ -AG-GCTTA--A-AG-A-C-G-AA--GCTT-A---A---TG--A---G--G-A--AA--T--AG--GGC-AG-TTATG--G-AG-A-G-A-AG--GCTT-A---A---TA--A---A--G-A--GA--T--AG--GAC-AG-CATGA--G-AG-A-G-A-AG--GCTC-C---A---TA--A---G--G-A--AA--C--AG--GAC-AG-TTATA--G-AG-A-G-A-AA--GTTT-A---A---TA--A---G--G-A--AA--T--AG--GAC-AG-GCATA--G-AG-G-G-A-MA--GCTT-A---A---CA--C---G--G-G--AT--T--AG--GAT-AG-TTATA--G-AG-A-G-A-AA--GGTT-A---A---CC--A---A--G-G--AT--T--AG--GAC-AG-TTATA--G-AG-A-G-G-GA--GTTT-A---A---CA--A---A--G-G--AT--T--AG--GAC-AA-TTATG--G-TG-A-G-G-AA--CTGT-A---A---TG--C---A--G-A--MA--C--GC--AGT-MA-TTATA--A-TG-A-G-G-AA--CTGT-A---A---TG--C---A--G-A--A---C--GC--GGT-AG-TTATA--G-TG-A-G-G-AA--CTGT-A---A---TG--C---A--G-A--AA--C--GL--AGT-AG-TTATA--G-TG-A-G-G-AA--CTGT-A---A---TG--C---A--G-A--M--C--GC--AGT-TG-ACTTA--G-TC-A-G-G-AA--GGGT-A---A---TA--A---G--G-G--MA--T--GC--GGT-

$$
\begin{aligned}
& \text { <- stop in some sIVs } \\
& \text { W } / / 3^{\prime} \text { aje } \\
& \text { I/3'sj }
\end{aligned}
$$

CONSENSUS HIV 2ROD HIV2NIHZ HIV2ISY HIV2ST HIV2BEN HIV2D194 HIV2GHI SIVMM251 SIVMA1 42 SIVMM239 SIVMNE SIVSRAH4 SIVSHAPBJ

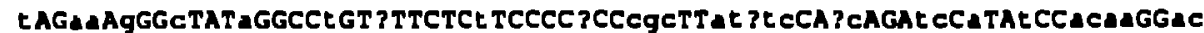

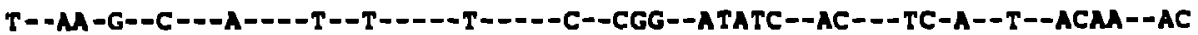
T--AA-G--C---A----T--T--2--T-1--C--CGG--ACATC--AC--TC-A--T--ACAA--AC T--AA-G--C---A----T--T-_--T---D-C--CGG--AmATT--AC---TC-A--T--ACAA--AC

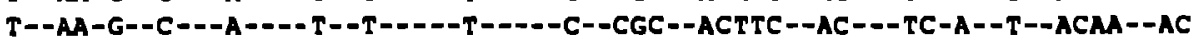

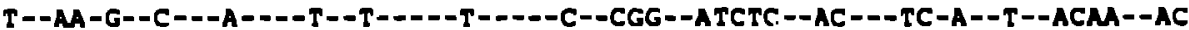
T--AA-G--C---A----T--T--D-C--- -C-CGG--ATCTC--AC--TC-A--T--ACAC--AC T--A-G--C---A----T--T---- T- - - T--CGG--ATCTC--AC---TC-A--T--ACAC--AC A--GC-G--G---A----A--G-----T----A--CTC--ATTTC--GT---CT-A--C--AACA--AC A--AC-G--G---A----A--G-----T----A--CTC--ATTTC--GT--CC-A--C--ACA--AT A--GC-G--G---A----A--G-----T-..--A--CTC--ATTTC--GC---CC-A--T--AACA--AC A--GC-A--G---A----A--G-----T----A--TTC--ATTTC--GT---CC-A--T--GACA--AC A--AC-G--T-D-A----A--G-----T--D-T--CGC--ATGTT--GC---TC-C--T--ACAA--GC

CONSENSUS HIVZROD HIV 2NIHZ HIVZISY HIV 25T HIV 2BEN HIV2DI94 HIV2GHI SIVMM251 SIVMMI 42 STVMM2 39 SIVMNE SIVSMMHA sivsmapos c- early stop In GHI

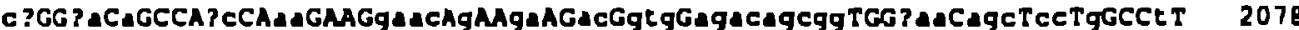
CG--GA-A----GC--AC----AAAC-G--GA--AF-GTG-AAGCAACGG---AGA-AGA-AC-G---C- 2248 CA--AA-A----GC--GA----NAC-G--GA--AC-TTG-AAGCAACGG--AGA-AGA-CT-G--T-T 2221 TG--AA-A----GA--GA----AAAC-G--GA--AC-TTG-GAACGACGT--.AAG-AGA-CC-G--T- 2224 CG--AA-A----GC--GA----AAC-G--GA--AC-TTG-AAACACCGT--AGA-AAT-GG-G---C- 2230 CG--GA-A----GC--AC---GAAC-G--GA--AC-TCG-ACGCGACAG--TTA-GAC-TG-G---T- 2245 AG--GA-A-- -GC--AC----AAC-G--GA--AC-CCG-AGACGACAG--TTT-GGC-TG-G---T- 2218 CG--GA-A----GC--AC----GMAC-G--GA--AC-ACA-AGACGACGA--TTA-GAC-TG**--T- 2221 CC--CA-T----AC--GA----GCAA-G--GG--AC-GTG-AGAAGGCGG--CAA-AGC-CC-G---T- 2296 CC--CT-T----AC--AA----GCAA-A--GG--AC-GTG-AGGCAGCGG---CAA-AGC-CC-G---T- 2299 CC--CA-T- - -AC - -GA- - - -GCAA-G- -AG-.AC-GTG-ALAAGGCGG--CAA-AGC-CE-G--T- 2290 LA--CA-T----AC - -AA----GAAC-G--GG--AC-CTG-AGGCAGCGG---CAA-AGC-CC-G---T- 2296 GA--AA-C----AC--AA---- GAGA-G--GG--AC-GTG-AGACAG.AGG--CAG-AGA-CT-G---T- 2308 CA--AF-T- - -AC - -AA- - -GAGA-G - -GG--AC-GTG-AGGCAGAGG---CAA-AGA-CT-G--T- 2320 
$<-$ tat

CONSENSUS GGC?gaTAgaAAtattCAtTTCCTGATCCgCCa?cTgataCGCCTCTTGAC?GgCTaTaCA?CA?CTg HIV 2ROD HIV 2 RIHZ FIV2ISY HIV 2ST HIV2BEN HIV2DI $\$ 4$ HIVZGHI

SIVMA251 SIVMM1 42 SIVMA239 SIVANE SIVSMAH4 SIVSMPBJ - -CGA--GCA--TATA--T---_----G--AGC-GATT---CGA--GCA--TATA--T--_-----G--TGC-GATT---------CCG-GC-A-A--AC-TC-G

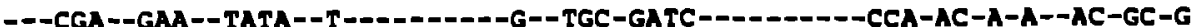
---CGA--AGA--TATA--T--_-_--G--AGC-GATT--_-_---ACA-AC-A-A--AC-TC-G ---CAA--AAC-TGTG--G--_-_--A--TAC-GACT---0----TCG-GC-A-A--AC-IC-G ---CAC--AAC--CATA--A---------A--TAC-GACT---------CCG-GC-A-A--AC-GC-G --CGA--AAC- -TATA--T---_----A--TAC-GACT--_--1---CCG-GC-A-A--AG-TC-G

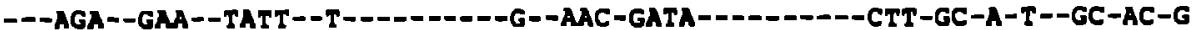
--AGA--GAA--TATT--T--------G--AAC-GATA--------CTT-GC-A-T--GC-AC-G $--A G A--G M--T A T T--T--0----G--A C-G A T A-----0--C T T-G C-A-T--G C-A C-G$ $--A G A--G A--T A T T--T-------G--M C-A A T A--------C T T-G T-A-T--G C-A C-G$ ---AGA--GAA--TATT--T-------G--AGT-GATA---------CTT-GC-A-T--GC-GC-G

CONSENSUS HIV2ROD HIVZNIH2 HIV2ISY HIV2ST HIV 2BEN HIV2D194 HIV2GH1

SIVMH2SI SIVHMl 42 SIVMK2]9 SIVMNE SIVSMMH4 SIVSIMPBJ

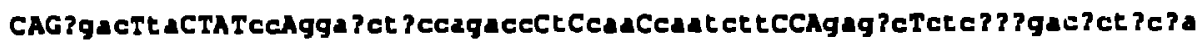
- -GGAC-TA----CC-GGAGCTTCCTGACC-T-CM-TCATCTA--GMTC-C . . . . . . . . . . ---GGAC-TA----CC-GGATCTCCCCGATC-T-CM-CNATCTY---GAGTC-CCAGNGAGCACTAACA - -GGAC-TA-_-CC-GA. . . . . . . . -T-TAC-TGATCCT--ACCAC-C. . . . . . . . ---GGAC-TA----CC-GGAGCTTCCAGACC-T-CA-TMTCTC--GAGTC-TCGGAGAGCATTGACA -.-AGAC-TA----CC-AGAACTCCCCGACC-G-CGA-TGATCTC---GAGTC-A. . . . . . . ACA ---GGGC-TA----CC-AGAACTCCCCGACC-G-CGA-TGATCTC--GAGTC-A. . . . . . . ACA

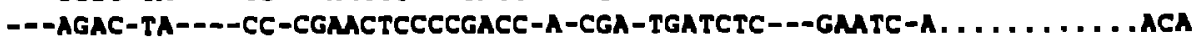
---AACC-TG----CG-GAGCATACCAGATC-T-CAA-CAATACT---GAGGC-CTCTGCGACCCTACGA ---AACC-TG----CG-GAGCATACCAGATC-T-CA-CANTAT---GAGGC-CTCTGCGACCGTACGG --MACC-TG----CG-GAGTATACCAGATC-T-CA-CMATACT--GAGGC-CTCTGCGACCCTACAG ---AAC-TG---CG-GAGCATACCAGATC-T-CAA-CAATATT---GAGAT-CTCCACGACCCTACAG ---GGAT-GG----TG-GGAGCTACCAGATC-T-CAA-CAGTGCT---GAGCC-CTCAAGACGTTGCAA --AGAT-GG----TG-GGACTGCCAAAC-T-CAG-CAGTGCT---GAGCC-CTCAAGGACGGTGCAG

$$
\text { <- rov naf } \rightarrow
$$

CONSENSUS agaatcagaGA????ctrAGacteda/cagCCtacctrCAaTATGGGtGcgagTggatCCAaGAaGCg? HIVZROD HIV2NIHZ H:V2ISY HIV23T HIV LEEN HIV2D194 HIV2GHI S IVMA251 SIVMM1 42 SIVMH239 SIVMNE SIVSMMH4 SIVSMAPBJ ..... A-A--CTGGC-G--ACTTAGAACAG--TTCT-G--A------T-CGAG-GGA---A-.-A--AT GCMTCA-A--CTGGC-G--GCTTAMGCAG--TACC-r--G------T-CGAG-GGA----A--A--rT . . . . A-A--CTGGC-G--ACTCAAGGCAG--TACC-G--G------T-CGAG-GGA----A--A--GT GCAGTCA-A--CTGGC-G--ATTTAACACAG--TACC-G--A------G-CGAG-GGA----A--A--GT GCAATCA-G--CTGGC-G--ACTTAGGCGG--CMC-G--A------T-CGAG-GGA-- --A--A--TT GCAATCA-G--CTGGC-G--ACTTAAGGCGG--TACC-G--A------T-CGAG-GGA----A--A--GT GCAATCA-G--CTGGC-G--ACTTAMGCGG--TACC-G--A------G-CGAG-GGA----A--A--AT AGGGTTC-A--AGTCC-C--GACTGMCTGA--TACC-A--A----_-T-GAGC-ATT----T--G--GG AGAATTC-A--AGTCC-C--GCTTGMCTGA--TACC-A--A------T-GAGC-ATT----A--A--GG AGGATTC-A--AGTCC-C--GACTGMATGA--TACC-A--A------T-GAGC-AIT----T--G--GG AGAATCC-A--AGTCC-C--GACTGMCTAA--TACC-A--A------T-GAGC-ACT----A--G--GG AGAGTCC-T--AGTCA-C--AATTGNATAG--TACC-A--A------T-GCGC-ATT----A--A--AG AGAGCCC-T--AGTCA-C--AGTTCAGATAG--TACC-A--G------T-GCGT-ACC----A--A--AG TC-AG-CCGCCGCG-GGGC-ACAAG---G---C--G-GGG--GT-CA-GGGCT-G--GAGGGTATTGiAA TC-MA-CCCTTGCA-GGAC-ACMG--G---C--G-AGG---GG-GA-AGACT-G--GAGGGCGCTGCA TC-AG-CCCTCGCG-GGGT-ACAAG---G---C--A-GAG---GG-GA-GAGCT-G--GGCGGCTCTGGG TC-GA-CCTTCGCG-GGGC-ACGGG-- -G---C--A-AM---CT-GA-AGGCT-C--GGCGACACTGGG TC-AA-CATTCGCG-GGAC-ACGAG---G---C--G-GGG---GT-GG-ATGGT-A--GGAGCAGCGCIi HIV2ST HIV 2BEN HIV2DI94 HIV2GHI SIVMM23I SIVMMI12. SIVMM239 SIVMAF

'iIV:iMmII4 TC-GA-CATTCGCA-GGAC-GCGAG---G---A--G-GGG---GT-GA-GGGGT-A--TGAAGCAGCFCA TC-AA-CAT TCGCA-AGAC-ACGAG---G---C--G-GAG---AT-GG-GGGGT-A--TGCAGCAGTGCA TC-AA-CCGGCTGG-GATC-GCGAC---A---C--G-G,GR---GT-GA-ACiACT-A--GGAGACTCTTAG

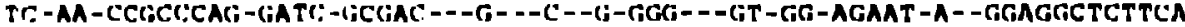

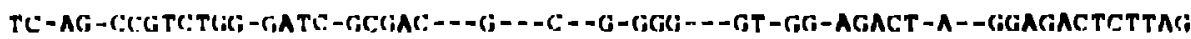

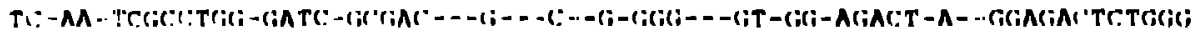

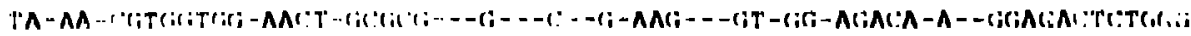


HIV-2/SIV NEF CDS

CONSENSUS ATGGGTG ???Tgg:TCCAaGAaGCggtcCaAGC??C??gAga? CTgCgagAGAgACTCTTgCgggCGC HIV2ROD -----T-CGAG-GGA----A--A--ATTC-AG-CCGCCGCG-GGGC-ACAAG---G---C--G-GGG--HIV2NIHZ -----T-CGPG-GGA----A--A--GTTC-AA-CCCTTGCA-GGAC-ACAAG---G---C--G-AGG--HIV2ISY HIV2ST HIVZBEN HIV2DI 94 HIV2GHI SIVMM25I SIVMAP 11 SIVMMI 42 SI VMM 39 SIVMNE SIVSMMH4 SIVSMMPBJ $----T-C G A G-G G A----A--A--G T T C-A G-C C C T C G C G-G G G T-A C A A G---G=-C--A-G A G--1$ - ---G-CGAG-GGA - - -A--A--GTTC-GA-CCTTCGCG-GGGC-ACGGG---G---C--A-AAA----- T-CGAG-GGA----A--A- - TTTC-AA-CA TTCGCG-GGAC-ACGAG---G---C--G-GGG--- - - T-CGAG-GGA--- -A--A--GTTC-GA-CA TTCGCA-GGAC-GCGAG---G---A--G-GGG-$----G-C G A G-G G A----A--A--A T T C-A A-C A T T C G C A-A G A C-A C G A G---G---C--G-G A G---$ $----T-G A G C-A T T----T--G--G G T C-A A-C C G G C T G G-G A T C-G C G A C---A---C--G-G G G---$ ---- T-GAGC-ATT-- - T--G--GGTC-AA-CCGACTGG-GATC-GCGAC---A---C--G-GGG------ T-GAGC-ATT----A--A--GGTC-AA-CCGCCCAG-GATC-GCGAC---G---C--G-GGG--$----T-G A G C-A T T---T--G--G G T C-A G-C C G T C T G G-G A T C-G C G A C---G--C--G-G G G---$ ----T-GAGC-ACT ---A--G--GGTC-AA-TCGCCTGG-GATC-GCGAC---G---C--G-GGG------ T-GCGC-ATT----A--A--AGTA-AA-CGTGGTGG-AACT-GCGCG---G---C--G-AAG--CONSENSUS HIV2ROD HIV2NIHZ HIV2ISY HIV2ST HIV $2 B E N$ HIV2DI 94 HIV $2 \mathrm{GHI}$ SIVMM2 5 I SIVMMP 11 SIVMMI 42 SIVMI239 SIVMNE SIVSMMH 4 SIVSMMPBJ

qtGgaGagact TaTGggagact ctg?gaagagt tGGaAGggGgATactcGCaaTcCCaagaaggATcAGg GT-CA-GGGCT-G--GAGGGTATTGGAACGAATC--G--GG-A--ACTC--GG-T--AA-AA-G--C--A GG-GA-AGACT-G--GAGGGCGCTGCAACGAATC--G--GG-G--ACTT--AG-C--AC-AA-G--C--G GG-GA-GAGCT-G--GGGGGCTCTGGGACGAATC--A--GG-G--ACTC--AG-T--AC-AA-G--C--G CT-GA-AGGCT-C--GGGGACACTGGGACAAAT'--G--GG-A--ACTT - -AG-C--AA-AA-G--C--G GT-GG-ATGGT-A--GGAAGCAGCGCGACGCATC--G--GG-A--ACTC--AG-T--AA-AA-A--C--G GT-GA-GGGGT-A--TGAAGCAGCGCAACGCATC--G--GG-A--CCTC--AG-C--AA-AA-G--C--G AT-GG-GGGGT-A--TGCAGCAGTGCAACGCGTC--G--GG-A--ACTC--AG-C--AA-AA-G--C--G GT-GA-AGACT-A--GGAGACTCTTAGGAGAGGT--A--AT-G--CCTC--AA-C--TA-GA-G--T--G GT-GG-AGACT-A--GGAGACTCTTAGGAGAGGT--A--AT-G--CCTC--AA-C--TA-GA-G--T--G GT-GG-AGAAT-A--GGAGGCTCTTCAAAGGGGT--A--AT-G--CCTC--AA-C--TA-GA-G--T--A GT-GG-AGACT-A--GGAGACTCTTAGGAGAGGT--A--AT-G--ACTC--AA-C--CA-GA-G--T--A GT-GG-AGACT-A--GGAGACTCTGGGAAGAGTT--A--AT-G--ACTC--AA-C--TA-GA-G--C--A GT-GG-AGACA-A--GGAGACTCTGGGAAGGGTT--A--AG-G--ACTC--AA-C--TA-GC-C--C--G GT-GA-AGACT-A--GGAGACTCTGGGAAGGGTT--A--GG-A--ACTC--AA-C--AA-AC-C--C--G

$$
\text { <- onv }
$$

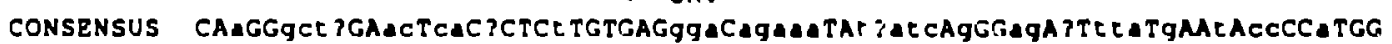
HIV2ROD HIVZNIHZ HIVZISY HIV2ST HIV2BEN HIV2O194 HIV $2 \mathrm{CH} I$ SIVMM2 51 SIVMHP ! ] SIVMH 42 SIVMH2 39 SI VMNE SIVSMMHA - -G - -AGCA- - MA-CG-C-- -C - - - -GGA-GGCAG - TCAGC-G--AG-C-TTA-S--T-CT--A----G--AGCA--AC-CG-C---C-----GGA-AGCGA--TCAGC-G--AG-T-TTG-A--T-CC--A--- -G- - AGCA- -AA - TG-C-- -C - - - -GGA-AGAGA - TCAGC-A - - AG-C - TTA-G--T-CC--A---

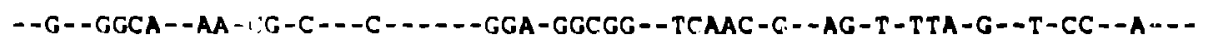
$--G--A G C A--A C-C G-C---C----$ GGA-AGCAG - - TCAGC-G--AG-G-ACA-G- - C-GC--A-- $--G--A G C A--A A-C G-C---C----G G A-A G C A G--$ TCAGC -G - -AG -G -TCA-G - - C-CC - -A--

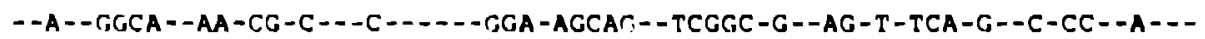
- - A--GCTT--GC-CA-G-- T--.--GGA-AGAAA--CAATC-G- -GC-G-ATA-G--T-CT--A--. $--A--G C T T--G C-C A-G---T--\infty-G G A-A G A M A-$ CAATC-G--GC-G-ATA-G--T-CT--A----A--GCTT--GC-CA-T---T-----GCC-AMAAA-- :AATC-G--AG-A-ACA-G--T-CT--A--- -A--GCTT - -GC-CA-T-- - T-----GGA-AGAAA--C.MATC-G--AC-G-ATA-G- - T-CT- -A----A--GCTC--GC-TA-T---T-----GGA-AGAAA--CAATC-G--AC-G-TTA-G--T-CT--A----A--GCTT--GC-CA-T---T'-----CCT-AGAAA- - TAGTI-G--AC-A- - TA-G--T-CC--C--SIVSMMPBJ - -A--GCTT--GC-CA-T---T--.---CCT-AGAAA--TTGTG-G--TC-A-TTA-G--T-CC--T-.. 
CONSENSUS AgaaACCCaGCagcagaaaggsaaAAa?ta?caTACAgacAaCAAAAtATgGATGATgTaGAT???ga?g HIV2ROD HIV2NIHZ HIV2ISY HIV2ST HIV2BEN HIV2DI 94 HIV2GH1 SIVMM251 SIVMMP 11 SIVMM] 42 SIVMA239 SIVMNE SIVSMMH4 SIVSMMPBJ

CONSENSUS HIVZROD HIV2NIHZ HIVZISY HIV2ST HIV2BEN HIV 2DI 94 HIV 2जH 1 S IVMM 251 SI VMMP 11 SIVMMI 42 SI J'MM2 39 SIVMNE SIVSMMH4 SIVSMMPBJ -AGGA---A--AGCAGAAAGGGAG--AAATTTG--C-GGC-A-----T--G------G-A---TCAGATG -GAAC -- -A-- AGCAGAAAGGGAG--AGAATTG--C-AAC-G-----T--G------G-A---CTAGATG -GAAC---A--AACAGAAAAGGAA--AGAATCG--C-GGC-A-----T--G---_-.G-A---TCAGATG -GAGC---A - - A . . GAAGGGGAG--AGGCTCG--C-AGC-A-----T--G------G-A---TCAGATG -GAAA---A--AACAGAAAGACAG--AGATTTG--T-GGC-G-----T--G-----G-A---TCTGATG -GAAC---A--AGCAATAGGGCAG--AAATTCA--T-AGC-G-----T--G------G-A---TCTGATG -GAAC---G--AATAGAAGGACAG--AAAGTTA--C-AGC-G-----T--G------A-A---TCTAGTG -GAAA-- -A--TGAAGAAAAAGAA--A TTAGCA--C-GAA-A-----T--G------A-A---GAGGAAG -GAAA---A-- TGAAGAAAAGGAA--ATTAGCA--C-GAA-A-----T--G-----A-A---GAGGAGG -GAAA---A--TGAAGAGAGGAAA - -ATTACCA--C-GAA-A-----T--A--1--A-A---GAGGAAG -GAAA -- -A-- TGAAGAGAGAGAA--ATTAGCA--C-GAA-A-----T--G-----A-A---GAGTAAG -AAAA---A - -TGGAGAGAGGGAA--ATTAGCA--C-GAA-A----T--A-----A-A---GAAGAAG -GGAA---A--AACAGAAAGAGCA--ATTAGGT--T-GAC-A-----C--G-----G-G---GATGAAG -GGAA-- A--AACAGAAAGAGCA--ATTAGAT--T-GAC-A-----C--G------G-G--_..... A

aTgaTgAcctagTagG ?gt ? CCaGt ? a ? cCaAgAGT ?CC ? ct aAGagcaATgAc?tacAaat TGGCAat A-GA-G-CCAAG-AA-AGTTT-T-TCACAC-AAA---A--ACTA--ACCA--G-CACAT-GAT--- -AT A-GA-G-CCAAG-AG-ATTCC-T-TCACAC-PAG---A--ATTA--ACCA--G-CATTC-AAT---- -GT A-GA-G-CCTAG-AG-GGTCT-T-ACACAT-AAG---A--ATTG--AGCA--G-CATAT-GAA----GT A-GA-G-CCTAG-AG-GGTCC-T-TCACAC-AAG---A--AT TA--AGAA--G-CATAT-GGT--- -AG A-GA-G-CCTAA-AG-AGT TC-T-T TACAC-LAG---A--ACGG--AGAA--G-CCTAT-AAT-----AT A-GA-G-CCTAG-GG-AGTTC-T-TTATGC-AAG---A--GCTG--AGAA--G-CCTAT-AAC---- -AT A-GA-G-CCTAG-GG-AGTTC-T-TTACAC-AAG---A--ACTA--AGCA--G-CCTAT-AAT--- - -GT A-GA-G-CTTGG-AG-GGTAT-A-TGAGGC-AAA---T--CCTA--AGCA- G-CTTAC-AAT---- -AT A-GA-G-CTTGG-AG-GGTAT-A-TGAGGC-AAA---T--CCTA--AGCA- -G- -TTAC-AAT--- - AT A-GA-G-CTTGG-AG-GATAC-A-TTGAGG-CAG---T--CCTA--AACA--G-GTTAC-AAT--2- AT A-GA-G-CTTGG-AC-GGTAT-A-TGAGGC-AAA---T-CCTA--AACA--G-GTTAC-AAT----AT A-AA-G-CTTGG-AG-GGTAC-A-IGAGGC-ACG---T--CTTA--AATA--A-GTTAC-AAT---- -GT A-GA-G-CTTAA-AG-TGTCT-A-TGCACC-AAG---C--CTTA--GGCC--G-CATAC-AAT---- -AT G-GC-G-CTTAG-AG-TTGTC-A-TGAGCC-AAG---C--CGTA--AATC--G-CATAC-AAT-----AT

LTR $\rightarrow$ (for further Information, see the LTR)

CONSENSUS

HIV2ROD

HIV2NIHZ

HIV2ISY

HIV2ST

HIV2BEN

HTV201:4

HIV 2GHI

SIVMM2 51

SIVMMP II

SIVMM 142

SIVMM2 39

SIVMNE

gGGCTGgA^GGGaTETaTTACAgTga? agaAGACaTAgA

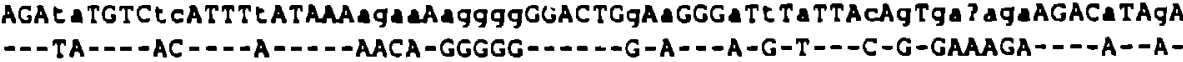
- TA---AC---A----AACA-GGGGG----G-A---A-G-T---C-G-GAAAGA--- A--A---CA----AG---A----AGAT-AGGGG-----G-A---A-G-A---C-G-GAGAGA----A--G-- TA----AC----G-..--AGAA-AGGGG-----G-A---C-G-A-- -C-G-GATAGG----G--G-

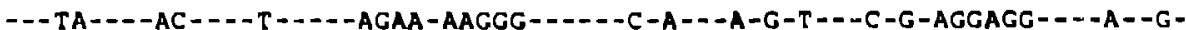

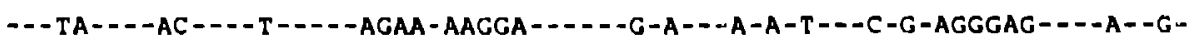

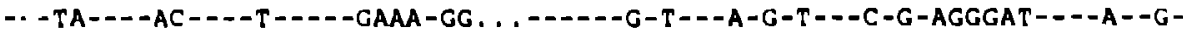

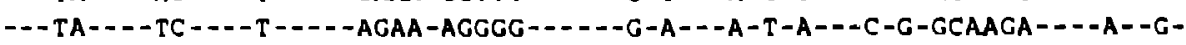

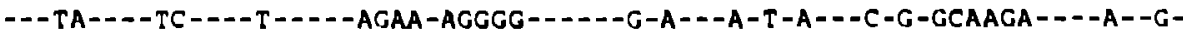
$---T A---T C---\tau-1--A G A A-A G G G G----G-A---A-T-A---C-G-G C A A G A----A=-G-$ ---CA----TC----T-----AGAA-AGGGG------G-A---A-T-A-- -C-G-GCAAGA----A--G -

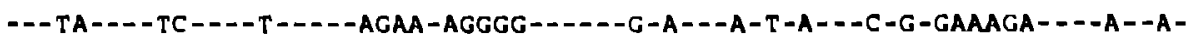
---CA----TC----T ----AGAA-AGGGG-----G-A---A-T-A--C-A-GAGAGA--- -A- -G-

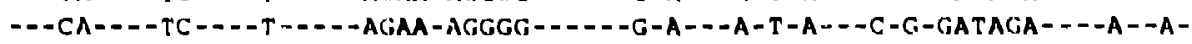




\section{HIV2/SIV NEF}

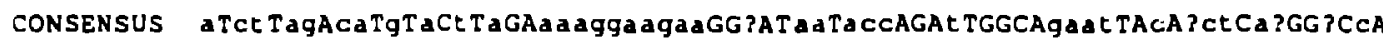
HIV2ROD HIV2NIH2 HIV2ISY HIV2ST HIV2BEN HIV2D194 HIV2GHI SIVMM25I SIVMMP 11 SIVMMI 42 SIVMA239 SIVMNE SIVSMMH 4 SIVSMMPBJ A-CT -AA-TA-A-A-T -A--AAAGGAAGAA--G- - AA-TGC-- T---- -GAAC- - C- . CT-AT--G-C

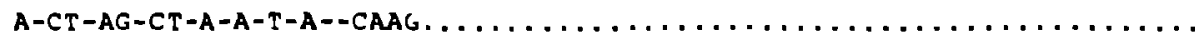
A-CC-AG-CA-A-A-T-A--AAAGGAAGAA--G--AA-TCC--- T-----GAAC--T-.CT-AT--G-TG-CC-AG-CA-A-A-T-A--AAAGGAAGAG--A--AA-TGG---C-----GAAC--T-. CT-AT--A-CA-CC-AG-CA-A-A-C-A--AAAAGAGGAA--G--AA-ACC---T-----GAAT--T-.CT-AT--G-CA-CC-AG-CT-G-T-C-A--AAAGGAGGAA--G--AA-ACC---T----GAAT--T-. CT-AT--G-CA-CC-AG-CT-G-A-C-h--AAAGGAGGAA--G--AA-ACC---T--..-GAAT--T-.CT-AT--G-CA-CT-AG-CA-G-A-T-A--AAAGGAAGAA--C--CA-ACC---T-----GGAT--C-CCT-A . --A-CA-CT-AG-CA-G-A-T-A--A........--C--CA-ACC-- T--.- -GGAT--C-CCT-A . - -A-CA-CT -AG-CA-A-A-T-A--AAAGGAAGAA--C--CA-ACC---T-----GA. .--C-.CT-C. --A-CA-CT-AG-CA-A-A-T-A--AAAGGAAGAA--C--CA-ACC---T---- -GGAT--C-CCT-A . - -A-CA-CT-AG-CA-G-A-T-A--AAAGGAAGAA--C--CA-GCC---T-----GAAT--C-CCT-G. --A-CA-AT-AG-TA-G-A-T-G--AAAGGAAGAA--A--AA-ACC---T-----AAAT--C-CAT-A, - -G-CCONSENSUS HIV2ROD HIV2NIHZ HIV2ISY HIV2ST HIV 2BEN HIV2DI 94 HIV 2GH I SI VMM2 51 SI VMMP II IIVMA1 42 SIVMA2 39 SIVMNE SIVSMMH 4 G-AATT--A--C-CT-T-CAC-AT--G---C-C-----AT--G-CC-A---GATG-CT--G-T--A-CTC A-AT-AG-TC-G-A-T-A--AAAGGAAGAA--A--AA-ACC---T-----AAAT--C-CAG-A. --G-C-

gGa t AAGaTACCcaA ?GE ? CTt tGGgTGGcTaTGGAAa ? TAgT ? CCaGTA ? atgTctCAgAtGAgGcaC G-AGTA--A--C-CA-T-TTC-TT--G---C-A-----GC--C-AC-A---GATG-CC--C-A--A-GGG

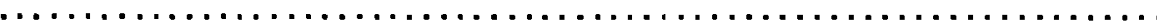
G-AGTA--G--C-CA-T-TTC-TT--G---T-A-----GC--G-AC-A---ACTG-CC--C-A--A-GGG G-AGTA--G--T-CA-A-TTC-TT--G---T-A-----GC--G-AC-A---GATG-CC--C-A--G-GAG G-AGTA--G--C-CA-T-TAC-TC--G---C-G-----GC--G-AT-A---GAAC-CT--C-A--G-CAG G-AACA--G--C-CA-T-TAC-TC--G---C-G-----AC--G-AC-A---GACA-CT--C-A--G-CAG G-AGTA--G--C-CA-T-TGT-TC--G---C-A----GC--G-AC-A---GATG-CT--C-A--G-CAG G-AATT--A--C-CA-A-ACA-TT--C---C-A-----AT--G-CC-T---AATG-AT--G-T--G-C C A-AATT--A--C-CA-A-ACA-TT--C---C-A-----AT--G-CC-T---AATG-AT--G-T--G-CAC G-AATT--A--C-TA-A-ATG-TT--C---C-A-----AT--A-CC-T-D-AATG-AT--G-T--G-CAC G-AATT--A--C-CA-A-ACA-TT--C---C-A-----AT--G-CC-T---NATG-AT--G-T--G-CAC G-ACCT--A--C-CA-A-ACA-TT--C---C-A-----AT--G-CC-T---NATG-AT--G-T--G-CAC SIVSHQPBJ G-AATC--A--C-CT-T-TTC-TT--A---C-C-----AT--G-CC-G--AATG-CT--G-T--A-CTC

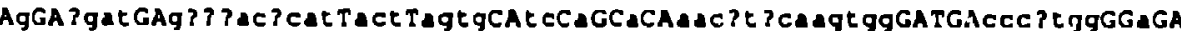
-G--CACT- -G. . ACTCAC-GCT-AGTA--TC-A--A--AACAAGCAAGTTT-----CCCGCAT--G--

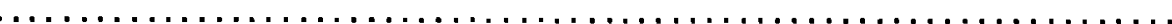
-G--CACT--G. . ACTCTC-GCT-AATG--CT-A--A--AGTAAGCAGATTT---_-CCCGCAT--G-T--CAGT--G. . ACTCAC-GCT-AGTG- - TC-A--A--AACAAGCAGGTTT-- - -CCCGCAT--A--G--AGAT - -G. . GCCAAC-GCT-AGTA--CC-A--A--MACAAGCAGACAT---- -TGAGCAT--G--G--AGTA--G. . . ACCAAC-GCT-AGTA--CC-A--A--AACAAGCAGATAT-----CGAGCAT--G--G--CGAT--G. . ACCAAC-ACT-AACA- - CC-A- - A--AACAAGCAGACAT- - - -CGAGCAT- -A--G--GGAT--GAGG . , CAT-ATT-AATG--GC-A--T--AACTTCCAAGTGG-- - -CCCTTGG--A--G--GGAT--GAGG . . СAT-ATT-AATG--GC-A--T--AACT TCCAAGTGG-----CCCTTGG--A-G--GGAT--GGAG . . CAT-ATT-AG'SG--CC-A--T--AACT TCCCAGTGG-----CCCTTGL--A--G--GGAT--GGAG . . . CA T-ATT-MATG--TC-A--T--AACT TCCCAGTGG-----CCCTTGG--A--G--GGGT--GGAG . . AAT-ATT-ACTG- - TC-A--T--AACT TCCCAGTGG- - - -CCCTTGG--A-SIVSMMPBJ 
CONSENSUS ggtacTage?TGGagTtTGAtcCaacgeTaGcctata?tTAtgaGgCaTt tat ?gatAcCCAGAaGAg HIV 2ROD HIV 2NIHZ HIV2ISY HIV 2ST HIV2BEN HIV2D194 HIV2GHI SIVMA251 SIVMMP II SIVMMI 42 SIVMM239 SIVMNE SIVSMMH 4 SIVSMMPBJ GACAC-AGTC---GAG-----TC-CTTGC-G--TTATAGT--CGA-G-T-TTAT-CGGT-C-----G--A

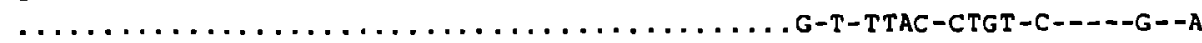
GACAC-AGTC---AAG----CC-CATGC-G--TCATGAG--CAC-A-C-TTAT-CTAT-C---- G--A AACAT-AGTT---AGG-----CC-CACGC-A--TTTTAGC--CGA-G-C-TTAT-CGAT-C-----G--G GACAT-AGTG---CAG-----CT-CATGC-G--CTATAAC--CAA-G-C-TCAC-CTGT-C-----A--G GACAC-AGTT---CGG-----CC-CATGC-G--CTATAGT--CAA-G-C-TCAT-CTGC-C-----A--A AACAC-ACTT---CGG----CC-CACGC-G--CTATGAT--CAA-G-T-TCAT-CTGC-T---- -A- - G GGTTC-AGCG---AAG-----TC-AACTC-A--CTACACT--TGA-G-A-ATGC-AGAT-C-----A--G GGTTC-AGCG---AAG-----TC-AACTC-A--CTACACT--TGA-G-A-ATGT-AGAT-C-----A--G GGTTC-AGCA---AAG----TC-AACTC-A--CTACACT--TGA-G-A-ATAT-AGAT-C-----A--G GGTTC-1ISA---AAG-----TC-AACTC-G--CTACACT--TGA-G-A-ATGT-AGAT-C-----A--G GGTTC-AGTA---AAG--_-TC-AACTC-A--CTACACT - TGA-G-A-ATAT-ACAT-C-..--A--G GGTAC-GGCA---AAG----TC-AGAAT-A--TTATAGL--TAA-G-A-TTAT-AAGT-C-- - -A--G GGTAC-GGCA---AAG-----TC-AAAGT-A--TTATAAC--TAA-G-A-TTGT-GAGC-C-----A--G

TTtGG ? ? CaAgTCAGGCCTG?CAgAggAaGAggtgaagagaAggCt ? a ? GCAAGaGGc ? ac ?at at HIV 2 NIHZ HIV2ISY HIV 2ST HIV 2BEN HIV2OI94 HIV2GHI SIVMM25I SIVMMP 11 SIVMH1 42 SIVMH239 SIUMNE SIVSMAH 4 SIVSMMPBJ --T--GCATA-T----AC--CC-G-GA-A--GTGGAAGGEG-GA-TGAAA---- -G--AA-ACCATT T- -T--GCACA-G----AA--GA-G-AG-T--CTGGAAGGCA-AA-TGAAA--- - - - -GA-ACCATTT- T--GTACA-G---- -CC--CC-G-GG-T--ATGGAAGGCA-GA-TGAAA-- - - - - GA-ACCGTT T- T--GCACA-G----AT--CC-G-GA-A--ATGGAAGGCA-AA-TGAAA-- - -A--GA-ACCATAT - T--GCACA-G-----AT--CC-G-GA-A--GTGGAAGGCA-AA-TGAAA--- -A--GA-ACCATAT- - T--GCACA-G----AT - -CC-G-GA-A--GTGGAAGGCA-AA - TGAAA---- -A- - GA-ACCATAT - . --AAGCA-G-----CC--TC-G-GG-A--GGTTAGAAGA-GG-\#"

--T--AAGCA-G----CC--TC-G-GG-A--GGTTAGAAGA-GG-TAACC--- -A--CC-TCTTAAC$--T--A A G C A-G----C C--T C-G-G A-A-$-GGTTAAAAGA-GG-TAGCC-----A--CC-TCTTGAA--T--AAGCA-G-----CC--TC-G-GG-A--GGTTAGAAGA-GG-TAACC--_--A--CC-TCTTAAC--T--AAGCA-G-----CC--TC-G-GG-A--GGTTAGAAGA-GG-TAACC-----A--CC-CTTAAAA- - T--TAGTA-G-----CT--TC-G-GG-A--GGTAAAGAGA-GG-TAACC--- - A--CC-T TT . AAA690

CONSENSUS HIV 2ROD HIV2NIHZ HIV 2 I $5 Y$ HIV 2ST HIV $2 \mathrm{BEN}$ HIV 2D1 94 HIV 2GH1 SIVMMP II SIVMM 42 SIVMM2 39 SIVMNE SIVSMMH4 - T--TAGTC-G-----CT--TC-A-GG-A--GGTGCAGAGA-GG-TAACC-----A--CC-TTTAAAA-

?gget gACA AGALGgAMACT AGC??? 737 GT*** 771 GT** $\quad 543$ GT** 771 GC*** 768 GTGAA**" 774 GTGAA*. 774 GTW 768 TGGCTG---A--G-G---TC-... 783 TGGSTG---G--A-G---TA-_.. 789 TGGCTG--A-A-G---TC--\#, 792 TGGCTG---A--G-G----TA--*, 792 TGGCTG---A--A-G---AA--TGA $: 929$ SIVSMMPBJ TGGCTC--A--A-A---AA--\# 186 




1 agtegctetg cggagagget ggcagattga gecetgggag gttctcteca gcactagcag

61 gtagagectg ggtgttccet qetagactet caccagtget tggeeggeac tgggeagacg

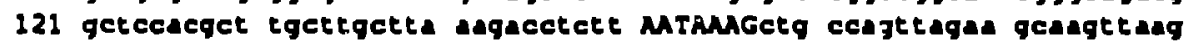
slgnal $\rightarrow$

281 tgtgtgttec eatetetect agtegcegec tggteatteg gtgtecatct gagtadada 241 secctggtet gttaggacce tteccgett gagatcca ggcaggaaa tecctagcag <- lys-tRNA pbs

301 gteggcgece gaacagggac tegaagagg actgagagge cetggaacac ggctgagtga 361 aggcagtaag ggcggeagga acauaccacg acggagtget cetagaaag egcgggecqa 421 gqeacegang eggegtgtgq agegggagtg augaggect ecgggtgaag gtaagtacet

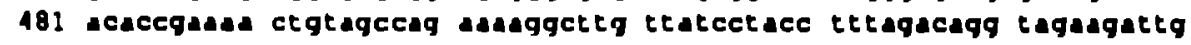
541 tgggagATGg gegcqugaad ctecgtettg agagggaua adgeagacga ateagaana gag start $\rightarrow$

601 gttaggttac ggcceascgq aAagaauga tacaggtcas aecatgttgt gtgggcagcq 661 aetgaaltag acagat.tegg attggcagaq agcetgttgq autcaaaga aggttgccae

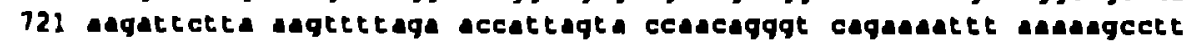
781 tteataccg tetgegteat ttggtgctg cacgeagag agasagtgas aquectgaa

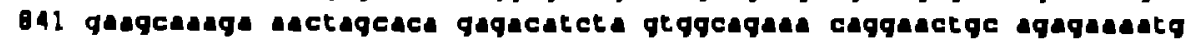
901 ccautatea qtagaccaec aqcaccacet agtgggaag gaggaabct cecegtgcae

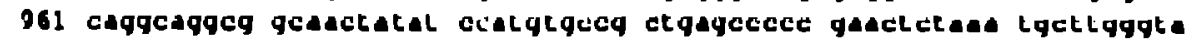
1021 aattagtag agquaagar gttcggggca gaaqtagtqc caggattcea ggcactctca 1081 qaagqctgca cqcectatqa tatcauca atgcteatt gtgtgqqequ tcaccagca

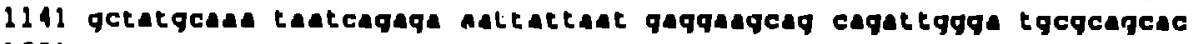

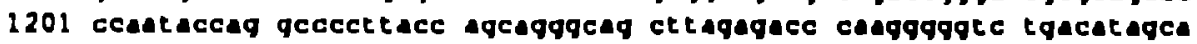

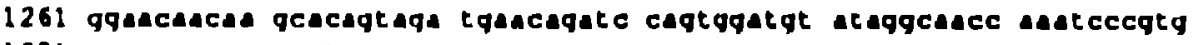

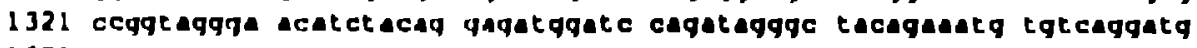

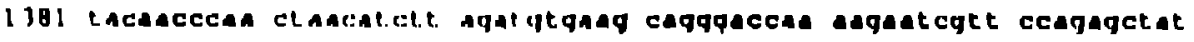

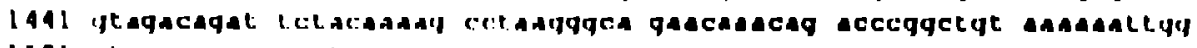

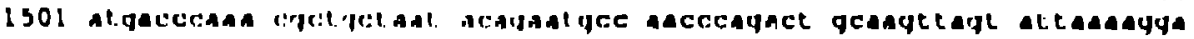

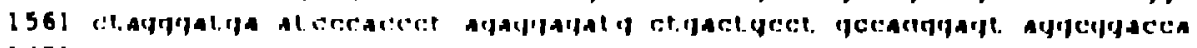

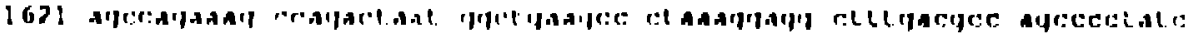

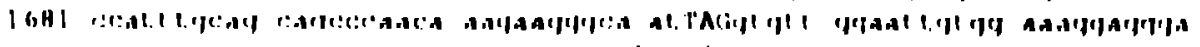
binl net - ,

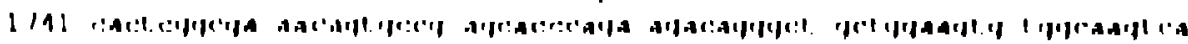

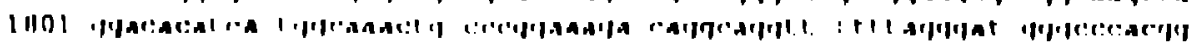


1861 ggaadgeage cecgeastt cecegcggce caagctecte aggggetgat accaacagea 1921 cecceatag atccageagt ggacetgttg gagaatata tgcagcagg gagaageag 1981 agagageaga gggagagace atacaaggag gtgacggagg actectgea cetegageag 2041 ggagagacgc cccacagagg ggcgacagag gacttgctac accteattc tetctetgga 2101 aadgaccagt agtcacagea ttcatcgagg atcagceggt agaagteted ctagacacag 2161 gagctgatga ctcautagta gcaggaatag agttagggga cauttacact ccauautag

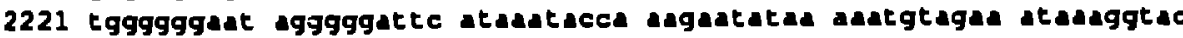
2281 taataaag agtaagagce accatarga caggagatac cccantcae attettggca

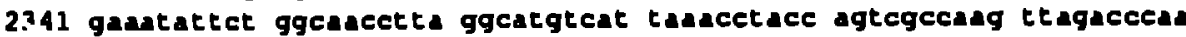
2401 taaagtace attgaageca gggaagatg gaccasgct gauacatgg cecetaca 2461 aggaauat agaugcacta aagaautt gtgauauat ggaugggag ggccactag

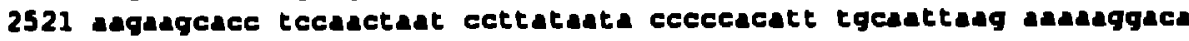
2581 agacasatg gagaatgeta atagattte gagadctaa cagggtgact caagatted 2641 cagauttca getaggaute ccacaccegg caggattagc cauadgad aggattactg 2701 tactagatgt aggggatgec tacttteca taccactaca tgaagattet aggcatata

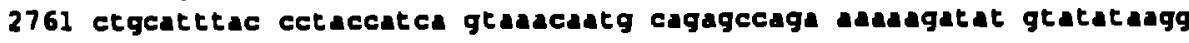
2821 tcttaccaca aggatgoasa ggatcaceag casctttca attcatgatg aggcaatet 2881 tagaucett cagaaagca aueceagacg teatetcat ccatacatg gatgatatet 2941 tautagctag tgacaggacg ggtttagagc atgacaagt agtcetgca ctaaagaec 3001 ttetgaatgg cetagggtte tetaceccag atgagaagt ceaaaggac cetecgtte 3061 atggatggg ctatgaattg tggccascta atggaadet geagaadata castacetc 3121 agaagaut atggacagte augacatce aAactagt aggagttttg aactgggegg 3181 egcagatcta tccagggata auaccasc atteatgta attgattaga ggaaatga 3241 cactracaga ggaagtncag tggacagagt tagcagaggc agauctaga gaaucaua 3301 ttatcteag teaggauca gagggatcct actatcagga agaugagad ctagaagea 3361 cagtcatcas angceagae auteagrggg catecauat acaceaggga gagagggte 3421 taaagtagg aagtatgeg asgatauas atacteatac cautggggte agactactag

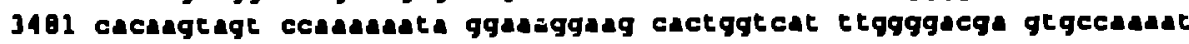
3541 ttcacctacc ggtagagaga gacacctggg agcastggtg ggatasetac tygcagtaa 3601 catgggtcec agagtgggac tecgtatcta ccecaccact ggteaggttg acatteact 3661 tggtaggaga tectataeca ggcacagaga cetttacac agatggatca tgcaatagac 3721 agtcauage aggaanagca ggatatgtae cagatagagg gagagacejg gtaugagtat

3781 tagagcaac atccateag caagcagaac tagaugectt tgegatggca etggcagact

3841 caggtcccas ggttatatc atagtagact cacagtatgt atggggata gtagcaggce

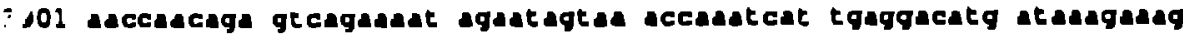

3961 asgcagtcta tgttgcatgg gtcccagece ateasgcat aggaggasec caggagtag

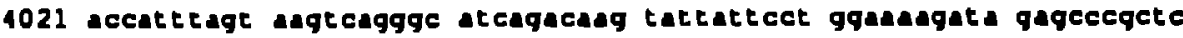

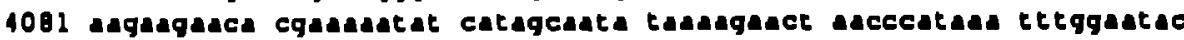

$\$ 141$ cccauctage ggcagacag atagtauca catgtgecca atgecaucag auagagaag

1201 ccatacatgg gcasgtaut gcagaatag gcgtthggea astggactgc acacacted

1261 aggaaar cartatagta gcagtgcatg ttgcagtgg attcatagas gcagastca

1321 teccacagga atcaggaagg cagacagcar tettcctatt aseactgqce agtaggtgge

4381 castacaca cttgeacaca gacastggec ccastteac ttcacaggas gtgagatgg

1441 tggcatggtg gataggtata gagcastcet teqgagtace thacasteca caangecagg

4501 gagtagtaga agcangat caccaccta auetcagat agtegatt agagaacagg

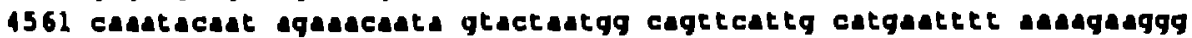

1621 gagpatagg ggatatgace ccagcagasa gactauta catgatcace acagaecasg

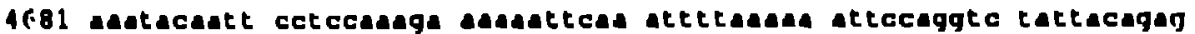

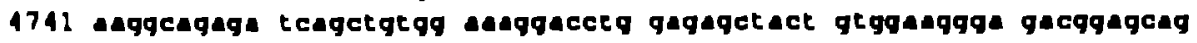

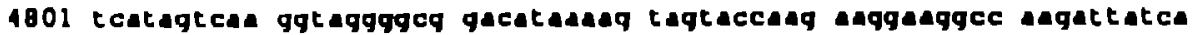

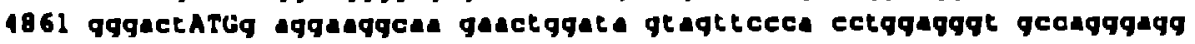
vif start $\rightarrow$

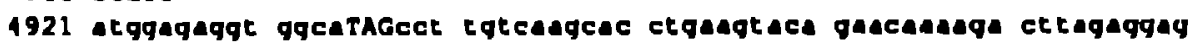
<- pol

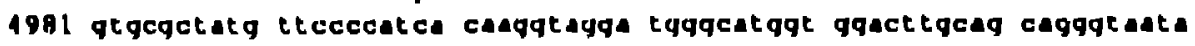

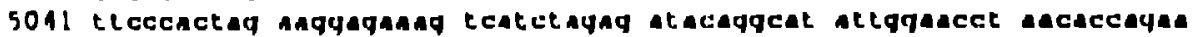

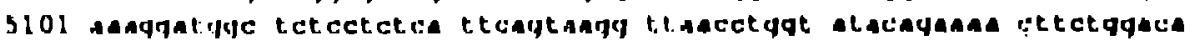

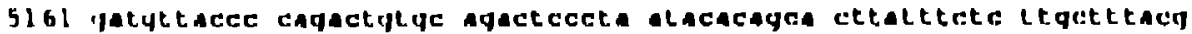

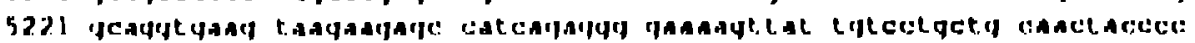

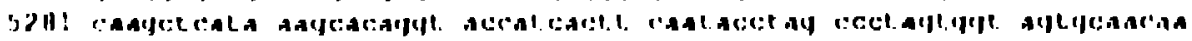

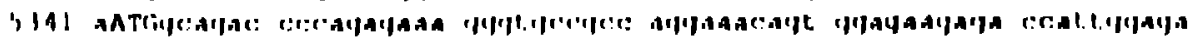

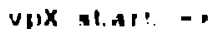

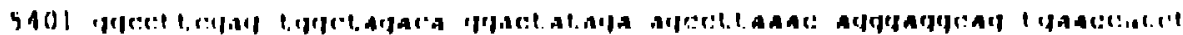

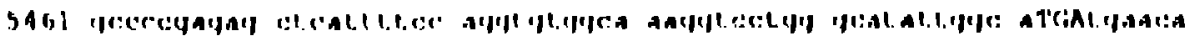


5521 agggatgtca acaagttaca cauagtatag atattegtge atautgeaga aagetgtgta 5581 tatacattc aagaaggggt gcactigect ggggagagga catggecegg gaggatggag 5641 aceaggaect ceccetectc cecetccagg tetagtcTM TGactgage accaacagag $<-v p x$

$$
\text { vpR } \rightarrow
$$

5701 tttcececag asgatgggac cecacggaga gagetaggga gtacetgggt autagauet 5761 ctgasggaaa teasggaaga agecteaaa catetrgate ectgettget aategctete 5821 ggcasctata tetatadag achrggagac accetrgang gagecagaga getcattaga tat start $\rightarrow$

5801 gtcetacac gagecetete cgtgeacatc agagegggat gtgaccgctc agaagggc 5941 cauacasga gaugagetce ttgcecagct geacegacec ctagajgcat geactacte

<- vpR

6001 atgctattgt asgeagtgca gttaceattg ceagctgtgt ttettgaada agggctcgg 6061 gatatggtat Geqcgacagg geagacgadg adgacteca agaasucta agacteatce rev etart $\rightarrow$

6121 gectectgea teagataagr aagearggag ectggtagga atcagetgct tgttgecatt S. If $N$ env $\rightarrow$

6181 ttattacta gtgettget acatatge aucatatg tgactgttet ctatggcata 6241 ceggegtgga gaatgcate tactceceta tettgtgca ccauatag agatact 6301 gggaccatce agtgettgec aqacautgat gattatcagg aatascet aategtgaca 6361 gaagettetg atgcatggga cautacagta acagaacang caacaganga tgtctggaga 6421 ctgtergaga catcatau accacgtgte apttgacge ccetacgtgt gqcgatgaut 6401 tgtatata cttcagggac tacegegace ecgagtceac caucattac autatagat 6541 gadatteta cetgtatagg egacancaec tgeacaggat taggqauga agaggtggtt 6601 gagtgegagt tcastatgac ggggctagaa caagataaga asaggaagta taatgacgea 6661 tggtactcas gagatgtggt ttgtgacadg acaucggad caggcacatg ttacatgaga 6721 cartgcauca catcagtcat caugagtca tgtgacaug actattggga tgctatgaag 6781 tttagatact gtgcaccace gggtetigce ctactasat geatgatac casctated 6041 ggctttgac ctaggtgete taugtagta getgctteat gcecaggat gatggaucq

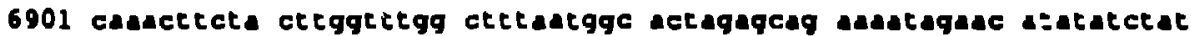
6961 tggcatghta aggatatag gactatcat agcteaca agtateata tetcacatg 7021 catcgtauga gaceaggaa tasgacagte gtaccata cactergte agggegaagg 7001 tetcactete ggceagteta caccausa cetgggcagg satggtgttg gtttesagge 7141 eactggatag asgecatgeg ggagqtgasg cauecettq cauacatce caggtacgga

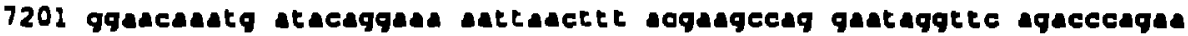
7261 gtgacataca tgtggacta ctgeagagga gastectct actgtatat gactggtec 7321 cteattggg tagaasata gacgaacca acacacggca actatgcgec atgecatata 7301 oggcagata ttacsectg gcataggta qqgacastg tatattggec tectagggas 7441 ggggagtiga cetgcaste ancagtauce agcatartg ctasattga cteagatgga 7301 atcagacea acattacett tagegcagas gtggeagane tgtacegate agaetrgggg 7561 gactacaset tgatagaagt ancaccatt cegt.tegcac etacaasaga gasagatat 7621 tecteggete eagtgaggas casuagaggt gtgttegtge tagggttett gggtetete 7681 gegecageag gttetgcast gggeggegeq tecthgege tgteggetca gteceggact 7741 teactggecg ggateqtgea geas;agca cagctgttgg acgtggtcas gagacacae 7901 gasergttgc gattgacogt ctyggquacg auautctce aggeagage cactgetate 1861 gagautect tauggacea ggcacagcta autcatggg gatglgegte taggeaggec 7921 tgccacacta ctgtaccatg gotaatgac tecteacac ctgactggas cautatgaca

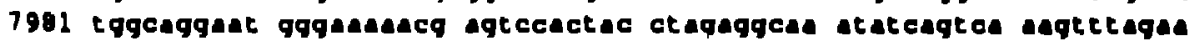

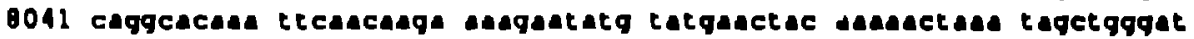
B101 gtettrggca actggtetga tttgacutce tgqatcauat atattcane tggagtetat

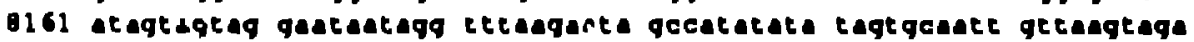

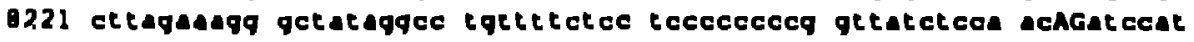
八 J'. I

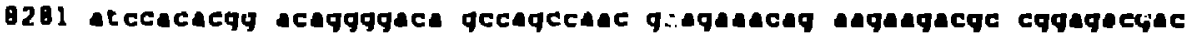

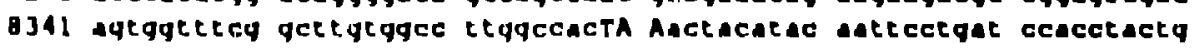
$<-t a t$

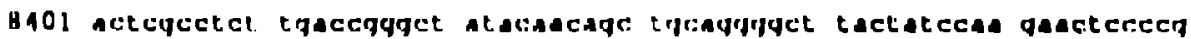

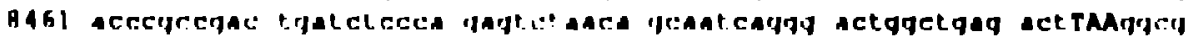
<- rav

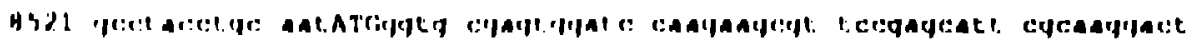
nel nliarl - ,

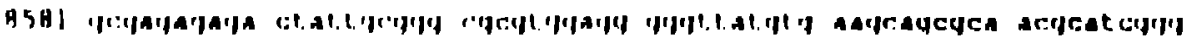

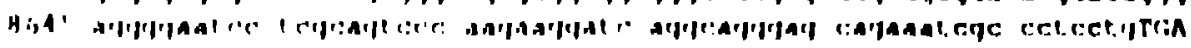


0701 gggacagcag tateagcagg gilgagttcat gaacacceca tggagaacec cagcagcaat

0761 aggcagaa aattcatata agcagcaaa tatggatgat gtugatictg atgatgatga

8821 cetagtggga gttcetgtta '-gccaagagt accgetgaga gauatgacet atauactgge

B881 atagatatg tcacattet: tauagaaa aggaggactg gaagggatat tetacagtag 3. LTR $\rightarrow$

0941 ggagagacat agaatcetag actegttcet agaaaggag gaagggatau taccagattg

9001 gcagaated seteatggge caggascag gtacccatg tacttegggt ggctgtggaa

9061 actagtacea gtagacatct cacagaggc agaggagta gagaceact gettagtuca

9121 cecagcaca acasgeagat atgatgacga gcatggggag acactagttt ggeggtttga

9181 cccatgetg gectatagtt acaaggect cattetgcac ccagaagat ttgggcaca

9241 gteaggateg ceagagaag agtggaggc auactgaua geaagaggga taceatatag

9301 tgaracag gaceacest acttggteag ggeaggaut agctactas ancagctgag <- naf

9361 actgcaggga etttceagaa ggggctgtaa ccasgggagg gacatgggag gagctggtgg

9421 ggaucgcect catatectct gtataacgt acecgetect tgeategtat te 
CONSENSUS SIVAGMTYO SIVAGM155 SIVAGM 3 SIVAGM 677 SIVAGM 692 SIVAGM9

CONSENSUS SIVAGMTYO SIVAGM155 SIVAGM3 SIVAGM677 SIVAGM 692 SIVAGM

CONSENSUS SIVAGHTYO SIVAGM155 SIVAGM 3 SIVAGM 677 SIVAGM 692 SIVAGM9

CONSENSUS SIVAGMTYO SIVAGM155 SIVAGM 3 SIVAGM 677 SIVAGM 692 SIVAGM9

CONSENSUS SIVAGMTYO SIVAGM1S5 SIVAGM3 SIVAGM 677 SIVAGM692 SIVAGM 9

CONSENSUS SIVAGMTYO SIVAGMISS SIVAGM3 S IVAGME77 SIVAGM 692 SIVAGM9

CONSENSUS SIVAGMTYO SIVAGM155 SIVAGM 3 SIVAGM 677 SIVAGM 692 SIVAGMS
TGGA TGGGATTATTACTCCGAAGgAG? aAtAAG TCCTgAA ? CTqTALGCCCT?ALGAATGGGGAT T-T-G-AA-TAAG--CC-G--TC-G--T--TC-T--T--A-----G-- -

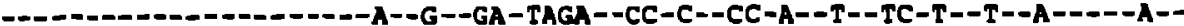

CATEGATGATTGGatGCt TggtCa? ?aGGaCCaGG?ATAAGATacCC?agatgCTTtGGcTTCTGcTTE

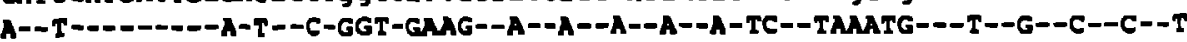
C--T--0-----A-C--A-GGT-AMM-A--T--G-A--A-AC--GAGGTG--T--C--C--C--C C--T-D-D-A-T--T-GGT-AGN--A--A--A--C--A-AC--AAGAT---C--C--C--C--T A--A--_---D-C-A--T-ACT-ACCA--C--G--G--A--G-AC--GAGAGT-D-T--C--C--C--T C--A--_---D-C-A-D-ATA-TCCA--T--A--C-DC-A-AT--AAGATG---T--G--C--T--T C--T-------A-T--A-GGT-AGCA--A--A--C--A--A-AT--CCGCTG---T--C--T--C--C

aAg? TaGTaCCaGTgGaccTgCaTGAgGAGCa aAaca TGTGA ?AGaCAt TGceTggTgCAtCCaGCaC A-GC-A--G--A--G-ACT-ACA---G--A--ACAMCA-----A--A--T--CC-AG-C--T--A--GA-GC-A--A--G--T-CCC-GCA---G--A--AGAAACA-----A--G--T--CT-GG-A--C--A--AA-AT-G--A--A--A-ACC-GCA---G--A--AGAGACT-----G--A--T--CC-GG-G--T--A--AA-GC-A--C--A--G-ACC-GCA---G--G--ACGCAAC-----G--A--C--TC-GA-G--T--A--AG-AT-A--G--A--G-ACC-TAG---G--A--GCAAGGA----A-A-G--C--TC-GG-C--T--T--TA-GT-A--A--G--A-AGA-GCA---A--G--AGAMCC-----G--A--T--CT-GG-G--T--T--A-

AA? Tg?? gGA GA ?CCE GATGGtATCA?tCATGGAGAAtCTTGGteTGGAagTTtgAtCC? ?AtGtTPG

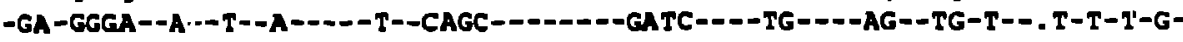

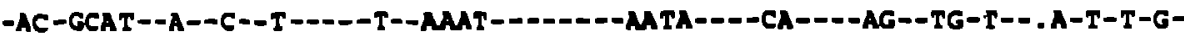
-AG-GAGG--A--C--T----A--CAAC-------AGTC----TC----AG--TG-T--.C-T-T-A-

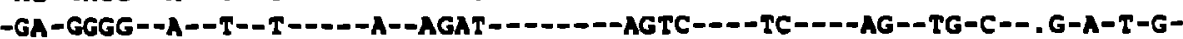

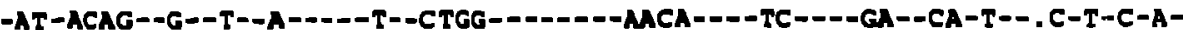
-AG-ANA--G--C--C----C--CAGT-------GACC---TC----AG--TG-C--CT-T-T-A-

\section{<- nef}

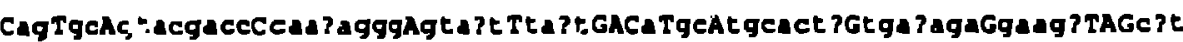
-AA-AC-GTACGACC-CATCGGG-GTACT-TACT---A-GC-TGCCCTG-TGA . AGA-GAAG . - -CCA -TG-TC-GTACGACC-CTCAAGGE-GTACT-TACA--T-AT-TTCAACA-TGGTACA-GAMC---CC. -AG-CC-ATATGACC-TACAGMA-ATATC-CACT---A-GC-TGATCTT-GCA . AGA-GAAG. ---C . T -GG-GG-GTACCGCC- . . . . . GG-CATGT-TAG--A-GC-CGNACAT-CAA . AGC-C. . . . . - . . T -A. -GC-AGGCCATG-CA. . . . GG-G. TGT-CAAT--A-GC-TGCAACA-TGG. . . . -GAAG. - - -C. . -AG-GC-GTATGACC-MACAGAC-GTATT-AGAA---A-GC-TGCACTG-TGA . AGA-GAAG , - -C . T

gaccgcaggct TGcGGTTa ?gc?cgTc?ce ?aggaGatgACatcaaaac ? ? ? ? ? ? 7TGCTgAc ? ? GACCGCNAGCC--C.---A.GMCA-CACCATGGA-ATG--ATTAMAAC . . . . . . .---G-C... . GACCACAGGCT--C-. - - T . CCTGG-TGCCTAGGA-ATG--ATTIAGAAC . . . . . . . - - -G-C. . . MACCCAGGCT--T-_-AMGCACA-CACCATGGT-ATG--ATTAMGAAC . . . . . . -..-G-C....

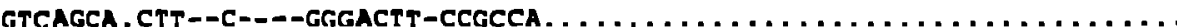
......... T--C-- -A. GCGCG-CCGGGACCT-TGT--CAMCCAGCATAGCAACCA----A-TGAGC MCCGCAGGCT - T-_--AMC : . CG-TGCCGGGGA-ATG--ATTTGMAC . . . . . . .---G-CAA. .

enhancer

onhancer

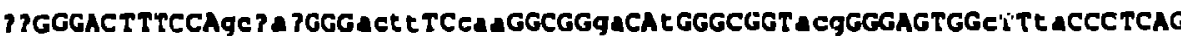

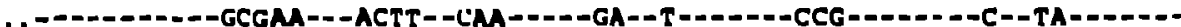
. . - , , . - -

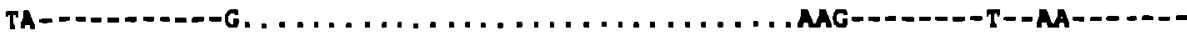
. . -

I-C-1

MAY 91 
glgnal $\rightarrow$ putative mRNA start $\rightarrow$

\begin{tabular}{|c|c|c|}
\hline CONSENSUS & A?gCTGCATAAAAGCAGATGCTCgCtGGCTTGTAACTCAGT ?CTCTTACTAGGAGACCAGCTLGAGCCTG & 512 \\
\hline SIVAGMTYO & 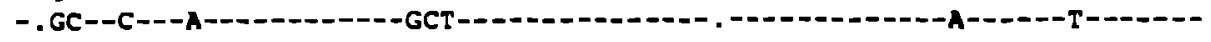 & 539 \\
\hline SIVAGM1 55 & 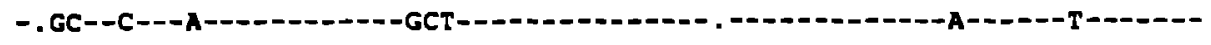 & 539 \\
\hline SIVAGM3 & $-----T------2$ & 538 \\
\hline SIVAGMG77 & $-----A-------$ & 502 \\
\hline SIVAGM692 & $-----A------$ & 501 \\
\hline SIVAGH9 & $--\mathbf{T}=-$ & 537 \\
\hline CONSENSUS & ACCtGGt? TGGCC ?aCCaGGGGTMG?GaCTCCt tGGCTT ?agAaAGCT? & 577 \\
\hline SIVAGMTYO & 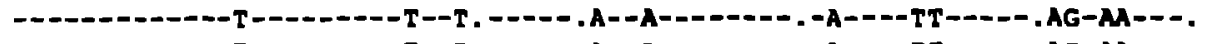 & 604 \\
\hline SIVAGM155 & 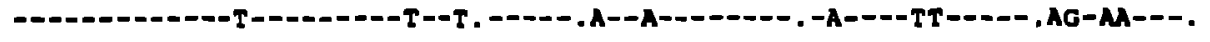 & 604 \\
\hline SIVAGM3 & $-----.-A----T T-----. A G-M---$ & 603 \\
\hline SIVAGM 677 & $1--G--------,-A----T T-----C A T-T A--C$ & 569 \\
\hline 5IVAGH692 & 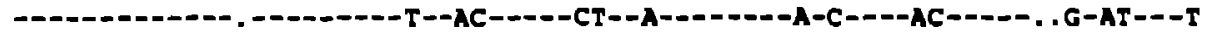 & 568 \\
\hline SIVAGM9 & 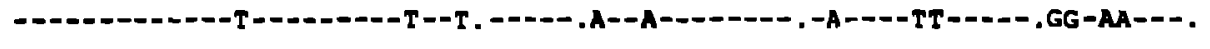 & 602 \\
\hline slgnal & $\rightarrow$ & \\
\hline CONSENSUS & 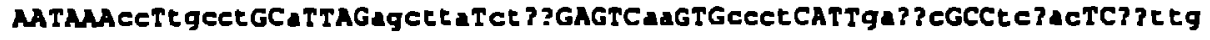 & 640 \\
\hline SIVAGMTYO & ----- C. -TGCCT--A----AGCTTA-CT. .----AA---CCCT----GA. . C---TC. AC--TCTTG & 668 \\
\hline SIVAGMI55 & 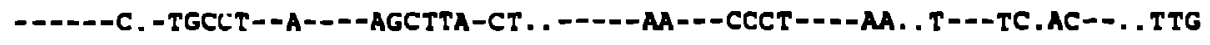 & 666 \\
\hline SIVAGM3 & ---- TC-TCGLT--A---AGCTTC-CT., - & 666 \\
\hline SIVAGM677 & ----- CC-.GCTC--.---TCGCTA-ATTG----AA---C..T---GCTGC--GAGCC--. TAG & 633 \\
\hline SIVAGM 692 & 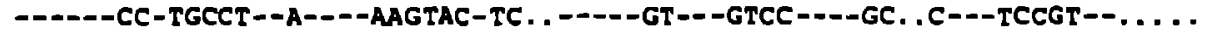 & 629 \\
\hline SIVAGM9 & ---- CA-TGCCT--A----AGCTTA-CC. . ----AA---CCCT---GA. .C $--T C . A C--\ldots$ MAG & 665 \\
\hline CONSENSUS & AACGgG? ?AA ? ?CTLCATTACTGGGTTCTCtCTCt? ACCCAGGeGaGAGAAACTCCAGCA & 695 \\
\hline SIVAGMTYO & A-C-G- . A-T.C-TCCT---- & 725 \\
\hline S?VAGM155 & 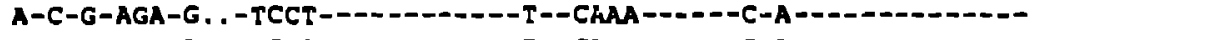 & 724 \\
\hline SIVAGMJ & 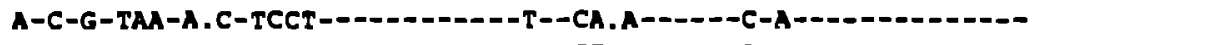 & 724 \\
\hline SIVAGM677 & 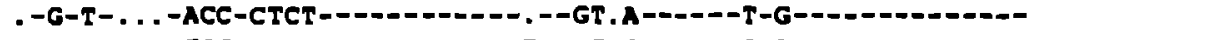 & 687 \\
\hline SIVAGM692 & 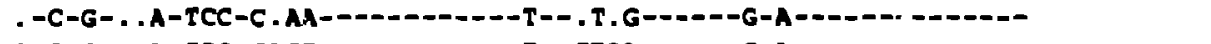 & 683 \\
\hline SIVAGM9 & 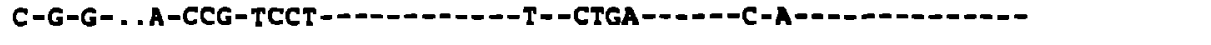 & 723 \\
\hline
\end{tabular}


AGM GAG CDS

CONSENSUS ATGGGggeGGCTACCTCAGCACTgaalaGGAGACaAT TAGAC?AATTEGAgCAtATaCGaCTtCGCCCGA SIVAGMTYO ----GGC--C-AC-------AMATA----CAAT-A--CCAA--T--GA-A--AC-A--T--C---SIVAGM155 -----GGC--C-AC--------GMTA----CAAT-A--TGMA--T--GC-T--AC-A--T--C---SIVAGM3 ----GGC--C-AC--------MATA--_-CAAT-A--CAAA--T--GC-T--AL-A--T--C---SIVAGH692 -----TTC--G-TC--------GTCAG-----MAT-A--CCAA--T--AC-T--AC-T--T--C---SIVAGM 677 -----CGG--G-CA--_-_-_-GTCAG--_-- AGCC-C--CACG--C--GA-G--TA-G--A--T----

CONSENSUS ICGGAAAgAAaAATTACCAAATEAAACATTTAATATGGGCAGGCAAggAaATGGAgCGCTT CGGCCTCCA SIVAGMTYO SIVAGMISS SIVAGM3

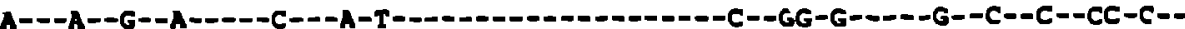

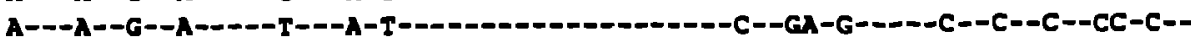

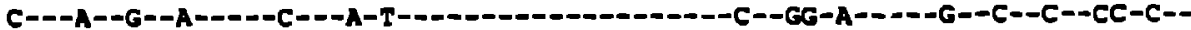

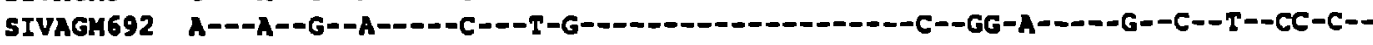

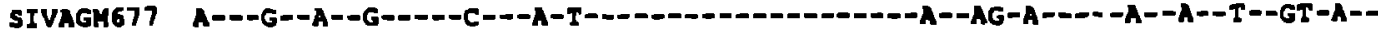

CONSENSUS TGAgALgtT?tTAGAACAgAaGAaGGPTGTAAAAAgATCATAGAMGT CTCE?CCCCCTAGACCAACa

SIVAGMTYO $---G-G G T-G T-G--G A--G-G--G--G--A-A-G A---1----C C-C T A C--C C-A----A--A$ SIVAGMISS SIVAGM3

SIVAGM 692 SIVAGH677

consensus SIVAGMTYO SIVAGM155 SIVAGH SIVAGM692 SIVAGM677

CONSENSUS SIVAGATYO SIVAGMI55 SIVAGM SIVAGM 692 SIVAGM677

CONSENSUS SIVAGRTYO SIVAGMI5S SIVAGM 3 SIVAGH6S2 SIVAGM 677

CONSENSUS SIVAGKTYO SIVAGMISS SIVAGM3 SIVAGM 692 SIVAGM677

CONSENSUS SIVAGMTYO SIVAGMISS SIVAGM3 SIVAGM692 SIVAGM 677 -- -G-GAT-AC-A--AT--G-A--A--A---A-G-AG----------AC-CTAC--GC-A-----A--A $---A-A G T-G T-A--M--G-A-\cdot G--G---A-A-A G--0--0---A T-G C T T-C T-A--2-A-C$

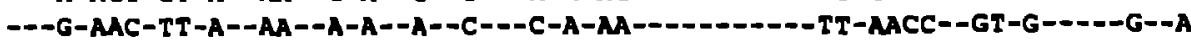

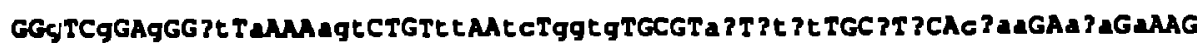
--A--G--G--CT-A---AGT----TC--TC-TGTG----AC-ATAT---T-G--CAAG--ACA-A----G--G--A--AA-G---AGT----AT--TC-GGTG-----AT-GCTT---G-C--CCAA--AA-A----G--G--G--CT-A---AGT----TT--CC-TGTG-----AT-GTTT---G-A--CAMA--TAA-G----G--G--A--TT-A---AGC----TC--TT-GACC-_-.-CA-TTGC---A-T--TCAG--AGC-A----C--C--G--GC-A---GCT----TT--TT-GTGC-----CA-TTGG---A-T--CGCA--ACA-A---

TGMAGACACAGgGMAGCagTAgCNA ?AgTAAgaCAMCACTFCCAtCTAGTGGACMAgaAaAAatGC -

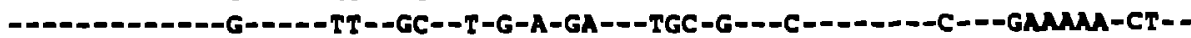
-

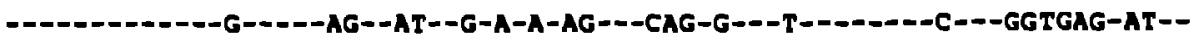

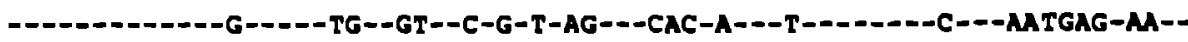

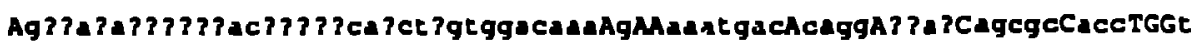
- . . . . . . . . . ACAGAGACATCTAGTGGACAM-G--AATGAC-AGGG-A TAG-AGCGC-ACC---G -GTT . . . . . . . ACG. . . CCACCTGGTGGACA JC-G--NAATAC-CAGG-GGAA-AGCGA-ACC---T -GAAAGMATACAACAGAGACATCTAGTGGACAM-G--MAATGAC-AGGG-GTAA-AGTGC-ACC---T -GCCAA. . . . . . . . . . . . . . GGAATAG-T--G. . . . . CAAC- . . C-GACAC-TAG--T -GCTAAA. . . . . . . . . . . . . . . A-G--MATGAG-CAAC-. . G-GCCAC-TCG-.-C

ggcagtcaAATTtcCCaGcacaAcA rCAggg7ATGcaTGGgTaCAtgtgCC?t TgtcaCC zGcACce TGCAGTCAG----TT--A-CGCA-C-A--AGGA-- -CC--G-A--TGTA --CT-GTCA--GC-C--CT GGCAGCCA----TT--C-CACA-C-G--AGGG----CA---G-G--TGTA--AC-TTCA--TC-C--CC GGCAGTCAA----TC--A-CACA-C-A--GGGA----CA---A-A--TGTG--CT-GTCA--AC-C--CT AGGAGTCAA----AC--G-CACA-C-G--GMT----TA---G-A--TGTG--AC-TAGC--CA-A--AT GATCNAGA---AC--A-TAGT-A-T--GNA----CC---G-A--CCAG--TT-GTCT--GC-C--GT -

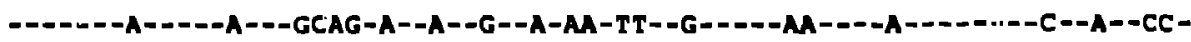

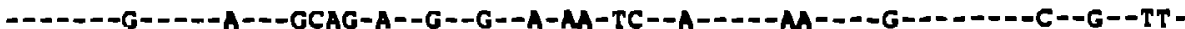

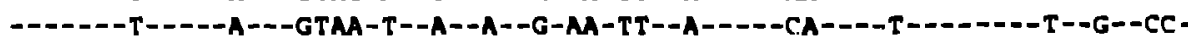


CONSENSUS ?TCAGAa JGTTGCACCCCTATGACAT?AAECAgATGCTtAATGT TCTaGGAGACCATCAgGGGC SIVAGMTYO A-----A--C--CACAC-C-----CA-T--T--G---C-T-----GC-A-----T-----A-----AT-A SIVAGM155 C-----A--C--CACCC-A-----CA-C--T--G---C-T-----CT-A-----T-----G-----CT-G SIVAGH3 A--.--A--G--CACAC-C-----CA-C--T--A---C-T-----CC-G-----C-----A----GC-A S:VAGM692 G-----A--A--TACCC-A-----TG-G--C--A---T-G-----TC-A-----C-----G-----CC-G

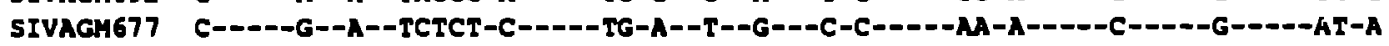

CONSENSUS SIVAGMTYO SIVAGM155 SIVAGM3

SIVAGM692 SIVAGH677

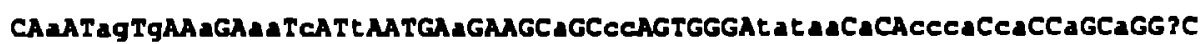
--A--AG-G--A--GA-C--T-----A-----A--CC------TGTAA-A--CCCA-TA--C--A--A--A--AG-G--A--AA-A--T-----G-----A--CC-------TGTAA-C--CCCA-CG--G--A--C--A--AG-A--A--MA-C--C-----G-----A--CC-------TATAG-T--CCCA-CA--A--A--A--G--AG-G--A--GG-C--C-----A-----T--CC----2--CATTA-A--TCCC-CA--A--A--G-

CONSENSUS SIVAGMTYO SIVAGM15S SIVAGM3

SIVAGM692 SIVAGM677 --A--TC-T--G--AG-C--T-----A-----A--AG------CAGGA-A--CAGA-CA--A--T--C-

C?t TaCCAGCaGgaCAgCTCAGaGACCC?ag PGG?TCagAtATAGCAGGgACcACCAGCaCagT?CAAGA -CC-A-----A--A--GC-C--G--C--TCGC--C--A--T-------G--C--C--CT-AG-A--A--CT-G-----G--A--GC-C--G--T--GGGG--A--A--T--D---O-G-D-C-T--TA-AG-G--A--AT-A-----A--A--AC-C--A--- - TAGA--C--T--C--------A--C--C--CA-AG-G--A--GC-C-----A--G--AT-G--A--T--MMGG--G--A--C----O--G--T--T--TA-CA-T--A--

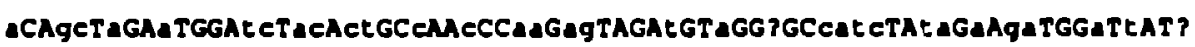

A--GT-A--A----TC-AT-CT--T--C--CC-GG----T--A--T--CATC--CC-G-GA---A-T--T G--GC-A--G----TC-AT-CT--T--C--AA-GG----T--A--G--CATC--TC-A-GA---A-C--C A--GC-G--A----TA-AC-CA--C--T--CA-AG----T--G--T--CATE--TA-A-GG---A-T--C A--AC-A--A----TT-AC-CA--C--C--MA-MA----C--G--A--TATC--TA-G-GA---G-A--A A--MA-A--G----CC-TC-AT--C--T--MA-MA----C--A--G--ACMA--CA-A-MA---G-T--T

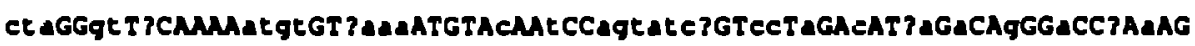
CTA--AC-T-----GTG'--CNAA-----C--C--AGTATCA--CC-A--C--TA-G--G--A--T-A-CTA--GT-A-----ATGT--AAA-----C--T--AGTGTCT--TT-A--T--CA-A--A--G--E-A-CTG--GT-G----ATGT--MMA-----C--C--AGTGTCT--CT-A--C--AM-A--G--G--C-A-SIVAGH677 GCA--GC-G-----ATGT--CAGA-----T--T--MCAGGG--TC-G--T--M-A--A--A--A-G--

CONSENSUS SIVAGMTYO SIVAGM155 SIVAGM3 SIVAGM692 SIVAGM677 ITG--CT-A-----GGTA--GCAG-----C--T--CCNAMG--CC-A--C--TC-A--G--A--T-A--

854 898

098

910

880

880

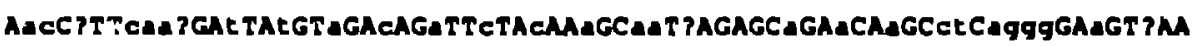
-GC-C-- CMAG--T--T--G--C--A--T--C--G--AA-T-----A--A--A--CT-AGGG--A--G--AC-A--CNM--T--T--A--C--A--C--T--A--AA-A-----A--A--A--TT-AGGA--A--C--AG-A--CAAA- E- C--A--T--G--C-.-C--A--AM-A-----T--G--G--CT-AGGA--A--A--AT-T--TAGC--T--T--A--T--A--C--C--G--CC-G-----A--A--A--CT-TCAG--T--T--

CONSENSUS SIVAGMTYO SIVAGM155 SIVAGM3 SIVAGH692 SIVAGM677 $-A C-C--C C A G--C--T--A--C--A--C--T=-A--C C-G----A--A--A--A C-A C A G--T--T--$ AC-A-D-D--G-AT-TT-GC-C--T--G--D--C--C--A--T--C--AG-A--TT-G--G--CC-A AC-G--D--D-G-AT-AT-AC-C--T--G-----T--T--A--C--T--AG-C--CC-A--G--CC-G GA-T-D-D--G-CA-TC-GT-G--T--A-D--T--C--A--G--T--GG-C--TC-G--A--GC-A 1038 1038 1050

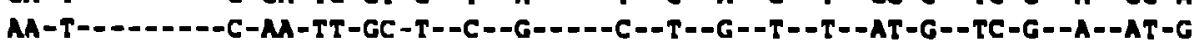

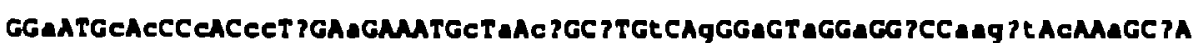
--A---C-C--C..-CC-T--A------T-A-CG--T--T--G--G--A--A--C--AACCT-C--A--A$--G---C-C--C=-T C-T--A------C-G-C A--C--T--A--G--G--A--C--A n G T T-C--A--C-$ $--A---C-T--C--T C-A--A------T-A-C T--C--T--A--A--G--\lambda--A--A A G T T-C--A--A-$ SIVAGH692 --C---C-C--T--CT-G--A------C-T-CG--A--C--G--A--A--G--A--CCMAT-C--A--CSIVAGM677 
CONSENSUS SIVAGMTYO SIVAGM155 SIVAGM 3 SIVAGM692 SIVAGM 677

CONSENSUS SIVAGHTYO SIVAGM155 SIVAGM3

SIVAGM692 SIVAGH 677

CONSENSUS SIVAGMTYO SIVAGM15S SIVASM3 SIVAGM692 SIVAGM677

pol orf $\rightarrow$

CONSENSUS SIVAGMTYO SIVAGM1 55 SIVAGM3

SIVAGM692

SIVAGM 677

CONSENSUS SIVAGHTYO SIVAGHIS5 SIVAGM 3

SIVAGM 692 SIVAGM671

CONSENSUS SIVAGHTYO SIVAGMISS SIVAGM] SIVAGM 692 SIVAGM 677

CONSENSUS SIVAGMTYO S IVAGMISS SIVAGM3

SIVAGM692 SIVAGM677
AagT rATGGCAGAMTGATGCa?aa?atgCa?ag?cagMACATGgTrCAgcagGgaggtc?aAgagg?aG -AG-A----C--.-----CAGACCA TGCAAAATCMA--C---G-G--GCAG-CAGGTCCAAAA. . . A. -AG-C----C-.-.----CAGMCCTGCAGAGTCAG--C---G-A--GCAG-GAGGTGGAGGGGAA-AG-G----C--.-.----CAAAATATGCNAGCCAG--C---A-G--ACAG-GCGGTCAGAGAGGMA-AT-G----T-..-_--_--NATCMATGCAGGGGGTC--C---G-A--GCM-CAGGAATAGGAGGTA-GC-A----T--.------AGTAAT. . . . . GGACAG--\$---G-C--AGTG-GACCTCAGAAAAAGG-

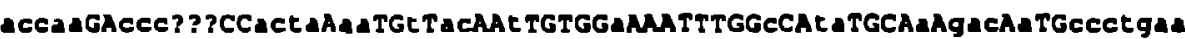
АСMA--CCC. .. --ACTA-GA--T-AT--T-----A--------C--TA----A-GAC-A--TCCGGAA АCCAA--CCC. . .--GCCA-AG--I-AC- -C-----A--------C--CA----G-GGC-G--TCCTGAG ACCM--CCC. .. --AGTA-AG--T-AC--T-----A----_---C--TA----A-GAC-A--CCCTGM AGGGA--GGMAGA--AGTT-MA--C-AC--A--_--A----_---G--TG----G-MA-T--CACTCMA CCCCC--GGG. . . --GCTA-MA--C-TT--T-----C--------A--TA----A-GGG-A--CMGGGA

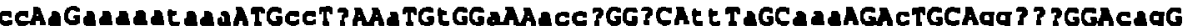
CC-A-GMAACMA---TC-A--G--T--A--ATTG--A--CC-A--AMA---C----GG. .. ---CAGCC-A-AMMATMA---TC-T--A--T--A--GCCA-.G--CT-A--AM---C----GG. ...--CAGCC-A-AMGATGAG---CT-G--A--T--G--ACCA--G--TT-A--AMA---T----GA. . .---CAGMA-G-GCCAGTAGT---CC-G--A--T--A--ACCT--C--TT-T--TCG---T-_--GA. . . --GCACC-A-ACAGATCM---CT-T--G--C--C--AATT--C--TA-G--AM---C-_--AGAT---CAG-

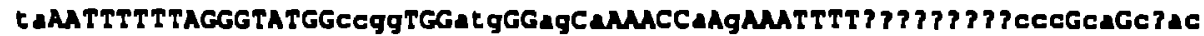

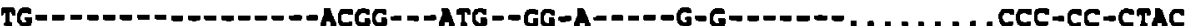

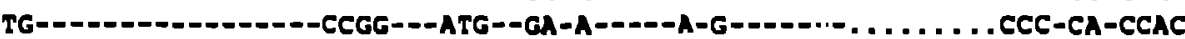

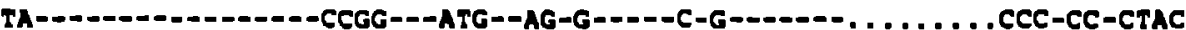
TA-_-_-_-_-_-CAGG --ATG--AG-A-_---A-A-_-_--TTAGMCACAGA-CA-CA. .

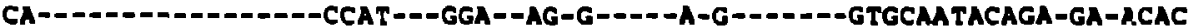

teTtqg?g?ggaACCaAct GCgCCeCCtecaecgarcarc7crac ?cC ?TACGAcccaGCaAagaaGCTC TC-TGGAGCGGM--GAGT--G--T--TCCACCGAGCGGCACCACCC-A-_-_-CCCA--A-AGM---C TC-TGGGGCGGAA--AAGT--G--C--TCCACCGAACACTCTACAC-T-----CCCA--A-AGMA---C TC-TGGGGTGGAG--AACT--G--C--TCCACCGAGT. . ...... C-A-----CCCT--A-AGAA---C -G-C........--CTCC--C--T--ACCGCCGCACACCCA: SGG-G-----CGM--C-CTCG--T AG-TGGTCTGGMA--MACA--C--C--A. . . . . . . ATGGRAACAG-T-----TCCA--A-AGM---C

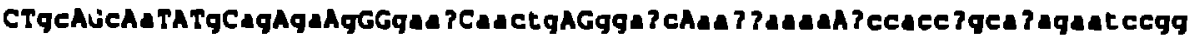
1422 --GC--C-A---G-AG-.GA-A--GAAA-MACTG--GGAGC-MAGAGGA-TCCACCGGCMATCAATCCGG --GC--C-G---G-AG-GA-A--GMA-AMATG--MATC-GAACAGM-CCCCCCAGCGMCAATCCAG --GC--C-A---G-AG-CA-G--GAAG-AGTTG--GGAAC-MGGMMA-ACCACCAGCAGTGMATCCCG --GG--A-A---A-CC-AG-G--AGCC-AACM--GAGAA-AGTAGAGA-GAGC. . . TCCCMAGCGGGGA 1313

1516 1492 1492

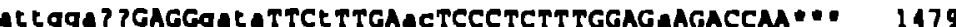

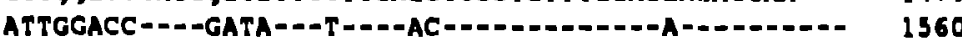

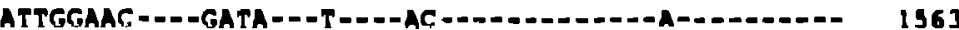

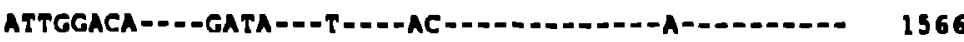

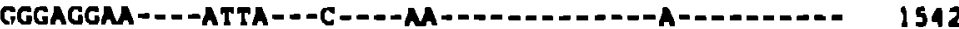

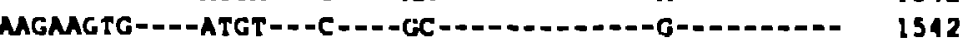


AGM POL CDS

gag cds $\rightarrow$

CONSENSUS TIG?GG?AAA?TTGGCCATATG??MAGACAATGTCCGGMCCAMG?AA ?A ?A ?ATG? ?TPAAATGTGGa

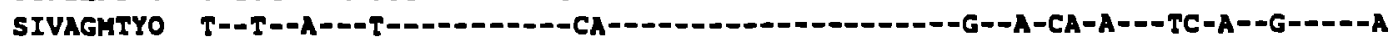
SIVAGM155

SIVAGM3 SIVAGM677 $-----A--G-T G-G---C T-G--A-----G$

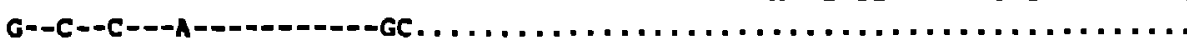

CONSENSUS SIVAGHTYO SIVAGH 55 SIVAGA3

SIVAGM677

CONSENSUS SIVAGRTYO SIVAGM155 SIVAGM 3

SIVAGM 67 ;

CONSENSUS SIVAGHTYO

SIVAGM155 SIVAGMJ

pol orf -s

MACEaggCACL TAGCAMAGACTGCAg? ? gGGACAGGE ?MATTTTTTAGGGTATGGCCggTGGaLgG

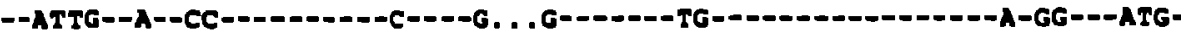

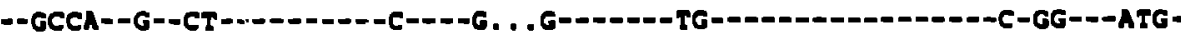

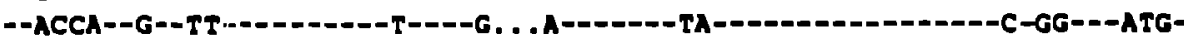

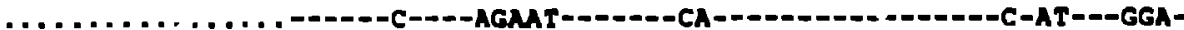

G?gC?MACCAAGMATTTTCCCgC?gC?ACtCt tGgggegGaACAagtGegCCCeCteCaCCgagCa? -GG-A--.--G-_-.----CCCGCCGCT-CTCTT-GAGCG-MCCGAGT-CG--TC-TC-A--GAGCGG -GA-A----A-1---D--CCCGCAGCC-CTCTT-GGGCG-MACCMGT-CG--CC-TC-A--GACM -AG-G--.--C-_.-_--CCCGCCGT-CTCTT-GGGTG-AGCCACT-CG--CC-TC-A--GAG. . . -AG-G--_--A-_--_--GTGCMATAC-GAGGA-ACACA-TTGGTCTG-M--AA-AG-C--CCCAAT

C?C? ACACC?TACGACCCAGCAAAGMGCTCCTgCAGCA PTATGCAGAgM PGGjAA PLAACTGAG ?gÄg CACCACCC--A-----C--A-------------G-----A------.-G--A--GA-A-MAC----GG-G

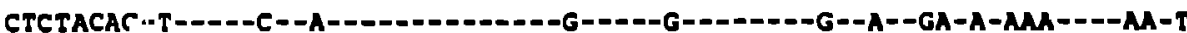

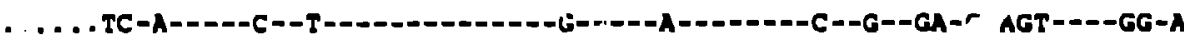
SIVAGM677

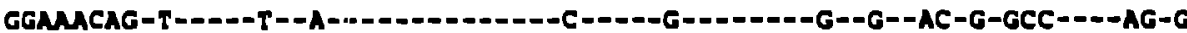

CONSEnSUS SIVAGMTYO SIVAGMI55 SIVAGM3

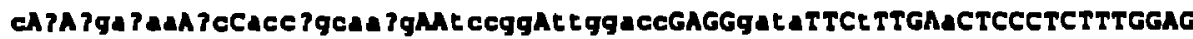
C-A-AGAGGA-TC-ACCGGCAATG--TCCGG-TTGGACC---GATA---T---A-_-_-_.

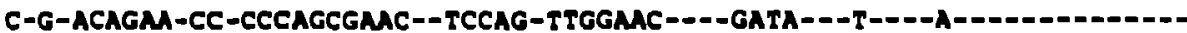
C-A-GGAMA-AC-ACCAGCAGTG--TCCCG-TTGGACA---GATA---T----A--1-_-1-_-_. SIVAGH677

G-G-GAGMC-GA-MGGAACAG--GGAGA-AGMGTG----ATGT---G----G--_-_-_-_-_-_.

$$
\text { <- gag }
$$

CONSENSUS SIVAGMTYO SIVAGMISS SIVAGK3 SIVAGM 677

CONSENGUS SIVAGMTYO SIVAGM155 SIVAGK3 SIVAGH 677

CONSENBUS BIVAGMTYO STVAGMI55 SIVAGH sIV.16M677

CONBENBUS SIVAGMTYO SYVAGM1S5 SIVAGM sivacimb\%

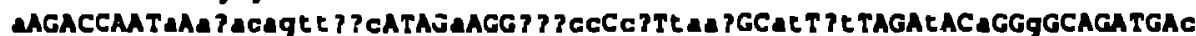
A---_--A-AGACAGTGTAT----A---GGTCC-CA-TAAG--AC-GC----C--A--G--.-.... C

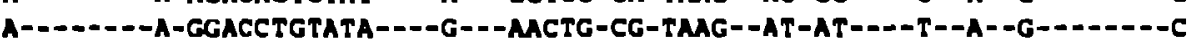

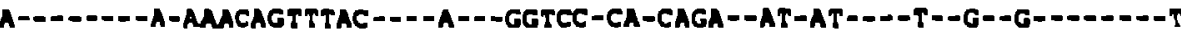
G--.----G-AACGAGTCATC-.--A---AACGC-AG-GCAA--CT-GT----T--A--A---.----C

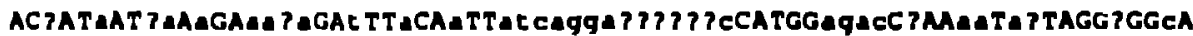
--C--A-- ТA-A--AMAT--T--A--A--ATCAGGT. ..... C--.--AGAC-C--AA-TA----G--C--T--A--AA-G--TACA--T--A--A--AAGGGGA. .... . T-----AGAC-A--MA-AG----A--A-

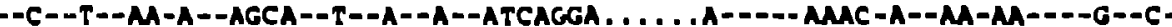
--T--A--TC-A--AMG--C--G--C--TCCCCCACATAMC-----CGTT-C--GG-AG ---A--T-

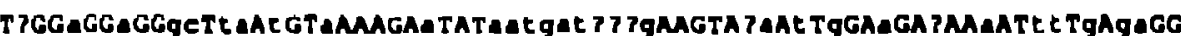
-A--A--A--CС-TA-T--A-----A---AMCGACAGCLG-----AA-A-A--A--T--A--TT-G-GA--T--G--A--GT-AA-C--A-----A---GATMATGTAG-----CA-T-G--A--C--G--AT-A-GA--T--A--G--AC-CA-T--A-----G---AGTGATAGGG-----AG-T-G--A--C--A--TT-G-GA--A--A--A--GA-TC-T--C-----A---CAGGGGGTAC-----CA-T-G--G--T--A--CA-C-CC--

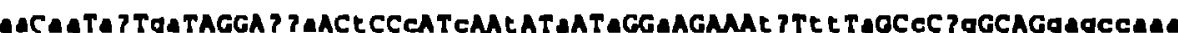
MA-MA-AT-GT---..-GCA--T--C--T--T--A--A--T-----TT-GC-G--C-CG----TGCCCCGG AA-AG-CC-CA-D--GCA--T--C--C--T--C--A--A-----CT-TT-A--C-AG---GAGCCAAA ('A-CA-AT-GA-----ACC--T--C--A--C,--A--T--A--.--TA-AT-A--A-CA--_-GAGCCAAA 
CONSENSUS SIVAGMTY SIVAR:1155 SIVAGM SIVAGM677

CONSENSUS SIVAGMTYO SIVAGMI 55 SIVAGM3 SIVAGM677

CONSENSUS SIVAGMTYO SIVAGMISS SIVAGM 3 SIVAGH677

CONSENSUS SIVAGMTYO SIVAGM155 SIVAGM 3 SIVAGM677

CONSENSUS SIVAGHTYO SIVAGMISS SIVAGK3 SIVAGK677

CONBENSUS SIVAGMTYO SIVAGM1SS SIVAGM 3 SIVAGM67?

CONSENSUS SIVAGMTYO SIVAGMIS5 S, [VAGM 3 SIVAGM 677

CONSENSUS SIVAGMTYO SIVAGMISS S [VAGH] SIVAGM6T7

CONSENSUS S IVAGMTYO SIVAGMIS5 SIVAGM 3 SIVAGMOTI

CONSENSUS S I VAGiMTYO SI VACiML b. :IVAcim I

:I IVACimoll

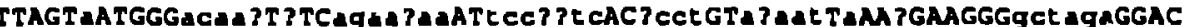
----A----ACAAT-A--AGAAMA--TCCTGTC--ACCT--CAMAT-G--G---_--GCTCGG--------G----GCAAT-G--GCAGACA--ACCMTC--CCCG--ACGCT-A--G------GCCAGA-------A----TCAAC-G--AGAACAA--TCCEATT--CCCT--GAAT-A--A------GCTAGA----_--T----AGTTC-A--TAGTCAS--TGAGGAA--AAAA--ACAAC-A--A-_-_-AAAGAT---_

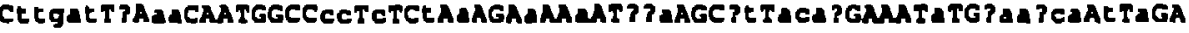
-CTGTG-A-GA-_-_.-TC-C--T-A---G--G--TGA---TT-ACAG--D--A--TTCCCA-T-A-ACGPT-G-AG-------AC-C--T-A---A--A--MAT---CC-GCAA--D-T--CAAMC-T-A--TTTCT-A-M--- - - -CC-C--C-A-- -A--A- MA-- -CT-ACAG----A--TGACCA-T-A--TANAT-G-AA-------CT-A--A-G---A--A--TGA---TT-AACA-----A--CAAACA-A-G--

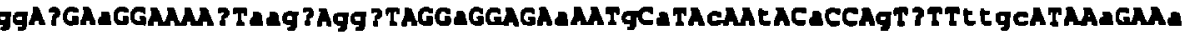
GC-G--A-----A-CAGT-GAG----A-----A---G-A--C--T--C---A-A--TTGC----A---G GG-A--A----D-T-AAGC-GGG----G-----C---G-A--C--T--A---G-A--CTGT----G---A GA-A--A-2----A-TAGC-AGA----A-----G---G-A--C--C--T---G-G--ITGC----A---A

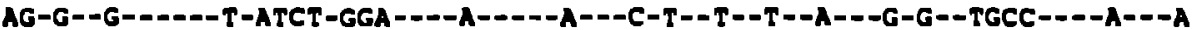

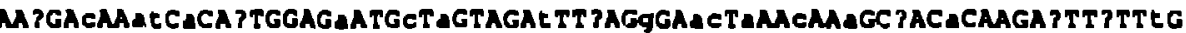

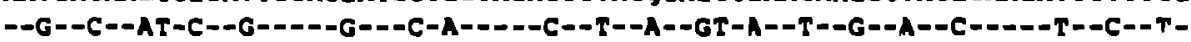

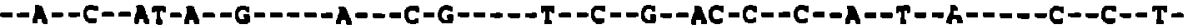
$--A--C--G T-A--A-----A--T-A-----T--T--G=-A C-A--C--A--A--A-----T--T--C-$ $--G--T--A A-A--A-----A-=-C-T----T--C--G--A C-A--C--A--T--T-----C--T--T-$

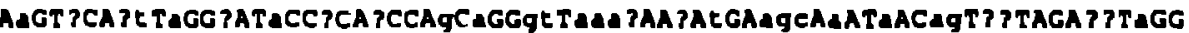
$-A--G=-A T-A--G--A--C--C---G-A--A T-M A G--G-T--G A C-G--A--A G-T T----T G-A-D$ $-A--C--A T-A--T--A--C--T---G-A--G T-M A G--A-T--A G C-A--A--C A-T A----T G-G=-$ $-A--A--G T-A--C--A--T--T---T-A--G T-C G A--G-T--C G G-A--A--A G-A T----C A-A-D$. $-G--T--G C-G--A--T--T--C---G-G--C C-T C A G--A-A--A G C-A--C--A G-M A----C A-A-$

gGAtGCcTATTATtc?ATACCAt 7ga 7cCagAgTTtAGaNATACACaGCtTTEACcAT 7CCt ?CaGT

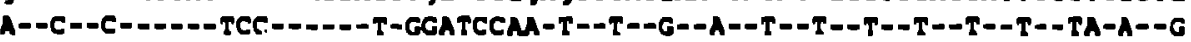
G--T--A-D--DAGC---D-C-GGATCCTG-G--T--A--A--C--A--T--C--C--C--TA-G--A

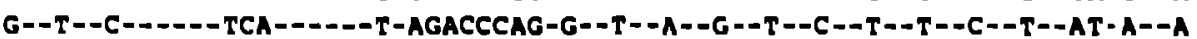

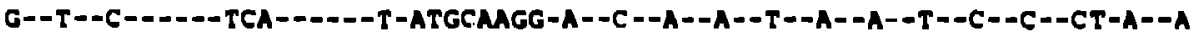

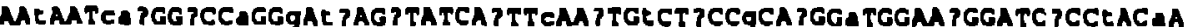
--T---CAG--A--C--G-TT--G---D-A--C--C--T--C--G--A--G-----A-----T--T--A--C---GAG--A--A--C-TA--A-----A--T--T--C--A--G--G--C-----G-----C--G--A $--T---C A A--G--A--T-C T--A-----G--C--C--T--T--A--A--A---7-G-----C--A--T-$ $--T---A C A--G--A--G-T A--G-----G--C--T--T--G=-T--G--A---D-A---D-T--T--A-$

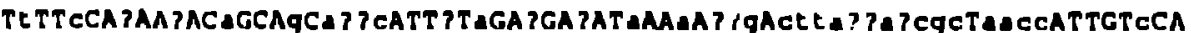
-C--C--A--T--A---G-ATCC---T-G--G--G--A--A-GMA-CTTGCCAGCAC-AACC-----A--T--C--A--C--A---T-AMA---C-A--A--A--A--G-AAG-ATTAMACAGC-GACG-----C--

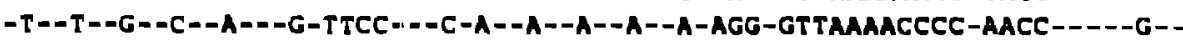
$-T--C--G--T--G--G-M A C---T-A--G--G--C--A-G G C-C A C T C C T G G G T-A G A A----C-$

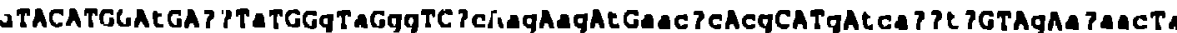
A--_--T--TT-A--G-A-GT--TC-AG-AA-T-AACAC-CC--G-CAMATTA--G-ACAGT-A G------ -T--CC-C---G-A-GA--AC-AG-AG-G-GTCCA-AG---G-TCAGCTA-- -C-AACAC-T A-------T--CC-A---G-A-GG--TC-GG-AG-T-AATAC-CG---G-TCGGTTG---G-ACAAC-A

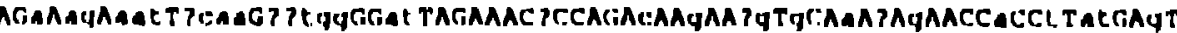

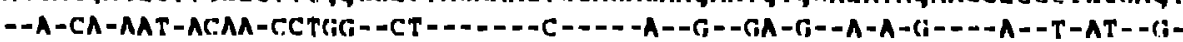

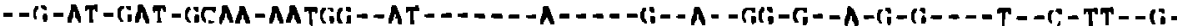

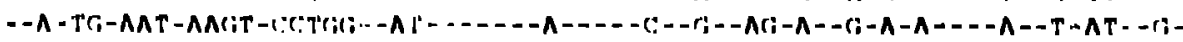


CONSENSUS SIVAGMTYO SIVAGMIS5 SI:AGM3 SIVAGM677

CONSENSUS SIVAGHTYO SIVAGH155 SIVAGM SIVAGH677

CONSENSUS SIVAGMTYO SIVAGMIS5 SIVAGM3 SIVAGH677

CONSENSUS SIVAGMTYO SIVAGMISS SIVAGH3 SIVAGM 677

CONSENSUS SIVAGHTYO SIVAGMI55 SIVAGM SIVAGH 677

CONSENGUS SIVAGMTYO SIVAGM15.5 SIVAGHJ SIVAGM677

CONgEnsus SIVAGHTYO SIVAGMISS SIVAGHJ SIVAGM677

CONSENSUS SIVAGMTYO SIVAGM155 SIVAGMJ SIVAGM677

CONSENGUS SIVAGHTYO SIVAGHISS S [VAGM] SIVAGMG7)

CONSENSUS SIVAGMTYOS :S IVAGMLIS DIVARial 'iIVAr:MhI'

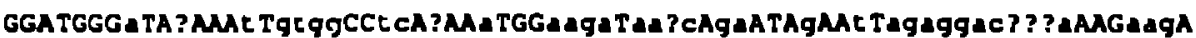
-------A--C---C-TTGG--TC-C--A---GMC-AMGC-GA---C--C-GGAGGA . . . A---ATG-.-1-h--T---T-ATGG--TC-T--A---AAGT-ACAA-GT---G--T-AGAGAMG. . A---AMC---1--A--C---T-GTGG--AC-C--G---CAGA-AAGC-GC---G--T-AGAAGAC. . A---AAG$------G--T--T-G C A T--G A-T--A---A C C A-T A A C-M A---G--T-A C C C C C C T T A G---G A G-$

ATGGAC?GT AMALgALATACAgAa?ETAGTAGggaACTMATTGGGCAgSACAAT TE TAECCAGGACTE

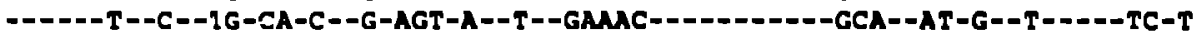
--O--A--G--TG-TC-T--G-AAT-G--A--GMAT-_-_-_-_-GCA--AT-A--T-_---AT-G

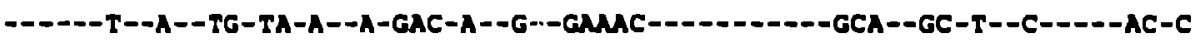

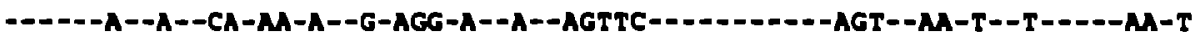

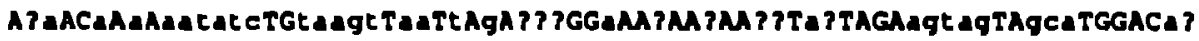
-AG--A-G-.. . ATA--CMAGT-A-T-E-GGA--A--G--A--TC-GT----GCTAG--GCT-----AC -GA--A-A-MATATC--TMGC-AC-T-G-... .--A--G--A--TT-AT--_-CGTGG--GM-----CC -GA--T-A-AACTTG--TAAAT-MA-C-G-. . . --A--A--G--CT-AC----MCAG--ACC-----AG -AA--C-A-CA TACC --TGCCA-GT-G-G-.., --G--A--G--CC-CC----AGAM--GTA--.--AG

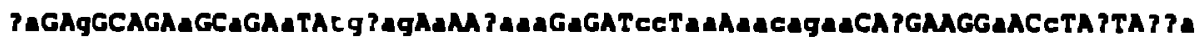
CT--G-----A--T--A--TGCAG-A--TGCA-A---TC-TA-AMCAGM--G-----A--C--T--CMA CA--G-----A--A--G--CGMG-A--CMAG-A---CC-MA-AACAGAG--A-----T--T--T--TGC AG--A-----A--A--A--TGCAG-A--CAM-A---CT-MA-AACGGM--G-----G--C--C--CM M--G-----G--A--A--TMGA-C--TCAA-G---AG-GC-GGMACA--A-----A--A--C--TGA

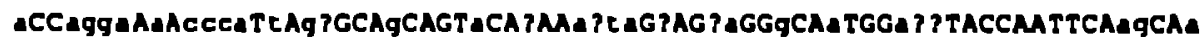
A--AGGA-T-CCTA-T-GG---G----A--G--ATTG-A--GA--A--G---AGT--_-_-_--AM--A A--AGM-A-CCCC-T-GG---G----A--G--ATTA-G--AT--G--A---TCA-_-------AG--G A--AGGA-G-CCCA-C-GA---G--_-G--A--ACTA-A--GA--T--A---AGT--_---1---AG--A C--TCTC-A-GMT-A-TA---A----T--A--GCMA-G--MA--G--A---ACA----------CC--A

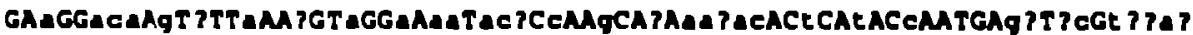
--A--ACA-G-C--G--A--A--A-MA-ACA-C--G--A-AGMAC--C--T--A--.--AC-TC-CACAT --A--AAA-A-C-..A--G--A--G-AG-TCG-C--A--G-MAGCT--T--C--C-----GT-GC-TGTAC --G--ACA-G-A--A--A--A--T-MA-ACA-A--G--G-AMAC--T--T--C-.---GT-CC-TGTAT --A--GCC-G-A--A--G--G--A-GA-ATG-C--G--A-GAGAA--T--T--T-----TC-A-GACTC

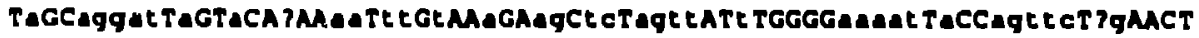
-A--TCGTT-A--G--G--GA-TT-C--A--AG-TC-AGTT--T-----GATAT-A--AGTTC-AG----A--AGGAG-A--.'--G--AA-AG-G--A--GG-CC-AGTA--T-----ACMT-A--CACTT-TG----G--AGGAT-A--A--A--AC-TT-T--A--AT-TT-AGTT--A-----AGAGT-G--ACTCC-TG-..-A--ACACC-T--C--A--AA-CT-T--G--AG-AC-TACC--T----AAGAC-T--ACGAG-AC--.-

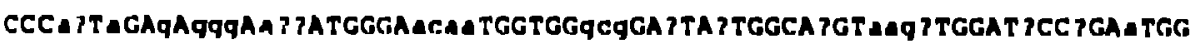

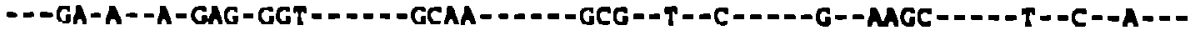

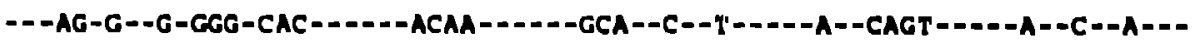

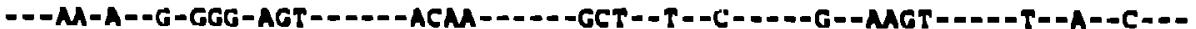

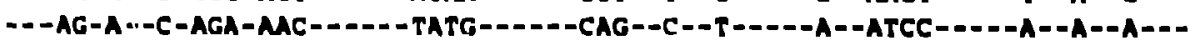

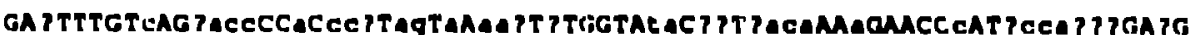
--T-----C--CACC --A-CTT-GC-C-MAC-A-----CA-AT-AACA--A-----C--ACCCAAO--G--C---_-C--TGTT -G-CCT-AG-A-CTT-G-.-.-TA-AC-GACT--G-----C--CCCGGGA--G--A-----C--TACC--A-CCC-AG-A-AAT-A--.--TA-CC-GACA--A---_-C--ACCAAAG--A--G--.-T-T-CACA--A-TCC-AG-A-AAC-G----TT-CT-AGTA--A-_-_-A--CMMAGGA--A-

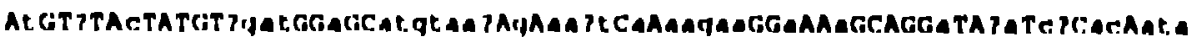
-C--T--C:----A. . .--A--ATGiCAnC: - (;-AATT-A-AACiAn--A--A-----A--CA-CT-AC-ATA

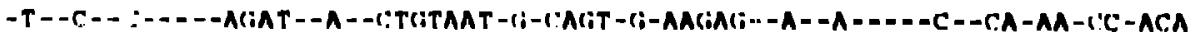

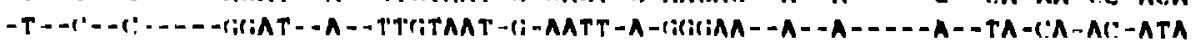

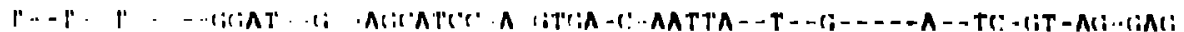


CONSENSUS SIVAGMTYO SIVAGM155 SIVAGM3 SIVAGM677

CONSENSUS SIVAGMTYO SIVAGHISS SIVAGM3 SIVAGM677

CONSENSUS SIVAGMTYO SIVAGM1SS SIVAGM3 SIVAGM677

CONSENSUS SIVAGMTYO SIVAGM155 SIVAGM SIVAGM677

consensus SIVAGMTYO SIVAGM155 SIVAGMJ SIVAGM677

COHSENSUS SIVAGMTYO SIVAGMIS! SIVAGMJ SIVAGM677

CONSENSUS SIVAGHTYO SIVAGHISS SIVAGM

SIVAGM677

CONBENSUS SIVAGHTYO SIVAOHISS SIVAGHJ SIVAGMG7!

CONSENSUS SIVAOMTYO giVAGMISS SIVAGMJ SIVAGMG\%

CONSF.NSUS Sivacimtyo :DIVAcimls

:AVACIMI

alVA:Mall

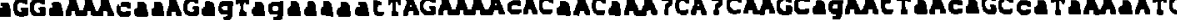
C--A---CAG--AG-AGAACAT--------C--T--C--T--G-----AA--T-A-CA--TA-A--A--A--C---CAA--AG-ACAACAGC--------C--A--A--T--A-----TG--C-G-CA--CA-A--A--T--G---CAA--GG-GGAMMAT-------T--A--A--C--G-----AG--T-A-TG--CA-A--A--A--A---AGT--AA-TAGGGAAT-------C--C--I--C--A-----AG--T-A-CA--AG-T--G---

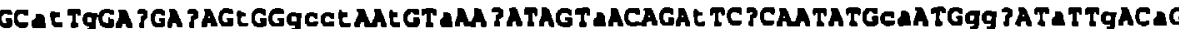
--TT-G--A--C--T--GCCT--T--G--C-----A-----C--T-------CA---GGA--T--G--A--CT-G--G--T--C--CCCT--A--C--T-----A-----T--A---D---CG---GGC--A--G--A--AC-A--A--T--T--GCCT--T--A--C-----A-----T--A-------CA---GGA--A--A--T-

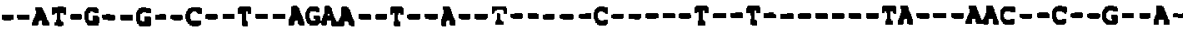

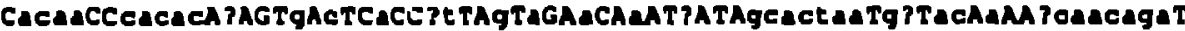
-ACMA--CACAC-A---G-T--A--AT--G-A--G--A--T---GCCTTMA-GA-AC-A--GCACAM-ACAG--CACAC-G---G-C--C--AC--G-A--A--A--A---GCACAGA-GG-AC-G--AGAAGCCA-CCMA--CACAC-G---G-C--A--CT--A-A--A--A--T---GCACTAA-GG-AC-A--ACATCAGA-ATGT--ACAGG-A---A-C--A--CT--G-G--A--G--A---CAMGCCC-M-GA-A--GAGGCAGG-

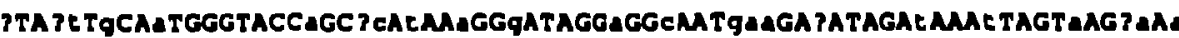
A--TT-G--G--------A--AC-T--A--A-----A--A---GAG--G-----T---T----G--TA-A

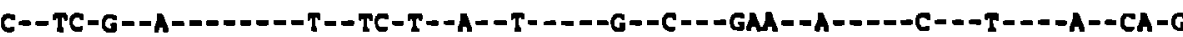

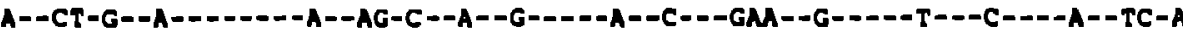
C--CT-A--A-------A--TC-T--G--G-----A--C---ACA--A--.--T---T----A--CA-A

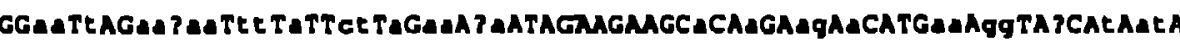
--CA-T--MGAG-TT-A--CT-A-M-AA---_-_-----T--A--MA-G-.--M-GA--T--T-AT--AG-T--MGMA-AT-G--CA-T-GC-GG--_--1---A--A--AG-A----AT-GG--T--C-GT--GA-G--GMMA-TT-A--TT-A-MA-MA--_-------C--G--GG-A----AA-GG--C--T-AT--MA-A--ACAGA-CC-C--CT-A-AT-GA---1-----A--A--TG-C----CA-AG--C--T-AC-

At TGGAgAAA ? CTAGCAgACACAT ?TGG? ? TACCAOAAATAGTAGCAMAGA PATAGTAGCCAEGTGECC -T----AA-ACC-A-C-G-TACA-A---GC-T--AC-A--A--A--A-----G--A--G--CATG--T--C----GA-ATC-A-C-G-CACA-T---AT-G--AC-A--A--A--T-----A--T--A--MATG--C--T----GG-ACT-A-C-G-CACT-A---GC-A--AC-A--T--G--A-----A--A--A--CATG--T--T----GA-GTA-G-T-C-GGA-T---AT-A--TA-T--A--A--A-----G--A--A--GGCA--T--

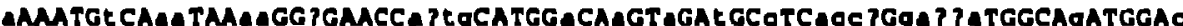

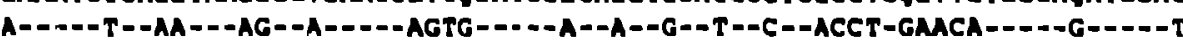

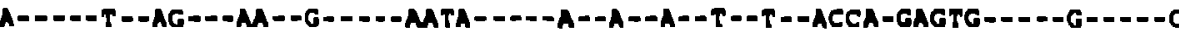

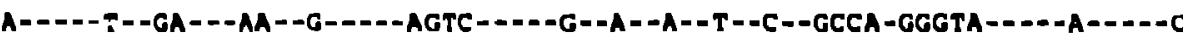
C-_..--C---MA---GA--A-_---TAAG-..---A--G--A--C--C--CATT-AAACT-..--G-----C

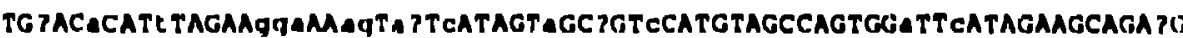
TG

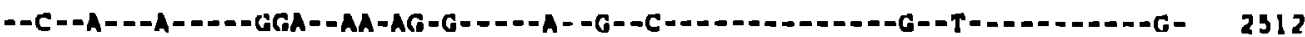

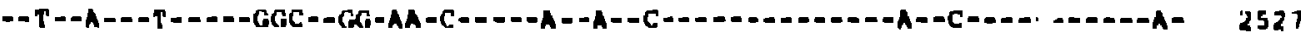

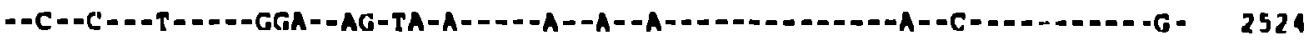

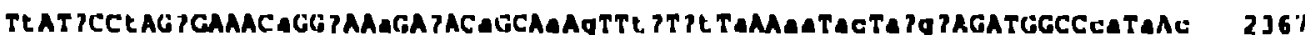
-C--A--T--0-----A--A--A--A--G-..-A-G--TC-AT-A--AA-AC-OAGT--.-----TII-A-C 2612

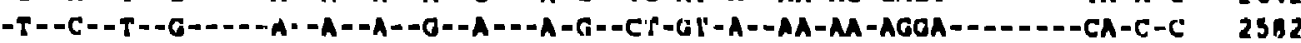

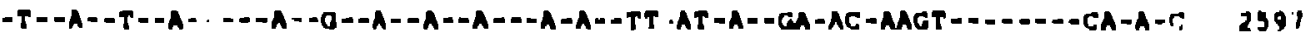

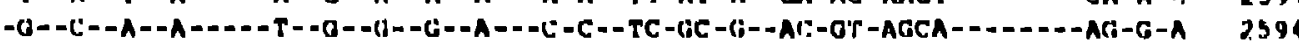

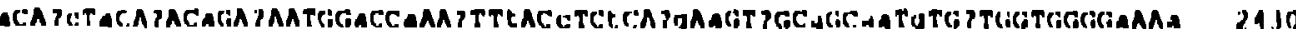

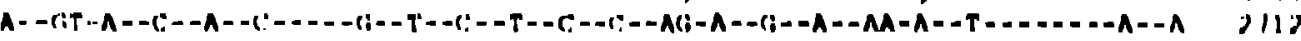

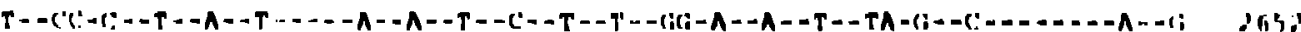

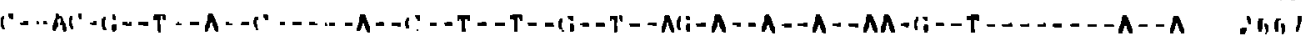

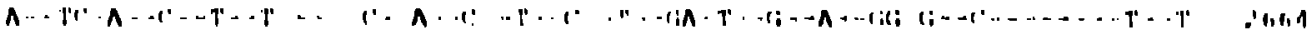


CONSENSUS SIVAGMTYO SIVAGK155 SIVAGM3 SIVAGM677

cONSENSUS SIVAGMTYO SIVAGM155 SIVAGH3 SIVAGM 677

CONSENSUS SIVAGHTYO SIVAGMLSS SIVAGH3 SIVAGH 677

consensus SIVAGHTYO SIVAGH1SS sIVAGHJ SIVAGH677

CONgENgUS SIVAGHTYO SIVAGH 55 SIVAGH 3 SIVAGH677

CONSENSUS SIVACMTYO 5 IVACH155 SIVAGH 3 SIVAGH677

CONSENGUS SIVAGATYO SIVAGMIS SIVAGM SIVAGM 677

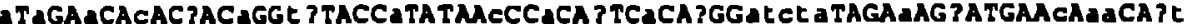
A-T--A--T--A--A--TA----A-----C--C--A--T--A--ATCAA----A--C-----C-AG--AT G-A--A--C--IY--G--GG----A-----T--A--G--C--G--ATCTA----A--T-----C-AA--AT A-A--A--C--C--A--TG----C-----C--T--A--A--A-OCTCTA----G--T-----T-AA--GT A-A--G--C--C--T--MA----T-----C--A--G--A--G--TAGTG--_-A--C-----C-GA--GC

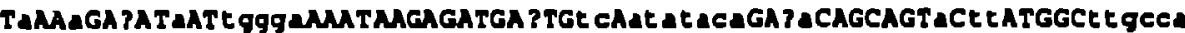

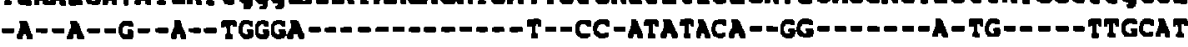

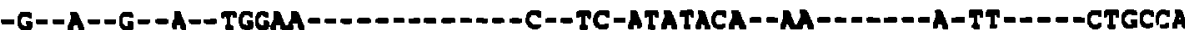

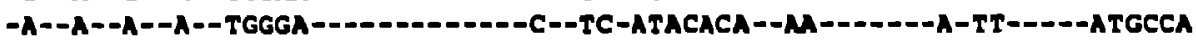
-C--G--A--C--CTCTC----_--------T--TG-GAGATTG--GA-------G-MA-----TACGCA

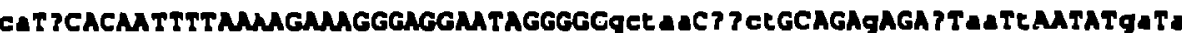
АC-T-10.--

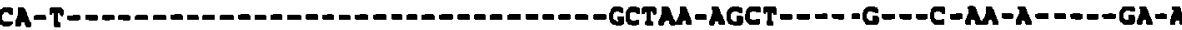

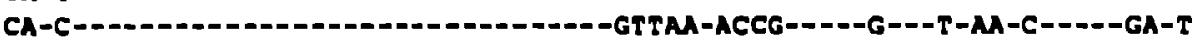

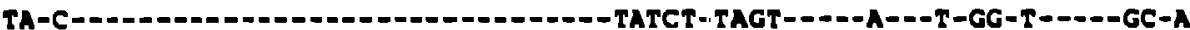

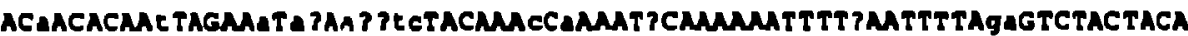

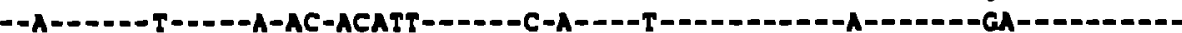

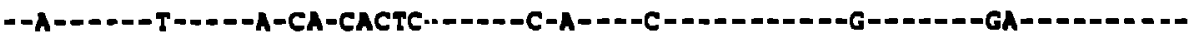

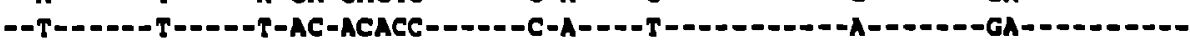

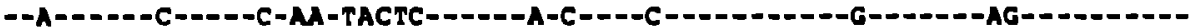

GAGMAGgIGAGACCCRGTqTGGMAGGACCAGOAC7A ITAATOTGGMMGG PGMGGCGC JGTGGTCRT -

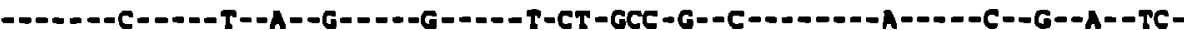

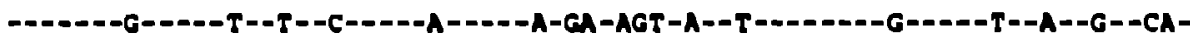

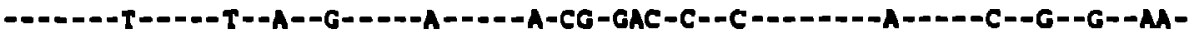

\section{vit eds $\rightarrow$}

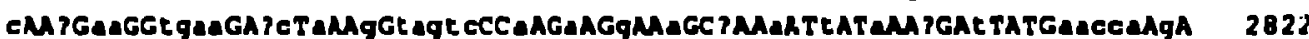
C--G-AC--MAGT--CC-A--G-TTGTA-OA--A--G--A--T--A--T--T--G--T--.-MCCC-A- 3132 C--G-MA--TGM--AC-G--G-TAGTT--G--A--G--A--A--A--C--A--A--C-.--AGCCA-G- 3072 C--A-GA--TGTG--AT-A--A-MTAC--A--A--G--A--A-A-A--T--A--G--T----MACCA-G- 3007 T--A-AG--GGM--CA-C--G-TAGTC--C--G--A--G--T--O--T--C--A--T-.--GAGAO-G- 3004

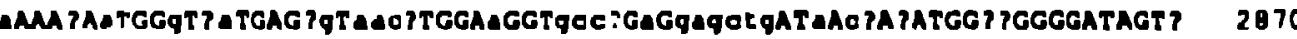
C---G-G---G-AA--...GG-GACG----A---ACCA-G-GATCTG--A-C\#** 3186

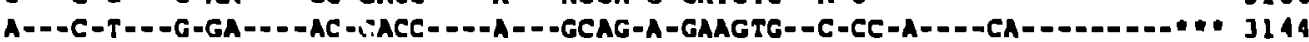
A---G-A---G-GA----AG-AACT----A---GCCG-A-GAGCTG--A-C\#..

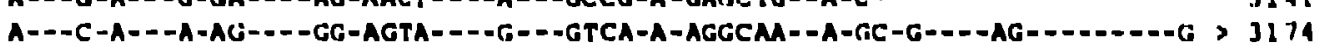


AGM VIF CDS

pol cda ->

CONSENSUS ATGAaCCAAGALAAA ?AATGGgT ?ATGAG?gTAAC?TGGAAGGTgCC?GAGgAgCLgATAAC?ALATGGC SIVAGMTYO ---AACCC-A-C---G-G---G-MA----GG-GACG----A---ACCA-G-GATCTG--A-CT-A----C SIVAGM155 ---AGCCA-G-A---C-T---G-GA----AC-CACC----A---GCAG-A-GAGTTG--C-CC-A----C SIVAGM3 ---AACCA-G-A---G-A---G-GA----AG-AACT----A---GCCG-A-GAGCTG--A-CT-A----C SIVAGM677 ---GAGAG-G-A---C-A---A-AG----GG-AGTA----G---GTCA-A-AGGCAA--A-GC-G----A

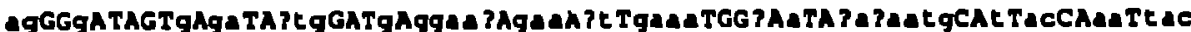
AG--A-----C-GA--TTG---G-GACAA-GMA-TT-GMA---A-C--TTATATG--T-AC--AA-TAC AG--G----T-GA--TTG---G-ATMM-GGA-TC-GMM---G-A--CNMAT--T-AT--MA-CAC MA--G-----G-GG--CTG---G-GGACT-GMA-AT-AGAC---A-A--TCGMATG--C-AC--AA-TAC SIVAGM677 GG--G-----G-CT--CAA---C-GGMT-MAC-AT-GCCT---G-A--CACACAT--T-GG--GG-GCA

CONSENSUS

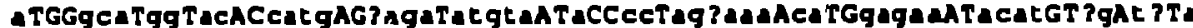
SIVAGMTYO SIVAGM155 SIVAGM3 A---GCT-GG-AC--CATG--TAGA-ATGTA--A--AA-AGGAAA-CA--GGGAA--ATGT--AG-CC-A T---GCA-GG-AC--TATG--CAGA-ATGTA--A--CC-CCCAGG-AG--GAGM--CCAT--GG-TA-C SIVAGH 677 A---GCA-GG-AC--MTG--TAGA-ATGAG--A--CC-AGGGCA-CA--GMGT--ACAT--AG-TC-A A---CAG-TI'-GG--CTAC--CCAG-TCATT--C--CT-ATCAMA-GA--ATTAC--AGA--GA-TA-T

CONSENSUS SIVAGHTYO SIVAGM155 SIVAGM3

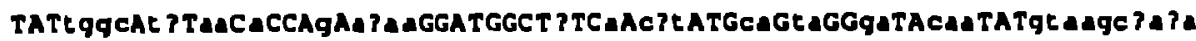
---TGGC-TT-MA-A---G-GCMA---_-----A--C-CAT---CA-TA--TA--CMA---GTAAGCAATT ---TGGC-TT-AG-T---A-ACAA---_----C--A-CTT---CA-TA--MA--CAA---GTTAGCCTAG ---TGGC-TC-GA-A---G-MAG---_----A--A-CAT---CT-AG--GA--CAG---CTMAGCAATA SIVAGM 677 ---CACA-CC-CA-C-.-G-MGA--.--.---C--A-GTC---GA-TA--GT--TCC---TACCATCMA

CONSENSUS SIVAGMTYO SIVAGMIS5 SIVAGM3 SIVAGM677

CONSENSUS SIVAGMYYO SIVAGMISS SIVAGM3 SIVAGM677

CONSENSUS SIVAGKTYO SIVAGMISS S IVAGMJ SIVAGM617

CONgEnsus SIVACKTYO SIVAGMISS SIVAGHJ sIVAGM67"

c:ONgRnsus SIVAGHTYO sivachl SS aI VAcim J GiVAcima I

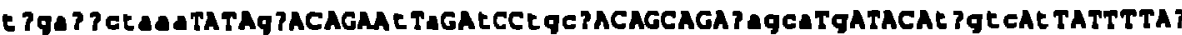

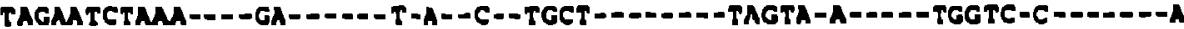

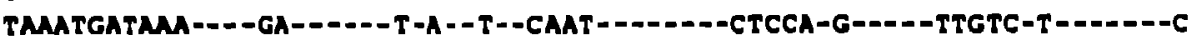

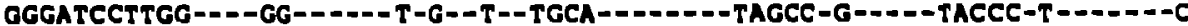

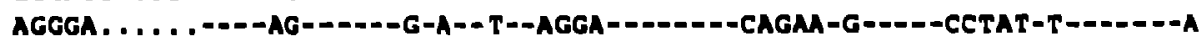

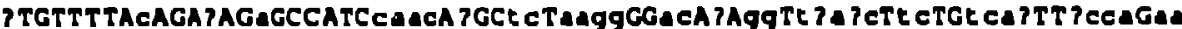
P--.-_-A---A--A--_---CMAC-A--TC-GAGG--CC-C.-GA-TTGTC-TC--TCAG--TCCA-AA C-....--C-.-T--A-.--_-CAAC-G--AC-MAGG--AA-C-GG-TCATC-TC--TCMA--TCCA-GA T--.---C---A--G-.----AGGA-A--CC-ATTG--AC-G-GG-TCACC-TC--TCAG--CCCC-AG C--.---C---T--A------CAAC-G--TA-CAGA--GG-G-AG-ATACG-GG--CACA--CMG-AA

vpX cds $\rightarrow$

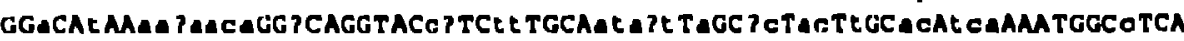

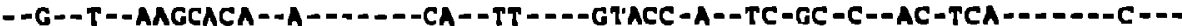
--A--T--ACTMCA--T-..--_--CC--CT----ATATT-A--AT-AC-A--CC-TCA-------C--. --A--C--GAAAACA--A---..---CC--TT----ATACT-A--TC-CC-T--AC-CCA---.----C-..

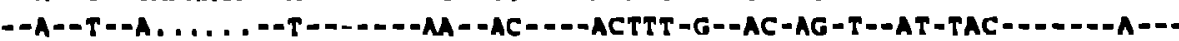

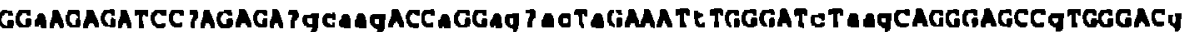
- -

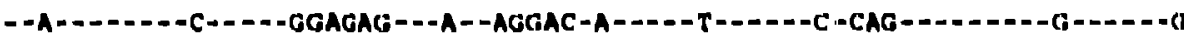

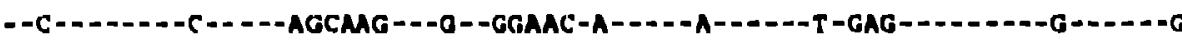

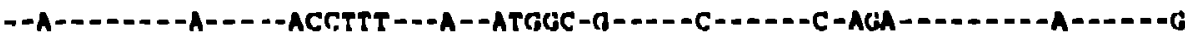

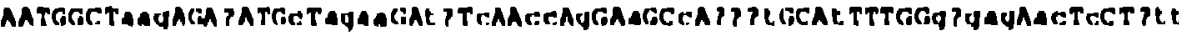

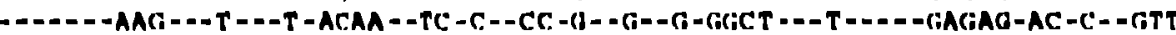

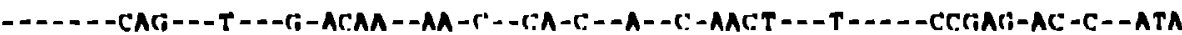

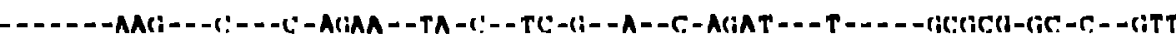

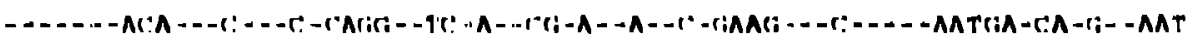

140

194 210 
AGM VIF

CONSENSUS CCAAGTATGGATTA?TGTCAgGAGGA?GGgaPAGACALgGPACtCCCATg?tgGAMGGGC?TA??? 63B

SIVAGMTYO --A--------C--C---CAG-----A--AGMA--AC-TG-T-CT----GATG-------C--CM $>70 B$

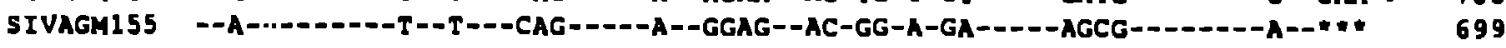

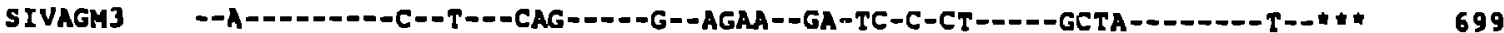

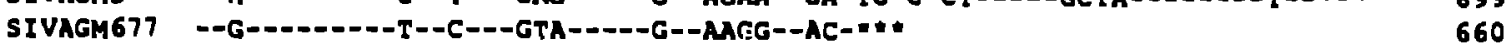

I.C:-12 
AGM VPX CDS

vif cds $\rightarrow$

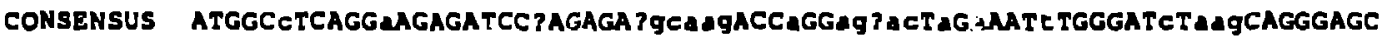
SIVAGMTYO -----C-----G--------A-----GGCMG---A--AGMG-A-----T------C-AAG--------

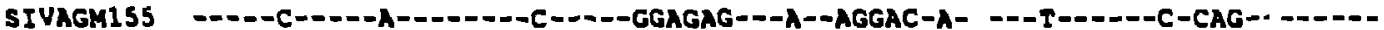

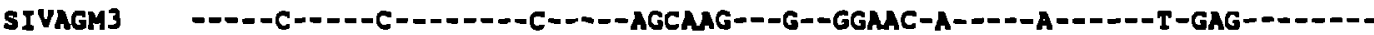

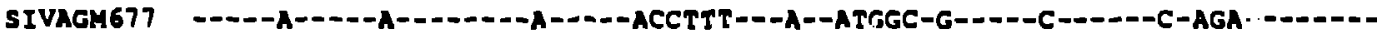

CONSENSUS

SIVAGMTYO

SIVAGM155

SIVAGM3

CgTGGGACgMTGGCTAaqAGA 7ATGCTAgAaGA 7T CMACCAgGA GCCA ? 7 tGCAETTTGGg?gagA

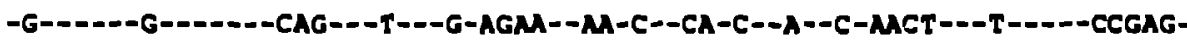

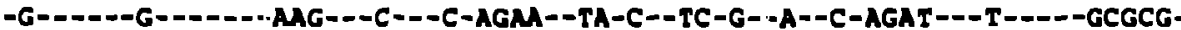

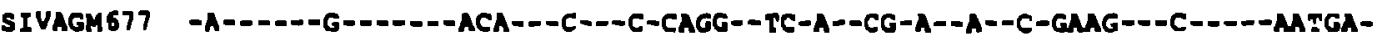

CONSENSUS

SIVAGMTYO

SIVARM155

SIVAGM3

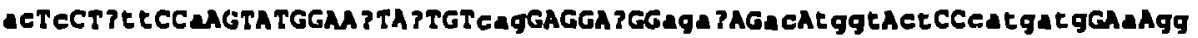
AC-C--GTT--A--_-_--_-C--C---CAG-----A--AGM--AC-TGGT-CT--CATGATG--A-GG AC-C--ATA--A-------_-T--T---CAG-----A--GGAG--AC-GGGA-GA--CATAGCG--A-GG GC-C--GTT--A-_-_.-_-_-C--T---CAG---_-G--AGMA--GA-TCGC-CT--CATGCTA--A-GC

SIVAGM677

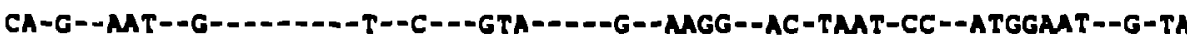

<- vil

CONSENSUS SIVAGMTYO SIVAGM1SS SIVAGM3 SIVAGM677

GccTA ?A ?TAE TAT -CC--C--A--T--.-AGGC-A--A--A--GG--C-C-----G-----TC-A-----G--CA-G--A--GC $-C A--T--G=-T---C G C T-A--T--G--A G--C-C-----G-----C C-G=----\lambda--T C-C--G--A C$ -CT--T--A--T---AMAT-G--G--A--AG--C-C--_--G-.-_-CC-G-----A--CC-C--A--AC $-G C--C=-G=-C---A G M-T--T--A--G T--A-G=----A=----C A-A-----T--T A-A--G=-A G$

tat eds $\rightarrow$

CONBENSUS SIVAGMTYO SIVAGMISS SIVAGH3 SIVAGM677

\section{Eat edagagaratgo}

DatGGACAAGggGagGangagCaggaCg?gteCCaCCAGg

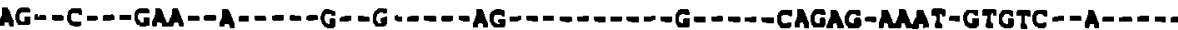

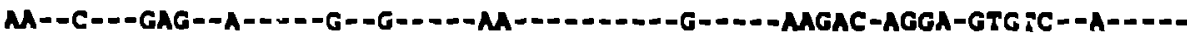

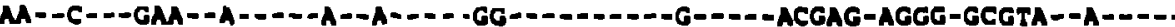

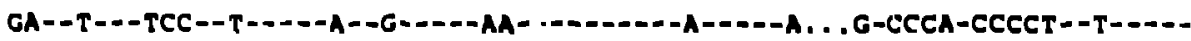

CONSENSUS SIVAGMTYO

ACTTGat... 336

-D-AG-D 360

SIVAGMISS -.-.-AT-2 300

SIVAGM] -----AT--- J60

SIVAGM677 --.-CA-C 337 


\section{AGM TAT}

AGH TAT CDS

\begin{tabular}{|c|c|c|}
\hline $\begin{array}{l}\text { CONSENSUS } \\
\text { SIVAGMTYO }\end{array}$ & 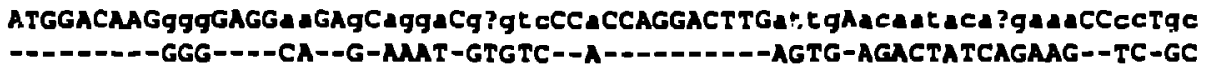 & $\begin{array}{l}68 \\
70\end{array}$ \\
\hline SIVAGM15S & --------GGG----MA--C-AGGA-GTGTC--A---------ATTA-ACAATACAGGAAA--CC-TG & 70 \\
\hline SIVAGM 3 & ---DG---AC--G-AGGG-GCGTA--A---------ATTG-ACMACTCAAGCA--CC-GA & 70 \\
\hline SIVAGM677 & - & 55 \\
\hline CONSENSUS & 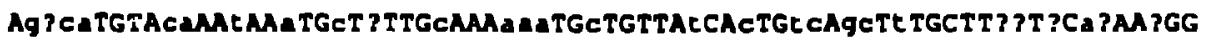 & \\
\hline SIVAGHTYO & -GRCT----MA--T--A--T-T---C---MAA--T-----C--C--TC-GC-T-----TC-G-AA--A-- & 140 \\
\hline SIVAGM155 & -GACA----CA--I--A--C-T---C---MAA--C-----T--T--CC-AT-C-----CT-A-ÜG- -A-- & 140 \\
\hline SIVAGH3 & -GCGG--- -CA--C--G--C-A---T---TGT--C-----T--C--TC-GC-T-----TT-A-AA--G-- & 140 \\
\hline \multirow[t]{2}{*}{ SIVAGM677 } & $-A C C A----C T--T--A--C-A---C---M A A--C-----T--C--T G-G C-T-----C C-G-A G--G--$ & 125 \\
\hline & rev cda $\rightarrow$ & \\
\hline ONSENSUS & ग7TAGGLgTEACCTATCATGCC?CTAGGACCAGMAGMAgAagAEtgCT?eg?tT?ATCgCgttCCTgEA & 195 \\
\hline SIVAGHTYO & 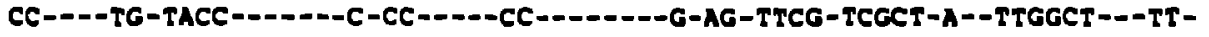 & 210 \\
\hline SIVAGM1SS & 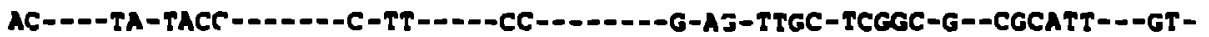 & 210 \\
\hline 5IVAGH3 & 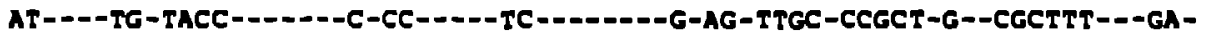 & 210 \\
\hline \multirow[t]{2}{*}{ SIVAGM677 } & CT---AG-GCGT-----T-TC----AA---D-A-CA-GCAC-CMAGA-A--CAMGAC---AT- & 195 \\
\hline & exon Y/exon & \\
\hline ONSENSUS & CAgCAgCAATCCATATCCAECCgt ? ? ?ggAAGGgAC?CCCAGACAACGCAG ?AG PGCCAG? AgAAGGTGG & 258 \\
\hline IVAGATYO & 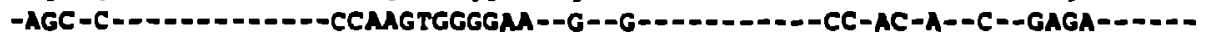 & 280 \\
\hline SIVAGM1SS & -CGC-C-1-CI & 277 \\
\hline SIVAGH3 & 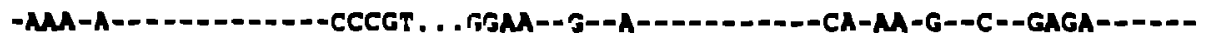 & 277 \\
\hline SIVAGH677 & 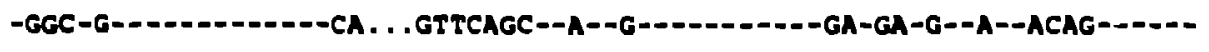 & 262 \\
\hline CONSENSUS & 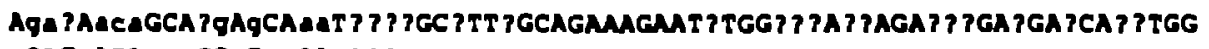 & 307 \\
\hline SIVAGMTYO & $-G A C-A C A---G G-T--M A-\cdots$ & 303 \\
\hline SIVAGM155 & 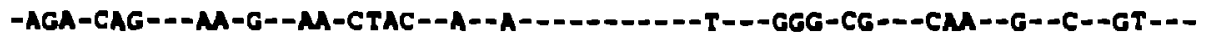 & 347 \\
\hline SIVAGM3 & 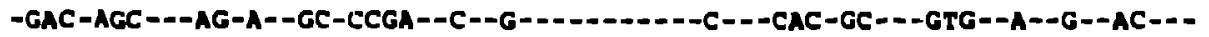 & 347 \\
\hline SIVAGH677 & $-G A A-G C A--G C-G--M A-\cdots$ & 285 \\
\hline 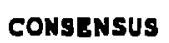 & CATGCTAT"* & \\
\hline SIVAGMISS & $---G--A--D-$ & \\
\hline SIVAGM3 & $----A--G-----$ & \\
\hline
\end{tabular}


AGM REV CDS

\begin{tabular}{|c|c|c|}
\hline & & \\
\hline DNEENSUS & ATGCC?CTAGGACCAGAAGAAAgAagAttgCT?Cg?tT?ATCgCgttCCTgtACagCAgCAATCCATATC & 66 \\
\hline LVAGMTYO & 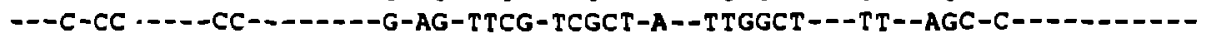 & 70 \\
\hline VAGM155 & 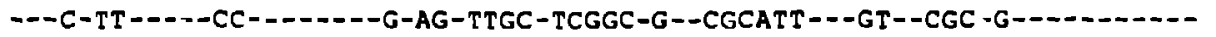 & 70 \\
\hline im3 & 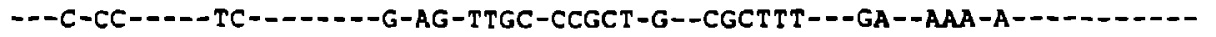 & 70 \\
\hline AGM677 & 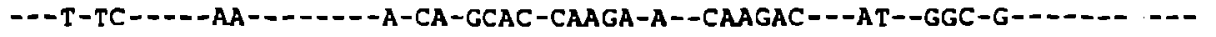 & 70 \\
\hline US & CAcccgtg???gaaGGgAC?GCCAGACAACGCaG ?aG?GCCAG?agaAGGTGGAga ?pr.CaGCA ?gAgCA & 127 \\
\hline VAGMTYO & --CCAAGTGGGGAA--G--G----1-----CC-AC-A--C--GAGA-------GAC-ACA---GG-T-- & 140 \\
\hline VAGM155 & --TCCGTG. . .GAA--G--A----1--1--CA-GA-A--C--AAGA-------AGA-CAG---AA-G-- & 137 \\
\hline AGM3 & -- CCCGTG. . GAA--G--A----1--1.-A-AA-G--C--GAGA------GAC-AGC---AG-A-- & 137 \\
\hline M677 & --CAGTTCAGC...--A--G---------GA-GA-G- -A--A & 137 \\
\hline NSUS & AaT?? ?agcet TgGCAGaAAGAatc? ? ? \&CaccagagaggAagAgCA ? CtgGTGcatgcadTTg & 189 \\
\hline AGMTYO & AA-TAGAGTCT-G-T--A---.... CTC. . . . . . . . C-AG-G--G...--TATGCAG--G-CCGC & 192 \\
\hline AGMI55 & AA-CTACGCAT-A-C--A----ATTTGGGGGACGAGACAAG-GG-C--GTTG---CAGGCAA--G-CCAA & 207 \\
\hline & GC-CCGAGCCT-G-C--A----ATCTGGCACAGCAGAGTGG-AG-G & 207 \\
\hline VAGM677 & TG---GCTCAAC--C-AGAG & 207 \\
\hline & 2TGgt & 243 \\
\hline ;MTYO & C--GCTGAC--GGCTC-AC-CT---CTAT-----GT-- & 255 \\
\hline M. & 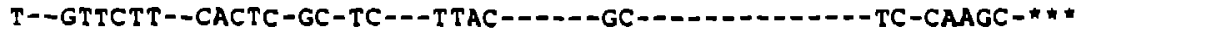 & 270 \\
\hline GM & 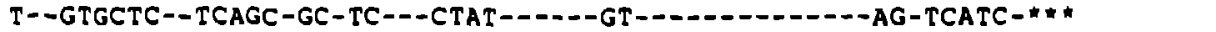 & 270 \\
\hline /AGM677 & 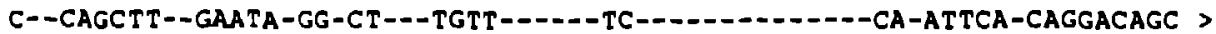 & 441 \\
\hline
\end{tabular}


AGM ENV CDS

CONSENSUS SIVAGMTYO SIVAGMI55 SIVAGM 3 SIVAGM677

CONSENSUS SIVAGMTYO SIVAGM155 SIVAGM3 SIVAGM 677

CONSENSUS SIVAGMTYO SIVAGM155 SIVAGM 3 SIVAGM 677

CONSENSUS SIVAGMTYO SIVAGMISS SIVAGM 3 SIVAGM 677

CONSENSUS SIVAGMTYO SIVAGM155 SIVAGM 3 SIVAGM 677

CONSENSUS SIVAGMTYO SIVAGM155 SIVAGM 3 SIVAGM 677

CONSENSUS SIVAGMTYO SIVAGMI 55 SIVAGM 3 SIVAGM 677

CONSENSUS SIVAGMTYO SIVAGM155 SIVAGM 3 SIVAGM 677

CONSENGUS SIVAGMTYO SIVAGM1SS SIVAGM 3 SIVAGM 677

CONSENSUS SIVAGMTYO SIVAGIMISS SIVAGM] SIVAGiM67"
ATGaggadg? ?at Taata???Tag?aaTAaTAgtaitAGGgATAgGadTAg?gatagtacadagca?C ---AGGTATACAA-AATAACCT-AGGA--A--GTG--.--A---G-GA---TGT-AAGT. . . AAG. . . ---ACAAAGTTCT-AGGAATTI-TATAG--T--GGA----G---G-AA---GGA-AAGTACAAAACAG---AAGCTGACAT-ACTG. . . A-AGGGA--C--TTA-----G---G-AG---TGC-TAA TACAAGGCAA---GGGAGATTGC-TATAAAAA-ACTAA--A--GCA-----G---A-TA---GAA-A. . GGT . . AAC-

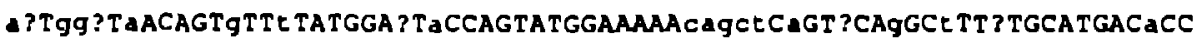

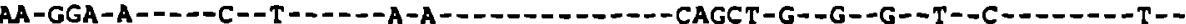

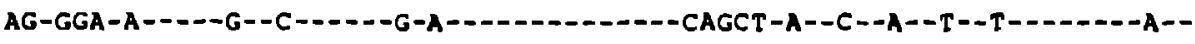

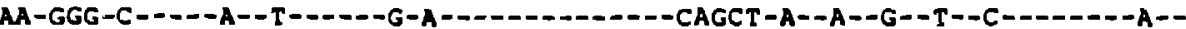

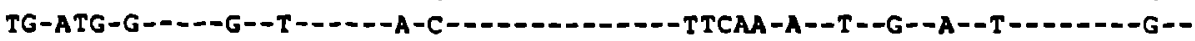

CAC ?ACCAg?t T?TGGGCAACLACLAA? TgCATACCAGATGATCATGACLALACAGA ?GT ACC? CTAAA?

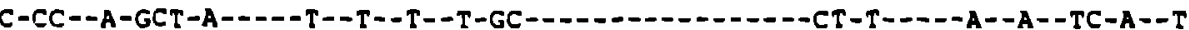

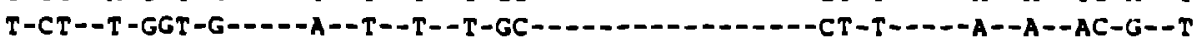

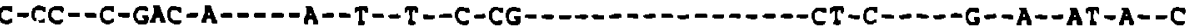

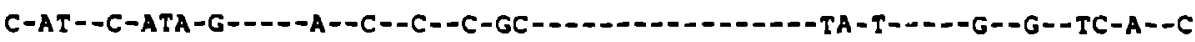

ATCAC?GAaCCaTTEGA ?GCaTGGgCtGA ?agaAA ?CCET TagTAgCACAAGCAGgaAGTAA ?ATaCA ?C --C--T--AC-A--T--G--A---GGT--TAGA--C- -AT -AA--GC--_-_-_-CC-----C--C- -TT --A--A--GC-A--T--A--A---GCA--CAGA--T--CT-AG--GC-_-_-_-GA-_-_-C--T--CC

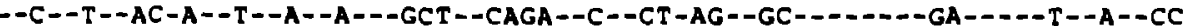

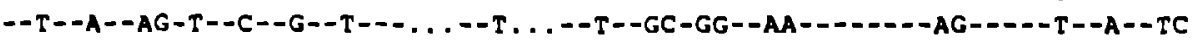

T?CTCTTTGA ?CA PACEATGA ACCE TGTGTAAAg ? T ATCACCLCTATG PAT?MAATG? ? ?TGTGTAGA -A--C-----G--A--TA-G-AA--T-----G--GT-A--A--AC-A--C--C--G---AAT---..--G--G--.--A--G--AT-A-AG--C-----A--GC-A--A--TC-A--T--C--A---AAT-1-1-...

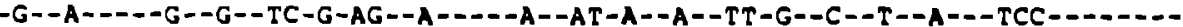

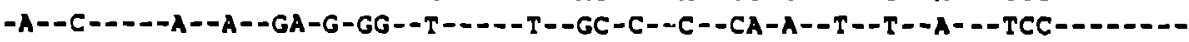

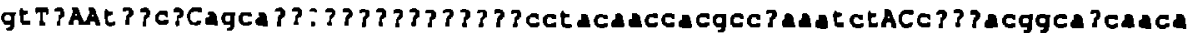
GT-A --TTCCA-AMGAGMAGGGCGACAACACCTACMACGACGCCGAATCT--C........... GT-A--AGGCT-CGCA. . . . . ACCTCTACCCCAGCAACC. . . . . . . TCT- T. . . ACGGCAGGAACC AT-G--CTCCT-TGAG........... CCTACCACCACTCSTAAAAGT--C... ACGCCCTCAACA

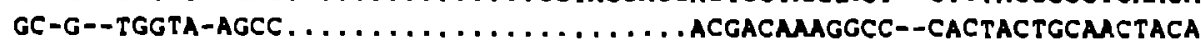

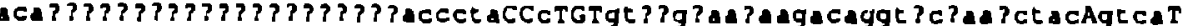
................. GGCCTA--C---gTAGGCCCGACGTCAGGTGAAAATCTAC-GTCC-

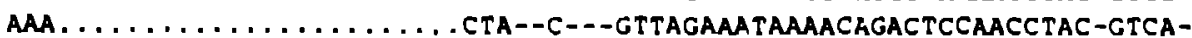
ACCAATATCACAGCC TCAACAACCACTTTG--G---GTCCAGAACAAGACAAGT'ACTGTGTTAG-ATCA-

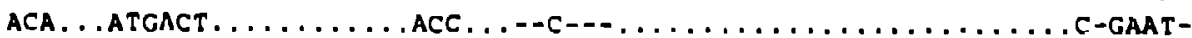

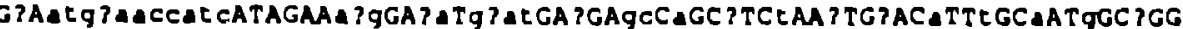
-T-ATGCAAGCATT-------AGG--GA-GGAG--T--GC-C--C- -T--T--T--A--C--A--G--T--C-ACGACACCATC------AAG--GA-GAAT--C--GG-A--G--A--C--C--C--T--T--G--T--

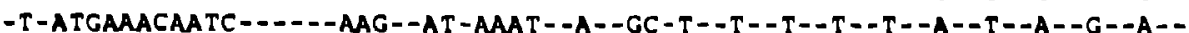
-C-GTACAGAGCAG-----GGA--AA-GGCA--G--AC-A--A--C--C--C--T--T--A--T--A--

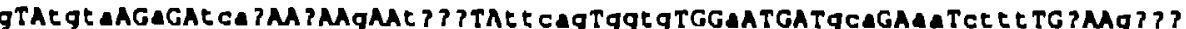
C--TGTA--A--TCAG--G--A--TTAT--TTCTG-GGTG---A-----GCA--AA-CTAT--C--AAAT G--CATT--G--CCAA--G--G--T. . --CTCAG-AGTA---A--.--GCA--MA-CTTT--T--G... G--TGTA--A--TCAG--A--G--G...--TTCAG-GGTG---A-----GCA--AA-CATG--T--G. . A--TCAA--A--TGTA--A--G--T. . --TAGCA-GACC---T----CAG--GT-AGTC--C--TAAT

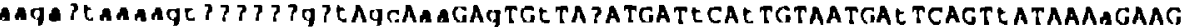

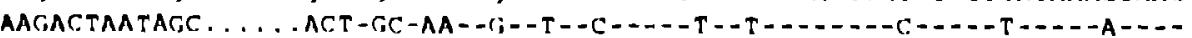

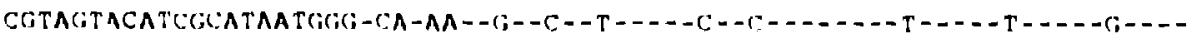

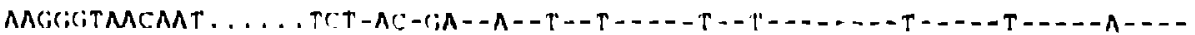

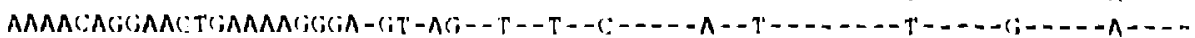


CONSENSUS SIVAGMTYO SIVAGM155 SIVAGM3 SIVAGH677

CONSENSUS SIVAGMTYO SIVAGM155 SIVAGM3 SIVAGH677

CONSENSUS SIVAGMTYO SIVAGM155 SIVAGM3 SIVAGM 677

CONSENSUS SIVAGMTYO SIVAGM155 SIVAGM3 SIVAGM677

CONSENSUS SIVAGMTYO SIVAGM155 SIVAGM3 SIVAGH 677

CONSENSUS SIVAGHTYO SIVAGMISS SIVAGH3 SIVAGM677

CONSENSUS SIVAGMTYO SIVAGH1S5 SIVAGM 3 sivach677

CONSENSUS SIVAGHTYO SIVAGHLSS SIVAGHJ SIVAGM 677

CONSENSUS SIVAGHTYO SIVAGHISS SIVAGMJ SIVAGM677

CONSENSUS SIVAGHTYO SIVAGM 155 S [VAC:M] GIVAGM6?

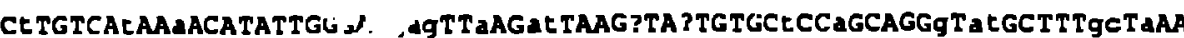

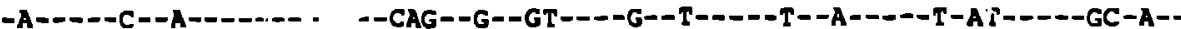

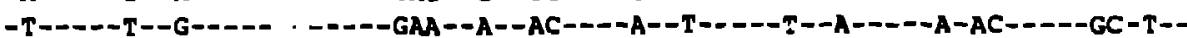

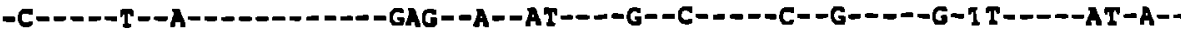

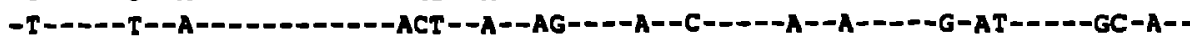

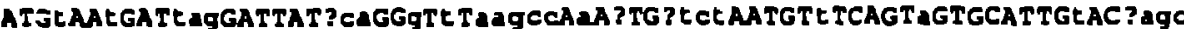
---T--T---GA------MAT--G-A-AMACA-A-T--CTCA-----A-----A--------T--AGGC -- T--T---TGG------GCA--A-T-AAGCC-G-A--TTCT-----T----A-------C--AACT ---C--C---TAT------GCA--G-T-AAGAC-A-C--TICT--_-T-_-_-G-_--_--T--TAAC

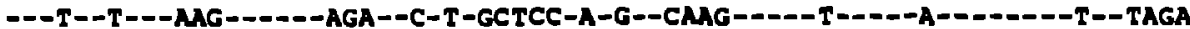

TTaATgMATACaCagT ?ACEAC rgggeTgtt ?t TgMAGGaAGCtat tCaGA ?AATCGACCCAGATaT --A--G-----A--AG-G--A--A--GT-GTTGC-G---.-A--CTATCAT--G---C----CC----A--A--G-----A--AG-A--C--T--TC-GTTAT-G-----A--CTATTCA--A---C----CC----C--G--A-----A--AG-G--T--T--AC-GTTGT-G-----G--CTACTCA--G---C----CC----A--A--C-----T--TA-A--T--A--GA-AGGAT-A----OT-OTAGATCA--A---A----AG----A-

GGCAgMAACat ?Gagt 2a ? ?MATgAC?CAGTgt TaATCt tgt TAMAMAgCat TA?MA ? CT ?aCAGTtAC ----G---CATA-GGTAAAT---A-CA----AT-GA-CTTGT-C--C--GCAC--T--TC-AT----C-C ----A---CATG-AGTGAGC---G-CT----GT-AA-CTTGC-C--T--GCAT--T--CC-GA----T-C ----G---CATA-AGTMAC---G-CT-_--GT-AG-GTTAT-T--T--ACAT--C--TC-MA----T-C ----G---GGAG-MATGAT---G-TA----TA-AA-MAGT-G--T--GTTT--C--CT-GA----G-G

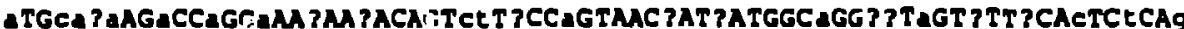
C--TAGG--A--A--A--C--G--.- CC-A--G-----G--A-----G--AC-G--T--C--C--T--A A--CAM--G--A--G--T--G-----CT-G--A-----G--A---D-A--AT-A--C--C--C--A--G

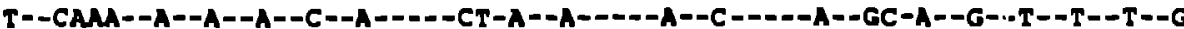
A--CCGA--A--T--T--T--A-----GT-G--A-----A--C-----A--GT-A--A--T--C--T--G

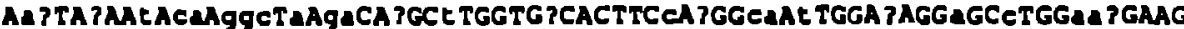
-M--C--C-TG-AGC-T-GA--G--T-----T------G-A--CA-T----G---T--C---CGG---AG--T--T-CA-GAC-A-GG- -G--C-----C------C-G--CA-T----A---A--T---AAG---

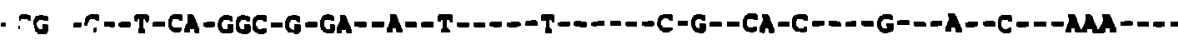

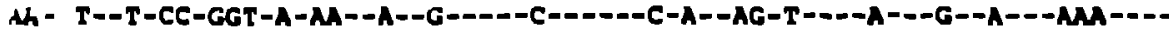

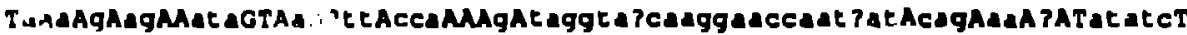
-AAA-C-AA--ATA---G-GTT-CCA---G-CAGGTATAMAGGAACCAATAAT-CAG-AC-C--ATACC-ACA-G-GG--ATA---A-ATT-CCA---G-ACGGTACCMGGCACCAATGAT-CAA-CA-A--CTTTT-MAA-A-TG--ATA---A-ATT-CCA---G-TAGATACCMGGAACCAATGAT-CTG-AG-G--TTATC-CAG-G-AG--. . . ---A-GMA-GTG---A-T. . . CTT. . . ACAGAG'MAGC-TAG-MA-T--ACATC-

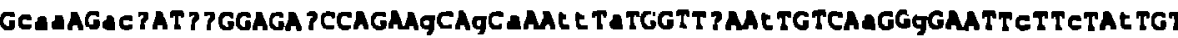
-CM--ACA--GG---.-C------G--T-C--CT-G-----T--T-----A--A-----C--T--T---

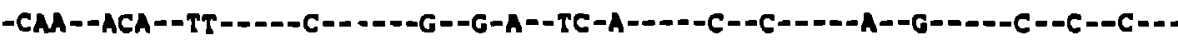

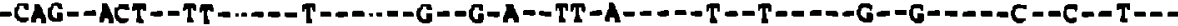

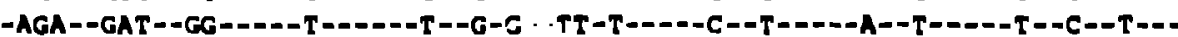

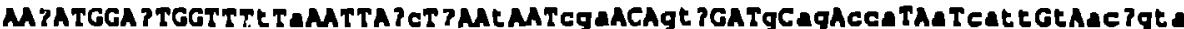
--G-----T---_--T-A--.--CT-A--T---AA---TGG---G-AT-CCA--_;-TTTT-T-GC...A --A-----C------T-A--.--TC-G--T---TTA---GTG---G-TG-TCA--A-CATT-T-MAMCA --A-----T---_--C-A-----CC-G--T---CGT---GTA---C-UG-CCA--A-CCGT-T-ATGGTA --G-_---C----_-A-C---_-TC-A--C---_'GA---GM---G-AG-AGG--C-MTA-G-CCTGTG

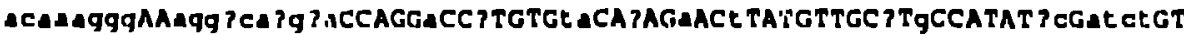

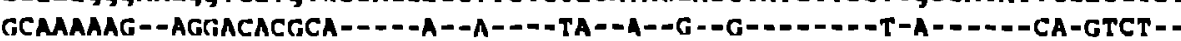

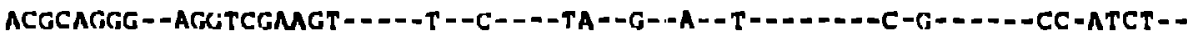

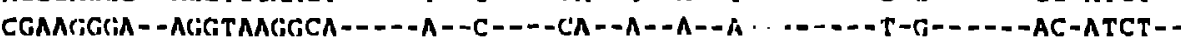

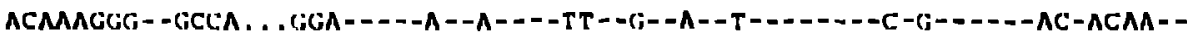


CONSENSUS SIVAGHTYO SIVAGH155 SIVAGH SIVAGM 677

CONSENSUS SIVAGMTYO SIVAGMISS SIVAGM3 SIVAGM 677

CONSENSUS SIYAGMTYO SIVAGMI55 SIVAGM 3 SIVAGM677

CONSENSUS SIVAGMTYO SIVAGMIS5 SIVAGM3 SIVAGM677

CONSENSUS SIVAGMTYO SIVAGHISS SIVAGH SIVAGH677

CONSENSUS SIVAGMTYO SIVAGM1SS SIVAGMJ SIVAGM677

CONSENSUS SIVAGMTYO SIVAGM155 SIVAGM 3 SIVAGM677

CONSENSUS SIVAGMTYO SIVAGMISS SIVAGM 3 SIVAGM 677

CONSENSUS SIVAGMTYO SIVAGMISS SIVAGM3 SIVAGM 677

CONSENSUS SIVAGMTYO SIVAGMISS SIVAGMJ S:VAG:467\%
?aT aAATGATTggTA ?ACL CT aTC AAagAadaCaTATGCtCC?CCAAGaGAAGGaCAt TTGCA ? TGCACg AA-A------CC--T--CC-A--A-AG--AACT-----T--G-----A-----A--T---C-A--C-GG

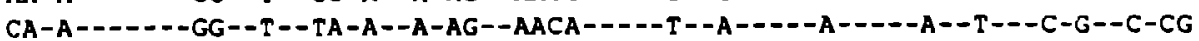
CA-T------GG--C--AC-A--A-GG--AACC-----A--G-----A-----G--C---C-A--C-CA AG-A--.---GG--C--TG-C--T-AA--GGTA-----T--A-----G-----T--T---G-G--T-AC

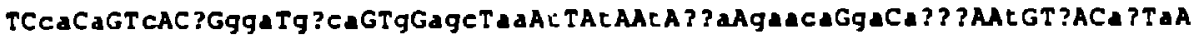
--CA-A--C--T-GGA-GACA--T-AGC-TA-T--T--T-GTA-AAACA-AA-C. . . --C--G--AC-A--CA-A--T--T-GGA-GACA--A-AGC-AA-C--T--T-ACC-GAACA-GA-A... --T--A--AT-G--CA-G--A--G-GTA-GTCA--G-AGC-AA-T--C--T-GTA-GAACA-GA-T. . - -T--A--AT-A--AT-A--C--G-CAC-ATAC--G-CAA-AG-T--T--C-ACA-GTCTG-CC-AATA--T--G--CC-A-

GTCCCCAGATAgAaCCAICTGGGCggCaGAATTGGGagaTACANTTAGTAGA ?ATEACACCAATTGG ----C---A--GAATCT--C-----GGCT---T----CAGA--C------A--G--A--C------------.--C---A--GAACC--C-----GGCP ---T----CAGA--C-----G--A--G--T-------------C---A--GAAACC--C -----AGCA---T----CAGG--C-----A--A--A--T--------T---G--CGCAGC--A-----GTAC---C----AGAC--T-----A--A--G--A----------

CTTEGCaCCCACAGAaGTAAG ? ?GaTATACgGGaggCCa ?gagAG?CAAAA ? ?GAGT CCCgTTCGTGCTA C--T--A--C-----A--A--GC-T--T--G--AGGACATGAG--GCA---GA-A--C--G--T-----A--T--A--C-----A--C--GC-A--C--G--AGGCCAAGAG--GCA---AC-A--C--G--C-----C--C--A--C-----A--A--AA-G--T--G--AGGTCATGAC--MAC---GS-A--C--G--C-----C--T--T--T-----T--A--AA-A--T--T--CCCCACA ... --AGA---AA-G--G--A--C-----.

GGGTICCTAGGCTTCTTGGGaGCTGCTGG ?ACTGCAATGGGaGCAGCGGCgACa TCCTGACgGTCCAGT

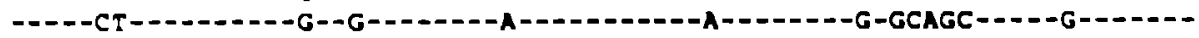

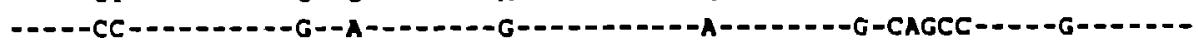

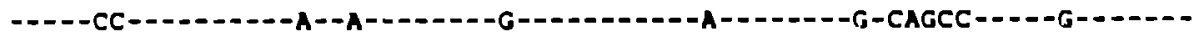

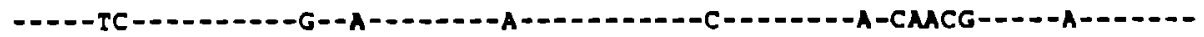

CLC PGCATTTPCTTGCTGGGATA PTGCAGCAGCAGMGAAETGCTGGCGGTGTGG ?gCLCAACAgCA

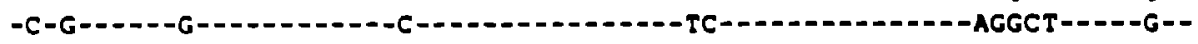

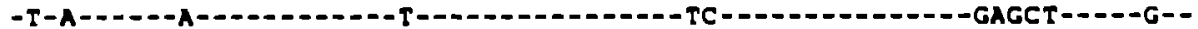

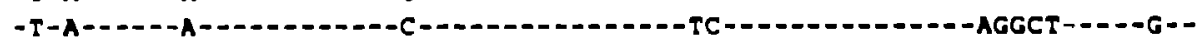

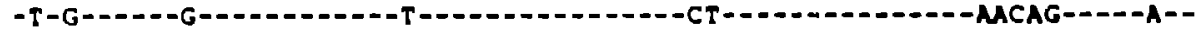

GaTGTTGAGCTGACCATTTGGGGTGGAMAACCTCMTGCCCGCGTCACAGCLCTLGAGAGTACCT.

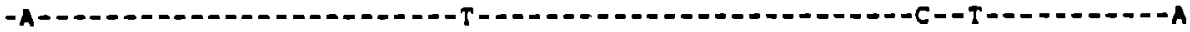

A-1

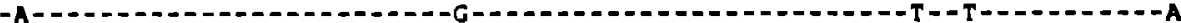

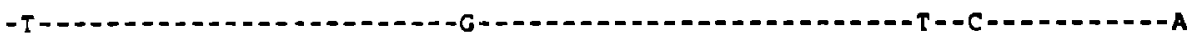

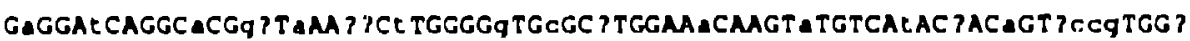
-A---T-----A--AC-A--CT-C-----G--C--A--.--A-----A-----T--C--A--GGAG---C

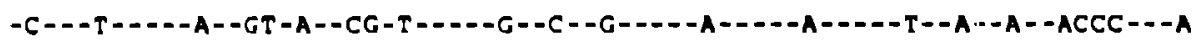

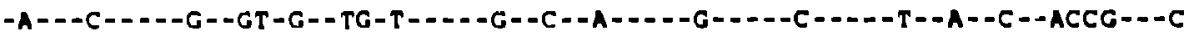

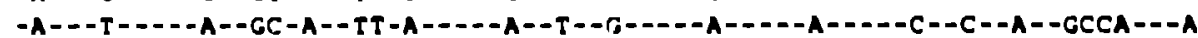

?gTggAaCAL ?? ?ACECCEgA?TGGAA AATATGACETGGETGGAgTGGGAAGACA?ATAg?tgat TT CC-GG-CA--TCGG--T--GG-T---C-A--------T---T----G-----A-G---A--AGCTGAT--

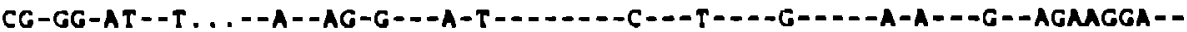

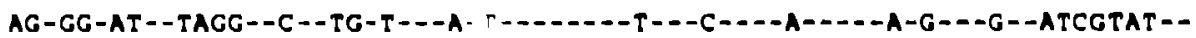

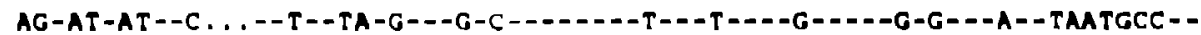

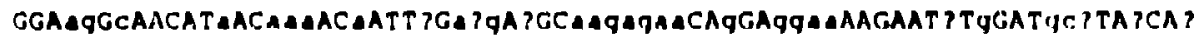

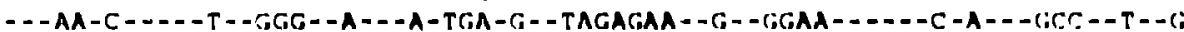

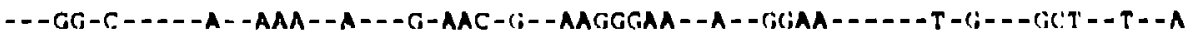

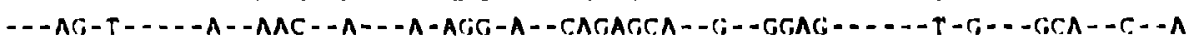

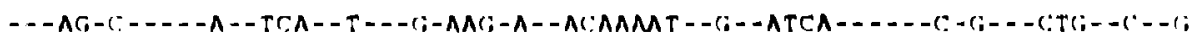


CONSENSUS SIVAGMTYO SIVAGM155 SIVAGM3 SIVAGM677

CONSENSUS SIVAGMTYO SIVAGM155 SIVAGM 3 SIVAGM 677

CONSENSUS SIVAGMTYO SIVAGM155 SIVAGM 3

SIVAGM 677

CONSENSUS SIVAGMTYO SIVAGMIS5 SIVAGM 3 SIVAGM677

CONSENSUS SIVAGMTYO SIVAGM? SIVAGM 677

CONSENSUS SIVAGMTYO SIVAGM 3

SIVAGM677

CONSENSUS SIVAGMTYO SIVAGM 3 SIVAGMG77

CONSENSUS SIVAGMTYO SIVAGMJ SIVAGM677

CONSENSUS SIVAGMTYO SIVAGMJ SIVAGM 67"
AA ?TT?aCt??tTGGTCag?tTTCTGGTCETGGTTCgatETCTCAAaATGGCTgaACat TLTaAAATaG --G--AACTAGT-----AGAT - -C------T------GATT-C----AA---C-TAA-AT-T-A--A--G--G--GTCAGAC-----GAGT--T-----T------GATT-T----AA---C-GAA-AT-T-A --G--A--A--AAGTAGT-----AGAT--C-----T-----GATT-C----AG---C-GAA-AT-C-A--A--A-

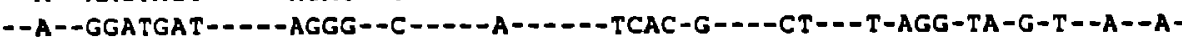

GaTTTTT?GLA?TAgT Aggt ATEATAGG ?TTAAGATTgCtLTaCaCA ?TAT atg? ?TG ?AT agctAgggT -A-----A-TAA--G-AGGAA-AA-----G--------ACTT-ACAC-G---ATGGA--T--AGTG-GGG-C-----G-CAG--A-AGGCG-TA----G--------GCTT-ACAC-T---ATACT--C--AGCT-GGG-A-----G-ATG--C-AGGTA-TA----A--------GCTT-ATAC-G---ATTCT--C--AGCT-GGG-A-----A-TGA--G-GATTA-TC----A--------TGCA-GGGT-T---GGGGA--T--CAGA-ATA-

TAGGCAGGG ?TA?tCTCCTCTALECCACAGATCCATATCCACCCgtgg? ? ?aAGGAC?GCCAGACAAC

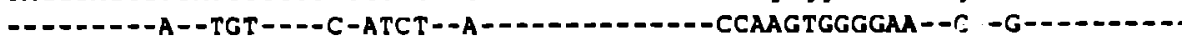
-

GCAG? \&G?GCCAG? agaAGGTGGAga ?A\&CAGCA ?gAgCAaA T? ? \&gCCE tgGCAGAAAGA ? TCC? ? ?A -CC-AC-A--C--GAGA-------GAC-ACA---GG-T--AA-TAGAGTCTTG-T--A--- : -C...-CA-GA-A--C--AAGA-..---AGA-CAG---AA-G- -AA-CTACGCAT $\cdots$

-CA-AA-G--C--GAGA------GAC-AGC---AG-A--GC-CCGAGCCTTG-C--A----A--TGGC-GA-GA-G--A--ACAG-------GAA-GCA---GC-G--AA-TGATAAGATT-C--G----G--CTCA-

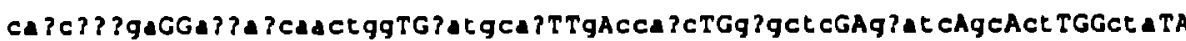
AG. . AGCA--...........-TATGCAG--G-CCGCC--GCTGAC--GGCTC-AC-CT---CTA-CAGCAGAGT--AAGAGCAACTGG--CAAGCGA--G-CCAAT--GTGCTC- -TCAGC-GC -TC---CTA-CACCTTCGA--ATCAACAACTGG--GCTCAAC--C-AGAGC--CAGCTT--GAATA-GG-CT---TGT--

$$
\text { <- rev net cda } \rightarrow
$$

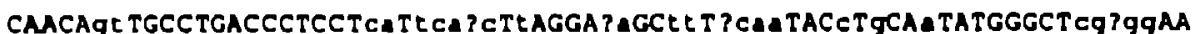

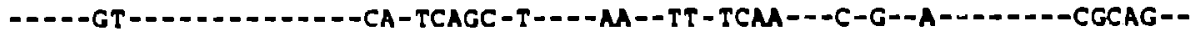

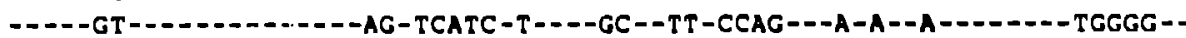
-

CTCAAAqCCGcaGCACAAGa rtagt t caAgCTCTGGCA rGcgt TGCacaAaAcGCrtG r raCcagatat ---A--ACC-GC---CA--AAATACTCCAAA--C - --AG-CGT---ACAAA-C - -AT-TCA-CAGA-AT ---A--GCC-CA---CA--AAGCAGTTGTCG--T----AC-CCT---ACAAA-C--GG-CTA-CAGA-AT ---C--GAG-CA-- -AC--GGCT TGCT -AAG--C - - -GA-GGC---GAGGG-A- -CT - GGG-AGAC-GG

GqceTqCTtqCaGATCCGCTTATaGGqCa aTCaTCAACAqTCCAAGAAGAGTGCGaCAAGGCCTTGAaqA

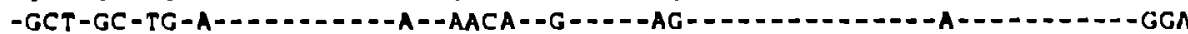

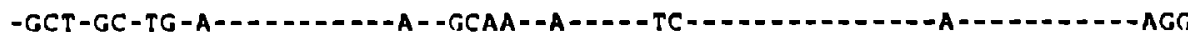

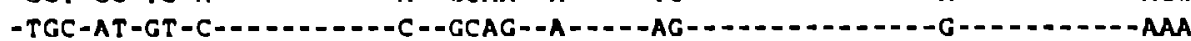


AGM NEF CDS

env cds $\rightarrow$ CONSENSUS SIVAGMTYO SIVAGM155 SIVAGM3 SIVAGM 677

ATGGGCT? ggC, JAaCTCAAAgCCGCaGCACAAGaAg?agttCa ?gCTCTGGC?aGcgtTGCaC? aAAGC ------CGCAG-A---A--ACC-GC---CA--AAA TACTCCAAAC-C----AG-CGT---ACAAA-C------ TGGGG-G---A--GCC-CA---CA--AAGCAGTTAACCA-C----GA-CTT---ACGCA-C-- - - TGGGG-----CCAGC-A---C--GAG-CA---AC--GGCTTGCTCAAGC-C----GA-GGC---GAGGG-A--

CONSENSUS SIVAGMTYO SIVAGM155 SIVAGM3 SIVAGM677

CONSENSUS SIVAGMTYO SIVAGM155 SIVAGMJ SIVAGM677

CONSENSUS SIVAGMTYO SIVAGMISS SIVAGM 3 SIVAGM 677

CONSENSU: SIVAGMTYO SIVAGMISS SIVAGM 3 SIVAGM 677

CONSENSUS SIVAGMTYO SIVAGM15S S [VAGM] SIVAGM677

CONSENSUS SIVAGMTYO SIVAGMI 35 S IVAGMJ SIVAGM677

CONSENSUS SIVAGMTYO SIVAGHISS S [VAGM] SIVAGHG77

CONSENSUS S IVACMTYO BIVACimISS S [VAGH]

a?VACimbl]
g?g?caCcagaTatGgceTgCTtgCaCATCCGCTTAT \}GGgCAATCaTCAAC? ? TCCAAGAAGAGTGCGa ATGTCA-CAGA-AT-GCT-GC-TG-A-_-_-_--A--AACA--G----AG----------

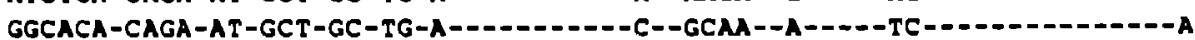

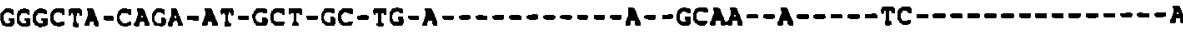

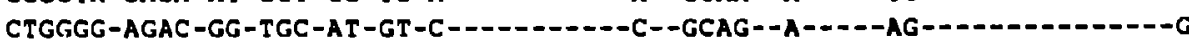

$$
\text { c- onv }
$$

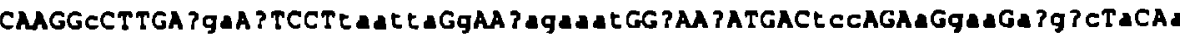
- - -C - - -GGA-A-_--TAATTA-G--ACAAAAT--C--C----GGA-- -G-NAA-GAGGC-T--A ----5---GGA-G----TAATTA-G--GAGAAAT--C--C-----TCC---A-GAA-ACGTC-A--G

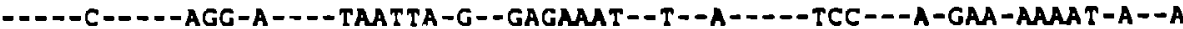

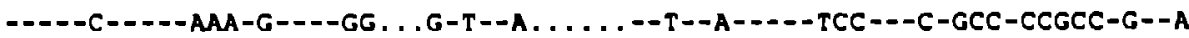

GAAGGAGACA ? ?TgGGAtGA ? TGGLCt GATGA ?GA\&GA ?GAAGT ?GGATTtCCAGT ? AgACCAAGAGTgC --A--A--CACC-GG--A--G-- -TCG-----T--G--A-----G--A--T--A--GAG---AA--- -A$--C--G--C C A A-G G--T--A---T C A-----A--A--T-----G--A--T--A--A A G---A A----G-$ $--A--A--$ TAAA-GG--T--A-- -TCT-----A--A--T-----A--A--T--A--AAG---AA----G$--A--A--C A C C-T T--T--G---G A T-----T--A--A-----A--C--C--T--G C A---T C---C-$

$L T R \rightarrow$

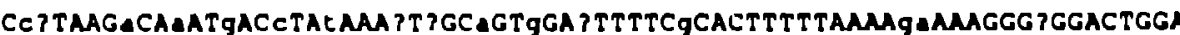

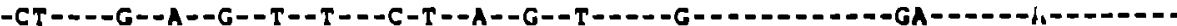

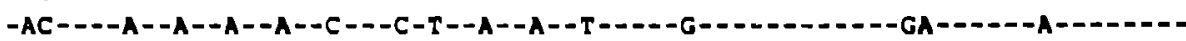

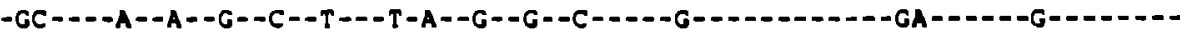

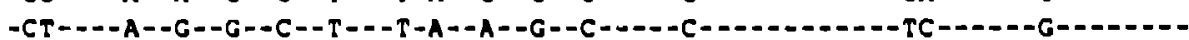

TGGGATE TATTACTCCGALAGGAGAAL AAGATCCTGAAT CTGTALGCCCTCMAGA PTGGGGAAT ?ATt

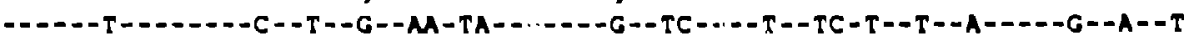

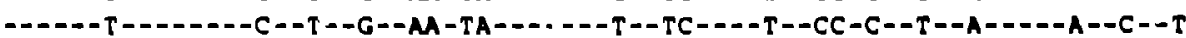

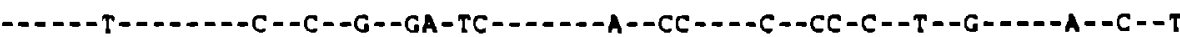

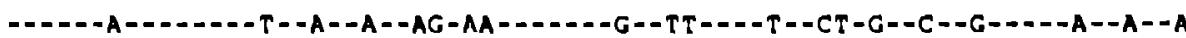

GATGATTGGAALGCET TgTCAAAGGACCAGG ?ATAAGATACCCgAgat qCTTEGGCTTCTGCTTEAAgC

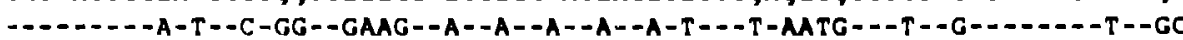
$-1-----A-C--A-G G--A A A A--A--T--G-\cdot A--A-A---G-G G T G---T--C-------C--G C$ $------A-T--T-G G--A G M A--A--A--A--C--A-A--A-G A T G---C--C---\ldots-T--A T$

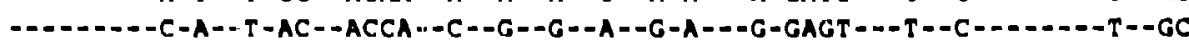

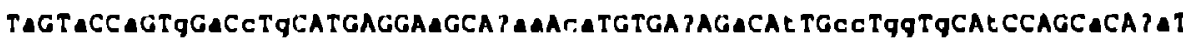

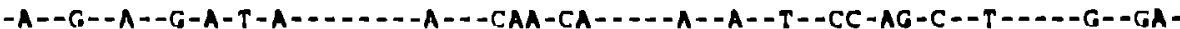
$-A--A--G--T-C-C-G------A---G A A-C A-----A--G--T--C T-G Q-A--C-----A--A C-$ $-G--A--A--A-A-C-G------A---G A G-C T----G--A--T--C C-G G-G--T--D-A--A G=$ $-A--C--A--G-A-C-G=----G---C G C-A C----G--A--C--T C-G A-G--T----A--Q A-$

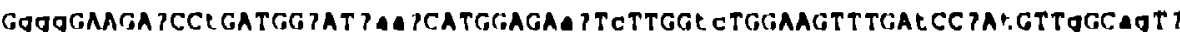

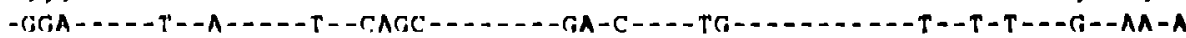

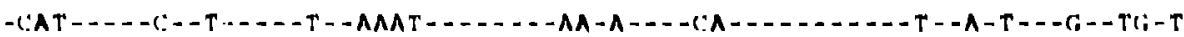

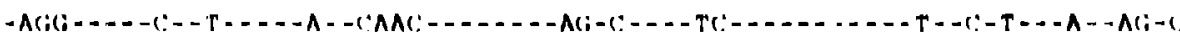

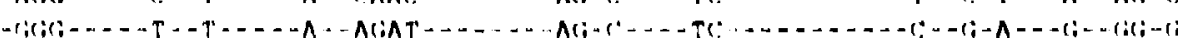


AGM NEF

CONE SUS CAgTACgaCCCCad?agggAgtaCtTLACtGACaTgCAtgaactt???gg?AdgaGgad"* *

646

SIVAGMTYO C-G--CGA---CAATCGGG-GTACT-T-CT---A-GC-TGCGCTGGTG...-AGA-GAAG---

690

SIVAGM 55 C-G--CGA---CTCAAGGG-GTAET-T-CA---T-AT-TTCAACAGTTGGT-CAG-AAAC---

SIVAGM3

C-A--TGA-.--TAACAGAA-ATATC-C-CT---A-GC-TGATCTT... GGC-AGA-GAAG---

693

SIVAGM 677 G-G--CCG---G......G-CATGT-T-AG---A-GC-CGAACAT...GCA-AGC-C**

690

672

I. ( .21

MAY 91 


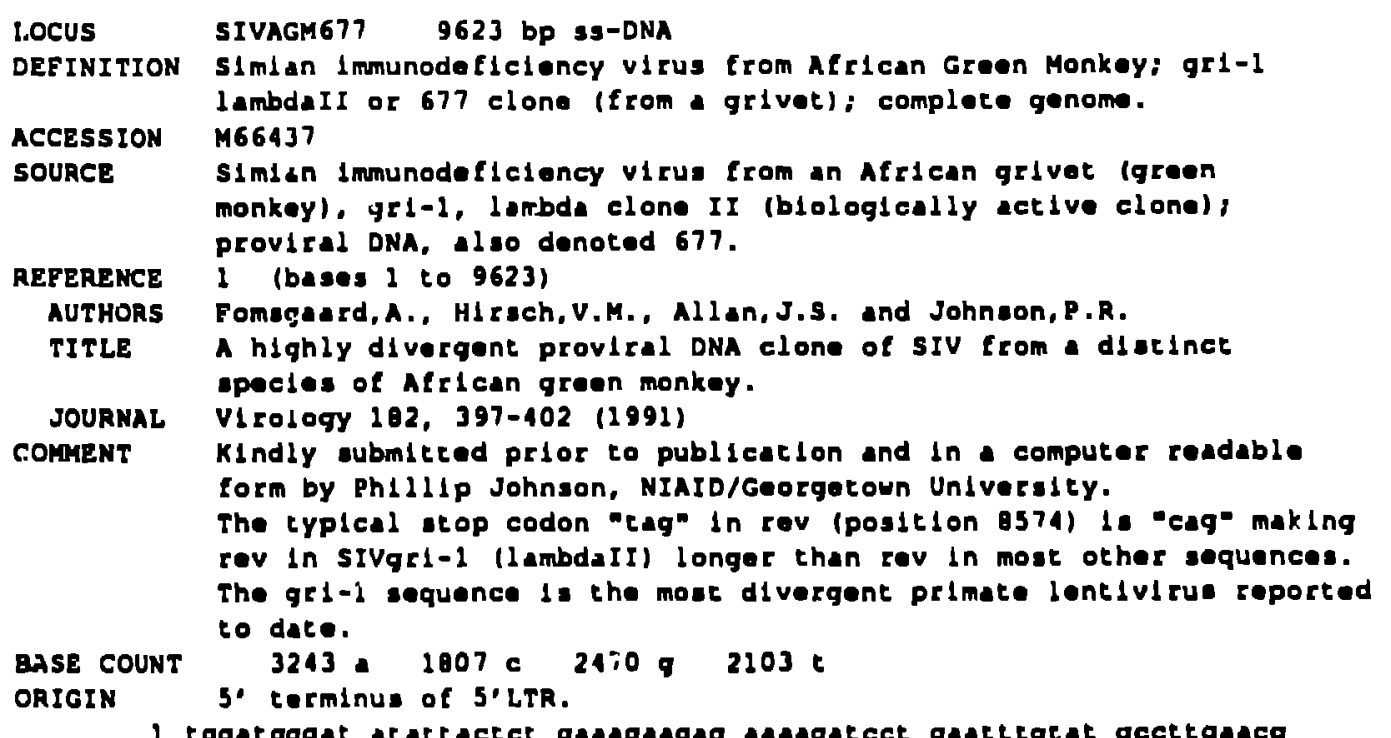

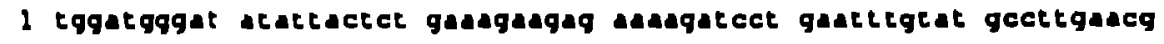
S. LTR $\rightarrow$

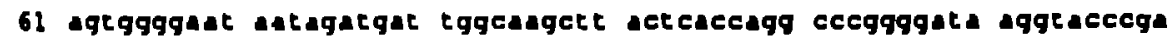

121 gagtctetgg ctectgctet adgetagtec eagtggacet geatgaggag geacgcaact

181 qtgagagaca ctgtctgatg catccagcac agatggggga agatccegae gqaetagatc

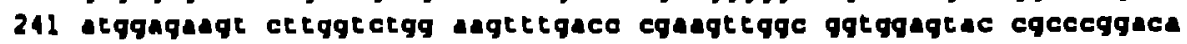

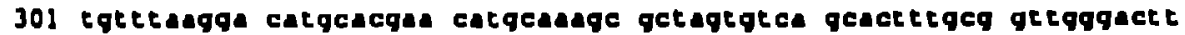

361 tecgesaggq acttecaca gtgggtggat cqgaggcggt acaggggegg tactgggagt

121 gqotetcoce teagagetge atasageag atgetegetg getgquact cagtctcte

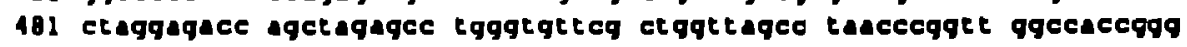

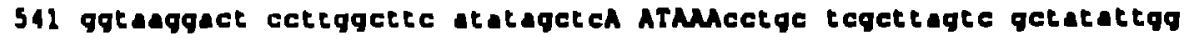
ulgnal $\rightarrow$ c- R rpe

601 agtcagtge teatgetge qeeqagecte tagagqtgaa cetetcted tgagtecec

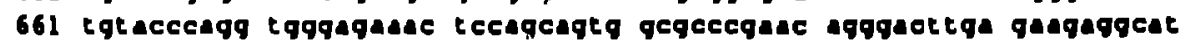
<- lya-tRNA pbe

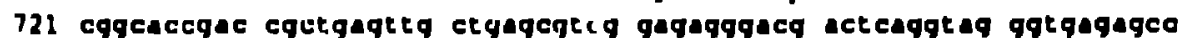

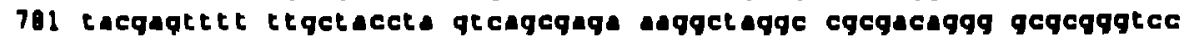

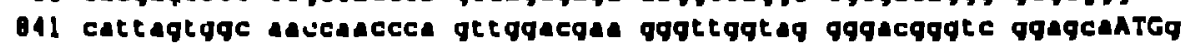
gag vetart $\rightarrow$

901 yeggggqtea ctcagcartg teagggaqae gcetequcac qtecqaqaaq at agqctac

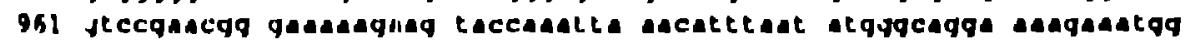

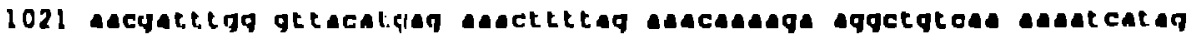

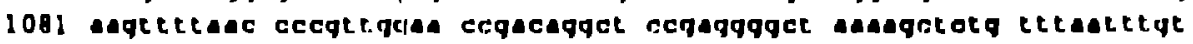

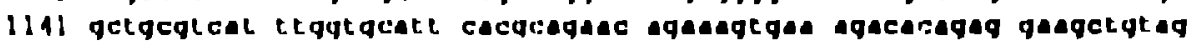

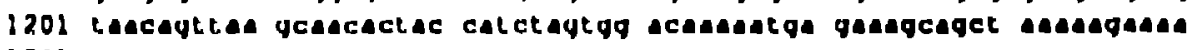

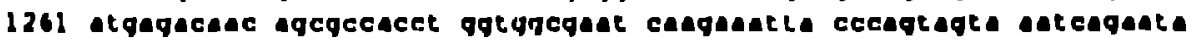

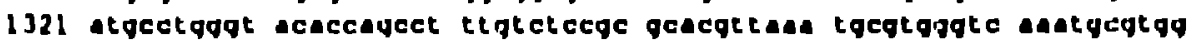

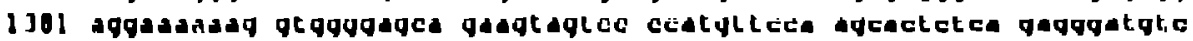

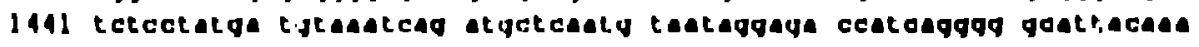

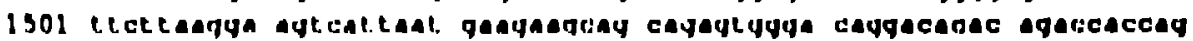

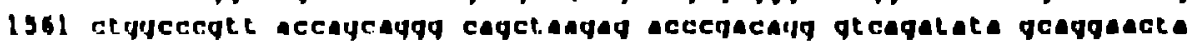

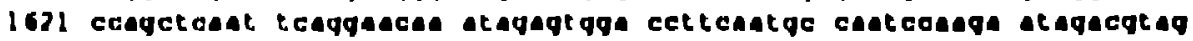

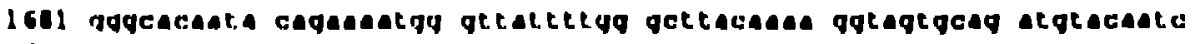

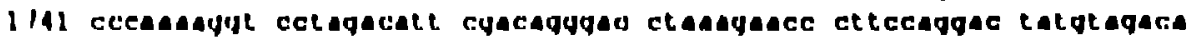

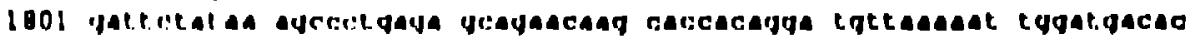

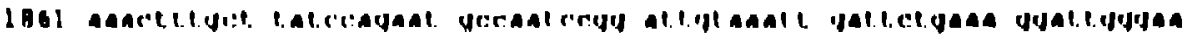

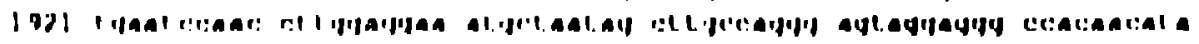

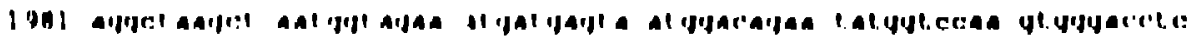

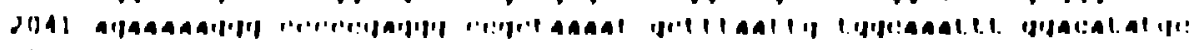

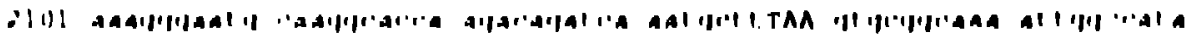
liil wi $\rightarrow$

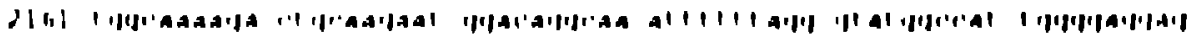

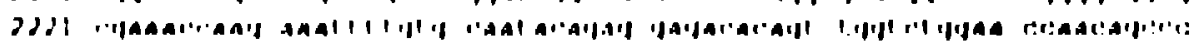

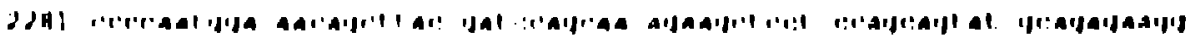


2341 garagcgect gagagaggag agagadeaga cadggaaca gaaggagaa gaagtggagg 2401 atgtetecte gagetecetc tetggaggag accastGaa cgagtcatca tagadggac $<-g a g$

2461 gecagtgcau gectgttag atacaggagc agatgacact atadteas auaggact 2521 gcactttcce ccacataac catggegttc caaggtagta ggaggtatag gaggagggat 2581 tcatgtcaa gatatcagg gggtacasgt acauttggag gataaatca tcaceggete 2641 attctaata ggagtacac caatcautat tataggadga atatettag etcaggeagg 2701 catgaadta gttatgggag ttctatctag tcagattgag gaadcaaag tacasctada 2761 agaaggaad gatggaccta adtgadaca atggccetta tcaagagaa aadtgasgc 2821 tttascaga atatgcasc aatgganga ggaggaad ttatctagge taggaggaga 2881 autccttat atacaccag tgtttgceat augauadg gatauacac auggagaet 2941 gettgtagat ttcagggac taacaage tactcagac tttettgagg ttcagctggg 3001 attcetcac ccagcgggcc ttcagaaas gaagcautc acagtalat acatagggga 3061 tgectattat tcataccat tatgeagga attcagaaa tatacagcat ttaccatcce 3121 ctcagtadat aatacaggge cagggatadg gtatcagtte aetgtctgc ctcagggatg 3101 gadaggatct cctacautt tccagadtac ggcagcaac attetagagg agatcaaag 3241 gcacactect gggttagaa tegr.ceata catggacgat ttgtggttgg cgtcagacca 3301 egatgagact agacatade aecaggtaga catagtaaga angagctgc tagaaargg 3361 tetagaacc ccagacasa astccasag agascegcca tgggatgga tggggtataa 3421 attgeatecg autautgga ccattuaca autagateta ccccettag aggagatg

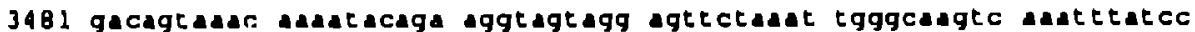

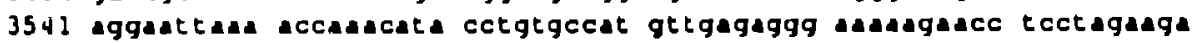
3601 atagtatgg acagaagagg cagaggcaga atataagac astcaggga tagtgcagga

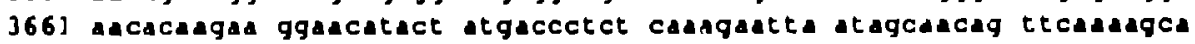
3721 aggagaaggg castggacat accattcac ccapaagg gcagtatta aggtgggadg

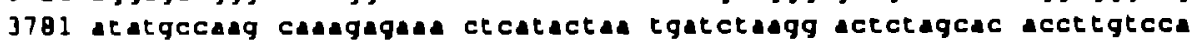
3841 aAautctgt asggaagcac teaccatetg gggaagact ccacgagtac auctcccagt 3901 agacasgas acatgggate tgeggtggce ggactattgg eagtatcet ggataccaga 3961 atgggagtt getagcacac cactcetagt asactgtgq tattcettag taaagaace 1021 aatcaugga gaagatgtte attatgtgga eggggcagca tccaugtga ccauttegg

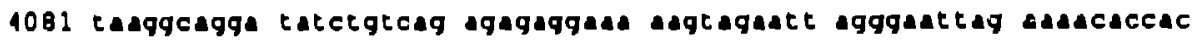

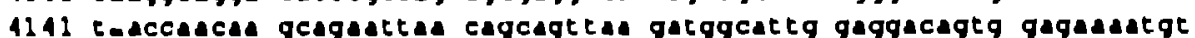

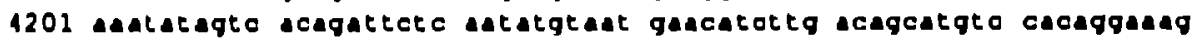

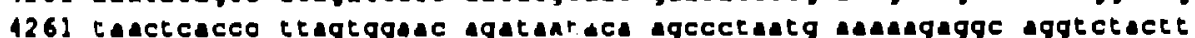

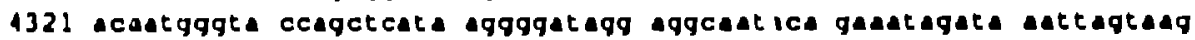

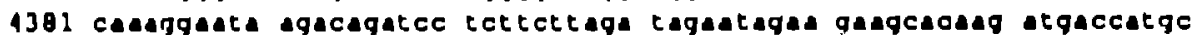

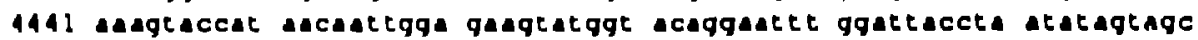

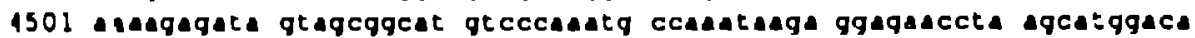

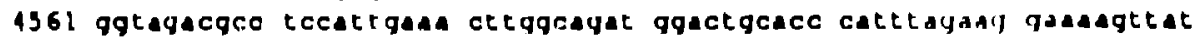

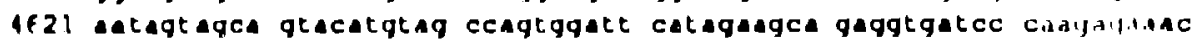
168: tgqgaeggag acagcacact tectgctgas actgttagca equtggceag tgaencatct

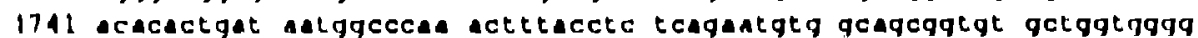

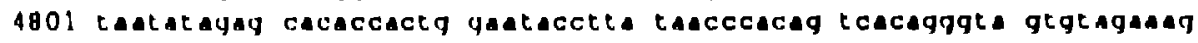

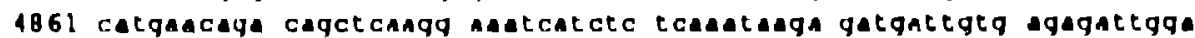

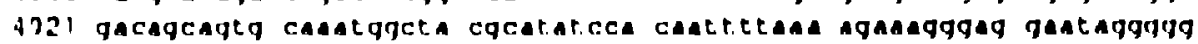

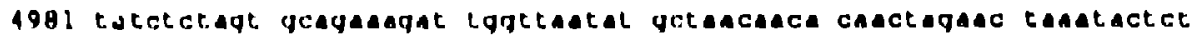

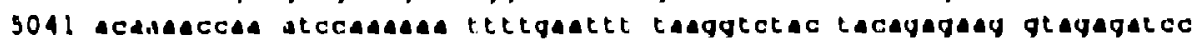

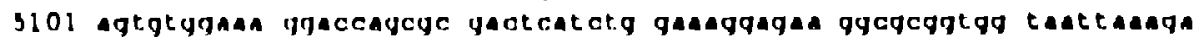

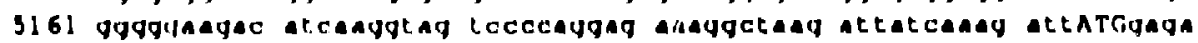
vil otart $\rightarrow$

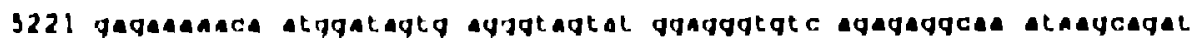

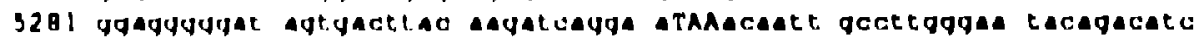
$<-$ pol

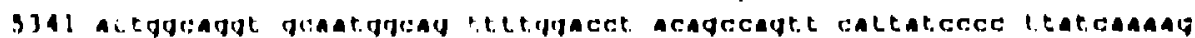

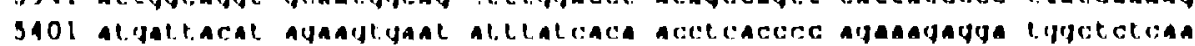

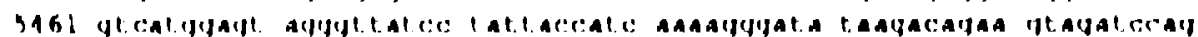

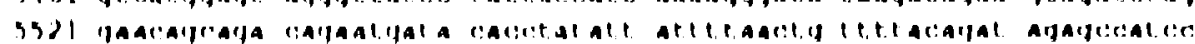

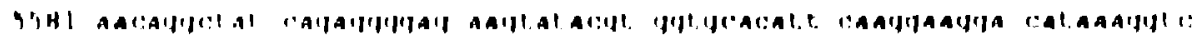

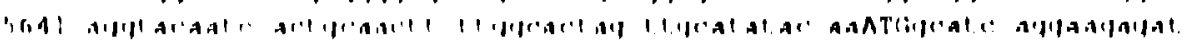
$\left.v_{1}\right)=1+11-\cdot$

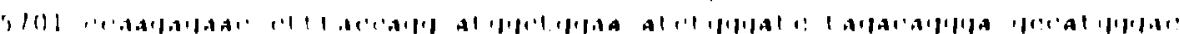

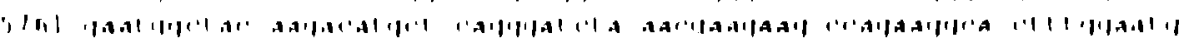

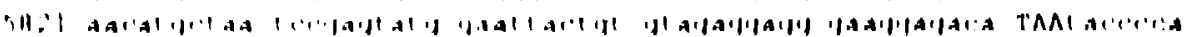
$\therefore$ - $v 11$ 
5881 tggatgaga taggctacad gtactataga attgttcada agtctatgtt tgtacattcc

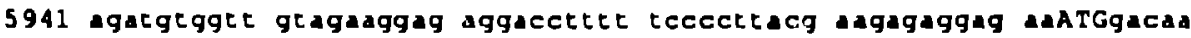
tat start $\rightarrow$

6001 ggaggaggag ccccacccec tcctccagga cttgcaragg ccattacade catgtactaa c- vpX end

6061 caacgetat tgcaaaaat getgttatca ctgtgagctt tgcttcctgc agaagggct

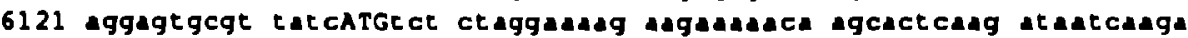
rev atart $\rightarrow$

6181 cccta:acgg cagcagTaag tATGgggaga thgettatad aatactade adagcaata 5. IJ $八$ env $\rightarrow$

6241 gggatagta taggaatagg tascctgtat gtgacagtgt tttatggaat crcagtatgg

6301 adaetrca cagttcaggc attetgcatg acgcccate ccaetatgtg ggcaecace

6361 actgcatac cagatgatca tgataataca gaggtgcctc taadattac agaagctto

6421 gaggcttggg ataatccgct gqtaauca gcagagagta atatacatct actctetgaa

6481 caacgatga ggccttgtgt taagctctcc cccatatgta thaaatgte ctgtgtagag

6541 ctgaatggta cagccacgac aaggccacc actactgca ctacaacat gactaccccc

6601 tgtcagatet gcagtacaga gcagatagaa ggagaaugg cagaggaacc agcatccaac

6661 tgcactettg caetgcagg atatcasaga gatgtauas agattatag catgacctgg

6721 tatgatcagg agt tagtctg cartataad acaggaagtg auagggaag taaggactgt

6781 tacatgatac attgtaatga tecagtgata aagaagct qtgatauac atatgggat

6041 actetaagag tagatactg tgcaccagca gggtatgctt tgctaaatg taatgataag

6901 gattatagag getttgetcc aagtgcas aatgttccag tugtgcattg tactagatea

6961 atcaatacte ctatauctac agggatagga ttaarggt. gtagatcaga aatagaaca

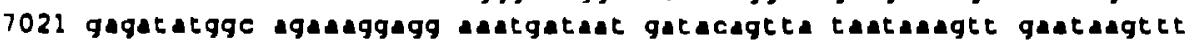

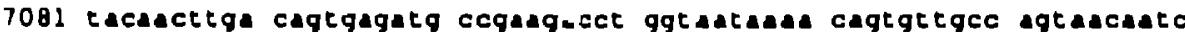

7141 atggcagggt tagtattcca ctctcagaa tatarecca ggttaasace agcgtggtgc

7201 cacteccasg gagattggaa aggggcatgg augagtca gagaegaagt augaagtg

7261 aasaccte caqaagtaaq catagasat atacitctga gaagqatatq ggqagatcca

7321 gateagega attetggtt caattgtcaa ggtgattte tctattgtae gatggactga

7381 cteaccaet atctaacaa tcgacaga gatgcagaag gtactaatag gacctgtgac

7441 aAggyaagc caggaccagg accatgtgtt cagagaect atgttgcetg coatatacga

7501 caagtagtaa atgattggta cactgtctct waaggtat atgctcoacc aegggaeggt

7561 cattrggagt gtaactcatc agtcacggca ctatacg:gq caatagatta taacaugag

7621 tetgqcccaa taatgtgac cctangtccl caggtacgoa goatatgogo gtaogaetg

7681 gqagactata aettagtaga gatauacce attggotttg otootsoaga tgtaegaage

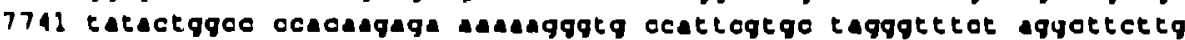

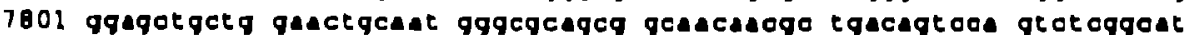

7861 tggettgctg ggatattgca gcagcagad ucttgotgq cqgetgtgga acugcasca

7921 cagtegttga agctqaccat trggggtgtq auascctca atgcecgegt cacagctctc

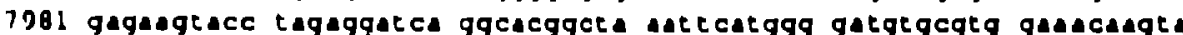

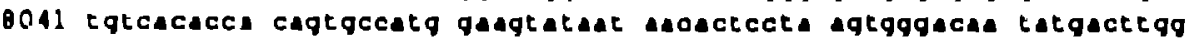

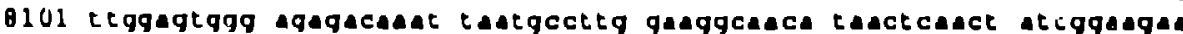

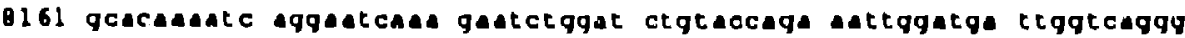

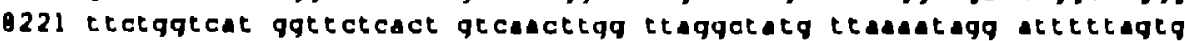

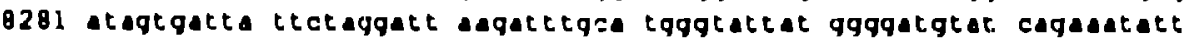

0341 eqgeagggat atalcctct cccccAGato catatccaca gttoagcgga aggecagac $113^{\circ}$ i

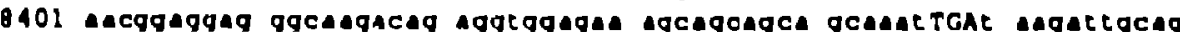
c- tac

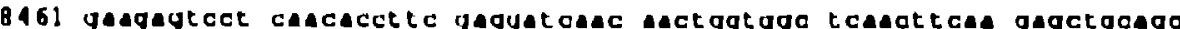

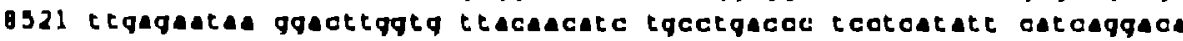

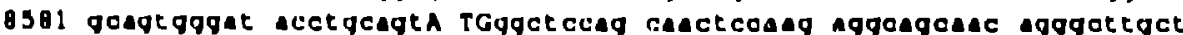
net etart $\rightarrow$

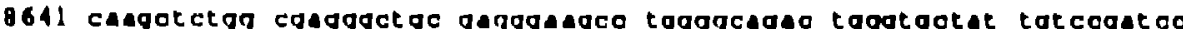

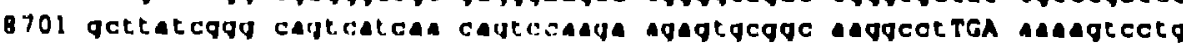
<- rev

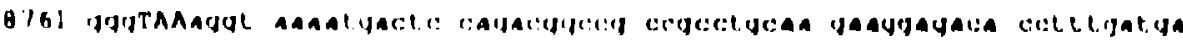
- anv

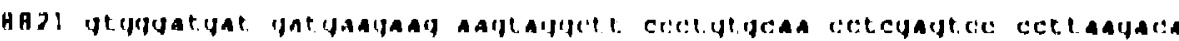

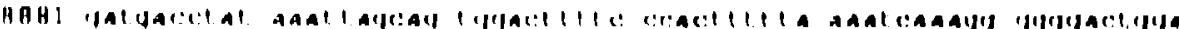

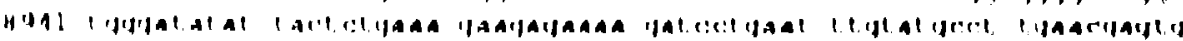

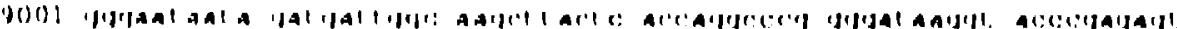

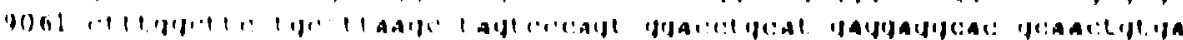

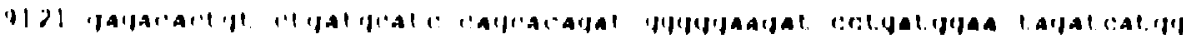

I.C.24

MAY 91 
9181 agaagettg gretggaagt ttgaccega gttggeggtg gagtacegec cggacatgtt 9241 taagacatg cacgadcatg caadgegcTA Gtgteagcac tetgeggteg ggactteceg $<-$ nef ond

9301 ccagggacte tecacagtgg gtggategga ggcggtacag gggcggtact gggagtggct 9361 ttececteag agctgeatad dagcagatgc tegctggct gtacteagt etctedetag Ilgnal $\rightarrow$ R IPt $\rightarrow$

9421 gagaccagct agagcetggg tgttcgctgg ttagcctaac ceggttggcc accgggggtd 9481 aggactcet ggct teatat agctcAATAA Acctgetegc teagtegcta tattggagte algnal $\rightarrow$

9541 agtgetcat tgctgegecg agcetetaga qgtgasete tettactggg ttetectgta

9601 cecaggtg7g agaacteca gca 





The amino acid sequence alignments that follow were generated by the PIMA program ("partern induced muluple alignment"; R. F.Smith and T .F.Smith, PNAS 87: 118-122, 1990). The alignments were also subject to some hand-editing performed with MASE. For unformation concerning both these UNIX tools, contact Dr. Randall Smith, MBCKR, Dana Farber Cancer Institute, Harvard School of Public Health, 44 Binney St., Boston MA 02115, (617)-732-3746.

With a few exceptions, only full-length protein sequences have been included in this com. pilation. One simian and one HIVI sequence were not ready for publication at this time, but are reflected in the consensus sequences and patierms. If you have a question about a particular stretch of alignment, contact us for help, as it has been sometimes possible in the past to obtain an author's approval to release sequence data over a limited region.

The reference sequences for the alignments are mixed case consensus sequences in which upper case leuers refer to amino acid residues which are conserved $100 \%$ and lower case letters represent amino acid residues conserved in at least 50\% of the sequences. For type 1 viral sequenres, separatc consensus sequences have been generated for the North American and African samples.

Within the sequences, "-"s are used to indicate residues conserved with respect to the reference sequence and "."'s represent actual gaps. Blank spaces within the alignment indicate lack of sequence information over that region. Annotation of the ENV amino acid sequences udlizes "*" (or somedmes " ")" for conserved cysteino residues and "w.." for potential N-linked glycosylation siles.

Following each alignment the consensus sequence for that allgnmeit is shown with the corresponding AACC ("amino acid class covering") PATTERN. The AACC pallems are described in the above-mentioned reference of Smith and Smith and the amino acid class hienurchy used to construct the paticms is shown in the diagram below. Each position in a pattern represents the minimum inclusive amino acld class that covers the range of residues observed or anticipated at that position for the entire set of sequences in the alignment (except that the SIVcpz. sequences havo not been included will the HIV-1 AACC patiems). Ordinary consensus sequences have the disadvantage of being "averages," thus they reduco the information represented in the variution; howover, they are relatively insensitive to sequenco errory and defective viral sequences. The AACC pauterns, someumes called "consensus-like" pall. ms retain more of the information, but they aro highly subject to ouliers and spurious variants. By presending both klnds of consensus sequences, wo cover the information from both diroctions.

In the AACC patterms, upper case letters indicate idenditles, l.c., 100\% conservation of a particular amino acid at that position, with exception of the wild-card " $X$ ". Oaps are represented by "."'s. Positions at which all die observed or andiciparad amino acid residurs belong to a paricular umino acid class aro indlcaled by 'm.'s, with all the members of that class listed vertically below that position. This listing does not imply that all such subsditutions have occurred at that site, nor does it proclude the possibillty of a non-conserved replacement occurring at that ste.

Tho AACC uralysis utility calculutes the information conient, or the number of amino ucid equivalents coneained in each pattem: toully variable slies (wild-card $X$ ) havo 0 information and cotally invartune sites (a specifle amino acid) have information of 1.0); all other sites, more or less variuble, take on values between 10 and 1.0 amiluo acid equivulents. For this calculation, all 20

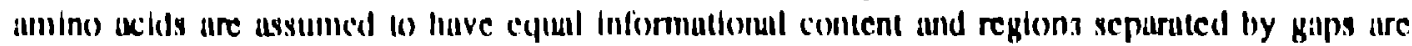
as sumed to be suntistically independent of one unother. An tadtitional calculation derives a length. inmulized information content, or informution densily, irom cach patiern. A program (subpat) 
recently provided by Dis. Randall Smith and Temple Smith, has been used to select maximally information dense subregions from the partems based upon a user defined threshold. For the most par, this analysis confirms the invariant stretches discovered through ordinary consensus sequences; however, it also identifies variable but conserved regions that might be otherwise overlooked.

The absolute information density thresholds used in this report are indicaled above each consensus/pattem allignment. The minimum information density required for each subpartem was chosen in order to select well defined regions with information densities significantly above the average for that pattem. The regions containing maximally information dense subpatterns we indicaled by "*n's placed ove the appropriace region of the alignment. Thus a calalog of conserved and invarian protein structures becomes possible. Such a calalog of HIV-1 proleins is included.

Because the AACC patuems are acutely sensitive Lo mare amino acld replacements, and sequencing errors, the istabog of inveriant and conserved paterns is highly stringent. The annotation of this catalog is. as the user can tell, in its carliest stage. The ennotstion of epitopes heavily relied upon notes provided by Dr. Jemes Bradrc, Veccine Branch, AIDS Division, NIAID.

The HIV.1 principal neutralization determinant (PND" or V3 loop) is not sufficiendy conserved to be included in the catalog; accordingly, its analysis and patuerns are found in Part $\mathrm{DI}$.

Al ignment score symbol

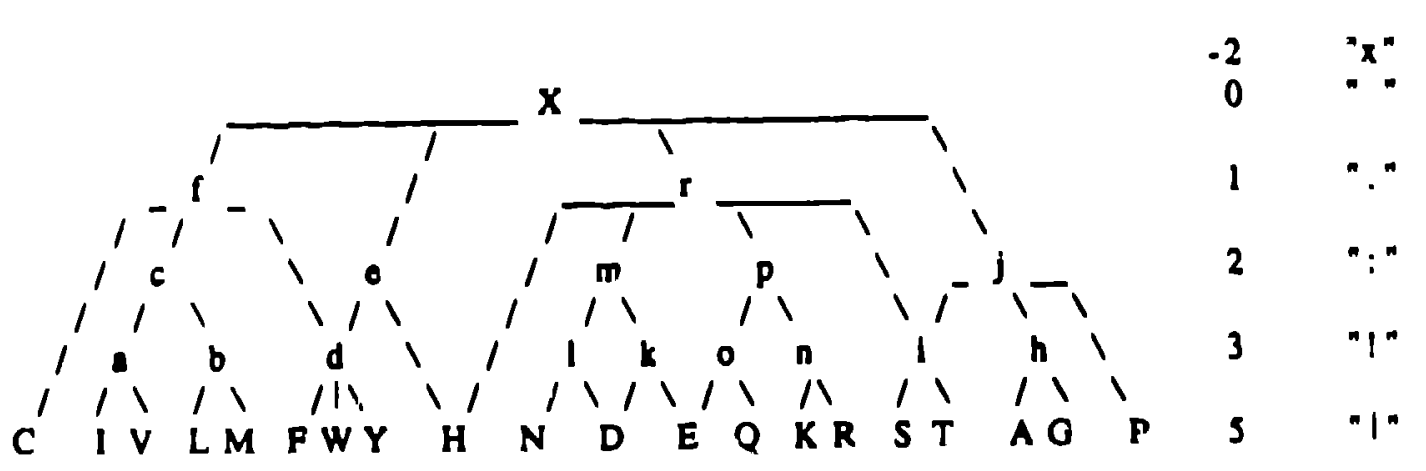


PART II. AMINO ACID SEQUENCES AND THEIR VARIATION

INTRODUCTION . . . . . . . . . . . . . . . . . . II-1

GAG

HIV-1 . . . . . . . . . . . . . . .

HIV-1 Consensus Sequences and Patuems . . . . . . . . . . . 8

HIV-2/SIV . . . . . . . . . . . . . . . . . 13

HIV-2/SIV Consensus Sequences and Pallems . . . . . . . . . . 16

SIVAGM . . . . . . . . . . . . . . . . . . 18

SIVAGM Consensus Sequences and Patterns . . . . . . . . . . . . 19

POL

HIV-1 .................... 21

HIV.1 Consensus Sequences and Patterns . . . . . . . . . . . . . 26

HIV-2/SIV . . . . . . . . . . . . . . . . . . 33

HIV-2/SIV Consensus Sequences and Patlems . . . . . . . . . . . 38

SIVAGM . . . . . . . . . . . . . . . . . . . 42

SIVAGM Consensus Sequences and Patterns . . . . . . . . . . . . 44

VIF

HIV-1 . . . . . . . . . . . . . . . . 48

HIV-1 Consensus Sequences and Patuerns . . . . . . . . . . . . . 49

HIV-2/SIV . . . . . . . . . . . . . . . . . . . 50

HIV-2/SIV Consensus Sequences and Patterns . . . . . . . . . . . 52

SIVAOM . . . . . . . . . . . . . . . . . 53

SIVAGM Consensus Sequences and Patterns . . . . . . . . . . 54

VPX

HIV-2/SIV . . . . . . . . . . . . . 55

HIV-2/SIV Cease.ssus Sequences end Pallems . . . . . . . . . . . 56

SIVAGM . . . . . . . . . . . . . . . . . . . . . . . . . 57

SIVAOM Consensus Sequences and Patterns . . . . . . . . . . . 58

VPR

HIV-I . . . . . . . . . . . . . . . . . . . 59

HIV-1 Consensus Sequences and Pallerns . . . . . . . . . . . . . 60

HIV-2uSIV . . . . . . . . . . . . . . . . . . . 61

HIV-2/SIV Consensus Sequences and Patlems . . . . . . . . . . . 62

TAT

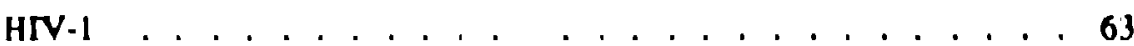

HIV-I Consensus Scquences and Patterns . . . . . . . . . . . . . 64

HIV-2/SIV . . . . . . . . . . . . . . . . . . 65

HIV-2/SIV Consensus Sequences and Pallems . . . . . . . . . . 66

SIVAGM ...................... 67

SIVAGM Consensus Sequences und Putlems . . . . . . . . . . . . 68 
REV

HIV-1 . . . . . . . . . . . . . . . . . . . . . . . . 69

HIV-1 Consensus Sequences and Patuerns . . . . . . . . . 70

HIV-2/SIV . . . . . . . . . . . . . . . 71

HIV-2/SIV Consensus Sequences and Paluems . . . . . . . . . . . 72

SIVAGM ................... . 73

SIVAGM Consensus Sequences and Patterns . . . . . . . . . . . 74

VPU

HIV-1 . . . . . . . . . . . . . . . 75

HIV-1 Consensus Sequences and Paterns . . . . . . . . . 76 ENV

HIV-1 . . . . . . . . . . . . . . . . . . 77

HIV-1 Consensus Sequences and Patterns . . . . . . . . . . . . . 84

HIV-2/SIV . . . . . . . . . . . . . . . . . . . . . . . . . . 90

HIV-2/SIV Consensus Sequences and Palterns . . . . . . . . . . . 94

SIVAGM ........................ . . . 98

SIVAOM Consensus Sequences and Patcerns . . . . . . . . . . 100

NEF

HIV-1 . . . . . . . . . . . . . . . . 103

HIV-1 Consensus Sequences and Pauterns . . . . . . . . . . . 105

HIV-2/SIV . . . . . . . . . . . . . . . . . . . . . . . . . . 107

HIV-2/SIV Consensus Sequences and Paltems . . . . . . . . . . . 108

SIVAGM . . . . . . . . . . . . . . . . . . . . . . . . . . 109

SIVAGM Consensus Sequences and Pallerns . . . . . . . . . . 110

SIVMND PROTEINS . . . . . . . . . . . . . . . . . . . . 111 
CONSENSUS1 MGARASVLSGG LDKWEK IRLRPGGKKKYKLKH IWWASROLERFAVNPgLLET SEGCROI I GOLOP ILQT

HIVLAI

HIVHXB2R

HIVMN

HIVJH3

HIVJRCSF

HIVOYI

HIVSF 2

HIVNYSCG

HIVNL43

HIVCDC4

HIVHAN

HIVRF

CONSENSU

HIVELI

HIVZ226

HIVNDK

HIVHAL

HIVU 455

SIVCPZ

CONSENSUS1

HIVLAI

HIVHXB2R

HIVMN

HIVJH 3

HIVJRCSF

HIVOYI

HIVSE 2

HIVNY5CG

HIVNL43

HIVCDCA

HIVHAN

HIVRF

CONSENSUS 2

HIVELI

HIV2226

HIVNDK

HIVMAL

HIVU 455

SIVCPZ

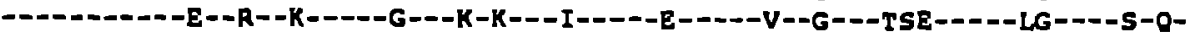

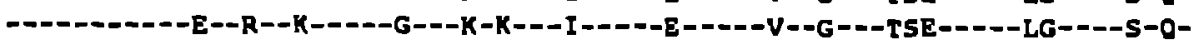

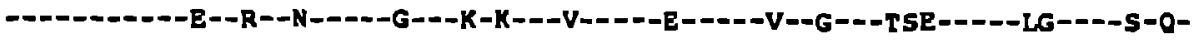

-

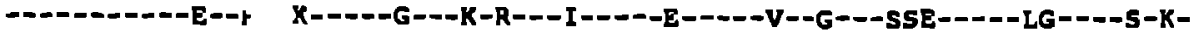

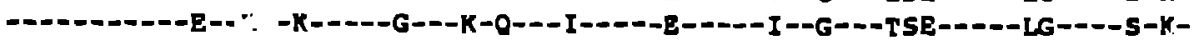

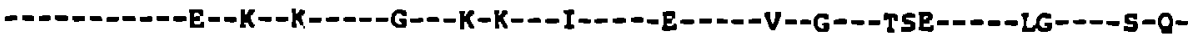

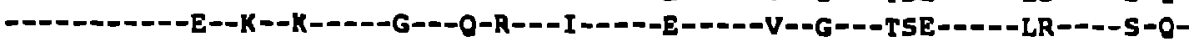

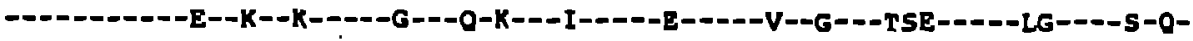

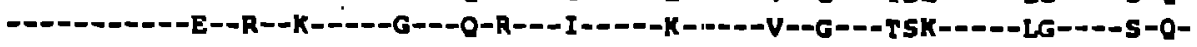

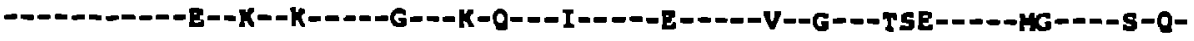

-

MGARASVLgGgKLD?WEK 1 RLRPGGKKKY I IKH I vWASRELEIfal IPGLlEt SQGCKq 1 gGLqPa ?qT

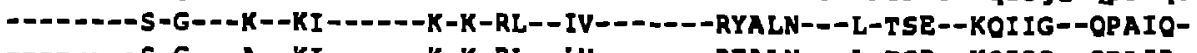

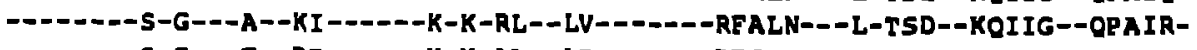

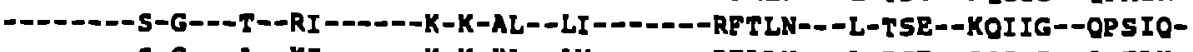

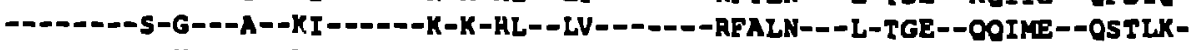

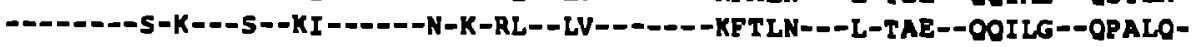
-

GSEEl ISLYNtVAt LYCVHOr JevkDTKEALdK1EEEOnKS . kKKAOO? ? . . . . . . AAAdt ? ? ?Gngs

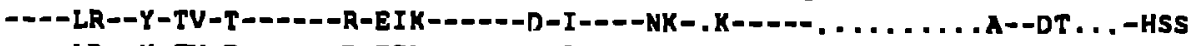

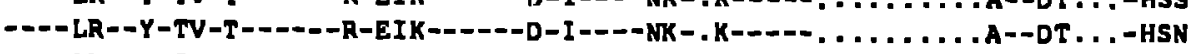

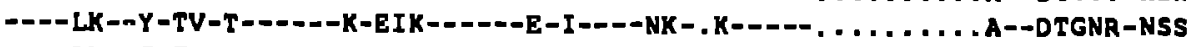

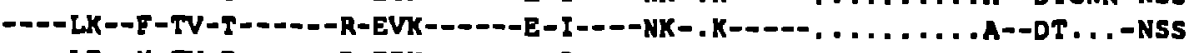

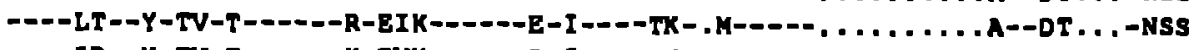

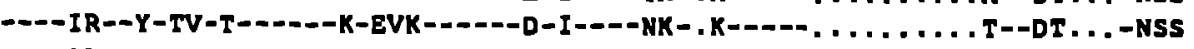

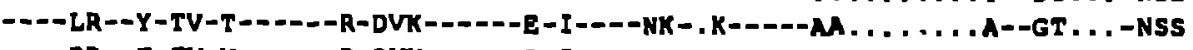

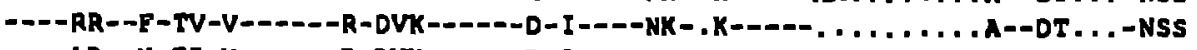

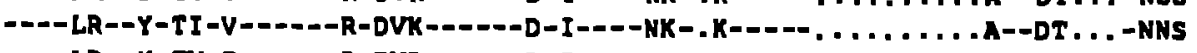

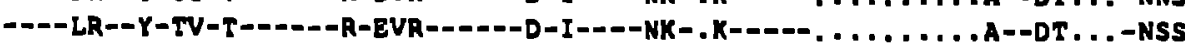

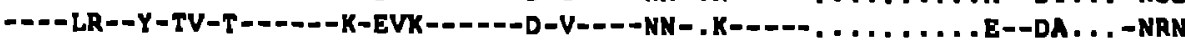

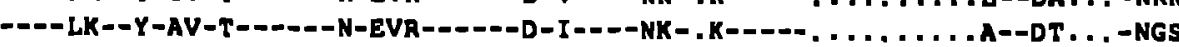

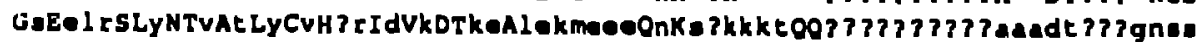
-T-ELR--Y- -V-T-Y-V-KG-D-K--KE-LEKMEEE-K-S.KKKA-C . . . . . . . ANADT. . GNKS -S-ELR-P-F-V-T-Y-V-ER-E-K--KE-LEKMEEE-N-SKNKKA-_ . . . . . . . AAADA. . GNNS -S-EIR--Y--V-T-Y-V-ER-E-K--KE-VEKPEEE-N-S.KKKT-_ . . . . . . . AMAD . . . . .SS -S-EIK--.Y--V-T-Y-V-OR-D-K--KE-LDKIEEI-N-S . ROKT --NAMAQO . . . AAMA . . . KNSS -T-ELR--Y--V-V-Y-V-OR-D-K--KE-LNKIEEM-N-N.KORT-_ . . . . . . . AAANT . . G.S. -S-GLR--F-L-V-W-I-SD-T-E--OK-L. . . . E-L-R. HHGE--SKTESNSGSREGGASQGASASA

TI7 $\backslash /$ p24

CONSENSUSI QVSONYPIVONI QGOMVHOAISPRTLNAWVKVUEEKAFSPEVIPMF IALEGATPODLNTMLNTVGGHOA HIVLAI HIVHXB2R HIVHN HIVJH3 HIVJACSF HIVOYI HIVSF 2 HIVNYSCG HIVNL43 HIVCDCA HIVHAN HIVRF CONSENSUS? HIVELI HIVZ2Z6 IIIVNDK IIIVMAL. HIVUabs sivcez

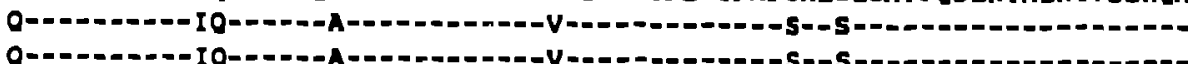

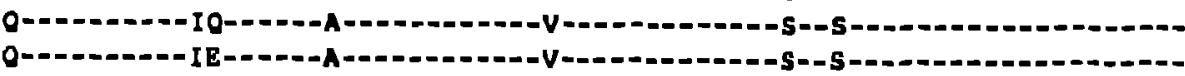

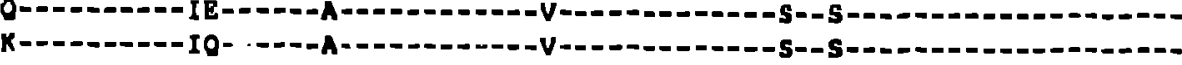

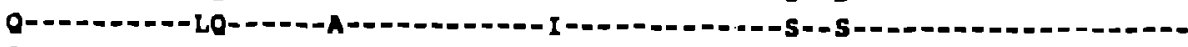

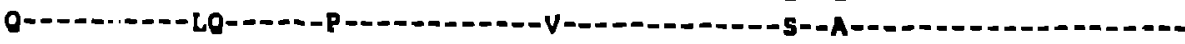

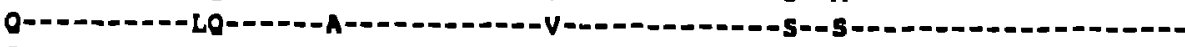

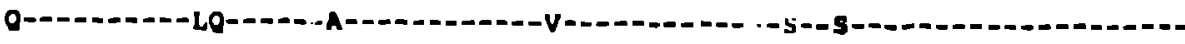

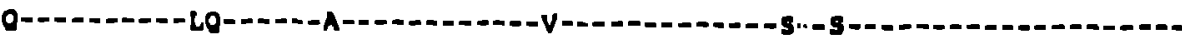

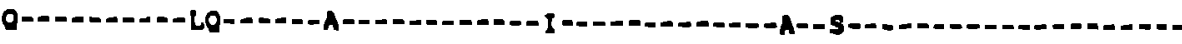

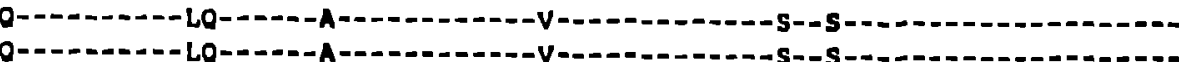
qUSqNYP IVON TCGOMUHOA I SPHTLNAKVKVI EOKAFSPEVIPMESALSEGAL POOINEHLNEVGGHOA

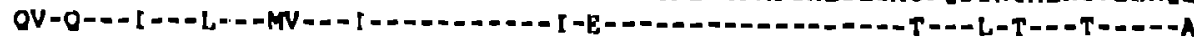

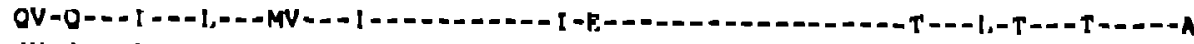

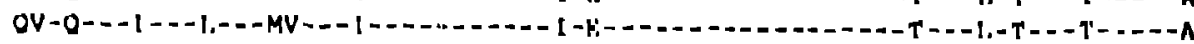

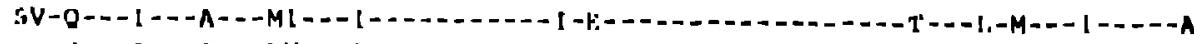

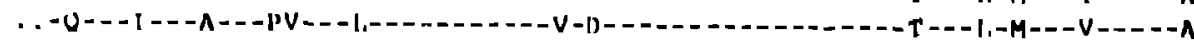

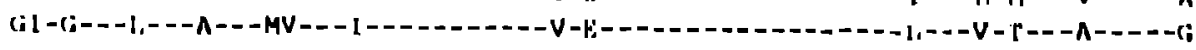


CONSENSJSI AMOHLKETI NEEANEWDRIHPUhAGPI APGQMREPRGSDIAGTTSTLOEOIGWHTNNPP IPVGEIYKRWI 266 HIVLAI HIVHXB2R - HIVIN

HIVЛH

HIVJRCSF -

HIVOYI -

HIVSF 2 -

HIVIY5CG -

HIVNL4 3 -

HIVCDCA

HIVHAN $\quad$ -

HIVRF

CONSENSUS2 AMOMLKOE I NEEAAOWDR1 HPUHAGP 1 APGOMREPRGSDTAGTTST IOEOI ?WMT ANPPIPVG 1 YKRW1 260

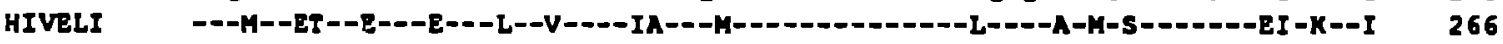

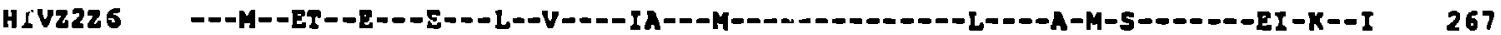

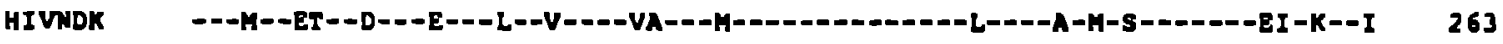

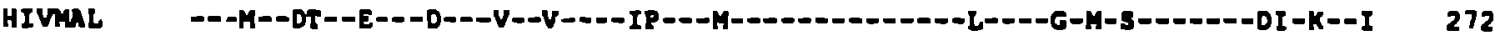

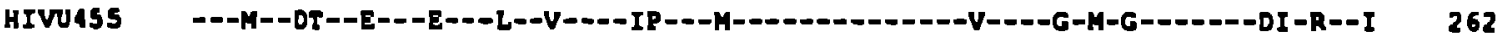

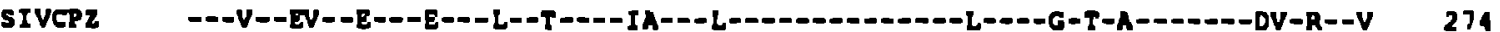

CONSENSUSI ILGLNKIVRAYSPESILDI IOGPKEPFRDYVDFYKTLRAEOA

HIVLAI

HIVHXB29

HIVMT -

-

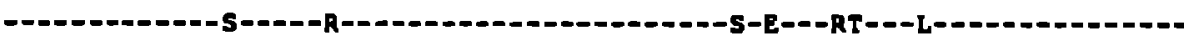

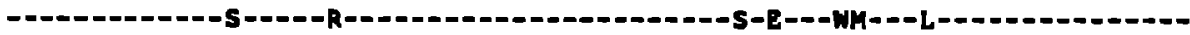

HIVJHJ

HIVJRESF

HIVOYI

-

- - -

-

- -

HIVNY5Ce

HIVNL43

- -

-

-

-

HIVHAN

HIVRF

I IGLNKIVRMY OPVSI LDIROGP KEPFRDYVORFYKTLRAEOA COTVKNWMTETLL : ONANPDCKE ILKA

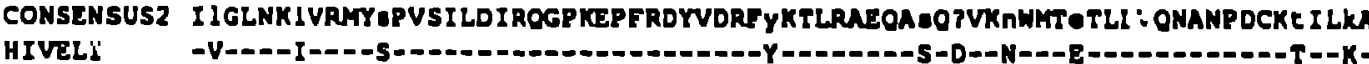

HIV2226

HIVNDK

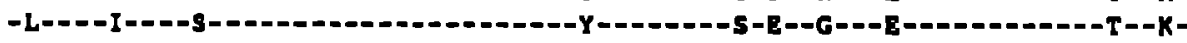

-L-1--I-- - -

HIVMAL

HIVUAS5

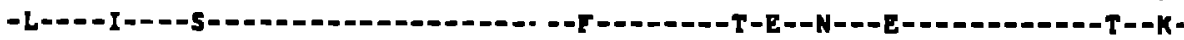

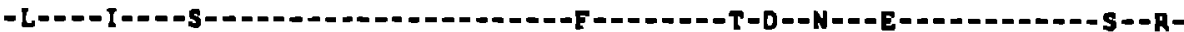

SIVCP 2

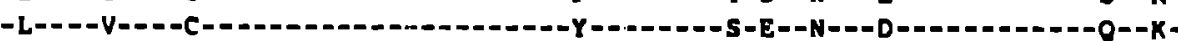

p24 VI $/ 1$

V p 7

CONSENSUSI LGP AATLEEMMTACQGVGGPGHKARULAEAMSOV. TnLAT . IMMOTGNF TnORKEUKCFNCGKOGHIAKN HIVLAI HIVHXB2R HIVMN HTVJH3 HIVJRCSF HIVOYI HIVSF 2 HIVTYSCG HIVNL43 HIVCDC4 HIVHAN

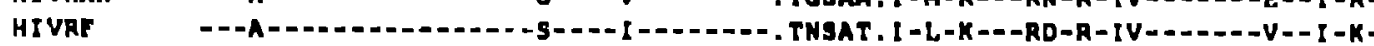
-

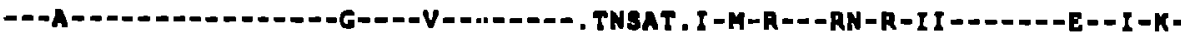
- -A-1

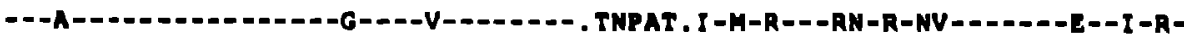

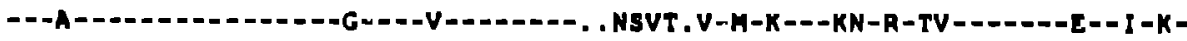
- - A-

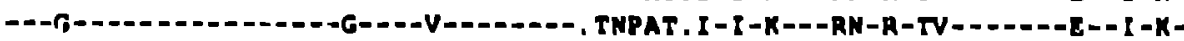
-A- - A-1.-1

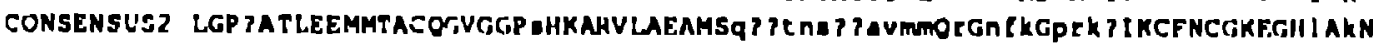
HIVELI HIV 27,6 HIVNDK -

IITVMAL.

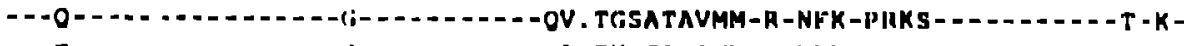
IIIVISAs - -

: i IVCP?. -

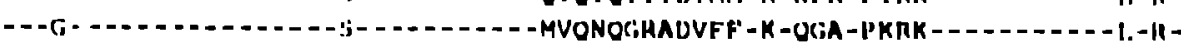


HIV1 GAG

pol cds $\rightarrow$

p $7 /$

CONSENSUS1 CIAPRKKGCWKCGKEGHOMKdCTE??ROANELGKIWPSHKGRPGN? ? ? ? ?? ? ? ?F 10:RPEP TAPPE

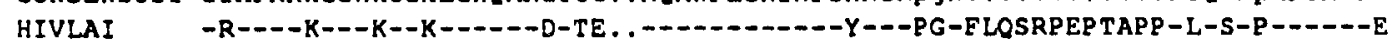

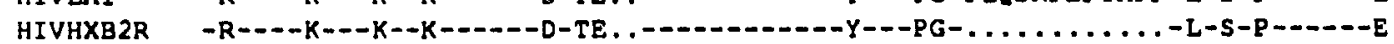

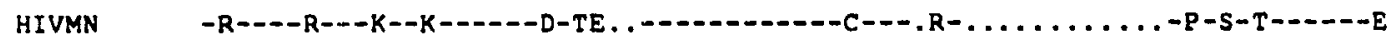

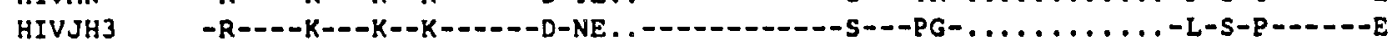

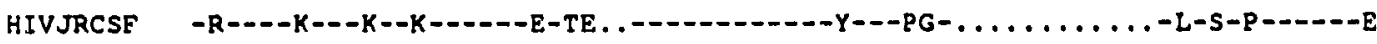

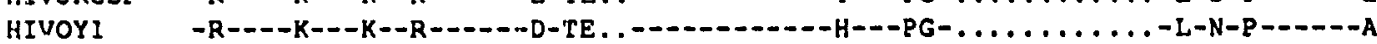

HIVSF 2 -R----K---R--R--D-D-D-TE. . - -

HIVNYSCG -R----K---K--K--D--D-TE...

HIVNL43 -R----K---K--K----D-D-TE...-- 3 -

HIVCDC4 -K----K---K--R--.---D-TE..-- 4

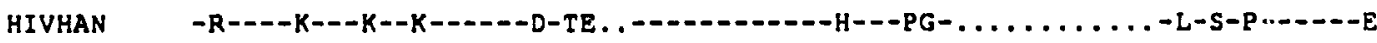

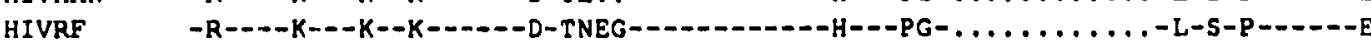

CONSENSUS2 CEAPRKKGCWKCGKEGHOMKDCTE. ROANFLGK1WPShKGRPGN. . . . . . . . F1OSRPEPTAPPA

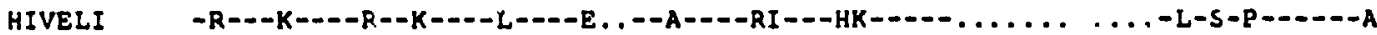

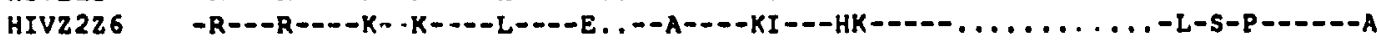

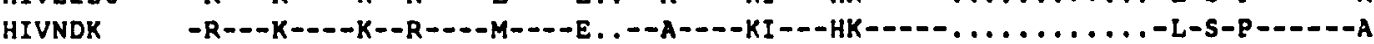

HIVMAL $\quad-R--K----K--K-\cdots-M--\ldots-E \ldots--A----K I---H K-----\ldots \ldots \ldots \ldots \ldots, L-S-P------A$

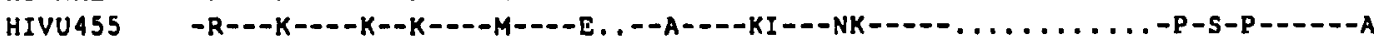

SIVCPZ

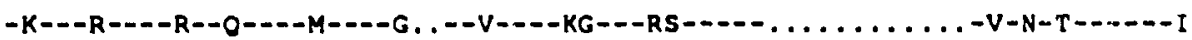

$$
\checkmark / \text { (minor) }
$$$$
1 / \text { (minor) }
$$

/ p6 terminun

$(808)$

CONSENSUSI ESF I fGEEt TPSOKOE? ? ? ? IOKALYPLASLISLFGNDPaSq $\quad 500$

HIVLAI $---R S-V-T T T-S---. . . P I=-E----T--R---N-S-Q-\quad 512$

HIVHXB2R -D-RS-V-TTT-P-D-....PI--E--D-T--R----N--S-Q- 500

HIVMN $\quad---R F-E-T T T-Y=---K K O E T I--D----A--K----N-D-O-\quad 506$

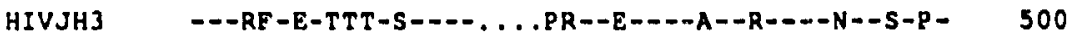

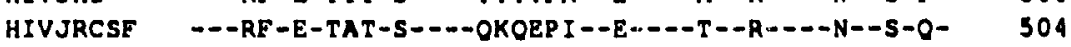

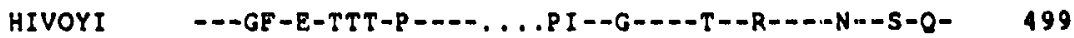

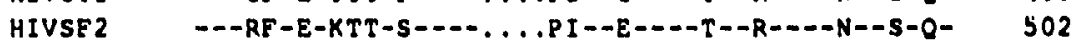

HIVNYSCG --KF-E-TTT-S-D.....PI--E----A--R----S-S-S-O- SOO

HIVNL43 ---RF-E-TTT-S-D.....PI--E----A--R---S-S-S-0- 300

HIVCDC4 -D-RE-D-TTT-S-D.....PR--E-D-A--R-D-N-S-O- 500

HIVHAN --RF-.-ATA-S-D.....PI--E----A--K-N--S--S-0- 499

HIVAT $\quad--R F-E-T . T-S---. . . K I--E----A--K----N--S-O-\quad S 01$

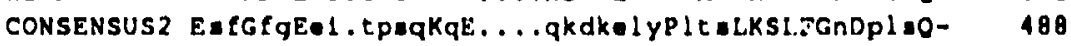

HIVELI -SF-FG-EI.TPSO-O-... OKDKELY-LTS-...-N-PLS-_ 500

HIVZ226 -SF-FG-EI.TPSO-O-...OKDKELY-STA-..-N-PLL-- 501

HIVNOK -SF-FG-EI.TPSQ-O-...OKDKELY-LAS-_.-N-N-PSS-- 497

HIVMAL $\quad$ SF-FG-EI.KPSO-O-...OKDKELY-LAS-.-.-N-OLS-D $50 S$

HIVU455 -IF-MG-KM. TSPA-O-...LLKDEOT-I.VS _....-N-PLS- - 493

SIVCPZ $\quad$ SY-YO-EE.KSOE-K-....G.ESSLY-PTS-..--S-PSS-D 508 
Information dense stretches 10.93 or greater) are Indlcated by ***.

CONSENSUSI MGARASULSGGQLDKWEKIRLRPGGKKKYKLKHIVWASROLERFAUNPGLLET SOGCROI I gOLQRSLqT 70 CONSENSUS2 -.-.---s-gK--?---1---G-k---r1--1V----E--rfaln-G-1------kq11---qPa?-- 68

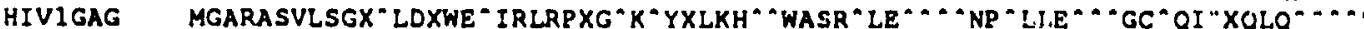

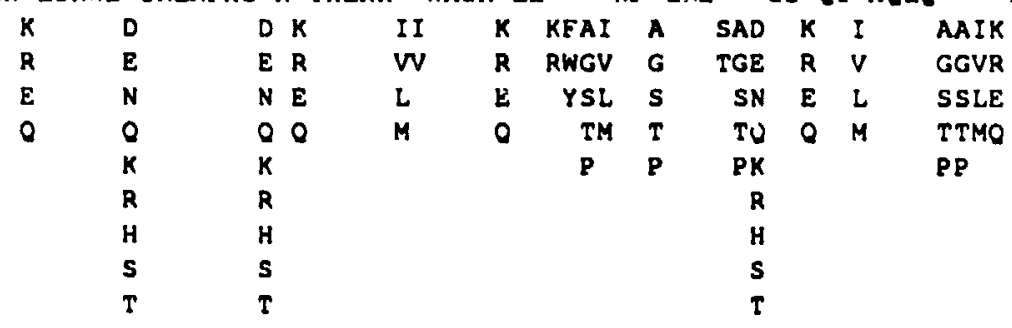

"W"*"

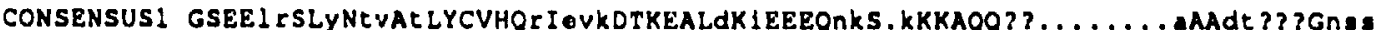

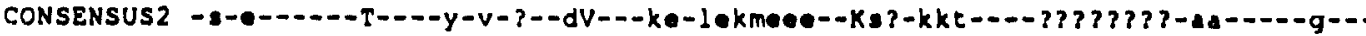

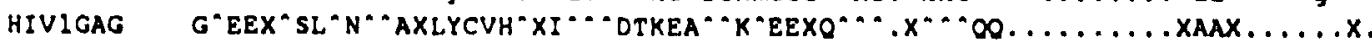
$S$ D F AI K DIK ID I DDD KKA T E W GV R EVR VEV EEE RRG $\begin{array}{llllllllll}N & Y & E & L N & L & N N N & E & S\end{array}$

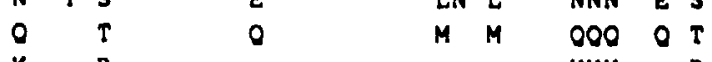
$\begin{array}{lll}\mathrm{P} P K K & \mathrm{P}\end{array}$ R RRR

$\begin{array}{ll}\text { H } & \text { HHH } \\ \text { S } & \text { SSS }\end{array}$

T

p17 \/ p24

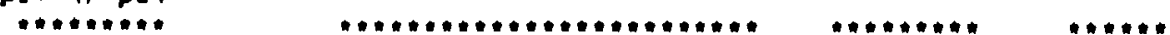

CONSENSUS1 GVSONYPIVON! QGOMVHOAISPRTLNAWVKL VEEKAFSPEVIPMF GALEGATPODLNTMLNTVGGHOA CONSENSUS2 -v-q---1- - - PO--mV--A1 - - -

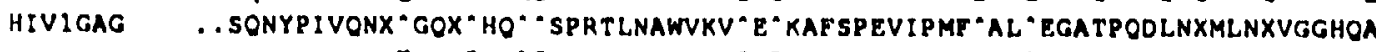

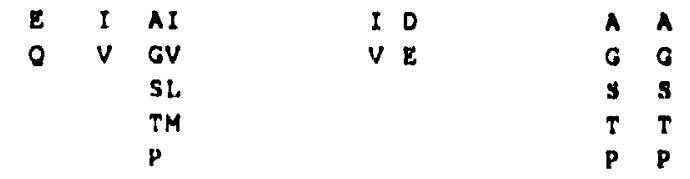

n...................

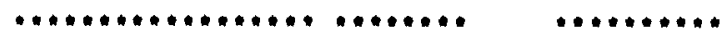

CONSENSUS! AMOMLKETI NEEAAEWORLHPULAGP I APGOMREPRGSDIAGTTSTLOEOIGWMTONPP IPVGEIYKRW

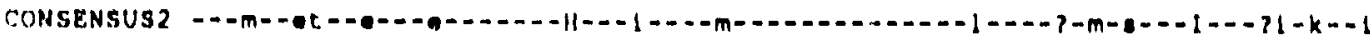
HIVIGAG

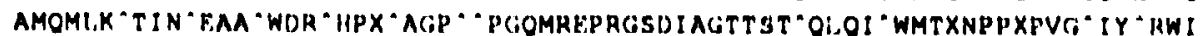

\begin{tabular}{|c|c|c|c|c|}
\hline 0 & 0 & $D$ & I & D \\
\hline $\mathbf{E}$ & $\boldsymbol{z}$ & E & $v$ & E. \\
\hline & & & I. & $\mathbf{N}$ \\
\hline & & & $M$ & 0 \\
\hline & & & & K \\
\hline & & & & $\mathbf{R}$ \\
\hline & & & & 11 \\
\hline & & & & s \\
\hline & & & & \\
\hline
\end{tabular}




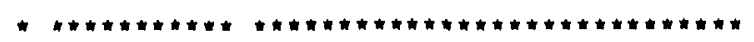

CONSENSUSI ILGLNKIVRMYSPTSILDI ROGPKEPFRDYVDRFYKTLRAEOASOEVKNWMTETILVONANP DCKT I LKA

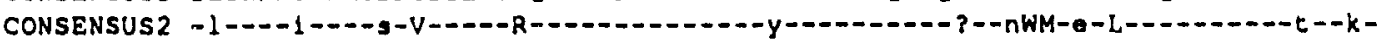

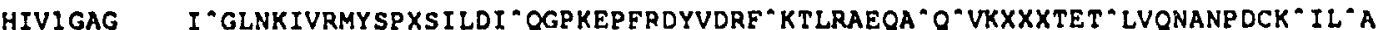

$\begin{array}{llllll}\text { I } & \text { K } & \text { F } & \text { S D } & \text { I } & \text { S } \\ \text { V } & \text { R } & \text { W } & \text { T E } & \text { V } & \text { T } \\ \text { L } & \text { Y } & & \text { L } & & \\ \text { M } & & & \text { M } & \\ & & & \text { F } & \\ & & & \text { W } & \end{array}$

$$
\text { p24 V/ V/ }
$$

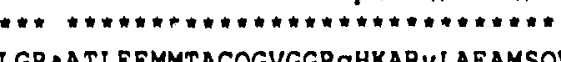

CONSENSUSI LGP AATLEEMMTACQGVGGP gHKARVLAEAMSOV. tnaA, IMmOrGNF rnOrKtUKCFNCGKnGHIAKN CONSENSUS2 - - ? - - -

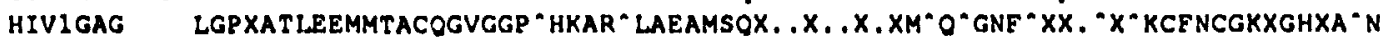

CONSENSUSI CIAPRKKGCWKCGKEGHOMKDCE ? ?RUANFLGKIWPSHKGRPGN ? ? ? ? ? ? ? ? ?E IOARPEPTAPPE

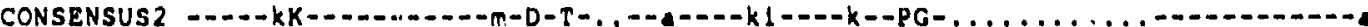

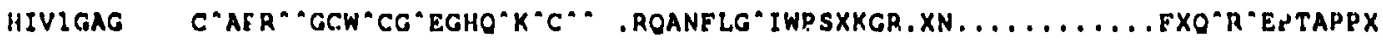

$$
\checkmark(m \operatorname{lnor}) \quad \quad 1 / \text { (minor) }
$$

f p6 terminun 18001

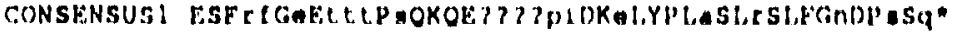

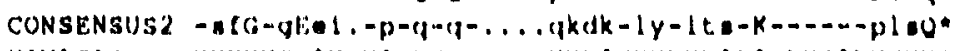

500

HIVIGAO

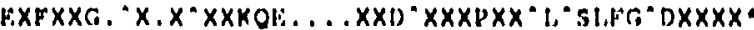

ABB

$\begin{array}{ll}K & \boldsymbol{A} \\ \mathrm{R} & \mathbf{0} \\ \mathrm{R} & \mathbf{s} \\ \mathbf{0} & \mathrm{T} \\ & \mathbf{p}\end{array}$

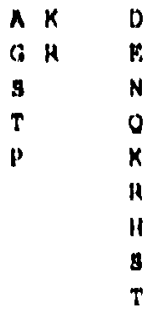




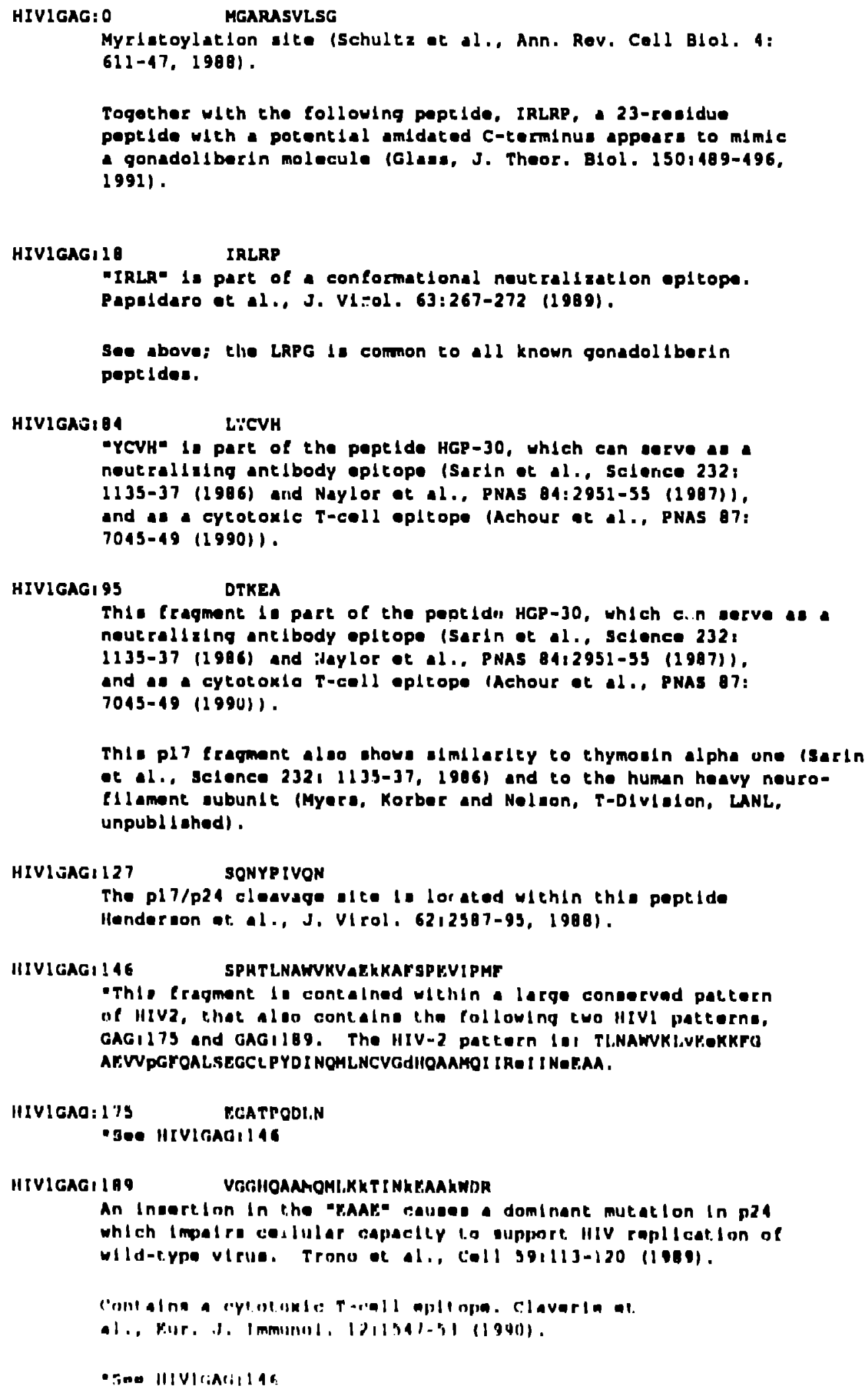




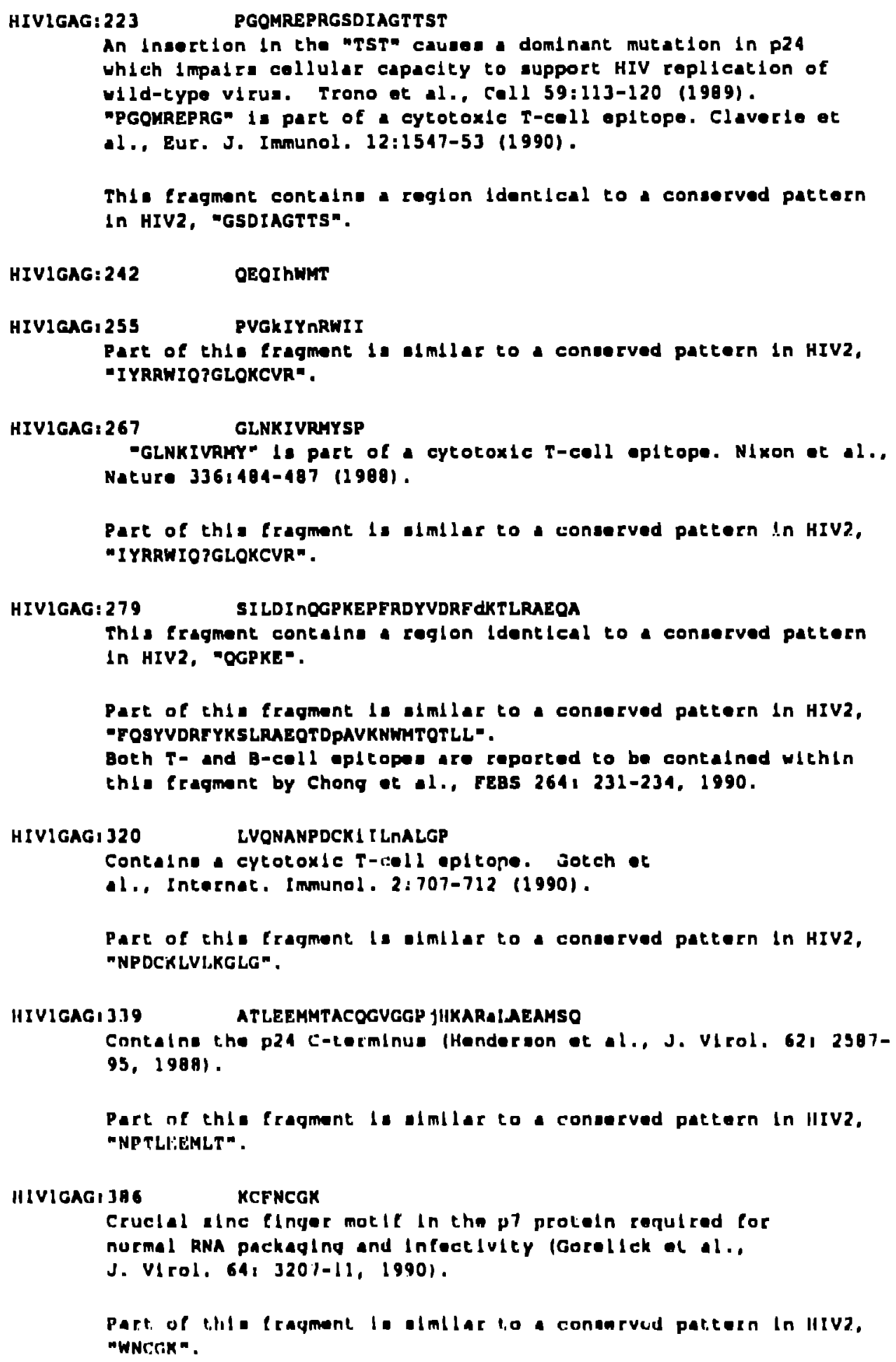




\section{HIVI GAG}

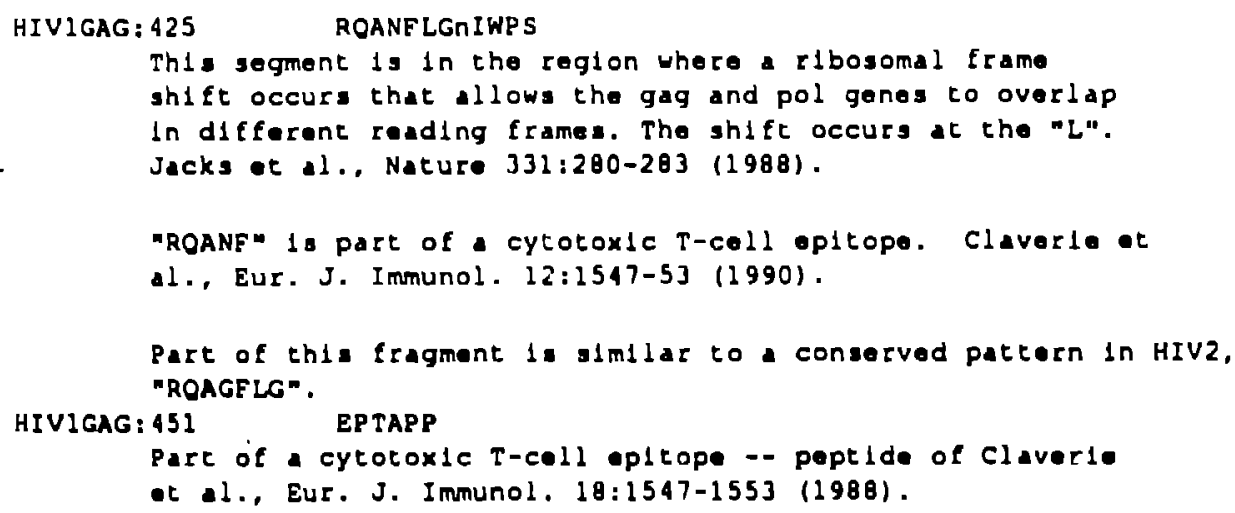




\section{HIV2/SIV GAG}

CONSENSUS MGATRSVL?GKKADELEK1RLRPGGKKKY?LKH?VWAaNELDTFGLAESLLESKEGCOKI I SVLAPIVPT HIVZROD HIV2NIHZ HIVZISY HIVZST HIV 2BEN HIV2DI94 HIV2CHI HIV2D205 SIVMM2 51 SIVMMI 42 SIVMM239 SIVMNE SIVSMMH 4 SIVSMMPBJ

CONSENSUS HIV2ROD HIV 2 NIHZ HIVZISY HIV $25 T$ HIV $2 B E N$ HIV2DI94 HIV2GHI HIV2D205 SIVHM231 SIVHMI 42 SIVHM239 SIVMNE SIVSMMH4 SIVSMMPBJ

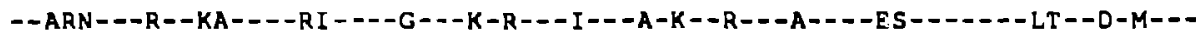

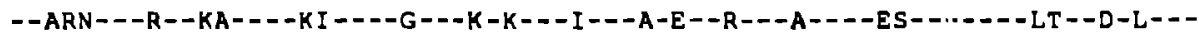
$--A K N=--R--K A----K I=---G---K-R---I---A-E--R=--T----E S------I S--E-L---$

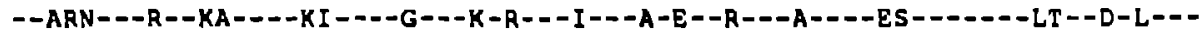

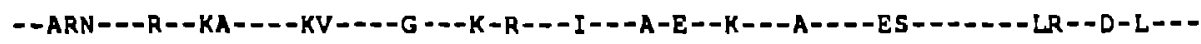

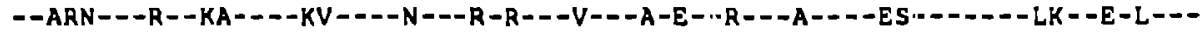

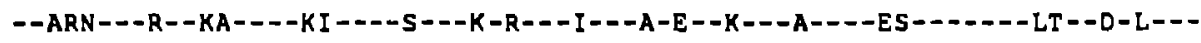

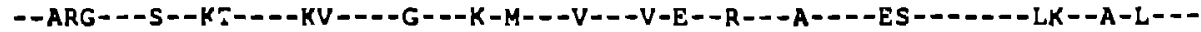
$--A R N=--S--K A----K I----G---K-M=--V---A-E--R---A----E N=------L 5--A-L-D$ $--A R N=--S--K A----K I----G=-K-M---V---A-E--R---A-\cdots=-E N---=--L S--A-L---$

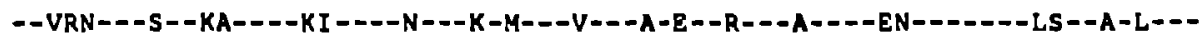

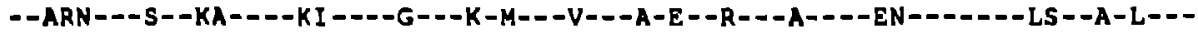
--ARN-- -S--EA----KV----N---K-M---V---A-E--R---A----DN-------LS--A-L-- $--A R N---S--K A----K I----G---R-Q---I---A-E--R---A----E N------L S--A-L-\cdots$

pl 6 I/p28

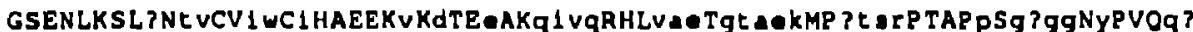
--_---OF-TV--IW-I-----V-D--G--OIVR---VAE-GTAEK--STSR----S-EKGG-Y---H.

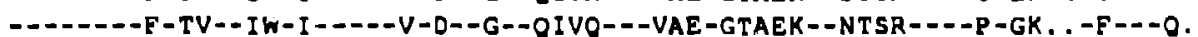

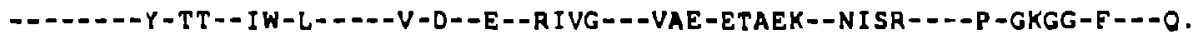
$------F-T V--I W-I-----A-D--E--O K V Q---V A E-K T T E K--S T S R---P-G N G G-F--0$. $------E-T V--I W-L-----V-D--E--K L A Q---V A E-G T A E K--N T S R----P-G K R G-Y---Q$.

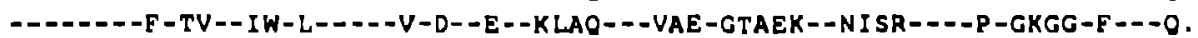

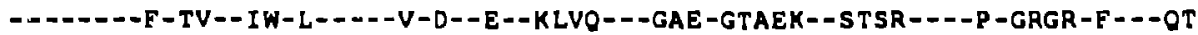
- - - - - -E-IV--IF-L-_-_-V-D--E--KIAQ---AAD- . . EK--AT:UK-_--P-G . . G-Y---O.

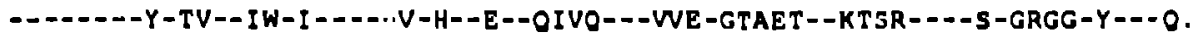

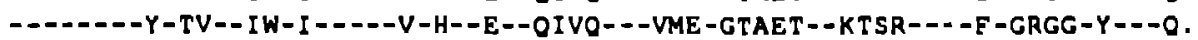

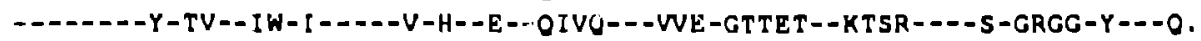
-------Y-TV--IW-I - - --V-H--E--OIVO-_-VVE-GTAET--KTSR-D-S-GRGG-Y--OO.

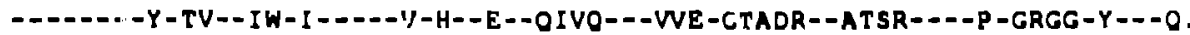

CONSENSUS HIV 2ROD HIV 2NIHZ HIVIISY HIV 2ST HIV2BEN HIV 20194 IIIV2GHI IIIV2D205 SIVMM25! SIVMMI 42 S[VMM2]9 SIVMNE SIVSMMHI SIVSMMPISJ TGGNY THIPLSPETLNAWVKLVEKKFGAEVVPGFOALSEGCLPYDINOMLNCVGDHQAAMOI I RE I ING

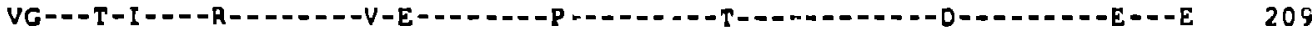

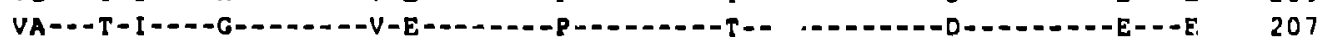

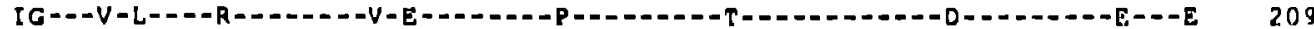

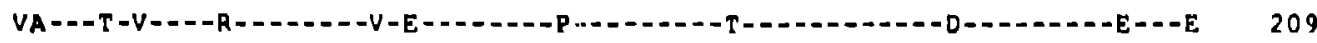

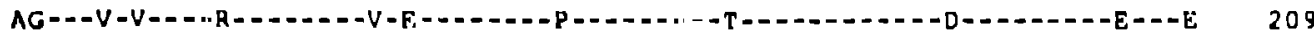

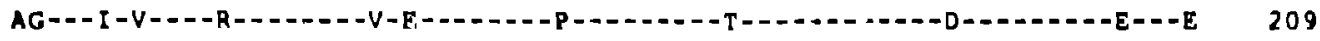

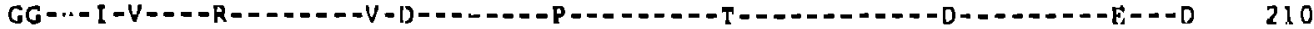

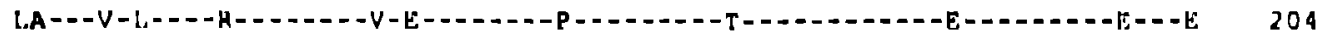

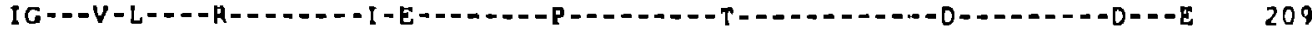

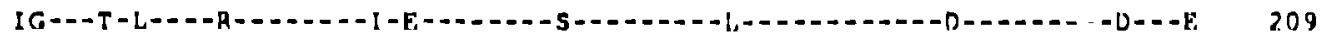

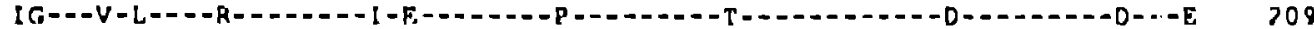

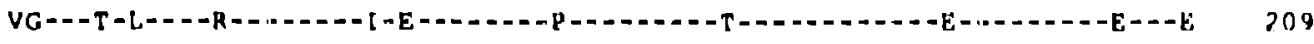

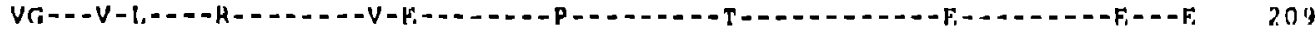

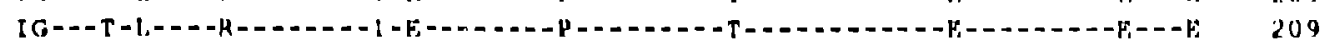


CONSENSUS EAADW ?QHPIPGPIPAGOLREPIGSDIAGTTSEV?EOI GWMYRQQNPUPVGEIYRRWIO?GLOKCVRMY HIV2ROD HIV2NIHZ HIV2ISY HIV25T HIV2BEN HIV2D194 HIV?GH 1 HIV2D205 SIVMM 251 SIVMMI 42 SIVMM239 SIVMNE SIVSMMH 4 SIVSMMPBJ

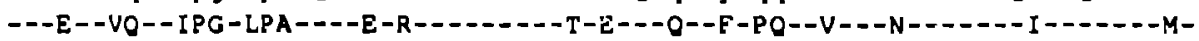

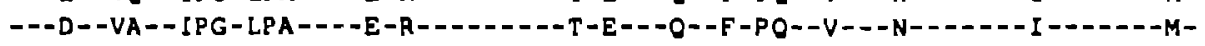

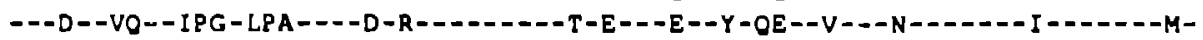

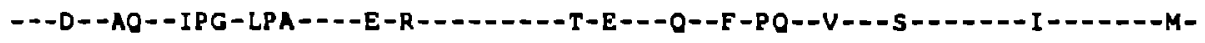

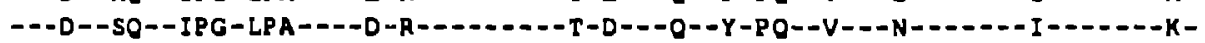

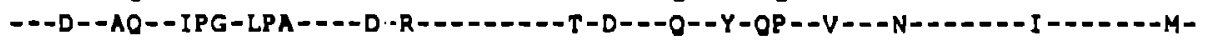

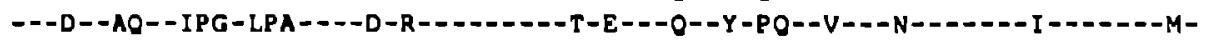

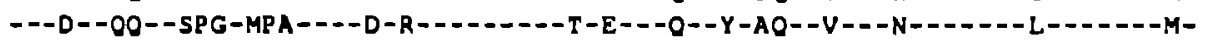

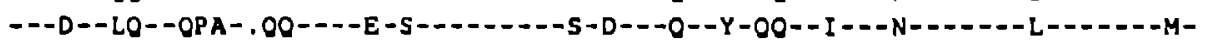

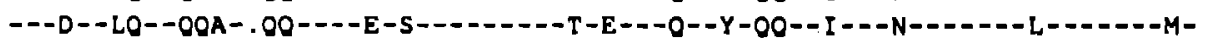

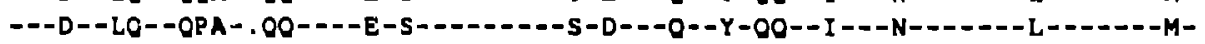

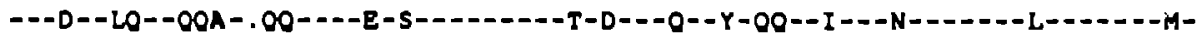

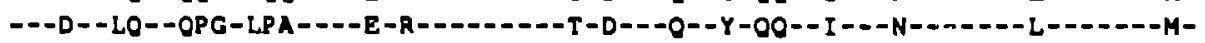

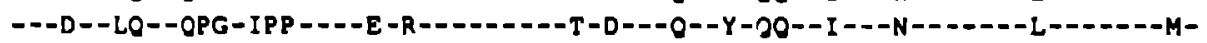

NPTn I LDVKOGPKEPFOSYVDRFYKS LAAEOTDPAVKNWMTOTLLIONANPDCKLVLKGLGMNP TLEEML

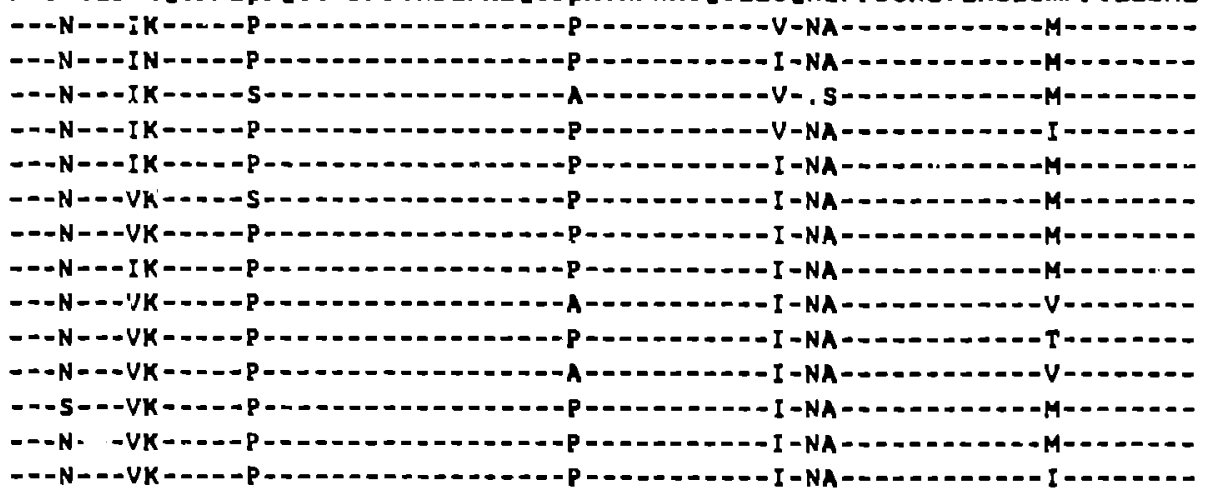

p28

$1 / p^{8}$

Consensus HIV 2 ROD HIVZNIHZ SIV ISY HIV2ST HIV 2 BEN HIV20194 HIV 2GHI HIV'020S SIVMH2 51 S IVMHI 42 SIVMM2 39 SIVMNE SIVSMMH 4 SIVSMMPBJ

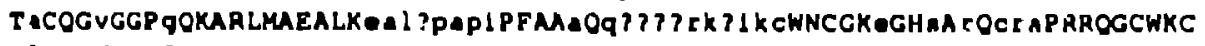

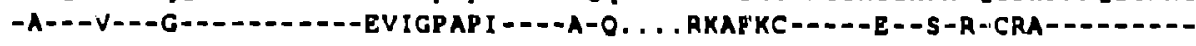

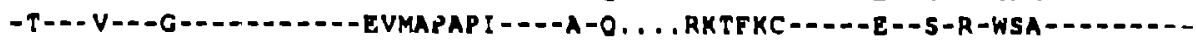

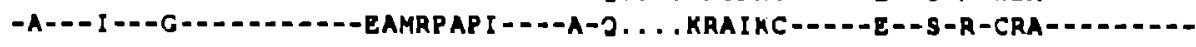

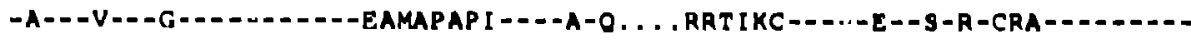

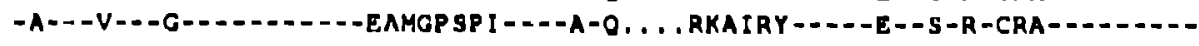

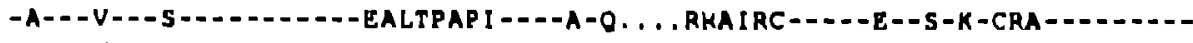

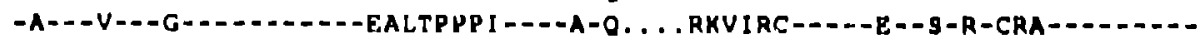

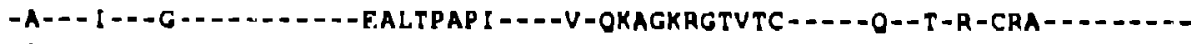

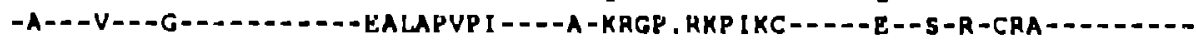

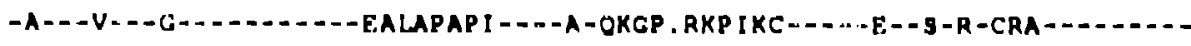

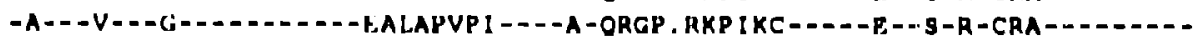

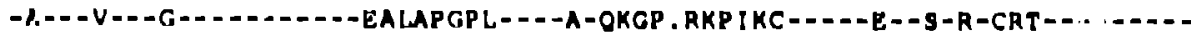

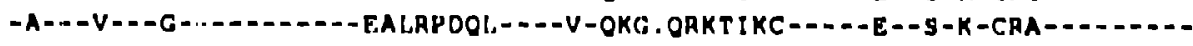

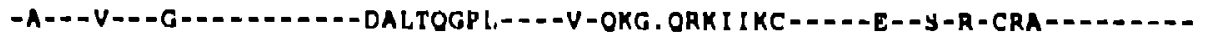

338

349

347

348

349

349

349

350

344

348

340

348

348

349

349

402

415

413

414

413

415

413

416

414

$41 \%$

417

417

417

418

418

II.14 
pol eds $\rightarrow$

$p 8 \backslash / p 1 \quad p 1 \backslash / p 6$

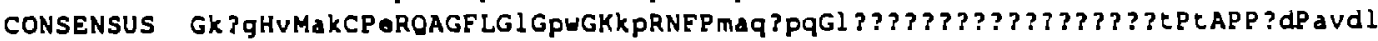

HIV2ROD -KPG-I-TN--D-_._-LL-PW--KP-_-VVAQVPO-L............ T-T---VD-AVDL

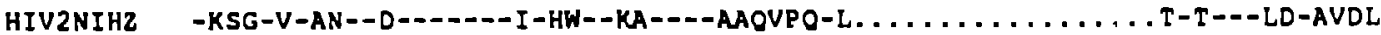

HIV2ISY -KSG-I -AN--D-...--L-PW--KP----VVPSSO-L........... T-T--MD-AVDL

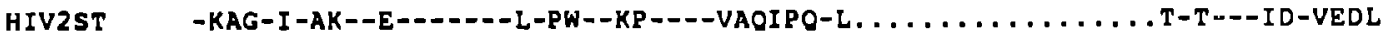

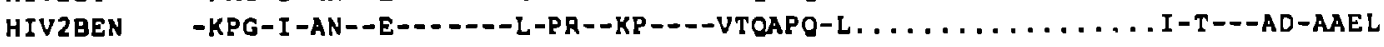

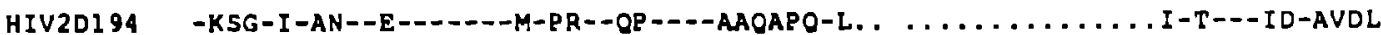

HIV2GHI -KTG-V-AK--E-_...-M-PW--KP-_-VAOAPP-L...........I-T---AD-AVDL

HIV20205 -KTG-I -SK--E-_-_-_L-PW--KP-_--MTOVPQ-VTPSAPPMNPAEGMTPRGAT-S---AD-AVEM

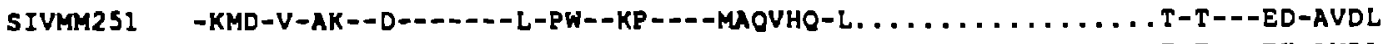

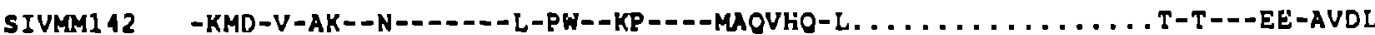

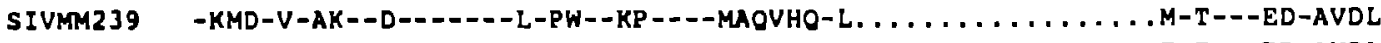

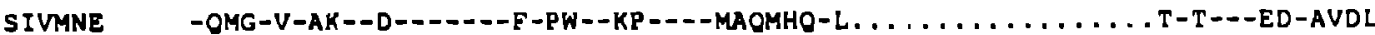

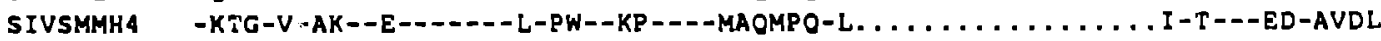

SIVSMMPBJ

-KAG-V-AK--E-_.-.-L-PW--KP----MAOMPO-L............T-T---ED-AVDL

451

467

465

466

467

467

467

468

484

469

469

469

469

470

470

I/ (HIV2 only; not mlnor)

$1 /$ (HIVZ only: not mlnor)/ p6 terminus

CONSENSUS

HIV 2ROD

HIV2NIHZ

HIV2ISY

HIV2ST

HIV2BEN

HIV2D194

HIV2GHI

HIV2D205

SIVMM251

SIVMMI 42

SIVMM 239

SIVMNE

SIVSMAH 4

SIVSMMPBJ

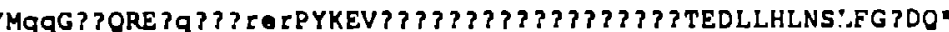

483

-EK - -OQ-KR - - . Q. . RER-- - - TEDLLHLEOGETP YREP P - - - -

522

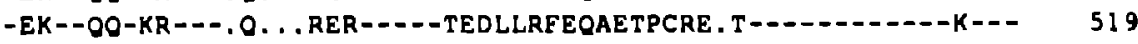

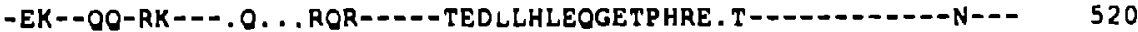

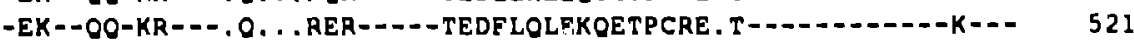

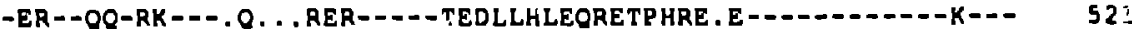

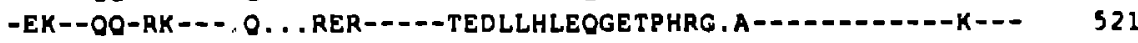

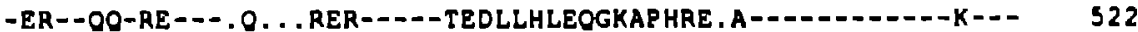

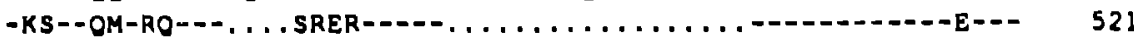

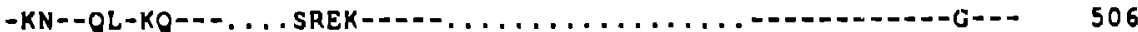

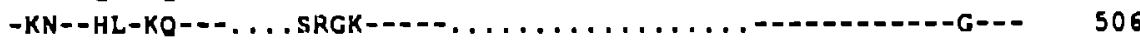

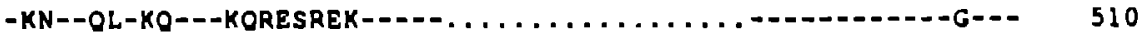

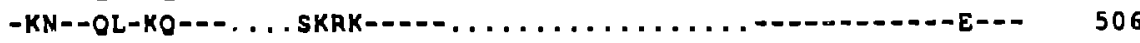

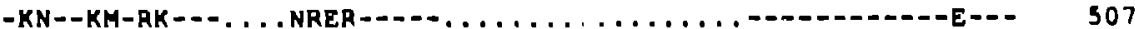

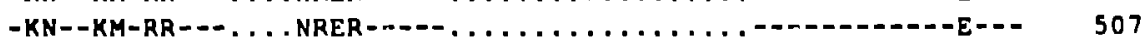


Information dense stretches $(0.93$ or greater) art indicated by ***.

CONSENSUS MGAINSVL?GKKADELEKIRLRPGGKKKY?LKH?VWAANELD.FGLAESLLESKEGCOKIISVLAPIVPT

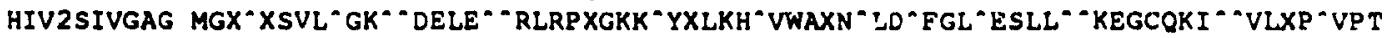

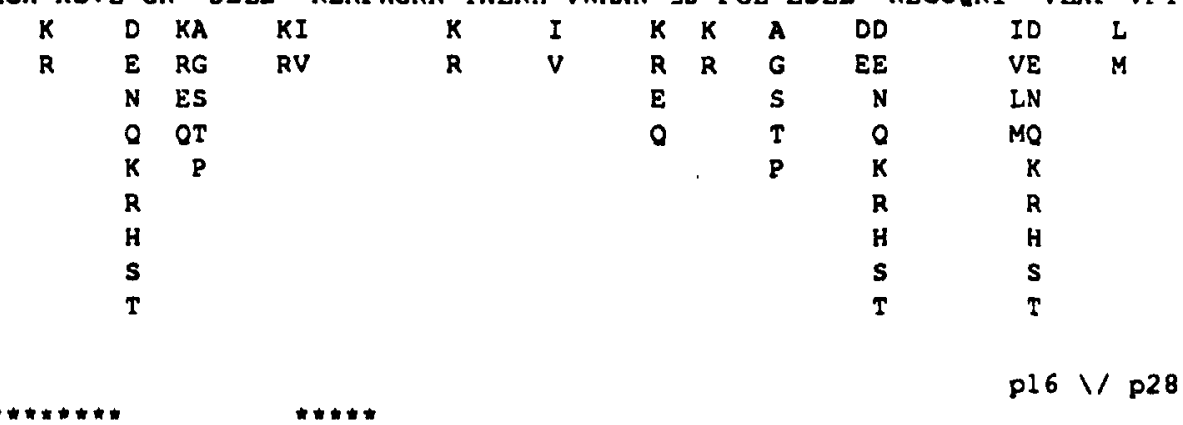

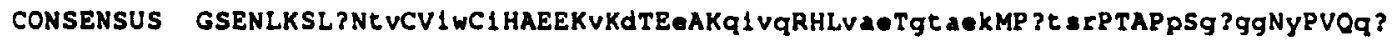

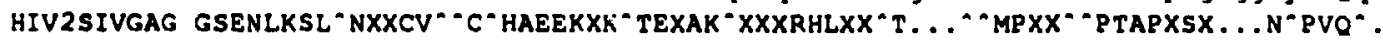

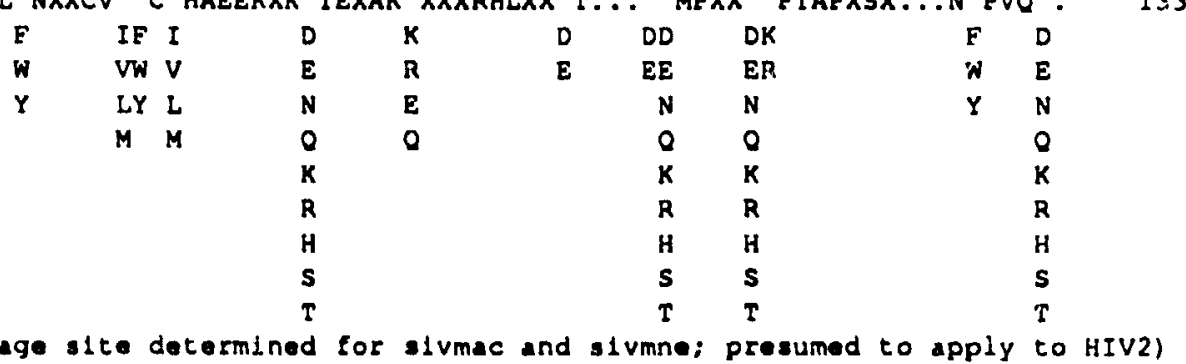

(p28 cleavage site determined for slvmac and sivmne; presumed to apply to HIV2)

PLSPTLNAWVKLVEKKFGAEVVPGFALSEGCEPYDINOMLNCVGdHQAAMOIIREIIN• 201

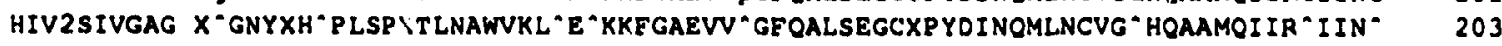

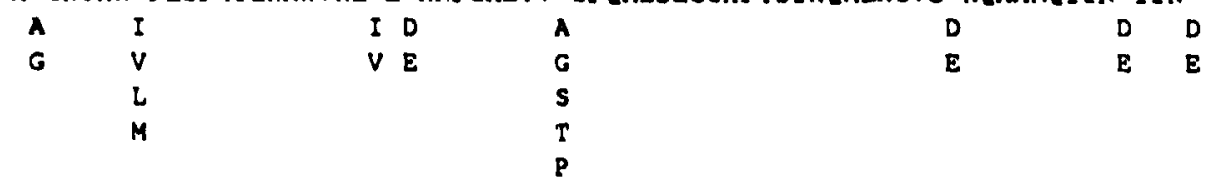

\section{*."}

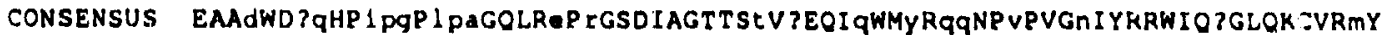
HIV2SIVGAG E.AA“WOXXHPXX"P.XXGOLR "P"GSOIAGTTS"V“EOI"WM"RXXNP "PVG"IYRRMIO"GLOKCVRXY

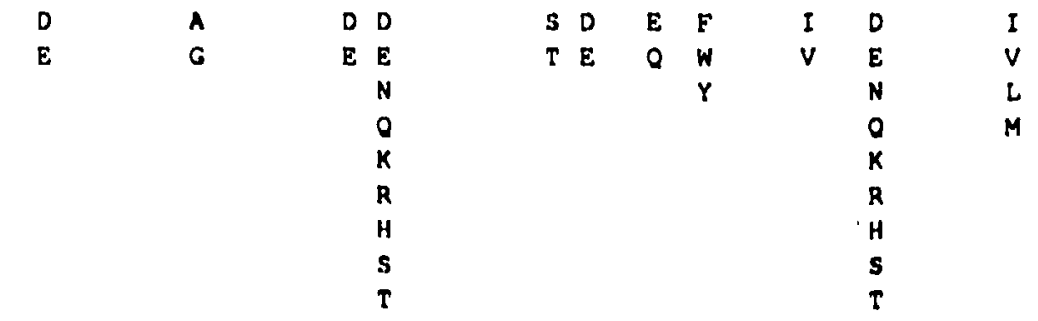


CONSENSUS NPTIILDUKOGPKEPFOSYVDRFYKSLRAEOTDPAVKNWMTQTLLIONANPDCKLVLIGLGMNPTLEEML HIV2SIVGAG NPT"ILD" OGPKE-FOSYVDRFYKSLRAEQTD"AVKNWMTOTLL"Q. "NPDCKLVLKGLGXNPTLEEML

338 341

$\begin{array}{lll}D & \text { ID } & \text { A } \\ \text { E } & \text { VE } & \text { G } \\ \text { N } & \text { N } & \text { S } \\ \text { O } & \text { O } & \text { T } \\ \text { K } & \text { K } & \text { P } \\ \text { R } & \text { R } & \\ \text { H } & \text { H } & \\ \text { S } & \text { S } \\ \text { T } & \text { T }\end{array}$

\section{Iacoc \\ p28 \\ $\backslash \mathrm{p \theta}$}

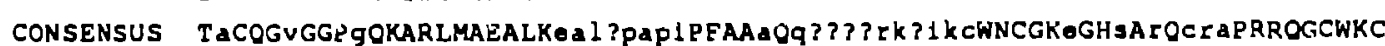

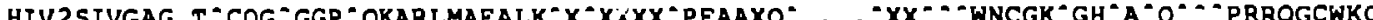

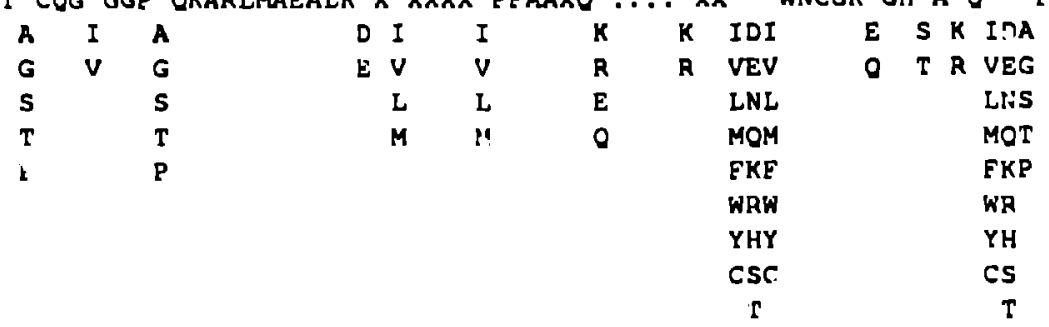

pol cds $\rightarrow$

$$
\text { pQ } \backslash / p 1 \quad p 1 \backslash p \in
$$

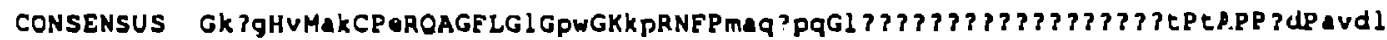

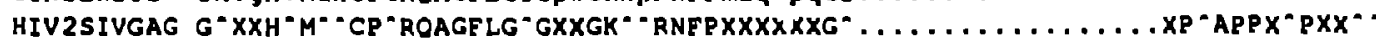

$\begin{array}{ll}\text { I } & \text { KA } \\ \text { V } & \text { RC } \\ \text { L } & \text { ES } \\ \text { M } & \text { OT } \\ \text { F } & \text { P } \\ \text { W } & \\ \text { Y } & \\ \text { C } & \end{array}$
Y/ (litv2 only; not minor)
V (HIV2 only; not m1nor)/ p6 term1nus

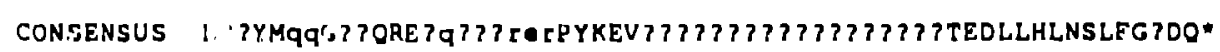

IIIVZSIVGAG L" "YM'XG" "ORE.....'X'PYKEV.

483

$\begin{array}{cccccc}\text { KD } & \text { D } & \text { KK } & & \text { K K } \\ \text { RE } & \text { E } & \text { RR } & & \text { R } & \text { R } \\ \text { EN } & \text { N } & \text { E } & & \\ \text { O } & \text { O } & \text { O } & & \\ \text { K } & \text { K } & & & \\ \text { R } & \text { R } & & & \\ H & H & & & \\ \text { 5 } & \text { S } & & & \\ \text { T } & \text { T } & & & \end{array}$


CONSENSUS SIVAGMTYO SIVAGMISS SIVAGM3 SIVAGM692 SIVAGM677

CONSENSUS SIVAGMTYO SIVAGM155 SIVAGM3 SIVAGM 692 SIVAGM 677

CONSENSUS SIVAGMTYO SIVAGM155 SIVAGM SIVAGM 692 SIVAGM 677

CONSENSUS SIVAGMTYO SIVAGMISS SIVAGM3 SIVAGM 692 SIVAGM 677

CONSENSUS SIVAGMTYO SIVAGMI5S SIVAGM3 SIVAGM692 SIVAGM 677

CONSENSUS SIVAGMTYO SIVAGMISS SIVAGM

SIVAGM692 SIVAGM677

CCNSENSUS SIVAGMTYO SIVAGMISS SIVAGM3

SIVAGM692 SIVAGM677

CONSENSUS SIVAGMTYO SIVAGMISS SIVAGM

SIVAGM692 SIVAGM677
MGaat SALnFRqLD?FEhIRLRP NGKKKYOIKHLIWAGKeMERFGLHEkLLEteEGCKK I IEVL ?P LEPT

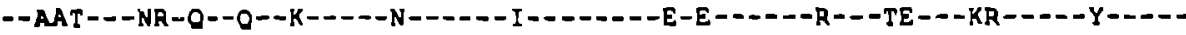

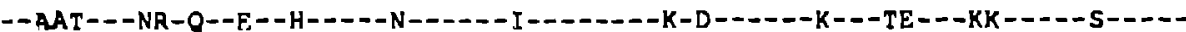

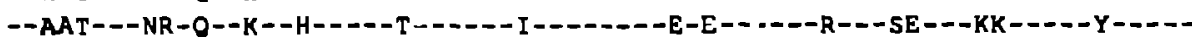

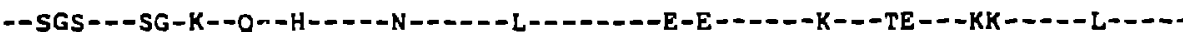

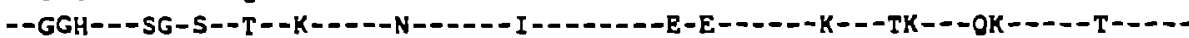

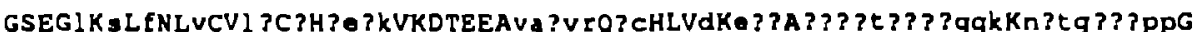
----L-S-F--V--LY-L-KEOK--..---VATVR-HC---E-EKS- . . . . TETSSGOK-NDKGIAAPP ----M-S-Y--V--LL-V-OEKK------OLAIVR-CC---D-EKT-V. . . I.PPGGOO-NNTGGTATP----L-S-F--V--LE-V-KDKE-------VAIVR-CC---E-ERN -ERNTTET SSGOK-NDKGVTVPP-

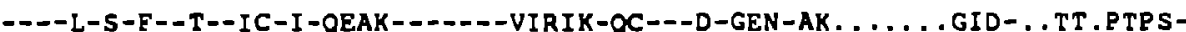
--O-L-A-F--C--IW-I -AEOK---.---WVTVK-HY---D-NEK-AK.........K-NETT . APPG-

?SQN EP \& QGOGNAWUHU PLSPRTLNAWVK AVEEKK FGAE I VPMFOA LSEGC TPYD I NOMLNV I GDHOGAL

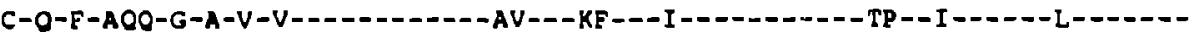

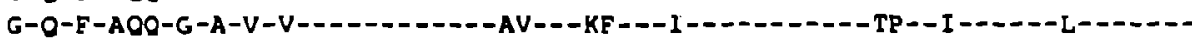
G-O-F-AOO-G-A-I-V-_-

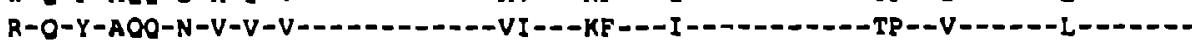

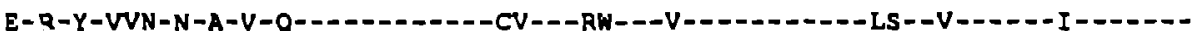

OI VKE I INEEAAQWD? THPPPAGP LPAGQLRDP IGSDIAGTT STVOEOI EWI YTANPRVDVGAI YR IW I I

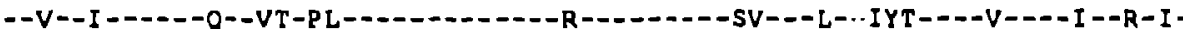

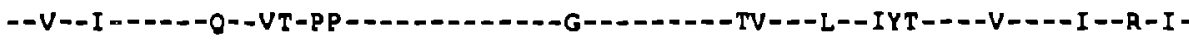

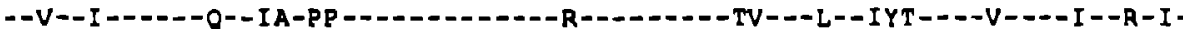

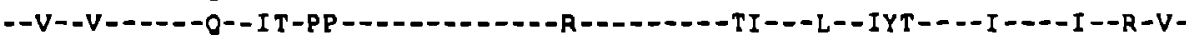

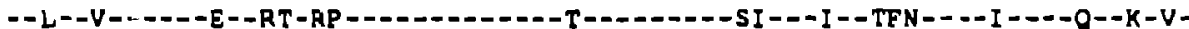

IGLOKGVKMYNPVSVLDIROGPKEPFKDYVDRFYKAI RAEOA SGOVKQWMT OLLLIONANP DCKVI LKGL

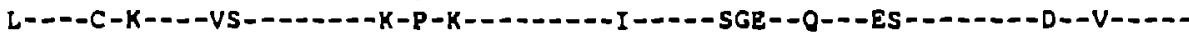

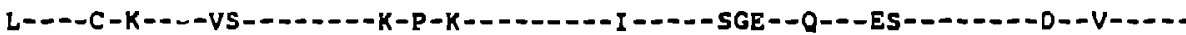

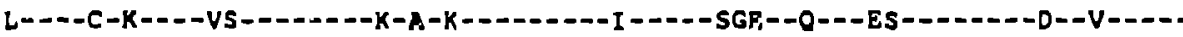

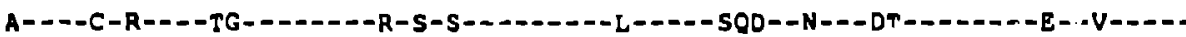

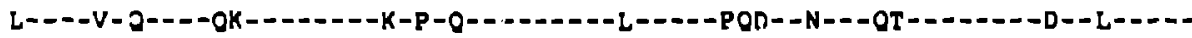

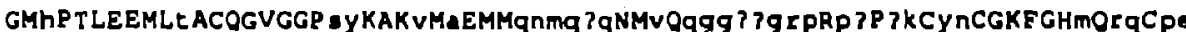

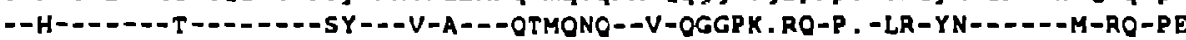

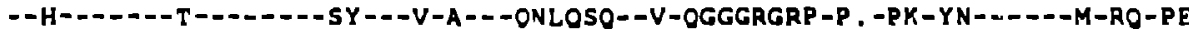

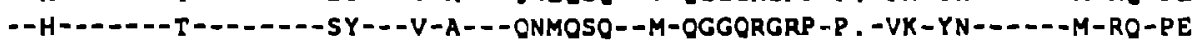

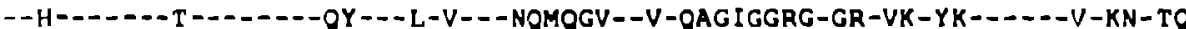

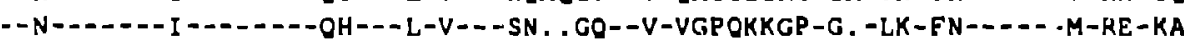

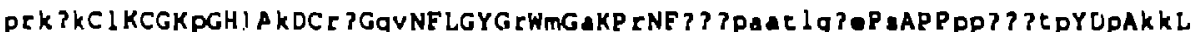
PRKTK-L----L--L-K--R. -OV-_.---R-M-A--R--... PAATLGAE-S---PPSGTTP--P-KK-

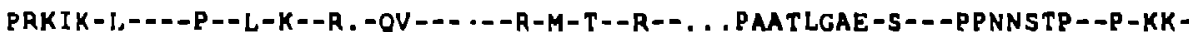

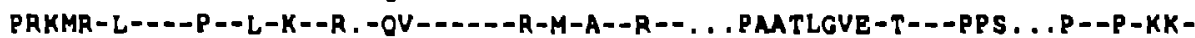
KGPVV-L----P--F-R--R, -AV------R-M-A--K--LEHPAA. Y. . -S---PPHNPGA--E-TRPROIK-F----I --M-K--KN-OA--.---H-S-A--R--VOYRGDTVGLE-T--- . . META--P-KK-

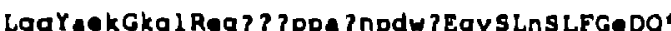
-OO-AEK-KOL-EOKRNPPAMNPDWT-GY - - N-D--E--. -OO-AEK-KOM-NONRNPPANNPDWN-GY--N-D-E-.

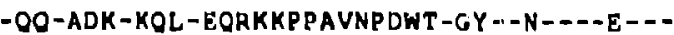
-EK-TOE-AOD-RKVEKS. SOAGREE-DY--K-- -E---OQ-AEK-ORL-EEREOTRKOKEKEV-DV--S---G---

\section{6}

519

520

521

513

311

$11 \cdot 18$ 
Information dense regions $(0.93$ or greater) are Indleated by ***.

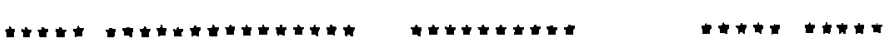

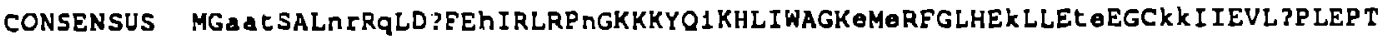

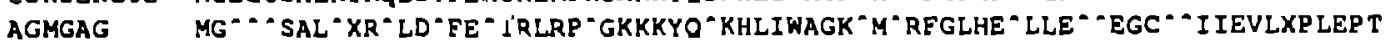

$\begin{array}{llllllllllll}\text { AAD } & \text { D } & \text { D } & \text { D } & \text { D } & \text { D } & \text { I } & \text { K } & \text { K } & \text { K } & \text { SK } & \text { KK } \\ \text { GGE } & \text { E } & \text { E } & \text { E } & \text { E } & \text { E } & \text { V } & \text { R } & E & \text { R } & \text { TR } & \text { RR } \\ \text { S N } & \text { N } & \text { N } & \text { N } & \text { N } & \text { N } & \text { L } & \text { E } & & \text { E } & \text { E } \\ \text { T } & 0 & 0 & 0 & 0 & 0 & 0 & M & 0 & & 0 & 0\end{array}$

$\begin{array}{llllllllll}P & K & K & K & K & K & K\end{array}$

$\begin{array}{lllllll}\mathbf{R} & \mathbf{R} & \mathbf{R} & \mathbf{R} & \mathbf{R} & \mathbf{R}\end{array}$

H $\quad H \quad H \quad H \quad H \quad H$

$\begin{array}{llllll}\mathbf{S} & \mathbf{S} & \mathbf{S} & \mathbf{S} & \mathbf{S} & \mathbf{S} \\ \mathbf{T} & \mathbf{T} & \mathbf{T} & \mathbf{T} & \mathbf{T} & \mathbf{T}\end{array}$

\#**艹

CONSENSUS GSEGIKsLENLUCVI?C?H?Q?KVKDTEEAVA TVIO?CHLVdKe??A????E????gqkKn?tg???PPG

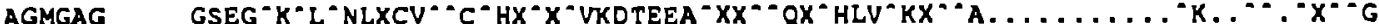

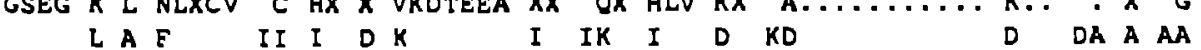
MG $W$ VV V E R $\quad$ V VR V $E$ RE

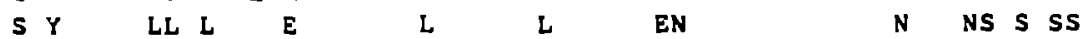

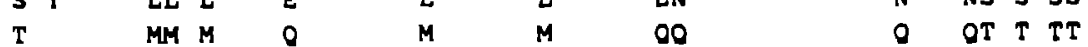
$\begin{array}{llllllll}P & F & F & K & K & K P & P & P P\end{array}$ $\begin{array}{lllll}\mathbf{F} & \mathbf{F} & \mathbf{K} & \mathbf{K} & \mathbf{R P} \\ \mathbf{W} & \mathbf{W} & \mathbf{R} & \mathbf{R} & \mathbf{R}\end{array}$

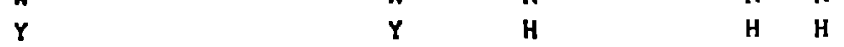

C C $\quad$ S $\quad$ S $\quad$ S

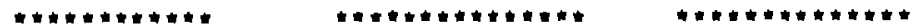

CONSENSUS TSQN EPAGGOGNAWVHVPLSPRTLNAWVKAVEEKK IGAEIVPMFOALSEGCCPYDINOMLNVIGDHOGAL AGMGAG

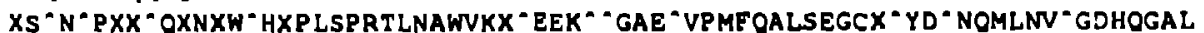

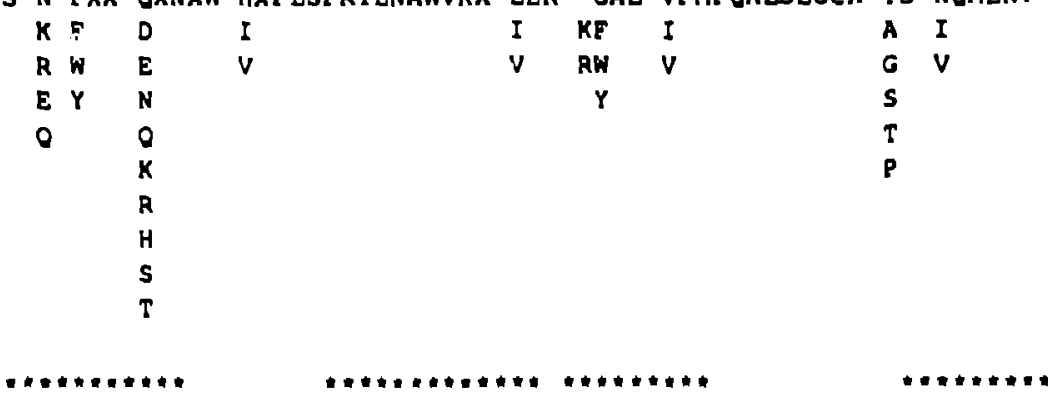

CONSENSUS

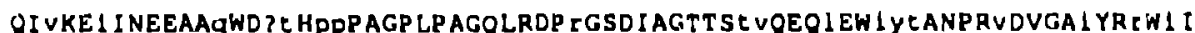
OI - KE" INEEAA " WDX HXXPAGP LPAGQLRDPXGSDIAGTTS " OEO"EWX " ANPR "DVGAXYR* "

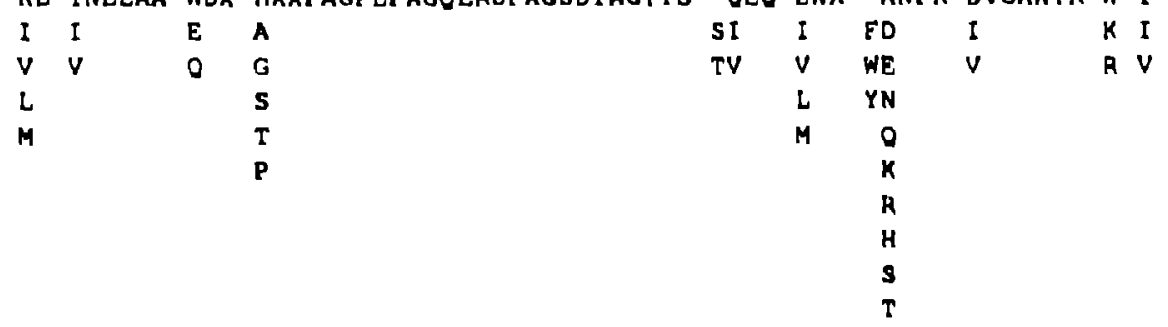




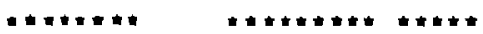

CONSENSUS IGLOKCVKMYNPVSVLDIROGPKEPFKDYVDRFYKAIRAEOASGOVKGWMTOSLLIONANP DCKVILKGL AGMGAG

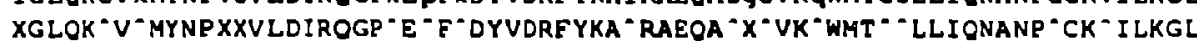

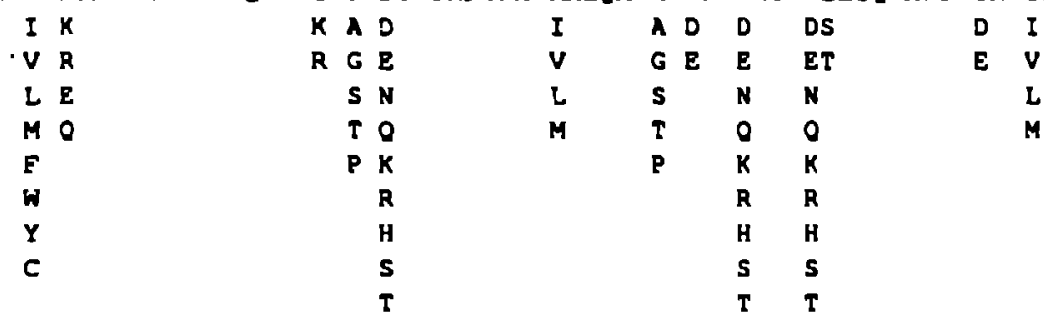

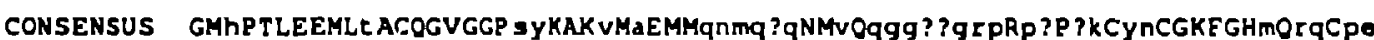
AGMGAG

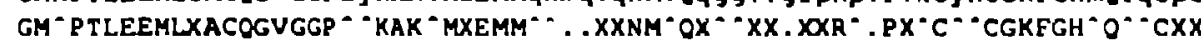

D
N
O
K
R
H
S
I

$\begin{array}{lll}\text { DE } & \text { I } & \text { D } \\ \text { EW } & \text { V } & \text { E } \\ \text { NY } & \text { L } & \text { N } \\ \text { OH } & \text { H } & \text { OQ } \\ \text { K } & & \text { K } \\ \text { R } & & \text { R } \\ \text { H } & & \text { H } \\ \text { S } & & \text { SS } \\ \text { T } & & \text { T }\end{array}$

$\begin{array}{rrrrrrrr}\text { DD } & \text { I } & \text { A } & \text { A } & \text { K } & \text { FD } & \text { I } & \text { KD } \\ \text { EE } & \text { V } & \text { GG } & \text { G } & \text { R } & \text { WE } & \text { V } & \text { RE } \\ \text { NN } & \text { L } & \text { S } & \text { S } & \text { YN } & \text { L } & \text { N } \\ \text { OO } & \text { M } & \text { T } & \text { T } & \text { O } & \text { H } & \text { O } \\ \text { KK } & & \text { P } & \text { P } & \text { K } & & \text { K } \\ \text { RR } & & & & \text { R } & & \text { R } \\ \text { HH } & & & & \text { H } & \text { H } \\ \text { SS } & & & & \text { S } & \text { S } \\ \text { TT } & & & & \text { T } & \text { T }\end{array}$

$\bullet \bullet \bullet *$

CONSENSUS prK?KClKCGKPGHLAKDCE?GqVNELGYGIWIGAKP ENF? ? PAAElq? PPAPPPP?7?LPYDPAKKL AGMGAG

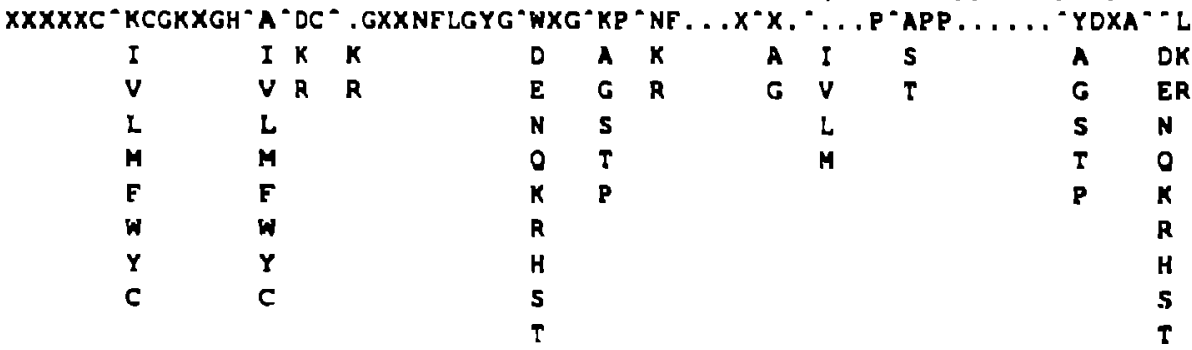

\begin{tabular}{|c|c|c|c|c|c|c|c|c|}
\hline \multirow{3}{*}{$\begin{array}{l}\text { CONSENSUS } \\
\text { AGMGAG }\end{array}$} & \multicolumn{8}{|c|}{ LqqYakkGkq L Req? ? ?ppa ? npdw ?EqYSLnSLFGeDQ" } \\
\hline & $L^{-\infty} Y^{*}$ & $\cdots G$ & $\mathbf{X P}$ & ${ }^{\prime} \mathrm{x}$ &.$x$ & $x y$ & $\mathbf{S L}$ & SLFGXDQ" \\
\hline & EK & ADK & $\mathbf{K}$ & OK & KDA & D & I & D \\
\hline & OR & GER & $\mathbf{R}$ & EA & REG & $\mathbf{E}$ & $v$ & $\mathbf{E}$ \\
\hline & $\mathbf{E}$ & SNE & $\mathbf{E}$ & NE & ENS & $\mathbf{N}$ & L & $\mathbf{N}$ \\
\hline & 0 & ToO & 0 & 00 & OOT & 0 & $M$ & 0 \\
\hline & & PK & & $K$ & $\mathbf{K P}$ & $K$ & $F$ & $\mathbf{K}$ \\
\hline & & $\mathbf{R}$ & & $R$ & $\mathbf{R}$ & $\mathbf{R}$ & 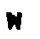 & $\mathbf{A}$ \\
\hline & & H & & H & H & II & $\mathbf{Y}$ & H \\
\hline & & 5 & & 5 & $S$ & 5 & c & $\mathbf{s}$ \\
\hline & & $T$ & & $T$ & $T$ & $\mathbf{T}$ & & $\mathbf{T}$ \\
\hline
\end{tabular}




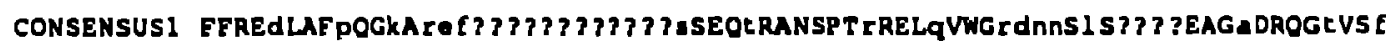

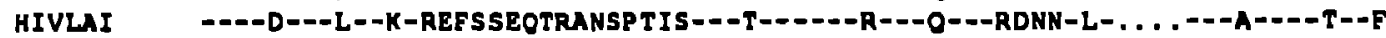

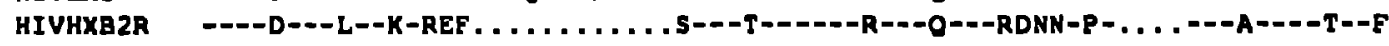

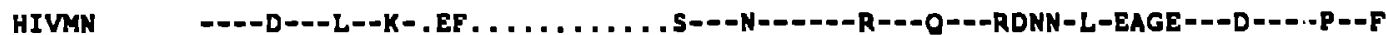

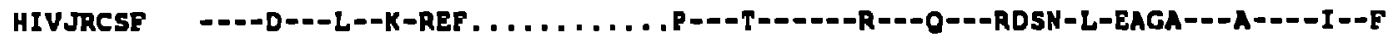

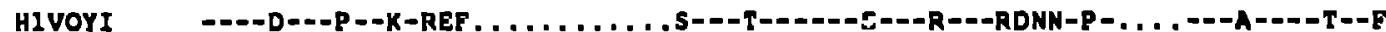

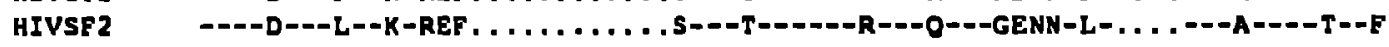

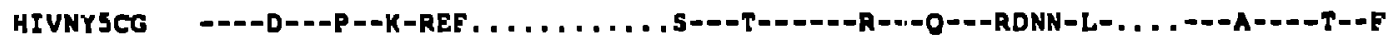

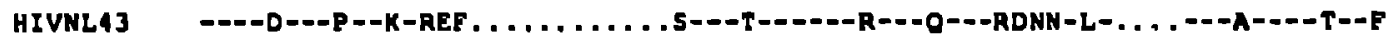

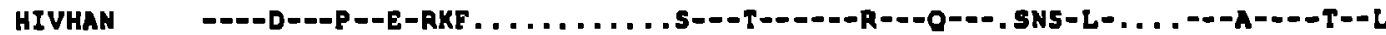

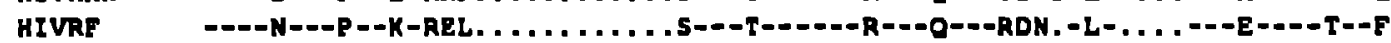

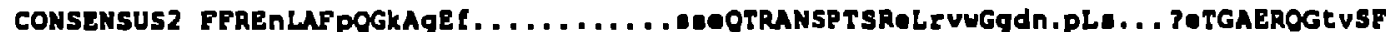

HIVELI ---N---P--K-G-L. . . . . . . . . SPK--...--E-RVW-RDN, P-S . . .K-.-.--TV--

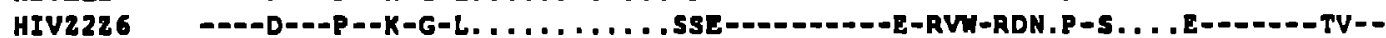

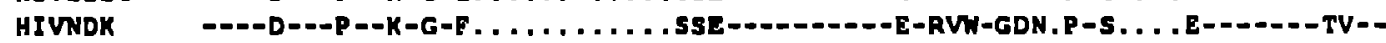

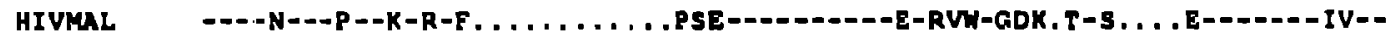

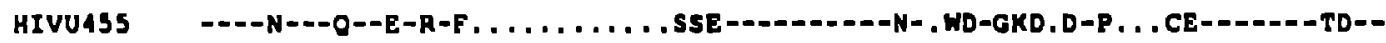

SIVCP2 ----R---P-RE-ROL. . . . . . . . . . CAE-N-T-G--D-E-WVP-GR . .EEP . . . GEERGRE - . SI -T

V/ protease e- gag ads end

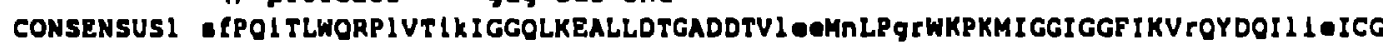
HIVLAI NF--I--.---L--IK-----_-

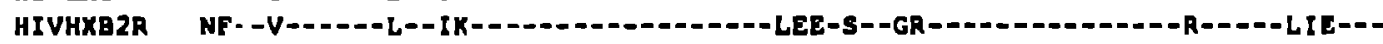
HIVMA

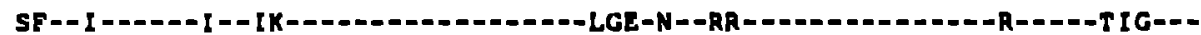

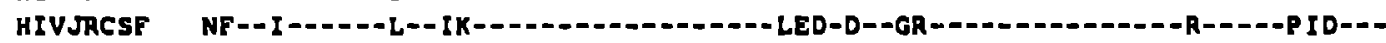
HIVOY: NL-I I -

HIVSF2 HIVNYSCG HIVNLA3 HIVHAN

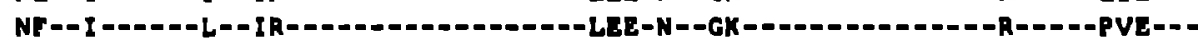

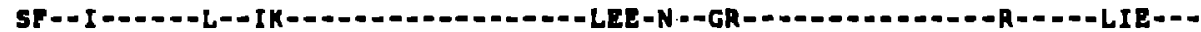

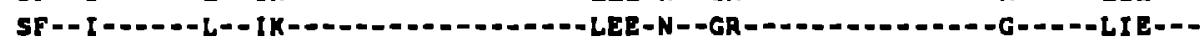

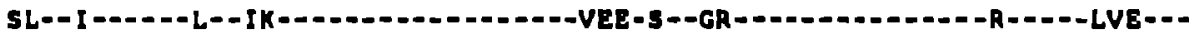

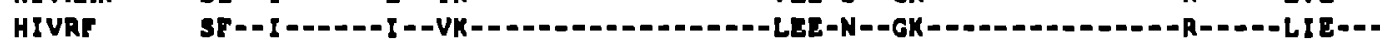
CONSENSUSZ IPOITLWORP IVE IKL GGOLKEALLDTGADDTVLEOI NLPGKWKPKIGGIGGTIKVROYDOI I IEICG HIVELI HIVZ226 HIVNDK HIVMAL HIVUA5S SIVCPZ MF-

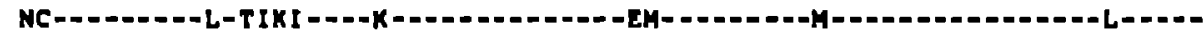

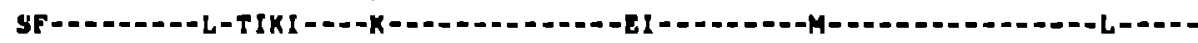

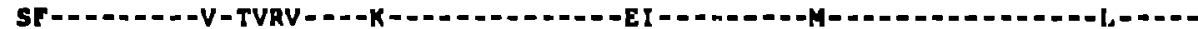

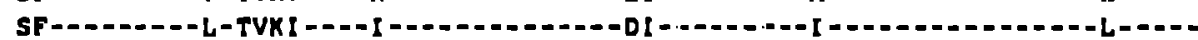

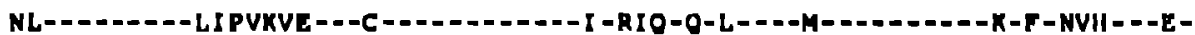

prolanase $/ /$ p66, p31

CONSENSUS! IKALGTVLVGPTPUNI I GRNLLTOIGCTLNFPISP I KTVIVKLKPGMOGIKVKOWP LTFKK IKALVEICT HIVLAT HIVHXO2R HIVMN HTVJRCSF HIVOYI HIVSF2 HIVNYSCG IIIVNLA3 IIIVHAN HIVRT CONSENSUS2 HIVELI IIIV7,226 HIVNDK HIVMal. Hivuass inciry

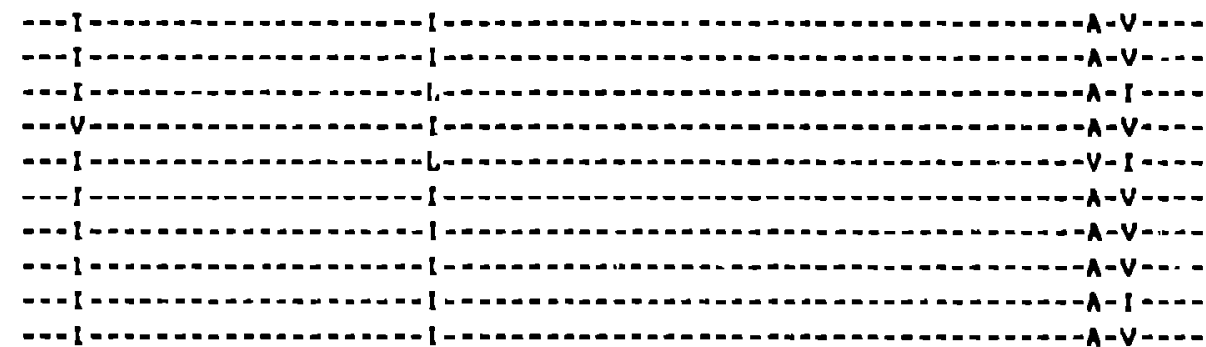

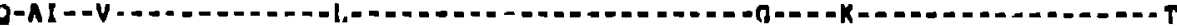
H-AI - -V - L

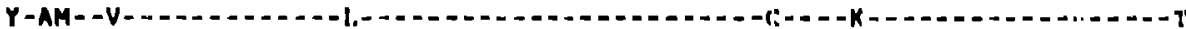

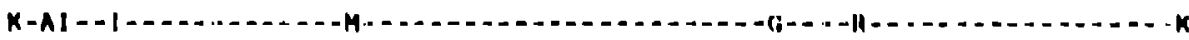

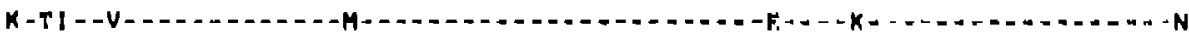

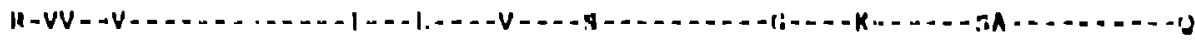

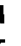


CONSENSUS1 EKEKEGKI SKLGPENPYNTPUFAIKKKDSTKHRKLVDFRELNK TTODFWEVOLGIPHPAGLKKKKSVTVL

HIVLAI HIVHXE2R -

HIVHM

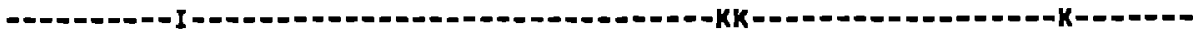

HIVJRCSF

HIVOYI

HIVS.' 2

HIVNYSCO

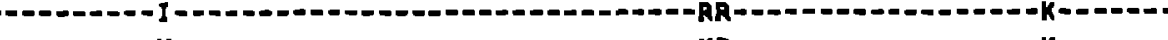

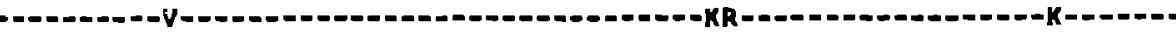

-

HIVNL43

HIVHAN

-

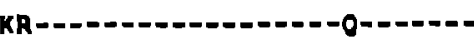

HIVRF

CONSENSUS2 ๑HEKEGKI I IGPENPYNTP IFAIKKKDSTKWRKLVAFRELNKRTODFWEVOLGIPHPAGLKKKKSVTVL

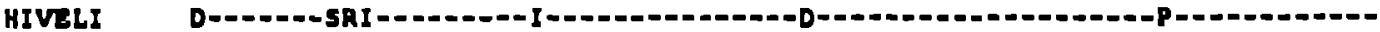

HIVZ226

HIVNDX

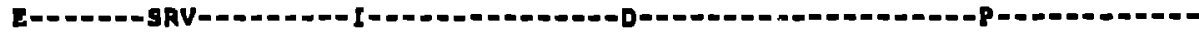

HIVMAL

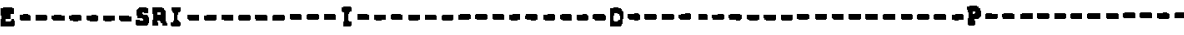

HIVUA5S

SIVCPZ

D-D-OKI -

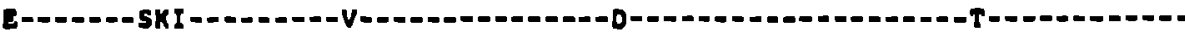

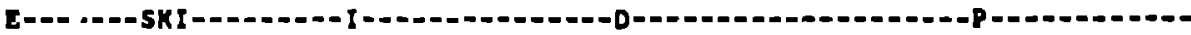

CONSENSUSI DVGDAYFSUPLDKAFRKYTAFTLPSINNETPG IRYOYNVLPOGWKGPAIFOASMTKILEPFEKONPAIV HIVLAI HIVHXB2R HIVMN HIVJRCSF HIVOYI HIVST2 HIVNYSCG HIVNLA 3 HIVHAN

HIVRT DVDAYFSVELK C._. -

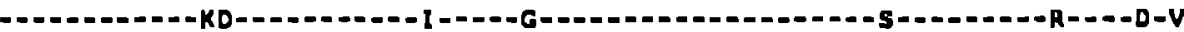

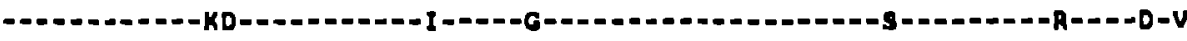
-

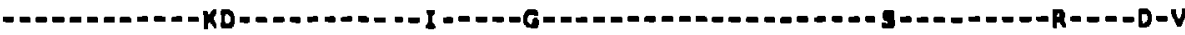

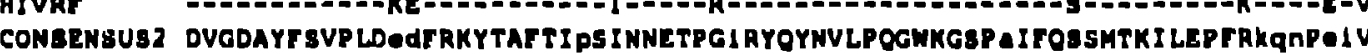
HIVELI HIV2226 HIVNOK HIVMAL HIVUASS SIVCPz -

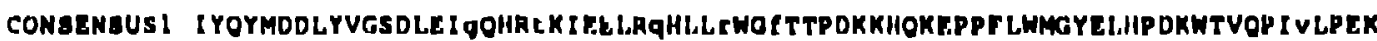
HIVLA: IIIVHXA2R IIIVMN HIVJRCgr IIIVOY I IIIVSF? HIVNYSCO HIVNLA J HIVHAN IIIVRT - - D. -

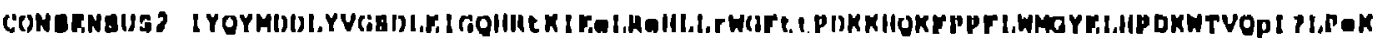
IITELI IItVร276 IIIVNOK IIIVMaL. IIIVU439 nivert.

-

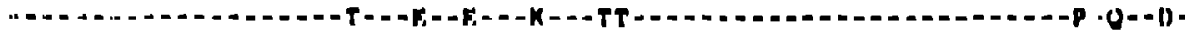

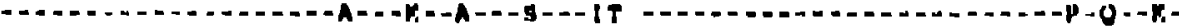

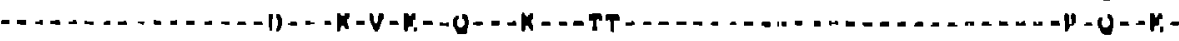


CONGENSUSI DSWTVNDIOKLVGKLNWASOIYAGIKVKQLCKLLRGEKALTEVI PLT TEAELELAENREI LKEPVHGVYY HIVIAI HIVHXB2R HIVHK HIVJRCSF HIVOYI HIVSF2 HIVNYSCG HIVNLA3 HIVHAN HIVRF CONBENSUS2 HIVELI HIVZ226 HIVNDK HIVMAL HIVUASs SIVCP 2

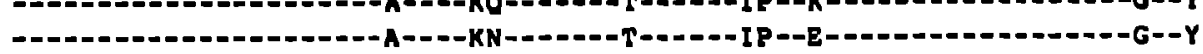

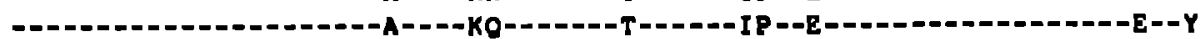
-

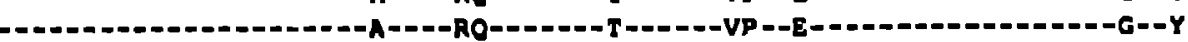

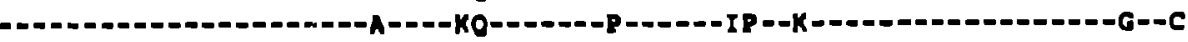

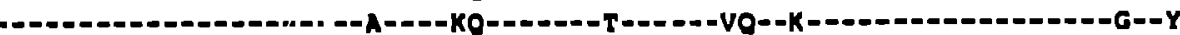

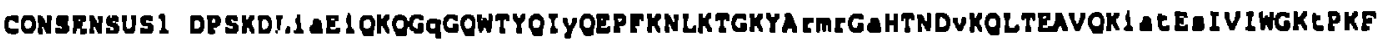
HIVLAI

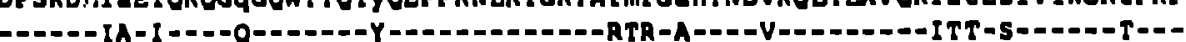
HIVHXB2R HIVMN HIVJRCST HIVOYI HIVSF? HIVNYSCG HIVNL43 IIVHAN HIVRT

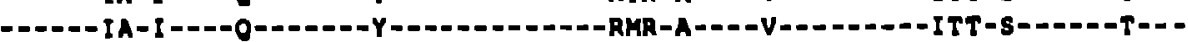

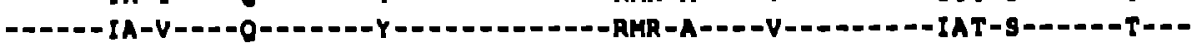
-

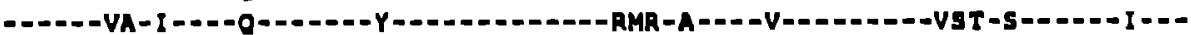

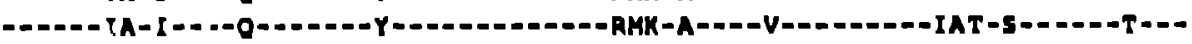
-

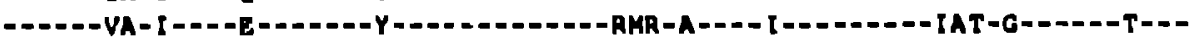

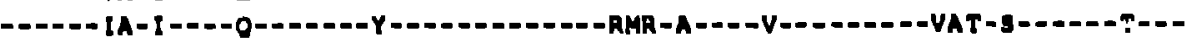
G')HSENSUS2 DPSKDLIAEI OKOG ? QOWTYOIYORP \&KNLK TGKYAR? RQAHTNOVKOLEEAVOK I IE ES IVIWGKEPKF HIVELI HIVZ226 HIVNDK

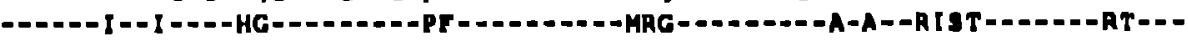

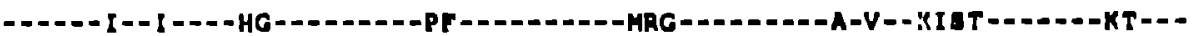
-

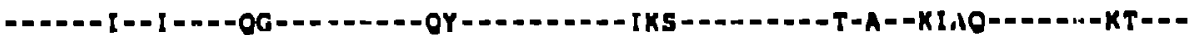
HIVMAL HIVUASs SIVCP2

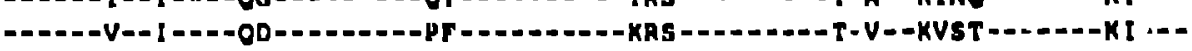

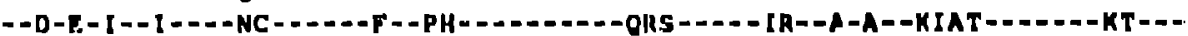

p5I V/

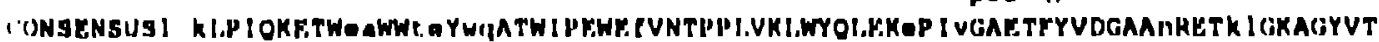
IIIVIAI HIVIIXIIZH IIIVMN IIIVJRCSF IIIVOYI IIIvar? MIVNTSCa IIIVNLA] IIVUAN IIIVAT

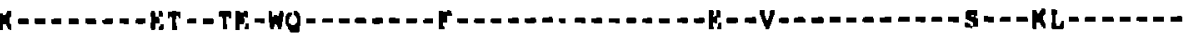

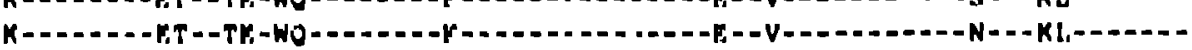
A = X-_._-KT-

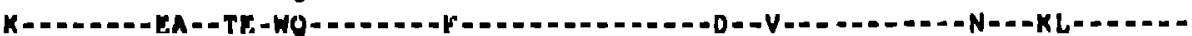

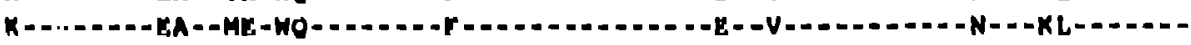

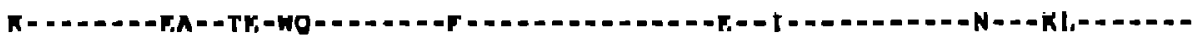
K-

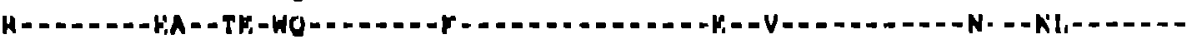

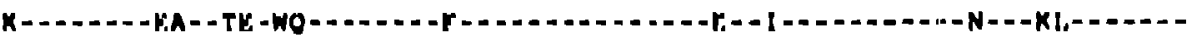

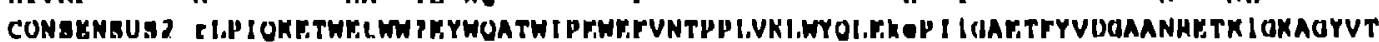
HIVFi,I IITVi2 276 IIIVNIBK

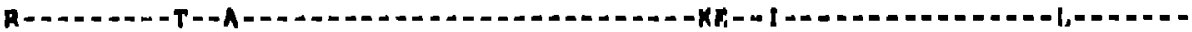
n-_._.

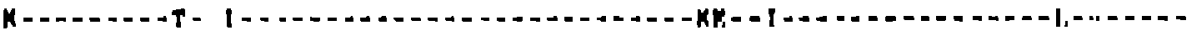

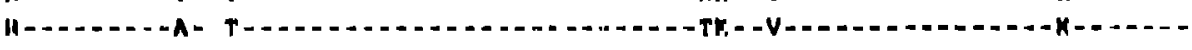
$\| 1-\ldots+1,-\Lambda \cdot M-\ldots \ldots$ IIIVAal,

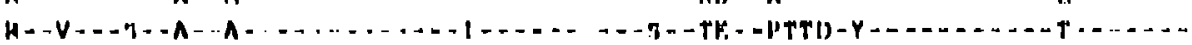


CONSENSUSI dRG?OKVVSI TDTTNOKTELOAI HLALODSGLEVNIVTDSOYALGI IOAOPDKSESELVSOIIEQLIKKE

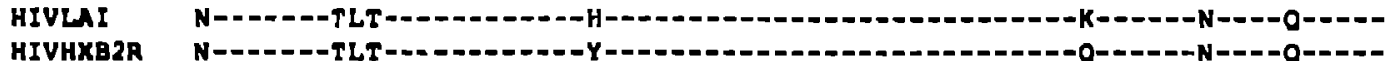

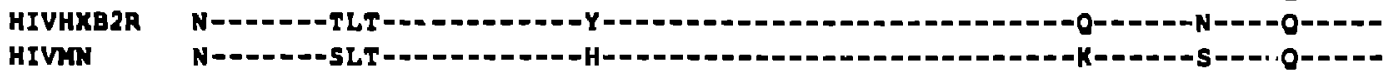

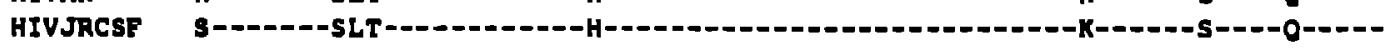

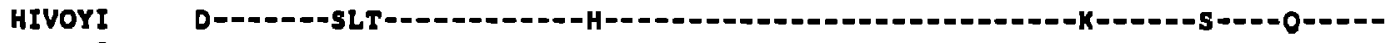

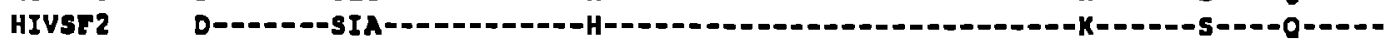

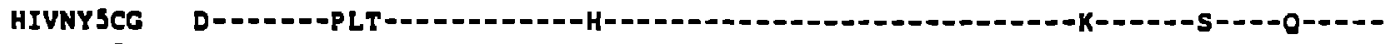

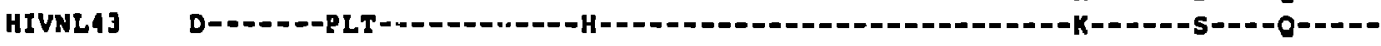

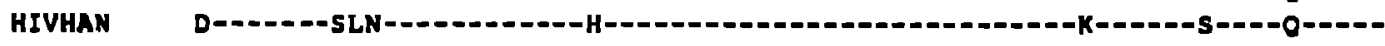

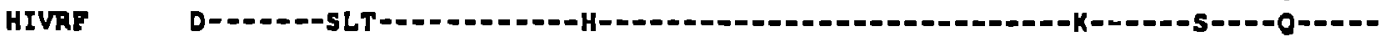
CONSENSUS2 DRGROKVWPI TdTTNOKTELqAI NLALODSGIEVNIVTDSOYALGI ICAOPDKSESEIVNOI IEqLIKKE HIVELI -

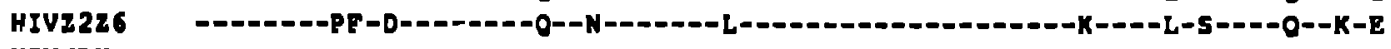

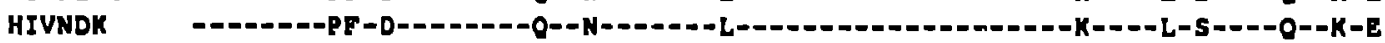

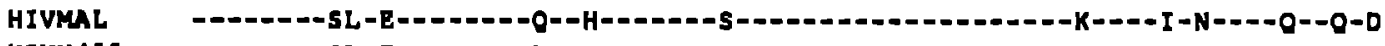

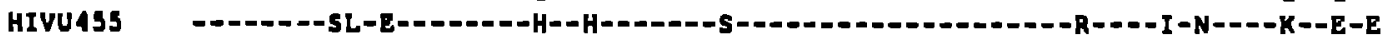

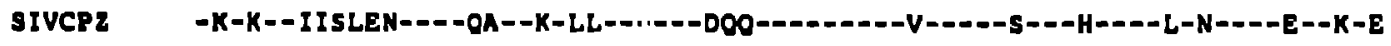

CONGENSUS 1 KVYLAWVPAHKGIGGNEOVDKLVSAGIRKVLFLDGIDKAOOOHEKYHSNHRAMA DEFNIPPUVAKEIVAS HIVLAI HIVHXB2R

HIVMN

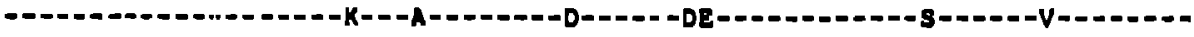
HIVJRCSF

HIVOYI

HIVSF 2

HIVNYSCG

HIVILA3

HIVHAN

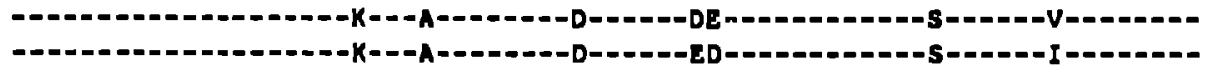

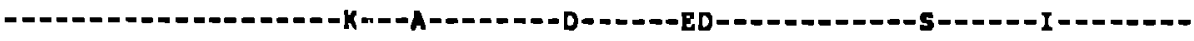
- - - - - - -

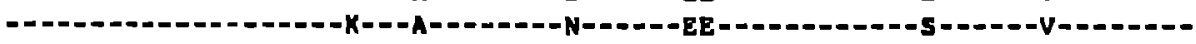
- HIVRF

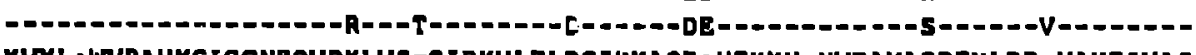
CONSENSUS2 KVYLAWVPAHKGIGGNEOVDKLVSqGIRKVLFLDGIUKAOEEHEKYHANIRAKASDFNLPPVVAKEIVAS HIVLLI HIV2226 HIVNDX HIVMAL HIVU455

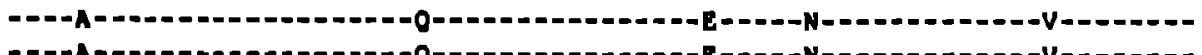

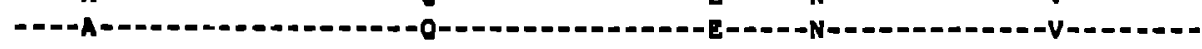

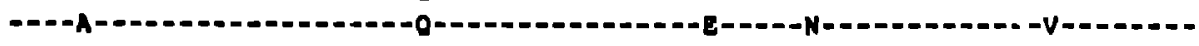

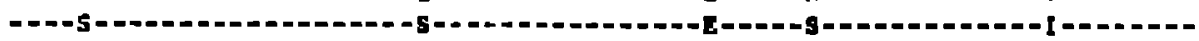
sIVCP2

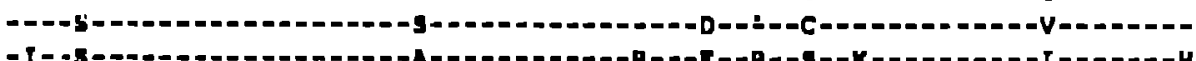

CONGENSUS! CDKCOLKGEAMHGOVDCSPGIWOLDCTHLEGKVILVAVHVASGYIEAEVIPAETGOETAYTILKLAGRAP HIVLAI HIVHXB2R IIIVMN HIVJRCSF HivoY! Hivsr? HIVNYSCo HIVNLAJ HIVHAN

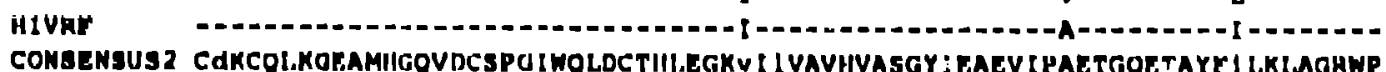
COKCOLKG

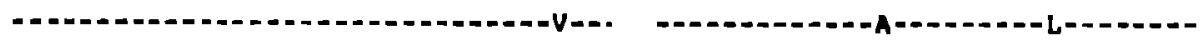

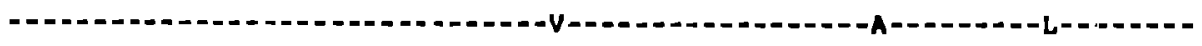

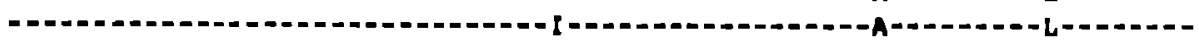
- - - - -

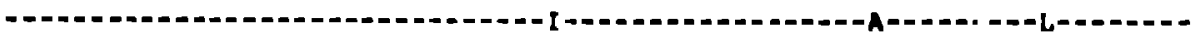

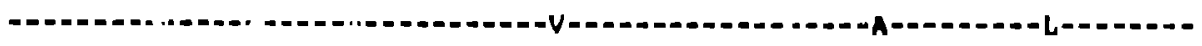

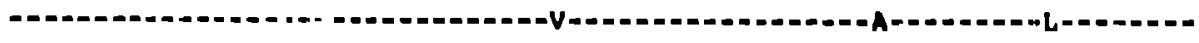

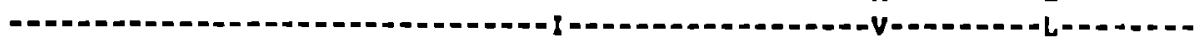
HIVELI HIVZ226 HIVNOK -D-_._- - IIVIAL. IIIVU453

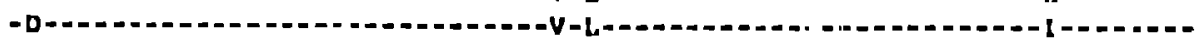

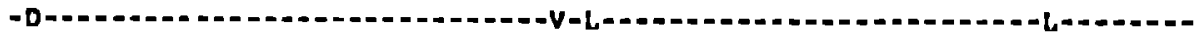

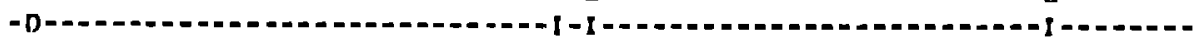

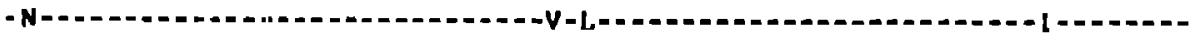
sivepz 
HIV1 POL

CONSENSUS I VkE 1 HTDNG SNE $A E T$ TVAACWWAG IKOEFGI PYNPOSOGVVESMWR LKK I IGOVRDOAEHLKEAVOMA

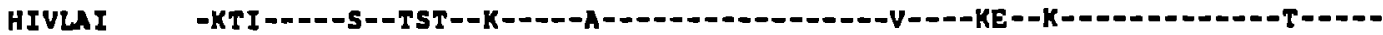

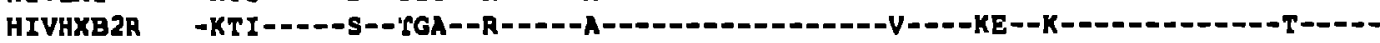
HIVMN

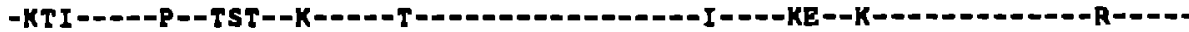

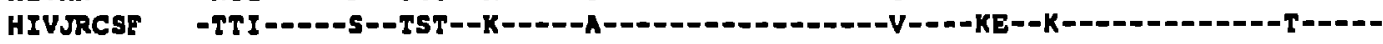
HIVOYI HIVSE 2 HIVWYSCG HIVNL43 HIVHAN HIVRF

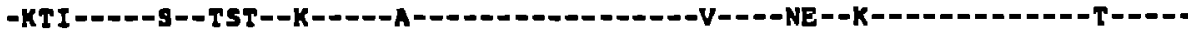

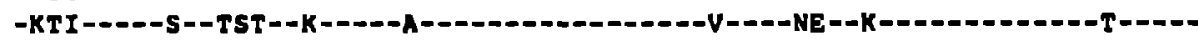

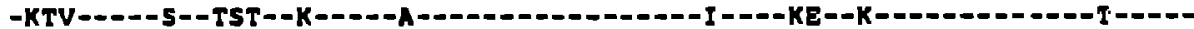

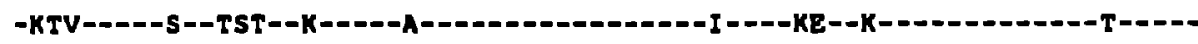

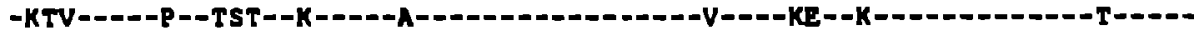

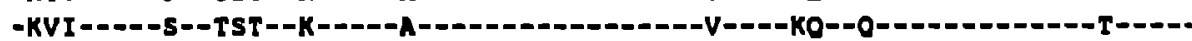
CONSENSUS 2 VKUVHTDNGSNFTSA_VKAACWHAgIKOEEGIPYNPOSOGVVESMNELKKI IGOVRAOAEHLKTAVOMA HIVELI --VV--------A---A---G-K-GE HIVZ22,6 HIVNDK

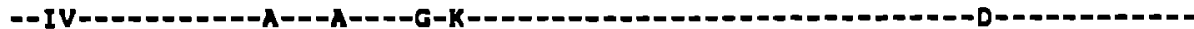

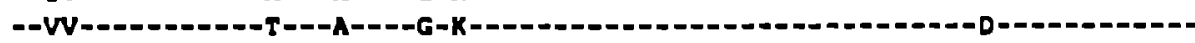

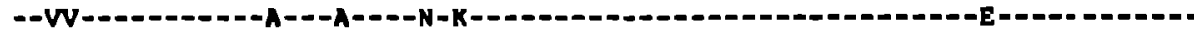
HIVHAL

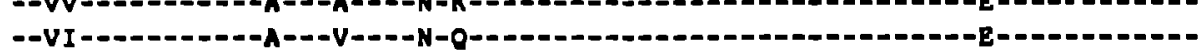
HIVUASS

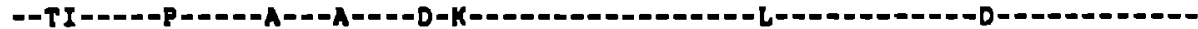

CONSENSUSI VFIHNFKRKGGIGGYSAGERIVAI IATDIOE KELOKOI TKIONFAVYYRDEIDP IWKGPAKLLWKGEGAV HIVLAI HIVHXB2R HIVMN C. -

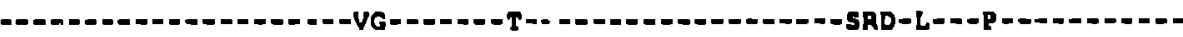
HIVJRCSF

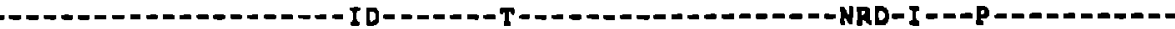

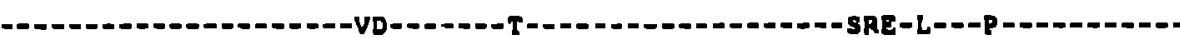
iIVOY I HIVSF 2 HIVNYSCG

HIVNL43 -

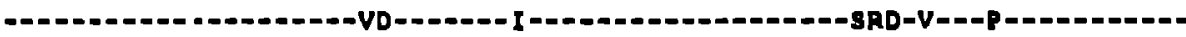
C. HIVHAN HIVRF VT I HNFKRKGGIGGYSAGERI I DI I ATDIOTKELOKOI ?KI ONFRVYYRDARDP IWKGPAKLLWKGEGAV

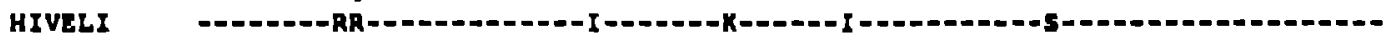
HIV2226 HIVNDK

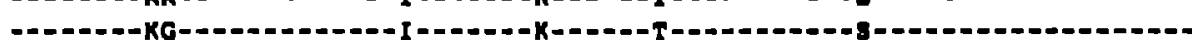
- . . HIVMAL HIVUA55

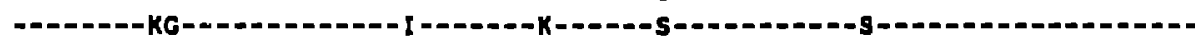
SIVCP 2 -

VIE cd: $\rightarrow$

CONSENSUSI VIODNADIKVVPRRKAKLIRDYGKOMATDDCVASRODED* 1003

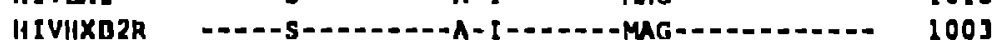

IIIVMN

HIVJACSF

HIVOY I

HIVIT2

HIVNY SCE

HIVNLA]

IIIVHAN

HIVRF

CONSENSUS?

IIIVELI

IIIVI2Z6

MIVNDK

HIVMAT.

IIIVUass

sivelp?

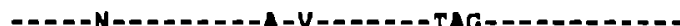

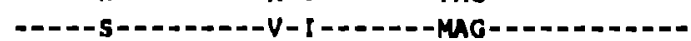

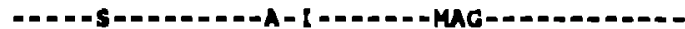

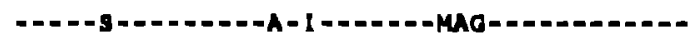

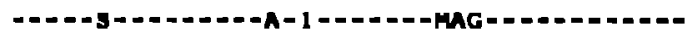

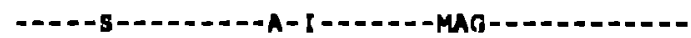

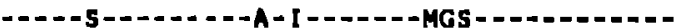

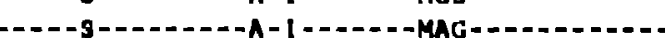

VIODNGD I KUVPARKVK I I RDYGKOMAGDDCVA I RODED* VIODSG

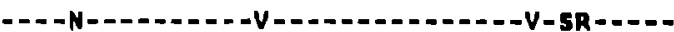

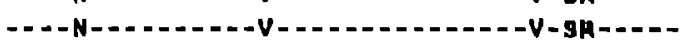

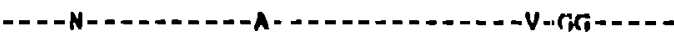

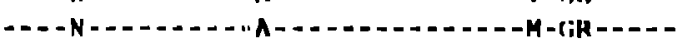

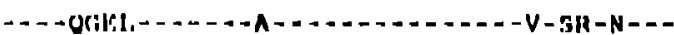

1006

1007

1003

1003

1003

1003

1002

1002

996

1002

1002

1002

1002

1002

1001 
V1 POL

Information dense reglons 10.95 or greacer) are Indicated by ***.

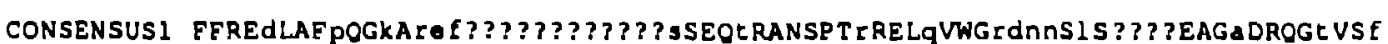
CONSENSUS2

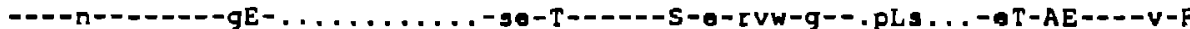
54 HIVIPOL FFRE LAFXOG A.

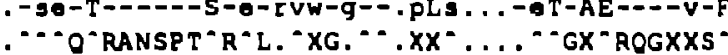
53

N

$\begin{array}{cc}K & K I \\ R & R V \\ E & E L \\ Q & O M \\ & F \\ & W \\ & Y \\ & C\end{array}$

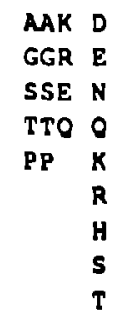

$\begin{array}{lll}D & D \\ E & E \\ N & N \\ O & \\ K & \\ R & \\ H & \\ S & \\ T & \end{array}$

$\begin{array}{llll}D D & A & K A & D \\ E E & G & R G & E \\ N N & S & E S & \end{array}$

RG

M $80 \mathrm{~T}$

F KK

W RR

Y HH
He

C ss

I/ procease <- gag eds end

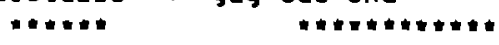

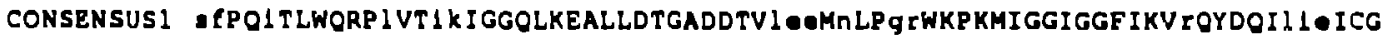

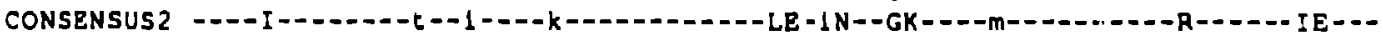
HIIVIPOL

\begin{tabular}{|c|c|c|c|}
\hline \\
\hline DI & $I$ & $I$ & AIKI \\
\hline EV & v & $v$ & GVRV \\
\hline NL & & 2 & 5 \\
\hline OM & & M & $\mathbf{T}$ \\
\hline $\mathbf{K F}$ & & & $\mathbf{P}$ \\
\hline RW & & & \\
\hline HY & & & \\
\hline sc & & & \\
\hline
\end{tabular}

protease $\backslash /$ p66, p51

n.4.

CONSENSUSI HKAIGTVLVGPTPUNI IGRNLLTOIGCTLNFPISP I ETVPUKLKPGMDGPKVKOWPLTEEKIKALVEICT CONSENSUS 2 ?-A---V HIVIPOL XK" GT'LVGPTPVNIIGRN"LTO'GCTLNFPISPIETVPUKLKPXMDGP "VKOWPLTEEKIKXLXEIC"

$\begin{array}{llll}A I & I & L & I \\ G V & V & M & V \\ \text { SL } & & & L \\ \text { IM } & & & M \\ P & & & \end{array}$




\section{HIV1 POL}

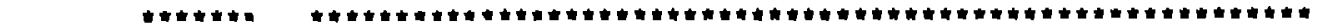

CONSENSUS1 EMEKEGKI SKIGPENPYNTPVFAIKKKDSTKARKLVDERELNKTTODFWEVOLGIPHPAGLKKKKSVTVL 264

-MEKEGKIX“"GPENPYNTP-FAIKKKDSTKWAKLV“FRELN"-TODFWEVOLGIPH'AGLK'KKSVTVL 260

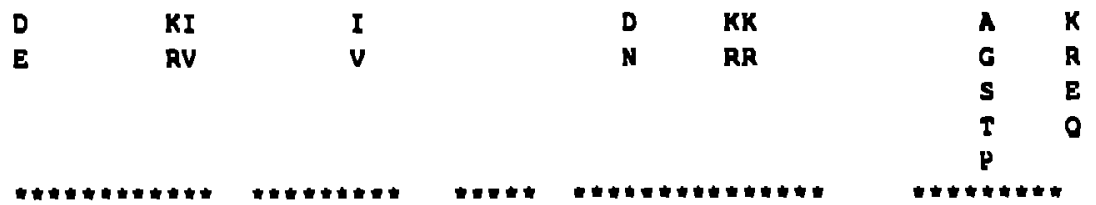

CONSENSUS $\perp$ DVGDAYFSUPLDKdFRKYTAFTIPS INNETPGIRYOYNVLPOGWKGSPAIFOASMTKILEPF IKONPdIV

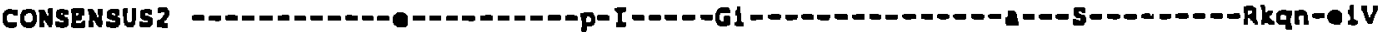
HIVIPOL DVGDAYFSVPLD"-FRKYTAFTI'SXNNETPX'RYOYNVLPOGWGSP'IFOXSMTKILEPF"...'

KD
RE
EN
OO
K
R
H
S
T

$\begin{array}{llll}\text { A } & \text { I } & \text { A } & \text { KDKD DII } \\ \text { G } & \text { V } & \text { C } & \text { RERE EVV } \\ \text { S } & & \text { NEN } & \text { L } \\ \text { T } & & \text { OOO } & \text { M } \\ \text { P } & \text { T } & \text { K K } \\ & & \text { R R } \\ & & \text { H H } \\ & & \text { S S } \\ & & \text { T T }\end{array}$

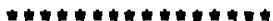

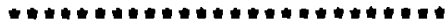

CONSENSUSI IYOYMDOLYVGS DLE I GOHRE K I EE LRQHLL LWG ITTPDKKHOKEP PF LWMGYELHP DKWTVOP I VLPEK

CONSENSUS2 -... - HIVIPOL

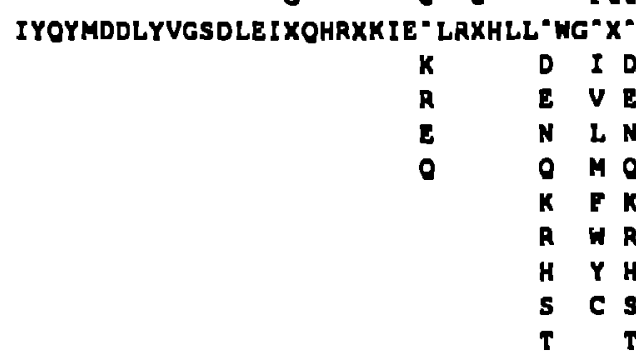

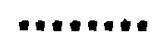

$\ldots \ldots \ldots+$

- D.e.

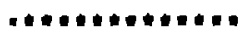

CONSENSUSI DSWTUND IOKLVGKLNWASOTYAGIKVKQLCKLLRGEKALTEVI PLTEEAELELAENREI LKEPVHIQVYY

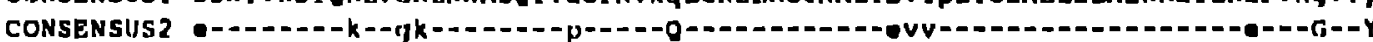

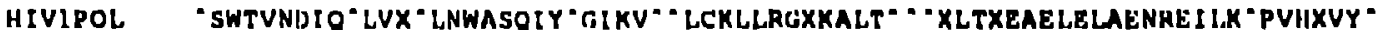

\begin{tabular}{|c|c|c|c|c|c|c|c|}
\hline$D$ & D & $n$ & $\mathbf{A}$ & KD & DII & D & $I$ \\
\hline $\mathbf{E}$ & $\mathbf{E}$ & $\mathbf{R}$ & G & HF: & EVV & $\mathbf{E}$ & $v$ \\
\hline & $\mathbf{N}$ & & $\mathbf{s}$ & $\mathbf{N}$ & & & $\mathbf{L}$ \\
\hline & 0 & & $\mathbf{T}$ & 0 & & & $M$ \\
\hline & $\mathbf{K}$ & & $p$ & $\mathbf{K}$ & & & $\mathbf{F}$ \\
\hline & $h$ & & & ค & & & $W$ \\
\hline & II & & & II & & & $Y$ \\
\hline & $\mathbf{s}$ & & & $\mathbf{5}$ & & & C \\
\hline & $T$ & & & $\mathbf{T}$ & & & \\
\hline
\end{tabular}


IIIV1 POL

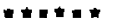

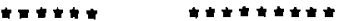

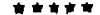

CONSENSUS 1 DPSKDL 1 AE 1 OKOGQGOWTYOIYOEP FKNLKTGKYARMYGaHTNDVKOLTEAVOK 1 atESIVIWGKTPKF 544

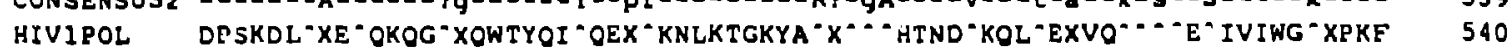

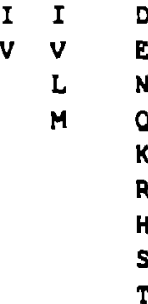

$\begin{array}{ll}\boldsymbol{E} & \mathbf{E} \\ \boldsymbol{W} & \boldsymbol{W} \\ \mathbf{Y} & \mathbf{Y}\end{array}$

$K$ KAA I $A$ KIADA K

R RGG $V$ G RVGE G R

SN S

$\begin{array}{lll}\text { TT } & \text { T } & \text { TO T } \\ \text { PP } & \text { P } & \text { PK P }\end{array}$

$\begin{array}{lll}\text { TT } & \text { T } & \text { TO T } \\ \text { PP } & \text { P } & \text { PK P }\end{array}$

R $R$

H H

T $T$

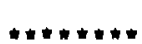

p51 \/

CONSENSUS I KLPIOKETWOAWT OYUQATHIPEWE FVNTPP LUKLWYOLEK P I VGAETFYVDGAANRETKIGKAGYY

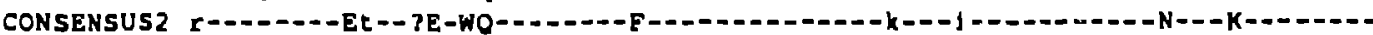

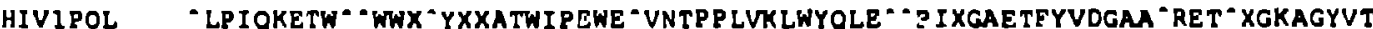

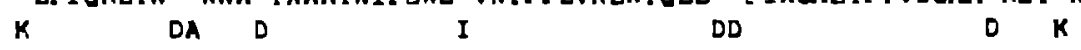

R EG E

$I$
$V$
$L$
$M$
$F$
$W$
$Y$
$C$

EE
N
O
$K$
$R$
$H$
S
$T$

E $\mathbf{R}$

$\mathbf{S}$

$\mathbf{T}$

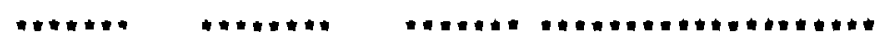

CONSENSUSI dRGROKVVEI IDTTNOKTELOAI HLALOOSGLEVNIVTDSOYALGIIGAOPDKSESELVMOIIEGLIKKE

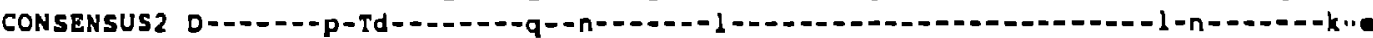

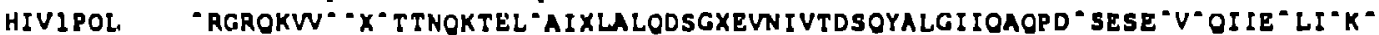

\begin{tabular}{|c|c|c|c|c|c|c|c|}
\hline D & $A I D$ & D & $\mathbf{K}$ & I & D & $K$ & $K$ \\
\hline $\mathbf{E}$ & GV E & $\boldsymbol{E}$ & A & v & $\mathbf{E}$ & $\mathbf{R}$ & R E \\
\hline $\mathbf{N}$ & SL & $N$ & $\mathbf{E}$ & $L$ & $N$ & $E$ & $\mathbf{z}$ \\
\hline 0 & $T M$ & 0 & 0 & M & 0 & 0 & 0 \\
\hline $\mathbf{K}$ & $\mathbf{P F}$ & $K$ & & & K & & \\
\hline $\mathbf{R}$ & $w$ & R & & & P & & \\
\hline H & $Y$ & $H$ & & & H & & \\
\hline 5 & c & 9 & & & $\mathbf{s}$ & & \\
\hline$T$ & & $T$ & & & $T$ & & \\
\hline
\end{tabular}

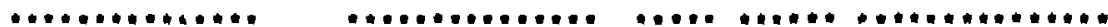

CONSENSUSI KVYLAWVPAHKG I GGNEOVDKLVSAG I HKVLFLdGIDKAOQEHEKYHSNWHAMARDENLPPUVAKEIVAS

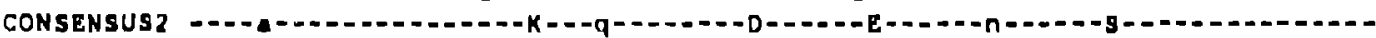
IIVIPOL KVYL-WVPAHKGIGGNEOVDXI.VSXGIHKVLFL-GIDKAO־ HEKYIXNWRAMA-DFNLPP-VAKE IVAS

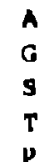

N DD 


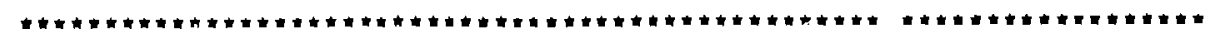
CONSENSUS 1 CDKCOLKGEAMHGOVDCSPGIWOLDCTHLEGKVILVAVHVASGYIEAEVIPAETGQETAYFI LKLAGRWP CONSENSUS2 -d- 2 HIVIPOL C`KCOLKGEAMHGQVDCSPGIWOLDCTHLEGK-I VAVHVASGY IEAEVIPXETGOETAYE LKLAGRWP

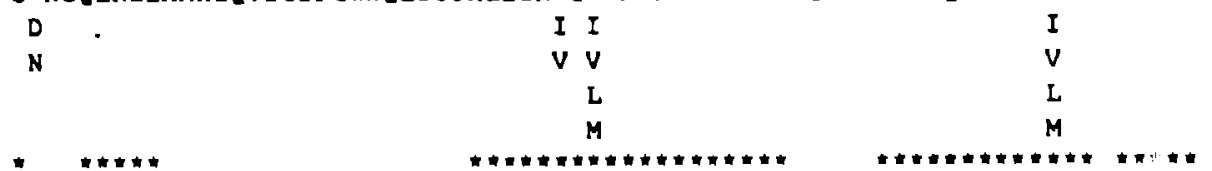

CONSENSUS 1 VkE IHTDNGsNF se TVKAACWWAGIKOEFG IPYNPQSOGVVESMNKELKKI IGUVRDOAEHLKLAVOMA CONSENSUS2 -KVV-----S--TSAa-K-A---Ag-K--_-

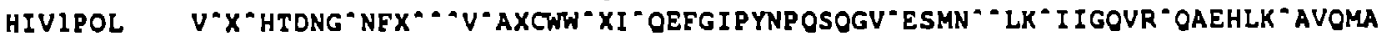

888

$\begin{array}{lllll}\text { D I } & \text { A } & \text { AAA K } & \text { A } & \text { K } \\ \text { E V } & \text { G } & \text { GGG R } & \text { G } & \text { A } \\ \text { N } & \text { S } & \text { SSS } & \text { S } & \text { E } \\ \text { O } & \text { T } & \text { TTT } & \text { T } & 0 \\ \text { K } & \text { P } & \text { PPD } & \text { P } & \end{array}$

I DE K

D D

EO R $R$ E E

N E

O O 0

$\mathbf{R}-\mathbf{P} \quad \mathbf{P P}$.

$\mathbf{H}$

S

$\begin{array}{ll}\mathbf{K} & \mathbf{K} \\ \mathbf{R} & \mathbf{R}\end{array}$

$\mathbf{T}$

$\begin{array}{ll}\text { H } & \text { H } \\ \mathbf{S} & \text { S } \\ \mathbf{T} & \end{array}$

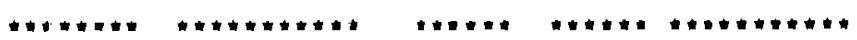

LONSENSUSI VFIHNFKRKGGIGGYSAGERIVDIIATDIOEKELOKOITKIONERVYYRDSIDP IWKGPAKLLWKGEGAV

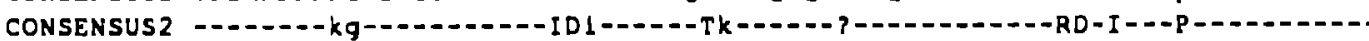
HIVIPOL VFIHNFKR-XGIGGYSAGERI-X`IATDIOX-ELOKOIXKIONFRVYYRD - " ${ }^{-}$-WKGXAKLLWKGEGAV

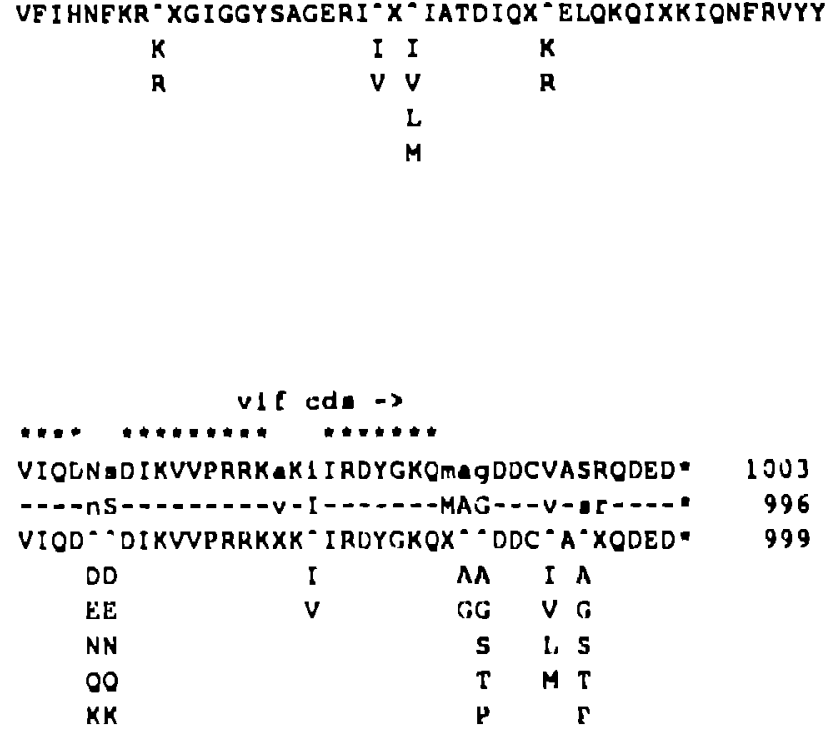

CONSENSUSI VIOLNGDIKVVPRRKAKIIRDYGKOMAgDOCVASRODED* 100]

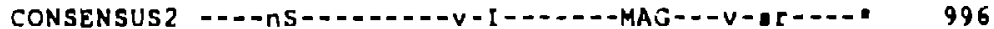

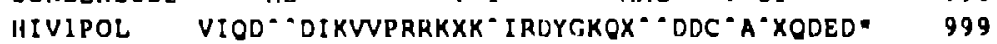

मP

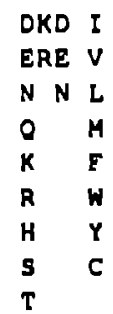

960

55

TT 


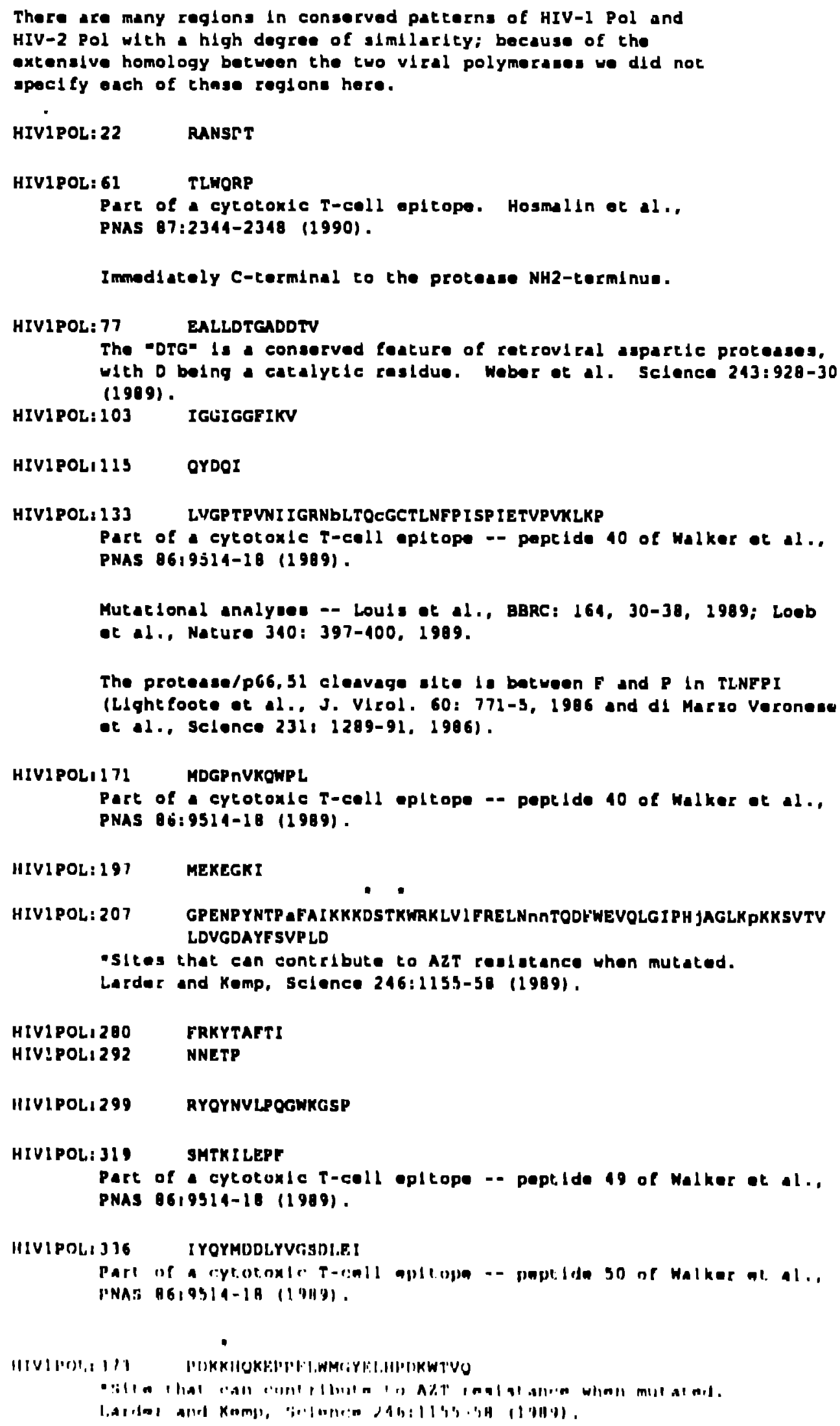




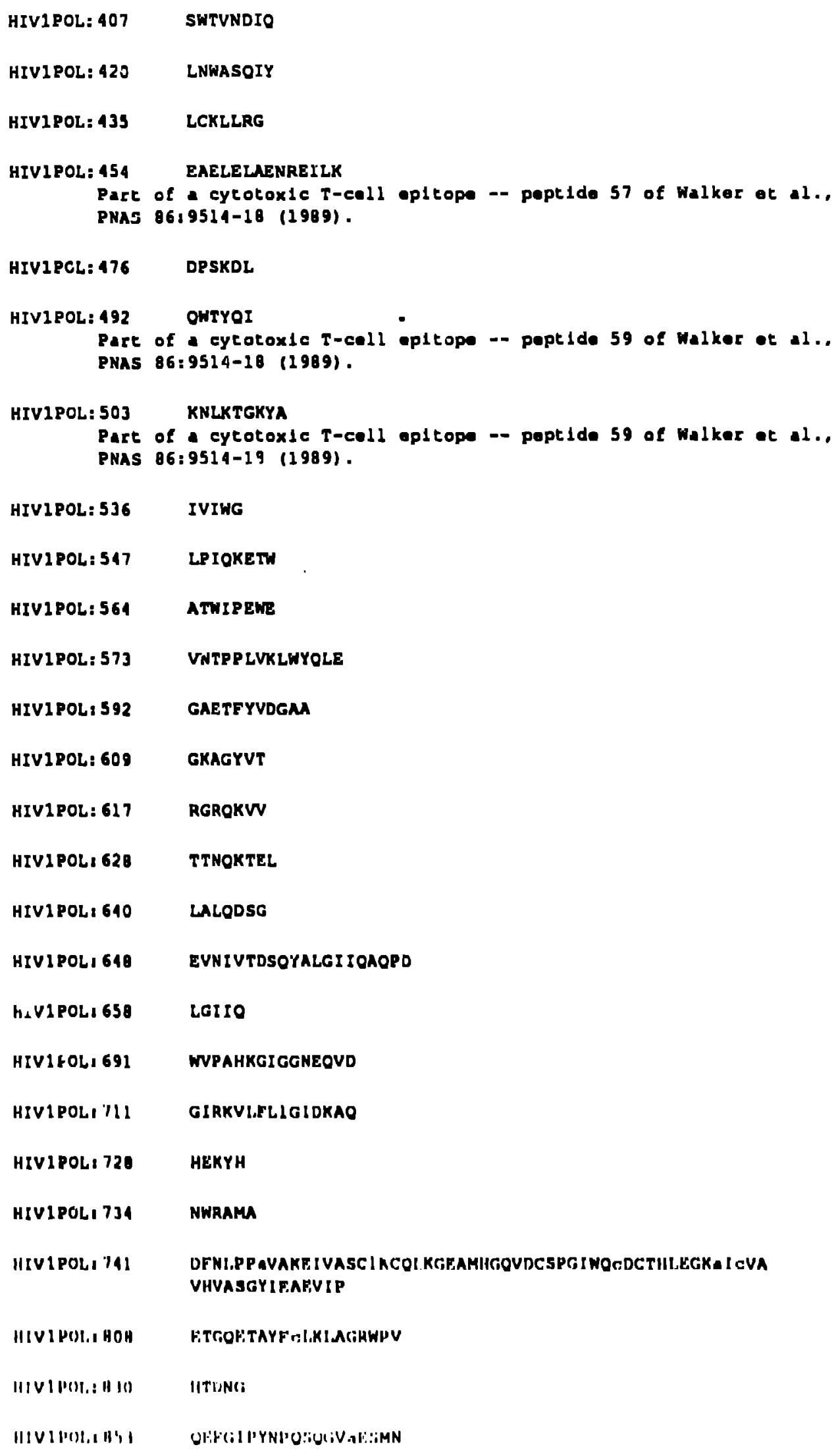


HIV1 POL

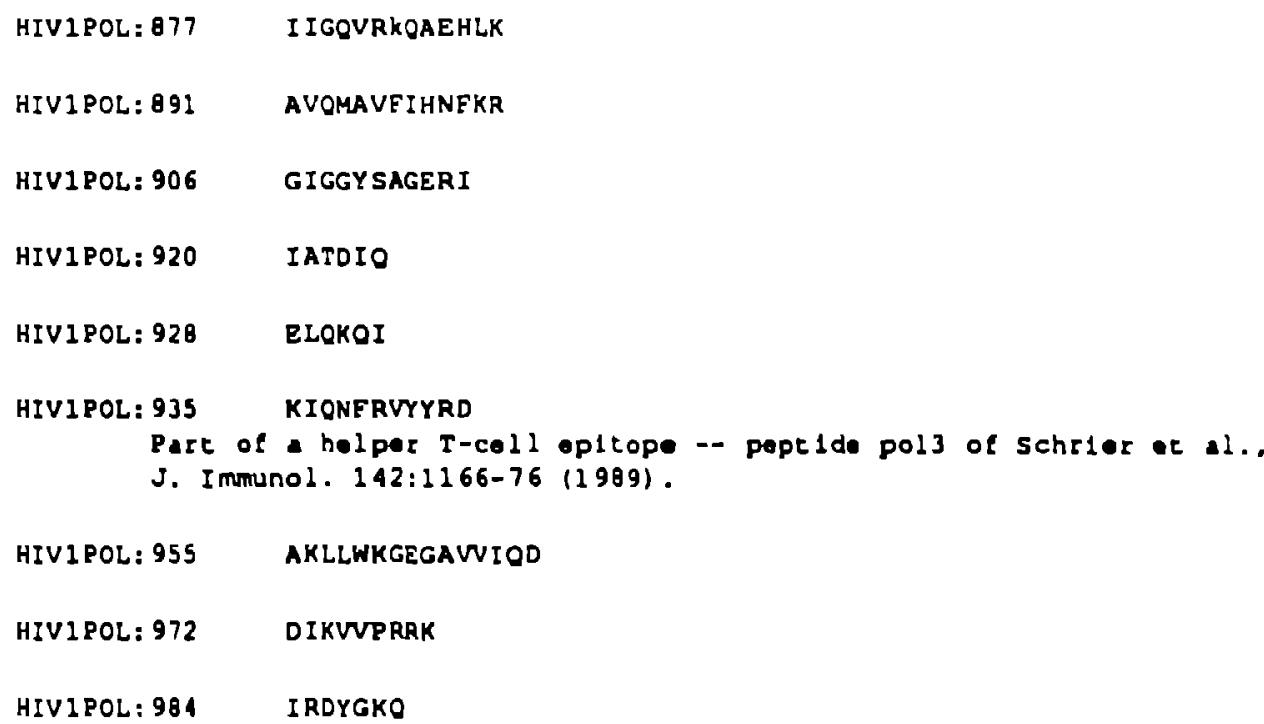


CONSENSUS TggFER?WPMGKeapOIPhGPs?sGa??????????????????dtnc?p?gSS?Gs??eIhadg?k??? HIV2ROD HIV 2NIHZ HIV 2 ISY HIV2ST HIV2BEN HIV2D1 94 HIV2GHI HIV2D20S SIVMM251

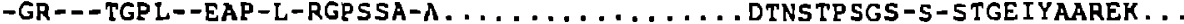
-GW--DWP L--EGP-L-RGPSPA-A . . . . . . . . . NTNSTP IGS-S-P TGE I YAARKK . . -GW-- -AWTM--EAP-L-RGPKEA-A . . . . . . . . . NTNSTPNGS-S-P TGEVHAAREK . . -GG- -VGPH--EAP-F-CGPNPA-A . . . . . . . . . . DTNSTPDRP-R-P TREVHAAREK... -GR-_-VGPT--EAS-L-RDPSPS-A............ DTNSTSGRS-S-TVGEIYAAREK ... -GR--DGPT--AAP-L-RGPSSS-A . . . . . . . . . DTNSTPNRS-S-PVGEIYAAREK... -GR---DGSM--EAP-L-RGPSSS-A . . . . . . . . . DTNSTPSRS-S-SIGKIYAAGER. . -GG---VRT L- -EAS-L-HDPSAS-SDT ICTP DEP SRGHDTSGGDTICAPCRS-S-DAEKLHADGET . . . -GG---PWSM--EAP-F-HGSSAS-A............ DANCSPRGP-C-SAKE LHAVGQA... -GG---PWPL--EAP-F-HGSSAS-A.............. DANCSPRRT-C-SAKELHALGOA...

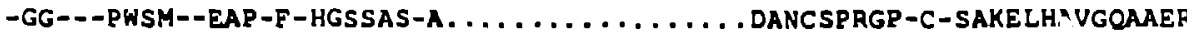
-GR-- - JWPM- -EAP-F-HGPNAS-A. . . . . . . . . DANCSPRGP -C-SAKKLHAVGQT . . SIVSHMH4 SIVSMMPBJ -GG-_-AWPM--EAP-F-HGPDAS-A............... DTNCSPRGS-C-STEELHEDGOK . . -SG---AWPM--EAP-F-HGPDAS-A............. DTNCSPRGS-C-STEELHEDGOK...

41

49

49

49

49

49

49

49

52

49

49

<- gag eds

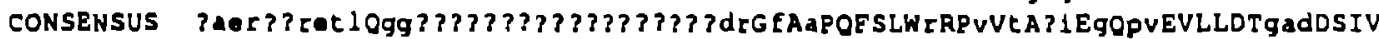
HIV2ROD HIV $2 \mathrm{NIHZ}$ HIV2ISY HIV2ST HIV2BEN HIV2D1 94 HIV2GH1 HIV2D20S SIVMA123I SI VMMI 42 SIVMM2 39 SIVMNE SIVSHMH4 SIVSHMPEJ

CONSENSUS HIV2ROD HIV 2 N HZ HIVZISY HIV2ST HIV2BEN HIV?DL 94 HIV2GHI HIV2D2OS S IVMH2SI SI VMMI 42 SIVMM2 39 SIVHNE SIVSHMH4 SIVSMMPUJ
. TERAERET I -GSDRGLTAPRAGGDT IOGATNR-L-A-----K--V-T-YI-G-PV-----GAD--. AKGAERETV-GSDRGLTAFRAGRDTMOGD, DR-L-A- - - - K--V-T-HI -G-PV- - - $-R A N-\cdots$ - TERAETKT I -RSDRGLAASRARRDTTQRD. DR-L-A------K--V-T-YI -D-PV-_---GAD-- . AERAEREA I -RSDRGLPAARE TRDTMORD . DR-L-A-- - - K - -V-T-HV-G-PV- - - -GAD-- . AEGAEGET I -KGDGGLAAPRAERDT SORG. DR-L-A---- K--V-T-YI-D-PV-----GAD-- -

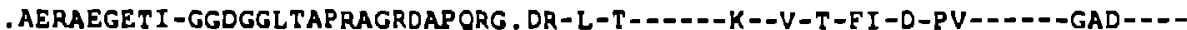

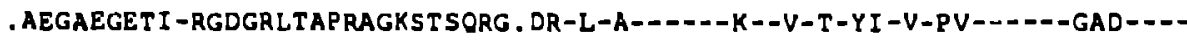

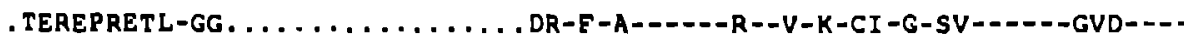

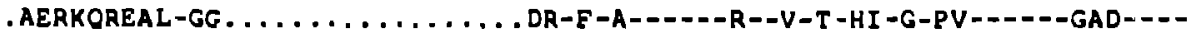
.AERKOREAL-GG. . . . . . . . . . . DR-E-A------R--V-T-HI -G-PV-----GAD----

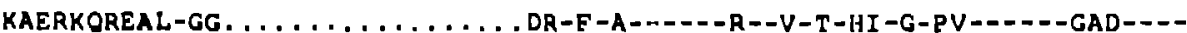

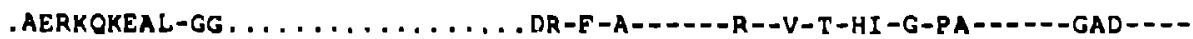

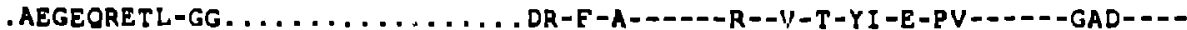

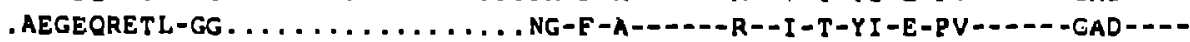

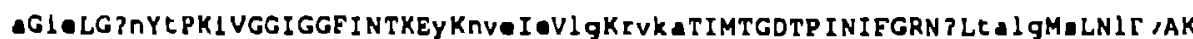

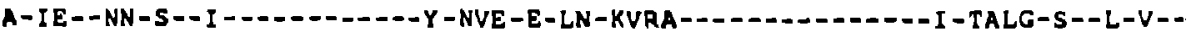

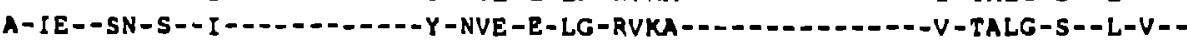

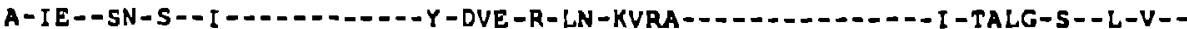
A-VE--SN-S- - I - A-IE - - CN - T - - I - . - - - -..Y - NVE-K-LN-RVRA-_.

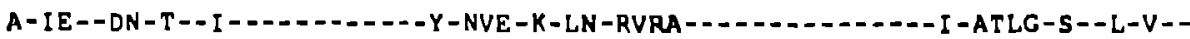
A-IO--DN-V--I -

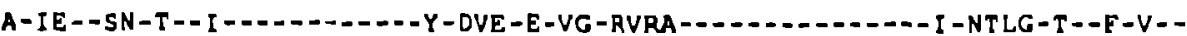
T-IE-PH-T-I IT-IE-PH-T-I - I-1-_T-IE--PH-T-I - I A-IE--PH-T--! - - A-IE-PN-T-I - I -

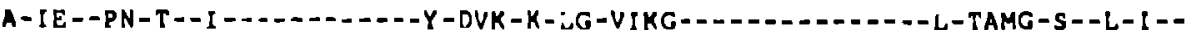




\section{HIV2/SIV POL}

CONSENSUS VOPIKVLLKPGKAGPKI FOWPLGKEKIELLIEICEKMEKEGCLEEAPPTNPYNTPTFAIKKKDKNKWRML HIV2ROD HIV2NIH2 HIVËSY HIV2ST HIV2BEN HIV2DI 94 HIV2GHI HIV2020S SIVMA25I SIVAMI 42 SIVMA239 SIVANE SIVSHMH4 SIVSMTPBJ VE-I - IM- - -D--KLR-- - TK- - E- -K- - - KE-IE-I -IM-----D--RLK----TK---E--K- - - - - KE- - - - - -

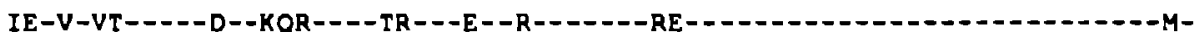

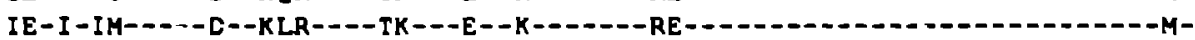

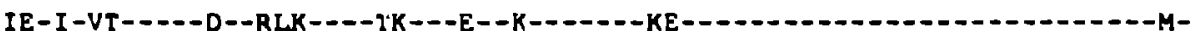

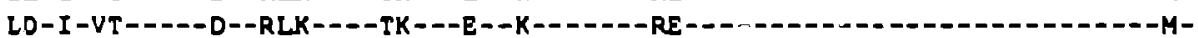

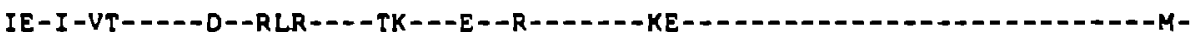

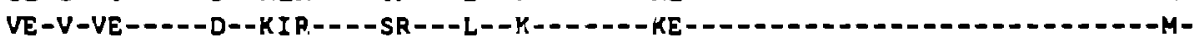

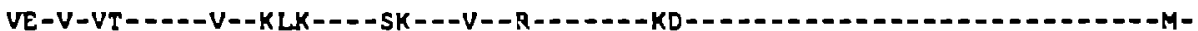

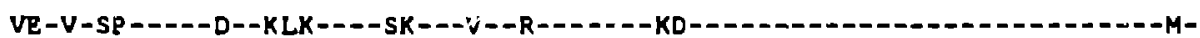

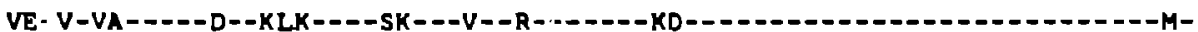

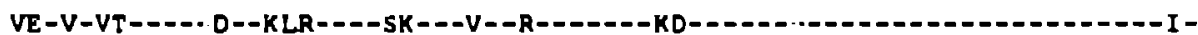

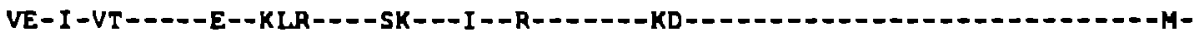

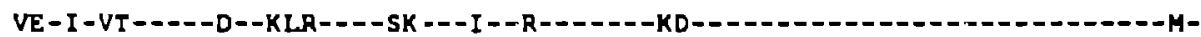

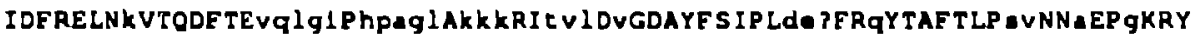
HIV?KOD HIVZNIHZ HIVZISY HIV25T HIV2BEN HIV2DI94 HIV2GHI HIV 20205 SIVAM 251 SIVMHI 42 S IVA4239 SIVANE SIVSMAH4 SIVSMRPBJ

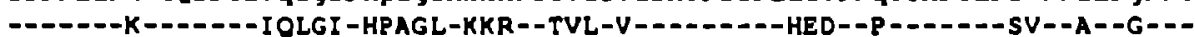

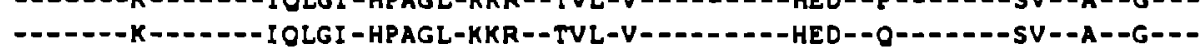

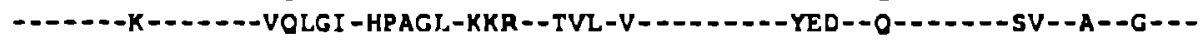

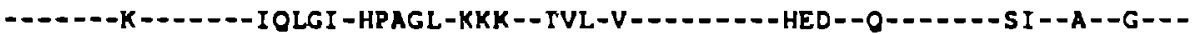
-

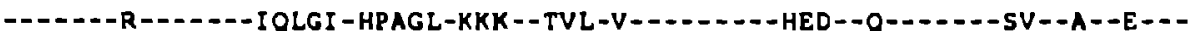

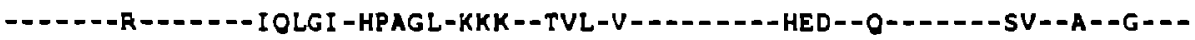

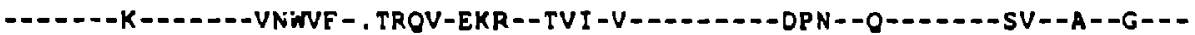

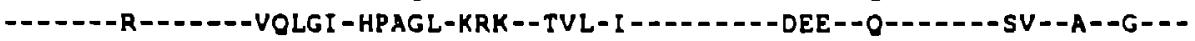

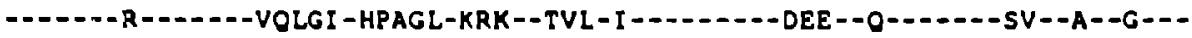

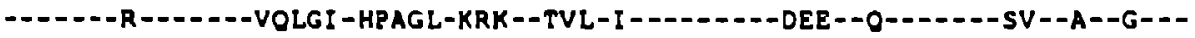

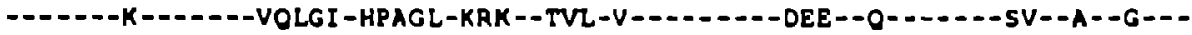

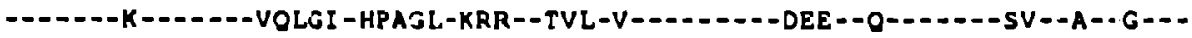

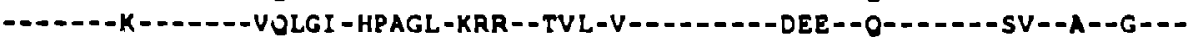

CONSENSUS HIV2ROD HIV2NIHZ HIVZISY HIV2ST HIV $2 B E N$ HIV2D 194 HIV2GH1 HIV2D205 SIVMM251 SIVMMI 12 S IVMH2 39 SIVHNE SIVSMMHA S IVSMMPBJ

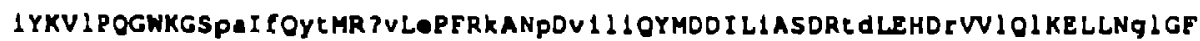

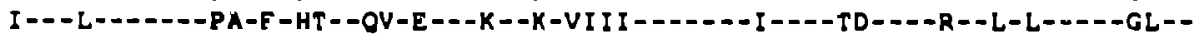

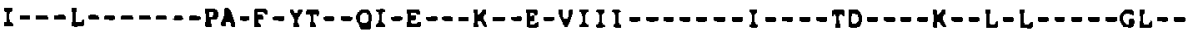

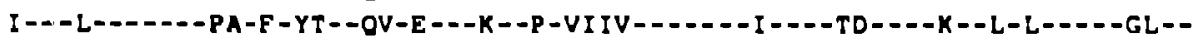

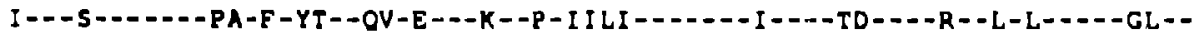

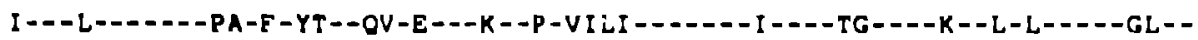

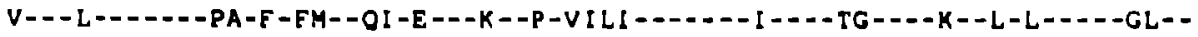

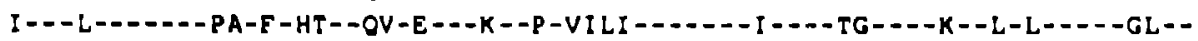

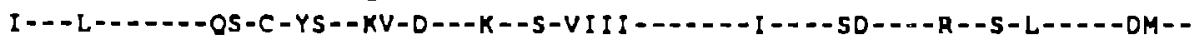

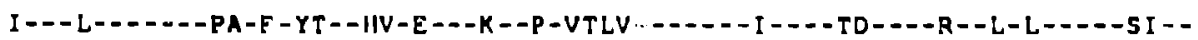

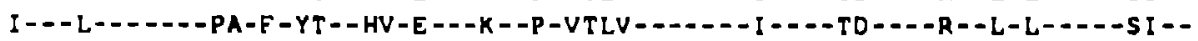

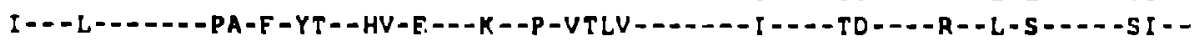

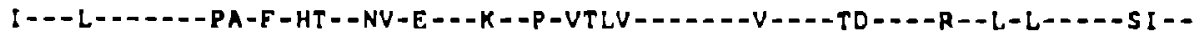

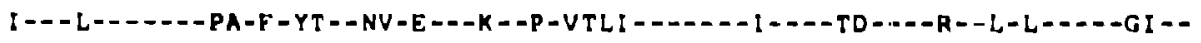




\section{HIV2/SIV POL}

CONSENSUS SLPEEKFOKDPI FQWMGYELWP EKWKLOK I QLPQKE ?WTVNA IOKLVGVLNWAOI YPGIKTKhICTLI HIV2ROD HIV2NIH2 HIV2ISY HIV25T HIV2BEN HIV2O194 HIV2GH1 HIV2D205 SIVMA2S1 SIVMinl 42 SIVMH239 SIVRE SIVSMMH 4 SIVSMAPBJ

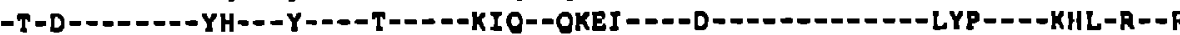

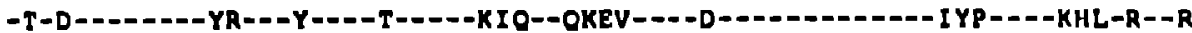

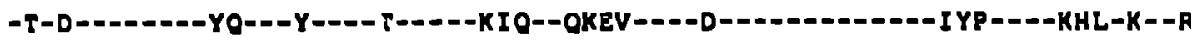

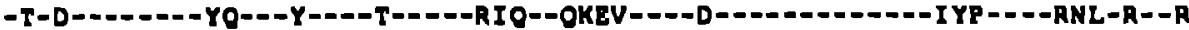

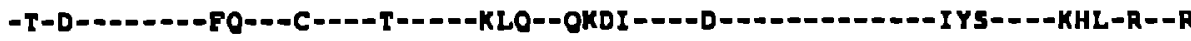

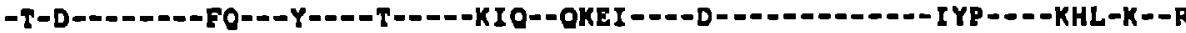

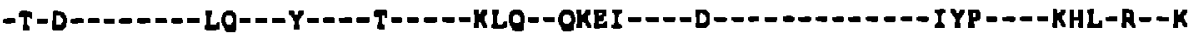

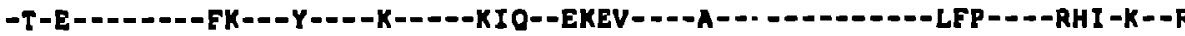

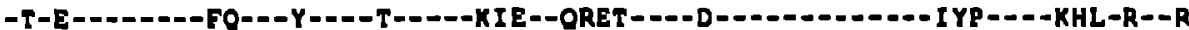

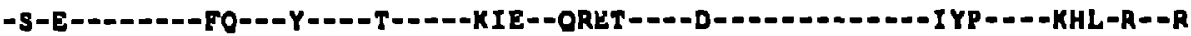

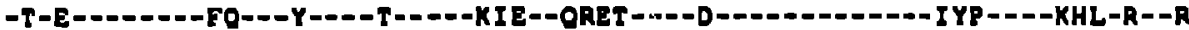

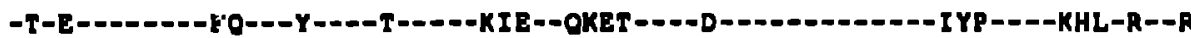

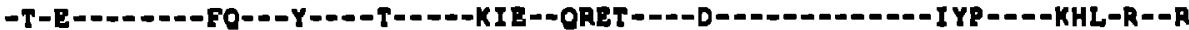

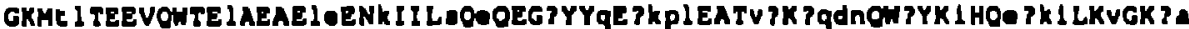

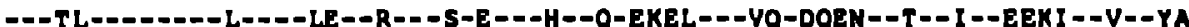

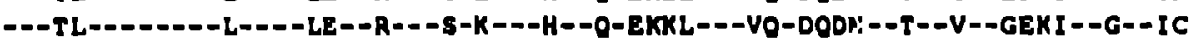

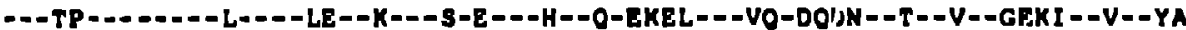

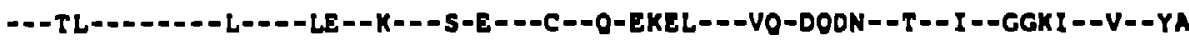

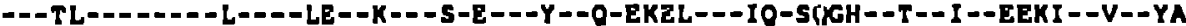

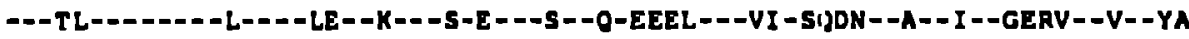

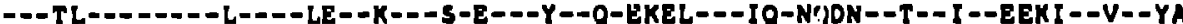

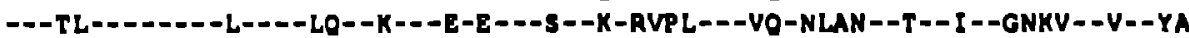

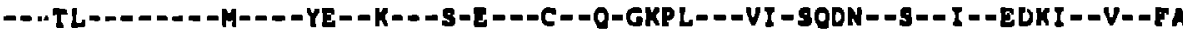

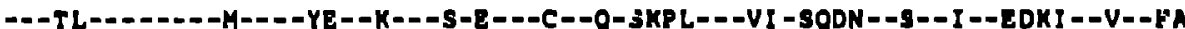

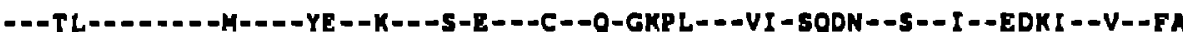

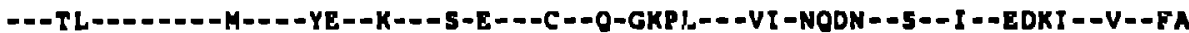

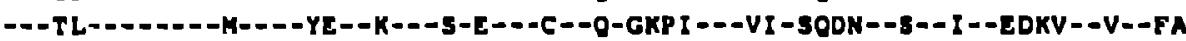

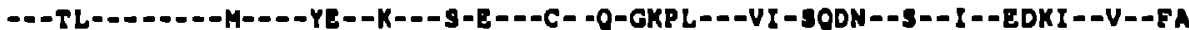

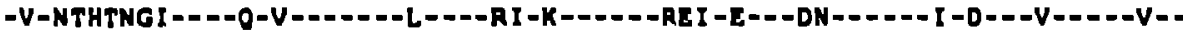
HIVINIHZ HIVISYY

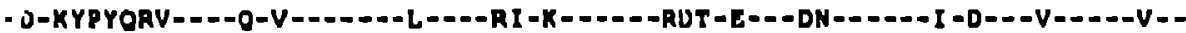
HIVIST

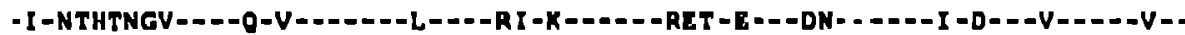

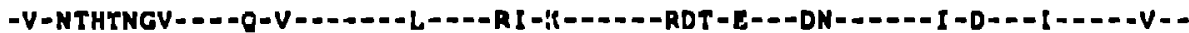
HIV2BEN HIV2OI94

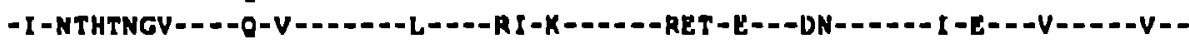

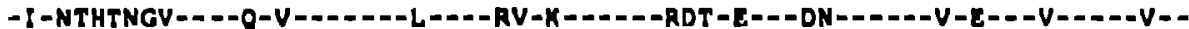
HIV2GHI HIV2D20S SIVMm2SI SIVMM1 12 JIVMM238 SIVMNE SIVSKHHA

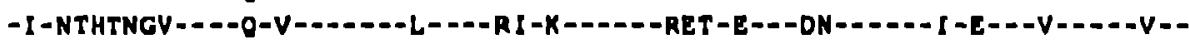

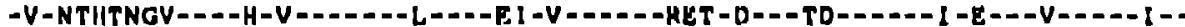

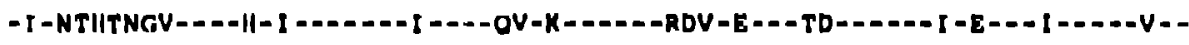
-I -NTHTNGV- - - - I- I - -

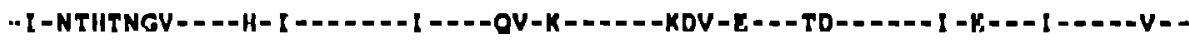

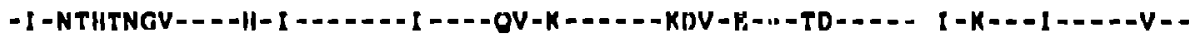

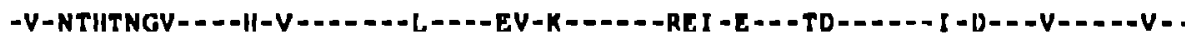




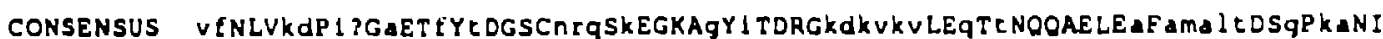
HIV2ROD HIVZNIHZ HIV2ISY HIV2ST HIV2BEN HIV 20194 HIV2GH 1 HIV2D205 SIVMM25I SIVMMI 42 5 IVAM2 39 SIVMNE SIVSMMH4 SIVSMAPBJ

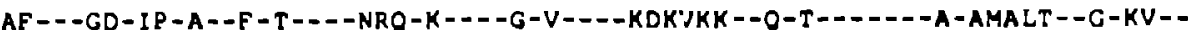
$A E=-G E-V P-A--F-T---N R Q-K=--G-I=--R D R V K V--Q-T-2---A-A M A L T-G-K A=$

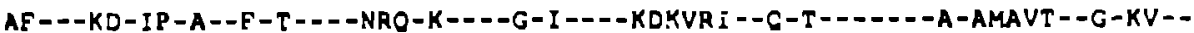

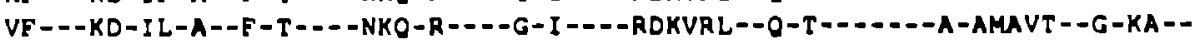

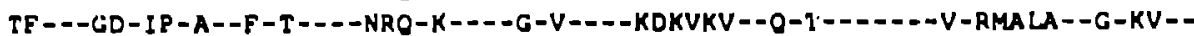

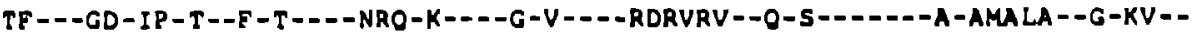

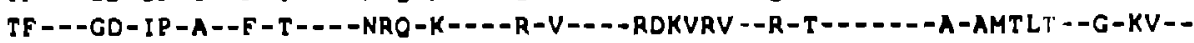
AY---KD-LE-R--Y-T--- -NRT-K----G-V--D-KDKVKV- -O-T-------A-ALALT - -E-QV--

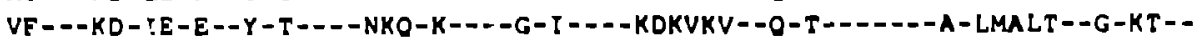

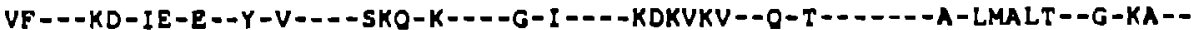

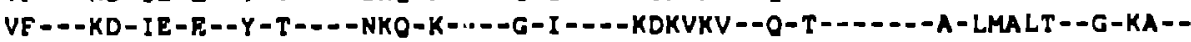

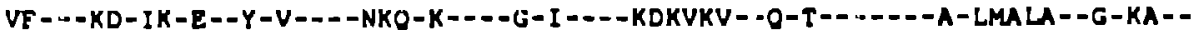
VF---KE-IQ-A--F-V-D-NRQ-R----G-V-D-RDKAKL--Q-T---D--A-YLALA--G-KA--

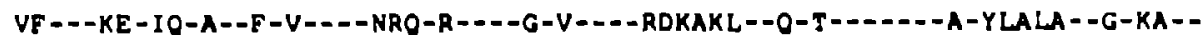

IVDSOYVMGI ? AqQPEE SE I I IVRAI IEAMIKK A I YVAWVPAHKG I GGNOEVDHLVSOGIROVLF LAK I

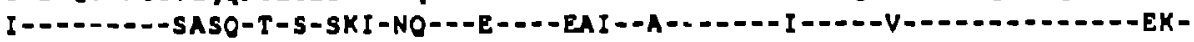

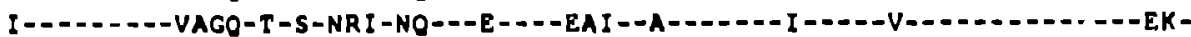

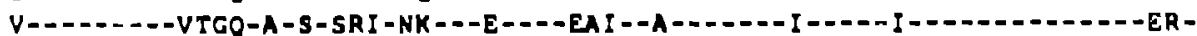

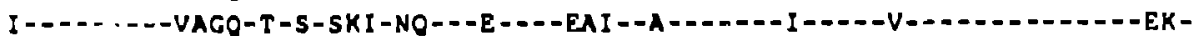

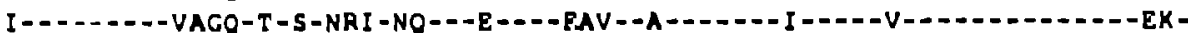

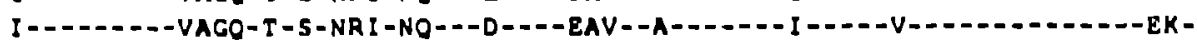

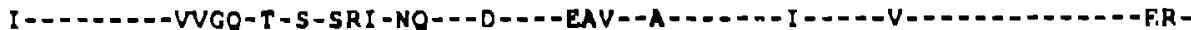
I -

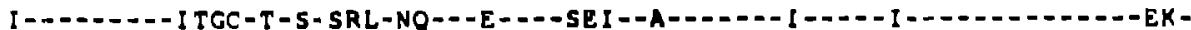

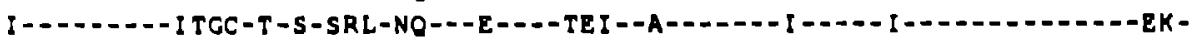

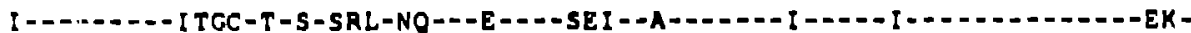

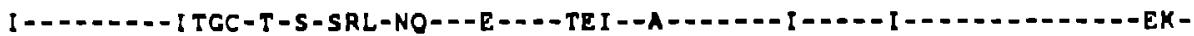
I - CONSENSUS HIVZROD HIV2NIHZ HIVZISY HIV2ST HIVZBEN III 20194 HIV2GHI HIV2020S S IVMMZ3I 5 IVMMI 42 S! VMM2 39 SIVMNE SIVIMHHA

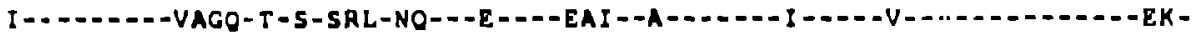

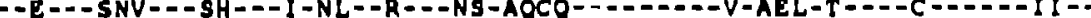

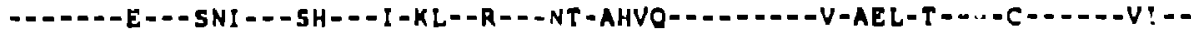

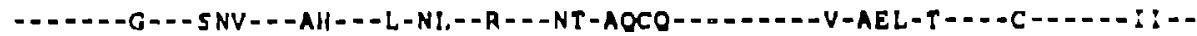

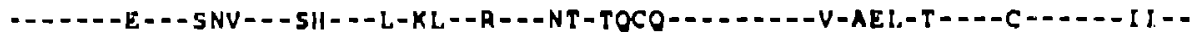

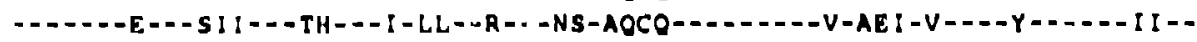

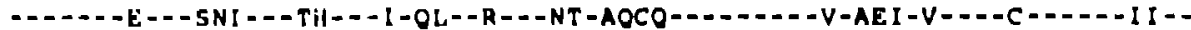

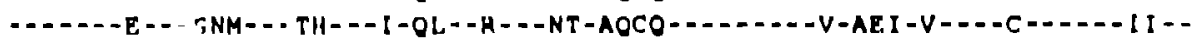

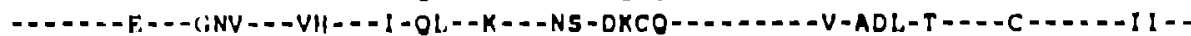

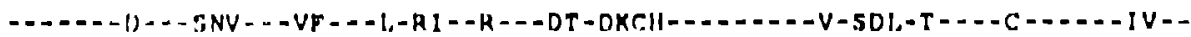

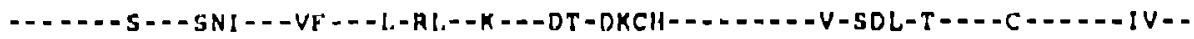

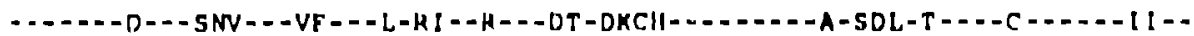

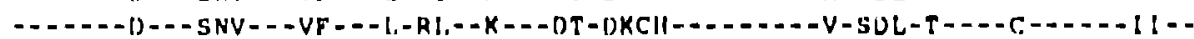

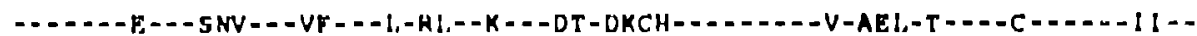




\begin{tabular}{|c|c|c|c|}
\hline CONSENSUS & AVHVASGFIEAEVIPC & EtGROTALE LLKLA ARWP I THIJHTDNGANF SqOVKHVAWW RG IEqt FG & 840 \\
\hline HIV2ROD & 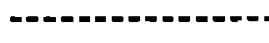 & $-2------------2---I--------A--1$ & 880 \\
\hline HIV2NIH2 & & $--V=--0 \mathrm{~T}-$ & 887 \\
\hline HIVZISY & --- & $--V---0 s$ & 887 \\
\hline HIV2ST & & $--I---Q S-$ & 887 \\
\hline HIV2BeN & & $--v---0 s$ & 887 \\
\hline HIV2DI 94 & --- & $---I---0 s-2$ & 887 \\
\hline HIV2GHI & --- & $--2=--I--$ & 887 \\
\hline HIV2D205 & $---\infty$ & $--S---I--$ & 887 \\
\hline SIVMM2S1 & --- & $--G--I--$ & 370 \\
\hline STVHH1 42 & --- & $---\mathrm{A}---\mathrm{HT}-2$ & 870 \\
\hline S IVH4239 & $=-0$ & $--\mathrm{A}---\mathrm{HT}-\mathrm{C}$ & 874 \\
\hline SIVNRE & $-\infty-$ & $---\mathrm{A}=--\mathrm{HT}-\mathrm{C}$ & 870 \\
\hline SIVSMMH4 & $\ldots$ & $--A---0 T-$ & 070 \\
\hline SIVSMMPBJ & -- & $1-6-1=-1$ & 870 \\
\hline CONSENSUS & OSOGVVEAMNHHLKn & PET I VLMAVHCMNFK RGG 1 GDMTP AER I INM 1 TEEOE IOF ?O? & 907 \\
\hline HIV2ROD & $---\infty-\infty$ & $--I----I------R---I-----S--L I--I-I-----L-A$ & 958 \\
\hline HI' $2 \mathrm{NIHZ}$ & $-\mathbf{n - n}$ & 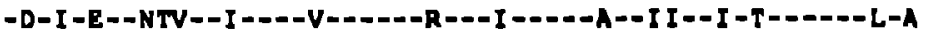 & 957 \\
\hline HIV2 ISY & 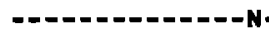 & $-I--=-V------R---I-----V--L V--I-T-=----L-A$ & 957 \\
\hline HIV2sT & 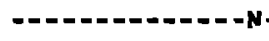 & 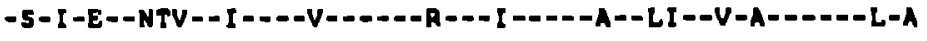 & 957 \\
\hline HIVZBEN & 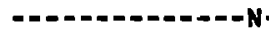 & 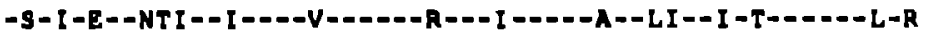 & 957 \\
\hline HIV20194 & $-\infty-\infty-N$ & 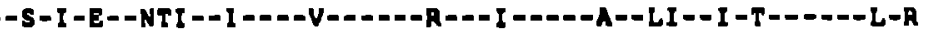 & 957 \\
\hline HIV7GHI & 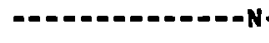 & 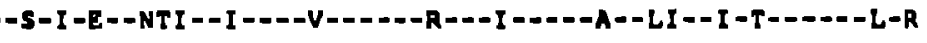 & 957 \\
\hline HIV 20205 & $-----N$ & 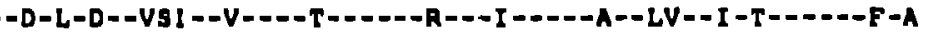 & 957 \\
\hline SIVHM2S1 & 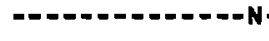 & $---A--L I--I-T------0-S$ & 940 \\
\hline SIVHMI 42 & $-----N$ & 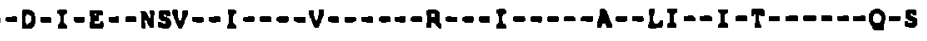 & 940 \\
\hline SIVMM239 & $-----N$ & $---A--L I--I-T------0-S$ & 944 \\
\hline SIVHNE & 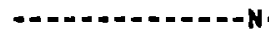 & $-D-I-E--N S H--I----V----\infty-R---I \sim----A--L L=-I-T------O-S$ & 940 \\
\hline SIVSMMH4 & $-2--2-2--2--2-2$ & 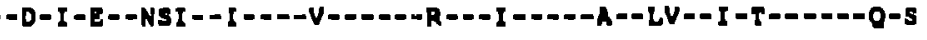 & $94 \mathrm{C}$ \\
\hline SIVSMMPBJ & 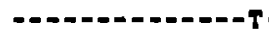 & 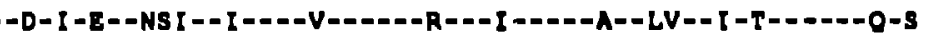 & 940 \\
\hline & & VIE cdv $\rightarrow$ & \\
\hline cONSENSUS & KNakfKnFrVY'YREG & IdOLWKGPGELLWKGGGAVI vKVGEd I KVVPRAKAK I I kdYGggke 70 anh I Fd & 976 \\
\hline HIV2ROD & -- SKLKD $-\mathrm{R}-\mathrm{P}-\boldsymbol{- O}$ & 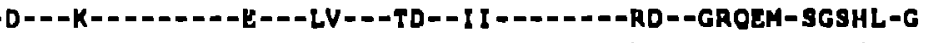 & 102 ค \\
\hline HIVZNIHZ & $--S K L K N-R--F=--$ & 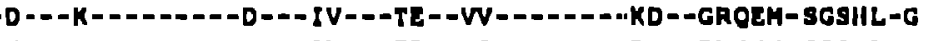 & 1027 \\
\hline HIVZISY & $--S K L, K N-A--B=--$ & 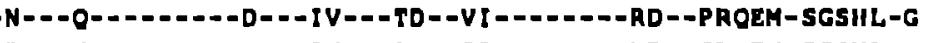 & 1027 \\
\hline HIV2s: & $--S K L O N-R--F=--$ & 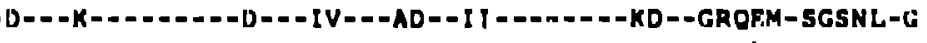 & 1027 \\
\hline HJ:2BEN & $--5 N F K N-0--Y---$ & 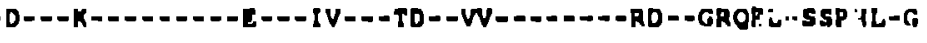 & 1027 \\
\hline IIIV20I 94 & -- SNFKK $-0--Y---$ & 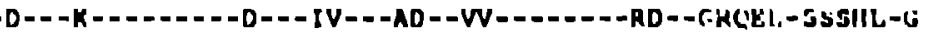 & 1027 \\
\hline HIV2GHI & -- SNFKN-O-O-Y-- & 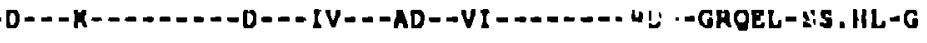 & 1026 \\
\hline IIIV2D203 & $=-L K F Q N-Q-\cdot-Y---$ & $D--K---2--2--E---I I---T E--V V-\cdots$ & 1027 \\
\hline SIVMH25I & -- SKFKN-K- -Y - - & 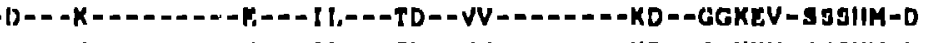 & 1010 \\
\hline S IVMH I 4) & -- SKFKN-R- -Y--- & 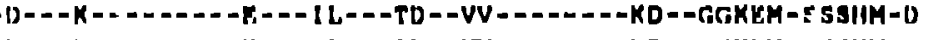 & 1010 \\
\hline S IVMM 239 & -- SKPKN $-\mathrm{K}--Y---$ & 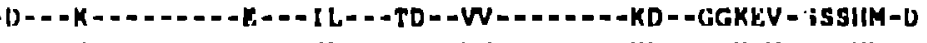 & 1014 \\
\hline SIVHNE & $--S K P^{\prime} K N-R=-Y--$. & 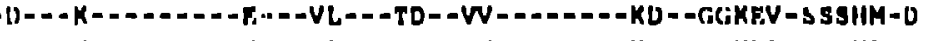 & 1010 \\
\hline S IVJMMII4 & -- SKF'KN-R- -Y--- & 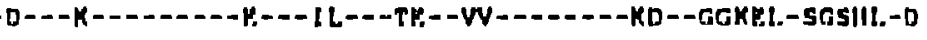 & 1010 \\
\hline SIVUMMPRJ & -- SKFKN-R--Y--- & 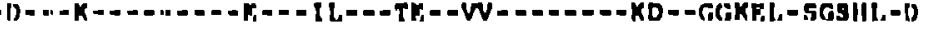 & 1010 \\
\hline CrJNSEMSUS & trrastivaeriz & 943 & \\
\hline HIVZHOD & AR. EUG-H=- & 1036 & \\
\hline HIVZNIIIZ & AR. . F, ();-H-- & 1033 & \\
\hline IIIVZISY & AH, $\mathrm{BDC} ;-\mathrm{H}=-$ & 10.34 & \\
\hline IIIV2ST & AR, Fil)i-V-- & 1033 & \\
\hline IIVIHFN & AK, Y,I)Ci-H-C.PCO ) & 1127 & \\
\hline IIIV7nI 94 & AH, F.ISK -V- - & 1033 & \\
\hline HIV)eall & AHF:Bur:.-V -. & 1013 & \\
\hline IIIVII)>ns & TH. UAH-M-U:HI= & $101 \mathrm{H}$ & \\
\hline :IVMMDSI & Tr: , F.AH ..V - - & $101 \mathrm{H}$ & \\
\hline :AIVMMIAJ & TH, I:AR-V ... & I1) II & \\
\hline : (YARIJ II & 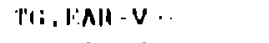 & 102 & \\
\hline :IVMNP: & T'ri, liAlI V V & 11111 & \\
\hline IIV:SMAIIA & I': I, ISAR.V.- & 11111 & \\
\hline “IV:IAMIII.I & l'ri.liAH.V & $111|1|$ & \\
\hline
\end{tabular}


Information dense stretches $(0.9$ or grester) are Indleated by tit.

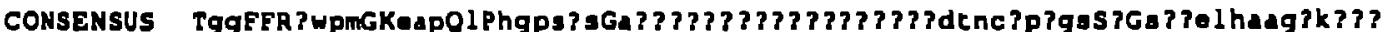

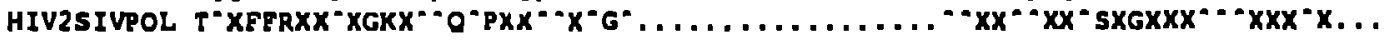

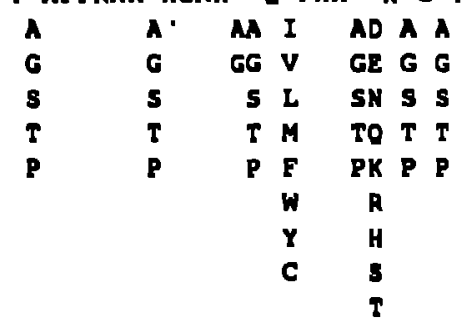

$\begin{array}{rrrrr}\text { DA } & \text { M } & \text { A } & \text { KIF } & K \\ \text { NG } & \text { GG } & \mathbf{G} & \text { RVW } & \text { R } \\ \text { S } & \text { SS } & \text { S } & \text { ELY } & \text { E } \\ \text { T } & \text { TT } & \text { T } & \text { OMH } & 0 \\ \text { P } & \text { PP } & \text { P } & & \end{array}$

\section{<- gaq cdo}

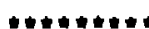

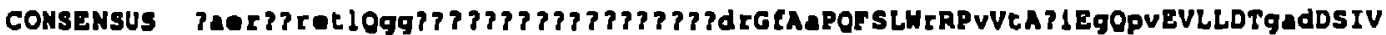

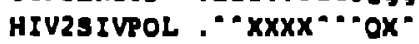
.XX'A*POFSLH'RP'V'AX'EXO'XEVLLDTXX"DSIV

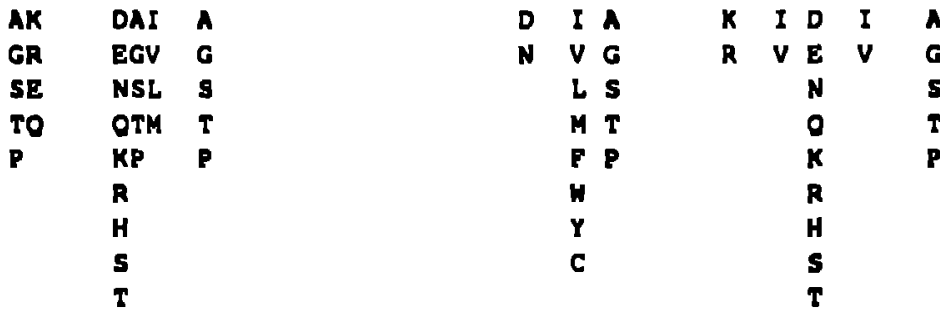

$\bullet \bullet \bullet \bullet \bullet \bullet \bullet \bullet \bullet \bullet \bullet \bullet \bullet \bullet$

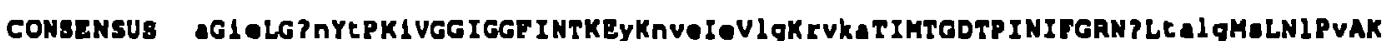

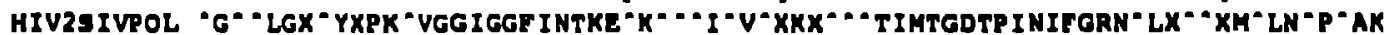

$$
\ldots \cdots
$$$$
\text { V NVE R V VRG }
$$$$
\text { G GH } T \text { V V }
$$$$
\text { L } \quad \mathbf{E} \text { I }
$$$$
\text { M } 0
$$$$
\text { F } \mathrm{K}
$$$$
\text { M } \mathbf{R}
$$$$
\text { Y H }
$$$$
\text { r } \mathbf{s}
$$

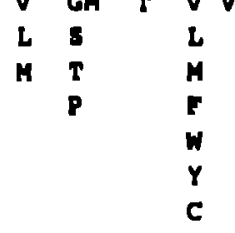

CONGENSUS VOP !KUL LKPGKUGPK I ROWP LOKEK I AALEE ICEKMEKOGOLEEAPPTNPYNTPTFA IKKKDKNKWHML

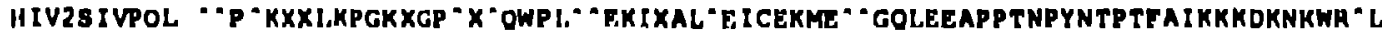

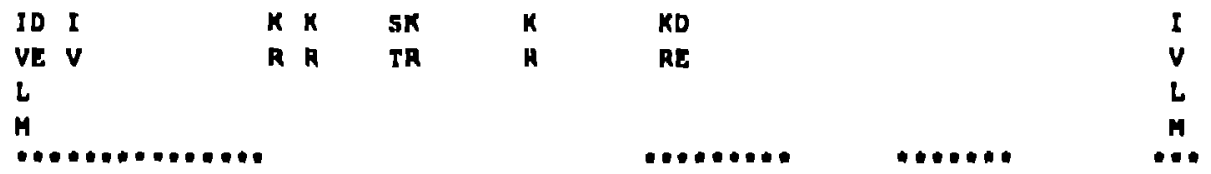

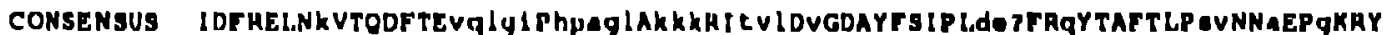

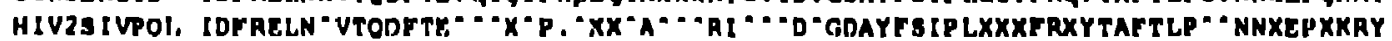

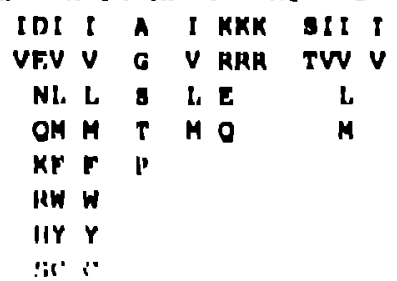




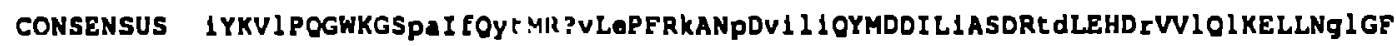

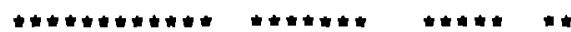

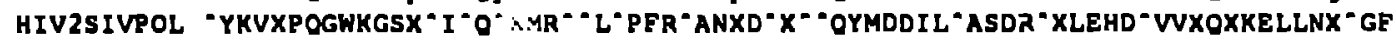

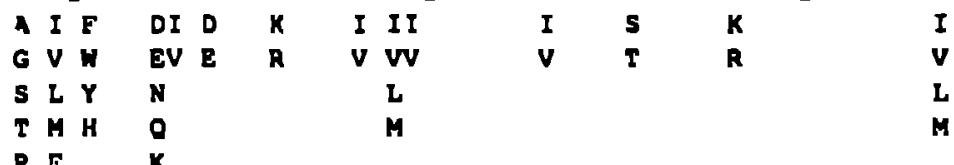

P F

W $R$

$\mathbf{Y} \quad \mathbf{H}$

C $\mathbf{S}$

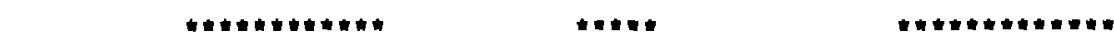

CONSENSUS StPEEKFOKDPP fqWMGYELWP EKWKLOK I qLPqke JWTVNdIOKLVGVLNWMOI YPGIKTKhICrLIr

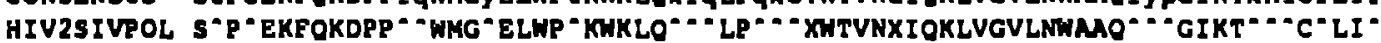

$\begin{array}{lcccccccc}\text { S D } & \text { ID } & \text { I } & \text { D } & \text { KIE } & \text { EKD } & \text { IFA } & \text { KDI K } & \text { K } \\ \text { T E } & \text { VE } & \text { V } & \text { E } & \text { RVO } & \text { ORE } & \text { VWG } & \text { REV R } & \text { R } \\ & \text { LN } & \text { L } & \text { N } & \text { L } & \text { LYS } & \text { NL } \\ & \text { MO } & \text { H } & \text { O } & \text { M } & \text { T T } & \text { OH } \\ & \text { FK } & \text { F } & \text { K } & & & \text { K } & \text { R } \\ & \text { WR } & \text { H } & \text { R } & & & & \text { H } \\ & \text { YH } & \text { Y } & \text { H } & & & & \text { S } \\ \text { CS } & \text { C } & \text { S } & & & & \text { T } \\ & \text { T } & & \text { T } & & & & \end{array}$

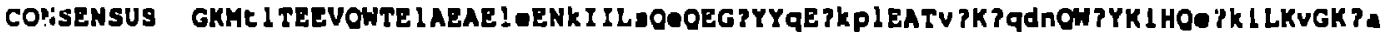

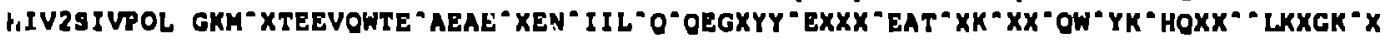

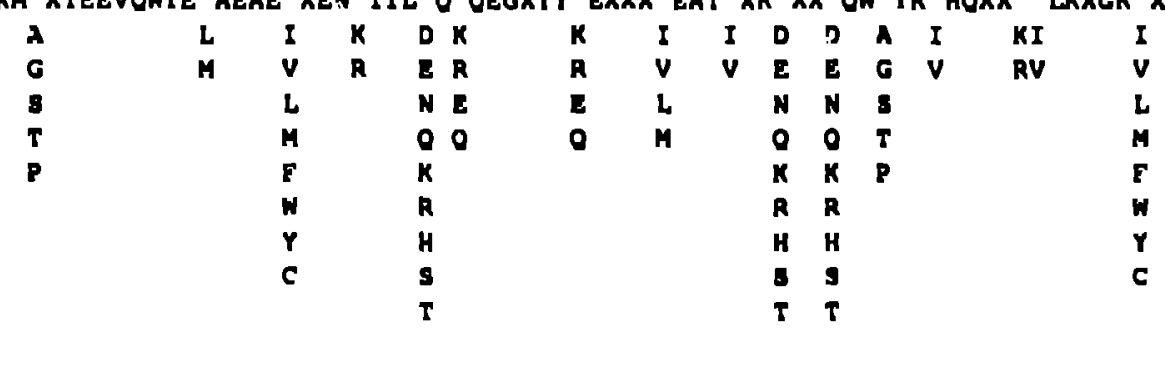

CONSENSUS K!KREhE nQQRLLAhVVOKIGKEAIVIWG? IPKFHLPVECE TWOOW ? dYWOVTWIP OHDPUSTPP LVIIL

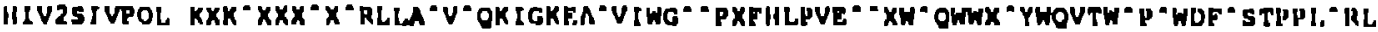

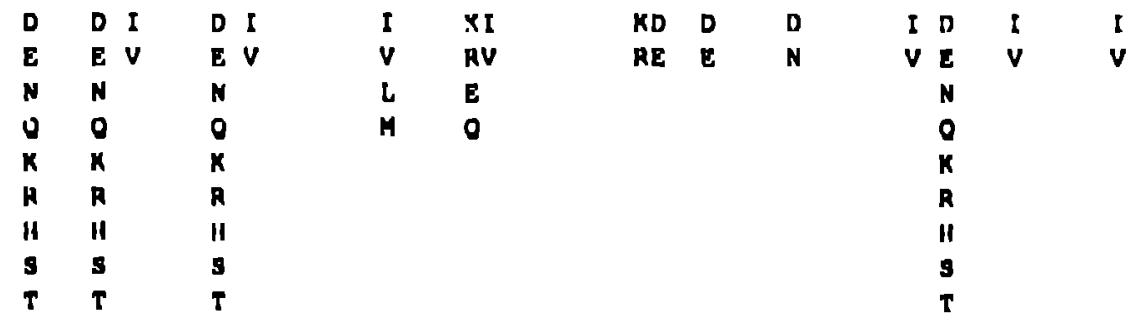




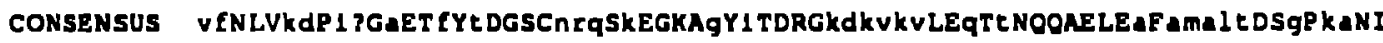

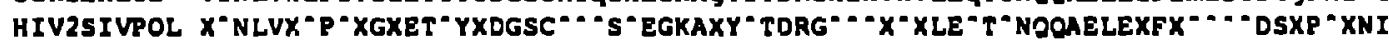

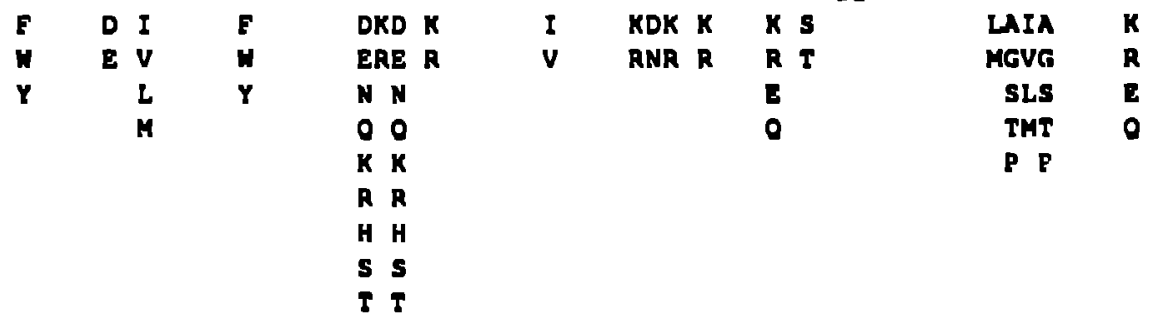

CONSENSUS IVDSOYVHGI I AGQPEE AEAI IVRAI IEAMIKKeA I YVAWVPAHKGI GGNOEYDHLVSOGIROVLFLAK I

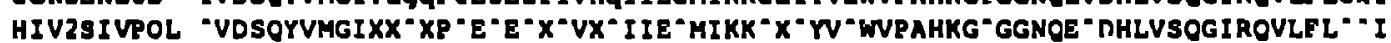

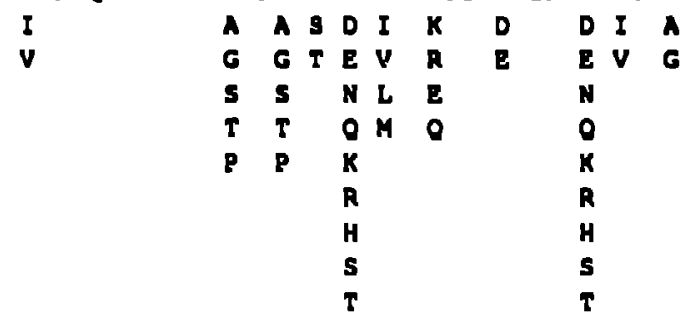

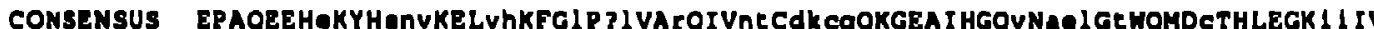

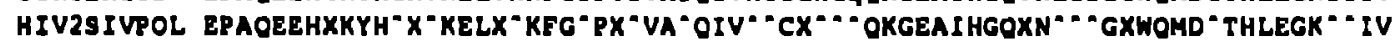

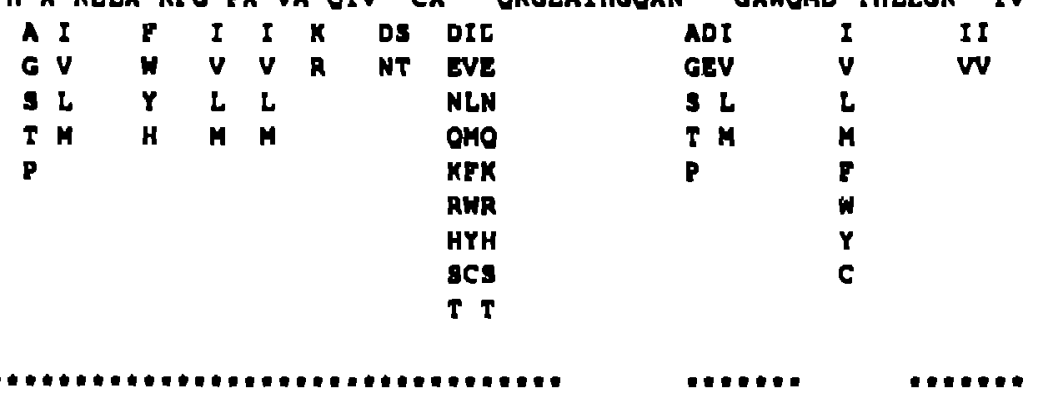

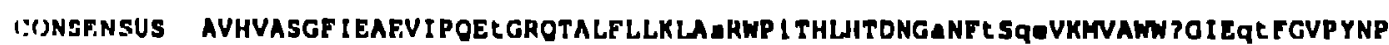

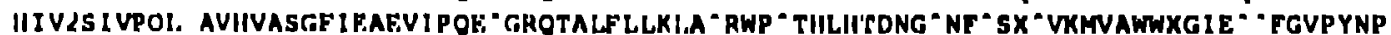

$\begin{array}{ll}\text { A } & \text { I } \\ \text { I } & \text { V } \\ \text { I } & \\ \text { P } & \end{array}$


CONSENSUS OSOGVVEAMNHHLKNOIdR IROOARE PET IVLMAVHCMNFK TRGG IGDMTP AERI I NMI TEEOEIOF ?O?

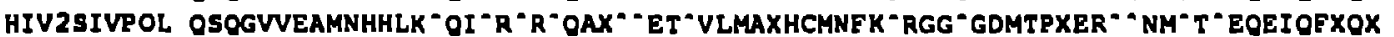

R V VV V G

L LL S

$\begin{array}{lll}V & \text { LL } & \text { S } \\ \text { M } & \text { MM } & \text { T }\end{array}$

0 O M M

$\mathbf{P}$

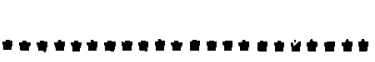

Vif eds $\rightarrow$

CONSENSUS KNSK fknF IVYYREGRdOLWKGPGELLWKG GAVI vKVGTdIKVUPRRKAKIIKdYGgqKeJDSsshIEd

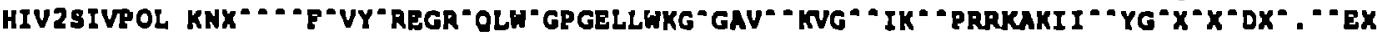

DIKD $K$ F D $K$

EVRE $R$ N $N$

NLEN E Y $Y$

OMOQ O

KF $\mathrm{K}$

D II AD II

KD A K I A OL

RW R

HY H

sc $s$

VV GE VV

LL $S$

0

$\mathbf{T}$

$\begin{array}{rrrrrrr}\text { RE } & \text { G } & \text { R } & \text { V } & \text { G } & \text { E } \\ \text { N } & \text { S } & \text { E } & \text { L } & \text { S } & \text { N } \\ \text { O } & \text { T } & \text { Q } & \text { M } & \text { T } & \text { Q } \\ \text { K } & \text { P } & & & \text { P } & \text { K } \\ \text { R } & & & & & \text { R } \\ \text { H } & & & & & \text { H } \\ \text { S } & & & & & & \text { S } \\ \text { T } & & & & & & \text { T }\end{array}$

CONSENSUS tr roagEvart7o- 985

HIV2SIVPOL 'X."XXE"A" 1016

$\begin{array}{lll}\text { A } & \text { E } & \text { I } \\ \text { S } & 0 & \text { V } \\ \text { S } & & \text { L } \\ \text { T } & & \text { M } \\ \text { P } & & \end{array}$


CONSENSUS SIVAGMTYO SIVAGM155 SIVAGM 3 SIVAGM677

CONSENSUS SIVAGMTYO SIVAGMIS5 SIVAGM3 SIVAGM677

CONSENSUS SIVAGHTYO SIVAGMISS SIVAGM3 SIVAGK677

CONSENSUS SIVAGMTYO SIVAGMISS SIVAGM SIVAGM677

CONSENSUS SIVAGHTYO SIVAGHISS SIVAGH3 SIVAGH677

CONSENSUS SIVAGHTYO SIVAGHISS SIVAGH3 SIVAGH67?

CONSENSUS SIVAGMTYO SIVAGHISS SIVAGM] SIVAGMGI!

CONSENSUS SIVAGMTYO SIVAGHLSS SIVAGH SIVACM677

CONSENSUS SIVAGHTYO SIVAGHISS SIVAGM] SIVAGH677

CONS KNGUS GIVAcimtyo sivacimiss TIVAC:MI :IVAI:MnI)

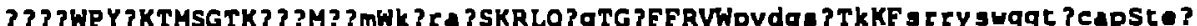
LWKI ---AKTMSGTKENKMSKVWKIGTPS----. G--E--_--TVDGGK-E--SRRY SWSGTECAS -TER . ................ MWKARALS----.G--E--_--PVDGDK-K--SRSHSWGGTKCAP - TEO ........... TKKDEMLEMWETRAFS----.R--K-----PVDGSE-0--SRRYSWGGANCAP -TE. VRON---G. . . . . . . . . . . . . -.-EW--K-_---PLGRSE-K--CAIVRRHSWSGTN-PRN

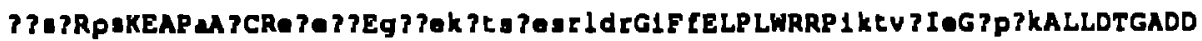
HHP I -PS--- -A-I =-ERETT -GAKEESTGNESGLDR-I -F------IKTVY-E-VPIK----_-LYTL-PS----A-V--ERETN-KSEOKPPSEQSRLER-I-F---_-_-IRTCI-G-TAVK--.--_--. .SI-PC----A-I --OGEAV-GTKEKTTSSESRI DR-I -F--------IKTVY-L-VP IR----_--GNSL-SS----P-V--EGTAP-RGERTDKETEGERSG-C-L-_-_-_-_MKRVI -E-TPVO-_._._.-_

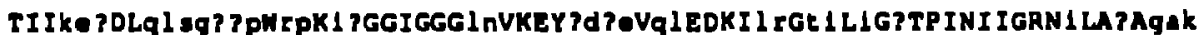
---KEN--OLSG. . P-RP-II--_---LN----NDRE-KI ----LR-TI-L-A-_---_-_-LL-P-VPR ---KDT --OLRG. . S-RP -IV--_-_-LN-_--DNVE-OL--_-LR-TV-I-A----_----F--O-GAK

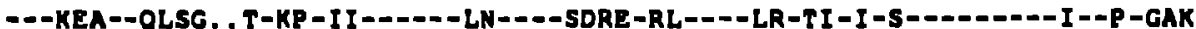

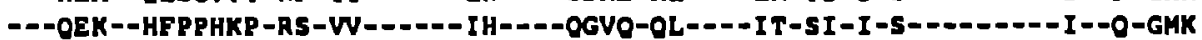

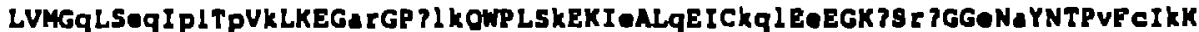
$----Q--E K-P V-P-K----A R--C V R-----K---E--0---S O L-0-D-I-R V--E-A----I-C-K-$

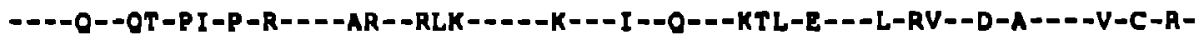

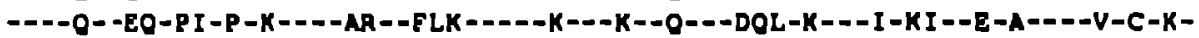

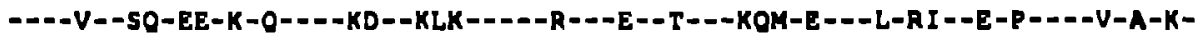

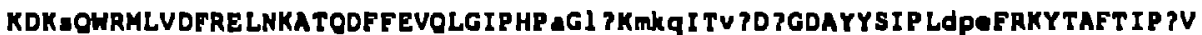
- - - S-

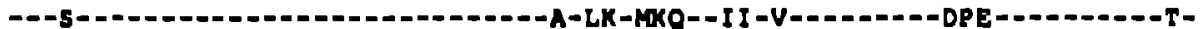

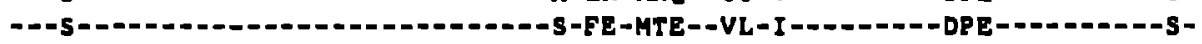

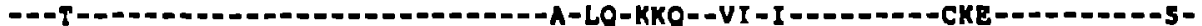

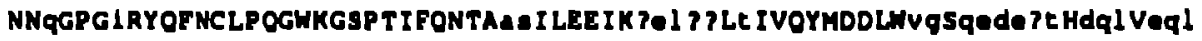
-O-_-I - - -

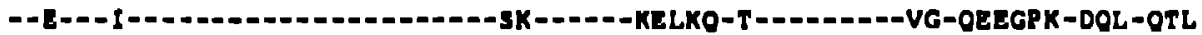

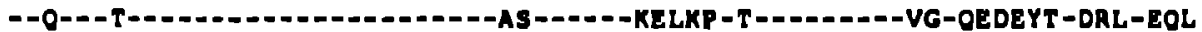

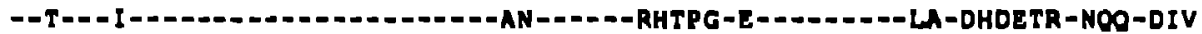

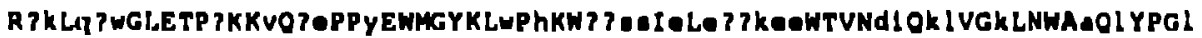

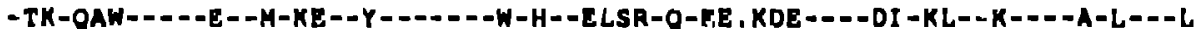

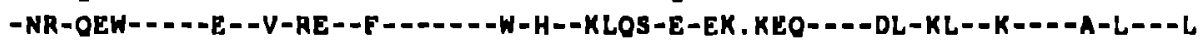

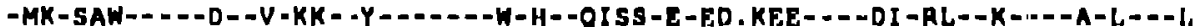

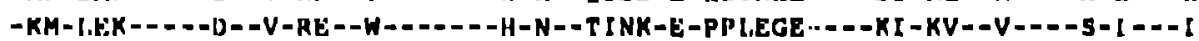

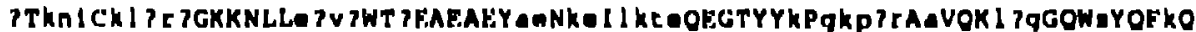

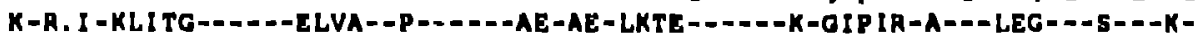

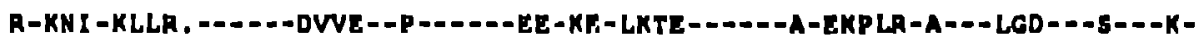

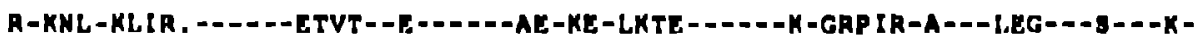

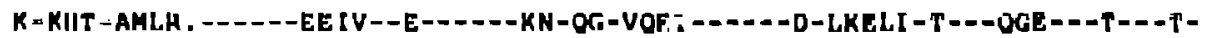

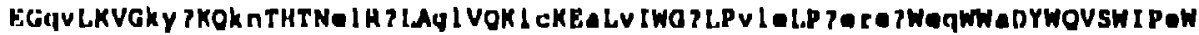

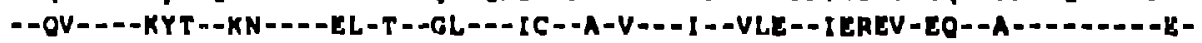

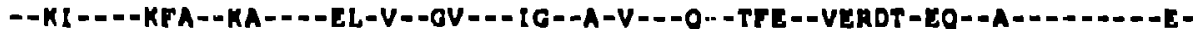

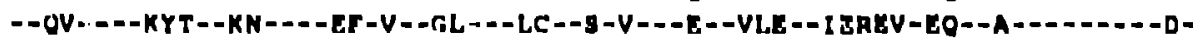

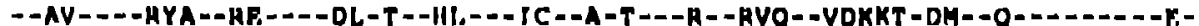

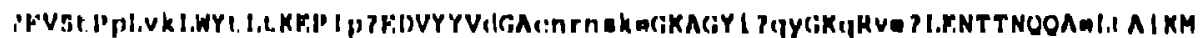

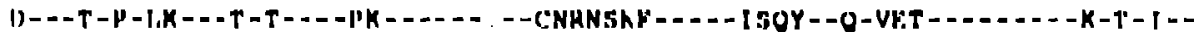

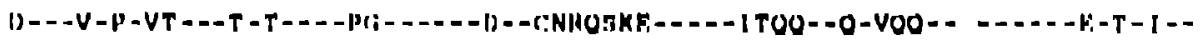

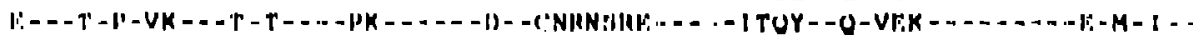

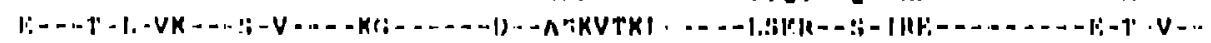


CONSENSUS SIVAGMTYO SIVAGM155 SIVAGM3 SIVAGM677

CONSENSUS SIVAGHTYO SIVAGM1 55 SIVAGH3

SIVAGH 677

CONSENSUS SIVAGHTYO SIVAGM155 SIVAGM3 SIVAGM 677

CONSENSUS SIVAGMTYO SIVAGMI55 SIVAGM 3 SIVAGM 677

CONSENSUS SIVAGHTYO SIVAGH15S SIVAGH SIVAGM 677

CUNSENSUS SIVACKTYO SIVAGH1SS SIVAGH SIVAOM 677
ALEDSGPnUNIVTDSOYAMgILTAqP qSASP LVEOI I I mVqK ? I I LOWVPAhKGIGGNEEI DKLVSK

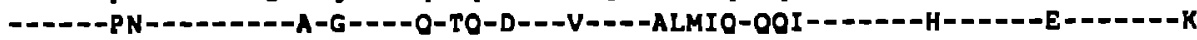

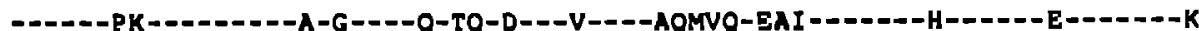
-

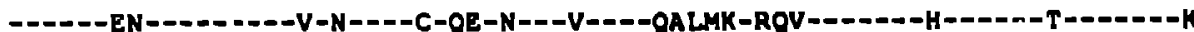

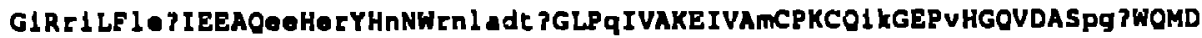
-I-RV--LEK-----EK-ER--N--KNLADTY---0-------M----IK---V------PGT--_

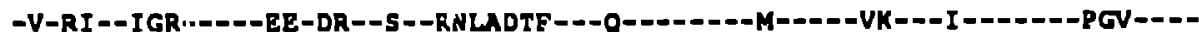

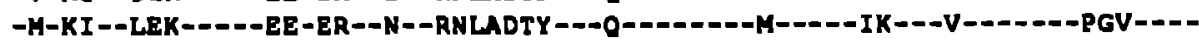

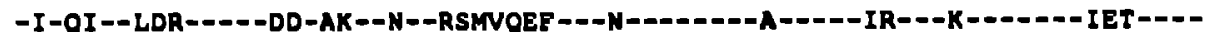

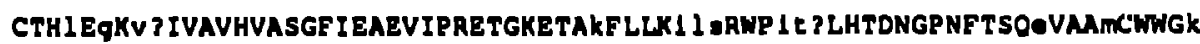
---L-K-VV-_._. --I-G-IV-_-_- - L-G-VI -. --LL-G-VI -._-

1EHTTG?PYNPOSOGS IESHNKOLKEIIGKIRDDCGYEETAVIMACh IHNFKRKGGIGGIL SAERLINm I

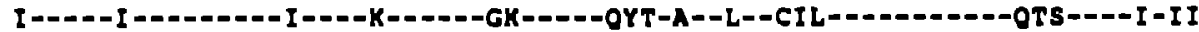

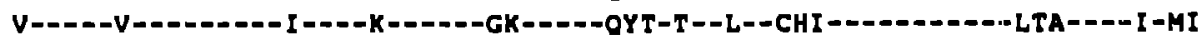

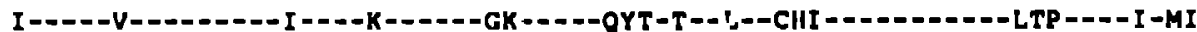
I

TTOLE ? ? R LOEKI OKI LNF TVYYREGRDPUWKGP A ?LIWKGEGAVV ?KOGO ? I KVVPRRKAKI IKDYAPT

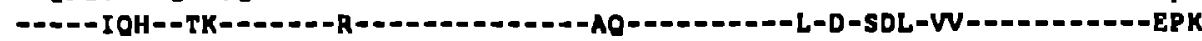
--_-INT--TK--_-_-R-1._-

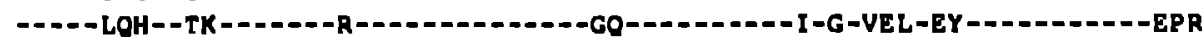

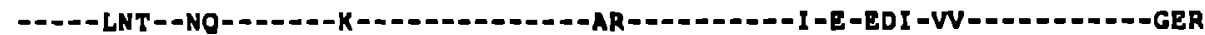

K7mgdEg7 IEGA?G?dn?MPGDS? 978 ORVGN-GDV--TRGSDN* 1061

KTLGD-THL--AGGSDHO-A-_-. 1047

KRMGD-SHL--AGGADN" 1046

KTMDS-GSM--VAEANKO-E---DLODOE * 1057 
Information dense reglons 10.95 or groater) are Indicated by wh".

ant m+*

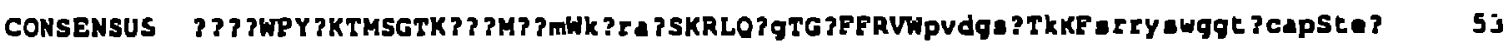
AGMPOL

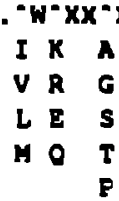

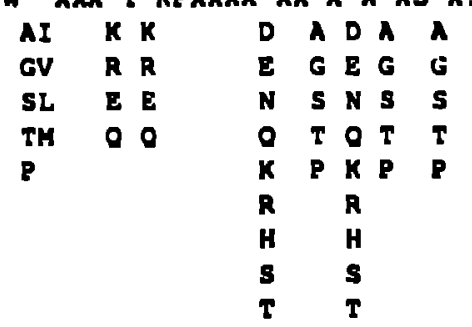

$\bullet \bullet \bullet \bullet \bullet \bullet$

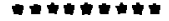

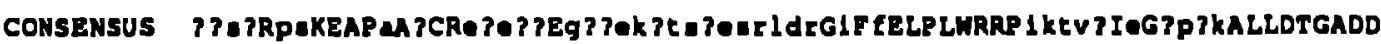
AGROL

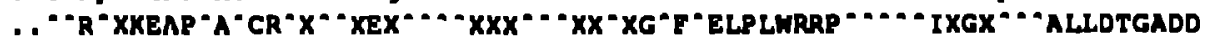

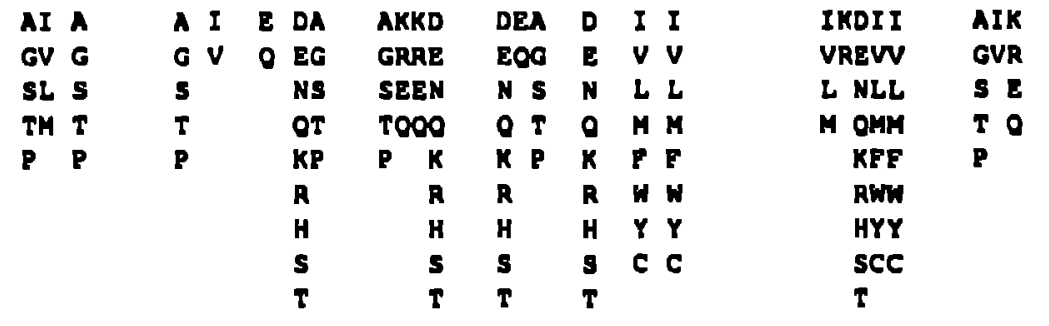

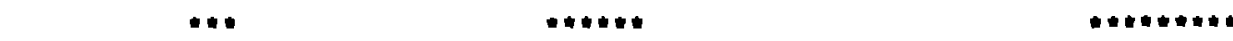

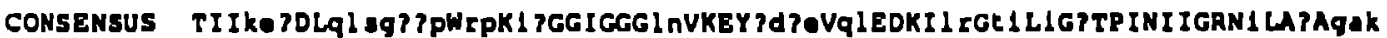
AGMPOL

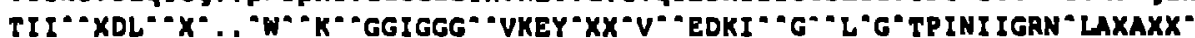

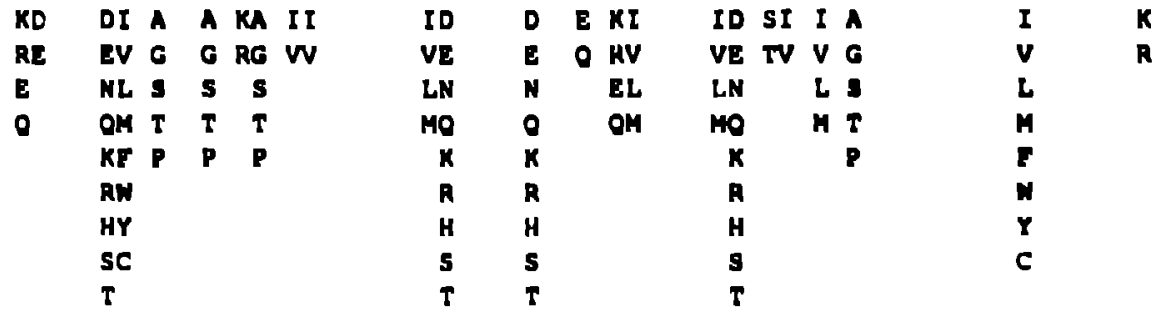

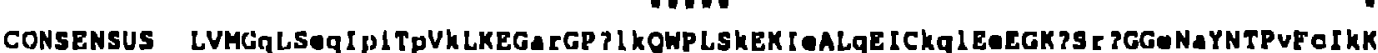
AGMPOL

\begin{tabular}{|c|c|c|c|c|c|c|c|c|c|c|c|c|}
\hline & & & & & & & & & & & & \\
\hline 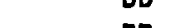 & $n$ & 0 & $I K$ & $\kappa$ & 0 & OUL & $n$ & & n & & & 1 \\
\hline EL & A & E & VR & R & B & EEM & $\mathbf{R}$ & $v$ & RV & 8 & G & $v$ \\
\hline NN & $E$ & $\mathbf{N}$ & L & & $N$ & NN & E & L & & & 8 & \\
\hline 90 & 0 & $?$ & H & & 0 & $\infty$ & 0 & M & & & t & \\
\hline KK & & K & & & K & KK & & & & & & \\
\hline AR & & R & & & H & $\begin{array}{l}\text { RA } \\
\text { HH }\end{array}$ & & & & & & \\
\hline HH & & H & & & 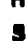 & $\begin{array}{l}\text { HH } \\
\text { gs }\end{array}$ & & & & & & \\
\hline $\begin{array}{l}88 \\
\mathrm{Tt}\end{array}$ & & $T$ & & & $T$ & TT & & & & & & \\
\hline
\end{tabular}


CONSENSUS

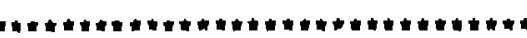

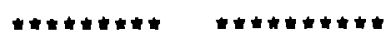

AGMPOL

KDK IOWRMLVDFRELNKATODFFEVOLGI P HP aGI 3KmkqIT $V$ ?D?GDAYYSIP LdPEFRKYTAFT IP ?V

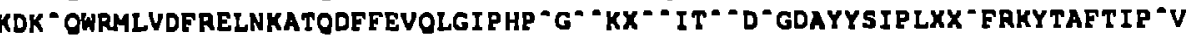

A IK DE II I

G VR EO VV V

S LE $N$ L

I MO $O$ M

P F K

U $\mathbf{R}$

$\mathbf{Y} \mathbf{H}$

c $\mathbf{s}$

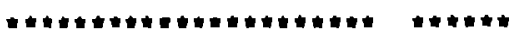

$\bullet \bullet \bullet \bullet+\bullet \bullet \bullet \bullet$

CONSENSUS NNGGPGIRYOFNCLPOGHKGSPTIFONTAASLEEIK ?OL ? ?LEIVOYMDDLWVGSqOde?tHdqIVEqI AGMPOL NN' GPGXRYOFNCLPUGWKGSTIFONTA " ILEEIK- XXXL-IVOYMDDLW

D
E
$\mathbf{N}$
$\mathbf{O}$
$\mathbf{K}$
$\mathbf{R}$
$\mathbf{H}$
$\mathbf{S}$
$\mathbf{T}$

$\begin{array}{rrr}\text { AD } & \text { KD } & \text { D } \\ \text { GE } & \text { RE } & \text { E } \\ \text { SN } & \text { N } & \text { N } \\ \text { TC } & \mathbf{O} & \mathbf{O} \\ \text { PK } & \text { K } & \text { K } \\ \text { R } & \text { R } & \text { R } \\ \text { H } & \text { H } & \text { H } \\ \text { S } & \text { S } & \text { S } \\ \text { T } & \text { T } & \text { T }\end{array}$

$\begin{array}{llllll}\text { IA } & \text { DDD } & \text { D } & \text { DK } & \text { D } & \text { I } \\ \text { VG } & \text { EEE } & \text { E } & \text { NR } & \text { E } & \text { V } \\ \text { L } & \text { NNN } & \text { N } & \text { E } & \text { N } & \text { L } \\ \text { M } & \text { OO } & \text { O } & \text { O } & \text { O } & \text { M } \\ \text { KK } & \text { K } & & \text { K } \\ \text { RR } & \text { R } & & \text { R } \\ \text { HH } & \text { H } & & \text { H } \\ \text { SS } & \text { S } & & \text { S } \\ \text { TT } & \text { T } & & \text { T }\end{array}$

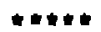

$\bullet \bullet \bullet \bullet \bullet \bullet \bullet$

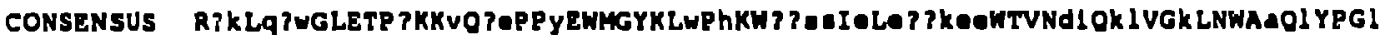
AGMPOL

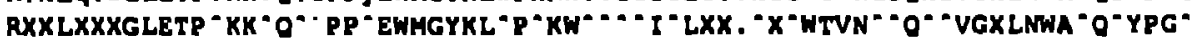

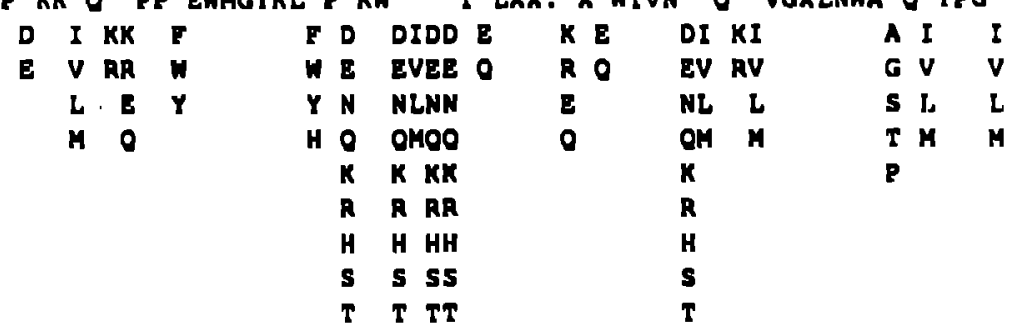

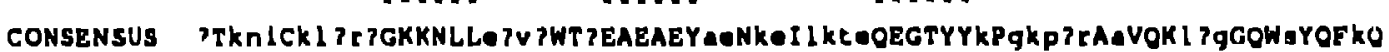
AGMPOL

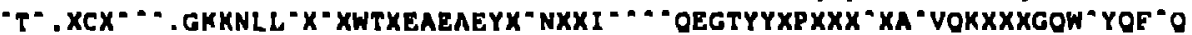

$\begin{array}{ccccc}\text { IKDD } & \text { I } & \text { A } & \text { S } & \text { D } \\ \text { VREE } & \text { V } & \text { G } & \text { T } & \text { E } \\ \text { LENN } & \text { L } & \text { S } & & \text { N } \\ \text { MOOO } & \text { M } & \text { T } & & \text { O } \\ \text { KK } & & \text { P } & & \text { K } \\ \text { RR } & & & & \text { R } \\ \text { HH } & & & & \text { H } \\ \text { SS } & & & & \text { S } \\ \text { TT } & & & & \text { T }\end{array}$




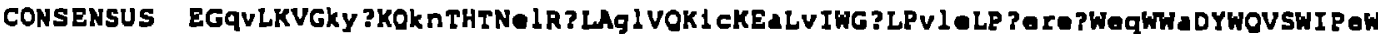
AGMPOL

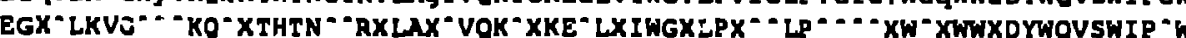

$\begin{array}{lrr}\text { IE } & \text { IDKD } & \text { D } \\ \text { VO } & \text { VERE } & \text { E } \\ \text { L } & \text { N } \\ \text { H } & \mathbf{O} \\ \mathbf{E} & \mathrm{K} \\ \mathbf{H} & \mathrm{R} \\ \mathbf{Y} & \mathrm{H} \\ \mathbf{C} & \mathbf{S} \\ \mathrm{T} & \mathbf{T}\end{array}$

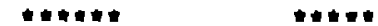

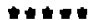

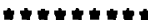

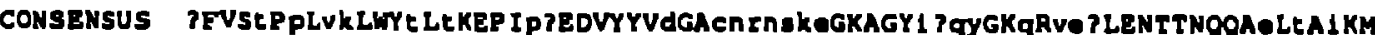
AGHPOL

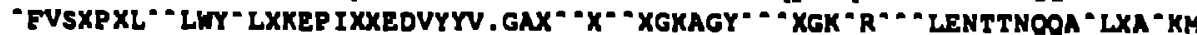

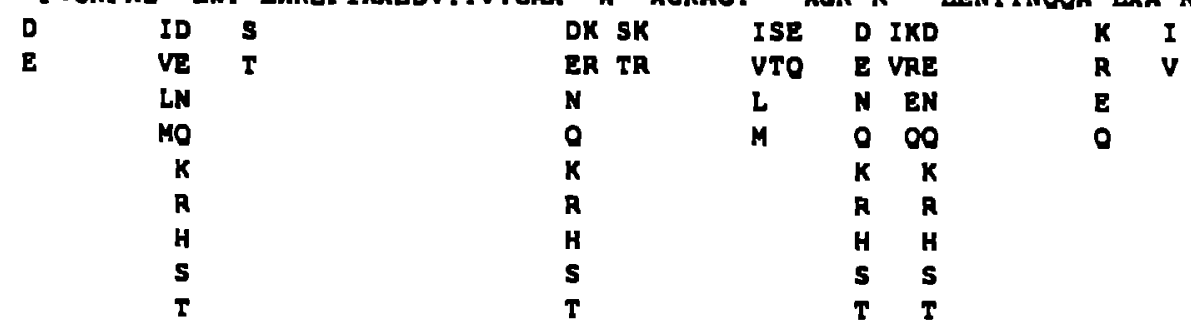

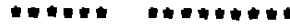

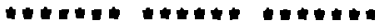

CONSENSUS ALEDSGPNVNIVTDSOYAMgILTAqPLqSASPLvEOIIAImVqK Zq1YLOWVAhKGIGGNEEI DKLVSK AGHPOL

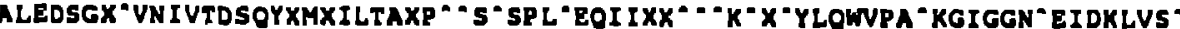

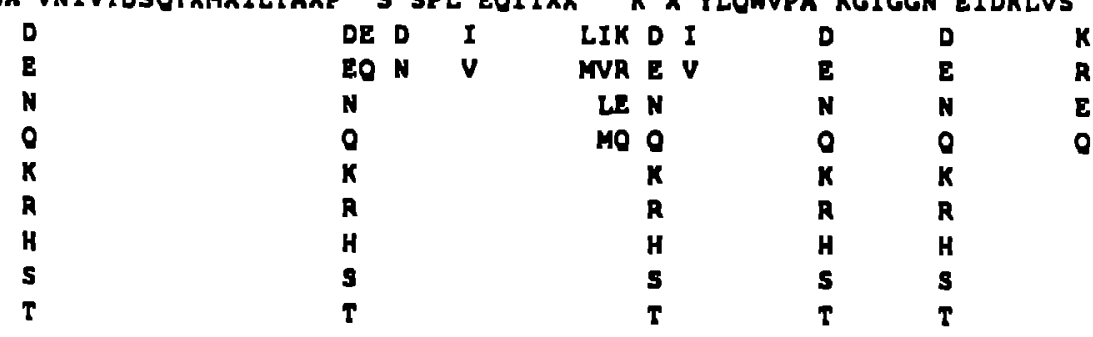

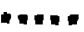

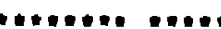

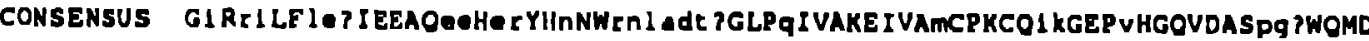
AGHPEL

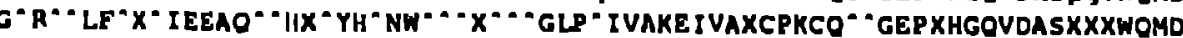

I KI I K

$\checkmark$ RV $V R$

L E L

M $O$ M

$\begin{array}{cccccc}\text { DD } & \text { K } & \text { D } & \text { KDL } & \text { DDF } & \text { D } \\ \text { EE } & \text { R } & \text { E } & \text { REM } & \text { EEH } & \text { E } \\ \text { N } & & \text { N } & \text { N } & \text { NNY } & \text { N } \\ 2 & & \mathbf{O} & \mathbf{O} & \mathbf{O O} & \mathbf{0} \\ \text { K } & & \text { K } & \text { K } & \text { KK } & \text { K } \\ \text { R } & \text { R } & \text { R } & \text { RR } & \text { R } \\ \text { H } & \text { H } & \text { H } & \text { HH } & \text { H } \\ \text { S } & \text { S } & \text { S } & \text { SS } & \mathbf{S} \\ \text { T } & \text { T } & \text { T } & \text { TT } & \text { T }\end{array}$


$\because \div$

CTH I EgKV ? IVAVHVASGF IEAEV IPRETGKETAKF LLK I I SRWP I $t$ ?LHTDNGPNFT5O॰VAAICWWGK

\begin{tabular}{|c|c|c|c|c|c|}
\hline I I I & D & IIA & IDD & D & I \\
\hline v $\quad \mathbf{v}$ & $\mathbf{E}$ & VVG & VEE & $\mathbf{E}$ & $\mathbf{v}$ \\
\hline$I$ & $\mathbf{N}$ & LLS & NN & $\mathbf{N}$ & $\mathbf{L}$ \\
\hline M & 0 & MMYT & 00 & & $M$ \\
\hline & $\mathbf{k}$ & $\mathbf{P}$ & $\mathbf{K X}$ & & \\
\hline & $\mathbf{R}$ & & $\mathbf{R R}$ & & \\
\hline & H & & Hн & & \\
\hline & $\mathbf{s}$ & & ss & & \\
\hline & $\mathbf{T}$ & & $\mathbf{T T}$ & & \\
\hline
\end{tabular}

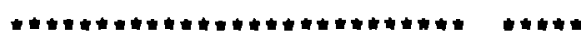

$\bullet \bullet \bullet \bullet \bullet+\bullet \bullet \bullet \bullet$

CONSENSUS AGMPOL

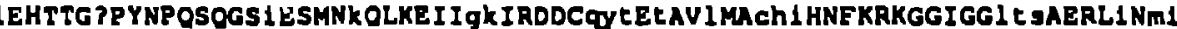
"EHTTG" PYNPQSOGS "ESHN" OLKEI I" IRDDC" XXE" AVXMAXX" HNFKRKGGIGGX" "AERL"

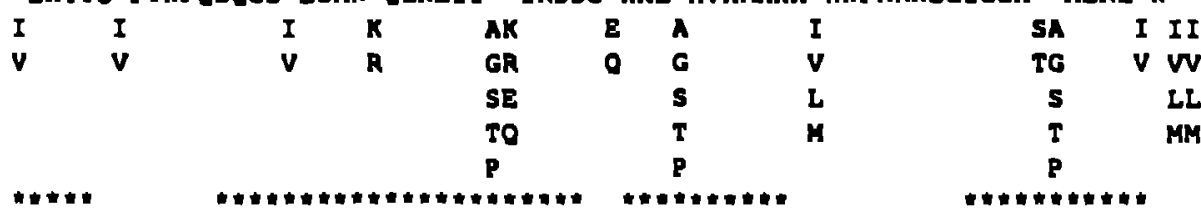

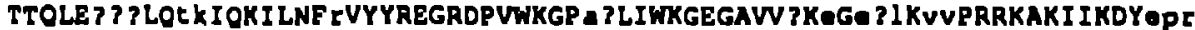
TTOLE"- LQ" - IOKI LNF"VYYREGRDPVWKGP " LIWKGEGAW" KXGX" -KX"PRRKAKI IKD“'XX"

$\begin{array}{ll}\text { IDD } & \text { DK } \\ \text { VEE } & \text { ER } \\ \text { LNN } & \text { NE } \\ \text { MOQ } & \text { OO } \\ \text { KK } & \text { K } \\ \text { RR } & \text { R } \\ \text { HH } & \text { H } \\ \text { SS } & \mathbf{S} \\ \text { TT } & \text { '? }\end{array}$

AK
GR
$\mathbf{E}$
$\mathbf{Q}$

$\begin{array}{ccc}\text { I } & \text { DI } & \text { I } \\ \text { V } & \text { EV } & \text { V } \\ \text { L } & \text { L } & \text { L } \\ \text { H } & \text { H } & \text { H } \\ & & \text { F } \\ & & \text { W } \\ & & \text { Y } \\ & & \text { C }\end{array}$

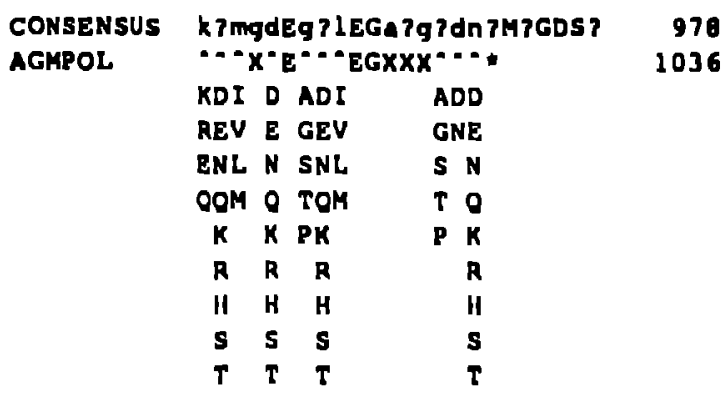


<- pol eda

CONSENSUS1 MENRWGVMIVWOVDRMRI ITWKSLVKHHMY IS?KAKGW IYIHHYESEhP I1SSEVhIP IGd. ATLVITTY HIVLAI ----WO-------V------R--KS-----M-V-G--RGWF-R-----PH-RI-- - H--L-D.AR--I --HIVHXB2R -.--WO------V-----R--KS-----M-V-G--RGMF-R---C-PH-RI----H--L-D.AR--I -.HIVMN $\quad-.-$-RO------A-----R--KS-----M-I-K--KGRT-R-----TH-RI-D--H--L-D. AR--I ---

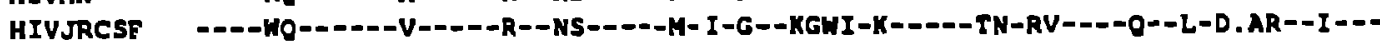
HIVOYI ----WO--.---VV-----R--KS-----M-V-K--KGWF-R-----TH-RI----H--L-D.AT--V---

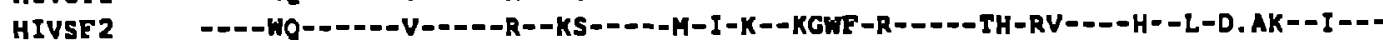

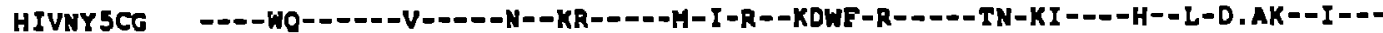

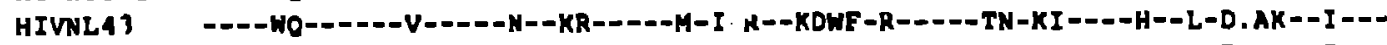

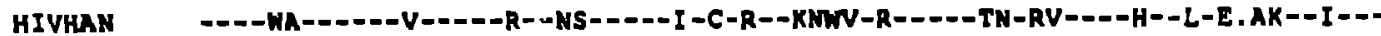
HIVRF ----WO--_.--V-----R--KS-----M-I-R--KGWF-R--_--TH-RI----H--P-D.ER--I --CONSENSUS2 MENRHOVHIVWOVDRMII ITWKSLVKhHMYUSKKA? WWEYRHHYOS?HPK ISSEVHIPLGE. ATLVV TTY HIVELI HIV2226 - 6 HIVNDK HIVRAL - -

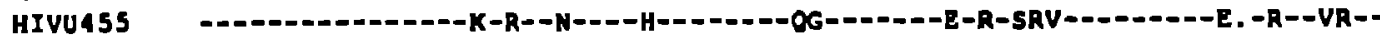
SIVCPZ -

CONSENSUS 1 WGLHTGER dWHLGOGV IEWRKKYY STOVDPdLADQLIHIYYFDCFSOSAI InAI IGIIVSP ICEYQAGH HIVLAI --------D-------VSI---KKR-S-----E---O---LY------D---RKALL-HI---R--YO---

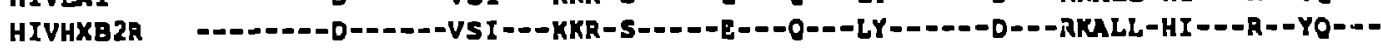
HTVMN HIVJRCSF HIVOYI

HIVSF 2 HIVNY5CG

HIVNL4J

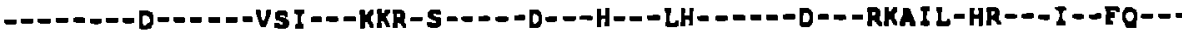

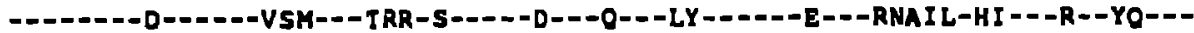

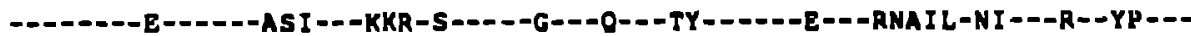

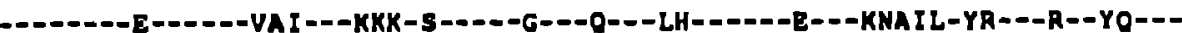

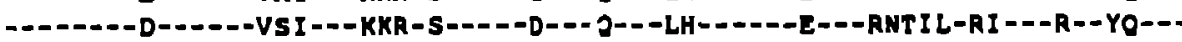

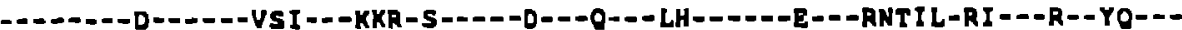
HIVHAN HIVT:

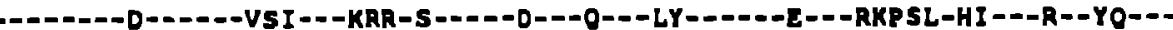
CONSENSUS2 WGLHTGEKdWHLGQGVS IEWRKTRY STOVDPGLADQLIHMYYFDCF AEAAIRKAILGhIVAPICOYOAGH HIVELI HIV2226 HIVNDK HIVMAL HIVU455 SIVCP Z ---H---RE----OQ--.----KR-..---V--G---Q---HY-.---S-S---K----D--SPR-E----

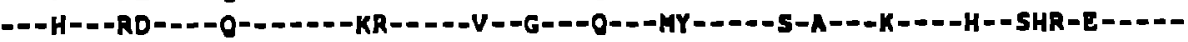

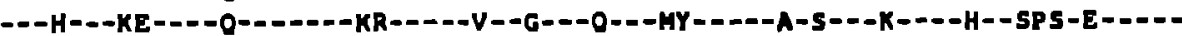

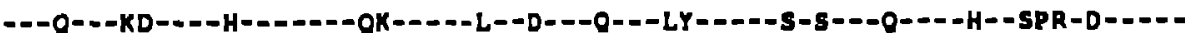

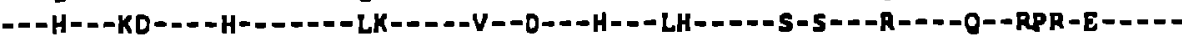

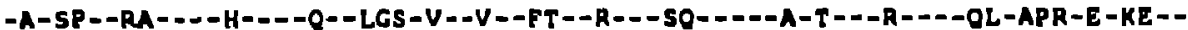

\section{UPR Cd: -}

CONSENSUSI AKVGELOYLALAALITPKKIKPP:P SVTKL.TEOAWNKPOKTKGHRGSHTMNOH* 189

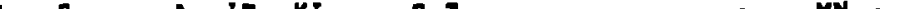

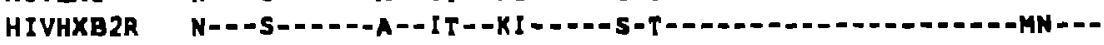
HIVMN HIVJRCSF HIVOYI HIVSF 2 HIVHY SCC HIVNL 43 HIVHAN HIVRF

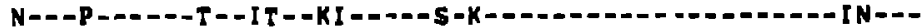

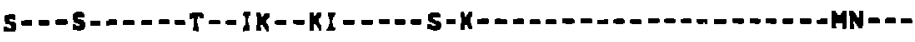

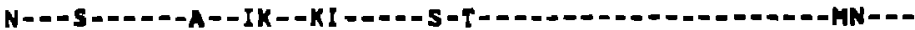

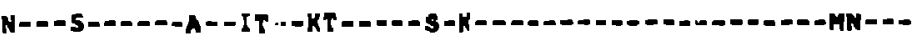

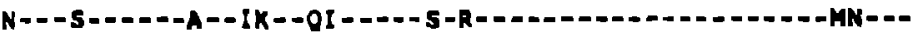

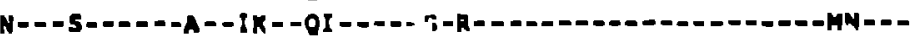

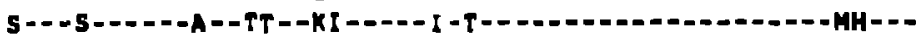

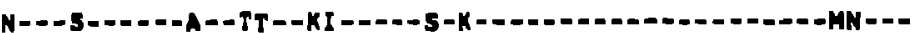
CONSENSUS 2 nKVGSLOYLALCALI APKKIKPPLP SV RKLTEDRWNKPOKTKGhrGShTmNGH•

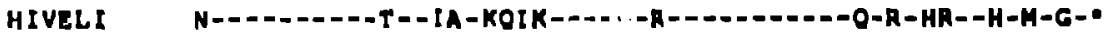
HIVZ22 6 HIVNDK HIVMAL HIVU455 SIVCP Z

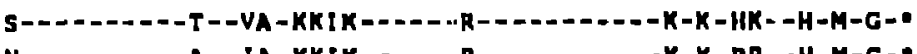

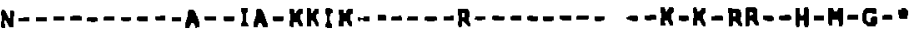
N-D-D-D-T - IA-KKTR-- - - -R-- - - - - - O-K-HR- -H-H-G-O

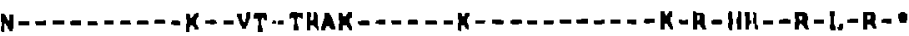

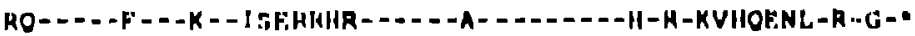


Information dense stretchea $(0.8$ or greater) are Indlcated by **..

$$
<- \text { pol eds }
$$

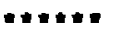

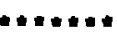

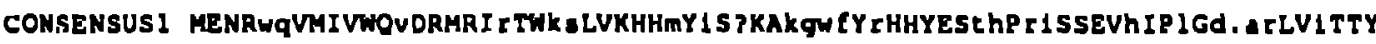

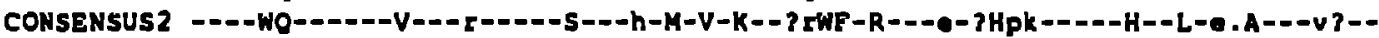

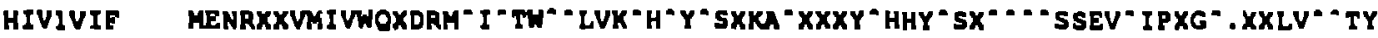

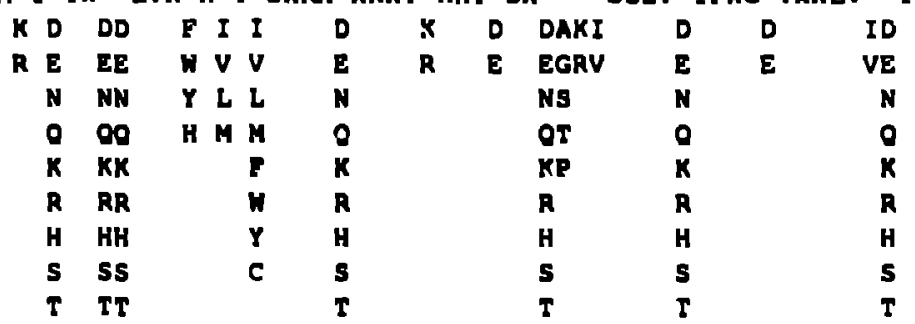

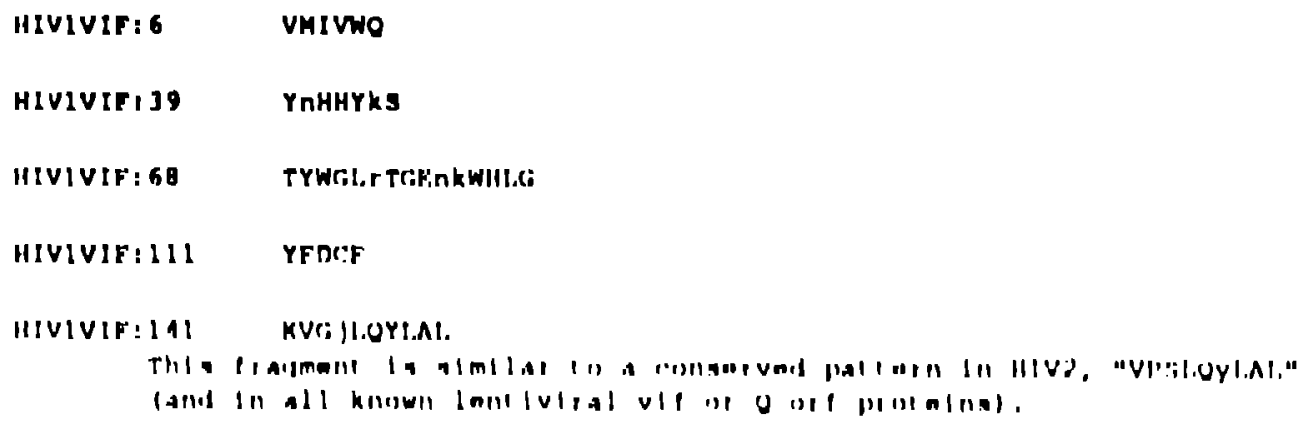

IIIVIVIF:IGH RI,THDHWNKPORTIN: 
c- pol cds

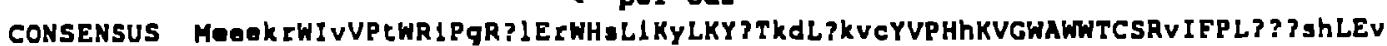
HIV2ROD -EEDKR--V--T--V-G-, M-K--S-V-Y---K-KD-EKVC----H-_-_...--VV----KGNSH--I

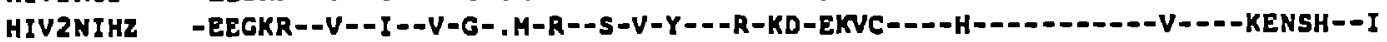

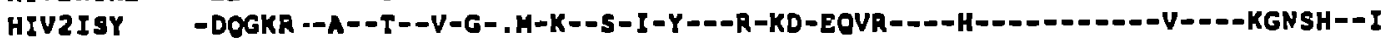

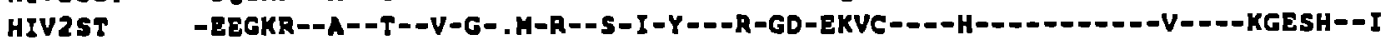

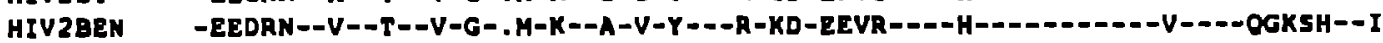
HIV2D194 HIV2GHI HIV2D205 SIVMH25I SIVMa1142 SIVM4239 SIVMNE

SIVSMRHA SIVSMMPBJ

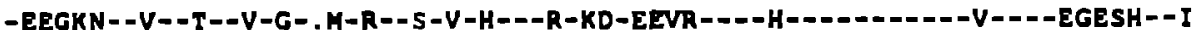

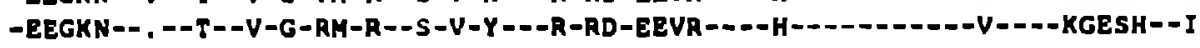

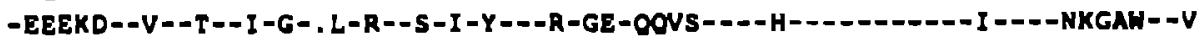

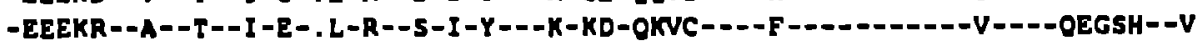

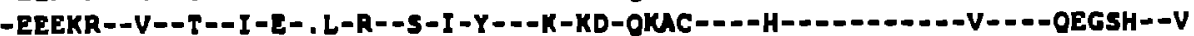

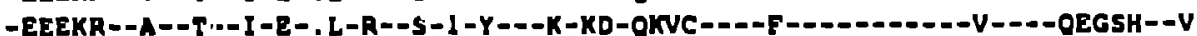

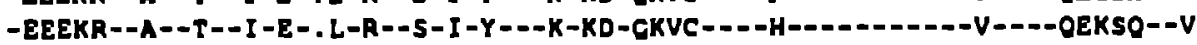

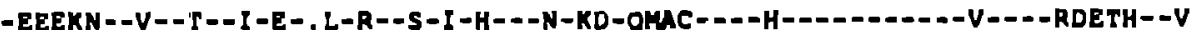
CONSENSUS HIV2ROD HIV2NIHZ HIVZ ISY HIV2ST HIV2BEN HIV20194 HIV2GH I HIV20205 SIVMm25I SIVMMI 12 SIVMA23 9 SIVHNE SIVSMHHA SIVSMRPBJ

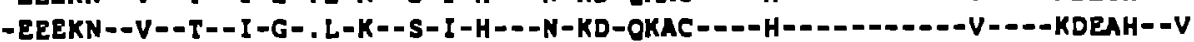

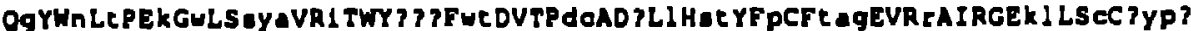

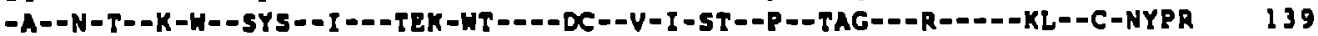

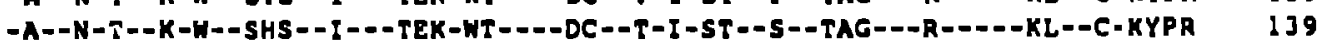

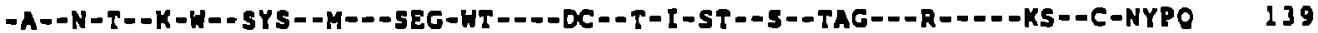

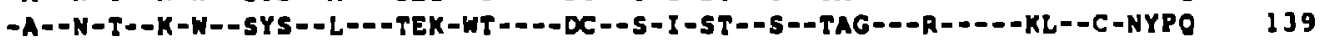

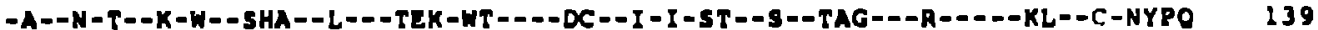
-A--N-T--K-H--SHS--L-D-TEK-HT---DC--S-I-5T--S--TAG---R-D--KL--C-NYPO 139

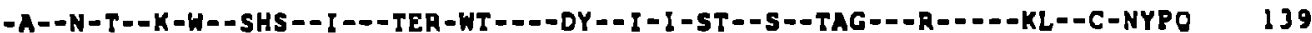
-G--N-T--R-F--SYA--L-D-ERN-YT---ODV--O-L-GS--S--SAN---R-----KI--Y-NYPS 139

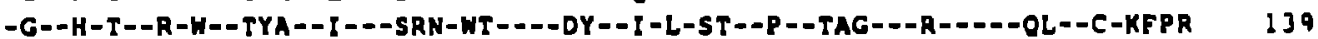

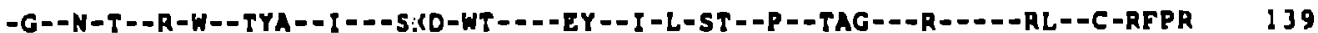
$-G--H-T--K-W--T Y A--I---S K N-W T-D-N Y--I-L-S T-P--T A G---R----O L--C-R F P R \quad 139$

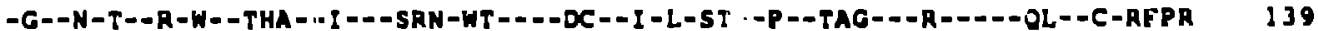

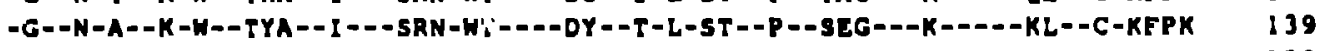
$-G--N-T--K-H--T Y A--I---S R N-H T--D-D Y--T-L-G T--P--S E G---R--D-K L--C-K F P K$

\section{vpx cdi $\rightarrow$}

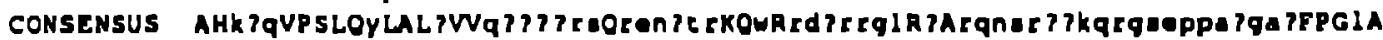
HIV 2POD HIVINIHZ HIVIISY HIVIST IIV2DEN HIV2DI 94 HIVZGHI HIV2020s S【VMH2s] SIVMHI 4 d S IVMM2 J9 SIVMNE SIVSMAH4 SIVSAMPBJ - -RAO--- - -F---V--OQ'D. RP-RDSTTR--R-ADYARGL-L-KODSRSHKORSSESPTPRTY-- -V - -RSO--_--E.--V- JOND.RS-GNSATR--R-GDYRRGL-M-RODSRGYKORGSESPPTRAH-- -L$--K S K=---F--V-$-UOND. KP-RONTTR--W-RNYARGL - L-RODGRSHKORGSEP PAOGAY -- -V -

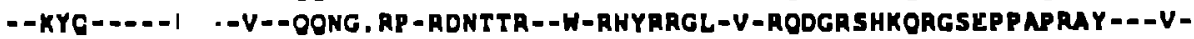
- -KAO- - - Y - - - - -OQND. RP - RKGTAR - -W-RDHWRGL - V-REOHRSLKOGGSEP SAPRAH - - V - KAO-- - -Y---V--CONG. RP. TKGAAR--W-RDHWRGL-V-ROOYRSLKOGGSEP SAPRAH-D-V- - KVO-- - - - - - -V - - COND, RP-.RKGTAR--W-RDHWRGL-V-ROOYRSLKORGSEP SAPRAH- - V- -EGO-_- - -F - - -R - -OEGKNGS-GESATR - -R-RNSRRS I - L-RKNNAKAOOGSGOPFAPATY-- -1. - -RYO- - - - Y - - K- - . SOV, R5 -GENPTW- -H-RDNRRGL - M-KONSKGDKORGSKPPTKGAD-- - I. -

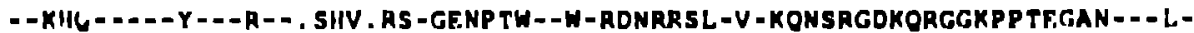

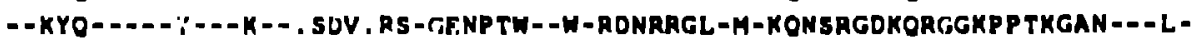
- -KTO-_._-Y - -R - . SYV, RS -RENPTW--N-RDNRASL-M-KONSRGDKORGSKPPTKGVD- - L -

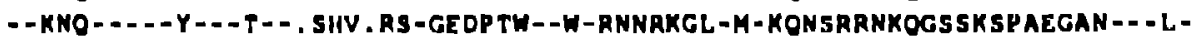


HIV2/SIV VIF

$\begin{array}{lll}\text { CONSENSUS } & \text { KVLGILA* } & 192 \\ \text { HIV2ROD } & \text { E--E---- } & 215 \\ \text { HIV2NIHZ } & \text { E--E---- } & 215 \\ \text { HIV2ISY } & \text { K--E---- } & 215 \\ \text { HIV2ST } & \text { K--E---- } & 215 \\ \text { HIV2BEN } & \text { K--E---- } & 215 \\ \text { HIV2DI94 } & \text { K--G---- } & 215 \\ \text { HIV2GHI } & \text { K--E---- } & 215 \\ \text { HIV2O205 } & \text { E--G---- } & 216 \\ \text { SIVMM251 } & \text { K--G---- } & 214 \\ \text { SIVMMI42 } & \text { K--G--- } & 214 \\ \text { SIVMM239 } & K--G--- & 214 \\ \text { SIVMNE } & K--G--- & 214 \\ \text { SIVSMMH4 } & K--G---- & 214 \\ \text { SIVSMMPBJ } & K--G---- & 214\end{array}$


Information dense scretches $(0.8$ or greater) are indicated by *...

$$
\text { e- pol cde }
$$

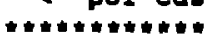

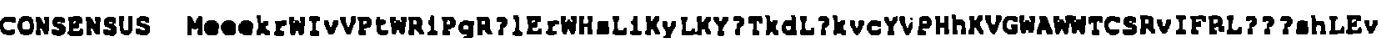

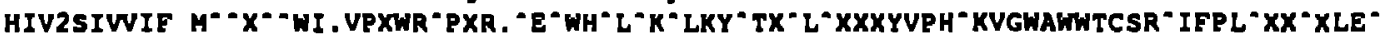

\begin{tabular}{|c|c|c|c|c|c|c|c|c|c|c|c|c|}
\hline DE & KD & $I$ & L K & A I & $F$ & D & D & D & $E$ & I & D & $\mathbf{A}$ \\
\hline EQ & $\mathbf{R E}$ & $v$ & M R & G V & $\mathbf{v}$ & $\mathbf{E}$ & $\boldsymbol{E}$ & $\mathbf{E}$ & $\boldsymbol{n}$ & $\mathbf{V}$ & $\mathbf{E}$ & G \\
\hline & $\mathbf{N}$ & & & $\mathbf{s}$ & $Y$ & $\mathbf{N}$ & & $\mathbf{N}$ & $Y$ & & $\mathbf{N}$ & $\mathbf{s}$ \\
\hline & 0 & & & $\mathbf{T}$ & H & 0 & & 0 & H & & 0 & $\mathbf{T}$ \\
\hline & $\mathbf{K}$ & & & $\mathbf{P}$ & & $\mathbf{K}$ & & $K$ & & & $\mathbf{K}$ & $\mathbf{P}$ \\
\hline & $\mathbf{R}$ & & & & & $\mathbf{A}$ & & $\mathbf{R}$ & & & $\mathbf{R}$ & \\
\hline & H & & & & & H & & H & & & H & \\
\hline & $\mathbf{s}$ & & & & & $\mathbf{s}$ & & $\mathbf{s}$ & & & $\mathbf{s}$ & \\
\hline & $T$ & & & & & $\mathbf{T}$ & & $\mathbf{T}$ & & & $\mathbf{T}$ & \\
\hline
\end{tabular}

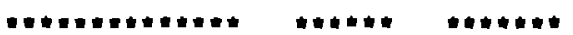

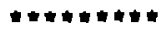

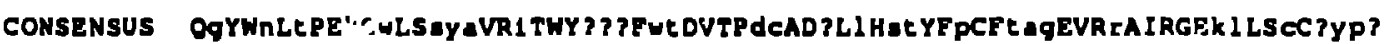

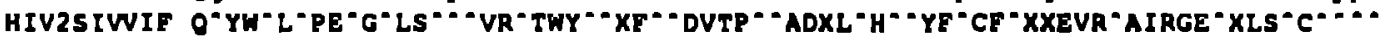

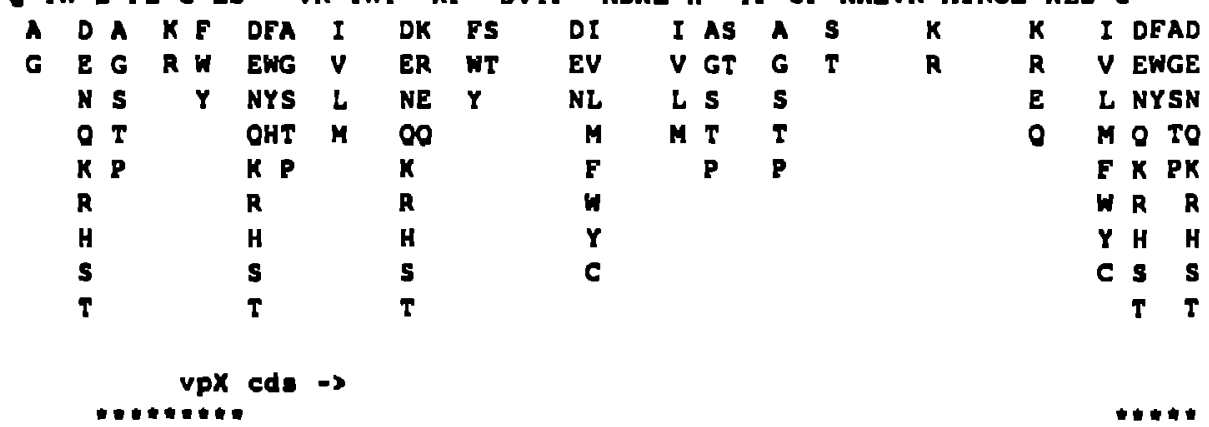

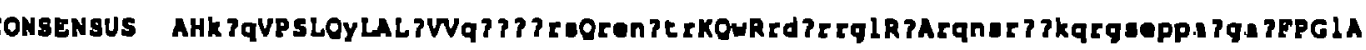

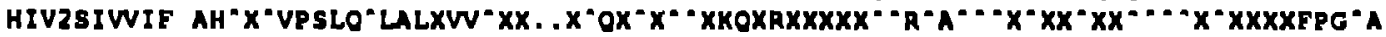

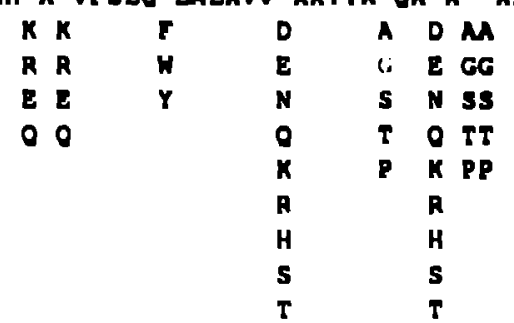

AI I DKD D $K$ MAKA A

GV $V$ ERN E $R$ GGRG $G$

SL L NE $N$ E SSES

TM M 00 O 0 TTOT

$\begin{array}{llllll}P & K & K & \text { PP } & P & P\end{array}$

$\cdots$

consensus kVLqILA 192

HIVZSIVIY "VLXILA. 21J

$\mathbf{R}$
$\mathbf{R}$
$\mathbf{Q}$


CONSENSUS SIVAGMTYO SIVAGMI5S SIVAGM3 SIVAGH677

CONSENSUS SIVAGMTYO SIVAGM15S SIVAGM3

SIVAGH677

CONSENSUS SIVAGHTYO SIVAGHISS SIVAGM3 SIVAGM677

CONSENSUS SIVAGHTYO SIVAGH 55 SIVAGM SIVAGA 677

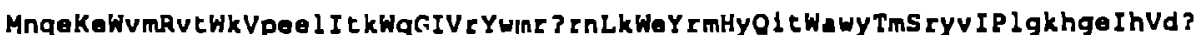
-NPN-E-VM-VT-K-PGDL-TK-O---R-WMRORN-K-N-YM-Y-IT-AWY-M-RYV--IGKHGE-C-DL -SOE-H-VM-LT-K-OEEV-TK-O---R-WMNKRN-K-E-KM-Y-I T-AWY-M-RYV--LPGSGE-H-DI -NOE-E-VM-VT -K-PEEL-TK-O---R-WMRTRK-D-K-RM-Y-IT-AWY-M-RYE--LGOHGS-H-DL -ERE-O-IV-VV-R-SERO-SR-R---T-KIRNKO-P-E-RH-W-VO-OFW-Y-OFI--LSKDDY-E-NI

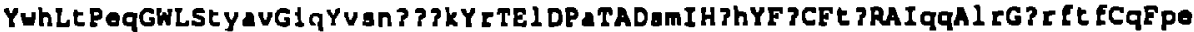
-WH-T-EO----TYAV-IO-VSNLESK-R--L--A---SI --GH--N--KE---CO-LR-HRFVF-O-PE -WH-A-KO--O-TYAV-IO-VSLVNDK-R--L--N---SH--CH-OT-OTD-.- -OO-LR-NRF IF-O-PG -WH-T-EK----TYAE-IQ-LSNRDPW-R--L--A---SL--TH--T--TE- --RK-LL-ORFTF-O-PE -HN-T-ER----SHGV-LS-YHOKG. . -K--V--G---RH- -LY--N--TD---DO-IR-EKYTW-T-KE

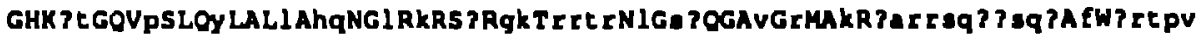
---ST---P---Y---L-HO--L-E--K-GK-RRSR-L-SK---V-O--K-YVTRSOPGGEA-F-ERTPV ---LT---P---Y---L-HQ--L-K--Q-GE-RRTR-L-SQ---V-R--Q-YGRRNOORSOT-F-PRTP I ---KT---P---Y---L-HO--L-O--O-SK-GGTR-M-FE---V-R--K-HARRYOSGSOD-F-ARAPV --- . .--OO---L---V-YT--I-K--K-TF-RMAG-L-SR---M-R-OT-HAOGSKRRSOK-L-NEHAN

PSMELLSGG? r ?E? ? \&H?gKG I? 
Information dense stretchss $(.7$ or greacar) are Indlcated by *t".

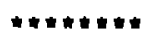

CONSEnSUS MnqeKeWVmRVtWkVpoel ItkWqGIVrYwmrienLkWoYrmHYO1tWaWyTmSryVIPlgkhgeIhVd? 68 AGIVIF

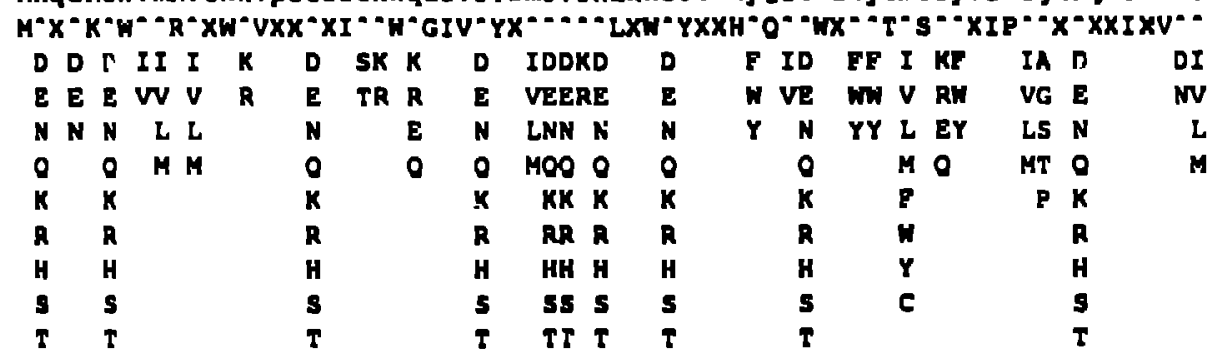

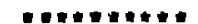

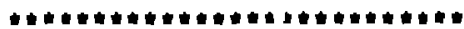

CONSENSUS

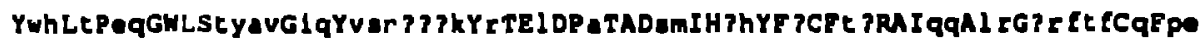
AGRVIF

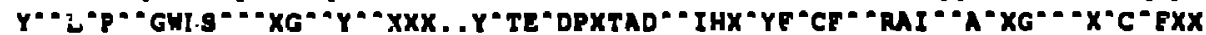

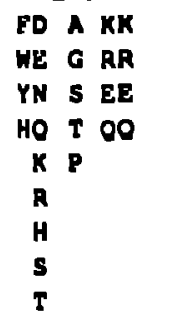

$\begin{array}{rr}\text { SFA ID ID } \\ \text { THG VE VE } \\ \text { Y } & \text { LN LN } \\ \text { H MO MO } \\ \text { K FK } \\ \text { R WR } \\ \text { H YH } \\ \text { S CS } \\ \text { T T }\end{array}$

$\begin{array}{ll}\mathbf{R} & \mathbf{I} \\ \mathbf{V} & \mathbf{L} \\ & \end{array}$

$\begin{array}{llll}\text { DI } & \text { F } & \text { D } & \text { DD } \\ \text { EV } & \text { W } & \text { E } & \text { EE } \\ \text { NL } & Y & \text { N } & \text { N } \\ \text { OH } & H & \text { O } & \text { O } \\ \text { K } & & \text { K } & \text { K } \\ \text { R } & & \text { R } & \text { R } \\ \text { H } & & \text { H } & \text { H } \\ \mathbf{S} & & \mathbf{S} & \mathbf{S} \\ \text { T } & & \mathbf{T} & \mathbf{T}\end{array}$

K.Y I DKE P D

AR V ERH $W E$

EE L N Y Y N

OQ 40

$\mathbf{N}$

K $\quad$ K

R $\mathrm{R}$

YH

T

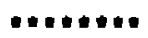

CONSENSUS AGMVIE

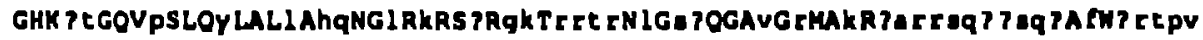

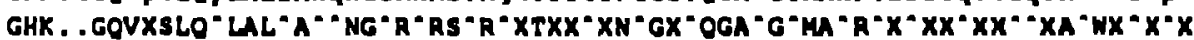

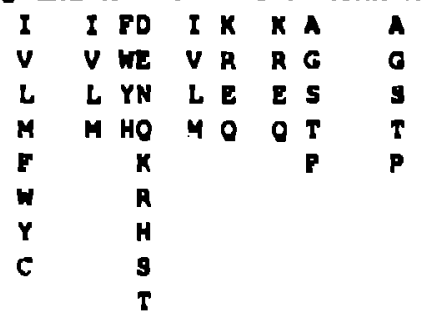

I K D F D $K$ AE I K A

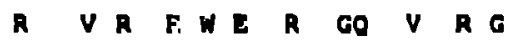
E L E N Y N E S L E I O M O O

CUNSENSUS AGMVIF $\bullet \bullet \bullet \bullet$

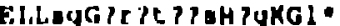

207

PSMELLXXGXX - *

$$
\begin{array}{r}
\text { KD } \\
\text { RE } \\
\text { rN } \\
\text { WO } \\
\mathbf{K} \\
\mathbf{A} \\
\mathbf{H} \\
\mathbf{S}
\end{array}
$$


<- vif eds

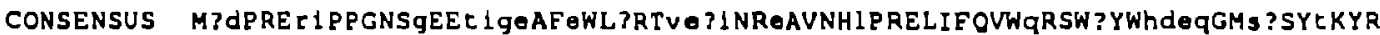
HIV2ROD HIV2NIHZ HIV2ISY HIV2ST HIV2BEN HIV2D194 HIV2GHI HIV2D 205 SIVMM25I SIVMM1 12 SIVMM?.39 SIVMNE SIVSMMH4 SIVSMMPBJ

CONSENSUS HIV2ROD HIV2NIHZ HIVZISY HIV2ST HIV2BEN HIV2O294 HIV 2GHI HIV2D20S SIVMM251 SIVMM 142 SIVMM239 SIVMNE SIVSMMH4 SIVSMMPBJ

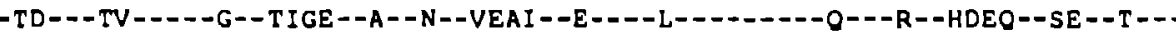

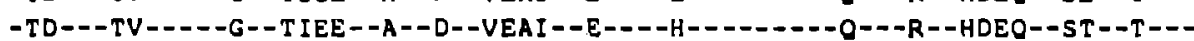

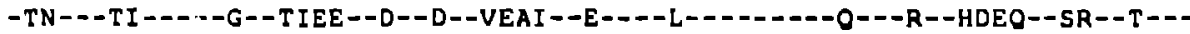

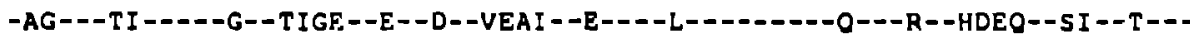

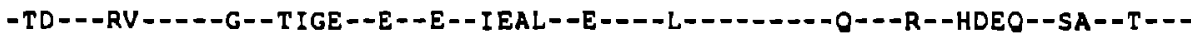

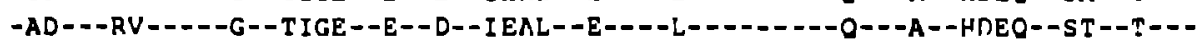

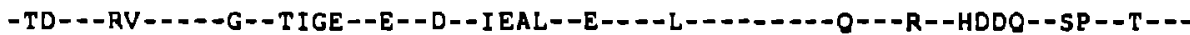

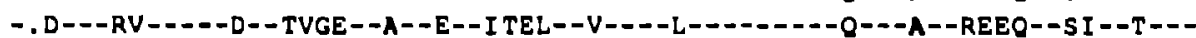

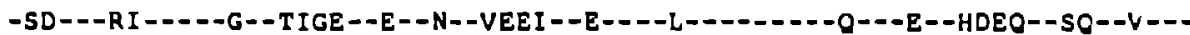

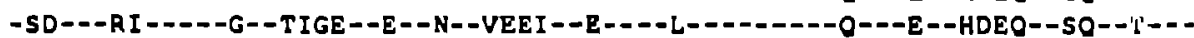

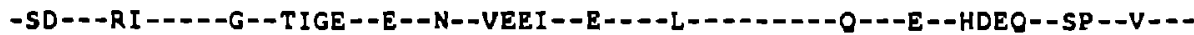

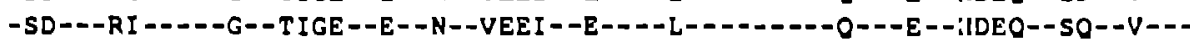
-SD---RI - - - -G--TIGK--E--N--VEEI - -A-- - L- - - - - - -R---E--HDEM--SE--T--

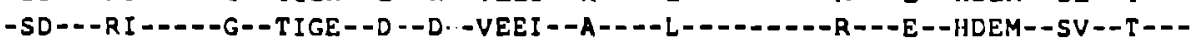

YLCl 1OkA ? fmH?kkgCrClgoghG?gGw ? IPGPPPPPPPGLA" --CI I-K-VYM-VRKGCT-LGRGH-PG-W. RP-------- -V- -CLI-M-MYM-AKRDGT-LGGGM-OK-, GDO---------V--CLM-K-VFM-EKKGCT-RGEGH-PG-W. RS--.---.--V - -CLM-K-MF I -SKRGCT - LGGGH-PG-W. RS---- - - - - V - - CLM-K-IFT-FKRGCT-WGEDM-RE-LEDO--_---- - - $--C I M-K-V Y I-F K K G C T-L G R G H-P G-W$. RP - - - - - - - -V -- CLM-K-VF I -EKRGCT-LGGGH-PG-W. RS--------V- - LLM-K-MFV-YTKGCR-LOEGH-PG-W. RS-------- -A- -CLM-K-LFM-CKKGCA-LGEGH-AG-W . RP---------A- -CLI-K-LFM-CKKGCR-LGEGH-AG-W . RP - - - -CLI-K-LFM-CKKGCR-LGEGH-AG-W . RP--------A$-C L I-K-L F M-C K K G C R-I . G E G H-A G-W . R P--\ldots+\ldots-A-$ $--C L I-K-L F V-C K K G C R-L G E E H-A G-W$. RT - - - - - - -A- 
Information dense strecches ( 0.7 or greater) are Indlcated by *t*."

$\because * * * * * * * *$

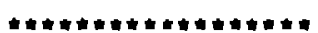

c- v1f cds

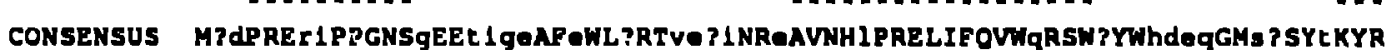

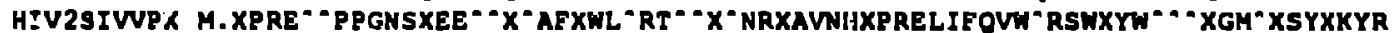

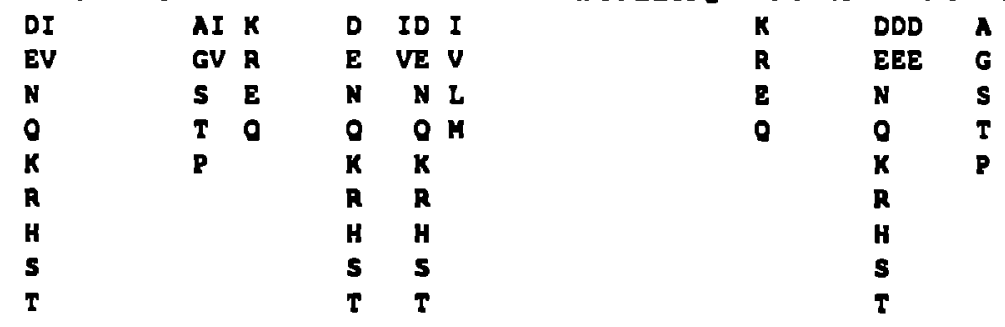

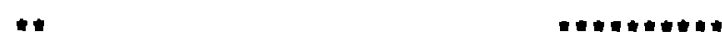

CONSENSUS YlcllokA?fmHzkkgcrClgeghG

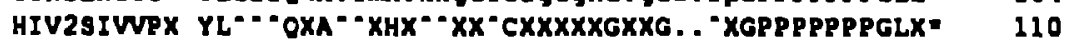

$\begin{array}{lllll}\text { III } & \text { IF } & \text { DK } & \text { D } & \text { D } \\ \text { WVV } & \text { VW } & \text { ER } & \text { E } & \text { E } \\ \text { LLL } & \text { LY } & \text { N } & \text { N } & \text { N } \\ \text { MMY } & \text { M } & \text { O } & \text { O } & \text { O } \\ \text { F } & & \text { K } & \text { K } & \text { K } \\ \text { H } & & \text { R } & \text { R } & \text { R } \\ \text { Y } & & \text { H } & \text { H } & \text { H } \\ \text { C } & & \text { S } & \text { S } & \mathbf{3}\end{array}$




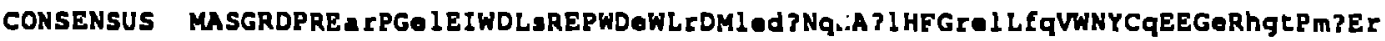
SIVAGMTYO -- - - - -AR--EV-----S----K--R--LODL-O--RL---REL-FO-----Q---E-HGT-MM-R

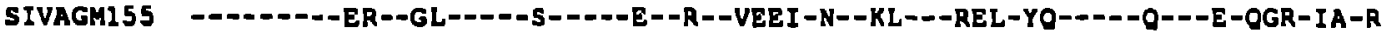

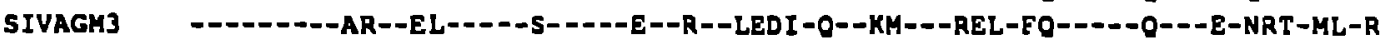

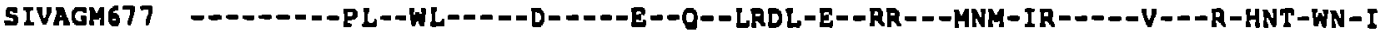




\section{IGMVPX}

Information denge strecches 10.9 or greater) are indicated by me.

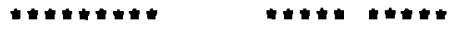

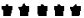

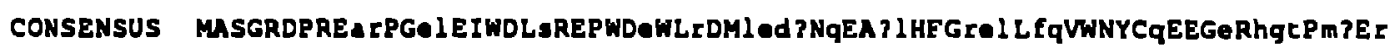
AGIVPX

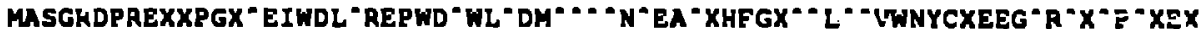
I $K K$ IKDI D $K$

$K$ D D I

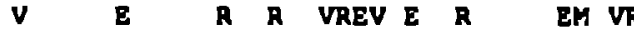
L N E E LELN L N LE

O O MO MO

MO

$\mathbf{F}$

H

$r$

H

R

H

s

c

$R E E V$

E N $\mathbf{L}$

$000 \mathrm{H}$

K $K F$

$R \mathbf{R}$

H $Y$

S $S C$

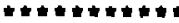

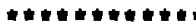

consensus

AYKYY I IVOKA IFVHFRCGCRRRQPE PYEERR TGOGGG I I IVPPGLd "

A KI AL

$\begin{aligned} \text { RV } & \text { GM } \\ \text { L } & \text { S } \\ \text { M } & \text { T } \\ & \text { P }\end{aligned}$

$\begin{array}{lll}\mathbf{D} & \mathbf{D} & \text { A } \\ \mathbf{E} & \mathbf{N} & \mathbf{G} \\ \mathbf{N} & & \text { S } \\ \text { O } & & \text { T } \\ \mathbf{K} & & \text { P } \\ \mathbf{R} & & \\ \text { H } & & \\ \mathbf{S} & & \\ \mathbf{T} & & \end{array}$




\section{HIV1 VPR}

$<-v i E$ cds

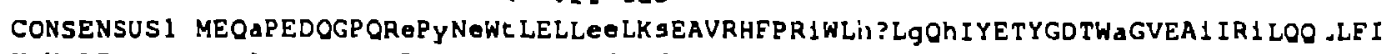

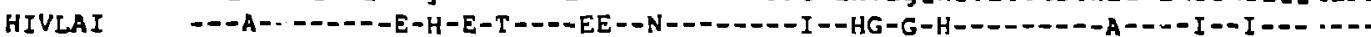

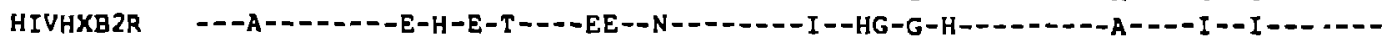

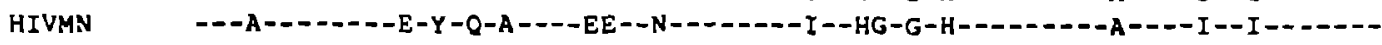

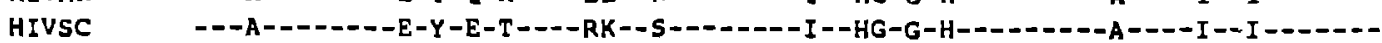

HIVJRCSF -- A-_-

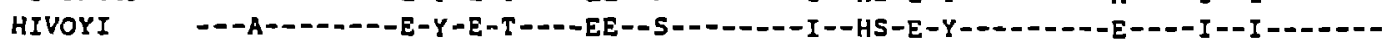

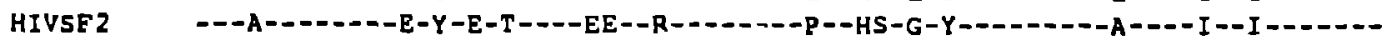

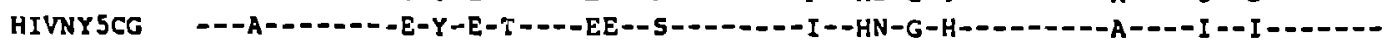

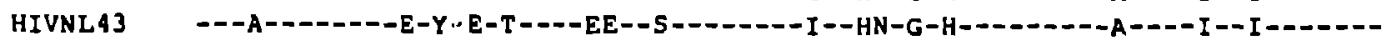

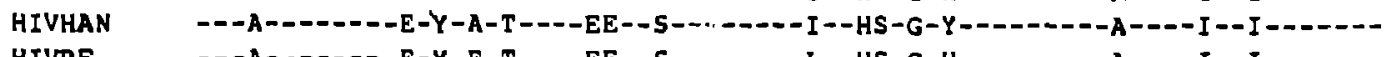

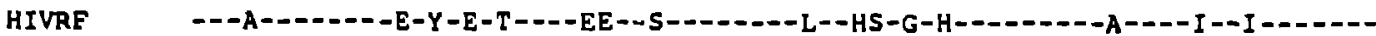

CONSENSUS 2 MEOAP DOGPOREPYNEUT LELLEELKSEAVRHEPRIWLHSLGOhIYOTYGDTW? GVEAIIR1 LOOLLF I

HIVELI -----A--_---YYN-KA - - -

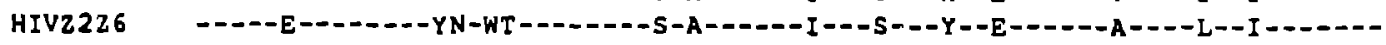

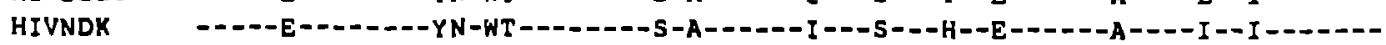

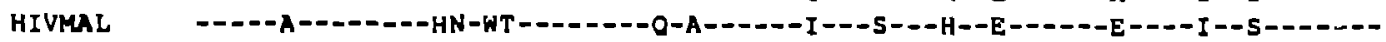

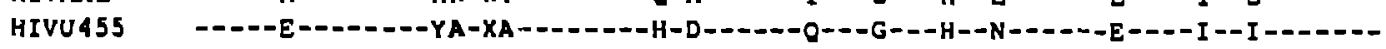

SIVCPZ

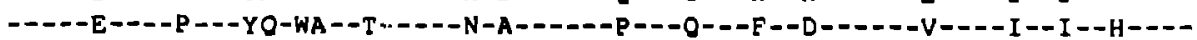

tat eds $\rightarrow$

CONSENSUS1 HFr1gCThSRIGITEORRA ZRNGaSRS*

HIVLAI --RIGCRH----VTO---A.---A----

HIVHXE2R - -ONWVET

HIVMN - -RIGCRH---IIIR---A, ---A--.-

HIVSC - RIGCRH--.-IIQ---S.---S-..-

HIVJRCSF --RIGCRH--.-ITR---A.--A--.-

HIVOYI - -RIGCOH----ITR---A.---A----

HIVSE2 --RIGCOH----IIO---AR---A---

HIVNY5CG --RIGCOH----IIR---A.---A-_--

HIVNL43 --RIGCRH----VTR---A.---A----

HIVHAN --RIGCRH----ITP---A.---A-_-

HIVRF --RIGCOH----ITR---A.---A---

CONSENSUS 2 HFRIGCOHSRIgITIQRRA.RNG SSRS"

HIVELI -...--...-G-IRO--A. -.-S-..-

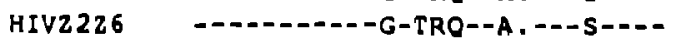

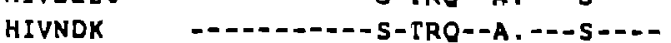

HIVMAL

HIVU455 -...-.-.-G-IPG--G.-.-A-..-

SIVCPZ ---L---N--G-LPO--R.S--SN--- 


\section{HIV1 VPR}

Informalion dense stretches 10.8 or greater) are indleated by $\# *$.

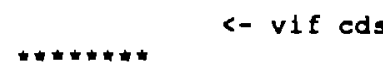

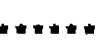

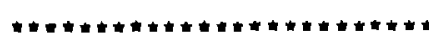

CONSF 'SUS1 MEOAPEDQGPOREPYNOWT LELLEOLK SEAVRHFPRIWLh?LgOhIYETYGDTWAGVEA1 IR1LOQLLF I

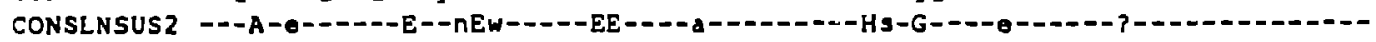

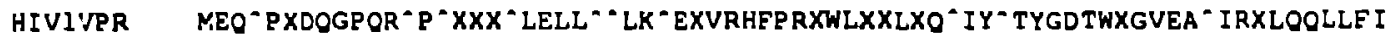

$$
\begin{array}{lll}
E & F & A \\
O & W & G \\
Y & S \\
H & T
\end{array}
$$$$
\text { KK D }
$$

RR E

\section{cac cds $\rightarrow$}

CONSENSUSI HEIIGCThSRIGI:-ORRA?RNGASRS CONSENSUS2 --RIGCQH---gI--q--..--S--D"

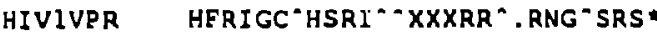
$K$ AI A A

R GV G G

E S S S

Q $\quad \mathrm{T} \quad \mathrm{T} T$

HIVIVPR: 6 DOGPOROP Soe Trlstem et al.. Nature 347:341-1, 1990.

HIVIVPR: $30 \quad$ VRHFPR Th1 e fragment la smilar to a conserved pattern in HIV2, "EALkHFDP". see Trlstem te al., Nature 347: 341-2, 1990.

HIVIVPR: 45 IYMTYGDTWXGVEACIRXLOOLLFIHERIGCPHSRI

Th1 a fragment contalna a region simllar to a conserved pattern in HIV2, "LORAL".

HIVIVPR: $90 \quad$ RNG JSRS 


\section{HIV2/SIV Y'PR}
<- vpX cds
tat cd: $\rightarrow$

\begin{tabular}{|c|c|c|}
\hline CONSENSUS & Mtea ? ?e?PPede?PqRepwdeWv ?e?L?E I kaEALKHFDP rLLt aLGnYIY?RHGD L l eGAgeLI I ILQ & 62 \\
\hline HIV2ROD & -AEAP TEL--VDGT -L-EPGDE-I IEI -R-IKE---K----R--IA--KY-YT-----LE--RE--KV-- & 70 \\
\hline HIV2NIHZ & -TEAP TEL--EDRT-P-EPGDA-VIEI-R-IEE---R----R--TA--RY-YT----LE--RE-RI-- & 70 \\
\hline HIV2ISY & -TEAPAEF--EDGT-P-EPGDE-VIEI-R-IKE---K----R--TA--YY-YT---- LE- RE--RV-- & 70 \\
\hline HIV2ST & -TEAPTES--EDRT-P-EPGDE-VIET-R-IKX---K----R--IT--NY-YA----LE--RG--RI-- & 70 \\
\hline HIV2BEN & -TEAP TEF--EDGT-R-DLGSD-VIET-R-IKE---R----R--IA--YY -HN & 70 \\
\hline HIV2D194 & -TEAPTEF--EDGT-A-ELGST-VIET-K-IKE---K----C-IA--NY-YN-----LE--RE--RV-- & 70 \\
\hline HIV2GHI & -TEAP TEF--EDGT-R-ELGGD-VIRI-G-IKE---K----R--IA--NY-HS-----PE--RE--RI - & 70 \\
\hline HIV2D205 & -AEAAPEI - -ENEN-O-EPWEE-IGEI-E-IKO---K----R--TA--NF-YS----LA--GE--KI-- & 70 \\
\hline SIVMH2SI & $-. \mathrm{E} .$. ER--ENEG-O-EPWDE-VVEV-E-LKE---K----R--TA--NH-YN----LE--GE--RI-- & 66 \\
\hline SIVMM1 42 & - E. . ER--ENEG-O-EPWDE-VVEV-K-LKE---K- $--R--T A--N H-Y N----L E--G E--K I--$ & 66 \\
\hline SIVMM239 &.$- E$. . ER--ENEG-Q-EPWDE-VVEV-E-LKE---K----R--TA--NH-YN-----LE--GE--RI-- & 66 \\
\hline SIVANE & - . E . . ER--EDEG-Q-EPWDE-VVEV-E-LKE---K----R--TA--NH-YN-----LE--GE--KI-- & 66 \\
\hline SIVSMMH4 & -A. . . ER--EDEA-O-EPWDE-VVEV-E-LKE---K----R--TA--NY-YD-----LE--GE--RI-- & 66 \\
\hline SIVSMMPBJ & 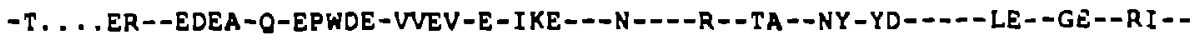 & 66 \\
\hline
\end{tabular}

CONSENSUS

HIV 2ROD

HIV 2 NIHZ

HIV2ISY

HIV2ST

HIV 28EN

HIV2DI 94

HIV2GH 1

HIV 20205

SIVMM2 51

STVMM1 42

SIVMM2 39

5 I VMNE

SIVSMMH4

SIVSMMPBJ

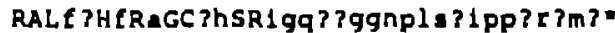

89

105

105

105

105

87

105

105

101

97

101

101

101

101 
HIV2/SIV VPR

Information dense stretches $(0.7$ or greater) are Indlcated by *"*.

$<-v p X$ cds

tat cds $\rightarrow$

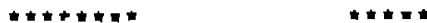

CONSENSUS Mtea??e?PPede?PqRepwdeWv?e?L?E1keEALkHFDP rLLtaLGnYIY?RHGDT leGAgeLI $\downarrow L O$

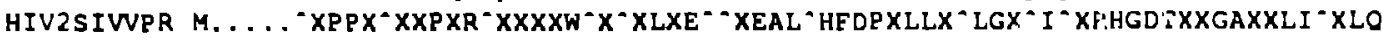

\begin{tabular}{|c|c|c|c|c|c|c|c|}
\hline 0 & $D$ & $D$ & I & $\mathbf{K}$ & IK & D & A \\
\hline$E$ & $\mathbf{N}$ & $\mathbf{E}$ & $v$ & $\mathbf{R}$ & $V_{R}$ & $\mathbf{E}$ & $G$ \\
\hline $\mathrm{N}$ & & & & $\mathbf{E}$ & LE & $\mathbf{N}$ & $\mathbf{s}$ \\
\hline 0 & & & & 0 & MO & 0 & $\mathrm{~T}$ \\
\hline K & & & & & & $\mathbf{K}$ & P \\
\hline $\mathbf{R}$ & & & & & & $\mathbf{A}$ & \\
\hline H & & & & & & H & \\
\hline $\mathbf{s}$ & & & & & & $\mathbf{s}$ & \\
\hline$T$ & & & & & & $T$ & \\
\hline
\end{tabular}

CONSENSUS RALE?HERAGC?hSRIgq??ggnpls?1pp?r?m?* HIV2SIVVPR RAL"X.H"A“GCX"SRX"*

$\begin{array}{lllll}\text { I } & \text { I } & \text { A } & \text { D } & \text { A } \\ \text { V } & \text { V } & \text { G } & \text { E } & \text { G } \\ \text { L } & \text { L } & \text { S } & \text { N } & \text { S } \\ \text { M } & \text { M } & \text { T } & \text { Q } & \text { T } \\ \text { E } & \text { F } & \text { P } & \text { K } & \text { P } \\ \text { W } & \text { W } & \text { R } & \\ \text { Y } & \text { Y } & \text { H } & \\ \text { C } & \text { C } & \text { S } & \\ & & & \text { T }\end{array}$


$1 / 3 \cdot \sin 1 / 3 \operatorname{si}$

rev eds $\rightarrow$

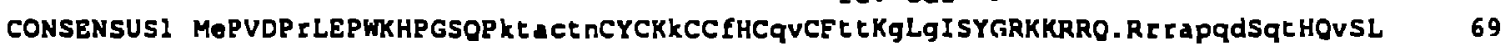

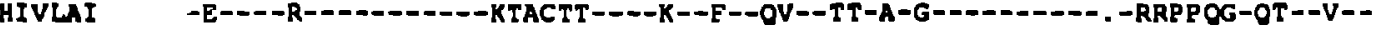

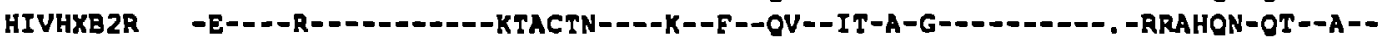

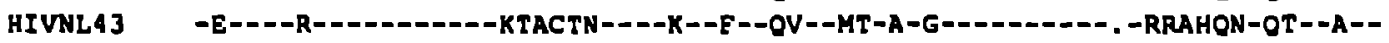

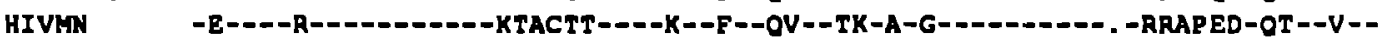

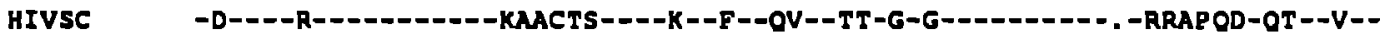

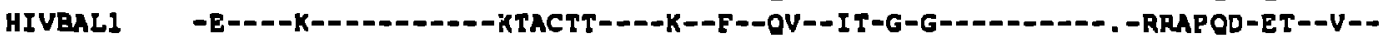

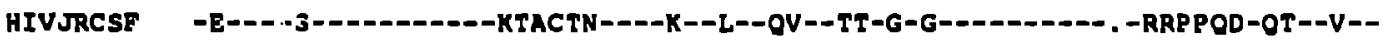

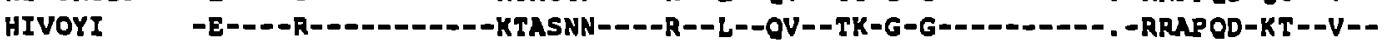

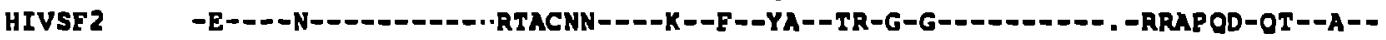

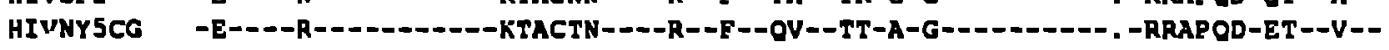

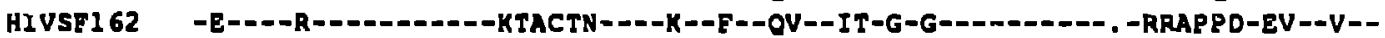

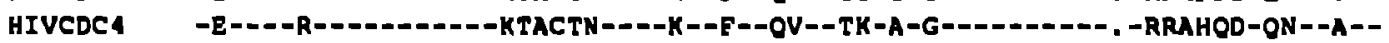

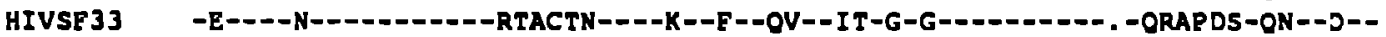

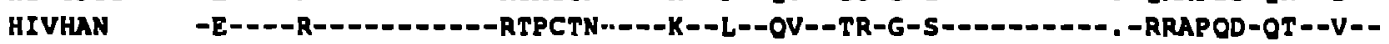

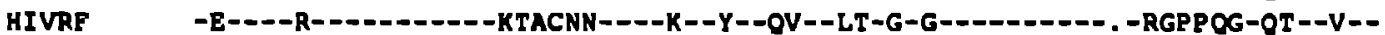

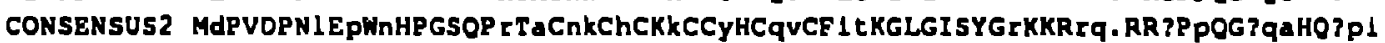

HIVELI -D-----L-P-N------R-P-NK-H--K--Y--PV--LN--------R---RQ. --G-P--GOA--VPI

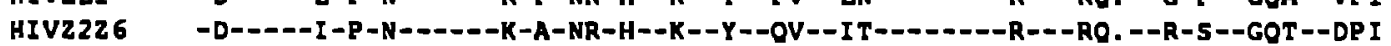

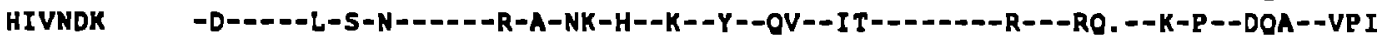

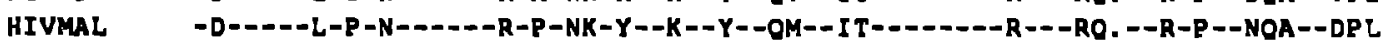

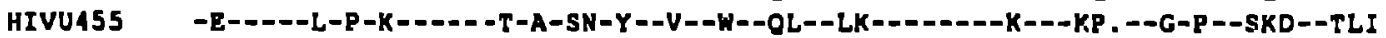

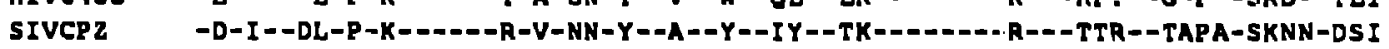

exon Vexon

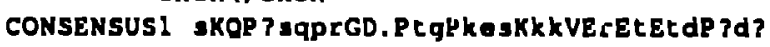

HIVLAI S--TSOPR--.-TG-KE* 86

HIVHXB2R

S-TSOPR-D. -TG-KE

HIVNLA3

S--TSOSR--. -TG-KE*

HIVAN

P---APOFR-D, -TG-KES-KK--R-T-TH-VD*

P---ASOAR--. -TG-KES-KK--R-T-TD-VD*

HIVBALI

HIVJRCSF

S---TSHPR... . -TG-CES-KK--R-T-TD-VH*

P---SSOOR--, -TS-KES-KK - -R-T-TD-LN"

S---ASOPR--. -TG-KES-KK--R-T TD -ED*

S---ASOSR--. -TG-TES-KK--R-T-TD-FO*

HIVSF 2

HIVNY5CG

S---ASOPR--, -TG-KES-KK--R-T-TD-DH*

P-C-ASOPQ--. -TG-KES-KX--R-T-TD-VH*

S--SSOTR--. -TG-KEP-KE--R-A-TD-LO*

S---SSOPR--. -TG-KES-KE--R-T-TD-LD*

P---SSOOR-- -DS-KKS-KK--R-T-AD-FD.

S---TSOPR--, -TG-KES-EK--R-T-TD-AVO*

HIVSF 3]

HIVHAN

HIVRF

PKOP s sO ? rgd? PEGPKe 3KKKVES ?A ?TD? ?A= 
Information dense stretches 10.8 or greater) are indlcated by *t*.

$$
\begin{aligned}
& \text { V/3.9y } 1 / 3^{\prime} 3 \mathrm{~s} \\
& \text { rev cds } \rightarrow
\end{aligned}
$$

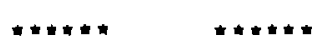

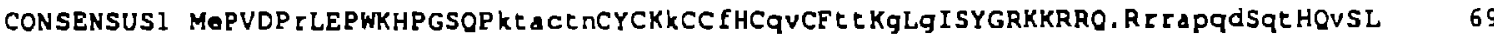

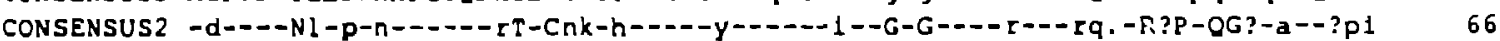

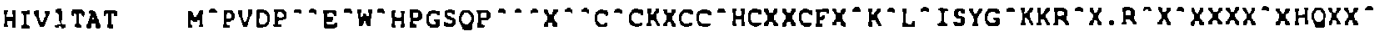

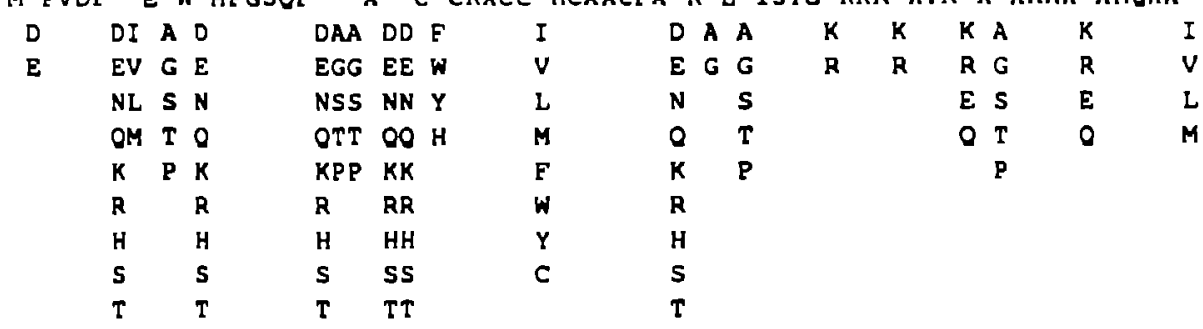

exon\/exon

CONSENSUS1 sKOP? sqprGD. PLgPkeskkkVErEtEtdP?d?" 99

CONSENSUS2 Pk--s-0?-gd?p-Gp--?-KK--S?A?TD?-A* 92

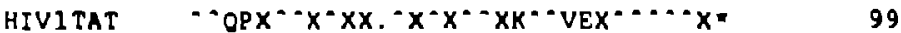

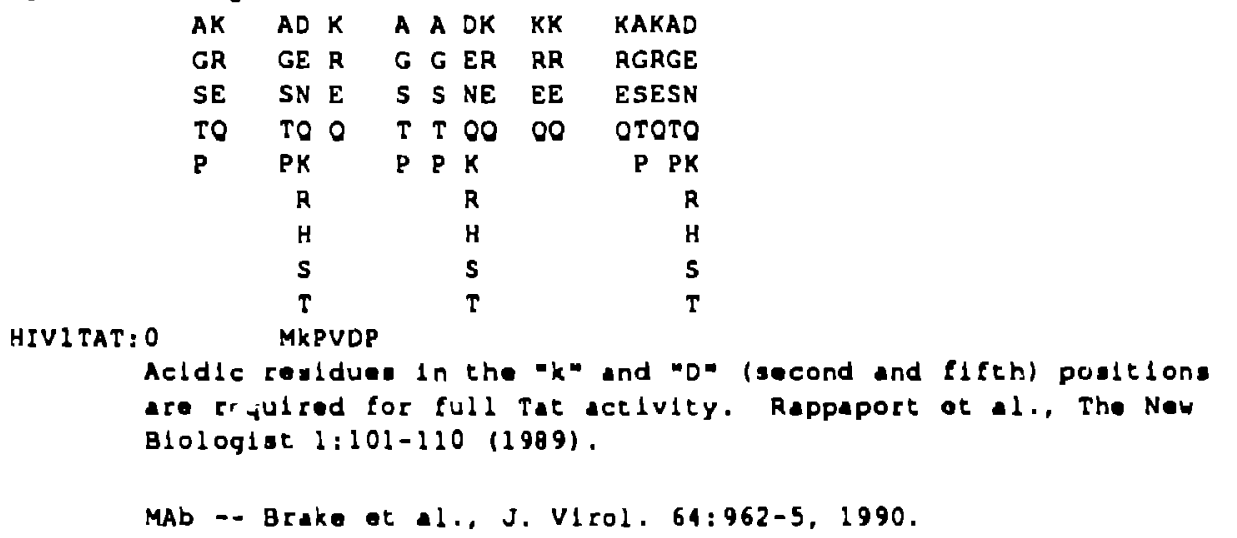

HIVITT:12 HPGSOP

HIVITAT: 40 KhLJISYGNKKR

The "GRKKR" has been Impllcated in nuclear locallzation.

Ruben ot al., J. VIrol. 63:1-8 (1989) and Hauber

et al... J. V1rol. 63:1181-87 (1989). Part of reglon III

as deflned by Green and Lowensteln, Cell:55, 1179-88, 1988 .

MAb - Brake et al., J. V1rol. 64:962-5, 1990.

Thle fragment contalne a roglon almilar to a conerved pattern

In HIV2, KGLGI". 
<- vpR eds

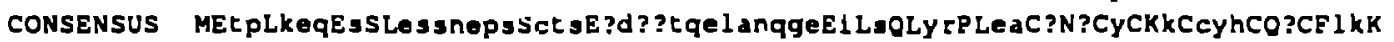
HIV2ROD --TP-KAP-S--KSCNEPE-RTS-ODVATQELARQGE-I-S--YR--ET-N-S-Y--R-CYH--M--LNHIV2NIHZ --TP-KAP-S--ESCNEPS-RTS-QDVATOELAROGE-I-S--YR--EA-T-S-Y--K-CYD--L--LOHIV2ISY --TP-KAP-S--GSYNEPS-RTS-ODVATOELANOGE-I-S--YR--ET-N-K-E--G-CFH--L--LNHIVEST --TP-KAP-G--GSYNEPS-CTS-ODAAAOGLVSPGD-I-Y--YO--EA-D-K-Y- -K-CYH-OM--LNHIV2BEN --TP-KAP-S--KPYNEPS-CTS-RDVTAOELAKOGE-L-A--HR--EP-T-K-Y--R-SEH--L--SKHIV2D1 94 -TP-KEP-S--ESYNEPS-CTS-RDVTAOERAKOGE-L-A--HR--EA-T-S-Y--Q-SYH--L--LKHIV2GH1 --TH-KAP-S--ESYNEPS-CTS-OGVTAOELAKOGE-L-5--HR--EA-T-5-Y--Q-SFH--L-LKHIV20205 P-IP-CEO-S--KSSNEPS-5TS-PVVNTOGLDNOGE-I-S--YR--KA-D-T-Y--K-CYH--L--LKSIVAL251 --TP-REO-N-OESSERS-CIL-ADATTPCSANLGE-I-S-YR--EA-Y-T-Y-OK-CYH--F--LKSIVMYP 11 - TP-REO-N--ESSNERS-CIL-ADATTPESANLGE-I-S--YR--EA-Y-T-Y- K-CYH--F--LKSIVMH1 42 --TP-REO-N--ESSNERS-YI S-MMAIPESANLGE-I-S--YR--EA-Y-T-Y--K-CYH--F--LKSIVMM239 --TP-REO-N--ESSNERS-CIS-ADASTPESANLGE-I-S--YR--EA-Y-T-Y--K-CYH--F--LKSIVMNE SIVSMMH4 SIVSMMPBJ --TP-REO-N--KSSNGRS-CTS-AAAPTLESANLEE-I -S--YR--EE-Y-T-Y--K-CYH- -F- LK--TP-KEO-N--ESCREHS-SI S-VDVPTPESANLEE-I-S--YR--EA-Y-K-Y--R-CYH--H--LK--TP-KEQ-S--ESSREHS-SIS-VDADTPESASLEE-I-S--YR--EA-Y-K-Y--R-CYH--H--LK-

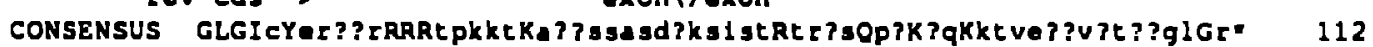
HIV2ROD ---C-ERKGR---TPKKT-THPSPTPD.KSIST-TGDS-PT-KO-KTVEATVETDTGP-R- 130 HIV2NIHZ ---W-DRKGR---TPKKT-AHPSSASD.KSIST-TRNS-PE-KO-KTLEATVETDLGL-R- 130 HIVZISY ---C-DRKGR---SPKKT-AHSSPASD.KSIST-TGNS-TE KO-KTLGTTLEADEGL-R- 130 HIV2ST ---H-ERKGR--TPKKT-AHSSSASD.KSIST-TGNS-PE-KO-KTLETALETIGGP-K- 130 HIV2BEN ----5-ERKGR---TPRKT-TPSPSAPD.KSIST-TGDS-PT-EO-KTGEATVVTTCGL-0- 130 HIV2D194 ---H-AROGR---TPRKT-THPPPASD.KSIST-TGDS-PT-KO-KTPETTVVSACGL-H- 130 HIV2GHI ---W-ARKSR---TPRKT-THSSSASD.KSIST-TGDS-PT-EO-KTTETTMVTTCSL-RHIV2D205 -.-C-DRS..---SAKRA-TTAPSAP (uxon not sequenced) 95 SIVMM251 ---C-EQSRK---TPKKA-ANTSSASN. KLIPN-TRHC-PE-AK-ETVEKAVATAPGL-R- 130 SIVMMP 11 - --C-EOSRK---TPKKA-ANTSSASN. KLIPN-TRHE-PE-AK-ETVEKAVATAPGL-R- 130 SIVMM1 42 ---5-EKSHR---TPKKA-ANTSSASN. EPIPN-IRLC-PK-AK-ETVEAAVATAPGL-R- 130 SIVMH239 ---C-EOSRK---TPKKA-ANTSSASN. KPISN-TRHC-PE-AK-ETVEKAVATAPGL-R- 130 SIVMNE ---C-EOSRR---TPKKA-ANTSSASN. KPISD-TRHC-PK-EQ-ETVENAVATAPGL-R- 130 SIVSMMH4 ---C-EO.H. --TPKKT-ANPFPASN. KSLST-ARNR-PK-EK-ETVETEVAADLGL-R- 128 SIVSMMPBJ -.-C-EQO...--TPKKT-ANTSSASD.KSLSR-ARNC-PK-EK-ETVEAEVATDLGL-R- 128 
Informacion dense stretches $(0.7$ or greater) are Indicated by "*.".

$$
c-v p R c d s
$$

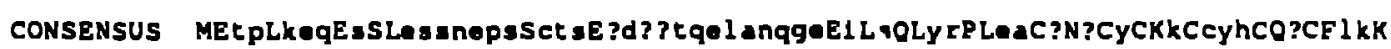

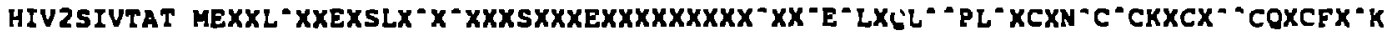

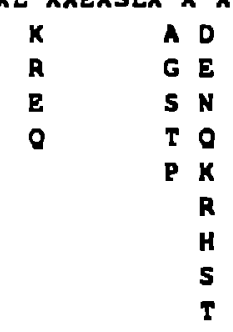

$\begin{array}{lllll}D & \text { D I } & \text { EX } & \text { K } \\ \text { E } & \text { E V } & \text { HR } & \text { P } \\ \text { N } & \text { L } & \text { YE } & \text { E } \\ \text { O } & \text { M } & \text { HO } & \text { O } \\ \text { K } & & & \\ \text { R } & & & \\ \text { H } & & & \\ \text { S } & & & \\ \text { T } & & & & \end{array}$

D
E
N
$\mathbf{O}$
$\mathbf{K}$
$\mathbf{R}$
$\mathbf{H}$
$\mathbf{S}$
$\mathbf{T}$
ED

WE YN

$\mathbf{N}$

0

H

$\mathbf{T}$

exon \/axon

ov cds $\rightarrow$

\#\#

CONSENSUS GLGICYer?\}rRRRtpkktKa?ssasdiksl stRtz?sOp?KrqKktve??v?t??glGr"

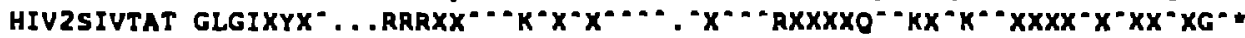

112

$\begin{array}{lr}K & \text { KKA A A NAD K IAD } \\ R & \text { RAG G G GGGN R VGE } \\ E & \text { S S S SSS E LSN } \\ 0 & \text { T T T TTT O MTO }\end{array}$

$\begin{array}{rrr}\text { AD } & \text { K } & \text { KD } \\ \text { GE } & \text { R } & \text { KE } \\ \text { SN } & \text { E } & \text { E } \\ \text { TO } & \mathbf{O} & \mathbf{O O} \\ \text { PK } & & K \\ \text { R } & & \\ H & & \\ \text { S } & & \\ \text { T } & & \end{array}$

$I A A$

$V G G E$

L $S S N$

M T $\mathrm{T} Q$

$\begin{array}{rrrrrr}\text { PK } & \text { PK } & K & \text { P } & \text { P } & \text { K } \\ \text { R } & \text { R } & \text { R } & & & \text { R }\end{array}$

$\begin{array}{llll}H & H & H & H\end{array}$

$\begin{array}{llll}\mathbf{T} & \mathbf{T} & \mathbf{S}\end{array}$ 
CONSENSUS MDKgEeeq?VShQDL 1 eqYrkPLqLCENKC?CKKCCYHCqLCFLqKGLGVtYHapRtRRKk1as I?r?P? SIVAGMTYO ---G-AEOIVSH---SEDYQK--OT-K---F--K-----QL---O----VT--AP - T- - KIRSLNLA-L SIVAGM155 ---G-EDODVSH---IKOYRK--ET-T---F--K-----OF---R----I T--AF-T---KIASADRI-V SIVAGM3 ---G-DEQGAYH---IEOLKA--KR-T---Y--C----OLL--OO---VT--AP-I ---KIAPLDRF -E

SIVAGM 677 --- , -EEPHPLL--- . H. . R. --OP-T---Y--K-.---EL---O----VR--VS-K--OTSTQDNQD-I

qqQSISE r?gRd?OTTqe? qekVEL?A? An?? ? ?RKL???? ??? ?GA??* QH----TKWG-DG---PTSOEK- -TT-GSN *

96

SIVAGMTYO

SIVAGM155

SIVAGM 3

PO---IR.G-DS---OESOKK--EO-KANLRIS----GDETRGPV--GN-

OK----TR.G-DS---OKGOEK--TS-RTAPSLG----AOOSGRAT--SD-

100

SIVAGM677

RQ--- T.VO-NG---EEGKTE--KA-AAN*

119

119 


\section{IGMTAT}

Information denge stretches (.7 or greater) are Indlcated by ***.

CONSENSUS MDKgEeeq?VshODLleqY5KPLqtCtNKC?CKKCCYHCqlCELqKGLGvtYHapRtRRKK1asl? r?P? AGMTAT

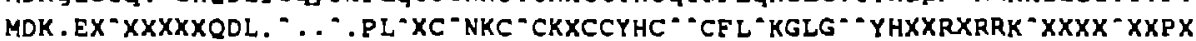

$\begin{array}{llll}D & K & K & D \\ E & R & R & E \\ \text { N } & \text { E } & \text { E } & \text { N } \\ O & O & O & O \\ \text { K } & & & K \\ \text { R } & & & \text { R } \\ \text { H } & & & \text { H } \\ \text { S } & & & \text { S } \\ \text { T } & & & \text { I }\end{array}$

$\begin{array}{rrr}\text { EI } & \text { K } & \text { ID } \\ \text { OV } & \text { R } & \text { VE } \\ \text { L } & \text { E } & \text { N } \\ \text { M } & \text { O } & \text { O } \\ \text { F } & & \text { K } \\ \text { W } & & \text { R } \\ \text { Y } & & \text { H } \\ \text { C } & & \text { S } \\ & & \text { T }\end{array}$

CONSENSUS qqQSIStr?gRd?OTTqE?qEkVEL?A?an????RKNL????????GA??" AGMTAT

\begin{tabular}{|c|c|c|c|c|c|}
\hline D & DA & \multicolumn{2}{|c|}{ DAKDK } & D & A \\
\hline E & NG & \multicolumn{2}{|c|}{ EGRER } & $\mathbf{E}$ & G \\
\hline $\mathrm{N}$ & $\mathbf{S}$ & \multicolumn{2}{|c|}{ NSENE } & $\mathbf{N}$ & $\mathbf{S}$ \\
\hline 0 & $\mathbf{T}$ & \multicolumn{2}{|c|}{$0 T 000$} & 0 & $\mathrm{~T}$ \\
\hline K & $\mathbf{P}$ & KP & $\mathbf{K}$ & K & $\mathbf{P}$ \\
\hline $\mathbf{R}$ & & $\mathbf{R}$ & $\mathbf{R}$ & $\mathbf{R}$ & \\
\hline H & & H & H & H & \\
\hline 5 & & $\mathbf{s}$ & $\mathbf{s}$ & $\mathbf{s}$ & \\
\hline$T$ & & $\mathbf{T}$ & $T$ & $T$ & \\
\hline
\end{tabular}


HIV1 REV

I/ J' if exon V/exon

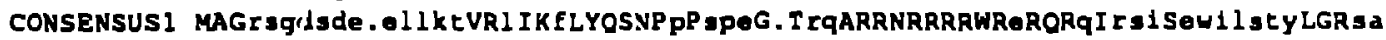
HIVLAI ---RSGDSDE. DLLKA--L--F-----P-NPE-. -RO----_----E---O-HSI-ERILSTY---SA

HIVHXB2R ---RSGDSDE. ELIRT--L--L------P-NPE-. -RO---------E---Q-HSI-ERILGTY---SA

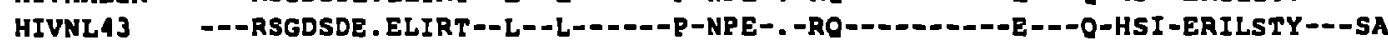

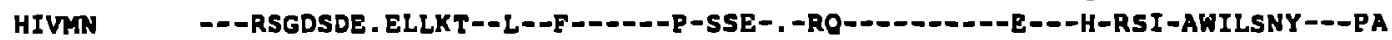

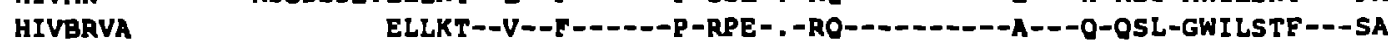

Hive

HIVJH3

---RSGDSEE . ELLKT--L--F------P-KPE-. -RQ--------E---O-RS I GWILSNY---LA

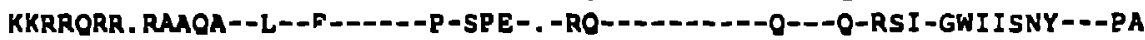

HIVBAL1

HIVJRCSF

HIVOYI

HIVSF 2

HIVNY 5CG

HIVSF 162

HIVCDC4

HIVSF 33

HIVHAN

HIVRF ---RSGDSDE. ELLKT--L--F------P-ILE-, -RO---_-_---E---O-RSI -ERILGAY---SA

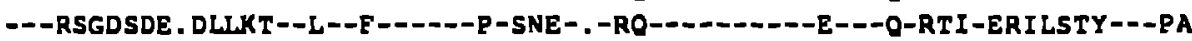

---RSGDSDE. ELLKT--L--F----..-P-NPE - . -RO---------E---OQ-RKI -GWILSTY---SA

---RSGDSDE . ELLRT- L--L--_--P-SPE-. -RO--_-_-_--E---O-RSI-GWILSTY---SA

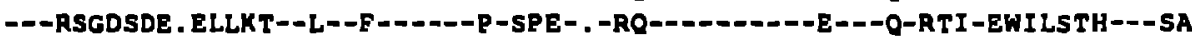

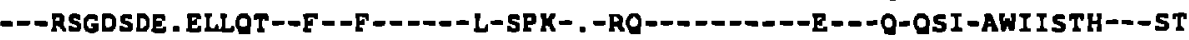

--- RSGDSDE . ELIKT--I--H------P-KPE-. -RO--------E---R-HSI -AWLLSTC---SA

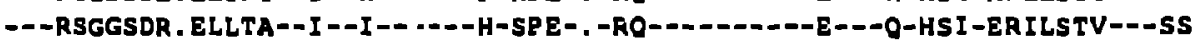

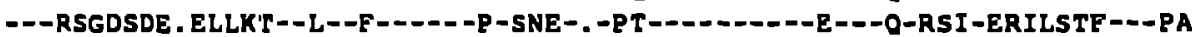

---RRGDSDE. DLLKA--L--S------P-SPE-.,-RO--_-_----E---O-RRC-EWILDTY---SV

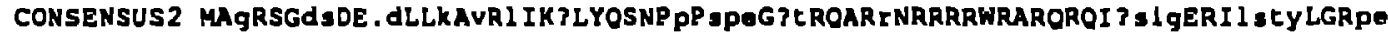

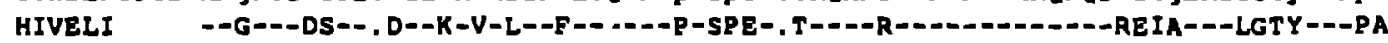

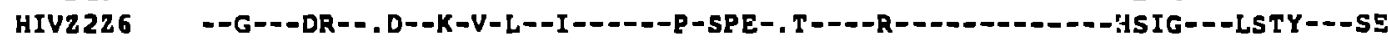

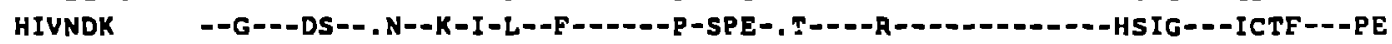

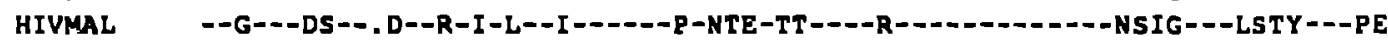

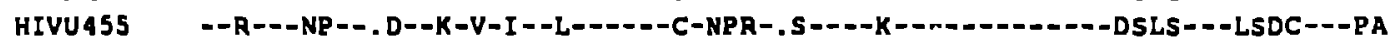

SIVCP 2

--G--EPQ-DAR--Q-VKI--I ------Y-SPE-.T-K--R--_-------K--SEISG-VLATY---PP

CONSENSUS 1 QRVPLOLPP I ORLLLDCSODCGESGTQ . . . . . 7GVGSPQLLVESP TVLEJGLKE* 115

HIVLAI E--P-----LE--T-CCNE---T--TO......--S-OI-V---TV-ES-T--- 116

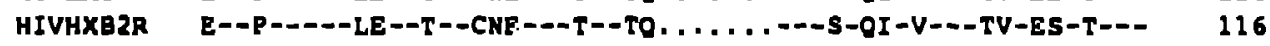

HIVNL43 E--P-.---LE--T--CNE---T--TO.......--S-OI-V---TV-ES-T--_ 116

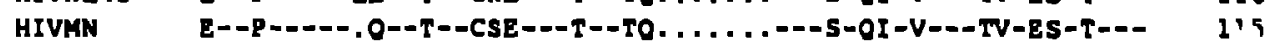

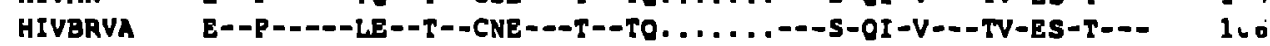

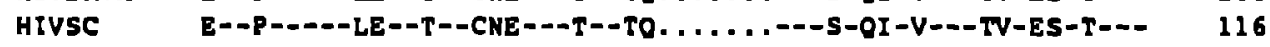

HIVJH3 E--P-D--LE-T-T-CNE--T-TO.......--S-S-OI-V-.-TI-ES-T-.. 113

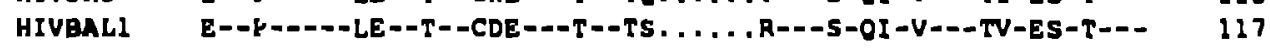

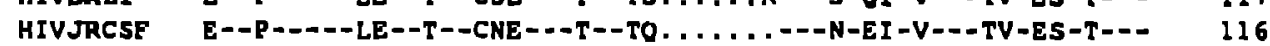

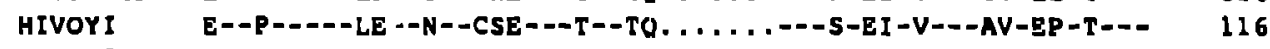

HIVSE 2 E--P-D--LE--T-CSE---N--AO......--S-OI-V---AV-DS-T-D $\quad 116$

HIVNYSCG E--P-.--LEE-T--CSE---T--TO......--S-OI-L-.-AV-GS-T-.- 116

HIVSF162 E--P-D--LE--N--CSE--T-T-TO......--S-OV-G---AV-DS-T--- 116

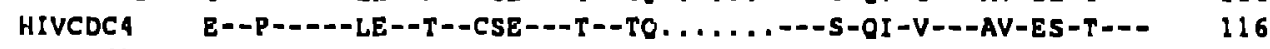

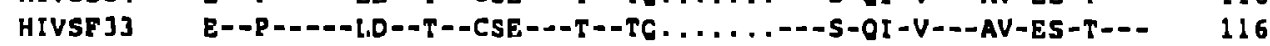

HIVHAN E--P-----LE--T--CSE---N--TO......--S-OV-V---AV-EP-T--- 116

HIVRF D--O-.---LE--T--SSE---T--TO......--S-OV-V---AV-ES-A-D 116

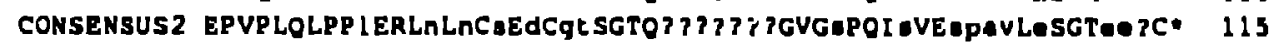

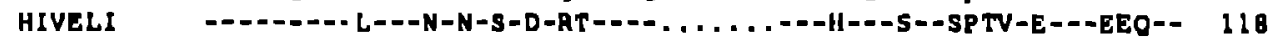

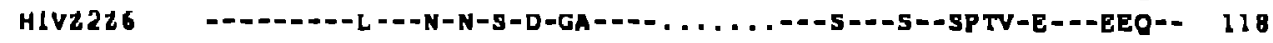

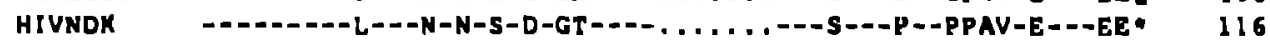

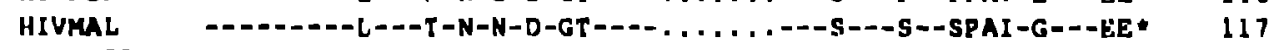

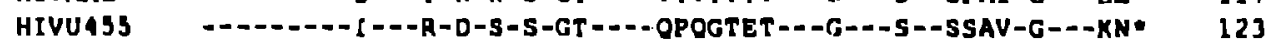

SIVCPZ K-GD-E--ELDK-S-O-V-TTODV--SNTSOPOTAT-ETVPAGCNYSI-GK-AKN* 124 
Information dense stretches 10.8 or greater) are indicated by ***.

$$
1 / 3 \text { sf exon }
$$

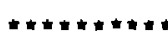

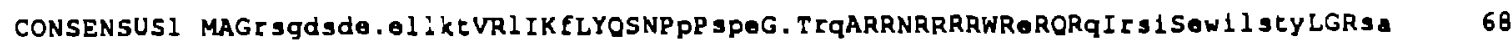

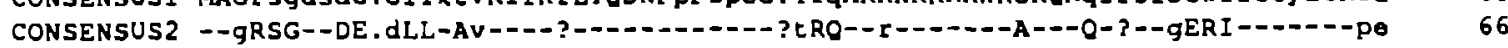

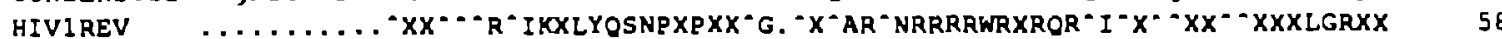

\begin{tabular}{|c|c|c|c|c|c|c|c|c|c|}
\hline$D$ & DAI & I & K & $s$ & D & K & $D$ & $D$ & IA \\
\hline$E$ & EGV & $\mathbf{V}$ & $\mathbf{R}$ & $T$ & $E$ & $\mathbf{R}$ & $E$ & $\mathbf{E}$ & VG \\
\hline $\mathbf{N}$ & NS & $L$ & $E$ & & $\mathbf{N}$ & & $\mathbf{N}$ & $\mathbf{N}$ & LS \\
\hline 0 & OT & $\mathbf{M}$ & 0 & & 0 & & 0 & 0 & $M T$ \\
\hline K & KP & $F$ & & & K & & $\mathbf{K}$ & $K$ & FP \\
\hline $\mathbf{R}$ & $\mathbf{R}$ & $\boldsymbol{w}$ & & & $\mathbf{R}$ & & $\mathbf{R}$ & R & W \\
\hline H & H & $\mathbf{Y}$ & & & H & & $\mathrm{H}$ & $\mathrm{H}$ & $\mathbf{Y}$ \\
\hline s & $\mathbf{s}$ & $c$ & & & $s$ & & $\mathbf{S}$ & $\mathbf{S}$ & C \\
\hline$T$ & $T$ & & & & $T$ & & $T$ & $T$ & \\
\hline
\end{tabular}

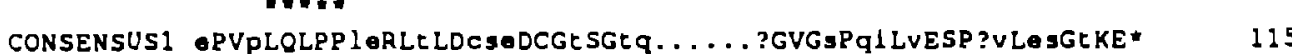

CONSENSUS2 E-P-D-----E--n-nC-Ed-g--TO??????---2-OIsV-spa---S-Tee?C 115

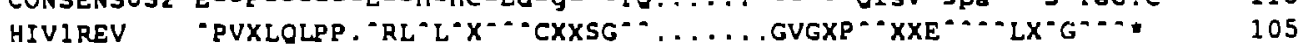

$\begin{aligned} & \text { HIVIREV: } 12 \text { "LYQSNP } \\ & \text { An Insertion proximal to the } L \text { - causes an over 100-fold }\end{aligned}$

$\begin{array}{lllllllll}D & D & D & D K D & \text { AD } & \text { EI } & \text { AAAI } & \text { A } & \text { AKD } \\ \text { E } & \text { E } & \text { N } & \text { ERE } & \text { GE } & \text { OV } & \text { GGGV } & \text { G GRE } \\ N & \text { N } & \text { NEN } & \text { SN } & & \text { SSS } & \text { S SEN } \\ \text { O } & \text { O } & \text { OOO } & \text { TO } & & \text { TTT } & \text { T 'I'O } \\ \text { K } & \text { K } & \text { K K } & \text { PK } & & \text { PPP } & \text { P } & \text { P }\end{array}$

$\begin{array}{lllll}D & \text { E E N ERE } & G E & \text { GV GGG G GRE }\end{array}$

N N NEN SN TS SS S SEN

$\begin{array}{lllllll}K & K & K & K & P K & P P P & P\end{array}$

R R R R R

H H H H H

$S \quad S \quad S \leq S$

decrease In structural geno expression. Trono et al.. Cell.

59:113-120 (1989). Mutations in the "Yo and "SN" also

cause a dramatic loos of function. Mallm et al.. Cell

58:205-214 (1989). See also Venkatesh ot al.. Virology 176:

$39-47,1990$.

HIVIREV: 28 ARTNRRRRWR

The "RFRRw" is Implicated In the localization of rev in the

nucleolus and 1 s requlred for function. Porklns ot al.. J.

Acquired Immune Deflclency Syndrome 2:256-263 (1989): Mallm

et al.. Coll 58:205-214 (1989): Venkatesh at al.. Virology 176:

39-47, 1990 .

Thlo fragment contalna a reglon simllar to a conserved pattern

In HIV2, "GtAnORRnTERR".

HIVIREV : 64 LQLE P*

-Mutatione proximal to the mpe cause a trane-domlnant

repression of Rev function. Malim at al., Cell 58:205-214 (1989).
} 
exon /exon

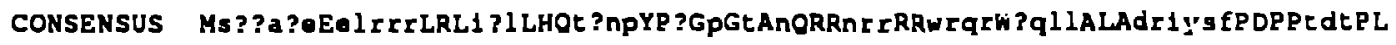
HIV2ROD -NERADE-GLQRK---IRL---T.NP--2-P-T-S---NRR--WKOR-ROIL---DSIYTF----ADS-HIV2NIHZ -TERADE-GLQRK--IRL---T.NP--O-P-T-S--NRR--WKOR-ROIL---DSIYTF----ADS-HIV2ISY -TERADE-GVRRK--OIRL--OT.NP--O-L-T-R---NRR--WERR-KOIL---DRIYTF----ADP-HIV25T HIV2BEN HIV2D1 94 HIV2GH1 SIVMA251 SIVMP11 SIVRAI 42 SIVA239 SIVINE -NERAEE-ELRRK---IRL---T. NP--Q-P-T-5---NRR--WKOR-RQLV---DKIYTF----ADS--SERADE-GLQGK---LRL---T . NP--Q-P-T-S---NRR--RRRO-LRLV---NKLCAV----TDS--RDRADE-GLOEK---IRL-- - I . NP--H-O-T-S---NRR--RRRC-FRLV---TKLHTI----TDS--HEKADG-EL QER---IRL---T . NP--H-P -T-S---NRR--ORRR-LRLV-- - DKLYTE--- -TDS--SSHERE-ELRKR---IHL---T . NS-T-P-T-N--OQRR--WRRR-GRLL---DRIYSF-_--TDT--SSHERE-ELRKR---IHL---T . NS--T-P -T-N- -ORR--WRRR-OQLL---DRIYSE--- - TDT--

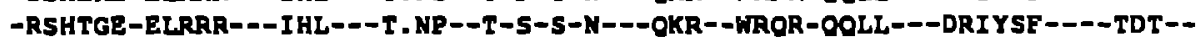
-SNHERE-ELRKR---IHL--T.NP--T-P-T-N--ODRK--WRRR-UQLL---DRIYSF----TDT--SSHAEE-ELRRR-- - IHL--T . NP-OT-P-T-N--NRR--WRQR-OQLL---DRI YSE----TNT--SST . . E-ELRRR-- - IHE-- -T. NR--Q-P-T-N---RRR--WRQR-QQIL---DRIYSF----VDT--

EOTI-H--G-T-OEL-D-CTNLPES . . . SESIDSSORL--I- 107

DRAI-H--R-T-OEL-D--TDLPES . . . . . . . NSNOGL--T- 103

DRA I-O-G-T-OEL-D-TDLPES . . . . . . . NSNOGL-T- 103

DRAI-D-A-T-HEL-D--TDLPES . . . . . . NSNOGL- -T- 103

DLAI-Q-N-A-ESI-D-ONTPEALCDPTKGSRSPOD* 107

DLAI-Q-N-A-ESI-D-OTTPEALCDPTKDSRSPOD* 107

OLAI-Q-N-A-ESI-D--TNIPEALCDPTENSRSPQA* 107

DLAI-O-N-A-ESI-D-TNTPEALCDPTEDSRSPOD* 107

DLVT -O-N-A-ESI-D-TNI PEILHDPTENPRSPOD* 107

$\begin{array}{ll}\text { OLVT -O-ON-A-ESI -D-TNI E I LHDPTENPRSPQD* } & 107 \\ \text { DLAI-Q-G-A-EEL-D--TSAPEPLNDVAK. . . SP* } & 100\end{array}$

SIVSMMPBJ OLAI-Q--R-A-EEL-N--ASAPEPLKDAAE...SP* 100 


\section{HIV2/SIV REV}

Information dense stretches $(0.7$ or greater) are Indlcated by *t*.

exon Vexon

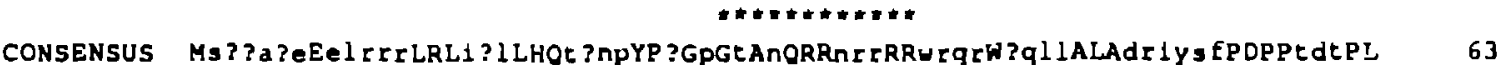

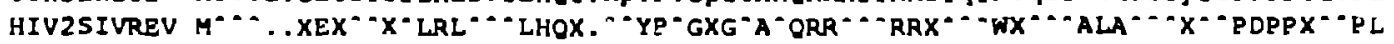

\begin{tabular}{|c|c|c|c|c|c|c|c|c|c|c|c|c|}
\hline DDD & IK K & IDI & DA & $D$ & 5 & 0 & DKK & KKK & KII & DDI & AI & DA \\
\hline$E E E$ & $v_{R} R$ & VEV & NG & 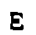 & $T$ & $E$ & ERR & RRA & RVV & EEV & $G V$ & EG \\
\hline NNN & LE & LNL & $\mathbf{s}$ & $\mathbf{N}$ & & $\mathbf{N}$ & $\mathbf{N}$ & EEE & ELL & NNL & SL & NS \\
\hline 090 & MO & MOM & $T$ & 0 & & 0 & 0 & 000 & OMM & $\infty M$ & $T M$ & OT \\
\hline KKK & & KF & P & $\mathbf{K}$ & & $K$ & K & & & KK & PF & $\mathrm{KP}$ \\
\hline RRR & & $\mathbf{R H}$ & & $\mathbf{R}$ & & $\mathbf{R}$ & $\mathbf{R}$ & & & RR & $\mathbf{W}$ & $\mathbf{R}$ \\
\hline HHH & & HY & & H & & $\mathrm{H}$ & H & & & $\mathrm{HH}$ & $\mathbf{Y}$ & $\mathrm{H}$ \\
\hline sss & & sc & & 5 & & $\mathbf{s}$ & $\mathbf{s}$ & & & SS & $c$ & $s$ \\
\hline TTT & & $T$ & & $T$ & & $T$ & $T$ & & & $\mathrm{TT}$ & & $T$ \\
\hline
\end{tabular}

CONSENSUS dlaloqLO?L?I??IPdPptn?pe?l????????s?q?IAEt"

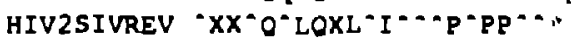

$D$ I $D$ A DDI $D$ AD

E V E G EEV N GE

N S NNL SN

O T OOH TO

K P KK PK

R RR R

H HH H

$\begin{array}{lll}S & \text { SS } & \text { S } \\ T & \text { TT } & T\end{array}$ 
CONSENSUS MpLGPEErIIIrlIafLY? SNPYPPV?EGTARQRRRARrRWrqqO?OIralaeR1??tr?eeQlVqaidq SIVAGMTYO -P--P--RREVRL-WL-YST----PSGE-_-_-_---R--ROQ-D-IRVLVE-.L. . .OE-. -YAVDR SIVAGM155 -P--P--RRLLRL-AF-YRS----SV.E--_-..----R--KNR-K-IYALAE-IWGTROED-L-QAIDQ SIVAGM 3 -P--S--RRLLRL-AF-NKN---PV.E--..----R--RQA-E-LRALAE-IWHSRVEE-L-OAIDO

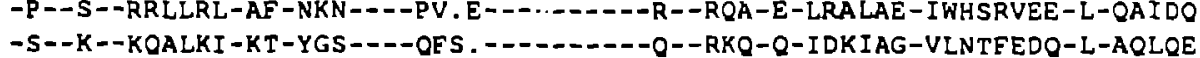

CONSENSUS LVI? ?qhL? 1OQLPDPP? Sa*
SIVAGMTYO -ADEAOH-AI-O----HSA-

SIVAGM155 -VLDTOH-VT-O-----SOA-

81

84

SIVAGM 3

-VLDOOH-AI -O-.---SSS-

89

SIVAGM 677

-OLENKD-VL-H-----HIHQDSSGIPAVWAPATPRGSNRACSSSGEGCEGS LGOTGCYCPIRLSGSHOO 


\section{AGMREV}

Information deng- screcche: 1.7 or greater) are Indicared by "**,

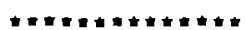

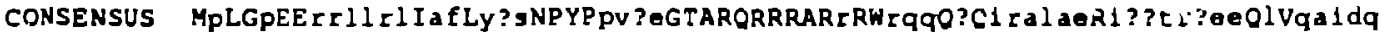

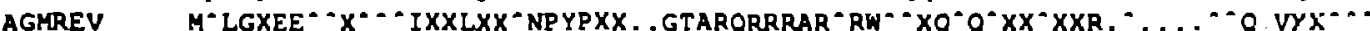

CONSENSUS LVI??qhL?10qLPDPR?sa" 81

ACMREV $\quad X X X^{*} x^{*} L X X 0^{*} L P D P P^{-} X X^{*}$

$\begin{array}{ccc}D \text { KD } & \text { D } & \text { D } \\ \text { E RE } & \text { E } & \text { E } \\ \text { EN } & \text { N } & \text { N } \\ O O & O & \text { O } \\ \text { K } & \text { K } & \text { K } \\ \text { R } & \text { R } & \text { R } \\ \text { H } & \text { H } & \text { H } \\ \text { S } & \text { S } & \text { S } \\ \text { T } & \text { T } & \text { T }\end{array}$


CONSENSUS1 Mqplqi?aIvaLVVaaI laIVWWs. Iv? ieyRkil rqr. . . kidRLIDri ?ERAEDSGNESeGdqeE. IS HIVLAT -QPIOI.A-AA---AI-IA-V--S. -VIIEY-KILRQR. . . KID--_-RLI-_-

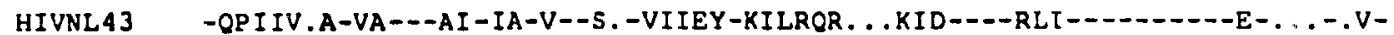
HIVMN -OPLVIAA-VA---AG-IA-V- S. -VFIEY-KIRROR. . .KID---.RIS---- HIVBRVA -OPLQISA-VA---AA-IA-V--S. -ALLEY-KLLRQR. . KID----RIR-- - - - - -E-DQE-.LHIVSC -OPLQILS-VA---AA-IA-V--S. -VEILI ENIKTK...KNRQVN NKRKSR-----E-IRK-.LHIVJH3 -QSL IAA-VA---VA-IA-V--S. -VFIEY-KILRQR. . KID----RIR----1----E-DQE-. LHIVBAL1; 2 -OALQISA-VG---AA-IA-V--T. -VFIEY-KILRQR, . KID----RIT---- - - -D-DQE-.LHIVSF 2 -QSLOILA-VS-D-VA-IA-V--T. -VLIEY-KILRORK. T.D --E EKKO.KTVAMKVK-TRRN. YO HIVJRCSF -QRLQILA-VA-- AG-IA-I--S, -VLIEY-KILRQR . . KID----KIR-- K HIVNYSCG -LSE.. ......... NSC-DH.SVHRIQESI.KTKK. SRODN. NKRKSRROWQ -RR-PGRI.IHIVSF162 -QPLQILA-VA- -AA-IA-V--T. -VYIEY-KILRQR . . KID----RIT-- 162 HIVHAN -LSLOIAA-VA---AA-IV-V--T. -VVIEY-KLLRQR. . . KIN----RLT--HIVRF -OSLEILA-VA---AA-LA-V--T. -VGIEI-KTLROKKK T.G --E EK-Q.KTVAMRVMEMRKN.CO CONSENSUS 2 mQP IVI IAI AALVVA I I IAIVVWT. IVEIEYRIIKrQR. . kIDCL1DRI IERAEDSGNESEGdrEE. LS

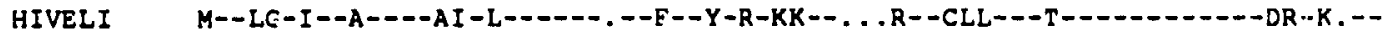

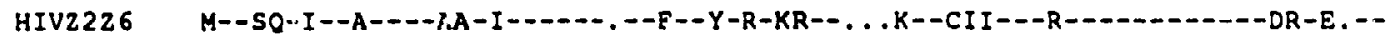

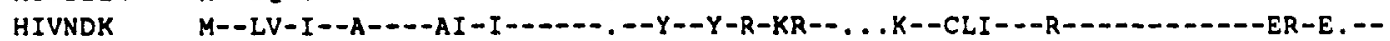

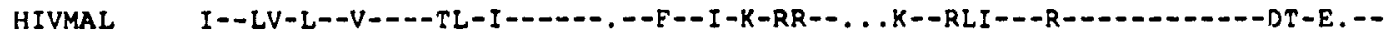
SIVCPZ MTLLVGL.VLI--GLIAWN-CI-GY-IKWGY-RYKRH-LETE-ERLNLILR---D-D-N-EE-ER-E

CONSENSUSI AL????VEm?GH?A.PWdIdDI? 77

HIVLAI --VEMGVEM, - -H-.,WDID-L* 81

HIVNL43 --VEMGVEM. $3-H-$. WDID-L* 81

HIVMN $\quad-\ldots$ VGM.--D-. -WVIN-L? 81

HIVBRVA $\quad-\ldots$ VEMG-D-. -WDID-L 82

HIVSC $\quad-\ldots$ VEM. $--D-,-G D: D-L$ ? 79

HIVJH3 $3 \ldots$...VEM. - -D-.-WDID-L 80

HIVBAL1:2 -....VEM. - -H-.,WDIN-L* 81

HIVSF 2 H-WRWGTLLL-MLMI?

HIVJRCSF $\quad-\ldots$..VER. - -L-, -WDIN-L* 81

HIVNY 5CG TCGDGARCSL-C S? 62

HIVSF 162 - ....VVER. - L-. -WOVD-L* 81

HIVHAN $\quad-\ldots$..VER.--L-. WDVN - ?

HIVRF H-WKWGTMLL-MLMI?

CONSENSUS 2 KL. . . VEM.GHAA.PWOTDDL * 80

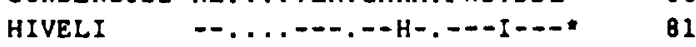

HIVZ2Z6 -.....-...-H-.,-I--- 82

HIVNDK $\quad-\ldots \ldots, \ldots,-H-, \ldots,-\ldots, 81$

HIVMAL $\quad-\ldots \ldots-\ldots,-D-. .--V-\ldots+81$

SIVCP $Z \quad Q-I H, N Y N H N N-F-N-M, F . \cdots \quad 88$ 
Information dense stretches 10.8 or greaterl are indlcated by ***.

env cds $\rightarrow$

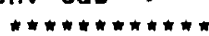

CONSENSUS1 Mqplq1?aIVaLVVaaI 1 aIVVWs.Iv? 1eyRk11 rqr...k1dRLIDI1?ERAEDSGNESEGdqeE. IS

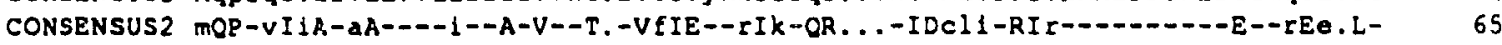

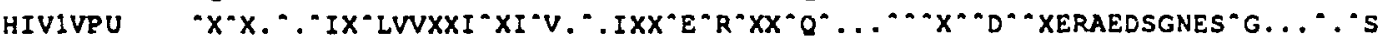

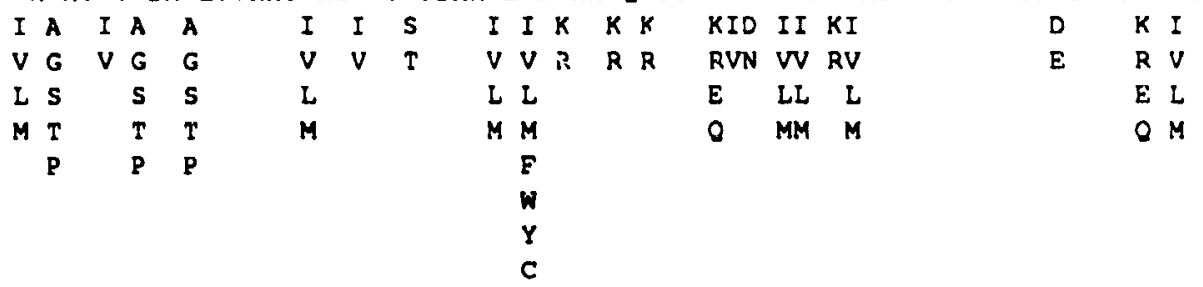

CONSENSUS1 AL????VEm?GH?A.PWdIdDI* 77

CONSENSUS $2 \mathrm{~K}-\ldots$...VEM. - - h-. -WDPD-L" 80

HIVIVPU XL.... XX.GHXA,PXX"DX* 75

ID

VN

HIVIVPU:49 ERAEOSGNESKG

See Strebel et al., science 241: 1221-4, 1988. 
HIVI ENV

c- upU cds

Ignal portide/gpl20

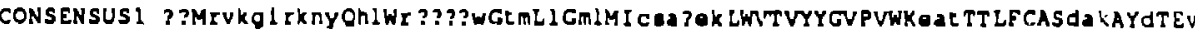

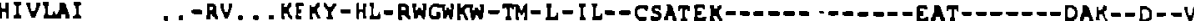

HIVHXB2R $\because-R V$. KEKY-HL-RKGWRW-TM-L-ML-CSATEK-_-

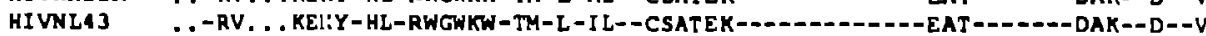

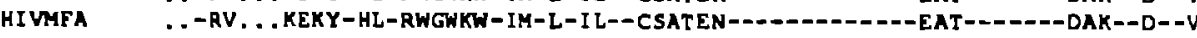

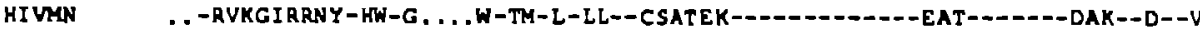

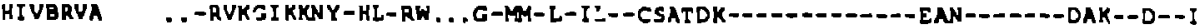

HTVC

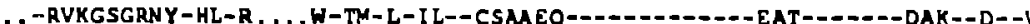

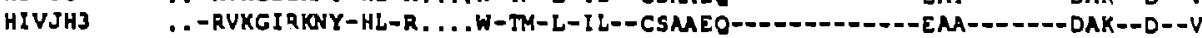

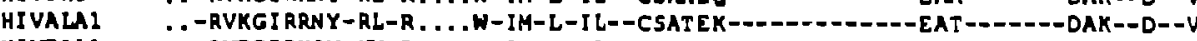

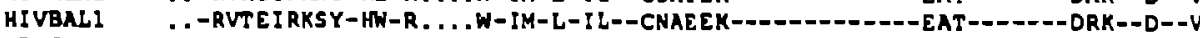

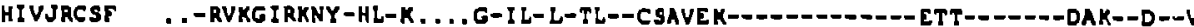

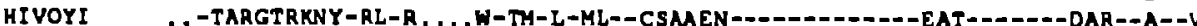

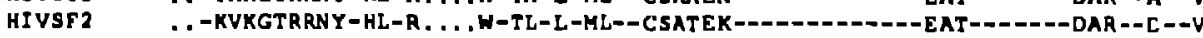

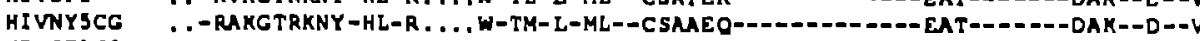

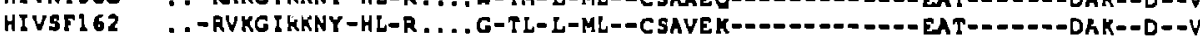

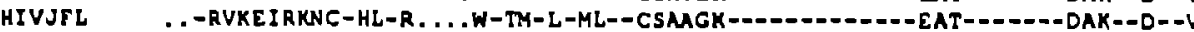

HI VCDCA MA-RAKGIRKNC-HL-R. ...W-TH-L-ML--CSMAAN-_-_-_.

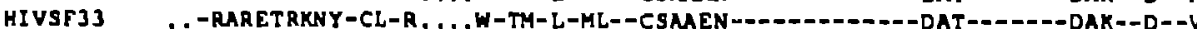

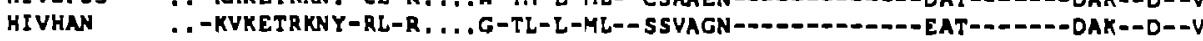

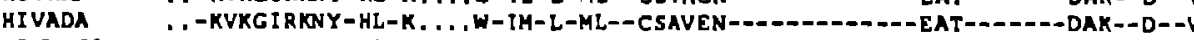

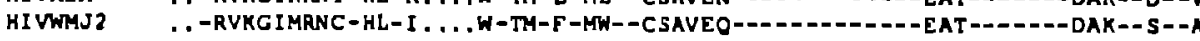

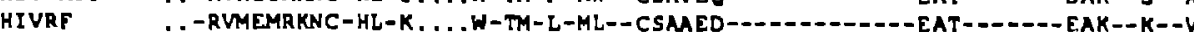

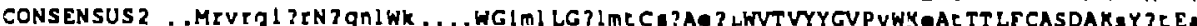

HIVELI .. -RARGIER-CONW-K.... --IML- I UMT-SA-DH-_-

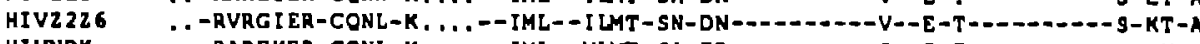

HIVNDK _. -RAREKER-CONL-K.... - IML- -MUMT-SA-ED-_-

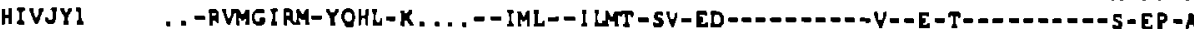

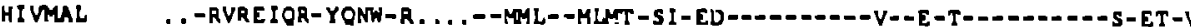

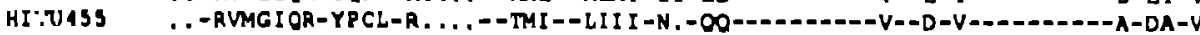

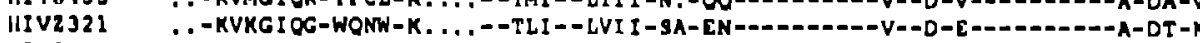

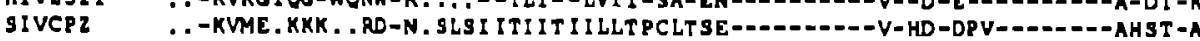

63

65

65

65

64

65

64

64

64

64

64

64

64

64

64

64

58

64

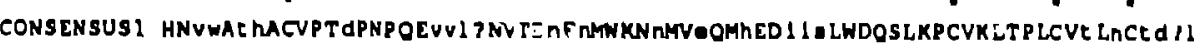

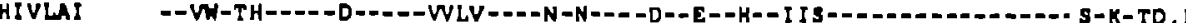

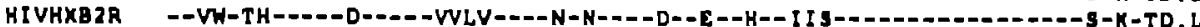

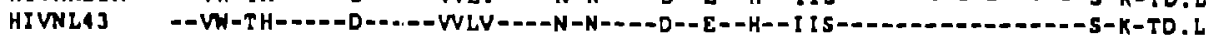

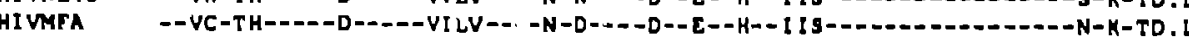

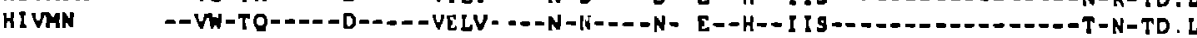

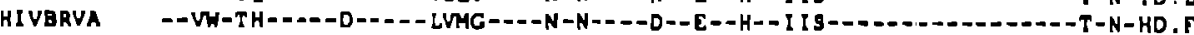

HIVSC

HIV

HIVALAI

HIVBALI

IIIVJRCSF

HIVOYI

HIVBT

HIVNYSCG

IIVSFI 62

HIVJTL

HIVCDCA

HIVSTJ J

HIVIIAN

HIVADA

HIVNMJ 2

HIVRT

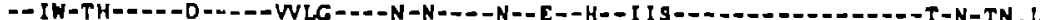

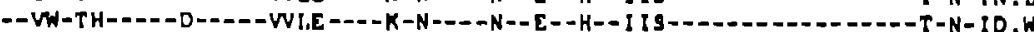

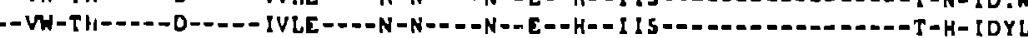

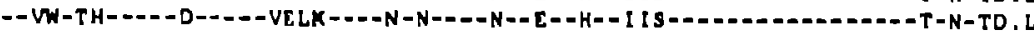

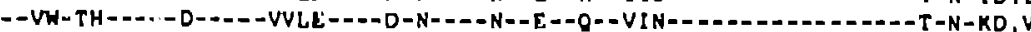

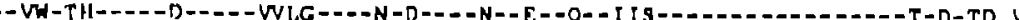

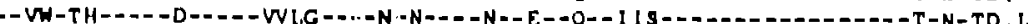

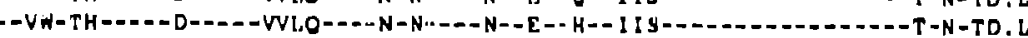

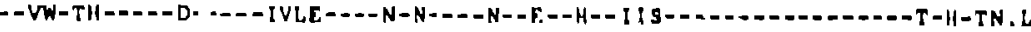

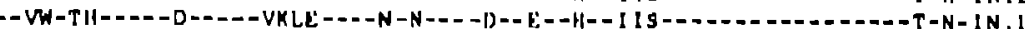

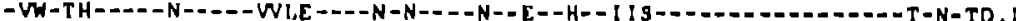

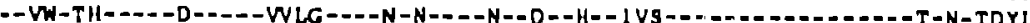

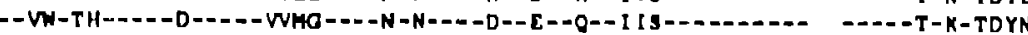

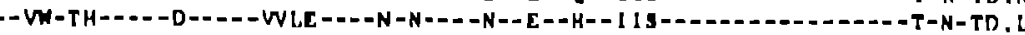

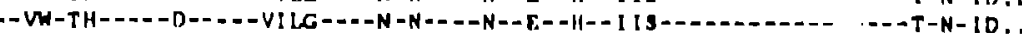

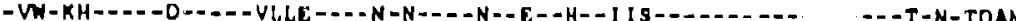

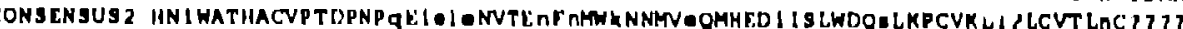

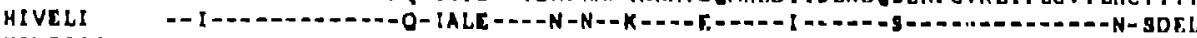

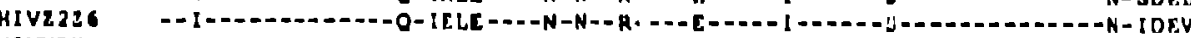

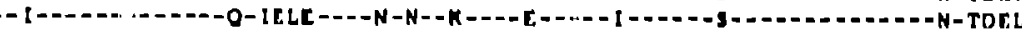

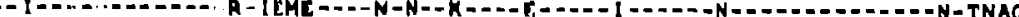

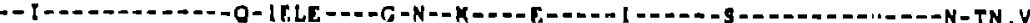

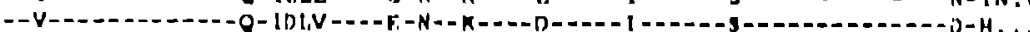

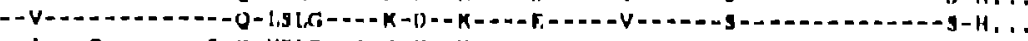

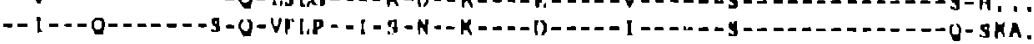

131

134

134

134

134

133

133

134

13]

3

13

133

נ3

is

1.34
1.14

[1]

132

134

124

134

134

134

111

10

111
111

11.77 
HIV 1 ENV

$$
\text { ....... }
$$

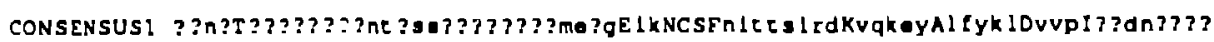
HIVLAI G.NA-NTNSS...NTNSSS... GEMMMEKG-IK----NISTSIRG-VQKEY-FFYKL-IIP-. . DN.... HIVHXB $2 R$ K.ND-........ NTNSSS... .GRMIMEKG-IK---NISTSIRG-VOKEY-FFYKL-IIP-..DN....

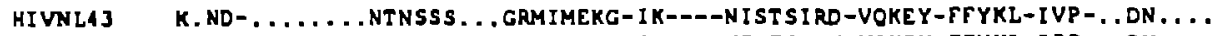
HIVMFA K.ND-........ NTNSSN. . .GRMIMEKG-IK--_-NISTSIRN-VOKEY-FFYKL-IRP-..DN .... HIVMN R.NT-NTNNS.TANNNSNS. .EGT..IKGG-MK----NITTSIRD-HOKEY-LLYKL-IVS-..DN . . . HIVBRVA ..NA-.........NATSNS...GK. MYMEGG-MK---NITTSIRD-MOKEY-LFYKL-IVP-.. DND... HIVSC $\quad$ R.ND-STNAT...NTTSSN...RG. OMEGG-MT---NITTSIRS-VOKEY-LFYKL-VVP-..DN.... HIVJH3 G.ND-SPNAT. ...NTTSSG...GE. MMEKG-MY----NITTSIRD-VOKEH-LFYKH-VVP-.. NNSTKD HIVALAI ..NA- .........NTTSSN. ..GT. KMERG-IK--- -NITTSI RD-MQKES-LFHKL-VVP-.. DN.... HIVBALI R.NA-NGNOT . . . NTTSSS. . RG.MVGGG-MK---NITTNIRG-VOKEY-LFYKL-IAP-. . DN . . . .

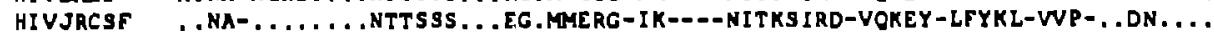
HIVOYI HIVSF2 G.KA-.........NTNSSN. .. WKEEI.KG-IK-..-NITTSIRD-IOKEN-LFRNL-VVP-..DNAST. HJVNYSCG T.NA-.Y....A.NGSSE.........ERG-IR----NVTTIIRN-IOKEY-LFYRL-IVP-..DK.... HIVST162 K.NA-.........NTKSSN...WKE. HDRG-IK----KVTTSIRN-HOKEY-LFYKL-WVP-..DN.... HIVJFL K, NV-.....NI.NNS .......WGK, HEEG-IK----NTTTSIKN-MOREY-LFYKL-VVP-.. DK.... HIVCDCA NTNN-T........NTTELSI IVWNEQRGKG-MR-_-_NITTSIRD-VQREY-LFYKL-VEP-.DDN .... HIVSF?3 G.NA- ........NTNNSS. . . GGTVEKE-IK-_-NITTGIRD-VOKAY-YFYKL-VVP-DDDN.... HIVHAN $\quad$. NA-.........NSS....WGR.MEKG-IO---KVTTNIRD-VOKES-LFYKT-VVP-..DNNKT.

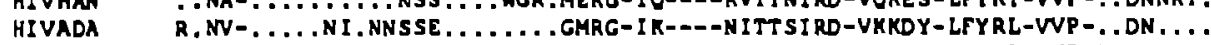
HIVWHJ 2 K. NI-DW. . . . KNTT ........... II GGG-VK----NITTSRRD-VHKEY-LFYKL-VVP-KGDN.... HIVRF L.NG-.........NVTSSS... GGTMMENG-IK----QVTT SRRO-TOKKY-LFYKL-VVP-EKGNISPK

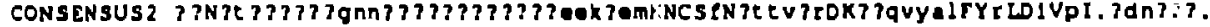
HIVELI RN-GT.M.... GNNVTT.........EEKG.M-..-F-VTTVLK--KOOVYAL--A-EI-P-..DNDSS. HIVZ226 ME-VT,M KNNNVT ........ EEIR,M-D-F-ITTVVR--TKOVHAL-R-DI-P-..DNDNS. HIVNDK R.-.S....KGN.......GKVEEEEKR...--F-V....R--REOVYAL--K-I-P-..DNHNR. HIVJY1 G.-KT....TNGNATTNO.... EEOMMEKGEH--.-F-ITTVIS--KKOVHAL--R--V-P-..DDDNS.

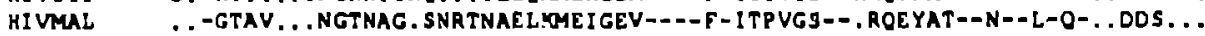

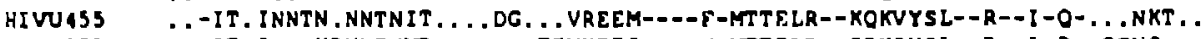

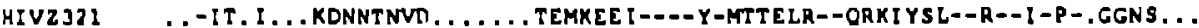
SIVCPZ $\quad$. -TS. . OAKNLTNOTSS.........PPLEM-_-F-VTTELR--KKOVYSL-VR-V-NL.. GNE...

pept lde $T$ reglon

$$
\text { -... }
$$

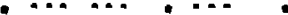

CONSENSUS I ? ? ? ? ?

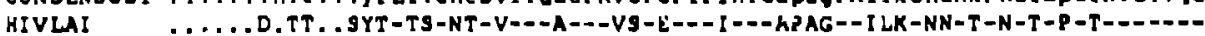

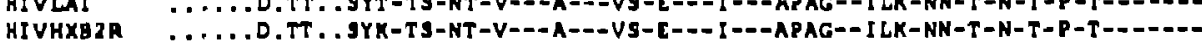

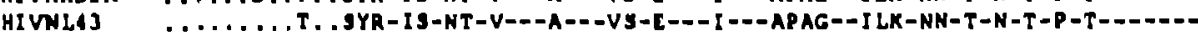

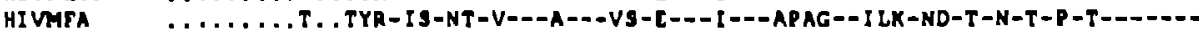
HIVAN HIVBRVA

HIVBRYA

HIVSC

HIVJH3
HIVALAI

HIVBAL!

HIVJaCSF

HIVOYI

HIVSF?

HIVNYSCG

HIVSFI 62

HIVJrL

HIVCDCA

Hiverg

HIVHAN

HIVADA

HIVWMJ?

HIVRT

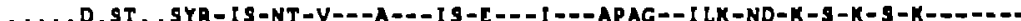

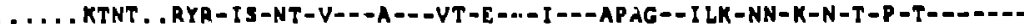

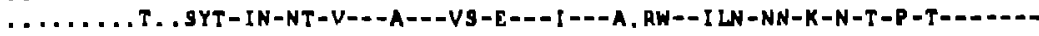

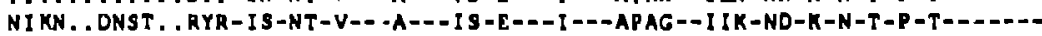

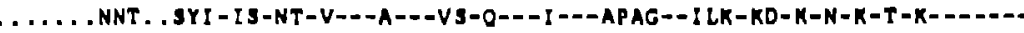

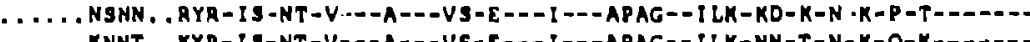

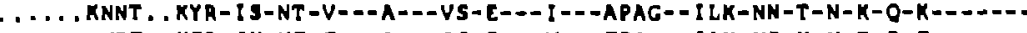

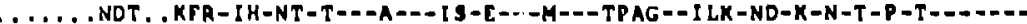

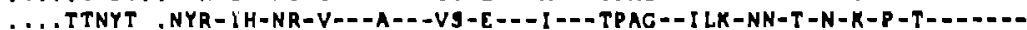

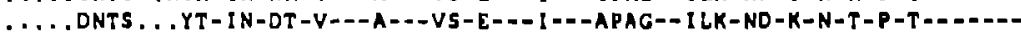

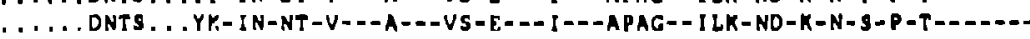

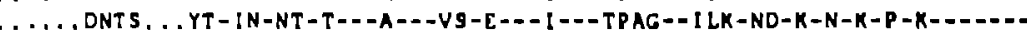

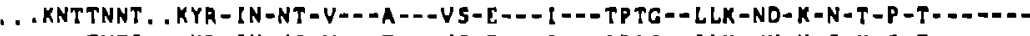

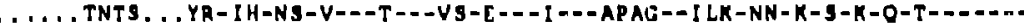

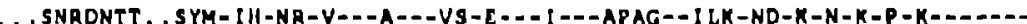

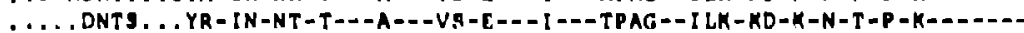

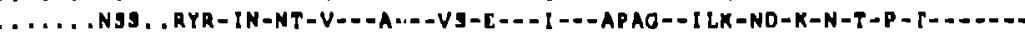

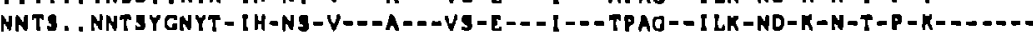

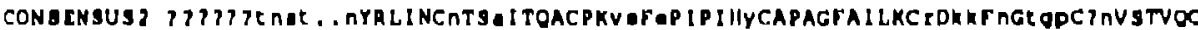

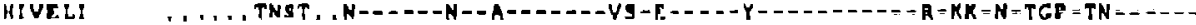

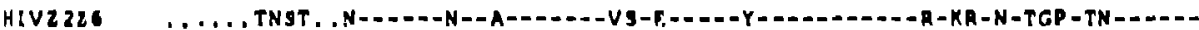
HIVJYI HIVMAL HIVU435 IIIVZ32I IIVCP2

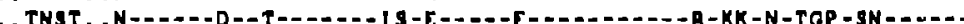

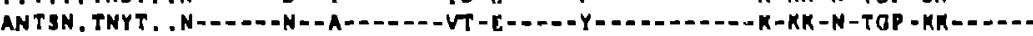

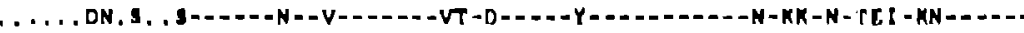

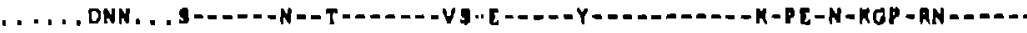

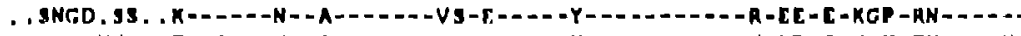

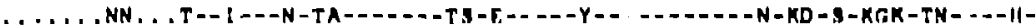


HIV1 ENV

... $\quad \ldots \quad \ldots \quad \ldots \quad$ v3 neucrallzation loop

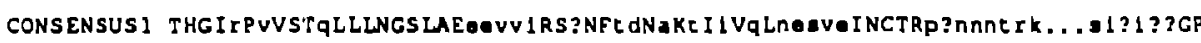
HIVLAI ---R-V---0-HIVHXB2R ----R-V---O---------EEVVI--V-OTD-A-T-I-O-NTSVE--.---P. NNNTRK. . RIRIQR-HIVNL43 ----R-V---O--..-----EDVVI --A--TD-A-T-I-Q-NT SVE--.--P. NNNTRK... SIRI QR HIVArA HIVMN

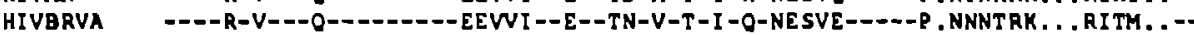
HIVSC HIVJHJ HIVALA1 HIVBALI HIVJRCSF

HIVOYI

HIVST2

HIVNYSCG

HIVSF1 62

HIVJFL

HIVCDCA

HIVSFJ3

HIVHAN

HIVADA

HIVIMJ 2

HIVRF

CONSENSUS

HIVELI

HIVZ226

HIVNDK

HIVJYI

HIVMAL

HIVUA5s

HIVZ321

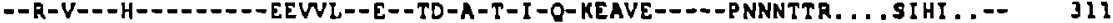
----K-V---O---------EEVVI --E--TD-A-T-I I-O-KEPVV---.-P. SKTTRR ...RIHI ...- 323

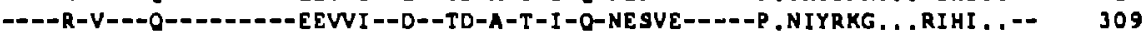
----R-V---O---------EEWVI--A--AD-A-V-I-O-NESVE-----P.NNNTRK...SIHI..-- 315

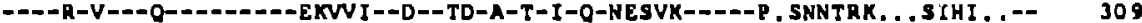

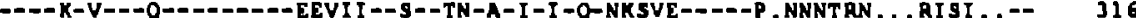

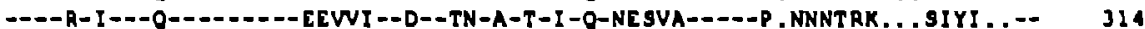

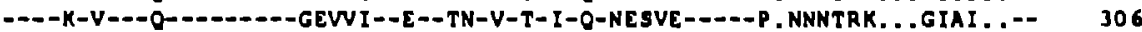

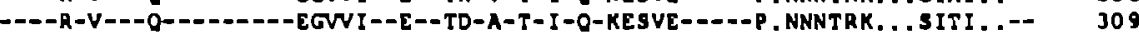

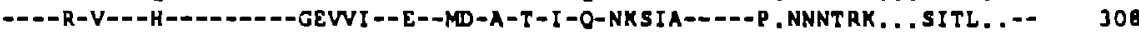

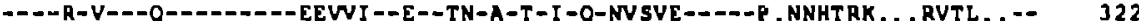

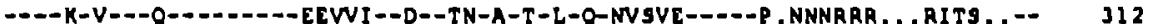

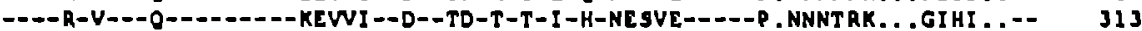

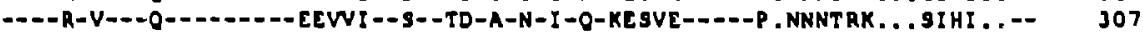
$---R-V---0--------E E I V I--E--T D-A-T-I-H-N E S V E--.--P . Y N N V R R . . . S L S I . .--300$

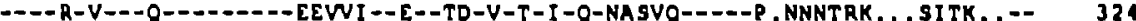

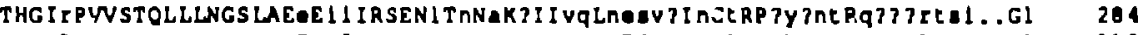

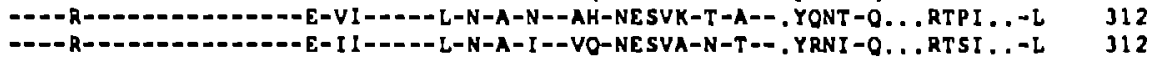
-

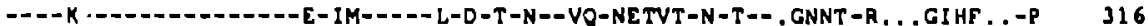
--K----

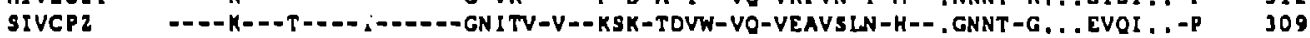

V3 neutrallation loop - s

$$
\text { -... } \quad \cdots \quad \quad \cdots
$$

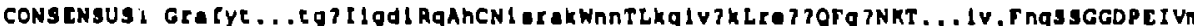

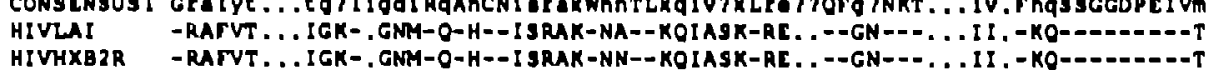

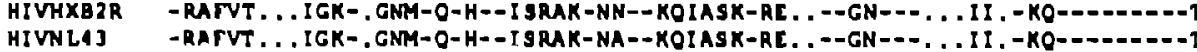

HIVMFA -RAFVT . . . IGK-, GNM-O-H--ISRAK-MS--KOIASK-RE. . --GN---, , . VI, -KO---.------T

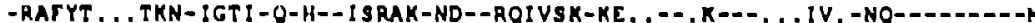
-RVYYT... TGO-IGDT-R-H- -LSRSK- CN- KOIVTK-HV... K

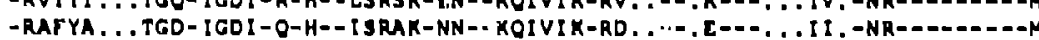
-RAFYT ... TKO-AGDL-O-H--INRAR-NA--KOIVGK-RK, . --.V-.-....IV, -NR-.-.....-M

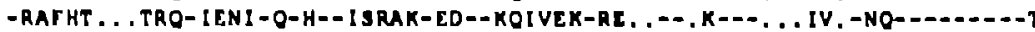

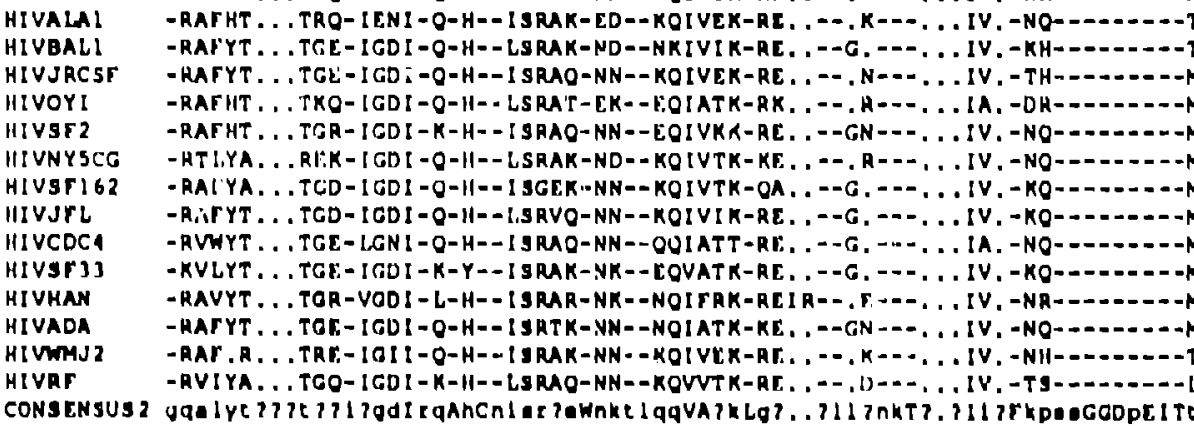

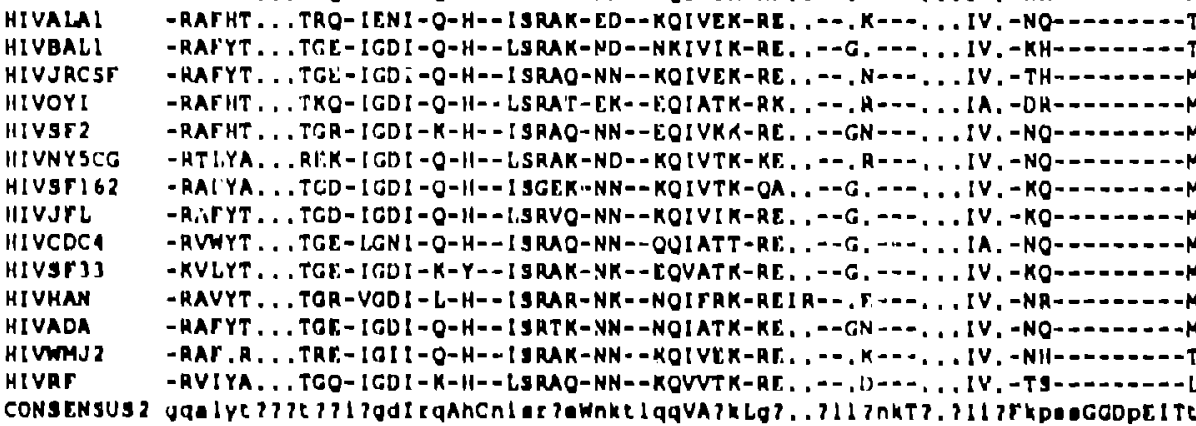

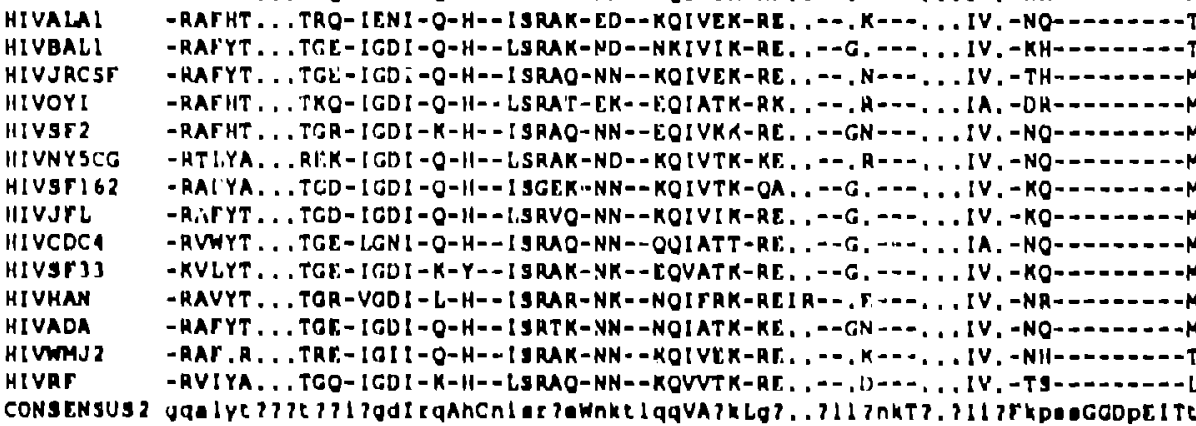

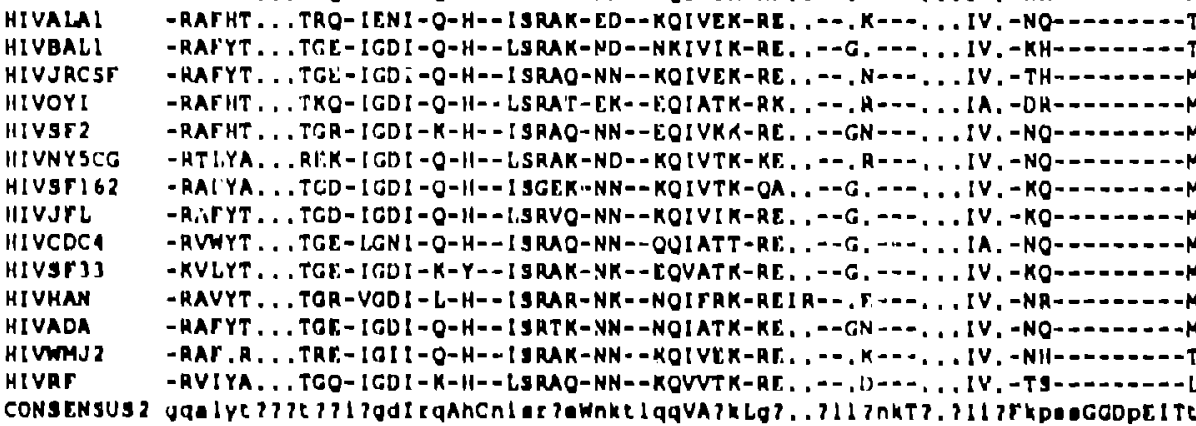

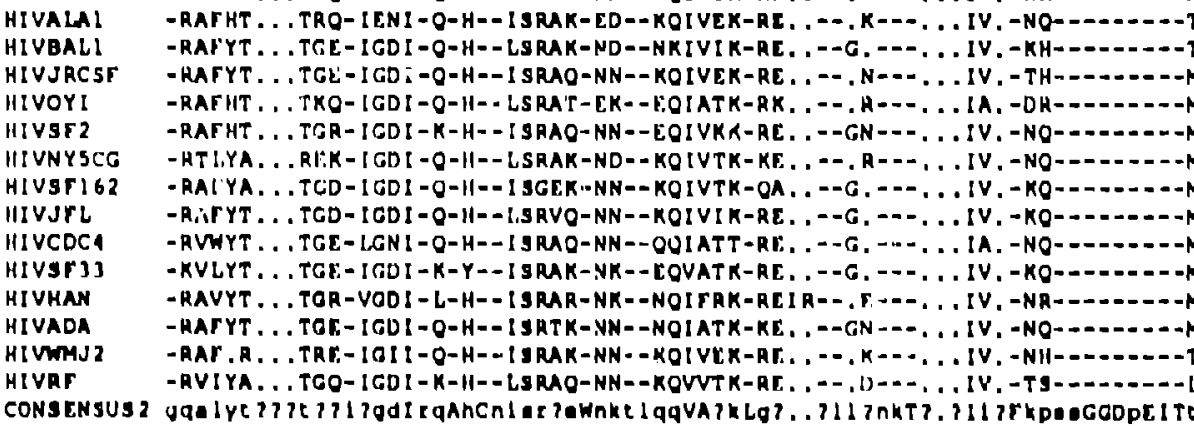

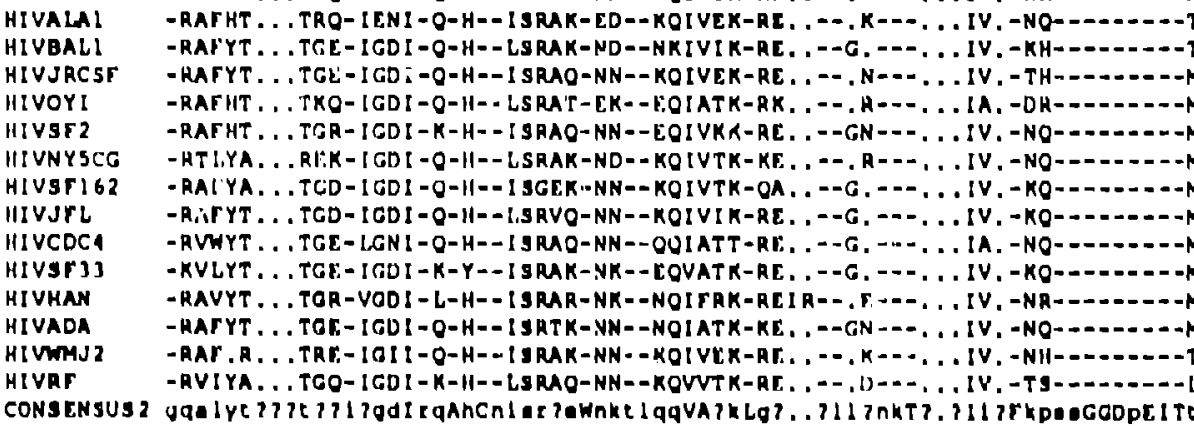

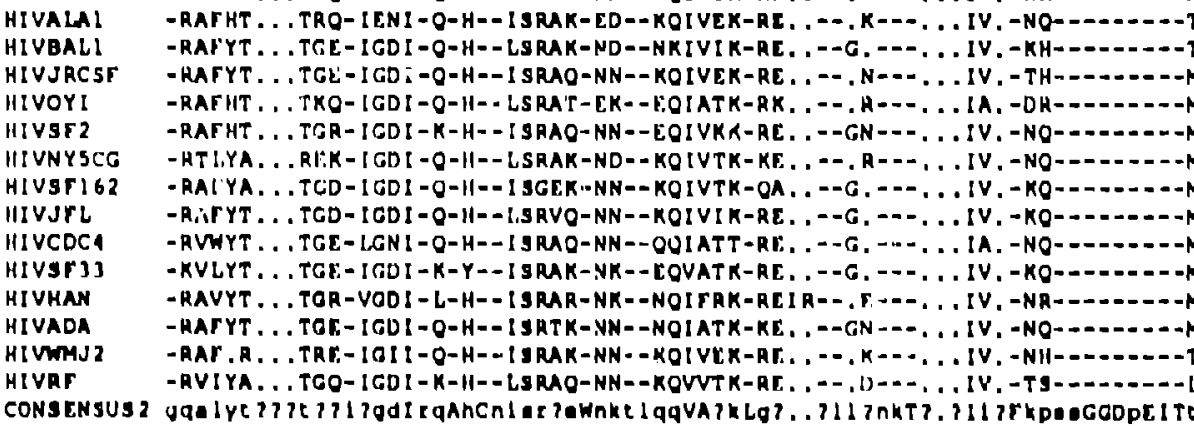

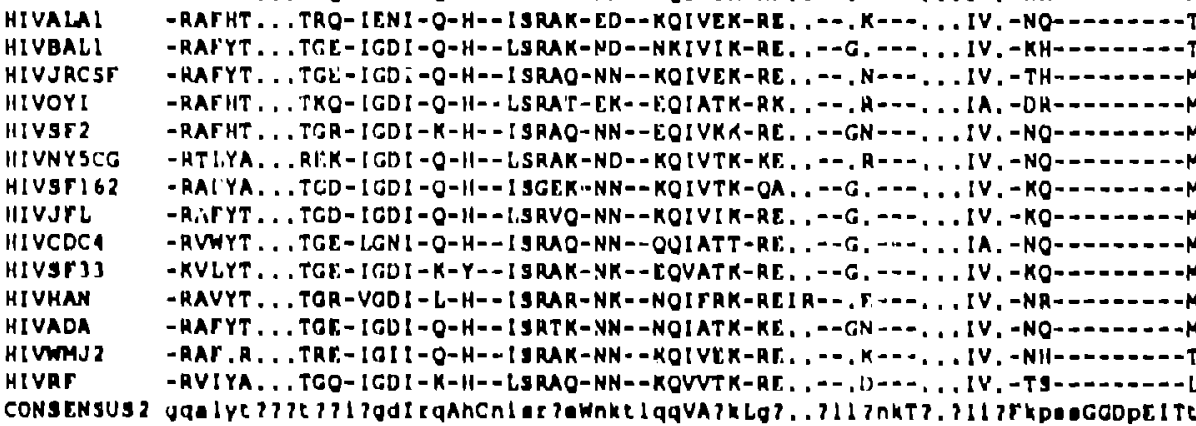

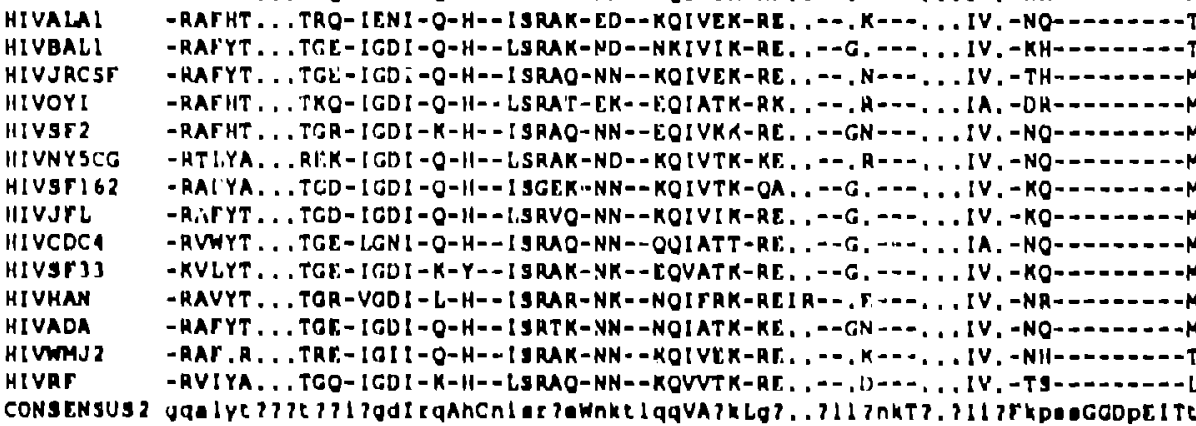

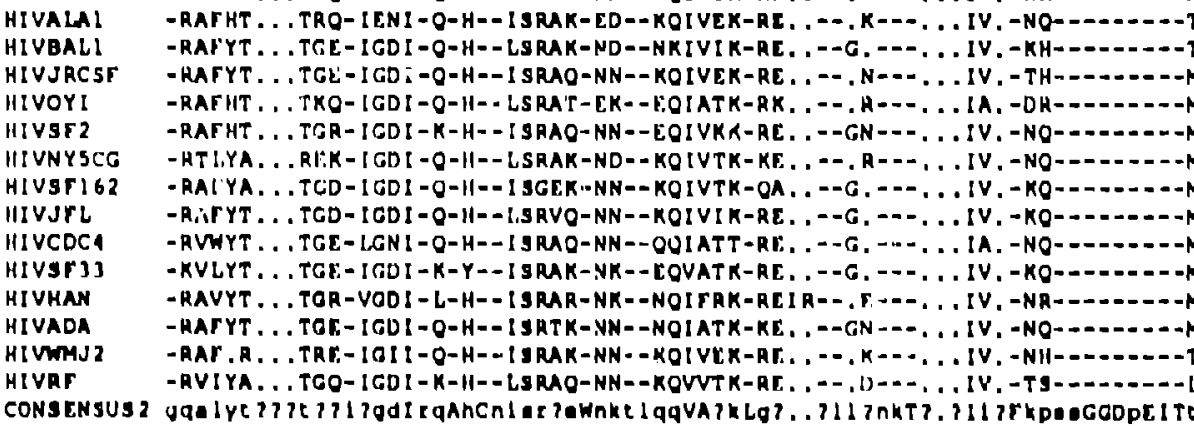

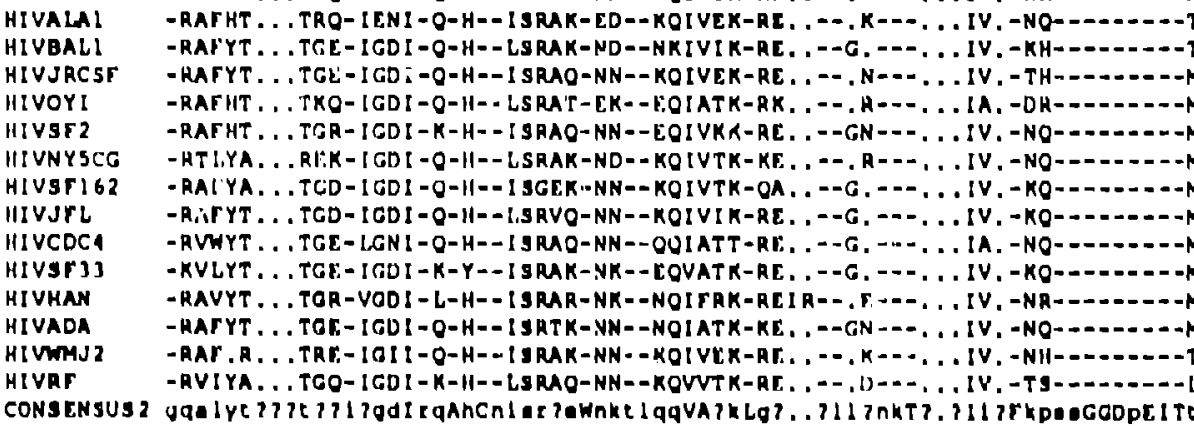

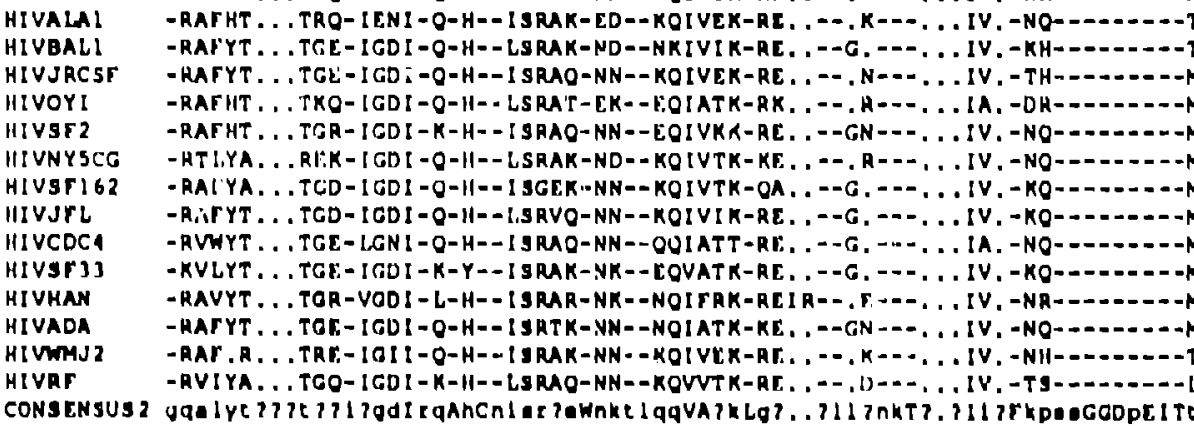

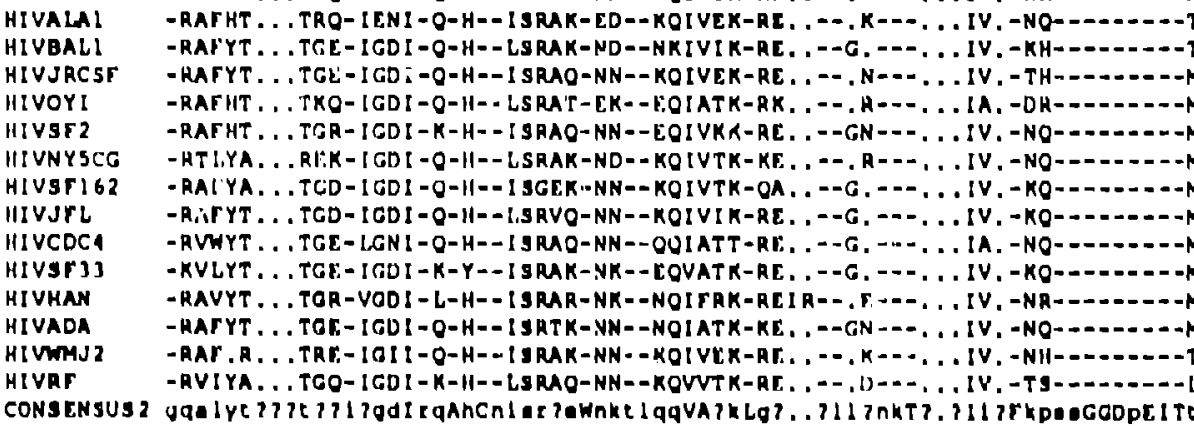

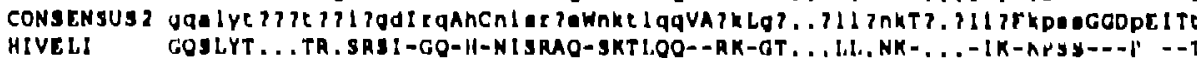

hiVHOK

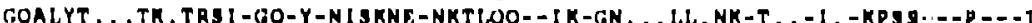

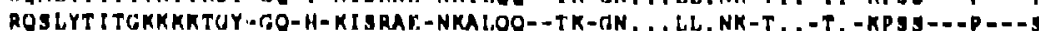

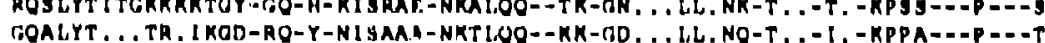

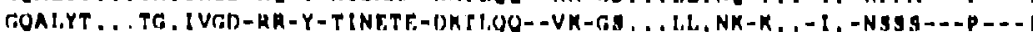

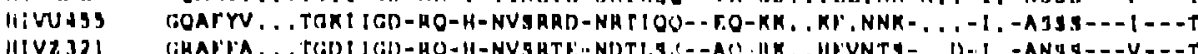

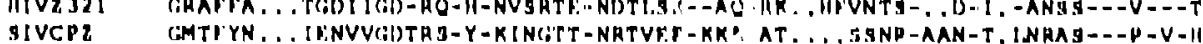

392 
HIVI ENV

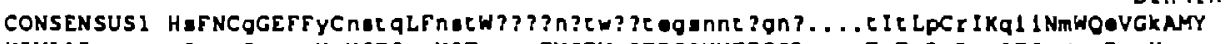

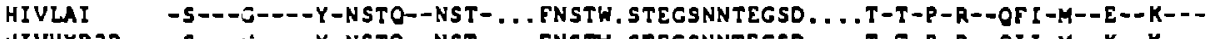
HIVHXB2R -S---G----Y-NSTO--NST- . . FNSTW. STEGSNNTEGSD. . . T-T-P-R--OII-M--K- -K--

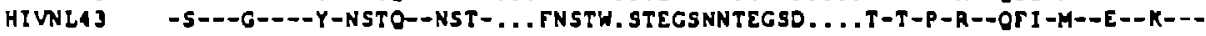
Hivata HIVMN -

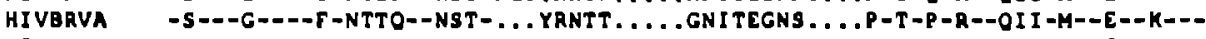
HIVSC HIVJH3 HIVAIAI HIVBALI HIVJRCSF HIVOYI HIVSF? HIVNYSCG HIVSFI 62 HIVJFL HIVCDCA HIVSFJ J HIVHAN

HIVADA

HIVIMJ 2 HIVRF NSTWNDTEGSN NSTE . GPKGSNNTEEN. . . -S-O-G----Y-NSTO-NST- . . . .NVT . . . EESNNTVENN . . . .T-T-P-R--OII M-M-E-E-R--.

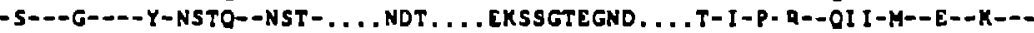

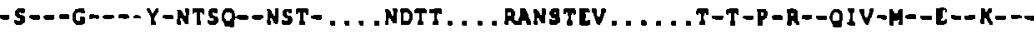

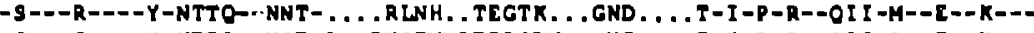

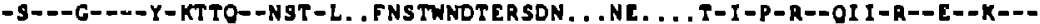

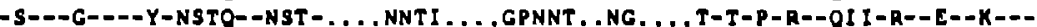

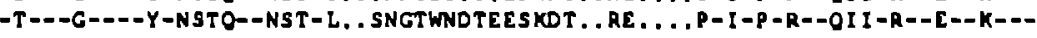
-S-O-G-D--Y-NSTO-NSA-NVT SNGTWSVT, RKOKDTGD. . . . I-T-P-R--OI I-R--V--K---S---R-D-Y-NTTK--NST-NE. . NSTWNAT . GND. . . . . . . TT-T-P-R-OII-M-

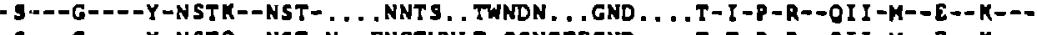
-S---G-.--Y-NSTO-NST-N. . FNGTWNLT. OSNGTEGND. . . .T-T-P-R--OII-M-OE-OK---9---G---YY-NSTO-NST- . . . . NGT . DIR, GDNKN, . ST . . . . L-T-P-R--OI I-M--G-OK-.-

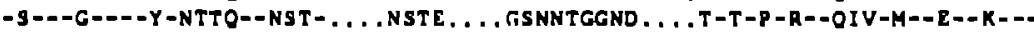

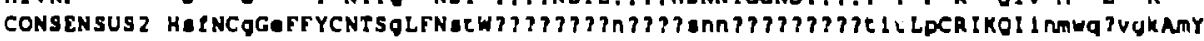

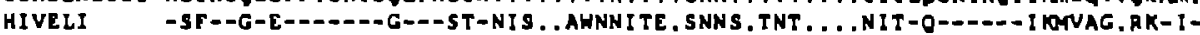

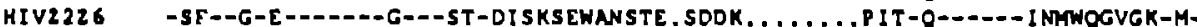
HIVNDK

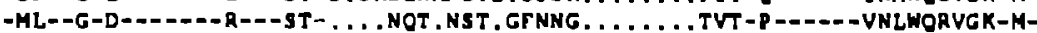

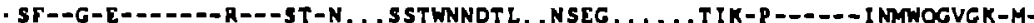

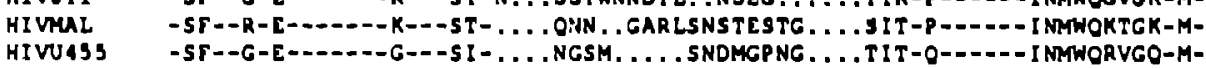
HIVU4SS
HIVIJ2!

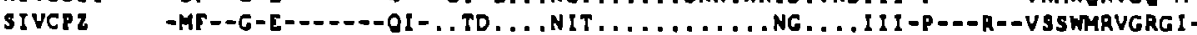

$$
\text { - } \cdots
$$

$$
\text { ... }
$$

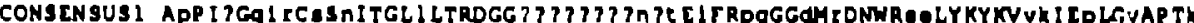

HIVLAI HIVHX82R HIVWLA3

HIVAFA

HIVMN

HIVBRVA

HIVIC

HIVJHJ

[IIVALA]

HIVBALI

HIVJRCSF

HIVOY!

HIVST2

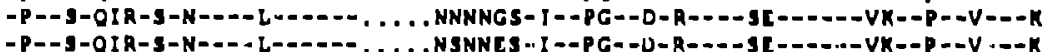

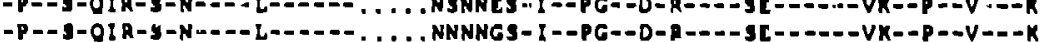

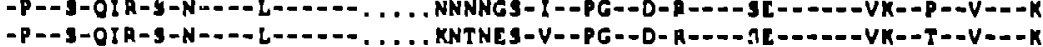

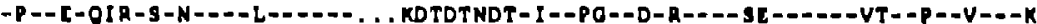

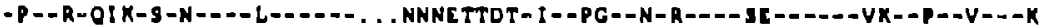

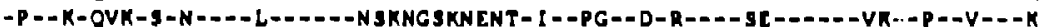

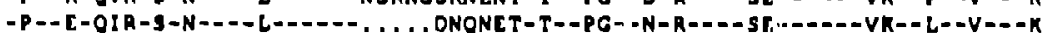

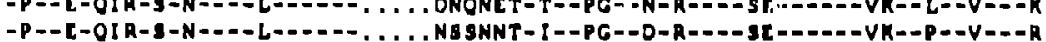

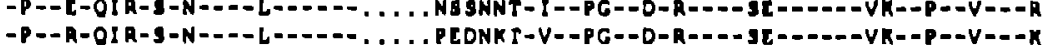

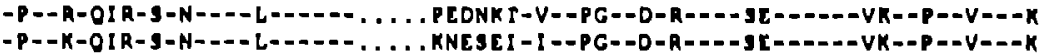

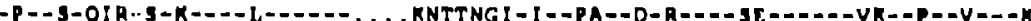

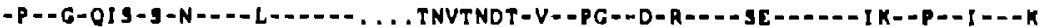

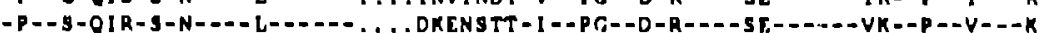

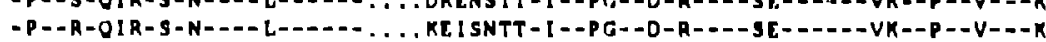

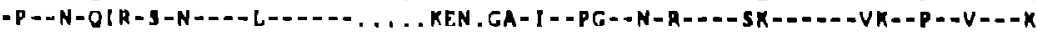

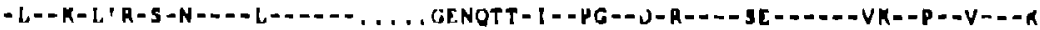
-P--I.-QI R-S-N=--

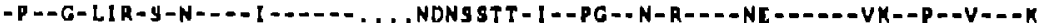

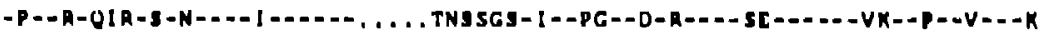

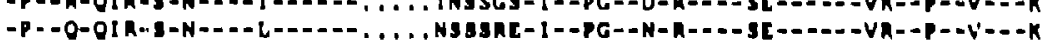

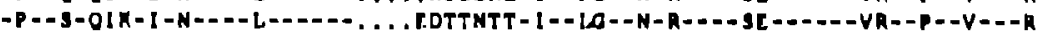

HIVCDCA

ilivergo

HIVHAN

HIVADA

HIVMAS

HIVRr

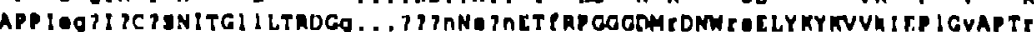

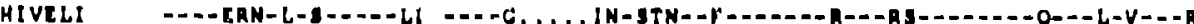

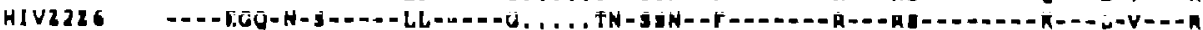

HIVNDK

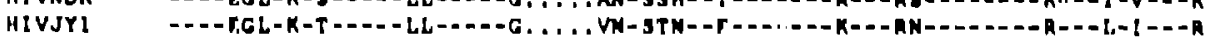

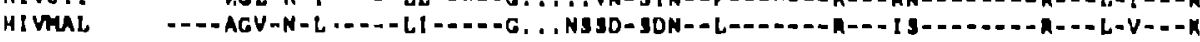

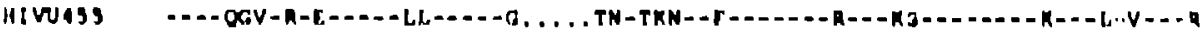

IIIVL12! - -

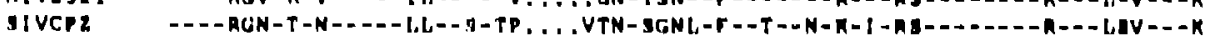


gp120/gp41

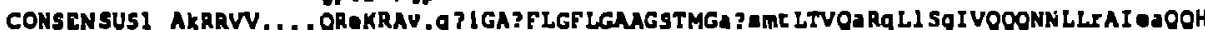
HIVLI

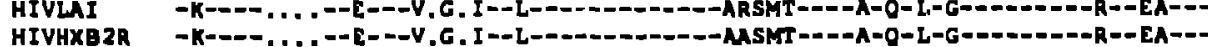

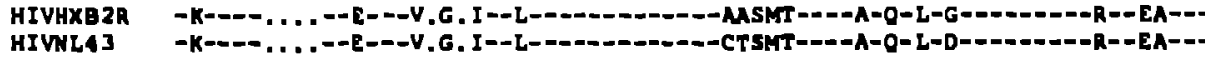

Hiva

HIVBRVA

HIVGC

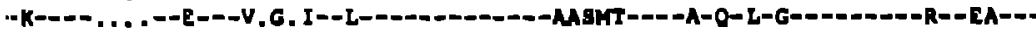

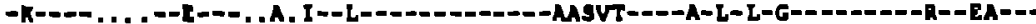

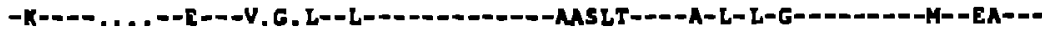

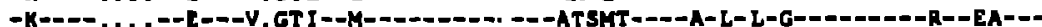

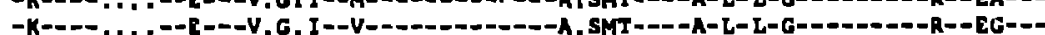

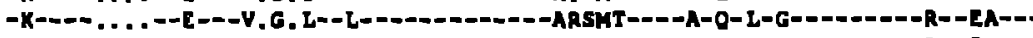

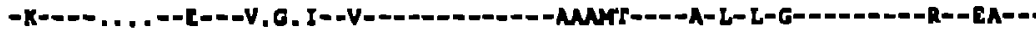

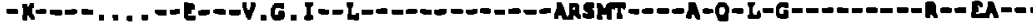

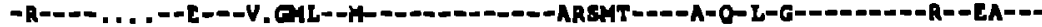

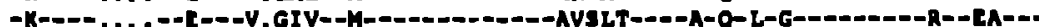

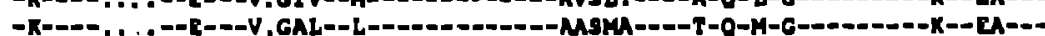

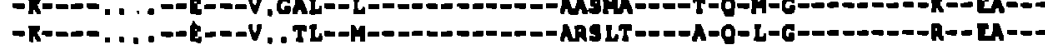

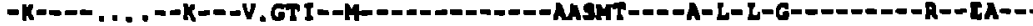

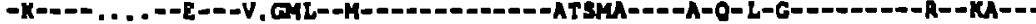

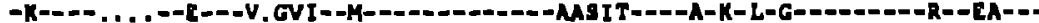

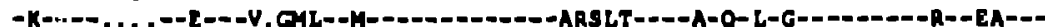

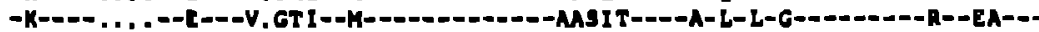

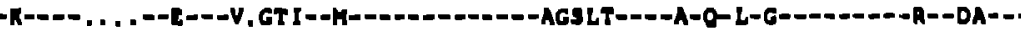

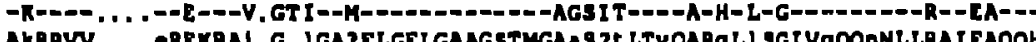

HIVF.PI 62

HIVJPL

HIYsr3is

HIVWN

HIYAD

HIVmisa

HIVRF

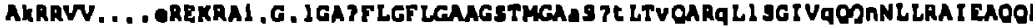

HIVLLI

HIVz226

HIVIDK

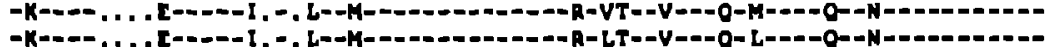

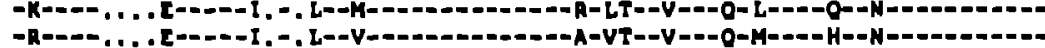

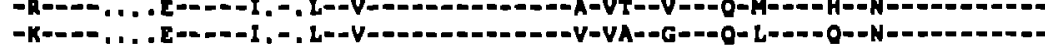

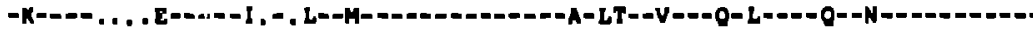

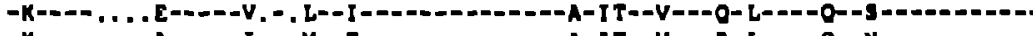

Hivuss

HIVIJ2

I

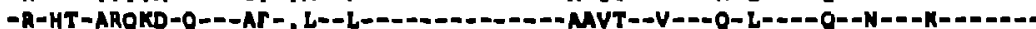

$$
\text { - } \quad \text { - } \quad \ldots . . .
$$

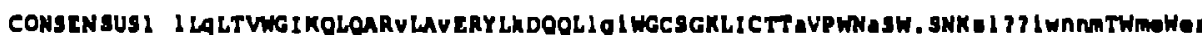
HIVLAI L-Q-DAC

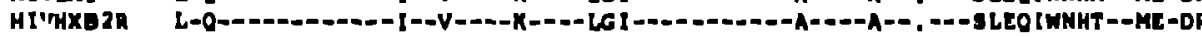

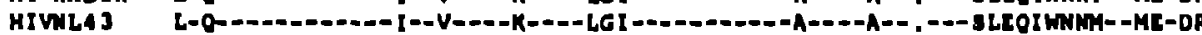

HIVATA

HIVA

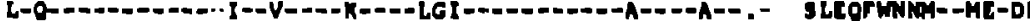

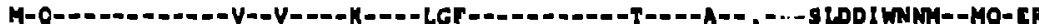
M-E-

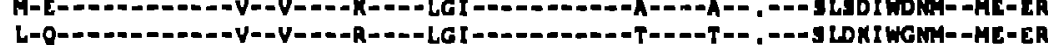

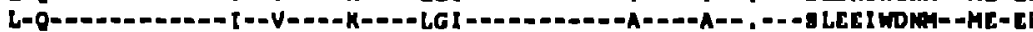

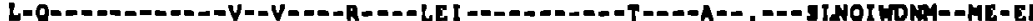

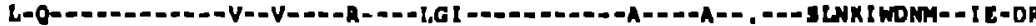

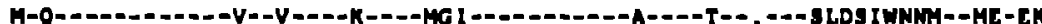

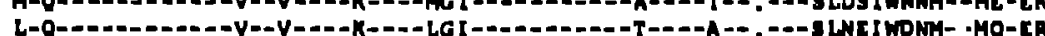

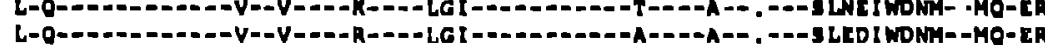
L-O--1,

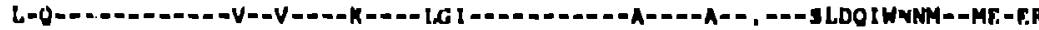

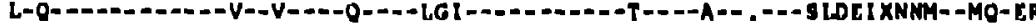
L-0-1-

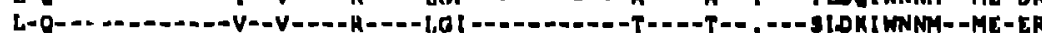

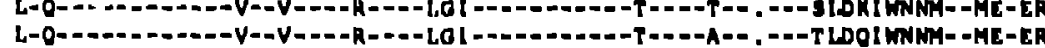
L-0-_-_- -

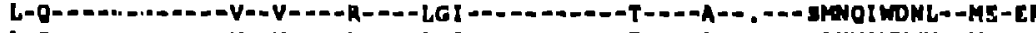

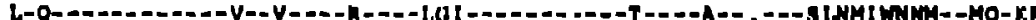

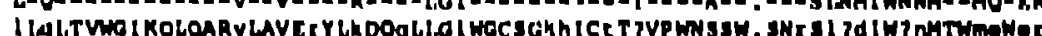

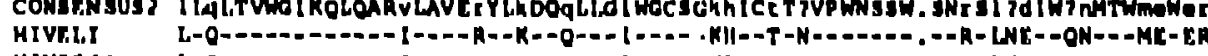

HIVI210

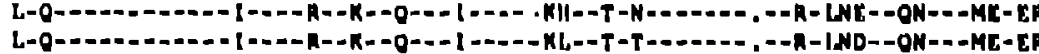
I. OM-O--D-- -

IIVSYI

HIVAL

IIIT645

IIVIJ)
alvipis

L-O-_-

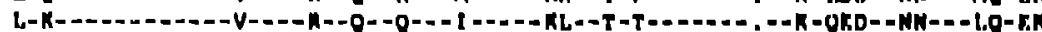

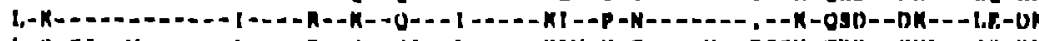

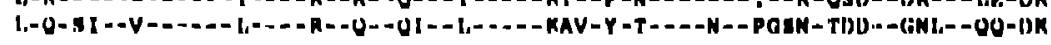




\section{HIV1 ENV}

...

a.

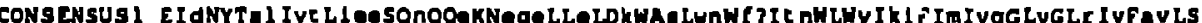
HIVAI

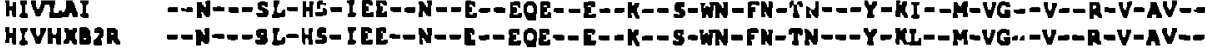
HIVNLA3 --N--SL-HS-IEC--N--E--EOE--C--K--S-WN-FH-TN---Y-KL--H-VG--V--R-V-AV-D

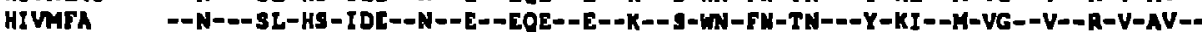
HIVIr HIVRA HIVSC

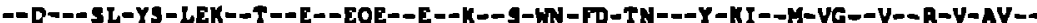

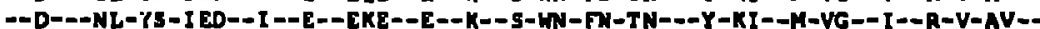

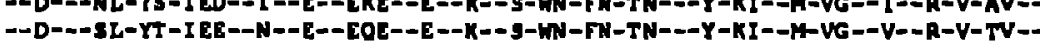

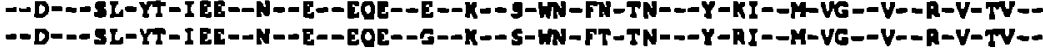

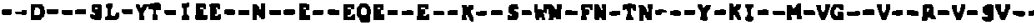

HIVALAI

HIVBAL1

HIVJACSP

HIVOYI

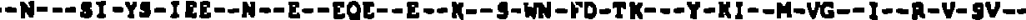

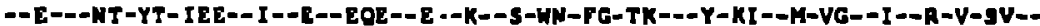

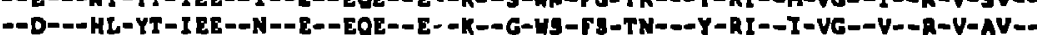

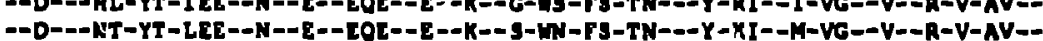

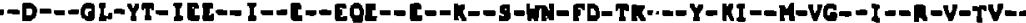

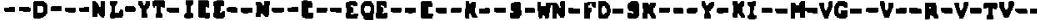

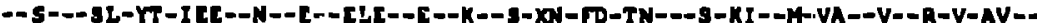

HIVTYSCe

HIVSTI62

HIVJFL

HIVCDC4

HIVSF33

HIVHN

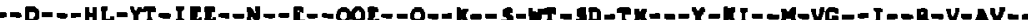

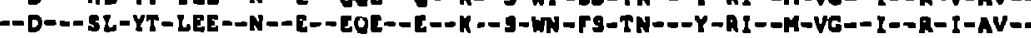

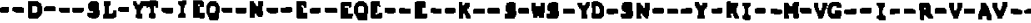

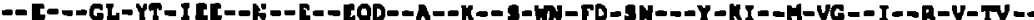

HIVADA

MIVIN2

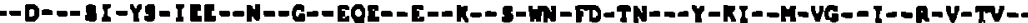

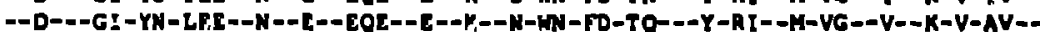

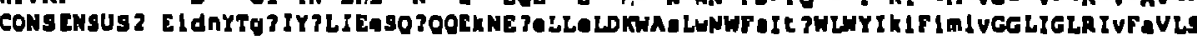

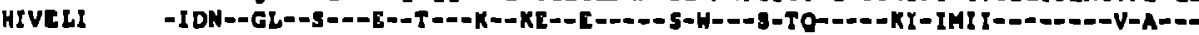

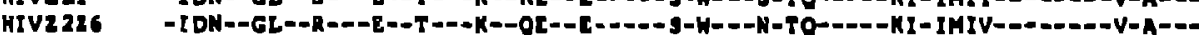

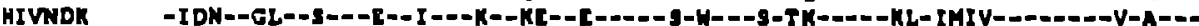

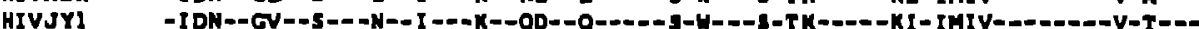

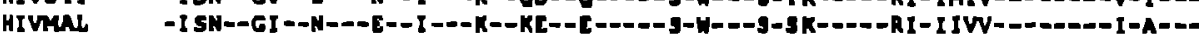

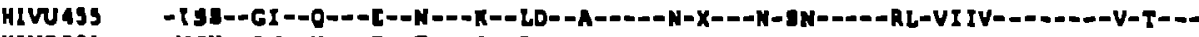

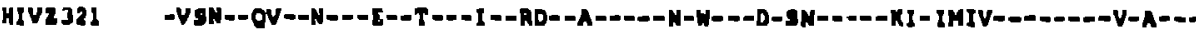

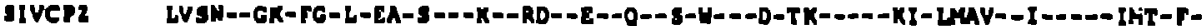

$$
1 / 3^{\circ} \text { : }
$$$$
\text { c- cac có }
$$

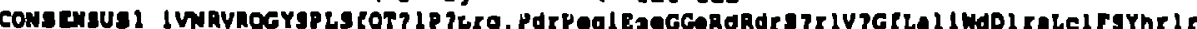
HIVLAI I-C..-.

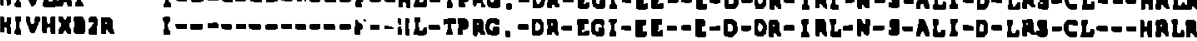
HIVNL4] I- 3 Mrima HIVRaYa HIVRAVA
hIVSC HIVSC HIVJH3 HIVALAI HIVEALI HIVJACar hIVoY! Hiverz Hivirsce HIVSFIG? HIVJTL IIVCDC IIIVST3! IIIVHAY HIVINI HIVRr l-_-

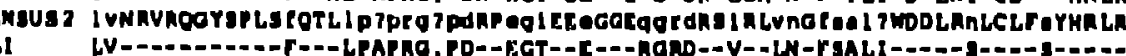

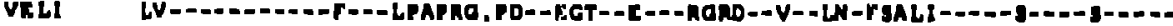
IIVZ226 LU-E-- W
HIVNDK HIVSYI HIVMAL HIVU459 Hivis2 IVCPI I - - -

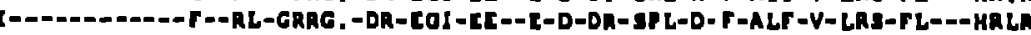
I -

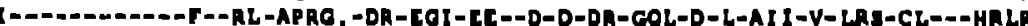

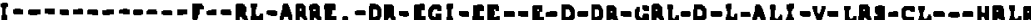

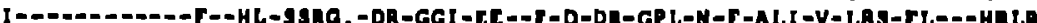
I I -

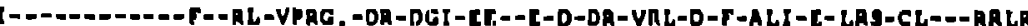
I - -

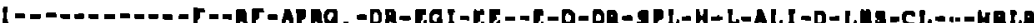
I-I -

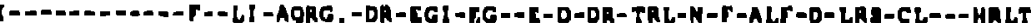

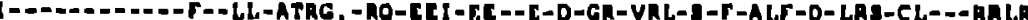
I - - 1-.......

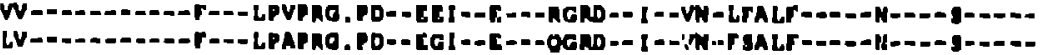

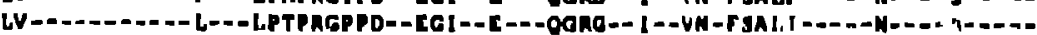
II - I. [ I -

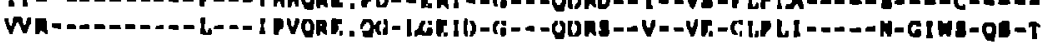


HIVI ENV

c- rev cde

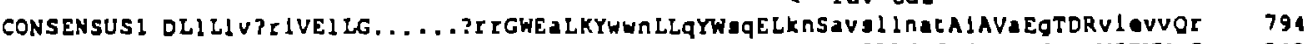

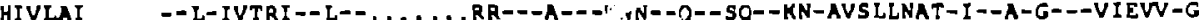
HIVHXB2R --L-IVTRI--I-_ .....RR---A-..WWN--O--SO--KN-AVSLLNAT-I--A-G--OVIEVV-G HIVNLA3 --L-IVTRI--L- -.......RR---A---WWN--0--SO--KN-AVNLLNAT-I--A-G---VIEVL-A

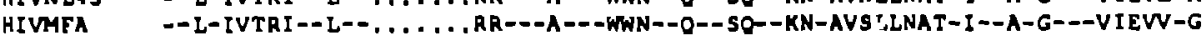
HIVMN --L-IMARI--L--......RR---V---WWN--O--SO--KS-AVSLLNAT-I--A-G---VIEVL-R HIVBRVA --L-IVTRI--L-.......RR---V---WWN--0--SO--KN-AVS: LNAT-I--G-R---AIEVV-P.

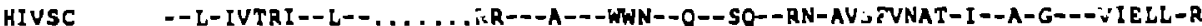
HIVJH3 --L-IVTRI - -L--......RR---A---WWN--0--SO-OKN-AVSLFNAI -I - -A-G---VLKIL-R HIVALA] --L-IVTRI--L-........RR---V---WWN--O--SO--KN-AVSLLNAT-I--A-G---VIEVL-R HIVBALI --L-IVMAI--L--......LAG---V---WWN--O--SG--KN-AVSLLNAT-V--A-G---VIEVL-R

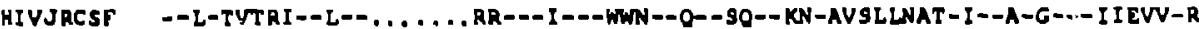

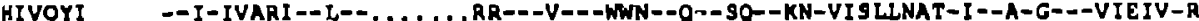

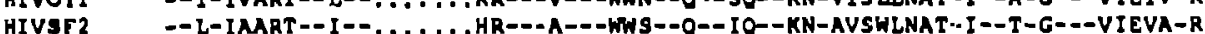
HIVNYSCG --L-IVARI--L--......RR---A---CWN--Q--GQ--KN-AISLLNAT-V--A-G-_-VI EVA-R HIVSFI 62 - I I IMARI--L--.......RR-- A---WGN--0--IO--KN-AVSLFDAI-I=-A-G---IIEVA-R

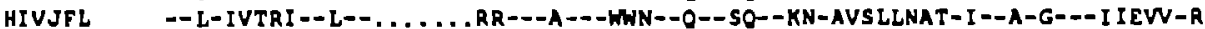
HIVCDC4 --L-IVARI-OL--......RR---V---WWN--0--SO--KN-AVSLUNVT-I--A-G---VIEV -R HIVSTJ3 --L-IVARI--L-_......RR---V---WWN--L--SO--KN-AVSLLNAT-I--A-G---VIEVW-R HIVHAN --L-IVAKI--T--.......RR---V---WWN--0--50--KN-AVS LFNTI-I--A-G---VIEWV-A HIVADA -L-IVARI--L--......RR---V---WWN--O-OSO-RN-AVS LLNAT-I --A-G-_-VIEVW-R HIVWMJ 2 --L-IVKRI--L--......RR---A---WWN--0--SK--KN-AVGLLNAI-I--A-G--DVI TWV-R

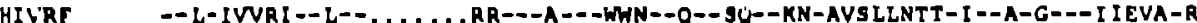

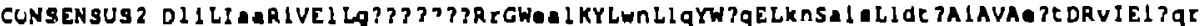

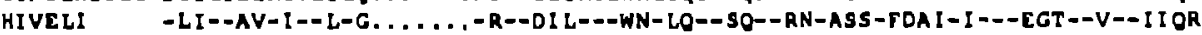
HIVZ226 -LI--MA-I--L-G.......-R--TAL---WN-LO--SR-OKN-ASS-LDTI-I---EGT--V--IVRR HIVNDK -SI--AA-I--L-G.......-R--EAL--WWN-LO--SQ--RN-ASS-LOTI-I---ERT--V-OVVOR HIVJYI HIVMAL HIVUass HIVZJ?!

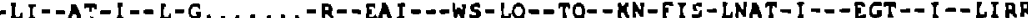

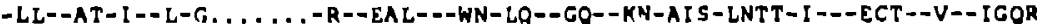
-FA--VA-A-- L-GRSSLKGL-L- - IGL---WN-LL--GR--KI-AIT-LDAV-V---GWI --V--IGOT -CA--AA-I--T-......I-R- -ETL---GN-VI --GQ- -KN-AIN-LDTV-I---DWT--V--VVOR SIVCPZ SLACNVH-OLKT-GHLILHSL-LLRERLCL-GGIIO--GK--KI-AIS-LDAT-I---EGT--I--AFOV

CONSENSUSL IRAIIHIPRRIROGLERALI. HIVLAI AC-AIRH-PR-I---L-II-LHIVHXB2A AC-AIRH-PR-I---L--I-1.HIVNLAJ AY-AIRH-PR-I - $-L-I-L$. HIVMFA AY-AIRH-PR-I-D-L-I-L-LHIVMN AG-AILH-PT-I--DL-A-LHIVERVA AF-AILH-PR-I-D-L-A-OHIVAC AT-AILH-PT-I-D-L-D-A-QHIVJHJ HIVALAI HIVBAL! HIVJACsr !I voYI irvor: HIVSF2
HIVNYSCG H!VST! 62 HIVJTL HIVCDCA hIVSiJj IIIVHAN HIVADA HIVMMJ? HIVRT $A F-A I L H-P T-I=--L--A-L-$ AF-AILH-PR-I--DF-A-A-LAV-AILH-PR-I-- L- - A-LVY-AILH-PT-I---L--A-LAY-ATLN-PK-I---L-DA-LAY-AILH-HR-I $=-L-L-L-L-$ IC-GILH-PR-I $=--L=-L-L-$ IG-ATLH-PR-! :--r--A-LIO-AILH-PV-I $=-L--A-L-$ IY-AFL,H-PR-I =--F--A-L$V G-A I L H-P T-I=--P--A-L-$ AC-AILH-PR-V-D-L--A-LIY-A ILH-P T- I - - L - - L-I. CONIENSUS2 7 RATLEIPRRIROG IERALL"

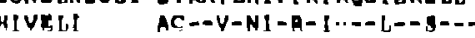
HIVZ226 AC-OV-HI-T-I---L-DL=IIIVNDK HIVJYI HIVMAI IIVU135 HIVZ32! $A C=-1-N V-R-I-=-1,-=L=-$ $A F-\cdot V-H I-R-V-=-I,--A-\cdot$

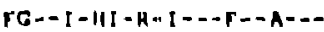
IO--I - NI - H-I I- - - L- $-A-D$

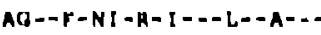

IVCPZ 
Information dense stretchos (0.8 or greater) are Indicated by *t*. Cygtelne resldues are indicated by and potential $\mathrm{N}-g$ lycosylation sites by $\cdots$. The SIVCPz env sequence has been Included in the ordinary CONSENSUS2 (african sequences) but not in the HIVIENV pattern.

\section{<- vpu cds \\ Ignal pepelde/gpl20}

-

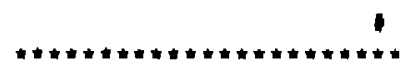

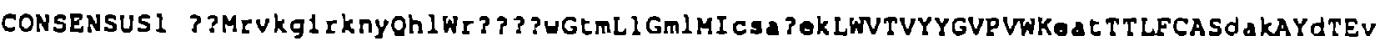
CONSENSUS? , - -

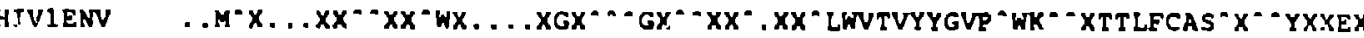

$\begin{array}{lllrlll}\text { D } & \text { DI } & \text { I } & \text { LII } & \text { II } & \text { D } & \text { D } \\ \text { E } & \text { EV } & \text { V } & \text { MVV } & \text { VV } & \text { E } & \text { E } \\ \text { N } & \text { NL } & \text { L } & \text { LL } & \text { LL } & \text { N } & \text { N } \\ \text { O } & \text { OM } & \text { M } & \text { MM } & \text { MM } & \text { O } & \text { O } \\ \text { K } & \text { KF } & \text { E } & \text { F } & \text { F } & \text { K } & \text { K } \\ \text { R } & \text { RW } & \text { W } & \text { W } & \text { W } & \text { R } & \text { R } \\ \text { H } & \text { HY } & \text { Y } & \text { Y } & \text { Y } & \text { H } & \text { H } \\ \text { S } & \text { SC } & \text { C } & \text { C } & \text { C } & \text { S } & \text { S } \\ \text { T } & \text { T } & & & & \text { I } & \text { T }\end{array}$

a.

KA

V EG

E RG

$\mathbf{s}$

T

$\mathbf{S}$

0

$\mathbf{K}$

$\mathbf{P}$

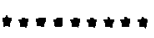

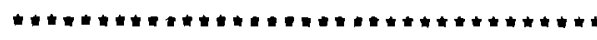

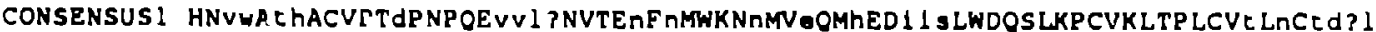

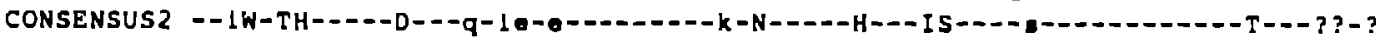
HIVIENV

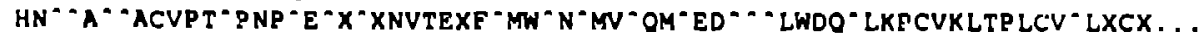

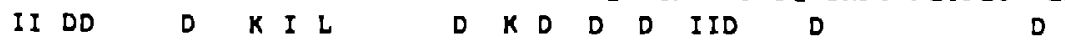

W $\mathrm{E}$

N R $V$ M

N R N E E VVE

E

L NN E L

M O $O$ M

F KK

$\begin{array}{lrr}\mathrm{N} & \text { VVE } & \mathrm{E} \\ \mathrm{Q} & \mathrm{N} & \mathrm{N}\end{array}$

W RR

$Y$ HH

C 55 $\mathrm{T}$ t

$\because \ldots$

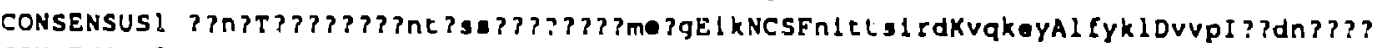

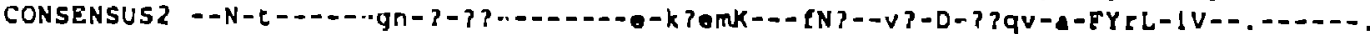

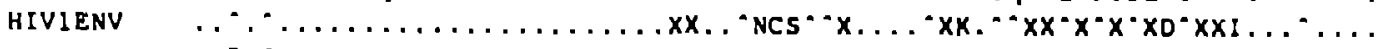

$\begin{array}{ll}D & S \\ E & T \\ N & \\ 0 & \\ K & \\ R & \\ H & \\ S & T\end{array}$

$\begin{array}{lr}D & \text { FD } \\ \text { E } & \text { WE } \\ \text { N } & \text { YN } \\ \text { O } & \text { O } \\ \text { K } & \text { K } \\ \text { R } & \text { R } \\ \text { H } & \text { H } \\ \text { S } & \text { S } \\ \text { T } & \text { T }\end{array}$

$D$
E
N
Q
K
R
H
S
T

$\begin{array}{lllll}\text { DK } & \text { A } & \text { I } & \text { O } & \text { I } \\ \text { ER } & \text { G } & V & \text { E } & \text { V } \\ \text { NE } & \text { S } & \text { L } & \text { N } & \text { L } \\ \text { OO } & \text { T } & \text { M } & \text { O } & \text { M } \\ \text { K } & \text { P } & \text { F } & \text { K } & \\ \text { R } & & \text { W } & \text { A } & \\ \text { H } & & \text { Y } & \text { H } & \\ \text { S } & & \text { C } & \text { S } & \\ \text { T } & & & T & \end{array}$

157 
Piptide T region $1 \cdots$,

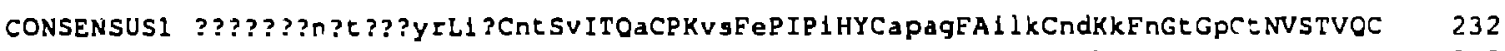

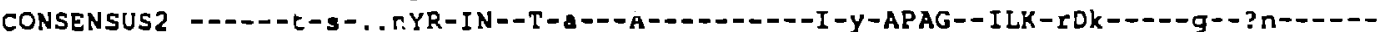
HIVIENV

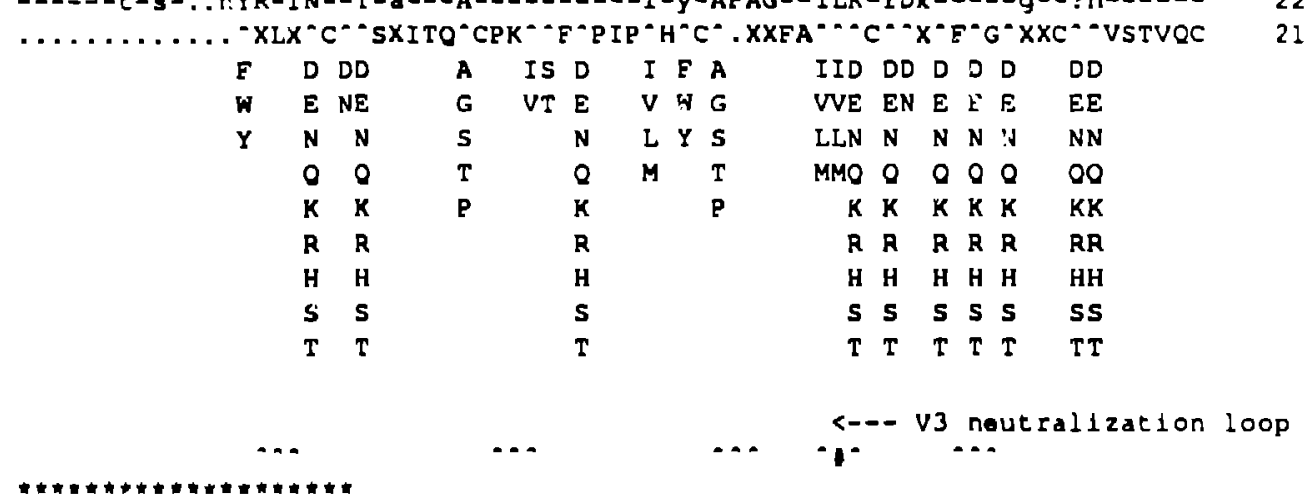

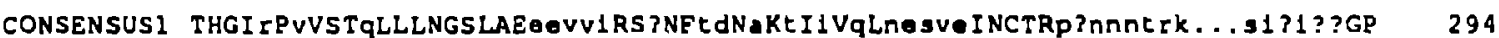

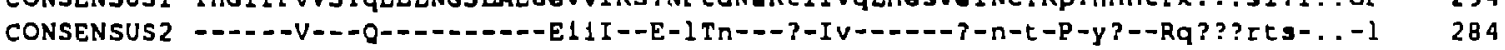

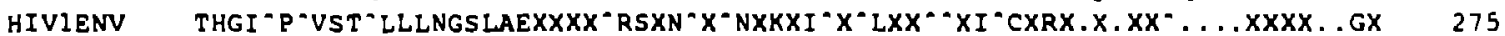

\begin{tabular}{|c|c|c|c|c|c|c|}
\hline \multirow{9}{*}{$\begin{array}{ll}K & I \\
R & V\end{array}$} & D & $I$ & I D & I & D & AI \\
\hline & $E$ & $v$ & $V \mathrm{~N}$ & v & $\mathbf{E}$ & GV \\
\hline & $\mathbf{N}$ & L & L & L & $\mathbf{N}$ & $S$ \\
\hline & 0 & $M$ & $\mathbf{M}$ & M & 0 & $I$ \\
\hline & K & & $\mathbf{F}$ & & K & $\mathbf{P}$ \\
\hline & $\mathbf{R}$ & & $\mathbf{W}$ & & $\mathbf{R}$ & \\
\hline & $\mathbf{H}$ & & $\mathbf{Y}$ & & H & \\
\hline & $\mathbf{s}$ & & C & & 5 & \\
\hline & $T$ & & & & $\mathbf{T}$ & \\
\hline
\end{tabular}

v3 neutralization loop $\rightarrow$

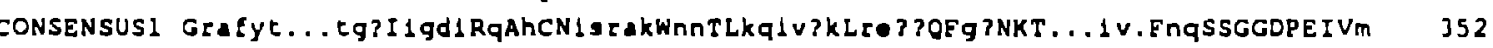

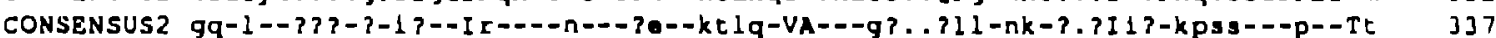

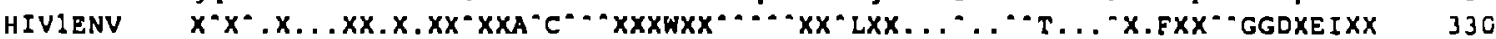

\begin{tabular}{|c|c|c|c|c|c|c|c|c|c|}
\hline$K$ & $I$ & $F$ & DID & AIDKI & $D$ & I & DD & I & AA \\
\hline$R$ & $v$ & $\boldsymbol{W}$ & EVE & GVERV & $E$ & v & EE & $v$ & GG \\
\hline $\mathbf{E}$ & L & $Y$ & NLN & SLNE & $\mathbf{N}$ & $L$ & NN & & ss \\
\hline 0 & M & $\mathrm{H}$ & OMO & TMOO & 0 & M & 00 & & $\mathrm{TT}$ \\
\hline & & & $K K$ & P K & $K$ & $E$ & KK & & PP \\
\hline & & & $R \quad R$ & $\mathbf{R}$ & $\mathbf{R}$ & W & RR & & \\
\hline & & & H H & $H$ & H & $\mathbf{Y}$ & $\mathrm{HH}$ & & \\
\hline & & & S 5 & 5 & $\mathbf{5}$ & C & ss & & \\
\hline & & & $T T$ & $T$ & $T$ & & TT & & \\
\hline
\end{tabular}

CD4

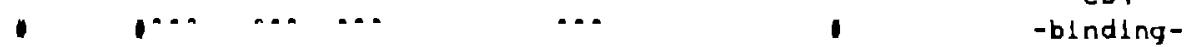

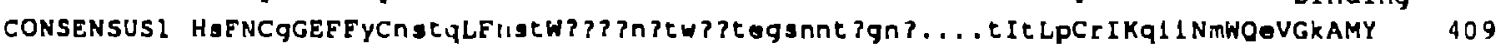

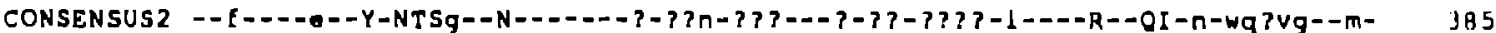

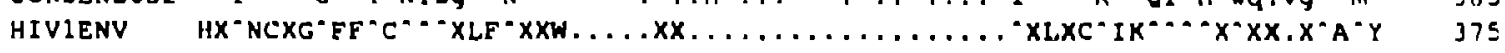

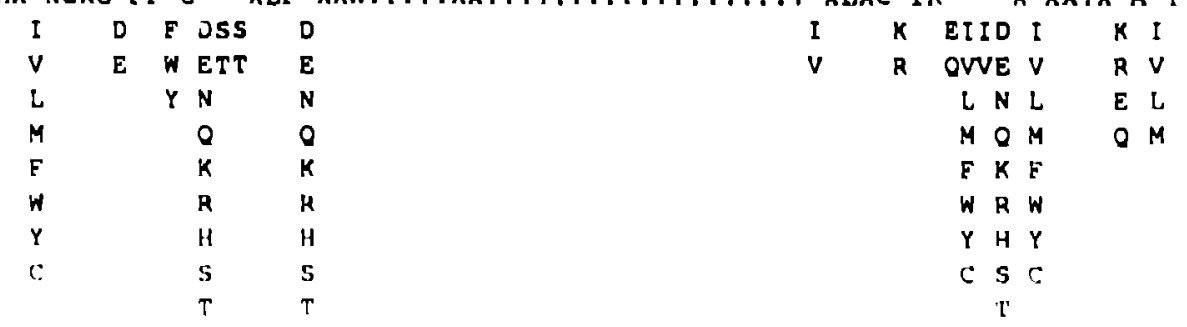




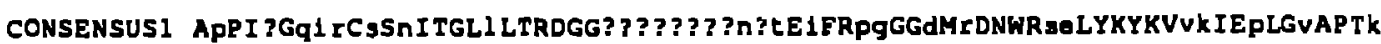
CONSENSUS2 -P--Ag?I ?-?-N---1------g...---nNS-n-Tf-PG- D------r-E------V---P1-----r HIV1ENV

\begin{tabular}{|c|c|c|c|c|c|c|c|c|c|c|}
\hline I & D & II & D & I & A & DK & DX & ID & I I & K \\
\hline $\mathrm{v}$ & $\bar{\varepsilon}$ & $\mathbf{v}$ & $E$ & $\vec{v}$ & G & N R & ER & VE & V v & $\mathbf{R}$ \\
\hline & $\mathbf{N}$ & LL & $\mathbf{N}$ & L & & & NE & N & L & \\
\hline & 0 & pas & 0 & $\mathbf{M}$ & & & $\infty$ & 0 & M & \\
\hline & $\mathbf{k}$ & & $x$ & $F$ & & & $\mathbf{K}$ & K & & \\
\hline & $\mathbf{R}$ & & $\mathbf{R}$ & " & & & $\mathbf{R}$ & R & & \\
\hline & H & & H & $Y$ & & & H & H & & \\
\hline & $\mathbf{s}$ & & $\mathbf{s}$ & c & & & $\mathbf{s}$ & S & & \\
\hline & $\mathbf{T}$ & & $\mathbf{T}$ & & & & $\mathbf{T}$ & $\mathbf{T}$ & & \\
\hline
\end{tabular}

CONSENSUSI AKRRVV. . . . OREKRAV.gP IGA PFLCELGMGSTMGA ? EmELTVOARQL1SGIVOOONNLLFAI EAOOH

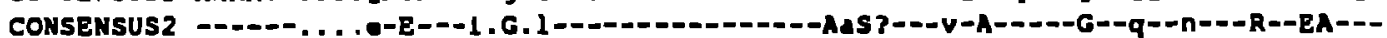
HIVIENY

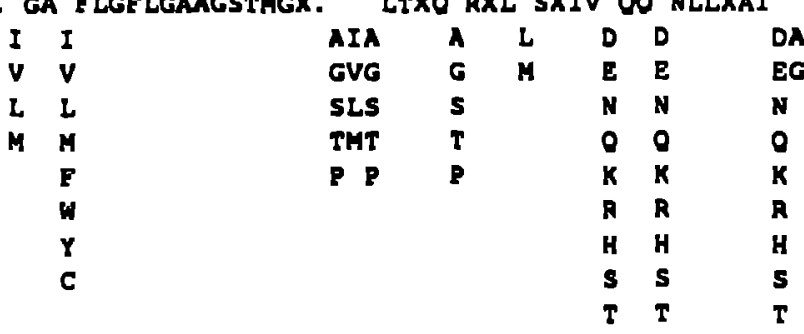

\section{1000}

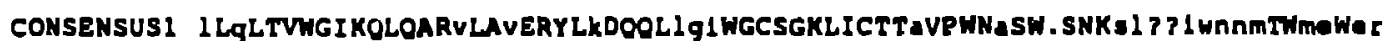
CONSENSUS2 -

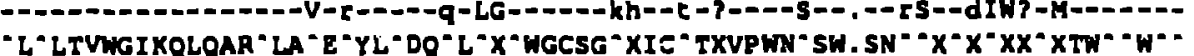

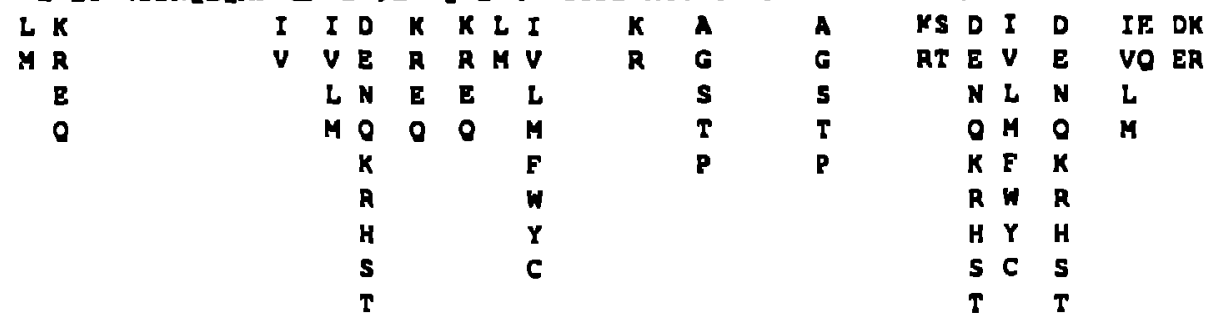

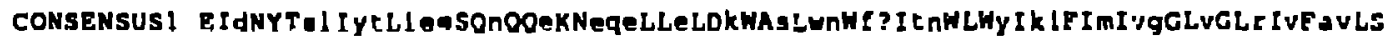

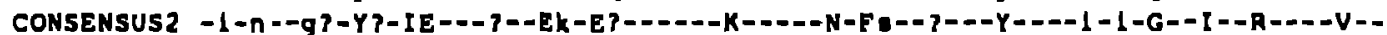
HIVIENV

\begin{tabular}{|c|c|c|c|c|c|c|c|c|c|c|c|}
\hline $\begin{array}{l}\text { IDD } \\
\text { VEE }\end{array}$ & $\begin{array}{l}\text { FD } \\
\text { WE }\end{array}$ & $\begin{array}{l}\text { IDD } \\
\text { VEE }\end{array}$ & $\begin{array}{ll}E & 0 \\
0 & B\end{array}$ & $\begin{array}{l}D \\
E\end{array}$ & $\begin{array}{l}\text { D } \\
\text { E }\end{array}$ & $\begin{array}{l}\text { SD } \\
\text { TE }\end{array}$ & $\begin{array}{l}\text { KI } \\
\text { RV }\end{array}$ & $\begin{array}{l}\text { I IIIA } \\
\text { VVVVG }\end{array}$ & $\begin{array}{l}\mathbf{I} \\
\mathbf{V}\end{array}$ & $\begin{array}{ll}K & I \\
\text { R } & V\end{array}$ & $\begin{array}{l}\text { A I } \\
\text { GV }\end{array}$ \\
\hline NN & $\mathbf{Y N}$ & $L N$ & & $\mathbf{N}$ & $\mathbf{N}$ & N & L & 2 & & & $\mathbf{s}$ \\
\hline$\infty$ & HO & MO & & 0 & 0 & 0 & M & M & & & $\mathbf{T}$ \\
\hline KK & K & $x$ & & $\mathbf{K}$ & K & K & & & & & $\mathbf{P}$ \\
\hline RR & R & $R$ & & $\mathbf{R}$ & $\mathbf{R}$ & $\mathbf{R}$ & & & & & \\
\hline HH & H & H & & H & H & H & & & & & \\
\hline ss & $\mathbf{s}$ & s & & $\mathbf{s}$ & $\mathbf{S}$ & $\mathbf{s}$ & & & & & \\
\hline TT & $\eta$ & $T$ & & $T$ & $T$ & $T$ & & & & & \\
\hline
\end{tabular}


$\backslash / 3^{\prime}$ sf $<-$ tat cds

$\pi * \pi+\pi * \pi+\cdots * *$

CONSENSUS 1 IVNRVROGYSPLSEOT?IP?PIg.PdrPeg1EeeGGeRdPdrS?rlV?GFLaliWdDIrsLelFSYhrlr 732 CONSENSUS2 $1 \mathrm{~V}----1 .------L-p----$ ?p-R-----E---Eqgr-R-1RLVn--s--?-D-LRn-CL-3-HRLR 705 HIVIENV

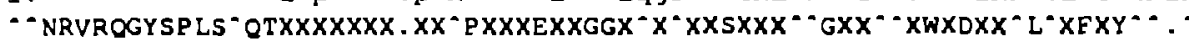

\begin{tabular}{|c|c|c|c|c|c|c|c|}
\hline II & I & K & $\mathrm{K}$ & ID & AI & $D$ & $D D$ \\
\hline vV & $v$ & $\mathbf{R}$ & $\mathbf{R}$ & VE & GV & $E$ & EE \\
\hline L & L & $E$ & $E$ & LN & SL & $\mathbf{N}$ & NN \\
\hline \multirow[t]{6}{*}{ M } & M & 0 & 0 & NiO & $T M$ & 0 & 00 \\
\hline & $F$ & & & $\mathrm{~K}$ & $P$ & K & KK \\
\hline & $\omega$ & & & $\mathbf{R}$ & & $\mathbf{R}$ & $\mathbf{R R}$ \\
\hline & $\mathbf{Y}$ & & & H & & $\mathbf{H}$ & $\mathrm{HH}$ \\
\hline & C & & & $\mathbf{s}$ & & $\mathbf{s}$ & SS \\
\hline & & & & T & & $T$ & TT \\
\hline
\end{tabular}

<- zov cds

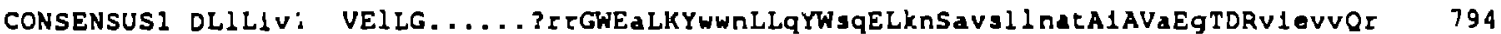

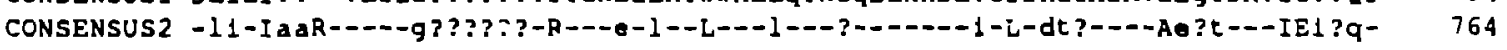

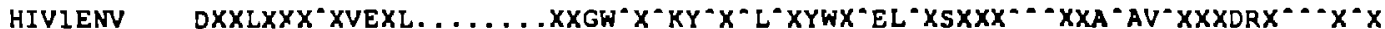

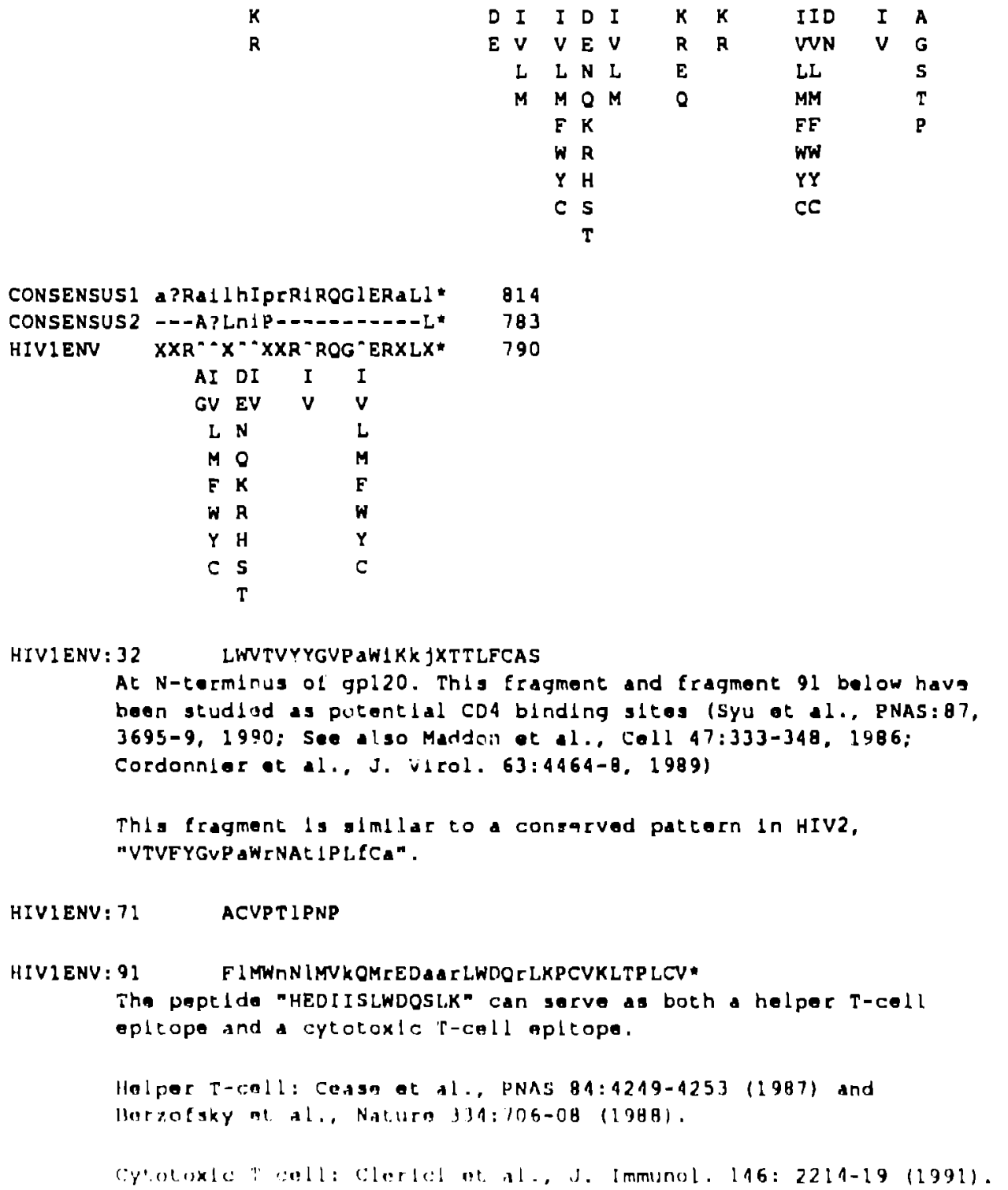







A small Insertion at "TMG" Inhibits gp120/gp4l association. Kowalsk1 et al.. Sclence 237:1351-55 (1987).

Th1a fragment is similar to a conserved pactern in HIV2. "fVLGELGELJTAGJAM".

HIVIENV: 530 QOHbLPLTVWGIKOLOARALA

A mutation In the last "A- can result in a noutralization oscape mutant. Rettz et al.. Coll 54:57-63 (1988). That -A- 1s the first residue of the so-called "AVERY- peptide that separatea thl. fragment from the following fragment.

The fragment is ombedded in a region of gp41 that is important for oligomeric assembly. Earl ot al., nNAS 87:648-52 (1990), and Doma ot al.. J. V1rol. 64:3537-40 (1990).

Thle Cragment ls amliar to a conserved pattern In HIVZ, "OOEbLRLTVWGJKNLOJRVIA".

HIVIENV: 564 WGCSG

Part of a 12 amino acid neutral1zing antibody opltope.

Schrler et al.. J. Virol. 62:2531-36 (1988). Also Gnann

et al., J. Yirol. 61:2639-41, 1987 and Sclence:37:1346-9, 1987.

The fragment is embedded In. region of gp4l that is Important

for ol lgomeric ascembly. Earl et al., PNAS 87:648-52 (1990),

and Doms ot al., J. Virol. 64:3537-40 (1990).

Antibody to thila fragment la croas-reactive with CNS tisaue, namely an atrocyte proteln of 43 kD at 111 to be Identified (Yamada ot al., J. Virol. 65:1370-6, 1991).

HIVIENV:S76 VPWNJSW

HIVIENV: 660 GLAGLIIAFJALSCANRVROGYSPLS Mutational etudy -- Heleeth et al., J.V1rol.6416314-8, 1990

Thlo fragment lo almilar to a congerved pattarn in HIV2, "NRDTWGT". 


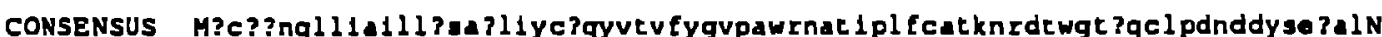
HIV2ROD HIV2NI 42 HIV2ISY HIV2SI HIV2BEN HIV2D194 HIV2GHI SIVMM251 SIVHM1 42 SIVRA239 5 IVMNE SIVSMAH 4 SIVSMAMPBJ HIV2D205

CONSENSUS HIV2ROD HIV2NIH2 HIV2 ISY HIV2ST HIV2BEN HIV2D1 94 HIV2GHI SIVMM25I SIVMM1 42 SIVHM2 39 STVHNE SIVSMMHA SIVSMMPQJ HIV2D205 - . M. . NOLLIAI LLASACLVYCTOYVTVFYGVP TWKNAT I LFCATRNRDTWGTIOCLP DNDDYOEITL-KGSKNOLLIAIVLASAYLIHCKOFVTVFYGIPAWRNASI PLFCATKNRDTAGTIOCLPDNDDYOEI TL- . SGK I OLLVAFLLTSACLI YCTKYVTVFYGVPVWKNAS I PLFCATKNRDTWGTIOCLPDNDDYOETPL- . CGRNOLFVASLLASACLI YCVOYVTVFYGUPVWRNAS I PLCATKNRDTWGTIOCLPDNDDYOEIAL-EPGRNOLFVILLTSACLVYCSOYVTVFYGIPAWKNAS IPLFCATKNRDTWGTIOCLPDNDDYOE I IL-EPGRNOLLVAI LLTSACLIYCKOYVTVFYGIPAWRNAS IPLFCATKNRDTWGTIOCLPDNDDYOEITL-. CGKSLLCVASLLASAYLVYCTOYVTVFYGVPVWRNAS I PLFCATKNRDTWGTIOCKPDNDDYOE ITL -GCLGNOLLIAILLLSVYGIYCTOYVTVFYGVPAWRNAT I PLCCATKNRDTWGTTOCLPDNGDYSELAL-GCLGNOLLIAILLESVYGIYCJOYVTVFYGVANRNATIPLFCATKNRDTWGTTOCLPDNDDYSELAL-GCLONOLLIAI LLLSVYGI YCTLYVTVEYGVPAWRNATI PLFCATKNRDTWGTTOCLPDNGDYSEVAL-GCLGNOLL IAI LFLSAYGIYCIOYVTVFYSVPAWRNAT IPLFCVIRNRDTWGTTOCLPDNDDYSELAL-GCLGNOLLIALLLVSVLEICCVOYVTVFYGVPAWKNATIPLFCATKNRDTWGTTOC'PDNDDYSELAI -GCLGNOLLIALLLLSASGIYCVOYVTVFYGIPAWRNATVPLFCATKNROTWGTTOCLPDNGDYSELAI -LLSSHAYPHGGTOOFP SFVOPOTE TPGELYSUSOTINTTLRSGTORLLMHG I IOHNROHMCGDS LKPP -

- -

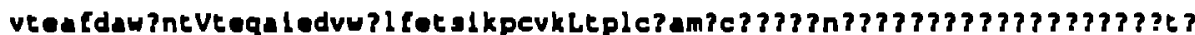
VTEAFDAWNNT-TEOAIEDVWHLETSIKPCVK-TPLCVAMKC.....SST. . . . . ESST . GNNTTS

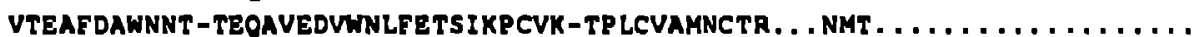
VTEAFDAWNNI - TEOAVEDVWNLFETSIKPCVK-TPLCVTMNC. . . . NAS. . . . . . . . . . VTEAFDAWNNT -TEOAVEDVWSLFETSIKPCVK-TP LCVAMRC . . . . NST . . . . . . . . . TAKNT VTEAFDAWNNT - TEOAVEDVWHLFETSIKPCVK-TPLCVAMNCSRVOGNTT . . . . . . TPNPR

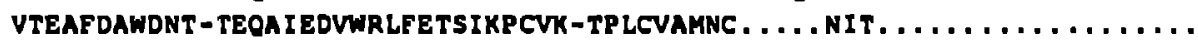

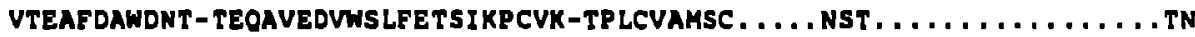
VTESFDAWENT - TEOAIEDVWOLFETS IKPCVK-SPLCI TMRC . . . . . NKSETDRWCLTKSST . . . . . . VTESF NAWENT-TEOA IEDVWOLFETSIKPCVK-SPLCI THRC . . . . . NKSETDKWGLTKSST . . . . TT VTES I WNNT - TEOAIEDVWOLFETS I KPCVK-SPLCI TMRC. . . . . NKSETDAWG LTKS I T . . . . TT ITESF DAWENT - TEOA IEDVWHLFETS IKPCVK-TPLCI THKC . . . . . NKSETDKWGLTKSST . . . . . TT VTEAFDAWONT - TEOA IEDVWNLFETSIKPCVK - TPLCIAMRC . . . . . NKTETDRWGLTGNAG . . . . TT VTEAFDAWDNT-TEOAIEDVWNLFETSIKPCVK-TPLEI THRC . . . . . NKSETDRWGLTGTPAPTTTOTT HVSNPHCVWOT-VKPKOTOGMPVVLPPLSLLPP-VGKRLTKOTHAKMTMAODEKRKCNVILVRDEEMSNR:

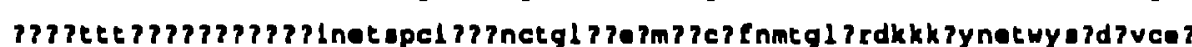
KSTSTTTTTPTDO . . EOEI SEDTPCARADNCSGLGEEET I NCOFNMTGLERDKKKOYNETWY SKDVVCET ... . TWTGRTDTO. NITI I NDTSH. ARADNCTG LKEEEMI DCOF SHTGLERDKRKOY TEAWY SKDVVCD. TESAVATTSPS . . , GPDMINDTDPCIOLNNCSGLREEDMVECOFNATOLELDKKKOY SETWY SKDVVCES TSTPTTTTTA . . . . NTT . IGENSSC I RTDNCTGLGEEEMVDCOFNMTGLERDKKKLY NETWY SKDVVCES TSSSTTS . RPPTS . AAS I INETSNC? ENNTCAGLGYEEMHOCEF NMKGLEODKKRRYKDTWY LEDVVCD. . SGTTATP SPP . . . NITI IDENSTC IGDNNCTG LGKEEVVECEF NMTGLEODKKRKY NDAWY SRDVVCDK NTTTTGSTT . . . . . GHSE INET SP SYS . DNCTGLGKEE I UNCOFYMTGLERDKKKOYNETWY SKDVVCES TI TTMAP TSAPVSEX I DMVNET TSC I AONNCTGLEOEOMI SCKF THTGLKRDKTKEYNETWY STDLVCLO ASTTTTTTAKSVE. TRDIVNETSPCWHDNCTGLEOEPMI SCKYNMTGLKRDKKKEYNEFHY SADI.VCRO ASTTSTTASA. . . . . KVUMYNET SSC I AOONCTGLEOEOMI SCKFNATGLKRDKKKEYNETHY SADI,VCEO A. PTAIPTKAE. . . AI AVVNENSPC I NKDNCTGLEOEPM I SCKFNMTGLKRDKRAEY NETWY SADLVCEO TTAI TT TATPSV. . AENVINESNPC I KNNSCAGLROEPMI GCKFNATGLNADKKKEYNETWY SHDLI ICEO TTOASTTP TSP I . . TAKVVNDSDPC I K INNCTGI.ROEPHVSCKFNHTGL,KRDKKAEYNHTWY SHUL,VËLO IKTPGTOKL SVI IPGSI PADA IEPATOOLSKSHVTNI IGTAGLG IVLIPAGFYYDVMIPTIOASCPTAVHA 


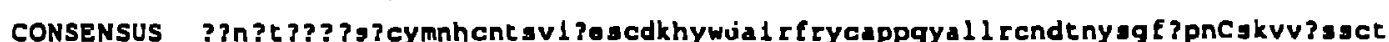
HIV2ROD HIV2NIHZ HIV2ISY HIV2ST HIV2BEN HIV2D1 94 HIV2GH1 SIVMM251 SIVMM1 42 SIVMA2 39 SIVMNE SIVSMMH4 SIVSMMPBJ HIV2D205 N.NST . NOTO. CYMNHCNT SVITESCDKHYWDA IRFRYCAPPGYALLRCNDTNYSGFAPN-SKVVASTCT ..NNTSSO.SKCYMNHCNTSVITESCDKHYWDAMRFRYCAPPGFALLRLNDTNYSGFAPN-SKVVAATCT D.NST.D. RKRCYMNHCNTSVITESCDKHYWDAMKFRYCAPPGFVLLRCNDTNYSGFEPN-SKVVASTCT . . NDT . KKEKTCYMNHCNTSVI TESCDKHYHDTMRPRYCAPPGFALLRCNDTNYSGFEPN-SKVVMATCT . . NTT . . . AGTCYHRHCNTS IIKESCDKHYWDAMRFRYCAPPGFAL LRCNDTNYSGFEPK-TKVVAASCT T. NGTG. . . . TCYMRHCNTSVIKESCDKHYWDAMKFRYCAPPGFAL LRCNDTNYSGFEPK-SKVVAMSCT . . NNTKDGKNRCYMNHCNTSVI TESCDKHYWDA IKFRYCAPPGYALLRCNDTNYSGFEPK-SKVVASTCT G. NSTD. NESRCYMNHCNTSVIOESCDKHYWDTI RFRYCAPPGYALLRCNDTNYSGFMPK-SKVVVSSCT G.NSTG. NESRCYMNHCNTSVIOECCDKDYWDA IRCRYCAPPGYALLRCNDTNYSGFMPN-SKVVVSSCT G. NNTG. NESRCYMNHCNTSVIOESCDKHYWDAIRFRYCAPPGYALLRCNDTNYSGFMPK-SKVVUSSCT G. NST . ENESRCYMNHCNTSVIOESCDKHYWDAIRYRYCAPPGYALLRCNDTNYSGTMPN-SKVVVSSCT SANES. . . ESKCYMHHCNTSVIOESCDKHYWDAIRFRYCAPPGYALLRCNDSNYLGFAPN-SKVVV: SCT NSNET . . . DSKCYMNHCNTSVIOESCDKHYWDA IRFRYCAP PGYALLRCNDSNYSGFAPN-TKVVVSSCT PPAOEWKHSP LHGLASMVOGORTGHIYIGMKKT I GPSAIHTI ICOYTVRGUETRRLYOEP-ODYFSIHSL

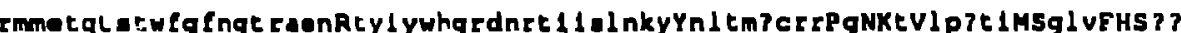
RMMETOTS TWFGFNGTRAEN-TY I YWHGRDNRT I I SLNKY-NLSLHCKR-G--I -KOIML- -GHV---HY RMMETOTS TWFGFNGTRAEN-TY I YWHGKDNRT I I SLNNF-NLTHHCKR-G--T-LP I TF--GFK---. . RMMETOPS TWLGENGTRAEN-TY I YWHGRDNRTI I SLNKY-NLTILCRA-E--T-VPITL--GRR--- . . RMMETOTS TWFGFNGTRAEN-TYI YWHGRDNRT I I SLNKF-NLTVHCKR-G--T-VP I TL--GLV--- . . RMMETOTSTWFGFNGTRAEN-TY I YWHGRDNRTI I SLNKY-NLTMRCKA-G--T-LP ITL--GLV--- . . RMMETOTS TWFGFNGTRAEN-TYI YWHGKDNRTI I SLNKY-NLTHHCKR-G--T-VPITL--GRR--- . . RMME TOTS TWFGFNGTRAEN-TY I YWHGRDNRT I I SLNKY-NLSI HCKR-G--T-VPITL--GLV--- . . RMMETOTS TWFGFNGTRAEN - TY I YWHGRDNRT I I SLNKY - NLTMKCRR-G-T-LPVT I--GLV-- - . RMME TOTS TWFRFNGTRAEN-TY I YWHGRDNRT I I SLNKH-NLTHKCRR-G- -T-LPVTI - -ALV-- - . . RMME TOTSTWFGENGTRAEN-TY I YWHGRDNRT I I SLNKY-NLTHKCRR-G-OT-LPVT I--GLV-- - . RHME TOTSTWFGFNGTRAEN -TY I YWHSKDNRT I I SLNKY-NLTMKCRR-G- -T-LPVT I - -GLV-- - . RMMETOTSTWFGFNCTRAEN-TYIYWHGKSNRT I I SLNKY-NLTHRCRR-E-T-LPVT I--GLV--- , . RMMETOTS TWFGFNGTRAEN - TYI YWHGRSNRT I I SLNKY - NLTMRCRR-G--T-LPVTI - -GLV-- . . SIRDPDKLGAGLRETGOKPR-KGPSNIPGIKEVOKI SOAS-ONAEKVOI

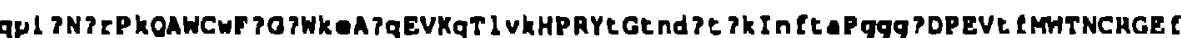

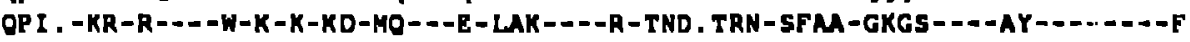

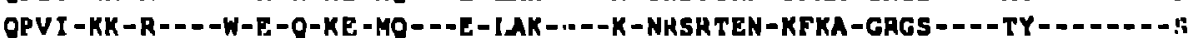

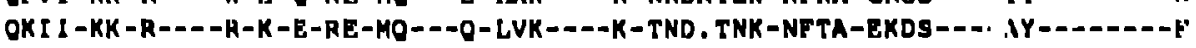

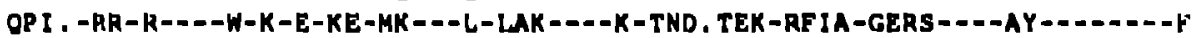

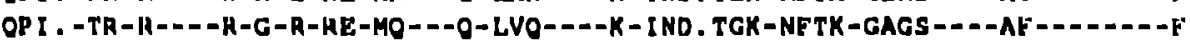

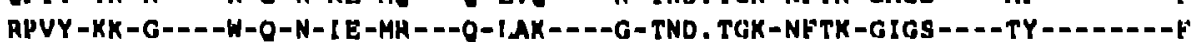

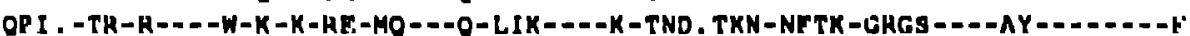

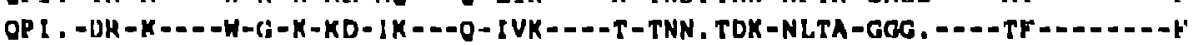

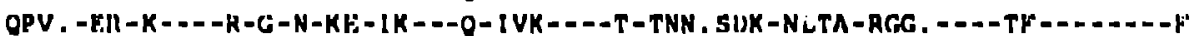

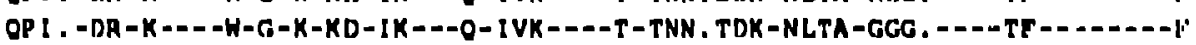

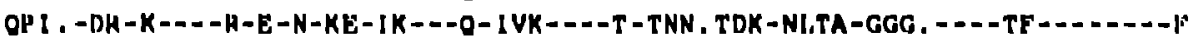

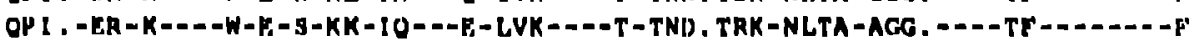

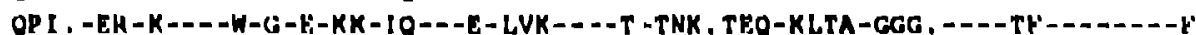


CONSENSUS HIV2ROD HIV2NI H2 HIV2ISY HIV2ST HIV2BEN HIV2D1 94 HIV2GHI SIVMA251 S IVMAI 42 5 IVNA2 39 SIVANE SIVSMMHA SIVSMMPBJ

CONSENSUS HIV2ROD HIV2NIH2 HIV2ISY HIV2ST HIV2BEN HIV2DI 94 HIV2GHI SIVMA251 SIVMMI 42 SIVMA2 39 SIVMNE SIVSMAH4 SIVSMMPBJ

CONSENSUS HIV2ROD HIVZNIHZ HIVZISY IIIV2ST HIV2BEN HIV2D1 94 HIVZGHI SIVMY23I SIVM41 42 SIVM42 39 SI UM.NE SI VSHAII4 i IVSAMPIJJ

CONSFNSUS HIVZADD IITVINIIIZ HIVZISY IIVZST IITZREH IIrVzo194 III vorill SI VMA2sI DI VHM I A? : I VMMว 19 i I VMNE: iI V:IMMIIA 'il V:IMmI'H.I
...

$-$

$\ldots$

LYC?M?WFLNWUEnT???????? ???kEq?? INYVPChI rO! INTWHKVGKNVYLPPROGdLECnSTVT

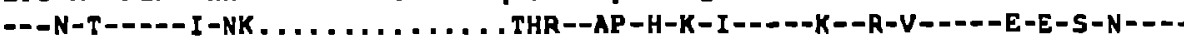

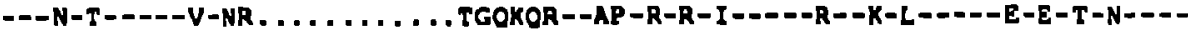

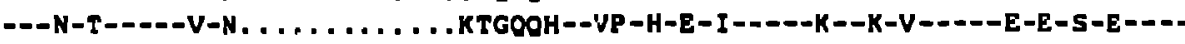

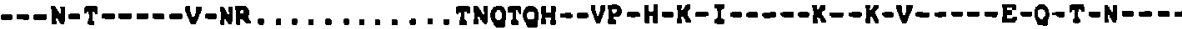

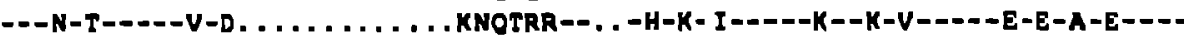

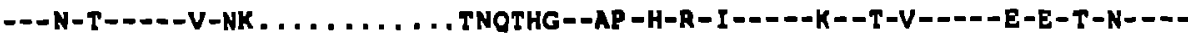

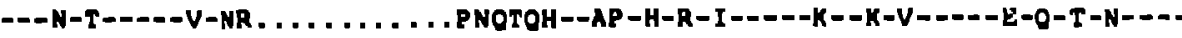

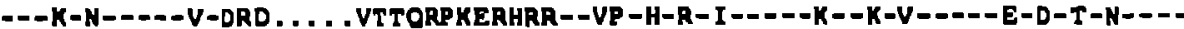

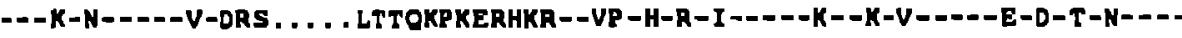

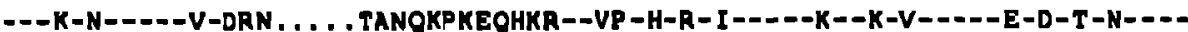
$---K-N-----V-D K N L . .$. TGTTOKPOERHKR--VP-H-R-I-D-..-K--R-V-D---E-D-T-N--

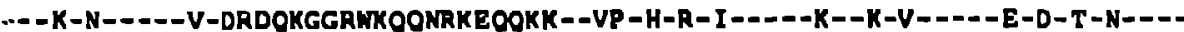

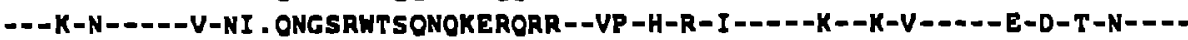

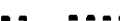

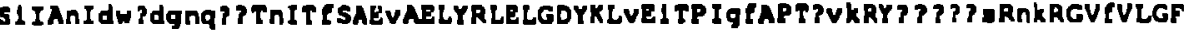

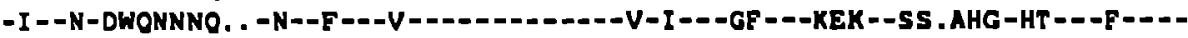

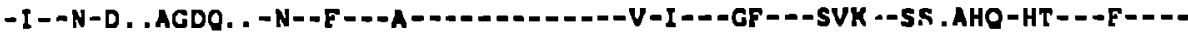

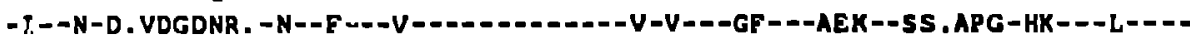
-I - -N-D. GGENO. . -N- -F---V-D-_-

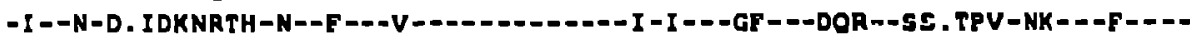

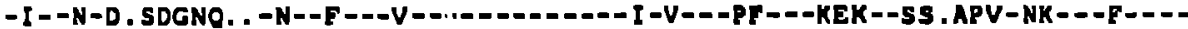
-I - -N-D. VNSNO. . -N--F---V-D-_-

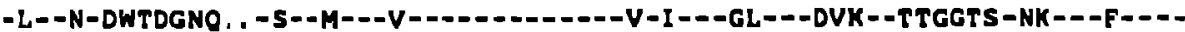
-L--N-NWTDGNO. , -S--M---V------------VV-I---GL---NVK--TTGGTS-NK---F---

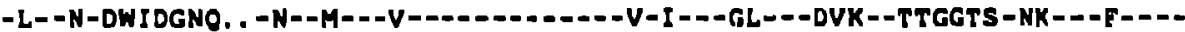

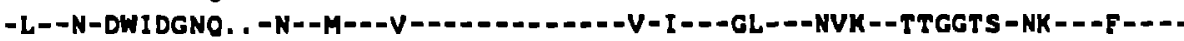

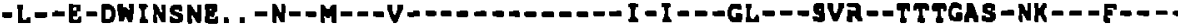

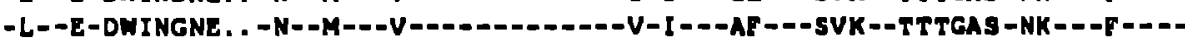

LGF LATAG AAMGAS ITI AAOSRTLI AGI VOOOOOLLOVKROOE I LRLTVWGE KNLO?RVEA IEKYLKD

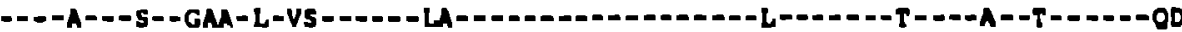

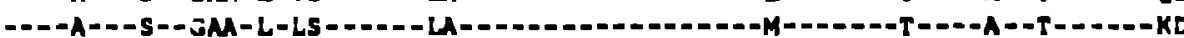

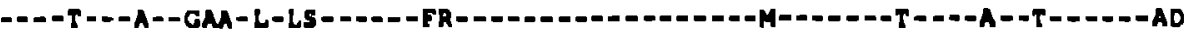

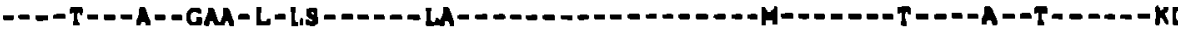

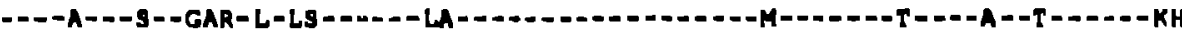

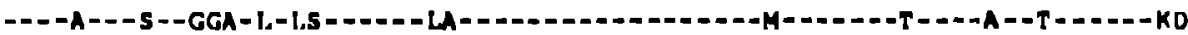

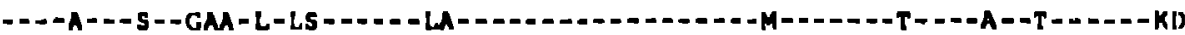

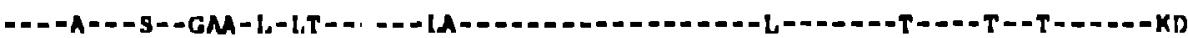

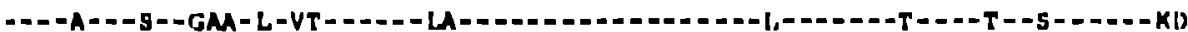

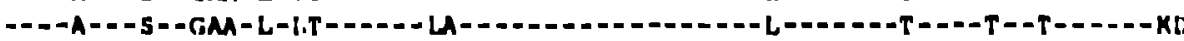
-.--A---S- -

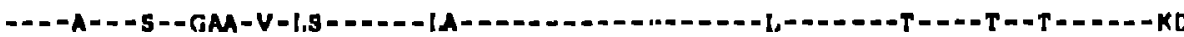

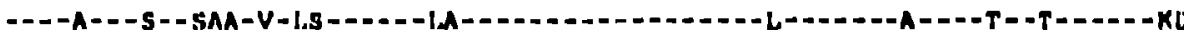

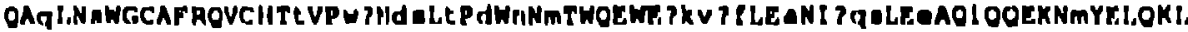

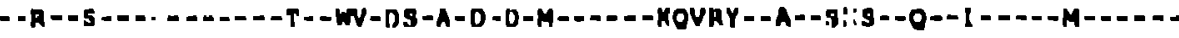

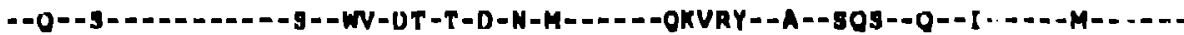

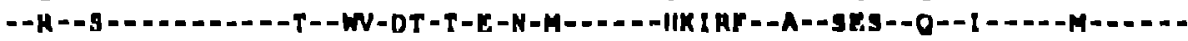

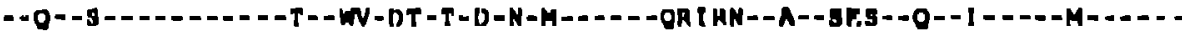
- -O- - I] - - -

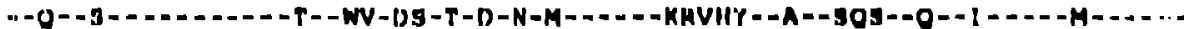

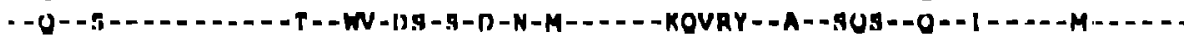

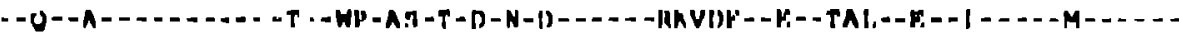

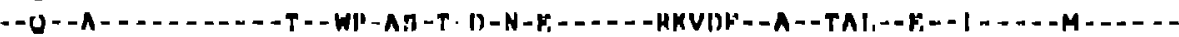

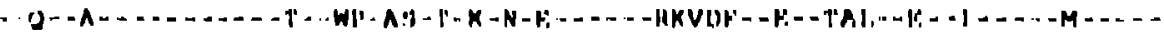

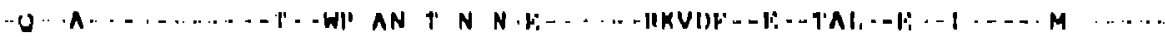

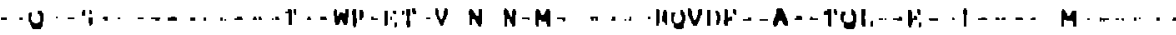
M- 
CONSENSUS HIV 2ROD HIV 2 NIHZ HIV2ISY HIV2ST HIV 2BEN HIV2DI 94 HIV 2GH1 SIVMM251 SIVMMI 42 SIVMM2 39 SIVMNE SIVSMMH4 SIVSMMPBJ

CONSENSUS HIV 2ROD HIV2NIHZ HIV 2ISY HIV2ST HIV 2BEN HIV2DI 94 HIV 2GHI SIVMM239 SIVMNE SIVSMMH4 SIVSMMPBJ

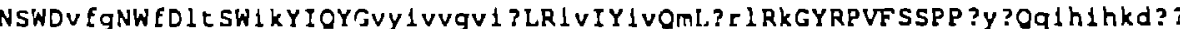

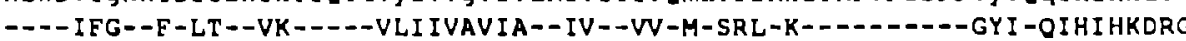

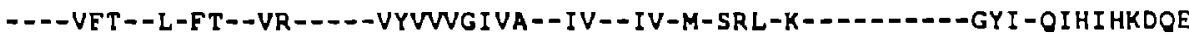
----VFG--F-LT--IK-----VMIVVGIVA--IV--VV-M-SRL-K----------GYI-OIHIHKDWE ----VEG--F-LT--IK---_-VYIVVGI IV--IV--VV-M-SRL-K---------AYF-OIHIHKDRE ----ILG--F-LT--VK-----VHIVVGI IA--IA--VV-L-SRF-K-_-_-_-_-GYL-OIHI HKDRG ----VFG--F-LT--IK-----VY IVIGI IG--IA--IV-L-SRL-K--_------GYL-QIHI I TDRG ----VFG--F-LT--IK-----VYIVVGVIV--IA--IV-L-SRL-K---------GYL-OIHIHTDRG ----VFG--F-LA--IK-----IYVVVGVIL--IV--IV-M-A:IL-0---.--0--SYF-" ----VFG--F-LT--IK-----IYI IVGVIL--IV--IV-M-ARL-0-_-...--_-SYF-* -.--VEG--F-LA--IK-...-VYY IVVGVIL--IV--IV-M-AKL-O--..------SYF-OTHIOODPA

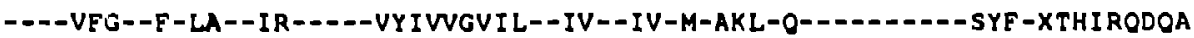
----IFG--F-LT--IR---_-VLIVLGVIG--IV--VV-M-ARL-O-_-_-_---AYV-OIP IHKGOE ---IFG--F-LT--IK-----VLIVLGVIG--IV--VV-M-ARL-O--_---_---AYV-OIPIOTGOE

<- tat cds

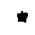

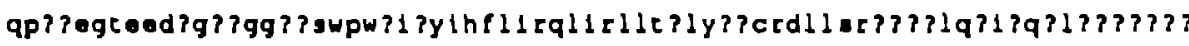
OPANEETEEDGGSNGGDRYWPWP I AYIHFLIROLIRLLTALYS ICRDLLSRSELTLOLI YONL . . . . . . OPAREETEEDVGSNGGDRSWPWP I AYI HF LI RLLIRLLTGLYNI CRDLLSR I SP ILOP IFOSLQRALTA I OPDREETEEDVGNDVGSRSWPWP I EY I HFLI ALLIR LLTALYNSCRDLLSR . . . LYLI LOP L . . . . . . OPAREE TEEDVGNSVGDNWWPWP I RY I HFL I ROL I R LLNRLYN I CRDLLSRSFOT LQL I SOSLRRALTAV OPANEGTEEDVGGDSGYDLWPWP I NYVQFLI HLLTRLLI GLYNI CRDLLSKNSPTRRLI SOSL . . . . TAI OPANEETEEDAGDDSGEGLWPWP LNYIOFL IHLLTRLLTGLYNSCRGLLSKNSPTRRLISOSL. . . . TA I OPANEGTEEDDRDDDGYDL*

LPTREGKERDGGEGGGNSSWPWOIEYIHFLI ROLIR LLTWLF SNCRTLLSRVYOI LOP I LORLSATLOR LPTKEGTEGDGGGSGGNSSWPWOIEY I HFLIROLIRLLTWLFSNCRTLLSRAYOILOP IFORFST TLOK : PPTKEGEEGDGGDRGGSRSWPWOIEYI HFLI ROLIRLLTWLFSSCRDWLLRSYOI LOPVLOSLSTTLORV LP TKEGEEGDGGGRGGNRSWPWOI EY I HFLI ROL I R LLTWLF SSCRDWLLRNCOTLOPVLOS LSRT LORA

$n \in f \rightarrow$ c- cov cde

CONSENSUS HIV 2ROD IIIV2NIHZ HIVZISY IIIV2ST HIV 2 BEN IIIV20194 SIVMM239 STVMNE SIVSMMHA SIVSMMPBS

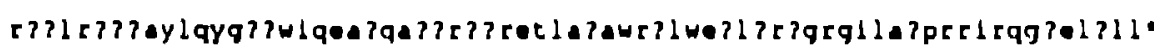
RDWLRLRTAF LOYC :EW IOEAFOAAARATKETIAGACRGLWRVLERIGRGI LAVPRR IROGAE I ALLRDWLRLKAAYLOYGCEW I GEAFOALARTTRETLAGAGRDLWRALOR I GRG I LAVPRR I ROGAE LA LL RDWL.HLKAAYLOYGCEW I OEAFOA LAHVTHETLTSAGR S LWGALGR IGRG I LAVPRR I ROGAE IALLRDWLRFNTAYLOYGGEW IOEAFRAFARATGETLTNAWRGFWGTLGOIGRG I LAVP HR IROGAE IAI.L RDWLRLKAAOLOYGCEW IOEAFOAI ARTTRETLAGAWGWLWEAARR IGRG I LAVPRR I ROGAE LAI.L.RDWLIRLKAAYLOYGCEW I OEAFRAFAHTAH:T I MIAWHGLCEAAOR I GRG ILAVPRR I ROGAE IALL REVI,RTELTYL.OYGWSYFHEAVOAVWRSATRTLAGAWGDL,WETL.HRGGRWILAIPRH IHOGLELTLL REVLATELTYLOYGWSYFOFAVOVAWASATH:TLAGAWGDL.WETLGRVGRW ILA I PRR I ROGLELTLLREVIHIE. IAYLOYGWHYFOEAVOAWWKI,AHETIASAWGI)IWETL,GRVGHG ILAIPRR IHOGLEI,TLI,-

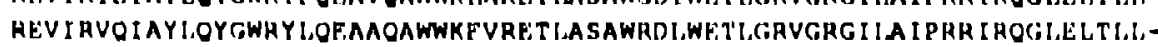


Information dense stretches 10.8 or greaterl are Indlcated by "w".

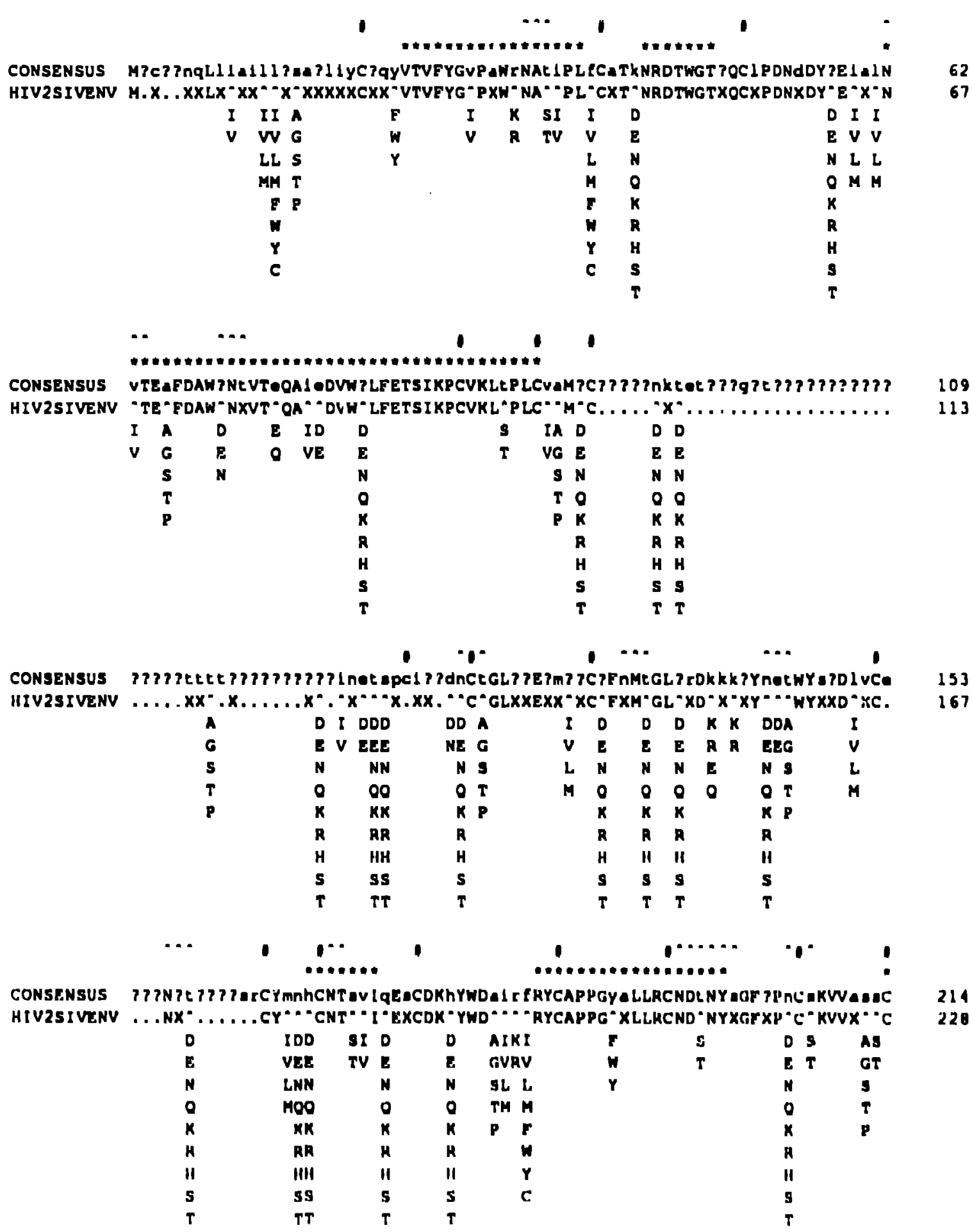




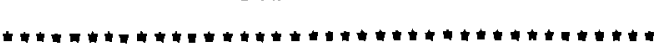

CONSENSUS TRMMETOLSTWfGFNGTRAENRTYIYWHgIDNRTIISLNKYYNLEM?CIRPGNKEVIPIt?mSGIVEHS?

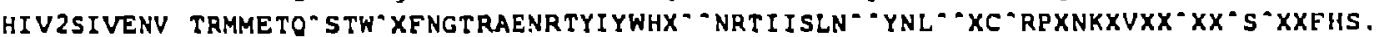

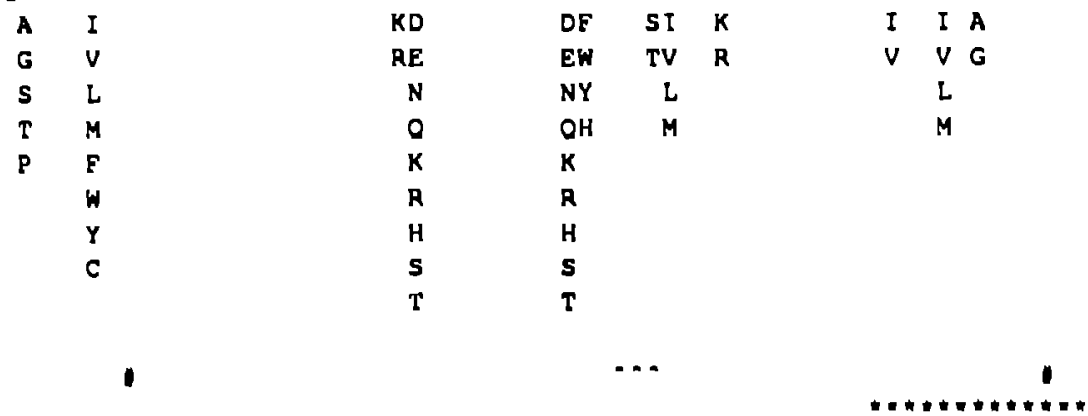

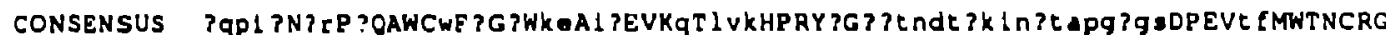

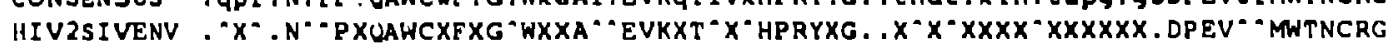

$\begin{array}{lll}K \text { I } & \text { DK } \\ R \text { V } & \text { ER } \\ \text { E } & \text { N } \\ \text { O } & O \\ & & K \\ & & R \\ & & H \\ & & \text { S } \\ & & \text { T }\end{array}$

$\begin{array}{ll}\text { IK } & I \\ \text { VR } & V \\ \text { LE } & \text { L } \\ \text { MO } & M\end{array}$

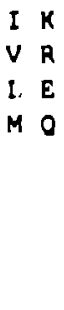

$\begin{array}{ll}\text { D } & S \\ \text { E } & T \\ \text { N } & \\ \text { O } & \\ \text { K } & \\ \text { R } & \\ \text { H } & \\ \text { S } & \\ \text { T } & \end{array}$

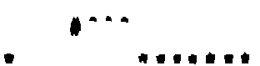

1

AF GW

$I$
V
L
$M$
$E$
$W$
$Y$
$C$

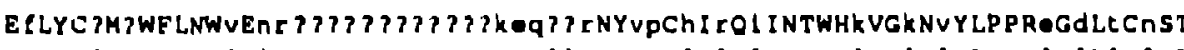

CONSENSUS

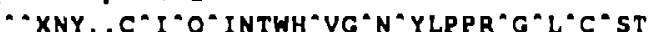
HIV2SIVENV EXLYC ${ }^{\circ}$ "WFLNW'E

$\begin{array}{ll}\text { DD } & \text { D K I } \\ \text { FE } & \text { E R V } \\ \text { NN } & \text { N E } \\ \text { OO } & O O \\ \text { KK } & K \\ \text { RR } & \text { R } \\ \text { IIH } & H \\ \text { SS } & \text { S } \\ \text { TT } & T\end{array}$

$\begin{array}{lll}K & D & I \\ R & E & V \\ N & L \\ O & M \\ K & \\ H & \\ & \text { II } \\ \text { S } & \\ \text { T }\end{array}$

E D A D
E E
N N
00
$H \mathrm{~B}$
II H
$5 \mathrm{~s}$

V $\mathrm{N}$

O E G E

TT

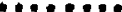

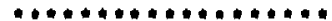

$N S N$

O T

K P

II

H

s

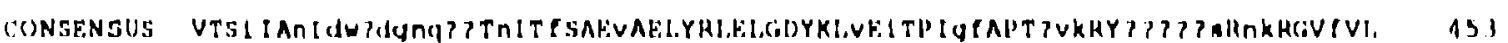

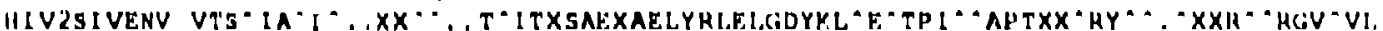

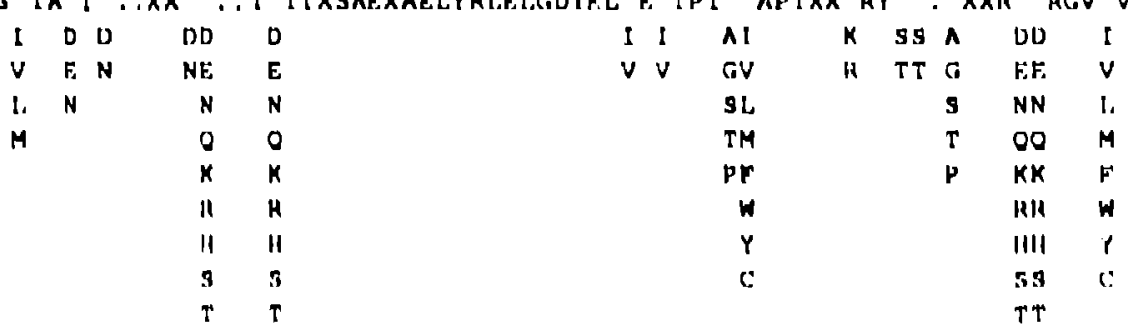




\section{HIV2/SIV ENV}

CONSENSUS GFLGFLATAGSAMgIASITISAOSRTLLAGIVOOOOOLLDVVKROOEILRLTVWGEKNLQ PAVLAIEKYL

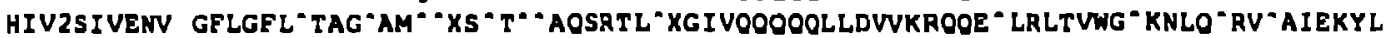

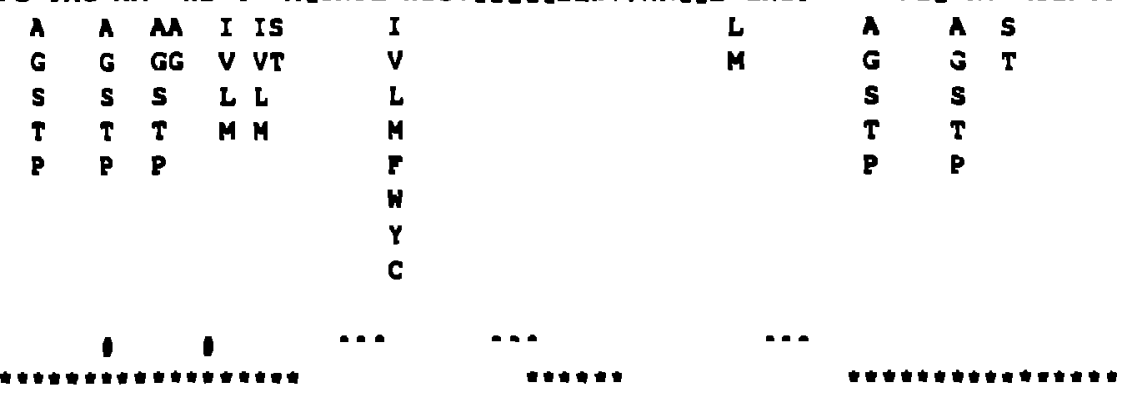

CONSENSUS KdOAQLNSWGCAFROVCHTEVPU ?NA SLEP DWNNmTWOEWE TKV? LLEANI TQSLECAOI OOEKNmYELO

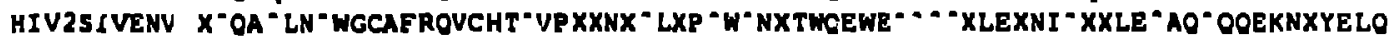

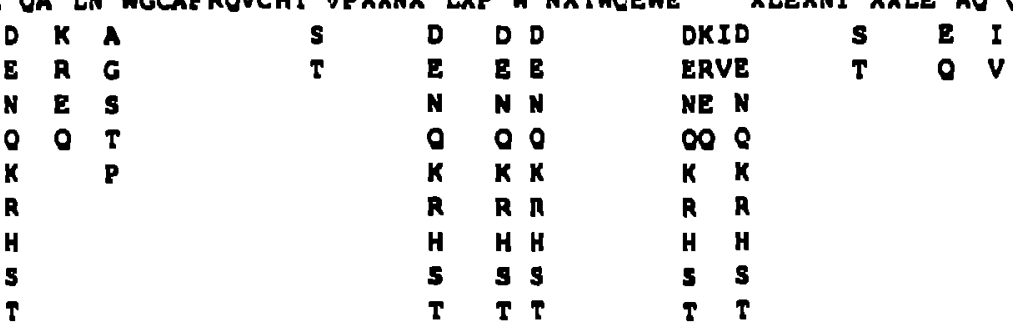

\#.....

learly otop

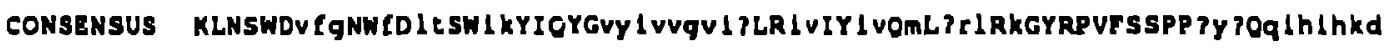

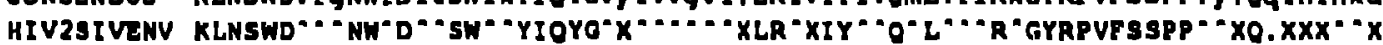

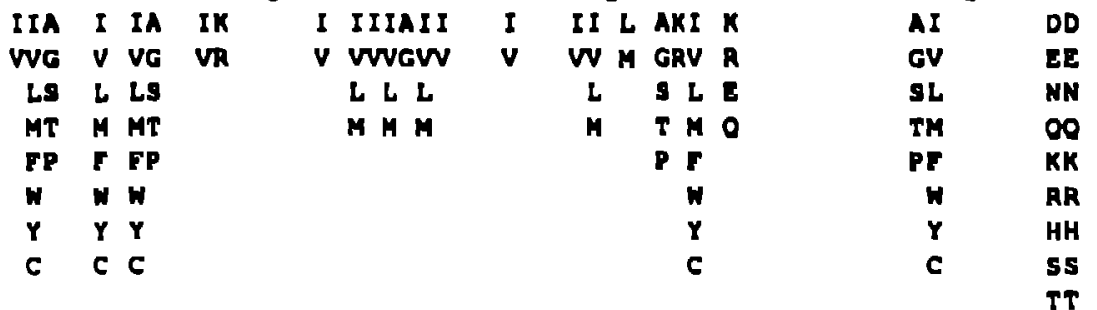

Cunsensus

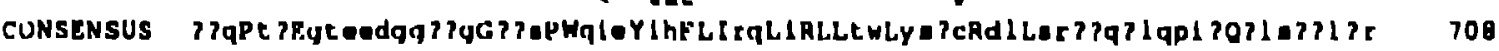

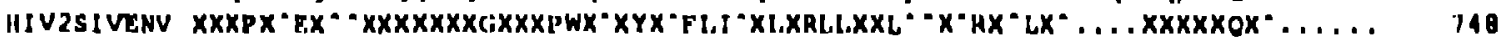

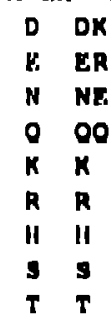

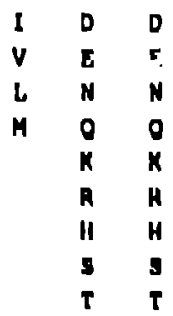

$\begin{array}{llll}\text { FO } & I & I & K \\ \text { WE } & V & V & H \\ \text { YN } & L & L & \\ \text { O } & \text { H } & \text { M } \\ \text { K } & \text { Y } & \text { Y } \\ \text { A } & \text { W } & \text { W } \\ \text { H } & Y & Y \\ \text { S } & C & C & \\ T & & \end{array}$

I
V
L
H
F
Y
C




\section{HIV2/SIV ENV}

$$
<-\operatorname{nef}->\text {, }
$$

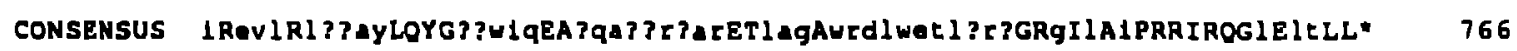

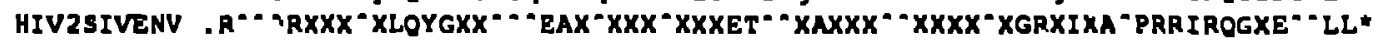

815

$\begin{array}{cccccccccc}\text { DII } & \text { A } & \text { FID } & \text { K } & \text { K } & \text { IA } & \text { II } & \text { K } & \text { I } & \text { IA } \\ \text { EVV } & \text { G } & \text { WVE } & \text { R } & \text { R } & \text { VG } & \text { WV } & \text { R } & \text { V } & \text { VG } \\ \text { LL } & \text { S } & \text { YLN } & \text { E } & & \text { LS } & \text { LL } & \text { E } & & \text { LS } \\ \text { MM } & \text { T } & \text { MO } & \text { O } & & \text { MT } & \text { MM } & \text { O } & & \text { MT } \\ \text { F } & \text { P } & \text { FK } & & & \text { P } & \text { FF } & & & \text { P } \\ \text { W } & & \text { WR } & & & & \text { WW } & & & \\ \text { Y } & & \text { YH } & & & \text { YY } & & & \\ \text { C } & & \text { CS } & & & \text { CC } & & & \end{array}$


CONSENSUS SIVAGMTYO SIVAGMIS5 SIVAGM 3 SIVAGH677

CONSENSUS SIVAGMTYO SIUAGMIS.5 SIVAGH 3 SIVAGM 677

CONSENSUS SIVAGMTYO SIVAGHIS5 SIVAGM SIVAGM 677

CONSENSUS SIVAGMTYO SIVAGH 55 SIVAGH3 SIVAGH 677

CONSENSUS SIVAGMTYO SIVAGK155 SIVAGA3 SIVAGK 677

CONSENSUS SIVAGATYO SIVAGMI 55 SIVAGM3 SIVAGM677

CONSENSUS SIVAGTTYO SIVAGMISS S[VAGM] SIVAGM677

CONSENSUS SIVAGMTYO SIVAGMISS SIVAGM3 SIVAGM677

CONSENSUS SIVAGMTYO SIVAGMI5S SIVAGM SIVAGH 677

CONSENSUS SIVAGMTYO SIVAGimiss GIVACIM 3 IIVACimg II
M??t11?1g1??IGIg1?? sEkqqu?TVEYG?PVWKNSSVOAFCMTPET I IWATTNCIPDDHDYTEVPLN

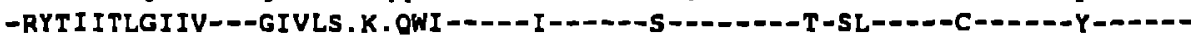

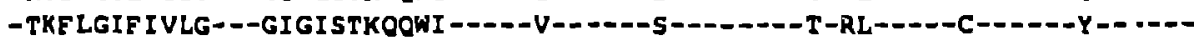

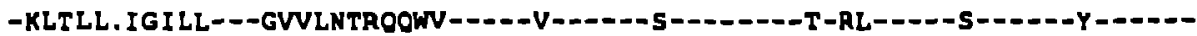

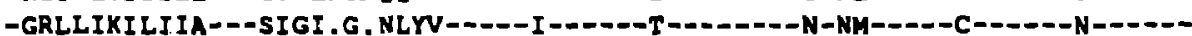

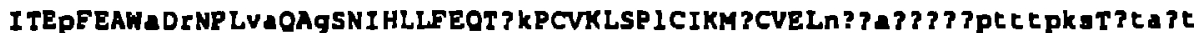
---P----G-R---1A--A----------MK------L----N----NSTRERATTPTTTPKS- . . . .

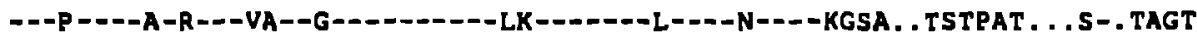

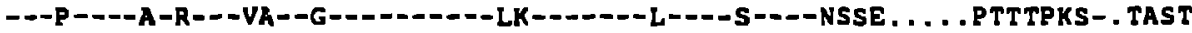

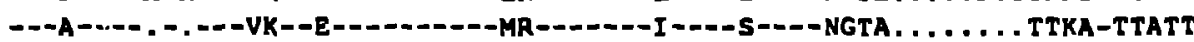

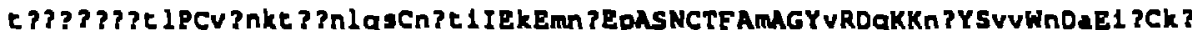
........GL--VGPTSGENLOS-NASI--R-MED-P--..---M---V--O--NY--VV-N-A-IY-KN

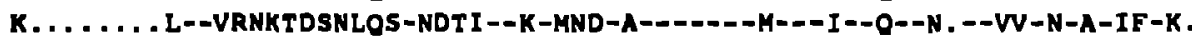
TNITASTTIL--VONKTSTVLES-NETI --K-LNE-P-D---OM---V--O--K. --VV-N-A-IM-K.

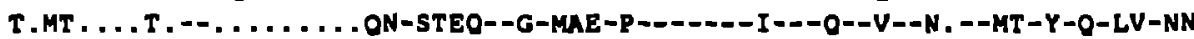

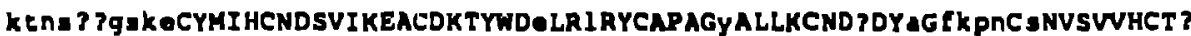
KTNS. . TSKE--_RSTSHNGTKE--_ -

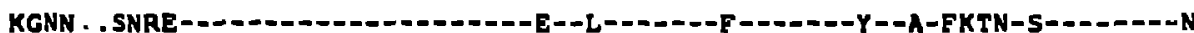
KTGSEKGSKD-_._.-_-_-_-_-_-

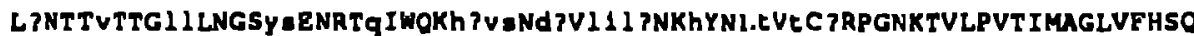

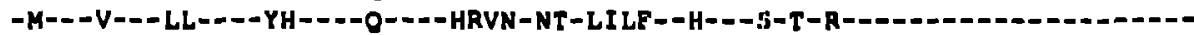

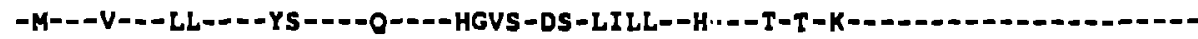

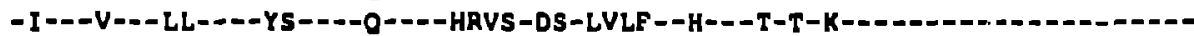

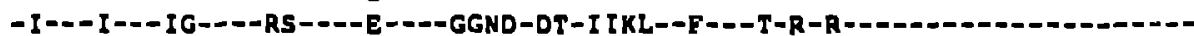

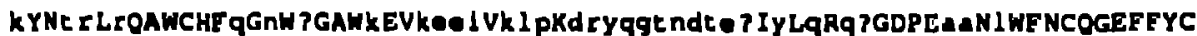
K--MK-R-------E-N-R---R--KOKI -ELP -DRYKGTNNTEH-Y-O-OW----AS-L- -

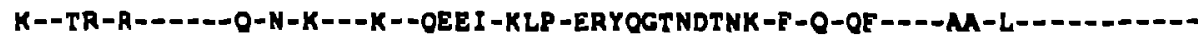

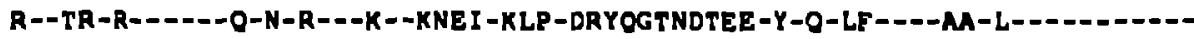
K--TR-K-- -- O-D-K---K--REE. -KKV-N. L. TEVSIEN-H-R-IW----SA-F--

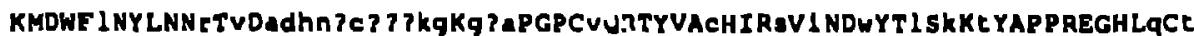

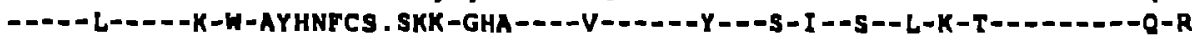

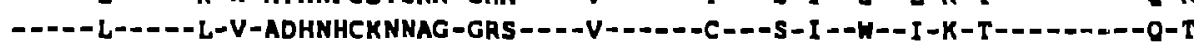

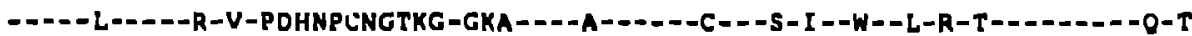

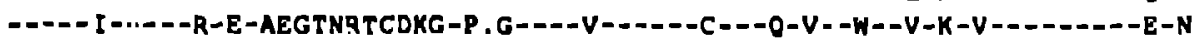

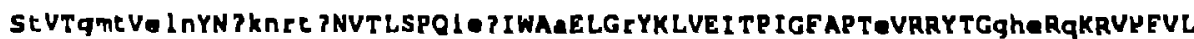

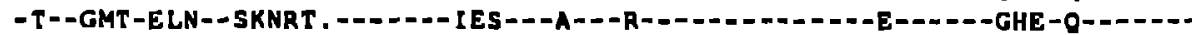

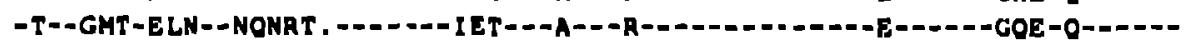

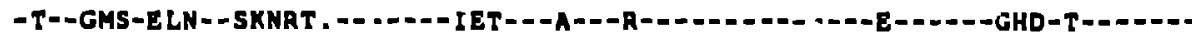

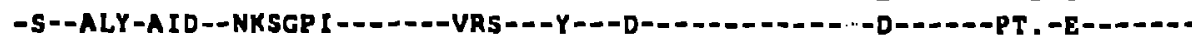

GF LGF LGAAGTAMGAAA ALTVOS ?HLLAGI LOOOKNLLAAVAOOOMLKLT IWGVKN:NARVTALEKYL C-

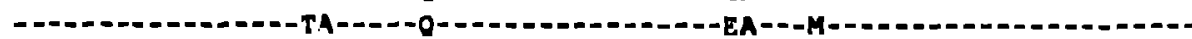

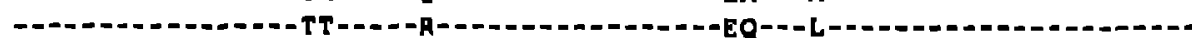

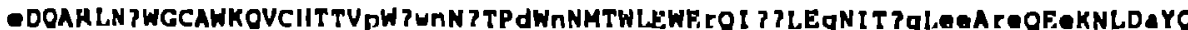
E.----S-D-1

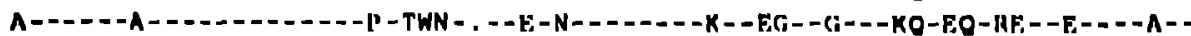
R:-- 
CONSENSUS SIVAGMTYO SIVAGM1 55 SIVAGM 3 SIVAGM677

CONSENSUS SIVAGMTYO SIVAGM155 SIVAGM3 SIVAGM 677

CONSENSUS SIVAGHTYO SIVAGMJ SIVAGH 677
KLs?WSdFWSWEd ESkWLn 1IK1 GFLV?Vg1 IGLRI I YE ?YgCI arVROGYSPLsPOIHIHPW?kg?PDN --TS--D-----DF-K--NIL-M---VIVGI I ---LLYTVYG--VRV----V--S------OVGKGR----SD--S----DE-K--NIL-I---AVIGVI---LLYTLYT--ARV----S--S---_--PW. KGO----SS--D-_---DE-K --NIL-I ---DVLGI I ---LLYTVYS--ARV----SS--S---_--PW. KGO----DD--G-----SL-T--GYV-I---VIVIIL---E AGVLWG--RNI----N--.,-----S.SA'R- --

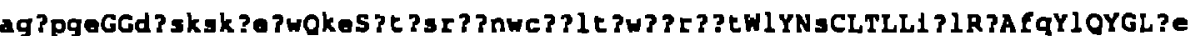
ADEPGE--DNSRIKLESW KO-.K.SR . . . CMOLTAWLTRLNT-L--S-----IOL-K-FO-L-_--AE AGEPEE--RTGKSKSTH*

AEGPGE--DKRKNSSEPW-KE-GTAEWKSNWCKRLTWWCSISSI-L--S-_-_-VHL-S-FO-I----GE GGGODR--ESSSSKLIRL-EE-STPSAINNWTLNFKSCSLRIRT-C--I-----IFI -T-VG-L--- -00

798

LK?AAqQ? ?qALAY ?AqRA ? ?qIWIACRSAYRA I INSPRRVROGLE? ILn"

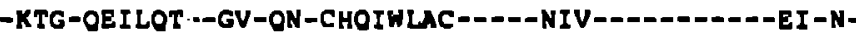
-KAA-OEAVVA- -RL-ON-GYOIWLAC-----AII-_-_--------GI -N-OEA-TGLAOA--RA-RE-WGRLGAIV-_-_-AVI-_.-_-_-_-_KV-G- 
Information dense gtretches $(0.9$ or groater) are Indicated by "*t.

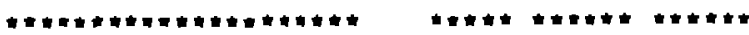

CONSENSUS M??tI1?lg1??IGIg1??3EkqqW?TVEY?PVAKNSIVOAFCHTPETrIWATTNCIPDDHDYTEVPLN

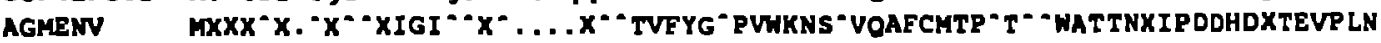

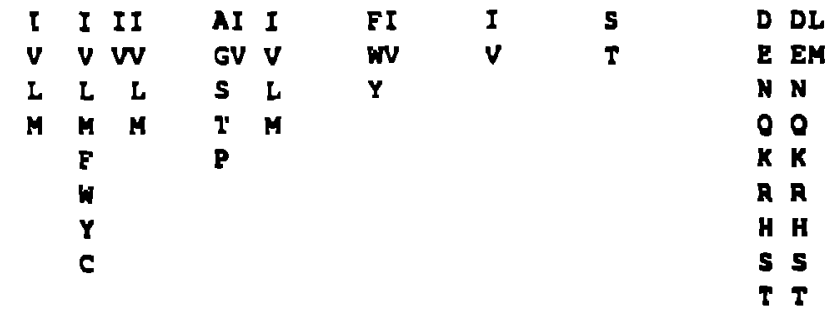

\section{- +*}

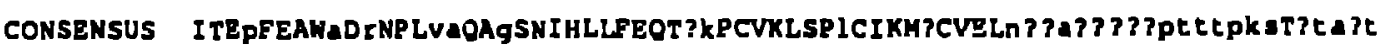
AGRENV ITE" FEAH.D.NPL- XOAXSNIHLLFEOT" - PCVKLSP-CIKH'CVEL"- $x$.

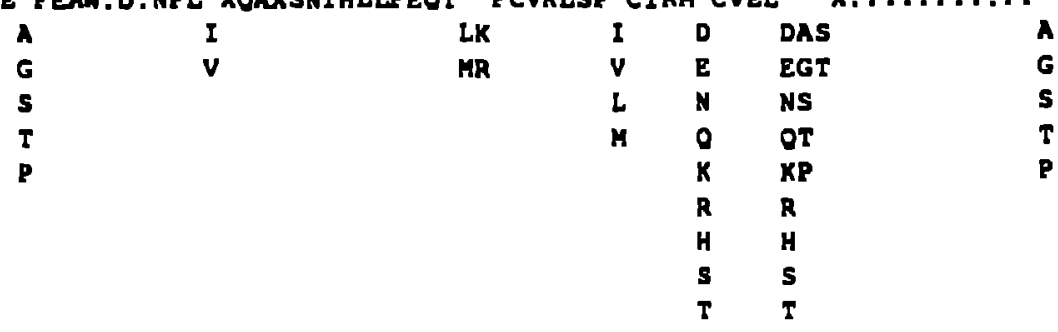
AGMBNV

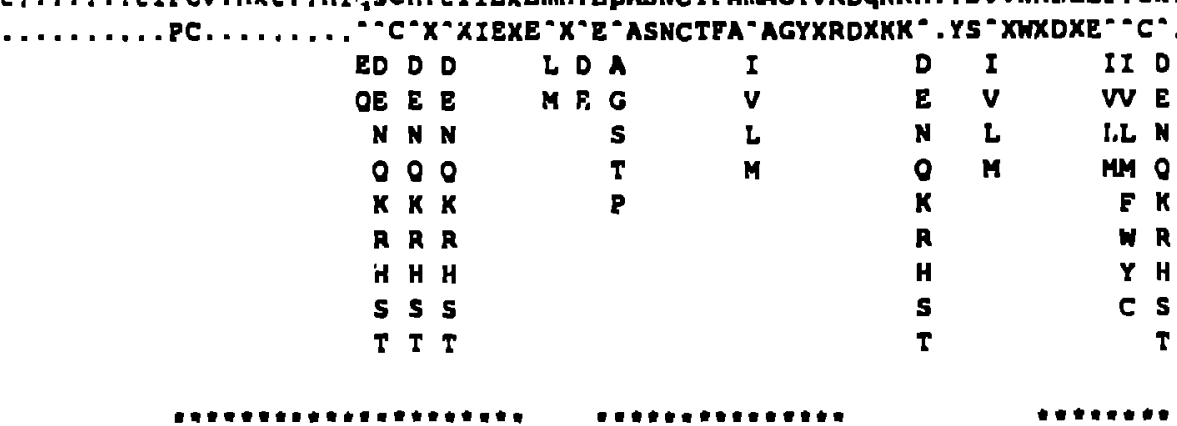

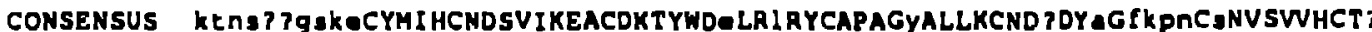
AGMENV

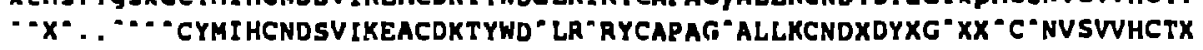


CONSENSUS L?NTTVTTGIILNGSYGFNRT IWOKh?VSND?VI 11 ?NKhYNLEVEC ?RPGNKTVLPVTIMAGLVFHSO

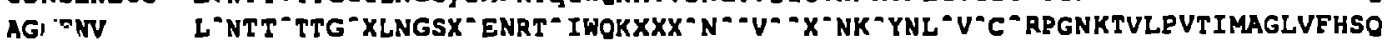

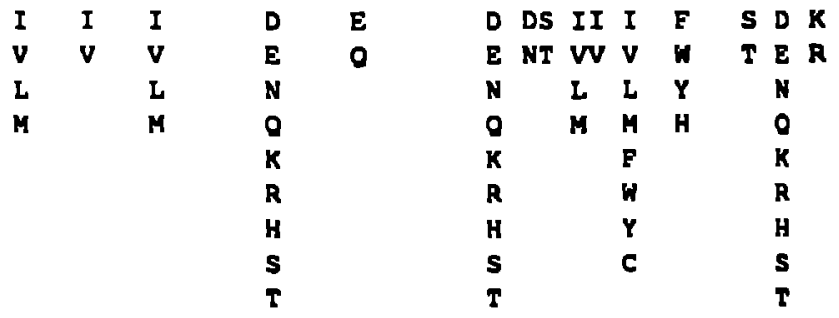

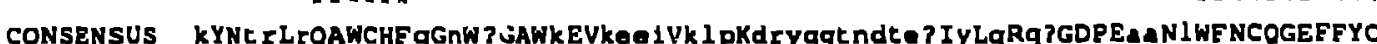
AGMENV

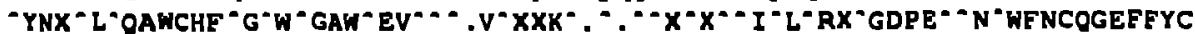
$K \quad K K$ $\begin{array}{llll}\mathbf{E} & \mathbf{D} & \mathbf{K} \\ \mathbf{O} & \mathbf{N} & \mathbf{R}\end{array}$

$\begin{array}{ccc}\text { K } & \text { KDK } & \text { K } \\ \text { R } & \text { RER } & \text { R } \\ \text { ENE } & \text { E } \\ \text { COO } & \mathbf{O} \\ \mathbf{K} & \\ \mathbf{R} & \\ \mathbf{H} & \\ \mathbf{S} & \\ \mathbf{T} & \end{array}$ D I AD D DD F K F
E V GE E EE W R N L SN N NN Y E Y F PK K $K$
H $R R R$
Y H H H
C $S \mathrm{~s} s$ T T $T$ AA I R $\quad \mathbf{R} R$ GG $V$ SS L M TO O O HO TT PP F w

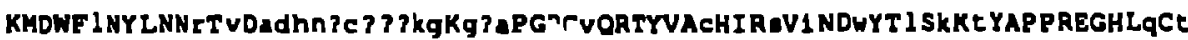

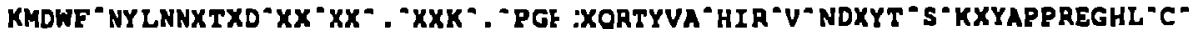

CONSENSUS AGMENV

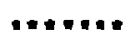

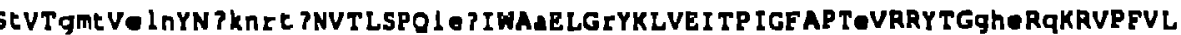

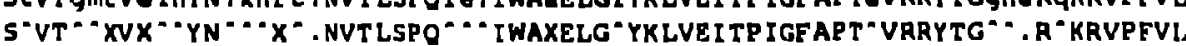

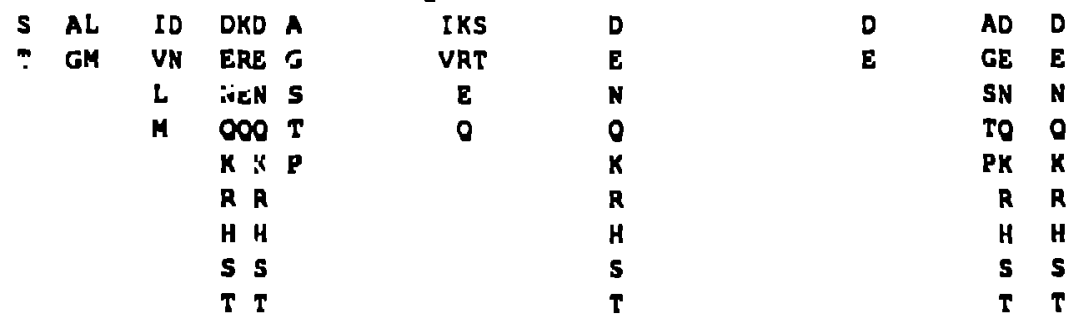




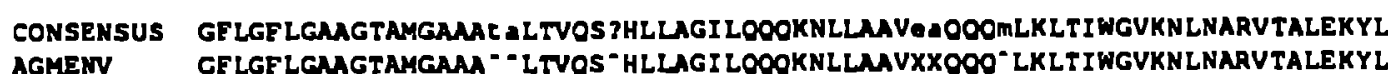
AGMENV

$\begin{array}{ccc}\text { SA } & K \\ \text { TG } & R \\ 5 & E \\ T & 0 \\ \text { P } & \end{array}$

$\mathbf{L}$

$\mathbf{K}$
$\mathbf{R}$
$\mathbf{E}$
$\mathbf{O}$

CONSENSUS AGMENV

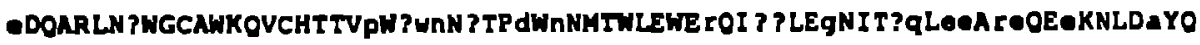

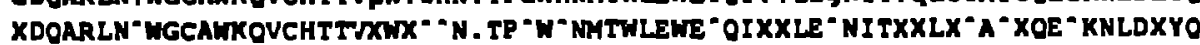

638 646

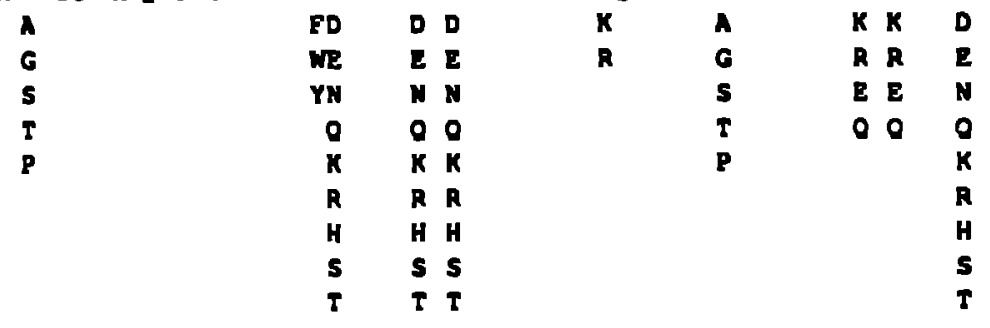

n...

-..... AGMENV

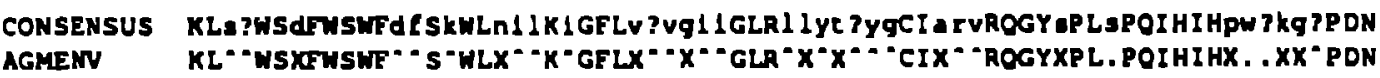

703

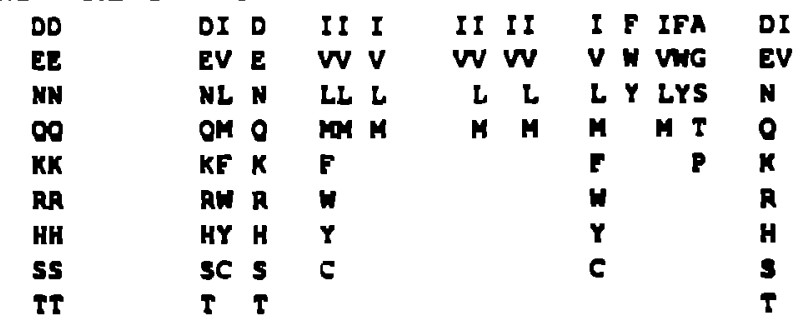

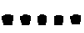
CONGEHSUS
AGNENV

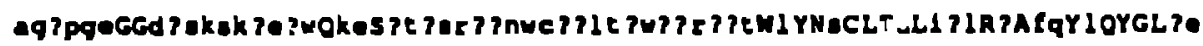

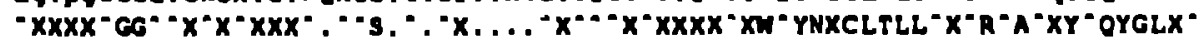

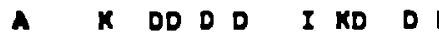

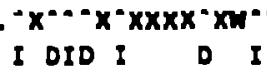

R EE E E V RE E E

$\checkmark$ EVE V

I I D I I

$E$ NN N $N$ LE N N

L NLN L

E v

- 000 MO 0 O

M OMO $\mathrm{M}$

KX $K \times$

V V E V V

L N L L

RA $R \mathbf{R}$

$F$ KFK F

M O M M

K F

HH H H Y

W RWR W

R F

$Y$ HYH Y

R i

C ses $C$ T $Y$

$\begin{array}{lll}H & Y & H Y \\ S & C & S C \\ T & & T\end{array}$

TT T T T T

$\mathbf{T}$

on.

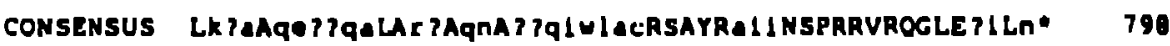
AGMENV

$\begin{array}{llllll}\text { K A } & \text { D } & \text { A } & \text { KO } & \text { KI } & \text { I } \\ \text { R G E E } & \text { G } & \text { RE } & \text { RV } & \text { V } \\ \text { E } & \text { N } & \text { S } & \text { EN } & \text { EL } & \text { L } \\ \text { O } & \text { O } & \text { T } & \text { O } & \text { OM } & \text { M } \\ & \text { K } & \text { P } & & & \text { F } \\ & \text { R } & & & & \text { W } \\ \text { H } & & & & \text { Y } \\ & \text { S } & & & & \text { C } \\ \text { T } & & & & \end{array}$

$\mathbf{8 2 6}$ 


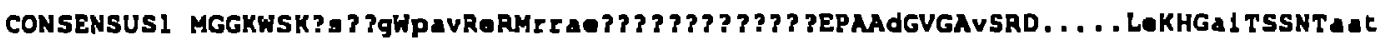
HIVLAI -

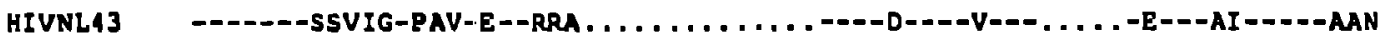

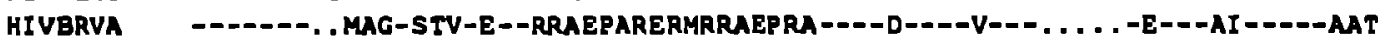

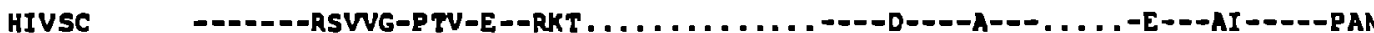

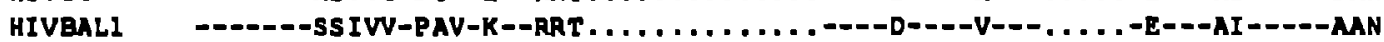

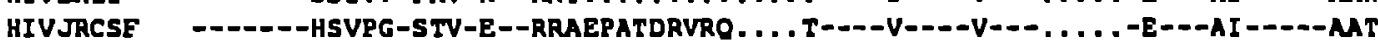

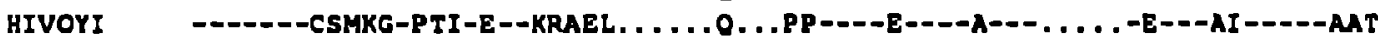

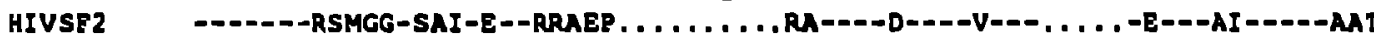

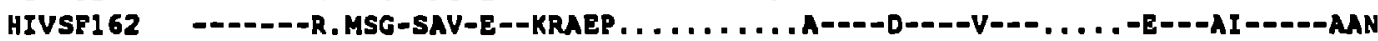
HIVSF33 - 3 -

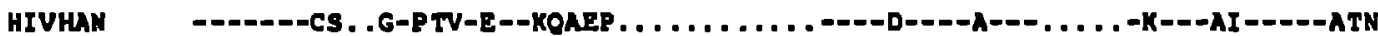

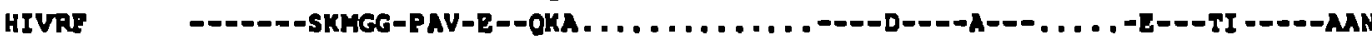

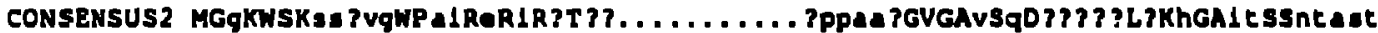

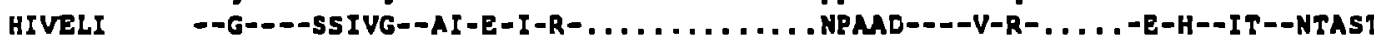

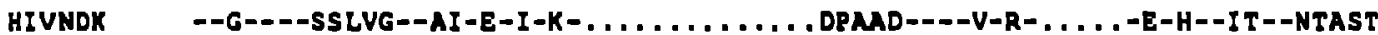

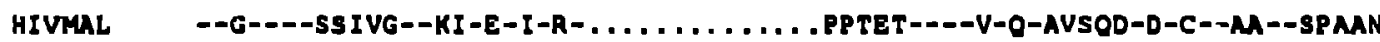

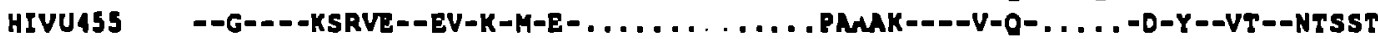

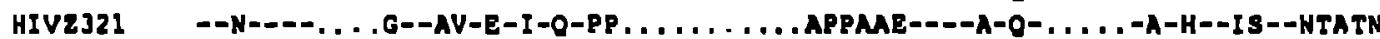

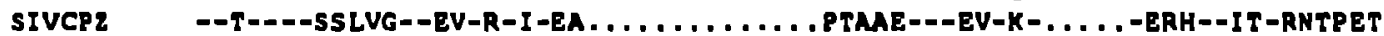

LTR $\rightarrow$

CONSENSUSI NAdCAWLEAq ? ?EdEOVGFPVIPOVPLRPMTYKAA ?DI SHF IKUKGGLEGII ?SqKROdI LDLW1 YHTOC

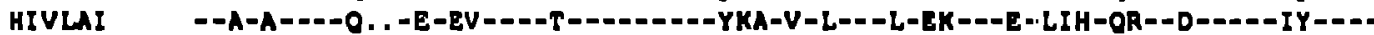

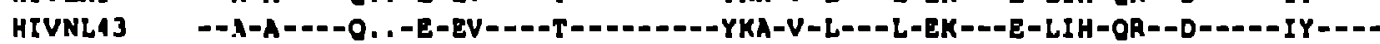

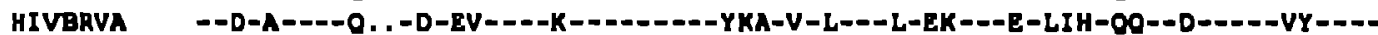

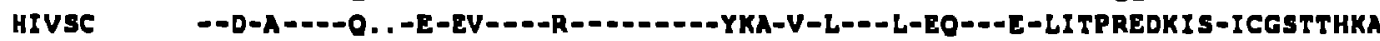

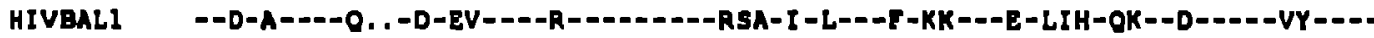

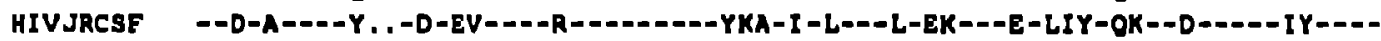

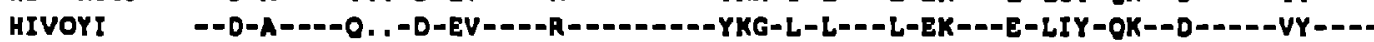

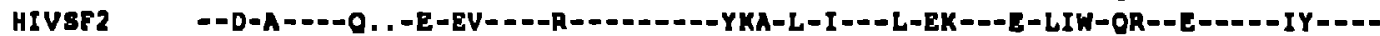

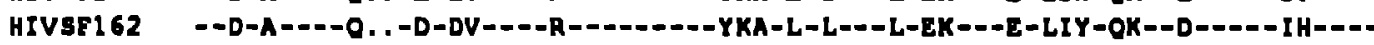
HIVBF 33 HIVHAN HIVRF

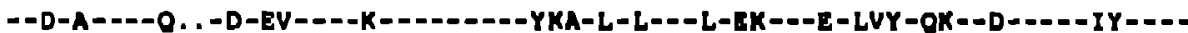

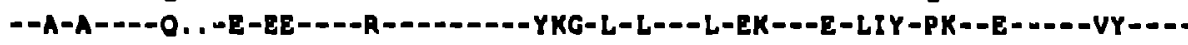

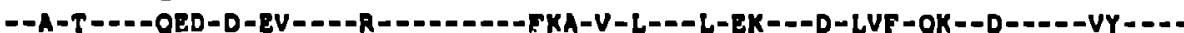

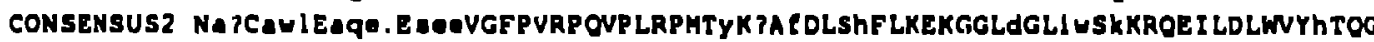
HIVELI HIVNDK HIVMAL HIVU455 HIVZ32!

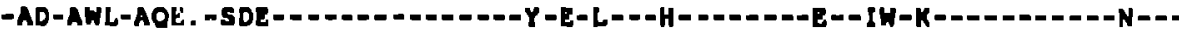

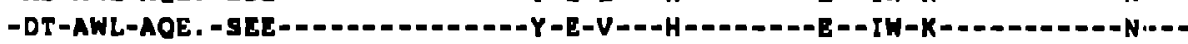

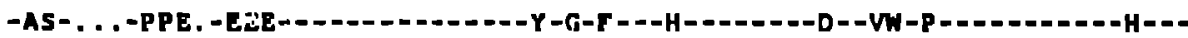

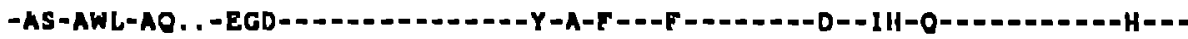

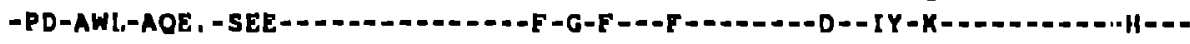
sivCPz 
-- max HIV2/SIV nef similaricy -- -- LTR NFAT-1 region --

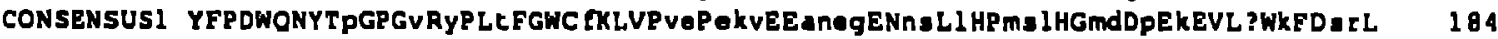

HIVLAI -

HIVNL4 3 --D-D-P---V-Y--T----Y----VE-DKV--ANKG--TS-L--VSL--MD-P-R---E-R--SR- 189

HIVBRVA - -------P---V-Y--T----F----VE-EKI--ANEG--NS-L--MSO--MD-P-R---O-R--SR-

HIVSC TSLIGRTTHO-O-SDI--C----F----VK-EKI--ANEG--NS-L--MSL--ME-P-R-.-E-R--NR-

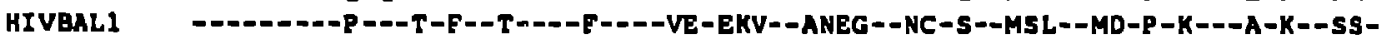

HIVJRCSF -

HIVOYI $\quad---D--P---I-Y--C----F----M D-D O V--A N E G--N 5-L--I S L--M D-P-K---V-K--S R-$

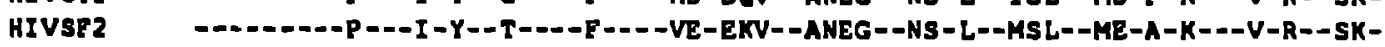

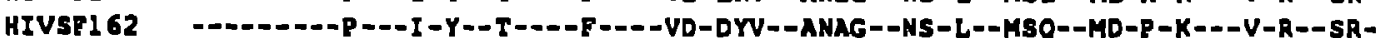

HIVSF33 --.-1--P---V-F--T----F----VE-EKV--ANEG--HS-L--MSL--ME-P-K---V-K--SH-

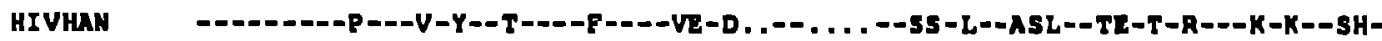

HIVRF - -

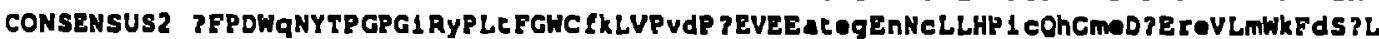

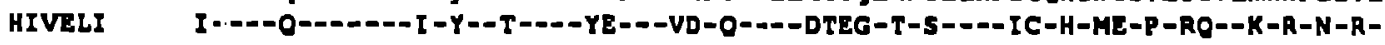

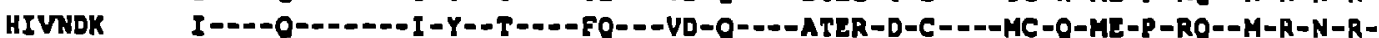

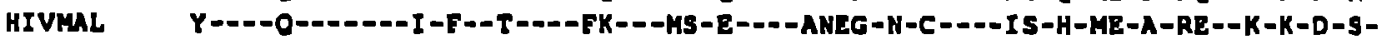

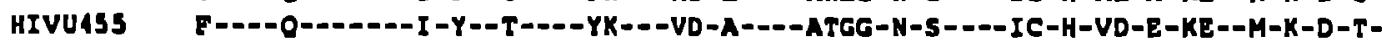

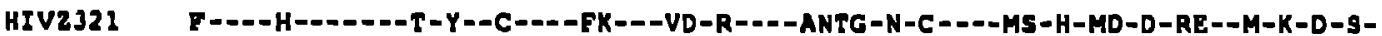
SIVCP2 F---O-O-T--T-F--C-N-FK---LTEEO--OANEGDN-C----IC-H-ME-EDKE--V-R-D-R-

-- NRF -.

CONSENSUSI A ThHMAROLHPEYYKdC? 201

HIVLAI -FH-V--EL--YY-N-E 206

HIVHLA3 -FH-V--EL-D-YF-N-O 206

HIVBRVA $-F H-M--E L,--Y Y-N-A \quad 218$

HIVSC $\quad$ FH-M-ODL-O-YY-D-L 207

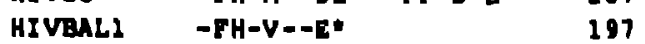

HIVJRCSF -LH-V--EL---YY-D-E 216

HIVOYI -FR-M--EV-D-YY-D-A 211

HIVST2 -FH-M--EL-DYYY-D-O 210

HIVSF1 62 -FH-M--EL-DYY-D-" 200

HIVSF3] -FR-M--EL-D-YY-D-D 205

HIVHAN $\quad$ FH-K- -EL-D-YY-D-D 200

HIVRF -FH-V--EK---YY-D-A 200

CONSENSUSZ AL PHTARETHPECYKAT* 193

HIVELI -FE-K-R-M-D-F-N"N 206

HIVNDK -LE-K-R-L-D-F-ODC 207

IIIVMAL $\quad-L R-R-R-O--Y--D C=\quad 209$

IIIVUASS $\quad-L K-R-Y-1,--P--D=\quad 205$

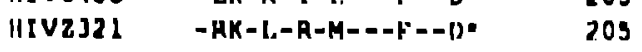

SIVCPZ $\quad-L R-I-R-O---Y--D$ Q 205 
Information dense etretches $(0.8$ or greater) are Indicated by *t".

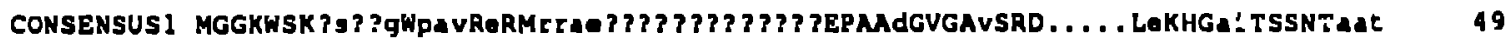

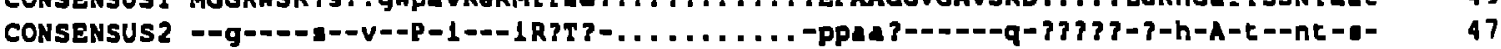

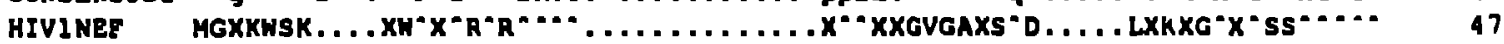

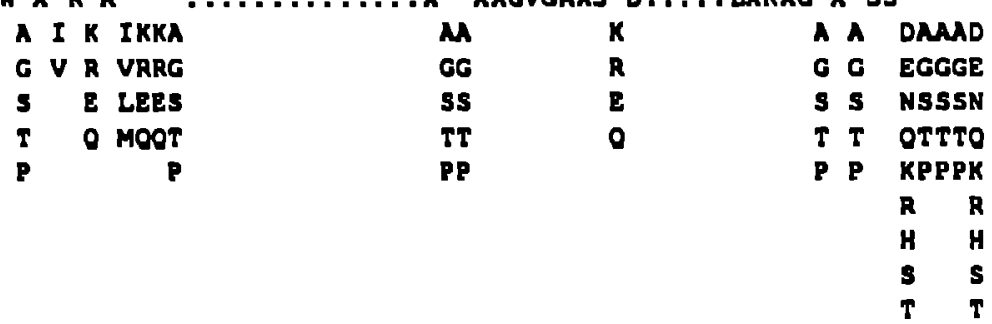

LTR $\rightarrow$

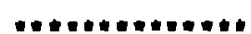

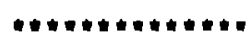

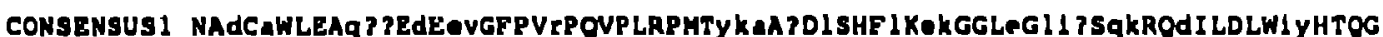

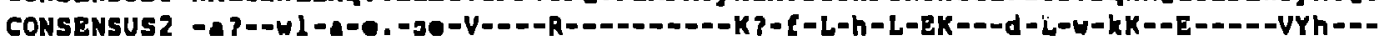

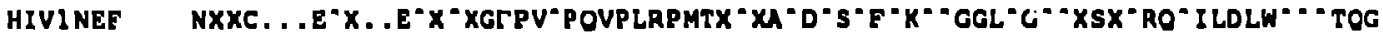

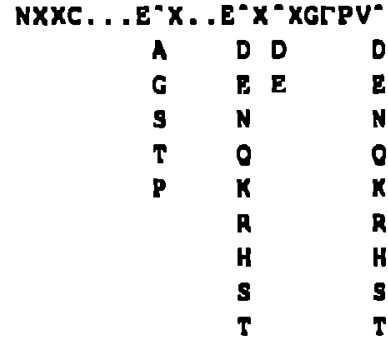

$\begin{array}{llllll}D & I & \text { I } & \text { F } & \text { I } & \text { KK } \\ \text { E } & \text { V } & V & \text { U } & \text { V } & \text { RR } \\ \text { N } & \text { L } & \text { L } & \text { Y } & \text { L } & \text { EE } \\ \text { O } & \text { H } & \text { H } & \text { H } & \text { M } & \text { OO } \\ \text { K } & \text { F } & & \text { T } \\ \text { R } & \text { W } & & \text { W } \\ \text { H } & \text { Y } & & \text { Y } \\ \text { S } & \text { C } & & \text { C } \\ \text { T } & & & \end{array}$

$\begin{array}{llll}\text { D I I } & \text { K } & \text { D } & \text { IFD } \\ \text { E W } & \text { R } & \text { E } & \text { VNE } \\ \text { L } & \text { E } & & \text { YN } \\ \text { M } & \text { Q } & & \text { HO } \\ & & & \text { R } \\ & & \text { H } \\ & & \text { S } \\ & & \end{array}$

\footnotetext{
- max HIVz/gIV nef elmllarlty --
}

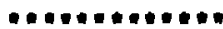

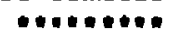

- LTR NFAT-1 reqlon --

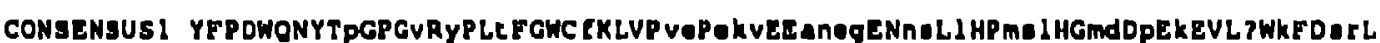

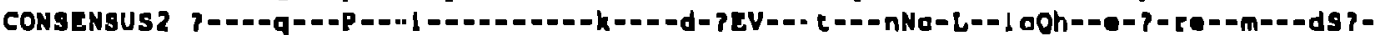
HIVINEF

\begin{tabular}{|c|c|c|c|c|c|c|c|c|c|c|c|}
\hline I & D & $\mathbf{A}$ & FA & FK & I0 & D & D & D & KE & $\mathbf{K}$ & DOD \\
\hline$v$ & $\boldsymbol{v}$ & 0 & WG & WA & VE & $\mathbf{E}$ & $\mathbf{E}$ & E & RO & $\mathbf{R}$ & NEE \\
\hline L & N & $\mathbf{J}$ & YS & YE & LN & $\mathbf{N}$ & $\mathbf{N}$ & & & & NN \\
\hline M & 0 & $T$ & $\mathbf{T}$ & 0 & MO & 0 & 0 & & & & 00 \\
\hline $\mathbf{r}$ & K & $\mathbf{P}$ & $\mathbf{P}$ & & K & $\mathbf{K}$ & K & & & & $\mathbf{K K}$ \\
\hline$w$ & II & & & & $\mathbf{R}$ & H & H & & & & HR \\
\hline 8 & 11 & & & & II & 11 & II & & & & IIII \\
\hline 6 & s & & & & 5 & $\mathbf{s}$ & 5 & & & & 55 \\
\hline & $T$ & & & & $T$ & $T$ & $T$ & & & & $T T$ \\
\hline
\end{tabular}

-- NAY -.

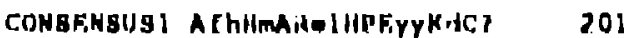

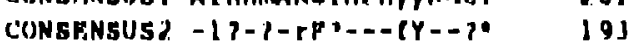

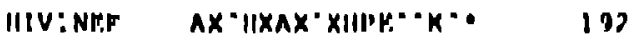

$\begin{array}{lll}\text { D } & \text { D } & \text { Fr D } \\ \text { F } & \text { N } & \text { WW N } \\ \text { N } & & \text { YY } \\ 0 & & \\ K & & \\ 11 & & \\ 11 & & \\ : 1 & & \\ 1 & \end{array}$




\section{IIVI NEF}

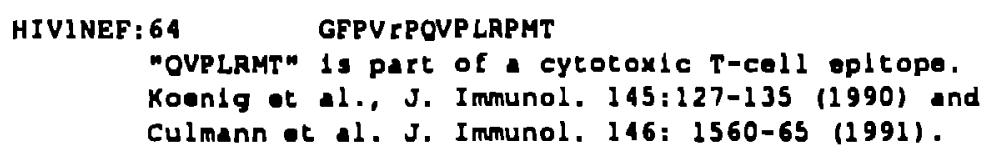


<- env cds

CONSENSUS MGasgSkkrskp???LrerLLrargetyg?l ? ?? ?ggYsqsqegsgkgq?spSCEgq?Y?qG? fmNtPW HIV 2ROD HIVZNIHZ HIV2ISY HIV23T HIV2BEN HIV2D1 94 HIV2GH 1 SIVMA2 51 SIVMAP 11 SIVMA1 42 SIVMNE SIVSMMH4 SIUSMMPBJ --ASG-KKHSRPPRG-OER--RARAGACGGYWNFSGGEYSRFOEGSDREOKSP - - -GRO-OQ-DFM-T- --ASG-KKRSKP LOG-OER--OARGETCGGRCNESGGGYLOSHEGSGREOMSP---GQR-OQ-DEV-T---ASG-KKRSRPSRG-OER--RARGGACGGLHDESEGGYSOFHEGSGREOKLP---GQR-OO-DFM-T---ASG-KKRSEP SRG-RER--OTPGEASGGHWDKLGGEYLOSOEGSGRGOKSP---GRR-OO-DEM-T---ASG-KKLSKHSRG-RER--RARGDGYGKORDASGGEYSOFOEESGREONSP - - -GOQ-OO-EYM-S---AST-KKRSEHSOG-RER--RARGGGYVKORNASGGESSOSOEGSGREOKSP---GOO-OO-E E M-T--ASG -KKHSKHSOR-RER--RAHGGGYVOOCNASGGEYSOSOEGSGKGOKSP - - -GOO-RO-DEH-T---GA I -I'RRSKPAGD-ROK--RARGETYGRLLGEVEDGSSOSLGGLGKGLSSR- - -GOK-NO-OYM-T--GAI -MURSKP TGD-ROK--RARGETYGRLLGEVEDGS3OSLGGLGKGLSSR---GOK-NO-OYM-T- --GA I -KK PSKP PRD-ROR--RARGENYGRLEKGVEDGSSOSLGGLDKGLSSL- - -GOK-NO-EYH-T---GAT-KRF SKSPGD-ROR--RARGETYGRLNEELEDGYSOSLGGSDKGSSLL- - GOK-NO-OFM-T---GA I -KKOYKRGGN-RER--OARGETYGRLWEGLEEGYSOSLGASGKGLSSL- - -POK-SE-OYM-T- --GVT-KKORRRGGN-YER--OARGETYGALWEGLEGEYSOSODASGKGLSSL-- -POK-CE-OEM-T--

LTR $\rightarrow$

CONSENSUS HIV2ROD HIV2NIHZ HIV2ISY HIV2st HIV2BEN HIV2D1 94 HIV2GH 1 SIVMM251 SIVMMP 11 SIVMAI 42 SIVMNE SIVSMMHA SIVSHAPBJ

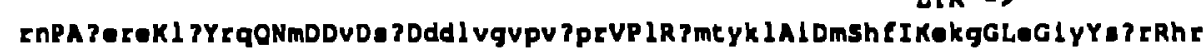
KD--AERE-NL-RQ--M--V-SD-DDQVRVSVTPK--L-PMTHRL-I-M-HL--TRG--E-HF-SER-HK RT-AERE-EL-KO--K--V-LD-DDOVGFPVTPR--L-PMTFKL -V-M-HF-EKG--E-LF $n$ SOR-HR RT--TEKE-ES-RO--M--V-SD-DDLVGVSDTSR--L-AMTYRM-V-M-DL-DKG--E-MY-SER-HR RA-- . EGE-GS-KO--H--V-SD-DDLVGVPVTPR-L-EMTYRL-R-M-HL- -EKG--E-LY-SDR-RR RN--TERO-DL-RQ--H--V-SD-DDLI GUPVTER--R-EMTYKL-I-M-HF--EKG--O-MF-SRR-HR RT--AIGO-NS-KO--H--V-SD-DDLVGVPVMPR--L-EMTYKL-I -M-HE-OEKG-E-IF -SRE-HR RT--I ECO-KL-KO--M- I-SS-DDLVGVPVTPR--L-AMTYKL-V-M-HP--KR . - D-MF-SRD-HR RN--EEKE-LA-RK--H- - I-EE-DDLVGVSVRPK--L-AMTYKL-I-H-HE--EKG-E-IY -SAR-HR RN--EEKE-LA-RK--H- I -EE-DDLVGVSVRPK--L-AMTYKL-I-M-HF--EKG--E-IY-SAR-HR RN--EERK-LP-RK- - I- - I-EE-CDLVGIPVEAR--L-THSYKL-I -H-HF - -EKG- -E - IY - SAR-HR KN--GERE - LA-RK- - I - I I-EE-NDLVGVPVRPR- - L-I I SYKL-V-M-HE--EKG- -E-IY - SER-HK RN--TERA-LG-RO--M -V-DE-DDLIGVSVHPR- - L-AMTYKL-I-M-HF-EKG--E-IY-NER- HR RN--TERA-LD-RQ--M--V-SA- , LUGCPVSPR- -V- I MTYKL-I-M-HF-EKG--E-IY-SDR-HK

- max HIVI NEF olmllardty =-

CONSENSUS HIV 2 ROO HIV2NIHZ HIVIISY HIV 2ST IIIV2BEN IIV 20194 IIIVZGH SI VMHZ 51 SIVMMP 11 SIVMHI 42 SI VMNE SIVSMHH4 IIVSMHPRJ CONgENSUS IIIV2ROD IIIVZNIHZ HIU2ISY IIV 2S":

\section{IIV2IHN}

IIIV20194

ITV2all gIVMazSI I! VMAP ! I :I I VMA| 42 II UMNI:

' I U:MPII I I V:MMIיII

57

70

70

70

70

70

70

70

70

70

70

70

70

70

121

140

140

140

139

140

140

139

140

140

140

140

140

138

18]

209

148

209

200

209

209

208

209

206

2011

209

209

207 


\section{HIV2/SIV NEF}

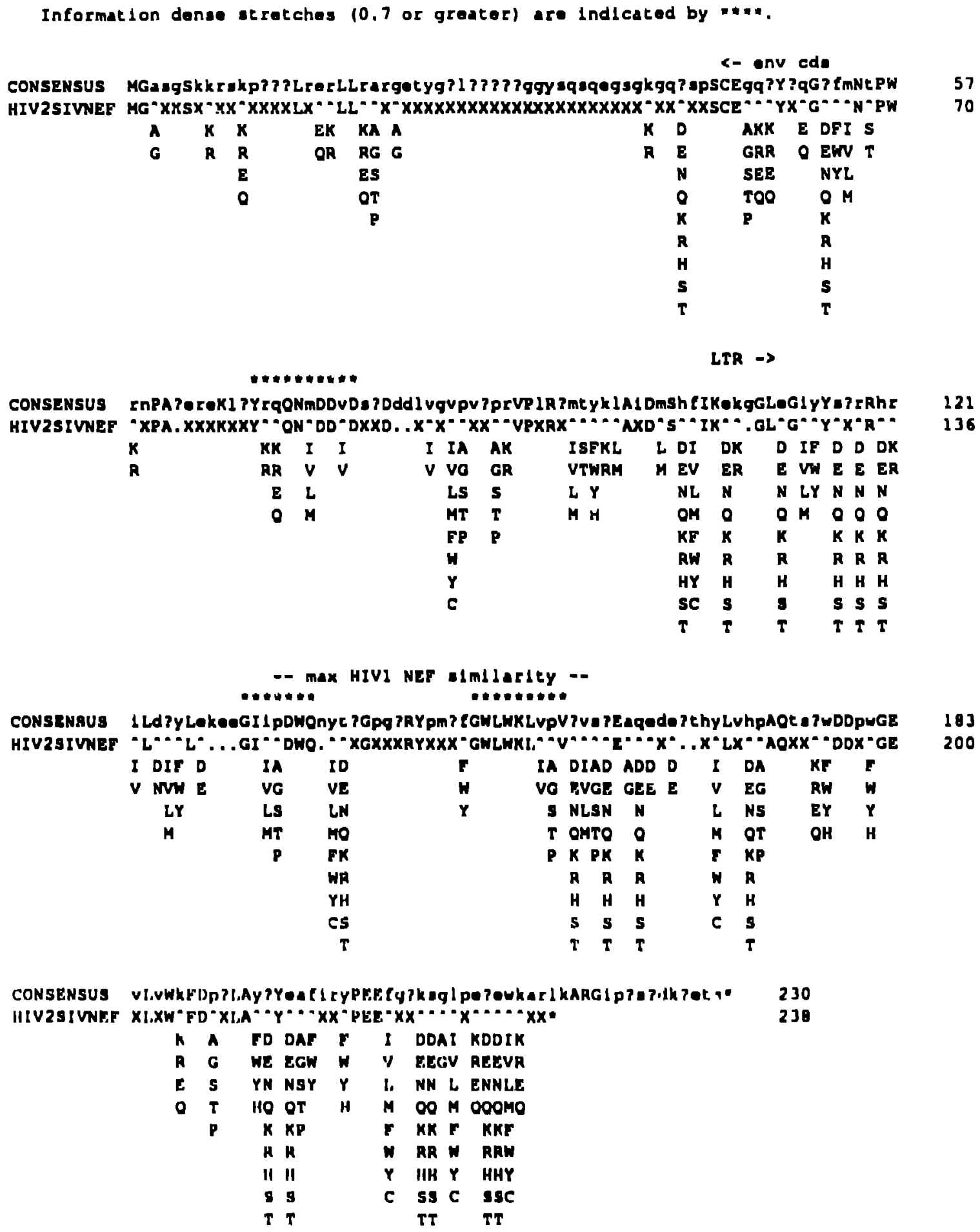




\begin{tabular}{|c|c|}
\hline CONSENSUS & MG ?gnSKpqhkkqlklWraLhktr?trygLLADPLIGqSST TOEECdKaL 7kSI IrKrnG JMTpagRrLO \\
\hline SIVAGMTYO & --SON--PAHKKYSKL-OA-HKTHVTRYG--A-----T---V----D-A-RK-LIR-ON-N--EEE-R-- \\
\hline SIVAGM155 & --LGS--POHKKOLTI-RA-HATRHTRYG--A-----O---L----D-G-RK-LIR-RN-N--PEG-R-- \\
\hline SIVAGM3 & --LGN--POHKKOLSL-HA-HKTRATRYG--A-----O---L----D-A-KE-LIR-RN-K--PEG-K-- \\
\hline SIVAGH677 & --SSN--ROOOGLLKL-RG-RGKPGADWV--S-----O----V----G-A-KK-W.G-.. -K--PDG-R-- \\
\hline CONSENSUS & -GDEWdEWAD TE?EVGFPVE PRVPLROMTYKLAVDFSHFLKAKGGLDG IYYSARRAK I LNLYALNEWG I I \\
\hline SIVAGRTYO & E--TWE--S-D-E-C-C- R- \\
\hline EIVAGH1.55 & 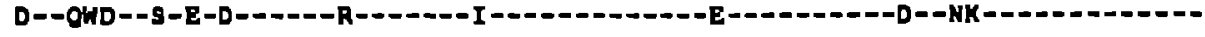 \\
\hline SIVAGM3 & 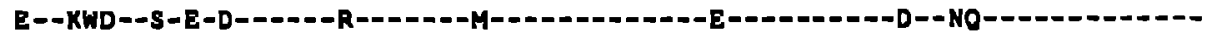 \\
\hline SIVAGH677 & 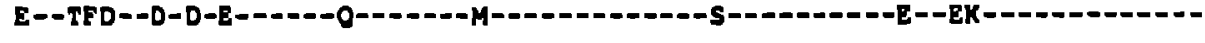 \\
\hline CONSENSUS & DDWnAWSKGPGIRYP rCFGFCFKLVPVdLHEEAOLCERHCLVHPAOMgEDPDG I NHCE ? LUWKFDP mLAV \\
\hline SIVAGHTYO & 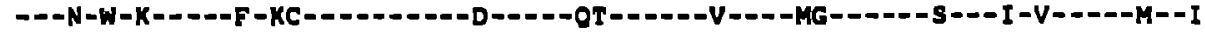 \\
\hline SIVAGHIS5 & 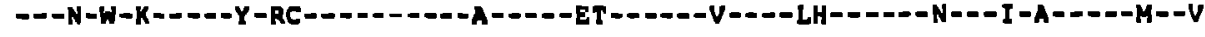 \\
\hline SIVAGM3 & 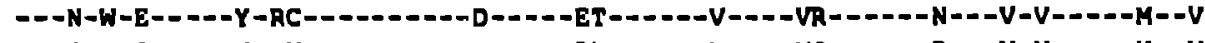 \\
\hline SIVAGH677 & 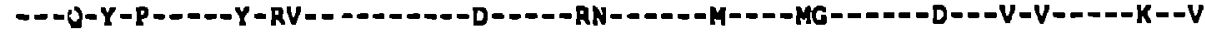 \\
\hline CONSENSUS & qYdPnreyftDmhl ligkrk* \\
\hline SIVAGMTYO & Q-D-NREYET-MHGLV.KRK- \\
\hline SIVAGH155 & Q-D-SREYFT-LYSTVGTGN- \\
\hline SIVAGH3 & Q-D-NRKYLT-MHDL.GKRK- \\
\hline SIVAGH677 & E-R-. . DMFK - MHEH . AKR" \\
\hline
\end{tabular}


Information dense acrotchea (.9 or greater) are Indlcated by ***.

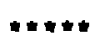

CONSENSUS MGrgnsKpqhkkqlk!WraLhktr?trygLLaDPLIGqSSTrOEECdKaL?kSI 1 rKrng?MTpOgRrLO AGMNEF

\begin{tabular}{|c|c|c|c|c|c|c|c|c|c|c|c|c|c|c|c|}
\hline D & DK & DI & DA & D & D & ADF & $\boldsymbol{A}$ & $D$ & $\mathbf{I}$ & $\boldsymbol{A}$ & $\mathbf{K K}$ & I & $D$ & D & $\mathbf{K}$ \\
\hline $\boldsymbol{E}$ & ER & EY & EG & $\mathbf{E}$ & $\mathbf{E}$ & GEN & $\boldsymbol{G}$ & $\mathbf{E}$ & $\mathbf{v}$ & $\boldsymbol{G}$ & RR & $\mathbf{v}$ & $\mathbf{E}$ & $\mathbf{E}$ & $\mathbf{R}$ \\
\hline $\mathbf{N}$ & NE & NL & $\mathbf{N}$ & $\mathbf{N}$ & $\mathbf{N}$ & SNY & $\mathbf{s}$ & $\mathbf{N}$ & L & & $E$ & L & $\mathbf{N}$ & & \\
\hline 0 & $\infty$ & OH & $\mathbf{0}$ & 0 & $\mathbf{0}$ & TO & $\mathbf{T}$ & 0 & $M$ & & 0 & M & 0 & & \\
\hline $\mathbf{K}$ & K & $K$ & $\mathbf{K}$ & $\mathbf{K}$ & $\mathbf{K}$ & PK & $\mathbf{P}$ & $\mathbf{K}$ & & & & $\mathbf{F}$ & $\mathbf{K}$ & & \\
\hline R & $\mathbf{R}$ & $\mathbf{R}$ & $\mathbf{R}$ & $\mathbf{R}$ & $\mathbf{R}$ & $\mathbf{R}$ & & $\mathbf{R}$ & & & & $\mathbf{N}$ & $\mathbf{R}$ & & \\
\hline H & H & H & H & $\mathbf{H}$ & H & H & & H & & & & $\mathbf{Y}$ & H & & \\
\hline $\mathbf{s}$ & $\mathbf{s}$ & $\mathbf{s}$ & $\mathbf{s}$ & $\mathbf{s}$ & $\mathbf{s}$ & $\mathbf{s}$ & & $\mathbf{s}$ & & & & C & $\mathbf{S}$ & & \\
\hline $\mathbf{T}$ & $\mathbf{T}$ & $\mathbf{T}$ & $\mathbf{T}$ & $\mathbf{T}$ & $\mathbf{P}$ & $\mathbf{T}$ & & $\mathbf{T}$ & & & & & $\mathbf{T}$ & & \\
\hline
\end{tabular}

CONSENSUS AGMNEP

GDEWdENGD?E?EVGEPVIPRVPLROMTYKLAVDFSHFLKOKGGLDGIYYSARRAK ILNLYALNEWGI

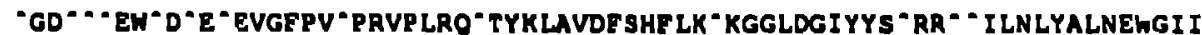

E EWE $\&$ E NY $N$

$0 \quad 0$

I

K I

I

E

L

D DK

E ER

$\begin{array}{lrr}D & D & \text { DK } \\ \text { E } & \text { E } & \text { ER } \\ N & & \text { NE } \\ 0 & & 0\end{array}$

R $R$

H H

$\begin{array}{ll}\mathbf{5} & \mathbf{5} \\ \mathbf{T} & \mathbf{T}\end{array}$

$\bullet \bullet \cdot *$

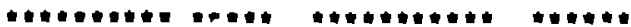

$\because * \bullet *$

CONSENSUS AGMNEF

DDWnAWSKGPGIRYP ICFGFCFKLVPVDLHEEA TCERHCLUHPAOMgEDPDGI IHGE ?LVHKEOPmLAV

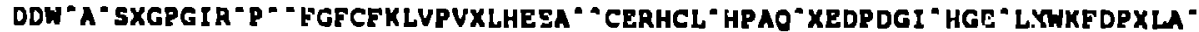

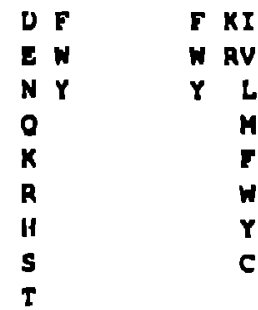

KD
R.
EN
OO
K
R
H
$\mathbf{s}$

CONSENSUS ATI INEF

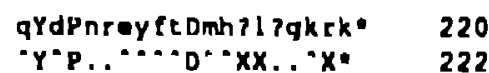

$\begin{array}{lllll}\text { E D } & \text { DIID IF } & \text { D } \\ \text { O E } & \text { EVWE } & \text { MW } & \text { E } \\ \text { N } & \text { NLLN } & \text { Y } & \text { N } \\ \text { O } & \text { OMMO } & \text { H } & \text { O } \\ \text { K } & \text { KTFK } & & \text { K } \\ \text { R } & \text { RINA } & & \text { R } \\ \text { II } & \text { HYYH } & \text { H } \\ \text { S } & \text { SCCS } & \text { S } \\ \text { T } & \text { T T } & \text { T }\end{array}$




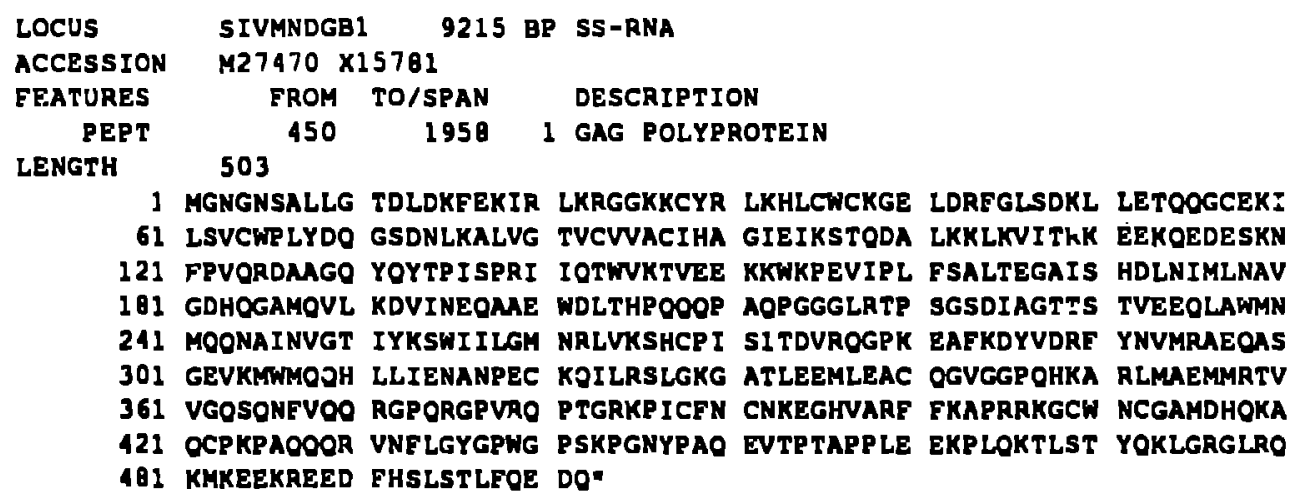

1 MGNGNSALLG TDLDKFEKIR LKRGGKKCYR LKHLCWCKGE LDRFGLSDKL LETOOGCEKI

61 LSVCWPLYDO GSDNLKALVG TVCVVACIHA GIEIKSTODA LKKLKVITKK EEKOEDESKN 121 FPVORDMAGO YOYTPISPRI IOTWVKTVEE KKWKPEVIPL FSALTEGAIS HDLNIMLNAV 101 GDHOGAMOVL KDVINEOME WDLTHPOOOP AOPGGGLRTP SGSDIAGTTS TVEEOLAWMN 241 MOONAINVGT IYKSWIILGH NRLVKSHCPI S1TDVROGPK EAFKDYVDRF YNVMRAEOAS 301 GEVKMWMOPH LLIENANPEC KOILRSLGKG ATLEEMLEAC OGVGGPOHKA RLMAEMMRTV 361 VGOSONFVOO RGPORGPVRO PTGRKPICFN CNKEGHVARF FKAPRRKGCW NCGAMDHOKA 421 OCPKPAOOOR UNFLGYGPWG PSKPGNYPAO EVTPTAPPLE EKPLOKTLST YOKLGRGLRO 481 KMKEEKREED FHSLSTLFOE DO"

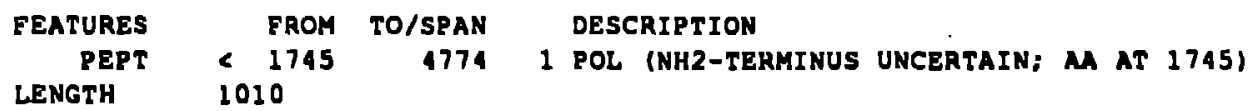

1 FFRVWPLGSL OTGELSGTRG DSNSSTIRGE ISAENSEHLS EIRERAOAED EGGEERGGFS

61 FPEYSLSRRP IEEVSVDGVT IRALLDTGAD DTIFNERNIK LKGNWOPKII GGIGGNLAVK 121 OYDNVYVEIR GKGTFGTVLI GPTPIDIIGR NIMEKLGGKL ILAOLSDKIP ITKVKLKPGV 181 DGPRIKOWPL SKEKIVGLOK ICDRLEEEGK ISRVDPGNNY NTPIFAIKKK DKNEWRKLID 241 ERELNKLTOD FHELOLGIPH PAGIKKCKRI TVLDIGDAYF SIPLDPDYRP YTAFTVPSVN 301 NOAPGKRYMY NILPOGWKGS PCIFOGTVAS LLEVFRKNHP TVOLYOYMDD LFVGSDYTAE 361 EHEKAIVELR ALLMTWNLET PEKKYOKEPP FHWMGYELHP DKWKIEKVOL PELAEOPTVN 121 EIOKLVGKLN WMOLYPGIK TKOLCKLIRG GLNITEKVTM TEEARLEYEO NKEILAEEOE 401 GSYYOPNKEL YVRFOKTTGG DISFOHKOGN KVLRAGKYGK OKTAHSNDLH KLAGATOKVG 541 RESIVIWGFV PKMOIPTTRE IWEDWHEYW $\propto$ CTWIPEVEF ISTPMLEREW YSLSPEPLEG 601 VETYYVDGAA NRDSKMGKAG YITDRGFORV EEYLNTTNOO TELHAVKLAL EDSGSYVNIV 661 TDSOYVUGIL ASRPTETDHP IVKEIIELMK GKEKIYLSWL PAHKGIGGNE OIOKLUSSGI 721 RKVLFLONIE PAOEEHEKYH SNEAOLREKF HLPALVAKOI VOSCSKCCHH GEPIKGOTDA 701 SLGWOIDCT HLENOIIIVA VHVASGFMKA EVITAETOKK TAEFLLKLAA OWPISKLHTD Q41 NGPNFTSOEV ETMCWWLGIE HTFGIPYNPO SUGVVNKNK YLKELIEKIR EDCKELKTAV 901 AMATFIHNFK GRGGLGGMTA GERIVNMIN'C ELEYOYOONO ISKNLNFKVY FREGRLOLWK 961 GPGILLWKGE GAWLKYOEE IKIVPRRKCK IIKDYGESGK NSOVNLSV*

$\begin{array}{lrrl}\text { FEATURES } & \text { FROM } & \text { TO/SPAN } & \text { DESCRIPTION } \\ \text { PEPT } & 4728 & 5246 & 1 \\ \text { LENGTH } & 113 & & \end{array}$

1 MERVERIVRL TWKVSSOHIE KWHWLURROM AWATANNEEG CWHLYPHFMA YNEWYTCSKV

61 VIIINRDIRL IVRSYWHLOI EVGCLSTYAV SIEAVURYPP FEKEWCTEIT PEVADHLIHL 121 HFYDCFMDSA VMKAIRGEEV LKVCRFYAGH MAQGVLSLOF LCLRVIYGPE ER*

$\begin{array}{lrrr}\text { FEATURES } & \text { TROM } & \text { TO/SPAN } & \text { DESCHIPTION } \\ \text { PEPT } & 5227 & \text { S541 } & 1 \\ \text { VPR PROTETH }\end{array}$

1 MGOKRDEOUS EDOGPPEPY NOWLADTHEE IKEEARKMFP LIILNAVSEY CVONTGSEEE

61 ACEKTITLMN RAIWVHLAOG CDGTFRERAP OLPPSGFRPR GDRL.

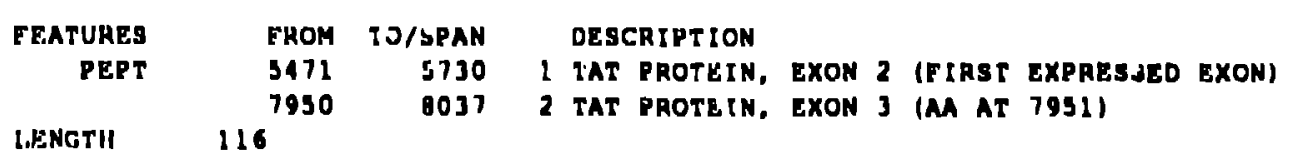

I MEPSGKEDHN CPPODSGOEF, IDYKOLLEEY YOPLOACENK CWCKKCCFHC MLCYOKKGLG

61 IAYIVYKKKV PGTHKKIPGS GEE IRAAID LSFHRTASHT YTANGOTTEK KKATA*

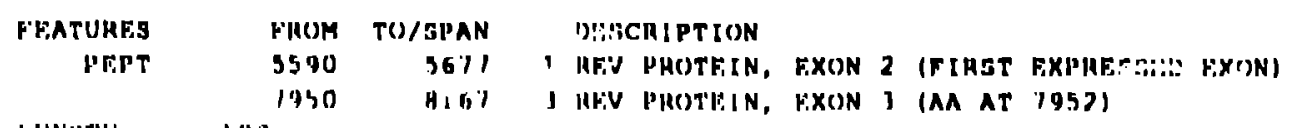
10)

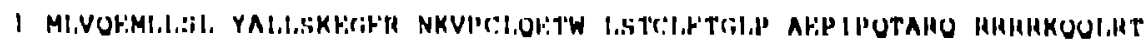

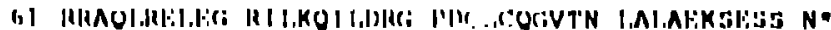




\section{SI VMND PROTEINS}

\begin{tabular}{rrrr} 
FEATURES & FROM & TO/SPAN & \multicolumn{1}{l}{ JESCRIPTION } \\
PEPT & 5661 & 8126 & 1 LNV POLYPROTEIN
\end{tabular}

1 MSTGNVYOEL IRRYLVVVKY LYEGKYEVSR SFSYTMFSLL VGIIGKOYVT VFYGUPVWKE

61 AKTHLICATD NSSLWVTT C IPELPOYDEV EIPDIKENFT GLIRENOIVY OAWHAMGSM 121 DTILKPCVKI NPYCVKMO:O ETENVSATTA KPITTPTTTS TVASSTEIYL DVDKNITEEK 181 VERNHVCRYN IIGLCRDSIKE EIVTNFRGDD VKCENNTCYM NHCNESVNTE DCOKGLLIRC 241 ILGCVPPGYV MLRYNEKLIN NKLCSNISAV OCTOHLVATV SSEFGFNGTM HKEGELIPID 301 DKYRGPEEFH ORKFVYKVPE KYGLKIECHR KGNRSVVSTP SATGLLFYHG LEPGKNLKKG 361 MCTFKGRWGL ALWSLAKELN KLNDSIKVNO TCKNFTSTGE ENKONTDKOK EFAKCIKTLK 421 IDNYTTSGDR AMEMMMATCO GEMFFCNVTR IMRAWNDPNE KXWYPYASCO IROIVDDWMO 101 VGRKIYLPPT SGFNNHIRCT HRVTEMYFEM OKIDSNETKM OIKFLPPSET SNOFVAYGAH 541 YKLVKIMPIG IAPTDVKRHT LPEHHKEKRG AVILGILGLL SLAGSAMGSV SVALTVOSOS 601 LVTGIVEOOK OLLKLIEOSS ELLKLTIWGV KNLOTRLTSL ENYIKDOALL SOWGCSWAOV 661 CHTSVENTNT SITPMUTSET WKEWETRTDY LONITEMLK OAYDREORNT YELOKLGDLT 721 SWASWFDFTW WVOYLKHGVF LVLGIIGLRI LLALWNTISR FROGYRPVFS ODCOONLYPK 781 RPDNGEEESN SLELGEHNSE NLKEESLMRS LIEDLTSFAR E*

$\begin{array}{rrrr}\text { FEATURES } & \text { FROM } & \text { TO/SPAN } & \text { DESCRIPTION } \\ \text { PEPT } & \text { B170 } & \text { BB14 } & \text { I NEF } \\ \text { LENGTH } & 215 & & \end{array}$

1 MGSSOSKKRS EAWVRYSSAL ROLVGGPVTP DGYKOIESSO GAEKOSLLRG RAYGTYSEGL 61 DKVONDPLTK DEKLDLTOOD PEEEEEVGFP VZROVSLRVP SYKDLIDFSH FIKEKGGLGG 121 IYYSRRREEI LDLYAENEWG FEPGWOOYTT GPGTRYPKTF GFLFKLEPUS RAIGDEYAAN 181 NHLLHSSOLC POEDPEGETL. MWSGTLILPM TLOH*

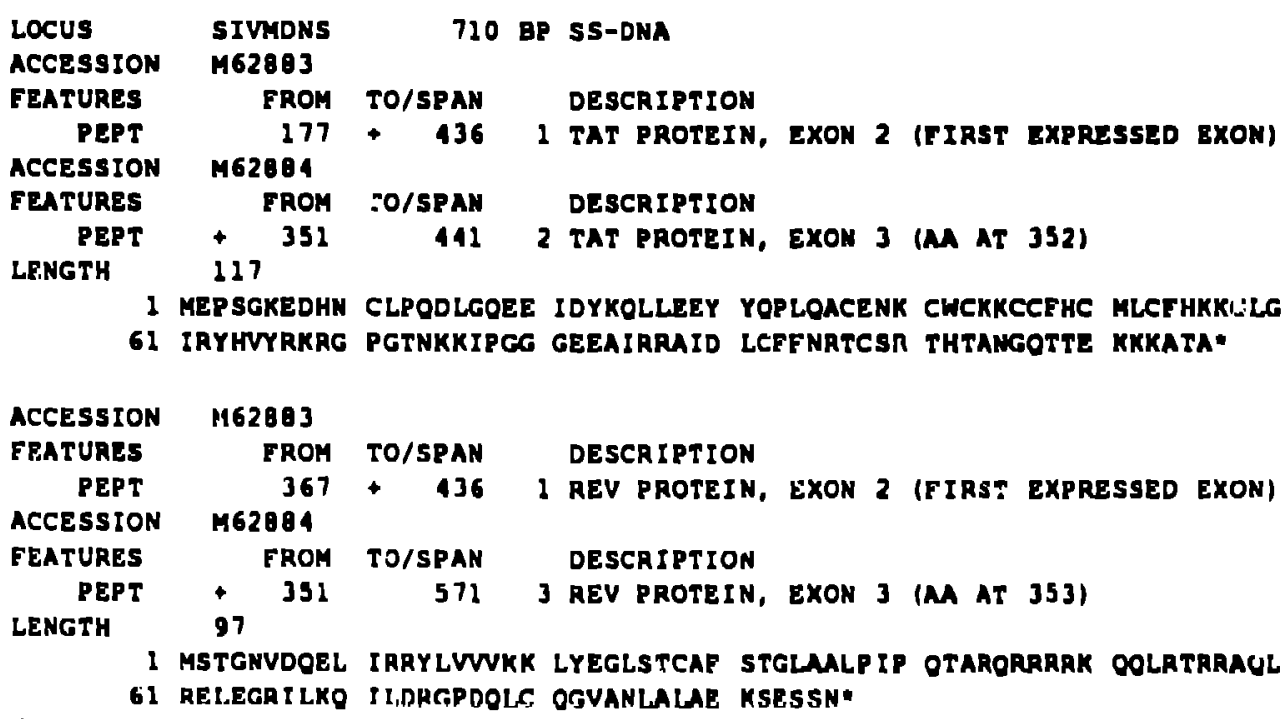

1 MEPSGKEDHN CLPQDLGOEE IDYKOLLEEY YOPLOACENK CWCKKCCFHC ALCFHKKLLLG

61 IRYHVYRKRG PGTNKKIPGG GEEAIRAID LCFFNATCSN THTANGOTTE KKKATA*

1 MSTGNVDOEL IRRYLVVVKK LYEGLSTCAF STGLMLPIP OTARORRARK OOLATARAGL 61 REL.EGRILKO II,DAGPDOLG OGVANLALAE KSESSN" 


\section{III}

ANALYSIS

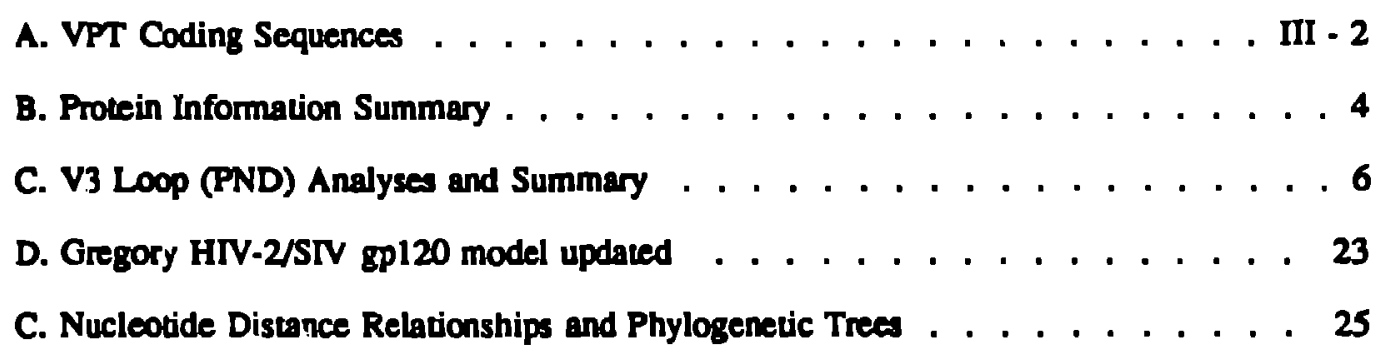


IIIA. VPT CODINO SEQUENCE

E. Cohen et al., J. AIDS 3,601-8, 1990, have uncovered an orf called T. Conservation of a putative ribosomal frameshift site, typically "aasaagtg", suggests that a $17 \mathrm{kD}$ fusion protein could be made: the first 29 AA residues of the 107 AA (typically) T product would come from the cat protein. A summary of the potential coding sequences and the amino acid alignment follow. A preliminary search of the PIR database converted to AACC pattems, using the PLSEARCH tool, revealed no obvious protein similarity or homology.

\begin{tabular}{|c|c|c|c|}
\hline SEQUENCE & $\begin{array}{l}\text { NUCLEOTIDE } \\
\text { COORDINATES }\end{array}$ & $\stackrel{\text { MA }}{\text { LENGTH }}$ & $\begin{array}{c}\text { SHIFT } \\
\text { SEOUENCE }\end{array}$ \\
\hline - - - - & - & $=-\infty-\infty$ & $--m-x-0$ \\
\hline LAI & $5412-5731$ & 106 & anasgeg \\
\hline HXB 2R & $5377-5699$ & 107 & dasagtg \\
\hline HIVNL43 & $5830-6149$ & 106 & asasagtg \\
\hline $\mathbf{M N}$ & $5845-6167=$ & 59 & anangtg \\
\hline sc & $541-875$ & 111 & asasgto \\
\hline BALI & $255-577 \times$ & 62 & acangtg \\
\hline JRCSF & $5842-6164$ & 107 & anasugtg \\
\hline JREL & $5147-5469$ & 107 & araadgtg \\
\hline OYI & $5373-5695$ & 62 & asaggtg \\
\hline SP2 & $5840-6162$ & 107 & arasgtg \\
\hline NY5CG & $5830-6108$ & 62 & alaggtg \\
\hline $5 F 162$ & $88-440^{\circ}$ & 62 & dasagtg \\
\hline $\operatorname{coc} 42$ & $88-410$ & 107 & Aasogtg \\
\hline SF 33 & $88-410$ & 59 & arasgtg \\
\hline HAN & $5296-5619$ & 107 & anasgeg \\
\hline $\mathbf{R F}$ & $5347-5669$ & 107 & sasigtg \\
\hline ELI & $5376-5699$ & 107 & arasgty \\
\hline 2226 & $5833-6155$ & 107 & arasgtg' \\
\hline NDK & $5365-5687$ & 107 & areagtg \\
\hline MAL & $5405-5727$ & 107 & asasgtg \\
\hline U455 & $5276-5600$ & 62 & argtgtg \\
\hline & Internal stop & & \\
\hline
\end{tabular}

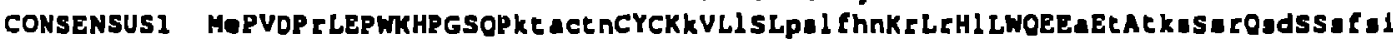

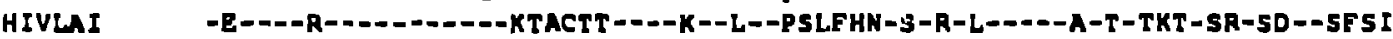

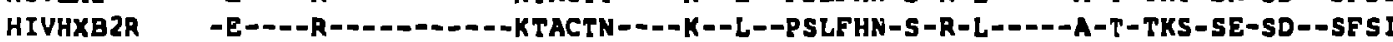

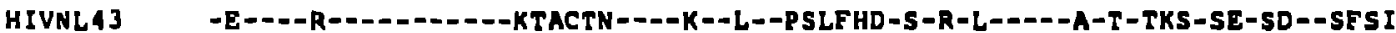

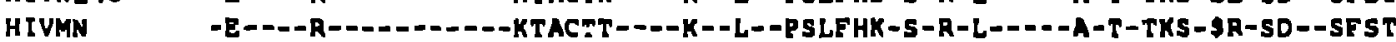

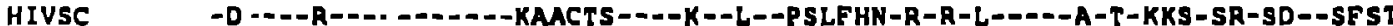

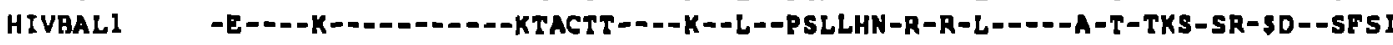

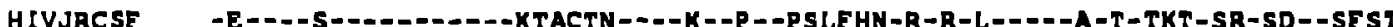

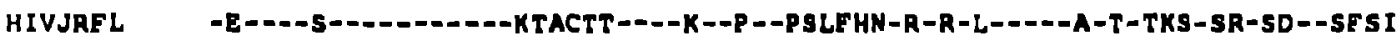

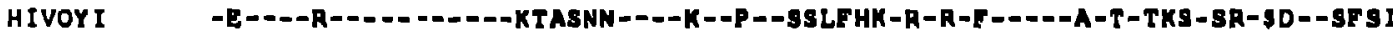
HIVSE HIVNY 5 CG H:VSF 162 HIVCDC42 HIVSF 33 HIVHAN HIVRE CONSENSUS 2 HIVELII HIVZ226 IIIVNOK IIIVMAL IIIVIAS

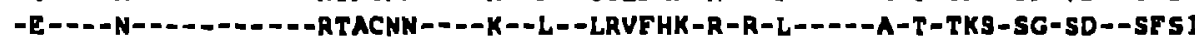

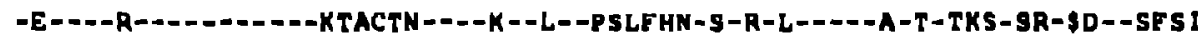

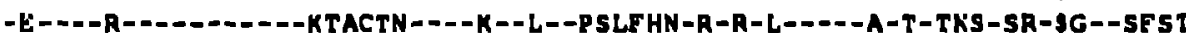

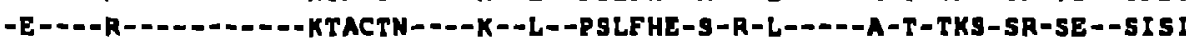

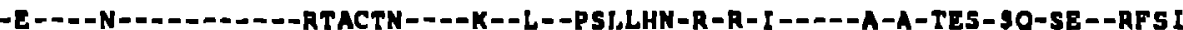
-E-D-R- - -

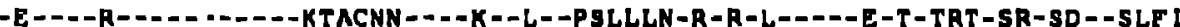

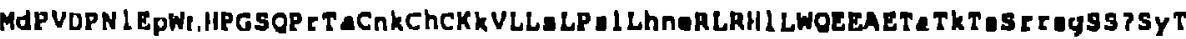

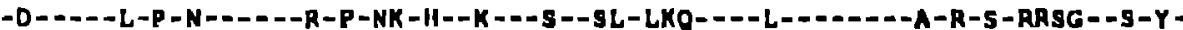

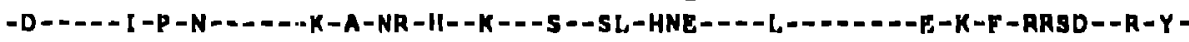

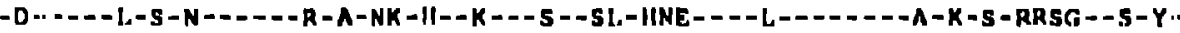

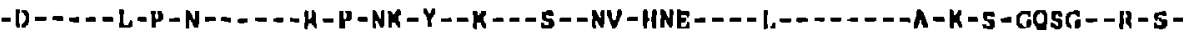

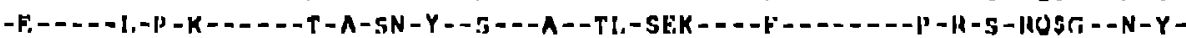


CONSENSUS1 KAVSatcna? ftnlsnSaISSSaNnSNacudhS?h? I1?

HIVLAI

KAVSstCna?ftnisnSaISSS sNnSNacVdhs ?h? Il?

HIVHXB2R -.--STCNATYTNSSN-SI--N-N--SCVVH-NH.RI*

HIVNL43

HIVHA

----STCNATY . NSSN-SI---N-N--SCVVH-NH.RI*

---STCNATESNSSN-SI---R-N--SCVIH-IH.RI *

HIVSe

----NTCNATE TNIIN-SI---S-N--SCWVY - IHINIGKY *

HIVBAL1

---STCNASI' TNISY-RI---S-N--SCVDH-IH. RI*

HIVJRCSF

----SACNATETNISN-SI---R-N--NCVVH-TH.RI* *

HIVJRFL

---SACNAIEIHISN-SI---N-N--SCWVH-TH.RI*

HIVOYI

---NTCNILETNISN-SI--ON-N--SCVDH-TL.RI*

HIVSF 2

---SKCNAIFTNISN-II---S-N--SCVDH-TH. RI*

HIVNYSCG

HIVSF 162

----STCNAFFOSLCGP \$C-\$NT

---SIFNATSSNISN-SI---S-N--SCVDH-VH.RIOENIKTKKNR*

HIVCDC 42

HIVSE 33

HIVHAN

---STCHAIFRNSSN-RI---S-N--SCVDH-IH.RI

116

----STCMVIFNNISN-SN---N-N--SYMDH-IN. KI *

----STCDVIETNSSN-SI---S-N--SCYDH-SH. RI *

HIVRF

----SICNAIERNISN-SI----S-T--SCVDH-WH. RN *

CONSENSUS2

HIVELI

HIV2226

HIVNDK

K---ST--A--R-N-_-_-_-SN-T-_-_-_.-.-H. -I*

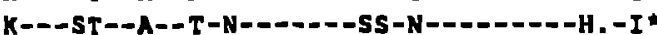

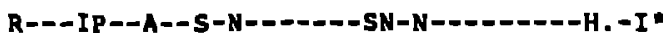

HIVMAL

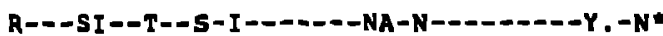

HIVU 455

K---NSLMSSHLWK-GOSOGS\$\$HS-SOS \$CGLSSV\$N

III-3 
Information contents (IC) and informacion densities (ID), expressed as amino acid equivalents, can be calculated from the dara sels presented in PART II; the equation for this calculation is discussed in Smich and Smich, PNAS 87, 118-122, 1990. In brief, an unveried amino acid residue in an AACC pattern will have 1.0 AA equivalents of information and a wild-card will have 0.0 AA equivalents of information; variable but conserved residues, e.g., Asp and Glu, will have intermediate values. Thus an ID of 0.3 implies that, on the average, a procein pautern has 0.3 AA equivalents at every position. The IC of a protein, by this way of thinking, is the number of residues in the molecule; for an AACC pautem, this value muat be corrected for the negative information resulting from gaps in an alignment. For example. the AACC patsem built from 62 myoglobin sequences has an IC of 68.3 and an ID of 0.45 . Typical IDs for patuerms derived from the PIR dalabase are 0.2-0.3. Obviously these quantities depend upon the number of different sequences involved in the computation. While this approach is less than ideal, it is clearly preferable in our minds to off-the-cuff judgments about "conservertion" and "homology."

The principal purpose of this analysis is to help clarify the problem of what is and what is not conserved By comparing the fraction of unvaried residves from the consezsus sequences in Part II to the IDs (c-a), some measure of the conservation over and above invariance is achieved. In the case of HIV-1s, inclusion of the SIV-cpz sequence in the AACC pattem but not in the ordinary consensus leads to a gross underestimation of this difference.

All of the figures that follow are approximate, based as they are upon changing data and averages: nevertheleas, some lendencies can be provisionally inferred: i) the smaller, regulatory proveins are the more variable proceins (a possible exception being HIV-1 VPR); ii) while there are fewer samples of HIV-2ASIV and, especially, AGM, the:C show remartable extents of vartacton (the AGM samples are for east African counuries only): iii) the fraction of unvaried residues represents, typically. 70\% to 85\% of the actual information provided by the data sel

\begin{tabular}{|c|c|c|c|c|c|c|c|}
\hline $\begin{array}{l}\text { HIV-I } \\
\text { PROTEIN }\end{array}$ & sequence & $\begin{array}{l}\text { approndmate } \\
\text { length }\end{array}$ & $\begin{array}{l}\text { unvarled } \\
\text { reeldues }\end{array}$ & $\begin{array}{c}\text { Eract Ion } \\
\text { (a) }\end{array}$ & $\begin{array}{c}p a c-J c \\
(b)\end{array}$ & $\begin{array}{c}\text { pet-ID } \\
\text { (c) }\end{array}$ & $\begin{array}{c}d d e t \\
(e-a)\end{array}$ \\
\hline 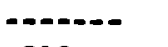 & $---0-2-0$ & $---\infty=$ & $----0-0 .-0$ & $--0-0-0$. & $-\infty-\infty$ & $-\infty-\infty$ & ----- \\
\hline GAG & 19 & 500 & 340 & 0,60 & 307 & 0.77 & 0.09 \\
\hline POL & 17 & 1000 & 784 & 0.70 & 864 & 0.86 & 0.08 \\
\hline vIF & 17 & 190 & 112 & 0.59 & 136 & 0.70 & 0.11 \\
\hline VPR & 10 & 95 & 67 & 0.71 & 75 & 0.77 & 0.06 \\
\hline TAT & 22 & 100 & 39 & 0.39 & 53 & 0.54 & 0.15 \\
\hline REV & 24 & 115 & 47 & 0.41 & 61 & 0.56 & 0.15 \\
\hline vpu & 19 & 75 & 30 & 0.40 & 43 & 0.31 & 0.11 \\
\hline ENV & 11 & 800 & 400 & 0.50 & 432 & 0.55 & 0.05 \\
\hline NEF & 19 & 200 & 90 & 0.49 & 123 & 0.62 & $0.1 \mathrm{~J}$ \\
\hline $\mathbf{T}$ orf & 22 & 105 & 39 & 0.37 & 51 & 0.47 & 0.10 \\
\hline HIV-2/SIV & $\bullet$ & approndmate & - unvarled & fract d on & pat-IC & pat-ID & $d ! f t$ \\
\hline PROTEIN & - equence. & Iongth & revidues & (a) & (b) & (c) & $(c-a)$ \\
\hline 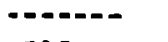 & $----x---0$ & $----\infty$ & $-0-0-0-0$ & 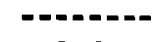 & $-\infty-\infty$ & - - - & $-=--$ \\
\hline GAG & 14 & 500 & 329 & 0.64 & 392 & 0.70 & 0.14 \\
\hline POL & 13 & 1000 & 647 & 0.65 & 794 & 0.77 & 0.12 \\
\hline vIF & 15 & 200 & 105 & 0.53 & 139 & 0.64 & 0.11 \\
\hline $\mathbf{v P x}$ & 15 & 110 & 62 & 0.56 & 72 & 0.65 & 0.09 \\
\hline VPN & 15 & 80 & 40 & 0.50 & 40 & 0.47 & -0.03 \\
\hline TAT & 16 & 130 & 39 & 0.30 & 35 & 0.43 & 0.13 \\
\hline REV & 15 & 90 & 36 & 0.43 & 53 & 0.50 & 0.07 \\
\hline ENV & 16 & OIS & 441 & 0.54 & 534 & 0.64 & 0.10 \\
\hline NEF & 14 & 240 & 11 & 0.3 & 113 & 0.41 & 0.11 \\
\hline
\end{tabular}


PROTEIN INFORMATION

\begin{tabular}{|c|c|c|c|c|c|c|c|}
\hline $\begin{array}{c}\text { AGM } \\
\text { PROTEIN }\end{array}$ & sequences & $\begin{array}{l}\text { approximate } \\
\text { langth }\end{array}$ & $\begin{array}{l}\text { unvarled } \\
\text { residues }\end{array}$ & $\begin{array}{c}\text { fraction } \\
\text { (a) }\end{array}$ & $\begin{array}{l}p a t-I C \\
\text { (b) }\end{array}$ & $\begin{array}{c}\text { pat - ID } \\
\text { (c) }\end{array}$ & $\begin{array}{l}d d f f \\
(c-a)\end{array}$ \\
\hline 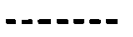 & - & --- & 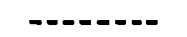 & - - & - & $-\ldots$ &.--- \\
\hline GAG & 5 & 520 & 317 & $0.6 !$ & 371 & 0.73 & 0.12 \\
\hline POL & 4 & 1050 & 642 & 0.61 & 773 & 0.73 & 0.12 \\
\hline VIF & 4 & & & & 130 & 0.56 & \\
\hline VPX & & & & & 91 & 0.76 & \\
\hline TAT & & & & & 54 & 0.55 & \\
\hline REV & & & & & 45 & 0.52 & \\
\hline ENV & 4 & 830 & 523 & 0.63 & 605 & 0.71 & 0.08 \\
\hline NEF & & & & & 168 & 0.74 & \\
\hline
\end{tabular}


IIIC: Analysis of the HIV-1 V3 Loop

Because of its capacity to stimulate potent immune responses, the HIV-I V 3 loop has been the focus of intense interest over the last few years. The V3 loop is localed in the third variable domain of the gp 120 protein and is bounded by two cysteine residues capable of forming a disulfide bridge. Located in a highly variable region of $\mathrm{gp} 120$, insertions and deletions are frequent, so that the size of the $\mathrm{V} 3$ loop ranges from 33 to 40 amino acids (most have 35 amino acid residues), including the cysteines that form its boundaries. The V3 loop is also known as the principal neutralizing domain (PND), because peptdes derived from this region are able to elicit type-specific neutralizing antibodies which can block viral infection in vito by blocking fusion (1-6). Peptides from this region are also able to serve as epitopes for both cytowxic $T$ cells $(7,8)$ and helper $T$ cells $(9,10)$. Finally, the PND has been implicated in determination of tropism (11).

Due to the blologically interesting features of the V3 loop, this region has ofien been included in PCR ampliticauton sequenco sudies. In the current HIV database there are approximately $700-800 \mathrm{~V} 3$ sequences, although many of these have not yet been released.

The intent of this section is to provide analysis of the potential for variabillity within the V3 loop. and to present the published V3 loop sequences In alignments that faclititote comparisons of sequences oblained from different sudies. This section includes:

I. A V3 loop consensus sequence and amino acid class covering pattem based on all of the sequences currently in the dalabaso.

2. Amino ecid alignments of published $V 3$ loop sequences.

3. The nucleic acld sequence alignments corresponding to the amino acid allgnmeits of section $\mathrm{C2}$.

4. Amino acid alignments of sets of sequences from the same Individual.

5. Histograms showing the distribution of similarity comparisons between V3 nucleodide sequences.

\section{References:}

1. Palker ot al. PNAS 85:1932-36 (1988)

2. Ooudumit el al., PNAS 85:4478-82 (1988)

3. Ho et al., J. Mrol. 61:2024-28 (1987)

4. Kenealy el al., AIDS Res. IIwn. Reiroviruses 5:1973 (1989)

3. Rusche et al., PNAS 85:3198-3202 (1988)

6. Javahertan el al., PNAS 86:8768-72 (1989)

7. Takahashl ef al., PNAS 85:3105-09 (1988)

8. Takahachl ef al., Sclence 246:118-121 (1989)

9. Palker et al.. J. Immunol. 142:3612-19 (1989)

10. Hart el al., JImmunol. 145:2677-85 (1990)

11. Westervell el al., PNAS \$:3097-101 (1991) 
IIIC1. V3 loop consensus and amino acid class covering pattern

The analysis in this section is bosed on 527 V3 loop amino acid sequences from N. America and Europe. The 52I sequences Include the sequences shown in the next sectlon (IIIC2), the LaRosa data sel (LaRosa et al., Science 249: 932-35 (1990)), and many addiluonal sequences graciously provided prior to publication by various groups. In paricular many V3 sequences were provided by Sleve Wolinsky's group al Northwestem University. Chicago, Ill,, and by Jaap Goudumit's group al the University of Amsterdam, the Netherlands. in cases where muluple identical sequences across the V3 loop occurred within a sequence set derived Irom a single individual, we included only one such representadve sequence in this set of 527 sequences (see section IIIC3).

The consensus sequence shows the most common anino acid found in a given position. The consersus presented here ts the same as the consensus derlved by LaRose et al., as well as the consensus from all soquences excluding the LaRose $a$ al. set. The gaps included to maintnin the alignment are not shown; insertions in these positions cccur in less than 2\% of the sequences (see section C2 for trle consensus with gaps). Directly beneath the consensus sequence the frequency of the most common amino acid found in a given posituon is shown as a percentage.

A modificallon of the amino acid clays covering (AACC) pattem, described at the beginning of section II, was used to generate an AACC pattern for the V3 loop. In AACC patierns, lower case letters represent related groups of amino acids. Because of the extensive vartability of the V3 loop and the large volume of sequences avallable in this region, a standard AACC patuern yields very liule information (see the AACC pattem generated when one considers $100 \%$ of the resldues possible at a glven postion In the allenment, where " $X$ " slyniftes a nonrelaced set of amino acids and " $\mathrm{g}$ " signifies that a gap can occur at a given poaluon). It is probable that sequence error or PCR ampllifeation of biologitenlly inactive mutent vinuses eccount for some of the very raro and unusual amino acids found al a given position in an allenment of the V3 loop. Considerable limitations on the amino ecid class typically found at a gi"en position result when only the most common set of the amino acids at that position are considered. An AACC patuem was genernted consldering 95\% of the total amino acids that can occur at a given position, excluding nro amino eclds thas yield a mors divergent amino acid class. Diroculy under thls pautern is the set of amino acids that were included to yteld the amino acid class, listed in order of thoir frequency, with the moat common at the top.

The 'OPOR' tip of the V3 loop is highly conserved, and is predicted to form a typo II bein-tum (Javahertan et al., PNAS 86:8768-72 (1989)). Among the 527 sequences analyzed hero, 416 have OPOR at the up (78.9\%). OPOK is the second most frequently observed, present in 41 sequences $(7.8 \%)$. OPOQ is the most common form found among the African isolntes. Both OPOK and OPOQ aro predicted structurally to make a type II betn-(um (W/lmol and Thonon, J. Mol. Blol. 203:221-232 (1984)). There are 24 vartants of the four amino acid tip found in the 527 sequences that make up tho $\mathrm{N}$. American/European dnte seli most of these vartants conserve the iypo II bets-tum predicied structuro. The up of Uti loop is importunt for the neutralizing antibody and T-cell epitopes described in the introduction in this soction. It may havo a funcional rolo in fusion (Freed ef al. J, Virol. 65:190-194 (1991)) and also as a protease cleavage alte (Hatlori et ul. FEDS Leths 248:48-52 (1989) and Clements ef al., AIDS Res. and llumen Reimviruses 7:3-16 (1991)). 
V3 consensus sequence, N. America and Europe:

CTRPNNNTRKSIHIGPGRAFYTTGEIIGDIRQAHC 100879996 o5 $939394867463944675949799860675825486753495809685949881 \quad 009199$

AACC pattern for $\geq 95 \%$ of sequences:

CXRPrrijrpXaXchPGpXfXjXg gIgGIcRpAeC $T \quad N N N T$ T K S I H I G

AACC pattern for $100 \%$ of sequences:

$C \times n \times X \times X \times g g \times X g \times X \times X g \times X g g g g g \times g X X X X A X X$ 
IIIC2. Pepude Alignments

The V3 loop amino acid alignments are divided into three sets: a North American/European set, and two smaller Africun sets.

The $\mathbf{N}$. American/European set is aligned with the consensus described in detail in section $\mathrm{C}$. The aligned amino acid sequences then begin with V3 sequences extracted from HIV envelope sequences presented in Parts I and II of the database. These sequences are identifable by the "HIV" prefix included in their names, and background information on these isolates can be found in Part 1 of the database. The next two sequences, labeled CANO, were provided by Chin-YI Ou's group at the CDC; these sequences are derived from one of the earliest $N$. American samples, taken from a Canadian airline steward (sometimes called "patient Zero"). The sequence FO is from an early tsolate and was kindly provided by $\mathrm{H}$. Guo, M. Reitz, and W. Blauner, NCI, LTCB. Following these sequences are $50 \%$ consensus sequences derived from PCR amplified sets of sequences from the same individual. More information on these sequencec can be found in Part I, and in sections C4 and C5. The Epstein sequences are consensus sequences from samples taken from the brain (B) or spleen (S) of three HIV infected children (Epstein et al., Virology 180: 583-590 (1991)). The Wolfs sequences are consensus sequences from a set of children infected by uransfusion from a single donor (Wolfs el al., PNAS 87: $9938-42$ (1990); the corresponding nucleutide sequences for this set should be available in the near future). The "Edin" sequences are consensus scquences from a cohort of hemophiliacs from Edinburgh, some of whom were infected by a common source (Balfe et al., J. Virol. 64: 6221-6233 (1990) and Simmonds et al., J. Virol. 64:5840-50)). The next sequence is Guo.JB.con, a consensus derived from samples collected in 1983 from the patient BRU (Guo et al., Narure 349:745-6 (1991)). The final sequences in this set are consensus sequences derived from direct sequencing of samples taken in 1983 from three patents: BRU (sequences from BRU are called "JB"), LAI, and DL (Wain-Hobson et al., Science 252:961-64 (1991)). These sequences have the prefix "W-H". For comparison HIVHXB2 and HIVLAI (formerly called HIVBRU) V3s are included.

Presentation of the LaRosa et al. data set (Science 249: 932-35 (1990)) in this dalabase is pending further reevaluation of the sequences by the authors; the nucleotide sequences are available through GenBank, accession numbers M61417-M61661. The original data set was revised (LaRosa et al., Sclence 251: 811 (1991)). The uncorrected version, as originally provided by the authors, appeared in the 1990 compendium (pages III-54-55); a summary of the corrections was provided in a dntabase mailing dated Jan. 1991.

The African V3 sequences are highly variable, and thus did not readily align with the N. American/European set. Therefore the aligiments of the African sequences wero generated using the PIMA program described in parts I and II of the database (a PIMA "cluster-score-cut-ofr" of 25 divlded the African sequences Into the two groups shown here). African sequenco alignments are shown with a $50 \%$ consensus sequence at the top, and an amino acld class covering (AACC) pautem generated by the PIMA progrum at the bottom (see part II). Both the consensus and the AACC pattem are based on sequences which are shown here as well as an wditional elght sequences kindly provided by soverul groups prior to publication. Most of the sequences shown here originaled in Zaire, although sequences from Ugunda (U455), Thnzania (CBL4), Uabon (OYI), and Rwanda (SF170) aro also shown. Further information concerning the African sequences can be found in parts I and II of the cuncabase. 
V3 Loop Amlno Acld Allgnment, N. America and Europe: CONSENSUS HIVSC HIVMN HIVJH32 HIVWMJ1 2 HIVWMJ22 HIVWMJ32 HIVSF2 HIVRF HIVBRVA HIVJRCSE HIVJRFL HIVCDC4 2 HIVHAN HIVNY5NEW HIVJFL HIVADA HIVSE 162 HIVSF 33 HIVALAI HIVBALI HIVBAL2

CANOA

CANOB

FO CTRPNNN . . TRKSIHI . . GPGRAFY. TTGEIIGDIRQAHC 35

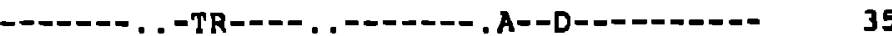

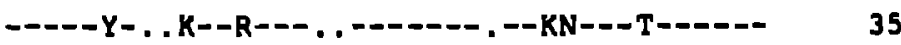

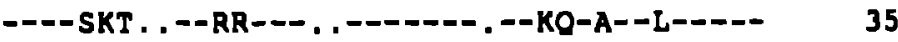

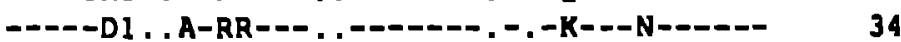

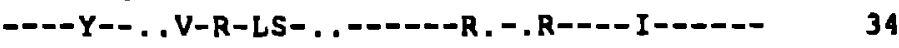

- - - DI . . A-RR-.. . . -

- - - . . .--O-Y . . - -

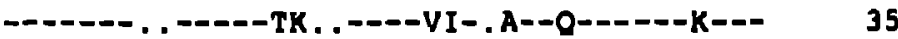

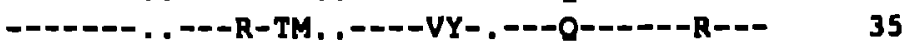

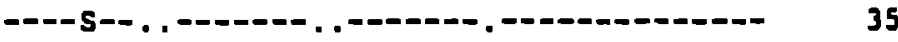

-C-O- . . . -

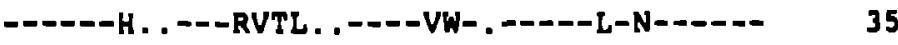

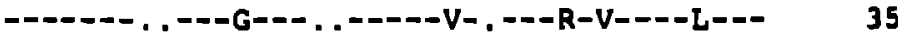

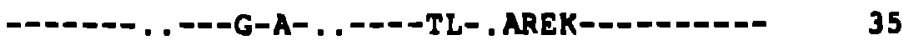

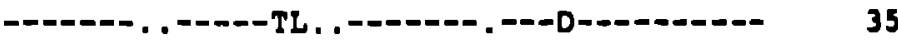

- -

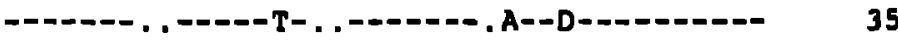

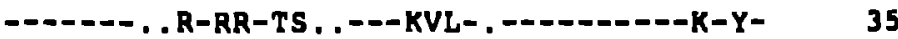

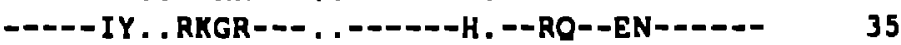

-_-D-O. . .

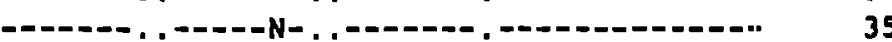

- - - H-O. . -

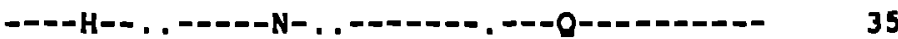

- - -

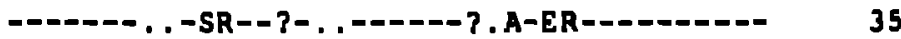

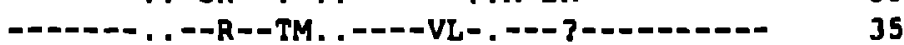

-2-D- . . .

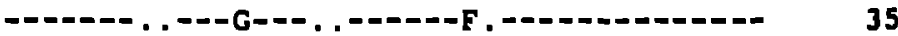

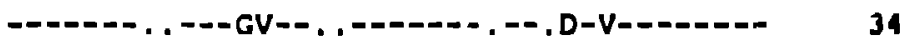

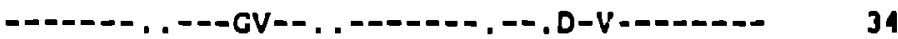

-

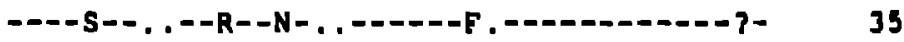

-

- -

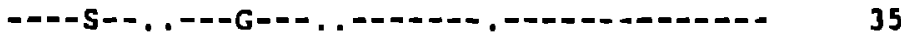

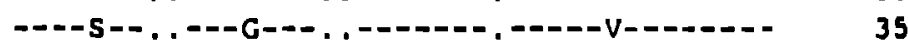

- -

- -

- -

--O-S-1, . -

-

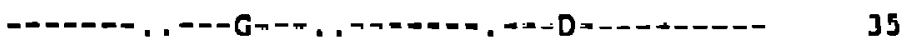

--O-S-- . . -

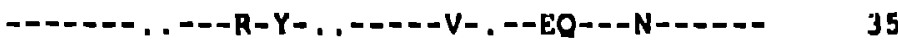

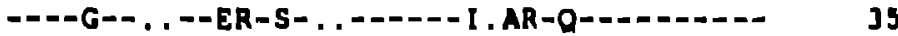

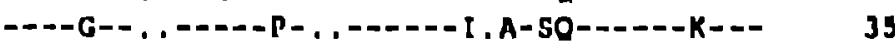

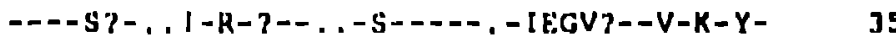

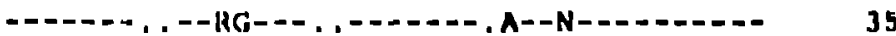

- - . . .

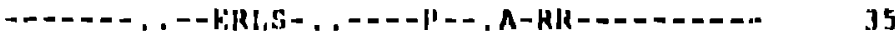

...--D., ,

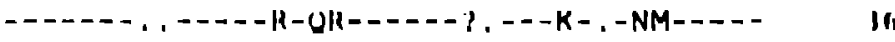

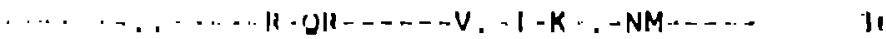

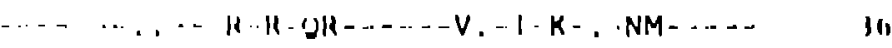






-The consensus sequence and amino acid class covering (AACC) pauern of the first set of African sequences (AfI) was derived from a total of 13 sequences. Eight of these sequences are not yet published so they cannot be presented in the database at this time. All sequences used firr the consensus sequences and AACC pattem of the second set of African sequences are shown. Lowercase letters in the AACC pattems designato umino acid classes, of groups of related amino acids. For more informution about AACC paltems, seo part II of the HIV database. Aligned diroctly beneath the AACC pattem is the set of all amino acids found in a given position in the alignment. AfI includes amino aclds from the elght sequences that are not shown as well as the five that are. The anino aclds are listed in order of their frequency, with the most frequent listed at the wip. 


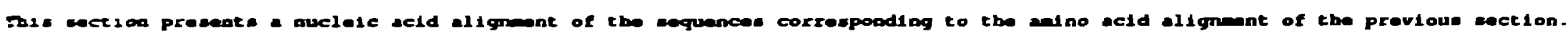

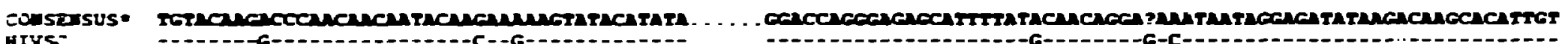
HIYS:

H:bres

HIVITiz

garimize

bivsre 2

ixing

LIT2:2

aITJRCSE

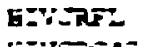

s:aces

Erras

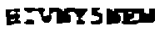

BIVT:

Bivasa

B:VTF: 62

ETVSE33

ETren:

EIVTa:

ETSaliz

3 E mavomus

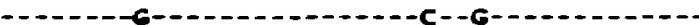

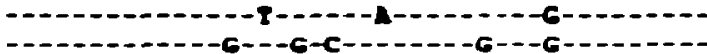

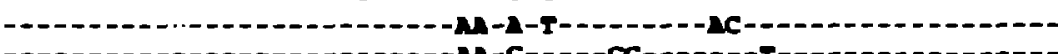

-

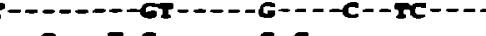

-

-

-

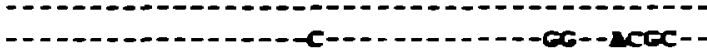

-

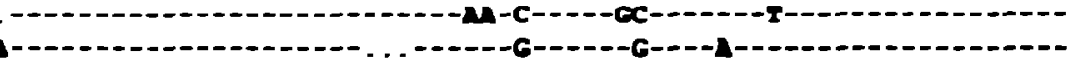
-

$c---\infty-\cdots$ -

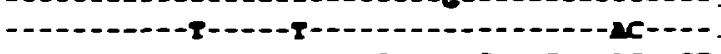
(n) con200 .

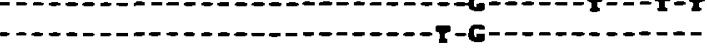

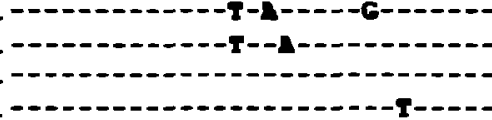

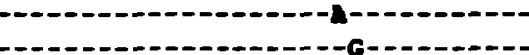
-

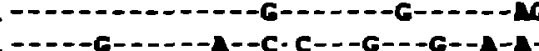

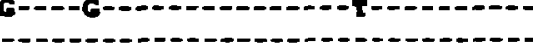
(n) (-

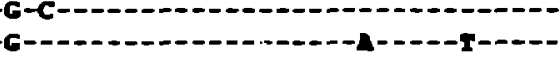
$-c-$

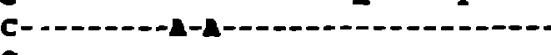
-

bis 5 incen

si.

si. siever

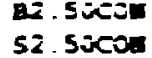

23.5000

53. $5000 \pi$

57.50000

207.5003

نr:- 50000

$\checkmark 22$. 5000

$\square 4.5000$

wo $\cos$. $J$

W. B. con. DL

W. B.cec. Sa

a. B. con - L

ErimenzR

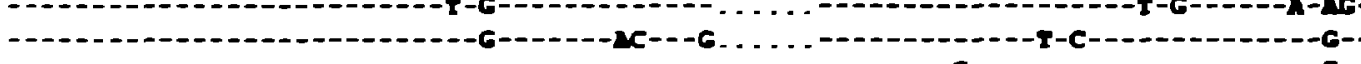

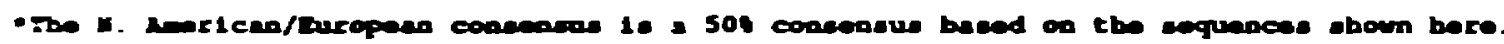
grotied togetber to create tbe conecens. 


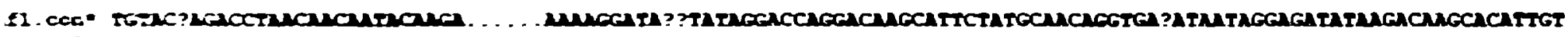

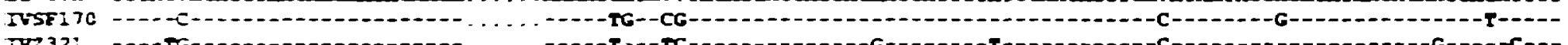

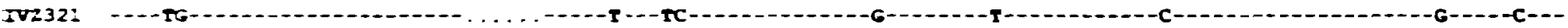

IVOTI C-

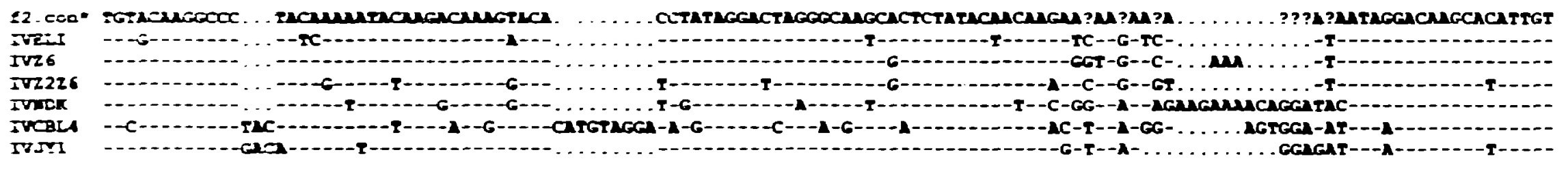

Ite consensis seouence for each wet is based on a 501 consenous of the sequences eboun bere. 


\section{HIV-1 V3 LOOP ANALYSIS}

\section{IIIC4. Intrapatient sequence sets}

Sibling sequences are derived from viruses obtained from the same individual. Sets of sequences frcm the same individual are referred to in this section as sibl, sib2 ... In order to facilitate comparisons between data sets, we have aligned the sequences presented here w the $\mathrm{N}$. American/European consensus, as in section C2.

The Edinburgh sequences (Balfe et al., J. Virol. 64:6221-6233 (1990) and Simmonds et al., J. Virol. 64:5840-50 (1990)) and the Epstein sequences (Epstein et al., Vrology 180:583-590 (1991)) often contained more than one sequence that was identical within the V3 loop. Whenever there was identity in V3 amino acid sequences between two or more sequences from a single individual, we included the sequence in this section only once. A tag was included on the sequence reference name to indicate how many sequences were identical within a sibling set to the one shown. For example, BI.DIR.con3seqs, the first sequence in the Epstein seh, was exactly the same as two additional BI sequences in the V3 loop: the fact that it was present three times is indicated by the "con3seqs". Note that identity w' thin the V3 loop does not imply identity in the surrounding sequences, nor at the nucleic acid level.

Also included in this section are sibling sequences from the patient BRU, designated "JB", and sequences from the patient LAI. Viral isolations from both of these patients were attempled at the Pasteur Institute in 1983 (Wain-Hobson el al, Science 252:961-64 (1991)). Apparent', virus from the patuent LAI contaminated a culture which was thought to be from the patent BRU, and hence LAI was the source of the virus previously known as HIVBRU, now called HIVLAI. Due to the high degree of similarity between the sequences of HIVLAI and HIV-IIIB, these viruses are thought to have originated from the same isolace. For further clarification of the origins of these viral sequences, see Wain-Hobson et al., Science 252:961-64 (1991), Guo et al., Natwe 349:745-6 (1991), Oallo, Narure 351:358 (1991).

The sibling sequence assignments previously made available for the LaRosa data set (LaRosa et al., Science 249:932-35 (1990)) in the database update daled Jan. 1991 and in GenBank, have been withdrawn by the authors, and are currently under reevaluation; therefore sibling assignments of the LaRosa data set are not presented here. 
V3 sequences originating from the patient BRU; the virus is now designated HIVJB:

Wain-Hobson et al., Science 252:961-64, (1991):

CONSENSUS
HIVJB.A0 46
HIVJB.A051
HIVJB.A0 45
HIVJB.A049
HIVJB.A053
HIVJB.A0 55
HIVJB.A0 50
HIVJB.A064
HIVJB.D24
HIVJB.D29
HIVJB.D30
HIVJB.B067

CTRPNNN . . TRKSIHI . . GPGRAEY . TTGEIIGDIRQAHC 35
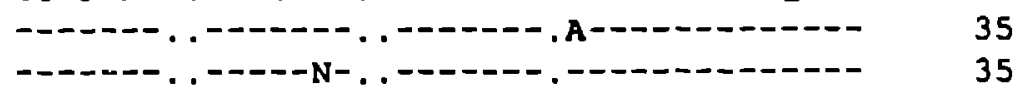

- - - - . . - - - . . - 35

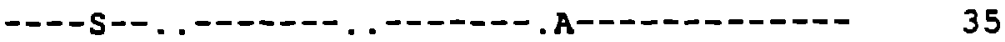

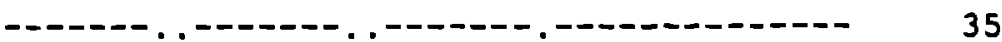

--1-- . . .

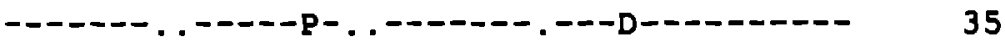

- - - - . . .

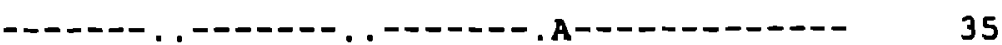

$----D$ D. . -

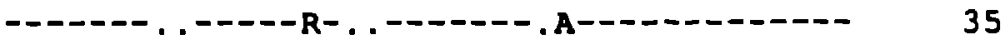

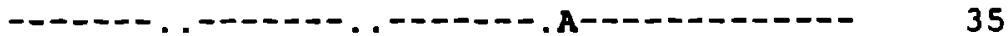

The "A" series was sequenced directly from a stored lymph node biopsy from the patient BRU, the " $D$ " series from a 198,3 culture of BRU virus with JBB donor cells, and B067 from a 1990 culture of the original isolate finm the man BRU.

Guo et al., Nature 349:745-46 (1991):

HIVJB.con6seqs

HIVJB31

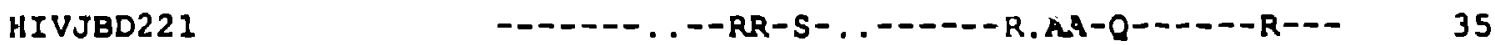

The Owo sequences are from a 1983 sample of the BRU-JBB donor cell culture sent from the Pasteur Institute to the Laboralory of Tumor Cell Biology at the NIH.

*Sequences are aligned with the $\mathrm{N}$. American/European consensus sequence. 
V3 sequences originating from the patient LAI, formerly called HIVBRU and originally called LAV-L, but now called HIVLAI:

Wain-Hobson et al., Science 252:961-64, (1991):

\begin{tabular}{|c|c|}
\hline CONSENSUS & CTRPNNN. . TRKS IHI . . GPGRAFY . TTGEI IGDIRQAHC \\
\hline HIVIAI . L7 & $-----. .----R-Q R------V .---K-.--M-----$ \\
\hline HIVLAI . L3 & $---\cdots-n,----R-Q R------V,-I-K-.-N M-----$ \\
\hline HIVIAI . L2 & $------\ldots----R-Q R------V .---K-.-N M-----$ \\
\hline HIVLAI. L6 & $-----.----R-Q R----T-V,-I-K-,-N M-\cdots$ \\
\hline HIVLAI. I9 & $--. .----R-H R-------.-I-K-., N M----$ \\
\hline HIVLAI . L4 & 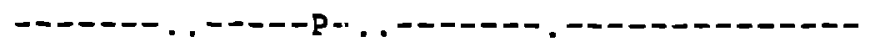 \\
\hline HIVLAI . L20 & 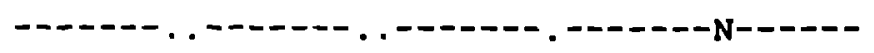 \\
\hline HIVIAI. LI6 & 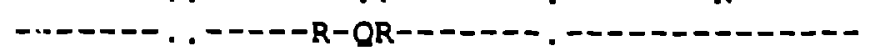 \\
\hline HIVLAI . E70 &.,$----------R-Q R------V ., I-K-,-N M-----$ \\
\hline HIVLAI .E79 & $------.,----R-Q R------V, I-K-.,-M-----$ \\
\hline HIVLAI . E69 & $------n .-----R-U R------V ., I-K-$ RNM--- - \\
\hline HIVLAI.E86 & $-------\ldots, G---R-Q R------V .-I-K-,-N M-\cdots$ \\
\hline HIVLAI.KI40 & $------n-----R-O R------V ., I-K-,-N M-\cdots--$ \\
\hline HIVLAI . K139 & $-----.,----R-Q R------V,-I-K-,-N V--\cdots$ \\
\hline HIVLAI . K138 &..-------- R-OEE-K---V. IRK- . EN----- \\
\hline HIVLAI.KI45 & $------. .----\mathrm{C}-\mathrm{QR}------\mathrm{V} .-\mathrm{I}-\mathrm{K}-., \mathrm{NM}-\cdots$ \\
\hline HIVIAI . K147 & $-----n,----R-Q R------V .-I-K-.-N-----Y$ \\
\hline HIVLAI.K155 & $------. .--G-R-O R------V ., I-K-., N M----$ \\
\hline HIVLAI.KI 44 & $------. .----R-Q R------V ., I-K-.,-M-\cdots--$ \\
\hline HIVLAI.K152 & 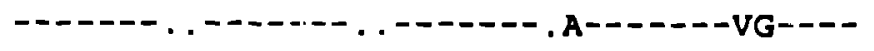 \\
\hline HIVLAI.HII7 &.,$----------R-Q R------V ., I-K-., N M----$ \\
\hline HIVLAI.HII6 &.,$---------R-Q R------V .-L-K-,-N M----$ \\
\hline HIVIAI.HI27 & $-----. .----R-O R------V$, I-K- . ENM---- \\
\hline HIVIAI . HI30 & $----D . .----R-Q R------V ., I-K-,-N M-----$ \\
\hline HIVLAI. H 335 & $----. . .---R-Q R------V ., I-K-,-N M----$ \\
\hline
\end{tabular}

The " $\mathrm{L}$ " series above is from a peripheral blood sample from the patient $L A I$ unken in 1983 and stored. The " $\mathrm{E}$ " series is from the original culture of $\mathrm{LAl}$ 's virus with donor JBB cells. The " $\mathrm{K}$ " series is from a bone marrow culture (M2T-/B) from which supemacant was sent to the Laboratory of Tumor Cell Biology at the NIH. Originally M2T-/B was thought to have been derived from cultured virus from the patieni BRU; apparently M2T-/B was contaminated by virus from the patient LAI. The "H" series is from a B-cell adopted culture that originated from M2T-/B.

For comparison, some of the early sequenced tsolates that are very similar to the sequences from LAI are shown:

Wain-Hobson el al., Cell 40:9-17 (1985):

LAV $\quad-\cdots$

Ruiner et al., Nature 313:277-284 (1985):

BH10 036

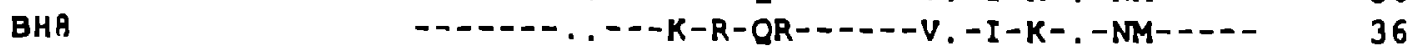

- Sequences are aligned with the N. American/Euronean consensus. 
EDINBURGH SIBLING SLQUENCES:

\begin{tabular}{|c|c|}
\hline $\begin{array}{l}\text { CONSENSUS } \\
\text { Sibl: }\end{array}$ & CTRPNNN . . TRKSIHI . . GPGRAEY . TTGEI IGDIRQAHC \\
\hline v77171. con2seqs & 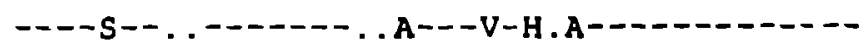 \\
\hline $\begin{array}{l}\text { v77173. con } 4 \text { segs } \\
\text { sib2: }\end{array}$ & $----S--\ldots------\ldots----V-H \cdot A-----------$ \\
\hline v872420.con 4 seqs & $----G-Y . .--E R-S-\ldots------$ I . ARRQ --------- \\
\hline V872421 & $----G--\ldots--E R-S-\ldots-----I$. AR-Q--n ------ \\
\hline $\begin{array}{l}\text { v872422.con } 4 \text { seqs } \\
\text { sib3: }\end{array}$ & 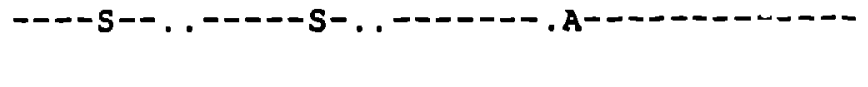 \\
\hline V91605 & $----G--\ldots----P-\ldots------I$. A-SQ-- $----K---$ \\
\hline V91609 & 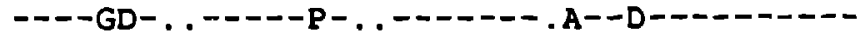 \\
\hline v91611. con2seqs & $----G--. .---R-S-\ldots------I, A-S Q------K---$ \\
\hline v91613 & 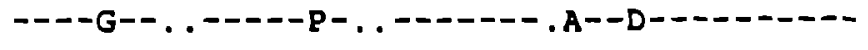 \\
\hline $\begin{array}{l}\text { sib4: } \\
\text { v74164. con3segs }\end{array}$ & 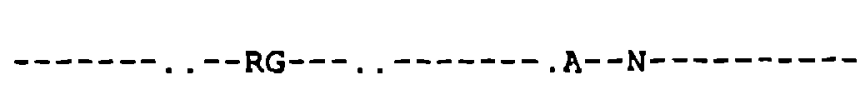 \\
\hline V74167 & 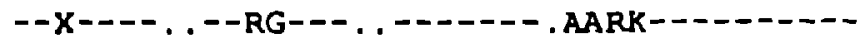 \\
\hline V74169 & 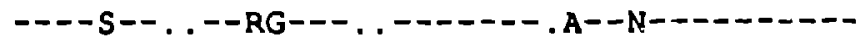 \\
\hline v74179 & 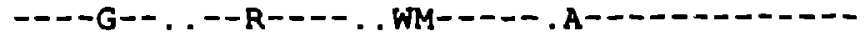 \\
\hline sib5: & \\
\hline V82711. con 2 seqs & $------. .--R-Y-\ldots-----V-.--E Q--\cdot N------$ \\
\hline v82712. con $2 \operatorname{segs}$ & 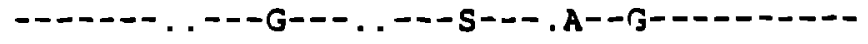 \\
\hline v82716 & 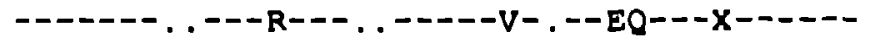 \\
\hline $\begin{array}{l}\text { V82717. con2seqs } \\
\text { sib6: }\end{array}$ & $-I----. .---R-Y-\ldots----U-,--E Q---N------$ \\
\hline V12834 & $----S K-\ldots I-R----\ldots-S-----$, I EGVA--V-K-Y- \\
\hline V12836 & $----S--\ldots K-R-M--\ldots-S-----$. I EGVT--V-K-Y- \\
\hline V12837 & $----S K-\ldots I-R-M--\ldots-S-----$, I IEGVA--V-K-Y- \\
\hline V12840 & $----\mathrm{S}-\mathrm{T} \ldots--\mathrm{R}----\ldots------, \ldots--\mathrm{VT}-\mathrm{V}$ VKK-Y- \\
\hline
\end{tabular}

The patient V12 is an HIV infected American nemophiliac. The other patients listed here are from a cohor of HIV infected hemophiliacs from Edinburgh, who had been exposed 10 an HIV contaminaled balch of factor VIII in 1984. Patients V77, V87, and V91 (sibs 1, 2, and 3) had closely related sequences, and were thought to be infected by related viruses. Balfe ct al., J. Virol. 64:6221-33 (1990), and Simmonds el al., J. Virol. 64:5840-50 (1990).

-The sequences are aligned with the N. American/European consensus. 


\section{HIV-1 V3 LOOP ANALYSIS}

EPSTEIN ET AL. SIBLING SEQUENCES:

\begin{tabular}{|c|c|}
\hline $\begin{array}{l}\text { CONSENSUS* } \\
\text { sibl: }\end{array}$ & CTRPNNN . . TRKS IHI . . GPGRAFY . TTGE I IGDIROAHC \\
\hline B1. DIR. con3seqs & $----U-, .---A----------$ \\
\hline B1. IAI. con 3 seqs & 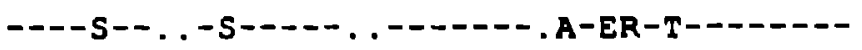 \\
\hline B1. IA2. consseqs & $----. .-S R R-R-\ldots-----$ V. ARER $---\cdots---$ \\
\hline B1. โB3 & 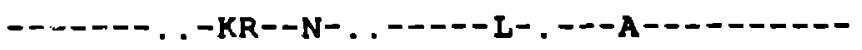 \\
\hline B1. IB5 & $-----\ldots$ SRR-S- . .-----V. ARER-- - - - - - \\
\hline B1. IC4 & 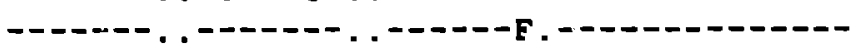 \\
\hline B1.I.BI & $--K----\ldots,-S R R-R-\ldots K--K--V . A R E R---N------$ \\
\hline SI.DIR & ------ . $^{--R--T M} . .----V L-.---A--------D-$ \\
\hline S1.IA10 & 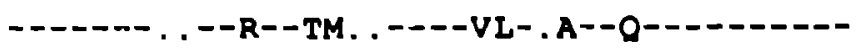 \\
\hline S1. IA2 & 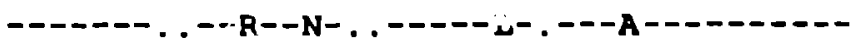 \\
\hline S1. IA6. con 4 segs &.,$--------R R-T M . .----V L-.,--Q------K---$ \\
\hline S1. IA9. con2 seqs & 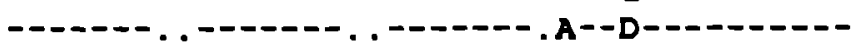 \\
\hline S1. IB2 & 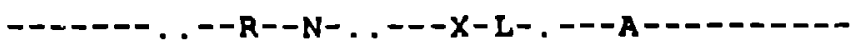 \\
\hline S1. IB4 &,$------ \ldots--R--T M . .----V L-.,--A---------$ \\
\hline S1.IB7 & 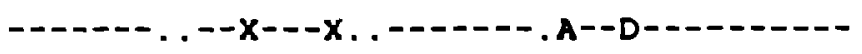 \\
\hline S1. IIA9 & $-------\ldots--R R-T M .,----V P-,---Q------K---$ \\
\hline $\begin{array}{l}\text { S1. IIB10 } \\
\text { sib2: }\end{array}$ & 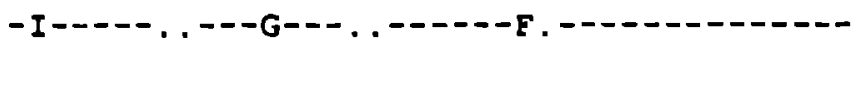 \\
\hline B2.DIR & 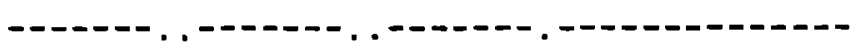 \\
\hline B2. I IB2 & 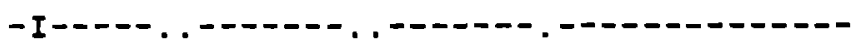 \\
\hline B2. I I B7 & 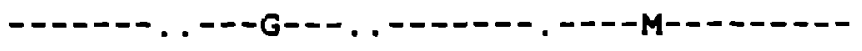 \\
\hline B2.I.BI & 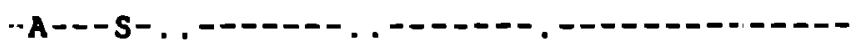 \\
\hline B2. I. B2. con 2 seqs & 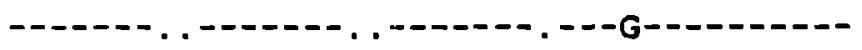 \\
\hline B2.I.B10 & 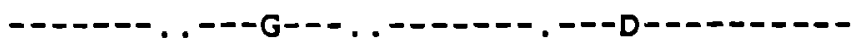 \\
\hline B2.I.B7 & 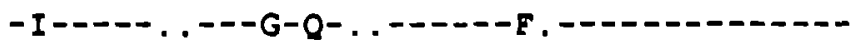 \\
\hline S2.DIR & 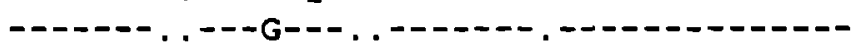 \\
\hline S2.IAS & 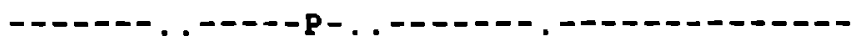 \\
\hline S2.IA 6 & 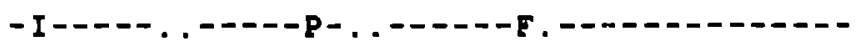 \\
\hline S2. IA 7 & 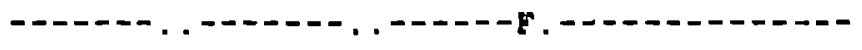 \\
\hline $52.1 \mathrm{~B} 3$ & 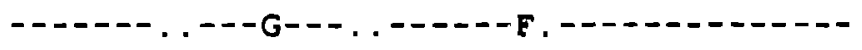 \\
\hline S2.I.A11 & 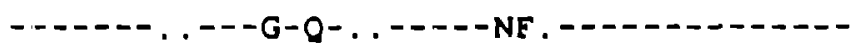 \\
\hline $\begin{array}{l}\text { S2.I.A3 } \\
\text { sib3: }\end{array}$ & 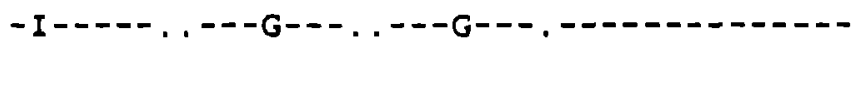 \\
\hline B3.DIR. con5seqs & 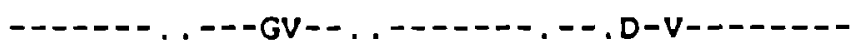 \\
\hline B3. IB3 & 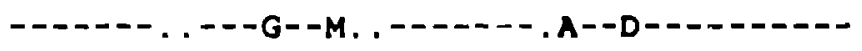 \\
\hline S3. I IC1 & 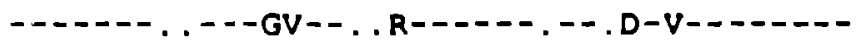 \\
\hline S3. IIC4. con $350 q$ s & $\cdot I----\ldots---G V--\ldots,-----.,-D-V-------$ \\
\hline S 3.IIDI & 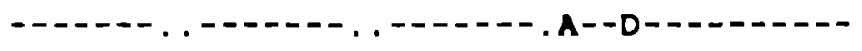 \\
\hline S3.II.D7 & 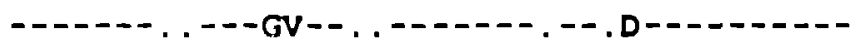 \\
\hline S3.II.C5 & 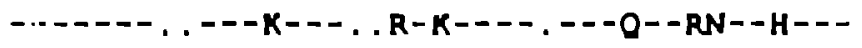 \\
\hline
\end{tabular}

B stands for brain and S for spleen; the number following refers to patients 1, 2 and 3 (Epstein et al., Vroloxy 180: 583-90 (1991)). "DIR" indicates that the sequencing was done directly from the samplo-the othei sequences were from clones. A direct sequence was obtained for samples B1, S1, B2, S2, und B3.

-The sequences are aligned with the $\mathbf{N}$. American/European consensus. 


\section{IIIC5. V3 Loop Similarity Distributions}

The similarity distributions in this section reflect the range of variation found among V3 loop nucleotide sequences. First, the similarity between 500 V3 loop nucleotide sequences was calculated. For these initial comparisons, no distinctions were made between intra- versus inserpatient sequences, or for the geographic origin of the sequences (N. American, European, and African sequences were included) (Fig. 1). This then was contrasted with intrapatient variation from non-cultured sequence sets (Fig. 2), and with the interpatient variation found among African sequences (Fig. 3). While the overall similarity (including both inter- and intra-patient sequence comparisons) ranged from identity (100\%) to 50\% similar, the average similarity was around $90 \%$. In contrast, sets of sequences from a single individual tended to be more highly conserved, and compartsons of 17 African viral sequences revealed much greater heterogeneity than is usually found between N. American and European isolates. Pairwise sequence similarity scores were calculated by dividing the number of homologous bases in two aligned sequences by the tovl number of bases in the aligned sequences. For the V3 losp nucleotides there are typically 105 bases. The percent similarity was rounded of for each pairwise comparison (98.4 and 97.7 would both be considered $98 \%$ similar) and the number of comparisons with a given percent similarity within a set was calculated. This number was converied to the fraction of the total ruinber of comparisons within the set to have a particular percent similarity, and is presented in a hisiograri. Generally for similarity comparisons of multiple aligned sequences, an entire column is eliminated from the alignment if any sequence included in the alignment hus a gap at a given position (Li et al., J. Mol. Blo . Evol. S:313-330 (1988)). Because of the limiled number of positions avalwuie in the $V 3$ loop, and the high frequency of insertions and deletions, we modifed this approach here and simply excluded positions in each pairwise sequence similarily comparison for which either of the two sequences under consideration had a gap inserted. 
Stmilarlty Distribution of v3 Loop Nucleotldes

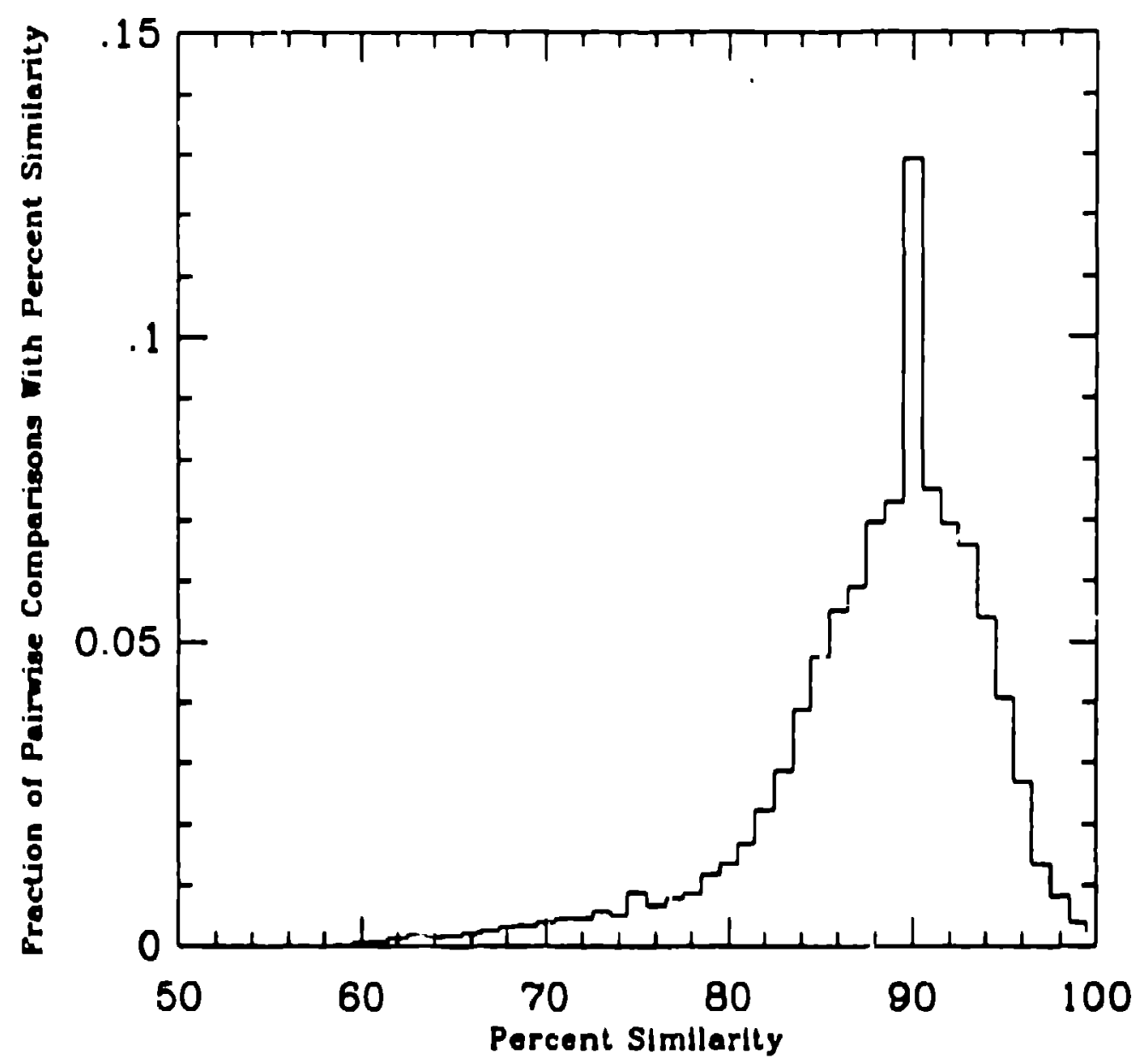

Pigure 1. Similarity Distribution of Nucleodde Sequences Encoding the V3 Loop. Similartity comparisons were made between all nucleodide sequences ancoding the roquences shown in soction C2. Including the $\mathrm{N}$. American/European sel and the African sols. a well a the nucloodde sequenies from LeRou of al. Sclence 249: 932-35 (1990)), and nucloodde equences from del that have not yol been published

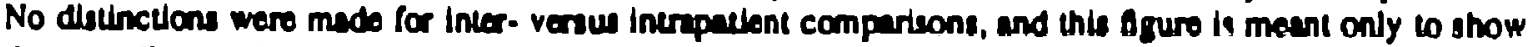
the overall potential vertation within the sequences encoding tis V3 boop. This distribution lis based on 124,750 peirwise sequenco compertisons ais 500 sequences. 
Simliarliy Distribution of Intrapatient v3 Loop Nucleolides

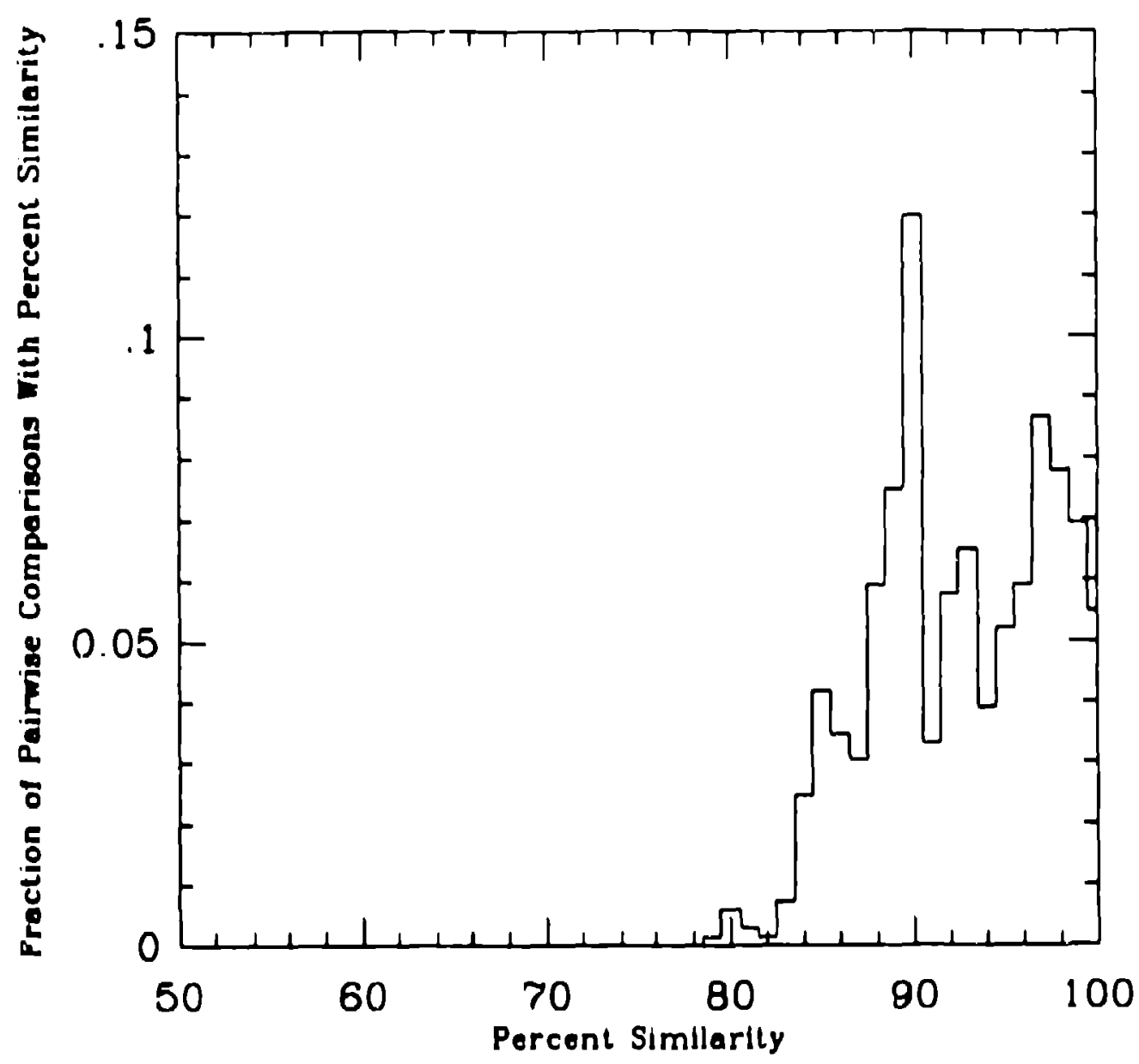

Figure 2. Similerity Diseribution of Uncultured Iritrygatient V3 Loop Nucleodide Sequences. This distribution is based on similarity comparisans of sibling requences from each palient in the hernophillac cohor (Balfo el al., J. Krol. 64:6221-6233 (1990) and Sim nonds al al., J. Virol. 64:5840-50 (1990)), and from brain and opleen dssue emplea (Epalein of al.s Mrology 100:583-90 (1991)). The sibling sequence sets ue shown in section $C_{4}$; the correaponding nu:leovide allignments are shown in par I of the HIV dutubase. This distribution is besed on 692 peirwise cornparisons of sequences derived from sibling viruses, from the nine individuals described in the iwo sludies silod above, with a range of 4-29 sequences avallable per albiling sea. 


\section{HIV2/SIV POL}

CONSENSUS aaATCCACETACCaGTaGAgAgaGA PatCTGGGAaCAgTGGTGGgCagAcTAtTGGCAAGTaACaTGGa HIV2ROD AAA--T--CC-A--A--A--G-GA--NATC-----G--G-----GATA-C--C-----A--G--A---A HIV2NIHZ AAA--T--CC-A--A--A--G-GA--TACC-----G--G-----GATA-C--C-----A--A--A---A HIV2ISY MAA--T--CC-A--A--A--A-GA--GACC----A--G----GATA-C--T-----A---G--A---A HIV2ST HIV2BEN MA--T--CC-G--A--G--G-GA--GACC-----G--G------GATA-C--C-----A--G--A---A HIV2D194 HIV2GHI HIV2D205 SIVMat251 SIVMH1 12 SIVMA239 SIVMNE SIVSHAH4 SIVSMMPBJ

CONSENSUS HIV 2ROD HIV2NIHZ HIV2 ISY HIV2ST HIV2BEN HIV2O194 HIV2GHI HIV2D205 SIVMM251 SIVMM1 12 SIVMM239 SIVMNE SIVSHAH4 SIVSMapaJ

CONSEnsus HIV2ROD HIV2NIHZ HIV2 ISY HIV2ST HIV 2BEN HIV20194 HIV 2GH] HIV20205 SIVMA25! SIVMM1 12 SIVMM239 SIVMNE MA--T--CC-G--A--G--G-GA--GACC-----G--G------GATA-C--C-----A--G--A---A MA--C--CT-A--G--G--G-GA--GACC-----G--G------GATA-C--C-----A--G--A---A GTG--C--TC-G--A--A--A-GA--GACA-----C--G------ACAG-T--C-----A--A--C---A MA--C--CT-A--A--T--G-GG--TGTh-----A--G------ACAG-C--T-----G--A--C---A MA--C--CT-A--A--T--G-AG--TGTA-----A--G------ACAG-C--T-----G--A--C---A MAA--C--CT-A--A--T--G-AG--TGTA-----A--G------ACAG-C--T-----G--A--C---A MAA--C--CT-A--A--T--G-AA--TGTA-----A--G------ACAG-C--T-----G--A--C---A AAA--C--TI-G--A--A--A-GA--AATT-----A--A------ACAG-T--T-----A--A--C---A AGA--T--TT-G--A--A--G-GA--MATT-----A--A----_-ACAG-T--T-----A--A--T---A

TCCCAgA rTGGGATTEgT ?TC ?ACeCCACCaCTagT CAGat Tagt ?TE TMACCTaGTaAaGA ?CCEAT -C--AG-C-----C--CG-G--T--C--A--AC-GG-C--GT-AGCG-TT--CC-G--AGGG--T--TA-C--AG-C--.--C--TG-A--T--C--A--AC-GG-C--GC-AGCA-TT--CC-G--AGGA--G--TG-C--AG-C-----C--CG-A--C--C--A--GT-GG-C--GT-AGCA-TT--CC-G--AMA--T--TA-C--AG-C-----C--CA-A--T--C--G--AC-GG-C--AT-AGTA-TT--CC-G--GAAA--T--CA-C--AG-G-----C--TG-A--T--C--A--AC-GG-C--GT-MACA-TT--CC-A--AGGA--T--TA-r--AG-G-----C--CG-A--T--C--A--AC-GG-C--GT-GACA-TT--CT-G--AGGA--T--TA-C--AG-G---O-C--CG-A--T--C--A--AC-AG-C--GT-MACA-TT--CC-A--AGGA--T--TA-C--AG-G-----C--TG-C--G--C--A--AT-AA-A--AC-AGCC-AC--CC-A--CAMA--C--CC-A--GG-G-----T--TA-C--A--G--A--AC-AG-A--AT-AGTC-TC--TC-A--GAAG--C--TA-A--GG-A-----T--CA-C--A--A--A--AT-AG-A--AT-AGTL-TC--TC-A--GMG--C--TA-A--GG-A-----T--TA-C--A--A--A--GC-AG-A--AT-AGTC-TC--TC-A--GAAG--C--TA-A--GA-A-----T--TA-C--A--A--A--AC-AG-A--AT-AGTC-TC--TC-G--MAG--C--TA-A--AG-T-----C--TG-G--A--A--T--CT-AG-C- -AT-AGTC-TC--CC-A--AMA--G--TA-A--AG-A-----C--TG-G--A--A--T--CT-AG-C--AC-AGTT-TC--CC-A--AMA--G--TA-

1850

1847

1847

1961

1907

1951

1907

1907

1910

1910

1922

1910

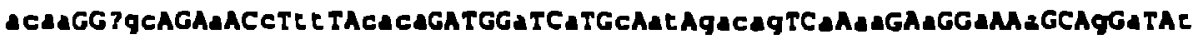
ACCA--TGC---G--C-TC--CACA-----A--C--C-AT-GGCAA--A-MA--A--A--A---G-A--T ACCA--CGC---A--T-TC--CACA-----A--C--C-AT-GGCAG--A-M--A--G--A---G-A--T ACCA--CCC---G--C-TC--CACG-----A--T--C-AT-GCCMA--A-AA--G--A--A---G-A--T ACTA--CGC---A--C-TC--CACA-----A--C--C-AT-AGCA--A-GA--A--A--A---G-A--C ACCA- -CGC---G--C-TC--CACA--.--A--A--C-AT-GACAG--A-MA--G--A--A---G-A--T ACCA--CAC---G--C-TT--CACA-----A--A--C-AT-GACAG--A-AA--A--A--A---G-A--T ACCA--CGC---G--C-TC--CACA-----A--A--C-AT-GACAG--A-AG--A--A--A---C-A--T AGM--GAG---A--C-AC--CACA-----G--C--C-AT-GMCC--A-AG--A--A--A---G-A--T AGAG--AGA---A--C-AT--TACA-----A--A--T-AT-AACAG--A-AA--A--G--A---G-A--T AGAG--AGA---A--C-AT--TGTA-----A--A--T-GT-AACAG--A-MA--A--A--A---G-A--T AGAG--AGA---A--C-AT- - TACA-----A--A--T-AT-AACAG--A-AA--A--G--A---G-A--T AMAG--AGA---A--C-AT--TGTA--.--A--A--T-AT-AACAG--A-AA--A--G--A---G-A--T 1920 1917 2917 2031 1977 
IIID. GP120 MODELS

In the SEP 90 release, on page III-52, a model for the HIV. 2 ap 120 protein structure was presented by $\mathrm{Tm}$ Gregory and colleagues. Since that time, those authors have improved their model (Gregory at al., "Structure and Function in Recombinant HIV-1 gp120 and Speculation About the Disulfide Bonding in the gp 120 Homologs of HIV.2 and SIV" in Advances in Experimental Medicine and Biology, in press. 1991), and those changes can be seen herein.

Again, these models have not been experimentally verified in the way that the HIV-1 gp 120 structure was verified (see III-51 of SEP 90 and Leonard ef al., J. Biol. Chem. 265: 10373-10382, 1990). For furcher information, contact Dr. Timathy Gregory, Genentech, (415)-266-1511.

\section{HIV-2/ROD gp120 (Proposed)}

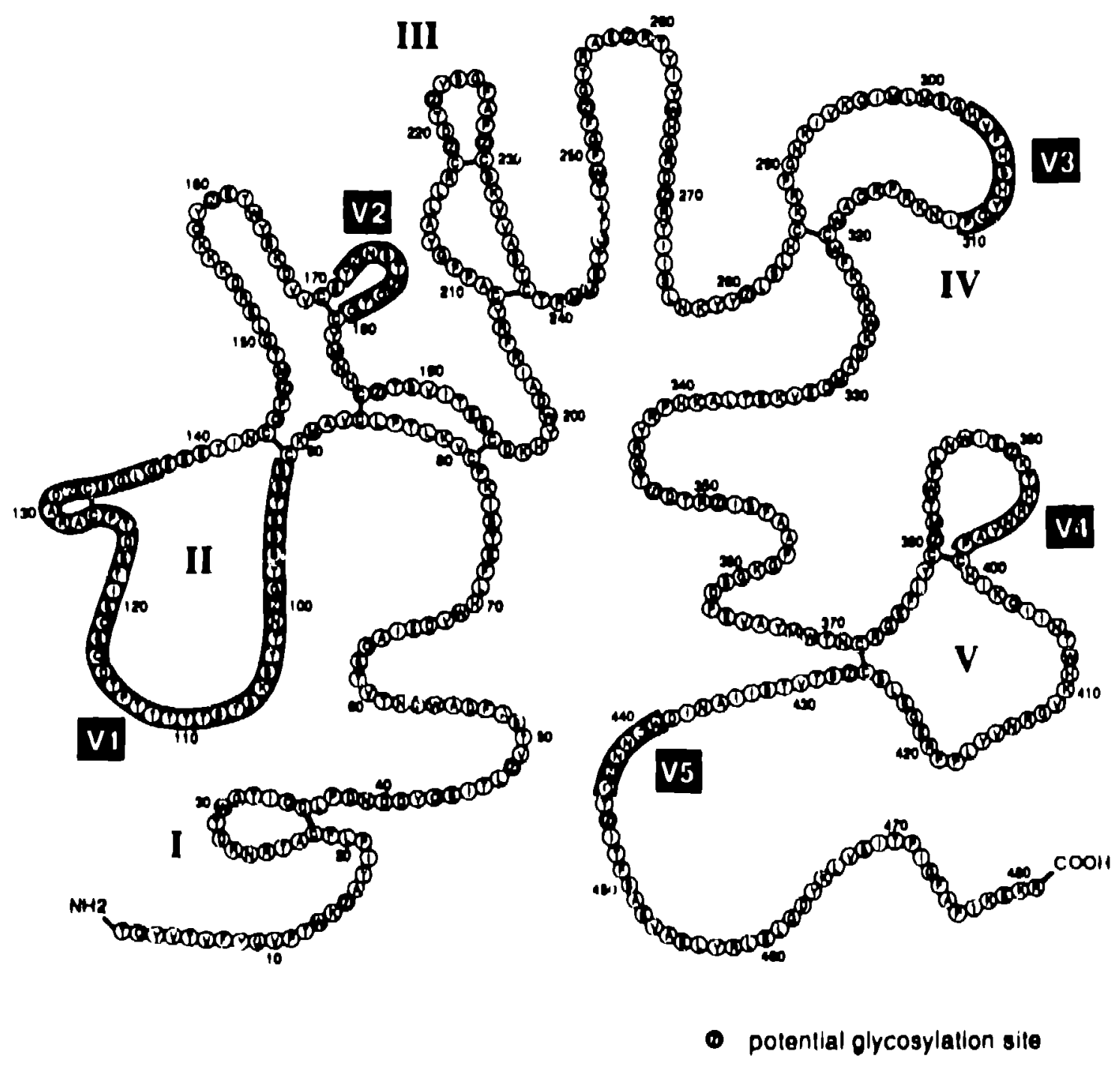




\section{SIV-MM142 gp120 (Proposed)}

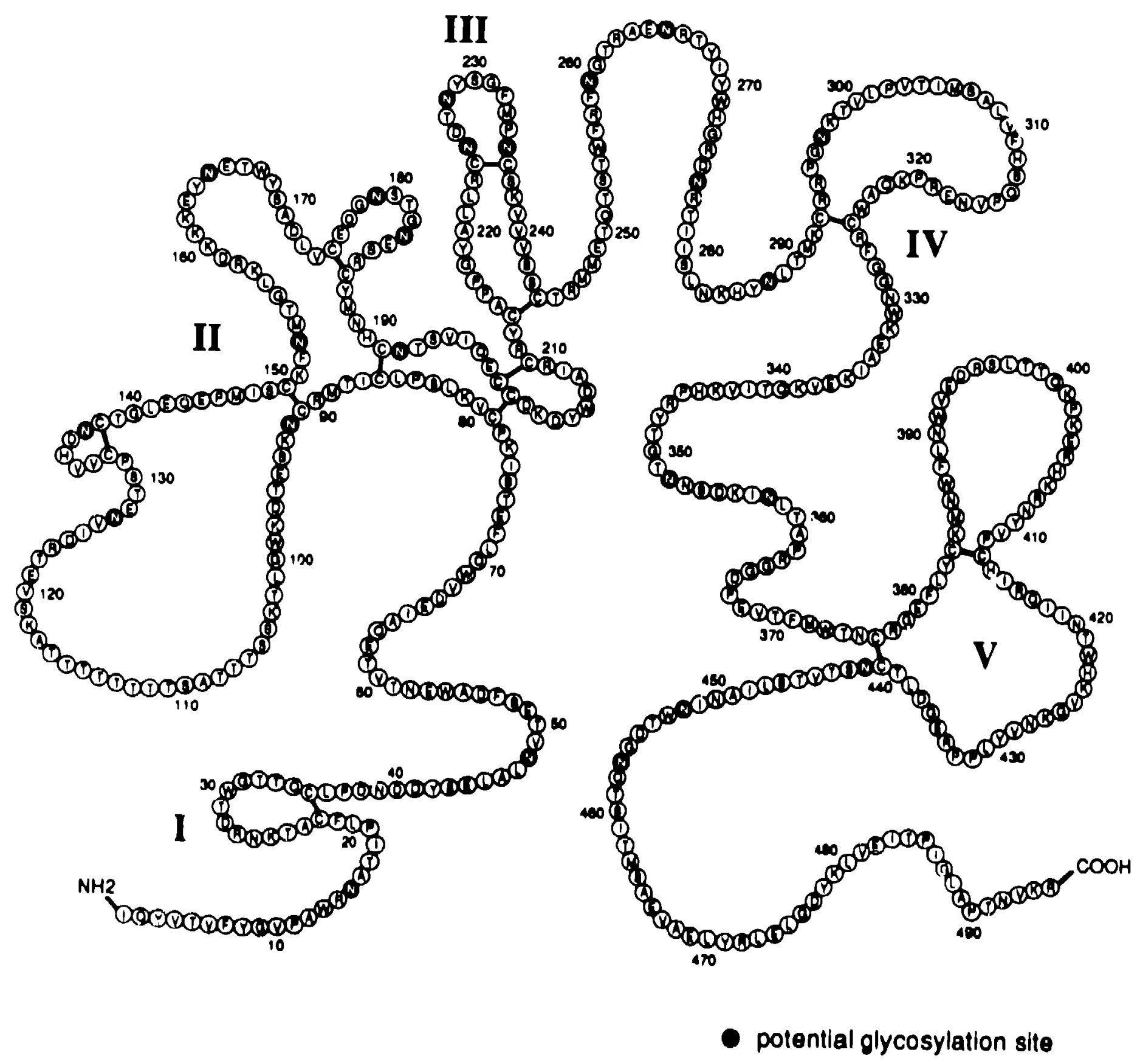




\section{SEQUENCE DISTANCES and PHYLOGENETIC TREES}

In the 1989 compendium (III-65 APR 89), a general discussion of HIV-I nucleotide sequence distance relationships was initiated. Al that time, sibling sequences representing viral forms taken from the same patient were found to differ from one another by up $103.3 \%$ in the env cds. Env sequences representing different HIV-1 infected persons in North America, sampled between 1983 and 1986, differed by $6 \%$ to 12\%, and comparable samples from Zaire covering the same time period differed by typically 16\%. The first result, representing intrapatient variation, was underestimated because it was based upon short-term or long-term cultured viral samples. In recent studies it is more commonly the case that sibling sequences differ by $4 \%$ 10 $6 \%$ when viral samples are direculy amplified and sequenced (sce Meyerhans ef al., Cell 58, 901, 1989; Balfe el al., J. Virol. 64, 6221, 1990; Delassus el al., J. Grol. 65, 225, 1990; Vartanian et al., J. Krol. 65, 1779, 1991: Wain-Hobson et al., Science 252, 961, 1991). Mulling and colleagues at Stanford have found a three-fold difference in the extent of variation, typically, between cultured and uncultured samples (in press, 1991); in contrast to these results, exceptionally homogencous sets of sibling and close cousin (linked) sequences have been reported by McNeamy ef al., PNAS 87, 1917, 1990 and by Kleim et al., AIDS Res. Ilum. Relroviruses 7, 417, 1991.

The second sampling result, representing interpatient variation, is not so eculcly affected by ex vivo selection, but it is subject to change in time, as a result of mutation, recombination and viral migration. Thus env V3 and V4V5 sequences from HIV-I infected persons in a single community of Florida in 1990 were found 10 differ by $13 \%$ on the average (see Morbldity and Mcrtality Weekly Repon of January 18 , 1991, Vol. 40, p.21). The extent of variation observed in this single study was nearly as great in 1990 as what had been scen in 1985 for North America and Znjre taken together.

These esumales are minimal cstimates for several reasons: when derived from phylogenetic urec unalyses, they represent only single base changes-insertions and deletions are not considered in most trec algorithms. Accordingly, when simple distance measurements are made in this databast, we have elected not to score gaps in order to achieve close comparison with the tree distances; but also to avold ambiguous alignments that gaps often entall and to sidesiep any artifactually Inseried (PCR-generated) stretches. If "gaps" are scored, all distence relationships must be asserted on the basis of similarly scored sequences ("upples" should not be compared with "oranges"), Moreover, it is most prudent with HIV lo score each gap as the equivalent of a single base substitution (Reitz et al., Nature 351, 358, 1991).

The uree analyses shown on the following pages were performed as described earlier (III-7 APR 90). They are of principal interest hecause they include the newly determined and relutively divergent U455 scquence from Uganda (Oram ef al., AIDS Res. IIwm. Retroviruses 6, 1073, 1990). The NOF scquonce wns kindly provided prior to publicalion by Dr. Michael Becter, University of Siellenbosch, South Africu. The sequence designaled CAM (Cambridge, U.K.) was kindly provitied by Allison Mclntosh, National Instivic for Medical Ressarch, MIII Hill. Both HIV.! urees shown-for gag and env-were rooled upon the SIV-cpr. sequence (not shown). Father than present branch lengths (only horizontal branch lengths are relevant wo distances), a bar-scalo is given. 
PHYLOGENETIC TREES

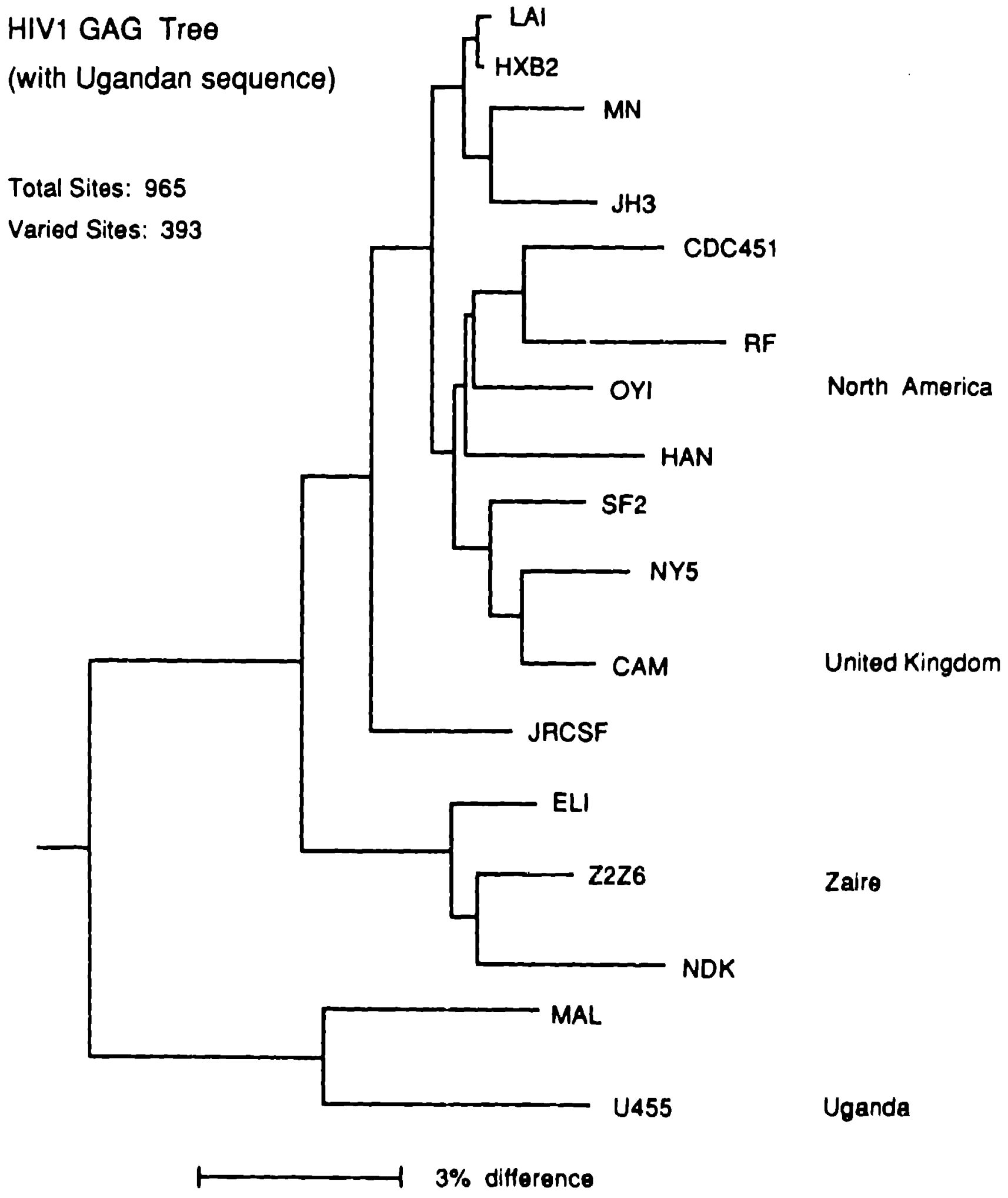


HIV1 ENV Tree (with Ugandan sequence)

Total Sites: 1144

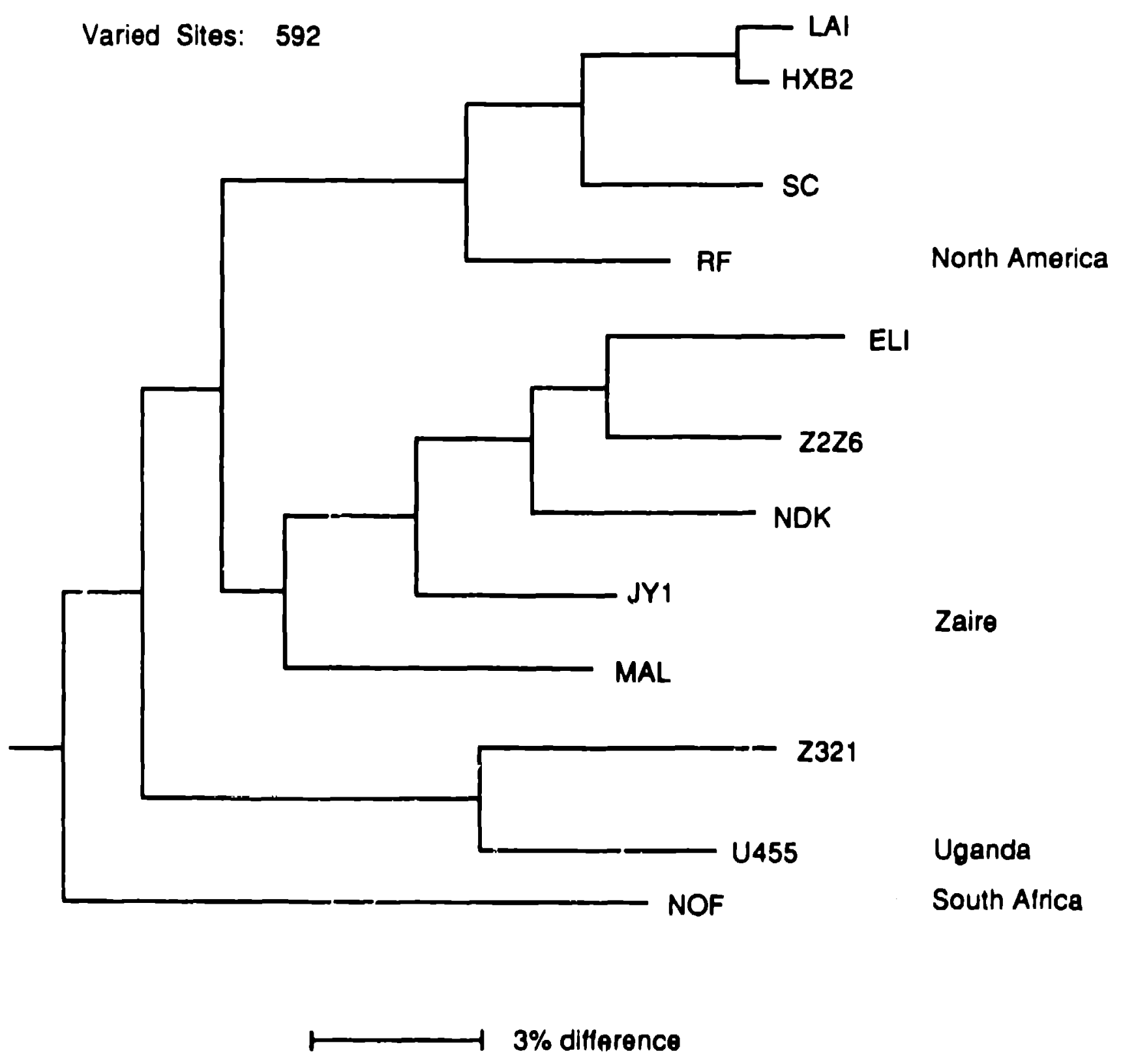




\section{Related Sequences}

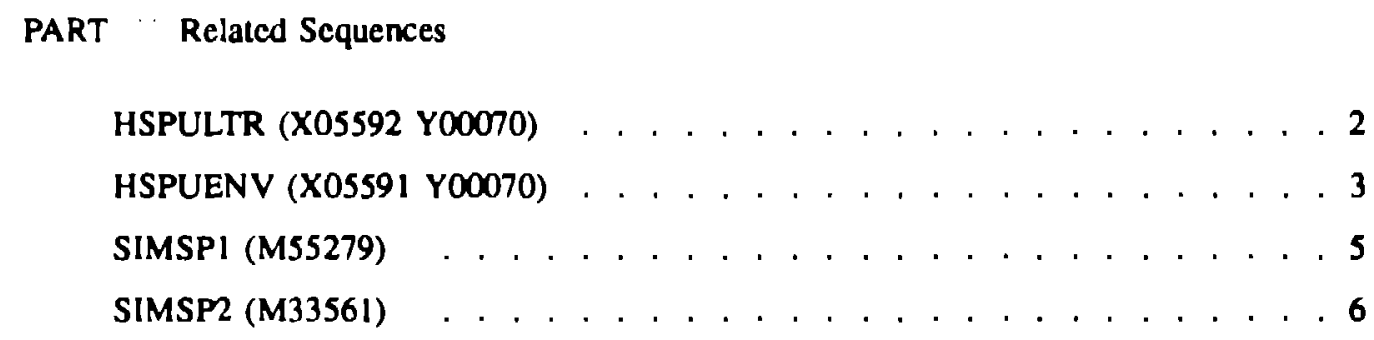


ZOCL's

CEFINITION ACCESSION

SOURCE REFERENCE

AUTHORS

$T$ ? TLE

jJURNAL COMMENT

BASE COUNT

IISPULTR 2206 bP SS-RNA

Human spumaretrovirus, HFV. DNA with 3 , LTR; bel-1, $-2,-3$. X05592 y0C070

Human spidmaretrovirus, HFV.

1 (bases I to 2206; onum. 4550 to 6755)

Fluegel, R.M., Rethul $1 \mathrm{~m}, \mathrm{~A}$. , Maurer,B. and Daral, G.

Nucleotide sequence analysis of the env gene and its flanking

reqloris of the human spumaretrovirus reveals two novel genes

EMBO J. 6, 2077-2084 (1987)

Recombinant clones that represent the J' part of the genome of the human spumaretrovirus (foamy virus) were established from cDNA (cione C55) and viral DNA (clone B52). See x0559) for C55

sequence. There are 12 octamer sequences, 6 nonamers and one decamer that form direct repeacs within the LTR. The Indirect repears include a decamer with a loop size of $78 \mathrm{bp}$ and cwo octamerg with a relatively small loop size of 34 and 5bp, respectlvely. - source: cell type-HSRV-infected HEL fibroblasts; clona-c55; Daca Kindly revlowed (02-SEP-1987) by Fluegal R.M.

OR:GIN

l atcctgaugt tggatetgg gTAAatata adcceteag aggadtget ggatergctg bel -2 orf $\rightarrow$

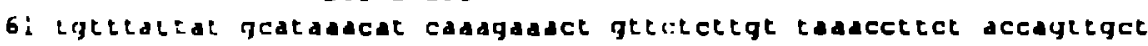

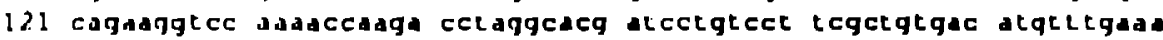

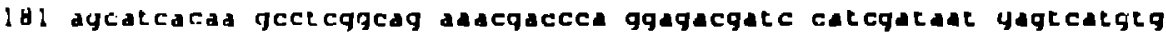
241 celccagtag eqacaccarg gicatigage catGatcace atgcaccad cetctetgga C- bel-1

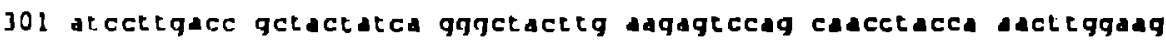
361 licacal.gtc aqgtggacce ttctgqgad aggtetatgy agactcatt ttgggtecce

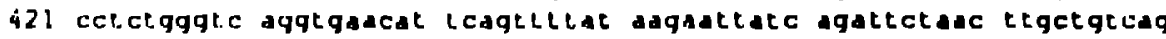

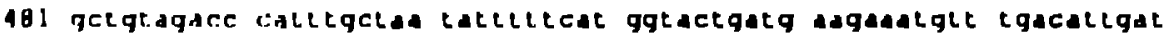

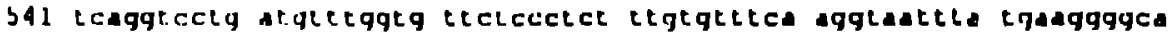

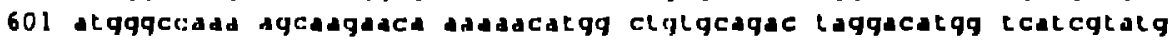

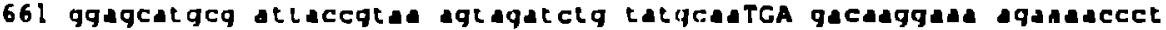
bel-3 orf $\rightarrow$

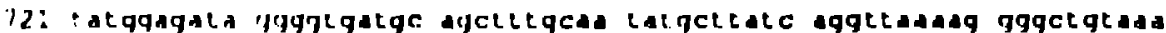

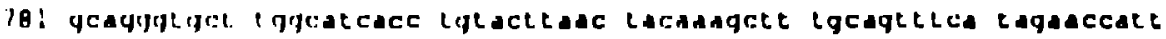

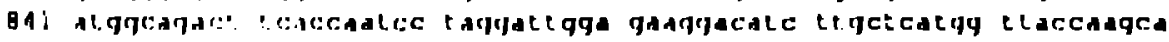

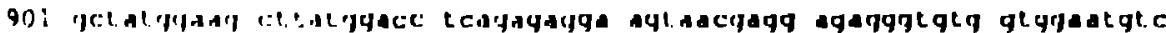

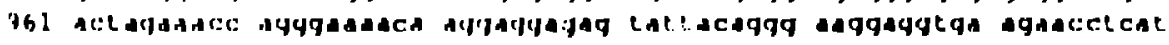

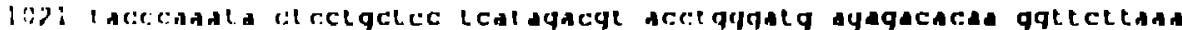

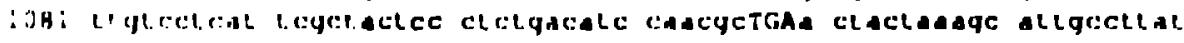
$<-b \in 1-2$

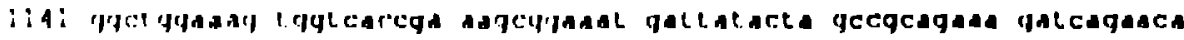

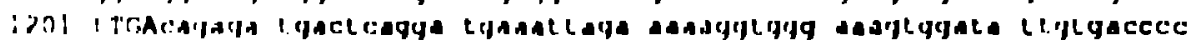
<- |je! - J

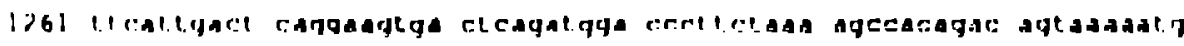

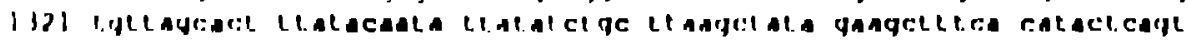

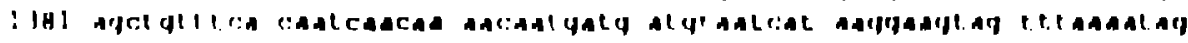

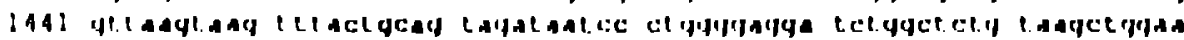

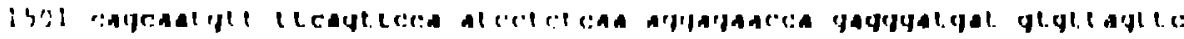

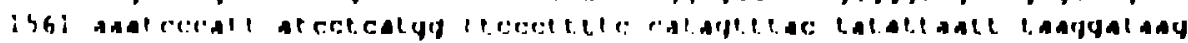

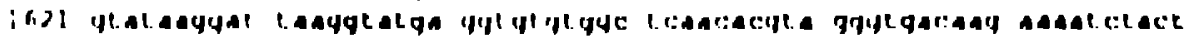

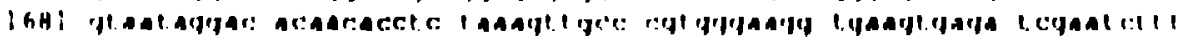

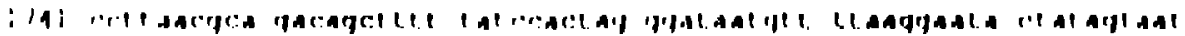

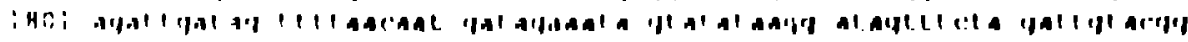

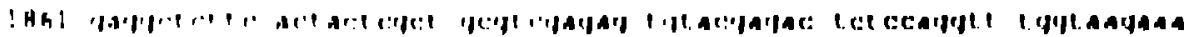

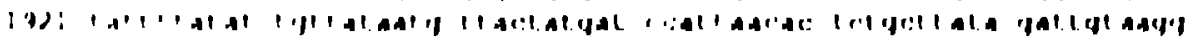

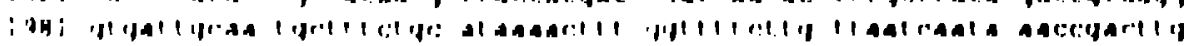

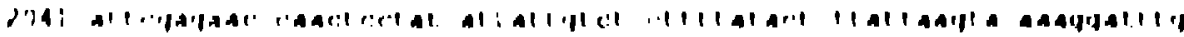

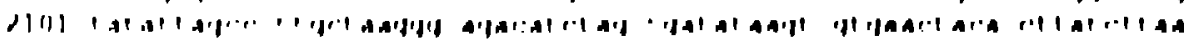

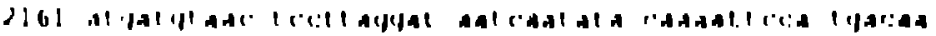


LOCUS HSP'JENV 4549 bP SS-RNA

JEEINITION Human spumaretrovirus (HFV or foamy virug) genomic RNA with env gene.

ACCESSION X05591 Y00070

SOURCE Human splmaretrovirug HFV.

REFERENCE 1 (baseg 1 to 4549; enum. 1 to 4549 )

AUTHORS Fluegel,R.M., Rethwilm,A., Maurer,B. and Daral,G.

TITLE Nucleotide sequence analysis of the env gene and lig flanking regions of the human spumaretrovirus reveals two novel genes

JOURNAL EMBO J. 6, 2077-2084 (:987)

COMMENT Recombinane clores that represent the $3^{\prime}$ part of the genome of the human spumarotrovirus (foamy $v$ lrus) were escablished from viral DNA (clone C55; see $\times 05592$ ) and CDNA (clone B52). The region common to both viral inserts comprlses $\theta 17$ bp (from the BamH at bp 4548 in X05591 to the HINDIII site at bp 816 In X05592 corresondig to bp 5365 in a fusloned viral sequence. The env precursor polypeptide contains 14 pot. N-1 inked glycosylation sites. "source: cell type-HSRV-infected HEL (lbroblasts; clone-B52;

Data kIndly reviewed (02-SEP-1987) by Fluegel R.M.

$$
\text { BASE COUNT Data kindly reviewed (02-SEP-1987) by }
$$

ORIGIN

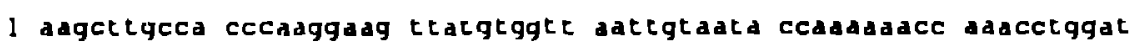
pol $\rightarrow$

61 gcagagtigg atcastett acagggleat tatataadg gatolcccan acastatea 121 tattettldg adgatggcad agtadaagtt tccagacctg aaggggtia aattattecc

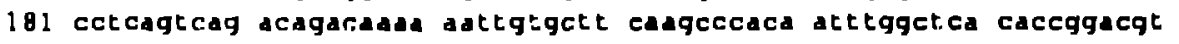
241 gaagccactc tettadad tgccaacete tattggtggc cadatatgag aaggatgtg 301 gttaacaac taqgacgetg teadagtgt thatcacad atgcttcca caagcetet 361 qqtectattc taagaccaga taggectcad adacetttg ataattett tattgactat 421 attggacct tgcedecte acagggatac ctatatgtat tagtagttg:- tgatggaatg 481 acaqgattca cttggttata ceccactade getcetteta etagcgcasc tgteadatct 541 ctcalgtac tcactagtat tgcautcca aaggtga'te actetgatca aqgtgcagca 601 thcactett cadectetgc tgaatgqgea aggasigag qtatacatt gqaatcagt 661 actect tatc acccceadag tggtagtadg gtggaagga adatagtga tataaacga 721 ctettaacta asctgetagt aygadgacce acasatgqt atgacctate gect.gtigta

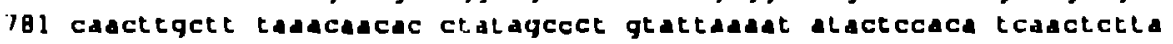

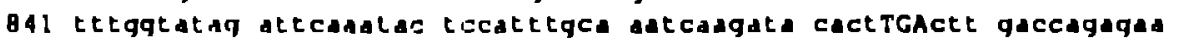
S1 orf $\rightarrow$

901 gangaactet cletellaca gjaaategt actlctelal accalccate caccecteca 961 gectectelc gttcctggtc tectgttglt ggceattgg teaggagagg gtgqctagge

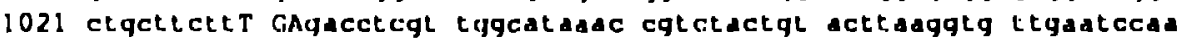
<- pol

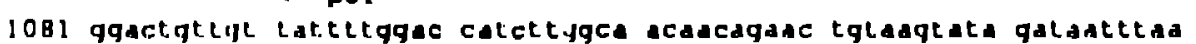

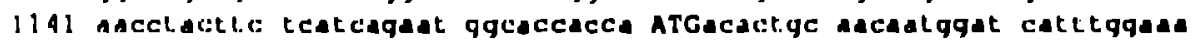
onv $\rightarrow$

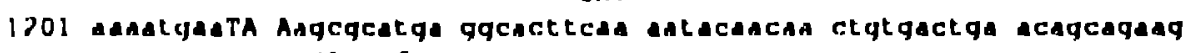
<- Sl ort

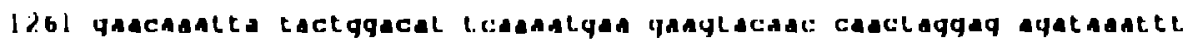

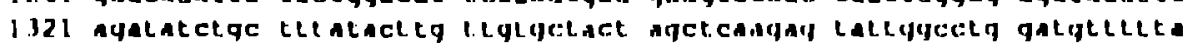

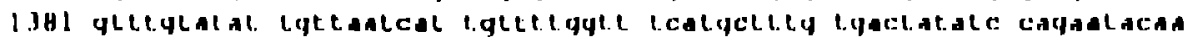

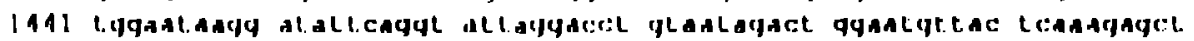

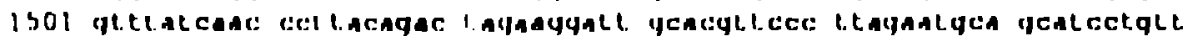

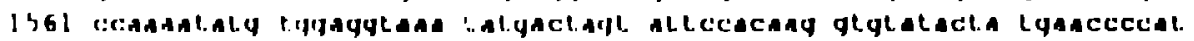

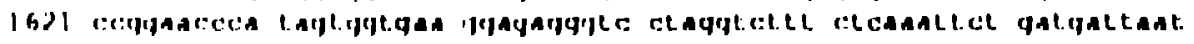

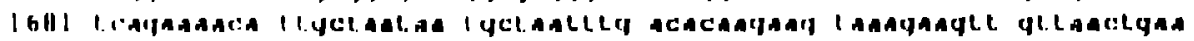

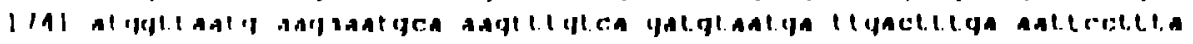

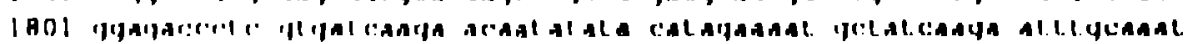

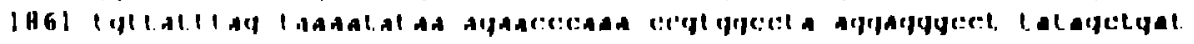

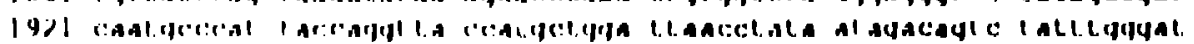

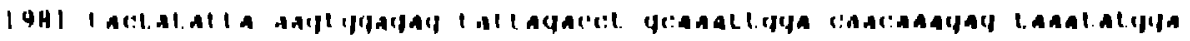

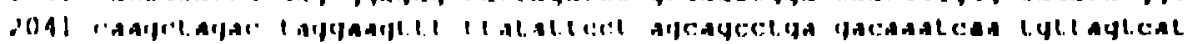

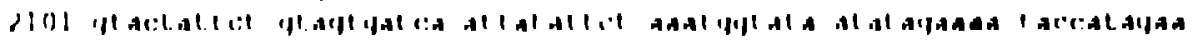

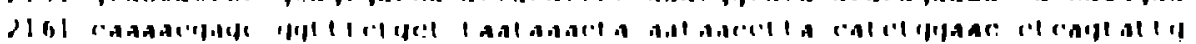

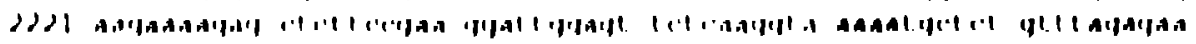


IISPUENV

2281 atcalgtgt tagatatctg cagtadacct gadetgtad tactatigad tacteatac

2341 tactccttct ctctacggga aggagattgt aattthacta adgatatgat ttctcagttg

2401 gttccagat gtgatggatt ctatadaat tetaagtgga tgcatatgca tccatatgct

2461 egtagatcet ggaydagta gadaatgaa adagagada ctadatgtag agatggggaa

2521 actagagat gtctgtatta tcctetatgg gacagtcceg aatctacata tgattetggt

2581 tatltagcat accaaagaa ttetcctcc cctatctgta tagaaca. ca gaaattaga

2641 gatcadgatt atgaagteta tcctctgtat cadgacgca adatagci.tc taadgatat

2701 ggat tgata cagttctact ctccetaag aatttctca attatacigg aactcetgta

2761 adtgaatge ctaatgcaag agctttcgta ggcetadag atcecaagt: tcctcettce

2821 tatcccaatg tcactaggga acattatact tcetgtata ataggadag agaagtgtt

2881 gataliaact atgctaagt aaggtctatg gggtatgcac ttacaggage agtgcaasce

2941 ttatctcad tatcagatat taatgatgas arctacagc a agaatata tteattaagg

3001 gatcatgcad taaccteat ggagctaca ttgcatgata tacctgttat ggaaggaatg

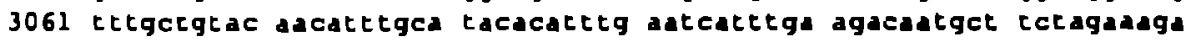

3121 agaatagact ggacctatat gtctagtact cggctacad adcattaca gaatctgat

3181 gatgagatga aagtadead gagategct agaggttgg tatattatgt taadeadec

3241 catagttctc ccacagctac agcetgggag attggateat atcatgate ggttatacet

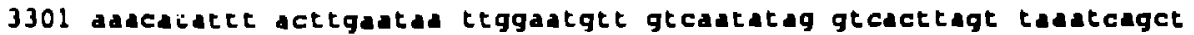

3361 ggacadtga ctcatgtade tatagctcat cettatgaad tadcaata ggatgtgta

34 'I gagactatat atctgcatct tgaggactgc acaggacag atcatgtcat atgtgatgtg

3481 gtaAagatag tgcagcctig tggeaatagc tcagacacga gtgattgtec tgtceggget

3541 gadgctgtad aagadccat tgtgcaagtc aucctctga adacggadg teatetggtt

3601 ctggcaagt c cacagactg tcagatccca ccatatgttc ctagcatcgt gactgt adt

3661 gaacadegt catgetttgg actgqacttt adaggecac tggttgcgga agaagattg

3721 agctttgac; cacgactgce adatctacad ctagattac cacattegjt tggadtate

3781 geadaatca agggatada adegadec acatcetctg gagadagtat adagagcag

384 :- attgaagag cadagctga getcetccga ctggacattc acgagggaga tactcetgcc

3901 tggatacade ayctagctgc agcadcaadg gacgtctggc cagcagcaga ttctgctcta

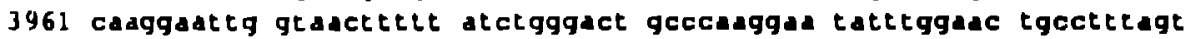

4021 itcttgggat acttaadgce tatcctata ggagtagggg tcattctct ggttattct

408 l atattiaga ttgtateatg galtcctacg aaAagaaga ateagraGce tccacctctg c- env

4141 gadtcagga cetgcagact ctgagtgage ttgttggece tgaaatgec ggagagggag 1201 agcTGActat tgctgaggas cetgaaga ascetegac gececagacg atataccaa bel -1 orf $\rightarrow$

4261 agagadgtca adgtgtgtc ttatcatgca tatasagaa tegaggacas acatcctcan 432 ] catattade tgcaggatey gatccccacd ccagaggaa tgagtaagtc actctgtada 1381 mgacrtatct tatgtggatt gtalagtgca qaadggcet cagagatt adggatgect

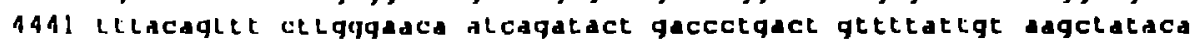
1501 I glatattel glealqctgt andealgal cccatgccca ladgalqqg bol -1 orf $\rightarrow$ 
cocus DEFINITION ACEESSION SOURCE SECMENT REFERENCE

AUTHORS

TITLE

JOURNAL COMMENT

FEATURES LTR

BASE COUNT ORIGIN

SIMSP! $\quad 1665$ bP 5S-RNA

Simian spumavirug, SFV-1, L.TR and flanking sequence. M552 79

Simlan spumavirus (SFV-1).

1 of 2 .

1 (bases 1 to 2665 )

Mergia,A., Shaw,K.E.S., Pracl-lowe,E., Barry,P.A. and luciw,P.A. Slmian foamy virus type l is d retrovlrus which encodes d iranscrlpelonal transacelvator

J. Vlr. L. 64, 3598-3604 (1990)

Kindly sisbmitted in computer readable form by K.Shaw and P.luclw. University of California at Davis.

from to/spen description

$1 \quad 1645$

506 a 303 c 347 g 509 t

l atalggtacj t.tgegaggag agqgtgtgge mgacagccac tadatgtata ggaccagacg

61 aggaadita elggtgtgaa latgatcace qlqgattet tcctatgqt ccadacade

121 tg*.ctcclac ctgggtgaga catgetgece cetactgtat ccaqaggttc gcaacaccat

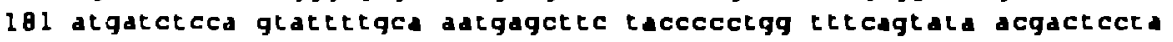

241 agggagtgag tedtaccagt gatcqaagge tccattatgg adatgadgga actctecagg

301 agtatarga gadetgtgat daggttaaga gaggatatga tgadutatct tccagtgat

361 actcagatga agattadag adattlacce agcaactgct tatgctegct tatgattcal

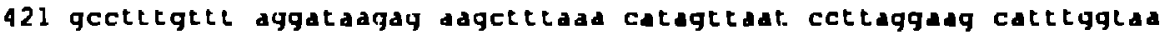

481 attctactad gcagacitgt teatetacta ceglgcttce gatggagade clagggacga

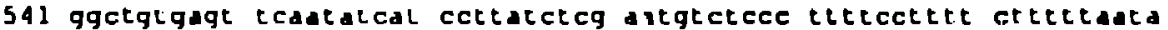

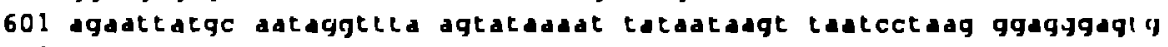

661 gadegtcctg atagadaca gacalgacgc tctcceatce ctcctttect cataclcada

72 I tctaagtaa yctegttgac cttctgccag ctqteagcat agadgratga taadacaga

781 daccacadac aggtgadggc tagclcaclg datadattga ctagtetttg cccayaac:

B 41 caggadgcay tgtiglgtgt tcadatctca aldatgcate ctggtcgtle teti addat

901 tatgtcatig lasacatcat atgadagcaa gadalgacte tgtadgacca cagagggaa

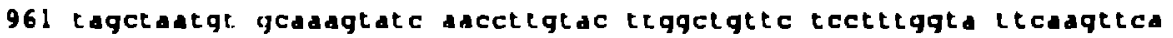

1021 adtcctgtad actgtqctac caccgatgla atatlqtgta atadgtgtt tectalatqa

1081 gtgtanate aact.celgat gactcacyaq glqaalggct cacaqtgadc gacgacljaa

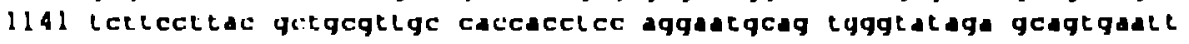

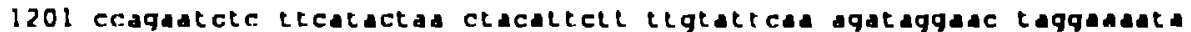

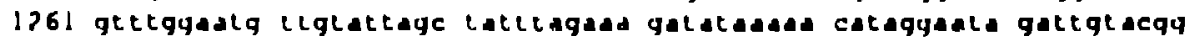

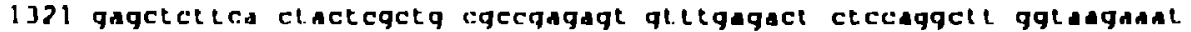

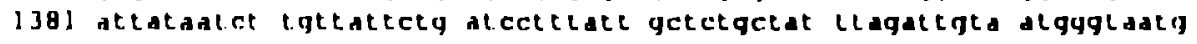

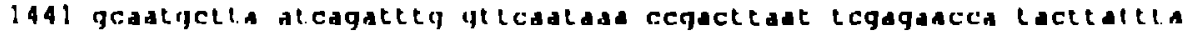

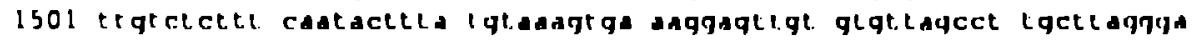

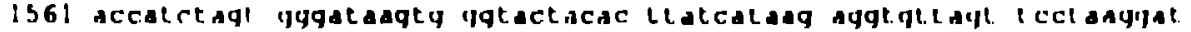

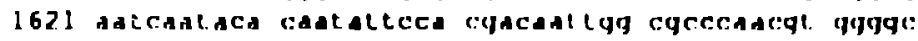


:OE:S S:MSP2 3534 bP IS-RNA

iffinit:on simian spidmavirus sFv-l, partal pol cos, complete env cos.

ACCESSION MJ356]

Si:.RCE Simian spumavirus (SFV-1).

ZIZUMENT 2 of 2 .

dU:H: NCE 1 (bases 1 to 3534)

AJrHons Mergla,A., Shaw,K.E.S., Lackner,J.E. and Luclu,P.A.

::REE Relalionstip of the env genes and the endonuclease domaln of the pol genes of simian loamy virus type 1 and human foamy virus

COURNAL J. Virol. 64, 406-410 (1990)

cOMMENT Kindly submited In computer readable form by K.Shaw and P.Luciu, University of Callfornia at Davls. The uuman (HFV) and aimian (stv) pol and env amino acid sequences at 2 , lcsenectively, about 84 and 641 similar.

ISASF CCUNT $\quad 1163$ a 648 c $\quad 674 \mathrm{~g} \quad 1047 \mathrm{t}$

JH:G:N

1 gadtcagla ctcetencca cccccaagt agtggtaang tggadaggad aadagtgac po: $->$

61 attadacgac tectadela detgctade qggagacctg ctaagtggta tgactacta

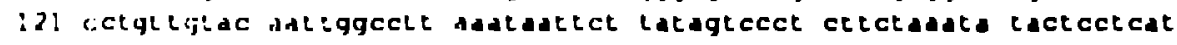

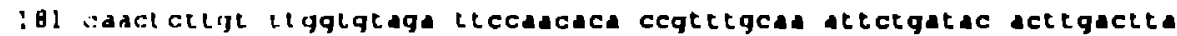

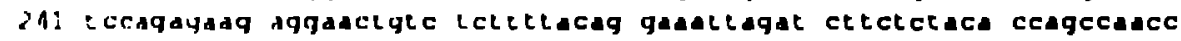

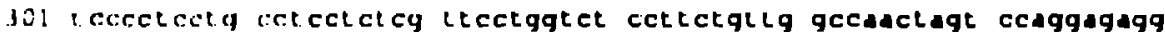

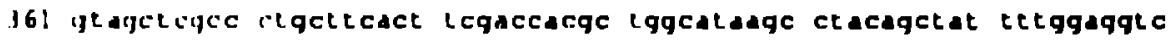

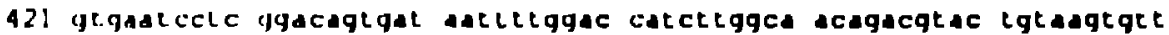

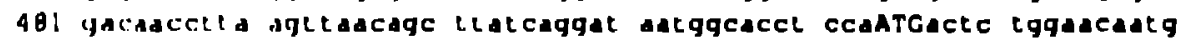
env atirt $\rightarrow$

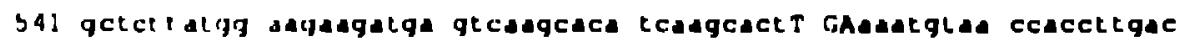
C- pol

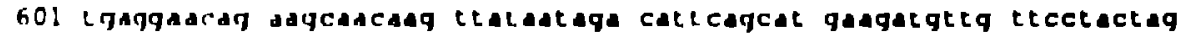

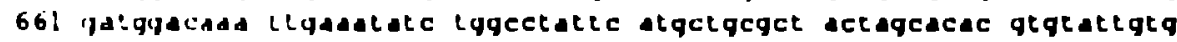

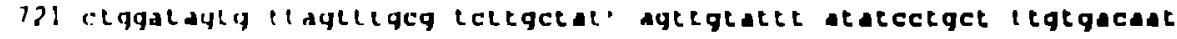

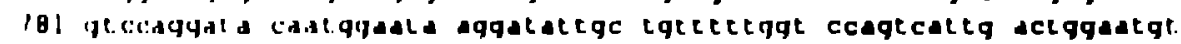

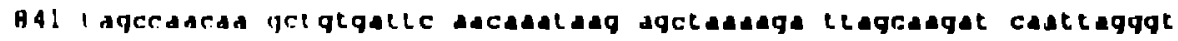

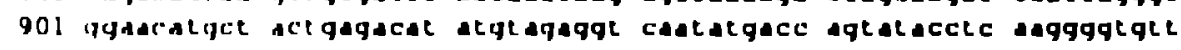

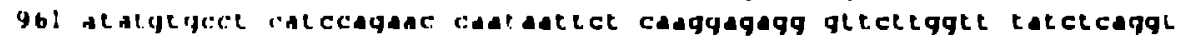

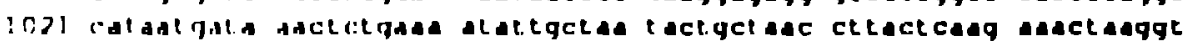

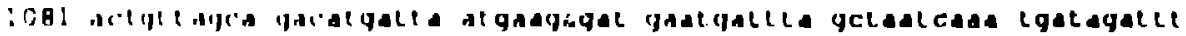

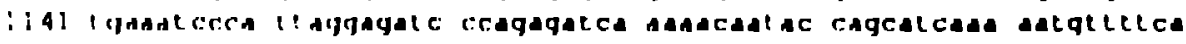

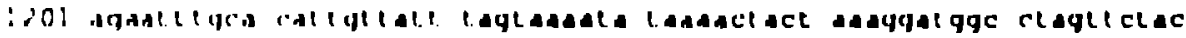

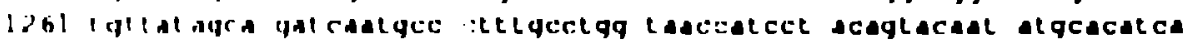

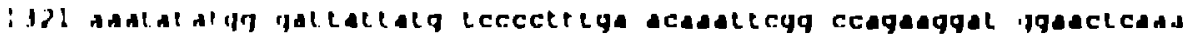

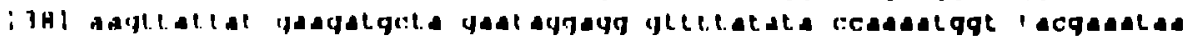

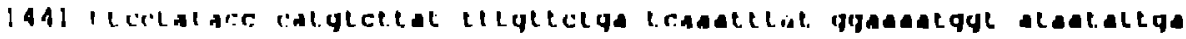

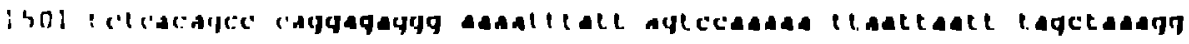

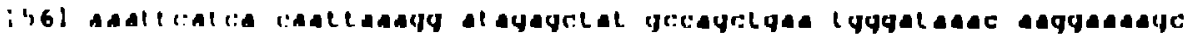

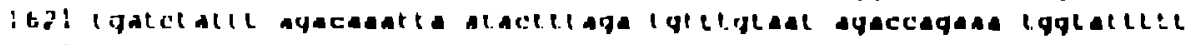

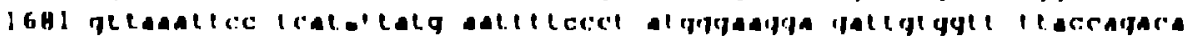

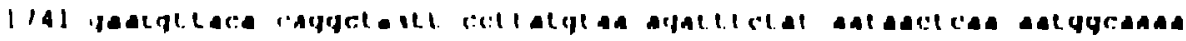

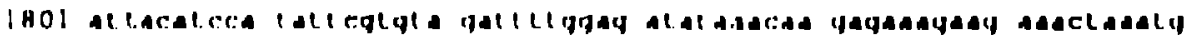

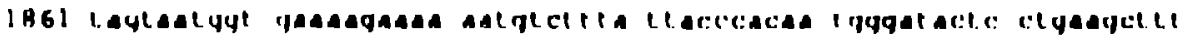

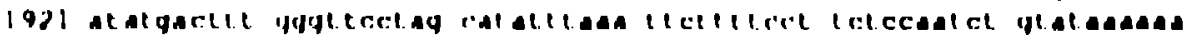

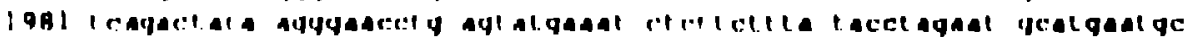

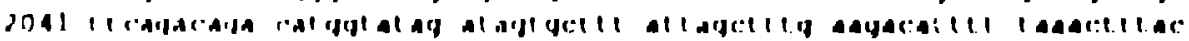

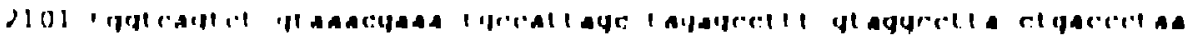

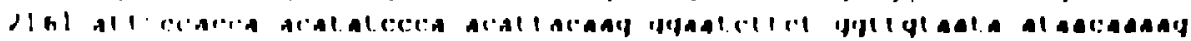

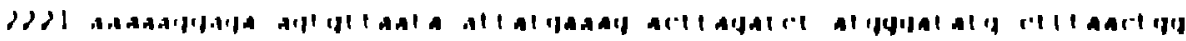

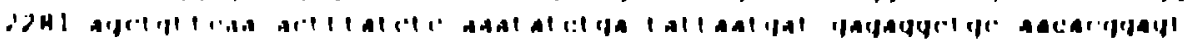

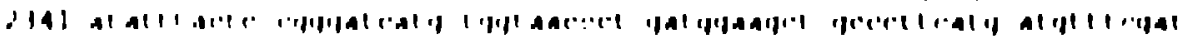

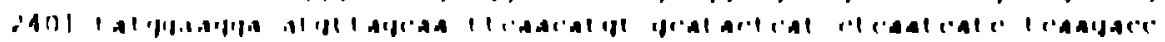

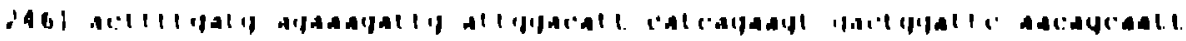

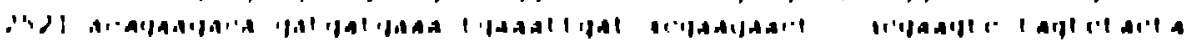

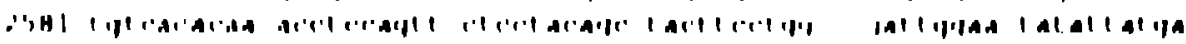

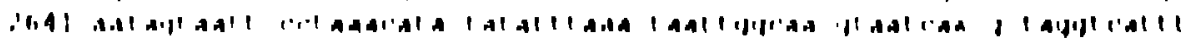


SIMSIP

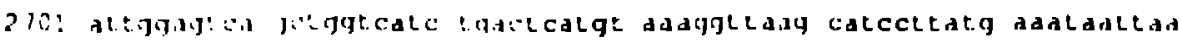

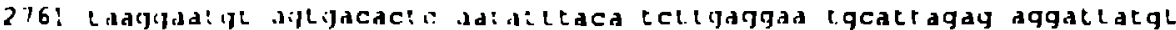

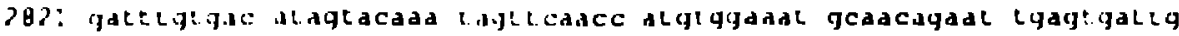

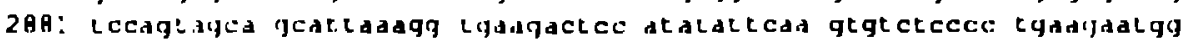

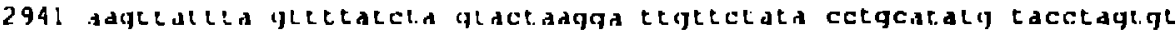

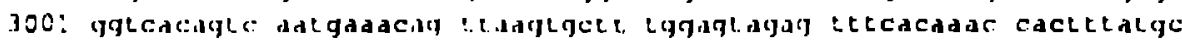

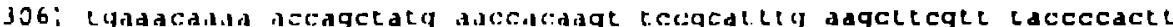

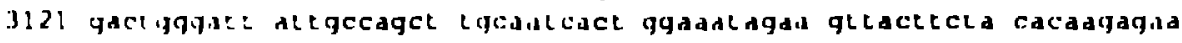

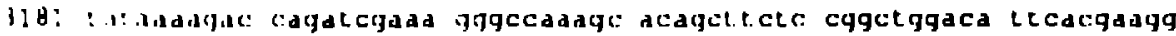

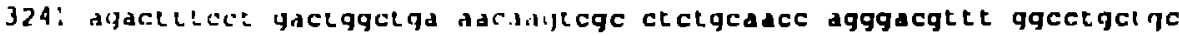

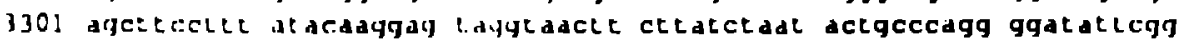

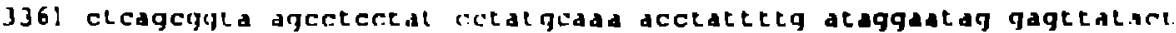

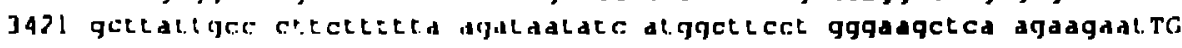

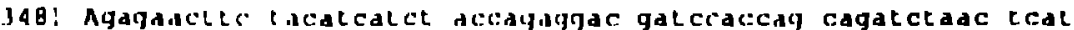

c- env 
The 1989 and 1990 releases of the Human Retroviruses and AIDS database contained a list of supplemental references captured from the molecular subset of MEDLINE as communicated through GENINFO at the National Center for Biomedical Communications, National Library of Medicine, National Insultutes of Health, Bethesda, Maryland. The reference set published in the 1990 compendium has not been updated at the time of publication and will not be included in this issue of the 1991 compendium. An updated list will be published later !his year. The complete list of references published in the 1989 and 1990 compendia can be made available upon request

The enclosed diskeltes comprise the clectronic portion of Pan V, providing HIV and SIV nucleic acid and protein sequences in ASCIl characters. As a general rule, American and nonEuropean users receive nucleic acid files in GenBank format and procein files in a reduced PIR format. European users receive these files in EMBL and Swiss-Prot formats. Both PC-DOS and Apple Maciniosh formulted diskeues are available; which format you reccive depends upon what you have requested. If you wish to change the diskeue format or the file formats, simply contact us at 505-665-1356 or fax a message to us at 505-665-3493. If you wish to contact us via the postal service, our address is: HIV Sequence Database, T-10, MS K710, LANL, Los Alamos, NM, 87545, U.S.A. The diskeltes contain READ. ME files to provide you with the most recent information regarding the ir contents.

GenBank, the EMBL. Data Library, and the DNA Dala Bank of Japan have, begun to implement a new. stundard FEATURE Table format which is incended wo increase the amount and type of machine-parsible information contained in the FEATURE table, and which will enhance data-sharing between the databases. As part of a gradual conversion to the new format, old entries from the AIDS dalubase will be presental with the new FEATURE table format and new or revised entrics will be presented in the old FEATURE wble format. By the end of 1991, all nuclootide sequence entries will be in the new format. 